Al1102 145694

NATL INST OF STANDARDS \& TECH R.I.C.

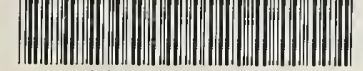

A11102145694

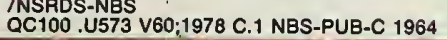

2.

NSRDS-NBS 60

U.S. DHBARTENT OF COMMERCL/Natonal Bureau of Standards

\title{
Atomic Energy Levels The Rare-Earth Elements
}






\section{Atomic Energy Levels-The Rare-Earth Elements \\ The Spectra of Lanthanum, Cerium, Praseodymium, Neodymium, Promethium, Samarium, Europium, Gadolinium, Terbium, Dysprosium, Holmium, Erbium, Thulium, Ytterbium, and Lutetium}

W.C. Martin, Romuald Zalubas, and Lucy Hagan

Institute for Basic Standards

National Bureau of Standards

Washington, D.C. 20234

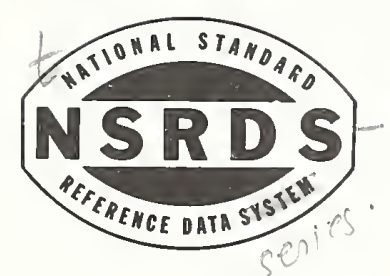

U.S. DEPARTMENT OF COMMERCE, Juanita M. Kreps, Secretary

Dr. Sidney Harman, Under Secretary

Jordan J. Baruch, Assistant Secretary for Science and Technology

NATIONAL BUREAU OF STANDARDS, Ernest Ambler, Director

Issued April 1978 


\section{Library of Congress Cataloging in Publication Data}

Martin, William Clyde, 1929-

Atomic energy levels-the rare-earth elements.

(Nat. stand. ref. data ser. ; NSRDS-NBS 60)

"National Standard Reference Data System."

Supt. of Docs. no.: C13.48:60

1. Earths, Rare-Spectra-Tables. 2. Atomic spectra-Tables, etc 3. Energy levels (Quantum mechanics)-Tables, etc. I. Zalubas, Romuald, joint author. 11. Hagan, Lucy, joint author. III. Title. IV Series: United States. National Bureau of Standards. National standard reference data series: NSRDS-NBS 60.

QC100.U573 no. 60 [QC462.R2] 602'.Is [539.7'2] 77-12195

\section{NSRDS-NBS 60}

Nat. Stand. Ref. Data Ser., Nat. Bur. Stand. (U.S.), 60, 422 pages (April 1978) CODEN: NSRDAP

(C) 1978 by the Secretary of Commerce on Behalf of the United States Government

\section{U.S. GOVERNMENT PRINTING OFFICE WASHINGTON: 1978}

For sale by the Superintendent of Documents, U.S. Government Printing Office, Washington, D.C. 20402 (Order by SD Catalog No. C13.48:60). Stock No. 003-003-01712-4 Price $\$ 9.50$ (Add 25 percent additional for other than U.S. mailing) 


\section{Foreword}

The National Standard Reference Data System provides access to the quantitative data of physical science, critically evaluated and compiled for convenience and readily accessible through a variety of distribution channels. The System was established in 1963 by action of the President's Office of Science and Technology and the Federal Council for Science and Technology, and responsibility to administer it was assigned to the National Bureau of Standards.

NSRDS receives advice and planning assistance from a Review Committee of the National Research Council of the National Academy of Sciences-National Academy of Engineering. A number of Advisory Panels, each concerned with a single technical area, meet regularly to examine major portions of the program, assign relative priorities, and identify specific key problems in need of further attention. For selected specific topics, the Advisory Panels sponsor subpanels which make detailed studies of users' needs, the present state of knowledge, and existing data resources as a basis for recommending one or more data compilation activities. This assembly of advisory services contributes greatly to the guidance of NSRDS activities.

The System now includes a complex of data centers and other activities in academic institutions and other laboratories. Components of the NSRDS produce compilations of critically evaluated data, reviews of the state of quantitative knowledge in specialized areas, and computations of useful functions derived from standard reference data. The centers and projects also establish criteria for evaluation and compilation of data and recommend improvements in experimental techniques. They are normally associated with research in the relevant field.

The technical scope of NSRDS is indicated by the categories of projects active or being planned: nuclear properties, atomic and molecular properties, solid state properties, thermodynamic and transport properties, chemical kinetics, and colloid and surface properties.

Reliable data on the properties of matter and materials are a major foundation of scicntific and technical progress. Such important activities as basic scientific research, industrial quality control, development of new materials for building and other technologies, measuring and correcting environmental pollution depend on quality reference data. In NSRDS, the Bureau's responsibility to support American science, industry, and commerce is vitally fulfilled.

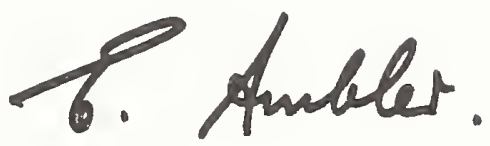

Ernest Ambler, Director 


\section{Preface}

The program on Atomic Energy Levels (AEL) initiated at the National Bureau of Standards in 1946 called for complete coverage of the Periodic Table. Three volumes prepared by Charlotte E. Moore and originally published as NBS Circular 467 were reprinted as NSRDS-NBS 35 in 1971. These volumes cover the elements Hydrogen through Actinium $(Z=$ 1-89) except for the rare earths, Cerium through Lutetium $(Z=58-71)$. The present compilation covering Lanthanum through Lutetium $(Z=57-71)$ has been prepared by W. C. Martin, Romuald Zalubas, and Lucy Hagan as part of a continuing program on the evaluation and compilation of atomic energy levels, spectral wavelengths and classifications. This work is being carried out in the Atomic Energy Levels Data Center of the Spectroscopy Section, Optical Physies Division, Institute for Basic Standards, and in the Office of Standard Reference Data. It represents one of the important activities of the National Standard Reference Data System.

A similar compilation for the actinide elements is planned. The preparation of a single volume covering both the rare-earth and the actinide elements, i.e., the originally planned Volume IV of AEL, would have unnecessarily delayed publication of the present tables. The somewhat different title of the present publication and the assignment of a separate NSRDSNBS series number are meant to recognize this change, as well as to alert readers to certain differences in the format of the tables from that of the previous AEL compilations.

The three AEL volumes now available as NSRDS-NBS 35 were originally issued during the period 1949-58, and the tables for many of the included spectra are now in need of revision. A part of this need is being met by new compilations for selected spectra. Moore's Selected Tables of Atomic Spectra [NSRDS-NBS 3, issued in sections] include H I, D, T [Sec. 6, 1972]; C I-VI [Sec. 3, 1970]; N I-III [Sec. 5, 1975]; N IV-VII [Sec. 4, 1971]; O I [Sec. 7, 1976]; Si I [Sec. 2, 1967]; and Si II-IV [Sec. 1, 1965]. A multiplet table as well as a table of energy levels is given for each spectrum in this series. Recent compilations of energy levels carried out in the Spectroscopy Section include ${ }^{4} \mathrm{He}$ I [W. C. Martin, J. Phys. Chem. Ref. Data 2, 257 (1973)], the 26 spectra of Fe, Fe I-xxvi [J. Reader and J. Sugar, J. Phys. Chem. Ref. Data 4, 353 (1975)], the 24 spectra of Cr, Cr I-xxIV [J. Sugar and C. H. Corliss, J. Phys. Chem. Ref. Data 6, 317 (1977)], and the 25 spectra of Mn, Mn I-XXv [C. H. Corliss and J. Sugar, J. Phys. Chem. Ref. Data 6, 1253 (1977)].

Many users need more extensive current data on atomic energy levels and spectra than are included in the critical compilations now available or planned for the near future. The NBS program thus includes publication of bibliographies covering the more recent literature in this area, with the references classified according to the types of data. The most recent such publication is the Bibliography on Atomic Energy Levels and Spectra, July 1971 through June 1975, by Lucy Hagan [NBS Spec. Publ. 363, Suppl. 1, 1977]. Earlier periods are covered in the bibliographies NBS Spec. Publ. 363 [L. Hagan and W. C. Martin, 1972] and NBS Spec. Publ. 306, Sections 1-4 [C. E. Moore, 1968-69].

The AEL program has from the beginning received the help and cooperation of spectroscopists in many laboratories who observe and analyze atomic spectra. I join the authors of the present compilation in expressing sincere gratitude for this assistance.

D. R. Lide, Jr., Chief

Office of Standard Reference Data 
Foreword

Preface

1. Introduction

1.1. Scope and Format of the Tables

1.2. Important Conventions

a. Naming of Levels

b. Incomplete Terms

2. Coupling Schemes, Allowed Terms, Percentage Composition,

Zeeman Effect

2.1. Coupling Types, Order of Coupling of Electrons, Ancestral Terms

a. LS Coupling (Russell-Saunders Coupling) ….............................

b. $J_{1}, j$ or $J_{1} J_{2}$ Coupling

c. $\quad j j$ Coupling of Equivalent Electrons

d. $\quad J_{1} l$ or $J_{1} L_{2}$ Coupling

e. $L S_{1}$ Coupling

f. Coupling Schemes and Term Symbols

2.2. Allowed Terms or Levels for Equivalent Electrons
a. LS Coupling (Table 1)
b. $\quad$ jj Coupling (Table 2)

2.3. Percentage Compositions of Levels

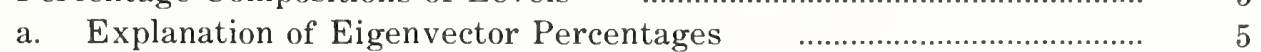

b. Need for Leading Percentages, and a Caveat …….......................... 6

2.4. Zeeman Effect (Tables 3-6)

3. Material Preceding Each Table-Summary Data, Comments, References $\quad . . . . . . . \quad 7$

4. Explanation of the Tables (by Column Heading) ……..................................... 8

4.1. Configuration

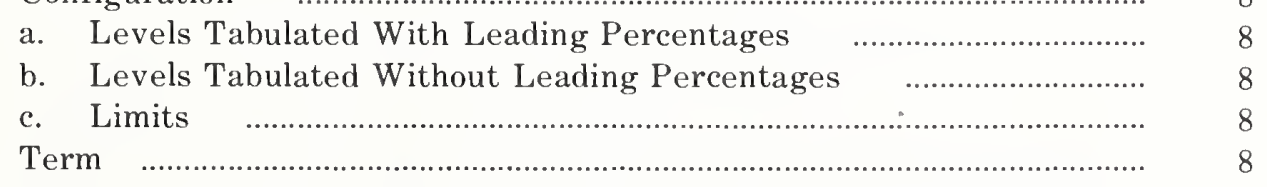

4.2. Term
a. Levels Tabulated With Leading Percentages: Naming of Levels,

Grouping of Levels Into Terms, Other Conventions $\quad$.................. 8

b. Levels Tabulated Without Leading Percentages $\quad$............................ 9

4.3. Total Electronic Angular-Momentum Quantum Number $J$...................... 10

4.4. Level ........................................................................................... 10

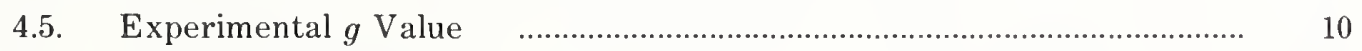

4.6. Leading Percentages $\quad$............................................................................. 10

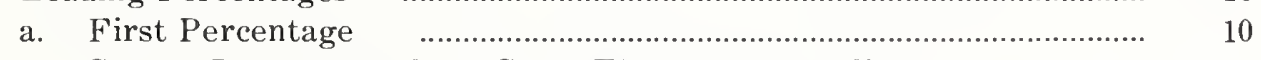

b. Second Percentage From Same Eigenvector as First
Percentage

c. Second Percentage Is for Leading Component of Eigenvector

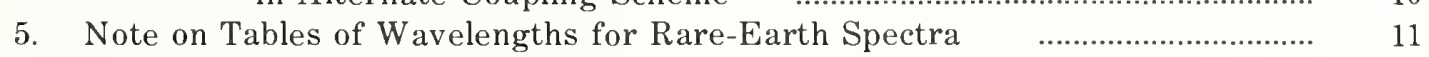

6. State of Analyses of Rare-Earth Spectra …………..................................... 12

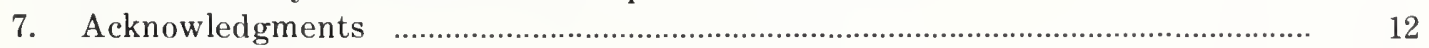

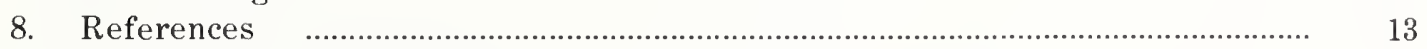

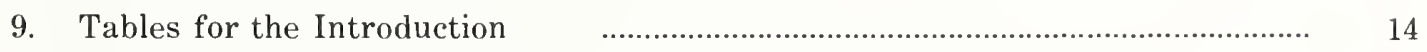

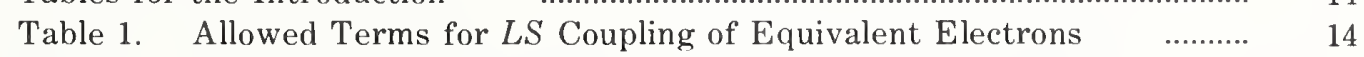

$\begin{array}{llll}\text { Table 2. } & \text { Allowed } J \text { Values for } l_{j}^{N} \text { Equivalent Electrons ( } j j \text { Coupling) } & \ldots \ldots . . & 17\end{array}$

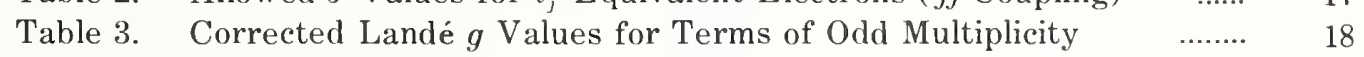

Table 4. Corrected Landé $g$ Values for Terms of Even Multiplicity $\quad \ldots . . . .21$

Table 5. Landé $g$ Values for Terms of Odd Multiplicity in Order of

Table 6. Landé $g$ Values for Terms of Even Multiplicity in Order of

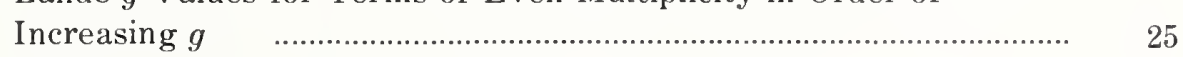

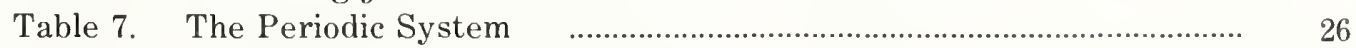


Index to Tables of Energy Levels

59

60

61

62

63

64

65
Cerium

\section{Element}

Lanthanum

Neodymium

Promethium

Samarium

Europium

Gadolinium

Terbium
Spectrum*

Page

La I

La II

La III

La IV

La V

Ce I

Ce II

Ce III

Ce IV

Ce V

Ce vi

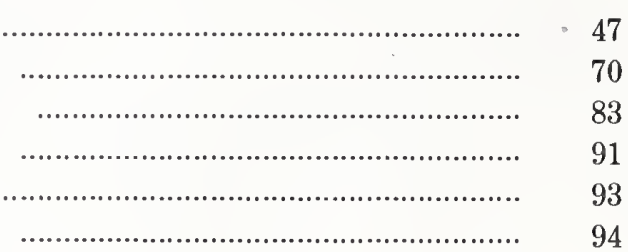

Pr I

Pr II

Pr III

Pr IV

Pr V

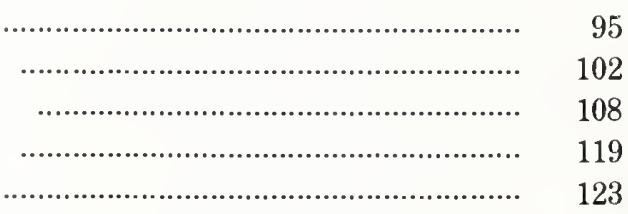

Nd I

Nd II

Nd III

[ Nd IV]

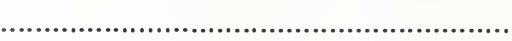

124

137

148

149

Pm I

Pm II

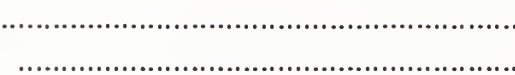

151

(Pm III)

156

[Pm IV]

160

161

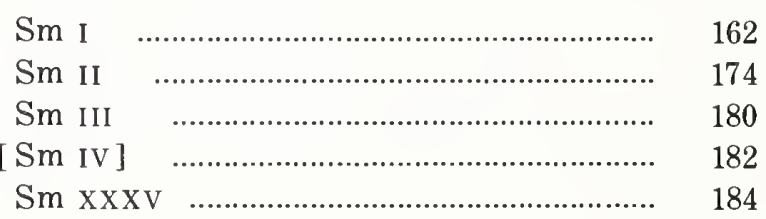

Eu I

185

Eu II

199

Eu III ……............................................. 204

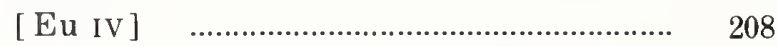

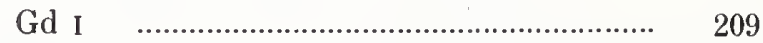

Gd II ................................................ 225

Gd III ……............................................ 235

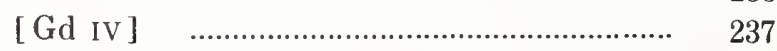

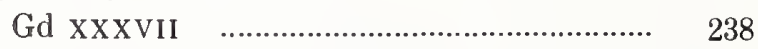

Tb I

Tb II _................................................... 250

Tb III, …................................................ 255

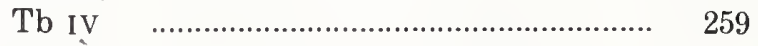


Index to Tables of Energy Levels-Continued

$Z$

66

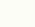

67

68

69

70
Erbium

Element

Dysprosium

Holmium

Thulium

Lutetium
Spectrum*

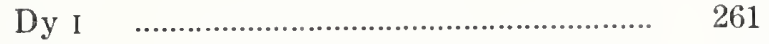

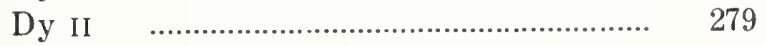

( Dy III ) ............................................. 292

[ Dy IV ] [........................................ 293

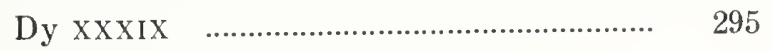

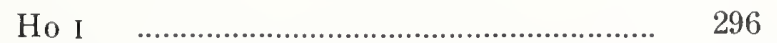

Ho II $\quad$.................................................... 305

Ho III $\quad$............................................... 308

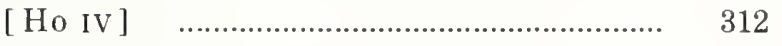

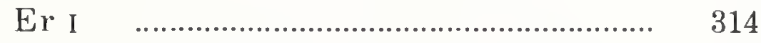

Er II $\quad$............................................. 332

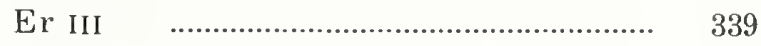

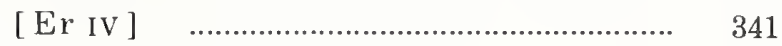

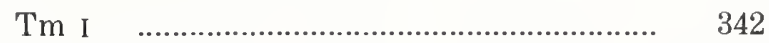

Tm II $\quad$................................................. 358

Tm III $\quad$............................................. " 368

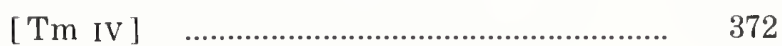

Yb I

Yb II $\quad$............................................... 382

Yb III $\quad$................................................ 391

Yb IV $\quad$............................................... 394

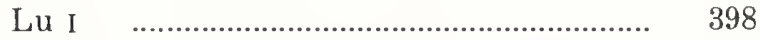

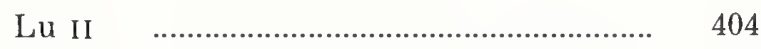

$\mathrm{Lu}$ III $\quad$............................................... 407

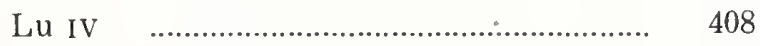

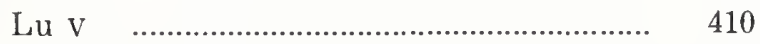

${ }^{*}$ Spectral symbols enclosed in brackets indicate data obtained from ions in crystals or solutions. No analyses are available for the spectra in parentheses. 



\title{
Atomic Energy Levels-The Rare-Earth Elements
}

\author{
The Spectra of Lanthanum, Cerium, Praseodymium, Neodymium, \\ Promethium, Samarium, Europium, Gadolinium, Terbium, \\ Dysprosium, Holmium, Erbium, Thulium, Ytterbium, and Lutetium
}

\author{
W. C. Martin, Romuald Zalubas, and Lucy Hagan
}

Institute for Basic Standards, National Bureau of Standards, Washington, D.C. 20234

\begin{abstract}
Energy level data are given for 66 atoms and atomic ions of the 15 elements lanthanum $(Z=57)$ through lutetium $(Z=71)$. These data have been critically compiled from published and unpublished material. Only experimentally determined energy levels are included, the energies being restricted to excitations of outer-shell electrons and to inner-shell excitations up to the soft $x$-ray range. The levels were taken from analyses of the spectra of atomic gases wherever possible; however, the levels for several of the triply ionized rare earths are from analyses of the spectra of the ions in crystals or solutions. In addition to the level value (usually in units of $\mathrm{cm}^{-1}$ ) and the parity, the $J$ value, configuration and term assignments, and the experimental $g$ value are listed wherever available. Leading percentages from the calculated eigenvector are also tabulated for each level if available. The levels are grouped into spectroscopic terms of appropriate coupling schemes where such groups appear meaningful. Ionization potentials are tabulated for most of the spectra. Complete references for the tabulated data are given for each spectrum.
\end{abstract}

Key words: Atomic energy levels; atomic spectroscopy; electron configurations; ionization potentials; lanthanides; rare earths; spectra; Zeeman effect.

\section{Introduction}

A program on the compilation of atomic energy levels was begun at the National Bureau of Standards in 1946 by Charlotte E. Moore under the direction of William F. Meggers [Moore, 1971].' The appearance of the third volume of Moore's Atomic Energy Levels (AEL) in 1958 completed the coverage of the elements through Ac $(Z=89)$ except for the rare earths $\mathrm{Ce}$ through $\mathrm{Lu}(Z=58-71)$. A planned Volume IV of AEL was to include the rare earths and the actinide elements; publication of such a volume was not feasible during the 1960 's, however, because many of the needed spectra had not been analyzed sufficiently, if at all. Atomic spectroscopists have greatly increased the available data for these elements during the past 15 years or so, and active work on the present compilation was begun in 1969 . As the compilation progressed we decided to publish the tables for the rare earths separately. A similar compilation for the actinide elements is planned.

\subsection{Scope and Format of the Tables}

The compiled data pertain to the energy levels of the atoms and ions of the 15 elements $\mathrm{La}(Z=57)$ through $\mathrm{Lu}$ $(Z=71)$. Although La I, La II, and La III were included in AEL Vol. III, the new and revised data obtained for La I through La V since 1958 warranted new compilations. Only experimentally determined values of the energy levels are tabulated, and the energies are restricted to excitations of outer-shell electrons and to inner-shell excitations up to the

${ }^{1}$ Names and dates in brackets indicate references at the end of this introduction. soft $x$-ray range. All but a relatively few of the levels have been obtained from analyses of optical spectra. The sources for most of these spectra were atomic gases; ideally, the levels are evaluated for free, unperturbed atoms and ions.

Reliable analyses were not available, however, for the (free-ion) spectra of triply ionized $\mathrm{Nd}, \mathrm{Pm}, \mathrm{Sm}, \mathrm{Eu}, \mathrm{Gd}, \mathrm{Dy}$, $\mathrm{Ho}, \mathrm{Er}$, and Tm. In each case we have therefore given a few levels as derived from the spectra of the ions in crystals or solutions. These levels are enclosed in brackets to indicate that the derived positions may differ from corresponding values for the unperturbed ions by several percent. No data of this type are included for species other than triply ionized.

An explanation of the data tabulated with the energy levels is given in section 4, arranged by column headings. The format of the AEL tables, which was based to a considerable extent on the earlier Atomic Energy States [Bacher and Goudsmit, 1932], is now probably the most familiar scheme for the presentation of energy level data. We have retained much of this format while adapting and extending it to give more detailed theoretical descriptions of levels for which such information is available. The main formal changes are the addition of the "Leading Percentages" (referring to the theoretical composition of the levels) and the deletion of the "Interval" column. Changes in the arrangement within some of the other columns are noted in section 4 .

We originally prepared a table for each spectrum with the levels of the two parities intermixed. In a number of cases, however, the complication added by intermixing two separately complex level structures appeared to outweigh 
any advantages of the arrangement. In such cases we give the levels in separate lists for the two parities, a practice now followed in most publications on complex spectra. (A deviation from the usual AEL arrangement of intermixed parities was in fact made for $H f$ I; see AEL Vol. III [Moore, 1971].)

The notations for several different coupling schemes and a convention bearing on the order of coupling of the electrons are used without comment in the tables. Explanations of the notations and of some other pertinent theoretical points are given in section 2 .

It should be emphasized that the familiarity of the format can be misleading unless certain new conventions are understood. We recommend the reading of section 4 of the Introduction (especially 4.1, 4.2, and 4.6) by all who plan to use this compilation in more than a casual way. The following points are based on the more detailed explanations given in sections 2 and 4.

\subsection{Important Conventions}

\section{a. Naming of Levels}

We have retained the practice of listing the levels according to spectroscopic terms, except where such groupings of levels would be meaningless as indications of atomic structure. In a note on coupling in rare-earth spectra, G. Racah wrote "... in many configurations of the rare earths there are interactions which are of the same order of magnitude, and it will be impossible to define the coupling scheme. This does not mean that it will be impossible to calculate the positions of the levels and even the exact compositions of the states; this only means that the composition will be such a complicated combination of different states, that it will be impossible to correlate it with a meaningful name or symbol" [Racah, 1960]. To make the compilation easier to use, we have tried to handle the question of term names in a reasonably consistent manner for all spectra. The criteria we followed in deciding whether to retain (or in some cases to assign) names for levels are explained in section 4.2 .

If a level is given with leading percentages and with a term symbol under the "Term" heading, the full set of symbols in the first two columns (plus the $J$ value) constitutes a unique name in this compilation. This also holds for levels given without leading percentages, provided the appropriate ancestral terms are given with the configuration. In most cases we have not given shortened designations for the levels; this is emphasized because in the AEL tables each term or interpreted level has a unique short designation under the column heading "Desig."

Levels not listed with a term symbol in the second column (under "Term") have no symbolic names for the purposes of this compilation. ${ }^{2}$ This should be noticed especially in connection with $J_{1} j$ and $J_{1} J_{2}$ coupling, since the symbols in the first column alone constitute complete theoretical term

\footnotetext{
${ }^{2}$ A convenient method for designating unnamed levels is to give the numerical value with the $J$ value as a subseript, and a superseript degree symbol for odd levels. It is usually sufficient to give the position to the nearest $\mathrm{cm}^{-1}$, or simply truncated at the decimal point.
}

descriptions in these schemes-such a term is a suggested name, however, only if it is followed by a $\left(J_{1}, j\right)$ or $\left(J_{1}, J_{2}\right)$ term symbol in the second column.

In cases of strong configuration interaction, an attempt to correlate each eigenvector with even a particular configuration can be meaningless. The configuration listed in the first column does not necessarily represent a configuration assignment for the level; the configuration for the first percentage is normally given in the "Configuration" column regardless of the magnitude of this percentage.

\section{b. Incomplete Terms}

Predicted levels that have not been found are usually not indicated notationally in these tables. The only exceptions occur as levels of partially known terms (shown as term groups), and the levels of such terms are grouped only under certain conditions (sec. 4.2). Some conventions regarding incomplete terms can be noticed in an example from the $4 f^{6}\left({ }^{7} \mathrm{~F}\right) 5 d$ subconfiguration of Eu III:

$\begin{array}{crc}\text { Term } & J & \text { Level } \\ & & \\ { }^{6} \mathrm{G} & 3 / 2 & \\ & 5 / 2 & 49905.64 \\ & 9 / 2 & \\ & 11 / 2 & 51650.77 \\ & 13 / 2 & 52099.87\end{array}$

The blank spaces under "Level" for $J=3 / 2$ and $9 / 2$ indicate that these levels have not been found in the analysis (a standard convention). It will be noticed, however, that no blank space or $J$ value appears for a third level "missing" from this term $(J=7 / 2)$. This omission signifies that none of the calculated eigenvectors is appropriate for the corresponding designation. By the omission we avoid the implication that a $4 f^{6}\left({ }^{7} \mathrm{~F}\right) 5 d^{6}{ }^{6} \mathrm{G}_{7 / 2}$ level is missing from the Eu III analysis. The best candidate levels for this designation are known and may be found among the levels near the ${ }^{6} \mathrm{G}$ term. (In this particular example, all of the calculated $4 f^{6}\left({ }^{7} \mathrm{~F}\right) 5 d{ }^{6} \mathrm{G}_{7 / 2}$ composition is distributed among known levels, since all twelve $J=7 / 2$ levels for the interacting $4 f^{6}\left({ }^{7} \mathrm{~F}\right) 5 d$ and $4 f^{6}\left({ }^{7} \mathrm{~F}\right) 6 s$ subconfigurations are known.)

\section{Coupling Schemes, Allowed Terms, Percentage Composition, Zeeman Effect}

We assume that the reader is familiar with the basic vocabulary and standard notations used in discussing atomic structure and spectra. ${ }^{3}$ However, the notations required for the full description of some of the theoretical states used in this compilation are necessarily more complex than for simpler spectra, and an explanation of certain adopted conventions is needed. The following outline of the notational scheme (sec. 2.1 and 2.2) includes all the coupling

\footnotetext{
${ }^{3}$ A pertinent summary is given in the introduction to Vol. I of AEL [Moore, 1971]. El'yashevich (1961) gives an extensive discussion and a number of useful tables relating to the theoretical properties of terms and levels of rare-earth configurations. In the case of words used differently by different authors (e.g., term, multiplet), we follow Condon and Shortley [1963, chaps. 7-9].
} 
types that now appear to be needed for Atomic Energy Levels compilations. ${ }^{4}$ This section is also in part an introductory supplement to section 4 , which gives a detailed explanation of the tables.

\subsection{Coupling Types, Order of Coupling of Electrons, Ancestral Terms}

In this section we give enough examples to make clear the meaning of the different coupling-scheme notations. Not all of the configurations in the examples have been identified experimentally, and some of the examples of a particular coupling scheme (given for heuristic purposes) may be physically inappropriate.

\section{a. LS Coupling (Russell-Saunders Coupling)}

Some examples of this familiar scheme are given below mainly to indicate a spacing convention bearing on the order of coupling of the electrons.

\section{Configuration}

1. $4 f^{7}\left({ }^{8} \mathrm{~S}^{\circ}\right) 6 s 6 p\left({ }^{3} \mathrm{P}^{\circ}\right)$

2. $4 \mathrm{f}^{7}\left({ }^{8} \mathrm{~S}^{\circ}\right) 6 s 6 p^{2}\left({ }^{4} \mathrm{P}\right)$

3. $4 f^{7}\left({ }^{8} \mathrm{~S}^{\circ}\right) 5 d^{2}\left({ }^{3} \mathrm{~F}\right)$

4. $4 f^{10}\left({ }^{3} \mathrm{~K} 2\right) 6 s 6 p\left({ }^{1} \mathrm{P}^{\circ}\right)$

5. $4 f^{7}\left({ }^{8} \mathrm{~S}^{\circ}\right) 5 d\left({ }^{7} \mathrm{D}^{\circ}\right) 6 p$

6. $4 f^{7}\left({ }^{8} \mathrm{~S}^{\circ}\right) 5 d\left({ }^{9} \mathrm{D}^{\circ}\right) 6 s\left({ }^{8} \mathrm{D}^{\circ}\right) 7 s$

7. $4 f^{7}\left({ }^{8} \mathrm{~S}^{\circ}\right) 5 d\left({ }^{9} \mathrm{D}^{\circ}\right) 6 s 6 p\left({ }^{3} \mathrm{P}^{\circ}\right)$

8. $4 f^{7}\left({ }^{8} \mathrm{~S}^{\circ}\right) 5 d^{2}\left({ }^{1} \mathrm{G}\right)\left({ }^{8} \mathrm{G}^{\circ}\right) 6 p$

9. $4 f\left({ }^{2} \mathrm{~F}^{\circ}\right) 5 d^{2}\left({ }^{1} \mathrm{G}\right) 6 s\left({ }^{2} \mathrm{G}\right)$

In the first example, the seven $4 f$ electrons couple to give an ${ }^{8} \mathrm{~S}^{\circ}$ term, and the $6 s$ and $6 p$ electrons couple to form the ${ }^{3} \mathrm{P}^{\circ}$ term; the final ${ }^{10} \mathrm{P}$ term is one of three possible terms obtained by coupling the ${ }^{8} \mathrm{~S}^{\circ}$ and ${ }^{3} \mathrm{P}^{\circ}$ parent terms. The next three examples are quite similar to the first. The meaning of the index number 2 following the ${ }^{3} \mathrm{~K}$ symbol in the fourth example is explained in section 2.2 .

The coupling in example five is appropriate if the interaction of the $5 d$ and $4 f$ electrons is sufficiently stronger than the $5 d-6 p$ interaction. The ${ }^{7} \mathrm{D}^{\circ}$ parent term results from coupling the $5 d$ electron to the ${ }^{8} \mathrm{~S}^{\circ}$ grandparent, and the $6 p$ electron is then coupled to the ${ }^{7} \mathrm{D}^{\circ}$ parent to form the final ${ }^{8} \mathrm{~F}$ term. A space is inserted between the $5 d$ electron and the ${ }^{7} \mathrm{D}^{\circ}$ parent to emphasize that the latter is formed by coupling to a term $\left({ }^{8} \mathrm{~S}^{\circ}\right)$ listed to the left of the space. The sixth example illustrates a similar coupling order carried to a further stage; the ${ }^{8} \mathrm{D}^{\circ}$ parent term results from the coupling of the $6 s$ electron to the ${ }^{9} \mathrm{D}^{\circ}$ grandparent.

The seventh example is similar to the first four cases, but in seven the first of the two terms that couple to form the

\footnotetext{
${ }^{4}$ Neither the $j j$ coupling scheme for equivalent electrons (2.1.c, 2.2.b) nor the $L S_{1}$ coupling scheme (2.1.e) is used in this volume.
}

final ${ }^{11} \mathrm{~F}$ term, i.e., the ${ }^{9} \mathrm{D}^{\circ}$ term, is itself formed by the coupling of the $5 d$ electron to the ${ }^{8} \mathrm{~S}^{\circ}$ core term. The eighth example shows an ${ }^{8} \mathrm{G}^{\circ}$ parent term formed by coupling the ${ }^{8} \mathrm{~S}^{\circ}$ and ${ }^{1} \mathrm{G}$ grandparent terms. A space is again used to emphasize that the following $\left({ }^{8} \mathrm{G}^{\circ}\right)$ term is formed by the coupling of terms listed before the space.

A different order of coupling is indicated in the final example, the $5 d^{2}{ }^{1} \mathrm{G}$ term being coupled first to the external $6 s$ electron instead of directly to the $4 f$ core electron. The $4 f\left({ }^{2} \mathrm{~F}^{\circ}\right)$ core term is isolated by a space to denote that it is coupled (to the $5 d^{2}\left({ }^{1} \mathrm{G}\right) 6 s^{2} \mathrm{G}$ term) only after the other electrons have been coupled. The notation in this particular case (with a single $4 f$ electron) could be simplified by writing the $4 f$ electron after the ${ }^{2} \mathrm{G}$ term to which it is coupled. It appears more important, however, to retain the convention of giving the core portion of the configuration first.

The notations in examples 1-4 are in the form recommended by Russell, Shenstone, and Turner [1929], and used in both the Atomic Energy States and $A E L$ compilations. The convention introduced in the remaining examples allows different orders of coupling of the electrons to be indicated without the use of additional parentheses, brackets, etc.

\section{b. $J_{1} j$ or $J_{1} J_{2}$ Coupling}

The symbol $J_{i}$ refers to the total angular momentum of an electron or of a group of electrons; a small $j$ indicates explicitly the angular momentum of one electron $(j=l \pm 1 / 2)$. In the configuration notations, $J_{i}$ values are written as subscripts.

Configuration

1. $4 f\left({ }^{2} \mathrm{~F}_{5 / 2}^{\circ}\right) 6 p_{3 / 2}$

2. $4 f^{2}\left({ }^{3} \mathrm{H}_{5}\right) 60 p_{1 / 2}$

3. $4 f^{11}\left({ }^{2} \mathrm{H}_{9 / 2}^{\circ} 2\right) 6 s 6 p\left({ }^{3} \mathrm{P}_{1}^{\circ}\right)$

4. $4 f^{9}\left({ }^{6} \mathrm{H}^{\circ}\right) 5 d\left({ }^{7} \mathrm{H}_{8}^{\circ}\right) 6 s 6 p\left({ }^{3} \mathrm{P}_{0}^{\circ}\right)$

5. $4 f^{12}\left({ }^{3} \mathrm{H}_{6}\right) 5 d\left({ }^{2} \mathrm{D}\right) 6 s 6 p\left({ }^{3} \mathrm{P}^{\circ}\right)\left({ }^{4} \mathrm{~F}_{3 / 2}^{\circ}\right)$

6. $5 f^{4}\left({ }^{5} \mathrm{I}_{4}\right) 6 d_{3 / 2}(4,3 / 2)_{11 / 2} 7 s 7 p\left({ }^{1} \mathrm{P}_{1}^{\circ}\right)$

These examples all have an $f$-electron core in $L S$ coupling. The $\left(J_{1}, j\right)$ and $\left(J_{1}, J_{2}\right)$ term symbols are used throughout this compilation. The configuration notation $4 f_{5 / 2} 6 p_{3 / 2}$ might well be used in the first example, but the notation shown emphasizes that the $4 f$ electron belongs more to the atomic core. This notation is also preferred because of its similarity to the more complex examples that follow.

\section{c. $j j$ Coupling of Equivalent Electrons}

This scheme has been used for calculations of some $5 f^{N}$ actinide configurations, and is often used in relativistic $a b$ initio calculations. Notations of the following types are consistent with those outlined above. 
Configuration

1. $6 d_{5 / 2} 6 d_{3 / 2}$

$(5 / 2,3 / 2)$

2. $6 d_{5 / 2}^{3}$

3. $5 f_{7 / 2}^{4} 5 f_{5 / 2}^{2}$

4. $5 f_{7 / 2}^{4} 5 f_{5 / 2}^{5}(8,5 / 2)_{21 / 2}^{\circ} 7 p_{3 / 2}$

5. $5 f_{7 / 2}^{3} 5 f_{5 / 2}^{3}(9 / 2,9 / 2)_{9} 7 s 7 p\left({ }^{3} \mathrm{P}_{2}^{\circ}\right)$

The configuration in the second example shows a general notation for equivalent electrons having the same $j$ value.

The third example shows a more general case of $j j$ coupling of equivalent electrons, with each of the two groups of electrons $\left(j_{1}=7 / 2, j_{2}=5 / 2\right.$ in this case) having more than one allowed $J$ value. The allowed $J$ values for $l_{j}^{N}$ equivalent-electron groups are given in table 2 through $j=7 / 2$. The $l_{7 / 2}^{4}$ group has two levels for each of the $J$ values 2 and 4 ; these are distinguished by seniority numbers given as preceding subscripts to the $J_{i}$ values. The $\left(J_{1}, J_{2}\right)$ notation under "Term" follows the convention that $J_{1}$ arises from the group of electrons on the left, $J_{2}$ from the group on the right. In pure $j j$ coupling, the levels of an entire subconfiguration such as $5 f_{7 / 2}^{4} 5 f_{5 / 2}^{2}$ are degenerate; thus the seven subconfigurations of $5 f^{6}$, for example, are the real "terms" in this scheme. The listing of the $\left(J_{1}, J_{2}\right)$ symbols under the "Term" heading is, however, a notational convenience implied by the fact that the final $\left(J_{1}, J_{2}\right)$, wavefunctions constitute the basis set in the $j j$ scheme (see 2.2.b).

The fourth and fifth examples are obvious extensions to configurations having external electrons, with a resultant $J_{2}$ coupled to the core $J_{1}$ to form a $\left(J_{1}, J_{2}\right)$ term.

\section{d. $J_{1} l$ or $J_{1} L_{2}$ Coupling 5}

Configuration

1. $4 f\left({ }^{2} \mathrm{~F}_{5 / 2}^{\circ}\right) 5 g$

Term

2. $4 f^{2}\left({ }^{3} \mathrm{H}_{4}\right) 5 g$

${ }^{2}[9 / 2]^{\circ}$

3. $4 f^{13}\left({ }^{2} \mathrm{~F}_{7 / 2}^{\circ}\right) 5 d^{2}\left({ }^{1} \mathrm{D}\right)$

4. $4 f^{13}\left({ }^{2} \mathrm{~F}_{5 / 2}^{\circ}\right) 5 d 6 s\left({ }^{3} \mathrm{D}\right)$

The first two terms result from coupling a parent-level $J_{1}$ to the orbital angular momentum of a $5 g$ electron $(l=4)$ to obtain a resultant $K$ value enclosed in brackets. The spin of the external electron is then coupled with the $K$ angular momentum to obtain a pair of $J$ values, $J=K \pm 1 / 2$ (for $K \neq 0$ ), not shown. The multiplicity (2) of such pair terms has usually been omitted from the term symbol; it is given explicitly in these tables, since other multiplicities occur in the more general $J_{1} L_{2}$ coupling (examples 3 and 4 ). The last two examples are straightforward extensions of $J_{1} l$ coupling, with the $L_{2}$ and $S_{2}$ momenta of the "external" term $\left({ }^{1} \mathrm{D}\right.$ and ${ }^{3} \mathrm{D}$ in examples 4 and 5 , respectively) replacing the $l$ and $s$ momenta of a single external electron.

Configuration

e. $L S_{1}$ Coupling ${ }^{6}$

$\begin{array}{ll}\text { 1. } 5 p^{5}\left({ }^{2} \mathrm{P}^{\circ}\right) 5 f \mathrm{G} & { }^{2}[7 / 2] \\ \text { 2. } 5 p^{3}\left({ }^{4} \mathrm{D}^{\circ}\right) 5 f^{2}\left({ }^{3} \mathrm{~F}\right) \mathrm{H}^{\circ} & { }^{3}[13 / 2]^{\circ}\end{array}$

In these hypothetícal examples from the $\mathrm{Xe} \mathrm{I}$ and I I isoelectronic sequences, the $5 f$ electrons are mainly "outside" the $5 p^{N}$ cores. The orbital angular momentum of the core is coupled with the orbital angular momentum of the external electron(s) to give the total orbital angular momentum $L$. The letter symbol for the final $L$ value is listed with the configuration because this angular momentum is then coupled with the spin of the core $\left(S_{1}\right)$ to obtain the resultant $K$ angular momentum of the final term (in brackets). The multiplicity of the $[K]$ term arises from the spin of the external electron(s).

\section{f. Coupling Schemes and Term Symbols}

The coupling schemes outlined above include those now most frequently used in calculations of atomic structure. Since the notations clearly distinguish the different schemes, it will not be necessary in our discussions of particular spectra to indicate the coupling schemes for the various configurations.

Each of the three types of term symbols gives the values of the two angular momenta that couple to give the total electronic angular momenta of the levels (indicated by the $J$ values). For configurations of more than one unfilled subshell, the angular momenta involved in the final coupling derive from two groups of electrons (either group may consist of only one electron). These are often an inner group of coupled electrons and an outer group of coupled electrons, respectively. In any case the quantum numbers for the two groups have been distinguished by subscripts 1 and 2, so that quantum numbers represented by capital letters without subscripts are total quantum numbers for both groups. Thus the quantum numbers for the two vectors that couple to give the final $J$ are related to the term symbol as follows:

Coupling

Scheme

$\begin{array}{lcc}L S & L, S & 2 S+1 L \\ J_{1} J_{2} & J_{1}, J_{2} & \left(J_{1}, J_{2}\right) \\ J_{1} L_{2}(\rightarrow K) & K, S_{2} & 2 S_{2}+1[K] \\ L S_{1}(\rightarrow K) & K, S_{2} & 2 S_{2}+1[K]\end{array}$

The parity is indicated by appended degree symbols on odd parity terms.

Quantum numbers for vectors that couple to give $J$

Term Symbol 


\subsection{Allowed Terms or Levels for Equivalent Electrons}

\section{a. LS Coupling (Table 1)}

Configurations with one or more groups of equivalent electrons are usually analyzed or calculated on the basis of $L S$ coupling of the equivalent electrons. The allowed terms of a configuration consisting of two inequivalent groups are obtained by coupling the $L$ and $S$ vectors of the groups in all possible ways, and the procedure may be extended to any number of such groups. Thus the allowed terms for any configuration can be obtained from a table of the allowed terms for groups of equivalent electrons. Table 1 is sufficient for this purpose for any configuration in this compilation.

The configuration $l^{N}$ has more than one allowed term of certain $L S$ types if $l>1$ and $2<N<4 l\left(d^{3}-d^{7}\right.$ and $f^{3}-f^{11}$ in table 1). Each recurring term of a particular type for one of these configurations in table 1 is immediately followed by a sequential index number [Nielson and Koster, 1963]; these index numbers stand for additional theoretical quantum numbers that differentiate the recurring terms except for a few terms of $f^{5}$ and $f^{9}, f^{6}$ and $f^{8}$, and $f^{7}$.

The first of the additional quantum numbers given with each term of the $d^{N}$ and $f^{N}$ configurations is the seniority number [Racah, 1942; 1943; 1949]. The seniority number of each $d^{N}$ term is followed by the group label of the term according to the irreducible representations of the $R_{5}$ group. This label consists of two integers $\left(w_{1} w_{2}\right)$ in parentheses, with $2 \geqslant w_{1} \geqslant w_{2} \geqslant 0$.

The theoretical classification of the terms of the $f^{N}$ configurations is according to the scheme of Racah [1949]. The seniority number (not needed, but given for completeness) is followed by the group labels $W$ (three integers $\left(w_{1} w_{2} w_{3}\right)$, with $2 \geqslant w_{1} \geqslant w_{2} \geqslant w_{3} \geqslant 0$ ) and $U$ (two integers $\left(u_{1} u_{2}\right)$, with $4 \geqslant u_{1}+u_{2}$ and $\left.u_{1} \geqslant u_{2} \geqslant 0\right)$. These identify respectively an irreducible representation of the $R_{7}$ group and an irreducible representation of its subgroup $G_{2}$. The few remaining duplicated terms are further labeled $A$ or $B$ to indicate Racah's separation of the two terms.

In the tables of energy levels, we use the index numbers assigned by Nielson and Koster to distinguish recurring terms. For the $d^{N}$ configurations, this procedure has the disadvantage of substituting an arbitrary number for a quantum number (the seniority) that itself distinguishes the recurring terms in all cases. The actual value of the seniority number is rarely needed, however, and we prefer a consistent notation for the $d^{N}$ and $f^{N}$ configurations.

\section{b. $j j$ Coupling (Table 2)}

The allowed $J$ values for a group of $N$ equivalent electrons having the same $j$ value, $l_{j}^{N}$, are given in table 2 for $j=1 / 2,3 / 2,5 / 2$, and $7 / 2$ (sufficient for $l \leqslant 3$ ). The $l_{7 / 2}^{4}$ group has two allowed levels for each of the $J$ values 2 and 4 . The subscripts distinguishing the two levels in each case are the seniority numbers [de-Shalit and Talmi, 1963].

The allowed levels of the configuration $n l^{P}$ may be obtained by dividing the electrons into sets of two groups $n l_{l+1 / 2}^{Q} n l_{l-1 / 2}^{R}, Q+R=P$. The possible sets run from $Q=P-2 l$ (or zero if $P<2 l$ ) up to $Q=P$ or $Q=2 l+2$, whichever is smaller. The (degenerate) levels for a set with both $Q$ and $R$ nonzero have wavefunctions defined by the quantum numbers $\left(\alpha J_{1}, \beta J_{2}\right)_{J}$, with the $J_{1}$ the resultant angular momentum of the $Q$ group, and $J_{2}$ from the $R$ group. The symbols $\alpha$ and $\beta$ represent any additional quantum numbers required to identify levels of the $Q$ and $R$ groups, respectively. The $J$ values of the allowed levels for each $\left(\alpha J_{1}, \beta J_{2}\right)$ subset are obtained by combining $J_{1}$ and $J_{2}$ in the usual way.

\subsection{Percentage Compositions of Levels}

\section{a. Explanation of Eigenvector Percentages}

The analyses of many rare-earth spectra could be carried out beyond a very limited stage only with the aid of calculations of the energy level structures. The calculations of interest here yield the wavefunctions of the levels expressed as eigenvectors of a certain type: the eigenvector for each level is a linear combination of single-configuration single-term wavefunctions. (The configuration and quantum numbers designating a level of a theoretical spectroscopic term determine the angular dependence of a corresponding wavefunction.) Thus the wavefunction $|\alpha J M\rangle$ of the $M$ sublevel (state) of a level labeled $\alpha J$ is expressed in terms of basis states; for example, if basis states $|\gamma S L J M\rangle$ are formed by Russell-Saunders coupling,

$$
|\alpha J M\rangle=\sum_{\gamma S L}|\gamma S L J M\rangle\langle\gamma S L J \mid \alpha J\rangle .
$$

The symbol $\gamma$ represents all the quantum numbers needed in addition to $S L J M$ to specify a basis state $|\gamma S L J M\rangle$ (configuration, parentage, group-theoretical label, etc.). The $\langle\gamma S L J \mid \alpha J\rangle$ are expansion coefficients, and

$$
\sum_{\gamma S L}|\langle\gamma S L J \mid \alpha J\rangle|^{2}=1
$$

The squared expansion coefficients for the various $y S L$ terms in the composition of the $\alpha J$ level are conveniently expressed as percentages, whose sum is $100 \%$. Thus the percentage contributed by the pure Russell-Saunders state $\gamma_{a} S_{b} L_{c} J$ is equal to $100 \cdot\left|\left\langle\gamma_{a} S_{b} L_{c} J \mid \alpha J\right\rangle\right|^{2}$. The notation for Russell-Saunders basis states has been used only for concreteness; the eigenvectors may be expressed in any coupling scheme, and the coupling schemes may be different for different configurations included in a single calculation (with configuration interaction).

Notations of the type $(A+B+\ldots)$, where $A, B, \ldots$ represent configurations, will be used in referring to calculations that include the interactions of the configurations listed between the parentheses.

The largest percentage in the composition of a level is called the "purity" of the level in that coupling scheme. The coupling scheme (or combination of coupling schemes if more than one configuration is involved) that results in the 
largest average purity for all the levels in a calculation is usually best for naming the levels. Calculations of very complex configurations are almost always carried out with Russell-Saunders basis states, however, and eigenvectors in this scheme are usually advantageous for most purposes. Transformations to other schemes of higher purity are often made as the last step of a calculation.

\section{b. Need for Leading Percentages, and a Caveat}

In the tables we give one or two leading percentages from the eigenvectors of the levels where these are available (see sec. 4). The percentages are important qualifiers of the configuration and term notations given with the levels. The need for some indication of low eigenvector purities seems evident. Even leading components too small to describe a level nevertheless serve to indicate the extent of term and/or configuration mixing in the eigenvectors. Percentages representing higher purities are quantitative confirmations of the term designations (but see below).

It should be emphasized that the eigenvectors are calculated quantities, dependent on particular theoretical models and subject to the inaccuracies of necessary approximations. Even within a particular approach such as, for example, the parametric treatment of Slater-Condon interactions, the results are affected by the inclusion or omission of explicit configuration interaction (and which configurations are included), effective interactions, explicitly term-dependent interactions, magnetic interactions in addition to the spin-orbit interaction, etc. The variation of the eigenvectors due to such effects can in some cases change the designations of particular levels. The calculations also vary considerably in the overall accuracy of the resulting eigenvectors, and thus in the accuracy of prediction of the observed quantities-energy levels, $g$ values, line strengths, etc. The reader is urged to consult the references given with the individual, tables for details about calculations of interest.

\subsection{Zeeman Effect (Tables 3-6)}

The Zeeman effect for "weak" magnetic fields is of interest here because of the importance of Zeeman data in the analysis of complex spectra. In the weak-field effect, the $J$ value of a level remains a good quantum number, although in general the level is split by the field into magnetic sublevels. (The field is weak only in the sense that the splittings must be small compared to the level separations. The nuclear magnetic moment is here assumed to be zero, or to have a negligible effect.) For our purpose, the $g$ value of such a level may be defined by the expression for the energy shift of its magnetic sublevel having magnetic quantum number $M$ (which has one of the $2 J+1$ values, $-J$, $-J+1, \ldots, J-1, J)$ :

$$
\Delta E=\operatorname{gM} \mu_{0} B
$$

The magnetic flux density is $B$ and $\mu_{0}$ is the Bohr magneton $\left(\mu_{11}=e \hbar / 2 m\right.$, in SI units). Since the $g$ value is a gyromagnetic ratio (expressed as a pure number), the concept is meaningless for $J=0$; levels having this $J$ value are excluded from the discussion.

The wavenumber shift $\Delta \sigma$ corresponding to the energy shift $g M \mu_{0} B$ is given by

$$
\Delta \sigma=g M\left(0.46686 \mathrm{~B} \mathrm{~cm}^{-1} / \mathrm{T}\right)
$$

Here the magnetic flux density $B$ is expressed in units of the tesla (symbol T, equivalent to $10^{4}$ gauss). The quantity in parentheses, the Lorentz unit, is of the order of $1 \mathrm{~cm}^{-1}$ for typical flux densities used to obtain the optical Zeeman effect. Most of the $g$ values tabulated here were derived by application of this formula (for each of the two combining levels) to measurements of optical Zeeman patterns. A single transverse-Zeeman-effect pattern (two polarizations, resolved components, and sufficiently complete) can yield the $J$ value and the $g$ value for each of the two levels involved [White, 1934; van den Bosch, 1957].

The highly accurate $g$ values tabulated for some low levels in neutral atoms have been obtained by atomic beam magnetic-resonance techniques; the analysis of this type of data usually involves nuclear moments, and in general is more complex than the weak-field optical effect [Marrus and Nierenberg, 1962; Wybourne, 1965, chap. 5].

The $g$ value of a level $\alpha J$ belonging to a pure $L S$-coupling term is given by the formula:

$$
g_{\alpha S L J}=1+\left(g_{s}-1\right) \frac{J(J+1)-L(L+1)+S(S+1)}{2 J(J+1)} .
$$

The independence of this expression from any other quantum numbers (represented by $\alpha$ ) such as the configuration, etc., is important. The expression is derived from vector coupling formulas by assuming a $g$ value of unity for a nure orbital angular momentum (singlet level) and writing the $g$ value for a pure electron spin ( $S$ level) as $g_{s}$ [Wybourne, 1965, p. 98]. A value of 2 for $g_{s}$ yields the Lande formula. If the anomalou's magnetic moment of the electron is taken into account, the value of $g_{s}$ is 2.002319 . "Corrected" Landé $g$ values ${ }^{7}$ obtained with this value for $g_{s}$ are given according to Russell-Saunders terms in tables 3 and 4. Tables 5 and 6 give Landé $g$ values in increasing order, the (three-place) values being from the uncorrected Landé formula.

The usefulness of Landé $g$ values is enhanced by their relation to the $g$ values in intermediate coupling. In the notation used in section 2.3 for the description of a level $\alpha J$ in intermediate coupling, the corresponding $g$ value is given by:

$$
g_{\alpha J}=\sum_{\gamma S L} g_{S L J}|\langle\gamma S L J \mid \alpha J\rangle|^{2}
$$

where the summation is over the same set of quantum numbers as for the wavefunction. The $g_{\alpha J}$ value is thus a weighted average of the Landé $g_{S L J}$ values, the weighting factors being just the corresponding component percentages

\footnotetext{
'The $g$ values in tables 3 and 4 are from an unpublished tabulation by Dr. Mark Fred of the Argonne National Laboratory. The arrangement here follows similar tables in Vol. I of AEL [Moore, 1971].
} 
from the eigenvector of the $\alpha J$ level in the $L S$-coupling representation. The range of $g$ values of interest can be obtained from the Lande formula; the practical range (table 6 ) is from -2.000 to 4.667 .

The Landé formula in general requires additional corrections if theoretical values accurate to three or more decimal places are needed. Relativistic and diamagnetic corrections have been calculated for the $g$ values of a number of low levels of neutral rare-earth atoms [Judd and Lindgren, 1961; Conway and Wybourne, 1963]. If the correction for intermediate coupling is excepted, the absolute value of the sum of the corrections to the Lande formula appears to be less than 0.002 for most of the $g$ values calculated by Judd and Lindgren.

The formulas for $g$ values in two other coupling schemes used in this compilation are:

$$
\begin{aligned}
& J_{1} J_{2} \text { coupling } \\
& g_{J}=g_{J 1} \frac{J(J+1)+J_{1}\left(J_{1}+1\right)-J_{2}\left(J_{2}+1\right)}{2 J(J+1)} \\
& \quad+g_{J_{2}} \frac{J(J+1)+J_{2}\left(J_{2}+1\right)-J_{1}\left(J_{1}+1\right)}{2 J(J+1)}
\end{aligned}
$$

$J_{1} L_{2}$ coupling

$$
\begin{aligned}
g_{J} & =2\left(g_{J_{1}}-1\right) \frac{K(K+1)+J_{1}\left(J_{1}+1\right)-L_{2}\left(L_{2}+1\right)}{(2 J+1)(2 K+1)} \\
& +\frac{3 J(J+1)-K(K+1)+S_{2}\left(S_{2}+1\right)}{2 J(J+1)}
\end{aligned}
$$

The $J_{1} L_{2}$-coupling formula is from Wybourne [1965, p. 100] with $g_{\mathrm{s}}$ set equal to 2. If $S_{2}=1 / 2$, so that $J=K \pm 1 / 2$, the second term simplifies to $(2 J+1) /(2 K+1)$, and the formula is equivalent to that given by Racah [1942a] for $J_{1} l$ coupling.

\section{Material Preceding Each Table-Summary Data, Comments, References}

Some basic data are collected at the beginning of the table for each spectrum. ${ }^{8}$ The configuration and term designation of the ground-state level are given, with the electrons of inactive closed subshells listed in parentheses. The wavenumber corresponding to the principal ionization energy is given, as well as this energy expressed in electron-volts $(\mathrm{eV})$. An equivalence of $1 \mathrm{eV}$ to $8065.479 \pm 0.021 \mathrm{~cm}^{-1}$ was used for the conversion [Cohen and Taylor, 1973]. The confidence levels of the quoted uncertainties for many of the ionization energies were not given in the original papers. Uncertainties quoted without comment are probably best taken as standard-deviation errors.

A summary list of the configurations to which experimental levels of each parity have been assigned is

${ }^{8}$ A single-page tabulation of the ground levels and ionization potentials for the neutral through triply ionized lanthanide atoms is given in a compilation of such data for the lanthanides and actinicles [Martin, Hagan, Reader, and Sugar, 1974]. A number of the ionization potentials are superseded by more accurate values in the present compilation. provided if these are not readily apparent from the table itself.

We have given the sources of the data for each spectrum, along with any necessary explanation of the theoretical interpretation. We have also tried to include in the references enough of the earlier papers to indicate the main line of progress on the energy-level analysis of the spectrum. References on energy levels of ions in crystals or solutions are mainly restricted to sources of the compiled data in cases where no free-ion data were available.

The extent of the observations of the spectra on which the analyses are based is usually indicated (wavelength ranges, approximate numbers of lines). The main references for line lists are given, except that we usually do not cite any of the extensive collections of atomic wavelengths that may be useful for a particular spectrum. References for several such collected tables are listed in section 5. The references for various other types of data not included in the tables (hyperfine structure, isotope shifts, etc.) are very incomplete, and in many cases are omitted entirely.

The abbreviations of periodical titles generally follow the Bibliographic Guide for Editors and Authors [1974]. The symbols following the citations indicate types of data or other content according to the following code:

\section{EL Energy Levels}

Experimental energy differences, except Hfs or IS. Includes references that suggest the rejection of previously reported levels or confirm previously doubtful levels.

\section{ND New Designations}

New or changed designations or $J$ values for known energy levels. This symbol is sometimes omitted, especially if the symbol EL occurs.

CL Classified Lines

Indicates the assignment of observed lines to transitions between energy levels that are specified by theoretical designations and/or by their positions in a known level scheme.

W Wavelengths (or wavenumbers)

New measurements, or wavelengths newly assigned to a particular spectrum. Also, measurements of other entities corresponding to energy differences between levels.

\section{ZE Zeeman Effect data or interpretation}

SE Stark Effect data or interpretation

Hfs Hyperfine structure Observations and theory.

IS Isotopic or Isomeric (Nuclear) Shifts Observations and theory.

IP Ionization Potential (Ionization Energy) 


\section{SF Series Formulae}

Evaluated series constants, including those appearing in polarization-theory formulae.

\section{PT Parametric Theory}

Calculations in which the energy parameters of Slater-Condon theory, or extensions thereof, were obtained by fitting the theory to experimental levels, $g$ values, and/or other observed quantities.

\section{AT Ab initio Theory}

These references are mostly to Hartree-Fock type calculations of energy parameters or levels.

Additional references for these types of data may be found in the NBS bibliographies [Moore, 1969; Hagan and Martin, 1972; Hagan, 1977]. A recent article on rare-earth atoms and ions by Blaise, Camus, and Wyart [1976] includes some types of data omitted from the present compilation, especially hyperfine structures and isotope shifts.

The date of submission of the manuscript for each spectrum is given in brackets following the list of references.

\section{Explanation of the Tables (By Column Heading)}

For most of the headings, it is convenient to discuss separately the cases of levels tabulated with and without leading percentages.

\subsection{Configuration}

\section{a. Levels Tabulated With Leading Percentages}

The electron configuration for the largest component in the calculated eigenvector for the level is normally given in this column. (The configuration is for the largest component to within the estimated uncertainty of the calculation; see 4.2.) Any ancestor terms or $J$ values appropriate to this component are normally included with the configuration, as in the examples in section 2.1.

A question mark after the configuration indicates that the assignment of the observed level to the calculated eigenvector is uncertain. This particular notation for an uncertain assignment usually implies that at least one other possible assignment of the level would give a different configuration or set of parent terms for the leading component.

The configuration is listed only once for a set of levels grouped into a term (see 4.2). All notations given with the configuration, including any question marks, apply to each level of such a term.

In cases of strong configuration interaction, more than half the percentage composition of a level may be due to components from a configuration different from that for the leading component; such an example makes it clear that the configuration given in the first column does not necessarily represent a configuration assignment of the level. A particular configuration may appear in the first column for more levels than the pure configuration is allowed (see Ce III for a relatively simple example).

\section{b. Levels Tabulated Without Leading Percentages}

The reliability and indeed the meaning of configuration assignments made without. supporting calculations vary greatly. Even in the most complex spectra, there may be some levels or groups of levels belonging largely to a single configuration. The observed structure, $g$ values, intensities, and other data can in such cases lead to unambiguous assignments representing high purity. (Examples are the lower levels of most $4 f^{N} 6 s^{2}, 4 f^{N} 6 s$, and $4 f^{N}$ configurations in the rare earths.) Quite often, however, the levels to be interpreted comprise a dense structure to which several configurations are known or expected to contribute. Some preliminary configuration assignments may be possible and useful in such cases, but such assignments for the bulk of the levels are often best regarded as tentative until confirmed by calculations. In many cases, meaningful singleconfiguration assignments do not exist.

We have tried to indicate doubtful features of the interpretations in the discussions of particular spectra and, to some extent, in the tables. A question mark following the configuration indicates explicitly that the assignment is doubtful. A doubtful ancestor term is indicated by a question mark after the term symbol within parentheses.

In some cases the configuration for a level may be known, although the appropriate ancestor terms (or even the preferred order of coupling of the electrons) cannot be determined without calculations. Such a configuration assignment (together with the final term and $J$ value) without full ancestry does not in general serve as a unique name for the level.

\section{c. Limits}

The wavenumber corresponding to the principal ionization energy is entered in proper sequence under "Level." The corresponding entry in the first column is the symbol for the next higher spectrum of the element, followed by the term designation and $J$ value for the ground level of this next ion. The configuration is normally omitted, and the word "Limit" appears in the "Term" column (the levels of the higher ion being limits for series in the lower spectrum). If the known levels of the lower ion (or atom) extend above the principal limit, one or more of the higher limits are also given at appropriate positions.

\subsection{Term}

a. Levels Tabulated With Leading Percentages: Naming of Levels, Grouping of Levels Into Terms, Other Conventions

A term symbol in the second column belongs to the eigenvector component whose configuration and ancestry appear in the first column. The assignment of a set of levels 
to a term is indicated by grouping the levels and by listing the configuration and term symbol for only the first (lowest) level of the group. ${ }^{9}$ The presence of both a configuration with all necessary ancestor terms in the first column and a term symbol in the second column guarantees that each of the levels grouped with the term is uniquely designated by the symbols in the first two columns, together with the $J$ value; i.e., such levels have names. This naming convention also applies to isolated levels not grouped into terms. The use of the $\left(J_{1}, J_{2}\right)$ term symbols in this connection should be noticed; a $J_{1} J_{2}$ configuration notation in the first column is indicated as a name for the associated level(s) only if a $\left(J_{1}, J_{2}\right)$ symbol appears in the second column.

We usually retained or formed a term (by grouping levels) if half or more of the candidate levels for the term were known and had leading components approximately $45 \%$ or larger. The $45 \%$ requirement was sometimes lowered significantly for levels having leading components sufficiently larger than the corresponding second components. Additional levels of lower purity $(\sim 30 \%$ to $45 \%$ ) were retained (or assigned) to help complete a multilevel term provided certain conditions were met. Thus the component for an assigned term name is normally the leading component in the eigenvector, and is also as large a percentage of the particular name as occurs in the eigenvector of any other level (known or not) with the same $J$ value. We have also tried to avoid a name for which the configuration contributes less to the total composition of the level than some other configuration. These are minimal requirements for avoiding completely inappropriate names.

One can assure more generally satisfactory names by disallowing any name representing significantly less than $50 \%$ eigenvector purity; and in the case of a leading percentage near $50 \%$, by requiring that the second percentage be significantly smaller (alternate designation clearly less appropriate), and that no other eigenvector should have a comparable leading percentage ( $\sim 50 \%)$ for the same designation. The weaker criteria for the grouping and naming of levels outlined above were adopted to allow practically any significant term structure within a single configuration to be exhibited in the tables. In cases of strong configuration interaction it sometimes happens that assignment to a particular term type remains appropriate for a level or level group for which no meaningful configuration assignment is possible. Such term names are not indicated by our scheme but may usually be deduced by examination of the two leading eigenvector percentages (see sec. 4.6).

Most of the calculated leading percentages for levels in question as to naming are probably uncertain by several percent. In order to facilitate term assignments, we have allowed a relatively few small deviations (by up to $\sim 4 \%$ ) from the above requirements on naming. These deviations are probably within the uncertainties of the calculations.

A group of levels (or a single level) constituting a term is always set off from neighboring terms or levels by full vertical spaces. For compactness, neighboring levels not belonging to terms are in many cases separated by less than full vertical spaces. Groups of such levels are not to be confused with the significantly more compact groups constituting terms.
Many of the resulting terms are incomplete, in the sense that no observed level is listed for one or more of the possible $J$ values of the term. A level "missing" from such a term may not have been found in the analysis; alternatively, it may be that no theoretical eigenvector is appropriate for the corresponding designation, even under the relaxed criteria described above. The first case is distinguished in the tables by printing the $J$ value of the level, and leaving a blank space in the "Level" column. The leading percentages for such a missing level are given if available. In the second case, the best candidate levels for the missing designation are usually known and lie in the same region as the levels assigned to the term.

Levels belonging to a term most of whose levels have not been found may nevertheless be grouped if the term appears to be an important one or lies in a region where most terms are more complete. The printed $J$ values of the missing levels explicitly indicate possible extensions of the analysis. No predicted term is shown, however, unless at least one level is known, and we emphasize that missing predicted levels are generally not indicated in these tables: No term symbols or $J$ values are listed for missing levels having low eigenvector purities or belonging to terms the levels of which are not grouped (for whatever reason). The reader is urged to consult the references to published calculations for additional predicted levels.

A level with the leading percentage $>45 \%$ from a singlelevel term (singlet, $S$ term, etc.) is usually so named (shown as a term) if the second percentage is significantly smaller, and if the other conditions outlined above are met. Isolated levels (those remaining after the formation of all terms) are named according to similar conditions.

Some levels of $f^{N}$ and $d^{N}$ configurations have large eigenvector components from two or more terms of the same LS type (sec. 2.2.a). Since the resultant lowering of the purities has no physical significance, we have retained the names of such levels having adequate total purity of a particular LS type and labeled them with the NielsonKoster index number for the term of the leading component. (Of course the corresponding group-theoretical numbers have little meaning for such a low-purity term.) Similar considerations have been applied in designating parent (or grandparent) terms arising from $f^{N}$ configurations.

A question mark after a term designation indicates that the assignment of the observed level(s) to the calculated eigenvector(s) is uncertain.

\section{b. Levels Tabulated Without Leading Percentages}

Most of the discussion in section 4.1.b of configuration assignments of levels with no calculated eigenvectors is pertinent to term assignments. Some of the additional difficulties often encountered in attempts to assign term names will be obvious from the preceding discussion.

We note here also that a term symbol in the second column does not guarantee that the symbols in the first two columns (and the $J$ value) uniquely name the level; the necessary nominal parentages may not be given. 


\subsection{Total Electronic Angular-Momentum Quantum Number $J$}

An uncertain $J$ value for a level is usually known to within two or three possible values; in such cases all the possible $J$ values are listed. Two or more $J$ values may also be listed at a single energy position to denote the unresolved levels of a term, etc. Three or more such $J$ values are indicated by listing the two extreme values separated by a dash.

\subsection{Level}

The levels are normally given in units of $\mathrm{cm}^{-1}$, with respect to the ground level at zero $\mathrm{cm}^{-1}$. Odd-parity levels are printed in italics. The uncertainty of the level positions in units of the last decimal place is not constant; it may vary by an order of magnitude even within an analysis. If no statement about the accuracy is made, it is usually safe to assume that the probable error is between 2 and $\sim 20$ units in the last place. A better estimate of the error in a particular case may sometimes be obtained by consulting the original paper(s) or line list.

Levels within terms are listed in order of position. Terms are listed in order of lowest (known) levels, with ungrouped levels being treated as terms. The $J$ value and blank space indicating a missing level of a term are given in the order of the corresponding calculated level if such a value is available.

A question mark following a level always indicates that the level may not be real.

An asterisk (*) following a level has the same meaning as a question mark following the assignment-the term assignment of the level is questionable. For configurations that have been calculated, this means that the correlation of the calculated eigenvector to the experimental level is questionable. The asterisk notation is used mainly for questionably assigned levels included in terms. An uncertain assignment of an isolated level may be indicated by an asterisk, but only if the configuration in the first column would be unchanged by any possible reassignment of the level (no question mark in the first column).

\subsection{Experimental $g$ Value}

These (dimensionless) magnetic splitting factors are usually obtained from measurements of weak-field Zeeman patterns (sec. 2.4). The relation between the uncertainty and the number of decimal places given differs according to the observer and the particular value; the range covered by this relation is similar to that for the level values (see above). More specific comments are made on the $g$ values for some spectra.

A colon following a $g$ value indicates that it may be significantly less accurate than values given to the same number of decimal places but not so marked. Values followed by a question mark are tentative, usually being based on assumptions made to allow reduction of the Zeeman patterns.

\subsection{Leading Percentages}

This column normally gives one or two percentages from the calculated eigenvector of the level. The space for the second percentage (on the right) is used in either of two ways, as explained below. All percentages are rounded off to the nearest percent, and the "\%" symbol is omitted.

Any use of this column in a manner not outlined below is explained in the tables for particular spectra.

\section{a. First Percentage}

If the level has an assigned name (see sec. 4.2), the first percentage is for this "name" component. A first percentage followed by a term symbol represents the largest component in the eigenvector of a level having no assigned name, the configuration and ancestry for this component being shown under "Configuration."

\section{b. Second Percentage From Same Eigenvector as First Percentage}

If two percentages are listed without the word "or" between them, the second percentage is the largest of the remaining percentages from the same eigenvector as the first percentage. If the second component belongs to the same configuration as the first, usually only the parent terms (or parent levels where appropriate) and final term for the second component are given after the percentage. Electron subshells common to different configurations for the first and second percentages may be omitted from the second configuration, the most frequent example being a common $4 f^{N}$ core. Term notations common to all second percentages listed for the levels of a term are usually given only once.

The coupling scheme for a second eigenvector component belonging to a different configuration may be different from the scheme for the first component; this should not be confused with case $c$ below, where the second listed percentage is the leading percentage in a different coupling scheme.

Since many authors list only the largest percentage for at least some levels, the absence of a second percentage in this compilation does not necessarily mean that it is less than the smallest percentage used here $(0.5 \%$, given as $1 \%)$.

The relative signs of the two eigenvector components are not given. These are often not given in the original publications. Furthermore, these signs depend on certain conventions, no one set of which has been accepted by all authors. The original articles can be consulted for (possibly) more complete eigenvectors with signs.

\section{c. Second Percentage Is for Leading Component of Eigenvector in Alternate Coupling Scheme}

Where the eigenvectors from a calculation are available in more than one coupling scheme, we often give in the second percentage space the leading percentage from the eigenvector calculated in an alternate scheme. This use of the second percentage space is indicated by the word "or" between the two percentages, since each is a leading 
percentage. Competitively high coupling purities for a configuration in different coupling schemes were, of course, regarded as an argument for giving leading percentages in alternate schemes, as was the case in which a second scheme for the configuration in question is the preferred scheme for a related configuration (two configurations connected by a strong transition array, etc.). We have favored $L S$ coupling as a second scheme in cases where another scheme was used for naming the levels.

It should be noted that the leading component in a secom. scheme is not necessarily a name for the level in that scheme; in cases of low purity, the eigenvectors of two (or more) levels of the same $J$ value may have the same leading component.

For some configurations, the alternate coupling schemes are both LS coupling, but with the electrons coupled differently in the two cases (see Eu I $4 f^{7} 5 d 6 s$, for example). In this case it is usually possible to set up terms in either scheme, as indicated by printing the term symbol for the second scheme only for the first level of each term.

\section{Note on Tables of Wavelengths for Rare-Earth Spectra}

Several of the more comprehensive collections of atomic spectral wavelengths that include the rare earths are listed here, since we have usually not included these publications in the references for particular spectra. The most complete existing lists of observed lines for many rare-earth spectra are at present unavailable in either published or report form.

Gatterer, A., and Junkes, J., Atlas der Restlinien, Vol. II, Spektren der Seltenen Erden, Specola Vaticana, 347 pp. (1945).

Wavelength range, 2265-7600 $\AA$. Mainly first and second spectra. Main tables have a total of 41605 lines arranged by element, with Sc, Y, $\mathrm{Zr}$, and Th included in addition to the lanthanides. Generally the most complete collection of rare-earth lines having tables separated according to element. Otherwise, these tables have been superseded. No energy level classifications.

Harrison, G. R., MIT Wavelength Tables, MIT Press, Cambridge, Mass., 429 pp. (1969).

Wavelength range, 2000-10000 $\AA$. Mainly first and second spectra. Main table has all elements and spectra together, arranged by wavelength. Includes original measurements of thousands of lines of the more complex rare-earth spectra, and was used extensively in the preparation of several other publications listed here. No energy level classifications.
Meggers, W. F., Corliss, C. H., and Scribner, B. F., Tables of Spectral-Line Intensities, Nat. Bur. Stand. (U.S.), Monogr. 145, Part I, 403 pp.; Part II, 228 pp. (1975). Wavelength range $2000-9000 \AA$. Mainly first and second spectra. Part $I$ is arranged by element. About 16000 of the strongest lines of the rare-earth spectra are included, and the tables for several of these spectra have lines and energy levels (for classified transitions) that have not been published elsewhere. Part II is arranged according to wavelength with all (70) elements together.

Moore, C. E., A Multiplet Table of Astrophysical Interest, Nat. Stand. Ref. Data Ser., Nat. Bur. Stand. (U.S.), 40, 253 pp. (1972). A reprint of the 1945 edition.

Wavelength range, $2950-13000$. Has multiplet tables (by spectrum) and a finding list (all included spectra together). Lines of several rare-earth spectra (as available in 1945) are listed in the multiplet format especially convenient for some users.

Wysocka-Lisek, J., Spectrum Lines of Rare Earths Arranged by Wavelengths, Lubelskie Towarzystwo Naukowe, Lublin, Poland, 583 pp. (1970).

Most complete published collection of spectrum lines of the neutral and singly ionized rare earths for the region $2000-10000 \AA$ (58000 lines including lines of $\mathrm{Se}, \mathrm{Y}, \mathrm{Zr}$, and $\mathrm{Th}$, in addition to the lanthanides). Arranged by wavelength with all elements together. No energy level classifications.

Zaidel', A. N., Prokof'ev, V. K., Raiskii, S. M., Slavnyi, V. A., and Schreider, E. Ya., Tables of Spectral Lines, IFI/Plenum, New York, 782 pp. (1970).

Part 1 has 52000 lines of 60 elements, including the rare earths. This part is arranged by wavelength, with all elements together (mainly first and second spectra, range 2000-8000 $\AA$ ). Part 2 has 38000 lines of 98 elements arranged by element, and includes lines from third and higher spectra. Both parts were revised to include material published in the 1960's. The wavelength range of Part 2 extends into the vacuum ultraviolet and further to the infrared. For the rare earths, the main advantage of Part 2 (relative to the other publications) is the tabulation of the stronger lines of some third and higher spectra. Also includes the energy of the upper level (to the nearest $0.01 \mathrm{eV}$ ) for classified lines. 


\section{State of Analyses of Rare-Earth Spectra}

The table ${ }^{10}$ below roughly summarizes the status of the analyses for the first six spectra of the rare earths, based on the data available for this compilation. Analyses of spectra of ions in crystals or solutions are included only to the extent of indicating (by brackets) nine triply ionized species for which the levels tabulated here are entirely derived from such data. Analyses of the atomic and free-ion spectra are indicated by numbers one to four, with the following approximate meanings:

1. The spectrum has been well excited and measured over the relevant optical range. For a first or second spectrum the Zeeman effect has been observed. Practically all of the strong lines are classified. (In practice the number of "strong" lines varies from less than 100 to several thousand, depending on the complexity of the spectrum.) The more important configurations have been calculated; all the lower levels and more than half of all the known levels have been interpreted according to the calculations. More or refined calculations might be needed, such as the inclusion of additional configuration interactions, use of effective interactions, recoupling transformations, or extension to additional configurations. Such additional calculations probably would allow some extension and refinement of the analysis, especially in the case of a first or second spectrum; however, the analysis is now one of the most complete for the type of spectrum.

2. These analyses are less complete than those described above. Important configurations are not known or have not been interpreted theoretically. The wavelength descriptions are usually satisfactory, but in some cases further observations are needed.

3. The analysis has progressed beyond the beginning stages, but important configurations are not known relative to the ground configuration; or the basic configurations have not been calculated. More observations may be needed.

4. Only a beginning analysis exists.

The number for a particular spectrum thus indicates roughly the completeness of the analysis relative to other spectra of the same type and level of complexity. The main tables should, of course, be perused for details on the analyses. Summary tables of "Observed Terms," of the type given in AEL Vols. I-III, have been omitted here as being impractical for many of the complex rare-earth spectra with extensive configuration interactions.

The energy levels for 57 of the atomic species in the present compilation are from analyses of atomic or free-ion spectra; ${ }^{11}$ before 1960 , there were no analyses at all for 35 of these spectra. Also since 1960, there has been additional work on all but 2 or 3 of the 22 spectra analyzed earlier. It is clear from the table, however, that this progress on the

\footnotetext{
${ }^{10}$ The table is a revised version of a similar summary made several years ago [Martin, 1972]. The separated group of spectra in the upper part of the table belong to the Xe I and I I isoelectronic sequences; the $4 f$-electron orbit is not collapsed (rare-earth like) in the low-ionization members of these sequences. The first three Lu spectra are also separated off, since the analyzed configurations include $4 f$ electrons only in the closed shell. Three additional spectra that have been analyzed, Sm XXXY, Gd XXXVII, and Dy XXXIX, are not shown.

"Analyses of crystal or solution spectra are excluded from consideration in the following remarks.
}

rare earths during the past 15 years or so has in most cases not included the fourth and higher spectra. (No analyses of third or fourth rare-earth spectra were carried out during the 20 years from the late 1930's to the late 1950's.) More complete data on energy levels and wavelengths for the rare earths are needed in several important areas of research. It is fortunate that work on a number of these difficult spectra is now continuing or planned.

Status of analyses of rare-earth spectra. See text for explanation.

\begin{tabular}{|c|c|c|c|c|c|c|}
\hline$Z$ & I & II & III & IV & $\mathrm{v}$ & $\mathrm{VI}$ \\
\hline $57 \mathrm{La}$ & 2 & 2 & 1 & 2 & 3 & \\
\hline $58 \mathrm{Ce}$ & 2 & 1 & 1 & 2 & 4 & 4 \\
\hline $59 \operatorname{Pr}$ & 2 & 3 & 1 & 1 & 2 & \\
\hline $60 \mathrm{Nd}$ & 2 & 2 & 3 & [ ] & & \\
\hline $61 \mathrm{Pm}$ & 3 & 3 & & [ ] & & \\
\hline $62 \mathrm{Sm}$ & 1 & 2 & 3 & [ ] & & \\
\hline $63 \mathrm{Eu}$ & 2 & 2 & 2 & [ ] & & \\
\hline $64 \mathrm{Gd}$ & 2 & 1 & 2 & [ ] & & \\
\hline $65 \mathrm{~Tb}$ & 2 & 3 & 2 & 2 & & \\
\hline $66 \mathrm{Dy}$ & 2 & 2 & 4 & [ ] & & \\
\hline $67 \mathrm{Ho}$ & 2 & 3 & 2 & [ ] & & \\
\hline $68 \mathrm{Er}$ & 2 & 2 & 2 & [ ] & & \\
\hline $69 \mathrm{Tm}$ & 1 & 1 & 1 & [ ] & & \\
\hline $70 \mathrm{Yb}$ & 2 & 1 & 1 & 1 & & \\
\hline $71 \mathrm{Lu}$ & 2 & 2 & 2 & 1 & 3 & \\
\hline
\end{tabular}

\section{Acknowledgments}

The international community of spectroscopists has been very generous in helping with this undertaking. We are especially grateful to those colleagues in other laboratories who have supplied unpublished data and theoretical results, the following being only the most outstanding in this regard: J. Blaise and his associates, especially J. F. Wyart, at the Laboratoire Aimé Cotton, Orsay; Zipora Goldschmidt and associates in the Racah Institute of Physics, the Hebrew University, Jerusalem; P. F. A. Klinkenberg, E. Meinders, and T. A. M. van Kleef, at the Zeeman Laboratory, Amsterdam; G. Smith, of the Department of Astrophysics, Oxford; H. M. Crosswhite, formerly at the Johns Hopkins University and now at the Argonne National Laboratory, and W. T. Carnall, of the Argonne National Laboratory. These and other contributors of unpublished material are cited in the discussions of particular spectra.

We are very grateful to Charlotte $E$. Moore for the example of her carefully done compilations of spectroscopic data, especially the Atomic Energy Levels volumes, and for the extensive bibliographic material that she maintained and kept current in this laboratory for more than 20 years.

Our colleagues in the Spectroscopy Section have generously assisted us whenever called upon. We especially thank C. H. Corliss, V. Kaufman, J. Reader, J. Sugar, and J. L. Tech for their expert help and advice on a variety of problems. Reader and Sugar have also supplied unpublished material. 
K. G. Kessler, Chief of the Optical Physics Division, and D. R. Lide, Jr.; Chief of the Office of Standard Reference Data, have supported this work over a period of years. Their encouragement and advocacy are much appreciated.

In the preparation of the tables, the data were first transferred to decks of punched cards which could be conveniently revised and expanded to include new material up to a cutoff date. After final checking, the coded punched card records were transferred to magnetic tape and edited to produce a final tape for automatic typesetting. The main editing code was obtained by suitable modifications of editinsertion programs of C. Messina and J. Hilsenrath [1970]. The modified code and its successful use to produce the tables via automatic photocomposition (Linotron) are largely due to Robert Thompson, of the Office of Standard Reference Data, and to Rebecca Morehouse, Anne Meininger, and the late Rubin Wagner of the Computer Assisted Printing Section. Kathy Strine and the late Ruth Peterson of the Spectroscopy Section did most of the keypunching of the original cards and helped with the checking. Mrs. Strine also typed most of the original manuscript; Marsha Ahalt and Arlene Musgrove did the later typing and keypunching, and helped with the proofreading. Miriam Oland of the Computer Assisted Printing Section retyped and coded the final manuscript for typesetting via photocomposition. We are very grateful to all these members of the NBS staff for this excellent assistance.

\section{References}

Bacher, R. F., and Goudsmit, S., Atomic Energy States, 562 pp. (McGrawHill Book Co., New York, N.Y., 1932).

Bibliographic Guide for Editors and Authors, 362 pp. (American Chemical Society, Washington, D.C., 1974).

Blaise, J., Camus, P., and Wyart, J. F., in Gmelin Handbuch der Anorganischen Chemie, Vol. 39, Part B4, pp. 124-334 (Springer-Verlag, Berlin, 1976).
Cohen, E. R., and Taylor, B. N., J. Phys. Chem. Ref. Data 2, 663 (1973).

Condon, E. U., and Shortley, G. H., The Theory of Atomic Spectra, 441 pp. (Cambridge University Press, 1935: reprinted 1951 and later years).

Conway, J. G., and Wybourne, B. G., Phys. Rev. 130, 2325 (1963).

de-Shalit, A., and Talmi, I., Nuclear Shell Theory, 573 pp. (Academic Press, New York, N.Y., 1963).

El'yashevich, M. A., Spectra of the Rare Earths, 586 pp. in 2 books, AEC-tr4403/LK (1961). Available from National Technical Information Service (U.S.), Springfield, VA 22161. [A translation of Spektry Redkikh Zemel (State Publishing House of Technical-Theoretical Literature, Moscow, 1953).]

Hagan, L., Nat. Bur. Stand. (U.S.), Spec. Publ. 363, Suppl. 1 (1977).

Hagan, L., and Martin, W. C., Nat. Bur. Stand. (U.S.), Spec. Publ. 363, 103 pp. (1972)

Judd, B. R., and Lindgren, I., Phys. Rev. 122, 1802 (1961).

Marrus, R. A., and Nierenberg, W. A., in Topics on Radiofrequency Spectroscopy, International School of Physies "Enrico Fermi," Course XVII, pp. 118-156, (Academic Press, New York, N.Y., 1962).

Martin, W. C., Opt. Pur. Apl. 5, 181 (1972).

Martin, W. C., Hagan, L., Reader, J., and Sugar, J., J. Phys. Chem. Ref. Data 3, 771 (1974).

Messina, C. G., and Hilsenrath, J., Edit-Insertion Programs for Automatic Typesetting of Computer Printout, Nat. Bur. Stand. (U.S.), Tech. Note 500, 50 pp. (1970).

Moore, C. E., Nat. Bur. Stand. (U.S.), Spec. Publ. 306, Sec. 4, 55 pp. (1969).

Moore, C. E., Atomic Energy Levels, Nat. Stand. Ref. Data Ser., Nat. Bur. Stand. (U.S.), 35, Vol. I, 359 pp., Vol. II, 259 pp., Vol. III, 282 pp. (1971). [Reprint of NBS Circular 467, which originally appeared in 1949 (Vol. I), 1952 (Vol. II), and 1958 (Vol. III).]

Nielson, C. W., and Koster, G. F., Spectroscopic Coefficients for the $p^{\prime \prime}, d^{n}$,

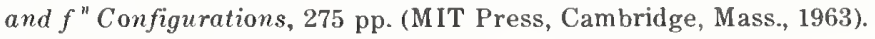

Racah, G., Phys. Rev. 61, 537 (1942a).

Racah, G., Phys. Rev. 62, 438 (1942b).

Racah, G., Phys. Rev. 63, 367 (1943).

Racah, G., Phys. Rev. 76, 1352 (1949).

Racah, G., in Transactions of the Joint Commission for Spectroscopy, J. Opt. Soc. Am. 50, 408 (1960).

Russell, H. N., Shenstone, A. G., and Turner, L. A., Phys. Rev. 33, 900 (1929).

van den Bosch, J. C., in Encyclopedia of Physics, S. Flügge, Ed., Vol. 28, 448 pp. (Springer-Verlag, Berlin, 1957).

White, H. E., Introduction to Atom ic Spectra, 457 pp. (McGraw-Hill Book Co., New York, N.Y., 1934).

Wybourne, B. G., Spectroscopic Properties of Rare Earths, 236 pp. (John Wiley \& Sons, New York, N.Y., 1965). 


\section{Tables for the Introduction}

TABLE 1. Allowed terms for $L S$ coupling of equivalent electrons ${ }^{\text {a }}$

\begin{tabular}{|c|c|c|c|c|c|c|c|c|c|c|c|c|c|c|c|c|}
\hline Config. & \multicolumn{16}{|c|}{ Allowed terms } \\
\hline$s$ & ${ }^{2} \mathrm{~S}$ & & & & & & & & & & & & & & & \\
\hline$s^{2}$ & ${ }^{1} \mathrm{~S}$ & & & & & & & & & & & & & & & \\
\hline$p$ and $p^{5}$ & ${ }^{2} \mathrm{P}^{\circ}$ & & & & & & & & & & & & & & & \\
\hline$p^{2}$ and $p^{4}$ & ${ }^{3} \mathrm{P}$ & ${ }^{1} \mathrm{D}$ & & is & & & & & & & & & & & & \\
\hline$p^{3}$ & ${ }^{4} \mathrm{~S}^{\circ}$ & ${ }^{2} \mathrm{D}^{\circ}$ & & $\mathrm{P}^{\circ}$ & & & & & & & & & & & & \\
\hline$p^{6}$ & ${ }^{1} \mathrm{~S}$ & & & & & & & & & & & & & & & \\
\hline$d$ and $d^{9}$ & ${ }^{2} \mathrm{D}$ & 1 & (10) & & & & & & & & & & & & & \\
\hline \multirow[t]{2}{*}{$d^{2}$ and $d^{8}$} & ${ }^{3} \mathbf{F}$ & 2 & (11) & ${ }^{1} \mathrm{G}$ & 2 & $(20)$ & ${ }^{1} \mathrm{~S}$ & 0 & $(00)$ & & & & & & & \\
\hline & ${ }^{3} \mathrm{P}$ & 2 & (11) & ${ }^{1} \mathrm{D}$ & 2 & $(20)$ & & & & & & & & & & \\
\hline \multirow[t]{2}{*}{$d^{3}$ and $d^{7}$} & ${ }^{4} \mathrm{~F}$ & 3 & (11) & ${ }^{2} \mathrm{H}$ & 3 & $(21)$ & ${ }^{2} \mathrm{~F}$ & 3 & $(21)$ & ${ }^{2} \mathrm{D} 2$ & 3 & $(21)$ & & & & \\
\hline & ${ }^{4} \mathrm{P}$ & 3 & (11) & ${ }^{2} \mathrm{G}$ & 3 & (21) & ${ }^{2} \mathrm{D}$ & 1 & $(10)$ & ${ }^{2} \mathrm{P}$ & 3 & (21) & & & & \\
\hline \multirow{4}{*}{$d^{4}$ and $d^{6}$} & ${ }^{5} \mathrm{D}$ & 4 & (10) & ${ }^{3} \mathrm{~F} 2$ & 4 & (21) & ${ }^{1} \mathrm{I}$ & 4 & $(22)$ & ${ }^{1} \mathrm{D} 1$ & 2 & $(20)$ & & & & \\
\hline & ${ }^{3} \mathrm{H}$ & 4 & (21) & ${ }^{3} \mathrm{D}$ & 4 & (21) & ${ }^{1} \mathrm{G}$ & G1 & (20) & ${ }^{1} \mathrm{D} 2$ & 4 & (22) & & & & \\
\hline & ${ }^{3} \mathrm{G}$ & 4 & (21) & ${ }^{3} \mathrm{P} 1$ & 2 & (11) & ${ }^{1} \mathrm{G}$ & G2 & (22) & ${ }^{1} \mathrm{~S} 1$ & 0 & (00) & & & & \\
\hline & ${ }^{3} \mathrm{~F} 1$ & 2 & (11) & ${ }^{3} \mathrm{P} 2$ & 4 & (21) & ${ }^{1} \mathrm{~F}$ & 4 & (22) & ${ }^{1} \mathrm{~S} 2$ & 4 & (22) & & & & \\
\hline \multirow[t]{4}{*}{$d^{5}$} & ${ }^{6} \mathrm{~S}$ & 5 & $(00)$ & ${ }^{4} \mathrm{P}$ & 3 & (11) & ${ }^{2} \mathrm{G}$ & 32 & (22) & ${ }^{2} \mathrm{D} 2$ & 3 & (21) & & & & \\
\hline & ${ }^{4} \mathrm{G}$ & 5 & (20) & ${ }^{2} \mathbf{I}$ & 5 & (22) & ${ }^{2} \mathrm{~F}$ & 81 & (21) & ${ }^{2} \mathrm{D} 3$ & 5 & (22) & & & & \\
\hline & ${ }^{4} \mathrm{~F}$ & 3 & (11) & ${ }^{2} \mathrm{H}$ & 3 & (21) & ${ }^{2} \mathrm{~F}$ & 2 & (22) & ${ }^{2} \mathrm{P}$ & 3 & (21) & & & & \\
\hline & ${ }^{4} \mathrm{D}$ & 5 & (20) & ${ }^{2} \mathrm{G} 1$ & 3 & (21) & ${ }^{2} \mathrm{D}$ & $\mathrm{D} 1$ & (10) & ${ }^{2} S$ & 5 & (22) & & & & \\
\hline$d^{10}$ & ${ }^{1} \mathrm{~S}$ & & & & & & & & & & & & & & & \\
\hline$f$ and $f^{13}$ & ${ }^{2} \mathrm{~F}^{\circ}$ & 1 & $(100)$ & (10) & & & & & & & & & & & & \\
\hline \multirow[t]{2}{*}{$f^{2}$ and $f^{12}$} & ${ }^{3} \mathrm{H}$ & 2 & (110) & (11) & ${ }^{3} \mathrm{P}$ & 2 & (110) & (11) & ${ }^{1} \mathrm{G}$ & 2 & $(200)$ & $(20)$ & ${ }^{1} \mathrm{~S}$ & 0 & $(000)$ & $(00)$ \\
\hline & ${ }^{3} \mathrm{~F}$ & 2 & (110) & (10) & ${ }^{1} \mathrm{I}$ & 2 & $(200)$ & (20) & ${ }^{1} \mathrm{D}$ & 2 & (200) & $(20)$ & & & & \\
\hline \multirow[t]{5}{*}{$f^{3}$ and $f^{11}$} & ${ }^{4} \mathrm{I}^{\circ}$ & 3 & (111) & (20) & ${ }^{2} \mathrm{~L}^{\circ}$ & 3 & (210) & (21) & ${ }^{2} \mathrm{G}^{\circ} 1$ & 3 & $(210)$ & $(20)$ & ${ }^{2} \mathrm{D}^{\circ} 2$ & 3 & $(210)$ & $(21)$ \\
\hline & ${ }^{4} \mathrm{G}^{\circ}$ & 3 & (111) & (20) & ${ }^{2} \mathrm{~K}^{\circ}$ & 3 & $(210)$ & (21) & ${ }^{2} \mathrm{G}^{\circ} 2$ & 3 & (210) & (21) & ${ }^{2} \mathrm{P}^{\circ}$ & 3 & $(210)$ & (11) \\
\hline & ${ }^{4} \mathrm{~F}^{\circ}$ & 3 & (111) & (10) & ${ }^{2} \mathrm{I}^{\circ}$ & 3 & $(210)$ & (20) & ${ }^{2} \mathrm{~F}^{\circ} 1$ & 1 & $(100)$ & $(10)$ & & & & \\
\hline & ${ }^{4} \mathrm{D}^{\circ}$ & 3 & (111) & (20) & ${ }^{2} \mathrm{H}^{\circ} \mathbf{1}$ & 3 & $(210)$ & (11) & ${ }^{2} \mathbf{F}^{\circ} 2$ & 3 & (210) & (21) & & & & \\
\hline & ${ }^{4} \mathrm{~S}^{\circ}$ & 3 & (111) & $(00)$ & ${ }^{2} \mathrm{H}^{\circ} 2$ & 3 & $(210)$ & (21) & ${ }^{2} \mathrm{D}^{\circ} 1$ & 3 & (210) & (20) & & & & \\
\hline \multirow[t]{12}{*}{$f^{4}$ and $f^{10}$} & ${ }^{5} \mathrm{I}$ & 4 & (111) & $(20)$ & ${ }^{3} \mathrm{H} 2$ & 4 & (211) & (11) & ${ }^{3} \mathrm{P} 1$ & 2 & (110) & (11) & ${ }^{1} \mathrm{G} 1$ & 2 & $(200)$ & (20) \\
\hline & ${ }^{5} \mathrm{G}$ & 4 & (111) & (20) & ${ }^{3} \mathrm{H} 3$ & 4 & (211) & (21) & ${ }^{3} \mathrm{P} 2$ & 4 & (211) & (11) & ${ }^{1} \mathrm{G} 2$ & 4 & (220) & (20) \\
\hline & ${ }^{5} \mathrm{~F}$ & 4 & (111) & (10) & ${ }^{3} \mathrm{H} 4$ & 4 & (211) & (30) & ${ }^{3} \mathrm{P} 3$ & 4 & (211) & (30) & ${ }^{1} \mathrm{G} 3$ & 4 & (220) & (21) \\
\hline & ${ }^{5} \mathrm{D}$ & 4 & (111) & (20) & ${ }^{3} \mathrm{G} 1$ & 4 & (211) & (20) & ${ }^{1} \mathrm{~N}$ & 4 & (220) & (22) & ${ }^{1} \mathrm{G} 4$ & 4 & (220) & (22) \\
\hline & ${ }^{5} \mathrm{~S}$ & 4 & (111) & $(00)$ & ${ }^{3} \mathrm{G} 2$ & 4 & (211) & (21) & ${ }^{1} \mathrm{~L} 1$ & 4 & (220) & (21) & ${ }^{1} \mathrm{~F}$ & 4 & $(220)$ & (21) \\
\hline & ${ }^{3} \mathrm{M}$ & 4 & (211) & $(30)$ & ${ }^{3} \mathrm{G} 3$ & 4 & (211) & (30) & ${ }^{1} \mathrm{~L} 2$ & 4 & (220) & (22) & ${ }^{1} \mathrm{D} 1$ & 2 & $(200)$ & (20) \\
\hline & ${ }^{3} \mathrm{~L}$ & 4 & (211) & (21) & ${ }^{3} \mathrm{~F} 1$ & 2 & (110) & (10) & ${ }^{1} \mathrm{~K}$ & 4 & $(220)$ & (21) & ${ }^{1} \mathrm{D} 2$ & 4 & $(220)$ & (20) \\
\hline & ${ }^{3} \mathrm{~K} 1$ & 4 & (211) & (21) & ${ }^{3} \mathrm{~F} 2$ & 4 & (211) & (10) & ${ }^{1} \mathrm{I} 1$ & 2 & $(200)$ & (20) & ${ }^{1} \mathrm{D} 3$ & 4 & $(220)$ & (21) \\
\hline & ${ }^{3} \mathrm{~K} 2$ & 4 & (211) & (30) & ${ }^{3} \mathrm{~F} 3$ & 4 & (211) & (21) & ${ }^{1} \mathrm{I} 2$ & 4 & (220) & (20) & ${ }^{1} \mathrm{D} 4$ & 4 & $(220)$ & (22) \\
\hline & ${ }^{3} \mathrm{I} 1$ & 4 & (211) & (20) & ${ }^{3} \mathrm{~F} 4$ & 4 & (211) & (30) & ${ }^{1} \mathrm{I} 3$ & 4 & (220) & (22) & ${ }^{1} \mathrm{~S} 1$ & 0 & $(000)$ & $(00)$ \\
\hline & ${ }^{3} \mathrm{I} 2$ & 4 & (211) & (30) & ${ }^{3} \mathrm{D} 1$ & 4 & (211) & (20) & ${ }^{1} \mathrm{H} 1$ & 4 & (220) & (21) & ${ }^{1} \mathrm{~S} 2$ & 4 & $(220)$ & (22) \\
\hline & ${ }^{3} \mathrm{H} 1$ & 2 & (110) & (11) & ${ }^{3} \mathrm{D} 2$ & 4 & (211) & (21) & ${ }^{1} \mathrm{H} 2$ & 4 & (220) & (22) & & & & \\
\hline
\end{tabular}


TABLE 1. Allowed terms for $L S$ coupling of equivalent electrons ${ }^{\text {a }}$ - Continued

\begin{tabular}{|c|c|c|c|c|c|c|c|c|c|c|c|c|c|c|c|c|}
\hline \multirow{2}{*}{$\frac{\text { Config. }}{f^{5} \text { and } f^{9}}$} & \multicolumn{16}{|c|}{ Allowed terms } \\
\hline & ${ }^{6} \mathrm{H}^{\circ}$ & 5 & (110) & (11) & ${ }^{4} \mathrm{~F}^{\circ} 3$ & 5 & (211) & (21) & ${ }^{2} \mathrm{~K}^{\circ} 5$ & 5 & $(221)$ & (31)B & ${ }^{2} \mathbf{F}^{\circ} 1$ & 1 & $(100)$ & (10) \\
\hline & ${ }^{6} \mathrm{~F}^{\circ}$ & 5 & (110) & (10) & ${ }^{4} \mathrm{~F}^{\circ} 4$ & 5 & (211) & (30) & ${ }^{2} I^{\circ} 1$ & 3 & $(210)$. & $(20)$ & ${ }^{2} \mathrm{~F}^{\circ} 2$ & 3 & $(210)$ & (21) \\
\hline & ${ }^{6} \mathrm{P}^{\circ}$ & 5 & $(110)$ & (11) & ${ }^{4} \mathrm{D}^{\circ} 1$ & 3 & (111) & (20) & ${ }^{2} \mathbf{I}^{\circ} 2$ & 5 & $(221)$ & (20) & ${ }^{2} \mathrm{~F}^{\circ} 3$ & 5 & (221) & (10) \\
\hline & ${ }^{4} \mathrm{M}^{\circ}$ & 5 & (211) & (30) & ${ }^{4} \mathrm{D}^{\circ} 2$ & 5 & (211) & $(20)$ & ${ }^{2} I^{\circ} 3$ & 5 & (221) & $(30)$ & ${ }^{2} \mathrm{~F}^{\circ} 4$ & 5 & (221) & (21) \\
\hline & ${ }^{4} \mathrm{~L}^{\circ}$ & 5 & (211) & (21) & ${ }^{4} \mathrm{D}^{\circ} 3$ & 5 & (211) & (21) & ${ }^{2} I^{\circ} 4$ & 5 & $(221)$ & (31)A & ${ }^{2} \mathbf{F}^{\circ} 5$ & 5 & (221) & $(30)$ \\
\hline & ${ }^{4} \mathrm{~K}^{\circ} 1$ & 5 & (211) & (21) & ${ }^{4} \mathrm{P}^{\circ} 1$ & 5 & (211) & (11) & ${ }^{2} I^{\circ} 5$ & 5 & (221) & $(31) \mathrm{B}$ & ${ }^{2} \mathrm{~F}^{\circ} 6$ & 5 & (221) & (31)A \\
\hline & ${ }^{4} \mathrm{~K}^{\circ} 2$ & 5 & (211) & (30) & ${ }^{4} \mathrm{P}^{\circ} 2$ & 5 & (211) & $(30)$ & ${ }^{2} \mathrm{H}^{\circ} 1$ & 3 & $(210)$ & (11) & ${ }^{2} \mathrm{~F}^{\circ} 7$ & 5 & (221) & (31)B \\
\hline & ${ }^{4} I^{\circ} 1$ & 3 & (111) & (20) & ${ }^{4} \mathrm{~S}^{\circ}$ & 3 & (111) & $(00)$ & ${ }^{2} \mathrm{H}^{\circ} 2$ & 3 & $(210)$ & (21) & ${ }^{2} \mathrm{D}^{\circ} 1$ & 3 & (210) & $(20)$ \\
\hline & ${ }^{4} \mathrm{I}^{\circ} 2$ & 5 & (211) & (20) & ${ }^{2} \mathrm{O}^{\circ}$ & 5 & (221) & (31) & ${ }^{2} \mathrm{H}^{\circ} 3$ & 5 & (221) & (11) & ${ }^{2} \mathrm{D}^{\circ} 2$ & 3 & $(210)$ & $(21)$ \\
\hline & ${ }^{4} I^{\circ} 3$ & 5 & (211) & (30) & ${ }^{2} \mathrm{~N}^{\circ}$ & 5 & (221) & (31) & ${ }^{2} \mathrm{H}^{\circ} 4$ & 5 & (221) & (21) & ${ }^{2} \mathrm{D}^{\circ} 3$ & 5 & (221) & $(20)$ \\
\hline & ${ }^{4} \mathrm{H}^{\circ} 1$ & 5 & (211) & (11) & ${ }^{2} \mathrm{M}^{\circ} 1$ & 5 & (221) & (30) & ${ }^{2} \mathrm{H}^{\circ} 5$ & 5 & (221) & (30) & ${ }^{2} \mathrm{D}^{\circ} 4$ & 5 & (221) & (21) \\
\hline & ${ }^{4} \mathrm{H}^{\circ} 2$ & 5 & (211) & (21) & ${ }^{2} \mathrm{M}^{\circ} 2$ & 5 & (221) & (31) & ${ }^{2} \mathrm{H}^{\circ} 6$ & 5 & $(221)$ & (31)A & ${ }^{2} \mathrm{D}^{\circ} 5$ & 5 & (221) & (31) \\
\hline & ${ }^{4} \mathrm{H}^{\circ} 3$ & 5 & (211) & (30) & ${ }^{2} \mathrm{~L}^{\circ} 1$ & 3 & (210) & (21) & ${ }^{2} \mathrm{H}^{\circ} 7$ & 5 & (221) & (31)B & ${ }^{2} \mathrm{P}^{\circ} 1$ & 3 & (210) & (11) \\
\hline & ${ }^{4} \mathrm{G}^{\circ} 1$ & 3 & (111) & (20) & ${ }^{2} \mathrm{~L}^{\circ} 2$ & 5 & (221) & (21) & ${ }^{2} \mathrm{G}^{\circ} 1$ & 3 & $(210)$ & $(20)$ & ${ }^{2} \mathbf{P}^{\circ} 2$ & 5 & (221) & (11) \\
\hline & ${ }^{4} \mathrm{G}^{\circ} 2$ & 5 & (211) & (20) & ${ }^{2} L^{\circ} 3$ & 5 & (221) & (31) & ${ }^{2} \mathrm{G}^{\circ} 2$ & 3 & $(210)$ & (21) & ${ }^{2} \mathrm{P}^{\circ} 3$ & 5 & (221) & (30) \\
\hline & ${ }^{4} \mathrm{G}^{\circ} 3$ & 5 & (211) & (21) & ${ }^{2} \mathrm{~K}^{\circ} 1$ & 3 & (210) & (21) & ${ }^{2} \mathrm{G}^{\circ} 3$ & 5 & (221) & (20) & ${ }^{2} \mathrm{P}^{\circ} 4$ & 5 & (221) & (31) \\
\hline & ${ }^{4} \mathrm{G}^{\circ} 4$ & 5 & (211) & (30) & ${ }^{2} \mathrm{~K}^{\circ} 2$ & 5 & (221) & (21) & ${ }^{2} \mathrm{G}^{\circ} 4$ & 5 & (221) & (21) & & & & \\
\hline & ${ }^{4} F^{\circ} 1$ & 3 & (111) & (10) & ${ }^{2} \mathrm{~K}^{\circ} 3$ & 5 & (221) & $(30)$ & ${ }^{2} \mathrm{G}^{\circ} 5$ & 5 & $(221)$ & (30) & & & & \\
\hline & ${ }^{4} \mathbf{F}^{\circ} 2$ & 5 & (211) & (10) & ${ }^{2} \mathrm{~K}^{\circ} 4$ & 5 & (221) & (31)A & ${ }^{2} \mathrm{G}^{\circ} 6$ & 5 & (221) & (31) & & & & \\
\hline \multirow[t]{30}{*}{$f^{6}$ and $f^{8}$} & ${ }^{7} \mathrm{~F}$ & 6 & $(100)$ & (10) & ${ }^{3} \mathrm{~K} 6$ & 6 & $(221)$ & (31)B & ${ }^{3} \mathrm{~F} 8$ & 6 & $(221)$ & (31)A & ${ }^{1} \mathrm{I} 6$ & 6 & $(222)$ & $(40) \mathrm{A}$ \\
\hline & ${ }^{5} \mathrm{~L}$ & 6 & (210) & (21) & ${ }^{3} \mathrm{I} 1$ & 4 & (211) & $(20)$ & ${ }^{3} \mathrm{~F} 9$ & 6 & $(221)$ & (31)B & ${ }^{1} \mathrm{I} 7$ & 6 & (222) & $(40) \mathrm{B}$ \\
\hline & ${ }^{5} \mathrm{~K}$ & 6 & $(210)$ & (21) & ${ }^{3} \mathrm{I} 2$ & 4 & (211) & $(30)$ & ${ }^{3} \mathrm{D} 1$ & 4 & $(211)$ & $(20)$ & ${ }^{1} \mathrm{H} 1$ & 4 & $(220)$ & (21) \\
\hline & ${ }^{5} \mathrm{I} 1$ & 4 & (111) & $(20)$ & ${ }^{3} \mathrm{I} 3$ & 6 & $(221)$ & (20) & ${ }^{3} \mathrm{D} 2$ & 4 & $(211)$ & (21) & ${ }^{1} \mathrm{H} 2$ & 4 & $(220)$ & (22) \\
\hline & ${ }^{5} \mathrm{I} 2$ & 6 & $(210)$ & $(20)$ & ${ }^{3} \mathrm{I} 4$ & 6 & (221) & (30) & ${ }^{3} \mathrm{D} 3$ & 6 & $(221)$ & (20) & ${ }^{1} \mathrm{H} 3$ & 6 & $(222)$ & (30) \\
\hline & ${ }^{5} \mathrm{H} 1$ & 6 & $(210)$ & (11) & ${ }^{3}$ I5 & 6 & (221) & (31)A & ${ }^{3} \mathrm{D} 4$ & 6 & (221) & (21) & ${ }^{1} \mathrm{H} 4$ & 6 & $(222)$ & $(40)$ \\
\hline & ${ }^{5} \mathrm{H} 2$ & 6 & $(210)$ & $(21)$ & ${ }^{3} \mathrm{I} 6$ & 6 & (221) & (31)B & ${ }^{3} \mathrm{D} 5$ & 6 & $(221)$ & (31) & ${ }^{1} \mathrm{G} 1$ & 2 & $(200)$ & $(20)$ \\
\hline & ${ }^{5} \mathrm{G} 1$ & 4 & (111) & (20) & ${ }^{3} \mathrm{H} 1$ & 2 & (110) & (11) & ${ }^{3} \mathrm{P} 1$ & 2 & $(110)$ & (11) & ${ }^{1} \mathrm{G} 2$ & 4 & $(220)$ & (20) \\
\hline & ${ }^{5} \mathrm{G} 2$ & 6 & $(210)$ & (20) & ${ }^{3} \mathrm{H} 2$ & 4 & (211) & (11) & ${ }^{3} \mathrm{P} 2$ & 4 & $(211)^{\circ}$ & (11) & ${ }^{1} \mathrm{G} 3$ & 4 & $(220)$ & (21) \\
\hline & ${ }^{5} \mathrm{G} 3$ & 6 & $(210)$ & (21) & ${ }^{3} \mathrm{H} 3$ & 4 & (211) & (21) & ${ }^{3} \mathrm{P} 3$ & 4 & $(211)$ & (30) & ${ }^{1} \mathrm{G} 4$ & 4 & $(220)$ & (22) \\
\hline & ${ }^{5} \mathrm{~F} 1$ & 4 & (111) & (10) & ${ }^{3} \mathrm{H} 4$ & 4 & (211) & (30) & ${ }^{3} \mathrm{P} 4$ & 6 & (221) & (11) & ${ }^{1} \mathrm{G} 5$ & 6 & $(222)$ & (20) \\
\hline & ${ }^{5} \mathrm{~F} 2$ & 6 & $(210)$ & (21) & ${ }^{3} \mathrm{H} 5$ & 6 & (221) & (11) & ${ }^{3} \mathrm{P} 5$ & 6 & $(221)$ & $(30)$ & ${ }^{1} \mathrm{G} 6$ & 6 & $(222)$ & $(30)$ \\
\hline & ${ }^{5} \mathrm{D} 1$ & 4 & (111) & (20) & ${ }^{3} \mathrm{H} 6$ & 6 & (221) & (21) & ${ }^{3} \mathrm{P} 6$ & 6 & $(221)$ & $(31)$ & ${ }^{1} \mathrm{G} 7$ & 6 & $(222)$ & $(40) \mathrm{A}$ \\
\hline & ${ }^{5} \mathrm{D} 2$ & 6 & (210) & (20) & ${ }^{3} \mathrm{H} 7$ & 6 & (221) & $(30)$ & ${ }^{1} \mathrm{Q}$ & 6 & (222) & $(40)$ & ${ }^{1} \mathrm{G} 8$ & 6 & $(222)$ & $(40) \mathrm{B}$ \\
\hline & ${ }^{5} \mathrm{D} 3$ & 6 & $(210)$ & (21) & ${ }^{3} \mathrm{H} 8$ & 6 & (221) & (31)A & ${ }^{1} \mathrm{~N} 1$ & 4 & $(220)$ & $(22)$ & ${ }^{1} \mathrm{~F} 1$ & 4 & $(220)$ & (21) \\
\hline & ${ }^{5} \mathrm{P}$ & 6 & (210) & (11) & ${ }^{3} \mathrm{H} 9$ & 6 & (221) & $(31) \mathrm{B}$ & ${ }^{1} \mathrm{~N} 2$ & 6 & (222) & $(40)$ & ${ }^{1} \mathrm{~F} 2$ & 6 & $(222)$ & (10) \\
\hline & ${ }^{5} \mathrm{~S}$ & 4 & (111) & $(00)$ & ${ }^{3} \mathrm{G} 1$ & 4 & (211) & (20) & ${ }^{1} \mathrm{M} 1$ & 6 & (222) & (30) & ${ }^{1} \mathrm{~F} 3$ & 6 & $(222)$ & (30) \\
\hline & ${ }^{3} \mathrm{O}$ & 6 & (221) & (31) & ${ }^{3} \mathrm{G} 2$ & 4 & (211) & (21) & ${ }^{1} \mathrm{M} 2$ & 6 & (222) & $(40)$ & ${ }^{1} \mathrm{~F} 4$ & 6 & $(222)$ & $(40)$ \\
\hline & ${ }^{3} \mathrm{~N}$ & 6 & (221) & (31) & ${ }^{3} \mathrm{G} 3$ & 4 & (211) & (30) & ${ }^{1} \mathrm{~L} 1$ & 4 & $(220)$ & (21) & ${ }^{1} \mathrm{D} 1$ & 2 & $(200)$ & $(20)$ \\
\hline & ${ }^{3} \mathrm{M} 1$ & 4 & $(211)$ & $(30)$ & ${ }^{3} \mathrm{G} 4$ & 6 & (221) & (20) & ${ }^{1} \mathrm{~L} 2$ & 4 & $(220)$ & $(22)$ & ${ }^{1} \mathrm{D} 2$ & 4 & $(220)$ & $(20)$ \\
\hline & ${ }^{3} \mathrm{M} 2$ & 6 & $(221)$ & (30) & ${ }^{3} \mathrm{G} 5$ & 6 & $(221)$ & (21) & ${ }^{1} \mathrm{~L} 3$ & 6 & $(222)$ & (40)A & ${ }^{1} \mathrm{D} 3$ & 4 & $(220)$ & (21) \\
\hline & ${ }^{3} \mathrm{M} 3$ & 6 & (221) & (31) & ${ }^{3} \mathrm{G} 6$ & 6 & (221) & (30) & ${ }^{1} \mathrm{~L} 4$ & 6 & $(222)$ & $(40) \mathrm{B}$ & ${ }^{1} \mathrm{D} 4$ & 4 & $(220)$ & (22) \\
\hline & ${ }^{3} \mathrm{~L} 1$ & 4 & (211) & (21) & ${ }^{3} \mathrm{G} 7$ & 6 & (221) & (31) & ${ }^{1} \mathrm{~K} 1$ & 4 & $(220)$ & (21) & ${ }^{1} \mathrm{D} 5$ & 6 & (222) & (20) \\
\hline & ${ }^{3} \mathrm{~L} 2$ & 6 & (221) & (21) & ${ }^{3} \mathrm{~F} 1$ & 2 & (110) & (10) & ${ }^{1} \mathrm{~K} 2$ & 6 & (222) & $(30)$ & ${ }^{1} \mathrm{D} 6$ & 6 & $(222)$ & (40) \\
\hline & ${ }^{3} \mathrm{~L} 3$ & 6 & (221) & (31) & ${ }^{3} \mathrm{~F} 2$ & 4 & (211) & (10) & ${ }^{1} \mathrm{~K} 3$ & 6 & $(222)$ & $(40)$ & ${ }^{1} \mathrm{P}$ & 6 & $(222)$ & (30) \\
\hline & ${ }^{3} \mathrm{~K} 1$ & 4 & (211) & (21) & ${ }^{3} \mathrm{~F} 3$ & 4 & (211) & (21) & ${ }^{1} \mathrm{I} 1$ & 2 & $(200)$ & (20) & ${ }^{1} \mathrm{~S} 1$ & 0 & $(000)$ & $(00)$ \\
\hline & ${ }^{3} \mathrm{~K} 2$ & 4 & (211) & (30) & ${ }^{3} \mathrm{~F} 4$ & 4 & (211) & (30) & ${ }^{1} \mathrm{I} 2$ & 4 & $(220)$ & (20) & ${ }^{1} \mathrm{~S} 2$ & 4 & $(220)$ & (22) \\
\hline & ${ }^{3} \mathrm{~K} 3$ & 6 & (221) & (21) & ${ }^{3} \mathrm{~F} 5$ & 6 & (221) & (10) & ${ }^{1} \mathrm{I} 3$ & 4 & $(220)$ & (22) & ${ }^{1} \mathrm{~S} 3$ & 6 & (222) & $(00)$ \\
\hline & ${ }^{3} \mathrm{~K} 4$ & 6 & $(221)$ & $(30)$ & ${ }^{3} \mathrm{~F} 6$ & 6 & (221) & (21) & ${ }^{1} \mathbf{I} 4$ & 6 & (222) & (20) & ${ }^{1} \mathrm{~S} 4$ & 6 & $(222)$ & $(40)$ \\
\hline & ${ }^{3} \mathrm{~K} 5$ & 6 & (221) & (31)A & ${ }^{3} \mathrm{~F} 7$ & 6 & (221) & (30) & ${ }^{1} \mathrm{I} 5$ & 6 & $(222)$ & $(30)$ & & & & \\
\hline
\end{tabular}


TABLE 1. Allowed terms for LS coupling of equivalent electrons a - Continued

\begin{tabular}{|c|c|c|c|c|c|c|c|c|c|c|c|c|c|c|c|c|}
\hline \multirow{2}{*}{ Config. } & \multicolumn{16}{|c|}{ Allowed terms } \\
\hline & ${ }^{8} \mathrm{~S}^{\circ}$ & 7 & $(000)$ & $(00)$ & ${ }^{4} \mathrm{G}^{\circ} 6$ & 7 & $(220)$ & (21) & ${ }^{2} \mathrm{~K}^{\circ} 1$ & 3 & $(210)$ & $(21)$ & ${ }^{2} \mathrm{G}^{\circ} 6$ & 5 & $(221)$ & (31) \\
\hline & ${ }^{6} \mathbf{I}^{\circ}$ & 7 & $(200)$ & $(20)$ & ${ }^{4} \mathrm{G}^{\circ} 7$ & 7 & $(220)$ & (22) & ${ }^{2} \mathrm{~K}^{\circ} 2$ & 5 & (221) & (21) & ${ }^{2} \mathrm{G}^{\circ} 7$ & 7 & $(222)$ & (20) \\
\hline & ${ }^{6} \mathrm{H}^{\circ}$ & 5 & (110) & (11) & ${ }^{4} \mathrm{~F}^{\circ} 1$ & 3 & (111) & (10) & ${ }^{2} \mathrm{~K}^{\circ} 3$ & 5 & (221) & $(30)$ & ${ }^{2} \mathrm{G}^{\circ} 8$ & 7 & $(222)$ & (30) \\
\hline & ${ }^{6} \mathrm{G}^{\circ}$ & 7 & $(200)$ & $(20)$ & ${ }^{4} \mathrm{~F}^{\circ} 2$ & 5 & (211) & $(10)$ & ${ }^{2} \mathrm{~K}^{\circ} 4$ & 5 & $(221)$ & (31)A & ${ }^{2} \mathrm{G}^{\circ} 9$ & 7 & $(222)$ & (40)A \\
\hline & ${ }^{6} \mathrm{~F}^{\circ}$ & 5 & (110) & (10) & ${ }^{4} \mathrm{~F}^{\circ} 3$ & 5 & (211) & (21) & ${ }^{2} \mathrm{~K}^{\circ} 5$ & 5 & (221) & (31)B & ${ }^{2} \mathrm{G}^{\circ} 10$ & 7 & $(222)$ & $(40) \mathrm{B}$ \\
\hline & ${ }^{6} \mathrm{D}^{\circ}$ & 7 & $(200)$ & $(20)$ & ${ }^{4} \mathrm{~F}^{\circ} 4$ & 5 & (211) & (30) & ${ }^{2} \mathrm{~K}^{\circ} 6$ & 7 & $(222)$ & $(30)$ & ${ }^{2} F^{\circ} 1$ & 1 & (100) & $(10)$ \\
\hline & ${ }^{6} \mathrm{P}^{\circ}$ & 5 & (110) & (11) & ${ }^{4} \mathrm{~F}^{\circ} 5$ & 7 & (220) & (21) & ${ }^{2} \mathrm{~K}^{\circ} 7$ & 7 & $(222)$ & $(40)$ & ${ }^{2} \mathbf{F}^{\circ} 2$ & 3 & $(210)$ & (21) \\
\hline & ${ }^{4} \mathrm{~N}^{\circ}$ & 7 & $(220)$ & (22) & ${ }^{4} \mathrm{D}^{\circ} 1$ & 3 & (111) & (20) & ${ }^{2} \mathrm{I}^{\circ} 1$ & 3 & $(210)$ & (20) & ${ }^{2} \mathrm{~F}^{\circ} 3$ & 5 & $(221)$ & (10) \\
\hline & ${ }^{4} \mathrm{M}^{\circ}$ & 5 & (211) & (30) & ${ }^{4} \mathrm{D}^{\circ} 2$ & 5 & (211) & $(20)$ & ${ }^{2} I^{\circ} 2$ & 5 & (221) & (20) & ${ }^{2} \mathrm{~F}^{\circ} 4$ & 5 & (221) & (21) \\
\hline & ${ }^{4} \mathrm{~L}^{\circ} 1$ & 5 & (211) & (21) & ${ }^{4} \mathrm{D}^{\circ} 3$ & 5 & (211) & $(21)$ & ${ }^{2} I^{\circ} 3$ & 5 & (221) & $(30)$ & ${ }^{2} \mathrm{~F}^{\circ} 5$ & 5 & $(221)$ & $(30)$ \\
\hline & ${ }^{4} \mathrm{~L}^{\circ} 2$ & 7 & $(220)$ & (21) & ${ }^{4} \mathrm{D}^{\circ} 4$ & 7 & $(220)$ & (20) & ${ }^{2} I^{\circ} 4$ & 5 & (221) & (31)A & ${ }^{2} \mathrm{~F}^{\circ} 6$ & 5 & (221) & (31)A \\
\hline & ${ }^{4} \mathrm{~L}^{\circ} 3$ & 7 & $(220)$ & $(22)$ & ${ }^{4} \mathrm{D}^{\circ} 5$ & 7 & $(220)$ & (21) & ${ }^{2} I^{\circ} 5$ & 5 & (221) & (31)B & ${ }^{2} \mathrm{~F}^{\circ} 7$ & 5 & (221) & (31)B \\
\hline & ${ }^{4} \mathrm{~K}^{\circ} 1$ & 5 & (211) & (21) & ${ }^{4} \mathrm{D}^{\circ} 6$ & 7 & (220) & (22) & ${ }^{2} I^{\circ} 6$ & 7 & $(222)$ & $(20)$ & ${ }^{2} \mathrm{~F}^{\circ} 8$ & 7 & $(222)$ & $(10)$ \\
\hline & ${ }^{4} \mathrm{~K}^{\circ} 2$ & 5 & (211) & $(30)$ & ${ }^{4} \mathrm{P}^{\circ} 1$ & 5 & (211) & (11) & ${ }^{2} I^{\circ} 7$ & 7 & (222) & (30) & ${ }^{2} \mathrm{~F}^{\circ} 9$ & 7 & $(222)$ & (30) \\
\hline & ${ }^{4} \mathrm{~K}^{\circ} 3$ & 7 & $(220)$ & (21) & ${ }^{4} \mathrm{P}^{\circ} 2$ & 5 & (211) & (30) & ${ }^{2} I^{\circ} 8$ & 7 & $(222)$ & $(40) \mathrm{A}$ & ${ }^{2} \mathrm{~F}^{\circ} 10$ & 7 & (222) & $(40)$ \\
\hline & ${ }^{4} \mathrm{I}^{\circ} 1$ & 3 & (111) & (20) & ${ }^{4} \mathrm{~S}^{\circ} 1$ & 3 & (111) & $(00)$ & ${ }^{2} I^{\circ} 9$ & 7 & $(222)$ & $(40) \mathrm{B}$ & ${ }^{2} \mathrm{D}^{\circ} 1$ & 3 & $(210)$ & (20) \\
\hline & ${ }^{4} I^{\circ} 2$ & 5 & (211) & (20) & ${ }^{4} \mathrm{~S}^{\circ} 2$ & 7 & $(220)$ & (22) & ${ }^{2} \mathrm{H}^{\circ} 1$ & 3 & $(210)$ & (11) & ${ }^{2} \mathrm{D}^{\circ} 2$ & 3 & $(210)$ & (21) \\
\hline & ${ }^{4} I^{\circ} 3$ & 5 & (211) & (30) & ${ }^{2} Q^{\circ}$ & 7 & (222) & (40) & ${ }^{2} \mathrm{H}^{\circ} 2$ & 3 & $(210)$ & (21) & ${ }^{2} \mathrm{D}^{\circ} 3$ & 5 & (221) & (20) \\
\hline & ${ }^{4} I^{\circ} 4$ & 7 & $(220)$ & $(20)$ & ${ }^{2} \mathrm{O}^{\circ}$ & 5 & (221) & (31) & ${ }^{2} \mathrm{H}^{\circ} 3$ & 5 & $(221)$ & (11) & ${ }^{2} \mathrm{D}^{\circ} 4$ & 5 & $(221)$ & (21) \\
\hline & ${ }^{4} \mathbf{I}^{\circ} 5$ & 7 & $(220)$ & $(22)$ & ${ }^{2} \mathrm{~N}^{\circ} 1$ & 5 & (221) & (31) & ${ }^{2} \mathrm{H}^{\circ} 4$ & 5 & (221) & (21) & ${ }^{2} \mathrm{D}^{\circ} 5$ & 5 & (221) & (31) \\
\hline & ${ }^{4} \mathrm{H}^{\circ} 1$ & 5 & (211) & (11) & ${ }^{2} \mathrm{~N}^{\circ} 2$ & 7 & (222) & (40) & ${ }^{2} \mathrm{H}^{\circ} 5$ & 5 & (221) & $(30)$ & ${ }^{2} \mathrm{D}^{\circ} 6$ & 7 & $(222)$ & $(20)$ \\
\hline & ${ }^{4} \mathrm{H}^{\circ} 2$ & 5 & (211) & (21) & ${ }^{2} \mathrm{M}^{\circ} 1$ & 5 & $(221)$ & $(30)$ & ${ }^{2} \mathrm{H}^{\circ} 6$ & 5 & (221) & (31)A & ${ }^{2} \mathrm{D}^{\circ} 7$ & 7 & $(222)$ & $(40)$ \\
\hline & ${ }^{4} \mathrm{H}^{\circ} 3$ & 5 & (211) & $(30)$ & ${ }^{2} \mathbf{M}^{\circ} 2$ & 5 & (221) & (31) & ${ }^{2} \mathrm{H}^{\circ} 7$ & 5 & (221) & (31)B & ${ }^{2} \mathrm{P}^{\circ} 1$ & 3 & (210) & (11) \\
\hline & ${ }^{4} \mathrm{H}^{\circ} 4$ & 7 & $(220)$ & (21) & ${ }^{2} \mathrm{M}^{\circ} 3$ & 7 & (222) & (30) & ${ }^{2} \mathrm{H}^{\circ} 8$ & 7 & $(222)$ & $(30)$ & ${ }^{2} \mathrm{P}^{\circ} 2$ & 5 & (221) & (11) \\
\hline & ${ }^{4} \mathrm{H}^{\circ} 5$ & 7 & $(220)$ & (22) & ${ }^{2} \mathrm{M}^{\circ} 4$ & 7 & $(222)$ & $(40)$ & ${ }^{2} \mathrm{H}^{\circ} 9$ & 7 & $(222)$ & (40) & ${ }^{2} \mathrm{P}^{\circ} 3$ & 5 & $(221)$ & (30) \\
\hline & ${ }^{4} \mathrm{G}^{\circ} 1$ & 3 & (111) & (20) & ${ }^{2} \mathrm{~L}^{\circ} 1$ & 3 & $(210)$ & (21) & ${ }^{2} G^{\circ} 1$ & 3 & (210) & (20) & ${ }^{2} \mathrm{P}^{\circ} 4$ & 5 & (221) & (31) \\
\hline & ${ }^{4} \mathrm{G}^{\circ} 2$ & 5 & (211) & (20) & ${ }^{2} \mathrm{~L}^{\circ} 2$ & 5 & (221) & (21) & ${ }^{2} \mathrm{G}^{\circ} 2$ & 3 & (210) & (21) & ${ }^{2} \mathrm{P}^{\circ} 5$ & 7 & $(222)$ & (30) \\
\hline & ${ }^{4} \mathrm{G}^{\circ} 3$ & 5 & (211) & (21) & ${ }^{2} \mathrm{~L}^{\circ} 3$ & 5 & $(221)$ & (31) & ${ }^{2} \mathrm{G}^{\circ} 3$ & 5 & (221) & (20) & ${ }^{2} \mathrm{~S}^{\circ} 1$ & 7 & $(222)$ & $(00)$ \\
\hline & ${ }^{4} \mathrm{G}^{\circ} 4$ & 5 & (211) & $(30)$ & ${ }^{2} \mathrm{~L}^{\circ} 4$ & 7 & $(222)$ & $(40) \mathrm{A}$ & ${ }^{2} \mathrm{G}^{\circ} 4$ & 5 & (221) & (21) & ${ }^{2} \mathrm{~S}^{\circ} 2$ & 7 & $(222)$ & (40) \\
\hline & ${ }^{4} \mathrm{G}^{\circ} 5$ & 7 & $(220)$ & $(20)$ & ${ }^{2} \mathrm{~L}^{\circ} 5$ & 7 & $(222)$ & $(40) \mathrm{B}$ & ${ }^{2} G^{\circ} 5$ & 5 & (221) & (30) & & & & \\
\hline$f^{14}$ & ${ }^{1} \mathrm{~S}$ & & & & & & & & & & & & & & & \\
\hline
\end{tabular}

a From Nielson and Koster (1963), with some rearrangement. (By permission of the M. I. T. Press.) 
TABLE 2. Allowed $J$ values for $l_{j}^{N}$ equivalent electrons (jj coupling).

\begin{tabular}{l|l}
\hline \multicolumn{1}{c|}{$l_{j}^{N}$} & \multicolumn{1}{c}{ Allowed $J$ values } \\
\hline$l_{1 / 2}$ & 0 \\
$l_{1 / 2}^{2}$ & \\
$l_{3 / 2}$ and $l_{3 / 2}^{3}$ & 0,2 \\
$l_{3 / 2}^{2}$ & 0 \\
$l_{3 / 2}^{4}$ & $5 / 2$ \\
$l_{5 / 2}$ and $l_{5 / 2}^{5}$ & $0,2,4$ \\
$l_{5 / 2}^{2}$ and $l_{5 / 2}^{4}$ & $3 / 2,5 / 2,9 / 2$ \\
$l_{5 / 2}^{3}$ & 0 \\
$l_{5 / 2}^{6}$ & \\
$l_{7 / 2}^{6}$ and $l_{7 / 2}^{7}$ & $7 / 2$ \\
$l_{7 / 2}^{2}$ and $l_{7 / 2}^{6}$ & $0,2,4,6$ \\
$l_{7 / 2}^{3}$ and $l_{7 / 2}^{5}$ & $3 / 2,5 / 2,7 / 2,9 / 2,11 / 2,15 / 2$ \\
$l_{7 / 2}^{4}$ & $0,{ }_{2} 2,{ }_{4} 2,{ }_{2} 4,{ }_{4} 4,5,6,8$ \\
$l_{7 / 2}^{8}$ & 0 \\
\hline
\end{tabular}


TABLE 3. Corrected Landé $g$-values for terms of odd multiplicity

\begin{tabular}{|c|c|c|c|c|c|c|c|c|c|c|c|c|}
\hline \multirow{3}{*}{ Term } & \multicolumn{12}{|c|}{ Multiplicity } \\
\hline & \multicolumn{2}{|c|}{$\begin{array}{c}\text { Singlets } \\
1\end{array}$} & \multicolumn{2}{|c|}{$\begin{array}{c}\text { Triplets } \\
3\end{array}$} & \multicolumn{2}{|r|}{$\begin{array}{c}\text { Quintets } \\
5\end{array}$} & \multicolumn{2}{|r|}{$\begin{array}{c}\text { Septets } \\
7\end{array}$} & \multicolumn{2}{|r|}{$\begin{array}{c}\text { Nonets } \\
9\end{array}$} & \multicolumn{2}{|r|}{$\begin{array}{c}\text { Undecets } \\
11\end{array}$} \\
\hline & $J$ & $g$ & $J$ & $g$ & $J$ & $g$ & $J$ & $g$ & $J$ & $g$ & $J$ & $g$ \\
\hline $\mathrm{S}$ & 0 & $\longrightarrow$ & 1 & 2.002319 & 2 & 2.002319 & 3 & 2.002319 & 4 & 2.002319 & 5 & 2.002319 \\
\hline $\mathrm{P}$ & 1 & 1.000000 & $\begin{array}{l}2 \\
1 \\
0\end{array}$ & $\begin{array}{r}1.501160 \\
1.501160 \\
\end{array}$ & $\begin{array}{l}3 \\
2 \\
1\end{array}$ & $\begin{array}{l}1.668213 \\
1.835266 \\
2.503479\end{array}$ & $\begin{array}{l}4 \\
3 \\
2\end{array}$ & $\begin{array}{l}1.751739 \\
1.918793 \\
2.336426\end{array}$ & $\begin{array}{l}5 \\
4 \\
3\end{array}$ & $\begin{array}{l}1.801855 \\
1.952203 \\
2.252899\end{array}$ & $\begin{array}{l}6 \\
5 \\
4\end{array}$ & $\begin{array}{l}1.835266 \\
1.968909 \\
2.202783\end{array}$ \\
\hline $\mathrm{D}$ & 2 & 1.000000 & $\begin{array}{l}3 \\
2 \\
1\end{array}$ & $\begin{array}{l}1.334106 \\
1.167053 \\
0.198840 \\
0.498840\end{array}$ & $\begin{array}{l}4 \\
3 \\
2 \\
1 \\
0\end{array}$ & $\begin{array}{l}1.501160 \\
1.501160 \\
1.501160 \\
1.501160 \\
\end{array}$ & $\begin{array}{l}5 \\
4 \\
3 \\
2 \\
1\end{array}$ & $\begin{array}{l}1.601392 \\
1.651507 \\
1.751739 \\
2.002319 \\
3.004638\end{array}$ & $\begin{array}{l}6 \\
5 \\
4 \\
3 \\
2\end{array}$ & $\begin{array}{l}1.668213 \\
1.735034 \\
1.851971 \\
2.085846 \\
2.670532\end{array}$ & $\begin{array}{l}7 \\
6 \\
5 \\
4 \\
3\end{array}$ & $\begin{array}{l}1.715942 \\
1.787537 \\
1.902087 \\
2.102551 \\
2.503479\end{array}$ \\
\hline $\mathrm{F}$ & 3 & 1.000000 & $\begin{array}{l}4 \\
3 \\
2\end{array}$ & $\begin{array}{l}1.250580 \\
1.083527 \\
0.665894\end{array}$ & $\begin{array}{l}5 \\
4 \\
3 \\
2 \\
1\end{array}$ & $\begin{array}{r}1.400928 \\
1.350812 \\
1.250580 \\
1.000000 \\
-0.002318\end{array}$ & $\begin{array}{l}6 \\
5 \\
4 \\
3 \\
2 \\
1 \\
0\end{array}$ & $\begin{array}{l}1.501160 \\
1.501160 \\
1.501160 \\
1.501160 \\
1.501160 \\
1.501160 \\
\end{array}$ & $\begin{array}{l}7 \\
6 \\
5 \\
4 \\
3 \\
2 \\
1\end{array}$ & $\begin{array}{l}1.572754 \\
1.596619 \\
1.634802 \\
1.701623 \\
1.835266 \\
2.169372 \\
3.505798\end{array}$ & $\begin{array}{l}8 \\
7 \\
6 \\
5 \\
4 \\
3 \\
2\end{array}$ & $\begin{array}{l}1.626450 \\
1.662247 \\
1.715942 \\
1.801855 \\
1.952203 \\
2.252899 \\
3.004638\end{array}$ \\
\hline G & 4 & 1.000000 & $\begin{array}{l}5 \\
4 \\
3\end{array}$ & $\begin{array}{l}1.200464 \\
1.050116 \\
0.749420\end{array}$ & $\begin{array}{l}6 \\
5 \\
4 \\
3 \\
2\end{array}$ & $\begin{array}{l}1.334106 \\
1.267285 \\
1.150348 \\
0.916473 \\
0.331787\end{array}$ & $\begin{array}{l}7 \\
6 \\
5 \\
4 \\
3 \\
2 \\
1\end{array}$ & $\begin{array}{r}1.429565 \\
1.405701 \\
1.367517 \\
1.300696 \\
1.167053 \\
0.832947 \\
-0.503478\end{array}$ & $\begin{array}{l}8 \\
7 \\
6 \\
5 \\
4 \\
3 \\
2 \\
1 \\
0\end{array}$ & $\begin{array}{l}1.501160 \\
1.501160 \\
1.501160 \\
1.501160 \\
1.501160 \\
1.501160 \\
1.501160 \\
1.501160 \\
\end{array}$ & $\begin{array}{l}9 \\
8 \\
7 \\
6 \\
5 \\
4 \\
3 \\
2 \\
1\end{array}$ & $\begin{array}{l}1.556844 \\
1.570765 \\
1.590652 \\
1.620483 \\
1.668213 \\
1.751739 \\
1.918793 \\
2.336426 \\
4.006958\end{array}$ \\
\hline $\mathrm{H}$ & 5 & 1.000000 & $\begin{array}{l}6 \\
5 \\
4\end{array}$ & $\begin{array}{l}1.167053 \\
1.033411 \\
0.799536\end{array}$ & $\begin{array}{l}7 \\
6 \\
5 \\
4 \\
3\end{array}$ & $\begin{array}{l}1.286377 \\
1.214783 \\
1.100232 \\
0.899768 \\
0.498840\end{array}$ & $\begin{array}{l}8 \\
7 \\
6 \\
5 \\
4 \\
3 \\
2\end{array}$ & $\begin{array}{r}1.375870 \\
1.340073 \\
1.286377 \\
1.200464 \\
1.050116 \\
0.749420 \\
-0.002318\end{array}$ & $\begin{array}{l}9 \\
8 \\
7 \\
6 \\
5 \\
4 \\
3 \\
2 \\
1\end{array}$ & $\begin{array}{r}1.445475 \\
1.431554 \\
1.411667 \\
1.381836 \\
1.334106 \\
1.250580 \\
1.083527 \\
0.665894 \\
-1.004637\end{array}$ & $\begin{array}{r}10 \\
9 \\
8 \\
7 \\
6 \\
5 \\
4 \\
3 \\
2 \\
1 \\
0\end{array}$ & $\begin{array}{l}1.501160 \\
1.501160 \\
1.501160 \\
1.501160 \\
1.501160 \\
1.501160 \\
1.501160 \\
1.501160 \\
1.501160 \\
1.501160 \\
\end{array}$ \\
\hline I & 6 & 1.000000 & $\begin{array}{l}7 \\
6 \\
5\end{array}$ & $\begin{array}{l}1.143188 \\
1.023865 \\
0.832947\end{array}$ & $\begin{array}{l}8 \\
7 \\
6 \\
5 \\
4\end{array}$ & $\begin{array}{l}1.250580 \\
1.178986 \\
1.071594 \\
0.899768 \\
0.599072\end{array}$ & $\begin{array}{l}9 \\
8 \\
7 \\
6 \\
5 \\
4 \\
3\end{array}$ & $\begin{array}{l}1.334106 \\
1.292343 \\
1.232681 \\
1.143188 \\
1.000000 \\
0.749420 \\
0.248261\end{array}$ & $\begin{array}{r}10 \\
9 \\
8 \\
7 \\
6 \\
5 \\
4 \\
3 \\
2\end{array}$ & $\begin{array}{r}1.400928 \\
1.378654 \\
1.348028 \\
1.304275 \\
1.238647 \\
1.133643 \\
0.949884 \\
0.582367 \\
-0.336425\end{array}$ & $\begin{array}{r}11 \\
10 \\
9 \\
8 \\
7 \\
6 \\
5 \\
4 \\
3 \\
2 \\
1\end{array}$ & $\begin{array}{r}1.455600 \\
1.446488 \\
1.434338 \\
1.417633 \\
1.393768 \\
1.357971 \\
1.300696 \\
1.200464 \\
1.000000 \\
0.498840 \\
-1.505797\end{array}$ \\
\hline
\end{tabular}


TABLE 3. Corrected Landé $g$-values for terms of odd multiplicity-Continued

\begin{tabular}{|c|c|c|c|c|c|c|c|c|c|c|c|c|}
\hline \multirow{3}{*}{ Term } & \multicolumn{12}{|c|}{ Multiplicity } \\
\hline & \multicolumn{2}{|c|}{$\begin{array}{c}\text { Singlets } \\
1\end{array}$} & \multicolumn{2}{|r|}{$\begin{array}{c}\text { Triplets } \\
3\end{array}$} & \multicolumn{2}{|c|}{$\begin{array}{c}\text { Quintets } \\
5\end{array}$} & \multicolumn{2}{|r|}{$\begin{array}{c}\text { Septets } \\
7\end{array}$} & \multicolumn{2}{|r|}{$\begin{array}{c}\text { Nonets } \\
9\end{array}$} & \multicolumn{2}{|r|}{$\begin{array}{c}\text { Undecets } \\
\qquad 11\end{array}$} \\
\hline & I & $g$ & $J$ & $g$ & J & $g$ & $J$ & $g$ &.$J$ & $g$ & $J$ & $g$ \\
\hline $\mathrm{K}$ & 7 & 1.000000 & $\begin{array}{l}8 \\
7 \\
6\end{array}$ & $\begin{array}{l}1.125290 \\
1.017899 \\
0.856812\end{array}$ & $\begin{array}{l}9 \\
8 \\
7 \\
6 \\
5\end{array}$ & $\begin{array}{l}1.222738 \\
1.153132 \\
1.053696 \\
0.904541 \\
0.665894\end{array}$ & $\begin{array}{r}10 \\
9 \\
8 \\
7 \\
6 \\
5 \\
4\end{array}$ & $\begin{array}{l}1.300696 \\
1.256148 \\
1.194895 \\
1.107391 \\
0.976135 \\
0.766126 \\
0.398608\end{array}$ & $\begin{array}{r}11 \\
10 \\
9 \\
8 \\
7 \\
6 \\
5 \\
4 \\
3\end{array}$ & $\begin{array}{r}1.364480 \\
1.337144 \\
1.300696 \\
1.250580 \\
1.178986 \\
1.071594 \\
0.899768 \\
0.599072 \\
-0.002318\end{array}$ & $\begin{array}{r}12 \\
11 \\
10 \\
9 \\
8 \\
7 \\
6 \\
5 \\
4 \\
3 \\
2\end{array}$ & $\begin{array}{r}1.417633 \\
1.402446 \\
1.382704 \\
1.356380 \\
1.320185 \\
1.268478 \\
1.190918 \\
1.066821 \\
0.849652 \\
0.415314 \\
-0.670531\end{array}$ \\
\hline $\mathrm{L}$ & 8 & 1.000000 & $\begin{array}{l}9 \\
8 \\
7\end{array}$ & $\begin{array}{l}1.111369 \\
1.013921 \\
0.874710\end{array}$ & $\begin{array}{r}10 \\
9 \\
8 \\
7 \\
6\end{array}$ & $\begin{array}{l}1.200464 \\
1.133643 \\
1.041763 \\
0.910507 \\
0.713623\end{array}$ & $\begin{array}{r}11 \\
10 \\
9 \\
8 \\
7 \\
6 \\
5\end{array}$ & $\begin{array}{l}1.273360 \\
1.227800 \\
1.167053 \\
1.083527 \\
0.964203 \\
0.785217 \\
0.498840\end{array}$ & $\begin{array}{r}12 \\
11 \\
10 \\
9 \\
8 \\
7 \\
6 \\
5 \\
4\end{array}$ & $\begin{array}{l}1.334106 \\
1.303733 \\
1.264248 \\
1.211601 \\
1.139211 \\
1.035797 \\
0.880676 \\
0.632483 \\
0.198145\end{array}$ & $\begin{array}{r}13 \\
12 \\
11 \\
10 \\
9 \\
8 \\
7 \\
6 \\
5 \\
4 \\
3\end{array}$ & $\begin{array}{r}1.385507 \\
1.366232 \\
1.341700 \\
1.309808 \\
1.267285 \\
1.208817 \\
1.125290 \\
1.000000 \\
0.799536 \\
0.448724 \\
-0.252898\end{array}$ \\
\hline M & 9 & 1.000000 & $\begin{array}{r}10 \\
9 \\
8\end{array}$ & $\begin{array}{l}1.100232 \\
1.011137 \\
0.888631\end{array}$ & $\begin{array}{r}11 \\
10 \\
9 \\
8 \\
7\end{array}$ & $\begin{array}{l}1.182240 \\
1.118456 \\
1.033411 \\
0.916473 \\
0.749420\end{array}$ & $\begin{array}{r}12 \\
11 \\
10 \\
9 \\
8 \\
7 \\
6\end{array}$ & $\begin{array}{l}1.250580 \\
1.205020 \\
1.145792 \\
1.066821 \\
0.958237 \\
0.803116 \\
0.570435\end{array}$ & $\begin{array}{r}13 \\
12 \\
11 \\
10 \\
9 \\
8 \\
7 \\
6 \\
5\end{array}$ & $\begin{array}{l}1.308406 \\
1.276280 \\
1.235393 \\
1.182240 \\
1.111369 \\
1.013921 \\
0.874710 \\
0.665894 \\
0.331787\end{array}$ & $\begin{array}{r}14 \\
13 \\
12 \\
11 \\
10 \\
9 \\
8 \\
7 \\
6 \\
5 \\
4\end{array}$ & $\begin{array}{r}1.357971 \\
1.335942 \\
1.308406 \\
1.273360 \\
1.227800 \\
1.167053 \\
1.083527 \\
0.964203 \\
0.785217 \\
0.498840 \\
-0.002318\end{array}$ \\
\hline $\mathrm{N}$ & 10 & 1.000000 & $\begin{array}{r}11 \\
10 \\
9\end{array}$ & $\begin{array}{l}1.091120 \\
1.009112 \\
0.899768\end{array}$ & $\begin{array}{r}12 \\
11 \\
10 \\
9 \\
8\end{array}$ & $\begin{array}{l}1.167053 \\
1.106307 \\
1.027336 \\
0.922042 \\
0.777262\end{array}$ & $\begin{array}{r}13 \\
12 \\
11 \\
10 \\
9 \\
8 \\
7\end{array}$ & $\begin{array}{l}1.231304 \\
1.186329 \\
1.129087 \\
1.054672 \\
0.955452 \\
0.819026 \\
0.624130\end{array}$ & $\begin{array}{r}14 \\
13 \\
12 \\
11 \\
10 \\
9 \\
8 \\
7 \\
6\end{array}$ & $\begin{array}{l}1.286377 \\
1.253333 \\
1.212029 \\
1.159460 \\
1.091120 \\
1.000000 \\
0.874710 \\
0.695725 \\
0.427246\end{array}$ & $\begin{array}{r}15 \\
14 \\
13 \\
12 \\
11- \\
10 \\
9 \\
8 \\
7 \\
6 \\
5\end{array}$ & $\begin{array}{l}1.334106 \\
1.310242 \\
1.280870 \\
1.244155 \\
1.197427 \\
1.136680 \\
1.055684 \\
0.944316 \\
0.785217 \\
0.546570 \\
0.164734\end{array}$ \\
\hline
\end{tabular}


TABIE 3. Corrected Landé $y$-values for terms of odd multiplicity - Continued

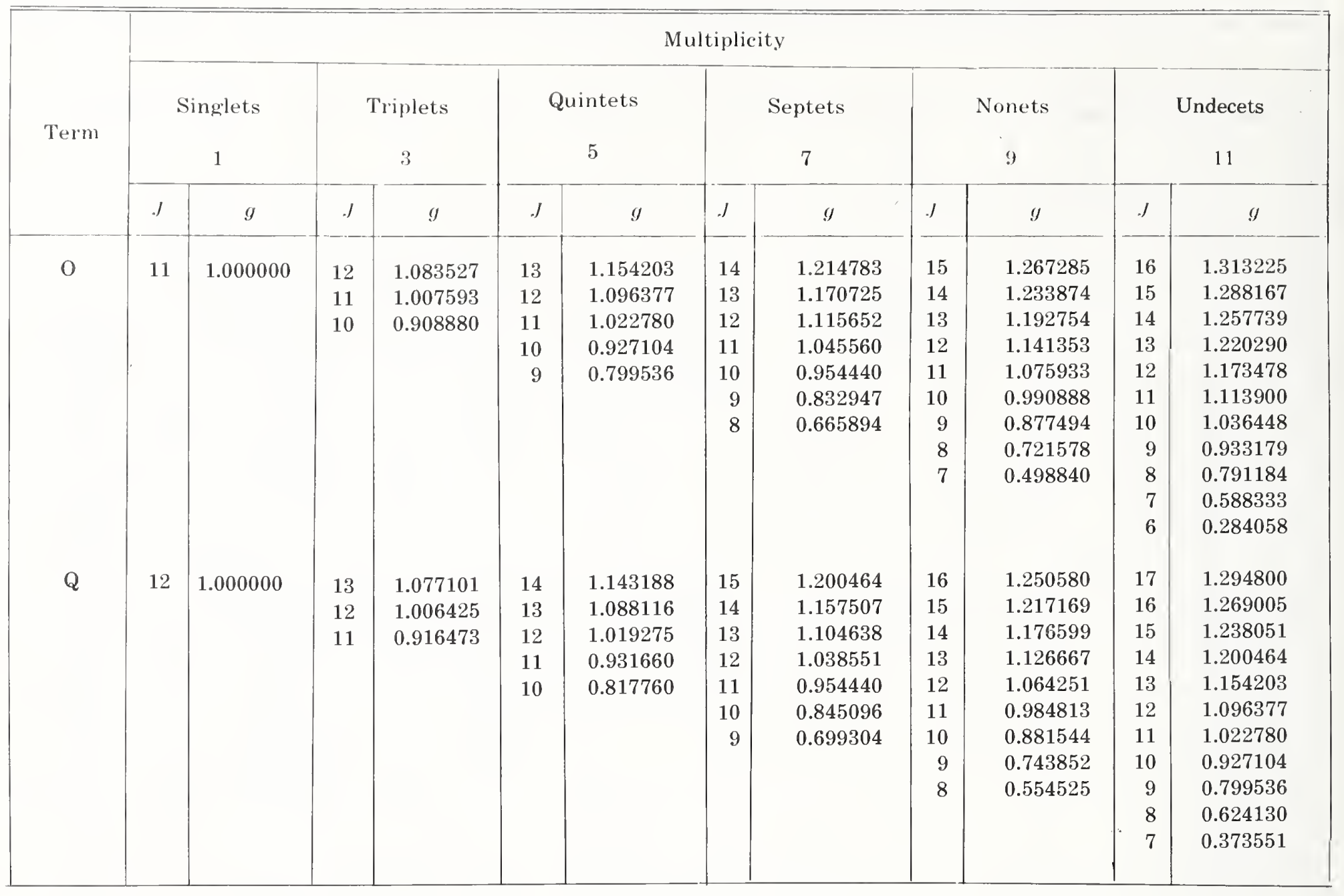


TABLE 4. Corrected Landé $g$-values for terms of even multiplicity

\begin{tabular}{|c|c|c|c|c|c|c|c|c|c|c|}
\hline \multirow{4}{*}{ Term } & \multicolumn{10}{|c|}{ Multiplicity } \\
\hline & \multirow{2}{*}{\multicolumn{2}{|c|}{$\begin{array}{c}\text { Doublets } \\
2\end{array}$}} & \multirow{2}{*}{\multicolumn{2}{|c|}{$\begin{array}{c}\text { Quartets } \\
4\end{array}$}} & \multirow{2}{*}{\multicolumn{2}{|c|}{$\begin{array}{c}\text { Sextets } \\
6\end{array}$}} & \multirow{2}{*}{\multicolumn{2}{|c|}{$\begin{array}{c}\text { Octets } \\
8\end{array}$}} & \multirow{2}{*}{\multicolumn{2}{|c|}{$\begin{array}{c}\text { Decets } \\
10\end{array}$}} \\
\hline & & & & & & & & & & \\
\hline & $J$ & $g$ & $J$ & $g$ & $J$ & $g$ & $J$ & $g$ & $J$ & $g$ \\
\hline S & $1 / 2$ & 2.002319 & $3 / 2$ & 2.002319 & $5 / 2$ & 2.002319 & $7 / 2$ & 2.002319 & $9 / 2$ & 2.002319 \\
\hline \multirow[t]{3}{*}{$\mathrm{P}$} & $3 / 2$ & 1.334106 & $5 / 2$ & 1.601392 & $7 / 2$ & 1.715942 & $9 / 2$ & 1.779582 & $11 / 2$ & 1.820079 \\
\hline & $1 / 2$ & 0.665894 & $3 / 2$ & 1.735034 & $5 / 2$ & 1.887768 & $7 / 2$ & 1.938680 & $9 / 2$ & 1.961821 \\
\hline & & & $1 / 2$ & 2.670532 & $3 / 2$ & 2.403247 & $5 / 2$ & 2.288696 & $7 / 2$ & 2.225057 \\
\hline \multirow[t]{5}{*}{$\mathrm{D}$} & $5 / 2$ & 1.200464 & $7 / 2$ & 1.429565 & $9 / 2$ & 1.556844 & $11 / 2$ & 1.637839 & $13 / 2$ & 1.693913 \\
\hline & $3 / 2$ & 0.799536 & $5 / 2$ & 1.372290 & $7 / 2$ & 1.588664 & $9 / 2$ & 1.698586 & $11 / 2$ & 1.764006 \\
\hline & & & $3 / 2$ & 1.200464 & $5 / 2$ & 1.658667 & $7 / 2$ & 1.811401 & $9 / 2$ & 1.880826 \\
\hline & & & $1 / 2$ & -0.002318 & $3 / 2$ & 1.868677 & $5 / 2$ & 2.059595 & $7 / 2$ & 2.097778 \\
\hline & & & & & $1 / 2$ & 3.338745 & $3 / 2$ & 2.804175 & $5 / 2$ & 2.575073 \\
\hline \multirow[t]{7}{*}{$\mathrm{F}$} & $7 / 2$ & 1.143188 & $9 / 2$ & 1.334106 & $11 / 2$ & 1.455600 & $13 / 2$ & 1.539710 & $15 / 2$ & 1.601392 \\
\hline & $5 / 2$ & 0.856812 & $7 / 2$ & 1.238647 & $9 / 2$ & 1.435351 & $11 / 2$ & 1.553729 & $13 / 2$ & 1.632232 \\
\hline & & & $5 / 2$ & 1.028638 & $7 / 2$ & 1.397746 & $9 / 2$ & 1.577093 & $11 / 2$ & 1.679895 \\
\hline & & & $3 / 2$ & 0.398608 & $5 / 2$ & 1.315015 & $7 / 2$ & 1.620483 & $9 / 2$ & 1.759330 \\
\hline & & & & & $3 / 2$ & 1.066821 & $5 / 2$ & 1.715942 & $7 / 2$ & 1.906860 \\
\hline & & & & & $1 / 2$ & -0.670531 & $3 / 2$ & 2.002319 & $5 / 2$ & 2.231421 \\
\hline & & & & & & & $1 / 2$ & 4.006958 & $3 / 2$ & 3.205102 \\
\hline \multirow[t]{8}{*}{$\mathrm{G}$} & $9 / 2$ & 1.111369 & $11 / 2$ & 1.273360 & $13 / 2$ & 1.385507 & $15 / 2$ & 1.467749 & $17 / 2$ & 1.530640 \\
\hline & $7 / 2$ & 0.888631 & $9 / 2$ & 1.172115 & $11 / 2$ & 1.343452 & $13 / 2$ & 1.457469 & $15 / 2$ & 1.538501 \\
\hline & & & $7 / 2$ & 0.984090 & $9 / 2$ & 1.273360 & $11 / 2$ & 1.441581 & $13 / 2$ & 1.549991 \\
\hline & & & $5 / 2$ & 0.570435 & $7 / 2$ & 1.143188 & $9 / 2$ & 1.415102 & $11 / 2$ & 1.567747 \\
\hline & & & & & $5 / 2$ & 0.856812 & $7 / 2$ & 1.365926 & $9 / 2$ & 1.597342 \\
\hline & & & & & $3 / 2$ & -0.002318 & $5 / 2$ & 1.257739 & $7 / 2$ & 1.652303 \\
\hline & & & & & & & $3 / 2$ & 0.933179 & $5 / 2$ & 1.773218 \\
\hline & & & & & & & $1 / 2$ & -1.338744 & $\begin{array}{l}3 / 2 \\
1 / 2\end{array}$ & $\begin{array}{l}2.135962 \\
4.675170\end{array}$ \\
\hline \multirow[t]{10}{*}{$\mathrm{H}$} & $11 / 2$ & 1.091120 & $13 / 2$ & 1.231304 & $15 / 2$ & 1.334106 & $17 / 2$ & 1.412720 & $19 / 2$ & 1.474783 \\
\hline & $9 / 2$ & 0.908880 & $11 / 2$ & 1.133175 & $13 / 2$ & 1.282705 & $15 / 2$ & 1.389136 & $17 / 2$ & 1.468576 \\
\hline & & & $9 / 2$ & 0.969627 & $11 / 2$ & 1.203268 & $13 / 2$ & 1.354667 & $15 / 2$ & 1.459888 \\
\hline & & & $7 / 2$ & 0.665894 & $9 / 2$ & 1.070871 & $11 / 2$ & 1.301397 & $13 / 2$ & 1.447189 \\
\hline & & & & & $7 / 2$ & 0.824992 & $9 / 2$ & 1.212613 & $11 / 2$ & 1.427563 \\
\hline & & & & & $5 / 2$ & 0.284058 & $7 / 2$ & 1.047729 & $9 / 2$ & 1.394853 \\
\hline & & & & & & & $5 / 2$ & 0.684985 & $7 / 2$ & 1.334106 \\
\hline & & & & & & & $3 / 2$ & -0.403246 & $5 / 2$ & 1.200464 \\
\hline & & & & & & & & & $3 / 2$ & 0.799536 \\
\hline & & & & & & & & & $1 / 2$ & -2.006957 \\
\hline \multirow[t]{10}{*}{ I } & $13 / 2$ & 1.077101 & $15 / 2$ & 1.200464 & $17 / 2$ & 1.294800 & $19 / 2$ & 1.369275 & $21 / 2$ & 1.429565 \\
\hline & $11 / 2$ & 0.922899 & $13 / 2$ & 1.107942 & $15 / 2$ & 1.239770 & $17 / 2$ & 1.338244 & $19 / 2$ & 1.414493 \\
\hline & & & $11 / 2$ & 0.964954 & $13 / 2$ & 1.159343 & $15 / 2$ & 1.294800 & $17 / 2$ & 1.394101 \\
\hline & & & $9 / 2$ & 0.726640 & $11 / 2$ & 1.035046 & $13 / 2$ & 1.231304 & $15 / 2$ & 1.365552 \\
\hline & & & & & $9 / 2$ & 0.827885 & $11 / 2$ & 1.133175 & $13 / 2$ & 1.323826 \\
\hline & & & & & $7 / 2$ & 0.443156 & $9 / 2$ & 0.969627 & $11 / 2$ & 1.259341 \\
\hline & & & & & & & $7 / 2$ & 0.665894 & $9 / 2$ & 1.151867 \\
\hline & & & & & & & $5 / 2$ & -0.002318 & $7 / 2$ & 0.952271 \\
\hline & & & & & & & & & $\begin{array}{l}5 / 2 \\
3 / 2\end{array}$ & $\begin{array}{r}0.513159 \\
-0.804174\end{array}$ \\
\hline & & & & & & & & & & \\
\hline
\end{tabular}


TABLE 4. Corrected Landé $g$-values for terms of even multiplicity-Continued

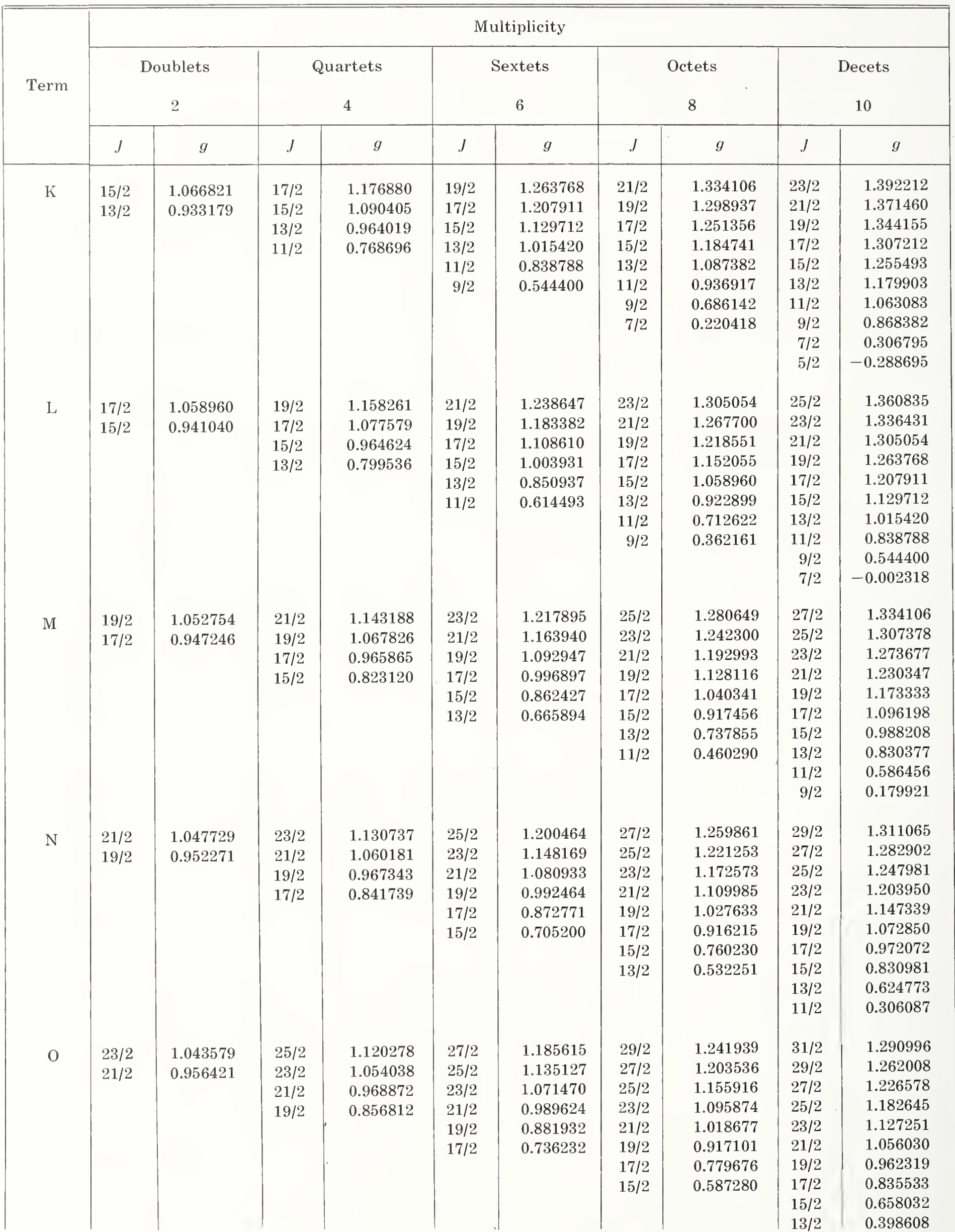


TABLE 4. Corrected Landé $g$-values for terms of even multiplicity - Continued

\begin{tabular}{|c|c|c|c|c|c|c|c|c|c|c|}
\hline \multirow{3}{*}{ Term } & \multicolumn{10}{|c|}{ Multiplicity } \\
\hline & \multicolumn{2}{|c|}{$\begin{array}{c}\text { Doublets } \\
2\end{array}$} & \multicolumn{2}{|c|}{$\begin{array}{c}\text { Quartets } \\
\qquad 4\end{array}$} & \multicolumn{2}{|c|}{$\begin{array}{c}\text { Sextets } \\
6\end{array}$} & \multicolumn{2}{|c|}{$\begin{array}{c}\text { Octets } \\
8\end{array}$} & \multicolumn{2}{|c|}{$\begin{array}{c}\text { Decets } \\
10\end{array}$} \\
\hline & $J$ & $g$ & $J$ & $g$ & $J$ & $g$ & $J$ & $g$ & $J$ & $g$ \\
\hline $\mathrm{Q}$ & $\begin{array}{l}25 / 2 \\
23 / 2\end{array}$ & $\begin{array}{l}1.040093 \\
0.959907\end{array}$ & $\begin{array}{l}27 / 2 \\
25 / 2 \\
23 / 2 \\
21 / 2\end{array}$ & $\begin{array}{l}1.111369 \\
1.049002 \\
0.970366 \\
0.869263\end{array}$ & $\begin{array}{l}29 / 2 \\
27 / 2 \\
25 / 2 \\
23 / 2 \\
21 / 2 \\
19 / 2\end{array}$ & $\begin{array}{l}1.172814 \\
1.124170 \\
1.063851 \\
0.987798 \\
0.890015 \\
0.761353\end{array}$ & $\begin{array}{l}31 / 2 \\
29 / 2 \\
27 / 2 \\
25 / 2 \\
23 / 2 \\
21 / 2 \\
19 / 2 \\
17 / 2\end{array}$ & $\begin{array}{l}1.226330 \\
1.188423 \\
1.142091 \\
1.084640 \\
1.012202 \\
0.919067 \\
0.796522 \\
0.630725\end{array}$ & $\begin{array}{l}33 / 2 \\
31 / 2 \\
29 / 2 \\
27 / 2 \\
25 / 2 \\
23 / 2 \\
21 / 2 \\
19 / 2 \\
17 / 2 \\
15 / 2\end{array}$ & $\begin{array}{l}1.273360 \\
1.243966 \\
1.208491 \\
1.165133 \\
1.111369 \\
1.043579 \\
0.956421 \\
0.841739 \\
0.686581 \\
0.469360\end{array}$ \\
\hline
\end{tabular}


TABLE 5. Landé $g$-values for terms of odd multiplicity in order of increasing $g$

\begin{tabular}{|c|c|c|c|c|c|c|c|c|c|c|c|c|c|}
\hline \multicolumn{2}{|c|}{$J=1$} & \multicolumn{2}{|c|}{$J=3-$ Cont } & \multicolumn{2}{|c|}{$J=5-$ Cont. } & \multicolumn{2}{|c|}{$J=6-$ Cont. } & \multicolumn{2}{|c|}{$J=8-$ Cont. } & \multicolumn{2}{|c|}{$J=10-$ Cont. } & \multicolumn{2}{|c|}{$J=12-$ Cont } \\
\hline-1.500 & ${ }^{11} \mathrm{I}$ & 1.500 & ${ }^{7} \mathbf{F}$ & 0.900 & ${ }^{9} \mathrm{~K}$ & 1.786 & ${ }^{11} \mathrm{D}$ & 1.153 & ${ }^{5} \mathrm{~K}$ & 1.000 & ${ }^{1} \mathrm{~N}$ & 1.141 & ${ }^{9} \mathrm{O}$ \\
\hline-1.000 & ${ }^{9} \mathrm{H}$ & & ${ }^{9} \mathrm{G}$ & 1.000 & ${ }^{1} \mathrm{H}$ & 1.833 & ${ }^{11} \mathrm{P}$ & 1.194 & ${ }^{7} \mathrm{~K}$ & 1.009 & ${ }^{3} \mathrm{~N}$ & 1.167 & ${ }^{5} \mathrm{~N}$ \\
\hline-0.500 & ${ }^{7} \mathrm{G}$ & & ${ }^{11} \mathrm{H}$ & & $\begin{array}{r}{ }^{7} \mathrm{I} \\
{ }^{3} \mathrm{H}\end{array}$ & \multicolumn{2}{|c|}{$J=7$} & 1.208 & ${ }^{11} \mathrm{~L}$ & 1.027 & ${ }^{5} \mathrm{~N}$ & 1.173 & ${ }^{11} \mathrm{O}$ \\
\hline 0.000 & $\begin{array}{l}{ }^{5} \mathrm{~F} \\
{ }^{3} \mathrm{D}\end{array}$ & 1.667 & ${ }^{5} \mathrm{P}$ & 1.033 & $\begin{array}{r}{ }^{3} \mathrm{H} \\
{ }_{11} \mathrm{~K}\end{array}$ & & & 1.250 & ${ }^{5} \mathbf{I}$ & 1.036 & ${ }^{11} \mathrm{O}$ & 1.186 & ${ }^{7} \mathrm{~N}$ \\
\hline 0.500 & ${ }^{3} \mathrm{D}$ & 1.750 & ${ }^{7} \mathrm{D}$ & 1.067 & ${ }^{11} \mathrm{~K}$ & 0.375 & ${ }^{11} \mathrm{Q}$ & & ${ }^{9} \mathrm{~K}$ & 1.055 & ${ }^{7} \mathrm{~N}$ & 1.212 & ${ }^{9} \mathrm{~N}$ \\
\hline 1.000 & ${ }_{3}^{1} \mathrm{P}$ & 1.833 & ${ }^{9} \mathrm{~F}$ & 1.100 & ${ }^{5} \mathrm{H}$ & 0.500 & ${ }^{9} \mathrm{O}$ & 1.292 & ${ }^{7} \mathbf{I}$ & 1.091 & ${ }^{9} \mathrm{~N}$ & 1.244 & ${ }^{11} \mathrm{~N}$ \\
\hline \multirow{5}{*}{1.500} & ${ }^{3} \mathrm{P}$ & \multirow{2}{*}{1.917} & ${ }^{7} \mathbf{P}$ & 1.133 & ${ }^{9} \mathrm{I}$ & 0.589 & ${ }^{11} \mathrm{O}$ & 1.319 & ${ }^{11} \mathrm{~K}$ & 1.100 & ${ }^{3} \mathrm{M}$ & 1.250 & ${ }^{7} \mathrm{M}$ \\
\hline & ${ }^{5} \mathrm{D}$ & & ${ }^{11} \mathrm{G}$ & 1.200 & ${ }^{3} \mathrm{G}$ & 0.625 & ${ }^{7} \mathrm{~N}$ & 1.347 & ${ }^{9} \mathrm{I}$ & 1.118 & ${ }^{5} \mathrm{M}$ & 1.276 & ${ }^{9} \mathrm{M}$ \\
\hline & ${ }^{7} \mathrm{~F}$ & 2.000 & ${ }^{7} \mathrm{~S}$ & & ${ }^{7} \mathrm{H}$ & 0.696 & ${ }^{9} \mathrm{~N}$ & 1.375 & ${ }^{7} \mathrm{H}$ & 1.136 & ${ }^{11} \mathrm{~N}$ & 1.308 & ${ }^{11} \mathrm{M}$ \\
\hline & ${ }^{9} \mathrm{G}$ & 2.083 & ${ }^{9} \mathrm{D}$ & 1.267 & ${ }^{5} \mathrm{G}$ & 0.750 & ${ }^{5} \mathrm{M}$ & 1.417 & ${ }^{11} I$ & 1.145 & ${ }^{7} \mathrm{M}$ & 1.333 & ${ }^{9} \mathrm{~L}$ \\
\hline & ${ }^{11} \mathrm{H}$ & 2.250 & ${ }^{9} \mathrm{P}$ & 1.300 & ${ }^{11} \mathrm{I}$ & 0.786 & ${ }^{11} \mathrm{~N}$ & 1.431 & ${ }^{9} \mathrm{H}$ & 1.182 & ${ }^{9} \mathrm{M}$ & 1.365 & ${ }^{11} \mathrm{~L}$ \\
\hline 2.000 & ${ }^{3} \mathrm{~S}$ & & ${ }^{11} \mathrm{~F}$ & 1.333 & ${ }^{9} \mathrm{H}$ & 0.804 & ${ }^{7} \mathrm{M}$ & 1.500 & ${ }^{9} \mathrm{G}$ & 1.200 & ${ }^{5} \mathrm{~L}$ & 1.417 & ${ }^{11} \mathrm{~K}$ \\
\hline 2.500 & ${ }^{5} \mathrm{P}$ & 2.500 & ${ }^{11} \mathrm{D}$ & 1.367 & ${ }^{7} \mathrm{G}$ & 0.875 & ${ }^{3} \mathrm{~L}$ & & ${ }^{11} \mathrm{H}$ & 1.227 & ${ }^{7} \mathrm{~L}$ & & \\
\hline $\begin{array}{l}3.000 \\
3.500\end{array}$ & $\begin{array}{l}{ }^{7} \mathrm{D} \\
{ }^{9} \mathrm{~F}\end{array}$ & $J=$ & & $\begin{array}{l}1.400 \\
1.500\end{array}$ & $\begin{array}{l}{ }^{5} \mathrm{~F} \\
{ }^{7} \mathrm{~F}\end{array}$ & & ${ }^{9} \mathrm{M}$ & 1.569 & ${ }^{11} \mathrm{G}$ & & ${ }^{11} \mathrm{M}$ & $J=$ & \\
\hline 4.000 & ${ }^{11} \mathrm{G}$ & & & & ${ }^{9} \mathrm{G}$ & $\begin{array}{l}0.911 \\
0.964\end{array}$ & ${ }^{7} \mathrm{~L}$ & 1.625 & ${ }^{11} \mathrm{~F}$ & 1.264 & ${ }^{9} \mathrm{~L}$ & 1.077 & ${ }^{3} \mathrm{Q}$ \\
\hline$J=$ & & $\begin{array}{l}0.000 \\
0.200\end{array}$ & $\begin{array}{r}{ }^{11} \mathrm{M} \\
{ }^{9} \mathrm{~L}\end{array}$ & 1.600 & $\begin{array}{c}{ }^{11} \mathrm{H} \\
{ }^{7} \mathrm{D}\end{array}$ & 1.000 & $\begin{array}{l}{ }^{11} \mathrm{M} \\
{ }^{1} \mathrm{~K}\end{array}$ & $J=$ & & $\begin{array}{l}1.309 \\
1.336\end{array}$ & $\begin{array}{l}{ }^{11} \mathrm{~L} \\
{ }^{9} \mathrm{~K}\end{array}$ & $\begin{array}{l}1.088 \\
1.104\end{array}$ & $\begin{array}{l}{ }^{5} \mathrm{Q} \\
{ }^{7} \mathrm{Q}\end{array}$ \\
\hline-0.667 & ${ }^{11} \mathrm{~K}$ & $\begin{array}{l}0.400 \\
0.450\end{array}$ & $\begin{array}{c}{ }^{7} \mathrm{~K} \\
{ }^{11} \mathrm{~L}\end{array}$ & $\begin{array}{l}1.633 \\
1.667\end{array}$ & $\begin{array}{r}{ }^{9} \mathrm{~F} \\
{ }^{11} \mathrm{G}\end{array}$ & $\begin{array}{l}1.018 \\
1.036\end{array}$ & ${ }^{3} \mathrm{~K}$ & 0.700 & ${ }^{7} \mathrm{Q}$ & 1.382 & ${ }^{11} \mathrm{~K}$ & 1.126 & $\begin{array}{l}{ }^{9} \mathrm{Q} \\
{ }^{5} \mathrm{O}\end{array}$ \\
\hline-0.333 & ${ }^{9} \mathbf{I}$ & 0.600 & ${ }^{5} \mathbf{I}$ & 1.733 & ${ }^{9} \mathrm{D}$ & $\begin{array}{l}1.036 \\
1.054\end{array}$ & $\begin{array}{l}{ }^{9} \mathrm{~L} \\
{ }^{5} \mathrm{~K}\end{array}$ & 0.744 & ${ }^{9} \mathrm{Q}$ & 1.400 & ${ }^{9} \mathbf{I}$ & 1.154 & 110 \\
\hline 0.000 & ${ }^{7} \mathrm{H}$ & & ${ }^{9} \mathrm{~K}$ & 1.800 & ${ }^{9} \mathrm{P}$ & 1.107 & ${ }^{{ }^{7} \mathrm{~K}}$ & 0.800 & ${ }^{5} \mathrm{O}$ & 1.445 & ${ }^{11} \mathrm{I}$ & 1.170 & ${ }^{7} \mathrm{O}$ \\
\hline 0.333 & ${ }^{5} \mathrm{G}$ & 0.750 & ${ }^{7} \mathbf{I}$ & & ${ }^{11} \mathrm{~F}$ & 1.125 & ${ }^{11} \mathrm{~L}$ & & ${ }^{11} \mathrm{Q}$ & 1.500 & ${ }^{11} \mathrm{H}$ & 1.192 & ${ }^{9} \mathrm{O}$ \\
\hline 0.500 & ${ }^{11} \mathbf{I}$ & 0.800 & ${ }^{3} \mathrm{H}$ & 1.900 & ${ }^{11} \mathrm{D}$ & 1.143 & ${ }^{3} \mathbf{I}$ & 0.833 & ${ }^{7} \mathrm{O}$ & $J=$ & & 1.220 & ${ }^{11} \mathrm{O}$ \\
\hline 0.667 & ${ }^{3} \mathrm{~F}$ & 0.850 & ${ }^{11} \mathrm{~K}$ & 1.967 & ${ }^{11} \mathrm{P}$ & 1.179 & ${ }^{5} \mathbf{I}$ & $\begin{array}{l}0.878 \\
0900\end{array}$ & ${ }^{9} \mathrm{O}$ & & & 1.231 & ${ }^{7} \mathrm{~N}$ \\
\hline & ${ }^{9} \mathrm{H}$ & 0.900 & ${ }^{5} \mathrm{H}$ & 2.000 & ${ }^{11} \mathrm{~S}$ & & ${ }^{9} \mathrm{~K}$ & 0.900 & ${ }^{3} \mathrm{~N}$ & 0.917 & ${ }^{3} \mathrm{Q}$ & 1.253 & ${ }^{9} \mathrm{~N}$ \\
\hline 0.833 & ${ }^{7} \mathrm{G}$ & 0.950 & ${ }^{9} \mathrm{I}$ & & & 1.232 & ${ }^{7} \mathbf{I}$ & 0.902 & ${ }^{5} \mathrm{~N}$ & 0.932 & ${ }^{5} \mathrm{Q}$ & 1.280 & ${ }^{11} \mathrm{~N}$ \\
\hline 1.000 & ${ }^{1} \mathrm{D}$ & 1.000 & ${ }^{1} \mathrm{G}$ & $J=$ & & 1.268 & ${ }^{11} \mathrm{~K}$ & $\begin{array}{l}0.906 \\
0.932\end{array}$ & ${ }^{7} \mathrm{~N}$ & 0.955 & ${ }^{7} \mathrm{Q}$ & 1.308 & ${ }^{9} \mathrm{M}$ \\
\hline & ${ }^{5} \mathrm{~F}$ & 1.050 & ${ }^{3} \mathrm{G}$ & & & 1.286 & ${ }^{5} \mathrm{H}$ & $\begin{array}{l}0.933 \\
1.000\end{array}$ & ${ }^{11} \mathrm{O}$ & 0.985 & ${ }^{9} \mathrm{Q}$ & 1.335 & ${ }^{11} \mathbf{M}$ \\
\hline 1.167 & ${ }^{3} \mathrm{D}$ & & ${ }^{7} \mathrm{H}$ & $\begin{array}{l}0.286 \\
0.429\end{array}$ & ${ }_{9}^{110} \mathrm{~N}$ & 1.304 & ${ }^{9} \mathbf{I}$ & 1.000 & ${ }^{1} \mathrm{M}$ & 1.000 & ${ }^{1} \mathrm{O}$ & 1.385 & ${ }^{11} \mathrm{~L}$ \\
\hline 1.500 & ${ }^{3} \mathrm{P}$ & 1.150 & ${ }^{5} \mathrm{G}$ & $\begin{array}{l}0.429 \\
0.548\end{array}$ & ${ }^{9} \mathrm{~N}$ & 1.339 & ${ }^{7} \mathrm{H}$ & 1011 & ${ }^{9} \mathrm{~N}$ & 1.008 & ${ }^{3} \mathrm{O}$ & & \\
\hline & ${ }^{5} \mathrm{D}$ & 1.200 & ${ }^{11} \mathrm{I}$ & $\begin{array}{l}0.548 \\
0.571\end{array}$ & $\begin{array}{l}{ }^{11} \mathrm{~N} \\
{ }_{7} \mathrm{M}\end{array}$ & 1.393 & ${ }^{11} \mathbf{I}$ & $\begin{array}{l}1.011 \\
1.033\end{array}$ & ${ }^{3} \mathrm{M}$ & 1.023 & ${ }^{5} \mathrm{O}$ & $J=$ & \\
\hline & ${ }^{7} \mathrm{~F}$ & 1.250 & ${ }^{3} \mathrm{~F}$ & & ${ }^{9} \mathrm{M}$ & 1.411 & ${ }^{9} \mathrm{H}$ & 1.056 & ${ }^{11} \mathrm{~N}$ & 1045 & $\begin{array}{r}{ }^{11} \mathrm{Q} \\
7 \mathrm{O}\end{array}$ & & \\
\hline & ${ }^{9} \mathrm{G}$ & & ${ }^{9} \mathrm{H}$ & $\begin{array}{l}0.667 \\
0.714\end{array}$ & ${ }^{5} \mathrm{~L}$ & 1.429 & ${ }^{7} \mathrm{G}$ & 1.067 & ${ }^{7} \mathrm{M}$ & $\begin{array}{l}1.045 \\
1.076\end{array}$ & ${ }^{9} \mathrm{O}$ & 1.143 & ${ }^{5} \mathrm{Q}$ \\
\hline & ${ }^{11} \mathrm{H}$ & 1.300 & ${ }^{7} \mathrm{G}$ & $\begin{array}{l}0.714 \\
0.786\end{array}$ & ${ }^{7} \mathrm{~L}$ & 1.500 & ${ }^{9} \mathrm{G}$ & 1.111 & ${ }^{3} \mathrm{~L}$ & 1.091 & ${ }^{3} \mathrm{~N}$ & 1.157 & ${ }^{7} \mathrm{Q}$ \\
\hline 1.833 & ${ }^{5} \mathrm{P}$ & 1.350 & ${ }^{5} \mathrm{~F}$ & & ${ }^{11} \mathrm{M}$ & & ${ }^{11} \mathrm{H}$ & & ${ }^{9} \mathrm{M}$ & 1.106 & $5 \mathrm{~N}$ & 1.176 & ${ }^{9} \mathrm{Q}$ \\
\hline 2.000 & ${ }^{5} \mathrm{~S}$ & 1.500 & ${ }^{5} \mathrm{D}$ & 0.857 & ${ }^{3} \mathrm{~K}$ & 1.571 & ${ }^{9} \mathrm{~F}$ & 1.133 & ${ }^{5} \mathrm{~L}$ & 1.114 & ${ }^{11} \mathrm{O}$ & 1.200 & ${ }^{11} \mathrm{Q}$ \\
\hline & ${ }^{7} \mathrm{D}$ & & ${ }^{7} \mathrm{~F}$ & $\begin{array}{l}0.857 \\
0.881\end{array}$ & ${ }^{9} \mathrm{~L}$ & 1.589 & ${ }^{11} \mathrm{G}$ & 1.167 & ${ }^{7} \mathrm{~L}$ & 1.129 & ${ }^{7} \mathrm{~N}$ & 1.214 & ${ }^{7} \mathrm{O}$ \\
\hline 2.167 & ${ }^{9} \mathrm{~F}$ & & ${ }^{9} \mathrm{G}$ & & ${ }^{5} \mathrm{~K}$ & 1.661 & ${ }^{11} \mathrm{~F}$ & 1.101 & ${ }^{11} \mathrm{M}$ & 1.159 & ${ }_{9}^{\mathrm{N}}$ & 1.233 & ${ }^{9} \mathrm{O}$ \\
\hline 2.333 & ${ }^{7} \mathrm{P}$ & & ${ }^{11} \mathrm{H}$ & $\begin{array}{l}0.905 \\
0.976\end{array}$ & ${ }^{7} \mathrm{~K}$ & 1.714 & ${ }^{11} \mathrm{D}$ & 1.211 & ${ }^{9} \mathrm{~L}$ & $\begin{array}{l}1.109 \\
1.182\end{array}$ & ${ }^{9} \mathrm{~N}$ & 1.257 & ${ }^{11} \mathrm{O}$ \\
\hline & ${ }^{11} \mathrm{G}$ & 1.650 & ${ }^{7} \mathrm{D}$ & $\begin{array}{l}0.976 \\
1.000\end{array}$ & ${ }^{1} \mathrm{I}$ & & & $\begin{array}{l}1.211 \\
1.222\end{array}$ & $5 \mathrm{~K}$ & $\begin{array}{l}1.182 \\
1.197\end{array}$ & ${ }^{5} \mathrm{M}$ & 1.286 & ${ }^{9} \mathrm{~N}$ \\
\hline 2.667 & ${ }^{9} \mathrm{D}$ & 1.700 & ${ }^{9} \mathrm{~F}$ & 1.000 & 11 & $J=$ & & & $\mathbf{h}$ & 1.197 & ${ }^{11} \mathrm{~N}$ & 1.310 & ${ }^{11} \mathrm{~N}$ \\
\hline 3.000 & ${ }^{11} \mathrm{~F}$ & 1.750 & ${ }^{7} \mathrm{P}$ & 1.024 & ${ }^{11} \mathrm{~L}$ & 0.556 & ${ }^{9} \mathrm{Q}$ & $\begin{array}{l}1.256 \\
1.267\end{array}$ & ${ }^{11} \mathrm{~K}$ & $\begin{array}{l}1.205 \\
1.235\end{array}$ & $\begin{array}{l}{ }^{7} \mathrm{M} \\
{ }^{9} \mathrm{M}\end{array}$ & 1.357 & ${ }^{11} \mathrm{M}$ \\
\hline$J=$ & & 1.850 & ${ }^{11} \mathrm{G}$ & 1.071 & ${ }^{5} \mathbf{I}$ & 0.625 & ${ }^{11} \mathrm{Q}$ & $\begin{array}{l}1.300 \\
1.333\end{array}$ & $\begin{array}{r}{ }^{9} \mathrm{~K} \\
{ }^{7} \mathrm{I}\end{array}$ & 1.273 & ${ }^{7} \mathrm{~L}$ & $J=$ & \\
\hline-0.250 & ${ }^{11} \mathrm{~L}$ & 1.950 & $\begin{array}{r}{ }^{9} \mathrm{P} \\
{ }^{11} \mathrm{~F}\end{array}$ & 1.143 & $\begin{array}{r}{ }^{9} \mathrm{~K} \\
{ }^{7} \mathrm{I}\end{array}$ & $\begin{array}{l}0.667 \\
0.722\end{array}$ & $\begin{array}{l}{ }^{7} \mathrm{O} \\
{ }^{9} \mathrm{O}\end{array}$ & $\begin{array}{l}1.333 \\
1.356\end{array}$ & ${ }^{11} \mathrm{~K}$ & 1.303 & $\begin{array}{l}{ }^{11} \mathrm{M} \\
{ }^{9} \mathrm{~L}\end{array}$ & 1.200 & ${ }^{7} \mathrm{Q}$ \\
\hline 0.000 & ${ }^{9} \mathrm{~K}$ & 2.000 & ${ }^{9} \mathrm{~S}$ & 1.167 & ${ }^{3} \mathrm{H}$ & 0.778 & ${ }^{5} \mathrm{~N}$ & 1.378 & ${ }^{9} \mathbf{I}$ & 1.341 & ${ }^{11} \mathrm{~L}$ & 1.217 & ${ }^{9} \mathrm{Q}$ \\
\hline 0.250 & ${ }^{7} \mathbf{I}$ & 2.100 & ${ }^{11} \mathrm{D}$ & 1.191 & ${ }^{11} \mathrm{~K}$ & 0.792 & ${ }^{11} \mathrm{O}$ & 1.433 & ${ }^{11} \mathrm{I}$ & 1.364 & ${ }^{9} \mathrm{~K}$ & 1.238 & ${ }^{11} \mathrm{Q}$ \\
\hline 0.417 & ${ }^{11} \mathrm{~K}$ & 2.200 & ${ }^{11} \mathrm{P}$ & 1.214 & ${ }^{5} \mathrm{H}$ & 0.819 & ${ }^{7} \mathrm{~N}$ & 1.444 & ${ }^{9} \mathrm{H}$ & 1.402 & ${ }^{11} \mathrm{~K}$ & 1.267 & ${ }^{9} \mathrm{O}$ \\
\hline 0.500 & ${ }^{5} \mathrm{H}$ & & & 1.238 & ${ }^{9} \mathrm{I}$ & 0.875 & ${ }^{9} \mathrm{~N}$ & 1.500 & ${ }^{11} \mathrm{H}$ & 1.455 & ${ }^{11} \mathrm{I}$ & 1.288 & ${ }^{11} \mathrm{O}$ \\
\hline 0.583 & ${ }^{9} \mathrm{I}$ & $J=$ & & 1.286 & ${ }^{7} \mathrm{H}$ & 0.889 & ${ }^{3} \mathrm{M}$ & 1.556 & ${ }^{11} \mathrm{G}$ & & & 1.333 & ${ }^{11} \mathrm{~N}$ \\
\hline 0.750 & ${ }^{3} \mathrm{G}$ & & & 1.333 & ${ }^{5} \mathrm{G}$ & 0.917 & ${ }^{5} \mathrm{M}$ & $I$ & & $J=$ & & & \\
\hline & ${ }^{7} \mathrm{H}$ & 0.167 & ${ }_{9}^{11} \mathrm{~N}$ & $\begin{array}{l}1.357 \\
1.381\end{array}$ & $\begin{array}{l}{ }^{11} \mathrm{I} \\
{ }^{9} \mathrm{H}\end{array}$ & $\begin{array}{l}0.944 \\
0.958\end{array}$ & $\begin{array}{l}{ }^{11} \mathrm{~N} \\
{ }^{7} \mathrm{M}\end{array}$ & $J=$ & & 1.000 & ${ }^{1} \mathrm{Q}$ & $J=$ & \\
\hline $\begin{array}{l}0.917 \\
1.000\end{array}$ & $\begin{array}{l}{ }^{5} \mathrm{G} \\
{ }^{1} \mathrm{~F}\end{array}$ & $\begin{array}{l}0.333 \\
0.500\end{array}$ & $\begin{array}{l}{ }^{9} \mathrm{M} \\
{ }^{7} \mathrm{~L}\end{array}$ & $\begin{array}{l}1.381 \\
1.405\end{array}$ & ${ }^{7} \mathrm{G}$ & $\begin{array}{l}0.958 \\
1.000\end{array}$ & $\begin{array}{l}1 \mathrm{MI} \\
{ }^{1} \mathrm{~L}\end{array}$ & 0.818 & ${ }^{5} \mathrm{Q}$ & 1.006 & ${ }^{3} \mathrm{Q}$ & & ${ }^{9} \mathrm{Q}$ \\
\hline 1.000 & ${ }^{11} \mathrm{I}$ & & ${ }^{11} \mathrm{M}$ & 1.500 & ${ }^{7} \mathrm{~F}$ & 1.014 & ${ }^{3} \mathrm{~L}$ & 0.845 & ${ }^{7} \mathrm{Q}$ & 1.019 & ${ }^{5} \mathrm{Q}$ & 1.268 & ${ }^{11} \mathrm{Q}$ \\
\hline 1.083 & ${ }^{3} \mathrm{~F}$ & 0.633 & ${ }^{9} \mathrm{~L}$ & & ${ }^{9} \mathrm{G}$ & & ${ }^{9} \mathrm{M}$ & 0.882 & ${ }^{9} \mathrm{Q}$ & 1.038 & ${ }^{7} \mathrm{Q}$ & 1.312 & ${ }^{11} \mathrm{O}$ \\
\hline & ${ }^{9} \mathrm{H}$ & 0.667 & ${ }^{5} \mathrm{~K}$ & & ${ }^{11} \mathrm{H}$ & 1.042 & ${ }^{5} \mathrm{~L}$ & 0.909 & ${ }^{3} \mathrm{O}$ & 1.064 & ${ }^{9} \mathrm{Q}$ & & \\
\hline 1.167 & ${ }^{7} \mathrm{G}$ & 0.767 & ${ }^{7} \mathrm{~K}$ & 1.595 & ${ }^{9} \mathrm{~F}$ & 1.083 & ${ }^{7} \mathrm{~L}$ & 0.927 & ${ }^{5} \mathrm{O}$ & 1.083 & ${ }^{3} \mathrm{O}$ & $J=$ & \\
\hline 1.250 & ${ }^{5} \mathrm{~F}$ & 0.800 & ${ }^{11} \mathrm{~L}$ & 1.619 & ${ }^{11} \mathrm{G}$ & & ${ }^{11} \mathrm{M}$ & & ${ }^{11} \mathrm{Q}$ & 1.096 & ${ }^{5} \mathrm{O}$ & & \\
\hline 1.333 & ${ }^{3} \mathrm{D}$ & 0.833 & ${ }^{3} \mathbf{I}$ & 1.667 & ${ }^{9} \mathrm{D}$ & 1.125 & ${ }^{3} \mathrm{~K}$ & 0.955 & ${ }^{7} \mathrm{O}$ & & ${ }^{11} \mathrm{Q}$ & 1.294 & ${ }^{11} \mathrm{Q}$ \\
\hline 1.500 & ${ }^{5} \mathrm{D}$ & 0.900 & ${ }^{5} \mathbf{I}$ & 1.714 & ${ }^{11} \mathrm{~F}$ & 1.139 & ${ }^{9} \mathrm{~L}$ & 0.991 & ${ }^{9} \mathrm{O}$ & 1.115 & ${ }^{7} \mathrm{O}$ & & \\
\hline
\end{tabular}


TABLE 6. Landé $g$-values for terms of even multiplicity in order of increasing $g$

\begin{tabular}{|c|c|c|c|c|c|c|c|c|c|c|c|}
\hline \multicolumn{2}{|c|}{$J=1 / 2$} & \multicolumn{2}{|c|}{$J=7 / 2-$ Cont. } & \multicolumn{2}{|c|}{$J=11 / 2-$ Cont. } & \multicolumn{2}{|c|}{$J=15 / 2-$ Cont. } & \multicolumn{2}{|c|}{$J=19 / 2-$ Cont. } & \multicolumn{2}{|c|}{$J=23 / 2-$ Cont. } \\
\hline-2.000 & ${ }^{10} \mathrm{H}$ & 0.508 & ${ }^{10} \mathrm{~K}$ & 0.615 & ${ }^{6} \mathrm{~L}$ & 0.659 & ${ }^{10} \mathrm{O}$ & 0.797 & ${ }^{8} \mathrm{Q}$ & 1.071 & ${ }^{6} \mathrm{O}$ \\
\hline-1.333 & ${ }^{8} \mathrm{G}$ & 0.667 & ${ }^{4} \mathrm{H}$ & 0.713 & ${ }^{8} \mathrm{~L}$ & 0.706 & ${ }^{6} \mathrm{~N}$ & 0.842 & ${ }^{10} \mathrm{Q}$ & 1.096 & ${ }^{8} \mathrm{O}$ \\
\hline-0.667 & ${ }^{6} \mathrm{~F}$ & & ${ }^{8} \mathbf{I}$ & 0.769 & ${ }^{4} \mathrm{~K}$ & 0.761 & ${ }^{8} \mathrm{~N}$ & 0.857 & ${ }^{4} \mathrm{O}$ & 1.127 & ${ }^{10} \mathrm{O}$ \\
\hline 0.000 & ${ }^{4} \mathrm{D}$ & 0.825 & ${ }^{6} \mathrm{H}$ & 0.839 & ${ }^{6} \mathrm{~K}$ & 0.824 & ${ }^{4} \mathrm{M}$ & 0.882 & ${ }^{6} \mathrm{O}$ & 1.130 & ${ }^{4} \mathrm{~N}$ \\
\hline 0.667 & ${ }^{2} \mathrm{P}$ & 0.889 & ${ }^{2} \mathrm{G}$ & & ${ }^{10} \mathrm{~L}$ & 0.831 & ${ }^{10} \mathrm{~N}$ & 0.917 & ${ }^{8} \mathrm{O}$ & 1.148 & ${ }^{6} \mathrm{~N}$ \\
\hline 2.000 & ${ }^{2} \mathrm{~S}$ & 0.952 & ${ }^{10} \mathrm{I}$ & 0.923 & ${ }^{2} \mathbf{I}$ & 0.863 & ${ }^{6} \mathrm{M}$ & 0.952 & ${ }^{2} \mathrm{~N}$ & 1.172 & ${ }^{8} \mathrm{~N}$ \\
\hline 2.667 & ${ }^{4} \mathrm{P}$ & 0.984 & ${ }^{4} \mathrm{G}$ & 0.937 & ${ }^{8} \mathrm{~K}$ & 0.918 & ${ }^{8} \mathrm{M}$ & 0.962 & ${ }^{10} \mathrm{O}$ & 1.203 & ${ }^{10} \mathrm{~N}$ \\
\hline 3.333 & ${ }^{6} \mathrm{D}$ & 1.048 & ${ }^{8} \mathrm{H}$ & 0.965 & ${ }^{4} I$ & 0.941 & ${ }^{2} \mathrm{~L}$ & 0.967 & ${ }^{4} \mathrm{~N}$ & 1.217 & ${ }^{6} \mathrm{M}$ \\
\hline 4.000 & ${ }^{8} \mathrm{~F}$ & 1.143 & ${ }^{2} \mathrm{~F}$ & 1.035 & ${ }^{6} \mathbf{I}$ & 0.965 & ${ }^{4} \mathrm{~L}$ & 0.992 & ${ }^{6} \mathrm{~N}$ & 1.242 & ${ }^{8} \mathrm{M}$ \\
\hline 4.667 & ${ }^{10} \mathrm{G}$ & & ${ }^{6} \mathrm{G}$ & 1.063 & ${ }^{10} \mathrm{~K}$ & 0.988 & ${ }^{10} \mathrm{M}$ & 1.028 & ${ }^{8} \mathrm{~N}$ & 1.273 & ${ }^{10} \mathrm{M}$ \\
\hline \multirow{2}{*}{\multicolumn{2}{|c|}{$J=3 / 2$}} & 1.238 & ${ }^{4} \mathrm{~F}$ & 1.091 & ${ }^{2} \mathrm{H}$ & 1.004 & ${ }^{6} \mathrm{~L}$ & 1.053 & ${ }^{2} \mathrm{M}$ & 1.304 & ${ }^{8} \mathrm{~L}$ \\
\hline & & 1.333 & ${ }^{10} \mathrm{H}$ & 1.133 & ${ }^{4} \mathrm{H}$ & 1.059 & ${ }^{8} \mathrm{~L}$ & 1.068 & ${ }^{4} \mathrm{M}$ & 1.336 & ${ }^{10} \mathrm{~L}$ \\
\hline $\begin{array}{l}-0.800 \\
-0.400\end{array}$ & ${ }^{10} I$ & 1.365 & ${ }^{8} \mathrm{G}$ & 1.203 & $\begin{array}{r}{ }^{8} \mathbf{I} \\
{ }^{6} \mathrm{H}\end{array}$ & $\begin{array}{l}1.067 \\
1.090\end{array}$ & $\begin{array}{l}{ }^{2} \mathrm{~K} \\
{ }^{4} \mathrm{~K}\end{array}$ & $\begin{array}{l}1.073 \\
1.093\end{array}$ & $\begin{array}{l}{ }^{10} \mathrm{~N} \\
{ }^{6} \mathrm{M}\end{array}$ & 1.391 & \\
\hline 0.000 & $\begin{array}{l}{ }^{8} \mathrm{H} \\
{ }^{6} \mathrm{G}\end{array}$ & $\begin{array}{l}1.397 \\
1.429\end{array}$ & $\begin{array}{l}{ }^{6} \mathrm{~F} \\
{ }^{4} \mathrm{D}\end{array}$ & $\begin{array}{l}1.20 \delta \\
1.259\end{array}$ & ${ }^{10} \mathrm{I}$ & 1.129 & ${ }^{6} \mathrm{~K}$ & 1.128 & ${ }^{8} \mathrm{M}$ & \multicolumn{2}{|c|}{$J=25 / 2$} \\
\hline 0.400 & ${ }^{4} \mathrm{~F}$ & 1.587 & ${ }^{6} \mathrm{D}$ & 1.273 & ${ }^{4} \mathrm{G}$ & & ${ }^{10} \mathrm{~L}$ & 1.158 & ${ }^{4} \mathrm{~L}$ & & \\
\hline \multirow[t]{2}{*}{0.800} & ${ }^{2} \mathrm{D}$ & 1.619 & ${ }^{8} \mathrm{~F}$ & 1.301 & ${ }^{8} \mathrm{H}$ & 1.184 & ${ }^{8} \mathrm{~K}$ & 1.173 & ${ }^{10} \mathrm{M}$ & 1.040 & ${ }^{2} \mathrm{Q}$ \\
\hline & ${ }^{10} \mathrm{H}$ & 1.651 & ${ }^{10} \mathrm{G}$ & 1.343 & ${ }^{6} \mathrm{G}$ & 1.200 & ${ }^{4} \mathbf{I}$ & 1.183 & ${ }^{6} \mathrm{~L}$ & 1.049 & ${ }^{4} \mathrm{Q}$ \\
\hline 0.933 & ${ }^{8} \mathrm{G}$ & 1.714 & ${ }^{6} \mathrm{P}$ & 1.427 & ${ }^{10} \mathrm{H}$ & 1.239 & ${ }^{6} \mathrm{I}$ & 1.218 & ${ }^{8} \mathrm{~L}$ & 1.064 & ${ }^{6} \mathrm{Q}$ \\
\hline 1.067 & ${ }^{6} \mathrm{~F}$ & 1.809 & ${ }^{8} \mathrm{D}$ & 1.441 & ${ }^{8} \mathrm{G}$ & 1.255 & ${ }^{10} \mathrm{~K}$ & 1.263 & ${ }^{6} \mathrm{~K}$ & 1.084 & ${ }^{8} \mathrm{Q}$ \\
\hline 1.200 & ${ }^{4} \mathrm{D}$ & 1.905 & ${ }^{10} \mathrm{~F}$ & 1.455 & ${ }^{6} \mathrm{~F}$ & 1.294 & ${ }^{8} \mathbf{I}$ & & ${ }^{10} \mathrm{~L}$ & 1.111 & ${ }^{10} \mathrm{Q}$ \\
\hline 1.333 & ${ }^{2} \mathrm{P}$ & 1.937 & ${ }^{8} \mathrm{P}$ & 1.552 & ${ }^{8} \mathrm{~F}$ & 1.333 & ${ }^{6} \mathrm{H}$ & 1.298 & ${ }^{8} \mathrm{~K}$ & 1.120 & ${ }^{4} \mathrm{O}$ \\
\hline 1.733 & ${ }^{4} \mathrm{P}$ & 2.000 & ${ }^{8} \mathrm{~S}$ & 1.566 & ${ }^{10} \mathrm{G}$ & 1.365 & ${ }^{10} \mathrm{I}$ & 1.343 & ${ }^{10} \mathrm{~K}$ & 1.135 & ${ }^{6} \mathrm{O}$ \\
\hline 1.867 & ${ }^{6} \mathrm{D}$ & 2.095 & ${ }^{10} \mathrm{D}$ & 1.636 & ${ }^{8} \mathrm{D}$ & 1.388 & ${ }^{8} \mathrm{H}$ & 1.368 & ${ }^{8} \mathrm{I}$ & 1.156 & ${ }^{8} \mathrm{O}$ \\
\hline 2.000 & ${ }^{4} \mathbf{S}$ & 2.222 & $\begin{array}{l}{ }^{10} \mathrm{D} \\
{ }^{10} \mathrm{P}\end{array}$ & 1.678 & ${ }^{10} \mathrm{~F}$ & 1.459 & ${ }^{10} \mathrm{H}$ & 1.414 & ${ }^{10} \mathrm{I}$ & 1.182 & ${ }^{10} \mathrm{O}$ \\
\hline & ${ }^{8} \mathrm{~F}$ & & ${ }^{10} \mathrm{P}$ & 1.762 & ${ }^{10} \mathrm{D}$ & 1.467 & ${ }^{8} \mathrm{G}$ & 1.474 & ${ }^{10} \mathrm{H}$ & 1.200 & ${ }^{6} \mathrm{~N}$ \\
\hline 2.133 & ${ }^{10} \mathrm{G}$ & \multicolumn{2}{|c|}{$J=9 / 2$} & \multirow{2}{*}{\multicolumn{2}{|c|}{$J=13 / 2$}} & 1.537 & ${ }^{10} \mathrm{G}$ & \multirow{2}{*}{\multicolumn{2}{|c|}{$J=21 / 2$}} & 1.221 & ${ }^{8} \mathrm{~N}$ \\
\hline 2.400 & ${ }^{6} \mathrm{P}$ & \multirow{4}{*}{$\begin{array}{l}0.182 \\
0.364 \\
0.545\end{array}$} & ${ }^{10} \mathrm{M}$ & & & & & & & $\begin{array}{l}1.247 \\
1.280\end{array}$ & ${ }^{8} \mathrm{M}$ \\
\hline 2.800 & $\begin{array}{l}{ }^{8} \mathrm{D} \\
{ }^{10} \mathrm{~F}\end{array}$ & & ${ }^{8} \mathrm{~L}$ & 0.400 & ${ }^{10} \mathrm{O}$ & & & 0.870 & ${ }^{4} \mathrm{Q}$ & 1.307 & ${ }^{10} \mathrm{M}$ \\
\hline & & & ${ }^{6} \mathrm{~K}$ & 0.533 & ${ }^{8} \mathrm{~N}$ & & & 0.890 & ${ }^{6} \mathrm{Q}$ & 1.360 & ${ }^{10} \mathrm{~L}$ \\
\hline \multirow{2}{*}{\multicolumn{2}{|c|}{$J=5 / 2$}} & & ${ }^{10} \mathrm{~L}$ & 0.626 & ${ }^{10} \mathrm{~N}$ & 0.632 & ${ }^{8} \mathrm{Q}$ & 0.919 & ${ }^{8} \mathbf{Q}$ & & \\
\hline & & 0.687 & ${ }^{8} \mathrm{~K}$ & 0.667 & ${ }^{6} \mathrm{M}$ & 0.687 & ${ }^{10} \mathrm{Q}$ & 0.957 & ${ }^{2} \mathrm{O}$ & $J=$ & \\
\hline-0.286 & ${ }^{10} \mathrm{~K}$ & 0.727 & ${ }^{4} \mathbf{I}$ & 0.738 & ${ }^{8} \mathrm{M}$ & 0.737 & ${ }^{6} \mathrm{O}$ & & ${ }^{10} \mathrm{Q}$ & & \\
\hline 0.000 & $\begin{array}{r}{ }^{8} \mathrm{I} \\
{ }_{6} \mathrm{H}\end{array}$ & 0.828 & ${ }^{6} \mathbf{I}$ & 0.800 & $\begin{array}{r}{ }^{4} \mathrm{~L} \\
{ }^{10} \mathrm{M}\end{array}$ & $\begin{array}{l}0.780 \\
0.836\end{array}$ & ${ }^{8} \mathrm{O}$ & 0.969 & ${ }^{4} \mathrm{O}$ & 1.111 & ${ }^{4} \mathrm{Q}$ \\
\hline 0.286 & $\begin{array}{l}{ }^{6} \mathrm{H} \\
{ }^{10} \mathrm{I}\end{array}$ & $\begin{array}{l}0.869 \\
0.909\end{array}$ & $\begin{array}{l}{ }^{10} \mathrm{~K} \\
{ }^{2} \mathrm{H}\end{array}$ & 0.831 & $\begin{array}{r}{ }^{10} \mathrm{M} \\
{ }^{6} \mathrm{~L}\end{array}$ & $\begin{array}{l}0.836 \\
0.842\end{array}$ & $\begin{array}{r}{ }^{10} \mathrm{O} \\
{ }^{4} \mathrm{~N}\end{array}$ & $\begin{array}{l}990 \\
019\end{array}$ & ${ }^{6} \mathrm{O}$ & 1.124 & $\begin{array}{l}{ }^{6} Q \\
{ }^{8} Q\end{array}$ \\
\hline $\begin{array}{l}0.514 \\
0.571\end{array}$ & $\begin{array}{l}{ }^{10} \mathrm{I} \\
{ }^{4} \mathrm{G}\end{array}$ & $\begin{array}{l}0.909 \\
0.970\end{array}$ & $\begin{array}{l}{ }^{2} \mathrm{H} \\
{ }^{4} \mathrm{H}\end{array}$ & $\begin{array}{l}0.851 \\
0.923\end{array}$ & ${ }^{8} \mathrm{~L}$ & $\begin{array}{l}842 \\
873\end{array}$ & ${ }^{6} \mathrm{~N}$ & 019 & $\begin{array}{l}{ }^{8} \mathrm{O} \\
{ }^{2} \mathrm{~N}\end{array}$ & $\begin{array}{l}1.142 \\
1.165\end{array}$ & ${ }^{10} \mathrm{Q}$ \\
\hline $\begin{array}{l}0.571 \\
0.686\end{array}$ & ${ }^{8} \mathrm{H}$ & 0.070 & ${ }^{8} \mathbf{I}$ & 0.933 & ${ }^{2} \mathrm{~K}$ & 0.916 & ${ }^{8} \mathrm{~N}$ & 056 & ${ }^{10} \mathrm{O}$ & 1.185 & ${ }^{6} \mathrm{O}$ \\
\hline 0.857 & ${ }^{2} \mathrm{~F}$ & 1.071 & ${ }^{6} \mathrm{H}$ & 0.964 & ${ }^{4} \mathrm{~K}$ & 0.947 & ${ }^{2} \mathrm{M}$ & 1.060 & ${ }^{4} \mathrm{~N}$ & 1.203 & ${ }^{8} \mathrm{O}$ \\
\hline & ${ }^{6} \mathrm{G}$ & 1.111 & ${ }^{2} \mathrm{G}$ & 1.015 & ${ }^{6} \mathrm{~K}$ & 0.966 & ${ }^{4} \mathrm{M}$ & 1.081 & ${ }^{6} \mathrm{~N}$ & 1.226 & ${ }^{10} \mathrm{O}$ \\
\hline 1.029 & ${ }^{4} \mathrm{~F}$ & 1.152 & ${ }^{10} \mathrm{I}$ & & ${ }^{10} \mathrm{~L}$ & 0.972 & ${ }^{10} \mathrm{~N}$ & 1.110 & ${ }^{8} \mathrm{~N}$ & 1.259 & ${ }^{8} \mathrm{~N}$ \\
\hline 1.200 & ${ }^{2} \mathrm{D}$ & 1.172 & ${ }^{4} \mathrm{G}$ & 1.077 & ${ }^{2} \mathbf{I}$ & 0.997 & ${ }^{6} \mathrm{M}$ & 1.143 & ${ }^{4} \mathrm{M}$ & 1.282 & ${ }^{10} \mathrm{~N}$ \\
\hline & ${ }^{10} \mathrm{H}$ & 1.212 & ${ }^{8} \mathrm{H}$ & 1.087 & ${ }^{8} \mathrm{~K}$ & 1.040 & ${ }^{8} \mathrm{M}$ & 1.147 & ${ }^{10} \mathrm{~N}$ & 1.333 & ${ }^{10} \mathrm{M}$ \\
\hline 1.257 & ${ }^{8} \mathrm{G}$ & 1.273 & ${ }^{6} \mathrm{G}$ & 1.108 & ${ }^{4} \mathbf{I}$ & 1.059 & ${ }^{2} \mathrm{~L}$ & 1.164 & ${ }^{6} \mathrm{M}$ & & \\
\hline 1.314 & ${ }^{6} \mathrm{~F}$ & 1.333 & ${ }^{4} \mathrm{~F}$ & 1.159 & ${ }^{6} \mathrm{I}$ & 1.077 & ${ }^{4} \mathrm{~L}$ & 1.193 & ${ }^{8} \mathrm{M}$ & & \\
\hline 1.371 & ${ }^{4} \mathrm{D}$ & 1.394 & ${ }^{10} \mathrm{H}$ & 1.179 & ${ }^{10} \mathrm{~K}$ & 1.096 & ${ }^{10} \mathrm{M}$ & 1.230 & ${ }^{10} \mathrm{M}$ & & \\
\hline 1.600 & ${ }^{4} \mathrm{P}$ & 1.414 & ${ }^{8} \mathrm{G}$ & 1.231 & ${ }^{4} \mathrm{H}$ & 1.108 & ${ }^{6} \mathrm{~L}$ & 1.238 & ${ }^{6} \mathrm{~L}$ & 1.172 & ${ }^{6} \mathrm{Q}$ \\
\hline 1.657 & ${ }^{6} \mathrm{D}$ & 1.434 & ${ }^{6} \mathrm{~F}$ & & ${ }^{8} \mathbf{I}$ & 1.152 & ${ }^{8} \mathrm{~L}$ & 1.267 & ${ }^{8} \mathrm{~L}$ & 1.188 & ${ }^{8} \mathrm{Q}$ \\
\hline 1.714 & ${ }^{8} \mathrm{~F}$ & 1.556 & ${ }^{6} \mathrm{D}$ & 1.282 & ${ }^{6} \mathrm{H}$ & 1.176 & ${ }^{4} \mathrm{~K}$ & 1.304 & ${ }^{10} \mathrm{~L}$ & 1.208 & ${ }^{10} \mathrm{Q}$ \\
\hline 1.772 & ${ }^{10} \mathrm{G}$ & 1.576 & ${ }^{8} \mathrm{~F}$ & 1.323 & ${ }^{10} \mathrm{I}$ & 1.207 & ${ }^{6} \mathrm{~K}$ & 1.333 & ${ }^{8} \mathrm{~K}$ & 1.241 & ${ }^{8} \mathrm{O}$ \\
\hline 1.886 & ${ }^{6} \mathrm{P}$ & 1.596 & ${ }^{10} \mathrm{G}$ & 1.354 & ${ }^{8} \mathrm{H}$ & & ${ }^{10} \mathrm{~L}$ & 1.371 & ${ }^{10} \mathrm{~K}$ & 1.261 & ${ }^{10} \mathrm{O}$ \\
\hline 2.000 & ${ }^{6} \mathrm{~S}$ & 1.697 & ${ }^{8} \mathrm{D}$ & 1.385 & ${ }^{6} \mathrm{G}$ & 1.251 & ${ }^{8} \mathrm{~K}$ & 1.429 & ${ }^{10} \mathrm{I}$ & 1.310 & ${ }^{10} \mathrm{~N}$ \\
\hline 2.057 & ${ }^{8} \mathrm{D}$ & 1.758 & ${ }^{10} \mathrm{~F}$ & 1.446 & ${ }^{10} \mathrm{H}$ & 1.294 & ${ }^{6} \mathrm{I}$ & & & & \\
\hline 2.229 & ${ }^{10} \mathrm{~F}$ & 1.778 & ${ }^{8} \mathrm{P}$ & 1.456 & ${ }^{8} \mathrm{G}$ & $\begin{array}{l}1.307 \\
1337\end{array}$ & ${ }^{10} \mathrm{~K}$ & & & & \\
\hline $\begin{array}{l}2.286 \\
2.572\end{array}$ & $\begin{array}{r}{ }^{8} \mathrm{P} \\
{ }^{10} \mathrm{D}\end{array}$ & $\begin{array}{l}1.879 \\
1.960\end{array}$ & $\begin{array}{l}{ }^{10} \mathrm{D} \\
{ }^{10} \mathrm{P}\end{array}$ & $\begin{array}{l}1.538 \\
1.549\end{array}$ & $\begin{array}{c}{ }^{8} \mathrm{~F} \\
{ }^{10} \mathrm{G}\end{array}$ & $\begin{array}{l}1.337 \\
1.393\end{array}$ & $\begin{array}{r}{ }^{8} \mathbf{I} \\
{ }^{10} \mathrm{I}\end{array}$ & 960 & ${ }^{2} \mathrm{Q}$ & 1.226 & ${ }^{8} \mathrm{Q}$ \\
\hline & & 2.000 & ${ }^{10} \mathrm{~S}$ & 1.631 & ${ }^{10} \mathrm{~F}$ & 1.412 & ${ }^{8} \mathrm{H}$ & 0.970 & ${ }^{4} \mathrm{Q}$ & 1.243 & ${ }^{10} \mathrm{Q}$ \\
\hline$J=7$ & & $J=$ & & 1.692 & ${ }^{10} \mathrm{D}$ & 1.467 & ${ }^{10} \mathrm{H}$ & 0.988 & ${ }^{6} \mathrm{Q}$ & 1.290 & ${ }^{10} \mathrm{O}$ \\
\hline 0.000 & ${ }^{10} \mathrm{~L}$ & 0.308 & ${ }^{10} \mathrm{~N}$ & $J=$ & & & & 1.012 & $\begin{array}{l}{ }^{8} \mathbf{Q} \\
{ }^{2} \mathrm{O}\end{array}$ & & \\
\hline 0.222 & ${ }^{8} \mathrm{~K}$ & 0.462 & ${ }^{8} \mathrm{M}$ & 0.471 & ${ }^{10} \mathrm{Q}$ & & & & ${ }^{10} \mathrm{Q}$ & & \\
\hline 0.444 & ${ }^{6} \mathbf{I}$ & 0.587 & ${ }^{10} \mathrm{M}$ & 0.588 & ${ }^{8} \mathrm{O}$ & 0.762 & ${ }^{6} \mathrm{Q}$ & 1.054 & ${ }^{4} \mathrm{O}$ & 1.273 & ${ }^{10} \mathrm{Q}$ \\
\hline
\end{tabular}




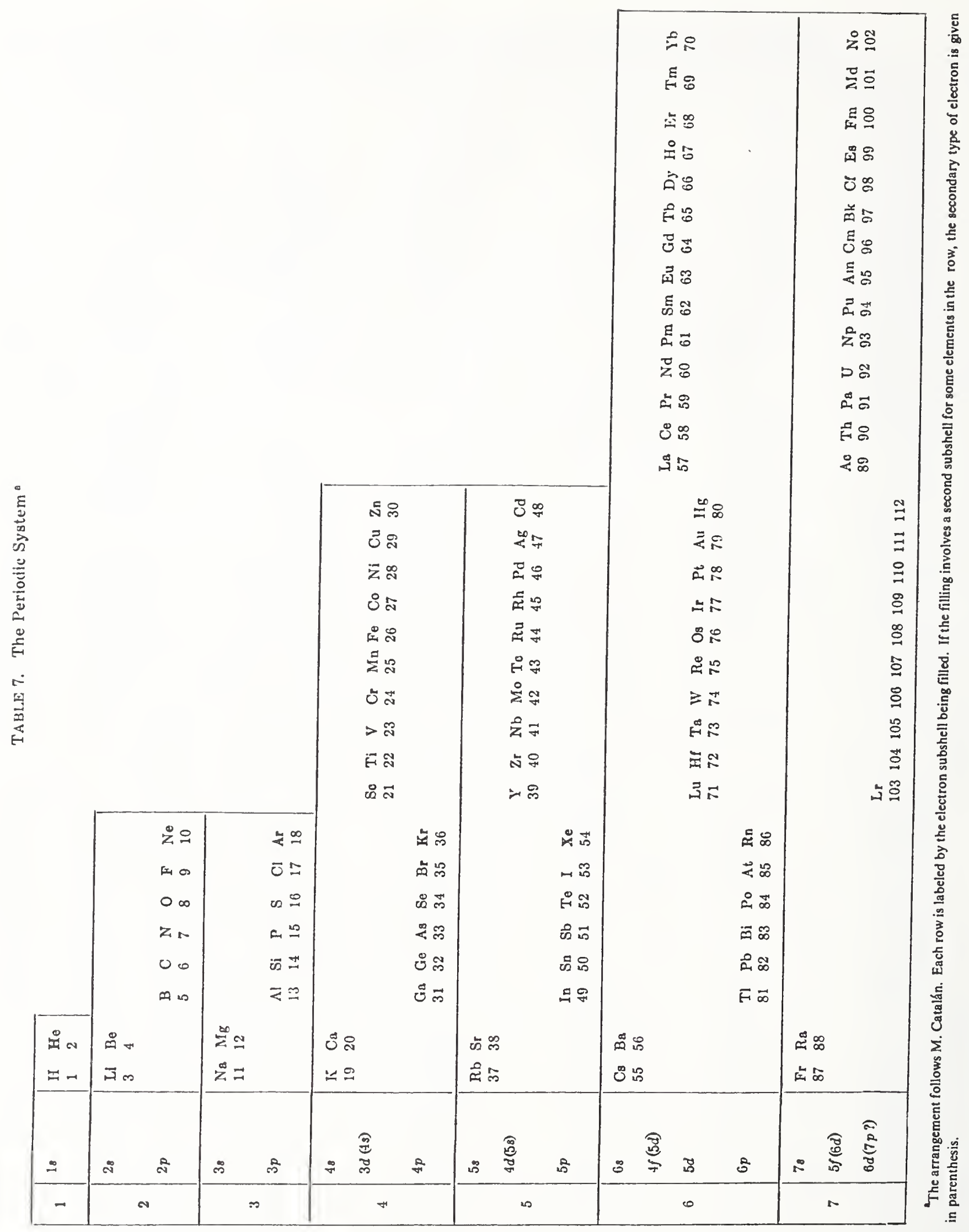




\section{LANTHANUM}

\section{La I}

57 electrons

$Z=57$

Ground state $\left(1 s^{2} 2 s^{2} 2 p^{6} 3 s^{2} 3 p^{6} 3 d^{10} 4 s^{2} 4 p^{6} 4 d^{10} 5 s^{2} 5 p^{6}\right) 5 d 6 s^{2}{ }^{2} \mathrm{D}_{3 / 2}$

Ionization energy $44981 \pm 5 \mathrm{~cm}^{-1}$

$5.5770 \pm 0.0006 \mathrm{eV}$

Identified even configurations

$5 d 6 s^{2}, 5 d^{2} 6 s, 5 d^{3}, 4 f 6 s 6 p, 5 d^{2} 7 s, 5 d 6 s 7 s, 5 d^{2} 6 d$

Identified odd configurations

$5 d 6 s 6 p, 5 d^{2} 6 p, 6 s^{2} 6 p, 4 f 5 d 6 s, 4 f 6 s^{2}, 4 f 5 d^{2} ?, 5 d^{2} 7 p ?$, $6 s^{2} 8 p-23 p$

\section{Emission Spectrum, Energy Levels, $g$ Values}

Meggers' [1932] list of wavelengths from are and spark sources has about 700 lines assigned to La I (2647-10952 $\AA$ ). The basic analysis of the spectrum by Russell and Meggers [1932] gave classifications for about 540 of these lines, and their list has not yet been superseded by publication of the more recent observations. Fisher, Knopf, and Kinney [1959] gave $14 \mathrm{La}$ lines in the infrared region 1.11-1.41 $\mu \mathrm{m}$, with 10 lines classified by known La I levels.

Stein [1967a, 1967b] was able to locate the $5 d^{3}{ }^{4} \mathrm{~F}$ term using modern computer techniques to calculate several important configurations of both parities and to make energy-level searches. The more comprehensive work on the analysis during the past 10 years is partly based on new but as yet unpublished observations carried out at the Argonne National Laboratory and at the Laboratoire Aimé Cotton in Orsay [Giacchetti and Wilson, 1968, 1976; Ben Ahmed, Bauche-Arnoult, and Wyart, 1974; Ben Ahmed, Verges, Wilson, and Giacchetti, 1976; Ben Ahmed, 1976, 1977]. Giacchetti and Wilson photographed the spectrum emitted from electrodeless lamps over the region 2500-9000 $\AA$, and Ben Ahmed and Verges obtained Fourier-transform spectra of such lamps in the range $6900 \AA$ to $2.9 \mu \mathrm{m}$. The list of stronger La lines given by Meggers, Corliss, and Scribner [1975] for the region 2187-8840 $\AA$ includes some energy-level classifications of La I lines by Giacchetti and Wilson, as well as classifications from the earlier analysis of Russell and Meggers.

All levels given here except the $6 s^{2} 8 p-23 p$ series members were reevaluated or newly derived in the extensions of the analysis mentioned above. The level positions are from Ben Ahmed et al. [1976a, 1976b], Ben Ahmed [1976, 1977], and Giacchetti and Wilson [1976]. The combination arrays show the positions of the low even levels to be consistent to within a few units in the third place [Giacchetti and Wilson, 1976].

Meggers' measurements of optical Zeeman-effect patterns in La I and La II were largely superseded by the more accurate determinations of Harrison, Rosen, and McNally [1945] at M.I.T. Zeeman data for $152 \mathrm{La}$ I lines and $506 \mathrm{La}$ II lines were given by Harrison et al., who noted that their $g$-value determinations from different lines "agree usually to within $0.004 \mathrm{~g}$ unit." New measurements of Zeeman-effect patterns obtained at the Argonne Laboratory enabled Ben Ahmed to determine many $J$ values and extend the analysis. The two- and threeplace $g$ values here are from these new data [Ben Ahmed et al., 1976a, 1976b; Ben Ahmed, 1976, 1977], or from Harrison et al. [1945], or are averages of values from these sources. Childs and Goodman [1971] used the atomic-beam magnetic-resonance technique to obtain the fiveplace $g$ values given for 13 low even levels. The stated uncertainties vary from 3 to 8 units in the fifth place except for the ${ }^{4} \mathrm{P}_{1 / 2}$ level and the two $5 d^{2}\left({ }^{1} \mathrm{D}\right) 6 s^{2} \mathrm{D}$ levels, for which the uncertainties are 2 to 3 units in the fourth place. 


\section{Theoretical Interpretation}

Russell and Meggers identified the lowest even and odd levels as belonging respectively to $5 d 6 s^{2}, 5 d^{2} 6 s$ and $5 d 6 s 6 p, 6 s^{2} 6 p$ terms. A number of their level assignments to higher configurations such as $5 d^{2} 7 s$ and $5 d^{2} 6 p$ have also been confirmed, but much of the present interpretation was possible only with modern calculations and extension of the analysis. The eigenvector percentages given here for the even levels are from the calculation of $\left(5 d 6 s^{2}+\right.$ $\left.5 d^{2} 6 s+5 d^{3}+4 f 6 s 6 p+5 d 6 s 7 s+5 d^{2} 7 s\right)$ by Ben Ahmed et al. [1976a]; these six configurations account for the known even levels up to about $33000 \mathrm{~cm}^{-1}$. Although configuration-interaction and intermediate-coupling effects are apparent in the eigenvectors, the purities nevertheless allow the grouping of most of these levels into Russell-Saunders terms. Ben Ahmed at al. [1976a] assigned six levels to two terms of the $5 d^{2} 6 d$ configuration, which was not included in the calculation. With regard to the uninterpreted even levels above $33000 \mathrm{~cm}^{-1}$ these authors note that "Several overlapping configurations are expected to lie in this energy region, in particular $6 p^{2} 5 d, 5 d^{2} 6 d, 5 d 6 s 6 d$ and $6 s^{2} 6 d$, not forgetting those levels above $35000 \mathrm{~cm}^{-1}$ belonging to the configurations already studied. Because of this confusion of levels (made worse by the large departures from LS coupling) we have not yet been able to classify the great majority of these levels."

Stein's [1967b] calculations for La I included the odd group $\left(5 d 6 s 6 p+6 s^{2} 6 p+5 d^{2} 6 p+4 f 6 s^{2}+\right.$ $4 f 5 d 6 s$ ). He found several of the configuration interactions to be very large (see below), and his eigenvector assignments correctly located the $4 f 6 s^{2}$ and $4 f 5 d 6 s$ configurations and showed the latter to be the main configuration for most of the levels previously assigned to $4 f 5 d^{2}$. Brewer [1971] also assigned or reassigned a number of the odd levels to $4 f 5 d 6 s$.

The percentages given here for the odd levels are from the thesis of Ben Ahmed [1977], who has calculated the five configurations listed above in connection with her extension of the analysis. We list the percentage for the largest single eigenvector component first, as usual. This number is, however, followed by the largest total percentage contribution to the eigenvector from a configuration different from that for the first percentage; if no such percentage from another configuration is $\geqslant 10 \%$, the second percentage space is blank. The large configuration mixing is apparent, and in some cases the second listed configuration is the largest total contributor to the eigenvector. Ben Ahmed regards the calculation as preliminary; a calculation including additional configurations is needed, since large deviations occur for some levels in the region above about $23000 \mathrm{~cm}^{-1}$. The inclusion of additional configurations, particularly $4 f 5 d^{2}$, will of course change some of the eigenvectors significantly. Ten of the eleven unidentified levels between 29466 and $33820 \mathrm{~cm}^{-1}$ can be assigned to doublet terms of the $4 f 5 d\left({ }^{3} \mathrm{G}^{\circ}\right) 6 s$ subconfiguration; however, these levels and the $5 d^{2}\left({ }^{3} \mathrm{P}\right) 6 p{ }^{4} \mathrm{~S}_{3 / 2}^{\circ}$ level were omitted from the calculation because of large deviations [Ben Ahmed, 1977]. The tentative assignments of several odd levels above $34000 \mathrm{~cm}^{-1}$ to $4 f 5 d^{2}, 5 d^{2} 7 p$, and $5 d 6 s 7 p$ terms are also due to Ben Ahmed. The $4 f 5 d^{2}$ configuration probably accounts for many of the unidentified levels in this region.

\section{$5 d 6 s^{2}{ }^{2} \mathbf{D}-6 s^{2} n p{ }^{2} \mathbf{P}^{\circ}$ Absorption Series and the Ionization Energy}

Garton and Wilson [1966] observed two series interpreted as $5 d 6 s^{2}{ }^{2} \mathrm{D}_{5 / 2}-6 s^{2} n p{ }^{2} \mathrm{P}_{3 / 2}^{\circ}$ and $5 d 6 s^{2}{ }^{2} \mathrm{D}_{3 / 2}-6 s^{2} n p{ }^{2} \mathrm{P}_{1 / 2,3 / 2}^{\circ}$, respectively $(n=8-23)$. The lines are broadened by autoionization for $n \geqslant 9$, and none of the (theoretical) doublets of the latter series were resolved. In some cases we have given the positions for the $6 s^{2} n p{ }^{2} \mathrm{P}_{3 / 2}^{\circ}$ levels as derived from the former series separately from the corresponding $n p{ }^{2} \mathrm{P}^{\circ}$ positions from the latter series, but the differences may not be significant. Garton and Wilson derived the quoted value for the La II $6 s^{2}{ }^{1} \mathrm{~S}_{0}$ limit from these series, and thus also the $5 d^{2}{ }^{3} \mathrm{~F}_{2}$ principal ionization energy.

\section{References}

Ben Ahmed, Z., unpublished material (1976). EL ZE PT

Ben Ahmed, Z., Thesis, Univ. Paris. Orsay (1977). EL CL ZE PT

Ben Ahmed, Z., Bauche-Arnoult, C., and Wyart, J. F., Physica (Utrecht) 77, 148 (1974). EL ZE Hfs PT

Ben Ahmed, Z, Verges, J, Wilson, M., and Giacchetti, A., Physica (Utrecht) 84C, 275 (1976a). EL ND ZE PT

Ben Ahmed, Z., Verges, J., Wilson, M., and Giacchetti, A., unpublished material (1976b). EL ZE

Brewer, L., J. Opt. Soc. Am. 61, 1101 (1971). ND 
Childs, W. J., and Goodman, L. S., Phys. Rev. A 3, 25 (1971). ZE Hfs PT

Fischer, W., Hühnermann, H., and Mandrek, K., Z. Phys. 269, 245 (1974). IS

Fisher, R. A., Knopf, Jr., W. C., and Kinney, F. E., Astrophys. J. 130, 683 (1959). CL W

Garton, W. R. S., and Wilson, M., Astrophys. J. 145, 333 (1966). EL CL W IP

Giacchetti, A., and Wilson, M., J. Opt. Soc. Am. 58, 740A (1968).

Giacehetti, A., and Wilson, M., unpublished material (1976). EL CL Hfs

Harrison, G. R., Rosen, N., and MeNally, Jr., J. R., J. Opt. Soc. Am. 35, 658 (1945). ZE

King, A. S., and Carter, E., Astrophys. J. 65,86 (1927). W

Meggers, W. F., J. Washington Acad. Sei. 17, 25 (1927). EL

Meggers, W. F., J. Res. Nat. Bur. Stand. (U.S.) 9, 239 (1932). W ZE

Meggers, W. F., and Burns, K., J. Opt. Soc. Am. 14, 449 (1927). EL Hfs

Meggers, W. F., Corliss, C. H., and Scribner, B. F., Nat. Bur. Stand. (U.S.), Monogr. 145, Part 1, 403 pp. (1975). CL

Moore, C. E., Atomic Energy Levels, Nat. Bur. Stand. Ref. Data Ser., Nat. Bur. Stand. (U.S.), 35, Vol. III, 282 pp. (1971). Compilation EL ZE IP

Russell, H. N., and Meggers, W. F., J. Res. Nat. Bur. Stand. (U.S.) 9, 625 (1932). EL CL ZE IP

Stein, J., J. Opt. Soc. Am. 57, 383 (1967a). EL ND CL PT

Stein, J., Thesis, Hebrew Univ. Jerusalem, Israel, 152 pp. (1967b). EL ND CL PT

Wilson, M., Phys. Rev. A 3, 45 (1971). AT

[May 1977]

La I, Even Parity

\begin{tabular}{|c|c|c|c|c|c|c|c|}
\hline Configuration & Term & $J$ & $\begin{array}{l}\text { Level } \\
\left(\mathrm{cm}^{-1}\right)\end{array}$ & $g$ & \multicolumn{3}{|c|}{ Leading percentages } \\
\hline $5 d 6 s^{2}$ & ${ }^{2} \mathrm{D}$ & $\begin{array}{l}3 / 2 \\
5 / 2\end{array}$ & $\begin{array}{r}0.000 \\
1053.164\end{array}$ & $\begin{array}{l}0.79755 \\
1.19907\end{array}$ & $\begin{array}{l}85 \\
82\end{array}$ & $\begin{array}{l}10 \\
13\end{array}$ & $5 d^{2}\left({ }^{1} \mathrm{D}\right) 6 s^{2} \mathrm{D}$ \\
\hline $5 d^{2}\left({ }^{3} \mathrm{~F}\right) 6 s$ & ${ }^{4} \mathrm{~F}$ & $\begin{array}{l}3 / 2 \\
5 / 2 \\
7 / 2 \\
9 / 2\end{array}$ & $\begin{array}{l}2668.188 \\
3010.002 \\
3494.526 \\
4121.572\end{array}$ & $\begin{array}{l}0.40446 \\
1.02940 \\
1.23742 \\
1.33278\end{array}$ & $\begin{array}{r}98 \\
98 \\
99 \\
100\end{array}$ & $\begin{array}{l}1 \\
1 \\
1\end{array}$ & $\begin{array}{l}5 d 6 s^{2}{ }^{2} \mathrm{D} \\
5 d 6 s^{2}{ }^{2} \mathrm{D} \\
\left({ }^{3} \mathrm{~F}\right)^{2 \mathrm{~F}}\end{array}$ \\
\hline $5 d^{2}\left({ }^{3} \mathrm{~F}\right) 6 s$ & ${ }^{2} \mathrm{~F}$ & $\begin{array}{l}5 / 2 \\
7 / 2\end{array}$ & $\begin{array}{l}7011.909 \\
8052.162\end{array}$ & $\begin{array}{l}0.89830 \\
1.13469\end{array}$ & $\begin{array}{l}86 \\
93\end{array}$ & $\begin{array}{l}5 \\
4\end{array}$ & $\begin{array}{l}\left({ }^{1} \mathrm{D}\right){ }^{2} \mathrm{D} \\
5 d^{3}{ }^{2} \mathrm{~F}\end{array}$ \\
\hline $5 d^{2}\left({ }^{3} \mathrm{P}\right) 6 s$ & ${ }^{4} \mathrm{P}$ & $\begin{array}{l}1 / 2 \\
3 / 2 \\
5 / 2\end{array}$ & $\begin{array}{l}7231.407 \\
7490.521 \\
7679.939\end{array}$ & $\begin{array}{l}2.65252 \\
1.70427 \\
1.50558\end{array}$ & $\begin{array}{l}99 \\
97 \\
82\end{array}$ & $\begin{array}{r}1 \\
2 \\
10\end{array}$ & $\begin{array}{l}\left({ }^{1} \mathrm{~S}\right){ }^{2} \mathrm{~S} \\
\left({ }^{1} \mathrm{D}\right){ }^{2} \mathrm{D} \\
\left({ }^{1} \mathrm{D}\right){ }^{2} \mathrm{D}\end{array}$ \\
\hline $5 d^{2}\left({ }^{1} \mathrm{D}\right) 6 s$ & ${ }^{2} \mathrm{D}$ & $\begin{array}{l}3 / 2 \\
5 / 2\end{array}$ & $\begin{array}{l}8446.044 \\
9183.797\end{array}$ & $\begin{array}{l}0.93603 \\
1.25449\end{array}$ & $\begin{array}{l}61 \\
67\end{array}$ & $\begin{array}{l}18 \\
16\end{array}$ & $\begin{array}{l}\left({ }^{3} \mathrm{P}\right){ }^{2} \mathrm{P} \\
\left({ }^{3} \mathrm{P}\right){ }^{4} \mathrm{P}\end{array}$ \\
\hline $5 d^{2}\left({ }^{3} \mathrm{P}\right) 6 s$ & ${ }^{2} \mathrm{P}$ & $\begin{array}{l}1 / 2 \\
3 / 2\end{array}$ & $\begin{array}{l}9044.214 \\
9719.439\end{array}$ & $\begin{array}{l}0.690 \\
1.220\end{array}$ & $\begin{array}{l}72 \\
54\end{array}$ & $\begin{array}{l}26 \\
21\end{array}$ & $5 d^{3}{ }^{2} \mathrm{P}$ \\
\hline $5 d^{2}\left({ }^{1} \mathrm{G}\right) 6 s$ & ${ }^{2} \mathrm{G}$ & $\begin{array}{l}9 / 2 \\
7 / 2\end{array}$ & $\begin{array}{l}9919.821 \\
9960.904\end{array}$ & $\begin{array}{l}1.107 \\
0.892\end{array}$ & $\begin{array}{l}91 \\
88\end{array}$ & $\begin{array}{l}8 \\
9\end{array}$ & $5 d^{32} \mathrm{G}$ \\
\hline $5 d^{3}$ & ${ }^{4} \mathrm{~F}$ & $\begin{array}{l}3 / 2 \\
5 / 2 \\
7 / 2 \\
9 / 2\end{array}$ & $\begin{array}{l}12430.609 \\
12787.404 \\
13238.323 \\
13747.276\end{array}$ & $\begin{array}{l}0.411 \\
1.026 \\
1.228\end{array}$ & $\begin{array}{l}98 . \\
99 \\
99 \\
97\end{array}$ & 1 & $\begin{array}{l}{ }^{2} \mathrm{D} 2 \\
{ }^{2} \mathrm{G}\end{array}$ \\
\hline $5 d^{3}$ & ${ }^{4} \mathrm{P}$ & $\begin{array}{l}1 / 2 \\
3 / 2 \\
5 / 2\end{array}$ & $\begin{array}{l}16617.30 \\
16735.14 \\
17099.38\end{array}$ & 1.698 & $\begin{array}{l}97 \\
94 \\
97\end{array}$ & $\begin{array}{l}2 \\
2 \\
2\end{array}$ & $\begin{array}{l}5 d^{2}\left({ }^{1} \mathrm{~S}\right) 6 s^{2} \mathrm{~S} \\
5 d^{2}\left({ }^{3} \mathrm{P}\right) 6 s^{2} \mathrm{P} \\
{ }^{2} \mathrm{D} 1\end{array}$ \\
\hline $5 d^{2}\left({ }^{1} \mathrm{~S}\right) 6 s$ & ${ }^{2} \mathrm{~S}$ & $1 / 2$ & 16991.42 & & 97 & 2 & $5 d^{34} \mathrm{P}$ \\
\hline $5 d^{3}$ & ${ }^{2} \mathrm{G}$ & $\begin{array}{l}7 / 2 \\
9 / 2\end{array}$ & $\begin{array}{l}17023.36 \\
17140.90\end{array}$ & 0.880 & $\begin{array}{l}90 \\
54\end{array}$ & $\begin{array}{r}9 \\
39\end{array}$ & $\begin{array}{l}5 d^{2}\left({ }^{1} \mathrm{G}\right) 6 s^{2} \mathrm{G} \\
{ }^{2} \mathrm{H}\end{array}$ \\
\hline $5 d^{3}$ & ${ }^{2} \mathrm{D} 2$ & $\begin{array}{l}3 / 2 \\
5 / 2\end{array}$ & $\begin{array}{l}18037.64 \\
18776.62\end{array}$ & & $\begin{array}{l}57 \\
68\end{array}$ & $\begin{array}{l}26 \\
21\end{array}$ & ${ }^{2} \mathrm{D} 1$ \\
\hline
\end{tabular}


La I, Even Parity-Continued

\begin{tabular}{|c|c|c|c|c|c|c|c|c|c|}
\hline Configuration & Term & $J$ & $\begin{array}{l}\text { Level } \\
\left(\mathrm{cm}^{-1}\right)\end{array}$ & $g$ & \multicolumn{4}{|c|}{ Leading percentages } & \\
\hline $5 d^{3}$ & ${ }^{2} \mathrm{H}$ & $\begin{array}{c}11 / 2 \\
9 / 2\end{array}$ & $\begin{array}{l}18310.92 \\
18315.88\end{array}$ & 0.970 & $\begin{array}{r}100 \\
61\end{array}$ & & 36 & ${ }^{2} \mathrm{G}$ & \\
\hline $5 d^{3}$ & ${ }^{2} \mathrm{P}$ & $\begin{array}{l}1 / 2 \\
3 / 2\end{array}$ & $\begin{array}{l}20392.60 \\
21037.30\end{array}$ & $\begin{array}{l}0.709 \\
1.316\end{array}$ & $\begin{array}{l}71 \\
67\end{array}$ & & $\begin{array}{l}26 \\
24\end{array}$ & $5 d^{2}\left({ }^{3} \mathrm{P}\right) 6 s^{2} \mathrm{P}$ & \\
\hline $5 d^{3}$ & ${ }^{2} \mathrm{~F}$ & $\begin{array}{l}7 / 2 \\
5 / 2\end{array}$ & $\begin{array}{l}21943.80 \\
21969.32\end{array}$ & & $\begin{array}{l}95 \\
93\end{array}$ & & $\begin{array}{l}4 \\
3\end{array}$ & $5 d^{2}\left({ }^{3} \mathrm{~F}\right) 6 s^{2} \mathrm{~F}$ & \\
\hline $5 d^{3}$ & ${ }^{2} \mathrm{D} 1$ & $\begin{array}{l}5 / 2 \\
3 / 2\end{array}$ & 25414.63 & & $\begin{array}{l}64 \\
56\end{array}$ & & $\begin{array}{l}23 \\
32\end{array}$ & ${ }^{2} \mathrm{D} 2$ & \\
\hline $4 f 6 s\left({ }^{3} \mathrm{~F}^{\circ}\right) 6 p$ & ${ }^{4} \mathrm{~F}$ & $\begin{array}{l}3 / 2 \\
5 / 2 \\
7 / 2 \\
9 / 2\end{array}$ & $\begin{array}{l}28742.34 \\
28754.96 \\
30055.05\end{array}$ & $\begin{array}{l}0.45 \\
0.93 \\
1.19\end{array}$ & $\begin{array}{l}96 \\
64 \\
54 \\
91\end{array}$ & & $\begin{array}{r}2 \\
15 \\
15 \\
3\end{array}$ & $\begin{array}{l}\left({ }^{3} \mathrm{~F}^{\circ}\right){ }^{2} \mathrm{D} \\
\left({ }^{1} \mathrm{~F}^{\circ}\right){ }^{2} \mathrm{~F} \\
\left({ }^{1} \mathrm{~F}^{\circ}\right){ }^{2} \mathrm{~F} \\
\left({ }^{3} \mathrm{~F}^{\circ}\right){ }^{2} \mathrm{G}\end{array}$ & \\
\hline $4 f 6 s\left({ }^{3} \mathrm{~F}^{\circ}\right) 6 p$ & & $7 / 2$ & 29045.86 & 1.15 & 40 & ${ }^{4} \mathrm{~F}$ & 33 & $\left({ }^{1} \mathrm{~F}^{\circ}\right)^{2} \mathrm{~F}$ & \\
\hline $4 f 6 s\left({ }^{3} \mathrm{~F}^{\circ}\right) 6 p$ & ${ }^{4} \mathrm{G}$ & $\begin{array}{l}5 / 2 \\
7 / 2 \\
9 / 2 \\
11 / 2\end{array}$ & $\begin{array}{l}29578.82 \\
30401.70 \\
30934.76\end{array}$ & $\begin{array}{l}0.78 \\
1.03 \\
1.16\end{array}$ & $\begin{array}{r}62 \\
77 \\
78 \\
100\end{array}$ & & $\begin{array}{l}23 \\
15 \\
18\end{array}$ & $\begin{array}{l}\left({ }^{3} \mathrm{~F}^{\circ}\right){ }^{4} \mathrm{~F} \\
\left({ }^{1} \mathrm{~F}^{\circ}\right){ }^{2} \mathrm{~F} \\
\left({ }^{1} \mathrm{~F}^{\circ}\right){ }^{2} \mathrm{G}\end{array}$ & \\
\hline $5 d^{2}\left({ }^{3} \mathrm{~F}\right) 7 s$ & & $3 / 2$ & 29874.97 & 0.70 & 42 & ${ }^{4} \mathrm{~F}$ & 30 & $5 d 6 s\left({ }^{3} \mathrm{D}\right) 7 s^{4} \mathrm{D}$ & \\
\hline $5 d^{2}\left({ }^{3} \mathrm{~F}\right) 7 s$ & & $5 / 2$ & 29905.33 & 1.14 & 20 & ${ }^{4} \mathrm{~F}$ & 20 & $5 d 6 s\left({ }^{1} \mathrm{D}\right) 7 s^{2} \mathrm{D}$ & - \\
\hline $5 d 6 s\left({ }^{3} \mathrm{D}\right) 7 s$ & ${ }^{4} \mathrm{D}$ & $\begin{array}{l}1 / 2 \\
3 / 2 \\
5 / 2 \\
7 / 2\end{array}$ & $\begin{array}{l}30019.24 \\
30169.82 \\
30354.28 \\
31287.59\end{array}$ & $\begin{array}{l}0.00 \\
0.91 \\
1.07 \\
1.41\end{array}$ & $\begin{array}{r}100 \\
58 \\
44 \\
85\end{array}$ & & $\begin{array}{r}39 \\
30 \\
7\end{array}$ & $\begin{array}{l}5 d^{2}\left({ }^{3} \mathrm{~F}\right) 7 s^{4} \mathrm{~F} \\
5 d^{2}\left({ }^{3} \mathrm{~F}\right) 7 s^{4} \mathrm{~F} \\
\left({ }^{3} \mathrm{~F}^{\circ}\right){ }^{4} \mathrm{D}\end{array}$ & \\
\hline $4 f 6 s\left({ }^{1} \mathrm{~F}^{\circ}\right) 6 p$ & ${ }^{2} \mathrm{~F}$ & $5 / 2$ & 30305.61 & 0.84 & 49 & & 25 & $\left({ }^{3} \mathrm{~F}^{\circ}\right){ }^{4} \mathrm{G}$ & \\
\hline $4 f 6 s\left({ }^{3} \mathrm{~F}^{\circ}\right) 6 p$ & ${ }^{4} \mathrm{D}$ & $\begin{array}{l}5 / 2 \\
3 / 2 \\
1 / 2 \\
7 / 2\end{array}$ & $\begin{array}{l}30908.86 \\
30988.36 \\
31061.85 \\
31925.00\end{array}$ & $\begin{array}{l}1.25 \\
1.12 \\
0.00 \\
1.27\end{array}$ & $\begin{array}{r}58 \\
85 \\
100 \\
63\end{array}$ & & $\begin{array}{r}23 \\
7 \\
14\end{array}$ & $\begin{array}{l}\left({ }^{1} \mathrm{~F}^{\circ}\right){ }^{2} \mathrm{D} \\
\left({ }^{1} \mathrm{~F}^{\circ}\right){ }^{2} \mathrm{D} \\
\left({ }^{1} \mathrm{~F}^{\circ}\right){ }^{2} \mathrm{G}\end{array}$ & \\
\hline $5 d^{2}\left({ }^{3} \mathrm{~F}\right) 7 s$ & ${ }^{4} \mathrm{~F}$ & $7 / 2$ & 31059.69 & 1.22 & 83 & & 17 & $\left({ }^{3} \mathrm{~F}\right)^{2} \mathrm{~F}$ & \\
\hline $5 d^{2}\left({ }^{3} \mathrm{~F}\right) 7 s$ & & $5 / 2$ & 31119.02 & 0.98 & 48 & ${ }^{4} \mathrm{~F}$ & 46 & $\left({ }^{3} \mathrm{~F}\right){ }^{2} \mathrm{~F}$ & \\
\hline $5 d 6 s\left({ }^{1} \mathrm{D}\right) 7 s$ & & $3 / 2$ & 31247.78 & 0.78 & 33 & ${ }^{2} \mathrm{D}$ & 30 & $5 d^{2}\left({ }^{1} \mathrm{D}\right) 7 s^{2} \mathrm{D}$ & \\
\hline $5 d 6 s\left({ }^{3} \mathrm{D}\right) 7 s$ & & $5 / 2$ & 31351.60 & 1.18 & 34 & ${ }^{4} \mathrm{D}$ & 21 & $\left({ }^{1} \mathrm{D}\right){ }^{2} \mathrm{D}$ & \\
\hline $5 d 6 s\left({ }^{3} \mathrm{D}\right) 7 \mathrm{~s}$ & ${ }^{2} \mathrm{D}$ & $\begin{array}{l}3 / 2 \\
5 / 2\end{array}$ & $\begin{array}{l}31688.66 \\
32872.94\end{array}$ & $\begin{array}{l}0.81 \\
1.19\end{array}$ & $\begin{array}{l}78 \\
74\end{array}$ & & $\begin{array}{r}9 \\
10\end{array}$ & $\begin{array}{l}5 d^{3}{ }^{2} \mathrm{D} 1 \\
5 d^{2}\left({ }^{1} \mathrm{D}\right) 7 s^{2} \mathrm{D}\end{array}$ & \\
\hline $5 d^{2}\left({ }^{3} \mathrm{~F}\right) 7 s$ & ${ }^{4} \mathrm{~F}$ & $9 / 2$ & 31923.96 & 1.34 & 99 & & 1 & $\left({ }^{1} \mathrm{G}\right){ }^{2} \mathrm{G}$ & \\
\hline $5 d^{2}\left({ }^{3} \mathrm{~F}\right) 7 s$ & ${ }^{2} \mathrm{~F}$ & $7 / 2$ & 32108.48 & 1.13 & 82 & & 17 & $\left({ }^{3} \mathrm{~F}\right){ }^{4} \mathrm{~F}$ & \\
\hline $4 f 6 s\left({ }^{1} \mathrm{~F}^{\circ}\right) 6 p ?$ & ${ }^{2} \mathrm{G}$ ? & $\begin{array}{l}7 / 2 \\
9 / 2\end{array}$ & 32219.53 & 1.06 & $\begin{array}{l}46 \\
50\end{array}$ & & & $\begin{array}{l}\left({ }^{3} \mathrm{~F}^{\circ}\right){ }^{4} \mathrm{D} \\
\left({ }^{3} \mathrm{~F}^{\circ}\right){ }^{2} \mathrm{G}\end{array}$ & \\
\hline $4 f 6 s\left({ }^{1} \mathrm{~F}^{\circ}\right) 6 p$ & & $\begin{array}{l}5 / 2 \\
1 / 2\end{array}$ & $\begin{array}{l}32348.34 \\
33143.55\end{array}$ & $\begin{array}{l}1.23 \\
1.39\end{array}$ & & ${ }^{2} \mathrm{D}$ & 36 & $\left({ }^{3} \mathrm{~F}^{\circ}\right){ }^{4} \mathrm{D}$ & \\
\hline $5 d^{2}\left({ }^{3} \mathrm{~F}\right) 6 d$ & ${ }^{4} \mathrm{H}$ & $\begin{array}{l}7 / 2 \\
9 / 2 \\
11 / 2 \\
13 / 2\end{array}$ & $\begin{array}{l}33286.50 \\
33753.41\end{array}$ & $\begin{array}{l}0.78 \\
1.02\end{array}$ & & & & & \\
\hline
\end{tabular}


La I, Even Parity_Continued

La I, Even Parity_Continued

\begin{tabular}{|c|c|c|c|c|c|c|c|c|c|}
\hline Configuration & Term & $J$ & $\begin{array}{l}\text { Level } \\
\left(\mathrm{cm}^{-1}\right)\end{array}$ & $g$ & Configuration & Term & $J$ & $\begin{array}{c}\text { Level } \\
\left(\mathrm{cm}^{-1}\right)\end{array}$ & $g$ \\
\hline \multirow[t]{41}{*}{$5 d^{2}\left({ }^{3} \mathrm{~F}\right) 6 d$} & ${ }^{4} \mathrm{G}$ & $5 / 2$ & 33350.00 & 0.68 & & & $3 / 2$ & 35875.99 & 1.78 \\
\hline & & $\begin{array}{l}7 / 2 \\
9 / 2\end{array}$ & $\begin{array}{l}33756.45 \\
34526.69\end{array}$ & 0.99 & & & $7 / 2$ & 35907.05 & 1.19 \\
\hline & & $11 / 2$ & 35236.20 & 1.21 & & & $7 / 2$ & 35931.75 & 1.28 \\
\hline & & $1 / 2$ & 33419.48 & 1.03 & & & $1 / 2$ & 36027.41 & 2.17 \\
\hline & & $3 / 2$ & 33657.05 & 1.17 & & & $9 / 2$ & 36034.60 & 1.10 \\
\hline & & $5 / 2$ & 33678.21 & 0.99 & & & $3 / 2$ & 36065.91 & 0.96 \\
\hline & & $7 / 2$ & 34032.72 & 0.90 & & & $5 / 2$ & 36109.65 & 1.28 \\
\hline & & $5 / 2$ & 34124.64 & 0.89 & & & $11 / 2$ & 36159.90 & 1.28 \\
\hline & & $1 / 2$ & 34249.24 & 0.97 & & & $3 / 2$ & 36172.80 & 1.24 \\
\hline & & $7 / 2$ & 34272.48 & 0.84 & & & $7 / 2$ & 36220.33 & 1.13 \\
\hline & & $3 / 2$ & 34369.05 & 1.62 & & & $5 / 2$ & 36258.93 & 1.40 \\
\hline & & $5 / 2$ & 34400.06 & 1.21 & & & $9 / 2$ & 36265.28 & 1.22 \\
\hline & & $7 / 2$ & 34482.28 & 1.21 & & & $7 / 2$ & 36292.96 & 1.23 \\
\hline & & $1 / 2$ & 34488.22 & 1.75 & & & $9 / 2$ & 36400.72 & 1.18 \\
\hline & & $5 / 2$ & 34529.88 & 0.88 & & & $7 / 2$ & 36792.36 & 0.89 \\
\hline & & $3 / 2$ & 34545.80 & 0.83 & & & $9 / 2$ & 36822.77 & 0.90 \\
\hline & & $1 / 2$ & 34590.09 & 1.32 & & & $1 / 2$ & 36840.70 & 2.02 \\
\hline & & $9 / 2$ & 34634.98 & 1.07 & & & $5 / 2$ & 36851.32 & 1.21 \\
\hline & & $7 / 2$ & 34663.99 & 1.10 & & & $3 / 2$ & 36853.58 & 1.13 \\
\hline & & $1 / 2$ & 34752.58 & 1.33 & & & $3 / 2$ & 37092.15 & 0.66 \\
\hline & & $3 / 2$ & 34758.54 & 1.28 & & & $3 / 2$ & 37248.61 & 1.38 \\
\hline & & $5 / 2$ & 34787.23 & 1.01 & & & $5 / 2$ & 37544.15 & 1.06 \\
\hline & & $1 / 2$ & 34860.96 & 0.95 & & & $3 / 2$ & 37612.91 & 1.43 \\
\hline & & $5 / 2$ & 34880.63 & 0.91 & & & $1 / 2$ & 37659.48 & 0.91 \\
\hline & & $3 / 2$ & 34906.89 & 1.07 & & & $7 / 2$ & 37833.78 & 0.90 \\
\hline & & $7 / 2$ & 34968.73 & 1.05 & & & $5 / 2$ & 37855.75 & 1.28 \\
\hline & & $3 / 2$ & 35009.99 & 1.22 & & & $5 / 2$ & 37903.24 & 0.93 \\
\hline & & $7 / 2$ & 35096.10 & 1.20 & & & $9 / 2$ & 38172.80 & 0.93 \\
\hline & & $5 / 2$ & 35117.70 & 1.20 & & & $7 / 2$ & 38178.00 & 0.99 \\
\hline & & $9 / 2$ & 35169.58 & 0.95 & & & $5 / 2$ & 38378.90 & 1.06 \\
\hline & & $3 / 2$ & 35280.42 & 0.94 & & & $7 / 2$ & 38391.75 & 1.04 \\
\hline & & $7 / 2$ & 35298.12 & 1.09 & & & $3 / 2$ & 38478.84 & 0.90 \\
\hline & & $3 / 2$ & 35308.40 & 1.04 & & & $9 / 2$ & 38755.72 & \\
\hline & & $5 / 2$ & 35393.38 & 1.28 & & & $1 / 2$ & 38921.15 & 0.22 \\
\hline & & $5 / 2$ & 35414.45 & 1.10 & & & $7 / 2$ & 38991.90 & 1.03 \\
\hline & & $9 / 2$ & 35445.98 & 1.11 & & & $3 / 2$ & 39110.34 & 0.87 \\
\hline & & $3 / 2$ & 35494.05 & 0.83 & & & $9 / 2$ & 39391.23 & 1.04 \\
\hline & & $9 / 2$ & 35552.32 & 1.15 & & & $9 / 2$ & 40084.07 & 1.25 \\
\hline & & $7 / 2$ & 35570.48 & 1.11 & & & $1 / 2$ & 40243.01 & 2.24 \\
\hline & & $1 / 2$ & 35581.78 & 1.80 & & & & & \\
\hline & & $3 / 2$ & 35628.33 & 1.13 & $\operatorname{La}$ II $\left({ }^{3} \mathrm{~F}_{2}\right)$ & Limit & & 44981 & \\
\hline
\end{tabular}


La I, Odd Parity

\begin{tabular}{|c|c|c|c|c|c|c|c|c|}
\hline Configuration & Term & $J$ & $\begin{array}{l}\text { Level } \\
\left(\mathrm{cm}^{-1}\right)\end{array}$ & $g$ & \multicolumn{4}{|c|}{ Leading percentages } \\
\hline $5 d 6 s\left({ }^{3} \mathrm{D}\right) 6 p$ & ${ }^{4} \mathrm{~F}^{\circ}$ & $\begin{array}{l}3 / 2 \\
5 / 2 \\
7 / 2 \\
9 / 2\end{array}$ & $\begin{array}{l}13260.38 \\
14804.08 \\
15019.51 \\
16243.17\end{array}$ & $\begin{array}{l}0.52 \\
1.09 \\
1.237\end{array}$ & $\begin{array}{l}71 \\
53 \\
89 \\
97\end{array}$ & & 10 & $5 d^{2} 6 p$ \\
\hline $5 d 6 s\left({ }^{3} \mathrm{D}\right) 6 p$ & & $5 / 2$ & 13631.04 & 1.096 & 43 & ${ }^{4} \mathrm{~F}^{\circ}$ & 10 & $5 d^{2} 6 p$ \\
\hline $5 d 6 s\left({ }^{3} \mathrm{D}\right) 6 p$ & ${ }^{4} \mathrm{D}^{\circ}$ & $\begin{array}{l}1 / 2 \\
3 / 2 \\
5 / 2 \\
7 / 2\end{array}$ & $\begin{array}{l}14095.69 \\
14708.92 \\
15503.64 \\
16099.29\end{array}$ & $\begin{array}{l}0.357 \\
1.01 \\
1.36 \\
1.37\end{array}$ & $\begin{array}{l}49 \\
57 \\
86 \\
81\end{array}$ & & 26 & $6 s^{2}\left({ }^{1} \mathrm{~S}\right) 6 p{ }^{2} \mathrm{P}^{\circ}$ \\
\hline $5 d 6 s\left({ }^{3} \mathrm{D}\right) 6 p$ & & $3 / 2$ & 15031.64 & 0.92 & 25 & ${ }^{2} \mathrm{D}^{\circ}$ & 11 & $5 d^{2} 6 p$ \\
\hline $4 f 6 s^{2}$ & ${ }^{2} \mathrm{~F}^{\circ}$ & $\begin{array}{l}5 / 2 \\
7 / 2\end{array}$ & $\begin{array}{l}15196.83 \\
16538.39\end{array}$ & $\begin{array}{l}0.906 \\
1.179\end{array}$ & $\begin{array}{l}53 \\
51\end{array}$ & & $\begin{array}{l}31 \\
29\end{array}$ & $5 d 6 s 6 p$ \\
\hline $5 d 6 s\left({ }^{3} \mathrm{D}\right) 6 p$ & & $1 / 2$ & 15219.89 & 0.313 & 46 & ${ }^{4} \mathrm{D}^{\circ}$ & 35 & $6 s^{2}\left({ }^{1} \mathrm{~S}\right) 6 p^{2} \mathrm{P}^{\circ}$ \\
\hline $6 s^{2}\left({ }^{1} \mathrm{~S}\right) 6 p$ & & $3 / 2$ & 16280.26 & 1.326 & 40 & ${ }^{2} \mathrm{P}^{\circ}$ & 51 & $5 d 6 s 6 p$ \\
\hline $5 d^{2}\left({ }^{3} \mathrm{~F}\right) 6 p$ & & $5 / 2$ & 16856.80 & 0.810 & 28 & ${ }^{4} \mathrm{G}^{\circ}$ & 24 & $4 f 6 s^{2}{ }^{2} \mathrm{~F}^{\circ}$ \\
\hline $5 d 6 s\left({ }^{3} \mathrm{D}\right) 6 p$ & ${ }^{4} \mathrm{P}^{\circ}$ & $\begin{array}{l}1 / 2 \\
3 / 2 \\
5 / 2\end{array}$ & $\begin{array}{l}17567.49 \\
17797.29 \\
18156.97\end{array}$ & $\begin{array}{l}2.63 \\
1.69 \\
1.175\end{array}$ & $\begin{array}{l}93 \\
82 \\
51\end{array}$ & & 31 & $5 d^{2} 6 p$ \\
\hline $5 d^{2}\left({ }^{3} F\right) 6 p$ & & $7 / 2$ & 17910.17 & 1.086 & 42 & ${ }^{4} \mathrm{G}^{\circ}$ & 19 & $4 f 6 s^{2}{ }^{2} \mathrm{~F}^{\circ}$ \\
\hline $5 d^{2}\left({ }^{3} \mathrm{~F}\right) 6 p$ & ${ }^{4} \mathrm{G}^{\circ}$ & $\begin{array}{l}5 / 2 \\
7 / 2 \\
9 / 2 \\
11 / 2\end{array}$ & $\begin{array}{l}17947.13 \\
18603.92 \\
19129.31 \\
20117.38\end{array}$ & $\begin{array}{l}1.061 \\
1.051 \\
1.173 \\
1.290\end{array}$ & $\begin{array}{l}42 \\
52 \\
96 \\
96\end{array}$ & & $\begin{array}{l}46 \\
23\end{array}$ & $\begin{array}{l}5 d 6 s 6 p \\
5 d 6 s 6 p\end{array}$ \\
\hline $5 d^{2}\left({ }^{3} \mathrm{~F}\right) 6 p$ & ${ }^{2} \mathrm{D}^{\circ}$ & $\begin{array}{l}3 / 2 \\
5 / 2\end{array}$ & $\begin{array}{l}18172.35 \\
19379.40\end{array}$ & $\begin{array}{l}0.799 \\
1.186\end{array}$ & $\begin{array}{l}60 \\
54\end{array}$ & & $\begin{array}{l}29 \\
30\end{array}$ & $5 d 6 s 6 p$ \\
\hline $5 d 6 s\left({ }^{1} \mathrm{D}\right) 6 p$ & & $3 / 2$ & 20018.99 & 1.01 & 37 & ${ }^{2} \mathrm{P}^{\circ}$ & 51 & $5 d^{2} 6 p$ \\
\hline $5 d^{2}\left({ }^{3} \mathrm{~F}\right) 6 p$ & ${ }^{4} \mathrm{~F}^{\circ}$ & $\begin{array}{l}3 / 2 \\
5 / 2 \\
7 / 2 \\
9 / 2\end{array}$ & $\begin{array}{l}20082.98 \\
20338.25 \\
20763.21 \\
21384.00\end{array}$ & $\begin{array}{l}0.724 \\
1.006 \\
1.178 \\
1.278\end{array}$ & $\begin{array}{l}72 \\
73 \\
63 \\
67\end{array}$ & & 11 & $5 d 6 s 6 p$ \\
\hline $5 d 6 s\left({ }^{1} \mathrm{D}\right) 6 p$ & & $1 / 2$ & 20197.34 & 0.63 & 47 & ${ }^{2} \mathrm{P}^{\circ}$ & 50 & $5 d^{2} 6 p$ \\
\hline $5 d^{2}\left({ }^{3} \mathrm{~F}\right) 6 p$ & & $5 / 2$ & 20972.17 & 0.890 & 41 & ${ }^{2} \mathbf{F}^{\circ}$ & 28 & $5 d 6 s 6 p$ \\
\hline $5 d^{2}\left({ }^{3} \mathrm{~F}\right) 6 p$ & & $7 / 2$ & 21447.86 & 1.103 & 31 & ${ }^{2} \mathrm{~F}^{\circ}$ & 16 & $5 d 6 s 6 p$ \\
\hline $5 d^{2}\left({ }^{3} \mathrm{~F}\right) 6 p$ & ${ }^{2} \mathrm{G}^{\circ}$ & $\begin{array}{l}7 / 2 \\
9 / 2\end{array}$ & $\begin{array}{l}21662.51 \\
22285.77\end{array}$ & $\begin{array}{l}0.995 \\
1.13\end{array}$ & $\begin{array}{l}51 \\
44\end{array}$ & & $\begin{array}{l}15 \\
32\end{array}$ & $4 f 5 d 6 s$ \\
\hline $5 d^{2}\left({ }^{3} \mathrm{~F}\right) 6 p$ & ${ }^{4} \mathrm{D}^{\circ}$ & $\begin{array}{l}1 / 2 \\
3 / 2 \\
5 / 2 \\
7 / 2\end{array}$ & $\begin{array}{l}22246.64 \\
22439.36 \\
22804.25 \\
2330326\end{array}$ & $\begin{array}{l}0.04 \\
1.192 \\
1.362 \\
1.178\end{array}$ & $\begin{array}{l}89 \\
92 \\
92 \\
80\end{array}$ & & & \\
\hline $5 d 6 s\left({ }^{3} \mathrm{D}\right) 6 p$ & & $7 / 2$ & 23221.10 & 1.078 & 23 & ${ }^{2} \mathrm{~F}^{\circ}$ & 47 & $4 f 5 d 6 s$ \\
\hline $5 d^{2}\left({ }^{3} \mathrm{P}\right) 6 p$ & ${ }^{2} \mathrm{~S}^{\circ}$ & $1 / 2$ & 23260.92 & 1.891 & 85 & & & \\
\hline $4 f 5 d\left({ }^{1} \mathrm{G}^{\circ}\right) 6 s$ & ${ }^{2} \mathrm{G}^{\circ}$ & $9 / 2$ & 23466.84 & 1.11 & 49 & & 31 & $5 d^{2} 6 p$ \\
\hline
\end{tabular}


La I, Odd Parity-Continued

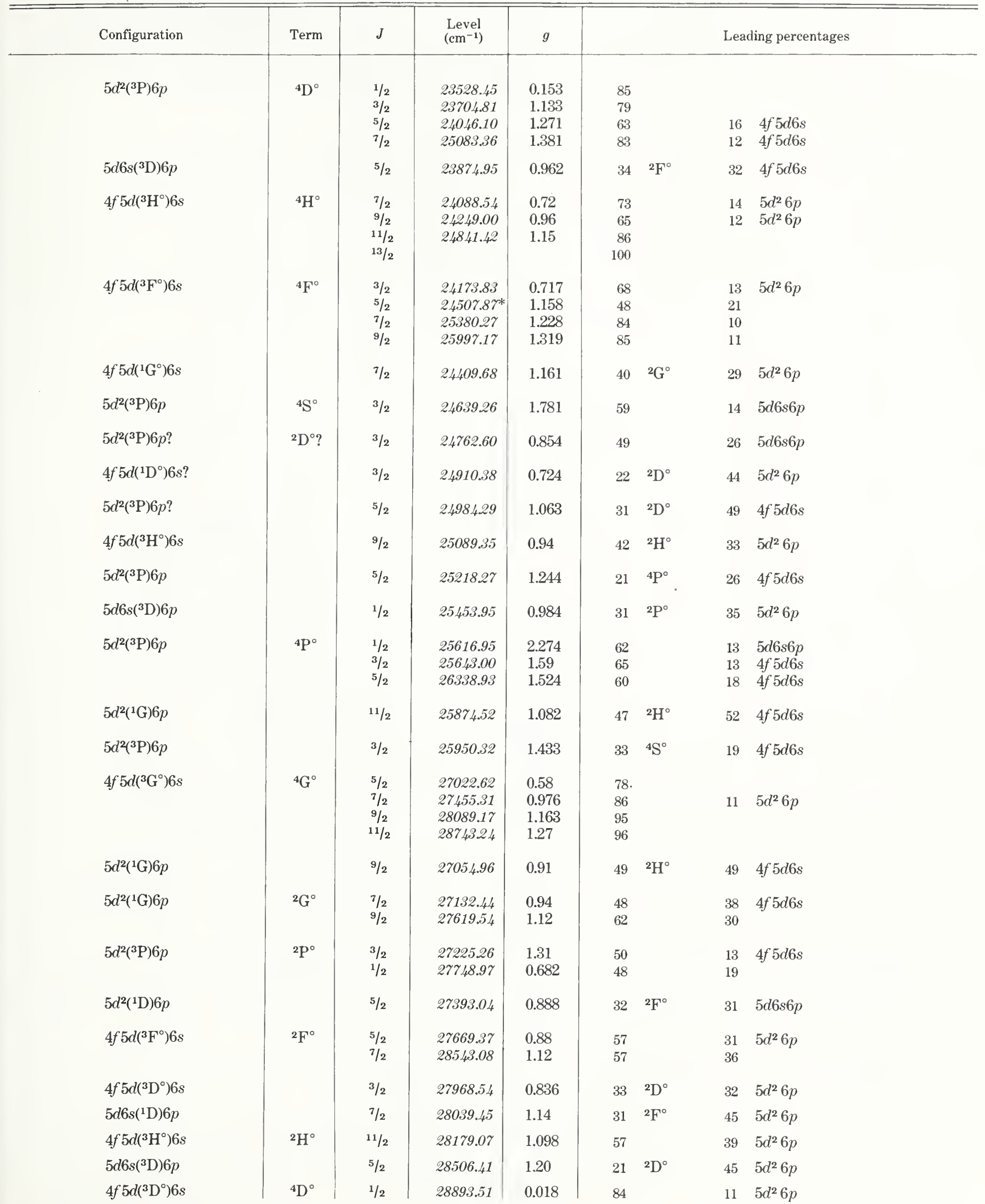


La I, Odd Parity-Continued

\begin{tabular}{|c|c|c|c|c|c|c|c|c|}
\hline Configuration & Term & $J$ & $\begin{array}{l}\text { Level } \\
\left(\mathrm{cm}^{-1}\right)\end{array}$ & $g$ & \multicolumn{4}{|c|}{ Leading percentages } \\
\hline $4 f 5 d\left({ }^{3} \mathrm{D}^{\circ}\right) 6 s$ & & $3 / 2$ & 28971.84 & 0.884 & 46 & ${ }^{4} \mathrm{D}^{\circ}$ & 22 & $5 d^{2} 6 p$ \\
\hline \multirow[t]{2}{*}{$4 f 5 d\left({ }^{3} \mathrm{D}^{\circ}\right) 6 s$} & & $3 / 2$ & 29199.57 & 1.106 & 36 & ${ }^{4} \mathrm{D}^{\circ}$ & 20 & $5 d^{2} 6 p$ \\
\hline & & $7 / 2$ & 29466.67 & 0.93 & & & & \\
\hline $4 f 5 d\left({ }^{3} \mathrm{D}^{\circ}\right) 6 s$ & & $5 / 2$ & $29502.18 *$ & 1.263 & 41 & ${ }^{4} \mathrm{D}^{\circ}$ & 19 & $5 d^{2} 6 p$ \\
\hline $4 f 5 d\left({ }^{3} \mathrm{P}^{\circ}\right) 6 s$ & & $1 / 2$ & 29564.70 & 0.78 & 35 & $2 \mathbf{P}^{\circ}$ & 19 & $5 d^{2} 6 p$ \\
\hline $4 f 5 d\left({ }^{3} \mathrm{D}^{\circ}\right) 6 s$ & & $5 / 2$ & $29775.58^{*}$ & 1.253 & 36 & ${ }^{4} \mathrm{D}^{\circ}$ & 20 & $5 d^{2} 6 p$ \\
\hline $4 f 5 d\left({ }^{3} \mathrm{D}^{\circ}\right) 6 s$ & & $7 / 2$ & 29894.91 & 1.352 & 34 & ${ }^{4} \mathrm{D}^{\circ}$ & 21 & $5 d^{2} 6 p$ \\
\hline $4 f 5 d\left({ }^{3} \mathrm{P}^{\circ}\right) 6 s$ & & $3 / 2$ & $29936.4^{*}$ & 1.492 & 44 & ${ }^{2} \mathrm{P}^{\circ}$ & 14 & $5 d 6 s 6 p$ \\
\hline \multirow[t]{9}{*}{$4 f 5 d\left({ }^{3} \mathrm{P}^{\circ}\right) 6 s$} & ${ }^{4} \mathrm{P}^{\circ}$ & $1 / 2$ & 29985.46 & 2.534 & 50 & & 17 & $5 d^{2} 6 p$ \\
\hline & & $3 / 2$ & $30417.46^{*}$ & 1.533 & 49 & & 15 & \\
\hline & & $5 / 2$ & 30896.84 & 1.424 & 67 & & 17 & \\
\hline & & $9 / 2$ & 30650.28 & 1.08 & & & & \\
\hline & & $5 / 2$ & 30788.45 & 1.04 & & & & \\
\hline & & $7 / 2$ & 30964.71 & 1.15 & & & & \\
\hline & & $5 / 2$ & 31477.22 & 0.90 & & & & \\
\hline & & $3 / 2$ & 31751.48 & 0.832 & & & & \\
\hline & & $7 / 2$ & 32140.55 & 1.15 & & & & \\
\hline \multirow[t]{7}{*}{$4 f 5 d\left({ }^{1} \mathrm{P}^{\circ}\right) 6 s$} & ${ }^{2} \mathrm{P}^{\circ}$ & $1 / 2$ & 32290.16 & 0.67 & 72 & & 13 & $5 d^{2} 6 p$ \\
\hline & & $\int / 2$ & & & 69 & & 16 & \\
\hline & & $9 / 2$ & 32415.73 & 0.92 & & & & \\
\hline & & $5 / 2$ & 32492.76 & 1.145 & & & & \\
\hline & & $3 / 2$ & 33204.05 & 1.324 & & & & \\
\hline & & $5 / 2$ & 33799.23 & 1.25 & & & & \\
\hline & & $1 / 2$ & 33820.31 & 0.617 & & & & \\
\hline \multirow[t]{3}{*}{$5 d^{2}\left({ }^{3} F\right) 7 p ?$} & ${ }^{4} \mathrm{~F}^{\circ} ?$ & $3 / 2$ & 34015.76 & 0.60 & & & & \\
\hline & & $\begin{array}{l}5 / 2 \\
7 / 2\end{array}$ & $\begin{array}{l}34213.53 \\
34988.17 ?\end{array}$ & & & & & \\
\hline & & $9 / 2$ & 35888.45 & & & & & \\
\hline \multirow[t]{3}{*}{$4 f 5 d\left({ }^{1} \mathrm{H}^{\circ}\right) 6 s$} & ${ }^{2} \mathrm{H}^{\circ}$ & $9 / 2$ & 34239.61 & 0.90 & 89 & & & \\
\hline & & $11 / 2$ & 34245.05 & 1.06 & 89 & & 10 & $5 d^{2} 6 p$ \\
\hline & & $3 / 2$ & 34299.11 & 1.01 & & & & \\
\hline \multirow[t]{3}{*}{$5 d^{2}\left({ }^{3} \mathrm{~F}\right) 7 p ?$} & ${ }^{4} \mathrm{G}^{\circ} ?$ & $5 / 2$ & 34358.60 & & & & & \\
\hline & & $\begin{array}{l}7 / 2 \\
9 / 2\end{array}$ & $\begin{array}{l}34380.86 \\
35236.06\end{array}$ & 1.13 & & & & \\
\hline & & $11 / 2$ & 36074.74 & & & & & \\
\hline $4 f\left({ }^{2} \mathrm{~F}^{\circ}\right) 5 d^{2}\left({ }^{3} \mathrm{~F}\right) ?$ & ${ }^{4} \mathrm{H}^{\circ} ?$ & $9 / 2$ & 34714.73 & 1.02 & & & & \\
\hline \multirow[t]{3}{*}{$5 d 6 s\left({ }^{3} \mathrm{D}\right) 7 p ?$} & ${ }^{4} \mathrm{~F}^{\circ} ?$ & $3 / 2$ & 34850.38 & 0.58 & & & & - \\
\hline & & $5 / 2$ & 34982.43 & 0.9 & & & & \\
\hline & & $5 / 2$ & 35232.81 & & & & & \\
\hline \multirow[t]{5}{*}{$4 f\left({ }^{2} \mathrm{~F}^{\circ}\right) 5 d^{2}\left({ }^{3} \mathrm{~F}\right) ?$} & ${ }^{2} \mathrm{G}^{\circ} ?$ & $\begin{array}{l}7 / 2 \\
9 / 9\end{array}$ & 35253.10 & 0.89 & & & & \\
\hline & & $8 / 2$ & & 1.09 & & & & \\
\hline & & $5 / 2$ & 35275.16 & 1.2 & & & & \\
\hline & & $3 / 2$ & 35470.85 & 1.42 & & & & \\
\hline & & $1 / 2$ & 35482.70 & & & & & \\
\hline
\end{tabular}


La I, Odd Parity-Continued

La I, Odd Parity-Continued

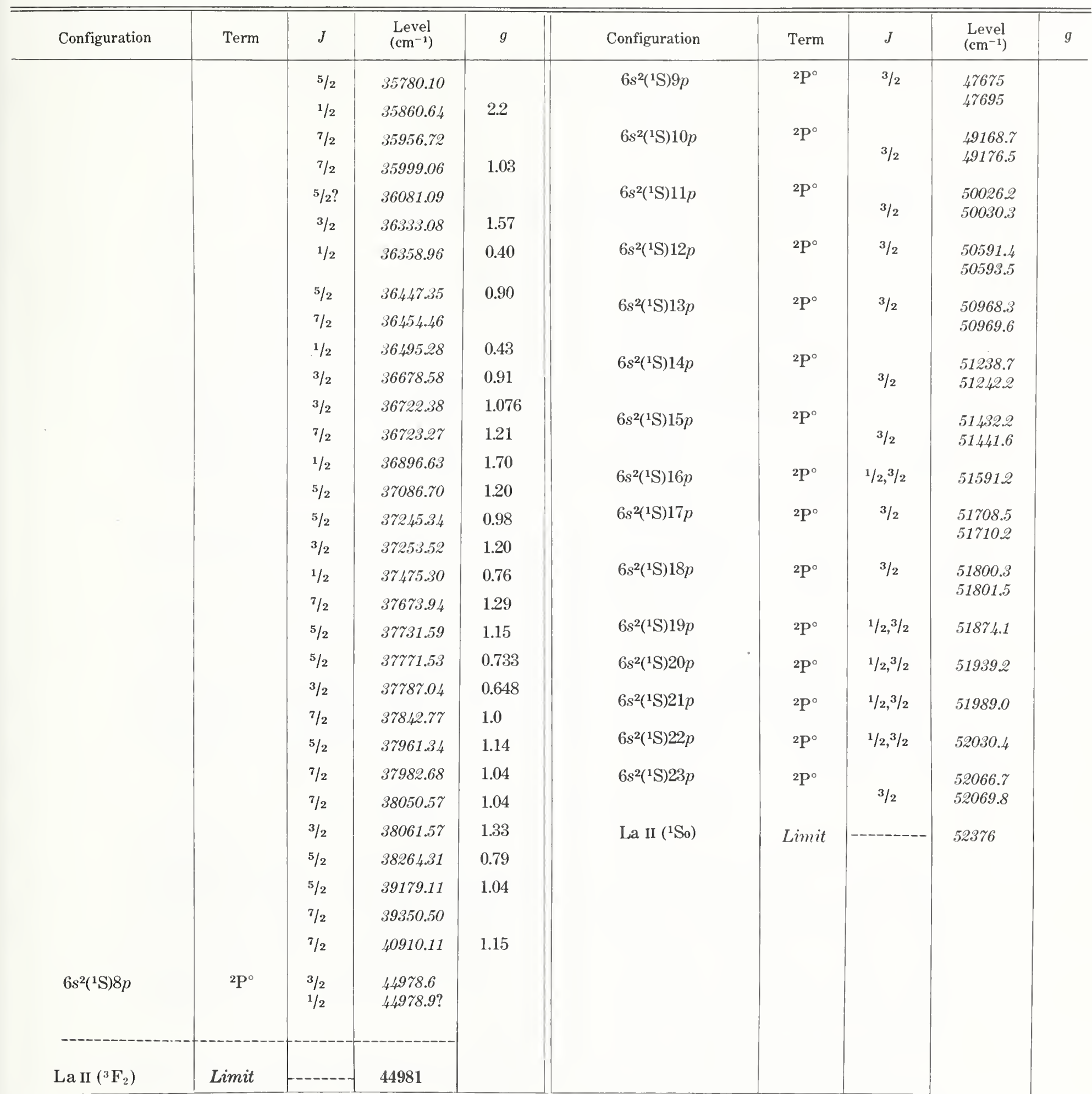


(Ba I sequence; 56 electrons)

Ground state $\left(1 s^{2} 2 s^{2} 2 p^{6} 3 s^{2} 3 p^{6} 3 d^{10} 4 s^{2} 4 p^{6} 4 d^{10} 5 s^{2} 5 p^{6}\right) 5 d^{2}{ }^{3} \mathrm{~F}_{2}$

Ionization energy $89200 \pm 80 \mathrm{~cm}^{-1}$

$11.060 \pm 0.010 \mathrm{eV}$

Identified even configurations

$5 d^{2}, 5 d 6 s, 6 s^{2}, 4 f 6 p, 4 f^{2}, 6 p^{2}, 5 d 6 d, 5 d 7 s$, $6 s 7 s ?, 6 s 6 d$

Identified odd configurations

$4 f 6 s, 4 f 5 d, 5 d 6 p, 6 s 6 p$

\section{Spectrum Observations, Zeeman Effect, Early Work on the Analysis}

Meggers [1927] and Russell and Meggers [1932] carried out most of the analysis of this spectrum, greatly extending the earlier work of Paulson [1914] and Goudsmit [1925]. Most of the energy levels and assignments are from the 1932 paper, which pointed out that observations of La II in the Schumann region were needed for extension of the analysis. No such observations appear to have been made to date. Meggers [1932] lists about 800 lines for La II in the range 2142-10955 $\AA$, with more than 700 having energy-level classifications [Russell and Meggers].

All except one of the $g$ values are from Harrison, Rosen, and McNally [1945]. (See La I for more detail on the Zeeman data.) These authors also give more accurate (3-place) wavelengths for some of the 507 La II lines for which they obtained Zeeman patterns.

La II was an important spectrum in early applications of the Slater-Condon theory of atomic structure. The identification of the complete $4 f 6 p, 4 f 5 d$, and $4 f^{2}$ configurations in La II led Condon and Shortley [1931] to extend Slater's formulas to configurations involving $f$ electrons. They showed that the La II $4 f 5 d$ and $4 f^{2}$ configurations fit the theory very well, and "their theoretical predictions led to the correct identifications of the difficult terms $4 f 5 d{ }^{1} \mathrm{H}^{\circ}$ and $4 f^{2}$ "I" [Russell and Meggers]. The agreement with theory for several other two-electron configurations in La II was less good because of neglect of strong configuration interactions and of the spin-orbit interaction.

\section{Designations and Calculations of the Even Levels}

More recent calculations have yielded quantitative details of the configuration interactions in both the even and odd groups. In a discussion of the $\left(5 d^{2}+5 d 6 s+6 s^{2}\right)$ group, Goldschmidt [1968b] notes the especially mixed configurational compositions of the $J=2$ levels at 1394 and $10095^{-1} \mathrm{~cm}^{-1}$. The ${ }^{1} \mathrm{D}$ character of both levels is apparent from the eigenvectors, but "the assignments of configurational quantum numbers . . . are meaningless." We indicate the lack of configuration assignments by listing the " $a$ ' $\mathrm{D}$ " and " $b$ i $\mathrm{D}$ " designations of these levels after the respective leading percentages, instead of under "Term." Russell-Saunders term notations preceded by small letters are in all cases as given by Russell and Meggers, and can be used in connection with line classifications given in their paper.

All eigenvector percentages for the even levels are from Goldschmidt's [1976] calculation of the group $\left(5 d^{2}+5 d 6 s+6 s^{2}+4 f^{2}+4 f 6 p+6 p^{2}+6 s 6 d\right)$. Her eigenvector assignments reverse the former configuration designations of the high $h^{1} \mathrm{D}_{2}$ and $i^{1} \mathrm{D}_{2}$ levels, a result not inconsistent with the intensities of the transitions from these levels. The $4 f 6 p$ levels are here arranged into four $j_{1} j_{2}$-coupling terms according to the leading percentages, the Russell-Saunders designations being listed after the word "or" in the last column. The four $5 d 7 \mathrm{~s}$ levels clearly form two $j_{1} j_{2}$ pairs, as listed, and again the less appropriate Russell-Saunders names are also shown.

The reality of the $5 d 6 d{ }^{3} \mathrm{P}_{0}$ level is questionable [Russell and Meggers], and the designations of the nominal $5 d 6 d{ }^{3} \mathrm{~S}_{1},{ }^{1} \mathrm{~S}_{0}$, and ${ }^{3} \mathrm{P}_{1}$ levels are given here as tentative. The inclusion of this configuration in the calculation of the even group is definitely needed. The $f^{3} \mathrm{~S}_{1}$ level given by Harrison et al. $\left(60660.18 \mathrm{~cm}^{-1}\right)$ classifies several lines listed by Russell and 


\section{La II-Continued}

Meggers, and is certainly real. The position is consistent with our suggested assignment to the $6 s 7 s$ configuration. The " $i{ }^{3} \mathrm{D}_{3}$ " level of Russell and Meggers $\left(69233.90 \mathrm{~cm}^{-1}\right)$ is still uninterpreted, and the tentative level at $64706.76 \mathrm{~cm}^{-1}$ is omitted here.

\section{Interpretation of the Odd Levels}

Goldschmidt [1968a; 1974] and Spector and Gotthelf [1970] have calculated the four identified odd configurations $(4 f 6 s+4 f 5 d+5 d 6 p+6 s 6 p)$, with the configuration interactions included. The eigenvector percentages here are from Goldschmidt's unpublished results [1974]. The $4 f 6 s$ levels are grouped into two $j_{1} j_{2}$-coupling pairs, with the Russell-Saunders names and leading percentages being given after the word "or" in the last column.

The designations $y{ }^{3} \mathrm{P}_{2}^{\circ}$ and $x{ }^{3} \mathrm{P}_{2}^{\circ}$ are retained for the levels at 29498 and $33204 \mathrm{~cm}^{-1}$, respectively, because the eigenvectors have high ${ }^{3} \mathrm{P}^{\circ}$ character; these designations are given after the leading percentages (and not under "Term"), however, to indicate that meaningful configuration assignments are prevented by the strong mixtures of $6 s 6 p$ and $5 d 6 p$ in the eigenvectors. Several $J=1$ levels in this group also have very mixed compositions. The eight odd levels lying above the interpreted group are listed with the ordinal designations used by Russell and Meggers.

\section{Ionization Energy}

Sugar and Reader [1965] related the $4 f 6 \mathrm{~s}$ levels, the $5 d 6 \mathrm{~s}$ and $5 d 7 \mathrm{~s}$ levels, and the $5 d 6 \mathrm{~s}$ and $6 s 6 d$ levels to known regularities for the three corresponding series, 4 fns, $5 d n s$, and $6 s n d$. The three values thus obtained for the ionization energy agreed to within $52 \mathrm{~cm}^{-1}$. We give their value with an error estimate conservatively based on this agreement.

\section{References}

Condon, E. U., and Shortley, G. H., Phys. Rev. 37, 1025 (1931). PT

Eremin, M. V., and Maryakhina, O. I., Opt. Spectrosc. (USSR) 26, 479 (1969). PT

Goldschmidt, Z. B., Thesis, Hebrew Univ. Jerusalem, Israel, 487 pp. (1968a). PT

Goldschmidt, Z. B., in Spectroscopic and Group Theoretical Methods in Physics, Ed. F. Block et al. (John Wiley and Sons, New York, N.Y., 1968b). PT

Goldschmidt, Z. B., unpublished material $(1974 ; 1976)$. ND PT

Goudsmit, S., Proc. Sect. Sci. Kon. Akad. Wet., Amsterdam, 28, 23 (1925). EL CL ZE

Harrison, G. R., Rosen, N., and McNally, Jr., J. R., J. Opt. Soc. Am. 35, 658 (1945). CL ZE

Meggers, W. F., J. Opt. Soc. Am. 14, 191 (1927). EL CL W

Meggers, W. F., J. Res. Nat. Bur. Stand. (U.S.) 9, 239 (1932). W ZE

Meggers, W. F., and Burns, K., J. Opt. Soc. Am. 14, 449 (1927). Hfs

Paulson, E., Ann. Phys. (Leipzig) 45, 1203 (1914). EL W

Russell, H. N., and Meggers, W. F., J. Res. Nat. Bur. Stand. (U.S.) 9, 625 (1932). EL ND CL W ZE IP

Spector, N., and Gotthelf, U., Opt. Pura Apl. (Spain) 3, 98 (1970). ND PT

Sugar, J., and Reader, J., J. Opt. Soc. Am. 55, 1286 (1965). IP

[January 1977] 
La II

\begin{tabular}{|c|c|c|c|c|c|c|c|c|}
\hline Configuration & Term & $J$ & $\begin{array}{l}\text { Level } \\
\left(\mathrm{cm}^{-1}\right)\end{array}$ & $g$ & \multicolumn{4}{|c|}{ Leading percentages } \\
\hline $5 d^{2}$ & $a^{3} \mathrm{~F}$ & $\begin{array}{l}2 \\
3 \\
4\end{array}$ & $\begin{array}{r}0.00 \\
1016.10 \\
1970.70\end{array}$ & $\begin{array}{l}0.721 \\
1.083 \\
1.248\end{array}$ & $\begin{array}{r}83 \\
100 \\
99\end{array}$ & & 11 & $\begin{array}{l}{ }^{1} \mathrm{D} \\
{ }^{1} \mathrm{G}\end{array}$ \\
\hline $5 d^{2}$ & & 2 & 1394.46 & 0.977 & 36 & $a{ }^{1} \mathrm{D}$ & 33 & $5 d 6 s^{1} \mathrm{D}$ \\
\hline $5 d 6 s$ & $a^{3} \mathrm{D}$ & $\begin{array}{l}1 \\
2 \\
3\end{array}$ & $\begin{array}{l}1895.15 \\
2591.60 \\
3250.35\end{array}$ & $\begin{array}{l}0.498 \\
1.133 \\
1.334\end{array}$ & $\begin{array}{r}100 \\
85 \\
100\end{array}$ & & 10 & $5 d^{2}{ }^{1} \mathrm{D}$ \\
\hline $5 d^{2}$ & $a^{3} \mathrm{P}$ & $\begin{array}{l}0 \\
1 \\
2\end{array}$ & $\begin{array}{l}5249.70 \\
5718.12 \\
6227.42\end{array}$ & $\begin{array}{l}1.497 \\
1.481\end{array}$ & $\begin{array}{l}92 \\
99 \\
96\end{array}$ & & $\begin{array}{l}4 \\
1 \\
3\end{array}$ & $\begin{array}{l}{ }^{1 \mathrm{~S}} \\
4 f^{2}{ }^{3} \mathrm{P} \\
5 d 6 s^{1} \mathrm{D}\end{array}$ \\
\hline $6 s^{2}$ & $a^{1} \mathrm{~S}$ & 0 & 7394.57 & & 75 & & 17 & $5 d^{2}{ }^{1} \mathrm{~S}$ \\
\hline $5 d^{2}$ & $a^{1} \mathrm{G}$ & 4 & 7473.32 & 1.000 & 98 & & 1 & $5 d^{2}{ }^{3} \mathrm{~F}$ \\
\hline $5 d 6 s$ & & 2 & 10094.86 & 1.005 & 53 & $b^{1} \mathrm{D}$ & 42 & $5 d^{2} 1 \mathrm{D}$ \\
\hline $4 f\left({ }^{2} \mathrm{~F}_{5 / 2}^{\circ}\right) 6 s_{1 / 2}$ & $(5 / 2,1 / 2)^{\circ}$ & $\begin{array}{l}2 \\
3\end{array}$ & $\begin{array}{l}14147.98 \\
14375.17\end{array}$ & $\begin{array}{l}0.664 \\
1.056\end{array}$ & $\begin{array}{l}96 \\
95\end{array}$ & $\begin{array}{l}\text { or } \\
\text { or }\end{array}$ & $\begin{array}{l}95 \\
64\end{array}$ & $z^{3} \mathrm{~F}^{\circ}$ \\
\hline $4 f\left({ }^{2} \mathrm{~F}_{7 / 2}\right) 6 s_{1 / 2}$ & $(7 / 2,1 / 2)^{\circ}$ & $\begin{array}{l}4 \\
3\end{array}$ & $\begin{array}{l}15698.74 \\
15773.77\end{array}$ & $\begin{array}{l}1.247 \\
1.017\end{array}$ & $\begin{array}{l}95 \\
93\end{array}$ & $\begin{array}{l}\text { or } \\
\text { or }\end{array}$ & $\begin{array}{l}97 \\
64\end{array}$ & $\begin{array}{l}z^{3} \mathrm{~F}^{\circ} \\
z^{1} \mathrm{~F}^{\circ}\end{array}$ \\
\hline $4 f 5 d$ & $z^{1} \mathrm{G}^{0}$ & 4 & 16599.17 & 0.969 & 73 & & 22 & ${ }^{3} \mathrm{H}^{\circ}$ \\
\hline $4 f 5 d$ & $y^{3} \mathrm{~F}^{\circ}$ & $\begin{array}{l}2 \\
3 \\
4\end{array}$ & $\begin{array}{l}17211.93 \\
18235.56 \\
19214.54\end{array}$ & $\begin{array}{l}0.754 \\
1.086 \\
1.232\end{array}$ & $\begin{array}{l}70 \\
97 \\
90\end{array}$ & & $\begin{array}{r}26 \\
8\end{array}$ & $\begin{array}{l}{ }^{1} \mathrm{D}^{\circ} \\
{ }^{1} \mathrm{G}^{\circ}\end{array}$ \\
\hline $4 f 5 d$ & $z^{3} \mathrm{H}^{\circ}$ & $\begin{array}{l}4 \\
5 \\
6\end{array}$ & $\begin{array}{l}17825.62 \\
18580.41 \\
19749.62\end{array}$ & $\begin{array}{l}0.846 \\
1.017 \\
1.178\end{array}$ & $\begin{array}{r}77 \\
100 \\
100\end{array}$ & & 19 & ${ }^{1} \mathrm{G}^{\circ}$ \\
\hline $4 f 5 d$ & $z^{1} \mathrm{D}^{\circ}$ & 2 & 18895.41 & 0.923 & 64 & & 28 & ${ }^{3} \mathrm{~F}^{\circ}$ \\
\hline $4 f 5 d$ & $z^{3} \mathrm{G}^{\circ}$ & $\begin{array}{l}3 \\
4 \\
5\end{array}$ & $\begin{array}{l}20402.82 \\
21331.60 \\
22282.90\end{array}$ & $\begin{array}{l}0.757 \\
1.049 \\
1.197\end{array}$ & $\begin{array}{r}96 \\
100 \\
99\end{array}$ & & & \\
\hline $4 f 5 d$ & $z^{3} \mathrm{D}^{\circ}$ & $\begin{array}{l}1 \\
2 \\
3\end{array}$ & $\begin{array}{l}21441.73 \\
22106.02 \\
22537.30\end{array}$ & $\begin{array}{l}0.542 \\
1.167 \\
1.288\end{array}$ & $\begin{array}{l}79 \\
83 \\
78\end{array}$ & & $\begin{array}{l}15 \\
13 \\
10\end{array}$ & $\begin{array}{l}5 d 6 p{ }^{3} \mathrm{D}^{\circ} \\
5 d 6 p{ }^{3} \mathrm{D}^{\circ} \\
4 f 5 d{ }^{1} \mathrm{~F}^{\circ}\end{array}$ \\
\hline $4 f 5 d$ & $z^{3} \mathrm{P}^{\circ}$ & $\begin{array}{l}0 \\
1 \\
2\end{array}$ & $\begin{array}{l}22683.70 \\
22705.15 \\
23246.93\end{array}$ & $\begin{array}{l}1.431 \\
1.459\end{array}$ & $\begin{array}{l}84 \\
79 \\
82\end{array}$ & & $\begin{array}{l}11 \\
11 \\
11\end{array}$ & $5 d 6 p^{3} \mathrm{P}^{\circ}$ \\
\hline $5 d 6 p$ & $y^{1} \mathrm{D}^{\circ}$ & 2 & 24462.66 & 0.887 & 56 & & 33 & ${ }^{3} \mathrm{~F}^{\circ}$ \\
\hline $4 f 5 d$ & $y^{1} \mathrm{~F}^{\circ}$ & 3 & 24522.70 & 1.034 & 84 & & 10 & ${ }^{3} \mathrm{D}^{\circ}$ \\
\hline $5 d 6 p$ & $y^{3} \mathrm{D}^{\circ}$ & $\begin{array}{l}1 \\
2 \\
3\end{array}$ & $\begin{array}{l}25973.37 \\
27388.11 \\
28315.25\end{array}$ & $\begin{array}{l}0.782 \\
1.168 \\
1.308\end{array}$ & $\begin{array}{l}44 \\
83 \\
81\end{array}$ & & $\begin{array}{l}27 \\
13 \\
10\end{array}$ & $\begin{array}{l}{ }^{1} \mathrm{P}^{\circ} \\
4 f 5 d^{3} \mathrm{D}^{\circ} \\
4 f 5 d^{3} \mathrm{D}^{\circ}\end{array}$ \\
\hline $5 d 6 p$ & $x^{3} \mathrm{~F}^{\circ}$ & $\begin{array}{l}2 \\
3 \\
4\end{array}$ & $\begin{array}{l}26414.01 \\
26837.66 \\
28565.40\end{array}$ & $\begin{array}{l}0.825 \\
1.088 \\
1.245\end{array}$ & $\begin{array}{l}59 \\
90 \\
95\end{array}$ & & 33 & ${ }^{1} \mathrm{D}^{\circ}$ \\
\hline $5 d 6 p$ & & 1 & 27423.91 & 0.876 & 37 & ${ }^{3} \mathrm{D}^{\circ}$ & 23 & ${ }^{1} \mathrm{P}^{\circ}$ \\
\hline $6 s 6 p$ & $y^{3} \mathrm{P}^{\circ}$ & 0 & 27545.85 & & 70 & & 19 & $5 d 6 p^{3} \mathrm{P}^{\circ}$ \\
\hline $6 s 6 p$ & & 1 & 28154.55 & 1.267 & 44 & ${ }^{3} \mathrm{P}^{\circ}$ & 30 & $4 f 5 d^{1} \mathrm{P}^{\circ}$ \\
\hline
\end{tabular}


La II-Continued

\begin{tabular}{|c|c|c|c|c|c|c|c|c|}
\hline Configuration & Term & $J$ & $\begin{array}{l}\text { Level } \\
\left(\mathrm{cm}^{-1}\right)\end{array}$ & $g$ & & & \multicolumn{2}{|c|}{ Leading percentages } \\
\hline $4 f 5 d$ & $z^{1} \mathrm{H}^{\circ}$ & 5 & 28525.71 & 1.004 & 99 & & & \\
\hline $6 s 6 p$ & & 2 & 29498.05 & 1.471 & 45 & $y^{3} \mathrm{P}^{\circ}$ & 40 & $5 d 6 p^{3} \mathrm{P}^{\circ}$ \\
\hline $4 f 5 d$ & $y^{1} \mathrm{P}^{\circ}$ & 1 & 30353.33 & 1.074 & 49 & & 22 & $5 d 6 p^{1} \mathrm{P}^{\circ}$ \\
\hline $5 d 6 p$ & $x^{3} \mathrm{P}^{\circ}$ & $\begin{array}{l}0 \\
1\end{array}$ & $\begin{array}{l}31785.82 \\
32160.99\end{array}$ & 1.492 & $\begin{array}{l}70 \\
65\end{array}$ & & $\begin{array}{l}26 \\
29\end{array}$ & $6 s 6 p{ }^{3} \mathrm{P}^{\circ}$ \\
\hline $5 d 6 p$ & $x^{1} \mathrm{~F}^{\circ}$ & 3 & 32201.05 & 1.005 & 91 & & & \\
\hline $6 s 6 p$ & & 2 & 33204.41 & 1.494 & 52 & $x^{3} \mathrm{P}^{\circ}$ & 45 & $5 d 6 p{ }^{3} \mathrm{P}^{\circ}$ \\
\hline $4 f\left({ }^{2} \mathrm{~F}_{5 / 2}^{\circ}\right) 6 p_{1 / 2}$ & $(5 / 2,1 / 2)$ & $\begin{array}{l}3 \\
2\end{array}$ & $\begin{array}{l}35452.66 \\
35787.53\end{array}$ & $\begin{array}{l}0.876 \\
0.719\end{array}$ & $\begin{array}{l}92 \\
80\end{array}$ & $\begin{array}{l}\text { or } \\
\text { or }\end{array}$ & & $\begin{array}{l}e^{3} \mathrm{G} \\
e^{3} \mathrm{~F}\end{array}$ \\
\hline $4 f\left({ }^{2} \mathrm{~F}_{7 / 2}^{\circ}\right) 6 p_{1 / 2}$ & $(7 / 2,1 / 2)$ & $\begin{array}{l}3 \\
4\end{array}$ & $\begin{array}{l}36954.65 \\
37172.79\end{array}$ & $\begin{array}{l}1.061 \\
1.127\end{array}$ & $\begin{array}{l}48 \\
86\end{array}$ & $\begin{array}{l}\text { or } \\
\text { or }\end{array}$ & & $\begin{array}{l}e^{3} \mathrm{~F} \\
e^{3} \mathrm{G}\end{array}$ \\
\hline $4 f\left({ }^{2} \mathrm{~F}_{5 / 2}^{\circ}\right) 6 p_{3 / 2}$ & $(5 / 2,3 / 2)$ & $\begin{array}{l}3 \\
4 \\
2 \\
1\end{array}$ & $\begin{array}{l}37209.71 \\
37790.57 \\
38221.49 \\
38534.11\end{array}$ & $\begin{array}{l}0.944 \\
1.113 \\
1.071 \\
0.497\end{array}$ & $\begin{array}{r}48 \\
82 \\
81 \\
100\end{array}$ & $\begin{array}{l}\text { or } \\
\text { or } \\
\text { or } \\
\text { or }\end{array}$ & & $\begin{array}{l}e^{1} \mathrm{~F} \\
e^{3} \mathrm{~F} \\
e^{3} \mathrm{D} \\
e^{3} \mathrm{D}\end{array}$ \\
\hline $4 f\left({ }^{2} \mathrm{~F}_{7 / 2}^{\circ}\right) 6 p_{3 / 2}$ & $(7 / 2,3 / 2)$ & $\begin{array}{l}5 \\
4 \\
3 \\
2\end{array}$ & $\begin{array}{l}39018.74 \\
39221.65 \\
39402.55 \\
40457.71\end{array}$ & $\begin{array}{l}1.21 \\
1.059 \\
1.274 \\
1.036\end{array}$ & $\begin{array}{r}100 \\
70 \\
80 \\
97\end{array}$ & $\begin{array}{l}\text { or } \\
\text { or } \\
\text { or } \\
\text { or }\end{array}$ & & $\begin{array}{l}e^{3} \mathrm{G} \\
e^{1} \mathrm{G} \\
e^{3} \mathrm{D} \\
e^{1} \mathrm{D}\end{array}$ \\
\hline $6 s 6 p$ & $x^{1} \mathrm{P}^{\circ}$ & 1 & 45692.17 & 0.999 & 77 & & 20 & $5 d 6 p^{1} \mathrm{P}^{\circ}$ \\
\hline $5 d\left({ }^{2} \mathrm{D}_{3 / 2}\right) 7 s_{1 / 2}$ & $(3 / 2,1 / 2)$ & $\begin{array}{l}1 \\
2\end{array}$ & $\begin{array}{l}49733.13 \\
49884.35\end{array}$ & $\begin{array}{l}0.500 \\
1.117\end{array}$ & & $\begin{array}{l}\text { or } \\
\text { or }\end{array}$ & & $\begin{array}{l}f^{3} \mathrm{D} \\
f^{3} \mathrm{D}\end{array}$ \\
\hline $5 d\left({ }^{2} \mathrm{D}_{5 / 2}\right) 7 s_{1 / 2}$ & $(5 / 2,1 / 2)$ & $\begin{array}{l}3 \\
2\end{array}$ & $\begin{array}{l}51228.57 \\
51523.86\end{array}$ & $\begin{array}{l}1.315 \\
1.036\end{array}$ & & $\begin{array}{l}\text { or } \\
\text { or }\end{array}$ & & $\begin{array}{l}f^{3} \mathrm{D} \\
f^{1} \mathrm{D}\end{array}$ \\
\hline $5 d 6 d$ & $f^{1} \mathrm{~F}$ & 3 & 52137.67 & 0.987 & & & & \\
\hline $5 d 6 d$ & $g^{3} \mathrm{D}$ & $\begin{array}{l}1 \\
2 \\
3\end{array}$ & $\begin{array}{l}52169.66 \\
52734.81 \\
53689.56\end{array}$ & $\begin{array}{l}0.621 \\
1.154 \\
1.218\end{array}$ & & & & \\
\hline $5 d 6 d$ & $f^{3} \mathrm{G}$ & $\begin{array}{l}3 \\
4 \\
5\end{array}$ & $\begin{array}{l}52857.88 \\
53333.37 \\
54434.65\end{array}$ & $\begin{array}{l}0.861 \\
1.036 \\
1.21\end{array}$ & & & & \\
\hline $5 d 6 d$ & $e^{1} \mathrm{P}$ & 1 & 53302.56 & 1.335 & & & & \\
\hline $5 d 6 d$ & $f^{3} \mathrm{~F}$ & $\begin{array}{l}2 \\
3 \\
4\end{array}$ & $\begin{array}{l}53885.24 \\
54840.04 \\
55321.35\end{array}$ & $\begin{array}{l}0.751 \\
1.088 \\
1.136\end{array}$ & & & & \\
\hline $5 d 6 d$ & $e^{3} \mathrm{~S} ?$ & 1 & 54365.80 & 1.455 & & & & \\
\hline $5 d 6 d ?$ & $e^{1} \mathrm{~S} ?$ & 0 & 54793.82 & & & & & \\
\hline $5 d 6 d$ & $e^{3} \mathrm{P}$ & $\begin{array}{l}0 \\
1 \\
2\end{array}$ & $\begin{array}{l}54964.19 ? \\
55230.33^{*} \\
56036.60\end{array}$ & $\begin{array}{l}1.552 \\
1.203\end{array}$ & & & & \\
\hline $4 f^{2}$ & $e^{3} \mathrm{H}$ & $\begin{array}{l}4 \\
5 \\
6\end{array}$ & $\begin{array}{l}55107.25 \\
55982.09 \\
56837.94\end{array}$ & $\begin{array}{l}0.883 \\
1.033 \\
1.14\end{array}$ & $\begin{array}{r}98 \\
100 \\
100\end{array}$ & & 2 & ${ }^{1} \mathrm{G}$ \\
\hline $5 d 6 d$ & $g^{1} \mathrm{D}$ & 2 & 55184.05 & 1.183 & & & & \\
\hline $5 d 6 d$ & $f^{1} \mathrm{G}$ & 4 & 56035.70 & 1.027 & & & & \\
\hline
\end{tabular}


La II-Continued

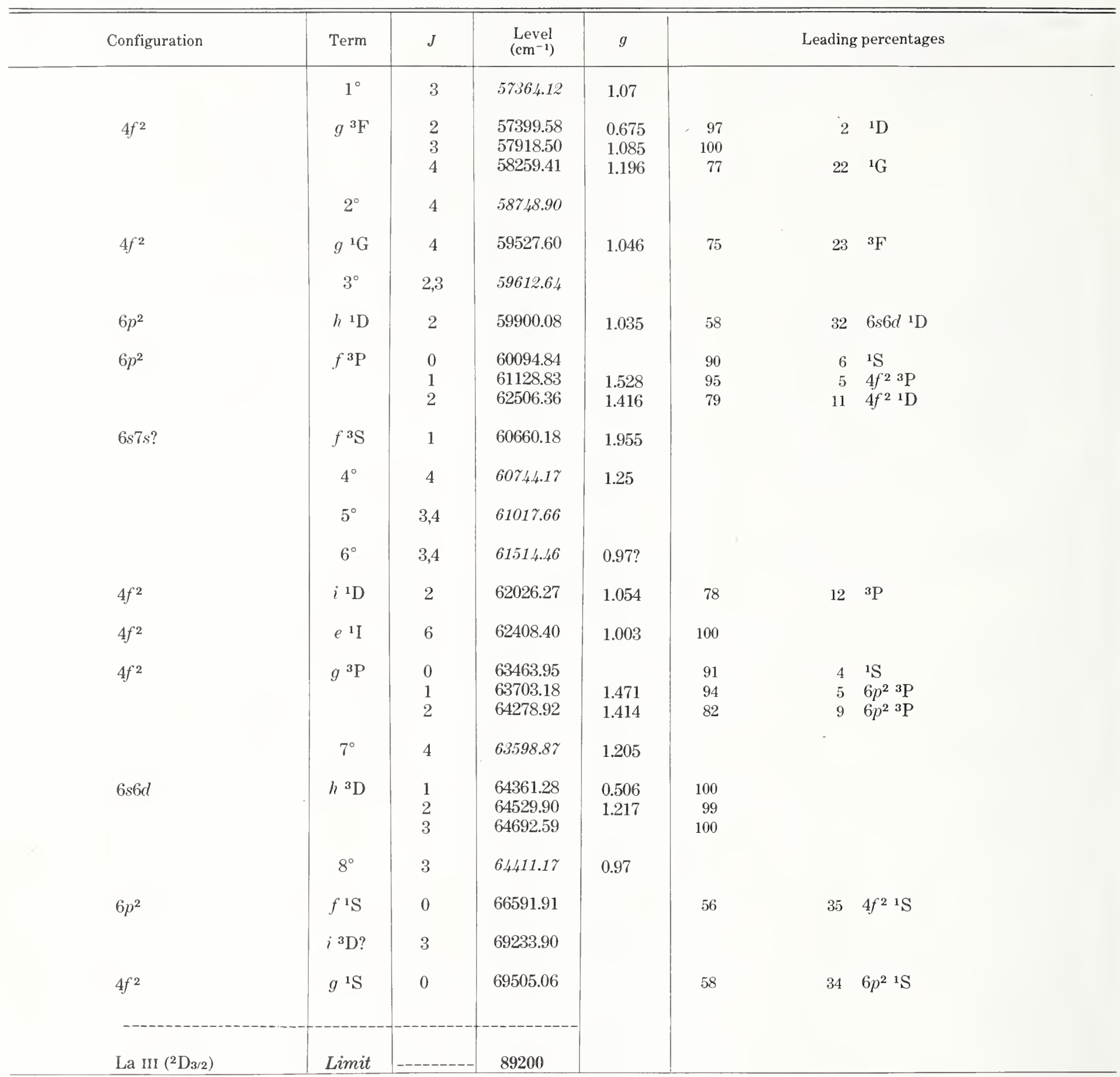


(Cs I sequence; 55 electrons)

Ground state $\left(1 s^{2} 2 s^{2} 2 p^{6} 3 s^{2} 3 p^{6} 3 d^{10} 4 s^{2} 4 p^{6} 4 d^{10} 5 s^{2} 5 p^{6}\right) 5 d^{2} \mathrm{D}_{3 / 2}$

Ionization energy $154675 \pm 5 \mathrm{~cm}^{-1}$

$19.1774 \pm 0.0006 \mathrm{eV}$

Identified even configurations

$5 p^{6} 5 d-9 d, 5 p^{6} 6 s-10 s, 5 p^{6} 5 g-8 g$

Identified odd configurations

$5 p^{6} 4 f-8 f, 5 p^{6} 6 p-9 p$

Gibbs and White first classified the La III $6 s-6 p$ and $5 d-6 p$ multiplets in their early work on one-electron spectra. Badami confirmed these terms in his 1931 paper and added the $6 d$ levels. The following year Meggers published new measurements of the La spectra, and Russell and Meggers gave new values for the known La III levels, as well as for the $7 s^{2} \mathrm{~S}$ level.

Lang's observations in the vacuum ultraviolet yielded the $5 f^{2} \mathrm{~F}^{\circ}$ term but not the $4 f$ term. The latter was located by Sugar and Kaufman, who found 13 new terms based on their measurements of 45 lines in the region 700-2000 $\AA$. Five of the highest terms were added by Odabasi [1967], who also obtained the intervals for the ${ }^{2} \mathrm{G}$ terms.

All levels except those of $5 d^{2} \mathrm{D}$ and $4 f^{2} \mathrm{~F}^{\circ}$ are from Odabasi's paper, and are accurate to about $\pm 0.2 \mathrm{~cm}^{-1}$. He gives 74 La III lines in the region $2200-11000 \AA$. The four lowest levels are from Johansson and Litzén, whose measurements of the three $5 d-4 f$ lines (in the infrared) yielded levels accurate to $\pm 0.02 \mathrm{~cm}^{-1}$. The ionization energy [Sugar and Reader, 1973] is based on the three values for the limit obtained by Kaufman and Sugar [1971] from the $n s, n f$, and $n g$ series.

Odabasi resolved the hfs of the $6 s^{2} \mathrm{~S}_{1 / 2}$ level, obtaining the sublevels $13590.56 \mathrm{~cm}^{-1}(F=3)$ and $13591.59 \mathrm{~cm}^{-1}(F=4)$. His value for the hyperfine splitting $\left(1.03 \pm 0.10 \mathrm{~cm}^{-1}\right)$ agrees well with measurements made with higher resolution; Wittke [1940], for example, obtained the value $1.076 \pm 0.01 \mathrm{~cm}^{-1}$.

Odabasi estimates that his values for the ${ }^{2} \mathrm{G}$ fine-structure intervals are accurate to \pm 0.10 $\mathrm{cm}^{-1}$, in which case the peculiar behavior of these intervals (an increase with increasing $n, n=5$ to 7 ) is an interesting theoretical problem.

\section{References}

Badami, J. S., Proc. Phys. Soc. London 43, 53 (1931). EL CL W

Gibbs, R. C., and White, H. E., Proc. Nat. Acad. Sci. (U.S.) 12, 551 (1926); Phys. Rev. 33, 157 (1929). EL CL W

Johansson, S., and Litzén, U., J. Opt. Soc. Am. 61, 1427 (1971). EL CL W

Kaufman, V., and Sugar, J., J. Opt. Soc. Am. 61, 1693 (1971). IP

Lang, R. J., Can. J. Res., Sect. A 13, 1 (1935). EL CL W

Meggers, W. F., J. Res. Nat. Bur. Stand. (U.S.) 9, 239 (1932). W ZE

Odabasi, H., J. Opt. Soc. Am. 57, 1459 (1967). EL CL W Hfs IP PT AT

Russell, H. N., and Meggers, W. F., J. Res. Nat. Bur. Stand. (U.S.) 9, 625 (1932). EL CL W ZE IP

Sugar, J., and Kaufman, V., J. Opt. Soc. Am. 55, 1283 (1965). EL CL W IP

Sugar, J., and Reader, J., J. Chem. Phys. 59, 2083 (1973). IP

Wittke, H., Z. Phys. 116, 547 (1940). Hfs

[October 1976] 
La III

\begin{tabular}{|c|c|c|c|c|}
\hline Configuration & Term & $J$ & $\begin{array}{l}\text { Level } \\
\left(\mathrm{cm}^{-1}\right)\end{array}$ & $g$ \\
\hline $5 p^{6}\left({ }^{1} \mathrm{~S}\right) 5 d$ & ${ }^{2} \mathrm{D}$ & $\begin{array}{l}3 / 2 \\
5 / 2\end{array}$ & $\begin{array}{r}0.00 \\
1603.23\end{array}$ & \\
\hline $5 p^{6}\left({ }^{1} \mathrm{~S}\right) 4 f$ & ${ }^{2} \mathrm{~F}^{\circ}$ & $\begin{array}{l}5 / 2 \\
7 / 2\end{array}$ & $\begin{array}{l}7195.14 \\
8695.41\end{array}$ & \\
\hline $5 p^{6}\left({ }^{1} \mathrm{~S}\right) 6 s$ & ${ }^{2} \mathrm{~S}$ & $1 / 2$ & 13591.14 & 2.10 \\
\hline $5 p^{6}\left({ }^{1} \mathrm{~S}\right) 6 p$ & ${ }^{2} \mathbf{P}^{\circ}$ & $\begin{array}{l}1 / 2 \\
3 / 2\end{array}$ & $\begin{array}{l}42015.04 \\
45110.94\end{array}$ & $\begin{array}{l}0.63 \\
1.37\end{array}$ \\
\hline $5 p^{6}\left({ }^{1} \mathrm{~S}\right) 7 s$ & ${ }^{2} \mathrm{~S}$ & $1 / 2$ & 82347.28 & \\
\hline $5 p^{6}\left({ }^{1} \mathrm{~S}\right) 6 d$ & ${ }^{2} \mathrm{D}$ & $\begin{array}{l}3 / 2 \\
5 / 2\end{array}$ & $\begin{array}{l}82380.76 \\
82814.27\end{array}$ & \\
\hline $5 p^{6}\left({ }^{1} \mathrm{~S}\right) 5 f$ & ${ }^{2} \mathrm{~F}^{0}$ & $\begin{array}{l}5 / 2 \\
7 / 2\end{array}$ & $\begin{array}{l}92454.54 \\
92534.73\end{array}$ & \\
\hline $5 p^{6}\left({ }^{1} \mathrm{~S}\right) 7 p$ & ${ }^{2} \mathbf{P}^{\circ}$ & $\begin{array}{l}1 / 2 \\
3 / 2\end{array}$ & $\begin{array}{l}93232.39 \\
94461.44\end{array}$ & \\
\hline $5 p^{6}\left({ }^{1} \mathrm{~S}\right) 8 s$ & ${ }^{2} \mathrm{~S}$ & $1 / 2$ & 110209.57 & \\
\hline $5 p^{6}\left({ }^{1} \mathrm{~S}\right) 7 d$ & ${ }^{2} \mathrm{D}$ & $\begin{array}{l}3 / 2 \\
5 / 2\end{array}$ & $\begin{array}{l}110534.20 \\
110738.31\end{array}$ & \\
\hline $5 p^{6}\left({ }^{1} \mathrm{~S}\right) 5 g$ & ${ }^{2} \mathrm{G}$ & $\begin{array}{l}7 / 2 \\
9 / 2\end{array}$ & $\begin{array}{l}114754.90 \\
114755.34\end{array}$ & \\
\hline $5 p^{6}\left({ }^{1} \mathrm{~S}\right) 6 f$ & ${ }^{2} \mathrm{~F}^{\circ}$ & $\begin{array}{l}5 / 2 \\
7 / 2\end{array}$ & $\begin{array}{l}114889.80 \\
114938.90\end{array}$ & \\
\hline $5 p^{6}\left({ }^{1} \mathrm{~S}\right) 8 p$ & ${ }^{2} \mathrm{P}^{\circ}$ & $\begin{array}{l}1 / 2 \\
3 / 2\end{array}$ & $\begin{array}{l}115602.26 \\
116225.92\end{array}$ & \\
\hline $5 p^{6}\left({ }^{1} \mathrm{~S}\right) 9 s$ & ${ }^{2} \mathrm{~S}$ & $1 / 2$ & 124504.10 & \\
\hline $5 p^{6}\left({ }^{1} \mathrm{~S}\right) 8 d$ & ${ }^{2} \mathrm{D}$ & $\begin{array}{l}3 / 2 \\
5 / 2\end{array}$ & $\begin{array}{l}124742.24 \\
124856.08\end{array}$ & \\
\hline $5 p^{6}\left({ }^{1} \mathrm{~S}\right) 6 g$ & ${ }^{2} \mathrm{G}$ & $\begin{array}{l}7 / 2 \\
9 / 2\end{array}$ & $\begin{array}{l}126952.47 \\
126953.16\end{array}$ & \\
\hline $5 p^{6}\left({ }^{1} \mathrm{~S}\right) 7 f$ & ${ }^{2} \mathrm{~F}^{\circ}$ & $\begin{array}{l}5 / 2 \\
7 / 2\end{array}$ & $\begin{array}{l}127042.58 \\
127075.60\end{array}$ & \\
\hline $5 p^{6\left({ }^{1} \mathrm{~S}\right) 9 p}$ & ${ }^{2} \mathrm{P}^{\circ}$ & $\begin{array}{l}1 / 2 \\
3 / 2\end{array}$ & $\begin{array}{l}127548.93 \\
127935.04\end{array}$ & \\
\hline $5 p^{6}\left({ }^{1} \mathrm{~S}\right) 10 s$ & ${ }^{2} \mathrm{~S}$ & $1 / 2$ & 132840.41 & \\
\hline $5 p^{6}\left({ }^{1} \mathrm{~S}\right) 9 d$ & ${ }^{2} \mathrm{D}$ & $\begin{array}{l}3 / 2 \\
5 / 2\end{array}$ & $\begin{array}{l}133006.65 \\
133076.90\end{array}$ & \\
\hline $5 p^{6}\left({ }^{1} \mathrm{~S}\right) 7 g$ & ${ }^{2} \mathrm{G}$ & $\begin{array}{l}7 / 2 \\
9 / 2\end{array}$ & $\begin{array}{l}134318.02 \\
134319.39\end{array}$ & \\
\hline $5 p^{6}\left({ }^{(} \mathrm{S}\right) 8 f$ & ${ }^{2} \mathrm{~F}^{\circ}$ & $\begin{array}{l}5 / 2 \\
7 / 2\end{array}$ & $\begin{array}{l}134373.83 \\
134399.63\end{array}$ & \\
\hline $5 p^{6}\left({ }^{1} \mathrm{~S}\right) 8 g$ & ${ }^{2} \mathrm{G}$ & $\begin{array}{l}7 / 2 \\
9 / 2\end{array}$ & $\begin{array}{l}139100.48 \\
139101.70\end{array}$ & \\
\hline La IV $\left({ }^{1} \mathrm{So}\right)$ & Limit & & 154675 & \\
\hline
\end{tabular}


La IV

(Xe I sequence; 54 electrons)

$Z=57$

Ground state $\left(1 s^{2} 2 s^{2} 2 p^{6} 3 s^{2} 3 p^{6} 3 d^{10} 4 s^{2} 4 p^{6} 4 d^{10} 5 s^{2}\right) 5 p^{6}{ }^{1} \mathrm{~S}_{0}$

Ionization energy $402900 \pm 500 \mathrm{~cm}^{-1}$

$49.95 \pm 0.06 \mathrm{eV}$

Reader and Epstein $[1975,1976]$ have observed this spectrum in the range $300-5200 \AA$ using sliding-spark sources. Ten resonance lines from upper $J=1$ levels were given in the 1975 paper, but about 200 lines are classified by the unpublished analysis used here. The connection of the system of excited levels to the ground level should be accurate to about $\pm 0.5 \mathrm{~cm}^{-1}$, and the relative positions of most of the excited levels are accurate to $\pm 0.2 \mathrm{~cm}^{-1}$.

All levels of the upper configurations are here designated in the $J_{1} l$ coupling scheme. Leading eigenvector percentages in the $L S$ scheme are also listed (after the word "or") except for the $5 p^{5} 4 f$ levels. The percentages are from Reader and Epstein [1976], whose calculations of $\left(5 p^{5} 5 d+5 p^{5} 6 s\right)$ and $\left(5 p^{5} 6 d+5 p^{5} 7 s\right)$ included the configuration interactions.

Reader and Epstein [1975] obtained the quoted ionization energy from the $5 p^{5} 6 s$ and $7 s$ levels by assuming a value for the difference in the quantum defects for these configurations.

\section{References}

Reader, J., and Epstein, G. L., J. Opt. Soc. Am. 65, 638 (1975). EL CL W IP

Reader, J., and Epstein, G. L., unpublished material (1976). EL CL W PT

[March 1977]

La IV

\begin{tabular}{|c|c|c|c|c|c|c|c|}
\hline Configuration & Term & $J$ & $\begin{array}{l}\text { Level } \\
\left(\mathrm{cm}^{-1}\right)\end{array}$ & \multicolumn{4}{|c|}{ Leading percentages } \\
\hline $5 p^{6}$ & ${ }^{1} \mathrm{~S}$ & 0 & 0.0 & & & & \\
\hline $5 p^{5}\left({ }^{2} \mathrm{P}_{3 / 2}^{\circ}\right) 4 f$ & $2[3 / 2]$ & $\begin{array}{l}1 \\
2\end{array}$ & $\begin{array}{l}143354.7 \\
145949.0\end{array}$ & & & & \\
\hline $5 p^{5}\left({ }^{2} \mathrm{P}_{3 / 2}^{\circ}\right) 4 f$ & $2[5 / 2]$ & $\begin{array}{l}3 \\
2\end{array}$ & $\begin{array}{l}149927.1 \\
160486.4\end{array}$ & & & & \\
\hline $5 p^{5}\left({ }^{2} \mathrm{P}_{3 / 2}^{\circ}\right) 4 f$ & $2[7 / 2]$ & $\begin{array}{l}3 \\
4\end{array}$ & 153339.1 & & & & \\
\hline $5 p^{5}\left({ }^{2} \mathrm{P}_{3 / 2}^{\circ}\right) 5 d$ & $2[1 / 2]^{\circ}$ & $\begin{array}{l}0 \\
1\end{array}$ & $\begin{array}{l}156100.3 \\
158412.6\end{array}$ & $\begin{array}{r}100 \\
65\end{array}$ & $\begin{array}{l}\text { or } \\
\text { or }\end{array}$ & $\begin{array}{r}100 \\
93\end{array}$ & $\begin{array}{l}{ }^{3} \mathrm{P}^{\circ} \\
{ }^{3} \mathrm{P}^{\circ}\end{array}$ \\
\hline $5 p^{5}\left({ }^{2} \mathrm{P}_{3 / 2}^{\circ}\right) 5 d$ & ${ }^{2}\left[{ }^{3} / 2\right]^{\circ}$ & $\begin{array}{l}2 \\
1\end{array}$ & $\begin{array}{l}162867.6 \\
181155.0\end{array}$ & $\begin{array}{l}90 \\
51\end{array}$ & $\begin{array}{l}\text { or } \\
\text { or }\end{array}$ & $\begin{array}{l}78 \\
88\end{array}$ & $\begin{array}{l}{ }^{3} \mathrm{P}^{\circ} \\
{ }^{3} \mathrm{D}^{\circ}\end{array}$ \\
\hline $5 p^{5}\left({ }^{2} \mathrm{P}_{3 / 2}^{\circ}\right) 5 d$ & ${ }^{2}\left[{ }^{7} / 2\right]^{\circ}$ & $\begin{array}{l}4 \\
3\end{array}$ & $\begin{array}{l}163693.3 \\
165070.7\end{array}$ & $\begin{array}{r}100 \\
78\end{array}$ & $\begin{array}{l}\text { or } \\
\text { or }\end{array}$ & $\begin{array}{r}100 \\
74\end{array}$ & $\begin{array}{l}{ }^{3} \mathrm{~F}^{\circ} \\
{ }^{3} \mathrm{~F}^{\circ}\end{array}$ \\
\hline $5 p^{5}\left({ }^{2} \mathrm{P}_{3 / 2}^{\circ}\right) 5 d$ & $2[5 / 2]^{\circ}$ & $\begin{array}{l}2 \\
3\end{array}$ & $\begin{array}{l}167921.7 \\
173335.5\end{array}$ & $\begin{array}{l}95 \\
80\end{array}$ & $\begin{array}{l}\text { or } \\
\text { or }\end{array}$ & $\begin{array}{l}42 \\
62\end{array}$ & $\begin{array}{l}{ }^{3} \mathrm{~F}^{\circ} \\
{ }^{3} \mathrm{D}^{\circ}\end{array}$ \\
\hline $5 p^{5}\left({ }^{2} \mathrm{P}_{1 / 2}^{\circ}\right) 4 f$ & $2[5 / 2]$ & $\begin{array}{l}3 \\
2\end{array}$ & 175013.8 & & & & \\
\hline $5 p^{5}\left({ }^{2} \mathrm{P}_{1 / 2}^{\circ}\right) 5 d$ & $2[5 / 2]^{\circ}$ & $\begin{array}{l}2 \\
3\end{array}$ & $\begin{array}{l}184885.7 \\
190831.7\end{array}$ & $\begin{array}{l}91 \\
96\end{array}$ & $\begin{array}{l}\text { or } \\
\text { or }\end{array}$ & $\begin{array}{l}55 \\
45\end{array}$ & $\begin{array}{l}{ }^{3} \mathrm{~F}^{\circ} \\
{ }^{1} \mathrm{~F}^{\circ}\end{array}$ \\
\hline
\end{tabular}


La IV-Continued

\begin{tabular}{|c|c|c|c|c|c|c|c|}
\hline Configuration & Term & $J$ & $\begin{array}{l}\text { Level } \\
\left(\mathrm{cm}^{-1}\right)\end{array}$ & \multicolumn{4}{|c|}{ Leading percentages } \\
\hline $5 p^{5}\left({ }^{2} \mathrm{P}_{1 / 2}^{\circ}\right) 5 d$ & $2[3 / 2]^{\circ}$ & $\begin{array}{l}2 \\
1\end{array}$ & $\begin{array}{l}188393.2 \\
215919.2\end{array}$ & $\begin{array}{l}85 \\
59\end{array}$ & $\begin{array}{l}\text { or } \\
\text { or }\end{array}$ & $\begin{array}{l}58 \\
93\end{array}$ & $\begin{array}{l}{ }^{3} \mathrm{D}^{\circ} \\
{ }^{1} \mathrm{P}^{\circ}\end{array}$ \\
\hline $5 p^{5}\left({ }^{2} \mathrm{P}_{3 / 2}^{\circ}\right) 6 s$ & $2[3 / 2]^{\circ}$ & $\begin{array}{l}2 \\
1\end{array}$ & $\begin{array}{l}198303.1 \\
200183.3\end{array}$ & $\begin{array}{l}98 \\
99\end{array}$ & $\begin{array}{l}\text { or } \\
\text { or }\end{array}$ & $\begin{array}{l}98 \\
59\end{array}$ & $\begin{array}{l}{ }^{3} \mathrm{P}^{\circ} \\
{ }^{1} \mathrm{P}^{\circ}\end{array}$ \\
\hline $5 p^{5}\left({ }^{2} \mathrm{P}_{1 / 2}^{\circ}\right) 6 s$ & ${ }^{2}[1 / 2]^{\circ}$ & $\begin{array}{l}0 \\
1\end{array}$ & $\begin{array}{l}219336.9 \\
220506.9\end{array}$ & $\begin{array}{r}100 \\
99\end{array}$ & $\begin{array}{l}\text { or } \\
\text { or }\end{array}$ & $\begin{array}{r}100 \\
59\end{array}$ & $\begin{array}{l}{ }^{3} \mathrm{P}^{\circ} \\
{ }^{3} \mathrm{P}^{\circ}\end{array}$ \\
\hline $5 p^{5}\left({ }^{2} \mathrm{P}_{3 / 2}^{\circ}\right) 6 p$ & $2[1 / 2]$ & $\begin{array}{l}1 \\
0\end{array}$ & $\begin{array}{l}231008.7 \\
244301.8\end{array}$ & $\begin{array}{l}86 \\
94\end{array}$ & $\begin{array}{l}\text { or } \\
\text { or }\end{array}$ & $\begin{array}{l}70 \\
57\end{array}$ & $\begin{array}{l}{ }^{3} \mathrm{~S} \\
{ }^{\mathrm{P}} \mathrm{P}\end{array}$ \\
\hline $5 p^{5}\left({ }^{2} \mathrm{P}_{3 / 2}^{\circ}\right) 6 p$ & $2[5 / 2]$ & $\begin{array}{l}2 \\
3\end{array}$ & $\begin{array}{l}233401.5 \\
236790.4\end{array}$ & $\begin{array}{r}85 \\
100\end{array}$ & $\begin{array}{l}\text { or } \\
\text { or }\end{array}$ & $\begin{array}{r}54 \\
100\end{array}$ & $\begin{array}{l}{ }^{3} \mathrm{D} \\
{ }^{3} \mathrm{D}\end{array}$ \\
\hline $5 p^{5}\left({ }^{2} \mathrm{P}_{3 / 2}^{\circ}\right) 6 p$ & $2[3 / 2]$ & $\begin{array}{l}1 \\
2\end{array}$ & $\begin{array}{l}237727.3 \\
239654.3\end{array}$ & $\begin{array}{l}88 \\
85\end{array}$ & $\begin{array}{l}\text { or } \\
\text { or }\end{array}$ & $\begin{array}{l}56 \\
69\end{array}$ & $\begin{array}{l}{ }^{1} \mathrm{P} \\
{ }^{3} \mathrm{P}\end{array}$ \\
\hline $5 p^{5}\left({ }^{2} \mathrm{P}_{1 / 2}^{\circ}\right) 6 p$ & $2[3 / 2]$ & $\begin{array}{l}1 \\
2\end{array}$ & $\begin{array}{l}254251.3 \\
259224.3\end{array}$ & $\begin{array}{r}91 \\
100\end{array}$ & $\begin{array}{l}\text { or } \\
\text { or }\end{array}$ & $\begin{array}{l}70 \\
45\end{array}$ & $\begin{array}{l}{ }^{3} \mathrm{D} \\
{ }^{3} \mathrm{D}\end{array}$ \\
\hline $5 p^{5}\left({ }^{2} \mathrm{P}_{1 / 2}^{\circ}\right) 6 p$ & $2[1 / 2]$ & $\begin{array}{l}1 \\
0\end{array}$ & $\begin{array}{l}259085.9 \\
260449.8\end{array}$ & $\begin{array}{l}90 \\
94\end{array}$ & $\begin{array}{l}\text { or } \\
\text { or }\end{array}$ & $\begin{array}{l}59 \\
57\end{array}$ & $\begin{array}{l}{ }^{3} \mathrm{P} \\
{ }^{1} \mathrm{~S}\end{array}$ \\
\hline $5 p^{5}\left({ }^{2} \mathrm{P}_{3 / 2}^{\circ}\right) 6 d$ & ${ }^{2}[1 / 2]^{\circ}$ & $\begin{array}{l}0 \\
1\end{array}$ & $\begin{array}{l}284205.8 \\
284981.9\end{array}$ & $\begin{array}{r}100 \\
65\end{array}$ & $\begin{array}{l}\text { or } \\
\text { or }\end{array}$ & $\begin{array}{r}100 \\
86\end{array}$ & $\begin{array}{l}{ }^{3} \mathrm{P}^{\circ} \\
{ }^{3} \mathrm{P}^{\circ}\end{array}$ \\
\hline $5 p^{5}\left({ }^{2} \mathrm{P}_{3 / 2}^{\circ}\right) 6 d$ & ${ }^{2}[7 / 2]^{\circ}$ & $\begin{array}{l}4 \\
3\end{array}$ & $\begin{array}{l}285856.2 \\
285951.0\end{array}$ & $\begin{array}{r}100 \\
94\end{array}$ & $\begin{array}{l}\text { or } \\
\text { or }\end{array}$ & $\begin{array}{r}100 \\
55\end{array}$ & $\begin{array}{l}{ }^{3} \mathrm{~F}^{\circ} \\
{ }^{3} \mathrm{~F}^{\circ}\end{array}$ \\
\hline $5 p^{5}\left({ }^{2} \mathrm{P}_{3 / 2}^{\circ}\right) 6 d$ & ${ }^{2}\left[{ }^{3} / 2\right]^{\circ}$ & $\begin{array}{l}2 \\
1\end{array}$ & $\begin{array}{l}286298.2 \\
290596.2\end{array}$ & $\begin{array}{l}97 \\
63\end{array}$ & $\begin{array}{l}\text { or } \\
\text { or }\end{array}$ & $\begin{array}{l}57 \\
52\end{array}$ & $\begin{array}{l}{ }^{3} \mathrm{P}^{\circ} \\
{ }^{1} \mathrm{P}^{\circ}\end{array}$ \\
\hline $5 p^{5}\left({ }^{2} \mathrm{P}_{3 / 2}^{\circ}\right) 6 d$ & $2[5 / 2]^{\circ}$ & $\begin{array}{l}2 \\
3\end{array}$ & $\begin{array}{l}287402.6 \\
287874.0\end{array}$ & $\begin{array}{l}98 \\
94\end{array}$ & $\begin{array}{l}\text { or } \\
\text { or }\end{array}$ & $\begin{array}{l}51 \\
71\end{array}$ & $\begin{array}{l}{ }^{1} \mathrm{D}^{\circ} \\
{ }^{3} \mathrm{D}^{\circ}\end{array}$ \\
\hline $5 p^{5}\left({ }^{2} \mathrm{P}_{3 / 2}^{\circ}\right) 7 s$ & $2[3 / 2]^{\circ}$ & $\begin{array}{l}2 \\
1\end{array}$ & $\begin{array}{l}292080.3 \\
292777.8\end{array}$ & $\begin{array}{r}100 \\
97\end{array}$ & $\begin{array}{l}\text { or } \\
\text { or }\end{array}$ & $\begin{array}{r}100 \\
62\end{array}$ & $\begin{array}{l}{ }^{3} \mathrm{P}^{\circ} \\
{ }^{1} \mathrm{P}^{\circ}\end{array}$ \\
\hline $5 p^{5}\left({ }^{2} \mathrm{P}_{1 / 2}^{\circ}\right) 6 d$ & $2[5 / 2]^{\circ}$ & $\begin{array}{l}2 \\
3\end{array}$ & $\begin{array}{l}307183.0 \\
308151.3\end{array}$ & $\begin{array}{r}97 \\
100\end{array}$ & $\begin{array}{l}\text { or } \\
\text { or }\end{array}$ & $\begin{array}{l}72 \\
41\end{array}$ & $\begin{array}{l}{ }^{3} \mathrm{~F}^{\circ} \\
{ }^{3} \mathrm{~F}^{\circ}\end{array}$ \\
\hline $5 p^{5}\left({ }^{2} \mathrm{P}_{1 / 2}^{\circ}\right) 6 d$ & ${ }^{2}[3 / 2]^{\circ}$ & $\begin{array}{l}2 \\
1\end{array}$ & $\begin{array}{l}307398.4 \\
310049.8\end{array}$ & $\begin{array}{l}97 \\
98\end{array}$ & $\begin{array}{l}\text { or } \\
\text { or }\end{array}$ & $\begin{array}{l}41 \\
45\end{array}$ & $\begin{array}{l}{ }^{3} \mathrm{P}^{\circ} \\
{ }^{1} \mathrm{P}^{\circ}\end{array}$ \\
\hline $5 p^{5}\left({ }^{2} \mathrm{P}_{1 / 2}^{\circ}\right) 7 s$ & $2[1 / 2]^{\circ}$ & $\begin{array}{l}0 \\
1\end{array}$ & $\begin{array}{l}313512.4 \\
313917.0\end{array}$ & $\begin{array}{l}100 \\
100\end{array}$ & $\begin{array}{l}\text { or } \\
\text { or }\end{array}$ & $\begin{array}{r}100 \\
64\end{array}$ & $\begin{array}{l}{ }^{3} \mathrm{P}^{\circ} \\
{ }^{3} \mathrm{P}^{\circ}\end{array}$ \\
\hline $\mathrm{La} \mathrm{v}\left({ }^{2} \mathrm{P}_{3 / 2}^{\circ}\right)$ & Limit & & 402900 & & & & \\
\hline
\end{tabular}




\section{La V}

(I I sequence; 53 electrons)

Ground state $\left(1 s^{2} 2 s^{2} 2 p^{6} 3 s^{2} 3 p^{6} 3 d^{10} 4 s^{2} 4 p^{6} 4 d^{10}\right) 5 s^{2} 5 p^{5}{ }^{2} \mathrm{P}_{3 / 2}^{\circ}$

Ionization energy $497000 \pm 5000 \mathrm{~cm}^{-1}$

$61.6 \pm 0.6 \mathrm{eV}$

Identified odd configuration $\quad 5 s^{2} 5 p^{5}$

Identified even configurations $\quad 5 s 5 p^{6}, 5 s^{2} 5 p^{4} 5 d, 5 s^{2} 5 p^{4} 6 s$

The levels and eigenvector percentages are from the analysis by Epstein and Reader [1976]. Their list of transitions from the even levels to the $5 p^{5}{ }^{2} \mathrm{P}^{\circ}$ ground-term levels extends over the range $389-825 \AA$. In addition to the $5 s 5 p^{6}{ }^{2} \mathrm{~S}_{1 / 2}$ level, most of the levels of the $5 p^{4} 5 d$ and $5 p^{4} 6 s$ configurations that can combine with the ground term have been found. The uncertainty of the $5 p^{5}{ }^{2} \mathrm{P}_{1 / 2}^{\circ}$ and $5 s 5 p^{6}{ }^{2} \mathrm{~S}_{1 / 2}$ levels relative to the ${ }^{2} \mathrm{P}_{3 / 2}^{\circ}$ ground level is $\pm 1 \mathrm{~cm}^{-1}$; the uncertainty increases for the higher levels to about $\pm 3 \mathrm{~cm}^{-1}$ at $250000 \mathrm{~cm}^{-1}$. We have rounded off the levels above $180000 \mathrm{~cm}^{-1}$ to the nearest $\mathrm{cm}^{-1}$.

Epstein and Reader based their interpretation on a calculation of the three known even configurations, with the configuration interactions being included. Eigenvector percentages for the $5 p^{4} 6 s$ configuration are given here in the $J_{1} j$ coupling scheme, the second percentages being from the unpublished complete eigenvectors [Epstein and Reader, 1975]. We have omitted names for several even levels, and some of the retained names have little or no meaning. For example, two $J=3 / 2$ levels with leading percentages well below $40 \%$ are assigned to terms. The two leading percentages for the nominal $5 p^{4}\left({ }^{3} \mathrm{P}\right) 5 d^{4} \mathrm{D}_{1 / 2}$ level $\left(169748.5 \mathrm{~cm}^{-1}\right)$ are almost identical to those for the unnamed level at $185115 \mathrm{~cm}^{-1}$. Epstein and Reader note strong configuration mixing in several of the eigenvectors. Although the interaction between the $5 s 5 p^{6}{ }^{2} \mathrm{~S}_{1 / 2}$ and $5 p^{4}\left({ }^{1} \mathrm{D}\right) 5 d^{2} \mathrm{~S}_{1 / 2}$ levels is less in La $\mathrm{V}$ than for lower sequence members, the La V $5 s 5 p^{6}{ }^{2} \mathrm{~S}_{1 / 2}$ level is displaced about $25000 \mathrm{~cm}^{-1}$ by this perturbation. The $5 p^{4} 5 d-5 p^{4} 6 s$ interaction is also strong (30-40\% admixture) for two pairs of levels.

Good agreement between the calculated and experimental even levels was necessary for the analysis, since the $J$ values could not be determined unambiguously before the levels were identified. In general the even levels having $J=1 / 2$ or $3 / 2$ are based on pairs of lines separated by the ${ }^{2} \mathrm{P}_{3 / 2}^{\circ}-{ }^{2} \mathrm{P}_{1 / 2}^{\circ}$ wavenumber interval. The questionable level at $169748 \mathrm{~cm}^{-1}$, designated $5 p^{4}\left({ }^{3} \mathrm{P}\right) 5 d{ }^{4} \mathrm{D}_{1 / 2}$, is an exception derived from a single line.

Each of the levels having $J=5 / 2$ is also based on a single allowed transition (to the ${ }^{2} \mathrm{P}_{3 / 2}^{\circ}$ ground level), but all such transitions clearly belong to La $\mathrm{V}$ and have wavelengths close to the predicted values. The level designated $5 p^{4}\left({ }^{3} \mathrm{P}_{2}\right) 6 s(2,1 / 2)_{5 / 2}^{\circ}$, at $225191 \mathrm{~cm}^{-1}$, is less certain because of the relative weakness of the transition. The assignment of the $J=3 / 2$ level for this term is also uncertain, because of ambiguity in the $J$ values of this level $\left(228545 \mathrm{~cm}^{-1}\right)$ and the nearby level at $228916 \mathrm{~cm}^{-1}$; it is possible that the $J$ values and eigenvector assignments of these two levels should be interchanged [Epstein and Reader, 1976].

Epstein and Reader obtained the ionization energy from the $5 p^{4}\left({ }^{3} \mathrm{P}_{2}\right) 6 s(2,1 / 2)$ term by using an estimated value of the effective quantum number $n^{*}(6 s)$. A correction for the perturbation of this term was included.

\section{References}

Epstein, G. L., and Reader, J., unpublished material (1975). PT

Epstein, G. L., and Reader, J., J. Opt. Soc. Am. 66, 590 (1976). EL CL W Hfs IP PT AT

[October 1976] 
La V

\begin{tabular}{|c|c|c|c|c|c|c|c|}
\hline Configuration & Term & $J$ & $\begin{array}{l}\text { Level } \\
\left(\mathrm{cm}^{-1}\right)\end{array}$ & \multicolumn{4}{|c|}{ Leading percentages } \\
\hline $5 s^{2}\left({ }^{1} \mathrm{~S}\right) 5 p^{5}$ & ${ }^{2} \mathrm{P}^{\circ}$ & $\begin{array}{l}3 / 2 \\
1 / 2\end{array}$ & $\begin{array}{r}0.0 \\
21634.1\end{array}$ & & & & \\
\hline $5 s 5 p^{6}$ & ${ }^{2} \mathrm{~S}$ & $1 / 2$ & 142970.1 & 70 & & 28 & $5 p^{4}\left({ }^{1} \mathrm{D}\right) 5 d^{2} \mathrm{~S}$ \\
\hline $5 s^{2}\left({ }^{1} \mathrm{~S}\right) 5 p^{4}\left({ }^{3} \mathrm{P}\right) 5 d$ & ${ }^{4} \mathrm{D}$ & $\begin{array}{l}5 / 2 \\
3 / 2 \\
7 / 2 \\
1 / 2\end{array}$ & $\begin{array}{r}166600.9 \\
167307.9 \\
169748.5 ?\end{array}$ & $\begin{array}{l}75 \\
65 \\
77 \\
52\end{array}$ & & $\begin{array}{r}7 \\
12 \\
16 \\
19\end{array}$ & $\begin{array}{l}\left({ }^{3} \mathrm{P}\right){ }^{4} \mathrm{~F} \\
\left({ }^{3} \mathrm{P}\right){ }^{4} \mathrm{P} \\
\left({ }^{3} \mathrm{P}\right) \\
\left({ }^{4} \mathrm{~F}\right. \\
{ }^{2} \mathrm{P}\end{array}$ \\
\hline $5 s^{2}\left({ }^{1} \mathrm{~S}\right) 5 p^{4}\left({ }^{3} \mathrm{P}\right) 5 d$ & ${ }^{4} \mathrm{P}$ & $\begin{array}{l}3 / 2 \\
1 / 2 \\
5 / 2\end{array}$ & $\begin{array}{l}182670 \\
183552 \\
189842\end{array}$ & $\begin{array}{l}33 \\
74 \\
52\end{array}$ & & $\begin{array}{l}20 \\
14 \\
18\end{array}$ & $\begin{array}{l}\left({ }^{1} \mathrm{~S}\right){ }^{2} \mathrm{D} \\
\left({ }^{3} \mathrm{P}\right){ }^{2} \mathrm{P} \\
\left({ }^{3} \mathrm{P}\right){ }^{2} \mathrm{~F}\end{array}$ \\
\hline $5 s^{2}\left({ }^{1} \mathrm{~S}\right) 5 p^{4}\left({ }^{3} \mathrm{P}\right) 5 d$ & & $1 / 2$ & 185115 & 48 & ${ }^{4} \mathrm{D}$ & 23 & (iD) ${ }^{2} \mathrm{P}$ \\
\hline $5 s^{2}\left({ }^{1} \mathrm{~S}\right) 5 p^{4}\left({ }^{3} \mathrm{P}\right) 5 d$ & ${ }^{4} \mathrm{~F}$. & $\begin{array}{l}9 / 2 \\
5 / 2 \\
3 / 2 \\
7 / 2\end{array}$ & $\begin{array}{l}187535 \\
188299\end{array}$ & $\begin{array}{l}83 \\
80 \\
67 \\
49\end{array}$ & & $\begin{array}{r}17 \\
7 \\
19 \\
23\end{array}$ & $\begin{array}{l}\left({ }^{1} \mathrm{D}\right){ }^{2} \mathrm{G} \\
\left({ }^{3} \mathrm{P}\right){ }^{4} \mathrm{D} \\
\left({ }^{3} \mathrm{P}\right){ }^{4} \mathrm{P} \\
\left({ }^{3} \mathrm{P}\right){ }^{2} \mathrm{~F}\end{array}$ \\
\hline $5 s^{2}\left({ }^{1} \mathrm{~S}\right) 5 p^{4}\left({ }^{3} \mathrm{P}\right) 5 d$ & & $3 / 2$ & 190217 & 29 & ${ }^{4} \mathrm{D}$ & 23 & $\left({ }^{1} \mathrm{D}\right){ }^{2} \mathrm{D}$ \\
\hline $5 s^{2}\left({ }^{1} \mathrm{~S}\right) 5 p^{4}\left(^{1} \mathrm{D}\right) 5 d$ & & $3 / 2$ & 196794 & 28 & ${ }^{2} \mathrm{P}$ & 28 & $\left({ }^{3} \mathrm{P}\right){ }^{2} \mathrm{P}$ \\
\hline $5 s^{2}\left({ }^{1} \mathrm{~S}\right) 5 p^{4}\left({ }^{1} \mathrm{D}\right) 5 d$ & & $5 / 2$ & 198577 & 26 & ${ }^{2} \mathrm{D}$ & 24 & $\left({ }^{3} \mathrm{P}\right){ }^{4} \mathrm{P}$ \\
\hline $5 s^{2}\left({ }^{1} \mathrm{~S}\right) 5 p^{4}\left({ }^{3} \mathrm{P}\right) 5 d$ & ${ }^{2} \mathrm{~F}$ & $\begin{array}{l}7 / 2 \\
5 / 2\end{array}$ & 200771 & $\begin{array}{l}43 \\
51\end{array}$ & & $\begin{array}{l}29 \\
23\end{array}$ & $\begin{array}{l}\left({ }^{3} \mathrm{P}\right){ }^{4} \mathrm{~F} \\
\left.\text { ( }{ }^{\mathrm{D}}\right)^{2} \mathrm{~F}\end{array}$ \\
\hline $5 s^{2}\left({ }^{1} \mathrm{~S}\right) 5 p^{4}\left({ }^{1} \mathrm{D}\right) 5 d$ & ${ }^{2} \mathbf{F}$ & $\begin{array}{l}5 / 2 \\
7 / 2\end{array}$ & 207398 & $\begin{array}{l}61 \\
78\end{array}$ & & $\begin{array}{l}27 \\
14\end{array}$ & $\begin{array}{l}\left({ }^{1} \mathrm{D}\right){ }^{2} \mathrm{D} \\
\left({ }^{\mathrm{B} P}\right){ }^{2} \mathrm{~F}\end{array}$ \\
\hline $5 s^{2}\left({ }^{1} \mathrm{~S}\right) 5 p^{4}\left({ }^{1} \mathrm{~S}\right) 5 d$ & ${ }^{2} \mathrm{D}$ & $\begin{array}{l}3 / 2 \\
5 / 2\end{array}$ & $\begin{array}{l}222023 \\
236365\end{array}$ & $\begin{array}{l}35 \\
64\end{array}$ & & $\begin{array}{l}28 \\
11\end{array}$ & $\begin{array}{l}\left.{ }^{(1 D}\right)^{2} \mathrm{P} \\
\left({ }^{(3 P}\right){ }^{2} \mathrm{~F}\end{array}$ \\
\hline $5 s^{2}\left({ }^{1} \mathrm{~S}\right) 5 p^{4}\left({ }^{3} \mathrm{P}_{2}\right) 6 s_{1 / 2}$ & $(2,1 / 2)$ & $\begin{array}{l}5 / 2 \\
3 / 2 ?\end{array}$ & $\begin{array}{l}225191 ? \\
228545^{*}\end{array}$ & $\begin{array}{l}81 \\
54\end{array}$ & & $\begin{array}{l}15 \\
15\end{array}$ & $\left({ }^{1} \mathrm{D}_{2}\right)\left(2,{ }^{1} / 2\right)$ \\
\hline $5 s^{2}\left({ }^{1} \mathrm{~S}\right) 5 p^{4}\left({ }^{1} \mathrm{D}\right) 5 d ?$ & ${ }^{2} \mathrm{~S} ?$ & $1 / 2 ?$ & 228916 & 49 & & 19 & $5 s 5 p^{6}{ }^{2} \mathrm{~S}$ \\
\hline $5 s^{2}\left({ }^{1} \mathrm{~S}\right) 5 p^{4}\left({ }^{3} \mathrm{P}\right) 5 d$ & ${ }^{2} \mathrm{D}$ & $\begin{array}{l}5 / 2 \\
3 / 2\end{array}$ & $\begin{array}{l}229740 \\
250921\end{array}$ & $\begin{array}{l}58 \\
43\end{array}$ & & $\begin{array}{l}24 \\
29\end{array}$ & $\begin{array}{l}\left({ }^{1} \mathrm{D}\right){ }^{2} \mathrm{D} \\
{ }^{\left({ }^{2} \mathrm{~S}\right)}{ }^{2} \mathrm{D}\end{array}$ \\
\hline $5 s^{2}\left({ }^{1} \mathrm{~S}\right) 5 p^{4}\left({ }^{3} \mathrm{P}\right) 5 d$ & & $3 / 2$ & 231424 & 24 & ${ }^{2} \mathbf{P}$ & 22 & $\left({ }^{1} \mathrm{D}\right){ }^{2} \mathrm{D}$ \\
\hline $5 s^{2}\left({ }^{1} \mathrm{~S}\right) 5 p^{4}\left({ }^{3} \mathrm{P}\right) 5 d$ & & $1 / 2$ & 237222 & 30 & ${ }^{2 P} \mathrm{P}$ & 28 & $\left({ }^{1} \mathrm{D}\right){ }^{2} \mathrm{P}$ \\
\hline $5 s^{2}\left({ }^{1} \mathrm{~S}\right) 5 p^{4}\left({ }^{3} \mathrm{P}_{0}\right) 6 s_{1 / 2}$ & & $1 / 2$ & 240308 & 38 & $(0,1 / 2)$ & 17 & $\left({ }^{1} \mathrm{D}\right) 5 d{ }^{2} \mathrm{~S}$ \\
\hline $5 s^{2}\left({ }^{1} \mathrm{~S}\right) 5 p^{4}\left({ }^{3} \mathrm{P}_{1}\right) 6 s_{1 / 2}$ & $(1,1 / 2)$ & $\begin{array}{l}3 / 2 \\
1 / 2\end{array}$ & $\begin{array}{l}246854 \\
250411\end{array}$ & $\begin{array}{l}96 \\
94\end{array}$ & & $\begin{array}{l}3 \\
3\end{array}$ & 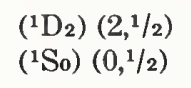 \\
\hline $5 s^{2}\left({ }^{1} \mathrm{~S}\right) 5 p^{4}\left({ }^{1} \mathrm{D}_{2}\right) 6 s_{1 / 2}$ & $(2,1 / 2)$ & $\begin{array}{l}5 / 2 \\
3 / 2\end{array}$ & $\begin{array}{l}255936 \\
257047\end{array}$ & $\begin{array}{l}83 \\
73\end{array}$ & & $\begin{array}{l}15 \\
20\end{array}$ & $\left({ }^{3} \mathrm{P}_{2}\right)(2,1 / 2)$ \\
\hline $\mathrm{La}$ VI $\left({ }^{3} \mathrm{P}_{2}\right)$ & Limit & $\ldots$ & 497000 & & & & \\
\hline
\end{tabular}




\section{CERIUM}

Ce I

58 electrons

$Z=58$

Ground state $\left(1 s^{2} 2 s^{2} 2 p^{6} 3 s^{2} 3 p^{6} 3 d^{10} 4 s^{2} 4 p^{6} 4 d^{10} 5 s^{2} 5 p^{6}\right) 4 f 5 d 6 s^{2}{ }^{1} \mathrm{G}_{4}^{\circ}$

Ionization energy $44672 \pm 3 \mathrm{~cm}^{-1}$

$\mathbf{5 . 5 3 8 7} \pm 0.0004 \mathrm{eV}$

Identified odd configurations

$4 f 5 d 6 s^{2}, 4 f 5 d^{2} 6 s, 4 f 5 d^{3} ?, 4 f^{2} 6 s 6 p$

Identified even configurations

$4 f^{2} 6 s^{2}, 4 f^{2} 5 d 6 s, 4 f 6 s^{2} 6 p, 4 f 5 d 6 s 6 p, 4 f 5 d^{2} 6 p, 4 f^{2} 5 d^{2}$

\section{Energy Levels, Wavelengths}

The levels are from Martin's unpublished tables for this spectrum [1975]; the low levels of both parities up to $10000 \mathrm{~cm}^{-1}$ have been published previously [Martin, 1971]. The odd levels are given here to $31000 \mathrm{~cm}^{-1}$, and the table of almost 700 even levels includes all those known below $30000 \mathrm{~cm}^{-1}$. Many of the levels in the working lists above these points are not yet well established.

The uncertainty for a number of the odd levels below $5000 \mathrm{~cm}^{-1}$ is \pm 0.001 to $\pm 0.003 \mathrm{~cm}^{-1}$, and most of the high even levels $\left(>15000 \mathrm{~cm}^{-1}\right)$ have uncertainties of less than $0.01 \mathrm{~cm}^{-1}$. The low even levels and the high odd levels have uncertainties of 0.01 to $0.02 \mathrm{~cm}^{-1}$.

About 15000 lines are classified as transitions from high even levels to the low odd group belonging mainly to $4 f 5 d 6 s^{2}$ and $4 f 5 d^{2} 6 s$. A relatively small part of the spectrum arises from transitions to the low even levels. The principal line list, a table of wavelengths for the region 3000-10000 $\AA$ measured by Martin, Corliss, and Blanc, is unpublished. However, almost 1000 of the strongest Ce I lines are listed in the NBS intensity tables [Meggers, Corliss, and Scribner], most with energy-level classifications from Martin's analysis, and a published Ce line list for the infrared $(0.82-2.4 \mu \mathrm{m})$ has about 1100 classified Ce I lines [Verges, Corliss, and Martin]. The older line lists of King [1928] and of Paul [1936] were useful for their definite assignments of lines to Ce I. Martin and Wilson have reobserved the absorption spectrum to below $3000 \AA$, but no list of the wavelengths has been prepared.

\section{Zeeman Data, Optical and Magnetic-Resonance Investigations}

The three-place (and two-place) $g$ values have been obtained from measurements of Zeeman patterns for several thousand lines [Martin, 1975]. Childs and Goodman used the atomic-beam magnetic-resonance technique to obtain the 5-place $g$ values quoted for 32 low odd levels and for the lowest even level. This appears to be the largest number of such atomicbeam $g$-value determinations made for any atom thus far. The uncertainties range from 2 to 10 units in the fifth place. The uncertainty for the optically determined values also varies, and in most cases it is less than 0.01 .

Smith and Spalding [1961] used the atomic-beam magnetic-resonance technique to make the first measurements of three $g$ values in Ce I. The corresponding levels were found and included in the 15 low odd levels located in the first successful analysis of the optical spectrum; they were shown to be the nominal $4 f 5 d 6 s^{2}{ }^{1} \mathrm{G}_{4}^{\circ},{ }^{3} \mathrm{~F}_{2}^{\circ}$ and ${ }^{3} \mathrm{~F}_{3}^{\circ}$ levels [Martin, 1963]. No simultaneous determinations of $J$ and $g$ values for Ce I levels have been made by the atomic-beam magnetic-resonance method. Using a beam of cerium-143 atoms, Maleh [1965] observed resonances that he associated with the three low levels for which Smith and Spalding had obtained $g$ values. Maleh's interpretation of the resonances for two of the levels is in agreement with the $J$ values obtained from analysis of the optical spectrum, but his interpretation of a third set of resonances as due to a low level having $g=0.7651$ and $J=4$ is inconsistent with the more detailed and highly redundant optical data [Martin, 1963; 1971]. 


\section{Theoretical Calculations, Status of the Analysis}

Most of the theoretical calculations of Ce I have been carried out by Z. Goldschmidt and her colleagues at the Hebrew University of Jerusalem. The eigenvector percentages for the low odd configuration group $\left(4 f 5 d 6 s^{2}+4 f 5 d^{2} 6 s+4 f 5 d^{3}\right)$ are from a calculation by Goldschmidt and Salomon [1970] that included configuration interactions and effective interactions. The levels having low term purities could be reliably interpreted only with such calculations, and Martin's [1971] arrangement of these levels was also based on the eigenvectors of Goldschmidt and Salomon. We have allowed fewer practically meaningless term names for low-purity levels in the arrangement here, since the two leading percentages are listed.

The levels are complete to above $10000 \mathrm{~cm}^{-1}$, but the assignments of two odd levels having $J=4\left(3100\right.$ and $\left.3312 \mathrm{~cm}^{-1}\right)$ might possibly be interchanged, as might those of two levels having $J=5\left(7715\right.$ and $\left.7842 \mathrm{~cm}^{-1}\right)$. Experimental $g$ values are lacking for most of the low odd levels above $10000 \mathrm{~cm}^{-1}$, and only a few odd levels above $10500 \mathrm{~cm}^{-1}$ have been assigned at this stage. It is almost certain, however, that the lowest predicted level of either parity not yet found is a ${ }^{3} \mathrm{P}_{0}^{\circ}$ level (mainly from $4 f 5 d^{2} 6 s$ ) calculated by Goldschmidt and Salomon to be near $12000 \mathrm{~cm}^{-1}$. The region below this point has 113 odd levels.

Goldschmidt notes that $4 f^{2} 6 s 6 p$ and $4 f^{2} 5 d 6 p$ have been added to the three odd configurations mentioned above in a more recent calculation of all five configurations with interaction. The assignments of several odd levels above $18000 \mathrm{~cm}^{-1}$ to $J_{1} J_{2}$-coupling terms of $4 f^{2} 6 s 6 p$ are taken from this preliminary work by Goldschmidt and Salomon [1973]. An extension of the experimental data (such as $g$ values) will probably be necessary before enough levels can be reliably assigned to the $4 f 5 d^{3}, 4 f^{2} 6 s 6 p$, and $4 f^{2} 5 d 6 p$ configurations to allow improvement of the theoretical parameters.

Only the lowest few terms of the system of even levels could be identified without extensive calculations [Martin, 1971]. Y. Oreg [1973], under the direction of Zipora Goldschmidt, has constructed and carried out the first diagonalizations of the necessary matrices. The eigenvector percentages for the even levels are from Oreg's calculation of the nine configurations $\left(4 f^{2} 6 s^{2}+4 f^{2} 5 d 6 s+4 f^{2} 5 d^{2}+4 f 5 d 6 s 6 p+4 f 5 d^{2} 6 p+4 f 6 s^{2} 6 p+5 d^{2} 6 s^{2}+5 d^{3} 6 s+5 d^{4}\right)$. Most of the eigenvectors are highly mixed with regard to both configuration and term type. (Both the direct configuration interactions and effective interactions were included.) Comparison of the calculated and experimental levels shows that the great majority of predicted levels have been found up to about $27000 \mathrm{~cm}^{-1}$, and that they belong primarily to a mixture of the first six of the above configurations. In many cases, however, the calculated levels and $g$ values are not sufficiently accurate to allow unambiguous correlations with the experimental levels of this dense system. We have not entered eigenvector percentages for the even levels above some particular level (depending on the $J$ value) at which uncertain assignments become too numerous. Most of the interpretation up to about $20000 \mathrm{~cm}^{-1}$ appears secure, however. the assignments here being in general those made by Oreg. Some of the more questionable assignments are so marked. A few assignments were changed on the basis of line intensities and/or $g$ values, and a number of additional assignments were made [Martin, 1975]. The interpretation at this stage establishes at least the lower portions of the first six of the above configurations except $4 f^{2} 5 d^{2}$, and a few of the assigned levels have large $4 f^{2} 5 d^{2}$ components. Practically all of the $4 f^{2} 6 s^{2}$ configuration except ${ }^{1} \mathrm{~S}_{0}$ is now accounted for.

\section{Isotope Shifts, Ionization Energy}

Champeau [1972] has derived values of the isotope shifts associated with the $4 f 5 d^{2} 6 s$, $4 f^{2} 6 s^{2}$, and $4 f^{2} 5 d 6 s$ configurations, taken with respect to zero shift for $4 f 5 d 6 s^{2}$. A tentative and rough comparison of Oreg's eigenvectors for a number of even levels for which Champeau [1969] has obtained isotope shifts indicates a general consistency of the results; the isotope shifts of several additional even configurations could be obtained.

Worden, Conway, Paisner, and Solarz [1977] have observed high members of two series in Ce I by using laser techniques. These data became available too late for inclusion here, but the quoted ionization energy is a new determination from these series. 


\section{References}

Champeau, R.-J., Thesis, Univ. Paris, Orsay, 83 pp. (1969). IS

Champeau, R.-J., Physica (Utrecht) 62, 209 (1972); ibid., 225 (1972). IS

Childs, W. J., and Goodman, L. S., Phys. Rev. A 1, 1290 (1970). ZE

Goldschmidt, Z. B., and Salomon, D., unpublished material $(1970 ; 1973)$. ND PT

King, A. S., Astrophys. J. 68, 194 (1928). W

Maleh, I., Phys. Rev. 138, B766 (1965). Hfs

Martin, W. C., J. Opt. Soc. Am. 53, 1047 (1963). EL ZE

Martin, W. C., Phys. Rev. A 3, 1810 (1971). EL ND ZE

Martin, W. C., unpublished material (1975). EL ND CL W ZE

Meggers, W. F., Corliss, C. H., and Seribner, B. F., Nat. Bur. Stand. (U.S.), Monogr. 145, Part I, 403 pp. (1975). CL W

Oreg, Y., Thesis, Hebrew Univ. Jerusalem, Israel, 201 pp. (1973). ND PT

Paul, F. W., Phys. Rev. 49, 156 (1936). W

Smith, K. F., and Spalding, I. J., Proc. R. Soc. London, Ser. A., 265, 133 (1961). ZE

Verges, J., Corliss, C. H., and Martin, W. C., J. Res. Nat. Bur. Stand. (U.S.) 76A, 285 (1972). CL W

Worden, E. F., Conway, J. G., Paisner, J. A., and Solarz, R. W., unpublished material (1977). IP

[July 1976]

Ce I, Odd Parity

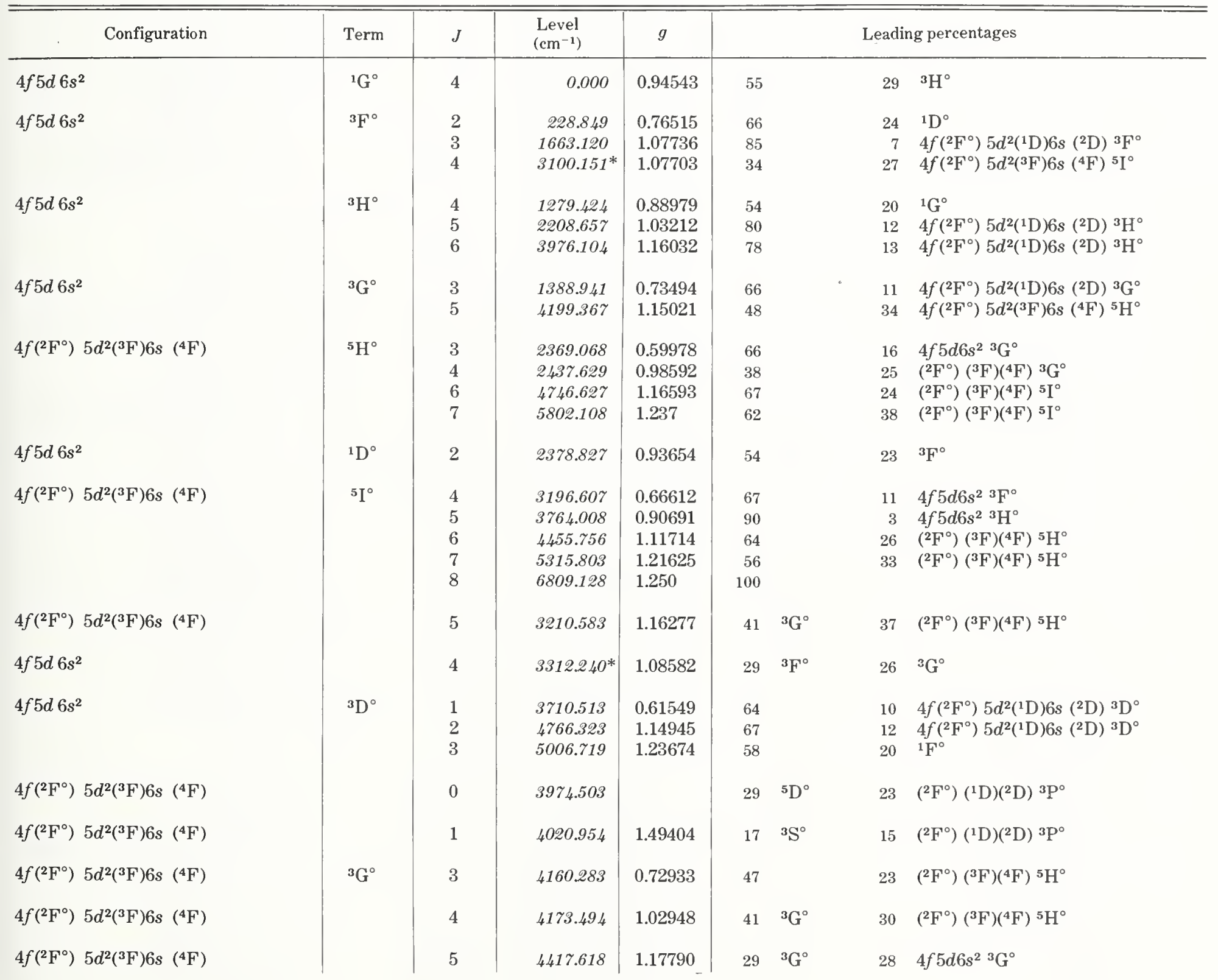


Ce I, Odd Parity_Continued

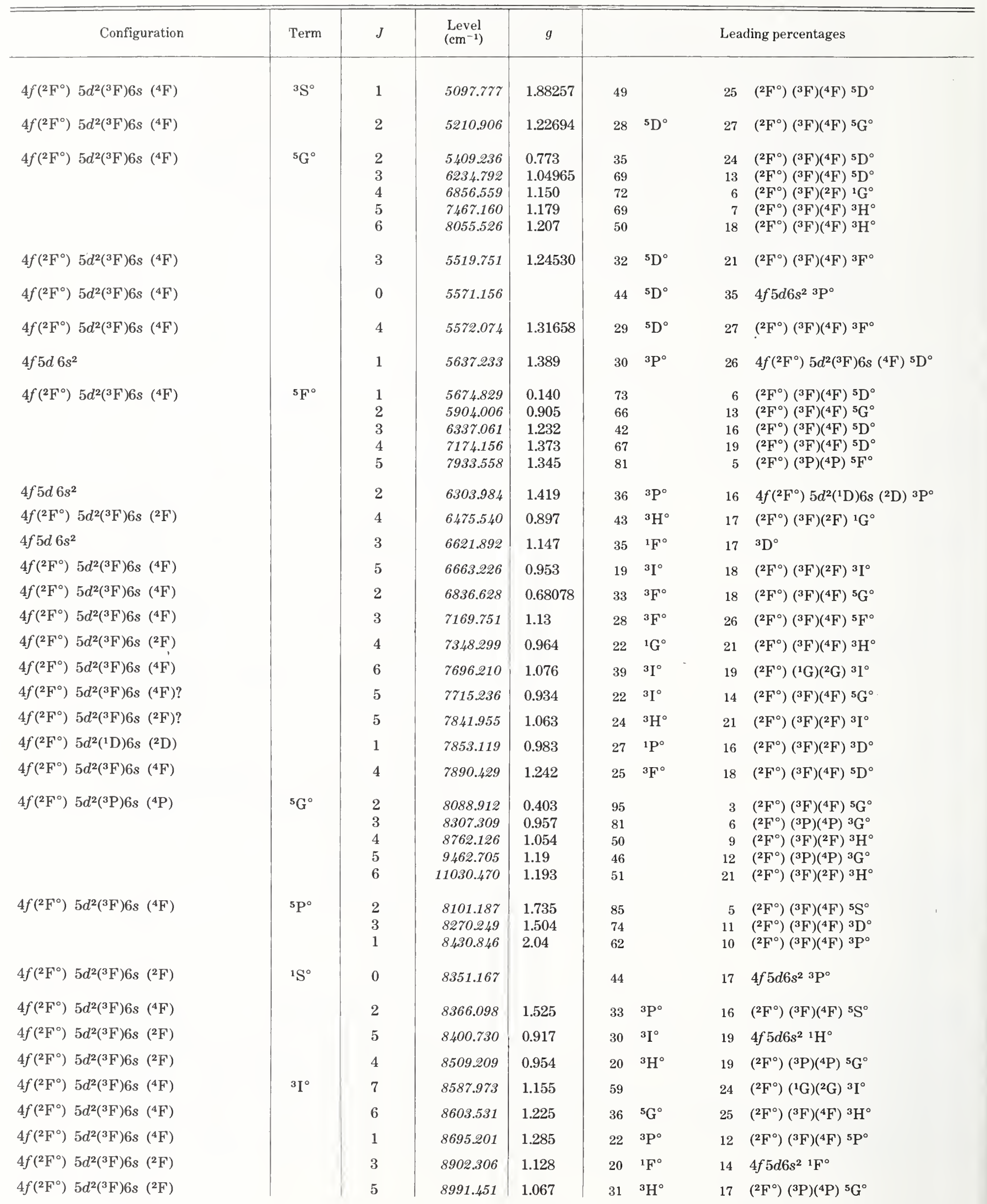


Ce I, Odd Parity_Continued

\begin{tabular}{|c|c|c|c|c|c|c|c|c|c|}
\hline & Configuration & Term & $J$ & $\begin{array}{l}\text { Level } \\
\left(\mathrm{cm}^{-1}\right)\end{array}$ & $g$ & \multicolumn{4}{|c|}{ Leading percentages } \\
\hline $4 f\left({ }^{2} \mathrm{~F}^{\circ}\right)$ & $5 d^{2}\left({ }^{3} \mathrm{~F}\right) 6 s\left({ }^{4} \mathrm{~F}\right)$ & \multirow{3}{*}{${ }^{3} \mathrm{D}^{\circ}$} & 0 & 9119.094 & & 40 & ${ }^{3} \mathrm{P}^{\circ}$ & 32 & $\left({ }^{2} \mathrm{~F}^{\circ}\right)\left({ }^{3} \mathrm{~F}\right)\left({ }^{2} \mathrm{~F}\right){ }^{1} \mathrm{~S}^{\circ}$ \\
\hline $4 f\left({ }^{2} \mathrm{~F}^{\circ}\right)$ & $5 d^{2}\left({ }^{3} \mathrm{P}\right) 6 s\left({ }^{4} \mathrm{P}\right)$ & & 3 & 9135.099 & 1.274 & 50 & & 12 & $\left({ }^{2} \mathrm{~F}^{\circ}\right)\left({ }^{3} \mathrm{~F}\right)\left({ }^{4} \mathrm{~F}\right){ }^{5} \mathrm{P}^{\circ}$ \\
\hline $4 f\left({ }^{2} \mathrm{~F}^{\circ}\right)$ & $5 d^{2}\left({ }^{3} \mathrm{~F}\right) 6 s \quad\left({ }^{2} \mathrm{~F}\right)$ & & 2 & 9200.707 & 1.376 & 24 & ${ }^{3} \mathrm{D}^{\circ}$ & 21 & $\left({ }^{2} \mathrm{~F}^{\circ}\right)\left({ }^{3} \mathrm{P}\right)\left({ }^{2} \mathrm{P}\right){ }^{3} \mathrm{D}^{\circ}$ \\
\hline $4 f\left({ }^{2} \mathrm{~F}^{\circ}\right)$ & $5 d^{2}\left({ }^{3} \mathrm{~F}\right) 6 s\left({ }^{2} \mathrm{~F}\right)$ & ${ }^{3} \mathbf{I}^{\circ}$ & $\begin{array}{l}6 \\
7\end{array}$ & $\begin{array}{r}9333222 \\
11061.551\end{array}$ & $\begin{array}{l}1.047 \\
1.141\end{array}$ & $\begin{array}{l}61 \\
79\end{array}$ & & $\begin{array}{l}20 \\
16\end{array}$ & $\begin{array}{l}\left({ }^{2} F^{\circ}\right)\left({ }^{1} G\right)\left({ }^{2} G\right){ }^{3} I^{\circ} \\
\left({ }^{2} F^{\circ}\right)\left({ }^{1} G\right)\left({ }^{2} G\right){ }^{3} I^{\circ}\end{array}$ \\
\hline $4 f\left({ }^{2} \mathrm{~F}^{\circ}\right)$ & $5 d^{2}\left({ }^{3} \mathrm{~F}\right) 6 s\left({ }^{4} \mathrm{~F}\right)$ & \multirow[b]{2}{*}{${ }^{3} \mathrm{D}^{\circ}$} & 1 & 9369.628 & 1.065 & 21 & ${ }^{3} \mathrm{P}^{0}$ & 17 & $\left({ }^{2} \mathrm{~F}^{\circ}\right)\left({ }^{3} \mathrm{~F}\right)\left({ }^{2} \mathrm{~F}\right){ }^{3} \mathrm{D}^{\circ}$ \\
\hline $4 f\left({ }^{2} \mathrm{~F}^{\circ}\right)$ & $5 d^{2}\left({ }^{3} \mathrm{P}\right) 6 s\left({ }^{4} \mathrm{P}\right)$ & & 2 & 9425.529 & 1.207 & 46 & & 8 & $\left({ }^{2} \mathrm{~F}^{\circ}\right)\left({ }^{3} \mathrm{~F}\right)\left({ }^{4} \mathrm{~F}\right){ }^{5} \mathrm{~S}^{\circ}$ \\
\hline $4 f\left({ }^{2} \mathrm{~F}^{\circ}\right)$ & $5 d^{2}\left({ }^{3} \mathrm{~F}\right) 6 s\left({ }^{2} \mathrm{~F}\right)$ & ${ }^{3} \mathrm{~F}^{\circ}$ & 2 & 9709.012 & 0.799 & 51 & & 13 & $\left({ }^{2} \mathrm{~F}^{\circ}\right)\left({ }^{3} \mathrm{P}\right)\left({ }^{2} \mathrm{P}\right){ }^{3} \mathrm{~F}^{\circ}$ \\
\hline $4 f\left({ }^{2} \mathrm{~F}^{\circ}\right)$ & $5 d^{2}\left({ }^{3} \mathrm{~F}\right) 6 s$ & \multirow{2}{*}{${ }^{3} \mathrm{G}^{\circ}$} & 3 & 9787.220 & 0.868 & 42 & & 26 & $\left({ }^{2} \mathrm{~F}^{\circ}\right)\left({ }^{3} \mathrm{P}\right)\left({ }^{2} \mathrm{P}\right){ }^{3} \mathrm{G}^{\circ}$ \\
\hline $4 f\left({ }^{2} \mathrm{~F}^{\circ}\right)$ & $5 d^{2}\left({ }^{1} \mathrm{G}\right) 6 s \quad\left({ }^{2} \mathrm{G}\right)$ & & 6 & 9830.608 & 1.081 & 31 & ${ }^{1} \mathrm{I}^{\circ}$ & 12 & $\left({ }^{2} \mathrm{~F}^{\circ}\right)\left({ }^{3} \mathrm{~F}\right)\left({ }^{2} \mathrm{~F}\right){ }^{1} \mathrm{I}^{\circ}$ \\
\hline $4 f\left({ }^{2} \mathrm{~F}^{\circ}\right)$ & $5 d^{2}\left({ }^{3} \mathrm{P}\right) 6 s\left({ }^{4} \mathrm{P}\right)$ & \multirow{3}{*}{${ }^{5} \mathrm{~S}^{\circ}$} & 1 & 9903.122 & 0.813 & 38 & ${ }^{3} \mathrm{D}^{\circ}$ & 14 & $\left({ }^{2} \mathrm{~F}^{\circ}\right)\left({ }^{3} \mathrm{P}\right)\left({ }^{4} \mathrm{P}\right){ }^{5} \mathrm{~F}^{\circ}$ \\
\hline $4 f\left({ }^{2} \mathrm{~F}^{\circ}\right)$ & $5 d^{2}\left({ }^{3} \mathrm{~F}\right) 6 s \quad\left({ }^{4} \mathrm{~F}\right)$ & & 2 & 9947.822 & 1.63 & 52 & & 18 & $\left({ }^{2} F^{\circ}\right)\left({ }^{3} F\right)\left({ }^{4} F\right){ }^{3} P^{\circ}$ \\
\hline $4 f\left({ }^{2} \mathrm{~F}^{\circ}\right)$ & $5 d^{2}\left({ }^{1} \mathrm{D}\right) 6 s\left({ }^{2} \mathrm{D}\right) ?$ & & 3 & 9996.647 & & 24 & ${ }^{3} \mathrm{G}^{\circ}$ & 16 & $\left({ }^{2} \mathrm{~F}^{\circ}\right)\left({ }^{3} \mathrm{P}\right)\left({ }^{2} \mathrm{P}\right){ }^{3} \mathrm{G}^{\circ}$ \\
\hline $4 f\left({ }^{2} \mathrm{~F}^{\circ}\right)$ & $5 d^{2}\left({ }^{1} \mathrm{D}\right) 6 s\left({ }^{2} \mathrm{D}\right)$ & \multirow{3}{*}{${ }^{5} \mathrm{D}^{\circ}$} & 4 & 10243.780 & & 18 & ${ }^{1} \mathrm{G}^{\circ}$ & 13 & $\left({ }^{2} \mathrm{~F}^{\circ}\right)\left({ }^{3} \mathrm{P}\right)\left({ }^{2} \mathrm{P}\right){ }^{1} \mathrm{G}^{\circ}$ \\
\hline $4 f\left({ }^{2} F^{\circ}\right)$ & $5 d^{2}\left({ }^{3} \mathrm{P}\right) 6 s\left({ }^{4} \mathrm{P}\right)$ & & 0 & 10249.014 & & 62 & & 23 & $\left({ }^{2} \mathrm{~F}^{\circ}\right)\left({ }^{3} \mathrm{~F}\right)\left({ }^{4} \mathrm{~F}\right){ }^{5} \mathrm{D}^{\circ}$ \\
\hline $4 f\left({ }^{2} \mathrm{~F}^{\circ}\right)$ & $5 d^{2}\left({ }^{3} F\right) 6 s\left({ }^{2} F\right)$ & & 3 & 10318.438 & & 20 & ${ }^{3} \mathrm{~F}^{\circ}$ & 18 & $\left({ }^{2} F^{\circ}\right)\left({ }^{3} F\right)\left({ }^{4} F\right){ }^{3} D^{\circ}$ \\
\hline \multirow[t]{4}{*}{$4 f\left({ }^{2} \mathrm{~F}^{\circ}\right)$} & $5 d^{2}\left({ }^{3} \mathrm{P}\right) 6 s \quad\left({ }^{4} \mathrm{P}\right)$ & & 1 & 10409.273 & & 39 & ${ }^{5} \mathrm{D}^{\circ}$ & 24 & $\left({ }^{2} \mathrm{~F}^{\circ}\right)\left({ }^{3} \mathrm{P}\right)\left({ }^{4} \mathrm{P}\right){ }^{5} \mathrm{~F}^{\circ}$ \\
\hline & & & 4 & 10586.772 & & & & & \\
\hline & & & 3 & 10604.889 & & & & & \\
\hline & & & 2 & 10612.867 & & & & & \\
\hline \multirow[t]{3}{*}{$4 f\left({ }^{2} \mathrm{~F}^{\circ}\right)$} & $5 d^{2}\left({ }^{3} \mathrm{P}\right) 6 s \quad\left({ }^{4} \mathrm{P}\right)$ & & 6 & 10673.847 & 1.201 & 40 & ${ }^{5} \mathrm{G}^{\circ}$ & 23 & $\left({ }^{2} \mathrm{~F}^{\circ}\right)\left({ }^{3} \mathrm{~F}\right)\left({ }^{2} \mathrm{~F}\right){ }^{3} \mathrm{H}^{\circ}$ \\
\hline & & & 4 & 10723.512 & & & & & \\
\hline & & & 3 & 10774.605 & & & & & \\
\hline \multirow[t]{6}{*}{$4 f\left({ }^{2} \mathrm{~F}^{\circ}\right)$} & $5 d^{2}\left({ }^{1} \mathrm{G}\right) 6 s \quad\left({ }^{2} \mathrm{G}\right)$ & & 5 & 10879.793 & $1.10 ?$ & 27 & ${ }^{1} \mathrm{H}^{\circ}$ & 20 & $\left({ }^{2} \mathrm{~F}^{\circ}\right)\left({ }^{1} \mathrm{G}\right)\left({ }^{2} \mathrm{G}\right){ }^{3} \mathrm{G}^{\circ}$ \\
\hline & & & 2 & 10901.971 & & & & & \\
\hline & & & 3 & 11131.710 & & & & & \\
\hline & & & 4 & 11271.088 & & & & & \\
\hline & & & 2 & 11301.537 & & & & & \\
\hline & & & 3 & 11337.859 & 0.860 & & & & \\
\hline \multirow[t]{8}{*}{$4 f 5 d 6 s^{2}$} & & & 5 & 11357.036 & & 20 & ${ }^{1} \mathrm{H}^{\circ}$ & 15 & $4 f\left({ }^{2} \mathrm{~F}^{\circ}\right) 5 d^{2}\left({ }^{1} \mathrm{G}\right) 6 s\left({ }^{2} \mathrm{G}\right){ }^{1} \mathrm{H}^{\circ}$ \\
\hline & & & 1 & 11517.044 & & & & & \\
\hline & & & 4 & 11545.398 & $1.174 ?$ & & & & \\
\hline & & & 1 & 11578.951 & & & & & \\
\hline & & & 1 & 11626.193 & & & & & \\
\hline & & & 2 & 11650.367 & & & & & \\
\hline & & & 4 & 11796.367 & & & & & \\
\hline & & & 4 & 11810.672 & & & & & \\
\hline \multirow[t]{4}{*}{$4 f\left({ }^{2} \mathrm{~F}^{\circ}\right)$} & $5 d^{2}\left({ }^{3} F\right) 6 s \quad\left({ }^{2} F\right)$ & ${ }^{3} \mathrm{G}^{\circ}$ & 5 & 11850.252 & & 60 & & 12 & $\left({ }^{2} \mathrm{~F}^{\circ}\right)\left({ }^{3} \mathrm{P}\right)\left({ }^{2} \mathrm{P}\right){ }^{3} \mathrm{G}^{\circ}$ \\
\hline & & & 3 & 11874.016 & & & & & \\
\hline & & & 2 & 12022.199 & & & & & \\
\hline & & & 1,2 & 12297.629 & & & & & \\
\hline \multirow[t]{2}{*}{$4 f\left({ }^{2} \mathrm{~F}^{\circ}\right)$} & $5 d^{2}\left({ }^{1} \mathrm{G}\right) 6 s \quad\left({ }^{2} \mathrm{G}\right)$ & & 5 & 12297.781 & $1.12 ?$ & 39 & ${ }^{3} \mathrm{H}^{\circ}$ & 9 & $\left({ }^{2} \mathrm{~F}^{\circ}\right)\left({ }^{3} \mathrm{P}\right)\left({ }^{4} \mathrm{P}\right){ }^{3} \mathrm{G}^{\circ}$ \\
\hline & & & 4 & 12351.320 & & & & & \\
\hline
\end{tabular}


Ce I, Odd Parity-Continued

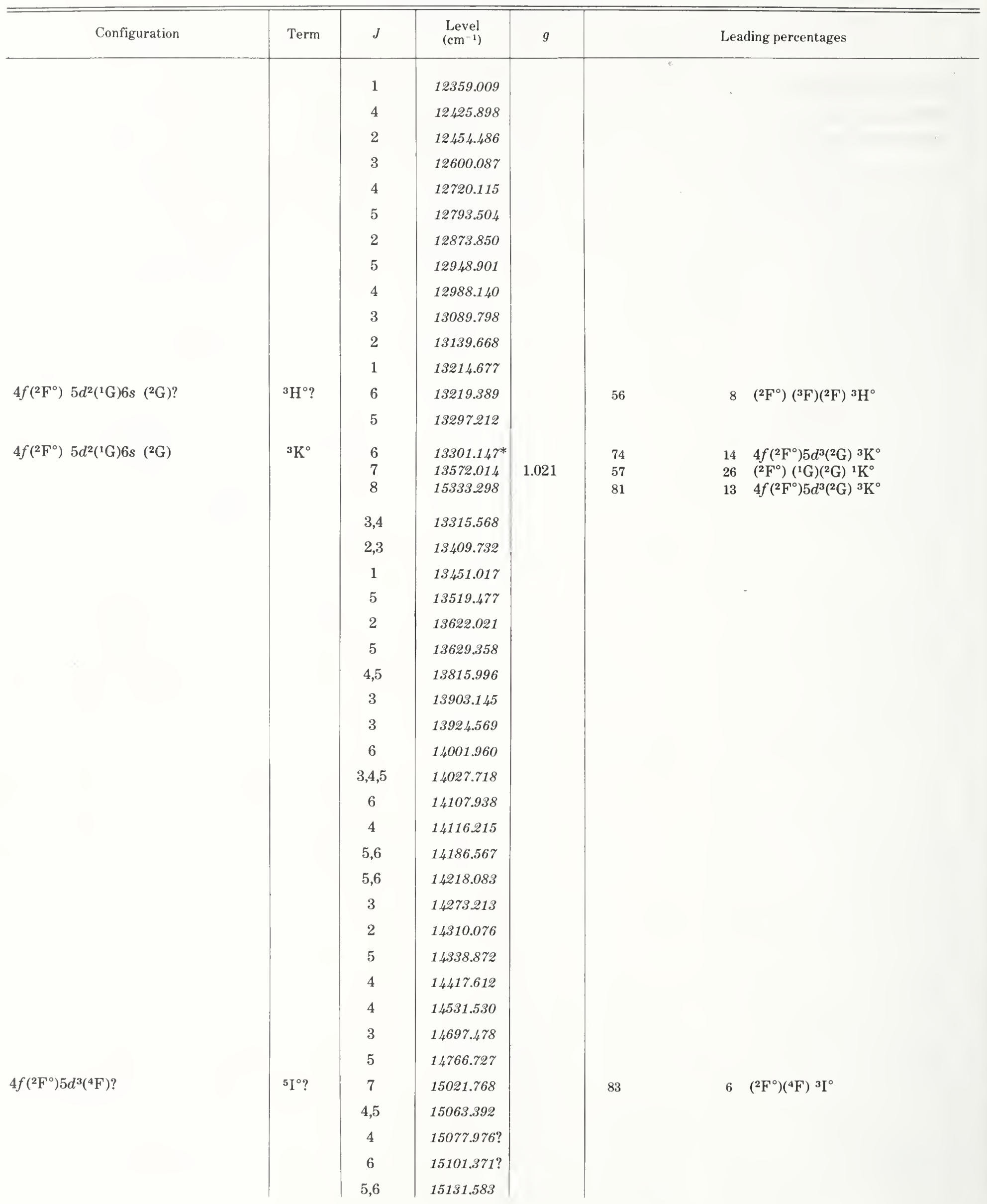


Ce I, Odd Parity-Continued

Ce I, Odd Parity_Continued

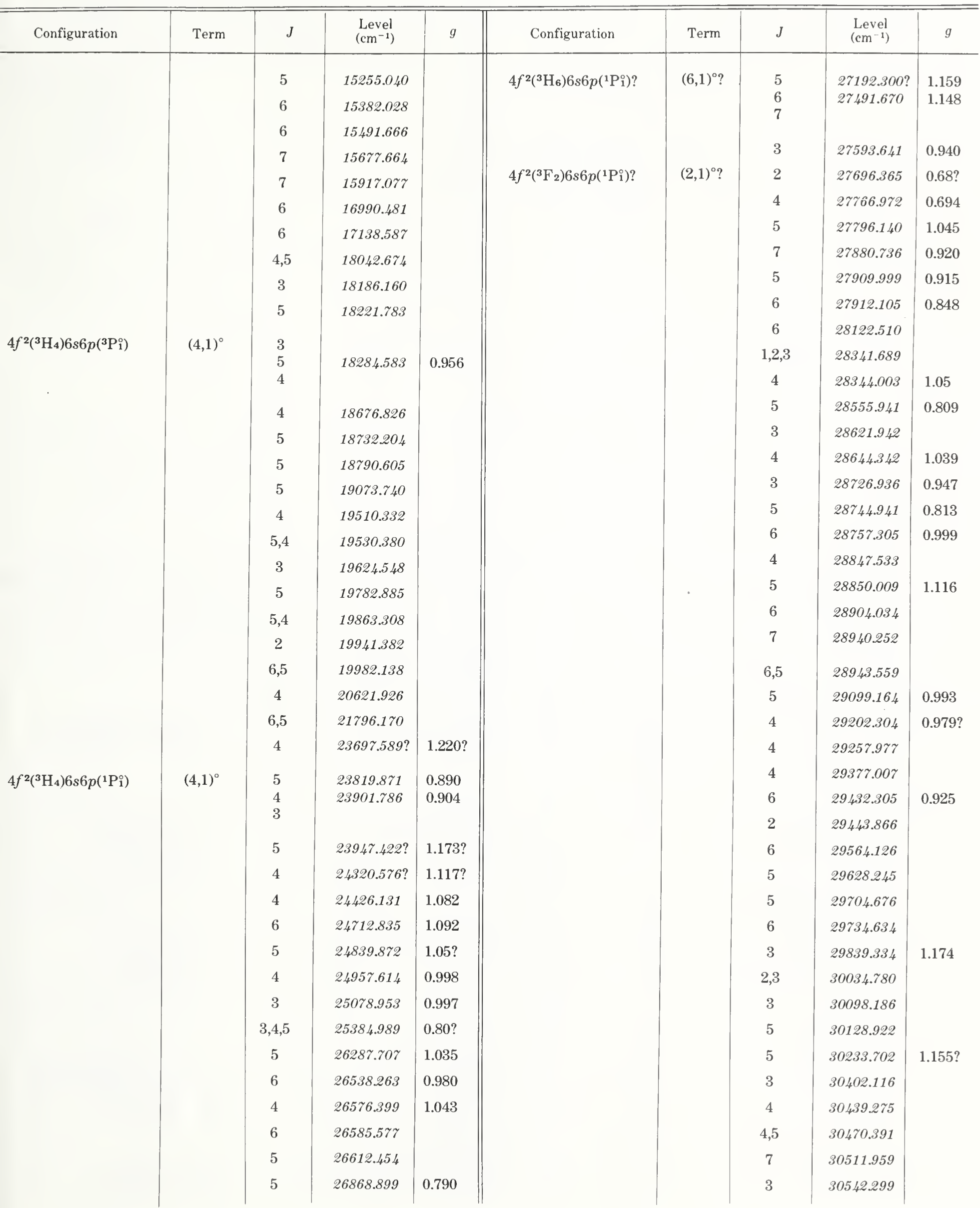




\begin{tabular}{|c|c|c|c|c|c|c|c|c|c|}
\hline Configuration & Term & $J$ & $\begin{array}{l}\text { Level } \\
\left(\mathrm{cm}^{-1}\right)\end{array}$ & $g$ & Configuration & Term & $J$ & $\begin{array}{l}\text { Level } \\
\left(\mathrm{cm}^{-1}\right)\end{array}$ & $g$ \\
\hline & & 4,5 & 30559.272 & & \multirow{8}{*}{ Ce II $\left({ }^{4} \mathrm{H}^{\circ} / 2\right)$} & & 4 & 30787.639 & $0.695 ?$ \\
\hline & & 5 & 30566.948 & & & & 3 & 30854.052 & \multirow[t]{7}{*}{0.737} \\
\hline & & 6 & 30603.359 & & & & 7 & 30876.234 & \\
\hline & & 4,3 & 30624.042 & & & & 2 & 30991.841 & \\
\hline & & 2 & 30706.313 & & & & & & \\
\hline & & 4 & 30739.270 & & & & & & \\
\hline & & 3 & 30740.884 & & & Limit & & 44672 & \\
\hline & & 5 & 30767.047 & & & & & & \\
\hline
\end{tabular}

Ce I, Even Parity

\begin{tabular}{|c|c|c|c|c|c|c|c|}
\hline Configuration & Term & $J$ & $\begin{array}{l}\text { Level } \\
\left(\mathrm{cm}^{-1}\right)\end{array}$ & $g$ & \multicolumn{3}{|c|}{ Leading percentages } \\
\hline $4 f^{2} 6 s^{2}$ & ${ }^{3} \mathrm{H}$ & $\begin{array}{l}4 \\
5 \\
6\end{array}$ & $\begin{array}{l}4762.718 \\
6238.934 \\
7780.202\end{array}$ & $\begin{array}{l}0.80508 \\
1.035 \\
1.169\end{array}$ & $\begin{array}{l}89 \\
92 \\
92\end{array}$ & $\begin{array}{l}4 \\
4 \\
4\end{array}$ & $4 f 5 d\left({ }^{3} \mathrm{G}^{\circ}\right) 6 s 6 p\left({ }^{1} \mathrm{P}^{\circ}\right){ }^{3} \mathrm{H}$ \\
\hline $4 f^{2} 6 s^{2}$ & ${ }^{3} \mathrm{~F}$ & $\begin{array}{l}2 \\
3 \\
4\end{array}$ & $\begin{array}{l}8235.605 \\
9206.305 \\
9379.148\end{array}$ & $\begin{array}{l}0.680 \\
1.083 \\
1.139\end{array}$ & $\begin{array}{l}88 \\
90 \\
55\end{array}$ & $\begin{array}{r}4 \\
4 \\
32\end{array}$ & $\begin{array}{l}4 f 5 d\left({ }^{3} \mathrm{~F}^{\circ}\right) 6 s 6 p\left({ }^{1} \mathrm{P}^{\circ}\right){ }^{3} \mathrm{~F} \\
4 f 5 d\left({ }^{3} \mathrm{~F}^{\circ}\right) 6 s 6 p\left({ }^{1} \mathrm{P}^{\circ}\right){ }^{3} \mathrm{~F} \\
{ }^{1} \mathrm{G}\end{array}$ \\
\hline $4 f^{2} 6 s^{2}$ & ${ }^{1} \mathrm{G}$ & 4 & 11361.895 & 1.101 & 52 & 35 & ${ }^{3} \mathrm{~F}$ \\
\hline $4 f^{2}\left({ }^{3} \mathrm{H}\right) 5 d\left({ }^{4} \mathrm{I}\right) 6 s$ & ${ }^{5} \mathrm{I}$ & $\begin{array}{l}4 \\
5 \\
6 \\
7 \\
8\end{array}$ & $\begin{array}{l}12114.115 \\
13124.010 \\
13605.000 \\
14609.088\end{array}$ & $\begin{array}{l}0.655 \\
0.957\end{array}$ & $\begin{array}{l}59 \\
50 \\
72 \\
78 \\
85\end{array}$ & $\begin{array}{r}17 \\
14 \\
8 \\
5 \\
5\end{array}$ & $\begin{array}{l}\left({ }^{3} \mathrm{H}\right)\left({ }^{2} \mathrm{H}\right){ }^{3} \mathrm{H} \\
\left({ }^{3} \mathrm{H}\right)\left({ }^{2} \mathrm{H}\right){ }^{1} \mathrm{H} \\
\left({ }^{3} \mathrm{H}\right)\left({ }^{4} \mathrm{I}\right){ }^{3} \mathrm{I} \\
\left({ }^{3} \mathrm{H}\right)\left({ }^{4} \mathrm{I}\right){ }^{3} \mathrm{I} \\
4 f\left({ }^{2} \mathrm{~F}^{\circ}\right) 5 d^{2}\left({ }^{3} \mathrm{~F}\right)\left({ }^{4} \mathrm{H}^{\circ}\right) 6 p{ }^{5} \mathrm{I}\end{array}$ \\
\hline $4 f^{2}\left({ }^{3} \mathrm{H}\right) 5 d\left({ }^{4} \mathrm{~K}\right) 6 s$ & ${ }^{5} \mathrm{~K}$ & $\begin{array}{l}5 \\
6 \\
7 \\
8 \\
9\end{array}$ & $\begin{array}{l}12366.834 \\
12960.950 \\
13908.826\end{array}$ & $\begin{array}{l}0.710 \\
0.90\end{array}$ & $\begin{array}{l}63 \\
75 \\
84 \\
89 \\
96\end{array}$ & $\begin{array}{l}9 \\
8 \\
7 \\
4 \\
4\end{array}$ & $\begin{array}{l}\left({ }^{3} \mathrm{H}\right)\left({ }^{2} \mathrm{H}\right){ }^{1} \mathrm{H} \\
\left({ }^{3} \mathrm{H}\right)\left({ }^{4} \mathrm{~K}\right){ }^{3} \mathrm{~K} \\
\left({ }^{3} \mathrm{H}\right)\left({ }^{4} \mathrm{~K}\right){ }^{3} \mathrm{~K} \\
\left({ }^{3} \mathrm{H}\right)\left({ }^{4} \mathrm{~K}\right){ }^{3} \mathrm{~K} \\
4 f\left({ }^{2} \mathrm{~F}^{\circ}\right) 5 d^{2}\left({ }^{3} \mathrm{~F}\right)\left({ }^{4} \mathrm{I}^{\circ}\right) 6 p{ }^{5} \mathrm{~K}\end{array}$ \\
\hline $4 f^{2}\left({ }^{3} \mathrm{H}\right) 5 d\left({ }^{4} \mathrm{~K}\right) 6 s$ & & 5 & 12467.827 & 0.948 & $22 \quad{ }^{5} \mathrm{~K}$ & 20 & $\left({ }^{3} \mathrm{H}\right)\left({ }^{2} \mathrm{H}\right){ }^{3} \mathrm{H}$ \\
\hline $4 f^{2}\left({ }^{3} \mathrm{H}\right) 5 d\left({ }^{4} \mathrm{G}\right) 6 s$ & ${ }^{5} \mathrm{G}$ & $\begin{array}{l}2 \\
3 \\
4 \\
5 \\
6\end{array}$ & $\begin{array}{l}12992.115 \\
13389.827 \\
14064.382 \\
16066.940\end{array}$ & $\begin{array}{l}0.388 \\
0.919\end{array}$ & $\begin{array}{l}38 \\
40 \\
46 \\
46 \\
44\end{array}$ & $\begin{array}{l}24 \\
15 \\
11 \\
12 \\
14\end{array}$ & $\begin{array}{l}4 f 5 d\left({ }^{3} \mathrm{~F}^{\circ}\right) 6 s 6 p\left({ }^{3} \mathrm{P}^{\circ}\right){ }^{5} \mathrm{G} \\
4 f 5 d\left({ }^{3} \mathrm{~F}^{\circ}\right) 6 s 6 p\left({ }^{3} \mathrm{P}^{\circ}\right){ }^{5} \mathrm{G} \\
\left({ }^{3} \mathrm{~F}\right)\left({ }^{4} \mathrm{G}\right){ }^{5} \mathrm{G} \\
\left({ }^{3} \mathrm{~F}\right)\left({ }^{4} \mathrm{G}\right){ }^{5} \mathrm{G} \\
\left({ }^{3} \mathrm{~F}\right)\left({ }^{4} \mathrm{G}\right){ }^{5} \mathrm{G}\end{array}$ \\
\hline $4 f^{2}\left({ }^{3} \mathrm{H}\right) 5 d\left({ }^{2} \mathrm{H}\right) 6 s$ & ${ }^{3} \mathrm{H}$ & $\begin{array}{l}4 \\
6\end{array}$ & $\begin{array}{l}13194.849 \\
13939.793\end{array}$ & $\begin{array}{l}0.775 \\
1.127 ?\end{array}$ & $\begin{array}{l}45 \\
46\end{array}$ & $\begin{array}{l}13 \\
10\end{array}$ & $\begin{array}{l}\left({ }^{3} \mathrm{H}\right)\left({ }^{4} \mathrm{I}\right){ }^{5} \mathrm{I} \\
\left({ }^{3} \mathrm{~F}\right)\left({ }^{2} \mathrm{H}\right){ }^{3} \mathrm{H}\end{array}$ \\
\hline
\end{tabular}


Ce I, Even Parity-Continued

\begin{tabular}{|c|c|c|c|c|c|c|c|c|}
\hline Configuration & Term & $J$ & $\begin{array}{l}\text { Level } \\
\left(\mathrm{cm}^{-1}\right)\end{array}$ & $g$ & \multicolumn{4}{|c|}{ Leading percentages } \\
\hline $4 f 5 d\left({ }^{1} \mathrm{G}^{\circ}\right) 6 s 6 p\left({ }^{3} \mathrm{P}^{\circ}\right)$ & & 4 & 13513.875 & 0.806 & 22 & ${ }^{3} \mathrm{H}$ & 18 & $\left({ }^{3} \mathrm{H}^{\circ}\right)\left({ }^{3} \mathrm{P}^{\circ}\right){ }^{3} \mathrm{H}$ \\
\hline $4 f 5 d\left({ }^{1} \mathrm{G}^{\circ}\right) 6 s 6 p\left({ }^{3} \mathrm{P}^{\circ}\right)$ & & 5 & 13784.151 & 1.023 & 19 & ${ }^{3} \mathrm{H}$ & 17 & $\left({ }^{3} \mathrm{H}^{\circ}\right)\left({ }^{3} \mathrm{P}^{\circ}\right){ }^{3} \mathrm{H}$ \\
\hline $4 f^{2}\left({ }^{3} \mathrm{H}\right) 5 d\left({ }^{2} \mathrm{H}\right) 6 s$ & & 5 & 13881.444 & 0.990 & 20 & ${ }^{3} \mathrm{H}$ & 20 & $\left({ }^{3} \mathrm{H}\right)\left({ }^{2} \mathrm{H}\right){ }^{1} \mathrm{H}$ \\
\hline $4 f^{2}\left({ }^{3} \mathrm{H}\right) 5 d\left({ }^{4} \mathrm{H}\right) 6 s$ & & 3 & 13902.760 & $0.665 ?$ & 42 & ${ }^{5} \mathrm{H}$ & 26 & $4 f 5 d\left({ }^{3} \mathrm{G}^{\circ}\right) 6 s 6 p\left({ }^{3} \mathrm{P}^{\circ}\right){ }^{5} \mathrm{H}$ \\
\hline $\left.4 f^{2(3} \mathrm{H}\right) 5 d\left({ }^{4} \mathrm{G}\right) 6 s$ & & 2 & 14098.305 & 0.484 & 27 & ${ }^{5} \mathrm{G}$ & 26 & $4 f 5 d\left({ }^{3} \mathrm{~F}^{\circ}\right) 6 s 6 p\left({ }^{3} \mathrm{P}^{\circ}\right){ }^{5} \mathrm{G}$ \\
\hline $4 f 5 d\left({ }^{3} \mathrm{H}^{\circ}\right) 6 s 6 p\left({ }^{3} \mathrm{P}^{\circ}\right)$ & & 3 & 14136.322 & 0.649 & 40 & ${ }^{5} \mathrm{H}$ & 20 & $\left({ }^{1} \mathrm{G}^{\circ}\right)\left({ }^{3} \mathrm{P}^{\circ}\right){ }^{3} \mathrm{G}$ \\
\hline $4 f 5 d\left({ }^{3} \mathrm{~F}^{\circ}\right) 6 s 6 p\left({ }^{3} \mathrm{P}^{\circ}\right)$ & & 3 & 14539.212 & 0.967 & 20 & ${ }^{5} \mathrm{G}$ & 20 & $\left({ }^{1} \mathrm{D}^{\circ}\right)\left({ }^{3} \mathrm{P}^{\circ}\right){ }^{3} \mathrm{~F}$ \\
\hline $4 f^{2}\left({ }^{3} \mathrm{H}\right) 5 d\left({ }^{4} \mathrm{H}\right) 6 s$ & & 4 & 14599.598 & 0.906 & 39 & ${ }^{5} \mathrm{H}$ & 18 & $4 f 5 d\left({ }^{3} \mathrm{G}^{\circ}\right) 6 s 6 p\left({ }^{3} \mathrm{P}^{\circ}\right){ }^{5} \mathrm{H}$ \\
\hline $4 f 5 d\left({ }^{3} \mathrm{~F}^{\circ}\right) 6 s 6 p\left({ }^{3} \mathrm{P}^{\circ}\right)$ & & 1 & 14635.243 & 0.407 & 44 & ${ }^{5} \mathrm{~F}$ & 16 & $\left({ }^{3} \mathrm{~F}^{\circ}\right)\left({ }^{3} \mathrm{P}^{\circ}\right){ }^{3} \mathrm{D}$ \\
\hline $4 f 5 d\left({ }^{3} \mathrm{~F}^{\circ}\right) 6 s 6 p\left({ }^{3} \mathrm{P}^{\circ}\right)$ & & 2 & 14646.445 & 1.152 & 19 & ${ }^{3} \mathrm{D}$ & 16 & $\left({ }^{3} \mathrm{~F}^{\circ}\right)\left({ }^{3} \mathrm{P}^{\circ}\right){ }^{5} \mathrm{~F}$ \\
\hline $4 f^{2}\left({ }^{3} \mathrm{H}\right) 5 d\left({ }^{4} \mathrm{~K}\right) 6 s$ & ${ }^{3} \mathrm{~K}$ & $\begin{array}{l}6 \\
7 \\
8\end{array}$ & $\begin{array}{l}14743.626 \\
16067.587\end{array}$ & $1.051 ?$ & $\begin{array}{l}67 \\
56 \\
84\end{array}$ & & $\begin{array}{r}10 \\
10 \\
5\end{array}$ & $\begin{array}{l}\left({ }^{3} \mathrm{H}\right)\left({ }^{4} \mathrm{~K}\right){ }^{5} \mathrm{~K} \\
\left({ }^{3} \mathrm{H}\right)\left({ }^{4} \mathrm{~K}\right){ }^{5} \mathrm{~K} \\
\left({ }^{3} \mathrm{H}\right)\left({ }^{2} \mathrm{~K}\right){ }^{3} \mathrm{~K}\end{array}$ \\
\hline $4 f 5 d\left({ }^{1} \mathrm{G}^{\circ}\right) 6 s 6 p\left({ }^{3} \mathrm{P}^{\circ}\right)$ & & 4 & 14748.945 & 0.992 & 19 & ${ }^{3} \mathrm{G}$ & 17 & $\left({ }^{3} \mathrm{H}^{\circ}\right)\left({ }^{3} \mathrm{P}^{\circ}\right){ }^{5} \mathrm{H}$ \\
\hline $4 f^{2}\left({ }^{3} \mathrm{H}\right) 5 d\left({ }^{4} \mathrm{I}\right) 6 s$ & ${ }^{3} \mathrm{I}$ & 5 & 14795.290 & & 53 & & 11 & $\left({ }^{3} \mathrm{H}\right)\left({ }^{2} \mathrm{H}\right){ }^{1} \mathrm{H}$ \\
\hline $4 f 5 d\left({ }^{1} \mathrm{G}^{\circ}\right) 6 s 6 p\left({ }^{3} \mathrm{P}^{\circ}\right) ?$ & & 3 & 15063.452 & 0.849 & 16 & ${ }^{3} \mathrm{G}$ & 13 & $\left({ }^{3} \mathrm{~F}^{\circ}\right)\left({ }^{3} \mathrm{P}^{\circ}\right)^{3} \mathrm{G}$ \\
\hline $4 f 5 d\left({ }^{1} \mathrm{G}^{\circ}\right) 6 s 6 p\left({ }^{3} \mathrm{P}^{\circ}\right)$ & & 4 & 15240.619 & 1.012 & 16 & ${ }^{3} \mathrm{G}$ & 14 & $\left({ }^{3} \mathrm{H}^{\circ}\right)\left({ }^{3} \mathrm{P}^{\circ}\right){ }^{5} \mathrm{H}$ \\
\hline $4 f^{2}\left({ }^{3} \mathrm{H}\right) 5 d\left({ }^{2} \mathrm{~F}\right) 6 s ?$ & & 3 & 15277.852 & 0.807 & 12 & ${ }^{1} \mathrm{~F}$ & 10 & $\left({ }^{3} \mathrm{H}\right)\left({ }^{2} \mathrm{~F}\right){ }^{3} \mathrm{~F}$ \\
\hline $4 f^{2}\left({ }^{3} \mathrm{H}\right) 5 d\left({ }^{4} \mathrm{H}\right) 6 s$ & & 5 & 15339.718 & 1.076 & 32 & ${ }^{5} \mathrm{H}$ & 11 & $4 f 5 d\left({ }^{3} \mathrm{G}^{\circ}\right) 6 s 6 p\left({ }^{3} \mathrm{P}^{\circ}\right){ }^{5} \mathrm{H}$ \\
\hline $4 f 5 d\left({ }^{3} \mathrm{H}^{\circ}\right) 6 s 6 p\left({ }^{3} \mathrm{P}^{\circ}\right)$ & & 4 & 15371.647 & 1.040 & 24 & ${ }^{5} \mathrm{H}$ & 19 & $\left({ }^{1} \mathrm{G}^{\circ}\right)\left({ }^{3} \mathrm{P}^{\circ}\right){ }^{3} \mathrm{~F}$ \\
\hline $4 f^{2}\left({ }^{3} \mathrm{H}\right) 5 d\left({ }^{4} \mathrm{I}\right) 6 s$ & & 6 & 15396.286 & & 37 & ${ }^{3} \mathrm{I}$ & 15 & $\left({ }^{3} \mathrm{H}\right)\left({ }^{2} \mathrm{I}\right){ }^{3} \mathrm{I}$ \\
\hline $4 f\left({ }^{2} \mathrm{~F}_{5 / 2}^{\circ}\right) 6 s^{2} 6 p_{1 / 2}$ & & 3 & 15555.992 & 1.005 & 14 & $(5 / 2,1 / 2)$ & 8 & $4 f^{2}\left({ }^{3} \mathrm{H}\right) 5 d\left({ }^{4} \mathrm{~F}\right) 6 s^{5} \mathrm{~F}$ \\
\hline $4 f 5 d\left({ }^{3} \mathrm{H}^{\circ}\right) 6 s 6 p\left({ }^{3} \mathrm{P}^{\circ}\right)$ & ${ }^{5} \mathrm{I}$ & $\begin{array}{l}5 \\
4 \\
6 \\
7 \\
8\end{array}$ & $\begin{array}{l}15561.346 \\
15843.449 \\
17066.246 \\
18027.669 \\
19567.423\end{array}$ & $\begin{array}{l}1.075 \\
0.883 \\
1.117 \\
1.179 \\
1.246\end{array}$ & $\begin{array}{l}27 \\
29 \\
56 \\
76 \\
93\end{array}$ & & $\begin{array}{r}25 \\
10 \\
8 \\
4 \\
5\end{array}$ & $\begin{array}{l}\left({ }^{1} \mathrm{G}^{\circ}\right)\left({ }^{3} \mathrm{P}^{\circ}\right){ }^{3} \mathrm{G} \\
\left({ }^{3} \mathrm{H}^{\circ}\right)\left({ }^{3} \mathrm{P}^{\circ}\right){ }^{3} \mathrm{H} \\
\left({ }^{3} \mathrm{H}^{\circ}\right)\left({ }^{3} \mathrm{P}^{\circ}\right){ }^{5} \mathrm{H} \\
4 f\left({ }^{2} \mathrm{~F}^{\circ}\right) 5 d^{2}\left({ }^{3} \mathrm{~F}\right)\left({ }^{4} \mathrm{I}^{\circ}\right) 6 p{ }^{5} \mathrm{I} \\
4 f\left({ }^{2} \mathrm{~F}^{\circ}\right) 5 d^{2}\left({ }^{3} \mathrm{~F}\right)\left({ }^{4} \mathrm{I}^{\circ}\right) 6 p{ }^{5} \mathrm{I}\end{array}$ \\
\hline $4 f\left({ }^{2} \mathrm{~F}_{5 / 2}^{\circ}\right) 6 s^{2} 6 p_{1 / 2}$ & & 2 & 15587.927 & 0.747 & 19 & $(5 / 2,1 / 2)$ & 9 & $4 f 5 d\left({ }^{3} \mathrm{G}^{\circ}\right) 6 s 6 p\left({ }^{3} \mathrm{P}^{\circ}\right)^{5} \mathrm{G}$ \\
\hline $4 f^{2}\left({ }^{3} \mathrm{H}\right) 5 d\left({ }^{2} \mathrm{~F}\right) 6 s$ & & 2 & 15607.981 & 0.839 & 21 & ${ }^{3} \mathrm{~F}$ & 17 & $\left({ }^{3} \mathrm{H}\right)\left({ }^{4} \mathrm{~F}\right){ }^{5} \mathrm{~F}$ \\
\hline $4 f 5 d\left({ }^{1} \mathrm{G}^{\circ}\right) 6 s 6 p\left({ }^{3} \mathrm{P}^{\circ}\right)$ & & 6 & 15644.943 & 1.171 & 36 & ${ }^{3} \mathrm{H}$ & 15 & $\left({ }^{3} \mathrm{H}^{\circ}\right)\left({ }^{3} \mathrm{P}^{\circ}\right){ }^{3} \mathrm{H}$ \\
\hline $4 f 5 d\left({ }^{3} \mathrm{~F}^{\circ}\right) 6 s 6 p\left({ }^{3} \mathrm{P}^{\circ}\right)$ & & 0 & 15649.774 & & 37 & ${ }^{5} \mathrm{D}$ & 23 & $\left({ }^{1} \mathrm{D}^{\circ}\right)\left({ }^{3} \mathrm{P}^{\circ}\right){ }^{3} \mathrm{P}$ \\
\hline $4 f 5 d\left({ }^{3} \mathrm{~F}^{\circ}\right) 6 s 6 p\left({ }^{3} \mathrm{P}^{\circ}\right)$ & & 4 & 15700.978 & 1.062 & 15 & ${ }^{5} \mathrm{G}$ & 10 & $4 f^{2}\left({ }^{3} \mathrm{H}\right) 5 d\left({ }^{4} \mathrm{G}\right) 6 s{ }^{5} \mathrm{G}$ \\
\hline $4 f 5 d\left({ }^{3} \mathrm{~F}^{\circ}\right) 6 s 6 p\left({ }^{3} \mathrm{P}^{\circ}\right)$ & & 1 & 15751.949 & 1.013 & 26 & ${ }^{5} \mathrm{D}$ & 16 & $\left({ }^{1} \mathrm{D}^{\circ}\right)\left({ }^{3} \mathrm{P}^{\circ}\right){ }^{3} \mathrm{P}$ \\
\hline $4 f 5 d\left({ }^{3} \mathrm{~F}^{\circ}\right) 6 s 6 p\left({ }^{3} \mathrm{P}^{\circ}\right)$ & & 2 & 15758.458 & 0.550 & 19 & ${ }^{5} \mathrm{G}$ & 15 & $\left({ }^{3} \mathrm{G}^{\circ}\right)\left({ }^{3} \mathrm{P}^{\circ}\right){ }^{5} \mathrm{G}$ \\
\hline $4 f^{2}\left({ }^{3} \mathrm{~F}\right) 5 d\left({ }^{4} \mathrm{H}\right) 6 s ?$ & & 3 & 15766.048 & 0.740 & 17 & ${ }^{5} \mathrm{H}$ & 12 & $\left({ }^{3} \mathrm{H}\right)\left({ }^{4} \mathrm{G}\right){ }^{5} \mathrm{G}$ \\
\hline $4 f\left({ }^{2} \mathrm{~F}_{5 / 2}^{\circ}\right) 6 s^{2} 6 p_{1 / 2} ?$ & & 3 & 15798.226 & 0.798 & 12 & $(5 / 2,1 / 2)$ & 11 & $4 f 5 d\left({ }^{1} \mathrm{G}^{\circ}\right) 6 s 6 p\left({ }^{3} \mathrm{P}^{\circ}\right)^{3} \mathrm{~F}$ \\
\hline $4 f 5 d\left({ }^{3} \mathrm{H}^{\circ}\right) 6 s 6 p\left({ }^{3} \mathrm{P}^{\circ}\right)$ & & 5 & 15879.778 & 0.952 & 16 & ${ }^{5} \mathrm{H}$ & 13 & $\left({ }^{3} \mathrm{H}^{\circ}\right)\left({ }^{3} \mathrm{P}^{\circ}\right){ }^{5} \mathrm{I}$ \\
\hline $4 f 5 d\left({ }^{3} \mathrm{~F}^{\circ}\right) 6 s 6 p\left({ }^{3} \mathrm{P}^{\circ}\right)$ & & 4 & 15945.111 & 0.987 & 25 & ${ }^{5} \mathrm{G}$ & 9 & $4 f^{2}\left({ }^{3} \mathrm{~F}\right) 5 d\left({ }^{4} \mathrm{H}\right) 6 s^{5} \mathrm{H}$ \\
\hline $4 f 5 d\left({ }^{3} \mathrm{~F}^{\circ}\right) 6 s 6 p\left({ }^{3} \mathrm{P}^{\circ}\right)$ & & 2 & 15967.087 & 0.901 & 23 & ${ }^{5} \mathrm{~F}$ & 11 & $\left({ }^{3} \mathrm{G}^{\circ}\right)\left({ }^{3} \mathrm{P}^{\circ}\right){ }^{5} \mathrm{G}$ \\
\hline $4 f 5 d\left({ }^{3} \mathrm{~F}^{\circ}\right) 6 s 6 p\left({ }^{3} \mathrm{P}^{\circ}\right)$ & & 3 & 16008.831 & 1.220 & 36 & ${ }^{5} \mathrm{~F}$ & 10 & $\left({ }^{3} \mathrm{~F}^{\circ}\right)\left({ }^{3} \mathrm{P}^{\circ}\right){ }^{3} \mathrm{D}$ \\
\hline $4 f 5 d\left({ }^{3} \mathrm{H}^{\circ}\right) 6 s 6 p\left({ }^{3} \mathrm{P}^{\circ}\right)$ & & 5 & 16016.409 & 1.071 & 43 & ${ }^{5} \mathrm{H}$ & 10 & $\left({ }^{3} \mathrm{H}^{\circ}\right)\left({ }^{3} \mathrm{P}^{\circ}\right){ }^{1} \mathrm{H}$ \\
\hline $4 f^{2}\left({ }^{3} \mathrm{~F}\right) 5 d\left({ }^{4} \mathrm{H}\right) 6 s$ & & 3 & 16017.358 & 0.863 & 25 & ${ }^{5} \mathrm{H}$ & 9 & $\left({ }^{3} \mathrm{H}\right)\left({ }^{4} \mathrm{G}\right){ }^{5} \mathrm{G}$ \\
\hline $4 f^{2}\left({ }^{3} \mathrm{H}\right) 5 d\left({ }^{4} \mathrm{H}\right) 6 s$ & & 4 & 16051.478 & 0.912 & 12 & ${ }^{3} \mathrm{H}$ & 10 & $\left({ }^{3} \mathrm{H}\right)\left({ }^{4} \mathrm{G}\right){ }^{5} \mathrm{G}$ \\
\hline $4 f^{2} 6 s^{2}$ & & 2 & 16080.882 & 1.010 & 34 & ${ }^{1} \mathrm{D}$ & 19 & $4 f 5 d\left({ }^{3} \mathrm{~F}^{\circ}\right) 6 s 6 p\left({ }^{3} \mathrm{P}^{\circ}\right)^{5} \mathrm{~F}$ \\
\hline $4 f 5 d\left({ }^{3} \mathrm{~F}^{\circ}\right) 6 s 6 p\left({ }^{3} \mathrm{P}^{\circ}\right)$ & & 3 & 16200.493 & 0.963 & 11 & ${ }^{5} \mathrm{G}$ & 11 & $\left({ }^{3} \mathrm{G}^{\circ}\right)\left({ }^{3} \mathrm{P}^{\circ}\right){ }^{5} \mathrm{G}$ \\
\hline
\end{tabular}


Ce I, Even Parity_Continued

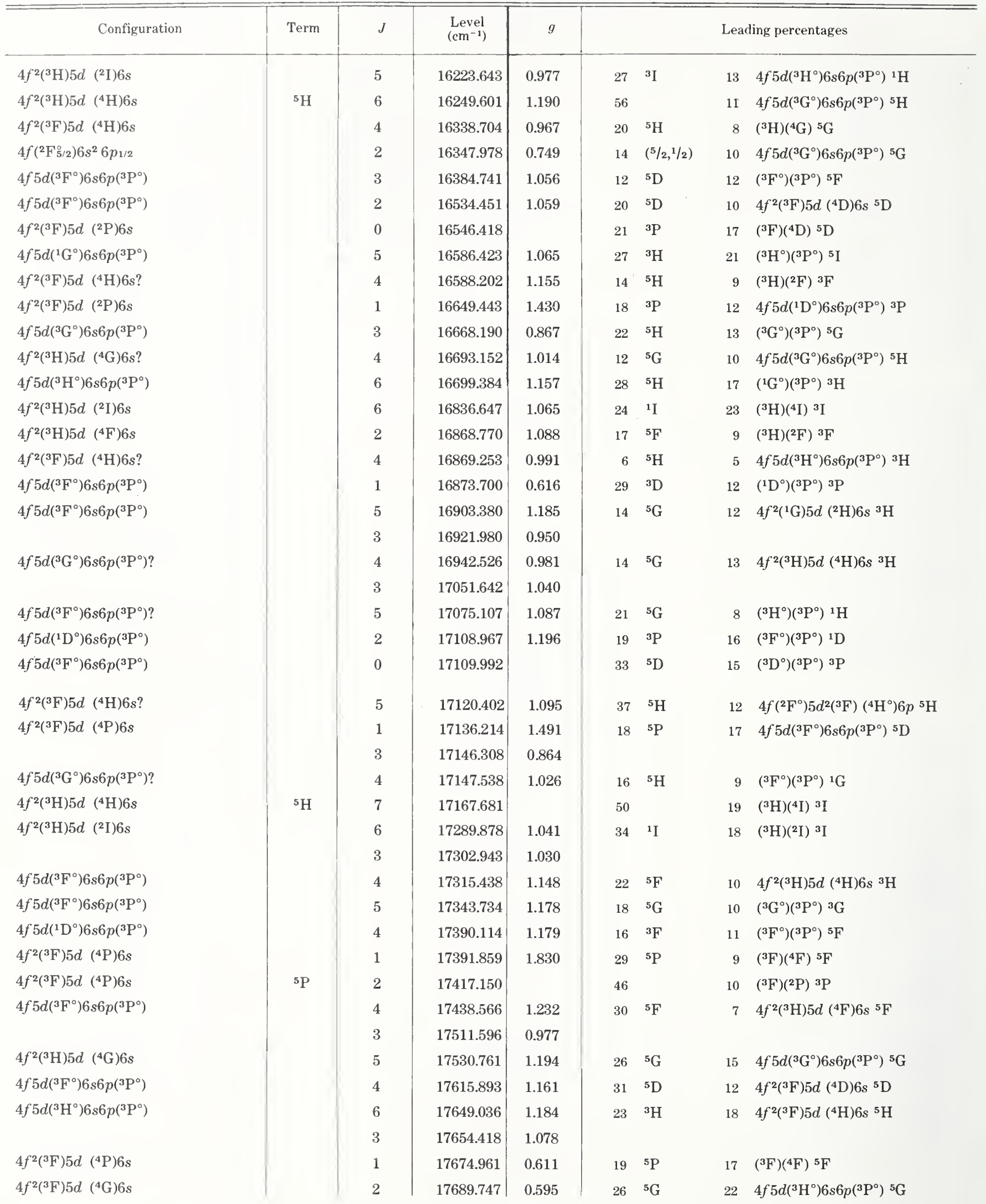


Ce I, Even Parity-Continued

\begin{tabular}{|c|c|c|c|c|c|c|c|c|}
\hline Configuration & Term & $J$ & $\begin{array}{l}\text { Level } \\
\left(\mathrm{cm}^{-1}\right)\end{array}$ & $g$ & \multicolumn{4}{|c|}{ Leading percentages } \\
\hline $4 f^{2} 6 s^{2}$ & & 0 & 17700.798 & & 20 & ${ }^{3} \mathrm{P}$ & 19 & $4 f 5 d\left({ }^{1} \mathrm{D}^{\circ}\right) 6 s 6 p\left({ }^{3} \mathrm{P}^{\circ}\right){ }^{3} \mathrm{P}$ \\
\hline $4 f 5 d\left({ }^{3} \mathrm{H}^{\circ}\right) 6 s 6 p\left({ }^{3} \mathrm{P}^{\circ}\right)$ & & 7 & 17708.707 & 1.206 & 28 & ${ }^{5} \mathrm{H}$ & 16 & $4 f^{2}\left({ }^{3} \mathrm{~F}\right) 5 d\left({ }^{4} \mathrm{H}\right) 6 s{ }^{5} \mathrm{H}$ \\
\hline 4f $5 d\left({ }^{3} \mathrm{G}^{\circ}\right) 6 s 6 p\left({ }^{3} \mathrm{P}^{\circ}\right)$ & & 5 & 17729.875 & 1.222 & 16 & ${ }^{5} \mathrm{H}$ & 9 & $4 f^{2}\left({ }^{3} \mathrm{H}\right) 5 d\left({ }^{4} \mathrm{H}\right) 6 s{ }^{3} \mathrm{H}$ \\
\hline $4 f 5 d\left({ }^{1} \mathrm{G}^{\circ}\right) 6 s 6 p\left({ }^{3} \mathrm{P}^{\circ}\right)$ & & 4 & 17731.228 & 1.110 & 10 & ${ }^{3} \mathrm{H}$ & 6 & $4 f^{2}\left({ }^{3} \mathrm{~F}\right) 5 d\left({ }^{4} \mathrm{~F}\right) 6 s^{5} \mathrm{~F}$ \\
\hline \multirow[t]{2}{*}{$4 f 5 d\left({ }^{1} \mathrm{P}^{\circ}\right) 6 s 6 p\left({ }^{3} \mathrm{P}^{\circ}\right)$} & & 2 & 17770.105 & 0.936 & 12 & ${ }^{3} \mathrm{~F}$ & 11 & $\left({ }^{3} \mathrm{G}^{\circ}\right)\left({ }^{3} \mathrm{P}^{\circ}\right){ }^{3} \mathrm{G}$ \\
\hline & & 3 & 17785.937 & 1.147 & & & & \\
\hline $4 f^{2}\left({ }^{3} \mathrm{~F}\right) 5 d\left({ }^{4} \mathrm{~F}\right) 6 s$ & & 2 & 17886.379 & 1.357 & 18 & ${ }^{5} \mathrm{~F}$ & 7 & $\left({ }^{3} \mathrm{~F}\right)\left({ }^{2} \mathrm{P}\right){ }^{3} \mathrm{P}$ \\
\hline $4 f^{2}\left({ }^{3} \mathrm{~F}\right) 5 d\left({ }^{4} \mathrm{H}\right) 6 s$ & & 4 & 17892.814 & 0.917 & 27 & ${ }^{3} \mathrm{H}$ & 12 & $4 f\left({ }^{2} \mathrm{~F}^{\circ}\right) 5 d^{2}\left({ }^{3} \mathrm{~F}\right)\left({ }^{4} \mathrm{H}^{\circ}\right) 6 p{ }^{3} \mathrm{H}$ \\
\hline \multirow[t]{2}{*}{$4 f^{2}\left({ }^{3} \mathrm{H}\right) 5 d\left({ }^{4} \mathrm{H}\right) 6 s$} & & 5 & 17895.539 & 1.087 & 23 & ${ }^{3} \mathrm{H}$ & 7 & $4 f 5 d\left({ }^{3} \mathrm{H}^{\circ}\right) 6 s 6 p\left({ }^{3} \mathrm{P}^{\circ}\right){ }^{5} \mathrm{H}$ \\
\hline & & 3 & 17921.087 & 1.222 & & & & \\
\hline $4 f^{2}\left({ }^{3} \mathrm{~F}\right) 5 d\left({ }^{4} \mathrm{H}\right) 6 s$ & & 6 & 17975.060 & 1.207 & 22 & ${ }^{5} \mathrm{H}$ & 14 & $\left({ }^{1} \mathrm{G}\right)\left({ }^{2} \mathrm{H}\right){ }^{3} \mathrm{H}$ \\
\hline \multirow[t]{2}{*}{$4 f\left({ }^{2} \mathrm{~F}^{\circ} / 2\right) 6 s^{2} 6 p_{1 / 2} ?$} & & 4 & 17998.974 & 1.040 & 13 & $(7 / 2,1 / 2)$ & 12 & $(5 / 2,3 / 2)$ \\
\hline & & 3 & 18005.652 & 1.136 & & & & \\
\hline $4 f 5 d\left({ }^{3} \mathrm{H}^{\circ}\right) 6 s 6 p\left({ }^{3} \mathrm{P}^{\circ}\right)$ & & 5 & 18008.442 & 1.139 & 9 & ${ }^{3} \mathrm{I}$ & 7 & $\left({ }^{3} \mathrm{~F}^{\circ}\right)\left({ }^{3} \mathrm{P}^{\circ}\right){ }^{5} \mathrm{~F}$ \\
\hline $4 f^{2}\left({ }^{3} \mathrm{~F}\right) 5 d\left({ }^{4} \mathrm{~F}\right) 6 s$ & & 2 & 18052.690 & 0.978 & 13 & ${ }^{5} \mathrm{~F}$ & 8 & $\left({ }^{3} \mathrm{~F}\right)\left({ }^{4} \mathrm{P}\right){ }^{5} \mathrm{P}$ \\
\hline $4 f 5 d\left({ }^{1} \mathrm{D}^{\circ}\right) 6 s 6 p\left({ }^{3} \mathrm{P}^{\circ}\right)$ & & 1 & 18066.931 & 0.719 & 19 & ${ }^{3} \mathrm{D}$ & 12 & $4 f^{2}\left({ }^{3} \mathrm{~F}\right) 5 d\left({ }^{4} \mathrm{~F}\right) 6 s^{5} \mathrm{~F}$ \\
\hline $4 f^{2}\left({ }^{3} \mathrm{H}\right) 5 d\left({ }^{2} \mathrm{G}\right) 6 s ?$ & & 4 & 18141.848 & 1.075 & 9 & ${ }^{1} \mathrm{G}$ & 9 & $\left({ }^{3} \mathrm{~F}\right)\left({ }^{2} \mathrm{~F}\right){ }^{3} \mathrm{~F}$ \\
\hline \multirow[t]{2}{*}{$4 f^{2}\left({ }^{3} \mathrm{H}\right) 5 d\left({ }^{4} \mathrm{~F}\right) 6 s$} & & 4 & 18162.311 & 1.161 & 15 & ${ }^{5} \mathrm{~F}$ & 11 & $4 f 5 d\left({ }^{3} \mathrm{G}^{\circ}\right) 6 s 6 p\left({ }^{3} \mathrm{P}^{\circ}\right){ }^{5} \mathrm{G}$ \\
\hline & & 3 & 18192.766 & 1.087 & & & & \\
\hline $4 f 5 d\left({ }^{3} \mathrm{G}^{\circ}\right) 6 s 6 p\left({ }^{3} \mathrm{P}^{\circ}\right)$ & & 5 & 18194.890 & 1.025 & 12 & ${ }^{5} \mathrm{H}$ & 11 & $\left({ }^{3} \mathrm{H}^{\circ}\right)\left({ }^{3} \mathrm{P}^{\circ}\right){ }^{1} \mathrm{H}$ \\
\hline $4 f^{2} 6 s^{2}$ & & 1 & 18201.970 & 1.302 & 19 & ${ }^{3} \mathrm{P}$ & 12 & $4 f 5 d\left({ }^{3} \mathrm{D}^{\circ}\right) 6 s 6 p\left({ }^{3} \mathrm{P}^{\circ}\right)^{5} \mathrm{D}$ \\
\hline $4 f^{2}\left({ }^{3} \mathrm{~F}\right) 5 d\left({ }^{2} \mathrm{P}\right) 6 s$ & & 1 & 18221.192 & 1.118 & 12 & ${ }^{1} \mathrm{P}$ & 12 & $4 f 5 d\left({ }^{3} \mathrm{~F}^{\circ}\right) 6 s 6 p\left({ }^{3} \mathrm{P}^{\circ}\right){ }^{5} \mathrm{D}$ \\
\hline $4 f^{2}\left({ }^{3} \mathrm{H}\right) 5 d\left({ }^{4} \mathrm{~F}\right) 6 s$ & & 2 & 18238.821 & 0.945 & 18 & ${ }^{3} \mathrm{~F}$ & 8 & $4 f 5 d\left({ }^{3} \mathrm{D}^{\circ}\right) 6 s 6 p\left({ }^{3} \mathrm{P}^{\circ}\right)^{5} \mathrm{~F}$ \\
\hline $4 f 5 d\left({ }^{3} \mathrm{~F}^{\circ}\right) 6 s 6 p\left({ }^{3} \mathrm{P}^{\circ}\right)$ & & 6 & 18294.451 & & 32 & ${ }^{5} \mathrm{G}$ & 10 & $\left({ }^{3} \mathrm{H}^{\circ}\right)\left({ }^{3} \mathrm{P}^{\circ}\right){ }^{3} \mathrm{H}$ \\
\hline \multirow[t]{2}{*}{$4 f 5 d\left({ }^{3} \mathrm{H}^{\circ}\right) 6 s 6 p\left({ }^{3} \mathrm{P}^{\circ}\right)$} & & 7 & 18294.645 & 1.224 & 34 & ${ }^{5} \mathrm{H}$ & 16 & $4 f^{2}\left({ }^{3} \mathrm{H}\right) 5 d\left({ }^{4} \mathrm{I}\right) 6 s^{3} \mathrm{I}$ \\
\hline & & 3 & 18313.861 & 1.235 & & & & \\
\hline $4 f 5 d\left({ }^{3} \mathrm{~F}^{\circ}\right) 6 s 6 p\left({ }^{3} \mathrm{P}^{\circ}\right)$ & & 2 & 18324.668 & 1.006 & 13 & ${ }^{1} \mathrm{D}$ & 12 & $\left({ }^{3} \mathrm{~F}^{\circ}\right)\left({ }^{3} \mathrm{P}^{\circ}\right){ }^{3} \mathrm{D}$ \\
\hline $4 f 5 d\left({ }^{3} \mathrm{~F}^{\circ}\right) 6 s 6 p\left({ }^{3} \mathrm{P}^{\circ}\right)$ & & 5 & 18365.725 & 1.158 & 18 & ${ }^{5} F$ & 14 & $\left({ }^{3} \mathrm{H}^{\circ}\right)\left({ }^{3} \mathrm{P}^{\circ}\right){ }^{3} \mathrm{H}$ \\
\hline \multirow[t]{2}{*}{$4 f 5 d\left({ }^{3} \mathrm{~F}^{\circ}\right) 6 s 6 p\left({ }^{3} \mathrm{P}^{\circ}\right)$} & & 4 & 18411.170 & 1.175 & 12 & ${ }^{3} \mathrm{G}$ & 12 & $4 f^{2}\left({ }^{3} \mathrm{~F}\right) 5 d\left({ }^{4} \mathrm{~F}\right) 6 s{ }^{5} \mathrm{~F}$ \\
\hline & & 3 & 18427.612 & & & & & \\
\hline $4 f^{2} 6 s^{2}$ & & 1 & 18535.575 & 0.602 & 22 & ${ }^{3} \mathrm{P}$ & 18 & $4 f 5 d\left({ }^{3} \mathrm{D}^{\circ}\right) 6 s 6 p\left({ }^{3} \mathrm{P}^{\circ}\right)^{5} \mathrm{~F}$ \\
\hline $4 f^{2}\left({ }^{3} \mathrm{H}\right) 5 d\left({ }^{2} \mathrm{G}\right) 6 s$ & & 4 & 18550.101 & 1.055 & 15 & ${ }^{3} \mathrm{G}$ & 6 & $4 f 5 d\left({ }^{3} \mathrm{G}^{\circ}\right) 6 s 6 p\left({ }^{3} \mathrm{P}^{\circ}\right){ }^{1} \mathrm{G}$ \\
\hline $4 f 5 d\left({ }^{3} \mathrm{~F}^{\circ}\right) 6 s 6 p\left({ }^{3} \mathrm{P}^{\circ}\right)$ & & 2 & 18587.749 & 1.135 & 12 & ${ }^{5} \mathrm{D}$ & 7 & $4 f^{2}\left({ }^{3} \mathrm{~F}\right) 5 d\left({ }^{4} \mathrm{P}\right) 6 s^{3} \mathrm{P}$ \\
\hline \multirow{2}{*}{$4 f 5 d\left({ }^{3} \mathrm{~F}^{\circ}\right) 6 s 6 p\left({ }^{3} \mathrm{P}^{\circ}\right)$} & & 6 & 18598.261 & 1.253 & 31 & ${ }^{5} \mathrm{G}$ & 17 & $\left({ }^{3} \mathrm{G}^{\circ}\right)\left({ }^{3} \mathrm{P}^{\circ}\right){ }^{5} \mathrm{H}$ \\
\hline & & 3 & 18611.386 & 1.028 & & & & \\
\hline \multirow[t]{2}{*}{$4 f 5 d\left({ }^{3} \mathrm{~F}^{\circ}\right) 6 s 6 p\left({ }^{3} \mathrm{P}^{\circ}\right)$} & & 5 & 18619.685 & 1.119 & 14 & ${ }^{5} \mathrm{~F}$ & 12 & $\left({ }^{3} \mathrm{G}^{\circ}\right)\left({ }^{3} \mathrm{P}^{\circ}\right){ }^{5} \mathrm{H}$ \\
\hline & & 3 & 18652.242 & 1.137 & & & & \\
\hline $4 f^{2}\left({ }^{3} \mathrm{H}\right) 5 d\left({ }^{4} \mathrm{~F}\right) 6 s$ & & 5 & 18661.289 & 1.237 & 25 & ${ }^{5} \mathrm{~F}$ & 12 & $4 f 5 d\left({ }^{3} \mathrm{~F}^{\circ}\right) 6 s 6 p\left({ }^{3} \mathrm{P}^{\circ}\right)^{5} \mathrm{~F}$ \\
\hline $4 f\left({ }^{2} \mathrm{~F}_{5 / 2}^{\circ}\right) 6 s^{2} 6 p 3 / 2$ & & 4 & 18692.793 & 1.110 & 11 & $(5 / 2,3 / 2)$ & 7 & $(7 / 2,3 / 2)$ \\
\hline $4 f^{2}\left({ }^{3} \mathrm{H}\right) 5 d\left({ }^{4} \mathrm{~F}\right) 6 s$ & & 2 & 18706.502 & 0.844 & 21 & ${ }^{3} \mathrm{~F}$ & 15 & $4 f 5 d\left({ }^{3} \mathrm{~F}^{\circ}\right) 6 s 6 p\left({ }^{3} \mathrm{P}^{\circ}\right)^{3} \mathrm{~F}$ \\
\hline \multirow[t]{2}{*}{$4 f 5 d\left({ }^{3} \mathrm{G}^{\circ}\right) 6 s 6 p\left({ }^{3} \mathrm{P}^{\circ}\right) ?$} & & 1 & 18727.755 & 0.625 & 13 & ${ }^{5} \mathrm{~F}$ & 12 & $4 f^{2}\left({ }^{3} \mathrm{~F}\right) 5 d\left({ }^{4} \mathrm{~F}\right) 6 s^{5} \mathrm{~F}$ \\
\hline & & 3 & 18758.513 & 1.047 & & & & \\
\hline $4 f^{2}\left({ }^{3} \mathrm{~F}\right) 5 d\left({ }^{4} \mathrm{G}\right) 6 s ?$ & & 5 & 18831.782 & 1.141 & 11 & ${ }^{5} \mathrm{G}$ & 9 & $4 f 5 d\left({ }^{3} \mathrm{G}^{\circ}\right) 6 s 6 p\left({ }^{3} \mathrm{P}^{\circ}\right){ }^{3} \mathrm{G}$ \\
\hline $4 f 5 d\left({ }^{3} \mathrm{G}^{\circ}\right) 6 s 6 p\left({ }^{3} \mathrm{P}^{\circ}\right)$ & & 4 & 18871.606 & 1.100 & 9 & ${ }^{3} \mathrm{G}$ & 9 & $\left({ }^{3} \mathrm{~F}^{\circ}\right)\left({ }^{3} \mathrm{P}^{\circ}\right){ }^{3} \mathrm{G}$ \\
\hline
\end{tabular}


Ce I, Even Parity-Continued

\begin{tabular}{|c|c|c|c|c|c|c|c|c|c|}
\hline Configuration & Term & $J$ & $\begin{array}{l}\text { Level } \\
\left(\mathrm{cm}^{-1}\right)\end{array}$ & $g$ & \multicolumn{5}{|c|}{ Leading percentages } \\
\hline $4 f 5 d\left({ }^{1} \mathrm{D}^{\circ}\right) 6 s 6 p\left({ }^{3} \mathrm{P}^{\circ}\right)$ & & 2 & 18880.662 & 0.934 & 14 & ${ }^{3} \mathrm{D}$ & 7 & $\left({ }^{3} \mathrm{G}^{\circ}\right)\left({ }^{3} \mathrm{P}^{\circ}\right){ }^{5} \mathrm{~F}$ & \\
\hline $4 f^{2}\left({ }^{3} \mathrm{~F}\right) 5 d\left({ }^{4} \mathrm{H}\right) 6 s$ & & 7 & 18942.478 & 1.257 & 39 & ${ }^{5} \mathrm{H}$ & 22 . & $4 f 5 d\left({ }^{3} \mathrm{H}^{\circ}\right) 6 s 6 p\left({ }^{3} \mathrm{P}^{\circ}\right){ }^{5} \mathrm{H}$ & \\
\hline $4 f^{2}\left({ }^{3} \mathrm{~F}\right) 5 d\left({ }^{4} \mathrm{H}\right) 6 s ?$ & & 5 & 18943.013 & 1.160 & 13 & ${ }^{3} \mathrm{H}$ & 5 & $\left({ }^{3} \mathrm{~F}\right)\left({ }^{4} \mathrm{~F}\right){ }^{5} \mathrm{~F}$ & \\
\hline $4 f^{2}\left({ }^{3} \mathrm{~F}\right) 5 d\left({ }^{2} \mathrm{P}\right) 6 s ?$ & & 1 & 18973.876 & 0.842 & 15 & ${ }^{3} \mathrm{P}$ & 12 & $\left({ }^{3} \mathrm{~F}\right)\left({ }^{2} \mathrm{P}\right){ }^{1} \mathrm{P}$ & \\
\hline $4 f^{2}\left({ }^{3} \mathrm{~F}\right) 5 d\left({ }^{2} \mathrm{D}\right) 6 s$ & & 2 & 18987.150 & 1.121 & 12 & ${ }^{1} \mathrm{D}$ & 9 & $\left({ }^{3} \mathrm{~F}\right)\left({ }^{2} \mathrm{D}\right){ }^{3} \mathrm{D}$ & \\
\hline $4 f\left({ }^{2} \mathrm{~F}^{\circ}\right) 5 d^{2}\left({ }^{3} \mathrm{~F}\right)\left({ }^{4} \mathrm{H}^{\circ}\right) 6 p$ & & 4 & 19014.863 & 0.911 & 26 & ${ }^{5} \mathrm{I}$ & 15 & $4 f^{2}\left({ }^{3} \mathrm{~F}\right) 5 d\left({ }^{4} \mathrm{H}\right) 6 s^{3} \mathrm{H}$ & \\
\hline $4 f 5 d\left({ }^{3} \mathrm{H}^{\circ}\right) 6 s 6 p\left({ }^{3} \mathrm{P}^{\circ}\right)$ & & 5 & 19062.498 & 1.097 & 12 & ${ }^{5} \mathrm{G}$ & 11 & $4 f^{2}\left({ }^{3} \mathrm{~F}\right) 5 d\left({ }^{4} \mathrm{H}\right) 6 s^{3} \mathrm{H}$ & \\
\hline \multirow[t]{2}{*}{$4 f^{2}\left({ }^{3} \mathrm{H}\right) 5 d\left({ }^{4} \mathrm{H}\right) 6 s$} & & 6 & 19072.103 & 1.122 & 17 & ${ }^{3} \mathrm{H}$ & 15 & $4 f 5 d\left({ }^{3} \mathrm{H}^{\circ}\right) 6 s 6 p\left({ }^{3} \mathrm{P}^{\circ}\right){ }^{3} \mathrm{I}$ & \\
\hline & & 3 & 19072.898 & 1.009 & & & & & \\
\hline $4 f^{2}\left({ }^{3} \mathrm{~F}\right) 5 d\left({ }^{2} \mathrm{D}\right) 6 s$ & & 2 & 19158.135 & 1.020 & 9 & ${ }^{1} \mathrm{D}$ & 9 & $4 f 5 d\left({ }^{1} \mathrm{D}^{\circ}\right) 6 s 6 p\left({ }^{3} \mathrm{P}^{\circ}\right){ }^{3} \mathrm{D}$ & \\
\hline $4 f 5 d\left({ }^{3} \mathrm{G}^{\circ}\right) 6 s 6 p\left({ }^{3} \mathrm{P}^{\circ}\right)$ & & 4 & 19220.360 & 1.137 & 14 & ${ }^{3} \mathrm{G}$ & 6 & $4 f^{2}\left({ }^{3} \mathrm{~F}\right) 5 d\left({ }^{4} \mathrm{~F}\right) 6 s^{5} \mathrm{~F}$ & \\
\hline $4 f 5 d\left({ }^{3} \mathrm{H}^{\circ}\right) 6 s 6 p\left({ }^{3} \mathrm{P}^{\circ}\right)$ & & 6 & 19235.098 & 1.097 & 16 & ${ }^{3} \mathrm{I}$ & 13 & $\left({ }^{3} \mathrm{G}^{\circ}\right)\left({ }^{3} \mathrm{P}^{\circ}\right){ }^{5} \mathrm{H}$ & \\
\hline \multirow[t]{2}{*}{$4 f^{2}\left({ }^{3} \mathrm{H}\right) 5 d\left({ }^{2} \mathrm{G}\right) 6 s$} & & 4 & 19244.787 & 1.000 & 13 & ${ }^{1} \mathrm{G}$ & 9 & $4 f\left({ }^{2} \mathrm{~F}^{\circ}\right) 5 d^{2}\left({ }^{3} \mathrm{~F}\right)\left({ }^{4} \mathrm{I}^{\circ}\right) 6 p^{3} \mathrm{H}$ & \\
\hline & & 3 & 19247.627 & 1.067 & & & & & \\
\hline \multirow[t]{3}{*}{$4 f\left({ }^{2} \mathrm{~F}^{\circ}\right) 5 d^{2}\left({ }^{3} \mathrm{~F}\right)\left({ }^{4} \mathrm{I}^{\circ}\right) 6 p$} & & 4 & 19296.353 & 0.926 & 22 & ${ }^{3} \mathrm{H}$ & 9 & $4 f 5 d\left({ }^{3} \mathrm{H}^{\circ}\right) 6 s 6 p\left({ }^{1} \mathrm{P}^{\circ}\right){ }^{3} \mathrm{H}$ & \\
\hline & & 2 & 19321.019 & 1.334 & & & & & \\
\hline & & 2 & 19330.215 & 0.961 & & & & & \\
\hline $4 f 5 d\left({ }^{3} \mathrm{H}^{\circ}\right) 6 s 6 p\left({ }^{3} \mathrm{P}^{\circ}\right)$ & & 5 & 19347.064 & 1.198 & 18 & ${ }^{5} \mathrm{G}$ & 14 & $\left({ }^{3} \mathrm{~F}^{\circ}\right)\left({ }^{3} \mathrm{P}^{\circ}\right){ }^{3} \mathrm{G}$ & $1=$ \\
\hline $4 f^{2}\left({ }^{3} \mathrm{H}\right) 5 d\left({ }^{4} \mathrm{H}\right) 6 s$ & & 6 & 19347.542 & 1.193 & 21 & ${ }^{3} \mathrm{H}$ & 9 & $\left({ }^{3} \mathrm{~F}\right)\left({ }^{4} \mathrm{H}\right){ }^{3} \mathrm{H}$ & \\
\hline \multirow[t]{4}{*}{$4 f^{2} 6 s^{2}$} & & 1 & 19362.490 & 1.392 & 15 & ${ }^{3} \mathrm{P}$ & 11 & $4 f 5 d\left({ }^{3} \mathrm{D}^{\circ}\right) 6 s 6 p\left({ }^{3} \mathrm{P}^{\circ}\right) \cdot{ }^{3} \mathrm{P}$ & \\
\hline & & 3 & 19406.157 & 1.158 & & & & & \\
\hline & & 2 & 19409.898 & 0.922 & & & & & \\
\hline & & 3 & 19457.393 & 0.972 & & & & & \\
\hline $4 f 5 d\left({ }^{3} \mathrm{D}^{\circ}\right) 6 s 6 p\left({ }^{3} \mathrm{P}^{\circ}\right)$ & & 4 & 19544.105 & 1.187 & 12 & ${ }^{5} \mathrm{~F}$ & 9 & $4 f^{2}\left({ }^{3} \mathrm{H}\right) 5 d\left({ }^{4} \mathrm{~F}\right) 6 s^{5} \mathrm{~F}$ & \\
\hline \multirow[t]{2}{*}{$4 f 5 d\left({ }^{1} \mathrm{D}^{\circ}\right) 6 s 6 p\left({ }^{3} \mathrm{P}^{\circ}\right) ?$} & & 1 & 19566.861 & 0.836 & 18 & ${ }^{3} \mathrm{D}$ & 12 & $4 f^{2}\left({ }^{3} \mathrm{~F}\right) 5 d\left({ }^{2} \mathrm{D}\right) 6 s^{3} \mathrm{D}$ & \\
\hline & & 2 & 19595.360 & 0.826 & & & & & \\
\hline \multirow[t]{2}{*}{$4 f 5 d\left({ }^{3} \mathrm{G}^{\circ}\right) 6 s 6 p\left({ }^{3} \mathrm{P}^{\circ}\right) ?$} & & 6 & 19627.275 & 1.075 & 23 & ${ }^{5} \mathrm{H}$ & 17 & $\left({ }^{3} G^{\circ}\right)\left({ }^{3} P^{\circ}\right){ }^{5} G$ & \\
\hline & & 3 & 19636.636 & 1.052 & & & & & \\
\hline $4 f\left({ }^{2} \mathrm{~F}^{\circ}\right) 5 d^{2}\left({ }^{3} \mathrm{~F}\right)\left({ }^{4} \mathrm{H}^{\circ}\right) 6 p ?$ & & 5 & 19661.076 & 1.047 & 22 & $5 I$ & 10 & $4 f^{2}\left({ }^{3} \mathrm{~F}\right) 5 d\left({ }^{4} \mathrm{H}\right) 6 s^{3} \mathrm{H}$ & \\
\hline $4 f^{2}\left({ }^{3} \mathrm{~F}\right) 5 d\left({ }^{4} \mathrm{D}\right) 6 s$ & & 4 & 19680.078 & 1.260 & 15 & ${ }^{5} \mathrm{D}$ & 10 & $4 f 5 d\left({ }^{1} \mathrm{G}^{\circ}\right) 6 s 6 p\left({ }^{3} \mathrm{P}^{\circ}\right){ }^{3} \mathrm{~F}$ & \\
\hline \multirow[t]{2}{*}{$4 f^{2}\left({ }^{3} \mathrm{H}\right) 5 d\left({ }^{2} \mathrm{~K}\right) 6 s ?$} & ${ }^{3} \mathrm{~K}$ ? & 6 & 19680.343 & 1.06 & 45 & & 7 & $\left({ }^{3} \mathrm{H}\right)\left({ }^{4} \mathrm{H}\right){ }^{3} \mathrm{H}$ & \\
\hline & & 3 & 19711.008 & 0.946 & & & & & \\
\hline $4 f 5 d\left({ }^{3} \mathrm{D}^{\circ}\right) 6 s 6 p\left({ }^{3} \mathrm{P}^{\circ}\right) ?$ & & 1 & 19768.832 & 0.813 & 9 & ${ }^{1} \mathrm{P}$ & 9 & $4 f^{2}\left({ }^{3} \mathrm{~F}\right) 5 d\left({ }^{4} \mathrm{D}\right) 6 s^{3} \mathrm{D}$ & \\
\hline \multirow[t]{6}{*}{$4 f\left({ }^{2} \mathrm{~F}^{\circ}\right) 5 d^{2}\left({ }^{3} \mathrm{~F}\right)\left({ }^{4} \mathrm{I}^{\circ}\right) 6 p$} & ${ }^{5} \mathrm{~K}$ & 5 & 19791.740 & 0.836 & 34 & & 18 & $4 f 5 d\left({ }^{3} \mathrm{H}^{\circ}\right) 6 s 6 p\left({ }^{3} \mathrm{P}^{\circ}\right)^{3} \mathrm{I}$ & \\
\hline & & 6 & 20591.937 & 0.961 & 51 & & 11 & $\left({ }^{2} \mathrm{~F}^{\circ}\right)\left({ }^{3} \mathrm{~F}\right)\left({ }^{4} \mathrm{H}^{\circ}\right)^{5} \mathrm{I}$ & \\
\hline & & 7 & 21654.060 & 1.053 & 66 & & 5 & $\left({ }^{2} \mathrm{~F}^{\circ}\right)\left({ }^{3} \mathrm{~F}\right)\left({ }^{4} \mathrm{H}^{\circ}\right){ }^{5} \mathrm{I}$ & \\
\hline & & 8 & 22864.055 & 1.164 & 59 & & 26 & $\left({ }^{2} \mathrm{~F}^{\circ}\right)\left({ }^{3} \mathrm{~F}\right)\left({ }^{4} \mathrm{H}^{\circ}\right){ }^{5} \mathrm{I}$ & \\
\hline & & 9 & 24350.503 & 1.222 & 95 & & 4 & $4 f^{2}\left({ }^{3} \mathrm{H}\right) 5 d\left({ }^{4} \mathrm{~K}\right) 6 s^{5} \mathrm{~K}$ & \\
\hline & & 2 & 19805.290 & 1.244 & & & & & \\
\hline $4 f^{2}\left({ }^{3} \mathrm{H}\right) 5 d\left({ }^{2} \mathrm{~K}\right) 6 s$ & & 7 & 19836.936 & & 40 & ${ }^{1} \mathrm{~K}$ & 30 & $\left({ }^{3} \mathrm{H}\right)\left({ }^{2} \mathrm{~K}\right){ }^{3} \mathrm{~K}$ & \\
\hline $4 f^{2}\left({ }^{3} \mathrm{~F}\right) 5 d\left({ }^{4} \mathrm{~F}\right) 6 s$ & & 4 & 19840.631 & 1.211 & 21 & ${ }^{5} \mathrm{~F}$ & 5 & $\left({ }^{3} \mathrm{~F}\right)\left({ }^{2} \mathrm{G}\right){ }^{1} \mathrm{G}$ & \\
\hline \multirow[t]{3}{*}{$4 f\left({ }^{2} \mathrm{~F}^{\circ}\right) 5 d^{2}\left({ }^{3} \mathrm{~F}\right)\left({ }^{4} \mathrm{I}^{\circ}\right) 6 p ?$} & & 5 & 19843.704 & 1.008 & 15 & ${ }^{5} \mathrm{~K}$ & 10 & $4 f^{2}\left({ }^{1} \mathrm{G}\right) 5 d\left({ }^{2} \mathrm{H}\right) 6 s^{3} \mathrm{H}$ & \\
\hline & & 2 & 19880.707 & 0.779 & & & & & \\
\hline & & 3 & 19928.468 & 1.157 & & & & & \\
\hline $4 f 5 d\left({ }^{3} \mathrm{P}^{\circ}\right) 6 s 6 p\left({ }^{3} \mathrm{P}^{\circ}\right) ?$ & & 1 & 19961.411 & & 15 & ${ }^{5} \mathrm{P}$ & 10 & $\left({ }^{3} \mathrm{D}^{\circ}\right)\left({ }^{3} \mathrm{P}^{\circ}\right){ }^{5} \mathrm{P}$ & \\
\hline
\end{tabular}


Ce I, Even Parity_Continued

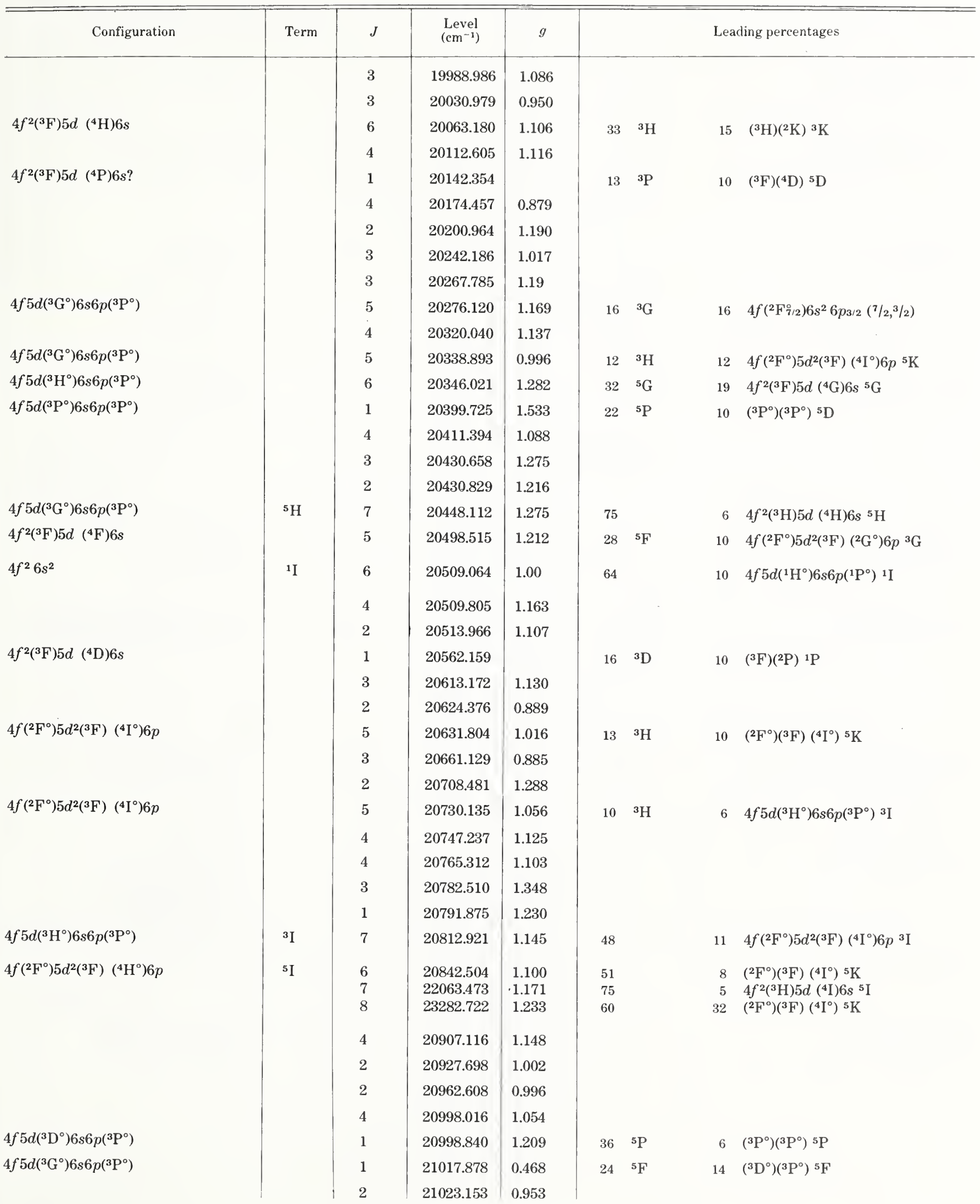


Ce I, Even Parity_Continued

\begin{tabular}{|c|c|c|c|c|c|c|c|c|}
\hline Configuration & Term & $J$ & $\begin{array}{l}\text { Level } \\
\left(\mathrm{cm}^{-1}\right)\end{array}$ & $g$ & \multicolumn{4}{|c|}{ Leading percentages } \\
\hline $4 f\left({ }^{2} \mathrm{~F}^{\circ}\right) 5 d^{2}\left({ }^{3} \mathrm{~F}\right)\left({ }^{4} \mathrm{H}^{\circ}\right) 6 p$ & & 5 & 21059.520 & 1.075 & 19 & ${ }^{5} \mathrm{I}$ & 10 & $\left({ }^{2} \mathrm{~F}^{\circ}\right)\left({ }^{3} \mathrm{~F}\right)\left({ }^{2} \mathrm{G}^{\circ}\right){ }^{3} \mathrm{H}$ \\
\hline \multirow[t]{2}{*}{$4 f 5 d\left({ }^{3} \mathrm{D}^{\circ}\right) 6 s 6 p\left({ }^{3} \mathrm{P}^{\circ}\right)$} & & 0 & 21068.470 & & 18 & ${ }^{3} \mathrm{P}$ & 11 & $\left({ }^{3} \mathrm{P}^{\circ}\right)\left({ }^{3} \mathrm{P}^{\circ}\right){ }^{5} \mathrm{D}$ \\
\hline & & 4 & 21076.477 & 0.949 & & & & \\
\hline $4 f\left({ }^{2} \mathrm{~F}^{\circ}\right) 5 d^{2}\left({ }^{3} \mathrm{~F}\right)\left({ }^{4} \mathrm{H}^{\circ}\right) 6 p$ & & 6 & 21100.065 & 1.069 & 11 & ${ }^{3} \mathrm{H}$ & 9 & $4 f^{2}\left({ }^{1} \mathrm{G}\right) 5 d\left({ }^{2} \mathrm{H}\right) 6 s^{3} \mathrm{H}$ \\
\hline \multirow[t]{3}{*}{$4 f 5 d\left({ }^{3} \mathrm{D}^{\circ}\right) 6 s 6 p\left({ }^{3} \mathrm{P}^{\circ}\right)$} & & 5 & 21153.703 & 1.250 & 15 & ${ }^{5} \mathrm{~F}$ & 12 & $\left({ }^{3} \mathrm{G}^{\circ}\right)\left({ }^{3} \mathrm{P}^{\circ}\right){ }^{5} \mathrm{~F}$ \\
\hline & & 3 & 21158.831 & 1.225 & & & & \\
\hline & & 4 & 21161.192 & 0.700 & & & & \\
\hline \multirow[t]{2}{*}{$4 f\left({ }^{2} \mathrm{~F}^{\circ}\right) 5 d^{2}\left({ }^{3} \mathrm{~F}\right)\left({ }^{4} \mathrm{D}^{\circ}\right) 6 p$} & & 1 & 21165.259 & 0.85 & 17 & ${ }^{5} \mathrm{~F}$ & 10 & $5 d^{3}\left({ }^{4} \mathrm{~F}\right) 6 s^{5} \mathrm{~F}$ \\
\hline & & 2 & 21168.987 & 1.128 & & & & \\
\hline \multirow[t]{3}{*}{$4 f 5 d\left({ }^{3} \mathrm{D}^{\circ}\right) 6 s 6 p\left({ }^{3} \mathrm{P}^{\circ}\right)$} & & 5 & 21226.361 & 1.134 & 14 & ${ }^{5} \mathrm{~F}$ & 13 & $4 f\left({ }^{2} \mathrm{~F}^{\circ}\right) 5 d^{2}\left({ }^{3} \mathrm{~F}\right)\left({ }^{2} \mathrm{G}^{\circ}\right) 6 p{ }^{3} \mathrm{H}$ \\
\hline & & 3 & 21237.916 & 1.010 & & & & \\
\hline & & 4 & 21267.903 & 1.107 & & & & \\
\hline $4 f^{2}\left({ }^{3} \mathrm{H}\right) 5 d\left({ }^{2} \mathrm{~K}\right) 6 s$ & & 7 & 21314.996 & 1.014 & 32 & ${ }^{1} \mathrm{~K}$ & 31 & $\left({ }^{3} \mathrm{H}\right)\left({ }^{2} \mathrm{~K}\right){ }^{3} \mathrm{~K}$ \\
\hline \multirow[t]{5}{*}{$4 f 5 d\left({ }^{3} \mathrm{H}^{\circ}\right) 6 s 6 p\left({ }^{3} \mathrm{P}^{\circ}\right)$} & & 6 & 21324.736 & 1.043 & 21 & ${ }^{\mathrm{I}} \mathrm{I}$ & 9 & $4 f\left({ }^{2} \mathrm{~F}^{\circ}\right) 5 d^{2}\left({ }^{3} \mathrm{~F}\right)\left({ }^{4} \mathrm{I}^{\circ}\right) 6 p^{3} \mathrm{I}$ \\
\hline & & 3 & 21340.864 & 1.108 & & & & \\
\hline & & 2 & 21375.475 & 0.950 & & & & \\
\hline & & 3 & 21399.014 & 1.265 & & & & \\
\hline & & 2 & 21420.772 & 1.114 & & & & \\
\hline $4 f^{2}\left({ }^{1} \mathrm{G}\right) 5 d\left({ }^{2} \mathrm{D}\right) 6 s$ & & 1 & 21431.568 & 0.640 & 13 & ${ }^{3} \mathrm{D}$ & 11 & $4 f\left({ }^{2} \mathrm{~F}^{\circ}\right) 5 d^{2}\left({ }^{3} \mathrm{~F}\right)\left({ }^{4} \mathrm{~F}^{\circ}\right) 6 p{ }^{3} \mathrm{D}$ \\
\hline \multirow[t]{3}{*}{$4 f 5 d\left({ }^{3} \mathrm{H}^{\circ}\right) 6 s 6 p\left({ }^{3} \mathrm{P}^{\circ}\right)$} & & 5 & 21456.528 & 1.200 & 21 & ${ }^{5} \mathrm{G}$ & 9 & $\left({ }^{3} \mathrm{G}^{\circ}\right)\left({ }^{3} \mathrm{P}^{\circ}\right){ }^{5} \mathrm{G}$ \\
\hline & & 3 & 21499.915 & 1.153 & & & & \\
\hline & & 4 & 21520.287 & 1.107 & & & & \\
\hline \multirow[t]{5}{*}{$4 f 5 d\left({ }^{3} \mathrm{H}^{\circ}\right) 6 s 6 p\left({ }^{3} \mathrm{P}^{\circ}\right)$} & & 5 & 21581.408 & 1.134 & 10 & ${ }^{5} \mathrm{G}$ & 7 & $4 f\left({ }^{2} \mathrm{~F}^{\circ}\right) 5 d^{2}\left({ }^{3} \mathrm{~F}\right)\left({ }^{2} \mathrm{I}^{\circ}\right) 6 p^{3} \mathrm{I}$ \\
\hline & & 3 & 21582.401 & 1.148 & & & & \\
\hline & & 4 & 21619.232 & 1.021 & & & & \\
\hline & & 2 & 21623.269 & $1.503 ?$ & & & & \\
\hline & & 3 & 21661.537 & 0.843 & & & & \\
\hline $4 f\left({ }^{2} \mathrm{~F}^{\circ}\right) 5 d^{2}\left({ }^{3} \mathrm{~F}\right)\left({ }^{2} \mathrm{~S}^{\circ}\right) 6 p$ & & 0 & 21664.821 & & 41 & ${ }^{3} \mathrm{P}$ & 8 & $\left({ }^{2} \mathrm{~F}^{\circ}\right)\left({ }^{3} \mathrm{~F}\right)\left({ }^{4} \mathrm{P}^{\circ}\right){ }^{3} \mathrm{P}$ \\
\hline \multirow[t]{2}{*}{$4 f^{2}\left({ }^{1} \mathrm{G}\right) 5 d\left({ }^{2} \mathrm{I}\right) 6 s$} & & 5 & 21683.722 & 0.934 & 9 & ${ }^{3} \mathrm{I}$ & 8 & $\left({ }^{3} \mathrm{~F}\right)\left({ }^{4} \mathrm{G}\right){ }^{3} \mathrm{G}$ \\
\hline & & 2 & 21712.611 & 1.395 & & & & \\
\hline $4 f^{2}\left({ }^{3} \mathrm{H}\right) 5 d\left({ }^{2} \mathrm{~K}\right) 6 s$ & ${ }^{3} \mathrm{~K}$ & 8 & 21722.069 & & 65 & & 10 & $\left({ }^{1} \mathrm{I}\right)\left({ }^{2} \mathrm{~K}\right){ }^{3} \mathrm{~K}$ \\
\hline \multirow{4}{*}{$4 f\left({ }^{2} \mathrm{~F}^{\circ}\right) 5 d^{2}\left({ }^{3} \mathrm{~F}\right)\left({ }^{4} \mathrm{I}^{\circ}\right) 6 p$} & ${ }^{5} I$ & 5 & 21725.348 & 0.997 & 45 & & 8 & $\left({ }^{2} \mathrm{~F}^{\circ}\right)\left({ }^{3} \mathrm{~F}\right)\left({ }^{2} \mathrm{G}^{\circ}\right){ }^{3} \mathrm{H}$ \\
\hline & & $\begin{array}{l}6 \\
7\end{array}$ & 22321.098 & 1.057 & 41 & & 5 & $\left({ }^{2} \mathrm{~F}^{\circ}\right)\left({ }^{3} \mathrm{~F}\right)\left({ }^{2} \mathrm{I}^{\circ}\right){ }^{3} \mathrm{I}$ \\
\hline & & $\begin{array}{l}7 \\
8\end{array}$ & $\begin{array}{l}23452.626 \\
24657.245\end{array}$ & 1.157 & 38 & & 8 & $4 f^{2}\left({ }^{1} \mathrm{G}\right) 5 d\left({ }^{2} \mathrm{I}\right) 6 s^{3} \mathrm{I}$ \\
\hline & & 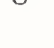 & 24657.245 & 1.238 & 70 & & 11 & $4 f^{2}\left({ }^{3} \mathrm{H}\right) 5 d^{2}\left({ }^{3} \mathrm{~F}\right)^{5} \mathrm{I}$ \\
\hline \multirow[t]{3}{*}{$4 f\left({ }^{2} \mathrm{~F}^{\circ}\right) 5 d^{2}\left({ }^{1} \mathrm{D}\right)\left({ }^{2} \mathrm{P}^{\circ}\right) 6 p$} & & 1 & 21725.865 & 1.337 & 10 & ${ }^{3} \mathrm{~S}$ & 7 & $\left({ }^{2} \mathrm{~F}^{\circ}\right)\left({ }^{3} \mathrm{~F}\right)\left({ }^{4} \mathrm{P}^{\circ}\right){ }^{3} \mathrm{~S}$ \\
\hline & & 2 & 21786.764 & 1.186 & & & & \\
\hline & & 3 & 21813.247 & 1.048 & & & & \\
\hline $4 f 5 d\left({ }^{1} \mathrm{~F}^{\circ}\right) 6 s 6 p\left({ }^{3} \mathrm{P}^{\circ}\right)$ & & 1 & 21823.516 & 0.653 & 17 & ${ }^{3} \mathrm{D}$ & 11 & $4 f^{2}\left({ }^{1} \mathrm{G}\right) 5 d\left({ }^{2} \mathrm{D}\right) 6 s^{3} \mathrm{D}$ \\
\hline \multirow[t]{6}{*}{$4 f\left({ }^{2} \mathrm{~F}^{\circ}\right) 5 d^{2}\left({ }^{3} \mathrm{~F}\right)\left({ }^{2} \mathrm{G}^{\circ}\right) 6 p$} & & 6 & 21877.490 & 1.128 & 24 & ${ }^{3} \mathrm{H}$ & 14 & $\left({ }^{2} \mathrm{~F}^{\circ}\right)\left({ }^{3} \mathrm{~F}\right)\left({ }^{4} \mathrm{H}^{\circ}\right)^{5} \mathrm{I}$ \\
\hline & & 4 & 21885.546 & 1.156 & & & & \\
\hline & & 5 & 21946.849 & 1.084 & & & & \\
\hline & & 3 & 21948.875 & 1.099 & & & & \\
\hline & & 4 & 21993.874 & 1.213 & & & & \\
\hline & & 2 & 21994.428 & 1.45 & & & & \\
\hline
\end{tabular}


Ce I, Even Parity_Continued

\begin{tabular}{|c|c|c|c|c|c|c|c|c|}
\hline Configuration & Term & $J$ & $\begin{array}{l}\text { Level } \\
\left(\mathrm{cm}^{-1}\right)\end{array}$ & $g$ & \multicolumn{4}{|c|}{ Leading percentages } \\
\hline & & 4 & 22063.708 & 1.156 & & & & \\
\hline \multirow[t]{3}{*}{$4 f 5 d\left({ }^{3} \mathrm{H}^{\circ}\right) 6 s 6 p\left({ }^{3} \mathrm{P}^{\circ}\right)$} & & 6 & 22064.966 & 1.150 & 17 & ${ }^{5} \mathrm{G}$ & 16 & $4 f\left({ }^{2} \mathrm{~F}^{\circ}\right) 5 d^{2}\left({ }^{3} \mathrm{~F}\right)\left({ }^{2} \mathrm{G}^{0}\right) 6 p{ }^{3} \mathrm{H}$ \\
\hline & & 2 & 22092.108 & 1.014 & & & & \\
\hline & & 3 & 22127.392 & 1.087 & & & & \\
\hline \multirow[t]{11}{*}{$4 f\left({ }^{2} \mathrm{~F}^{\circ}\right) 5 d^{2}\left({ }^{3} \mathrm{~F}\right)\left({ }^{2} \mathrm{~S}^{\circ}\right) 6 p$} & & 1 & 22162.504 & 0.806 & 11 & ${ }^{3} \mathrm{P}$ & 9 & $\left({ }^{2} \mathrm{~F}^{\circ}\right)\left({ }^{3} \mathrm{~F}\right)\left({ }^{4} \mathrm{~F}^{\circ}\right){ }^{5} \mathrm{~F}$ \\
\hline & & 2 & 22170.124 & 0.745 & & & & \\
\hline & & 5 & 22184.376 & 1.092 & & & & \\
\hline & & 3 & 22190.760 & 0.938 & & & & \\
\hline & & 4 & 22210.585 & 1.082 & & & & \\
\hline & & 2 & 22219.750 & 1.179 & & & & \\
\hline & & 1 & 22246.080 & 1.322 & & & & \\
\hline & & 3 & 22291.251 & 1.233 & & & & \\
\hline & & 4 & 22325.164 & 1.106 & & & & \\
\hline & & 5 & 22355.991 & 1.083 & & & & \\
\hline & & 2 & 22360.158 & 1.165 & & & & \\
\hline $4 f 5 d\left({ }^{3} \mathrm{P}^{\circ}\right) 6 s 6 p\left({ }^{3} \mathrm{P}^{\circ}\right) ?$ & & 1 & 22392.029 & 1.500 & 37 & ${ }^{5} \mathrm{D}$ & 19 & $\left({ }^{3} \mathrm{D}^{\circ}\right)\left({ }^{3} \mathrm{P}^{\circ}\right){ }^{5} \mathrm{D}$ \\
\hline \multirow[t]{5}{*}{$4 f 5 d\left({ }^{3} \mathrm{H}^{\circ}\right) 6 s 6 p\left({ }^{3} \mathrm{P}^{\circ}\right)$} & & 6 & 22438.967 & 1.126 & 9 & ${ }^{1} \mathrm{I}$ & 7 & $\left({ }^{3} \mathrm{H}^{\circ}\right)\left({ }^{3} \mathrm{P}^{\circ}\right){ }^{5} \mathrm{G}$ \\
\hline & & 3 & 22477.386 & 0.990 & & & & \\
\hline & & 4 & 22503.719 & 1.049 & & & & \\
\hline & & 5 & 22518.695 & 1.080 & & & & \\
\hline & & 2 & 22549.057 & 1.185 & & & & \\
\hline $4 f\left({ }^{2} \mathrm{~F}^{\circ}\right) 5 d^{2}\left({ }^{3} \mathrm{~F}\right)\left({ }^{4} \mathrm{I}^{\circ}\right) 6 p$ & & 6 & 22558.596 & 1.180 & 17 & ${ }^{5} \mathrm{I}$ & 11 & $4 f 5 d\left({ }^{3} \mathrm{H}^{\circ}\right) 6 s 6 p\left({ }^{3} \mathrm{P}^{\circ}\right)^{5} \mathrm{G}$ \\
\hline \multirow[t]{3}{*}{$4 f\left({ }^{2} \mathrm{~F}^{\circ}\right) 5 d^{2}\left({ }^{3} \mathrm{~F}\right)\left({ }^{2} \mathrm{~S}^{\circ}\right) 6 p ?$} & & 1 & 22567.448 & 1.476 & 14 & ${ }^{3} \mathrm{P}$ & 8 & $\left({ }^{2} \mathrm{~F}^{\circ}\right)\left({ }^{1} \mathrm{D}\right)\left({ }^{2} \mathrm{P}^{\circ}\right){ }^{3} \mathrm{~S}$ \\
\hline & & 3 & 22600.482 & 1.250 & & & & \\
\hline & & 2 & 22660.085 & 0.955 & & & & \\
\hline \multirow[t]{2}{*}{$4 f\left({ }^{2} \mathrm{~F}^{\circ}\right) 5 d^{2}\left({ }^{3} \mathrm{~F}\right)\left({ }^{2} \mathrm{P}^{\circ}\right) 6 p$} & & 0 & 22678.015 & & 14 & ${ }^{1} \mathrm{~S}$ & 13 & $\left({ }^{2} \mathrm{~F}^{\circ}\right)\left({ }^{1} \mathrm{D}\right)\left({ }^{2} \mathrm{P}^{\circ}\right){ }^{1} \mathrm{~S}$ \\
\hline & & 3 & 22713.073 & 1.155 & & & & \\
\hline \multirow[t]{6}{*}{$4 f\left({ }^{2} \mathrm{~F}^{\circ}\right) 5 d^{2}\left({ }^{3} \mathrm{~F}\right)\left({ }^{4} \mathrm{I}^{\circ}\right) 6 p$} & & 7 & 22739.355 & 1.135 & 15 & ${ }^{3} \mathrm{I}$ & 13 & $4 f^{2}\left({ }^{1} \mathrm{G}\right) 5 d\left({ }^{2} \mathrm{I}\right) 6 s^{3} \mathrm{I}$ \\
\hline & & 5 & 22740.645 & 1.093 & & & & \\
\hline & & 4 & 22741.485 & 1.021 & & & & \\
\hline & & 4 & 22781.169 & 1.156 & & & & \\
\hline & & 2 & 22798.685 & 1.266 & & & & \\
\hline & & 3 & 22844.779 & 1.036 & & & & \\
\hline \multirow[t]{3}{*}{$4 f\left({ }^{2} \mathrm{~F}^{\circ}\right) 5 d^{2}\left({ }^{3} \mathrm{~F}\right)\left({ }^{4} \mathrm{I}^{\circ}\right) 6 p$} & & 6 & 22851.015 & 1.141 & 28 & ${ }^{3} \mathrm{H}$ & 14 & $4 f 5 d\left({ }^{3} \mathrm{H}^{\circ}\right) 6 s 6 p\left({ }^{1} \mathrm{P}^{\circ}\right){ }^{3} \mathrm{H}$ \\
\hline & & 5 & 22882.755 & 1.147 & & & & \\
\hline & & 4 & 22908.586 & 1.176 & & & & \\
\hline \multirow[t]{8}{*}{$4 f\left({ }^{2} \mathrm{~F}^{\circ}\right) 5 d^{2}\left({ }^{3} \mathrm{~F}\right)\left({ }^{4} \mathrm{D}^{\circ}\right) 6 p$} & & 1 & 22933.990 & 0.488 & 17 & ${ }^{5} \mathrm{~F}$ & 7 & $\left({ }^{2} \mathrm{~F}^{\circ}\right)\left({ }^{3} \mathrm{P}\right)\left({ }^{4} \mathrm{D}^{\circ}\right){ }^{5} \mathrm{~F}$ \\
\hline & & 4 & 22949.615 & 1.119 & & & & \\
\hline & & 2 & 22970.284 & 0.890 & & & & \\
\hline & & 3 & 22972.499 & 1.052 & & & & \\
\hline & & 2 & 23049.867 & 0.953 & & & & \\
\hline & & 5 & 23083.233 & 1.101 & & & & \\
\hline & & 5 & 23117.060 & 1.087 & & & & \\
\hline & & 3 & 23139.791 & 0.951 & & & & \\
\hline
\end{tabular}


Ce I, Even Parity-Continued

\begin{tabular}{|c|c|c|c|c|c|c|c|c|}
\hline Configuration & Term & $J$ & $\begin{array}{l}\text { Level } \\
\left(\mathrm{cm}^{-1}\right)\end{array}$ & $g$ & \multicolumn{4}{|c|}{ Leading percentages } \\
\hline & & 3 & 23154.693 & 0.696 & & & & \\
\hline $4 f\left({ }^{2} \mathrm{~F}^{\circ}\right) 5 d^{2}\left({ }^{3} \mathrm{~F}\right)\left({ }^{4} \mathrm{I}^{\circ}\right) 6 p$ & & 7 & 23184.096 & 1.151 & 27 & $5 \mathrm{I}$ & 17. & $\left({ }^{2} \mathrm{~F}^{\circ}\right)\left({ }^{3} \mathrm{~F}\right)\left({ }^{2} \mathrm{I}^{\circ}\right){ }^{3} \mathrm{I}$ \\
\hline \multirow[t]{5}{*}{$4 f\left({ }^{2} \mathrm{~F}^{\circ}\right) 5 d^{2}\left({ }^{3} \mathrm{~F}\right)\left({ }^{4} \mathrm{H}^{\circ}\right) 6 p$} & & 6 & 23190.384 & 1.169 & 38 & ${ }^{5} \mathrm{H}$ & 8 & $4 f 5 d\left({ }^{1} \mathrm{H}^{\circ}\right) 6 s 6 p\left({ }^{3} \mathrm{P}^{\circ}\right){ }^{3} \mathrm{H}$ \\
\hline & & 3 & 23240.982 & 0.865 & & & & \\
\hline & & 2 & 23244.973 & 0.993 & & & & \\
\hline & & 4 & 23251.722 & 1.053 & & & & \\
\hline & & 5 & 23292.136 & 0.975 & & & & \\
\hline \multirow[t]{2}{*}{$4 f 5 d\left({ }^{1} \mathrm{~F}^{\circ}\right) 6 s 6 p\left({ }^{3} \mathrm{P}^{\circ}\right)$} & & 1 & 23299.807 & 1.527 & 6 & ${ }^{3} \mathrm{D}$ & 6 & $4 f\left({ }^{2} \mathrm{~F}^{\circ}\right) 5 d^{2}\left({ }^{3} \mathrm{~F}\right)\left({ }^{4} \mathrm{D}^{\circ}\right) 6 p^{5} \mathrm{~F}$ \\
\hline & & 3 & 23301.906 & 1.272 & & & & \\
\hline \multirow[t]{2}{*}{$4 f\left({ }^{2} \mathrm{~F}^{\circ}\right) 5 d^{2}\left({ }^{3} \mathrm{~F}\right)\left({ }^{2} \mathrm{I}^{\circ}\right) 6 p$} & & 6 & 23370.168 & 1.034 & 13 & ${ }^{1} \mathbf{I}$ & 11 & $\left({ }^{2} \mathrm{~F}^{\circ}\right)\left({ }^{1} \mathrm{G}\right)\left({ }^{2} \mathrm{I}^{\circ}\right){ }^{1} \mathrm{I}$ \\
\hline & & 4 & 23391.896 & 1.067 & & & & \\
\hline \multirow[t]{4}{*}{$4 f\left({ }^{2} \mathrm{~F}^{\circ}\right) 5 d^{2}\left({ }^{3} \mathrm{~F}\right)\left({ }^{4} \mathrm{D}^{\circ}\right) 6 p$} & & 0 & 23392.754 & & 12 & ${ }^{5} \mathrm{D}$ & 11 & $4 f 5 d\left({ }^{3} \mathrm{P}^{\circ}\right) 6 s 6 p\left({ }^{3} \mathrm{P}^{\circ}\right){ }^{3} \mathrm{P}$ \\
\hline & & 5 & 23430.776 & 1.293 & & & & \\
\hline & & 4 & 23513.352 & 1.166 & & & & \\
\hline & & 3 & 23535.757 & 1.011 & & & & \\
\hline \multirow[t]{18}{*}{$4 f 5 d\left({ }^{3} \mathrm{D}^{\circ}\right) 6 s 6 p\left({ }^{3} \mathrm{P}^{\circ}\right)$} & & 1 & 23554.940 & 0.519 & 9 & ${ }^{3} \mathrm{D}$ & 7 & $4 f\left({ }^{2} \mathrm{~F}^{\circ}\right) 5 d^{2}\left({ }^{1} \mathrm{D}\right)\left({ }^{2} \mathrm{P}^{\circ}\right) 6 p^{3} \mathrm{D}$ \\
\hline & & 4 & 23596.416 & 1.043 & & & & \\
\hline & & 2 & 23620.200 & 1.047 & & & & \\
\hline & & 6 & 23627.278 & 1.012 & & & & \\
\hline & & 1 & 23673.890 & 1.604 & & & & \\
\hline & & 3 & 23701.801 & 1.008 & & & & \\
\hline & & 6 & 23709.980 & 0.943 & & & & \\
\hline & & 4 & 23722.196 & 1.051 & & & & \\
\hline & & 2 & 23725.770 & 1.088 & & & & \\
\hline & & 3 & 23727.513 & 1.193 & & & & \\
\hline & & 5 & 23749.896 & 1.148 & & & & \\
\hline & & 4 & 23811.369 & 1.113 & & & & \\
\hline & & 2 & 23860.329 & 0.962 & & & & \\
\hline & & 2 & 23869.048 & 1.236 & & & & \\
\hline & & 5 & 23885.162 & 1.16 & & & & \\
\hline & & 1 & 23895.211 & 0.813 & & & & \\
\hline & & 3 & 23909.764 & 1.186 & & & & \\
\hline & & 6 & 23944.962 & 0.889 & & & & \\
\hline \multirow[t]{7}{*}{$4 f\left({ }^{2} \mathrm{~F}^{\circ}\right) 5 d^{2}\left({ }^{3} \mathrm{~F}\right) \quad\left({ }^{4} \mathrm{~F}^{\circ}\right) 6 p$} & & 0 & 23951.456 & & 26 & ${ }^{5} \mathrm{D}$ & 12 & $4 f 5 d\left({ }^{3} \mathrm{D}^{\circ}\right) 6 s 6 p\left({ }^{1} \mathrm{P}^{\circ}\right){ }^{3} \mathrm{P}$ \\
\hline & & 5 & 23962.481 & 1.196 & & & & \\
\hline & & 4 & 23978.411 & 1.154 & & & & \\
\hline & & 5 & 24009.430 & 1.074 & & & & \\
\hline & & 2 & 24024.353 & 1.393 & & & & \\
\hline & & 3 & 24041.686 & 1.133 & & & & \\
\hline & & 2 & 24071.835 & 1.163 & & & & \\
\hline \multirow[t]{3}{*}{$4 f\left({ }^{2} \mathrm{~F}^{\circ}\right) 5 d^{2}\left({ }^{3} \mathrm{~F}\right)\left({ }^{4} \mathrm{D}^{\circ}\right) 6 p$} & & 1 & 24087.022 & 2.042 & 18 & ${ }^{5} \mathrm{P}$ & 11 & $4 f^{2}\left({ }^{3} \mathrm{P}\right) 5 d\left({ }^{4} \mathrm{P}\right) 6 s{ }^{5} \mathrm{P}$ \\
\hline & & 3 & 24131.943 & 1.155 & & & & \\
\hline & & 3 & 24135.490 & 1.195 & & & & \\
\hline $4 f\left({ }^{2} \mathrm{~F}^{\circ}\right) 5 d^{2}\left({ }^{3} \mathrm{~F}\right)\left({ }^{4} \mathrm{H}^{\circ}\right) 6 p$ & ${ }^{5} \mathrm{H}$ & 7 & 24148.194 & 1.216 & 51 & & 10 & $4 f^{2}\left({ }^{1} \mathrm{G}\right) 5 d\left({ }^{2} \mathrm{I}\right) 6 s^{3} \mathrm{I}$ \\
\hline
\end{tabular}


Ce I, Even Parity-Continued

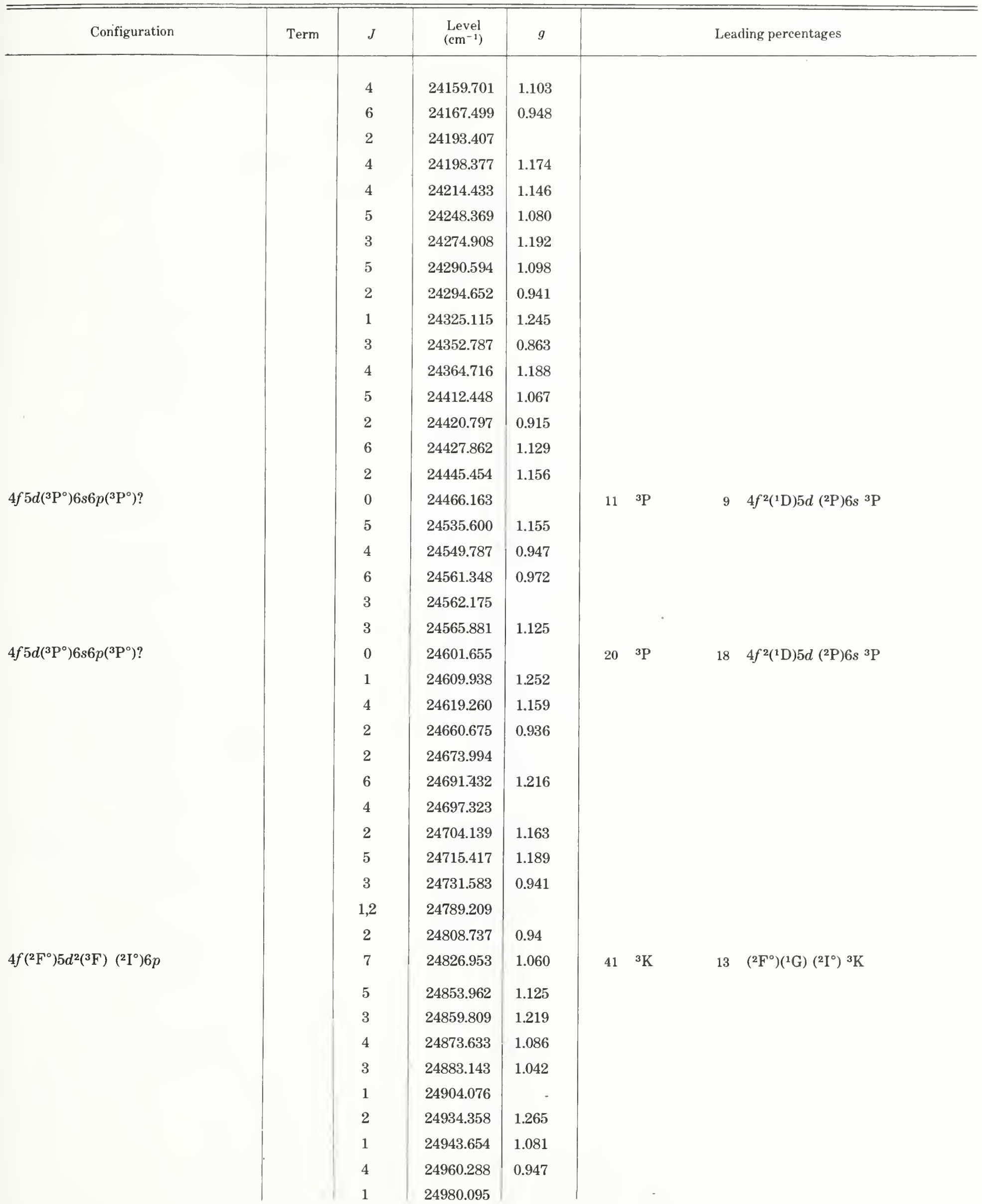


Ce I, Even Parity_Continued

\begin{tabular}{|c|c|c|c|c|c|c|}
\hline Configuration & Term & $J$ & $\begin{array}{l}\text { Level } \\
\left(\mathrm{cm}^{-1}\right)\end{array}$ & $g$ & & Leading percentages \\
\hline \multirow{12}{*}{$4 f\left({ }^{2} \mathrm{~F}^{\circ}\right) 5 d^{2}\left({ }^{3} \mathrm{~F}\right)\left({ }^{4} \mathrm{I}^{\circ}\right) 6 p ?$} & & 3 & 25044.233 & 0.997 & & \\
\hline & & 6 & 25058.207 & 1.150 & & \\
\hline & & 2 & 25059.621 & 1.113 & & \\
\hline & & 4 & 25077.395 & 1.187 & & \\
\hline & & 5 & 25093.105 & 1.146 & & \\
\hline & & 3 & 25134.962 & 1.135 & & \\
\hline & & 1 & 25174.542 & 1.906 & & \\
\hline & & 1 & 25185.870 & 0.704 & & \\
\hline & & 2 & 25193.242 & 1.578 & & \\
\hline & & 4 & 25208.286 & 1.332 & & \\
\hline & & 7 & 25260.320 & 1.129 & $25 \quad{ }^{3} \mathrm{~K}$ & $12\left({ }^{2} \mathrm{~F}^{\circ}\right)\left({ }^{3} \mathrm{~F}\right)\left({ }^{2} \mathrm{H}^{\circ}\right){ }^{3} \mathrm{I}$ \\
\hline & & 5 & 25266.806 & 1.112 & & \\
\hline \multirow[t]{7}{*}{$4 f\left({ }^{2} \mathrm{~F}^{\circ}\right) 5 d^{2}\left({ }^{3} \mathrm{~F}\right)\left({ }^{4} \mathrm{D}^{\circ}\right) 6 p$} & & 0 & 25287.107 & & $315 \mathrm{D}$ & $13 \quad\left({ }^{2} \mathrm{~F}^{\circ}\right)\left({ }^{3} \mathrm{~F}\right)\left({ }^{4} \mathrm{~F}^{\circ}\right)^{5} \mathrm{D}$ \\
\hline & & 3 & 25297.222 & 1.225 & & \\
\hline & & 4 & 25331.885 & 0.836 & & \\
\hline & & 6 & 25344.548 & 1.126 & & \\
\hline & & 2 & 25346.707 & 1.158 & & \\
\hline & & 4 & 25365.812 & 0.941 & & \\
\hline & & 1 & 25431.677 & 1.086 & & \\
\hline \multirow[t]{19}{*}{$4 f\left({ }^{2} \mathrm{~F}^{\circ}\right) 5 d^{2}\left({ }^{3} \mathrm{~F}\right)\left({ }^{4} \mathrm{H}^{\circ}\right) 6 p ?$} & & 7 & 25434.458 & 1.086 & $26{ }^{3} \mathrm{I}$ & $14 \quad\left({ }^{2} \mathrm{~F}^{\circ}\right)\left({ }^{3} \mathrm{~F}\right)\left({ }^{2} \mathrm{H}^{\circ}\right){ }^{3} \mathrm{I}$ \\
\hline & & 5 & 25438.378 & 1.135 & & \\
\hline & & 3 & 25441.048 & 1.399 & & \\
\hline & & 2 & 25454.305 & 1.453 & & \\
\hline & & 3 & 25492.072 & 0.956 & & \\
\hline & & 6 & 25544.009 & 1.278 & & \\
\hline & & 2 & 25567.838 & 0.909 & & \\
\hline & & 1 & 25577.169 & & & \\
\hline & & 2 & 25635.648 & 1.311 & & \\
\hline & & 3 & 25640.598 & 1.009 & & \\
\hline & & 4 & 25668.215 & 1.069 & & \\
\hline & & 5 & 25686.084 & 0.885 & & \\
\hline & & 6 & 25730.382 & 1.179 & & \\
\hline & & 3 & 25734.430 & 1.199 & & \\
\hline & & 1 & 25740.465 & 1.319 & & \\
\hline & & 4 & 25742.736 & 1.029 & & \\
\hline & & 5 & 25774.246 & 1.11 & & \\
\hline & & 6 & 25825.193 & 0.949 & & \\
\hline & & 3 & 25854.290 & 1.09 & & \\
\hline \multirow[t]{6}{*}{$4 f\left({ }^{2} \mathrm{~F}^{\circ}\right) 5 d^{2}\left({ }^{3} \mathrm{~F}\right)\left({ }^{2} I^{\circ}\right) 6 p$} & ${ }^{3} \mathrm{~K}$ & 8 & 25859.876 & 1.144 & 55 & $11 \quad\left({ }^{2} \mathrm{~F}^{\circ}\right)\left({ }^{1} \mathrm{G}\right)\left({ }^{2} \mathrm{I}^{\circ}\right)^{3} \mathrm{~K}$ \\
\hline & & 4 & 25863.180 & 1.042 & & \\
\hline & & 5,6 & 25866.350 & & & \\
\hline & & 5 & 25868.082 & 1.057 & & \\
\hline & & 2 & 25881.592 & & & \\
\hline & & 5 & 25928.289 & 0.931 & & \\
\hline
\end{tabular}


Ce I, Even Parity-Continued

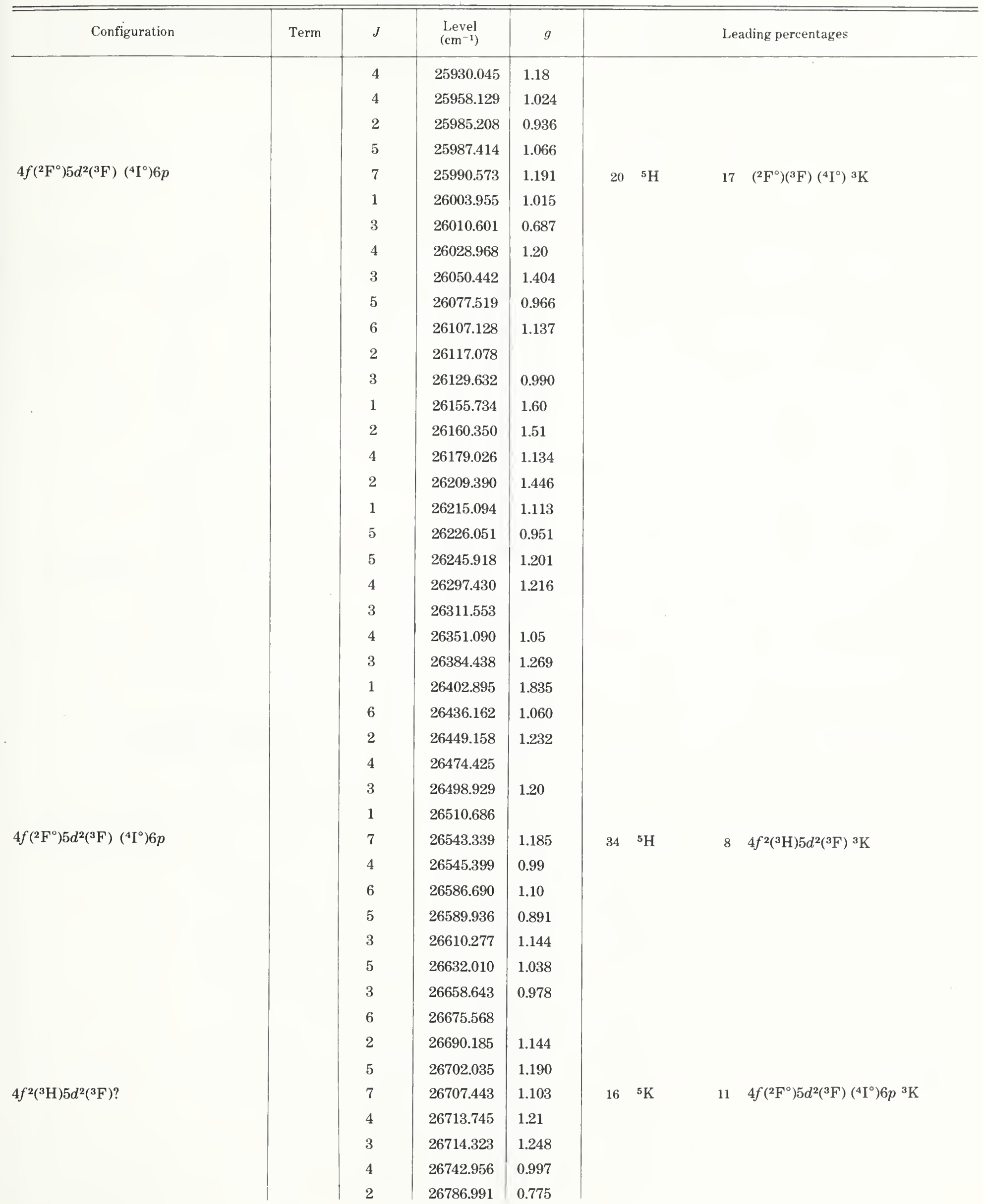


Ce I, Even Parity-Continued

\begin{tabular}{|c|c|c|c|c|c|c|}
\hline Configuration & Term & $J$ & $\begin{array}{l}\text { Level } \\
\left(\mathrm{cm}^{-1}\right)\end{array}$ & $g$ & & Leading percentages \\
\hline & & 6 & 26808.109 & 1.066 & & \\
\hline & & 4 & 26836.434 & 1.217 & & \\
\hline & & 3 & 26843.555 & & & \\
\hline & & 1 & 26853.642 & 1.605 & & \\
\hline & & 6 & 26883.075 & & & \\
\hline & & 1 & 26913.605 & & & \\
\hline & & 4 & 26929.306 & 1.075 & & \\
\hline & & 3 & 26934.381 & 1.270 & & \\
\hline & & 2 & 26943.447 & 1.026 & & \\
\hline & & 6 & 26972.051 & 1.186 & & \\
\hline & & 2 & 27013.438 & 0.94 & & \\
\hline & & 6 & 27038.349 & & & \\
\hline & & 6 & 27046.095 & & & \\
\hline & & 5 & 27059.164 & 0.989 & & \\
\hline & & 2 & 27076.477 & & & \\
\hline & & 5 & 27079.267 & 1.129 & & \\
\hline & & 4 & 27091.644 & 1.002 & & \\
\hline & & 4,3 & 27103.499 & & & \\
\hline & & 4 & 27114.140 & 1.25 & & \\
\hline & & 4 & 27159.064 & 0.83 & & \\
\hline & & 3 & 27172.959 & & & \\
\hline & & 1 & 27183.504 & 1.456 & & \\
\hline & & 3 & 27230.482 & & & \\
\hline & & 2 & 27254.752 & & & \\
\hline & & 4 & 27269.619 & & & \\
\hline & & 3 & 27269.831 & 0.855 & & \\
\hline \multirow[t]{13}{*}{$4 f\left({ }^{2} \mathrm{~F}^{\circ}\right) 5 d^{2}\left({ }^{3} \mathrm{~F}\right)\left({ }^{4} \mathrm{G}^{\circ}\right) 6 p$} & ${ }^{5} \mathrm{H}$ & 7 & 27271.854 & 1.239 & 54 & $8 \quad 4 f^{2}\left({ }^{1} \mathrm{I}\right) 5 d\left({ }^{2} \mathrm{I}\right) 6 \mathrm{~s}^{3} \mathrm{I}$ \\
\hline & & 6 & 27313.506 & 1.044 & & \\
\hline & & 5 & 27323.672 & 1.278 & & \\
\hline & & 2 & 27323.740 & & & \\
\hline & & 1 & 27334.124 & & & \\
\hline & & 2 & 27384.387 & 1.058 & & \\
\hline & & 4 & 27394.344 & 1.20 & & \\
\hline & & 5 & 27400.533 & 1.165 & & \\
\hline & & 4 & 27419.445 & & & \\
\hline & & 3 & 27438.661 & 1.143 & & \\
\hline & & 1 & 27474.071 & 0.537 & & \\
\hline & & 4 & 27483.038 & & & \\
\hline & & 2 & 27488.295 & & & \\
\hline \multirow[t]{6}{*}{$4 f^{2}\left({ }^{3} \mathrm{H}\right) 5 d^{2}\left({ }^{3} \mathrm{~F}\right)$} & ${ }^{5} \mathrm{I}$ & 7 & 27499.170 & & 44 & $22 \quad\left({ }^{3} F\right)\left({ }^{3} F\right)^{5} I$ \\
\hline & & 2 & 27520.602 & & & \\
\hline & & 4 & 27539.493 & & & \\
\hline & & 5 & 27541.881 & 1.065 & & \\
\hline & & 6 & 27551.129 & 1.138 & & \\
\hline & & 3 & 27575.557 & 1.131 & & \\
\hline
\end{tabular}


Ce I, Even Parity_Continued

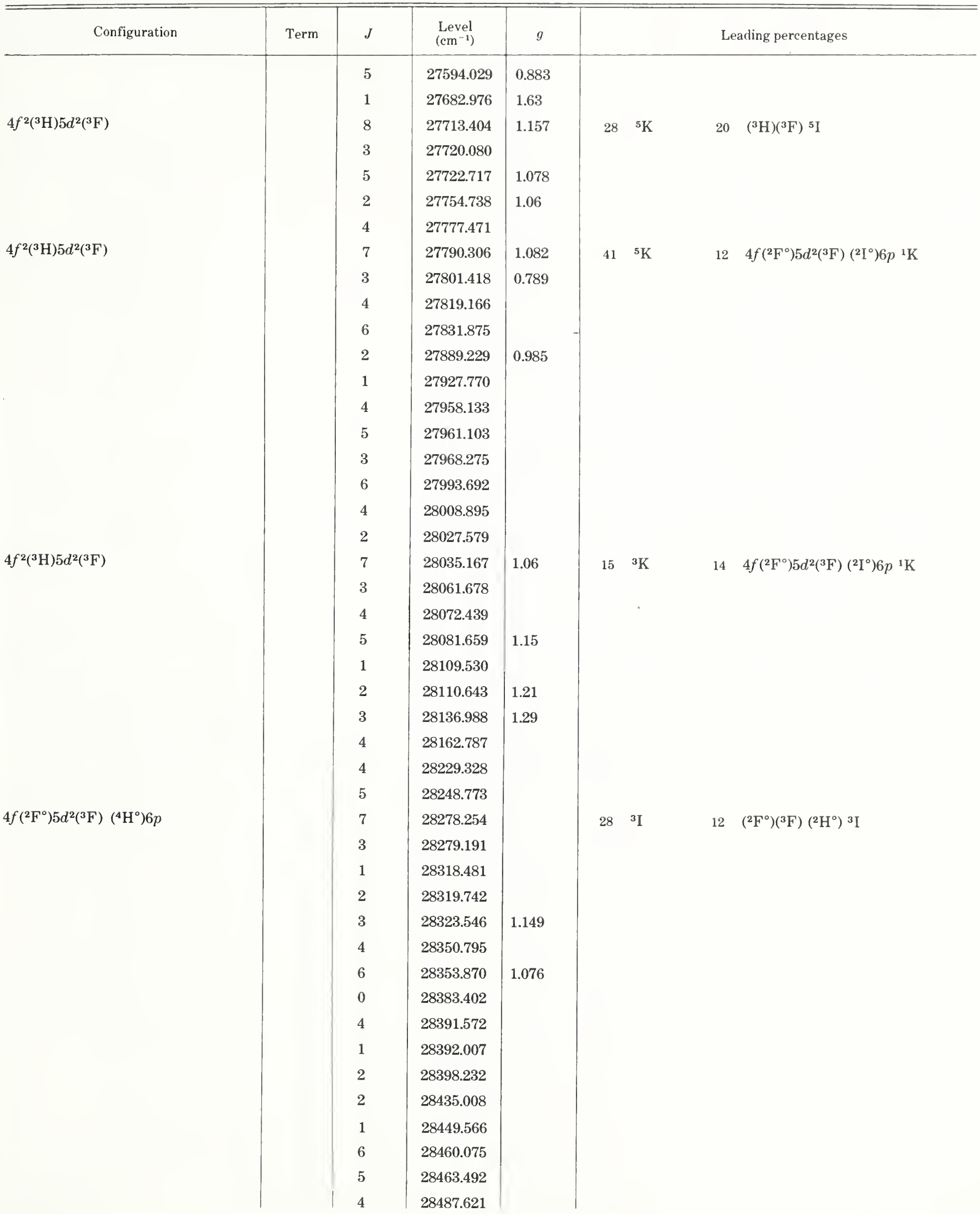


Ce I, Even Parity—Continued

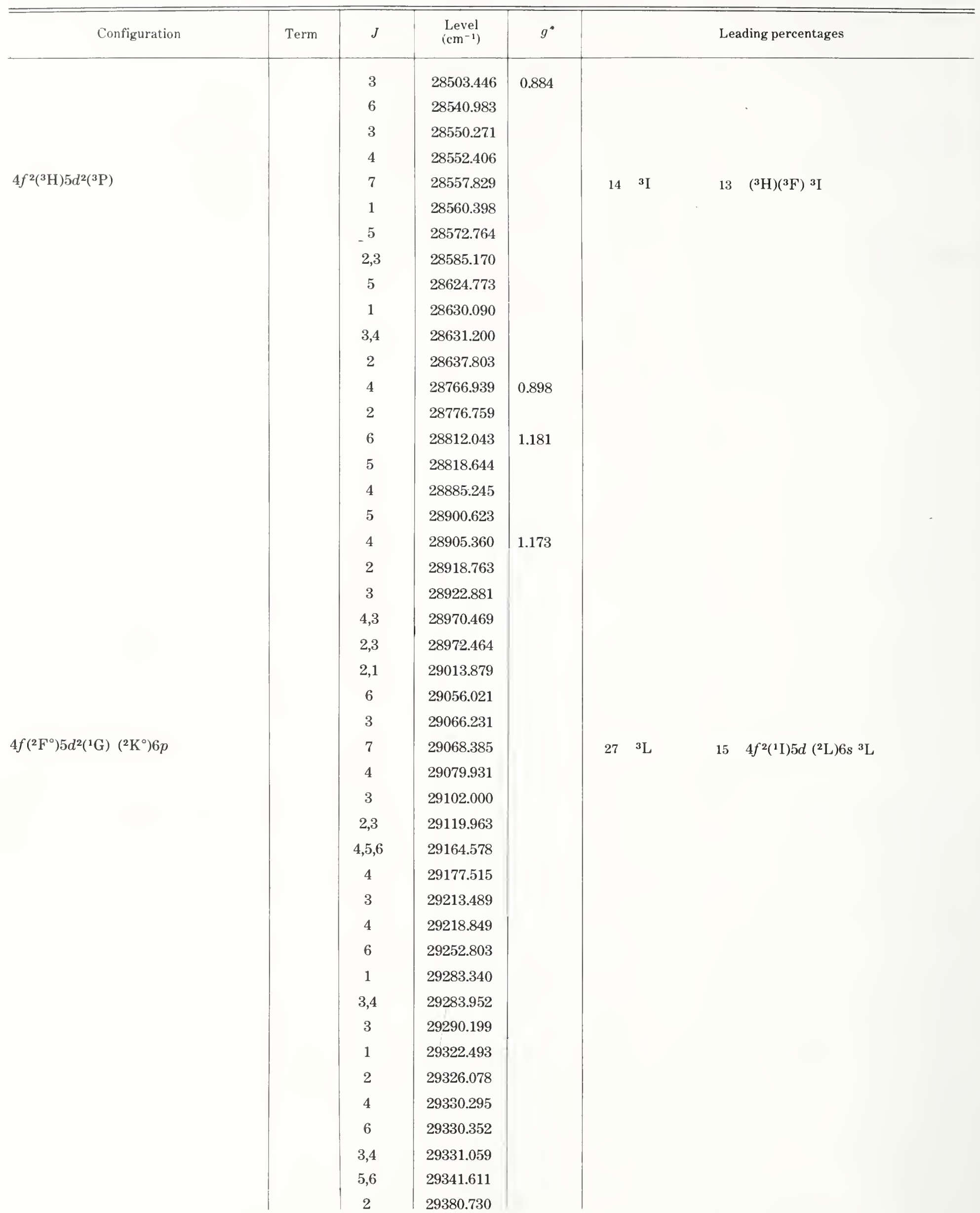


Ce I, Even Parity-Continued

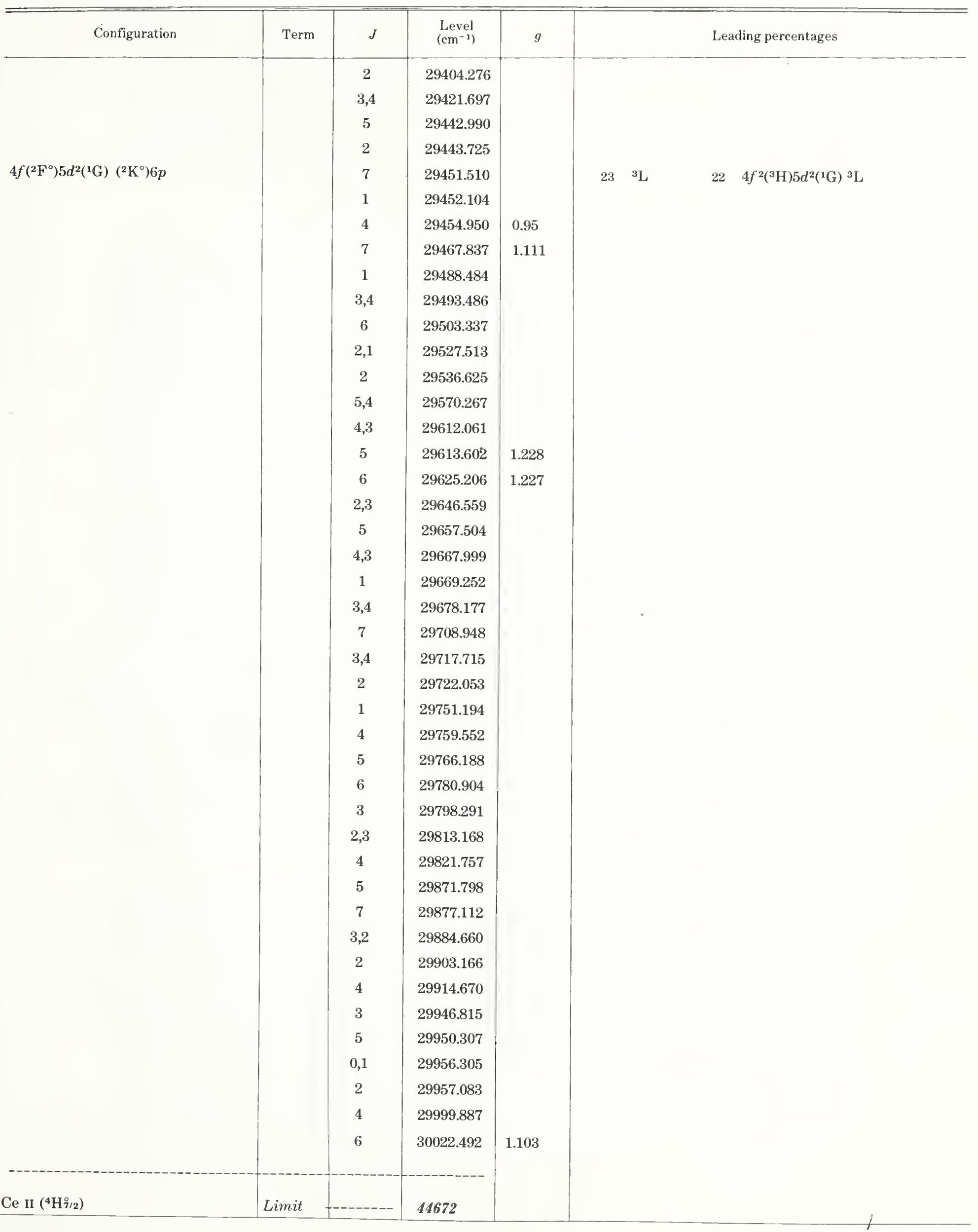




\section{Ce II}

(La I sequence; 57 electrons)

$Z=58$

Ground state $\left(1 s^{2} 2 s^{2} 2 p^{6} 3 s^{2} 3 p^{6} 3 d^{10} 4 s^{2} 4 p^{6} 4 d^{10} 5 s^{2} 5 p^{6}\right) 4 f 5 d^{2}{ }^{4} \mathrm{H}_{7 / 2}^{\circ}$

Ionization energy $87500 \pm 600 \mathrm{~cm}^{-1}$

$10.85 \pm 0.08 \mathrm{eV}$

Identified odd configurations

Identified even configurations
$4 f 5 d^{2}, 4 f 5 d 6 s, 4 f 6 s^{2}, 4 f^{2} 6 p, 4 f^{3}$

$4 f^{2} 6 s, 4 f^{2} 5 d, 4 f 5 d 6 p, 5 d^{3}, 4 f 6 s 6 p, 5 d^{2} 6 s, 5 d 6 s^{2} ?$

The preliminary analysis of this spectrum by Albertson and Harrison in 1937 gave a scheme of 31 lower and 51 upper energy levels classifying 584 lines. The basic methods of the analysis-searching a list of the most accurate obtainable wavelengths for repeating wavenumber differences by automatic techniques, and using Zeeman data to confirm, develop, and interpret the level scheme-were successfully applied to several other spectra during this period at MIT, and more recently the modern version of this method became the standard approach to analyses of the most complex and featureless atomic spectra. Such spectra are generally observed for atomic species having a large number of very low energy levels. Albertson and Harrison noted in this connection that for Ce II they had found "more than two dozen low energy levels within $6000 \mathrm{~cm}^{-1}$ of the lowest." (In fact Ce II has 46 levels below 6000 $\mathrm{cm}^{-1}$.) They suggested correctly that the low levels belonged to the $4 f 5 d 6 s$ and $4 f 5 d^{2}$ configurations.

This interpretation was confirmed by Harrison, Albertson, and Hosford [1941], who extended the original system of energy levels and also found a second, unconnected system of levels based on the lowest even configurations, $4 f^{2} 6 s$ and $4 f^{2} 5 d$. The 39 low levels of this second system were given configuration and term designations, as were the 40 high odd $\left(4 f^{2} 6 p\right.$ and $4 f^{3}$ ) levels. Term names were also assigned for many of the levels of the first system. Some 3600 of the approximately 7000 lines in their unpublished list $(2512-7000 \AA)$ were classified by a total of 316 levels. Zeeman measurements for 427 lines gave $g$ (and $J$ ) values for 280 of these levels. (This analysis and the data on which it was based superseded results reported in the three 1939 papers by, respectively, Gerasimov, Tsien, and van de Vliet; only the last of these was known to Harrison, Albertson, and Hosford.) Albertson worked intermittently on the analysis until 1949 and added designations for many of the levels.

G. Racah began calculations for Ce II at the Hebrew University in the 1950's, and in 1955 he found the connection between the two systems of levels. An extraordinarily complete theoretical interpretation of Ce II has resulted from Z. Goldschmidt's continuation of this work. Her 1968 thesis included all seven even configurations, with their interactions, in one calculation, and also gave her results for the four odd configurations $4 f^{2} 6 p$ and $\left(4 f 5 d^{2}+\right.$ $4 f 5 d 6 s+4 f 6 s^{2}$ ). Working with Albertson's unpublished line list, Goldschmidt also found some 50 real new levels and revised portions of the analysis.

Beginning in 1970, Corliss used new measurements of Ce II made at NBS to further extend the analysis [Corliss, 1973]. He assigned a number of levels to the $4 f^{3}$ configuration and also found many of the missing levels of configurations that had been calculated by Goldschmidt. In 1971-72, Corliss and Goldschmidt again extended and revised the analysis, making use of Goldschmidt's new calculations for the levels of both parities.

The levels are from Corliss's paper, which lists 192 odd and 288 even levels classifying about 7500 lines. He gives a total of about 11000 lines in the range $2512 \AA$ to $2.423 \mu \mathrm{m}$, with most of the wavelengths below $3300 \AA$ being taken from Albertson's list. The wavelengths longer than $1.1 \mu \mathrm{m}$ were taken from the 1972 list of Verges et al. Corliss notes that the strongest Ce II lines lie between 3800 and $4200 \AA$, and arise from the transition arrays $\left(4 f 5 d^{2}+\right.$ $4 f 5 d 6 s)-4 f 5 d 6 p$, and $4 f^{2} 6 s-4 f^{2} 6 p$.

The eigenvector percentages are from Goldschmidt's [1972] calculations. Every known level of a particular parity was assigned to an eigenvector from a calculation that included all 
known configurations of that parity. All configuration interactions and appropriate effective interactions were included [Goldschmidt, 1973].

The arrangement of the levels here results in a number of almost meaningless designations (in order to complete certain terms), but no name is assigned that is clearly less appropriate than an alternate name.

The ionization energy is from Sugar and Reader.

More than 150 lines of Ce II have been identified in the solar spectrum [Moore, Minnaert, and Houtgast, 1966], Ce being one of the most abundant rare-earth elements in the sun [Grevesse and Blanquet, 1969].

\section{References}

Albertson, W. E., and Harrison, G. R., Phys. Rev. 52, 1209 (1937). EL CL W

Albertson, W. E., unpublished material $(1949,1955)$. EL CL W ZE

Corliss, C. H., J. Res. Nat. Bur. Stand. (U.S.) 77A, 419 (1973). EL ND CL W PT

Gerasimov, F. M., Zh. Eksp. Teor. Fiz., 9, 1036 (1939). ZE

Goldschmidt, Z. B., Thesis, Hebrew Univ. Jerusalem, Israel, 487 pp. (1968). ND CL PT

Goldschmidt, Z. B., unpublished calculations (1972). ND PT

Goldschmidt, Z. B., Atomic Physies 3, S. J. Smith and G. K. Walters, Eds., pp. 221-246 (Plenum Press, New York, 1973). ND PT

Grevesse, N., and Blanquet, G., Sol. Phys. 8, 5 (1969).

Harrison, G. R., Albertson, W. E., and Hosford, N. F., J. Opt. Soc. Am. 31, 439 (1941). EL CL W ZE

Kiess, C. C., Hopkins, B. S., and Kremers, H. C., Sci. Papers Bur. Stand. 17, 317 (1921). W

King, A. S., Astrophys. J. 68, 194 (1928). W

Moore, C. E., Minnaert, M. G. J., and Houtgast, J., Nat. Bur. Stand. (U.S.), Monogr. 61, 349 pp. (1966).

Racah, G., Bull. Res. Council Israel 5A, 78 (1955). E L

Sugar, J., and Reader, J., J. Opt. Soc. Am. 5, 1286 (1965). IP

Tsien, Wei-Zang, Chinese J. Phys. 3,89 (1939). EL CL ZE

van de Vliet, H. J., Thesis, Zeeman Lab., Amsterdam, 85 pp. (1939). ZE

Verges, J., Corliss, C. H., and Martin, W. C., J. Res. Nat. Bur. Stand. (U.S.) 76A, 285 (1972). CL W

[July 1976]

\section{Ce II, Odd Parity}

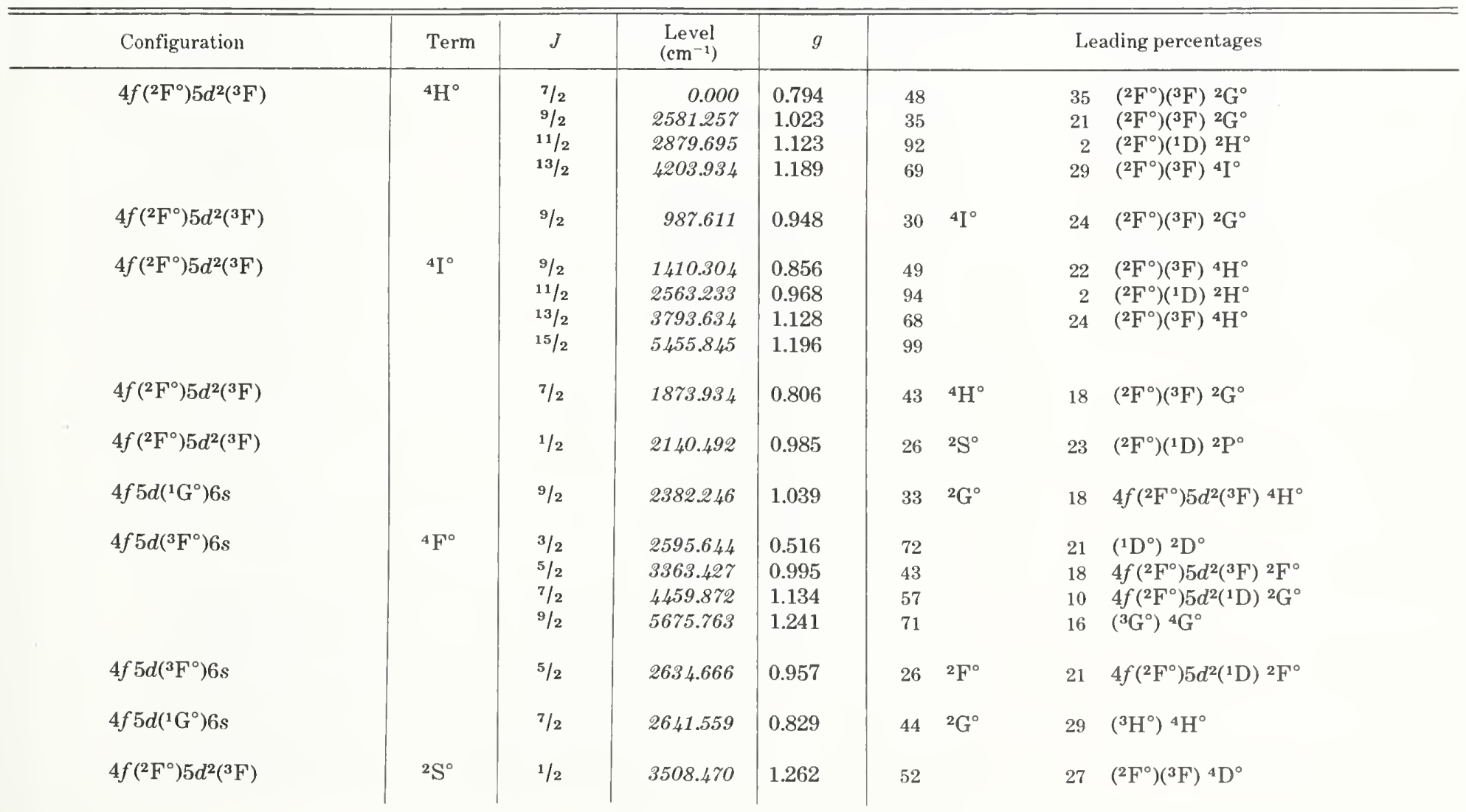


Ce II, Odd Parity—Continued

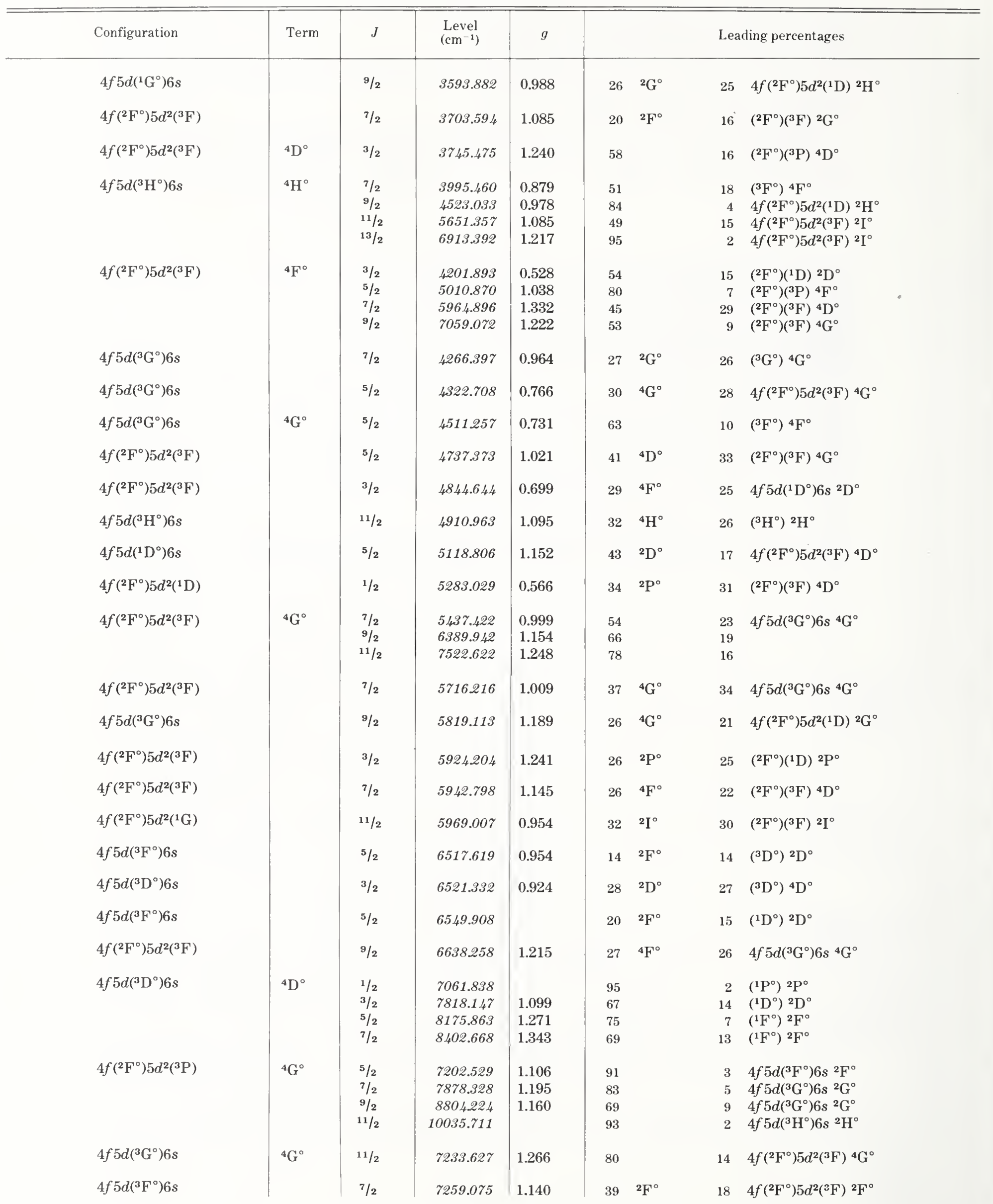


Ce II, Odd Parity_Continued

\begin{tabular}{|c|c|c|c|c|c|c|c|c|}
\hline Configuration & Term & $J$ & $\begin{array}{l}\text { Leve! } \\
\left(\mathrm{cm}^{-1}\right)\end{array}$ & $g$ & \multicolumn{4}{|c|}{ Leading percentages } \\
\hline $4 f\left({ }^{2} \mathrm{~F}^{\circ}\right) 5 d^{2}\left({ }^{3} \mathrm{~F}\right)$ & ${ }^{4} \mathrm{P}^{\circ}$ & $\begin{array}{l}3 / 2 \\
1 / 2 \\
5 / 2\end{array}$ & $\begin{array}{l}7278.922 \\
7522.458 \\
7746.185\end{array}$ & & $\begin{array}{l}85 \\
84 \\
77\end{array}$ & & $\begin{array}{r}6 \\
12 \\
7\end{array}$ & $\begin{array}{l}\left({ }^{2} \mathrm{~F}^{\circ}\right)\left({ }^{3} \mathrm{~F}\right){ }^{2} \mathrm{P}^{\circ} \\
\left({ }^{2} \mathrm{~F}^{\circ}\right)\left({ }^{3} \mathrm{~F}\right){ }^{2} \mathrm{~S}^{\circ} \\
4 f 5 d\left({ }^{3} \mathrm{D}^{\circ}\right) 6 s^{2} \mathrm{D}^{\circ}\end{array}$ \\
\hline $4 f\left({ }^{2} \mathrm{~F}^{\circ}\right) 5 d^{2}\left({ }^{3} \mathrm{~F}\right)$ & ${ }^{2} I^{\circ}$ & $13 / 2$ & 7293.938 & 1.100 & 48 & & 37 & $\left({ }^{2} \mathrm{~F}^{\circ}\right)\left({ }^{1} \mathrm{G}\right){ }^{2} \mathrm{I}^{\circ}$ \\
\hline $4 f\left({ }^{2} \mathrm{~F}^{\circ}\right) 5 d^{2}\left({ }^{1} \mathrm{G}\right)$ & & $9 / 2$ & 7713.089 & 0.950 & 28 & ${ }^{2} \mathrm{H}^{\circ}$ & 26 & $\left({ }^{2} \mathrm{~F}^{\circ}\right)\left({ }^{3} \mathrm{~F}\right){ }^{2} \mathrm{H}^{\circ}$ \\
\hline $4 f\left({ }^{2} \mathrm{~F}^{\circ}\right) 5 d^{2}\left({ }^{3} \mathrm{~F}\right)$ & ${ }^{4} \mathrm{~S}^{\circ}$ & $3 / 2$ & 8169.698 & & 66 & & 14 & $\left({ }^{2} \mathrm{~F}^{\circ}\right)\left({ }^{1} \mathrm{D}\right){ }^{2} \mathrm{P}^{\circ}$ \\
\hline $4 f\left({ }^{2} \mathrm{~F}^{\circ}\right) 5 d^{2}\left({ }^{3} \mathrm{P}\right)$ & ${ }^{2} \mathrm{D}^{\circ}$ & $\begin{array}{l}5 / 2 \\
3 / 2\end{array}$ & $\begin{array}{l}8280.946 \\
8702.444\end{array}$ & 1.189 & $\begin{array}{l}48 \\
50\end{array}$ & & $\begin{array}{r}7 \\
11\end{array}$ & $\begin{array}{l}\left({ }^{2} F^{\circ}\right)\left({ }^{3} F\right){ }^{4} D^{\circ} \\
\left({ }^{2} F^{\circ}\right)\left({ }^{3} F\right){ }^{2} D^{\circ}\end{array}$ \\
\hline $4 f 5 d\left({ }^{3} \mathrm{H}^{\circ}\right) 6 s$ & & $11 / 2$ & 8927.514 & 1.071 & 36 & ${ }^{2} \mathrm{H}^{\circ}$ & 31 & $4 f\left({ }^{2} \mathrm{~F}^{\circ}\right) 5 d^{2}\left({ }^{3} \mathrm{~F}\right){ }^{2} \mathrm{H}^{\circ}$ \\
\hline $4 f\left({ }^{2} \mathrm{~F}^{\circ}\right) 5 d^{2}\left({ }^{1} \mathrm{G}\right)$ & ${ }^{2} \mathrm{G}^{\circ}$ & $7 / 2$ & 9198.326 & & 64 & & 16 & $\left({ }^{2} \mathrm{~F}^{\circ}\right)\left({ }^{3} \mathrm{P}\right){ }^{2} \mathrm{G}^{\circ}$ \\
\hline $4 f\left({ }^{2} \mathrm{~F}^{\circ}\right) 5 d^{2}\left({ }^{3} \mathrm{P}\right)$ & & $1 / 2$ & 9269.826 & & 41 & ${ }^{4} \mathrm{D}^{\circ}$ & 35 & $\left({ }^{2} \mathrm{~F}^{\circ}\right)\left({ }^{3} \mathrm{~F}\right){ }^{2} \mathrm{P}^{\circ}$ \\
\hline $4 f 5 d\left({ }^{3} \mathrm{P}^{\circ}\right) 6 s$ & ${ }^{4} \mathrm{P}^{\circ}$ & $\begin{array}{l}1 / 2 \\
3 / 2 \\
5 / 2\end{array}$ & $\begin{array}{r}9491.493 \\
9634.186 \\
10641.442\end{array}$ & $\begin{array}{l}1.690 \\
1.526\end{array}$ & $\begin{array}{l}97 \\
87 \\
86\end{array}$ & & $\begin{array}{l}1 \\
4 \\
3\end{array}$ & $\begin{array}{l}\left({ }^{1} \mathrm{P}^{\circ}\right){ }^{2} \mathrm{P}^{\circ} \\
\left({ }^{1} \mathrm{P}^{\circ}\right)^{2} \mathrm{P}^{\circ} \\
4 f\left({ }^{2} \mathrm{~F}^{\circ}\right) 5 d^{2}\left({ }^{3} \mathrm{P}\right){ }^{4} \mathrm{D}^{\circ}\end{array}$ \\
\hline $4 f\left({ }^{2} \mathrm{~F}^{\circ}\right) 5 d^{2}\left({ }^{1} \mathrm{G}\right)$ & & $9 / 2$ & 9723.335 & 1.348 & 29 & ${ }^{2} \mathrm{G}^{\circ}$ & 18 & $\left({ }^{2} \mathrm{~F}^{\circ}\right)\left({ }^{3} \mathrm{P}\right){ }^{4} \mathrm{G}^{\circ}$ \\
\hline $4 f\left({ }^{2} \mathrm{~F}^{\circ}\right) 6 s^{2}$ & ${ }^{2} \mathbf{F}^{\circ}$ & $5 / 2$ & 9778.986 & & 73 & & 13 & $4 f\left({ }^{2} \mathrm{~F}^{\circ}\right) 5 d^{2}\left({ }^{1} \mathrm{~S}\right){ }^{2} \mathrm{~F}^{\circ}$ \\
\hline $4 f\left({ }^{2} \mathrm{~F}^{\circ}\right) 5 d^{2}\left({ }^{3} \mathrm{~F}\right)$ & & $3 / 2$ & 10088.640 & & 23 & ${ }^{2} \mathrm{P}^{\circ}$ & 17 & $\left({ }^{2} \mathrm{~F}^{\circ}\right)\left({ }^{3} \mathrm{P}\right){ }^{4} \mathrm{D}^{\circ}$ \\
\hline $4 f 5 d\left({ }^{1} \mathrm{~F}^{\circ}\right) 6 \mathrm{~s}$ & ${ }^{2} \mathrm{~F}^{\circ}$ & $\begin{array}{l}5 / 2 \\
7 / 2\end{array}$ & $\begin{array}{l}10114.883 \\
10274.971\end{array}$ & $\begin{array}{l}0.938 \\
1.195\end{array}$ & $\begin{array}{l}54 \\
52\end{array}$ & & $\begin{array}{r}8 \\
22\end{array}$ & $\begin{array}{l}4 f\left({ }^{2} \mathrm{~F}^{\circ}\right) 5 d^{2}\left({ }^{1} \mathrm{D}\right){ }^{2} \mathrm{~F}^{\circ} \\
\left({ }^{3} \mathrm{D}^{\circ}\right){ }^{4} \mathrm{D}^{\circ}\end{array}$ \\
\hline $4 f\left({ }^{2} \mathrm{~F}^{\circ}\right) 5 d^{2}\left({ }^{3} \mathrm{P}\right)$ & & $3 / 2$ & 10454.272 & & 31 & ${ }^{4} \mathrm{D}^{\circ}$ & 19 & $\left({ }^{2} \mathrm{~F}^{\circ}\right)\left({ }^{3} \mathrm{P}\right){ }^{4} \mathrm{~F}^{\circ}$ \\
\hline $4 f\left({ }^{2} \mathrm{~F}^{\circ}\right) 5 d^{2}\left({ }^{3} \mathrm{P}\right)$ & & $1 / 2$ & 10684.441 & & 36 & ${ }^{4} \mathrm{D}^{\circ}$ & 29 & $\left({ }^{2} \mathrm{~F}^{\circ}\right)\left({ }^{3} \mathrm{~F}\right){ }^{2} \mathrm{P}^{\circ}$ \\
\hline $4 f\left({ }^{2} \mathrm{~F}^{\circ}\right) 5 d^{2}\left({ }^{3} \mathrm{P}\right)$ & & $5 / 2$ & 10798.555 & 0.971 & 37 & ${ }^{4} \mathrm{D}^{\circ}$ & 27 & $\left({ }^{2} \mathrm{~F}^{\circ}\right)\left({ }^{3} \mathrm{P}\right){ }^{4} \mathrm{~F}^{\circ}$ \\
\hline $4 f\left({ }^{2} \mathrm{~F}^{\circ}\right) 5 d^{2}\left({ }^{1} \mathrm{G}\right)$ & ${ }^{2} \mathrm{H}^{\circ}$ & $\begin{array}{l}9 / 2 \\
11 / 2\end{array}$ & $\begin{array}{l}10924.876 \\
11742.245\end{array}$ & 1.081 & $\begin{array}{l}44 \\
51\end{array}$ & & $\begin{array}{l}22 \\
20\end{array}$ & $\begin{array}{l}\left({ }^{2} \mathrm{~F}^{\circ}\right)\left({ }^{1} \mathrm{G}\right){ }^{2} \mathrm{G}^{\circ} \\
4 f 5 d\left({ }^{1} \mathrm{H}^{\circ}\right) 6 s^{2} \mathrm{H}^{\circ}\end{array}$ \\
\hline $4 f\left({ }^{2} \mathrm{~F}^{\circ}\right) 5 d^{2}\left({ }^{3} \mathrm{P}\right)$ & ${ }^{4} \mathrm{~F}^{\circ}$ & $\begin{array}{l}3 / 2 \\
5 / 2 \\
7 / 2 \\
9 / 2\end{array}$ & $\begin{array}{l}11007.799 \\
11325.781 \\
11340.598 \\
12762.641\end{array}$ & $\begin{array}{l}1.123 \\
1.275\end{array}$ & $\begin{array}{l}58 \\
55 \\
38 \\
65\end{array}$ & & $\begin{array}{r}12 \\
19 \\
12 \\
9\end{array}$ & $\begin{array}{l}\left({ }^{2} \mathrm{~F}^{\circ}\right)\left({ }^{3} \mathrm{P}\right){ }^{4} \mathrm{D}^{\circ} \\
\left({ }^{2} \mathrm{~F}^{\circ}\right)\left({ }^{3} \mathrm{P}\right){ }^{4} \mathrm{D}^{\circ} \\
4 f 5 d\left({ }^{3} \mathrm{G}^{\circ}\right) 6 s^{2} \mathrm{G}^{\circ} \\
4 f 5 d\left({ }^{3} \mathrm{G}^{\circ}\right) 6 s^{2} \mathrm{G}^{\circ}\end{array}$ \\
\hline $4 f\left({ }^{2} \mathrm{~F}^{\circ}\right) 5 d^{2}\left({ }^{3} \mathrm{P}\right)$ & ${ }^{4} \mathrm{D}^{\circ}$ & $7 / 2$ & 11387.731 & & 60 & & 17 & $\left({ }^{2} \mathrm{~F}^{\circ}\right)\left({ }^{3} \mathrm{~F}\right){ }^{4} \mathrm{D}^{\circ}$ \\
\hline $4 f 5 d\left({ }^{3} \mathrm{G}^{\circ}\right) 6 s$ & & $7 / 2$ & 11949.189 & 1.029 & 22 & ${ }^{2} \mathrm{G}^{\circ}$ & 19 & $4 f\left({ }^{2} \mathrm{~F}^{\circ}\right) 5 d^{2}\left({ }^{1} \mathrm{D}\right){ }^{2} \mathrm{G}^{\circ}$ \\
\hline $4 f\left({ }^{2} \mathrm{~F}^{\circ}\right) 5 d^{2}\left({ }^{3} \mathrm{~F}\right)$ & ${ }^{2} \mathrm{D}^{\circ}$ & $\begin{array}{l}5 / 2 \\
3 / 2\end{array}$ & $\begin{array}{l}12057.107 \\
12466.430\end{array}$ & & $\begin{array}{l}47 \\
59\end{array}$ & & $\begin{array}{l}17 \\
18\end{array}$ & $\begin{array}{l}\left({ }^{2} \mathrm{~F}^{\circ}\right)\left({ }^{1} \mathrm{G}\right){ }^{2} \mathrm{~F}^{\circ} \\
\left({ }^{2} \mathrm{~F}^{\circ}\right)\left({ }^{3} \mathrm{P}\right){ }^{2} \mathrm{D}^{\circ}\end{array}$ \\
\hline $4 f\left({ }^{2} \mathrm{~F}^{\circ}\right) 6 s^{2}$ & & $7 / 2$ & 12260.088 & 1.168 & 42 & ${ }^{2} \mathrm{~F}^{\circ}$ & 35 & $4 f\left({ }^{2} \mathrm{~F}^{\circ}\right) 5 d^{2}\left({ }^{3} \mathrm{P}\right){ }^{4} \mathrm{~F}^{\circ}$ \\
\hline $4 f\left({ }^{2} \mathrm{~F}^{\circ}\right) 5 d^{2}\left({ }^{1} \mathrm{G}\right)$ & ${ }^{2} \mathrm{~K}^{\circ}$ & $\begin{array}{l}13 / 2 \\
15 / 2\end{array}$ & $\begin{array}{l}12326.417 \\
14404.40\end{array}$ & & $\begin{array}{l}97 \\
99\end{array}$ & & $\begin{array}{l}2 \\
1\end{array}$ & $\begin{array}{l}\left({ }^{2} \mathrm{~F}^{\circ}\right)\left({ }^{3} \mathrm{~F}\right){ }^{2} \mathrm{I}^{\circ} \\
4 f^{3}{ }^{2} \mathrm{~K}^{\circ}\end{array}$ \\
\hline $4 f\left({ }^{2} \mathrm{~F}^{\circ}\right) 5 d^{2}\left({ }^{3} \mathrm{~F}\right)$ & ${ }^{2} \mathrm{H}^{\circ}$ & $9 / 2$ & 12365.806 & 0.921 & 46 & & 23 & $4 f 5 d\left({ }^{1} \mathrm{H}^{\circ}\right) 6 s^{2} \mathrm{H}^{\circ}$ \\
\hline $4 f\left({ }^{2} \mathrm{~F}^{\circ}\right) 5 d^{2}\left({ }^{3} \mathrm{~F}\right)$ & & $11 / 2$ & 12751.782 & & 33 & ${ }^{2} \mathrm{H}^{0}$ & 23 & $4 f 5 d\left({ }^{1} \mathrm{H}^{\circ}\right) 6 s^{2} \mathrm{H}^{\circ}$ \\
\hline $4 f\left({ }^{2} \mathrm{~F}^{\circ}\right) 5 d^{2}\left({ }^{1} \mathrm{G}\right)$ & ${ }^{2} \mathrm{~F}^{\circ}$ & $\begin{array}{l}5 / 2 \\
7 / 2\end{array}$ & $\begin{array}{l}13012.095 \\
13515.853\end{array}$ & & $\begin{array}{l}44 \\
73\end{array}$ & & $\begin{array}{r}14 \\
9\end{array}$ & $\begin{array}{l}\left({ }^{2} \mathrm{~F}^{\circ}\right)\left({ }^{3} \mathrm{~F}\right){ }^{2} \mathrm{D}^{\circ} \\
\left({ }^{2} \mathrm{~F}^{\circ}\right)\left({ }^{3} \mathrm{~F}\right){ }^{2} \mathrm{~F}^{\circ}\end{array}$ \\
\hline $4 f\left({ }^{2} \mathrm{~F}^{\circ}\right) 5 d^{2}\left({ }^{3} \mathrm{P}\right)$ & & $9 / 2$ & 13659.329 & & 23 & ${ }^{4} \mathrm{~F}^{\circ}$ & 23 & $4 f 5 d\left({ }^{3} \mathrm{G}^{\circ}\right) 6 s^{2} \mathrm{G}^{\circ}$ \\
\hline
\end{tabular}


Ce II, Odd Parity_Continued

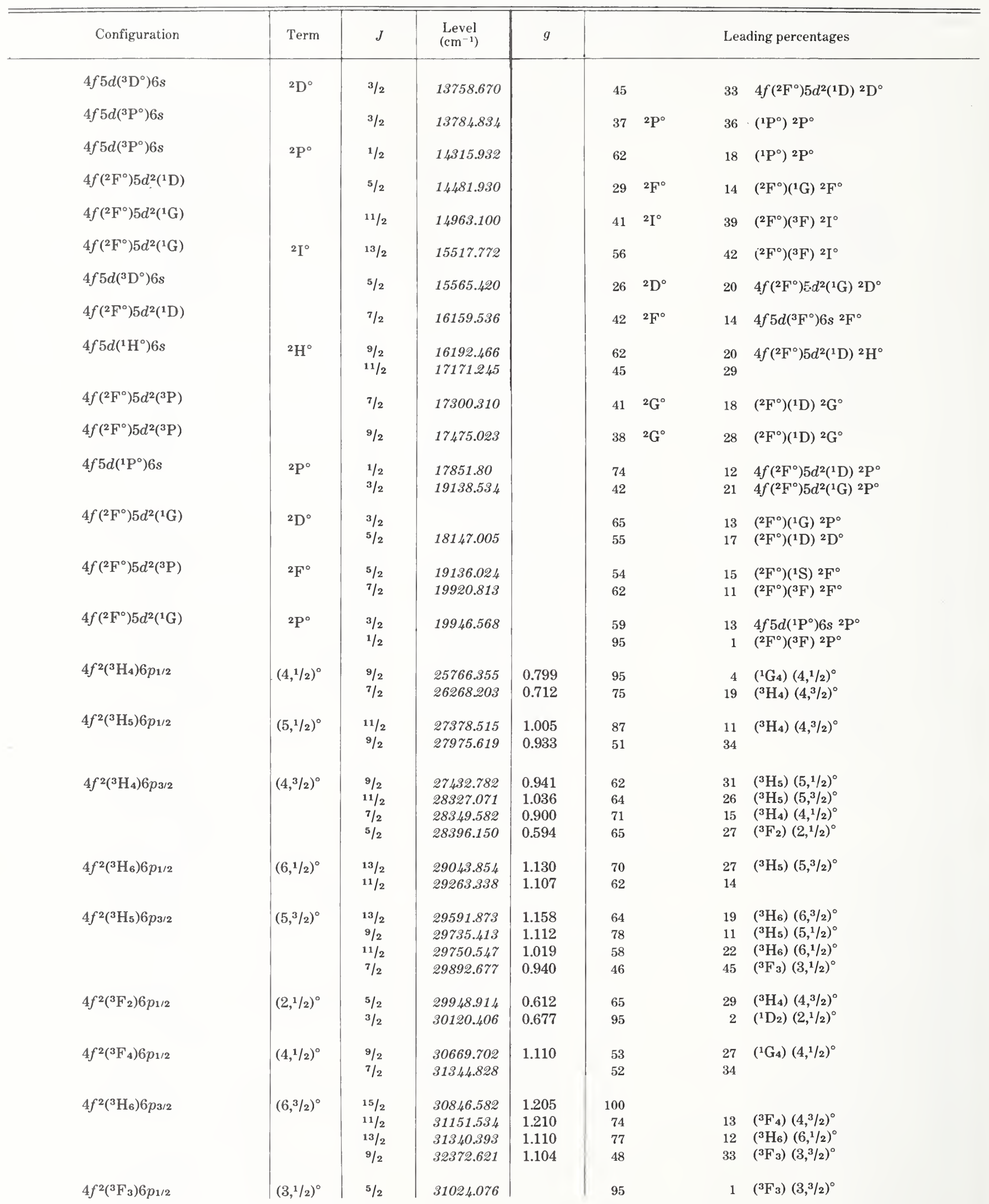


Ce II, Odd Parity_Continued

\begin{tabular}{|c|c|c|c|c|c|c|c|c|}
\hline Configuration & Term & $J$ & $\begin{array}{l}\text { Level } \\
\left(\mathrm{cm}^{-1}\right)\end{array}$ & $g$ & \multicolumn{4}{|c|}{ Leading percentages } \\
\hline $4 f^{2}\left({ }^{3} \mathrm{H}_{5}\right) 6 p_{3 / 2}$ & & $7 / 2$ & 31130.983 & 0.975 & 37 & $\left(5,{ }^{3} / 2\right)^{\circ}$ & 33 & $\left({ }^{3} \mathrm{~F}_{3}\right)\left(3,{ }^{1} / 2\right)^{\circ}$ \\
\hline $4 f^{2}\left({ }^{3} \mathrm{~F}_{2}\right) 6 p_{3 / 2}$ & $(2,3 / 2)^{\circ}$ & $\begin{array}{l}1 / 2 \\
3 / 2 \\
5 / 2 \\
7 / 2\end{array}$ & $\begin{array}{l}31613.833 \\
31747.243 \\
31966.748 \\
32235.239\end{array}$ & $\begin{array}{l}0.860 \\
1.015 \\
1.111\end{array}$ & $\begin{array}{l}95 \\
95 \\
87 \\
52\end{array}$ & & $\begin{array}{r}2 \\
2 \\
8 \\
15\end{array}$ & $\begin{array}{l}\left({ }^{1} \mathrm{D}_{2}\right)\left(2,{ }^{3} / 2\right)^{\circ} \\
\left({ }^{1} \mathrm{D}_{2}\right)(2,3 / 2)^{\circ} \\
\left({ }^{3} \mathrm{~F}_{3}\right)\left(3,{ }^{3} / 2\right)^{\circ} \\
\left({ }^{3} \mathrm{~F}_{3}\right)\left(3,{ }^{1} / 2\right)^{\circ}\end{array}$ \\
\hline $4 f^{2}\left({ }^{3} \mathrm{~F}_{3}\right) 6 p_{3 / 2}$ & $(3,3 / 2)^{\circ}$ & $\begin{array}{l}9 / 2 \\
3 / 2 \\
5 / 2 \\
7 / 2\end{array}$ & $\begin{array}{l}32616.019 \\
32826.668 \\
32885.367 \\
32989.218\end{array}$ & $\begin{array}{l}1.102 \\
1.10\end{array}$ & $\begin{array}{l}47 \\
95 \\
47 \\
46\end{array}$ & & $\begin{array}{r}28 \\
1 \\
28 \\
22\end{array}$ & $\begin{array}{l}\left({ }^{1} \mathrm{G}_{4}\right)\left(4,{ }^{1} / 2\right)^{\circ} \\
4 f 5 d\left({ }^{3} \mathrm{D}^{\circ}\right) 6 s{ }^{2} \mathrm{D}^{\circ} \\
\left({ }^{3} \mathrm{~F}_{4}\right)\left(4,{ }^{3} / 2\right)^{\circ} \\
\left({ }^{1} \mathrm{G}_{4}\right)(4,1 / 2)^{\circ}\end{array}$ \\
\hline $4 f^{2}\left({ }^{3} \mathrm{~F}_{4}\right) 6 p_{3 / 2}$ & $(4,3 / 2)^{\circ}$ & $11 / 2$ & 32864.278 & & 52 & & 29 & $\left({ }^{1} \mathrm{G}_{4}\right)(4,3 / 2)^{\circ}$ \\
\hline $4 f^{2}\left({ }^{3} \mathrm{~F}_{4}\right) 6 p_{3 / 2}$ & & $9 / 2$ & 33040.353 & 1.106 & 36 & $(4,3 / 2)^{\circ}$ & 34 & $\left({ }^{1} \mathrm{G}_{4}\right)\left(4,{ }^{3} / 2\right)^{\circ}$ \\
\hline $4 f^{2}\left({ }^{3} \mathrm{~F}_{4}\right) 6 p 3 / 2$ & & $7 / 2$ & 33111.163 & 1.086 & 29 & $(4,3 / 2)^{\circ}$ & 27 & $\left({ }^{3} \mathrm{~F}_{3}\right)(3,3 / 2)^{\circ}$ \\
\hline $4 f^{2}\left({ }^{1} \mathrm{G}_{4}\right) 6 p_{1 / 2}$ & & $7 / 2$ & 33535.636 & 0.966 & 31 & $\left(4,{ }^{1} / 2\right)^{\circ}$ & 23 & $\left({ }^{3} \mathrm{~F}_{4}\right)(4,1 / 2)^{\circ}$ \\
\hline $4 f^{2}\left({ }^{3} \mathrm{~F}_{3}\right) 6 p_{3 / 2}$ & & $5 / 2$ & 33594.152 & & 41 & $(3,3 / 2)^{\circ}$ & 34 & $\left({ }^{3} \mathrm{~F}_{4}\right)\left(4,{ }^{3} / 2\right)^{\circ}$ \\
\hline $4 f^{2}\left({ }^{3} \mathrm{~F}_{4}\right) 6 p_{1 / 2}$ & & $9 / 2$ & 33908.193 & 1.11 & 30 & $(4,1 / 2)^{\circ}$ & 21 & $\left({ }^{1} \mathrm{G}_{4}\right)\left(4,{ }^{2} / 2\right)^{\circ}$ \\
\hline $4 f^{2}\left({ }^{1} \mathrm{G}_{4}\right) 6 p_{3 / 2}$ & $(4,3 / 2)^{\circ}$ & $11 / 2$ & 34861.526 & & 63 & & 34 & $\left({ }^{3} \mathrm{~F}_{4}\right)(4,3 / 2)^{\circ}$ \\
\hline $4 f^{2}\left({ }^{3} \mathrm{~F}_{4}\right) 6 p_{3 / 2}$ & $(4,3 / 2)^{\circ}$ & $9 / 2$ & 35026.944 & 1.142 & 50 & & 41 & $\left({ }^{1} \mathrm{G}_{4}\right)\left(4,{ }^{3} / 2\right)^{\circ}$ \\
\hline $4 f^{2}\left({ }^{1} \mathrm{G}_{4}\right) 6 p_{3 / 2}$ & $\left(4,{ }^{3} / 2\right)^{\circ}$ & $5 / 2$ & 35298.190 & & 59 & & 32 & $\left({ }^{3} \mathrm{~F}_{4}\right)\left(4,{ }^{3} / 2\right)^{\circ}$ \\
\hline $4 f^{2}\left({ }^{3} \mathrm{~F}_{4}\right) 6 p_{3 / 2}$ & & $7 / 2$ & 35625.700 & 0.923 & 48 & $(4,3 / 2)^{\circ}$ & 46 & $\left({ }^{1} \mathrm{G}_{4}\right)\left(4,{ }^{3} / 2\right)^{\circ}$ \\
\hline $4 f^{3}$ & ${ }^{4} I^{\circ}$ & $\begin{array}{c}9 / 2 \\
11 / 2 \\
13 / 2 \\
15 / 2\end{array}$ & $\begin{array}{l}38194.728 \\
39079.298 \\
40039.524 \\
41058.614\end{array}$ & $\begin{array}{l}0.720 \\
0.964 \\
1.104 \\
1.197\end{array}$ & $\begin{array}{l}97 \\
99 \\
99 \\
99\end{array}$ & . & $\begin{array}{l}2 \\
1 \\
1\end{array}$ & $\begin{array}{l}{ }^{2} \mathrm{H}^{\circ} 2 \\
{ }^{2} \mathrm{H}^{\circ} 2 \\
{ }^{2} \mathrm{~K}^{\circ}\end{array}$ \\
\hline $4 f^{2}\left({ }^{1} \mathrm{D}_{2}\right) 6 p_{1 / 2}$ & $(2,1 / 2)^{\circ}$ & $\begin{array}{l}3 / 2 \\
5 / 2\end{array}$ & $\begin{array}{l}38661.520 \\
38835.734\end{array}$ & & $\begin{array}{l}82 \\
83\end{array}$ & & $\begin{array}{l}7 \\
7\end{array}$ & $\left({ }^{3} \mathrm{P}_{2}\right)(2,1 / 2)^{\circ}$ \\
\hline $4 f^{2}\left({ }^{1} \mathrm{D}_{2}\right) 6 p_{3 / 2}$ & $\left(2,{ }^{3} / 2\right)^{\circ}$ & $\begin{array}{l}1 / 2 \\
7 / 2 \\
3 / 2 \\
5 / 2\end{array}$ & $\begin{array}{l}40475.925 \\
40720.122 \\
41100.932\end{array}$ & & $\begin{array}{l}82 \\
88 \\
79 \\
89\end{array}$ & & $\begin{array}{l}9 \\
7 \\
7 \\
5\end{array}$ & $\left({ }^{3} \mathrm{P}_{2}\right)(2,3 / 2)^{\circ}$ \\
\hline $4 f^{2}\left({ }^{3} \mathrm{P}_{1}\right) 6 p_{1 / 2}$ & & $3 / 2$ & 42533.459 & & 42 & $(1,1 / 2)^{\circ}$ & 22 & $\left({ }^{3} \mathrm{P}_{2}\right)\left(2,{ }^{1} / 2\right)^{\circ}$ \\
\hline $4 f^{2}\left({ }^{1} \mathrm{I}_{6}\right) 6 p_{1 / 2}$ & $(6,1 / 2)^{\circ}$ & $\begin{array}{l}13 / 2 \\
11 / 2\end{array}$ & $\begin{array}{l}42712.810 \\
42729.447\end{array}$ & & $\begin{array}{l}79 \\
69\end{array}$ & & $\begin{array}{l}21 \\
30\end{array}$ & $\left({ }^{1} \mathrm{I}_{6}\right)\left(6,{ }^{3} / 2\right)^{\circ}$ \\
\hline $4 f^{2}\left({ }^{3} \mathrm{Po}_{0}\right) 6 p 3 / 2$ & $\left(0,,^{3} / 2\right)^{\circ}$ & $3 / 2$ & 44423.575 & & 69 & & 24 & $\left({ }^{3} \mathrm{P}_{2}\right)\left(2,{ }^{1} / 2\right)^{\circ}$ \\
\hline $4 f^{2}\left({ }^{1} \mathrm{I}_{6}\right) 6 p_{3 / 2}$ & $\left(6,,^{3} / 2\right)^{\circ}$ & $\begin{array}{l}13 / 2 \\
15 / 2 \\
11 / 2 \\
9 / 2\end{array}$ & $\begin{array}{l}44637.548 \\
45443.86 \\
45864.950 \\
46697.084\end{array}$ & & $\begin{array}{l}79 \\
99 \\
59 \\
89\end{array}$ & & $\begin{array}{r}20 \\
24 \\
4\end{array}$ & $\begin{array}{l}\left({ }^{1} I_{6}\right)(6,1 / 2)^{\circ} \\
\left({ }^{1} I_{6}\right)(6,1 / 2)^{\circ} \\
4 f^{3}{ }^{2} \mathrm{H}^{0} 2\end{array}$ \\
\hline $4 f^{3}$ & ${ }^{4} \mathrm{~F}^{\circ}$ & $\begin{array}{l}3 / 2 \\
5 / 2 \\
7 / 2 \\
9 / 2\end{array}$ & $\begin{array}{l}44651.303 \\
45130.529 \\
45598.301 \\
46162.692\end{array}$ & & $\begin{array}{l}62 \\
97 \\
92 \\
77\end{array}$ & & $\begin{array}{r}25 \\
2 \\
4 \\
9\end{array}$ & $\begin{array}{l}4 f^{2}\left({ }^{3} \mathrm{P}_{1}\right) 6 p_{3 / 2}\left(1,{ }^{3} / 2\right)^{\circ} \\
{ }^{2} \mathrm{D}^{\circ} 1 \\
{ }^{2} \mathrm{G}^{\circ} 1 \\
{ }^{2} \mathrm{H}^{\circ} 2\end{array}$ \\
\hline $4 f^{2}\left({ }^{3} \mathrm{P}_{2}\right) 6 p_{3 / 2}$ & $(2,3 / 2)^{\circ}$ & $\begin{array}{l}7 / 2 \\
5 / 2 \\
1 / 2 \\
3 / 2\end{array}$ & 44893.939 & & $\begin{array}{l}92 \\
91 \\
39 \\
70\end{array}$ & & $\begin{array}{r}7 \\
5 \\
40 \\
15\end{array}$ & $\begin{array}{l}\left({ }^{1} \mathrm{D}_{2}\right)(2,3 / 2)^{\circ} \\
\left({ }^{1} \mathrm{D}_{2}\right)(2,3 / 2)^{\circ} \\
\left({ }^{3} \mathrm{P}_{1}\right)(1,3 / 2)^{\circ} \\
\left({ }^{3} \mathrm{P}_{2}\right)\left(2,{ }^{3} / 2\right)^{\circ}\end{array}$ \\
\hline
\end{tabular}


Ce II, Odd Parity—Continued

\begin{tabular}{|c|c|c|c|c|c|c|c|c|}
\hline Configuration & Term & $J$ & $\begin{array}{l}\text { Level } \\
\left(\mathrm{cm}^{-1}\right)\end{array}$ & $g$ & \multicolumn{4}{|c|}{ Leading percentages } \\
\hline $4 f^{3}$ & ${ }^{2} \mathrm{H}^{\circ} 2$ & $\begin{array}{c}9 / 2 \\
11 / 2\end{array}$ & $\begin{array}{l}44949.011 \\
46588.131\end{array}$ & & $\begin{array}{l}61 \\
66\end{array}$ & & $\begin{array}{r}9 \\
12\end{array}$ & ${ }^{2} \mathrm{H}^{\circ} 1$ \\
\hline $4 f^{3}$ & & $7 / 2$ & 47430.184 & & 35 & ${ }^{2} \mathrm{G}^{\circ} 1$ & 31 & ${ }^{4} \mathrm{G}^{\circ}$ \\
\hline $4 f^{3}$ & ${ }^{4} \mathrm{G}^{\circ}$ & $\begin{array}{c}5 / 2 \\
7 / 2 \\
9 / 2 \\
11 / 2\end{array}$ & $\begin{array}{l}47459.894 \\
48330.79 \\
48549.99 \\
49617.70\end{array}$ & & $\begin{array}{l}98 \\
67 \\
65 \\
93\end{array}$ & & $\begin{array}{r}1 \\
18 \\
11 \\
4\end{array}$ & $\begin{array}{l}4 f\left({ }^{2} \mathrm{~F}^{\circ}\right) 5 d^{2}\left({ }^{3} \mathrm{P}\right){ }^{4} \mathrm{G}^{\circ} \\
{ }^{2} \mathrm{G}^{\circ} 1 \\
{ }^{2} \mathrm{G}^{\circ} 1 \\
{ }^{2} \mathrm{H}^{\circ} 1\end{array}$ \\
\hline $4 f^{3}$ & ${ }^{2} \mathrm{~K}^{\circ}$ & $\begin{array}{l}13 / 2 \\
15 / 2\end{array}$ & $\begin{array}{l}48045.10 \\
49087.88\end{array}$ & & $\begin{array}{l}98 \\
94\end{array}$ & & $\begin{array}{l}1 \\
4\end{array}$ & $\begin{array}{l}{ }^{2} \mathrm{I}^{\circ} \\
{ }^{2} \mathrm{~L}^{\circ}\end{array}$ \\
\hline $4 f^{3}$ & & $9 / 2$ & 49267.982 & & 33 & ${ }^{2} \mathrm{G}^{\circ} 1$ & 31 & ${ }^{4} \mathrm{G}^{\circ}$ \\
\hline Ce III $\left({ }^{3} \mathrm{H}_{4}\right)$ & Limit & 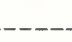 & 87500 & & & & & \\
\hline
\end{tabular}

Ce III, Even Parity

\begin{tabular}{|c|c|c|c|c|c|c|c|}
\hline Configuration & Term & $J$ & $\begin{array}{c}\text { Level } \\
\left(\mathrm{cm}^{-1}\right)\end{array}$ & $g$ & & \multicolumn{2}{|c|}{ Leading percentages } \\
\hline $4 f^{2}\left({ }^{3} \mathrm{H}\right) 6 s$ & ${ }^{4} \mathrm{H}$ & $\begin{array}{c}7 / 2 \\
9 / 2 \\
11 / 2 \\
13 / 2\end{array}$ & $\begin{array}{l}3854.012 \\
4165.550 \\
5513.709 \\
6967.547\end{array}$ & $\begin{array}{l}0.669 \\
0.949 \\
1.125 \\
1.225\end{array}$ & $\begin{array}{l}95 \\
55 \\
82 \\
98\end{array}$ & $\begin{array}{r}3 \\
40 \\
17 \\
1\end{array}$ & $\begin{array}{l}\left({ }^{1} \mathrm{G}\right){ }^{2} \mathrm{G} \\
\left({ }^{3} \mathrm{H}\right){ }^{2} \mathrm{H} \\
\left({ }^{3} \mathrm{H}\right){ }^{2} \mathrm{H} \\
4 f 5 d\left({ }^{3} \mathrm{G}^{\circ}\right) 6 p\end{array}$ \\
\hline $4 f^{2}\left({ }^{3} \mathrm{H}\right) 6 s$ & ${ }^{2} \mathrm{H}$ & $\begin{array}{c}9 / 2 \\
11 / 2\end{array}$ & $\begin{array}{l}5616.739 \\
7341.007\end{array}$ & $\begin{array}{l}0.934 \\
1.089\end{array}$ & $\begin{array}{l}56 \\
81\end{array}$ & $\begin{array}{l}42 \\
17\end{array}$ & $\left({ }^{3} \mathrm{H}\right){ }^{4} \mathrm{H}$ \\
\hline $4 f^{2}\left({ }^{3} \mathrm{H}\right) 5 d$ & ${ }^{2} \mathrm{H}$ & $\begin{array}{c}9 / 2 \\
11 / 2\end{array}$ & $\begin{array}{l}7011.804 \\
8278.054\end{array}$ & $\begin{array}{l}0.889 \\
0.957\end{array}$ & $\begin{array}{l}65 \\
56\end{array}$ & $\begin{array}{l}19 \\
14\end{array}$ & $\begin{array}{l}\left({ }^{3} \mathrm{H}\right){ }^{4} \mathrm{I} \\
\left({ }^{3} \mathrm{~F}\right){ }^{2} \mathrm{H}\end{array}$ \\
\hline $4 f^{2}\left({ }^{3} \mathrm{H}\right) 5 d$ & ${ }^{4} \mathrm{~K}$ & $\begin{array}{l}11 / 2 \\
13 / 2 \\
15 / 2 \\
17 / 2\end{array}$ & $\begin{array}{r}7092.265 \\
8423.672 \\
9771.956 \\
11165.796\end{array}$ & $\begin{array}{l}0.803 \\
0.980 \\
1.090 \\
1.187\end{array}$ & $\begin{array}{r}83 \\
96 \\
100 \\
100\end{array}$ & $\begin{array}{l}7 \\
2\end{array}$ & $\begin{array}{l}\left({ }^{3} \mathrm{H}\right){ }^{2} \mathrm{I} \\
\left({ }^{3} \mathrm{H}\right){ }^{2} \mathrm{I}\end{array}$ \\
\hline $4 f^{2}\left({ }^{3} \mathrm{~F}\right) 6 s$ & ${ }^{4} \mathrm{~F}$ & $\begin{array}{l}3 / 2 \\
5 / 2 \\
7 / 2 \\
9 / 2\end{array}$ & $\begin{array}{l}7454.951 \\
7722.285 \\
8531.678 \\
8774.064\end{array}$ & $\begin{array}{l}0.407 \\
0.986 \\
1.213 \\
1.247\end{array}$ & $\begin{array}{l}95 \\
68 \\
89 \\
61\end{array}$ & $\begin{array}{r}2 \\
27 \\
4 \\
34\end{array}$ & $\begin{array}{l}\left({ }^{1} \mathrm{D}\right){ }^{2} \mathrm{D} \\
\left({ }^{3} \mathrm{~F}\right){ }^{2} \mathrm{~F} \\
\left({ }^{3} \mathrm{~F}\right){ }^{2} \mathrm{~F} \\
\left({ }^{1} \mathrm{G}\right){ }^{2} \mathrm{G}\end{array}$ \\
\hline $4 f^{2}\left({ }^{3} \mathrm{H}\right) 5 d$ & ${ }^{4} \mathrm{I}$ & $\begin{array}{l}9 / 2 \\
11 / 2 \\
13 / 2 \\
15 / 2\end{array}$ & $\begin{array}{r}8131.217 \\
8896.729 \\
10058.226 \\
11309.972\end{array}$ & $\begin{array}{l}0.751 \\
0.961 \\
1.105 \\
1.193\end{array}$ & $\begin{array}{l}78 \\
92 \\
97 \\
98\end{array}$ & $\begin{array}{r}11 \\
4 \\
2 \\
1\end{array}$ & $\begin{array}{l}\left({ }^{3} \mathrm{H}\right){ }^{2} \mathrm{H} \\
\left({ }^{3} \mathrm{H}\right){ }^{2} \mathrm{I} \\
\left({ }^{3} \mathrm{H}\right){ }^{2} \mathrm{I} \\
\left({ }^{3} \mathrm{H}\right){ }^{2} \mathrm{~K}\end{array}$ \\
\hline $4 f^{2}\left({ }^{3} \mathrm{H}\right) 5 d$ & ${ }^{4} \mathrm{G}$ & $\begin{array}{c}5 / 2 \\
7 / 2 \\
9 / 2 \\
11 / 2\end{array}$ & $\begin{array}{r}8448.641 \\
9316.912 \\
10314.162 \\
11458.353\end{array}$ & $\begin{array}{l}0.633 \\
0.992 \\
1.04 \\
1.235\end{array}$ & $\begin{array}{l}49 \\
54 \\
55 \\
48\end{array}$ & $\begin{array}{l}30 \\
32 \\
34 \\
35\end{array}$ & $\left({ }^{3} \mathrm{~F}\right){ }^{4} \mathrm{G}$ \\
\hline $4 f^{2}\left({ }^{3} \mathrm{~F}\right) 6 s$ & ${ }^{2} \mathrm{~F}$ & $\begin{array}{l}5 / 2 \\
7 / 2\end{array}$ & $\begin{array}{l}8789.380 \\
9053.629\end{array}$ & $\begin{array}{l}0.905 \\
1.026\end{array}$ & $\begin{array}{l}69 \\
54\end{array}$ & $\begin{array}{l}28 \\
41\end{array}$ & $\begin{array}{l}\left({ }^{3} F\right){ }^{4} F \\
\left({ }^{1} G\right){ }^{2} G\end{array}$ \\
\hline $4 f^{2}\left({ }^{3} \mathrm{H}\right) 5 d$ & ${ }^{4} \mathrm{H}$ & $\begin{array}{c}7 / 2 \\
9 / 2 \\
11 / 2 \\
13 / 2\end{array}$ & $\begin{array}{r}9725.733 \\
10703.305 \\
11759.467 \\
13027.758\end{array}$ & $\begin{array}{l}0.694 \\
0.969 \\
1.124 \\
1.210\end{array}$ & $\begin{array}{l}63 \\
62 \\
64 \\
59\end{array}$ & $\begin{array}{l}25 \\
27 \\
28 \\
22\end{array}$ & $\left({ }^{3} \mathrm{~F}\right){ }^{4} \mathrm{H}$ \\
\hline
\end{tabular}


Ce II, Even Parity-Continued

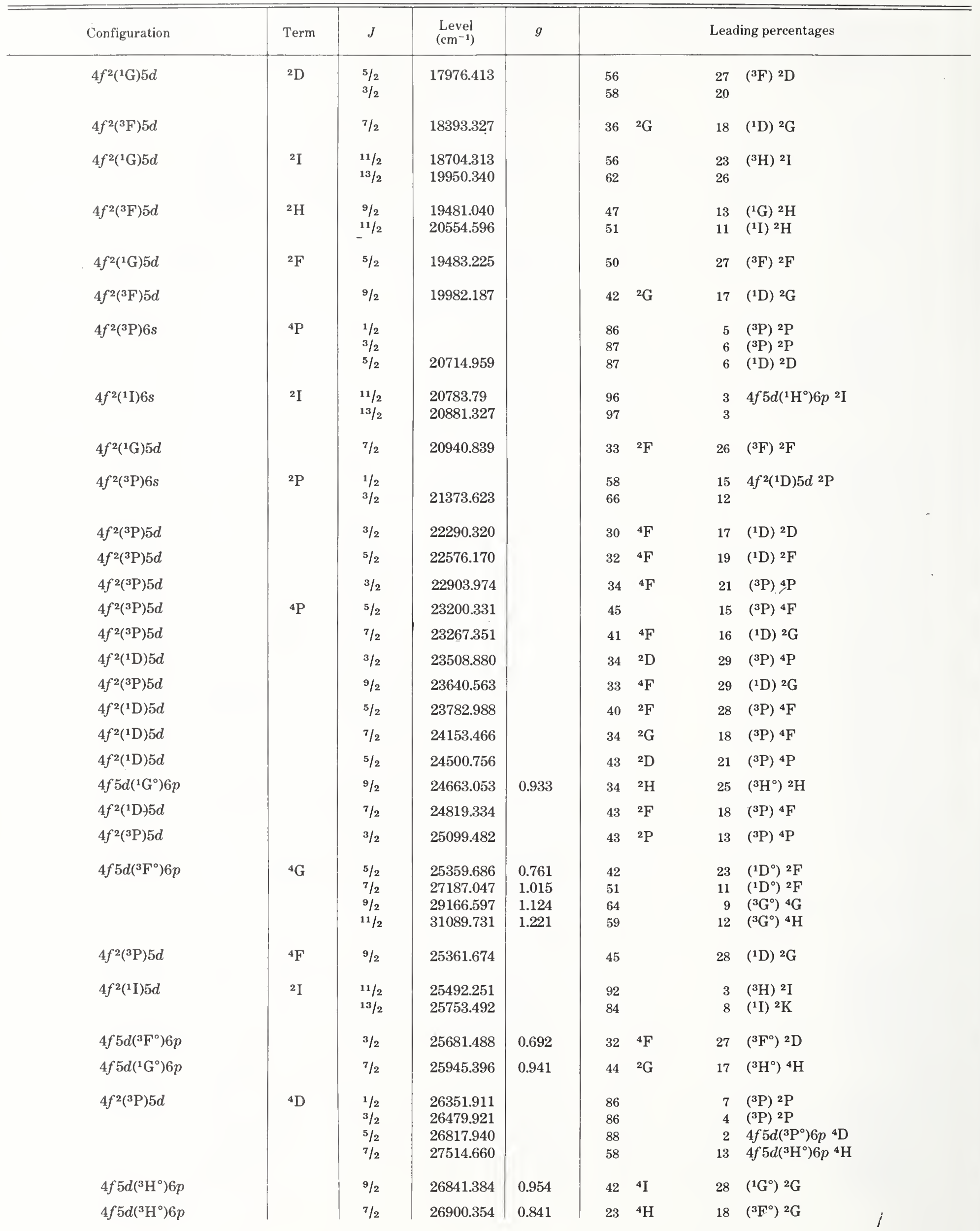


Ce II, Even Parity—Continued

\begin{tabular}{|c|c|c|c|c|c|c|c|c|}
\hline Configuration & Term & $J$ & $\begin{array}{l}\text { Level } \\
\left(\mathrm{cm}^{-1}\right)\end{array}$ & $g$ & \multicolumn{4}{|c|}{ Leading percentages } \\
\hline $4 f 5 d\left({ }^{3} \mathrm{~F}^{\circ}\right) 6 p$ & ${ }^{4} \mathrm{~F}$ & $5 / 2$ & 27249.669 & 1.061 & 55 & & 13 & $\left({ }^{3} \mathrm{~F}^{\circ}\right)^{2} \mathrm{D}$ \\
\hline $4 f^{2}\left({ }^{1} \mathrm{I}\right) 5 d$ & ${ }^{2} \mathbf{L}$ & $\begin{array}{l}15 / 2 \\
17 / 2\end{array}$ & $\begin{array}{l}27353.67 \\
28096.58\end{array}$ & & $\begin{array}{r}98 \\
100\end{array}$ & & 1 & $\left({ }^{3} \mathrm{H}\right){ }^{2} \mathrm{~K}$ \\
\hline $4 f 5 d\left({ }^{1} \mathrm{G}^{\circ}\right) 6 p$ & & $11 / 2$ & 27379.949 & 1.052 & 40 & ${ }^{2} \mathrm{H}$ & 33 & $\left({ }^{3} \mathrm{H}^{\circ}\right){ }^{4} \mathrm{I}$ \\
\hline $4 f^{2}\left({ }^{1} \mathrm{I}\right) 5 d$ & ${ }^{2} \mathrm{~K}$ & $\begin{array}{l}13 / 2 \\
15 / 2\end{array}$ & $\begin{array}{l}27706.631 \\
28117.519\end{array}$ & & $\begin{array}{l}75 \\
81\end{array}$ & & $\begin{array}{l}15 \\
17\end{array}$ & $\left({ }^{3} \mathrm{H}\right){ }^{2} \mathrm{~K}$ \\
\hline $4 f^{2}\left({ }^{3} \mathrm{P}\right) 5 d$ & & $7 / 2$ & 27811.496 & & 26 & ${ }^{4} \mathrm{D}$ & 24 & $4 f 5 d\left({ }^{3} \mathrm{H}^{\circ}\right) 6 p^{4} \mathrm{H}$ \\
\hline $4 f 5 d\left({ }^{3} \mathrm{~F}^{\circ}\right) 6 p$ & & $5 / 2$ & 27812.398 & & 40 & ${ }^{4} \mathrm{G}$ & 17 & $\left({ }^{3} G^{\circ}\right)^{2} \mathrm{~F}$ \\
\hline $4 f 5 d\left({ }^{3} \mathrm{~F}^{\circ}\right) 6 p$ & & $3 / 2$ & 27835.233 & 0.833 & 32 & ${ }^{4} \mathrm{~F}$ & 26 & $\left({ }^{1} \mathrm{D}^{\circ}\right)^{2} \mathrm{P}$ \\
\hline $4 f^{2}\left({ }^{1} \mathrm{I}\right) 5 d$ & ${ }^{2} \mathrm{H}$ & $\begin{array}{r}9 / 2 \\
11 / 2\end{array}$ & $\begin{array}{l}27905.157 \\
27950.889\end{array}$ & 0.920 & $\begin{array}{l}77 \\
81\end{array}$ & & $\begin{array}{l}7 \\
8\end{array}$ & $\left({ }^{3} \mathrm{~F}\right){ }^{2} \mathrm{H}$ \\
\hline $4 f 5 d\left({ }^{3} \mathrm{H}^{\circ}\right) 6 p$ & & $9 / 2$ & 27934.638 & 0.969 & 35 & ${ }^{4} \mathrm{H}$ & 27 & $\left({ }^{1} \mathrm{G}^{\circ}\right)^{2} \mathrm{H}$ \\
\hline $4 f 5 d\left({ }^{3} \mathrm{G}^{\circ}\right) 6 p$ & ${ }^{4} \mathrm{H}$ & $\begin{array}{c}7 / 2 \\
9 / 2 \\
11 / 2 \\
13 / 2\end{array}$ & $\begin{array}{l}28297.473 \\
29908.904 \\
31738.484 \\
33531.388\end{array}$ & $\begin{array}{l}0.906 \\
1.074 \\
1.140 \\
1.257\end{array}$ & $\begin{array}{l}46 \\
44 \\
64 \\
95\end{array}$ & & $\begin{array}{r}13 \\
15 \\
13 \\
2\end{array}$ & $\begin{array}{l}\left({ }^{3} \mathrm{G}^{\circ}\right)^{2} \mathrm{G} \\
\left({ }^{3} \mathrm{G}^{\circ}\right){ }^{2} \mathrm{G} \\
\left({ }^{3} \mathrm{~F}^{\circ}\right){ }^{4} \mathrm{G} \\
\left({ }^{3} \mathrm{H}^{\circ}\right){ }^{4} \mathrm{H}\end{array}$ \\
\hline $4 f 5 d\left({ }^{3} \mathrm{H}^{\circ}\right) 6 p$ & & $9 / 2$ & 28334.756 & 0.891 & 38 & ${ }^{2} \mathrm{H}$ & 31 & $\left({ }^{3} \mathrm{H}^{\circ}\right){ }^{4} \mathrm{I}$ \\
\hline $4 f 5 d\left({ }^{3} \mathrm{G}^{\circ}\right) 6 p$ & & $5 / 2$ & 28337.814 & 0.916 & 26 & ${ }^{4} \mathrm{G}$ & 16 & $\left({ }^{3} \mathrm{G}^{\circ}\right)^{2} \mathrm{~F}$ \\
\hline $4 f 5 d\left({ }^{1} \mathrm{D}^{\circ}\right) 6 p$ & & $1 / 2$ & 28345.313 & 0.387 & 44 & ${ }^{2} \mathrm{P}$ & 43 & $\left({ }^{3} \mathrm{~F}^{\circ}\right)^{4} \mathrm{D}$ \\
\hline $4 f 5 d\left({ }^{(} \mathrm{G}^{\circ}\right) 6 p$ & & $11 / 2$ & 28634.516 & 1.080 & 37 & ${ }^{2} \mathrm{H}$ & 27 & $\left({ }^{3} \mathrm{H}^{\circ}\right){ }^{4} \mathrm{I}$ \\
\hline $4 f 5 d\left({ }^{1} \mathrm{G}^{\circ}\right) 6 p$ & & $5 / 2$ & 28685.758 & 0.827 & 37 & ${ }^{2} \mathrm{~F}$ & 14 & $\left({ }^{3} \mathrm{H}^{\circ}\right){ }^{4} \mathrm{G}$ \\
\hline $4 f 5 d\left({ }^{3} \mathrm{H}^{\circ}\right) 6 p$ & & $9 / 2$ & 28725.148 & 1.002 & 36 & ${ }^{4} \mathrm{H}$ & 18 & $\left({ }^{1} \mathrm{G}^{\circ}\right)^{2} \mathrm{H}$ \\
\hline $4 f 5 d\left({ }^{3} \mathrm{~F}^{\circ}\right) 6 p$ & ${ }^{4} \mathrm{~F}$ & $7 / 2$ & 28730.712 & 1.124 & 54 & & 7 & $\left({ }^{3} \mathrm{~F}^{\circ}\right){ }^{4} \mathrm{G}$ \\
\hline $4 f 5 d\left({ }^{3} \mathrm{~F}^{\circ}\right) 6 p$ & & $3 / 2$ & 29029.353 & 0.980 & 31 & ${ }^{2} \mathrm{D}$ & 29 & $\left({ }^{1} \mathrm{D}^{\circ}\right)^{2} \mathrm{P}$ \\
\hline $4 f 5 d\left({ }^{3} \mathrm{~F}^{\circ}\right) 6 p$ & & $5 / 2$ & 29281.374 & 1.052 & 23 & ${ }^{2} \mathrm{D}$ & 22 & $\left({ }^{3} \mathrm{~F}^{\circ}\right)^{4} \mathrm{D}$ \\
\hline $4 f^{2}\left({ }^{3} \mathrm{P}\right) 5 d$ & & $7 / 2$ & 29364.740 & 0.894 & 17 & ${ }^{2} \mathrm{~F}$ & 14 & $4 f 5 d\left({ }^{3} \mathrm{G}^{\circ}\right) 6 p^{2} \mathrm{~F}$ \\
\hline $4 f 5 d\left({ }^{3} \mathrm{H}^{\circ}\right) 6 p$ & & $11 / 2$ & 29438.817 & 1.041 & 39 & ${ }^{4} I$ & 31 & $\left({ }^{3} \mathrm{H}^{\circ}\right)^{2} \mathrm{H}$ \\
\hline $4 f 5 d\left({ }^{3} \mathrm{G}^{\circ}\right) 6 p$ & & $3 / 2$ & 29449.778 & 0.723 & 36 & ${ }^{4} \mathrm{~F}$ & 23 & $\left({ }^{3} \mathrm{~F}^{\circ}\right)^{4} \mathrm{D}$ \\
\hline $4 f^{2}\left({ }^{3} \mathrm{P}\right) 5 d$ & ${ }^{2} \mathrm{~F}$ & $\begin{array}{l}5 / 2 \\
7 / 2\end{array}$ & $\begin{array}{l}29673.771 \\
29807.078\end{array}$ & 1.055 & $\begin{array}{l}51 \\
39\end{array}$ & & $\begin{array}{r}13 \\
8\end{array}$ & $5 d^{32} \mathrm{~F}$ \\
\hline $4 f 5 d\left({ }^{3} \mathrm{D}^{\circ}\right) 6 p$ & & $1 / 2$ & 29790.270 & 0.45 & 39 & ${ }^{2} \mathrm{P}$ & 26 & $\left({ }^{3} \mathrm{~F}^{\circ}\right)^{4} \mathrm{D}$ \\
\hline $4 f 5 d\left({ }^{3} \mathrm{~F}^{\circ}\right) 6 p$ & & $7 / 2$ & 29794.517 & 1.052 & 24 & ${ }^{2} \mathrm{~F}$ & 15 & $\left({ }^{3} \mathrm{~F}^{\circ}\right)^{4} \mathrm{D}$ \\
\hline $4 f 5 d\left({ }^{3} \mathrm{~F}^{\circ}\right) 6 p$ & & $3 / 2$ & 29984.052 & 0.773 & 38 & ${ }^{4} \mathrm{D}$ & 14 & $\left({ }^{3} G^{0}\right)^{4} F$ \\
\hline $4 f 5 d\left({ }^{3} \mathrm{G}^{\circ}\right) 6 p$ & & $5 / 2$ & 29994.041 & & 34 & ${ }^{4} \mathrm{G}$ & 21 & $\left({ }^{3} G^{\circ}\right){ }^{4} F$ \\
\hline $4 f 5 d\left({ }^{3} \mathrm{~F}^{\circ}\right) 6 p$ & & $7 / 2$ & 30065.164 & 1.075 & 29 & ${ }^{4} \mathrm{D}$ & 12 & $\left({ }^{3} \mathrm{H}^{\circ}\right)^{2} \mathrm{G}$ \\
\hline $4 f 5 d\left({ }^{3} \mathrm{H}^{\circ}\right) 6 p$ & ${ }^{4} \mathrm{H}$ & $11 / 2$ & 30134.910 & 1.146 & 53 & & 22 & $\left({ }^{3} \mathrm{H}^{\circ}\right)^{2} \mathrm{H}$ \\
\hline $4 f 5 d\left({ }^{3} \mathrm{G}^{\circ}\right) 6 p$ & & $7 / 2$ & 30166.057 & 1.036 & 30 & ${ }^{4} \mathrm{G}$ & 16 & $\left({ }^{1} \mathrm{D}^{\circ}\right)^{2} \mathrm{~F}$ \\
\hline $4 f 5 d\left({ }^{3} \mathrm{H}^{\circ}\right) 6 p$ & ${ }^{4} I$ & $13 / 2$ & 30180.096 & 1.115 & 91 & & 9 & $\left({ }^{3} \mathrm{H}^{\circ}\right)^{4} \mathrm{H}$ \\
\hline $4 f 5 d\left({ }^{3} \mathrm{~F}^{\circ}\right) 6 p$ & & $9 / 2$ & 30245.878 & 1.225 & 41 & ${ }^{4} \mathrm{~F}$ & 21 & $\left({ }^{1} \mathrm{G}^{0}\right)^{2} \mathrm{G}$ \\
\hline $4 f 5 d\left({ }^{3} \mathrm{H}^{\circ}\right) 6 p$ & & $5 / 2$ & 30425.349 & 0.831 & 37 & ${ }^{4} \mathrm{G}$ & 15 & $4 f 6 s\left({ }^{3} \mathrm{~F}^{\circ}\right) 6 p{ }^{4} \mathrm{G}$ \\
\hline $4 f 5 d\left({ }^{3} F^{\circ}\right) 6 p$ & & $5 / 2$ & 30637.157 & 1.125 & 10 & ${ }^{2} \mathrm{D}$ & 10 & $\left({ }^{3} G^{0}\right)^{2} F$ \\
\hline $4 f 5 d\left({ }^{3} \mathrm{G}^{\circ}\right) 6 p$ & & $9 / 2$ & 30702.610 & 1.079 & 30 & ${ }^{4} \mathrm{H}$ & 15 & $\left({ }^{3} \mathrm{~F}^{\circ}\right)^{2} \mathrm{G}$ \\
\hline $4 f 6 s\left({ }^{3} \mathrm{~F}^{\circ}\right) 6 p$ & & $3 / 2$ & 30745.286 & & 29 & ${ }^{4} \mathrm{~F}$ & 28 & $4 f 5 d\left({ }^{3} \mathrm{D}^{\circ}\right) 6 p^{4} \mathrm{~F}$ \\
\hline
\end{tabular}


Ce II, Even Parity-Continued

\begin{tabular}{|c|c|c|c|c|c|c|c|c|}
\hline Configuration & Term & $J$ & $\begin{array}{l}\text { Level } \\
\left(\mathrm{cm}^{-1}\right)\end{array}$ & $g$ & \multicolumn{4}{|c|}{ Leading percentages } \\
\hline $4 f 5 d\left({ }^{3} \mathrm{G}^{\circ}\right) 6 p$ & & $7 / 2$ & 30829.124 & 1.016 & 25 & ${ }^{4} \mathrm{Gr}$ & 12 & $\left({ }^{1} \mathrm{D}^{\circ}\right)^{2} \mathrm{~F}$ \\
\hline $4 f 5 d\left({ }^{1} \mathrm{D}^{\circ}\right) 6 p$ & & $3 / 2$ & 30961.518 & 1.043 & 25 & ${ }^{2} \mathrm{D}$ & 17 & $\left({ }^{3} \mathrm{~F}^{\circ}\right){ }^{4} \mathrm{D}$ \\
\hline $4 f 5 d\left({ }^{1} \mathrm{D}^{\circ}\right) 6 p$ & & $1 / 2$ & 31032.611 & 0.692 & 31 & ${ }^{2} \mathbf{P}$ & 24 & $\left({ }^{3} \mathrm{~F}^{0}\right){ }^{4} \mathrm{D}$ \\
\hline $4 f 5 d\left({ }^{3} \mathrm{~F}^{\circ}\right) 6 p$ & & $5 / 2$ & 31043.110 & 1.014 & 29 & ${ }^{2} \mathrm{~F}$ & 26 & $\left({ }^{3} \mathrm{~F}^{0}\right){ }^{4} \mathrm{D}$ \\
\hline $4 f 5 d\left({ }^{3} \mathrm{G}^{\circ}\right) 6 p$ & & $9 / 2$ & 31075.603 & 1.207 & 24 & ${ }^{4} \mathrm{~F}$ & 23 & $\left({ }^{3} \mathrm{G}^{\circ}\right){ }^{4} \mathrm{G}$ \\
\hline $4 f 5 d\left({ }^{3} \mathrm{H}^{\circ}\right) 6 p$ & ${ }^{4} \mathrm{H}$ & $13 / 2$ & 31155.623 & 1.203 & 80 & & 9 & $\left({ }^{3} \mathrm{H}^{\circ}\right)^{4} \mathrm{I}$ \\
\hline $4 f 5 d\left({ }^{3} \mathrm{D}^{\circ}\right) 6 p$ & & $3 / 2$ & 31170.645 & 1.118 & 31 & ${ }^{2} \mathbf{P}$ & 19 & $\left({ }^{3} \mathrm{D}^{\circ}\right)^{4} \mathrm{D}$ \\
\hline $4 f 5 d\left({ }^{3} \mathrm{~F}^{\circ}\right) 6 p$ & & $7 / 2$ & 31207.927 & 1.123 & 15 & ${ }^{4} \mathrm{D}$ & 12 & $\left({ }^{3} \mathrm{G}^{\circ}\right){ }^{4} \mathrm{~F}$ \\
\hline $4 f 5 d\left({ }^{1} \mathrm{D}^{\circ}\right) 6 p$ & & $5 / 2$ & 31234.878 & 0.975 & 20 & ${ }^{2} \mathrm{~F}$ & 16 & $\left({ }^{1} \mathrm{D}^{\circ}\right)^{2} \mathrm{D}$ \\
\hline $4 f 5 d\left({ }^{3} \mathrm{D}^{\circ}\right) 6 p$ & & $5 / 2$ & 31369.093 & & 15 & ${ }^{4} \mathrm{~F}$ & 15 & $5 d^{3}{ }^{4} \mathrm{~F}$ \\
\hline $4 f 5 d\left({ }^{3} \mathrm{G}^{\circ}\right) 6 p$ & & $7 / 2$ & 31558.626 & 1.167 & 13 & ${ }^{4} \mathrm{~F}$ & 12 & $\left({ }^{3} \mathrm{D}^{\circ}\right)^{4} \mathrm{~F}$ \\
\hline $4 f^{2}\left({ }^{1} \mathrm{I}\right) 5 d$ & & $9 / 2$ & 31568.019 & 1.138 & 33 & ${ }^{2} \mathrm{G}$ & 17 & $4 f 5 d\left({ }^{3} \mathrm{G}^{\circ}\right) 6 p{ }^{4} \mathrm{G}$ \\
\hline $4 f^{2}\left({ }^{3} \mathrm{P}\right) 5 d$ & & $3 / 2$ & 31766.109 & & 32 & ${ }^{2} \mathrm{D}$ & 11 & $5 d^{3}{ }^{2} \mathrm{D} 2$ \\
\hline $4 f 5 d\left({ }^{3} \mathrm{D}^{\circ}\right) 6 p$ & & $5 / 2$ & 31851.397 & 1.238 & 20 & ${ }^{4} \mathrm{P}$ & 18 & $\left({ }^{3} \mathrm{D}^{\circ}\right)^{4} \mathrm{D}$ \\
\hline $4 f 5 d\left({ }^{3} \mathrm{G}^{\circ}\right) 6 p$ & & $9 / 2$ & 31930.936 & 1.149 & 31 & ${ }^{4} \mathrm{Gr}$ & 20 & $4 f^{2}\left({ }^{1} \mathrm{I}\right) 5 d^{2} \mathrm{G}$ \\
\hline $4 f 5 d\left({ }^{3} \mathrm{H}^{\circ}\right) 6 p$ & & $7 / 2$ & 31937.653 & & 29 & ${ }^{4} \mathrm{G}$ & 23 & $\left({ }^{3} \mathrm{H}^{\circ}\right)^{2} \mathrm{G}$ \\
\hline $4 f 6 s\left({ }^{3} \mathrm{~F}^{\circ}\right) 6 p$ & & $5 / 2$ & 32138.698 & 1.008 & 23 & ${ }^{4} \mathrm{~F}$ & 11 & $4 f 5 d\left({ }^{3} \mathrm{G}^{\circ}\right) 6 p{ }^{2} \mathrm{~F}$ \\
\hline $4 f^{2}\left({ }^{1} \mathrm{I}\right) 5 d$ & & $9 / 2$ & 32197.982 & 1.195 & 28 & ${ }^{2} \mathrm{G}$ & 16 & $4 f 5 d\left({ }^{3} \mathrm{G}^{\circ}\right) 6 p{ }^{4} \mathrm{~F}$ \\
\hline $4 f 5 d\left({ }^{3} \mathrm{H}^{\circ}\right) 6 p$ & ${ }^{4} \mathrm{I}$ & $15 / 2$ & 32269.252 & 1.195 & 100 & & & \\
\hline $4 f 5 d\left({ }^{3} \mathrm{D}^{\circ}\right) 6 p$ & ${ }^{4} \mathrm{P}$ & $\begin{array}{l}1 / 2 \\
3 / 2\end{array}$ & $\begin{array}{l}32314.466 \\
33050.291\end{array}$ & 1.426 & $\begin{array}{l}57 \\
48\end{array}$ & & $\begin{array}{l}20 \\
14\end{array}$ & $\begin{array}{l}\left({ }^{3} \mathrm{D}^{\circ}\right)^{4} \mathrm{D} \\
\left({ }^{3} \mathrm{D}^{\circ}\right)^{2} \mathrm{P}\end{array}$ \\
\hline $4 f 5 d\left({ }^{3} \mathrm{~F}^{\circ}\right) 6 p$ & & $7 / 2$ & 32318.175 & 1.118 & 15 & ${ }^{2} \mathrm{~F}$ & 14 & $\left({ }^{3} G^{\circ}\right)^{2} \mathrm{G}$ \\
\hline $4 f^{2}\left({ }^{1} \mathrm{I}\right) 5 d$ & ${ }^{2} \mathrm{G}$ & $7 / 2$ & 32413.346 & 1.019 & 68 & & 7 & $4 f 5 d\left({ }^{1} \mathrm{H}^{\circ}\right) 6 p{ }^{2} \mathrm{G}$ \\
\hline $4 f 5 d\left({ }^{3} \mathrm{G}^{\circ}\right) 6 p$ & & $11 / 2$ & 32492.038 & 1.221 & 43 & ${ }^{4} \mathrm{G}$ & 28 & $\left({ }^{3} \mathrm{H}^{\circ}\right){ }^{4} \mathrm{G}$ \\
\hline $5 d^{3}$ & & $3 / 2$ & 32507.442 & 0.643 & 26 & ${ }^{4} \mathrm{~F}$ & 22 & 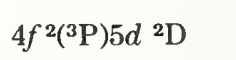 \\
\hline $4 f 5 d\left({ }^{3} \mathrm{D}^{\circ}\right) 6 p$ & & $3 / 2$ & 32638.165 & 1.070 & 37 & ${ }^{4} \mathrm{D}$ & 12 & $\left({ }^{3} \mathrm{P}^{\circ}\right){ }^{4} \mathrm{D}$ \\
\hline $4 f^{2}\left({ }^{3} \mathrm{P}\right) 5 d$ & & $5 / 2$ & 32716.647 & 1.151 & 28 & ${ }^{2} \mathrm{D}$ & 8 & $5 d^{3}{ }^{4} \mathrm{~F}$ \\
\hline $4 f 5 d\left({ }^{3} \mathrm{H}^{\circ}\right) 6 p$ & $2 \mathrm{I}$ & $\begin{array}{l}11 / 2 \\
13 / 2\end{array}$ & $\begin{array}{l}32802.165 \\
34513.468\end{array}$ & $\begin{array}{l}0.948 \\
1.079\end{array}$ & $\begin{array}{l}83 \\
84\end{array}$ & & $\begin{array}{l}3 \\
8\end{array}$ & $\begin{array}{l}\left({ }^{1} \mathrm{H}^{\circ}\right){ }^{2} \mathrm{I} \\
\left({ }^{3} \mathrm{H}^{\circ}\right){ }^{4} \mathrm{H}\end{array}$ \\
\hline $4 f 5 d\left({ }^{3} \mathrm{P}^{\circ}\right) 6 p$ & ${ }^{4} \mathrm{P}$ & $\begin{array}{l}1 / 2 \\
3 / 2\end{array}$ & $\begin{array}{l}32860.353 \\
33876.377\end{array}$ & 1.535 & $\begin{array}{l}56 \\
68\end{array}$ & & $\begin{array}{l}9 \\
8\end{array}$ & $\begin{array}{l}\left({ }^{3} \mathrm{D}^{\circ}\right)^{2} \mathrm{P} \\
5 d^{2}\left({ }^{3} \mathrm{P}\right) 6 s{ }^{4} \mathrm{P}\end{array}$ \\
\hline $4 f 5 d\left({ }^{3} \mathrm{~F}^{\circ}\right) 6 p$ & & $7 / 2$ & 32862.767 & 1.009 & 21 & ${ }^{2} \mathrm{Gr}$ & 17 & $\left({ }^{3} G^{\circ}\right)^{2} \mathrm{~F}$ \\
\hline $4 f^{2}\left({ }^{3} \mathrm{P}\right) 5 d$ & & $5 / 2$ & 33045.095 & 1.188 & 35 & ${ }^{2} \mathrm{D}$ & 15 & $4 f 5 d\left({ }^{3} \mathrm{D}^{\circ}\right) 6 p{ }^{4} \mathrm{P}$ \\
\hline $5 d^{3}$ & & $7 / 2$ & 33079.512 & 1.257 & 27 & ${ }^{4} \mathrm{~F}$ & 17 & $4 f 6 s\left({ }^{3} \mathrm{~F}^{\circ}\right) 6 p{ }^{4} \mathrm{~F}$ \\
\hline $5 d^{3}$ & & $5 / 2$ & 33148.589 & 1.098 & 21 & ${ }^{4} \mathrm{~F}$ & 16 & $4 f 5 d\left({ }^{3} \mathrm{D}^{\circ}\right) 6 p{ }^{4} \mathrm{P}$ \\
\hline $4 f 5 d\left({ }^{3} \mathrm{P}^{\circ}\right) 6 p$ & & $1 / 2$ & 33177.196 & 1.484 & 21 & ${ }^{4} \mathbf{P}$ & 18 & $\left({ }^{3} \mathrm{D}^{\circ}\right){ }^{4} \mathrm{P}$ \\
\hline $4 f 5 d\left({ }^{1} \mathrm{~F}^{\circ}\right) 6 p$ & & $9 / 2$ & 33296.805 & & 18 & ${ }^{2} \mathrm{G}$ & 18 & $\left({ }^{3} G^{\circ}\right)^{2} G$ \\
\hline $4 f 5 d\left({ }^{3} \mathrm{D}^{\circ}\right) 6 p$ & & $7 / 2$ & 33409.897 & & 15 & ${ }^{4} \mathrm{D}$ & 14 & $\left({ }^{3} \mathrm{G}^{\circ}\right)^{2} \mathrm{~F}$ \\
\hline $4 f 6 s\left({ }^{3} \mathrm{~F}^{\circ}\right) 6 p$ & & $5 / 2$ & 33552.582 & 0.920 & 13 & ${ }^{2} \mathrm{~F}$ & 11 & $\left({ }^{1} \mathrm{~F}^{\circ}\right)^{2} \mathrm{~F}$ \\
\hline $4 f 6 s\left({ }^{3} \mathrm{~F}^{\circ}\right) 6 p$ & & $3 / 2$ & 33574.145 & 0.675 & 20 & ${ }^{4} \mathrm{~F}$ & 15 & $4 f 5 d\left({ }^{3} \mathrm{D}^{\circ}\right) 6 p{ }^{4} \mathrm{~F}$ \\
\hline $4 f 5 d\left({ }^{3} \mathrm{H}^{\circ}\right) 6 p$ & & $11 / 2$ & 33659.968 & 1.249 & 41 & ${ }^{4} \mathrm{G}$ & 21 & $\left({ }^{3} \mathrm{G}^{\circ}\right){ }^{4} \mathrm{G}$ \\
\hline $4 f 5 d\left({ }^{3} \mathrm{D}^{\circ}\right) 6 p$ & & $5 / 2$ & 33808.317 & 1.230 & 44 & ${ }^{4} \mathrm{D}$ & 16 & $\left({ }^{3} \mathrm{P}^{\circ}\right){ }^{4} \mathrm{D}$ \\
\hline $4 f 5 d\left({ }^{3} \mathrm{G}^{\circ}\right) 6 p$ & ${ }^{2} \mathrm{H}$ & $\begin{array}{l}9 / 2 \\
11 / 2\end{array}$ & $\begin{array}{l}33811.567 \\
35716.083\end{array}$ & $\begin{array}{l}1.090 \\
1.096\end{array}$ & $\begin{array}{l}43 \\
61\end{array}$ & & $\begin{array}{r}16 \\
9\end{array}$ & $\begin{array}{l}\left({ }^{3} \mathrm{G}^{\circ}\right){ }^{2} \mathrm{G} \\
\left({ }^{3} \mathrm{H}^{\circ}\right)^{2} \mathrm{H}\end{array}$ \\
\hline
\end{tabular}


Ce II, Even Parity-Continued

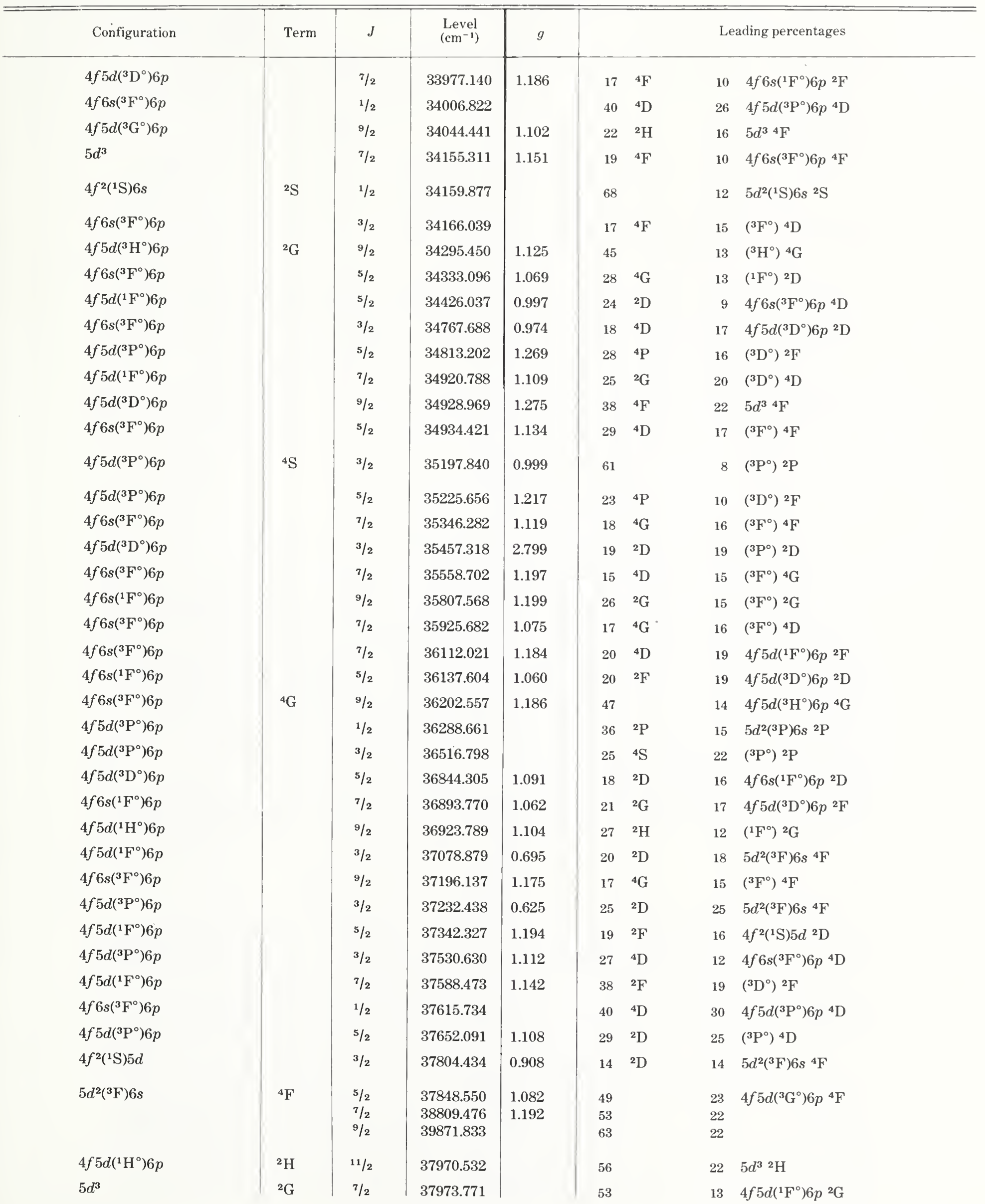


Ce II, Even Parity-Continued

\begin{tabular}{|c|c|c|c|c|c|c|c|c|}
\hline Configuration & Term & $J$ & $\begin{array}{l}\text { Level } \\
\left(\mathrm{cm}^{-1}\right)\end{array}$ & $g$ & \multicolumn{4}{|c|}{ Leading percentages } \\
\hline $4 f 5 d\left({ }^{1} \mathrm{~F}^{\circ}\right) 6 p$ & \multirow{10}{*}{${ }^{4} \mathrm{G}$} & $9 / 2$ & 38134.400 & 0.621 & 25 & ${ }^{2} \mathrm{G}$ & 22 & $\left({ }^{1} \mathrm{H}^{\circ}\right){ }^{2} \mathrm{H}$ \\
\hline $4 f 6 s\left({ }^{3} \mathrm{~F}^{\circ}\right) 6 p$ & & $11 / 2$ & 38137.682 & & 71 & & 13 & $4 f 5 d\left({ }^{3} \mathrm{H}^{\circ}\right) 6 p{ }^{4} \mathrm{G}$ \\
\hline $4 f 5 d\left({ }^{3} \mathrm{P}^{\circ}\right) 6 p$ & & $1 / 2$ & 38269.974 & & 33 & ${ }^{2} \mathrm{~S}$ & 23 & $5 d^{3}{ }^{4} \mathrm{P}$ \\
\hline $4 f 6 s\left({ }^{1} \mathrm{~F}^{\circ}\right) 6 p$ & & $3 / 2$ & 38452.750 & & 24 & ${ }^{2} \mathrm{D}$ & 15 & $\left({ }^{3} F^{\circ}\right){ }^{4} D$ \\
\hline $4 f 5 d\left({ }^{3} \mathrm{D}^{\circ}\right) 6 p$ & & $5 / 2$ & 38529.021 & 1.169 & 19 & ${ }^{2} \mathrm{D}$ & 12 & $4 f^{2}\left({ }^{1} \mathrm{~S}\right) 5 d^{2} \mathrm{D}$ \\
\hline $5 d^{3}$ & & $9 / 2$ & 38541.873 & 1.097 & 35 & ${ }^{2} \mathrm{G}$ & 10 & $4 f 5 d\left({ }^{1} \mathrm{H}^{\circ}\right) 6 p{ }^{2} \mathrm{G}$ \\
\hline $4 f 6 s\left({ }^{3} \mathrm{~F}^{\circ}\right) 6 p$ & & $5 / 2$ & 38832.709 & 1.273 & 35 & ${ }^{4} \mathrm{D}$ & 13 & $\left({ }^{1} \mathrm{~F}^{\circ}\right){ }^{2} \mathrm{D}$ \\
\hline $4 f 5 d\left({ }^{3} \mathrm{P}^{\circ}\right) 6 p$ & & $1 / 2$ & 38892.501 & & 30 & ${ }^{2} \mathrm{~S}$ & 24 & $5 d^{3}{ }^{4} \mathrm{P}$ \\
\hline $5 d^{3}$ & & $3 / 2$ & 38937.812 & 1.399 & 34 & ${ }^{4} \mathrm{P}$ & 9 & $4 f 5 d\left({ }^{3} \mathrm{P}^{\circ}\right) 6 p{ }^{2} \mathrm{P}$ \\
\hline $4 f 5 d\left({ }^{3} \mathrm{P}^{\circ}\right) 6 p$ & & $7 / 2$ & 39394.990 & 1.396 & 42 & ${ }^{4} \mathrm{D}$ & 23 & $4 f 6 s\left({ }^{3} \mathrm{~F}^{\circ}\right) 6 p{ }^{4} \mathrm{D}$ \\
\hline $5 d^{3}$ & \multirow[t]{3}{*}{${ }^{4} \mathrm{P}$} & $5 / 2$ & 39838.445 & 1.577 & \multicolumn{2}{|l|}{67} & 16 & $4 f^{2}\left({ }^{3} \mathrm{P}\right) 5 d{ }^{4} \mathrm{P}$ \\
\hline $5 d^{3}$ & & $1 / 2$ & 39855.395 & & 30 & ${ }^{2} \mathrm{P}$ & 22 & ${ }^{4} \mathrm{P}$ \\
\hline $5 d^{3}$ & & $3 / 2$ & 40511.127 & & 27 & ${ }^{2} \mathrm{P}$ & 22 & ${ }^{4} \mathrm{P}$ \\
\hline $4 f 5 d\left({ }^{1} \mathrm{H}^{\circ}\right) 6 p$ & ${ }^{2} \mathbf{I}$ & $\begin{array}{l}11 / 2 \\
13 / 2\end{array}$ & $\begin{array}{l}40673.575 \\
41992.356\end{array}$ & & \multicolumn{2}{|l|}{$\begin{array}{l}74 \\
90\end{array}$} & $\begin{array}{r}13 \\
6\end{array}$ & $\begin{array}{l}5 d^{3}{ }^{2} \mathrm{H} \\
\left({ }^{3} \mathrm{H}^{\circ}\right)^{2} \mathrm{I}\end{array}$ \\
\hline $4 f 5 d\left({ }^{1} \mathrm{H}^{\circ}\right) 6 p$ & ${ }^{2} \mathrm{G}$ & $\begin{array}{l}9 / 2 \\
7 / 2\end{array}$ & $\begin{array}{l}40858.205 \\
41085.208\end{array}$ & & \multicolumn{2}{|l|}{$\begin{array}{l}45 \\
57\end{array}$} & $\begin{array}{l}14 \\
14\end{array}$ & $\begin{array}{l}\left({ }^{1} \mathrm{H}^{\circ}\right)^{2} \mathrm{H} \\
5 d^{2}\left({ }^{1} \mathrm{G}\right) 6 s^{2} \mathrm{G}\end{array}$ \\
\hline $5 d^{3}$ & ${ }^{2} \mathrm{H}$ & $\begin{array}{c}9 / 2 \\
11 / 2\end{array}$ & $\begin{array}{l}41083.294 \\
41289.971\end{array}$ & 1.090 & \multicolumn{2}{|l|}{$\begin{array}{l}49 \\
53\end{array}$} & $\begin{array}{l}16 \\
27\end{array}$ & $4 f 5 d\left({ }^{1} \mathrm{H}^{\circ}\right) 6 p^{2} \mathrm{H}$ \\
\hline $5 d^{2}\left({ }^{3} \mathrm{~F}\right) 6 s$ & ${ }^{2} \mathrm{~F}$ & $\begin{array}{l}5 / 2 \\
7 / 2\end{array}$ & $\begin{array}{l}41198.309 \\
42573.191\end{array}$ & 1.143 & \multicolumn{2}{|l|}{$\begin{array}{l}51 \\
52\end{array}$} & $\begin{array}{r}9 \\
19\end{array}$ & $5 d^{3}{ }^{2} \mathrm{~F}$ \\
\hline $4 f 5 d\left({ }^{1} \mathrm{P}^{\circ}\right) 6 p$ & ${ }^{2} \mathrm{P}$ & $\begin{array}{l}1 / 2 \\
3 / 2\end{array}$ & $\begin{array}{l}41589.12 \\
42458.743\end{array}$ & & \multicolumn{2}{|l|}{$\begin{array}{l}73 \\
40\end{array}$} & $\begin{array}{l}13 \\
10\end{array}$ & $\begin{array}{l}\left({ }^{3} \mathrm{P}^{\circ}\right){ }^{2} \mathrm{~S} \\
5 d^{3}{ }^{2} \mathrm{D} 2\end{array}$ \\
\hline $4 f 5 d\left({ }^{1} \mathrm{P}^{\circ}\right) 6 p$ & & $3 / 2$ & 41748.279 & 0.880 & 23 & ${ }^{2} \mathrm{D}$ & 16 & $5 d^{2}\left({ }^{1} \mathrm{D}\right) 6 s^{2} \mathrm{D}$ \\
\hline $5 d^{2}\left({ }^{1} \mathrm{D}\right) 6 s$ & & $5 / 2$ & 42033.653 & 1.161 & 21 & ${ }^{2} \mathrm{D}$ & 20 & $4 f^{2}\left({ }^{1} \mathrm{~S}\right) 5 d^{2} \mathrm{D}$ \\
\hline $4 f 5 d\left({ }^{1} \mathrm{P}^{\circ}\right) 6 p$ & & $3 / 2$ & 43167.878 & & 35 & ${ }^{2} \mathrm{D}$ & 32 & $\left({ }^{1} \mathrm{P}^{\circ}\right)^{2} \mathrm{P}$ \\
\hline $4 f 5 d\left({ }^{1} \mathrm{P}^{\circ}\right) 6 p$ & ${ }^{2} \mathrm{~S}$ & $1 / 2$ & 43334.332 & & \multicolumn{2}{|l|}{66} & 15 & $\left({ }^{3} \mathrm{P}^{\circ}\right){ }^{2} \mathrm{~S}$ \\
\hline $4 f 5 d\left({ }^{1} \mathrm{P}^{\circ}\right) 6 p$ & ${ }^{2} \mathrm{D}$ & $5 / 2$ & 43460.533 & 1.170 & \multicolumn{2}{|l|}{64} & 7 & $5 d^{32} \mathrm{D} 2$ \\
\hline $5 d^{2}\left({ }^{3} \mathrm{P}\right) 6 s$ & ${ }^{4} \mathrm{P}$ & $\begin{array}{l}1 / 2 \\
3 / 2 \\
5 / 2\end{array}$ & $\begin{array}{l}43643.230 \\
44081.375 \\
44594.928\end{array}$ & 1.573 & $\begin{array}{l}75 \\
77 \\
68\end{array}$ & & $\begin{array}{l}9 \\
7 \\
9\end{array}$ & $\begin{array}{l}4 f 5 d\left({ }^{3} \mathrm{D}^{\circ}\right) 6 p{ }^{4} \mathrm{P} \\
4 f 5 d\left({ }^{3} \mathrm{D}^{\circ}\right) 6 p{ }^{4} \mathrm{P} \\
5 d^{3}{ }^{2} \mathrm{D} 1\end{array}$ \\
\hline $5 d^{3}$ & & $3 / 2$ & 46987.371 & & 31 & ${ }^{2} \mathrm{D} 2$ & 24 & $5 d^{2}\left({ }^{1} \mathrm{D}\right) 6 s^{2} \mathrm{D}$ \\
\hline $5 d^{2}\left({ }^{1} \mathrm{D}\right) 6 s$ & & $5 / 2$ & 47466.445 & & 34 & ${ }^{2} \mathrm{D}$ & 29 & $5 d^{32} \mathrm{D} 2$ \\
\hline $5 d^{2}\left({ }^{1} \mathrm{G}\right) 6 s$ & ${ }^{2} \mathrm{G}$ & $\begin{array}{l}7 / 2 \\
9 / 2\end{array}$ & $\begin{array}{l}47489.280 \\
48151.739\end{array}$ & & \multicolumn{2}{|l|}{$\begin{array}{l}48 \\
67\end{array}$} & $\begin{array}{r}22 \\
8\end{array}$ & $\begin{array}{l}4 f 6 s\left({ }^{3} \mathrm{~F}^{\circ}\right) 6 p^{2} \mathrm{G} \\
4 f 6 s\left({ }^{1} \mathrm{~F}^{\circ}\right) 6 p^{2} \mathrm{G}\end{array}$ \\
\hline $4 f 6 s\left({ }^{3} \mathrm{~F}^{\circ}\right) 6 p$ & & $5 / 2$ & 48024.675 & & 36 & ${ }^{2} \mathrm{~F}$ & 28 & $5 d^{32} \mathrm{~F}$ \\
\hline $4 f 6 s\left({ }^{3} \mathrm{~F}^{\circ}\right) 6 p$ & & $3 / 2$ & 48630.464 & & 26 & ${ }^{2} \mathrm{D}$ & 22 & $5 d^{2}\left({ }^{1} \mathrm{D}\right) 6 s^{2} \mathrm{D}$ \\
\hline $5 d^{3}$ & ${ }^{2} \mathrm{~F}$ & $7 / 2$ & 48657.336 & & \multicolumn{2}{|l|}{49} & 19 & $5 d^{2}\left({ }^{3} \mathrm{~F}\right) 6 s^{2} \mathrm{~F}$ \\
\hline $5 d^{3}$ & & $5 / 2$ & 49089.486 & & 38 & ${ }^{2} \mathrm{~F}$ & 18 & $4 f 6 s\left({ }^{3} \mathrm{~F}^{\circ}\right) 6 p{ }^{2} \mathrm{~F}$ \\
\hline $4 f 6 s\left({ }^{3} \mathrm{~F}^{\circ}\right) 6 p$ & & $7 / 2$ & 50368.336 & & 24 & ${ }^{2} \mathrm{~F}$ & 15 & $\left({ }^{3} \mathrm{~F}^{\circ}\right){ }^{2} \mathrm{G}$ \\
\hline $5 d\left({ }^{2} \mathrm{D}\right) 6 s^{2} ?$ & & $5 / 2$ & 50564.294 & & 32 & ${ }^{2} \mathrm{D}$ & 26 & $4 f 6 s\left({ }^{3} \mathrm{~F}^{\circ}\right) 6 p^{2} \mathrm{D}$ \\
\hline $4 f 6 s\left({ }^{3} \mathrm{~F}^{\circ}\right) 6 p$ & & $7 / 2$ & 51171.581 & & 23 & ${ }^{2} \mathrm{G}$ & 20 & $\left({ }^{3} \mathrm{~F}^{\circ}\right)^{2} \mathrm{~F}$ \\
\hline $4 f 6 s\left({ }^{3} \mathrm{~F}^{\circ}\right) 6 p$ & ${ }^{2} \mathrm{G}$ & $9 / 2$ & 52058.587 & & \multirow{2}{*}{\multicolumn{2}{|c|}{51}} & \multirow[t]{2}{*}{16} & $\left({ }^{1} \mathrm{~F}^{\circ}\right)^{2} \mathrm{G}$ \\
\hline Ce III $\left({ }^{3} \mathrm{H}_{4}\right)$ & Limit & --------- & 87500 & & & & & \\
\hline
\end{tabular}




\section{Ce III}

(Ba I sequence; 56 electrons)

$Z=58$

Ground state $\left(1 s^{2} 2 s^{2} 2 p^{6} 3 s^{2} 3 p^{6} 3 d^{10} 4 s^{2} 4 p^{6} 4 d^{10} 5 s^{2} 5 p^{6}\right) 4 f^{2}{ }^{3} \mathrm{H}_{4}$

Ionization energy $162903 \pm 20 \mathrm{~cm}^{-1}$

$20.198 \pm 0.003 \mathrm{eV}$

Identified even configurations $\quad 4 f^{2}, 5 d^{2}, 4 f 6 p, 5 d 6 s, 4 f 5 f, 4 f 7 p, 4 f 6 f, 6 p^{2} ?, 5 d 6 d$

Identified odd configurations $\quad 4 f 5 d, 4 f 6 s, 4 f 6 d, 4 f 7 s, 5 d 6 p, 4 f 8 s, 4 f 7 d, 4 f 5 g$

Most of the energy levels found by Kalia are based on real wavenumber differences, but the first correctly designated levels were given by de Bruin, Lier, and van de Vliet [1937], and by Russell, King, and Lang [1937]. The latter analysis gave nearly all the levels of seven configurations. These did not include, however, the $4 f^{2}$ ground configuration, which Sugar located in his extension of the analysis. His observations of the sliding-spark spectrum (1700 new lines, 757-11091 $\AA$ ) increased the known lines by a factor of 7 and yielded 126 new levels. Sugar also gave improved values for the previously known levels and assigned designations in appropriate coupling schemes. More recently Johansson and Litzén observed 31 infrared lines of Ce III $(1.1-2.6 \mu \mathrm{m})$ and completed the $4 f^{2}$ configuration by finding the ${ }^{3} \mathrm{P}$ term.

\section{Levels, Ionization Energy, $g$ Values}

Most of the energy levels and designations are from Sugar's paper. The $4 f^{2}{ }^{3} \mathrm{P}$ levels are from Johansson and Litzén, as are some improved values for other $4 f^{2}$ levels. Wyart recently located the $5 \mathrm{~d}^{2}{ }^{1} \mathrm{~S}_{0}$ level. The analysis is remarkably complete, almost all the expected levels having been found for 14 of the 17 identified configurations. Five of the 26 possible $4 f 6 f$ levels are missing. Sugar noted that his assignments of eight high even levels to $6 p^{2}$ and $5 d 6 d$ were not positive; we give the $6 p^{2}$ identification as tentative, as only one level was assigned to this configuration. Sugar's level for $5 d 6 p{ }^{1} \mathrm{P}_{1}^{\circ}\left(102249.70 \mathrm{~cm}^{-1}\right)$ does not agree well with the position calculated by Spector $\left(103780 \mathrm{~cm}^{-1}\right)$. Since the experimental evidence for the level is not strong, it is listed as questionable.

Sugar and Reader derived the ionization potential from the $4 f 6 s-8 s$ series, using a correction for the estimated deviation from the Ritz formula.

The $g$ values are mainly from Russell et al., from van de Vliet, or from our reductions of patterns given by these authors. An additional few values were taken from de Bruin et al. or from our reductions of patterns they measured. Most of the $g$ values not followed by a colon are probably accurate to \pm 0.02 . Values given with a colon are based on poor or unresolved patterns, and are in general less accurate.

\section{Theory}

Goldschmidt's [1968a] calculations have been used for the $4 f^{2}$ configuration and for the $\left(4 f 6 p+5 d^{2}+5 d 6 s\right)$ group. The effects of the $\left(4 f 6 p+5 d^{2}\right)$ configuration interaction on the four $J=4$ levels between 50000 and $55000 \mathrm{~cm}^{-1}$ are noteworthy. Since each of these levels has well over $50 \% 4 f 6 p$ configurational purity, all four are listed with this configuration in the first column, even though a pure $f p$ configuration has only three $J=4$ levels. The good $j_{1} j_{2}$ coupling shown by the $4 f 6 p$ levels having other $J$ values cannot hold for these four levels sharing three designations, and their leading $j_{1} j_{2}$ components range from $41 \%$ to $53 \%$. The level with smallest leading percentage $\left(51289.38 \mathrm{~cm}^{-1}\right)$ has no assigned $j_{1} j_{2}$ name, but it has a higher total $4 f 6 p$ configurational purity than two of the $J=4$ levels having such names. The basic reason for the low purities of the four levels is the interaction of $4 f 6 p$ with $5 d^{2}$ [Goldschmidt, 1968b], as evidenced by the large second percentages from $5 d^{2}{ }^{1} \mathrm{G}$ listed for two of the levels. Goldschmidt notes that the composition of each of these two levels (named $4 f\left({ }^{2} \mathrm{~F}_{7 / 2}^{\circ}\right) 6 p_{1 / 2}$ and $4 f\left({ }^{2} \mathrm{~F}_{7 / 2}^{\circ}\right) 6 p_{3 / 2}$ in the table) has a total of about $80 \%{ }^{1} \mathrm{G}$ character if the percentages from both configurations are given in $L S$ coupling. The strong interaction of $4 f 6 p^{1} \mathrm{G}$ and $5 d^{2}{ }^{1} \mathrm{G}$ causes 
the $5 d^{2}{ }^{1} \mathrm{G}$ components to be distributed among the four levels in such a way that $5 d^{2}{ }^{1} \mathrm{G}$ is missing as a designation. This case furnishes one of the simplest examples of some effects of configuration interaction having many analogues in more complex rare-earth spectra.

The percentages for the $4 f 5 d$ levels are from a calculation by Goldschmidt and Salomon. The inclusion of effective interactions in this calculation significantly improved the eigenvectors for several of the levels, as shown by comparisons of observed with calculated $g$ values [Goldschmidt, 1973].

The four levels of $4 f 6 s$ have very high purity in the $j_{1} j_{2}$ coupling scheme [Goldschmidt, 1967].

Spector's results have been used for the percentages of the $5 d 6 p, 4 f 6 d, 4 f 7 d$, and $4 f 5 g$ odd levels. The leading component for each of the $4 f 6 d$ levels is given in both the $j_{1} l$ and $L S$ coupling schemes, and leading components for the $4 f 7 d$ levels are given in the $j_{1} l$ and $j_{1} j_{2}$ schemes. The percentages for the $4 f 5 f, 4 f 6 f$, and $4 f 7 p$ even configurations are also from Spector. Most of Sugar's LS designations for the $4 f 5 f$ levels, made on the evidence of the intensities of the $4 f 5 d-4 f 5 f$ lines, were confirmed by Spector's calculations. The $L S$ scheme does not furnish satisfactory names for 6 of the $264 f 5 f$ levels, however, and the $j_{1} l$ scheme is definitely preferred.

\section{References}

Crosswhite, H. M., Phys. Rev. A 4, 485 (1971). PT

de Bruin, T. L., Lier, J. N., and van de Vliet, H. J., Proc. K. Ned. Akad. Wet. 40, 334 (1937). EL CL W ZE

Goldschmidt, Z. B., Colloq. Int. C.N.R.S., No. 164, 365 (1967). ND PT

Goldschmidt, Z. B., Thesis, Hebrew Univ. Jerusalem, Israel, 487 pp. (1968a). ND PT

Goldschmidt, Z. B., Spectroscopic and Group Theoretical Methods in Physics, Ed. F. Bloch et al., pp. $411-457$ (John Wiley \& Sons, Inc., New York, N.Y., 1968b). ND PT

Goldschmidt, Z. B., Atomic Physics 3, Ed. S. J. Smith and G. K. Walters, pp. 221-246 (Plenum Press, New York, N.Y., 1973). PT

Goldschmidt, Z. B., and Salomon, D., unpublished material (1974). PT

Johansson, S., and Litzén, U., Phys. Scr. 6, 139 (1972). EL CL W

Judd, B. R., Crosswhite, H. M., and Crosswhite, H., Phys. Rev. 169, 130 (1968). PT

Kalia, P. N., Indian J. Phys. 8, 137 (1933). E L CL

King, A. S., and King, R. B., Astrophys. J. 75, 40 (1932). W

Russell, H. N., King, R. B., and Lang, R. J., Phys. Rev. 52, 456 (1937). EL CL W ZE IP

Spector, N., J. Opt. Soc. Am. 55, 492 (1965). ND PT

Sugar, J., J. Opt. Soc. Am. 55, 33 (1965). E L N D CL W IP

Sugar, J., and Reader, J., J. Chem. Phys. 59, 2083 (1973). IP

van de Vliet, H. J., Thesis, Univ. Amsterdam, 85 pp. (1939). ZE

Wyart, J. F., private communication (1976). EL

[October 1976] 
Ce III, Even Parity_Continued

\begin{tabular}{|c|c|c|c|c|c|c|c|c|}
\hline Configuration & Term & $J$ & $\begin{array}{l}\text { Level } \\
\left(\mathrm{cm}^{-1}\right)\end{array}$ & $g$ & \multicolumn{4}{|c|}{ Leading percentages } \\
\hline $4 f\left({ }^{2} \mathrm{~F} \stackrel{\circ}{2}\right) 5 f$ & $2[9 / 2]$ & $\begin{array}{l}5 \\
4\end{array}$ & $\begin{array}{r}99604.30 \\
100814.08\end{array}$ & & $\begin{array}{l}69 \\
86\end{array}$ & $\begin{array}{l}\text { or } \\
\text { or }\end{array}$ & $\begin{array}{l}40 \\
77\end{array}$ & ${ }^{1} \mathrm{H}$ \\
\hline$" \prime$ & $2[5 / 2]$ & $\begin{array}{l}3 \\
2\end{array}$ & $\begin{array}{r}99708.39 \\
101354.33\end{array}$ & & $\begin{array}{l}67 \\
72\end{array}$ & $\begin{array}{l}\text { or } \\
\text { or }\end{array}$ & $\begin{array}{l}30 \\
69\end{array}$ & $\begin{array}{l}{ }^{3} \mathrm{D} \\
{ }^{3} \mathrm{~F}\end{array}$ \\
\hline$" \prime$ & $2[1 / 2]$ & $\begin{array}{l}1 \\
0\end{array}$ & $\begin{array}{l}100189.69 \\
102502.41\end{array}$ & & $\begin{array}{l}62 \\
94\end{array}$ & $\begin{array}{l}\text { or } \\
\text { or }\end{array}$ & $\begin{array}{l}33 \\
81\end{array}$ & $\begin{array}{l}{ }^{3} \mathrm{D} \\
{ }^{3} \mathrm{P}\end{array}$ \\
\hline $4 f\left({ }^{2} \mathrm{~F}_{5 / 2}^{\circ}\right) 7 p_{1 / 2}$ & $(5 / 2,1 / 2)$ & $\begin{array}{l}3 \\
2\end{array}$ & $\begin{array}{l}100662.63 \\
100734.04\end{array}$ & & $\begin{array}{l}98 \\
94\end{array}$ & & 6 & $(5 / 2,3 / 2)$ \\
\hline $4 f\left({ }^{2} \mathrm{~F}_{7 / 2}^{\circ}\right) 5 f$ & $2[9 / 2]$ & $\begin{array}{l}5 \\
4\end{array}$ & $\begin{array}{l}101178.46 \\
102566.29\end{array}$ & & $\begin{array}{l}83 \\
74\end{array}$ & $\begin{array}{l}\text { or } \\
\text { or }\end{array}$ & $\begin{array}{l}77 \\
42\end{array}$ & $\begin{array}{l}{ }^{3} \mathrm{G} \\
{ }^{1} \mathrm{G}\end{array}$ \\
\hline "' & $2[5 / 2]$ & $\begin{array}{l}3 \\
2\end{array}$ & $\begin{array}{l}101343.93 \\
103231.23\end{array}$ & & $\begin{array}{l}66 \\
62\end{array}$ & $\begin{array}{l}\text { or } \\
\text { or }\end{array}$ & $\begin{array}{l}55 \\
36\end{array}$ & $\begin{array}{l}{ }^{3} \mathrm{D} \\
{ }^{3} \mathrm{P}\end{array}$ \\
\hline " & ${ }^{2}[13 / 2]$ & $\begin{array}{l}7 \\
6\end{array}$ & $\begin{array}{l}101564.83 \\
103676.13\end{array}$ & & $\begin{array}{r}100 \\
67\end{array}$ & $\begin{array}{l}\text { or } \\
\text { or }\end{array}$ & $\begin{array}{r}100 \\
81\end{array}$ & $\begin{array}{l}{ }^{3} \mathrm{I} \\
{ }^{1} \mathrm{I}\end{array}$ \\
\hline$"$, & $2[3 / 2]$ & $\begin{array}{l}1 \\
2\end{array}$ & $\begin{array}{l}101647.49 \\
104177.07\end{array}$ & & $\begin{array}{l}46 \\
74\end{array}$ & $\begin{array}{l}\text { or } \\
\text { or }\end{array}$ & $\begin{array}{l}50 \\
55\end{array}$ & $\begin{array}{l}{ }^{3} \mathrm{~S} \\
{ }^{3} \mathrm{P}\end{array}$ \\
\hline $4 f\left({ }^{2} \mathrm{~F}_{5 / 2}^{\circ}\right) 7 p_{3 / 2}$ & $\left(5 / 2,{ }^{3} / 2\right)$ & $\begin{array}{l}3 \\
2 \\
4 \\
1\end{array}$ & $\begin{array}{l}101821.97 \\
102173.68 \\
102221.92 \\
102369.48\end{array}$ & & $\begin{array}{r}98 \\
94 \\
98 \\
100\end{array}$ & & 6 & $(5 / 2,1 / 2)$ \\
\hline $4 f\left({ }^{2} \mathrm{~F}_{/ / 2}^{\circ}\right) 5 f$ & $2[11 / 2]$ & $\begin{array}{l}5 \\
6\end{array}$ & $\begin{array}{l}102408.70 \\
102897.68\end{array}$ & & $\begin{array}{l}72 \\
77\end{array}$ & $\begin{array}{l}\text { or } \\
\text { or }\end{array}$ & $\begin{array}{l}83 \\
83\end{array}$ & ${ }^{3} \mathrm{H}$ \\
\hline " & $2[7 / 2]$ & $\begin{array}{l}3 \\
4\end{array}$ & $\begin{array}{l}102649.22 \\
103351.21\end{array}$ & & $\begin{array}{l}55 \\
83\end{array}$ & $\begin{array}{l}\text { or } \\
\text { or }\end{array}$ & $\begin{array}{l}83 \\
67\end{array}$ & ${ }^{3} \mathrm{~F}$ \\
\hline $4 f\left({ }^{2} \mathrm{~F}_{7 / 2}^{\circ}\right) 7 p_{1 / 2}$ & $(7 / 2,1 / 2)$ & $\begin{array}{l}3 \\
4\end{array}$ & $\begin{array}{l}102961.29 \\
103079.67\end{array}$ & & $\begin{array}{l}94 \\
92\end{array}$ & & $\begin{array}{l}4 \\
5\end{array}$ & $\left({ }^{7} / 2,3 / 2\right)$ \\
\hline $4 f\left({ }^{2} \mathrm{~F}_{7 / 2}^{\circ}\right) 5 f$ & $2[1 / 2]$ & $\begin{array}{l}1 \\
0\end{array}$ & 103612.68 & & $\begin{array}{l}58 \\
94\end{array}$ & $\begin{array}{l}\text { or } \\
\text { or }\end{array}$ & $\begin{array}{l}85 \\
81\end{array}$ & $\begin{array}{l}{ }^{3} \mathrm{P} \\
{ }^{1} \mathrm{~S}\end{array}$ \\
\hline $4 f\left({ }^{2} \mathrm{~F}_{7 / 2}^{\circ}\right) 7 p_{3 / 2}$ & $(7 / 2,3 / 2)$ & $\begin{array}{l}4 \\
3 \\
5 \\
2\end{array}$ & $\begin{array}{l}104289.06 \\
104293.20 \\
104351.04 \\
104840.50\end{array}$ & & $\begin{array}{r}94 \\
94 \\
100 \\
100\end{array}$ & & $\begin{array}{l}5 \\
6\end{array}$ & $\begin{array}{l}(7 / 2,1 / 2) \\
(7 / 2,1 / 2)\end{array}$ \\
\hline $4 f\left({ }^{2} \mathrm{~F}_{5 / 2}^{\circ}\right) 6 f$ & $2[7 / 2]$ & $\begin{array}{l}3 \\
4\end{array}$ & $\begin{array}{l}122160.10 \\
122628.94\end{array}$ & & $\begin{array}{l}79 \\
80\end{array}$ & & 21 & $\left({ }^{2} \mathrm{~F}_{5 / 2}^{\circ}\right)^{2}\left[{ }^{5} / 2\right]$ \\
\hline$" \prime$ & $2[11 / 2]$ & $\begin{array}{l}5 \\
6\end{array}$ & $\begin{array}{l}122289.31 \\
122870.19\end{array}$ & & $\begin{array}{l}75 \\
92\end{array}$ & & 25 & $\left({ }^{2} \mathrm{~F}_{5 / 2}^{\circ}\right){ }^{2}\left[{ }^{9} / 2\right]$ \\
\hline " & $2[9 / 2]$ & $\begin{array}{l}5 \\
4\end{array}$ & $\begin{array}{l}122611.07 \\
123201.62\end{array}$ & & $\begin{array}{l}70 \\
83\end{array}$ & & 25 & $\left({ }^{2} \mathrm{~F}_{5 / 2}^{\circ}\right)^{2}[11 / 2]$ \\
\hline " & $2[5 / 2]$ & $\begin{array}{l}3 \\
2\end{array}$ & $\begin{array}{l}122688.60 \\
123555.41\end{array}$ & & $\begin{array}{l}74 \\
67\end{array}$ & & $\begin{array}{l}20 \\
31\end{array}$ & $\begin{array}{l}\left({ }^{2} \mathrm{~F}_{5 / 2}^{\circ}\right){ }^{2}\left[{ }^{7} / 2\right] \\
\left({ }^{2} \mathrm{~F}_{5 / 2}^{\circ}\right)^{2}\left[{ }^{3} / 2\right]\end{array}$ \\
\hline$" \prime$ & $2[3 / 2]$ & $\begin{array}{l}1 \\
2\end{array}$ & 122807.84 & & $\begin{array}{l}88 \\
59\end{array}$ & & 32 & $\left({ }^{2} \mathrm{~F}_{5 / 2}^{\circ}\right){ }^{2}\left[{ }^{5} / 2\right]$ \\
\hline$" \prime$ & $2[1 / 2]$ & $\begin{array}{l}1 \\
0\end{array}$ & 122980.12 & & $\begin{array}{l}83 \\
99\end{array}$ & & & \\
\hline $4 f\left({ }^{2} \mathrm{~F}_{7 / 2}^{\circ}\right) 6 f$ & $2[9 / 2]$ & $\begin{array}{l}5 \\
4\end{array}$ & $\begin{array}{l}124433.38 \\
125091.24\end{array}$ & & $\begin{array}{l}80 \\
74\end{array}$ & & $\begin{array}{l}20 \\
20\end{array}$ & $\begin{array}{l}\left({ }^{2} \mathrm{~F}_{7 / 2}^{\circ}\right)^{2}\left[{ }^{11} / 2\right] \\
\left({ }^{2} \mathrm{~F}_{7 / 2}^{\circ}\right)^{2}{ }^{2}[/ / 2]\end{array}$ \\
\hline
\end{tabular}


Ce III, Even Parity—Continued

\begin{tabular}{|c|c|c|c|c|c|c|}
\hline Configuration & Term & $J$ & $\begin{array}{l}\text { Level } \\
\left(\mathrm{cm}^{-1}\right)\end{array}$ & $g$ & & Leading percentages \\
\hline $4 f\left({ }^{2} \mathrm{~F}_{7 / 2}^{\circ}\right) 6 f$ & $2[5 / 2]$ & $\begin{array}{l}3 \\
2\end{array}$ & $\begin{array}{l}124510.28 \\
125399.50\end{array}$ & & $\begin{array}{l}64 \\
74\end{array}$ & $\begin{array}{ll}36 & \left({ }^{2} \mathrm{~F}_{7 / 2}^{\circ}\right)^{2}\left[{ }^{2}[/ 2]\right. \\
18 & \left({ }^{2} \mathrm{~F}_{7 / 2}^{\circ}\right)^{2}\left[{ }^{2}[1 / 2]\right.\end{array}$ \\
\hline$"$ & ${ }^{2}[13 / 2]$ & $\begin{array}{l}7 \\
6\end{array}$ & $\begin{array}{l}124609.73 \\
125710.16\end{array}$ & & $\begin{array}{r}100 \\
59\end{array}$ & $36 \quad\left({ }^{2} \mathrm{~F}^{\circ} / / 2\right){ }^{2}\left[{ }^{11 / 2}\right]$ \\
\hline$"$ & ${ }^{2}[11 / 2]$ & $\begin{array}{l}5 \\
6\end{array}$ & $\begin{array}{l}125006.22 \\
125301.13\end{array}$ & & $\begin{array}{l}80 \\
62\end{array}$ & 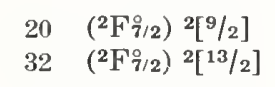 \\
\hline$"$ & $2[7 / 2]$ & $\begin{array}{l}3 \\
4\end{array}$ & $\begin{array}{l}125132.28 \\
125615.94\end{array}$ & & $\begin{array}{l}60 \\
80\end{array}$ & $\begin{array}{ll}35 & \left({ }^{2} \mathrm{~F}_{7 / 2}^{\circ}\right)^{2}[5 / 2] \\
20 & \left({ }^{2} \mathrm{~F}_{7 / 2}^{\circ}\right)^{2}[9 / 2]\end{array}$ \\
\hline $4 f\left({ }^{2} \mathrm{~F}_{7 / 2}\right) 6 f$ & $2[3 / 2]$ & $\begin{array}{l}1 \\
2\end{array}$ & 126052.62 & & $\begin{array}{l}66 \\
77\end{array}$ & $\begin{array}{lll}31 & \left({ }^{2} \mathrm{~F}_{\mathrm{T} / 2}^{\circ}\right) & { }^{2}[1 / 2] \\
21 & \left({ }^{2} \mathrm{~F}^{7} / 2\right) & { }^{2}[5 / 2]\end{array}$ \\
\hline $6 p^{2} ?$ & ${ }^{3} \mathrm{P} ?$ & $\begin{array}{l}0 \\
1 \\
2\end{array}$ & 130713.26 & & & \\
\hline $5 d 6 d$ & ${ }^{3} \mathrm{D}$ & $\begin{array}{l}1 \\
2 \\
3\end{array}$ & $\begin{array}{l}136321.39 \\
137902.90\end{array}$ & & & \\
\hline $5 d 6 d$ & ${ }^{1} \mathrm{~F}$ & 3 & 136447.67 & & & \\
\hline $5 d 6 d$ & ${ }^{3} \mathrm{~F}$ & $\begin{array}{l}2 \\
3 \\
4\end{array}$ & $\begin{array}{l}138264.72 \\
139477.67\end{array}$ & & & \\
\hline $5 d 6 d$ & ${ }^{3} \mathrm{P}$ & $\begin{array}{l}0 \\
1 \\
2\end{array}$ & 141104.03 & & & \\
\hline $5 d 6 d$ & ${ }^{1} \mathrm{D}$ & 2 & 141803.07 & & & \\
\hline Ce IV $\left({ }^{2} \mathrm{~F}_{5 / 2}^{\circ}\right)$ & Limit & & 162903 & & & \\
\hline
\end{tabular}

Ce III, Odd Parity

\begin{tabular}{|c|c|c|c|c|c|c|c|}
\hline Configuration & Term & $J$ & $\begin{array}{c}\text { Level } \\
\left(\mathrm{cm}^{-1}\right)\end{array}$ & $g$ & \multicolumn{3}{|c|}{ Leading percentages } \\
\hline $4 f 5 d$ & ${ }^{1} \mathrm{G}^{\circ}$ & 4 & 3276.66 & 0.99 & 72 & 22 & ${ }^{3} \mathrm{H}^{\circ}$ \\
\hline $4 f 5 d$ & ${ }^{3} \mathrm{~F}^{\circ}$ & $\begin{array}{l}2 \\
3 \\
4\end{array}$ & $\begin{array}{l}3821.53 \\
5502.37 \\
7150.05\end{array}$ & $\begin{array}{l}0.76 \\
1.10 \\
1.30\end{array}$ & $\begin{array}{l}72 \\
96 \\
86\end{array}$ & $\begin{array}{r}27 \\
3 \\
8\end{array}$ & $\begin{array}{l}{ }^{1} \mathrm{D}^{\circ} \\
{ }^{3} \mathrm{G}^{\circ} \\
{ }^{1} \mathrm{G}^{\circ}\end{array}$ \\
\hline $4 f 5 d$ & ${ }^{3} \mathrm{H}^{\circ}$ & $\begin{array}{l}4 \\
5 \\
6\end{array}$ & $\begin{array}{l}5127.27 \\
6361.27 \\
8349.99\end{array}$ & $\begin{array}{l}0.87 \\
1.07 \\
1.17\end{array}$ & $\begin{array}{r}77 \\
100 \\
100\end{array}$ & 18 & ${ }^{1} \mathrm{G}^{\circ}$ \\
\hline $4 f 5 d$ & ${ }^{3} \mathrm{G}^{\circ}$ & $\begin{array}{l}3 \\
4 \\
5\end{array}$ & $\begin{array}{l}6265.21 \\
7836.72 \\
9325.51\end{array}$ & $\begin{array}{l}0.76 \\
1.06 \\
1.22:\end{array}$ & $\begin{array}{l}93 \\
94 \\
99\end{array}$ & $\begin{array}{l}4 \\
4 \\
1\end{array}$ & $\begin{array}{l}{ }^{3} \mathrm{~F}^{\circ} \\
{ }^{3} \mathrm{~F}^{\circ} \\
{ }^{1} \mathrm{H}^{\circ}\end{array}$ \\
\hline $4 f 5 d$ & ${ }^{1} \mathrm{D}^{\circ}$ & 2 & 6571.36 & 0.88 & 67 & 27 & ${ }^{3} \mathrm{~F}^{\circ}$ \\
\hline $4 f 5 d$ & ${ }^{3} \mathrm{D}^{\circ}$ & $\begin{array}{l}1 \\
2 \\
3\end{array}$ & $\begin{array}{r}8922.05 \\
9900.49 \\
10126.53\end{array}$ & $\begin{array}{l}0.52: \\
1.18 \\
1.34\end{array}$ & $\begin{array}{l}97 \\
95 \\
74\end{array}$ & $\begin{array}{r}2 \\
4 \\
26\end{array}$ & $\begin{array}{l}{ }^{1} \mathrm{P}^{\circ} \\
{ }^{1} \mathrm{D}^{\circ} \\
{ }^{1} \mathrm{~F}^{\circ}\end{array}$ \\
\hline
\end{tabular}


Ce III, Odd Parity-Continued

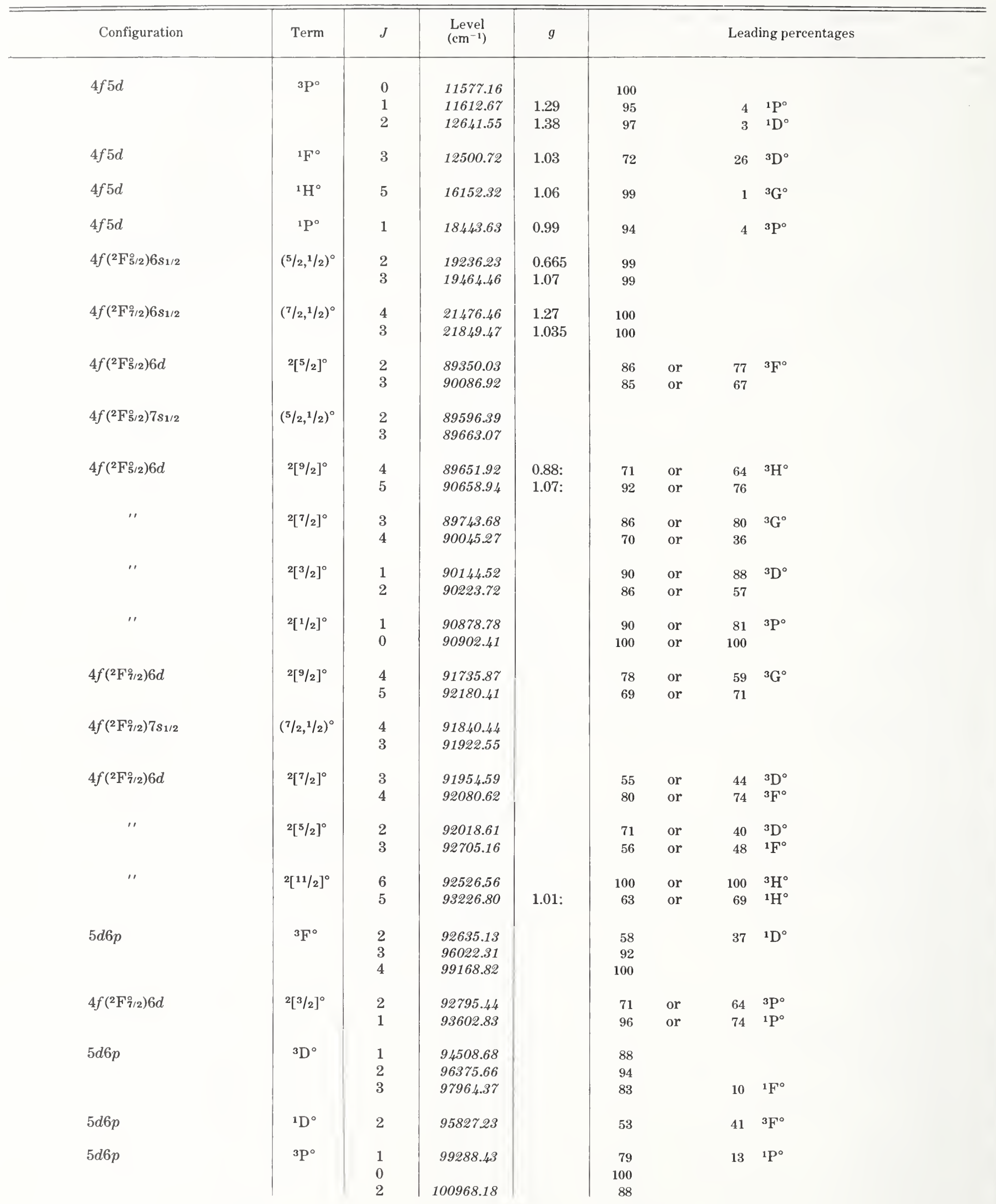


Ce III, Odd Parity-Continued

\begin{tabular}{|c|c|c|c|c|c|c|c|c|}
\hline Configuration & Term & $J$ & $\begin{array}{l}\text { Level } \\
\left(\mathrm{cm}^{-1}\right)\end{array}$ & $g$ & \multicolumn{4}{|c|}{ Leading percentages } \\
\hline $5 d 6 p$ & ${ }^{1} \mathrm{P}^{0}$ & 1 & $102249.70 ?$ & & 79 & & 18 & ${ }^{3} \mathrm{P}^{\circ}$ \\
\hline $5 d 6 p$ & ${ }^{1} \mathrm{~F}^{\circ}$ & 3 & 102369.16 & & 90 & & 10 & ${ }^{3} \mathrm{D}^{\circ}$ \\
\hline $4 f\left({ }^{2} \mathrm{~F}_{5 / 2}^{\circ}\right) 8 s_{1 / 2}$ & $\left(5 / 2,{ }^{1} / 2\right)^{\circ}$ & $\begin{array}{l}2 \\
3\end{array}$ & $\begin{array}{l}117949.15 \\
117986.06\end{array}$ & & & & & $\cdot$ \\
\hline $4 f\left({ }^{2} \mathrm{~F}_{5 / 2}^{\circ}\right) 7 d$ & ${ }^{2}[5 / 2]^{\circ}$ & $\begin{array}{l}2 \\
3\end{array}$ & $\begin{array}{l}118290.69 \\
118588.24\end{array}$ & & $\begin{array}{l}83 \\
97\end{array}$ & $\begin{array}{l}\text { or } \\
\text { or }\end{array}$ & $\begin{array}{l}95 \\
88\end{array}$ & $\begin{array}{l}\left({ }^{2} \mathrm{~F}_{5 / 2}^{\circ}\right) 7 d_{3 / 2} \\
\left({ }^{2} \mathrm{~F}_{5 / 2}^{\circ}\right) 7 d_{5 / 2}\end{array}$ \\
\hline$" \prime$ & ${ }^{2}[9 / 2]^{\circ}$ & $\begin{array}{l}4 \\
5\end{array}$ & $\begin{array}{l}118312.48 \\
118794.12\end{array}$ & & $\begin{array}{l}50 \\
98\end{array}$ & $\begin{array}{l}\text { or } \\
\text { or }\end{array}$ & $\begin{array}{l}81 \\
98\end{array}$ & $\begin{array}{l}\left({ }^{2} \mathrm{~F}_{5 / 2}^{\circ}\right) 7 d_{3 / 2} \\
\left({ }^{2} \mathrm{~F}_{5 / 2}^{\circ}\right) 7 d_{5 / 2}\end{array}$ \\
\hline$\prime \prime$ & ${ }^{2}\left[{ }^{7} / 2\right]^{\circ}$ & $\begin{array}{l}3 \\
4\end{array}$ & $\begin{array}{l}118318.07 \\
118476.74\end{array}$ & & $\begin{array}{l}97 \\
50\end{array}$ & $\begin{array}{l}\text { or } \\
\text { or }\end{array}$ & $\begin{array}{l}88 \\
81\end{array}$ & $\begin{array}{l}\left({ }^{2} \mathrm{~F}_{5 / 2}^{\circ}\right) 7 d_{3 / 2} \\
\left({ }^{2} \mathrm{~F}_{5 / 2}^{\circ}\right) 7 d_{5 / 2}\end{array}$ \\
\hline$"$ & ${ }^{2}[3 / 2]^{\circ}$ & $\begin{array}{l}2 \\
1\end{array}$ & $\begin{array}{l}118665.91 \\
118682.98\end{array}$ & & $\begin{array}{l}83 \\
98\end{array}$ & $\begin{array}{l}\text { or } \\
\text { or }\end{array}$ & $\begin{array}{l}95 \\
56\end{array}$ & $\begin{array}{l}\left({ }^{2} \mathrm{~F}_{5 / 2}^{\circ}\right) 7 d_{5 / 2} \\
\left({ }^{2} \mathrm{~F}_{5 / 2}^{\circ}\right) 7 d_{3 / 2}\end{array}$ \\
\hline$" \prime$ & ${ }^{2}[1 / 2]^{\circ}$ & $\begin{array}{l}0 \\
1\end{array}$ & 119043.35 & & $\begin{array}{r}100 \\
98\end{array}$ & $\begin{array}{l}\text { or } \\
\text { or }\end{array}$ & $\begin{array}{r}100 \\
57\end{array}$ & $\left({ }^{2} \mathrm{~F}_{5 / 2}^{\circ}\right) 7 d_{5 / 2}$ \\
\hline $4 f\left({ }^{2} \mathrm{~F}_{7 / 2}\right) 8 s_{1 / 2}$ & $(7 / 2,1 / 2)^{\circ}$ & $\begin{array}{l}4 \\
3\end{array}$ & $\begin{array}{l}120199.11 \\
120249.22\end{array}$ & & & & & \\
\hline $4 f\left({ }^{2} \mathrm{~F}_{7 / 2}^{\circ}\right) \tau d$ & ${ }^{2}[9 / 2]^{\circ}$ & $\begin{array}{l}4 \\
5\end{array}$ & $\begin{array}{l}120467.78 \\
120646.18\end{array}$ & & $\begin{array}{l}86 \\
84\end{array}$ & $\begin{array}{l}\text { or } \\
\text { or }\end{array}$ & $\begin{array}{l}97 \\
52\end{array}$ & $\begin{array}{l}\left({ }^{2} \mathrm{~F}_{7 / 2}^{\circ}\right) 7 d_{3 / 2} \\
\left({ }^{2} \mathrm{~F}_{7 / 2}^{\circ}\right) 7 d_{5 / 2}\end{array}$ \\
\hline$"$ & $2[7 / 2]^{\circ}$ & $\begin{array}{l}3 \\
4\end{array}$ & $\begin{array}{l}120652.44 \\
120685.11\end{array}$ & & $\begin{array}{l}55 \\
86\end{array}$ & $\begin{array}{l}\text { or } \\
\text { or }\end{array}$ & $\begin{array}{l}99 \\
97\end{array}$ & $\begin{array}{l}\left({ }^{2} \mathrm{~F}_{7 / 2}^{\circ}\right) 7 d_{3 / 2} \\
\left({ }^{2} \mathrm{~F}_{7 / 2}^{\circ}\right) 7 d_{5 / 2}\end{array}$ \\
\hline$"$ & ${ }^{2}[5 / 2]^{\circ}$ & $\begin{array}{l}2 \\
3\end{array}$ & $\begin{array}{l}120739.95 \\
121001.44\end{array}$ & & $\begin{array}{l}95 \\
55\end{array}$ & $\begin{array}{l}\text { or } \\
\text { or }\end{array}$ & $\begin{array}{l}58 \\
99\end{array}$ & $\begin{array}{l}\left({ }^{2} \mathrm{~F}_{7 / 2}^{\circ}\right) 7 d d_{3 / 2} \\
\left({ }^{2} \mathrm{~F}_{7 / 2}^{\circ}\right) 7 d_{5 / 2}\end{array}$ \\
\hline$" \prime$ & ${ }^{2}[11 / 2]^{\circ}$ & $\begin{array}{l}6 \\
5\end{array}$ & $\begin{array}{l}120845.67 \\
121056.71\end{array}$ & & $\begin{array}{r}100 \\
83\end{array}$ & $\begin{array}{l}\text { or } \\
\text { or }\end{array}$ & $\begin{array}{r}100 \\
50\end{array}$ & $\begin{array}{l}\left({ }^{2} \mathrm{~F}_{7 / 2}^{\circ}\right) 7 d_{5 / 2} \\
\left({ }^{2} \mathrm{~F}_{7 / 2}^{\circ}\right) 7 d_{3 / 2}\end{array}$ \\
\hline "' & ${ }^{2}[3 / 2]^{\circ}$ & $\begin{array}{l}2 \\
1\end{array}$ & $\begin{array}{l}121095.78 \\
121559.51\end{array}$ & & $\begin{array}{l}95 \\
98\end{array}$ & $\begin{array}{l}\text { or } \\
\text { or }\end{array}$ & $\begin{array}{l}58 \\
98\end{array}$ & $\left({ }^{2} \mathrm{~F}_{7 / 2}^{\circ}\right) 7 d_{5 / 2}$ \\
\hline $4 f\left({ }^{2} \mathrm{~F}_{5 / 2}^{\circ}\right) 5 g$ & ${ }^{2}[9 / 2]^{\circ}$ & $\begin{array}{l}4 \\
5\end{array}$ & $\begin{array}{l}122905.69 \\
122908.89\end{array}$ & & $\begin{array}{l}100 \\
100\end{array}$ & & & \\
\hline " & $2[11 / 2]^{\circ}$ & $\begin{array}{l}6 \\
5\end{array}$ & $\begin{array}{l}122919.83 \\
122922.37\end{array}$ & & $\begin{array}{l}100 \\
100\end{array}$ & & & \\
\hline$"$ & $2[7 / 2]^{\circ}$ & $\begin{array}{l}4 \\
3\end{array}$ & $\begin{array}{l}122932.21 \\
122933.38\end{array}$ & & $\begin{array}{l}100 \\
100\end{array}$ & & & \\
\hline$" \prime$ & ${ }^{2}[5 / 2]^{\circ}$ & $\begin{array}{l}2 \\
3\end{array}$ & $\begin{array}{l}122976.30 \\
122978.36\end{array}$ & & $\begin{array}{l}100 \\
100\end{array}$ & & & \\
\hline$" \prime$ & $2[13 / 2]^{\circ}$ & $\begin{array}{l}6 \\
7\end{array}$ & $\begin{array}{l}123010.29 \\
123017.02\end{array}$ & & $\begin{array}{l}100 \\
100\end{array}$ & & & \\
\hline " & ${ }^{2}[3 / 2]^{\circ}$ & $\begin{array}{l}2 \\
1\end{array}$ & $\begin{array}{l}123028.39 \\
123029.01\end{array}$ & & $\begin{array}{l}100 \\
100\end{array}$ & & & \\
\hline $4 f\left({ }^{2} \mathrm{~F}_{7 / 2}^{\circ}\right) 5 g$ & $2[11 / 2]^{\circ}$ & $\begin{array}{l}6 \\
5\end{array}$ & $\begin{array}{l}125155.89 \\
125158.97\end{array}$ & & $\begin{array}{l}100 \\
100\end{array}$ & & & \\
\hline$"$ & $2[9 / 2]^{\circ}$ & $\begin{array}{l}4 \\
5\end{array}$ & $\begin{array}{l}125164.86 \\
125168.37\end{array}$ & & $\begin{array}{l}100 \\
100\end{array}$ & & & \\
\hline$"$ & $2[13 / 2]^{\circ}$ & $\begin{array}{l}6 \\
7\end{array}$ & $\begin{array}{l}125181.54 \\
125186.61\end{array}$ & & $\begin{array}{l}100 \\
100\end{array}$ & & & \\
\hline
\end{tabular}


Ce III, Odd Parity-Continued

\begin{tabular}{|c|c|c|c|c|c|c|}
\hline Configuration & Term & $J$ & $\begin{array}{l}\text { Level } \\
\left(\mathrm{cm}^{-1}\right)\end{array}$ & $g$ & & Leading percentages \\
\hline $4 f\left({ }^{2} \mathrm{~F}_{7 / 2}^{\circ}\right) 5 g$ & ${ }^{2}\left[{ }^{7} / 2\right]^{\circ}$ & $\begin{array}{l}4 \\
3\end{array}$ & $\begin{array}{l}125193.91 \\
125196.03\end{array}$ & & $\begin{array}{l}100 \\
100\end{array}$ & \\
\hline "' & ${ }^{2}[5 / 2]^{\circ}$ & $\begin{array}{l}2 \\
3\end{array}$ & $\begin{array}{l}125230.90 \\
125232.67\end{array}$ & & $\begin{array}{l}100 \\
100\end{array}$ & \\
\hline " & ${ }^{2}[3 / 2]^{\circ}$ & $\begin{array}{l}2 \\
1\end{array}$ & $\begin{array}{l}125268.40 \\
125269.29\end{array}$ & & $\begin{array}{l}100 \\
100\end{array}$ & \\
\hline$"$ & $2[15 / 2]^{\circ}$ & $\begin{array}{l}8 \\
7\end{array}$ & $\begin{array}{l}125270.97 \\
125279.58\end{array}$ & & $\begin{array}{l}100 \\
100\end{array}$ & \\
\hline$"$ & $2[1 / 2]^{\circ}$ & $\begin{array}{l}0 \\
1\end{array}$ & $\begin{array}{l}125295.21 \\
125296.65\end{array}$ & & $\begin{array}{l}100 \\
100\end{array}$ & \\
\hline Ce IV $\left({ }^{2} \mathrm{~F}_{5 / 2}^{\circ}\right)$ & Limit & & 162903 & & & \\
\hline
\end{tabular}




\section{Ce IV}

(Cs I sequence; 55 electrons)

$Z=58$

Ground state $\left(1 s^{2} 2 s^{2} 2 p^{6} 3 s^{2} 3 p^{6} 3 d^{10} 4 s^{2} 4 p^{6} 4 d^{10} 5 s^{2} 5 p^{6}\right) 4 f^{2} \mathrm{~F}_{5 / 2}^{\circ}$

Ionization energy $296470 \pm 40 \mathrm{~cm}^{-1}$

$36.758 \pm 0.005 \mathrm{eV}$

Gibbs and White [1929] and Badami [1931] classified the $6{ }^{2} \mathrm{~S}-6 p{ }^{2} \mathrm{P}^{\circ}$ transitions, but Badami's extension of the analysis proved incorrect. The levels and interpretation given here are due to Lang [1936a], who reobserved the spectrum with spark sources. He lists 31 classified lines in the range $443-2778 \AA$.

Lang commented that the $6 d^{2} \mathrm{D}$ levels are "puzzling, as there seems to be no reason for their inversion, but no other possibility could be found in the spectrum." Reader has confirmed the correctness of these levels. He suggests that their perturbation is probably due to interaction with one or more configurations of the type $5 p^{5} 4 f n l$ (probably $5 p^{5} 4 f 5 d$ and/or $\left.5 p^{5} 4 f 6 s\right)$.

On the basis of the accurate ionization potential [Sugar and Reader] and the general behavior of $\mathrm{ng}$ series (in La III, for example), we find Lang's level for $6 g^{2} \mathrm{G}$ to be about 850 $\mathrm{cm}^{-1}$ above the expected position. Since a perturbation of this magnitude seems unlikely and the level is based on two weak lines, we have omitted it here. The $5 g^{2} \mathrm{G}$ level is based on four combinations, and its quantum defect of 0.02 is in accord with similar spectra.

The four Zeeman patterns observed by van de Vliet for the $6 s^{2} \mathrm{~S}-6 p{ }^{2} \mathrm{P}^{\circ}$ and $5 f^{2} \mathrm{~F}^{\circ}-$ $7 d^{2} \mathrm{D}$ lines are consistent with these classifications. The three $g$ values we have derived from his data are close to the theoretical single-electron values.

Edwin and King resolved the ${ }^{140} \mathrm{Ce}-{ }^{142} \mathrm{Ce}$ isotope shift for each of the $6 s{ }^{2} \mathrm{~S}-6 p{ }^{2} \mathrm{P}^{\circ}$ lines in Ce IV, using a pulsed hollow-cathode source; “... we believe this is the first time a fourth spectrum has been obtained with emission lines narrow enough for high-resolution measurements to be made on them."

\section{References}

Badami, J. S., Proc. Phys. Soc. London 43, 53 (1931). EL CL

Edwin, R. P., and King, W. H., J. Phys. B 2, 260 (1969). IS

Gibbs, R. C., and White, H. E., Phys. Rev. 33, 157 (1929). EL CL

Lang, R. J., Can. J. Res., Sect. A 13, 1 (1935); Sect. A 14, 127 (1936a). EL CL W IP

Lang, R. J., Phys. Rev. 49, 552 (1936b). EL CL W

Reader, J., private communication (1975).

Sugar, J., and Reader, J., J. Chem. Phys. 59, 2083 (1973). IP

van de Vliet, H. J., Thesis, Univ. Amsterdam, 85 pp. (1939). ZE

[July 1976] 
Ce IV

\begin{tabular}{|c|c|c|c|c|}
\hline Configuration & Term & $J$ & $\begin{array}{l}\text { Level } \\
\left(\mathrm{cm}^{-1}\right)\end{array}$ & $g$ \\
\hline $5 p^{6}\left({ }^{1} \mathrm{~S}\right) 4 f$ & ${ }^{2} \mathrm{~F}^{\circ}$ & $\begin{array}{l}5 / 2 \\
7 / 2\end{array}$ & $\begin{array}{r}0 \\
2253\end{array}$ & \\
\hline $5 p^{6}\left({ }^{1} \mathrm{~S}\right) 5 d$ & ${ }^{2} \mathrm{D}$ & $\begin{array}{l}3 / 2 \\
5 / 2\end{array}$ & $\begin{array}{l}49737 \\
52226\end{array}$ & \\
\hline $5 p^{8}\left({ }^{1} \mathrm{~S}\right) 6 s$ & ${ }^{2} \mathrm{~S}$ & $1 / 2$ & 86602 & 1.95 \\
\hline $5 p^{6\left({ }^{(} \mathrm{S}\right) 6 p}$ & ${ }^{2} \mathrm{P}^{\circ}$ & $\begin{array}{l}1 / 2 \\
3 / 2\end{array}$ & $\begin{array}{l}122585 \\
127292\end{array}$ & $\begin{array}{l}0.65 \\
1.36\end{array}$ \\
\hline $5 p^{6}\left({ }^{1} \mathrm{~S}\right) 6 d$ & ${ }^{2} \mathrm{D}$ & $\begin{array}{l}5 / 2 \\
3 / 2\end{array}$ & $\begin{array}{l}177198 \\
178913\end{array}$ & \\
\hline $5 p^{6}\left({ }^{1} \mathrm{~S}\right) 7 s$ & ${ }^{2} \mathrm{~S}$ & $1 / 2$ & 183502 & \\
\hline $5 p^{6}\left({ }^{1} \mathrm{~S}\right) 5 f$ & ${ }^{2} \mathrm{~F}^{\circ}$ & $\begin{array}{l}5 / 2 \\
7 / 2\end{array}$ & $\begin{array}{l}184545 \\
184746\end{array}$ & \\
\hline $5 p^{6}\left({ }^{1} \mathrm{~S}\right) 7 d$ & ${ }^{2} \mathrm{D}$ & $\begin{array}{l}3 / 2 \\
5 / 2\end{array}$ & $\begin{array}{l}221661 \\
221838\end{array}$ & \\
\hline $5 p^{6}\left({ }^{1} \mathrm{~S}\right) 8 s$ & ${ }^{2} \mathrm{~S}$ & $1 / 2$ & 225128 & \\
\hline $5 p^{6}\left({ }^{1} \mathrm{~S}\right) 5 g$ & ${ }^{2} \mathrm{G}$ & $7 / 2,9 / 2$ & 225625 & \\
\hline $\mathrm{Ce} v\left({ }^{1} \mathrm{~S}_{0}\right)$ & Limit & & 296470 & \\
\hline
\end{tabular}




\section{Ce V}

(Xe I sequence; 54 electrons)

$Z=58$

Ground state $\left(1 s^{2} 2 s^{2} 2 p^{6} 3 s^{2} 3 p^{6} 3 d^{10} 4 s^{2} 4 p^{6} 4 d^{10} 5 s^{2}\right) 5 p^{6}{ }^{1} \mathrm{~S}_{0}$

Ionization energy $528700 \pm 2000 \mathrm{~cm}^{-1}$

$65.55 \pm 0.25 \mathrm{eV}$

The five excited levels derive from five transitions to the ground level (365 to $552 \AA$ ). Reader and Ekberg based the identifications on comparisons along the $\mathrm{Xe} \mathrm{I}$ isoelectronic sequence through La IV. We have listed the $J=1$ upper levels as belonging to $J_{1} l$-coupling pairs, by analogy with La IV. Alternative Russell-Saunders names are given in the last column, the leading percentages for the $5 p^{5} 5 d$ levels being from a preliminary calculation by Hansen [1971].

Reader and Epstein derived the quoted ionization energy by using the $5 p^{5} 6 s$ levels, an estimated position for the $5 p^{5} 7 \mathrm{~s}$ levels, and an assumed value for the difference between the Rydberg denominators $n^{*}(7 s)-n^{*}(6 s)$.

\section{References}

Hansen, J., unpublished material (1971). PT

Reader, J., and Ekberg, J. 0., J. Opt. Soc. Am. 62, 464 (1972). EL CL W

Reader, J., and Epstein, G. L., J. Opt. Soc. Am. 65, 638 (1975). IP

[July 1976]

Ce $v$

\begin{tabular}{|c|c|c|c|c|c|c|}
\hline Configuration & Term & $J$ & $\begin{array}{l}\text { Level } \\
\left(\mathrm{cm}^{-1}\right)\end{array}$ & \multicolumn{3}{|c|}{ Leading percentages } \\
\hline $5 p^{6}$ & ${ }^{1 S}$ & 0 & 0 & & & \\
\hline $5 p^{5}\left({ }^{2} \mathrm{P}_{3 / 2}^{\circ}\right) 5 d$ & ${ }^{2}[1 / 2]^{\circ}$ & $\begin{array}{l}0 \\
1\end{array}$ & 181116 & $\begin{array}{l}\text { or } \\
\text { or }\end{array}$ & $\begin{array}{r}100 \\
93\end{array}$ & $\begin{array}{l}{ }^{3} \mathrm{P}^{\circ} \\
{ }^{3} \mathrm{P}^{\circ}\end{array}$ \\
\hline $5 p^{5}\left({ }^{2} \mathrm{P}_{3 / 2}^{\circ}\right) 5 d$ & $2[3 / 2]^{\circ}$ & $\begin{array}{l}2 \\
1\end{array}$ & 207057 & $\begin{array}{l}\text { or } \\
\text { or }\end{array}$ & $\begin{array}{l}75 \\
87\end{array}$ & $\begin{array}{l}{ }^{3} \mathrm{P}^{\circ} \\
{ }^{3} \mathrm{D}^{\circ}\end{array}$ \\
\hline $5 p^{5}\left({ }^{2} \mathrm{P}_{3 / 2}^{\circ}\right) 6 s$ & ${ }^{2}[3 / 2]^{\circ}$ & $\begin{array}{l}2 \\
1\end{array}$ & 247396 & $\begin{array}{l}\text { or } \\
\text { or }\end{array}$ & & $\begin{array}{l}{ }^{3} \mathrm{P}^{\circ} \\
{ }^{1} \mathrm{P}^{\circ}\end{array}$ \\
\hline $5 p^{5}\left({ }^{2} \mathrm{P}_{1 / 2}^{\circ}\right) 5 d$ & ${ }^{2}\left[{ }^{3} / 2\right]^{\circ}$ & $\begin{array}{l}2 \\
1\end{array}$ & 250399 & $\begin{array}{l}\text { or } \\
\text { or }\end{array}$ & $\begin{array}{l}51 \\
93\end{array}$ & $\begin{array}{l}{ }^{3} \mathrm{D}^{\circ} \\
{ }^{1} \mathrm{P}^{\circ}\end{array}$ \\
\hline $5 p^{5}\left({ }^{2} \mathrm{P}_{1 / 2}^{\circ}\right) 6 s$ & ${ }^{2}\left[{ }^{1} / 2\right]^{\circ}$ & $\begin{array}{l}0 \\
1\end{array}$ & 273477 & $\begin{array}{l}\text { or } \\
\text { or }\end{array}$ & & $\begin{array}{l}{ }^{3} \mathrm{P}^{\circ} \\
{ }^{3} \mathrm{P}^{\circ}\end{array}$ \\
\hline Ce vi $\left({ }^{2} \mathrm{P}_{3 / 2}^{\circ}\right)$ & Limit & & 528700 & & & \\
\hline
\end{tabular}




\section{Ce VI}

(I I sequence; 53 electrons)

Ground state $\left(1 s^{2} 2 s^{2} 2 p^{6} 3 s^{2} 3 p^{6} 3 d^{10} 4 s^{2} 4 p^{6} 4 d^{10}\right) 5 s^{2} 5 p^{5}{ }^{2} \mathrm{P}_{3 / 2}^{\circ}$

Ionization energy $626000 \pm 10000 \mathrm{~cm}^{-1}$

$77.6 \pm 1.2 \mathrm{eV}$

Reader and Ekberg classified the two transitions from the $5 s 5 p^{6}{ }^{2} \mathrm{~S}_{1 / 2}$ level to the ${ }^{2} \mathrm{P}^{\circ}$ ground-term levels, using comparisons along this isoelectronic sequence through La V. The value for the separation of the ${ }^{2} \mathrm{P}^{\circ}$ levels "is supported by additional measurements for transitions to $5 p^{5}{ }^{2} \mathrm{P}^{\circ}$ from as yet uninterpreted upper levels." The authors state that the $5 s^{2} 5 p^{4} 4 f$ configuration is expected "not far above the ground configuration" and suggest that the ground term is probably perturbed by interaction with this configuration.

Epstein and Reader derived the quoted ionization energy by an extrapolation based on the data for this sequence through La $\mathrm{V}$.

\section{References}

Epstein, G. L., and Reader, J., J. Opt. Soc. Am. 66, 590 (1976). IP

Reader, J., and Ekberg, J. O., J. Opt. Soc. Am. 62, 464 (1972). EL CL W

[July 1976]

Ce vi

\begin{tabular}{c|c|c|c}
\hline \hline Configuration & Term & $J$ & $\begin{array}{r}\text { Level } \\
\left(\mathrm{cm}^{-1}\right)\end{array}$ \\
\hline $5 s^{2}\left({ }^{1} \mathrm{~S}\right) 5 p^{5}$ & $2 \mathrm{P}^{\circ}$ & $\begin{array}{c}3 / 2 \\
1 / 2\end{array}$ & $\begin{array}{c}0 \\
25984\end{array}$ \\
$5 s 5 p^{6}$ & & $1 / 2$ & 160416 \\
& $2 \mathrm{~S}$ & $1 / 2$ & \\
\hline Ce VII ${ }^{\left(3 \mathrm{P}_{2}\right)}$ & Limit & & 626000 \\
\hline
\end{tabular}




\section{PRASEODYMIUM}

\section{$\operatorname{Pr}$ I}

59 electrons

$Z=59$

Ground state $\left(1 s^{2} 2 s^{2} 2 p^{6} 3 s^{2} 3 p^{6} 3 d^{10} 4 s^{2} 4 p^{6} 4 d^{10} 5 s^{2} 5 p^{6}\right) 4 f^{3} 6 s^{2}{ }^{4} \mathrm{I}_{9 / 2}^{\circ}$

Ionization energy $44070 \pm 40 \mathrm{~cm}^{-1}$

$5.464 \pm 0.005 \mathrm{eV}$

Identified odd configurations

$4 f^{3} 6 s^{2}, 4 f^{3} 5 d 6 s, 4 f^{2} 6 s^{2} 6 p ?, 4 f^{2} 5 d 6 s 6 p, 4 f^{2} 5 d^{2} 6 p ?$

Identified even configurations

$$
4 f^{2} 5 d 6 s^{2}, 4 f^{2} 5 d^{2} 6 s, 4 f^{3} 6 s 6 p, 4 f^{3} 5 d 6 p
$$

The levels are from unpublished tables of Blaise, Verges, Wyart, Camus, and Zalubas [1973a; 1973b], and Blaise and Ginibre [1976], who are extending this analysis. Zalubas and Borchardt [1973] reported the lowest three levels of the $4 f^{3} 6 s^{2}{ }^{4} I^{\circ}$ ground term and about 60 upper even levels.

The accuracy of the wavelength measurements for $\mathrm{Pr}$ I and $\mathrm{Pr}$ II is limited in general by unresolved hyperfine structure. The line list for the region 4000 to $12000 \AA$ has about 25000 $\operatorname{Pr} I$ lines [unpublished list for $\operatorname{Pr} I$ and $\operatorname{Pr}$ II; see Zalubas and Borchardt]. The list has been extended to $3 \mu \mathrm{m}$ at the Laboratoire Aimé Cotton, Orsay. Zalubas and Wilson [1964] give 3532 Pr I lines obtained in absorption in the range 1741-5839 $\AA$, and King's [1928] list has 1018 lines of $\operatorname{Pr} I$ and $\operatorname{Pr}$ II between 3111 and $6828 \AA$ with temperature classifications. Most of the 1460 stronger lines of $\operatorname{Pr} I$ and $\operatorname{Pr}$ II given by Meggers, Corliss, and Scribner [1975] belong to $\operatorname{Pr} I I$; the range is $2558-8715 \AA$, and energy-level classifications are listed for both spectra.

The Zeeman patterns are also affected by hyperfine structure, with a resultant lowering of the accuracy of the derived $g$ values [Blaise et al., 1973b; Blaise and Ginibre, 1976]. Except for the ${ }^{4} I^{\circ}$ ground term these are basically two-place values, although some values are given to the nearest $\mathbf{0 . 0 0 5}$. The more poorly determined $g$ values are followed by colons, and values obtained from ambiguous reductions of patterns are given with question marks.

Very accurate $g$ values for the levels of the ground term have been determined by the atomic-beam magnetic-resonance method. The values for the lowest three levels are from Böklen, Bossert, Foerster, Fuchs, and Nachtsheim [1975], and the value for the ${ }^{4}{ }_{15 / 2}^{\circ}$ level is from Lew [1970]. The values are rounded off to five decimal places from values given in the references with uncertainties from 1.5 to 4 units in the sixth place. Lew's [1953] original determination of the $J$ and $g$ values for the ${ }^{4} I_{9 / 2}^{\circ}$ level were important in establishing $4 f^{3} 6 s^{2}$ as the ground configuration of $\operatorname{Pr} \mathrm{I}$. The leading percentages for the ground-term levels are from Conway and Wybourne [1963].

Although the lower levels of several of the most important configurations have been identified, the interpretation of this spectrum is incomplete. The designations followed by question marks are regarded as tentative. Blaise, Verges, Wyart, and Camus [1975] have begun calculations for $\operatorname{Pr} \mathrm{I}$, and the percentages for the $4 f^{3} 5 d 6 \mathrm{~s}$ levels are from their results. The interpretation of some of the low even levels is also based in part on preliminary calculations. A more complete analysis and theoretical interpretation for $\operatorname{Pr}$ I will probably involve calculations in alternate coupling schemes, e.g., $J_{1} J_{2}$ coupling for the $4 f^{3} 6 s 6 p$ configuration. We have included parentages for some of the designations given without percentages. In some cases these are unique for the indicated order of coupling and final term, in other cases only probable.

The hyperfine structures of the levels have to some extent proved useful in the analysis [Blaise et al., 1973b]. Ginibre and Gerstenkorn [1970] began the theoretical and experimental work at Orsay on this aspect of the Pr I spectrum. Murakawa [1960] classified the strong $\operatorname{Pr}$ I line at $4951.37 \AA$ and analyzed its hyperfine structure. 
Worden, Conway, Paisner, and Solarz [1977] obtained the ionization energy from two-step photoionization threshold determinations carried out with laser techniques.

\section{References}

Blaise, J., and Ginibre, A., unpublished material (1976). EL ND ZE

Blaise, J., Verges, J., Wyart, J. F., and Camus, P., unpublished material (1975). PT

Blaise, J., Verges, J., Wyart, J. F., Camus, P., and Zalubas, R., J. Opt. Soc. Am. 63, 1315A (1973a). EL

Blaise, J., Verges, J., Wyart, J. F., Camus, P., and Zalubas, R., unpublished material (1973b). EL ND CL ZE Hfs

Böklen, K. D., Bossert, T., Foerster, W., Fuchs, H. H., and Nachtsheim, G., Z. Phys. A274, 195 (1975). ZE Hfs

Cabezas, A. Y., Lindgren, I. P. K., Marrus, R., and Nierenberg, W. A., Phys. Rev. 126, 1004 (1962). ZE Hfs

Conway, J. G., and Wybourne, B. G., Phys. Rev. 130, 2325 (1963). PT

Ginibre, A., and Gerstenkorn, S., unpublished material (1970). Hfs PT

King, A. S., Astrophys. J. 68, 194 (1928). W

Lew, H., Phys. Rev. 91, 619 (1953). ZE Hfs

Lew, H., Bull. Am. Phys. Soc. 15, 795 (1970). ZE Hfs

Meggers, W. F., Corliss, C. H., and Seribner, B. F., Nat. Bur. Stand. (U.S.), Monogr. 145, Part I, 403 pp. (1975). CL

Murakawa, K., J. Phys. Soc. Japan 15, 2306 (1960). EL CL Hfs

Worden, E. F, Conway, J. G., Paisner, J. A., and Solarz, R. W., unpublished material (1977). IP

Zalubas, R., and Borchardt, B. R., J. Opt. Soc. Am. 63, 102 (1973). EL ZE

Zalubas, R., and Wilson, M., J. Res. Nat. Bur. Stand. (U.S.) 69A, 59 (1965). W

[January 1977]

Pr I, Odd Parity

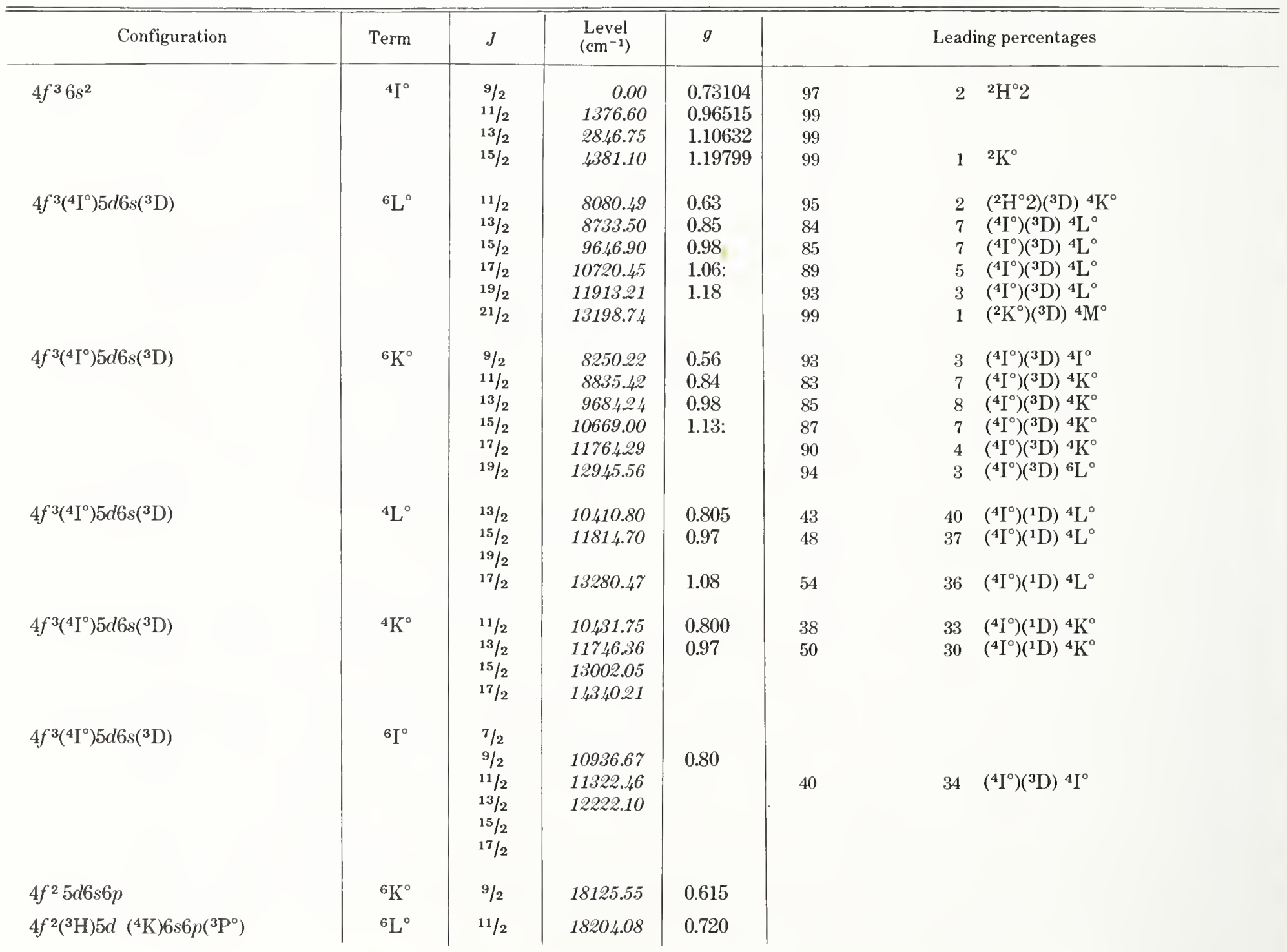


Pr I, Odd Parity-Continued

Pr I, Odd Parity-Continued

\begin{tabular}{|c|c|c|c|c|c|c|c|c|c|}
\hline Configuration & Term & $J$ & $\begin{array}{l}\text { Level } \\
\left(\mathrm{cm}^{-1}\right)\end{array}$ & $g$ & Configuration & Term & $J$ & $\begin{array}{l}\text { Level } \\
\left(\mathrm{cm}^{-1}\right)\end{array}$ & $g$ \\
\hline & \multirow{3}{*}{${ }^{6} \mathrm{~L}^{\circ}$} & $11 / 2$ & 18808.05 & 0.985 & & & $9 / 2$ & 23430.39 & \\
\hline \multirow[t]{2}{*}{$4 f^{2}\left({ }^{3} \mathrm{H}\right) 5 d\left({ }^{4} \mathrm{~K}\right) 6 s 6 p\left({ }^{3} \mathrm{P}^{\circ}\right)$} & & $13 / 2$ & 18829.41 & 0.97 & & & $11 / 2$ & 23475.11 & 0.965 \\
\hline & & $9 / 2$ & 18886.11 & 0.80 & & & $15 / 2$ & 23488.64 & 1.02 \\
\hline $4 f^{2} 5 d 6 s 6 p$ & ${ }^{6} \mathrm{~K}^{\circ}$ & $11 / 2$ & 18934.89 & 0.885 & & & $11 / 2$ & 23611.65 & 1.17 \\
\hline $4 f^{2} 5 d 6 s 6 p$ & ${ }^{6} \mathrm{I}^{\circ}$ & $7 / 2$ & 18987.10 & 0.64 & & & $11 / 2$ & 23767.22 & 0.925 \\
\hline $4 f^{2} 5 d 6 s 6 p$ & ${ }^{6} \mathrm{I}^{\circ}$ & $9 / 2$ & 19303.53 & 0.840 & & & $11 / 2$ & 23923.09 & 1.04 \\
\hline $4 f^{2} 5 d 6 s 6 p$ & & $13 / 2$ & 19315.96 & & & & $7 / 2$ & 23984.06 & 0.88 \\
\hline \multirow[t]{4}{*}{$4 f^{2}\left({ }^{3} \mathrm{H}\right) 6 s^{2} 6 p ?$} & \multirow[t]{4}{*}{${ }^{4} I^{\circ}$} & $9 / 2$ & 19339.86 & 0.77 & & & $13 / 2$ & 24116.09 & $1.00 ?$ \\
\hline & & $11 / 2$ & 19910.13 & 0.85 & & & $15 / 2$ & 24156.54 & 1.09 \\
\hline & & $11 / 2$ & 20249.54 & 1.035 & & & $13 / 2$ & 24390.15 & 1.020 \\
\hline & & $11 / 2$ & 20315.28 & 1.07 & & & $11 / 2$ & 24406.45 & 0.86 \\
\hline \multirow[t]{2}{*}{$4 f^{2} 5 d 6 s 6 p ?$} & \multirow[t]{2}{*}{${ }^{6} \mathrm{~K}^{\circ} ?$} & $13 / 2$ & 20339.47 & 1.05 & & & $15 / 2$ & 24528.91 & 1.105 \\
\hline & & $9 / 2$ & 20396.31 & 0.83 & & & $11 / 2$ & 24534.39 & 1.00 \\
\hline \multirow[t]{16}{*}{$4 f^{2} 5 d 6 s 6 p ?$} & \multirow[t]{16}{*}{${ }^{4} \mathrm{~L}^{\circ}$} & $13 / 2$ & 20439.49 & 0.915 & & & $7 / 2$ & 25013.78 & 0.960 \\
\hline & & $7 / 2$ & 20542.98 & 0.76 & & & $9 / 2$ & 25209.16 & 1.05 \\
\hline & & $11 / 2$ & 20583.92 & 1.06 & & & $13 / 2$ & 25236.52 & 1.005 \\
\hline & & $5 / 2$ & 20621.72 & 0.79 & & & $11 / 2$ & 25250.67 & 0.980 \\
\hline & & $15 / 2$ & 20652.01 & 1.135 & & & $11 / 2$ & 25280.40 & 1.11 \\
\hline & & $7 / 2$ & 20873.94 & $0.84:$ & & & $13 / 2$ & 25312.15 & 0.99 \\
\hline & & $11 / 2$ & 21013.27 & 0.84 & & & $11 / 2$ & 25366.96 & 1.07 \\
\hline & & $13 / 2$ & 21231.04 & & & & $13 / 2$ & 25382.49 & 1.145 \\
\hline & & $11 / 2$ & 21301.02 & $0.96:$ & & & $11 / 2$ & 25461.73 & 1.07 \\
\hline & & $5 / 2$ & 21369.79 & 0.63 & & & $11 / 2$ & 25551.27 & 1.02 \\
\hline & & $7 / 2$ & 21424.11 & 0.93 & & & $13 / 2$ & 25614.30 & 1.11 \\
\hline & & $13 / 2$ & 21466.44 & 1.05 & & & $9 / 2$ & 25657.85 & 0.98 \\
\hline & & $11 / 2$ & 21470.50 & 1.00 & & & $9 / 2$ & 25704.78 & 1.020 \\
\hline & & $15 / 2$ & 21655.15 & 1.14 & & & $9 / 2$ & 25729.03 & 1.025 \\
\hline & & $13 / 2$ & 21709.83 & 1.065 & & & $7 / 2$ & 25745.61 & 0.930 \\
\hline & & $11 / 2$ & 21813.11 & 1.05 & & & $9 / 2$ & 25814.85 & 1.020 \\
\hline \multirow{3}{*}{$\begin{array}{l}4 f^{2} 5 d^{2} 6 p ? \\
4 f^{2}\left({ }^{3} \mathrm{H}\right) 5 d^{2}\left({ }^{3} \mathrm{~F}\right)\left({ }^{5} \mathrm{~L}\right) 6 p ?\end{array}$} & \multirow{3}{*}{$\begin{array}{l}{ }^{6} \mathrm{~L}^{\circ} \\
{ }^{6} \mathrm{M}^{\circ}\end{array}$} & $11 / 2$ & 21969.49 & 0.64 & & & $13 / 2$ & 25843.56 & 1.055 \\
\hline & & $13 / 2$ & 21999.38 & 0.915 & & & $13 / 2$ & 25902.38 & 1.165 \\
\hline & & $11 / 2$ & 22577.25 & 1.085 & & & $9 / 2$ & 26006.18 & 1.055 \\
\hline \multirow{11}{*}{$4 f^{2} 5 d^{2} 6 p ?$} & \multirow{11}{*}{${ }^{6} \mathrm{~L}^{\circ}$} & $\begin{array}{l}13 / 2 \\
13 / 2\end{array}$ & $\begin{array}{l}22670.12 \\
22786.54\end{array}$ & $\begin{array}{l}0.92 \\
0.95\end{array}$ & & & $9 / 2$ & 26083.73 & 0.98 \\
\hline & & $11 / 2$ & 22811.26 & 1.02 & & & $5 / 2$ & 26197.80 & \\
\hline & & $13 / 2$ & 22831.71 & 1.105 & & & $11 / 2$ & 26310.93 & 1.04 \\
\hline & & $11 / 2$ & 22850.99 & 1.025 & & & $13 / 2$ & 26448.18 & \\
\hline & & $11 / 2$ & 22881.44 & 0.98 & & & $11 / 2$ & 26757.35 & 0.99 \\
\hline & & $11 / 2$ & 22949.56 & 0.85 & & & $11 / 2$ & 26766.20 & 1.26 \\
\hline & & $13 / 2$ & 22974.55 & 1.080 & & & $11 / 2$ & 26824.75 & 0.825 \\
\hline & & $9 / 2$ & 23087.27 & 0.950 & & & $9 / 2$ & 26828.82 & 1.02 \\
\hline & & $11 / 2$ & 23207.53 & 1.050 & & & $7 / 2$ & 26908.65 & 0.945 \\
\hline & & $9 / 2$ & 23280.09 & 1.060 & & & $11 / 2$ & $\begin{array}{l}27026.50 \\
2730998\end{array}$ & 0.83 \\
\hline & & $11 / 2$ & 23309.48 & 1.005 & & & $9 / 2$ & 27331.71 & 1.00 \\
\hline
\end{tabular}




\begin{tabular}{|c|c|c|c|c|c|c|c|c|c|}
\hline Configuration & Term & $J$ & $\begin{array}{l}\text { Level } \\
\left(\mathrm{cm}^{-1}\right)\end{array}$ & $g$ & Configuration & Term & $J$ & $\begin{array}{l}\text { Level } \\
\left(\mathrm{cm}^{-1}\right)\end{array}$ & $g$ \\
\hline & & $11 / 2$ & 27449.24 & 1.070 & & & $9 / 2$ & 28675.30 & 1.050 \\
\hline & & $11 / 2$ & 27571.91 & 1.065 & & & $11 / 2$ & 31728.49 & 1.040 \\
\hline & & $11 / 2$ & 27602.42 & 0.99 & & & $11 / 2$ & 31787.07 & 1.01 \\
\hline & & $13 / 2$ & 27991.76 & 1.050 & & & $9 / 2$ & 32704.29 & \\
\hline & & $11 / 2$ & 28168.72 & 1.20 & & & & & \\
\hline & & $9 / 2$ & 28501.82 & 1.120 & $\operatorname{Pr}$ II $\left({ }^{4} I_{9 / 2}^{\circ}\right) 6 s_{1 / 2} \quad(9 / 2,1 / 2)_{4}^{\circ}$ & Limit & & 44070 & \\
\hline
\end{tabular}

Pr I, Even Parity

Pr I, Even Parity

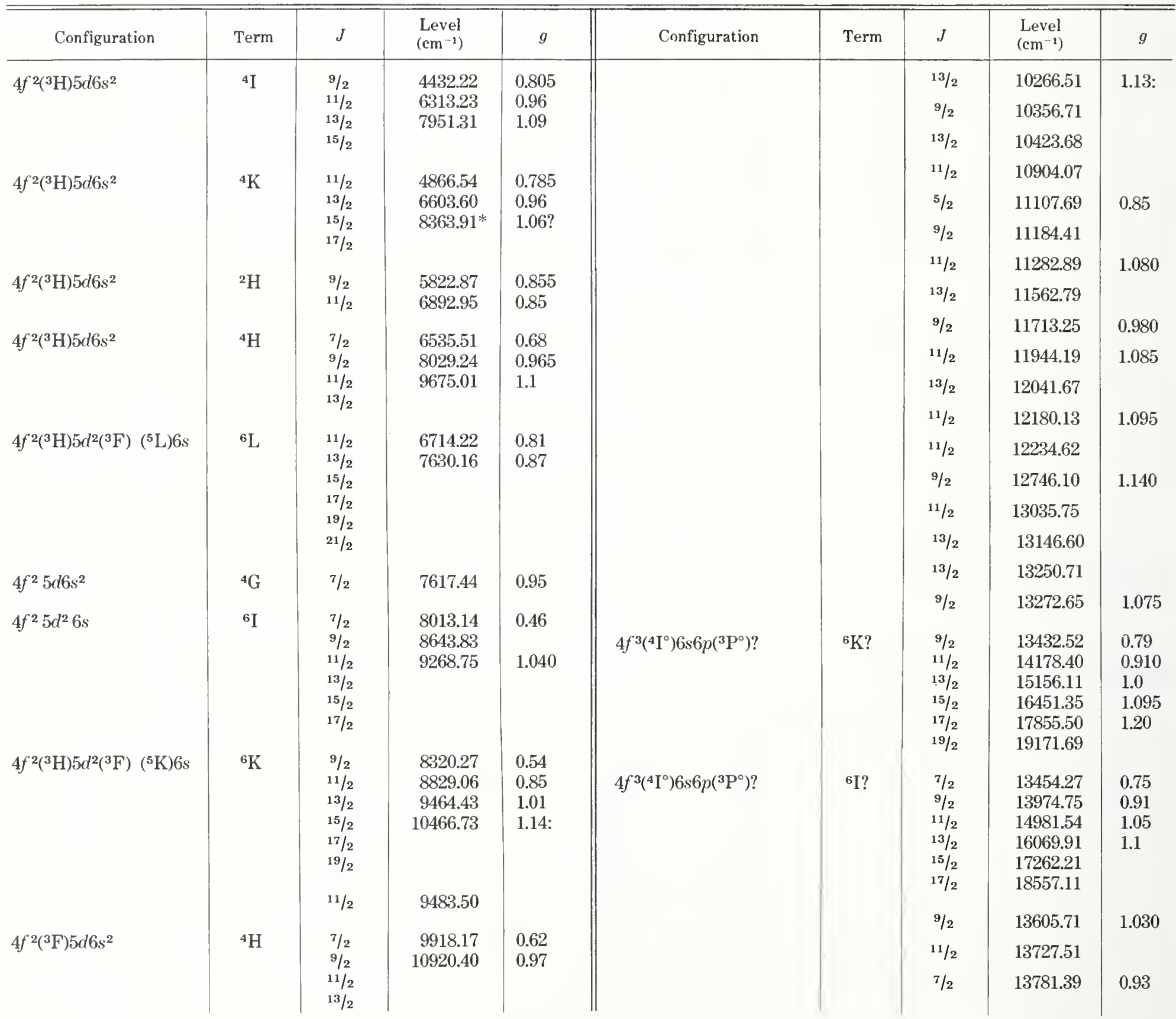


Pr I, Even Parity-Continued

Pr I, Even Parity-Continued

\begin{tabular}{|c|c|c|c|c|c|c|c|c|c|}
\hline Configuration & Term & $J$ & $\begin{array}{l}\text { Level } \\
\left(\mathrm{cm}^{-1}\right)\end{array}$ & $g$ & Configuration & Term & $J$ & $\begin{array}{l}\text { Level } \\
\left(\mathrm{cm}^{-1}\right)\end{array}$ & $g$ \\
\hline & & $9 / 2$ & 13822.51 & 1.170 & & & $17 / 2$ & 17695.16 & \\
\hline & & $7 / 2$ & 13867.20 & 0.71 & & & $11 / 2$ & 18144.32 & 0.945 \\
\hline & & $11 / 2$ & 13872.32 & 1.075 & & & $9 / 2$ & 18189.22 & \\
\hline & & $13 / 2$ & 14087.60 & 1.065 & & & $11 / 2$ & 18237.29 & 0.990 \\
\hline & & $9 / 2$ & 14139.33 & 1.020 & & & $15 / 2$ & 18277.91 & \\
\hline & & $9 / 2$ & 14186.39 & 1.00 & & & $11 / 2$ & 18309.12 & 0.905 \\
\hline & & $9 / 2$ & 14261.56 & 1.11 & & & $17 / 2$ & 18380.34 & \\
\hline & & $9 / 2$ & 14272.91 & 1.01 & & & $15 / 2$ & 18466.97 & 1.30 \\
\hline & & $9 / 2$ & 14468.33 & 1.00 & & & $15 / 2$ & 18548.53 & $1.14 ?$ \\
\hline & & $13 / 2$ & 14470.09 & & & & $9 / 2,11 / 2$ & 18578.24 & \\
\hline & & $11 / 2$ & 14505.11 & 0.97 & & & $9 / 2$ & 18636.18 & 1.145 \\
\hline & & $11 / 2$ & 14660.64 & 0.985 & & & $11 / 2$ & 19122.57 & 1.025 \\
\hline & & $11 / 2$ & 14760.32 & & & & $13 / 2$ & 19212.72 & 1.145 \\
\hline & & $9 / 2$ & 14764.32 & 0.880 & & & $11 / 2$ & 19343.25 & 1.06 \\
\hline & & $15 / 2$ & 14800.71 & & & & $15 / 2$ & 19364.58 & \\
\hline & & $11 / 2$ & 15111.50 & & & & $13 / 2$ & 19390.42 & 1.13 \\
\hline & & $11 / 2$ & 15238.16 & & $4 f^{3} 6 s 6 p ?$ & ${ }^{4} \mathrm{I} ?$ & $11 / 2$ & 19474.75 & 0.94 \\
\hline & & $13 / 2$ & 15372.31 & 1.055 & & & $15 / 2$ & 19566.82 & $1.08 ?$ \\
\hline \multirow[t]{3}{*}{$4 f^{2} 5 d 6 s^{2} ?$} & ${ }^{2} \mathrm{I} ?$ & $11 / 2$ & 15469.58 & 1.045 & & & $9 / 2,11 / 2$ & 19620.04 & \\
\hline & & $9 / 2$ & 15531.58 & & & & $7 / 2$ & 19654.58 & 0.95 \\
\hline & & $11 / 2$ & 15567.30 & 1.13 & & & $7 / 2$ & 19749.10 & 1.075 \\
\hline \multirow[t]{12}{*}{$4 f^{3}\left({ }^{4} \mathrm{I}^{\circ}\right) 6 s 6 p\left({ }^{3} \mathrm{P}^{\circ}\right) ?$} & ${ }^{4} \mathrm{~K} ?$ & $13 / 2$ & 15677.69 & 0.95 & & & $11 / 2$ & 19820.69 & 0.995 \\
\hline & & $13 / 2$ & 15772.58 & & & & $11 / 2$ & 19861.89 & 0.89 \\
\hline & & $13 / 2$ & 15850.49 & & & & $9 / 2$ & 19871.16 & 1.12 \\
\hline & & $11 / 2$ & 15882.93 & & & & $11 / 2$ & 19920.36 & 1.040 \\
\hline & & $11 / 2$ & 15904.93 & 1.00 & & & $9 / 2$ & 19963.96 & 1.010 \\
\hline & & $15 / 2$ & 15994.81 & & & & $11 / 2$ & 20089.26 & 1.035 \\
\hline & & $11 / 2$ & 16121.23 & & & & $9 / 2$ & 20154.60 & 1.225 \\
\hline & & $13 / 2$ & 16250.92 & & & & $11 / 2$ & 20171.75 & 1.09 \\
\hline & & $15 / 2$ & 16294.40 & & $4 f^{3}\left({ }^{4} \mathrm{I}^{\circ}\right) 6 s 6 p\left({ }^{1} \mathrm{P}^{\circ}\right) ?$ & ${ }^{4} \mathrm{I} ?$ & $9 / 2$ & 20190.85 & 0.81 \\
\hline & & $11 / 2$ & 16316.49 & & & & $11 / 2$ & 20243.72 & 1.095 \\
\hline & & $11 / 2$ & 16377.47 & & & & $7 / 2$ & 20269.46 & 1.035 \\
\hline & & $13 / 2$ & 16626.91 & & & & $11 / 2$ & 20271.23 & \\
\hline \multirow[t]{11}{*}{$4 f^{3} 6 s 6 p ?$} & & $15 / 2$ & 16650.65 & & & & $13 / 2$ & 20290.58 & 1.15: \\
\hline & & $11 / 2$ & 16778.49 & & & & $9 / 2$ & 20344.28 & 1.005 \\
\hline & & $13 / 2$ & 16823.81 & & & & $7 / 2$ & 20467.38 & 0.88 \\
\hline & & $11 / 2$ & 16935.88 & & & & $9 / 2$ & 20476.66 & 1.08 \\
\hline & & $13 / 2$ & 16979.04 & 1.10 & & & $9 / 2$ & 20620.80 & 1.235 \\
\hline & & $11 / 2$ & 17405.54 & 1.05 & & & $13 / 2$ & 20622.75 & 1.03 \\
\hline & ${ }^{4} \mathrm{~K} ?$ & $15 / 2$ & 17429.98 & & & & $7 / 2$ & 20653.36 & 1.185 \\
\hline & & $11 / 2$ & 17470.75 & 1.150 & & & $11 / 2$ & 20731.90 & $1.17 ?$ \\
\hline & & $17 / 2$ & 17494.45 & & & & $7 / 2$ & 20792.13 & 0.975 \\
\hline & & $13 / 2$ & 17504.18 & 1.040 & & & $13 / 2$ & 20798.25 & 1.05 \\
\hline & ${ }^{4} \mathrm{~K}$ ? & $11 / 2$ & 17577.91 & 0.76 & & & $13 / 2$ & 20827.15 & 1.005 \\
\hline
\end{tabular}


Pr I, Even Parity-Continued

Pr I, Even Parity-Continued

\begin{tabular}{|c|c|c|c|c|c|c|c|c|c|}
\hline Configuration & Term & $J$ & $\begin{array}{l}\text { Level } \\
\left(\mathrm{cm}^{-1}\right)\end{array}$ & $g$ & Configuration & Term & $J$ & $\begin{array}{l}\text { Level } \\
\left(\mathrm{cm}^{-1}\right)\end{array}$ & $g$ \\
\hline \multirow{44}{*}{$4 f^{3}\left({ }^{4} I^{\circ}\right) 6 s 6 p ?$} & \multirow{16}{*}{${ }^{4} \mathrm{~K}$ ? } & $9 / 2$ & 20878.48 & 1.04 & $4 f^{3} 6 s 6 p ?$ & ${ }^{4} \mathrm{~K}$ ? & $15 / 2$ & 22762.55 & 1.03 \\
\hline & & $9 / 2$ & 20938.62 & 1.07 & \multirow{7}{*}{$4 f^{3}\left({ }^{4} \mathrm{I}^{\circ}\right) 5 d\left({ }^{5} \mathrm{~L}^{\circ}\right) 6 p ?$} & \multirow[t]{7}{*}{${ }^{6} \mathrm{M}$ ? } & $13 / 2$ & 22785.33 & 0.71 \\
\hline & & $11 / 2$ & 20943.42 & 1.180 & & & $15 / 2$ & 23842.67 & 0.87 \\
\hline & & $11 / 2$ & 20982.95 & 1.135 & & & $\begin{array}{c}17 / 2 \\
19 / 2 ?\end{array}$ & $\begin{array}{l}25025.55 \\
26313.78\end{array}$ & 1.075 \\
\hline & & $7 / 2$ & 21072.64 & 0.950 & & & $21 / 2$ & & \\
\hline & & $7 / 2$ & 21105.88 & 0.865 & & & $23 / 2 ?$ & 29011.25 & \\
\hline & & $11 / 2$ & 21120.69 & 1.135 & & & $9 / 2$ & 22921.34 & 1.08 \\
\hline & & $13 / 2$ & 21159.76 & 1.120 & & & $9 / 2$ & 22924.39 & 1.115 \\
\hline & & $7 / 2$ & 21211.48 & 1.045 & \multirow[t]{5}{*}{$4 f^{3}\left({ }^{4} \mathrm{I}^{\circ}\right) 5 d\left({ }^{5} \mathrm{~L}^{\circ}\right) 6 p ?$} & \multirow[t]{5}{*}{${ }^{6} \mathrm{~L} ?$} & $11 / 2$ & 23067.34 & 0.76 \\
\hline & & $13 / 2$ & 21263.71 & & & & $13 / 2$ & 24073.41 & 0.99 \\
\hline & & $15 / 2$ & 21398.47 & $1.20:$ & & & $\begin{array}{c}15 / 2 \\
17 / 2 ?\end{array}$ & $\begin{array}{l}25168.99 \\
26252.14\end{array}$ & 1.12 \\
\hline & & $13 / 2$ & 21470.05 & & & & $19 / 2$ ? & 28613.84 & \\
\hline & & $11 / 2$ & 21471.81 & 1.010 & & & ${ }^{21} / 2 ?$ & 28809.03 & \\
\hline & & $7 / 2$ & 21513.28 & 1.18 & $4 f^{3} 6 s 6 p ?$ & ${ }^{4} I ?$ & $13 / 2$ & 23085.08 & 1.11 \\
\hline & & $7 / 2$ & 21565.83 & 0.96 & $4 f^{3} 6 s 6 p ?$ & ${ }^{4} \mathrm{G} ?$ & $9 / 2$ & 23242.13 & 1.185 \\
\hline & & $11 / 2$ & 21612.66 & 1.07 & $4 f^{3} 6 s 6 p ?$ & ${ }^{4} \mathrm{~K}$ ? & $17 / 2$ & 23793.67 & 1.09 \\
\hline & \multirow[t]{28}{*}{${ }^{4} \mathrm{~L}$ ? } & $13 / 2$ & 21618.49 & 0.850 & & & $9 / 2$ & 23818.60 & 0.90 \\
\hline & & $15 / 2$ & 21629.20 & 1.06: & & & $13 / 2$ & 23891.25 & 0.995 \\
\hline & & $7 / 2$ & 21649.64 & 0.88 & $4 f^{3} 5 d 6 p ?$ & ${ }^{6} \mathrm{~K} ?$ & $9 / 2$ & 23976.05 & 0.845 \\
\hline & & $11 / 2$ & 21677.15 & 1.00 & & & $13 / 2$ & 23997.59 & 1.090 \\
\hline & & $7 / 2$ & 21720.97 & 0.970 & $4 f^{3} 5 d 6 p ?$ & ${ }^{4} \mathrm{M} ?$ & $15 / 2$ & 24125.97 & 0.93 \\
\hline & & $13 / 2$ & 21746.01 & 1.115 & & & $11 / 2$ & 24136.57 & 1.105 \\
\hline & & $11 / 2$ & 21765.16 & 1.02 & $4 f^{3} 6 s 6 p ?$ & ${ }^{4} \mathrm{~K} ?$ & $17 / 2$ & 24195.13 & 1.16 \\
\hline & & $13 / 2$ & 21766.96 & 1.12 & $4 f^{3} 5 d 6 p ?$ & ${ }^{4} \mathrm{~L}$ ? & $13 / 2$ & 24235.31 & 0.87 \\
\hline & & $7 / 2$ & 21789.99 & 1.150 & & & $11 / 2$ & 24251.54 & 1.12 \\
\hline & & $9 / 2$ & 21795.17 & 1.06 & & & $13 / 2$ & 24267.84 & 1.10 \\
\hline & & $7 / 2$ & 21961.01 & 1.025 & & & $11 / 2$ & 24307.52 & 0.97 \\
\hline & & $7 / 2$ & 22014.11 & 1.165 & & & $11 / 2$ & 24312.05 & 0.86 \\
\hline & & $7 / 2$ & 22057.52 & 1.140 & & & $11 / 2$ & 24333.76 & 1.10 \\
\hline & & $15 / 2$ & 22088.82 & 1.09: & & & $9 / 2$ & 24441.81 & 0.995 \\
\hline & & $13 / 2$ & 22156.21 & 1.157 & & & $15 / 2$ & 24472.97 & 1.11 \\
\hline & & $7 / 2$ & 22159.67 & 0.885 & & & $13 / 2$ & 24555.04 & 1.035 \\
\hline & & $7 / 2$ & 22272.48 & 1.165 & & & $11 / 2$ & 24584.76 & 0.93 \\
\hline & & $13 / 2$ & 22416.15 & 1.11 & $4 f^{3} 5 d 6 p ?$ & ${ }^{6} \mathrm{~L} ?$ & $11 / 2$ & 24590.84 & 0.680 \\
\hline & & $7 / 2$ & 22453.16 & 1.11 & & & $15 / 2$ & 24634.79 & 1.075 \\
\hline & & $11 / 2$ & 22463.47 & 1.14: & $4 f^{3} 5 d 6 p ?$ & & $11 / 2$ & 24720.82 & 1.035 \\
\hline & & $15 / 2$ & 22498.81 & 1.02 & & & $15 / 2$ & 24754.55 & 1.075 \\
\hline & & $9 / 2$ & 22509.40 & 1.105 & $4 f^{3} 5 d 6 p ?$ & ${ }^{6} \mathrm{~K} ?$ & $11 / 2$ & 24821.53 & 0.96 \\
\hline & & $7 / 2$ & 22566.39 & 1.030 & & & $11 / 2$ & 24906.70 & 1.07 \\
\hline & & $9 / 2$ & 22694.60 & 1.03 & $4 f^{3} 5 d 6 p ?$ & & $11 / 2$ & 25006.96 & 1.030 \\
\hline & & $17 / 2$ & 22700.89 & 1.175 & $4 f^{3} 5 d 6 p ?$ & & $11 / 2$ & 25097.82 & 1.01 \\
\hline & & $13 / 2$ & 22725.88 & 1.14 & & & $11 / 2$ & 25279.18 & 1.10 \\
\hline & & $11 / 2$ & 22742.62 & & $4 f^{3} 5 d 6 p ?$ & ${ }^{4} \mathrm{~L}$ ? & $15 / 2$ & 25321.60 & 1.03 \\
\hline & & $9 / 2$ & 22761.90 & 1.110 & $4 f^{3} 5 d 6 p ?$ & & $11 / 2$ & 25333.72 & 0.91 \\
\hline
\end{tabular}


Pr I, Even Parity_Continued

Pr I, Even Parity_Continued

\begin{tabular}{|c|c|c|c|c|c|c|c|c|c|}
\hline Configuration & Term & $J$ & $\begin{array}{l}\text { Level } \\
\left(\mathrm{cm}^{-1}\right)\end{array}$ & $g$ & Configuration & Term & $J$ & $\begin{array}{l}\text { Level } \\
\left(\mathrm{cm}^{-1}\right)\end{array}$ & $g$ \\
\hline \multirow{5}{*}{$4 f^{3} 5 d 6 p ?$} & \multirow{7}{*}{${ }^{6} \mathrm{~L}$ ? } & $13 / 2$ & 25357.39 & 1.01 & & & $15 / 2$ & 26932.88 & \\
\hline & & $13 / 2$ & 25454.41 & 1.040 & & & $15 / 2$ & 26998.57 & 1.07: \\
\hline & & $9 / 2$ & 25473.75 & 0.915 & & & $11 / 2$ & 27153.94 & 0.96 \\
\hline & & $13 / 2$ & 25498.90 & 0.975 & $4 f^{3} 5 d 6 p$ & & $17 / 2$ & 27201.91 & \\
\hline & & $13 / 2$ & 25587.92 & 1.15 & $4 f^{3} 5 d 6 p$ & & $13 / 2$ & 27213.98 & \\
\hline \multirow[t]{2}{*}{$4 f^{3} 5 d 6 p ?$} & & $13 / 2$ & 25608.77 & 1.050 & $4 f^{3} 5 d 6 p ?$ & & $19 / 2$ ? & 27404.55 & \\
\hline & & $17 / 2$ & 25664.75 & & $4 f^{3} 5 d 6 p ?$ & & $17 / 2 ?$ & 27523.18 & \\
\hline $4 f^{3} 5 d 6 p ?$ & \multirow{10}{*}{$\begin{array}{l}{ }^{4} \mathrm{M} \text { ? } \\
{ }^{6} \mathrm{~K} ?\end{array}$} & $15 / 2$ & 25781.55 & 0.97 & & & $15 / 2$ & 27620.01 & 1.02 \\
\hline \multirow[t]{9}{*}{$4 f^{3} 5 d 6 p ?$} & & $13 / 2$ & 25840.18 & 1.04 & & & $11 / 2$ & 27634.52 & 0.94 \\
\hline & & $15 / 2$ & 25861.92 & 0.98 & $4 f^{3} 5 d 6 p ?$ & & $21 / 2 ?$ & 27634.53 & \\
\hline & & $13 / 2$ & 25962.72 & 1.15 & & & $13 / 2$ & 27721.26 & 0.96 \\
\hline & & $13 / 2$ & 26107.27 & 1.040 & $4 f^{3} 5 d 6 p ?$ & & $17 / 2$ & 27784.56 & 1.11 \\
\hline & & $13 / 2$ & 26123.37 & 1.10 & & & $11 / 2$ & 27869.73 & 1.27 \\
\hline & & $\begin{array}{l}13 / 2 \\
13 / 6\end{array}$ & 26258.65 & 1.08 & & & $11 / 2$ & 28054.35 & 1.060 \\
\hline & & $13 / 2$ & $\begin{array}{l}26297.91 \\
26352.84\end{array}$ & 0.99 & & & $11 / 2$ & 28149.78 & 0.98 \\
\hline & & $11 / 2$ & 26357.24 & 1.04 & & & $15 / 2$ & 28378.49 & 1.12 \\
\hline & & $9 / 2$ & 26392.39 & 1.035 & & & $11 / 2$ & 28560.68 & 0.97 \\
\hline $4 f^{3} 5 d 6 p ?$ & ${ }^{6} \mathrm{~L} ?$ & $15 / 2$ & 26410.25 & 1.12 & & & $15 / 2$ & 28906.27 & \\
\hline \multirow[t]{4}{*}{$4 f^{3} 5 d 6 p ?$} & \multirow[t]{5}{*}{${ }^{6} \mathrm{~K}$ ? } & $13 / 2$ & 26455.31 & 1.04 & & & $13 / 2$ & 28917.29 & 1.10 \\
\hline & & $9 / 2$ & 26528.81 & 0.83 & 4f $300 p ?$ & & $21 / 2$ ? & 28964.07 & \\
\hline & & $17 / 2$ & 26565.32 & 1.13: & 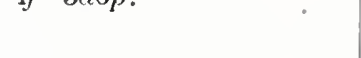 & & $17 / 2$ & 29805.84 & \\
\hline & & $15 / 2$ & 26654.48 & 1.02 & $4 f^{3} 5 d 6 p ?$ & & $19 / 2 ?$ & 31172.71 & \\
\hline $4 f^{3} 5 d 6 p ?$ & & $15 / 2$ & 26710.33 & & & & & & \\
\hline \multirow[t]{3}{*}{$4 f^{3} 5 d 6 p ?$} & \multirow[t]{3}{*}{${ }^{4} \mathrm{~K}$ ? } & $13 / 2$ & 26715.43 & 0.97 & & & & & \\
\hline & & $11 / 2$ & 26784.33 & 0.89 & $\operatorname{Pr}$ II $\left({ }^{4} I_{9 / 2}^{\circ}\right) 6 s_{1 / 2}\left(9 / 2,{ }^{1} / 2\right)_{4}^{\circ}$ & Limit & & 44070 & \\
\hline & & $11 / 2$ & 26844.01 & & & & & & \\
\hline
\end{tabular}


(Ce I sequence; 58 electrons)

Ground state $\left(1 s^{2} 2 s^{2} 2 p^{6} 3 s^{2} 3 p^{6} 3 d^{10} 4 s^{2} 4 p^{6} 4 d^{10} 5 s^{2} 5 p^{6}\right) 4 f^{3}\left({ }^{4} \mathrm{I}_{9 / 2}^{\circ}\right) 6 s(9 / 2,1 / 2)_{4}^{\circ}$

Ionization energy $85100 \pm 600 \mathrm{~cm}^{-1}$

$10.55 \pm 0.08 \mathrm{eV}$

Identified odd configurations

Identified even configurations
$4 f^{3} 6 s, 4 f^{3} 5 d, 4 f^{2} 5 d 6 p ?$

$4 f^{2} 5 d^{2}, 4 f^{2} 5 d 6 s, 4 f^{3} 6 p$

In their 1941 paper Rosen, Harrison, and McNally noted the difficulty in analyzing the Pr spectra, "both because of the complexity of this rare earth atom, and because many of its lines show hyperfine structure difficult to measure." Their successful analysis of Pr II was helped greatly by Zeeman-effect data obtained at magnetic fields up to 9.5 teslas (95000 gauss). They gave 74 levels, including all the low odd levels of $4 f^{3}\left({ }^{4} \mathrm{I}^{\circ}\right) 6 s$ and of the $4 f^{3}\left({ }^{4} \mathrm{I}^{\circ}\right) 5 d^{5} \mathrm{~L}^{\circ}$ and ${ }^{5} \mathrm{~K}^{\circ}$ terms. A number of the upper even levels were assigned to $4 f^{3} 6 p$. Schuurmans' [1946] independent analysis also yielded the low odd terms mentioned above.

Rosen at al. list more than 300 classified lines of Pr II (3650-7100 ̊), with Zeeman data for about half of them. The wavelengths were taken from the M.I.T. tables [Harrison, 1969], which have 2708 lines from the arc and spark spectra of Pr. The other main line lists are described in the preceding text for Pr I. About 13000 of the lines in an unpublished NBSOrsay list are believed to belong to $\operatorname{Pr}$ II.

The levels here are mainly from Blaise, Verges, Wyart, Camus, and Zalubas [1973], who extended the analysis to include about 200 levels. Blaise and Ginibre [1976] have supplied a number of additional levels from their more recent work on this spectrum.

The data of Rosen et al., Blaise et al., and Blaise and Ginibre were used in compiling the $g$ values, with many of the values being averages of values from the first two sources. The accuracy is usually limited by hyperfine structure; the most poorly determined $g$ values are followed by colons. Values derived from uncertain reductions of Zeeman patterns are indicated by question marks.

Wyart, Blaise, and Camus [1974] calculated $\left(4 f^{3} 6 s+4 f^{3} 5 d\right)$ for $\operatorname{Pr}$ II, and the percentages for the $4 f^{3} 5 d$ levels are from their unpublished eigenvectors [1975]. The pairing of the eight $4 f^{3}\left({ }^{4} I^{\circ}\right) 6 s$ levels into four $J_{1} j$-coupling terms was first noted by Rosen et al.; we have arranged the levels accordingly and listed the corresponding percentages from an unpublished calculation of $4 f^{3} 6 s$ [Sugar and Hagan, 1971]. The leading percentages in LS coupling from this calculation are also given, following the word "or." The two separate calculations we have used for $4 f^{3} 6 s$ and $4 f^{3} 5 d$ (only one of which includes the configuration interaction) are not very inconsistent, because the configuration interaction is relatively small.

Blaise et al. [1973] have shown that the lowest even levels belong to the $\left(4 f^{2} 5 d^{2}+4 f^{2} 5 d 6 s\right)$ group, but a detailed interpretation with assignments to calculated eigenvectors has not yet been made. Ginibre [1975] made a preliminary calculation $4 f^{3} 6 p$ without configuration interaction; the percentages for some of the higher even levels are from her results. Most of the even levels are unassigned or have only tentative assignments, pending more complete calculations and interpretation.

White's [1929] measurements of the hyperfine structures of $173 \mathrm{Pr}$ II lines enabled him to deduce a nuclear spin of $5 / 2$ and the occurrence of a single 6 s electron in a low configuration. Rosen et al. made some use of White's data in their analysis, and most of the subsequent work on hyperfine structure in $\operatorname{Pr}$ II has dealt with transitions to the $4 f^{3}\left({ }^{4} I^{\circ}\right) 6 s$ levels. The hyperfine patterns of a much larger number of Pr II lines could be resolved and conveniently recorded with modern spectroscopic techniques; such data would very likely prove highly useful in obtaining a more complete analysis and interpretation of this spectrum.

Sugar and Reader derived the quoted ionization energy. 


\section{References}

Belyanin, V. B., Opt. Spektrosk. 4, 264 (1958). CL W Hfs

Blaise, J., and Ginibre, A., unpublished material (1976). EL ND ZE

Blaise, J., Verges, J., Wyart, J. F., Camus, P., and Zalubas, R., unpublished material (1973). EL ZE

Brix, P., Phys. Rev. 89, 1245 (1953). Hfs

Ginibre, A., unpublished material (1975). PT

Harrison, G. R., M.I.T. Wavelength Tables, 2d Edition, 429 pp. (M.I.T. Press, Cambridge, Mass., 1969). W

King, A. S., Astrophys. J. 68, 194 (1928). W

Murakawa, K., J. Phys. Soc. Japan 15, 2306 (1960). Hfs PT

Rosen, N., Harrison, G. R., and MeNally, J. R., Jr., Phys. Rev. 60, 722 (1941). EL CL W ZE Hfs

Schuurmans, P., Physica (Utrecht) 11, 419 (1946). EL

Sugar, J., and Hagan, L., unpublished material (1971). PT

Sugar, J., and Reader, J., J. Opt. Soc. Am. 55, 1286 (1965). IP

White, H. E., Phys. Rev. 34, 1397 (1929). W Hfs

Wyart, J. F., Blaise, J., and Camus, P., Phys. Scr. 9, 325 (1974). PT

Wyart, J. F., Blaise, J., and Camus, P., unpublished material (1975). PT

[January 1977]

Pr II

\begin{tabular}{|c|c|c|c|c|c|c|c|c|}
\hline Configuration & Term & $J$ & $\begin{array}{l}\text { Level } \\
\left(\mathrm{cm}^{-1}\right)\end{array}$ & $g$ & \multicolumn{4}{|c|}{ Leading percentages } \\
\hline $4 f^{3}\left({ }^{4} \mathrm{I}_{9 / 2}^{\circ}\right) 6 s_{1 / 2}$ & $(9 / 2,1 / 2)^{\circ}$ & $\begin{array}{l}4 \\
5\end{array}$ & $\begin{array}{r}0.00 \\
441.95\end{array}$ & $\begin{array}{l}0.604 \\
0.875\end{array}$ & $\begin{array}{l}97 \\
90\end{array}$ & $\begin{array}{l}\text { or } \\
\text { or }\end{array}$ & $\begin{array}{l}97 \\
58\end{array}$ & $\left({ }^{4} I^{\circ}\right)^{5} I^{\circ}$ \\
\hline $4 f^{3}\left({ }^{4} \operatorname{Ii1}_{112}\right) 6 s_{1 / 2}$ & $(11 / 2,1 / 2)^{\circ}$ & $\begin{array}{l}6 \\
5\end{array}$ & $\begin{array}{l}1649.01 \\
1743.72\end{array}$ & $\begin{array}{l}1.064 \\
0.860\end{array}$ & $\begin{array}{l}94 \\
91\end{array}$ & $\begin{array}{l}\text { or } \\
\text { or }\end{array}$ & $\begin{array}{l}77 \\
59\end{array}$ & $\begin{array}{l}\left({ }^{4} I^{\circ}\right)^{5} I^{\circ} \\
\left({ }^{4} I^{\circ}\right)^{3} I^{\circ}\end{array}$ \\
\hline $4 f^{3}\left({ }^{4} \mathrm{I}_{13 / 2}^{\circ}\right) 6 s_{1 / 2}$ & $(13 / 2,1 / 2)^{\circ}$ & $\begin{array}{l}7 \\
6\end{array}$ & $\begin{array}{l}2998.36 \\
3403.21\end{array}$ & $\begin{array}{l}1.177 \\
1.037\end{array}$ & $\begin{array}{l}98 \\
95\end{array}$ & $\begin{array}{l}\text { or } \\
\text { or }\end{array}$ & $\begin{array}{l}90 \\
77\end{array}$ & $\begin{array}{l}\left({ }^{4} I^{\circ}\right)^{5} I^{\circ} \\
\left({ }^{4} I^{\circ}\right)^{3} I^{\circ}\end{array}$ \\
\hline $4 f^{3}\left({ }^{4} I^{\circ}\right) 5 d$ & ${ }^{5} \mathrm{~L}^{0}$ & $\begin{array}{c}6 \\
7 \\
8 \\
9 \\
10\end{array}$ & $\begin{array}{l}3893.46 \\
5108.40 \\
6417.83 \\
7805.61 \\
9255.14\end{array}$ & $\begin{array}{l}0.726 \\
0.908 \\
1.05 \\
1.12 \\
1.20\end{array}$ & $\begin{array}{l}94 \\
95 \\
97 \\
98 \\
99\end{array}$ & . & $\begin{array}{l}3 \\
1 \\
1 \\
1 \\
1\end{array}$ & $\begin{array}{l}\left({ }^{2} \mathrm{H}^{\circ} 2\right){ }^{3} \mathrm{~K}^{\circ} \\
\left({ }^{2} \mathrm{H}^{\circ} 2\right){ }^{3} \mathrm{~K}^{\circ} \\
\left({ }^{4} \mathrm{I}^{\circ}\right){ }^{5} \mathrm{~K}^{\circ} \\
\left({ }^{4} \mathrm{I}^{\circ}\right)^{5} \mathrm{~K}^{\circ} \\
\left({ }^{2} \mathrm{~K}^{\circ}\right){ }^{3} \mathrm{M}^{\circ}\end{array}$ \\
\hline $4 f^{3}\left({ }^{4} I^{\circ}\right) 5 d$ & ${ }^{5} \mathrm{~K}^{\circ}$ & $\begin{array}{l}5 \\
6 \\
7 \\
8 \\
9\end{array}$ & $\begin{array}{l}4097.60 \\
5226.52 \\
6413.93 \\
7659.76 \\
8958.49\end{array}$ & $\begin{array}{l}0.686 \\
0.911 \\
1.05 \\
1.15 \\
1.220\end{array}$ & $\begin{array}{l}90 \\
95 \\
96 \\
96 \\
96\end{array}$ & & $\begin{array}{l}6 \\
2 \\
1 \\
1 \\
1\end{array}$ & $\begin{array}{l}\left({ }^{4} I^{\circ}\right) 6 s^{3} I^{\circ} \\
\left({ }^{4} I^{\circ}\right){ }^{3} I^{\circ} \\
\left({ }^{4} I^{\circ}\right){ }^{5} L^{\circ} \\
\left({ }^{4} I^{\circ}\right){ }^{5} L^{\circ} \\
\left({ }^{4} I^{\circ}\right)^{3} L^{\circ}\end{array}$ \\
\hline $4 f^{3}\left({ }^{4} \mathrm{I}_{15 / 2}^{\circ}\right) 6 s_{1 / 2}$ & $(15 / 2,1 / 2)^{\circ}$ & $\begin{array}{l}8 \\
7\end{array}$ & $\begin{array}{l}4437.15 \\
5079.35\end{array}$ & $\begin{array}{l}1.250 \\
1.143\end{array}$ & $\begin{array}{l}99 \\
97\end{array}$ & $\begin{array}{l}\text { or } \\
\text { or }\end{array}$ & $\begin{array}{l}99 \\
89\end{array}$ & $\begin{array}{l}\left({ }^{4} I^{\circ}\right)^{5} I^{\circ} \\
\left({ }^{4} I^{\circ}\right)^{3} I^{\circ}\end{array}$ \\
\hline $4 f^{2}\left({ }^{3} \mathrm{H}\right) 5 d^{2}\left({ }^{3} \mathrm{~F}\right)$ & ${ }^{5} \mathrm{~L}$ & 6 & 5854.61 & 0.71 & & & & \\
\hline $4 f^{2}\left({ }^{3} \mathrm{H}\right) 5 d^{2}\left({ }^{3} \mathrm{~F}\right) ?$ & $5 \mathrm{I} ?$ & 4 & 7227.99 & 0.62 & & & & \\
\hline $4 f^{3}\left({ }^{4} I^{\circ}\right) 5 d$ & ${ }^{3} \mathrm{I}^{\circ}$ & $\begin{array}{l}5 \\
6\end{array}$ & $\begin{array}{l}7438.23 \\
8465.04\end{array}$ & $\begin{array}{l}0.880 \\
1.035\end{array}$ & $\begin{array}{l}59 \\
47\end{array}$ & & $\begin{array}{l}11 \\
19\end{array}$ & $\begin{array}{l}\left({ }^{4} I^{\circ}\right)^{5} I^{\circ} \\
\left({ }^{4} I^{\circ}\right)^{5} I^{\circ}\end{array}$ \\
\hline $4 f^{3}\left({ }^{4} \mathrm{I}^{\circ}\right) 5 d$ & ${ }^{5} \mathrm{I}^{\circ}$ & $\begin{array}{l}4 \\
5 \\
6 \\
7 \\
8\end{array}$ & $\begin{array}{r}7446.43 \\
8489.87 \\
9646.67 \\
11005.57 \\
11611.05\end{array}$ & $\begin{array}{l}0.631 \\
0.93 \\
1.076 \\
1.152 \\
1.234\end{array}$ & $\begin{array}{l}89 \\
83 \\
78 \\
49 \\
87\end{array}$ & & $\begin{array}{r}8 \\
8 \\
8 \\
28 \\
9\end{array}$ & $\begin{array}{l}\left({ }^{4} I^{\circ}\right){ }^{3} H^{\circ} \\
\left({ }^{4} I^{\circ}\right){ }^{3} H^{\circ} \\
\left({ }^{4} I^{\circ}\right)^{3} I^{\circ} \\
\left({ }^{4} I^{\circ}\right){ }^{3} I^{\circ} \\
\left({ }^{4} I^{\circ}\right){ }^{3} K^{\circ}\end{array}$ \\
\hline $4 f^{3}\left({ }^{4} \mathrm{I}^{\circ}\right) 5 d$ & ${ }^{5} \mathrm{H}^{\circ}$ & $\begin{array}{l}3 \\
4 \\
5 \\
6 \\
7\end{array}$ & $\begin{array}{r}7744.27 \\
8099.72 \\
9378.63 \\
10729.75 \\
12243.49\end{array}$ & $\begin{array}{l}0.52 \\
0.830 \\
1.05 \\
1.11 \\
1.18\end{array}$ & $\begin{array}{l}73 \\
40 \\
55 \\
50 \\
41\end{array}$ & & $\begin{array}{l}12 \\
35 \\
13 \\
23 \\
24\end{array}$ & $\begin{array}{l}\left({ }^{4} I^{\circ}\right){ }^{3} G^{\circ} \\
\left({ }^{4} I^{\circ}\right){ }^{3} H^{\circ} \\
\left({ }^{4} I^{\circ}\right){ }^{3} I^{\circ} \\
\left({ }^{4} I^{\circ}\right){ }^{3} I^{\circ} \\
\left({ }^{4} I^{\circ}\right){ }^{3} I^{\circ}\end{array}$ \\
\hline $4 f^{2} 5 d 6 s ?$ & 5I & 4 & 7832.43 & 0.61 & & & & \\
\hline
\end{tabular}


Pr II-Continued

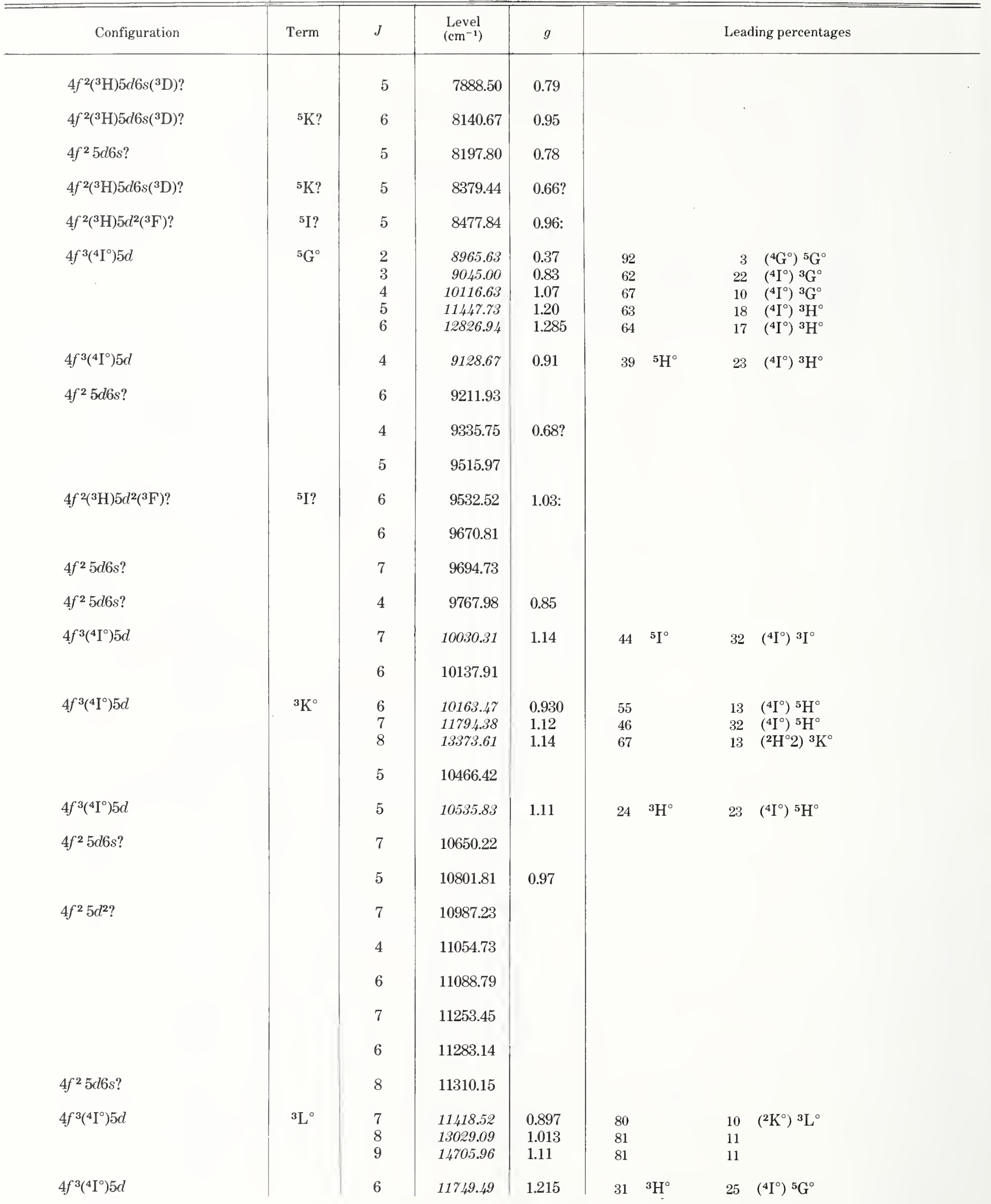


Pr II-Continued

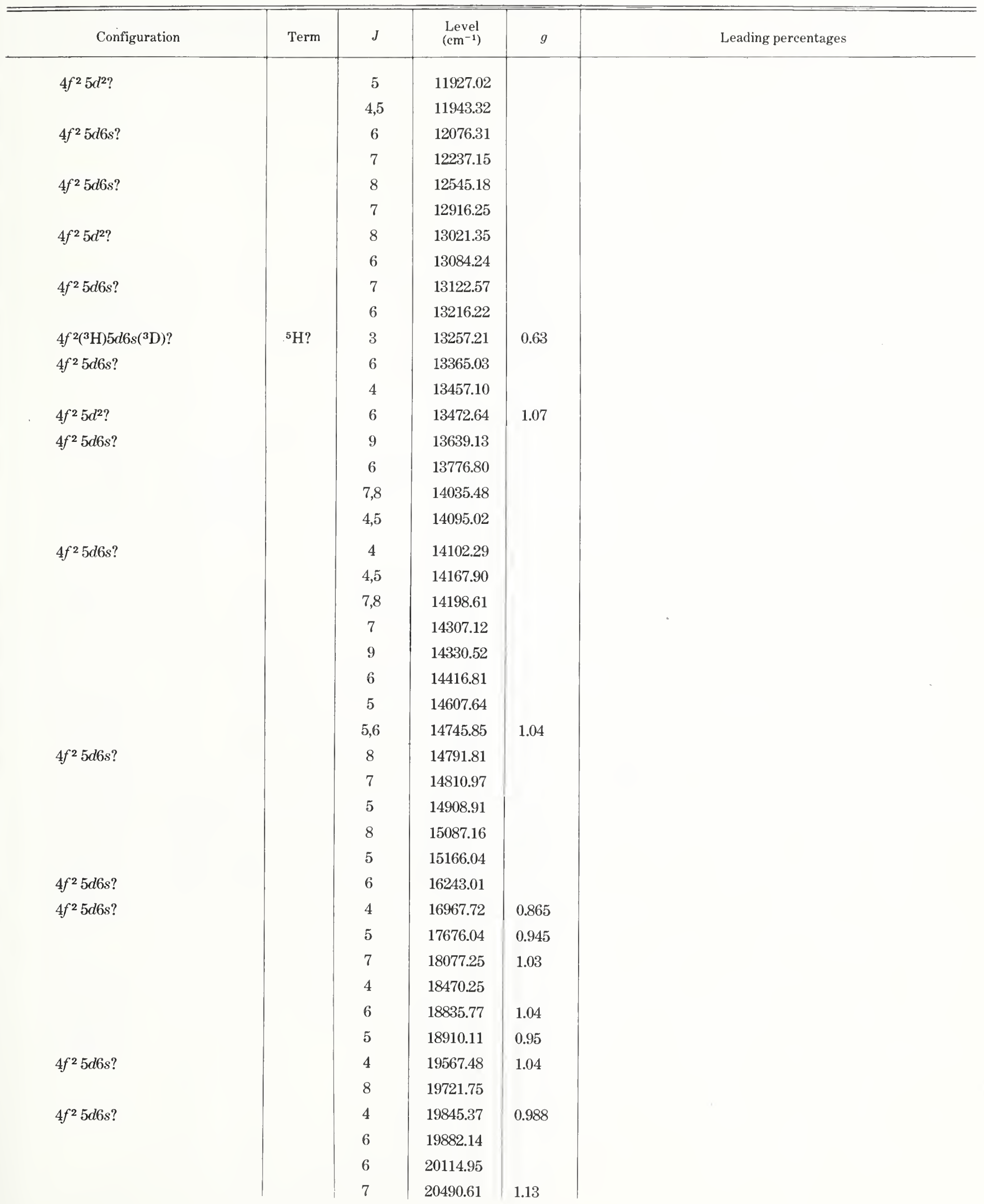


Pr II-Continued

\begin{tabular}{|c|c|c|c|c|c|c|}
\hline Configuration & Term & $J$ & $\begin{array}{l}\text { Level } \\
\left(\mathrm{cm}^{-1}\right)\end{array}$ & $g$ & & Leading percentages \\
\hline \multirow{5}{*}{$4 f^{2} 5 d 6 s ?$} & & 6 & 20554.30 & & & \\
\hline & & 5,6 & 21061.01 & & & \\
\hline & & 5,6 & 21131.26 & & & \\
\hline & & 4 & 21462.00 & & & \\
\hline & & 6 & 21494.75 & & & \\
\hline \multirow[t]{9}{*}{$4 f^{2} 5 d 6 s ?$} & & 5 & 21676.12 & 1.000 & & \\
\hline & & 6 & 21781.06 & & & \\
\hline & & 4 & 21971.46 & 0.84 & & \\
\hline & & 5 & 22040.05 & 0.998 & & \\
\hline & & 8 & 22184.27 & & & \\
\hline & & 5 & 22317.07 & 1.18 & & \\
\hline & & 6 & 22472.04 & & & \\
\hline & & 5 & 22571.48 & 1.166 & & \\
\hline & & 7 & 22660.77 & 1.03 & & \\
\hline \multirow[t]{14}{*}{$4 f^{3}\left({ }^{4} I^{\circ}\right) 6 p$} & ${ }^{5} \mathrm{~K}$ & 5 & 22675.44 & 0.830 & & \\
\hline & & 6 & 24115.50 & 0.959 & 88 & $7 \quad\left({ }^{4} I^{\circ}\right)^{3} \mathrm{I}$ \\
\hline & & 7 & 25569.19 & 1.07 & 91 & $5 \quad\left({ }^{4} \mathrm{I}^{\circ}\right)^{5} \mathrm{I}$ \\
\hline & & 8 & 27128.00 & 1.15 & 93 & $5 \quad\left({ }^{4} \mathrm{I}^{\circ}\right)^{5} \mathrm{I}$ \\
\hline & & 9 & 28816.25 & 1.221 & 99 & $1 \quad\left({ }^{2} \mathrm{~K}^{\circ}\right){ }^{3} \mathrm{~L}$ \\
\hline & & 4 & 22686.69 & & & \\
\hline & & 6 & 22718.35 & 1.08 & & \\
\hline & & 5 & 22885.59 & 0.997 & & \\
\hline & & 6 & 23141.44 & 1.061 & & \\
\hline & & 5 & 23261.36 & 0.920 & & \\
\hline & & 6 & 23505.46 & & & \\
\hline & & 4 & 23527.90 & 1.025 & & \\
\hline & & 5 & 23616.86 & $1.01 ?$ & & \\
\hline & & 5 & 23652.25 & 0.98 & & \\
\hline \multirow[t]{10}{*}{$4 f^{3}\left({ }^{4} I^{\circ}\right) 6 p$} & ${ }^{5} \mathrm{I}$ & 4 & $236 \dot{60} .20$ & 0.67 & 64 & $\left({ }^{4} \mathrm{I}^{\circ}\right)^{3} \mathrm{H}$ \\
\hline & & 5 & 24716.04 & 0.913 & 74 & $\left({ }^{4} \mathrm{I}^{\circ}\right)^{3} \mathrm{H}$ \\
\hline & & 6 & $25656.69^{*}$ & 1.046 & & \\
\hline & & 7 & 26860.95 & 1.119 & 60 & $\left({ }^{4} I^{\circ}\right)^{3} \mathrm{~K}$ \\
\hline & & 8 & 28201.95 & 1.160 & 65 & $27 \quad\left({ }^{4} \mathrm{I}^{\circ}\right)^{3} \mathrm{~K}$ \\
\hline & & 5 & 23712.63 & & & \\
\hline & & 7 & 23898.36 & 1.104 & & \\
\hline & & 8 & 23970.47 & 1.14 & & \\
\hline & & 6 & 23977.83 & 1.156 & & \\
\hline & & 6 & 24393.73 & 0.97 & & \\
\hline \multirow[t]{5}{*}{$4 f^{3}\left({ }^{4} \mathrm{I}^{\circ}\right) 6 p ?$} & & 4 & 24754.95 & 0.908 & & \\
\hline & & 7 & 24818.54 & 1.14 & & \\
\hline & & 6 & 24835.03 & 1.01 & & \\
\hline & & 7 & 25248.69 & 1.07 & & \\
\hline & & 4 & 25467.47 & 0.90 & & \\
\hline \multirow[t]{2}{*}{$4 f^{3}\left({ }^{4} \mathrm{I}^{\circ}\right) 6 p ?$} & & 5 & 25499.52 & 0.990 & & \\
\hline & & 4 & 25545.04 & & & \\
\hline
\end{tabular}


Pr II-Continued

\begin{tabular}{|c|c|c|c|c|c|c|}
\hline Configuration & Term & $J$ & $\begin{array}{l}\text { Level } \\
\left(\mathrm{cm}^{-1}\right)\end{array}$ & $g$ & & Leading percentages \\
\hline \multirow[t]{7}{*}{$4 f^{3}\left({ }^{4} I^{\circ}\right) 6 p$} & \multirow[t]{7}{*}{${ }^{5} \mathrm{H}$} & 3 & 25578.49 & 0.55 & \multirow[t]{24}{*}{97} & $2 \quad\left({ }^{2} \mathrm{H}^{\circ} 2\right){ }^{3} \mathrm{G}$ \\
\hline & & 6 & 25610.20 & 1.118 & & \\
\hline & & 3 & 25762.81 & 0.86 & & \\
\hline & & 4 & 25814.44 & 0.95 & & \\
\hline & & 5 & 25842.41 & 1.000 & & \\
\hline & & 5 & 26062.38 & & & \\
\hline & & 8 & 26139.77 & & & \\
\hline $4 f^{3}\left({ }^{4} I^{\circ}\right) 6 p ?$ & & 5 & 26146.01 & 1.05 & & \\
\hline $4 f^{3}\left({ }^{4} \mathrm{I}^{\circ}\right) 6 p ?$ & & 4 & 26226.56 & 0.91 & & \\
\hline \multirow[t]{5}{*}{$4 f^{3}\left({ }^{4} I^{\circ}\right) 6 p ?$} & & 6 & 26398.52 & 0.990 & & \\
\hline & & 7 & 26445.11 & 1.065 & & \\
\hline & & 6 & 26524.02 & 1.02 & & \\
\hline & & 5 & 26640.86 & 1.05 & & \\
\hline & & 5 & 26707.31 & 1.07 & & \\
\hline \multirow[t]{2}{*}{$4 f^{3}\left({ }^{4} I^{\circ}\right) 6 p ?$} & & 6 & 26961.96 & 1.076 & & \\
\hline & & 5 & 26973.49 & 1.11 & & \\
\hline \multirow[t]{2}{*}{$4 f^{3}\left({ }^{4} I^{\circ}\right) 6 p ?$} & & 5 & 27198.24 & 1.07 & & \\
\hline & & 5 & 27380.45 & 1.06 & & \\
\hline \multirow[t]{2}{*}{$4 f^{3}\left({ }^{4} I^{\circ}\right) 6 p ?$} & & 6 & 27604.94 & 1.13 & & \\
\hline & & 8 & 27781.69 & 1.075 & & \\
\hline \multirow[t]{3}{*}{$4 f^{3}\left({ }^{4} I^{\circ}\right) 6 p ?$} & & 7 & 28009.80 & 1.12 & & \\
\hline & & 6 & 28034.08 & & & \\
\hline & & 7 & 28172.96 & 1.14: & & \\
\hline $4 f^{3}\left({ }^{4} I^{\circ}\right) 6 p$ & ${ }^{3} \mathrm{I}$ & 6 & 28508.79 & 1.11 & & $37 \quad\left({ }^{4} I^{0}\right)^{5} \mathrm{H}$ \\
\hline $4 f^{3}\left({ }^{4} \mathrm{I}^{\circ}\right) 6 p ?$ & ${ }^{3} \mathrm{I} ?$ & 7 & 28577.79 & 1.19 & \multirow{17}{*}{51} & \\
\hline $4 f^{3}\left({ }^{4} I^{\circ}\right) 6 p ?$ & & 8 & 29723.97 & 1.15 & & \\
\hline $4 f^{3}\left({ }^{4} I^{\circ}\right) 6 p ?$ & ${ }^{5} \mathrm{H} ?$ & 7 & 30018.10 & 1.215 & & \\
\hline $4 f^{2}\left({ }^{3} \mathrm{H}\right) 5 d 6 p ?$ & ${ }^{5} \mathrm{~K}^{\circ} ?$ & 5 & 30844.63 & $0.74 ?$ & & \\
\hline $4 f^{2} 5 d 6 p ?$ & & 4 & 31775.93 & 0.78 & & \\
\hline $4 f^{2} 5 d 6 p ?$ & & 5 & 32590.69 & $0.69:$ & & \\
\hline $4 f^{2} 5 d 6 p ?$ & & 5 & 33277.53 & 0.91 & & \\
\hline $4 f^{2} 5 d 6 p ?$ & & 5 & 33387.62 & 0.93 & & \\
\hline \multirow[t]{2}{*}{$4 f^{2} 5 d 6 p ?$} & & 4 & 33440.49 & 0.83 & & \\
\hline & & 6 & 33616.69 & 0.98 & & \\
\hline $4 f^{2} 5 d 6 p ?$ & & 5 & 33674.55 & 0.97 & & \\
\hline $4 f^{2} 5 d 6 p ?$ & & 4 & 33981.23 & & & \\
\hline $4 f^{2} 5 d 6 p ?$ & & 6 & 34031.30 & 0.96 & & \\
\hline \multirow[t]{4}{*}{$4 f^{2} 5 d 6 p ?$} & & 4 & 34322.69 & 0.80 & & \\
\hline & & 6 & 35133.62 & & & \\
\hline & & 5 & 35220.91 & 0.89 & & \\
\hline & & 5 & 35497.57 & & & \\
\hline $\operatorname{Pr} I I I\left({ }^{4} \mathrm{I}_{9 / 2}^{\circ}\right)$ & Limit & ---- & 85100 & & & \\
\hline
\end{tabular}


Ground state $\left(1 s^{2} 2 s^{2} 2 p^{6} 3 s^{2} 3 p^{6} 3 d^{10} 4 s^{2} 4 p^{6} 4 d^{10} 5 s^{2} 5 p^{6}\right) 4 f^{34}{ }_{9 / 2}^{\circ}$

Ionization energy $174407 \pm 20 \mathrm{~cm}^{-1}$

$21.624 \pm 0.003 \mathrm{eV}$

Identified odd configurations

Identified even configurations
$4 f^{3}, 4 f^{2} 6 p, 4 f^{2} 5 f, 4 f 5 d^{2}, 4 f 5 d 6 s$

$4 f^{2} 5 d, 4 f^{2} 6 d, 4 f^{2} 6 s, 4 f^{2} 7 s, 4 f^{2} 8 s$

The levels are from Sugar's analysis $[1963,1969]$, with a few additions and revisions [Crosswhite, Crosswhite, and Judd, 1968; Sugar, 1974; Wyart, Blaise, and Camus, 1974]. There are more than 3300 classified lines in the range $821 \AA$ to $10717 \AA$. About 4400 lines are included in Sugar's list [1974] for the range above $2107 \AA$, and his 1969 paper has a table of 2948 lines in the range below this wavelength.

Rajnak's eigenvectors were used to obtain $L S$ percentages for the $4 f^{3}$ configuration. The compositions for $\left(4 f^{2} 6 p+4 f 5 d^{2}+4 f 5 d 6 s\right), 4 f^{2} 5 f$, and $4 f^{2} 6 d$ are from Sugar's calculations [1969], and those for $4 f^{2} 6 s$ and $4 f^{2} 5 d$ are from Goldschmidt's thesis [1968]. The second component given in the last column is from the same eigenvector as that used for the designation, except for the levels of the $4 f^{2} 5 f$ configuration. Although $J_{1} l$ coupling is the preferred scheme for this configuration, Sugar found the LS character of the levels useful in making his first identifications. Hence the leading $L S$ component for each $4 f^{2} 5 f$ level is listed instead of a second component from the $J_{1} l$ scheme.

The strong configuration interactions within the $\left(4 f^{2} 6 p+4 f 5 d^{2}+4 f 5 d 6 s\right)$ group [Sugar, 1969; Pasternak, 1970] are analogous to the interactions within Ce III $\left(4 f 6 p+5 d^{2}+5 d 6 s\right)$ found by Goldschmidt.

The extent of the observed structure for Pr III $4 f^{3}$ ( 39 of the 41 expected levels have been found) and the relative freedom from perturbation by other low configurations have made possible the evaluation of other deviations from the intermediate-coupling approximation; a number of "effective interactions" and additional magnetic interactions applicable to $f^{N}$ configurations were evaluated by fitting the observed $4 f^{3}$ levels [Rajnak, 1965; Crosswhite et al., 1968; Goldschmidt et al., 1968; Pasternak, 1970].

The energy parameters derived by Feneuille and Pelletier-Allard [1968] for the Pr III $\left(4 f^{2} 5 d+4 f^{2} 6 s\right)$ and $4 f^{2} 6 p$ configurations include effective interactions corresponding to "all two-particle operators, acting only upon the orbit of the electrons." Wyart et al. [1974] calculated $\left(4 f^{2} 5 d+4 f^{2} 6 s\right)$ with improved values for the parameters, and found two missing $4 f^{2}\left({ }^{3} \mathrm{~F}\right) 5 d{ }^{4} \mathrm{D}$ levels. These levels also agree well with the predictions of Goldschmidt [1968].

Judd [1968] cited the regularity of the observed intervals within each of several low $\mathrm{Pr}$ III $4 f^{2} 5 d$ quartets as an example of a theorem he derived. The Landé rule is well obeyed by these intervals despite overlapping of the terms. The smallness of the interactions between the terms is due to the approximate equality of the spin-orbit parameters $\zeta(4 f)$ and $\zeta(5 d)$. This equality also accounts for the regularity of the low lying $4 f 5 d^{2}$ quartets [Sugar, 1969].

Reader and Sugar [1965] were able to derive a value for the nuclear magnetic moment of ${ }^{141} \mathrm{Pr}$ from the wide hyperfine structure of transitions to $4 f^{2} 6 s$ levels.

Sugar obtained a value of $174284 \mathrm{~cm}^{-1}$ for the $\operatorname{Pr}$ IV $4 f^{2}{ }^{3} \mathrm{H}_{4}$ limit by fitting a Ritz formula to the $4 f^{2}\left({ }^{3} \mathrm{H}\right) 6 \mathrm{~s}, 7 \mathrm{~s}$, and $8 \mathrm{~s}$ levels. Sugar and Reader [1973] derived the quoted limit by applying a correction known to be needed in this region of the periodic table.

\section{References}

Crosswhite, H., Crosswhite, H. M., and Judd, B. R., Phys. Rev. 174, 89 (1968). EL PT

Feneuille, S., and Pelletier-Allard, N., Physica (Utrecht) 40, 347 (1968). PT

Goldschmidt, Z. B., Thesis, Hebrew Univ. Jerusalem, Israel, 487 pp. (1968). ND PT

Goldschmidt, Z. B., Pasternak, A., and Goldschmidt, Z. H., Phys. Lett. 28A, 265 (1968). PT

Judd, B. R., J. Opt. Soc. Am. 58, 1311 (1968). PT

Pasternak, A., Thesis, Hebrew Univ. Jerusalem, Israel, 219 pp. (1970). PT 
Rajnak, K., J. Opt. Soc. Am. 55, 126 (1965). ND PT

Reader, J., and Sugar, J., Phys. Rev. 137, B784 (1965). CL Hfs

Spector, N., J. Opt. Soc. Am. 54, 1359 (1964). ND PT

Sugar, J., Johns Hopkins Spectroseopic Rep. No. 22, 87 pp. (1961). EL CL W IP PT

Sugar, J., J. Opt. Soc. Am. 53, 831 (1963). EL Hfs IP PT

Sugar, J., J. Res. Nat. Bur. Stand. (U.S.) 73A, 333 (1969). EL ND CL W IP PT

Sugar, J., J. Res. Nat. Bur. Stand. (U.S.) 78A, 555 (1974). EL CL W

Sugar, J., and Reader, J., J. Chem. Phys. 59, 2083 (1973). IP

Trees, R. E., J. Opt. Soc. Am. 54, 651 (1964). PT

Wyart, J. F., Blaise, J., and Camus, P., Phys. Ser. 9, 325 (1974). EL ND CL PT

[July 1976]

Pr III, Odd Parity

\begin{tabular}{|c|c|c|c|c|c|c|c|}
\hline Configuration & Term & $J$ & $\begin{array}{l}\text { Level } \\
\left(\mathrm{cm}^{-1}\right)\end{array}$ & \multicolumn{4}{|c|}{ Leading percentages } \\
\hline $4 f^{3}$ & ${ }^{4} \mathrm{I}^{\circ}$ & $\begin{array}{c}9 / 2 \\
11 / 2 \\
13 / 2 \\
15 / 2\end{array}$ & $\begin{array}{r}0.00 \\
1398.34 \\
2893.14 \\
4453.76\end{array}$ & $\begin{array}{r}97 \\
99 \\
100 \\
99\end{array}$ & & $\begin{array}{l}2 \\
1 \\
1\end{array}$ & $\begin{array}{l}{ }^{2} \mathrm{H}^{\circ} 2 \\
{ }^{2} \mathrm{H}^{\circ} 2 \\
{ }^{2} \mathrm{~K}^{\circ}\end{array}$ \\
\hline $4 f^{3}$ & ${ }^{4} \mathrm{~F}^{\circ}$ & $\begin{array}{l}3 / 2 \\
5 / 2 \\
7 / 2 \\
9 / 2\end{array}$ & $\begin{array}{r}9370.66 \\
10138.18 \\
10859.06 \\
11761.69\end{array}$ & $\begin{array}{l}95 \\
98 \\
93 \\
79\end{array}$ & & $\begin{array}{r}5 \\
2 \\
4 \\
15\end{array}$ & $\begin{array}{l}{ }^{2} \mathrm{D}^{\circ} 1 \\
{ }^{2} \mathrm{D}^{\circ} 1 \\
{ }^{2} \mathrm{G}^{\circ} 1 \\
{ }^{2} \mathrm{H}^{\circ} 2\end{array}$ \\
\hline $4 f^{3}$ & ${ }^{2} \mathrm{H}^{\circ} 2$ & $\begin{array}{r}9 / 2 \\
11 / 2\end{array}$ & $\begin{array}{l}10032.92 \\
12494.63\end{array}$ & $\begin{array}{l}60 \\
82\end{array}$ & & $\begin{array}{l}10 \\
13\end{array}$ & $\begin{array}{l}{ }^{2} \mathrm{G}^{\circ} 1 \\
{ }^{2} \mathrm{H}^{\circ} 1\end{array}$ \\
\hline $4 f^{3}$ & ${ }^{4} \mathrm{~S}^{\circ}$ & $3 / 2$ & 10950.24 & 95 & & 4 & ${ }^{2} \mathrm{P}^{\circ}$ \\
\hline $4 f^{3}$ & & $7 / 2$ & 13887.60 & 41 & ${ }^{2} \mathrm{G}^{\circ} 1$ & 29 & ${ }^{2} \mathrm{G}^{\circ} 2$ \\
\hline $4 f^{3}$ & ${ }^{4} \mathrm{G}^{\circ}$ & $\begin{array}{c}5 / 2 \\
7 / 2 \\
9 / 2 \\
11 / 2\end{array}$ & $\begin{array}{l}14187.35 \\
15443.48 \\
15705.13 \\
17409.58\end{array}$ & $\begin{array}{l}99 \\
75 \\
55 \\
95\end{array}$ & & $\begin{array}{r} \\
15 \\
17 \\
3\end{array}$ & $\begin{array}{l}{ }^{2} G^{\circ} 1 \\
{ }^{2} G^{\circ} 1 \\
{ }^{2} H^{\circ} 1\end{array}$ \\
\hline $4 f^{3}$ & ${ }^{2} \mathrm{~K}^{\circ}$ & $\begin{array}{l}13 / 2 \\
15 / 2\end{array}$ & $\begin{array}{l}16089.14 \\
17642.06\end{array}$ & $\begin{array}{l}99 \\
96\end{array}$ & & $\begin{array}{l}1 \\
3\end{array}$ & $\begin{array}{l}{ }^{2} \mathrm{I}^{\circ} \\
{ }^{2} \mathrm{~L}^{\circ}\end{array}$ \\
\hline $4 f^{3}$ & & $9 / 2$ & 16763.98 & 43 & ${ }^{4} \mathrm{G}^{\circ}$ & 30 & ${ }^{2} G^{\circ} 1$ \\
\hline $4 f^{3}$ & ${ }^{2} \mathrm{D}^{\circ} 1$ & $\begin{array}{l}3 / 2 \\
5 / 2\end{array}$ & $\begin{array}{l}17095.63 \\
19046.09\end{array}$ & $\begin{array}{l}50 \\
98\end{array}$ & & $\begin{array}{r}40 \\
2\end{array}$ & $\begin{array}{l}{ }^{2} \mathrm{P}^{\circ} \\
{ }^{4} \mathrm{~F}^{\circ}\end{array}$ \\
\hline $4 f^{3}$ & ${ }^{2} \mathrm{P}^{\circ}$ & $\begin{array}{l}1 / 2 \\
3 / 2\end{array}$ & $\begin{array}{l}18693.65 \\
20856.86\end{array}$ & $\begin{array}{l}95 \\
53\end{array}$ & & $\begin{array}{r}5 \\
40\end{array}$ & $\begin{array}{l}{ }^{4} \mathrm{D}^{\circ} \\
{ }^{2} \mathrm{D}^{\circ} 1\end{array}$ \\
\hline $4 f^{3}$ & ${ }^{4} \mathrm{D}^{\circ}$ & $\begin{array}{l}3 / 2 \\
5 / 2 \\
1 / 2 \\
7 / 2\end{array}$ & $\begin{array}{l}23091.70 \\
23245.99 \\
23465.43 \\
24886.51\end{array}$ & $\begin{array}{r}82 \\
81 \\
95 \\
100\end{array}$ & & $\begin{array}{r}15 \\
18 \\
5\end{array}$ & $\begin{array}{l}{ }^{2} \mathrm{D}^{\circ} 2 \\
{ }^{2} \mathrm{D}^{\circ} 2 \\
{ }^{2} \mathrm{P}^{\circ}\end{array}$ \\
\hline $4 f^{3}$ & ${ }^{2} \mathrm{I}^{\circ}$ & $\begin{array}{l}11 / 2 \\
13 / 2\end{array}$ & $\begin{array}{l}24357.98 \\
25391.75\end{array}$ & $\begin{array}{l}84 \\
99\end{array}$ & & $\begin{array}{r}14 \\
1\end{array}$ & $\begin{array}{l}{ }^{2} \mathrm{H}^{\circ} 1 \\
{ }^{2} \mathrm{~K}^{\circ}\end{array}$ \\
\hline $4 f^{3}$ & ${ }^{2} \mathrm{~L}^{\circ}$ & $\begin{array}{l}15 / 2 \\
17 / 2\end{array}$ & $\begin{array}{l}25244.61 \\
26447.88\end{array}$ & $\begin{array}{r}97 \\
100\end{array}$ & & 3 & ${ }^{2} \mathrm{~K}^{\circ}$ \\
\hline $4 f^{3}$ & ${ }^{2} \mathrm{D}^{\circ} 2$ & $\begin{array}{l}3 / 2 \\
5 / 2\end{array}$ & $\begin{array}{l}26921.49 \\
27597.13\end{array}$ & $\begin{array}{l}80 \\
77\end{array}$ & & $\begin{array}{l}16 \\
19\end{array}$ & ${ }^{4} \mathrm{D}^{\circ}$ \\
\hline $4 f^{3}$ & ${ }^{2} \mathrm{H}^{\circ} 1$ & $\begin{array}{c}9 / 2 \\
11 / 2\end{array}$ & $\begin{array}{l}26979.66 \\
28101.77\end{array}$ & $\begin{array}{l}87 \\
70\end{array}$ & & $\begin{array}{l}11 \\
16\end{array}$ & $\begin{array}{l}{ }^{2} \mathrm{H}^{\circ} 2 \\
{ }^{\circ} \mathrm{I}^{\circ}\end{array}$ \\
\hline $4 f^{3}$ & ${ }^{2} \mathrm{~F}^{\circ} 2$ & $\begin{array}{l}7 / 2 \\
5 / 2\end{array}$ & 31787.93 & $\begin{array}{l}82 \\
73\end{array}$ & & $\begin{array}{l}17 \\
21\end{array}$ & ${ }^{2} F^{\circ} 1$ \\
\hline
\end{tabular}


Pr III, Odd Parity_Continued

\begin{tabular}{|c|c|c|c|c|c|c|c|}
\hline Configuration & Term & $J$ & $\begin{array}{l}\text { Level } \\
\left(\mathrm{cm}^{-1}\right)\end{array}$ & \multicolumn{4}{|c|}{ Leading percentages } \\
\hline $4 f^{3}$ & ${ }^{2} \mathrm{G}^{\circ} 2$ & $\begin{array}{l}9 / 2 \\
7 / 2\end{array}$ & $\begin{array}{l}39225.60 \\
39940.72\end{array}$ & $\begin{array}{l}59 \\
59\end{array}$ & & $\begin{array}{l}41 \\
40\end{array}$ & ${ }^{2} \mathrm{G}^{\circ} 1$ \\
\hline $4 f^{3}$ & ${ }^{2} \mathrm{~F}^{\circ} 1$ & $\begin{array}{l}7 / 2 \\
5 / 2\end{array}$ & 53092.80 & $\begin{array}{l}82 \\
77\end{array}$ & & $\begin{array}{l}17 \\
23\end{array}$ & ${ }^{2} \mathrm{~F}^{\circ} 2$ \\
\hline $4 f^{2}\left({ }^{3} \mathrm{H}_{4}\right) 6 p_{1 / 2}$ & $(4,1 / 2)^{\circ}$ & $\begin{array}{l}7 / 2 \\
9 / 2\end{array}$ & $\begin{array}{l}58158.08 \\
58174.15\end{array}$ & $\begin{array}{l}82 \\
95\end{array}$ & & 6 & $4 f\left({ }^{2} \mathrm{~F}^{\circ}\right) 5 d^{2}\left({ }^{3} \mathrm{~F}\right){ }^{4} \mathrm{H}^{\circ}$ \\
\hline $4 f^{2}\left({ }^{3} \mathrm{H}_{5}\right) 6 p_{1 / 2}$ & $(5,1 / 2)^{\circ}$ & $\begin{array}{c}9 / 2 \\
11 / 2\end{array}$ & $\begin{array}{l}60166.20 \\
60419.73\end{array}$ & $\begin{array}{l}79 \\
94\end{array}$ & & 7 & $\left({ }^{3} \mathrm{H}_{4}\right)(4,3 / 2)^{\circ}$ \\
\hline $4 f\left({ }^{2} \mathrm{~F}^{\circ}\right) 5 d^{2}\left({ }^{3} \mathrm{~F}\right)$ & & $7 / 2$ & 60520.32 & 43 & ${ }^{4} \mathrm{H}^{\circ}$ & 33 & $\left({ }^{3} \mathrm{~F}\right){ }^{2} \mathrm{G}^{\circ}$ \\
\hline $4 f^{2}\left({ }^{3} \mathrm{H}_{4}\right) 6 p_{3 / 2}$ & $(4,3 / 2)^{\circ}$ & $\begin{array}{l}9 / 2 \\
7 / 2 \\
5 / 2 \\
11 / 2\end{array}$ & $\begin{array}{l}61357.04 \\
61605.66 \\
61717.98 \\
62240.83\end{array}$ & $\begin{array}{l}53 \\
80 \\
72 \\
40\end{array}$ & & $\begin{array}{r}19 \\
7 \\
18 \\
35\end{array}$ & $\begin{array}{l}4 f\left({ }^{2} \mathrm{~F}^{\circ}\right) 5 d^{2}\left({ }^{3} \mathrm{~F}\right){ }^{4} \mathrm{I}^{\circ} \\
4 f\left({ }^{2} \mathrm{~F}^{\circ}\right) 5 d^{2}\left({ }^{3} \mathrm{~F}\right){ }^{2} \mathrm{G}^{\circ} \\
\left({ }^{3} \mathrm{~F}_{2}\right)(2,1 / 2)^{\circ} \\
\left({ }^{3} \mathrm{H}_{6}\right)\left(6,{ }^{\circ} / 2\right)^{\circ}\end{array}$ \\
\hline $4 f\left({ }^{2} \mathrm{~F}^{\circ}\right) 5 d^{2}\left({ }^{3} \mathrm{~F}\right)$ & ${ }^{4} I^{\circ}$ & $\begin{array}{l}9 / 2 \\
11 / 2 \\
13 / 2 \\
15 / 2\end{array}$ & $\begin{array}{l}62062.63 \\
63816.91 \\
65967.15 \\
68305.1\end{array}$ & $\begin{array}{l}44 \\
50 \\
86 \\
95\end{array}$ & & $\begin{array}{r}32 \\
24 \\
5\end{array}$ & $\begin{array}{l}4 f^{2}\left({ }^{3} \mathrm{H}_{4}\right) 6 p_{3 / 2} \\
4 f^{2}\left({ }^{3} \mathrm{H}_{5}\right) 6 p_{3 / 2} \\
\left({ }^{3} \mathrm{~F}\right){ }^{4} \mathrm{H}^{\circ}\end{array}$ \\
\hline $4 f\left({ }^{2} \mathrm{~F}^{\circ}\right) 5 d^{2}\left({ }^{3} \mathrm{~F}\right)$ & & $9 / 2$ & 62535.62 & 33 & ${ }^{2} \mathrm{G}^{\circ}$ & 27 & $\left({ }^{3} \mathrm{~F}\right){ }^{4} \mathrm{H}^{\circ}$ \\
\hline $4 f^{2}\left({ }^{3} \mathrm{H}_{6}\right) 6 p_{1 / 2}$ & $(6,1 / 2)^{\circ}$ & $\begin{array}{l}11 / 2 \\
13 / 2\end{array}$ & $\begin{array}{l}62558.67 \\
62678.70\end{array}$ & $\begin{array}{l}55 \\
89\end{array}$ & & $\begin{array}{r}37 \\
6\end{array}$ & $\begin{array}{l}\left({ }^{3} \mathrm{H}_{4}\right)(4,3 / 2)^{\circ} \\
\left({ }^{3} \mathrm{H}_{6}\right)(6,3 / 2)^{\circ}\end{array}$ \\
\hline $4 f^{2}\left({ }^{3} \mathrm{~F}_{2}\right) 6 p_{1 / 2}$ & $(2,1 / 2)^{\circ}$ & $\begin{array}{l}3 / 2 \\
5 / 2\end{array}$ & $\begin{array}{l}63221.28 \\
63576.27\end{array}$ & $\begin{array}{l}91 \\
75\end{array}$ & & 20 & $\left({ }^{3} \mathrm{H}_{4}\right)(4,3 / 2)^{\circ}$ \\
\hline $4 f\left({ }^{2} \mathrm{~F}^{\circ}\right) 5 d^{2}\left({ }^{3} \mathrm{~F}\right)$ & ${ }^{4} \mathrm{H}^{\circ}$ & $\begin{array}{l}7 / 2 \\
9 / 2 \\
11 / 2 \\
13 / 2\end{array}$ & $\begin{array}{l}63232.14 \\
64235.61 \\
64865.15 \\
66735.88\end{array}$ & $\begin{array}{l}42 \\
46 \\
73 \\
53\end{array}$ & & $\begin{array}{l}20 \\
24 \\
15 \\
32\end{array}$ & $\begin{array}{l}\left({ }^{3} \mathrm{~F}\right){ }^{2} \mathrm{G}^{\circ} \\
4 f^{2}\left({ }^{3} \mathrm{H}_{5}\right) 6 p_{3 / 2} \\
4 f^{2}\left({ }^{3} \mathrm{H}_{5}\right) 6 p_{3 / 2} \\
4 f^{2}\left({ }^{3} \mathrm{H}_{6}\right) 6 p_{3 / 2}\end{array}$ \\
\hline $4 f^{2}\left({ }^{3} \mathrm{H}_{5}\right) 6 p_{3 / 2}$ & $(5,3 / 2)^{\circ}$ & $\begin{array}{l}9 / 2 \\
7 / 2 \\
13 / 2\end{array}$ & $\begin{array}{l}63593.35 \\
63768.66 \\
64215.45\end{array}$ & $\begin{array}{l}67 \\
47 \\
86\end{array}$ & & $\begin{array}{r}11 \\
34 \\
8\end{array}$ & $\begin{array}{l}4 f\left({ }^{2} \mathrm{~F}^{\circ}\right) 5 d^{2}\left({ }^{3} \mathrm{~F}\right){ }^{4} \mathrm{H}^{\circ} \\
\left({ }^{3} \mathrm{~F}_{3}\right)(3,1 / 2)^{\circ} \\
\left({ }^{3} \mathrm{H}_{6}\right)(6,3 / 2)^{\circ}\end{array}$ \\
\hline $4 f\left({ }^{2} \mathrm{~F}^{\circ}\right) 5 d^{2}\left({ }^{3} \mathrm{~F}\right)$ & & $11 / 2$ & 64150.94 & 42 & ${ }^{4} I^{\circ}$ & 39 & $4 f^{2}\left({ }^{3} \mathrm{H}_{5}\right) 6 p_{3 / 2}$ \\
\hline $4 f^{2}\left({ }^{3} \mathrm{~F}_{3}\right) 6 p_{1 / 2}$ & $(3,1 / 2)^{\circ}$ & $\begin{array}{l}5 / 2 \\
7 / 2\end{array}$ & $\begin{array}{l}64401.03 \\
65295.90\end{array}$ & $\begin{array}{l}75 \\
45\end{array}$ & & $\begin{array}{r}7 \\
39\end{array}$ & $\begin{array}{l}4 f\left({ }^{2} \mathrm{~F}^{\circ}\right) 5 d^{2}\left({ }^{3} \mathrm{~F}\right){ }^{4} \mathrm{D}^{\circ} \\
\left({ }^{3} \mathrm{H}_{5}\right)(5,3 / 2)^{\circ}\end{array}$ \\
\hline $4 f\left({ }^{2} \mathrm{~F}^{\circ}\right) 5 d^{2}\left({ }^{3} \mathrm{~F}\right)$ & & $5 / 2$ & 64817.53 & 31 & ${ }^{2} \mathrm{~F}^{\circ}$ & 27 & $\left({ }^{3} \mathrm{~F}\right){ }^{4} \mathrm{G}^{\circ}$ \\
\hline $4 f^{2}\left({ }^{3} \mathrm{~F}_{4}\right) 6 p_{1 / 2}$ & $(4,1 / 2)^{\circ}$ & $\begin{array}{l}9 / 2 \\
7 / 2\end{array}$ & $\begin{array}{l}64857.15 \\
64979.55\end{array}$ & $\begin{array}{l}57 \\
46\end{array}$ & & $\begin{array}{l}24 \\
17\end{array}$ & $\left({ }^{1} \mathrm{G}_{4}\right)\left(4,{ }^{1} / 2\right)^{\circ}$ \\
\hline $4 f\left({ }^{2} \mathrm{~F}^{\circ}\right) 5 d^{2}\left({ }^{3} \mathrm{~F}\right)$ & ${ }^{4} \mathrm{G}^{\circ}$ & $\begin{array}{l}5 / 2 \\
7 / 2 \\
9 / 2 \\
11 / 2\end{array}$ & $\begin{array}{l}65909.44 \\
66852.87 \\
68544.19 \\
69978.08\end{array}$ & $\begin{array}{l}47 \\
47 \\
50 \\
62\end{array}$ & & $\begin{array}{l}18 \\
18 \\
35 \\
11\end{array}$ & $\begin{array}{l}\left({ }^{3} \mathrm{~F}\right){ }^{4} \mathrm{D}^{\circ} \\
4 f^{2}\left({ }^{3} \mathrm{~F}_{2}\right) 6 p_{3 / 2} \\
4 f^{2}\left({ }^{3} \mathrm{~F}_{3}\right) 6 p_{3 / 2} \\
\left({ }^{1} \mathrm{D}\right){ }^{2} \mathrm{H}^{\circ}\end{array}$ \\
\hline $4 f^{2}\left({ }^{3} \mathrm{H}_{6}\right) 6 p_{3 / 2}$ & $\left(6,{ }^{3} / 2\right)^{\circ}$ & $\begin{array}{c}11 / 2 \\
13 / 2 \\
15 / 2 \\
9 / 2\end{array}$ & $\begin{array}{l}65922.42 \\
66148.40 \\
66301.35 \\
67049.18\end{array}$ & $\begin{array}{l}88 \\
51 \\
96 \\
60\end{array}$ & & $\begin{array}{r}4 \\
25 \\
12\end{array}$ & $\begin{array}{l}4 f\left({ }^{2} \mathrm{~F}^{\circ}\right) 5 d^{2}\left({ }^{3} \mathrm{~F}\right){ }^{4} \mathrm{H}^{\circ} \\
4 f\left({ }^{2} \mathrm{~F}^{\circ}\right) 5 d^{2}\left({ }^{3} \mathrm{~F}\right){ }^{4} \mathrm{H}^{\circ} \\
\left({ }^{1} \mathrm{G}_{4}\right)\left(4,{ }^{1} / 2\right)^{\circ}\end{array}$ \\
\hline $4 f\left({ }^{2} \mathrm{~F}^{\circ}\right) 5 d^{2}\left({ }^{3} \mathrm{~F}\right)$ & & $7 / 2$ & 65935.25 & 22 & ${ }^{2} \mathrm{~F}^{\circ}$ & 12 & $\left({ }^{3} \mathrm{~F}\right){ }^{4} \mathrm{D}^{\circ}$ \\
\hline $4 f^{2}\left({ }^{3} \mathrm{~F}_{2}\right) 6 p_{3 / 2}$ & $(2,3 / 2)^{\circ}$ & $\begin{array}{l}1 / 2 \\
3 / 2 \\
7 / 2 \\
5 / 2\end{array}$ & $\begin{array}{l}66324.84 \\
66867.09 \\
67240.35 \\
67395.32\end{array}$ & $\begin{array}{l}81 \\
76 \\
58 \\
44\end{array}$ & & $\begin{array}{r}4 \\
7 \\
27 \\
22\end{array}$ & $\begin{array}{l}4 f\left({ }^{2} \mathrm{~F}^{\circ}\right) 5 d^{2}\left({ }^{3} \mathrm{~F}\right){ }^{2} \mathrm{~S}^{\circ} \\
4 f\left({ }^{2} \mathrm{~F}^{\circ}\right) 5 d^{2}\left({ }^{3} \mathrm{~F}\right){ }^{4} \mathrm{~F}^{\circ} \\
4 f\left({ }^{2} \mathrm{~F}^{\circ}\right) 5 d^{2}\left({ }^{3} \mathrm{~F}\right){ }^{4} \mathrm{G}^{\circ} \\
4 f\left({ }^{2} \mathrm{~F}^{\circ}\right) 5 d^{2}\left({ }^{3} \mathrm{~F}\right){ }^{4} \mathrm{~F}^{\circ}\end{array}$ \\
\hline
\end{tabular}


Pr III, Odd Parity_Continued

\begin{tabular}{|c|c|c|c|c|c|c|c|}
\hline Configuration & Term & $J$ & $\begin{array}{l}\text { Level } \\
\left(\mathrm{cm}^{-1}\right)\end{array}$ & \multicolumn{4}{|c|}{ Leading percentages } \\
\hline $4 f\left({ }^{2} \mathrm{~F}^{\circ}\right) 5 d^{2}\left({ }^{3} \mathrm{~F}\right)$ & ${ }^{4} \mathrm{~F}^{\circ}$ & $\begin{array}{l}3 / 2 \\
5 / 2 \\
7 / 2 \\
9 / 2\end{array}$ & $\begin{array}{l}66681.14 \\
67679.21 \\
69686.26\end{array}$ & $\begin{array}{l}76 \\
52 \\
32 \\
65\end{array}$ & & $\begin{array}{r}10 \\
30 \\
32 \\
8\end{array}$ & $\begin{array}{l}4 f^{2}\left({ }^{3} \mathrm{~F}_{2}\right) 6 p_{3 / 2} \\
4 f^{2}\left({ }^{3} \mathrm{~F}_{2}\right) 6 p_{3 / 2} \\
4 f^{2}\left({ }^{1} \mathrm{G}_{4}\right) 6 p_{1 / 2} \\
4 f^{2}\left({ }^{3} \mathrm{~F}_{4}\right) 6 p_{1 / 2}\end{array}$ \\
\hline $4 f\left({ }^{2} \mathrm{~F}^{\circ}\right) 5 d^{2}\left({ }^{3} \mathrm{~F}\right)$ & & $5 / 2$ & 66943.04 & 24 & ${ }^{4} \mathrm{D}^{\circ}$ & 14 & $4 f^{2}\left({ }^{3} \mathrm{~F}_{2}\right) 6 p_{3 / 2}$ \\
\hline $4 f\left({ }^{2} \mathrm{~F}^{\circ}\right) 5 d^{2}\left({ }^{1} \mathrm{D}\right)$ & & $9 / 2$ & 67398.79 & 29 & ${ }^{2} \mathrm{H}^{\circ}$ & 20 & $\left({ }^{3} \mathrm{~F}\right){ }^{2} \mathrm{H}^{\circ}$ \\
\hline $4 f^{2}\left({ }^{3} \mathrm{~F}_{3}\right) 6 p_{3 / 2}$ & $(3,3 / 2)^{\circ}$ & $\begin{array}{l}9 / 2 \\
3 / 2 \\
7 / 2 \\
5 / 2\end{array}$ & $\begin{array}{l}67870.76 \\
67965.47 \\
68492.43 \\
68978.55\end{array}$ & $\begin{array}{l}48 \\
91 \\
37 \\
41\end{array}$ & & $\begin{array}{l}26 \\
17 \\
35\end{array}$ & $\begin{array}{l}4 f\left({ }^{2} \mathrm{~F}^{\circ}\right) 5 d^{2}\left({ }^{3} \mathrm{~F}\right){ }^{4} \mathrm{G}^{\circ} \\
4 f\left({ }^{2} \mathrm{~F}^{\circ}\right) 5 d^{2}\left({ }^{3} \mathrm{~F}\right){ }^{4} \mathrm{~F}^{\circ} \\
\left({ }^{3} \mathrm{~F}_{4}\right)\left(4,{ }^{3} / 2\right)^{\circ}\end{array}$ \\
\hline $4 f\left({ }^{2} \mathrm{~F}^{\circ}\right) 5 d^{2}\left({ }^{3} \mathrm{~F}\right)$ & ${ }^{2} I^{\circ}$ & $\begin{array}{l}11 / 2 \\
13 / 2\end{array}$ & $\begin{array}{l}68238.12 \\
70381.48\end{array}$ & $\begin{array}{l}51 \\
53\end{array}$ & & $\begin{array}{l}36 \\
29\end{array}$ & $\left({ }^{1} \mathrm{G}\right){ }^{2} \mathrm{I}^{\circ}$ \\
\hline $4 f\left({ }^{2} \mathrm{~F}^{\circ}\right) 5 d^{2}\left({ }^{3} \mathrm{~F}\right)$ & & $7 / 2$ & 68331.73 & 22 & ${ }^{4} \mathrm{~F}^{\circ}$ & 21 & $4 f^{2}\left({ }^{3} \mathrm{~F}_{3}\right) 6 p_{3 / 2}$ \\
\hline $4 f^{2}\left({ }^{1} \mathrm{G}_{4}\right) 6 p_{1 / 2}$ & & $9 / 2$ & 68374.54 & 41 & $(4,1 / 2)^{\circ}$ & 22 & $\left({ }^{3} \mathrm{~F}_{4}\right)(4,1 / 2)^{\circ}$ \\
\hline $4 f^{2}\left({ }^{3} \mathrm{~F}_{4}\right) 6 p_{3 / 2}$ & $(4,3 / 2)^{\circ}$ & $11 / 2$ & 68525.62 & 53 & & 20 & $\left({ }^{1} \mathrm{G}_{4}\right)(4,3 / 2)^{\circ}$ \\
\hline $4 f^{2}\left({ }^{3} \mathrm{~F}_{4}\right) 6 p_{3 / 2}$ & & $7 / 2$ & 68801.67 & 29 & $(4,3 / 2)^{\circ}$ & 23 & $\left({ }^{1} \mathrm{G}_{4}\right)(4,3 / 2)^{\circ}$ \\
\hline $4 f\left({ }^{2} \mathrm{~F}^{\circ}\right) 5 d^{2}\left({ }^{3} \mathrm{~F}\right)$ & & $7 / 2$ & 68987.07 & 19 & ${ }^{4} \mathrm{D}^{\circ}$ & 15 & $\left({ }^{3} \mathrm{P}\right){ }^{4} \mathrm{G}^{\circ}$ \\
\hline $4 f^{2}\left({ }^{3} \mathrm{~F}_{4}\right) 6 p_{3 / 2}$ & & $9 / 2$ & 69138.28 & 36 & $(4,3 / 2)^{\circ}$ & 32 & $\left({ }^{1} \mathrm{G}_{4}\right)(4,3 / 2)^{\circ}$ \\
\hline $4 f\left({ }^{2} \mathrm{~F}^{\circ}\right) 5 d^{2}\left({ }^{3} \mathrm{~F}\right)$ & & $11 / 2$ & 69408.51 & 37 & ${ }^{2} \mathrm{H}^{\circ}$ & 25 & $\left({ }^{1} \mathrm{D}\right){ }^{2} \mathrm{G}^{\circ}$ \\
\hline $4 f\left({ }^{2} \mathrm{~F}^{\circ}\right) 5 d^{2}\left({ }^{3} \mathrm{~F}\right)$ & & $7 / 2$ & 69431.00 & 18 & ${ }^{4} \mathrm{D}^{\circ}$ & 17 & $\left({ }^{3} \mathrm{P}\right){ }^{4} \mathrm{G}^{\circ}$ \\
\hline $4 f\left({ }^{2} \mathrm{~F}^{\circ}\right) 5 d^{2}\left({ }^{3} \mathrm{P}\right)$ & ${ }^{4} \mathrm{G}^{\circ}$ & $\begin{array}{l}5 / 2 \\
9 / 2 \\
7 / 2 \\
11 / 2\end{array}$ & $\begin{array}{l}69681.6 \\
71021.1 \\
71385.6 \\
73609.1\end{array}$ & $\begin{array}{l}83 \\
43 \\
58 \\
76\end{array}$ & & $\begin{array}{r}6 \\
17 \\
24 \\
9\end{array}$ & $\begin{array}{l}\left({ }^{3} \mathrm{~F}\right){ }^{4} \mathrm{P}^{\circ} \\
\left({ }^{3} \mathrm{P}\right){ }^{2} \mathrm{G}^{\circ} \\
\left({ }^{1} \mathrm{D}\right){ }^{2} \mathrm{G}^{\circ} \\
\left({ }^{3} \mathrm{~F}\right){ }^{2} \mathrm{H}^{\circ}\end{array}$ \\
\hline $4 f\left({ }^{2} \mathrm{~F}^{\circ}\right) 5 d^{2}\left({ }^{3} \mathrm{P}\right)$ & & $3 / 2$ & 71501.0 & 32 & ${ }^{2} \mathrm{D}^{\circ}$ & 25 & $\left({ }^{3} F\right){ }^{4} \mathrm{~S}^{\circ}$ \\
\hline $4 f^{2}\left({ }^{1} \mathrm{G}_{4}\right) 6 p_{3 / 2}$ & $(4,3 / 2)^{\circ}$ & $\begin{array}{c}5 / 2 \\
9 / 2 \\
11 / 2 \\
7 / 2\end{array}$ & $\begin{array}{l}71536.38 \\
71592.34 \\
71736.45 \\
71978.52\end{array}$ & $\begin{array}{l}61 \\
51 \\
65 \\
48\end{array}$ & & $\begin{array}{l}28 \\
37 \\
29 \\
38\end{array}$ & $\left({ }^{3} \mathrm{~F}_{4}\right)(4,3 / 2)^{\circ}$ \\
\hline $4 f\left({ }^{2} \mathrm{~F}^{\circ}\right) 5 d^{2}\left({ }^{1} \mathrm{D}\right)$ & & $5 / 2$ & 71994.81 & 26 & ${ }^{2} \mathrm{D}^{\circ}$ & 17 & $\left({ }^{3} \mathrm{P}\right){ }^{4} \mathrm{D}^{\circ}$ \\
\hline $4 f\left({ }^{2} \mathrm{~F}^{\circ}\right) 5 d^{2}\left({ }^{3} \mathrm{P}\right)$ & & $9 / 2$ & 73029.9 & 36 & ${ }^{4} \mathrm{G}^{\circ}$ & 25 & $\left({ }^{1} \mathrm{G}\right){ }^{2} \mathrm{H}^{\circ}$ \\
\hline $4 f\left({ }^{2} \mathrm{~F}^{\circ}\right) 5 d^{2}\left({ }^{1} \mathrm{G}\right)$ & ${ }^{2} \mathrm{G}^{\circ}$ & $\begin{array}{l}7 / 2 \\
9 / 2\end{array}$ & $\begin{array}{l}73378.42 \\
75762.23\end{array}$ & $\begin{array}{l}65 \\
38\end{array}$ & & $\begin{array}{r}9 \\
21\end{array}$ & $\begin{array}{l}\left({ }^{3} \mathrm{P}\right){ }^{2} \mathrm{G}^{\circ} \\
\left({ }^{1} \mathrm{G}\right){ }^{2} \mathrm{H}^{\circ}\end{array}$ \\
\hline $4 f\left({ }^{2} \mathrm{~F}^{\circ}\right) 5 d^{2}\left({ }^{1} \mathrm{D}\right)$ & ${ }^{2} \mathrm{G}^{\circ}$ & $9 / 2$ & 73606.17 & 42 & & 12 & $\left({ }^{1} \mathrm{G}\right){ }^{2} \mathrm{H}^{\circ}$ \\
\hline $4 f\left({ }^{2} \mathrm{~F}^{\circ}\right) 5 d^{2}\left({ }^{3} \mathrm{P}\right)$ & & $5 / 2$ & 74105.2 & 27 & ${ }^{4} \mathrm{D}^{\circ}$ & 23 & $\left({ }^{3} \mathrm{P}\right){ }^{4} \mathrm{~F}^{\circ}$ \\
\hline $4 f\left({ }^{2} \mathrm{~F}^{\circ}\right) 5 d^{2}\left({ }^{3} \mathrm{P}\right)$ & ${ }^{4} F^{\circ}$ & $\begin{array}{l}3 / 2 \\
5 / 2 \\
7 / 2 \\
9 / 2\end{array}$ & $\begin{array}{l}74463.7 \\
75294.0\end{array}$ & $\begin{array}{l}45 \\
62 \\
74 \\
85\end{array}$ & & $\begin{array}{r}26 \\
12 \\
7 \\
6\end{array}$ & $\begin{array}{l}\left({ }^{3} \mathrm{~F}\right){ }^{2} \mathrm{D}^{\circ} \\
\left({ }^{3} \mathrm{P}\right){ }^{4} \mathrm{D}^{\circ} \\
\left({ }^{3} \mathrm{P}\right){ }^{4} \mathrm{D}^{\circ} \\
\left({ }^{3} \mathrm{~F}\right){ }^{4} \mathrm{~F}^{\circ}\end{array}$ \\
\hline $4 f^{2}\left({ }^{1} \mathrm{D}_{2}\right) 6 p_{1 / 2}$ & $(2,1 / 2)^{\circ}$ & $\begin{array}{l}3 / 2 \\
5 / 2\end{array}$ & $\begin{array}{l}75409.58 \\
75614.81\end{array}$ & $\begin{array}{l}79 \\
60\end{array}$ & & $\begin{array}{r}8 \\
10\end{array}$ & $\begin{array}{l}\left({ }^{3} \mathrm{P}_{2}\right)(2,1 / 2)^{\circ} \\
4 f\left({ }^{2} \mathrm{~F}^{\circ}\right) 5 d^{2}\left({ }^{1} \mathrm{D}\right){ }^{2} \mathrm{D}^{\circ}\end{array}$ \\
\hline $4 f\left({ }^{2} \mathrm{~F}^{\circ}\right) 5 d^{2}\left({ }^{3} \mathrm{~F}\right)$ & & $5 / 2$ & 75560.75 & 35 & ${ }^{2} \mathrm{D}^{\circ}$ & 18 & $4 f^{2}\left({ }^{1} \mathrm{D}_{2}\right) 6 p_{1 / 2}$ \\
\hline $4 f\left({ }^{2} \mathrm{~F}^{\circ}\right) 5 d^{2}\left({ }^{3} \mathrm{~F}\right)$ & & $11 / 2$ & 75640.00 & 32 & ${ }^{2} \mathrm{H}^{\circ}$ & 18 & $\left({ }^{1} \mathrm{D}\right){ }^{2} \mathrm{H}^{\circ}$ \\
\hline
\end{tabular}


Pr III, Odd Parity-Continued

\begin{tabular}{|c|c|c|c|c|c|c|c|}
\hline \multirow{2}{*}{$\frac{\text { Configuration }}{4 f\left({ }^{2} \mathrm{~F}^{\circ}\right) 5 d^{2}\left({ }^{3} \mathrm{~F}\right)}$} & \multirow[t]{2}{*}{ Term } & \multirow{2}{*}{$J$} & \multirow{2}{*}{$\begin{array}{c}\begin{array}{c}\text { Level } \\
\left(\mathrm{cm}^{-1}\right)\end{array} \\
76892.4\end{array}$} & \multicolumn{4}{|c|}{ Leading percentages } \\
\hline & & & & 30 & ${ }^{2} \mathrm{D}^{\circ}$ & 27 & $\left({ }^{1} \mathrm{D}\right){ }^{2} \mathrm{P}^{\circ}$ \\
\hline \multirow[t]{2}{*}{$4 f\left({ }^{2} \mathrm{~F}^{\circ}\right) 5 d^{2}\left({ }^{1} \mathrm{G}\right)$} & ${ }^{2} \mathrm{~F}^{\circ}$ & $5 / 2$ & 77822.00 & 70 & & 5 & $\left({ }^{3} \mathrm{~F}\right){ }^{2} \mathrm{~F}^{\circ}$ \\
\hline & & $7 / 2$ & 78463.6 & 58 & & 20 & $4 f^{2}\left({ }^{1} \mathrm{D}_{2}\right) 6 p_{3 / 2}$ \\
\hline \multirow[t]{4}{*}{$4 f^{2}\left({ }^{1} \mathrm{D}_{2}\right) 6 p 3 / 2$} & $(2,3 / 2)^{\circ}$ & $1 / 2$ & 78312.91 & 68 & & 11 & $\left({ }^{3} \mathrm{P}_{2}\right)\left(2,{ }^{3} / 2\right)^{\circ}$ \\
\hline & & $3 / 2$ & 78889.14 & 76 & & 8 & $\left({ }^{3} \mathrm{P}_{2}\right)(2,3 / 2)^{\circ}$ \\
\hline & & $5 / 2$ & 79366.65 & 79 & & 7 & $\left({ }^{3} \mathrm{P}_{2}\right)(2,3 / 2)^{\circ}$ \\
\hline & & $7 / 2$ & 79395.55 & 66 & & 14 & $4 f\left({ }^{2} \mathrm{~F}^{\circ}\right) 5 d^{2}\left({ }^{1} \mathrm{G}\right){ }^{2} \mathrm{~F}^{\circ}$ \\
\hline \multirow[t]{2}{*}{$4 f\left({ }^{2} \mathrm{~F}^{\circ}\right) 5 d^{2}\left({ }^{1} \mathrm{G}\right)$} & ${ }^{2} I^{\circ}$ & $11 / 2$ & 78694.57 & 35 & & 25 & $\left({ }^{3} \mathrm{~F}\right)^{2} \mathrm{I}^{\circ}$ \\
\hline & & $13 / 2$ & 79136.23 & 51 & & 26 & \\
\hline \multirow[t]{2}{*}{$4 f^{2}\left({ }^{3} P_{1}\right) 6 p_{1 / 2}$} & $(1,1 / 2)^{\circ}$ & $1 / 2$ & 79742.36 & 79 & & 12 & $\left({ }^{3} \mathrm{P}_{0}\right)\left(0,{ }^{1} / 2\right)^{\circ}$ \\
\hline & & $3 / 2$ & 80164.04 & 88 & & 5 & $\left({ }^{1} \mathrm{D}_{2}\right)\left(2,{ }^{3} / 2\right)^{\circ}$ \\
\hline \multirow[t]{2}{*}{$4 f^{2}\left({ }^{1} \mathrm{I}_{6}\right) 6 p_{1 / 2}$} & $(6,1 / 2)^{\circ}$ & $11 / 2$ & 80360.82 & 80 & & 6 & $4 f\left({ }^{2} \mathrm{~F}^{\circ}\right) 5 d^{2}\left({ }^{3} \mathrm{~F}\right){ }^{2} \mathrm{I}^{\circ}$ \\
\hline & & $13 / 2$ & 80988.76 & 70 & & 12 & $4 f\left({ }^{2} \mathrm{~F}^{\circ}\right) 5 d^{2}\left({ }^{1} \mathrm{G}\right){ }^{2} \mathrm{I}^{\circ}$ \\
\hline \multirow[t]{2}{*}{$4 f^{2}\left({ }^{3} \mathrm{P}_{2}\right) 6 p_{1 / 2}$} & $(2,1 / 2)^{\circ}$ & $3 / 2$ & 80897.85 & 75 & & 7 & $\left({ }^{1} \mathrm{D}_{2}\right)\left(2,{ }^{1} / 2\right)^{\circ}$ \\
\hline & & $5 / 2$ & 81404.52 & 83 & & 8 & \\
\hline $4 f^{2}\left({ }^{3} \mathrm{P}_{0}\right) 6 p_{3 / 2}$ & $(0,3 / 2)^{\circ}$ & $3 / 2$ & 82492.4 & 44 & & 35 & $4 f\left({ }^{2} \mathrm{~F}^{\circ}\right) 5 d^{2}\left({ }^{1} \mathrm{G}\right){ }^{2} \mathrm{D}^{\circ}$ \\
\hline \multirow[t]{3}{*}{$4 f^{2}\left({ }^{3} \mathrm{P}_{1}\right) 6 p_{3 / 2}$} & $(1,3 / 2)^{\circ}$ & $3 / 2$ & 83025.91 & 43 & & 23 & $\left({ }^{3} \mathrm{P}_{0}\right)(0,3 / 2)^{\circ}$ \\
\hline & & $1 / 2$ & & 51 & & 35 & $\left({ }^{3} \mathrm{P}_{2}\right)\left(2,3^{3} / 2\right)^{\circ}$ \\
\hline & & $5 / 2$ & 83426.55 & 79 & & 10 & $4 f\left({ }^{2} \mathrm{~F}^{\circ}\right) 5 d^{2}\left({ }^{1} \mathrm{G}\right){ }^{2} \mathrm{D}^{\circ}$ \\
\hline \multirow[t]{4}{*}{$4 f^{2}\left({ }^{1} \mathbf{I}_{6}\right) 6 p_{3 / 2}$} & $\left(6,{ }^{3} / 2\right)^{\circ}$ & $11 / 2$ & 83607.32 & 87 & & 7 & $\left({ }^{1} \mathbf{I}_{6}\right)\left(6,{ }^{1} / 2\right)^{\circ}$ \\
\hline & & $13 / 2$ & 83703.43 & 87 & & 11 & $\left({ }^{1} \mathrm{I}_{6}\right)\left(6,{ }^{1} / 2\right)^{\circ}$ \\
\hline & & $9 / 2$ & 84410.63 & 78 & & 8 & $4 f\left({ }^{2} \mathrm{~F}^{\circ}\right) 5 d^{2}\left({ }^{1} \mathrm{H}\right){ }^{2} \mathrm{H}^{\circ}$ \\
\hline & & $15 / 2$ & 84992.04 & 98 & & & \\
\hline \multirow[t]{4}{*}{$4 f 5 d\left({ }^{3} \mathrm{~F}^{\circ}\right) 6 \mathrm{~s}$} & ${ }^{4} \mathrm{~F}^{\circ}$ & $3 / 2$ & 84409.93 & 62 & & 11 & $4 f^{2}\left({ }^{3} \mathrm{P}_{1}\right) 6 p_{3 / 2}$ \\
\hline & & $5 / 2$ & & 56 & & 8 & $4 f\left({ }^{2} \mathrm{~F}^{\circ}\right) 5 d^{2}\left({ }^{3} \mathrm{P}\right)^{2} \mathrm{~F}^{\circ}$ \\
\hline & & $7 / 2$ & & 70 & & 15 & $\left({ }^{3} \mathrm{G}^{\circ}\right){ }^{4} \mathrm{G}^{\circ}$ \\
\hline & & $9 / 2$ & 88948.6 & 39 & & 35 & $\left({ }^{3} \mathrm{G}^{\circ}\right){ }^{4} \mathrm{G}^{\circ}$ \\
\hline \multirow[t]{4}{*}{$4 f^{2}\left({ }^{3} \mathbf{P}_{2}\right) 6 p_{3 / 2}$} & $(2,3 / 2)^{\circ}$ & $7 / 2$ & 84430.98 & 85 & & 9 & $\left({ }^{1} \mathrm{D}_{2}\right)(2,3 / 2)^{\circ}$ \\
\hline & & $1 / 2$ & & 47 & & 37 & $\left({ }^{3} \mathrm{P}_{1}\right)(1,3 / 2)^{\circ}$ \\
\hline & & $3 / 2$ & & 65 & & 16 & $4 f\left({ }^{2} \mathrm{~F}^{\circ}\right) 5 d^{2}\left({ }^{1} \mathrm{G}\right){ }^{2} \mathrm{P}^{\circ}$ \\
\hline & & $5 / 2$ & 85306.10 & 52 & & 19 & $4 f\left({ }^{2} \mathrm{~F}^{\circ}\right) 5 d^{2}\left({ }^{1} \mathrm{G}\right){ }^{2} \mathrm{D}^{\circ}$ \\
\hline \multirow[t]{4}{*}{$4 f 5 d\left({ }^{3} \mathrm{G}^{\circ}\right) 6 s$} & ${ }^{4} \mathrm{G}^{\circ}$ & $5 / 2$ & & 87 & & 7 & $\left({ }^{3} \mathrm{~F}^{\circ}\right){ }^{4} \mathrm{~F}^{\circ}$ \\
\hline & & $7 / 2$ & 87511.6 & 52 & & 26 & $\left({ }^{1} G^{0}\right)^{2} G^{\circ}$ \\
\hline & & $9 / 2$ & 88220.2 & 57 & & 35 & $\left({ }^{3} F^{\circ}\right){ }^{4} F^{\circ}$ \\
\hline & & $11 / 2$ & 90119.8 & 96 & & & \\
\hline \multirow[t]{2}{*}{$4 f 5 d\left({ }^{3} \mathrm{H}^{\circ}\right) 6 s$} & ${ }^{2} \mathrm{H}^{\circ}$ & $9 / 2$ & 90629.2 & 79 & & 5 & $4 f\left({ }^{2} \mathrm{~F}^{\circ}\right) 5 d^{2}\left({ }^{1} \mathrm{D}\right){ }^{2} \mathrm{H}^{\circ}$ \\
\hline & & $11 / 2$ & 93296.5 & 80 & & 9 & $\left({ }^{1} \mathrm{H}^{\circ}\right)^{2} \mathrm{H}^{\circ}$ \\
\hline \multirow[t]{2}{*}{$4 f 5 d\left({ }^{3} \mathrm{G}^{\circ}\right) 6 s$} & ${ }^{2} \mathrm{G}^{\circ}$ & $7 / 2$ & 92441.7 & 53 & & 21 & $\left({ }^{3} \mathrm{D}^{\circ}\right)^{4} \mathrm{D}^{\circ}$ \\
\hline & & $9 / 2$ & 95147.9 & 77 & & 8 & $4 f\left({ }^{2} \mathrm{~F}^{\circ}\right) 5 d^{2}\left({ }^{1} \mathrm{D}\right){ }^{2} \mathrm{G}^{\circ}$ \\
\hline \multirow[t]{2}{*}{$4 f\left({ }^{2} \mathrm{~F}^{\circ}\right) 5 d^{2}\left({ }^{1} \mathrm{~S}\right)$} & ${ }^{2} \mathrm{~F}^{\circ}$ & $5 / 2$ & 92554.8 & 54 & & 13 & $4 f 5 d\left({ }^{1} \mathrm{~F}^{\circ}\right) 6 s^{2} \mathrm{~F}^{\circ}$ \\
\hline & & $7 / 2$ & 93967.4 & 74 & & 4 & $4 f 5 d\left({ }^{3} \mathrm{G}^{\circ}\right) 6 s^{2} \mathrm{G}^{\circ}$ \\
\hline \multirow[t]{2}{*}{$4 f 5 d\left({ }^{1} \mathrm{~F}^{\circ}\right) 6 s$} & ${ }^{2} \mathrm{~F}^{\circ}$ & $5 / 2$ & & 50 & & 10 & $\left({ }^{3} \mathrm{D}^{\circ}\right)^{2} \mathrm{D}^{\circ}$ \\
\hline & & $7 / 2$ & 96830.5 & 51 & & 23 & $\left({ }^{3} \mathrm{~F}^{\circ}\right)^{2} \mathrm{~F}^{\circ}$ \\
\hline $4 f^{2}\left({ }^{3} \mathrm{H}_{4}\right) 5 f$ & ${ }^{2}[6]^{\circ}$ & $11 / 2$ & 110295.1 & 83 & or & 74 & $\left({ }^{3} \mathrm{H}\right){ }^{4} \mathrm{~K}^{0}$ \\
\hline & & $13 / 2$ & 111110.3 & 87 & or & 59 & \\
\hline$"$ & ${ }^{2}[4]^{\circ}$ & $7 / 2$ & 110333.5 & 91 & or & 81 & $\left({ }^{3} \mathrm{H}\right){ }^{4} \mathrm{H}^{\circ}$ \\
\hline & & $9 / 2$ & 110922.5 & 66 & or & 70 & $\left({ }^{3} \mathrm{~F}\right){ }^{4} \mathrm{H}^{\circ}$ \\
\hline$" \prime$ & ${ }^{2}[7]^{\circ}$ & $13 / 2$ & 110530.9 & 97 & or & 89 & $\left({ }^{3} \mathrm{H}\right){ }^{4} \mathrm{~L}^{\circ}$ \\
\hline & & $15 / 2$ & 111335.1 & 82 & or & 67 & \\
\hline
\end{tabular}


Pr III, Odd Parity_Continued

\begin{tabular}{|c|c|c|c|c|c|c|c|c|}
\hline Configuration & Term & $J$ & $\begin{array}{l}\text { Level } \\
\left(\mathrm{cm}^{-1}\right)\end{array}$ & \multicolumn{5}{|c|}{ Leading percentages } \\
\hline $4 f^{2}\left({ }^{3} \mathrm{H}_{4}\right) 5 f$ & ${ }^{2}[5]^{\circ}$ & $\begin{array}{l}11 / 2 \\
9 / 2\end{array}$ & $\begin{array}{l}110881.1 \\
111993.0\end{array}$ & $\begin{array}{l}78 \\
72\end{array}$ & $\begin{array}{l}\text { or } \\
\text { or }\end{array}$ & & $\begin{array}{l}41 \\
58\end{array}$ & $\begin{array}{l}\left({ }^{3} \mathrm{H}\right)^{2} \mathrm{I}^{\circ} \\
\left({ }^{3} \mathrm{~F}\right)^{2} \mathrm{I}^{\circ}\end{array}$ \\
\hline " & ${ }^{2}[2]^{\circ}$ & $\begin{array}{l}3 / 2 \\
5 / 2\end{array}$ & $\begin{array}{l}111268.7 \\
111494.8\end{array}$ & $\begin{array}{l}92 \\
64\end{array}$ & $\begin{array}{l}\text { or } \\
\text { or }\end{array}$ & & $\begin{array}{l}80 \\
59\end{array}$ & $\left({ }^{3} \mathrm{H}\right){ }^{4} \mathrm{~F}^{\circ}$ \\
\hline ", & $2[3]^{\circ}$ & $\begin{array}{l}7 / 2 \\
5 / 2\end{array}$ & 111342.8 & $\begin{array}{l}87 \\
63\end{array}$ & $\begin{array}{l}\text { or } \\
\text { or }\end{array}$ & & $\begin{array}{l}40 \\
52\end{array}$ & $\begin{array}{l}\left({ }^{3} \mathrm{H}\right){ }^{2} \mathrm{G}^{\circ} \\
\left({ }^{3} \mathrm{H}\right){ }^{4} \mathrm{G}^{\circ}\end{array}$ \\
\hline $4 f^{2}\left({ }^{3} \mathrm{H}_{5}\right) 5 f$ & ${ }^{2}[5]^{\circ}$ & $\begin{array}{l}11 / 2 \\
9 / 2\end{array}$ & $\begin{array}{l}112643.2 \\
113158.8\end{array}$ & $\begin{array}{l}66 \\
62\end{array}$ & or & & 61 & $\left({ }^{3} \mathrm{H}\right){ }^{4} \mathrm{H}^{\circ}$ \\
\hline " & ${ }^{2}[7]^{\circ}$ & $\begin{array}{l}13 / 2 \\
15 / 2\end{array}$ & $\begin{array}{l}112769.7 \\
112896.3\end{array}$ & $\begin{array}{l}51 \\
88\end{array}$ & $\begin{array}{l}\text { or } \\
\text { or }\end{array}$ & & $\begin{array}{l}39 \\
72\end{array}$ & $\begin{array}{l}\left({ }^{3} \mathrm{H}\right){ }^{2} \mathrm{I}^{\circ} \\
\left({ }^{3} \mathrm{H}\right){ }^{4} \mathrm{~K}^{\circ}\end{array}$ \\
\hline$"$ & ${ }^{2}[8]^{\circ}$ & $\begin{array}{l}17 / 2 \\
15 / 2\end{array}$ & $\begin{array}{l}113023.5 \\
113833.9\end{array}$ & $\begin{array}{l}94 \\
79\end{array}$ & $\begin{array}{l}\text { or } \\
\text { or }\end{array}$ & & $\begin{array}{l}94 \\
76\end{array}$ & $\begin{array}{l}\left({ }^{3} \mathrm{H}\right){ }^{4} \mathrm{~L}^{\circ} \\
\left({ }^{3} \mathrm{H}\right){ }^{2} \mathrm{~L}^{\circ}\end{array}$ \\
\hline$"$ & ${ }^{2}[4]^{\circ}$ & $\begin{array}{l}7 / 2 \\
9 / 2\end{array}$ & $\begin{array}{l}113291.8 \\
113780.5\end{array}$ & $\begin{array}{l}50 \\
66\end{array}$ & $\begin{array}{l}\text { or } \\
\text { or }\end{array}$ & & $\begin{array}{l}44 \\
35\end{array}$ & $\begin{array}{l}\left({ }^{3} \mathrm{H}\right){ }^{4} \mathrm{~F}^{\circ} \\
\left({ }^{3} \mathrm{H}\right){ }^{2} \mathrm{H}^{\circ}\end{array}$ \\
\hline$" \prime$ & $2[3]^{\circ}$ & $\begin{array}{l}5 / 2 \\
7 / 2\end{array}$ & $\begin{array}{l}113556.8 \\
113630.9\end{array}$ & $\begin{array}{l}45 \\
46\end{array}$ & $\begin{array}{l}\text { or } \\
\text { or }\end{array}$ & & $\begin{array}{l}23 \\
51\end{array}$ & $\begin{array}{l}\left({ }^{3} \mathrm{H}\right){ }^{4} \mathrm{D}^{\circ} \\
\left({ }^{3} \mathrm{H}\right){ }^{4} \mathrm{G}^{\circ}\end{array}$ \\
\hline "' & ${ }^{2}[6]^{\circ}$ & $\begin{array}{l}13 / 2 \\
11 / 2\end{array}$ & $\begin{array}{l}113600.3 \\
113664.3\end{array}$ & $\begin{array}{l}49 \\
61\end{array}$ & $\begin{array}{l}\text { or } \\
\text { or }\end{array}$ & & $\begin{array}{l}43 \\
67\end{array}$ & $\begin{array}{l}\left({ }^{3} \mathrm{H}\right){ }^{2} \mathrm{~K}^{\circ} \\
\left({ }^{3} \mathrm{H}\right){ }^{4} \mathrm{I}^{\circ}\end{array}$ \\
\hline$" \prime$ & ${ }^{2}[2]^{\circ}$ & $\begin{array}{l}3 / 2 \\
5 / 2\end{array}$ & $\begin{array}{l}113825.5 \\
113914.1\end{array}$ & $\begin{array}{l}93 \\
46\end{array}$ & $\begin{array}{l}\text { or } \\
\text { or }\end{array}$ & & $\begin{array}{l}45 \\
38\end{array}$ & $\begin{array}{l}\left({ }^{3} \mathrm{H}\right){ }^{2} \mathrm{D}^{\circ} \\
\left({ }^{3} \mathrm{H}\right){ }^{2} \mathrm{~F}^{\circ}\end{array}$ \\
\hline $4 f^{2}\left({ }^{3} \mathrm{H}_{6}\right) 5 f$ & ${ }^{2}[8]^{\circ}$ & $\begin{array}{l}17 / 2 \\
15 / 2\end{array}$ & $\begin{array}{l}114725.6 \\
115672.2\end{array}$ & $\begin{array}{l}95 \\
71\end{array}$ & $\begin{array}{l}\text { or } \\
\text { or }\end{array}$ & & $\begin{array}{l}95 \\
57\end{array}$ & $\begin{array}{l}\left({ }^{3} \mathrm{H}\right){ }^{4} \mathrm{~K}^{\circ} \\
\left({ }^{3} \mathrm{H}\right){ }^{2} \mathrm{~K}^{\circ}\end{array}$ \\
\hline "' & ${ }^{2}[6]^{\circ}$ & $\begin{array}{l}13 / 2 \\
11 / 2\end{array}$ & $\begin{array}{l}1147972 \\
115403.3\end{array}$ & $\begin{array}{l}69 \\
64\end{array}$ & $\begin{array}{l}\text { or } \\
\text { or }\end{array}$ & & $\begin{array}{r}48 \\
\cdot 34\end{array}$ & $\begin{array}{l}\left({ }^{3} \mathrm{H}\right){ }^{4} \mathrm{H}^{\circ} \\
\left({ }^{3} \mathrm{H}\right){ }^{2} \mathrm{H}^{\circ}\end{array}$ \\
\hline " & ${ }^{2}[9]^{\circ}$ & $\begin{array}{l}19 / 2 \\
17 / 2\end{array}$ & $\begin{array}{l}114970.7 \\
116489.8\end{array}$ & $\begin{array}{r}100 \\
89\end{array}$ & $\begin{array}{l}\text { or } \\
\text { or }\end{array}$ & & $\begin{array}{r}100 \\
89\end{array}$ & $\begin{array}{l}\left({ }^{3} \mathrm{H}\right){ }^{4} \mathrm{~L}^{\circ} \\
\left({ }^{3} \mathrm{H}\right){ }^{2} \mathrm{~L}^{\circ}\end{array}$ \\
\hline$" \prime$ & ${ }^{2}[4]^{\circ}$ & $9 / 2$ & 115324.3 & 46 & or & & 35 & $\left({ }^{3} \mathrm{H}\right){ }^{4} \mathrm{~F}^{\circ}$ \\
\hline$\prime \prime$ & ${ }^{2}[7]^{\circ}$ & $\begin{array}{l}13 / 2 \\
15 / 2\end{array}$ & $\begin{array}{l}115408.4 \\
116238.8\end{array}$ & $\begin{array}{l}64 \\
78\end{array}$ & $\begin{array}{l}\text { or } \\
\text { or }\end{array}$ & & $\begin{array}{l}60 \\
72\end{array}$ & $\begin{array}{l}\left({ }^{3} \mathrm{H}\right){ }^{4} \mathrm{I}^{\circ} \\
\left({ }^{3} \mathrm{H}\right){ }^{4} \mathrm{I}^{\circ}\end{array}$ \\
\hline$" \prime$ & & $7 / 2$ & 115420.8 & 36 & ${ }^{2}[3]^{\circ}$ & or & 35 & $\left({ }^{3} \mathrm{H}\right){ }^{4} \mathrm{D}^{\circ}$ \\
\hline " & ${ }^{2}[5]^{\circ}$ & $\begin{array}{c}9 / 2 \\
11 / 2\end{array}$ & $\begin{array}{l}115499.8 \\
115933.2\end{array}$ & $\begin{array}{l}59 \\
50\end{array}$ & $\begin{array}{l}\text { or } \\
\text { or }\end{array}$ & & $\begin{array}{l}54 \\
41\end{array}$ & $\begin{array}{l}\left({ }^{3} \mathrm{H}\right){ }^{2} \mathrm{G}^{\circ} \\
\left({ }^{3} \mathrm{H}\right){ }^{4} \mathrm{G}^{\circ}\end{array}$ \\
\hline $4 f^{2}\left({ }^{3} \mathrm{~F}_{2}\right) 5 f$ & ${ }^{2}[4]^{\circ}$ & $\begin{array}{l}7 / 2 \\
9 / 2\end{array}$ & $\begin{array}{l}115670.0 \\
116453.2\end{array}$ & $\begin{array}{l}89 \\
52\end{array}$ & $\begin{array}{l}\text { or } \\
\text { or }\end{array}$ & & $\begin{array}{l}82 \\
54\end{array}$ & $\begin{array}{l}\left({ }^{3} \mathrm{~F}\right){ }^{4} \mathrm{H}^{\circ} \\
\left({ }^{3} \mathrm{P}\right){ }^{4} \mathrm{H}^{\circ}\end{array}$ \\
\hline $\begin{array}{c}4 f^{2}\left({ }^{3} \mathrm{H}_{6}\right) 5 f \\
.\end{array}$ & ${ }^{2}[3]^{\circ}$ & $\begin{array}{l}5 / 2 \\
7 / 2\end{array}$ & $\begin{array}{c}115800.0 \\
116021.8\end{array}$ & $\begin{array}{l}48 \\
41\end{array}$ & $\begin{array}{r}\text { or } \\
2[3]^{\circ}\end{array}$ & or & $\begin{array}{l}47 \\
42\end{array}$ & $\begin{array}{l}\left({ }^{3} \mathrm{H}\right){ }^{2} \mathrm{D}^{\circ} \\
\left({ }^{3} \mathrm{H}\right){ }^{2} \mathrm{~F}^{\circ}\end{array}$ \\
\hline $4 f^{2}\left({ }^{3} \mathrm{~F}_{2}\right) 5 f$ & ${ }^{2}[5]^{\circ}$ & $\begin{array}{c}9 / 2 \\
11 / 2\end{array}$ & 1163092 & $\begin{array}{l}87 \\
63\end{array}$ & $\begin{array}{l}\text { or } \\
\text { or }\end{array}$ & & $\begin{array}{l}71 \\
31\end{array}$ & $\begin{array}{l}\left({ }^{3} \mathrm{H}\right){ }^{4} \mathrm{H}^{\circ} \\
\left({ }^{3} \mathrm{~F}\right){ }^{4} \mathrm{I}^{\circ}\end{array}$ \\
\hline$\prime \prime$ & ${ }^{2}[3]^{\circ}$ & $\begin{array}{l}5 / 2 \\
7 / 2\end{array}$ & $\begin{array}{l}116727.1 \\
117044.6\end{array}$ & $\begin{array}{l}66 \\
57\end{array}$ & $\begin{array}{l}\text { or } \\
\text { or }\end{array}$ & & $\begin{array}{l}50 \\
21\end{array}$ & $\left({ }^{3} \mathrm{~F}\right){ }^{4} \mathrm{G}^{\circ}$ \\
\hline $4 f^{2}\left({ }^{3} \mathrm{~F}_{3}\right) 5 f$ & ${ }^{2}[6]^{\circ}$ & $\begin{array}{l}13 / 2 \\
11 / 2\end{array}$ & $\begin{array}{l}117248.2 \\
117323.1\end{array}$ & $\begin{array}{l}56 \\
35\end{array}$ & $\begin{array}{l}\text { or } \\
\text { or }\end{array}$ & & $\begin{array}{l}54 \\
28\end{array}$ & $\left({ }^{3} \mathrm{~F}\right){ }^{4} \mathrm{H}^{\circ}$ \\
\hline " & & $3 / 2$ & 117540.5 & 26 & ${ }^{2}[1]^{\circ}$ & or & 24 & $\left({ }^{3} \mathrm{~F}\right)^{2} \mathrm{P}^{\circ}$ \\
\hline
\end{tabular}


Pr III, Odd Parity_Continued

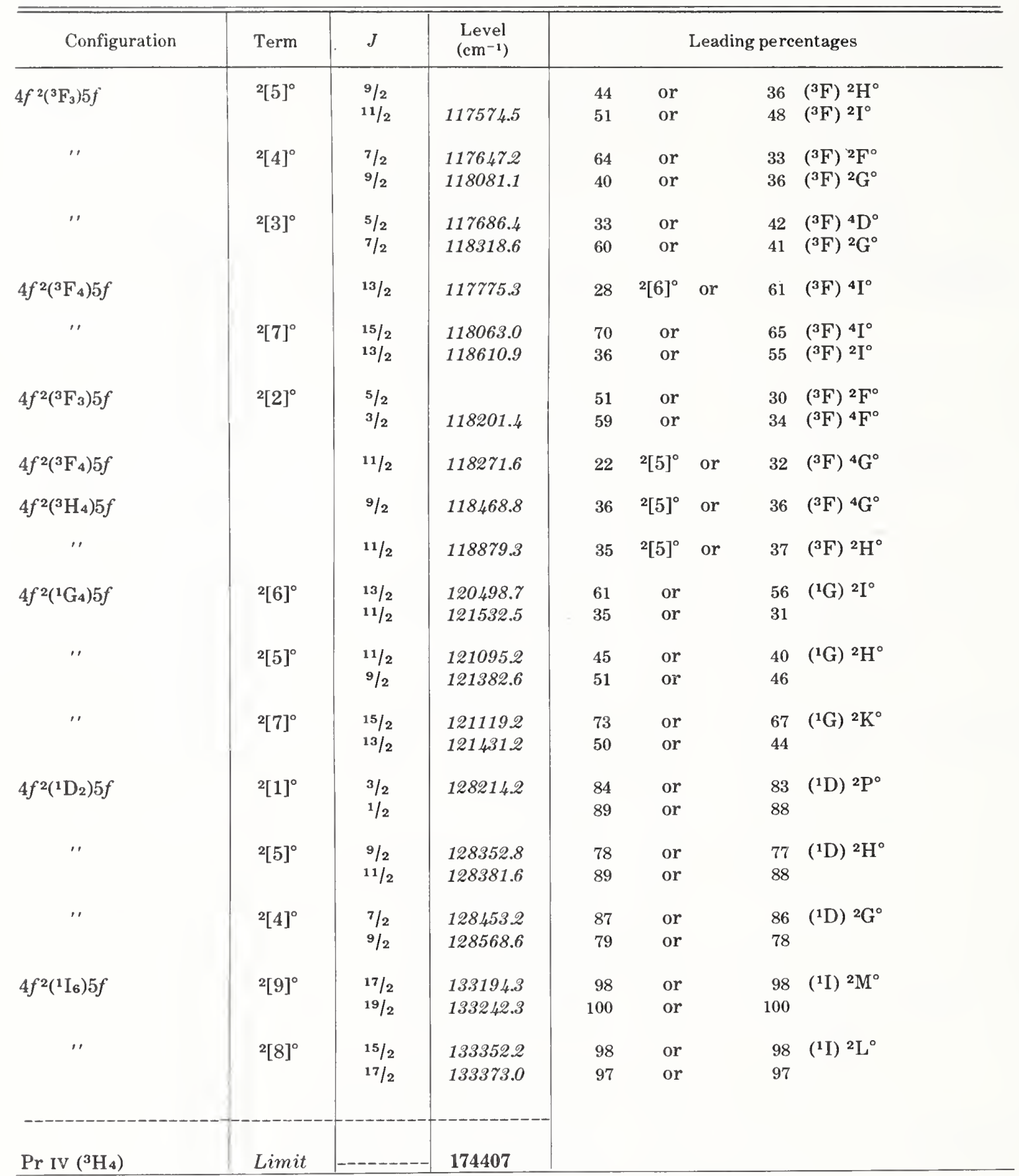


Pr III, Even Parity

\begin{tabular}{|c|c|c|c|c|c|c|c|}
\hline Configuration & Term & $J$ & $\begin{array}{l}\text { Level } \\
\left(\mathrm{cm}^{-1}\right)\end{array}$ & \multicolumn{4}{|c|}{ Leading percentages } \\
\hline $4 f^{2}\left({ }^{3} \mathrm{H}\right) 5 d$ & ${ }^{2} \mathrm{H}$ & $\begin{array}{l}9 / 2 \\
11 / 2\end{array}$ & $\begin{array}{l}12846.66 \\
14859.96\end{array}$ & $\begin{array}{l}52 \\
46\end{array}$ & & $\begin{array}{l}38 \\
27\end{array}$ & $\left({ }^{3} \mathrm{H}\right){ }^{4} \mathrm{I}$ \\
\hline $4 f^{2}\left({ }^{3} \mathrm{H}\right) 5 d$ & ${ }^{4} \mathrm{~K}$ & $\begin{array}{l}11 / 2 \\
13 / 2 \\
15 / 2 \\
17 / 2\end{array}$ & $\begin{array}{l}13352.10 \\
15454.16 \\
17534.76 \\
19649.67\end{array}$ & $\begin{array}{r}80 \\
95 \\
100 \\
100\end{array}$ & & & \\
\hline $4 f^{2}\left({ }^{3} \mathrm{H}\right) 5 d$ & ${ }^{4} I$ & $\begin{array}{c}9 / 2 \\
11 / 2 \\
13 / 2 \\
15 / 2\end{array}$ & $\begin{array}{l}14558.82 \\
15525.50 \\
17113.19 \\
18921.24\end{array}$ & $\begin{array}{l}59 \\
68 \\
98 \\
98\end{array}$ & & 26 & $\left({ }^{3} \mathrm{H}\right){ }^{2} \mathrm{H}$ \\
\hline $4 f^{2}\left({ }^{3} \mathrm{H}\right) 5 d$ & ${ }^{4} \mathrm{G}$ & $\begin{array}{l}5 / 2 \\
7 / 2 \\
9 / 2 \\
11 / 2\end{array}$ & $\begin{array}{l}15045.80 \\
16516.15 \\
18063.36 \\
19700.90\end{array}$ & $\begin{array}{l}50 \\
57 \\
56 \\
52\end{array}$ & & $\begin{array}{l}25 \\
25 \\
25 \\
29\end{array}$ & $\left({ }^{3} \mathrm{~F}\right){ }^{4} \mathrm{G}$ \\
\hline $4 f^{2}\left({ }^{3} \mathrm{H}\right) 5 d$ & ${ }^{4} \mathrm{H}$ & $\begin{array}{c}7 / 2 \\
9 / 2 \\
11 / 2 \\
13 / 2\end{array}$ & $\begin{array}{l}16135.97 \\
17627.00 \\
19308.93 \\
21238.78\end{array}$ & $\begin{array}{l}76 \\
76 \\
85 \\
77\end{array}$ & & $\begin{array}{l}10 \\
13\end{array}$ & $\begin{array}{l}\left({ }^{3} \mathrm{H}\right){ }^{4} \mathrm{G} \\
\left({ }^{3} \mathrm{H}\right){ }^{2} \mathrm{I}\end{array}$ \\
\hline $4 f^{2}\left({ }^{3} \mathrm{H}\right) 5 d$ & & $5 / 2$ & 18211.76 & 34 & ${ }^{2} \mathrm{~F}$ & 21 & $\left({ }^{3} \mathrm{~F}\right){ }^{2} \mathrm{~F}$ \\
\hline $4 f^{2}\left({ }^{3} \mathrm{H}\right) 5 d$ & ${ }^{2} I$ & $\begin{array}{l}11 / 2 \\
13 / 2\end{array}$ & $\begin{array}{l}18241.54 \\
19360.10\end{array}$ & $\begin{array}{l}47 \\
50\end{array}$ & & $\begin{array}{l}16 \\
19\end{array}$ & $\begin{array}{l}\left({ }^{1} \mathrm{G}\right){ }^{2} \mathrm{I} \\
\left({ }^{3} \mathrm{H}\right){ }^{4} \mathrm{H}\end{array}$ \\
\hline $4 f^{2}\left({ }^{3} F\right) 5 d$ & ${ }^{4} \mathrm{H}$ & $\begin{array}{l}7 / 2 \\
9 / 2 \\
11 / 2 \\
13 / 2\end{array}$ & $\begin{array}{l}18990.08 \\
20315.65 \\
21755.84 \\
23175.09\end{array}$ & $\begin{array}{l}88 \\
89 \\
88 \\
71\end{array}$ & & 15 & $\left({ }^{3} \mathrm{H}\right){ }^{2} \mathrm{~K}$ \\
\hline $4 f^{2}\left({ }^{3} \mathrm{H}\right) 5 d$ & & $7 / 2$ & 19872.43 & 33 & ${ }^{2} \mathrm{~F}$ & 23 & $\left({ }^{3} \mathrm{~F}\right)^{2} \mathrm{~F}$ \\
\hline $4 f^{2}\left({ }^{3} \mathrm{H}\right) 5 d$ & ${ }^{4} \mathrm{~F}$ & $\begin{array}{l}3 / 2 \\
5 / 2 \\
9 / 2\end{array}$ & $\begin{array}{l}20160.36 \\
20848.39 \\
22747.69\end{array}$ & $\begin{array}{l}78 \\
41 \\
49\end{array}$ & & $\begin{array}{l}16 \\
17 \\
13\end{array}$ & $\begin{array}{l}\left({ }^{3} \mathrm{~F}\right){ }^{4} \mathrm{~F} \\
\left({ }^{3} \mathrm{~F}\right){ }^{4} \mathrm{G} \\
\left({ }^{1} \mathrm{G}\right){ }^{2} \mathrm{H}\end{array}$ \\
\hline $4 f^{2}\left({ }^{3} \mathrm{~F}\right) 5 d$ & ${ }^{4} \mathrm{P}$ & $\begin{array}{l}3 / 2 \\
1 / 2 \\
5 / 2\end{array}$ & $\begin{array}{l}21148.43 \\
21611.19\end{array}$ & $\begin{array}{l}60 \\
75 \\
39\end{array}$ & & $\begin{array}{l}17 \\
13 \\
14\end{array}$ & $\begin{array}{l}\left({ }^{3} \mathrm{~F}\right){ }^{2} \mathrm{P} \\
\left({ }^{3} \mathrm{~F}\right){ }^{4} \mathrm{D} \\
\left({ }^{3} \mathrm{H}\right){ }^{4} \mathrm{~F}\end{array}$ \\
\hline $4 f^{2}\left({ }^{3} \mathrm{H}\right) 5 d$ & ${ }^{2} \mathrm{G}$ & $\begin{array}{l}7 / 2 \\
9 / 2\end{array}$ & $\begin{array}{l}21294.89 \\
24788.02\end{array}$ & $\begin{array}{l}38 \\
48\end{array}$ & & 15 & $\left({ }^{3} \mathrm{~F}\right){ }^{4} \mathrm{~F}$ \\
\hline $4 f^{2}\left({ }^{3} \mathrm{~F}\right) 5 d$ & ${ }^{4} \mathrm{G}$ & $\begin{array}{c}5 / 2 \\
7 / 2 \\
9 / 2 \\
11 / 2\end{array}$ & $\begin{array}{l}21418.84 \\
22080.94 \\
23844.58 \\
26095.23\end{array}$ & $\begin{array}{l}40 \\
40 \\
48 \\
42\end{array}$ & & $\begin{array}{l}16 \\
27 \\
14 \\
24\end{array}$ & $\begin{array}{l}\left({ }^{3} \mathrm{~F}\right){ }^{4} \mathrm{P} \\
\left({ }^{3} \mathrm{H}\right){ }^{4} \mathrm{~F} \\
\left({ }^{3} \mathrm{H}\right){ }^{4} \mathrm{G} \\
\left({ }^{1} \mathrm{G}\right){ }^{2} \mathrm{H}\end{array}$ \\
\hline $4 f^{2}\left({ }^{1} \mathrm{G}\right) 5 d$ & & $9 / 2$ & 21535.17 & 33 & ${ }^{2} \mathrm{H}$ & 13 & $\left({ }^{3} \mathrm{~F}\right){ }^{4} \mathrm{G}$ \\
\hline $4 f^{2}\left({ }^{3} \mathrm{~F}\right) 5 d$ & & $3 / 2$ & 22277.66 & 30 & ${ }^{4} \mathrm{D}$ & 27 & $\left({ }^{3} \mathrm{~F}\right){ }^{2} \mathrm{D}$ \\
\hline $4 f^{2}\left({ }^{3} \mathrm{H}\right) 5 d$ & & $7 / 2$ & 22527.70 & 22 & ${ }^{4} \mathrm{~F}$ & 16 & $\left({ }^{3} F\right){ }^{4} \mathrm{G}$ \\
\hline $4 f^{2}\left({ }^{3} \mathrm{~F}\right) 5 d$ & ${ }^{4} \mathrm{D}$ & $\begin{array}{l}3 / 2 \\
5 / 2 \\
1 / 2 \\
7 / 2\end{array}$ & $\begin{array}{l}23050.61 \\
23751.57 \\
23955.37 \\
24470.90\end{array}$ & $\begin{array}{l}30 \\
76 \\
66 \\
69\end{array}$ & & $\begin{array}{l}23 \\
10 \\
28\end{array}$ & $\begin{array}{l}\left({ }^{3} \mathrm{~F}\right){ }^{4} \mathrm{P} \\
\left({ }^{1} \mathrm{G}\right){ }^{2} \mathrm{~F} \\
\left({ }^{3} \mathrm{~F}\right){ }^{2} \mathrm{P}\end{array}$ \\
\hline $4 f^{2}\left({ }^{1} \mathrm{G}\right) 5 d$ & & $11 / 2$ & 23442.25 & 35 & ${ }^{2} \mathrm{H}$ & 22 & $\left({ }^{3} \mathrm{~F}\right){ }^{4} \mathrm{G}$ \\
\hline $4 f^{2}\left({ }^{3} F\right) 5 d$ & & $5 / 2$ & 23532.86 & 23 & ${ }^{4} \mathrm{~F}$ & 15 & $\left({ }^{3} \mathrm{H}\right){ }^{2} \mathrm{~F}$ \\
\hline $4 f^{2}\left({ }^{3} \mathrm{H}\right) 5 d$ & ${ }^{2} \mathrm{~K}$ & $\begin{array}{l}13 / 2 \\
15 / 2\end{array}$ & $\begin{array}{l}23647.77 \\
25979.34\end{array}$ & $\begin{array}{l}71 \\
86\end{array}$ & & $\begin{array}{l}19 \\
11\end{array}$ & $\begin{array}{l}\left({ }^{3} \mathrm{~F}\right){ }^{4} \mathrm{H} \\
\left({ }^{1} \mathrm{I}\right){ }^{2} \mathrm{~K}\end{array}$ \\
\hline
\end{tabular}


Pr III, Even Parity-Continued

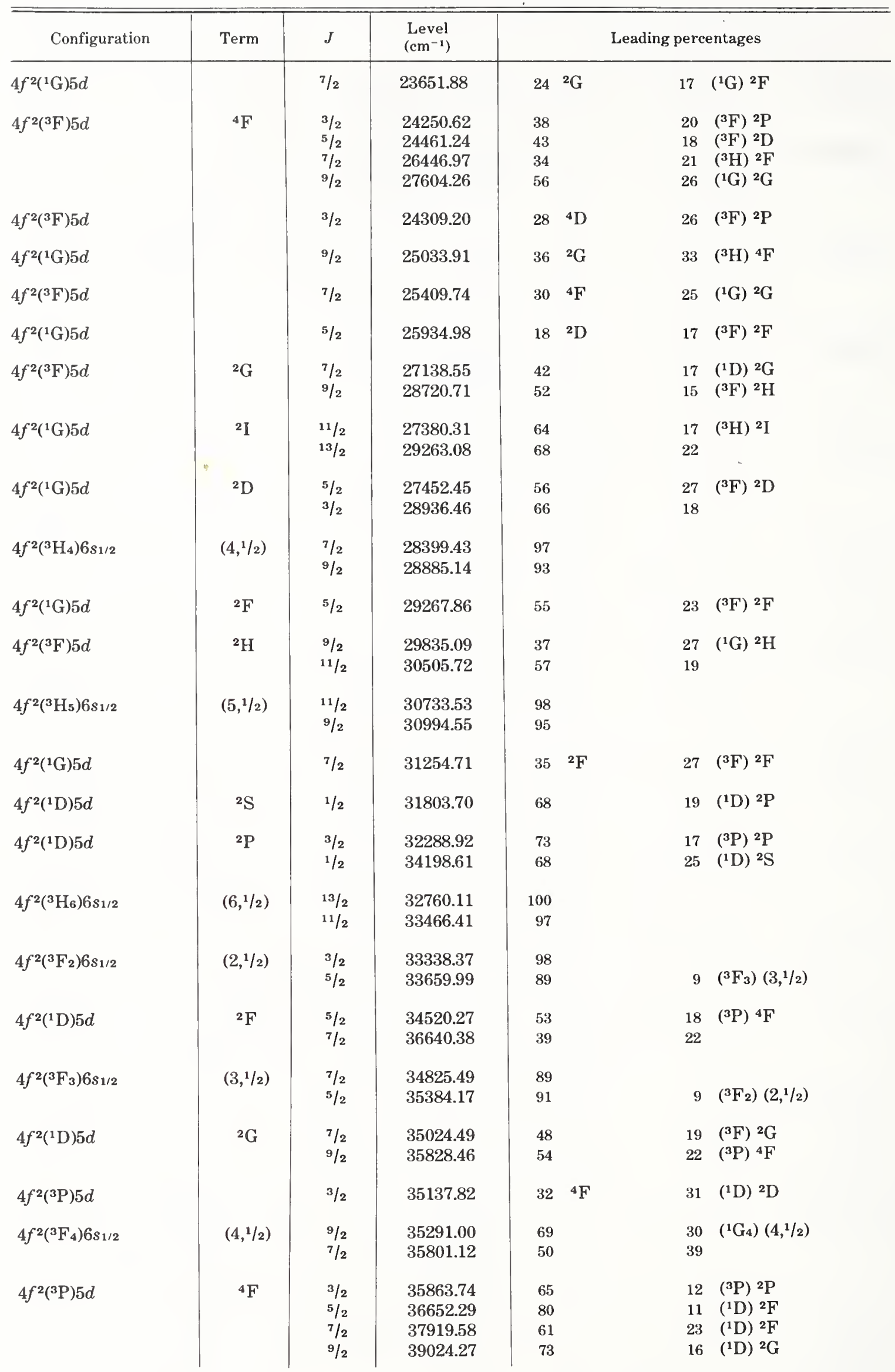


Pr III, Even Parity-Continued

\begin{tabular}{|c|c|c|c|c|c|c|c|}
\hline Configuration & Term & $J$ & $\begin{array}{l}\text { Level } \\
\left(\mathrm{cm}^{-1}\right)\end{array}$ & \multicolumn{4}{|c|}{ Leading percentages } \\
\hline $4 f^{2}\left({ }^{1} \mathrm{I}\right) 5 d$ & ${ }^{2} I$ & $\begin{array}{l}13 / 2 \\
11 / 2\end{array}$ & $\begin{array}{l}36642.13 \\
36670.57\end{array}$ & $\begin{array}{l}69 \\
92\end{array}$ & & 26 & $\left({ }^{1} \mathrm{I}\right){ }^{2} \mathrm{~K}$ \\
\hline $4 f^{2}\left({ }^{3} \mathrm{P}\right) 5 d$ & ${ }^{4} \mathrm{P}$ & $\begin{array}{l}1 / 2 \\
3 / 2 \\
5 / 2\end{array}$ & $\begin{array}{l}36683.21 \\
37197.64 \\
38694.51\end{array}$ & $\begin{array}{l}57 \\
40 \\
50\end{array}$ & & $\begin{array}{l}35 \\
32 \\
23\end{array}$ & $\begin{array}{l}\left({ }^{3} \mathrm{P}\right){ }^{2} \mathrm{P} \\
\left({ }^{1} \mathrm{D}\right)^{2} \mathrm{D} \\
\left({ }^{1} \mathrm{D}\right)^{2} \mathrm{D}\end{array}$ \\
\hline $4 f^{2}\left({ }^{3} \mathrm{P}\right) 5 d$ & & $5 / 2$ & 37011.92 & 41 & ${ }^{4} \mathrm{P}$ & 39 & $\left({ }^{1} \mathrm{D}\right){ }^{2} \mathrm{D}$ \\
\hline $4 f^{2}\left({ }^{1} \mathrm{G}_{4}\right) 6 s_{1 / 2}$ & $(4,1 / 2)$ & $\begin{array}{l}9 / 2 \\
7 / 2\end{array}$ & $\begin{array}{l}38448.38 \\
38726.50\end{array}$ & $\begin{array}{l}68 \\
57\end{array}$ & & $\begin{array}{l}30 \\
42\end{array}$ & $\left({ }^{3} \mathrm{~F}_{4}\right)(4,1 / 2)$ \\
\hline $4 f^{2}\left({ }^{3} \mathrm{P}\right) 5 d$ & & $1 / 2$ & 38549.90 & 35 & ${ }^{4} \mathrm{D}$ & 30 & $\left({ }^{3} \mathrm{P}\right){ }^{4} \mathrm{P}$ \\
\hline $4 f^{2}\left({ }^{1} \mathrm{I}\right) 5 d$ & ${ }^{2} \mathrm{~K}$ & $\begin{array}{l}13 / 2 \\
15 / 2\end{array}$ & $\begin{array}{l}38701.59 \\
38785.76\end{array}$ & $\begin{array}{l}63 \\
72\end{array}$ & & $\begin{array}{l}28 \\
16\end{array}$ & $\begin{array}{l}\left({ }^{1} \mathrm{I}\right){ }^{2} \mathrm{I} \\
\left({ }^{1} \mathrm{I}\right){ }^{2} \mathrm{~L}\end{array}$ \\
\hline $4 f^{2}\left({ }^{3} \mathrm{P}\right) 5 d$ & ${ }^{4} \mathrm{D}$ & $\begin{array}{l}3 / 2 \\
1 / 2 \\
5 / 2 \\
7 / 2\end{array}$ & $\begin{array}{l}39120.49 \\
39485.48 \\
40098.66 \\
41023.98\end{array}$ & $\begin{array}{l}56 \\
57 \\
71 \\
85\end{array}$ & & $\begin{array}{l}22 \\
34 \\
19 \\
11\end{array}$ & $\begin{array}{l}\left({ }^{3} \mathrm{P}\right){ }^{4} \mathrm{P} \\
\left({ }^{3} \mathrm{P}\right){ }^{2} \mathrm{P} \\
\left({ }^{1} \mathrm{D}\right)^{2} \mathrm{D} \\
\left({ }^{1} \mathrm{D}\right){ }^{2} \mathrm{~F}\end{array}$ \\
\hline $4 f^{2}\left({ }^{1} \mathrm{I}\right) 5 d$ & ${ }^{2} \mathrm{~L}$ & $\begin{array}{l}15 / 2 \\
17 / 2\end{array}$ & $\begin{array}{l}39725.99 \\
41026.87\end{array}$ & $\begin{array}{r}83 \\
100\end{array}$ & & 16 & $\left({ }^{1} \mathrm{I}\right){ }^{2} \mathrm{~K}$ \\
\hline $4 f^{2}\left({ }^{1} \mathrm{I}\right) 5 d$ & ${ }^{2} \mathrm{H}$ & $\begin{array}{l}11 / 2 \\
9 / 2\end{array}$ & $\begin{array}{l}39732.99 \\
39870.17\end{array}$ & $\begin{array}{l}86 \\
81\end{array}$ & & & \\
\hline $4 f^{2}\left({ }^{3} \mathrm{P}\right) 5 d$ & & $3 / 2$ & 40205.74 & 32 & ${ }^{2} \mathrm{P}$ & 29 & $\left({ }^{3} \mathrm{P}\right){ }^{4} \mathrm{D}$ \\
\hline $4 f^{2}\left({ }^{3} \mathrm{P}\right) 5 d$ & ${ }^{2} \mathrm{~F}$ & $\begin{array}{l}7 / 2 \\
5 / 2\end{array}$ & $\begin{array}{l}44679.98 \\
44903.42\end{array}$ & $\begin{array}{l}77 \\
71\end{array}$ & & $\begin{array}{l}10 \\
12\end{array}$ & $\begin{array}{l}\left({ }^{3} \mathrm{~F}\right){ }^{2} \mathrm{~F} \\
\left({ }^{1} \mathrm{D}\right)^{2} \mathrm{~F}\end{array}$ \\
\hline $4 f^{2}\left({ }^{1} \mathrm{I}\right) 5 d$ & ${ }^{2} \mathrm{G}$ & $\begin{array}{l}9 / 2 \\
7 / 2\end{array}$ & $\begin{array}{l}45805.95 \\
46673.78\end{array}$ & $\begin{array}{l}94 \\
98\end{array}$ & & . & \\
\hline $4 f^{2}\left({ }^{1} \mathrm{D}_{2}\right) 6 s_{1 / 2}$ & $\left(2,{ }^{1} / 2\right)$ & $\begin{array}{l}5 / 2 \\
3 / 2\end{array}$ & $\begin{array}{l}45807.1 \\
45844.65\end{array}$ & $\begin{array}{l}88 \\
90\end{array}$ & & $\begin{array}{l}9 \\
8\end{array}$ & $\left({ }^{3} \mathrm{P}_{2}\right)\left(2,{ }^{1 / 2}\right)$ \\
\hline $4 f^{2}\left({ }^{3} \mathrm{P}\right) 5 d$ & ${ }^{2} \mathrm{D}$ & $\begin{array}{l}3 / 2 \\
5 / 2\end{array}$ & $\begin{array}{l}48401.64 \\
48745.48\end{array}$ & $\begin{array}{l}90 \\
87\end{array}$ & & & \\
\hline $4 f^{2}\left({ }^{3} \mathrm{P}_{1}\right) 6 s_{1 / 2}$ & $(1,1 / 2)$ & $\begin{array}{l}3 / 2 \\
1 / 2\end{array}$ & $\begin{array}{l}50227.79 \\
50869.3\end{array}$ & $\begin{array}{l}97 \\
86\end{array}$ & & 14 & $\left({ }^{3} \mathrm{P}_{0}\right)(0,1 / 2)$ \\
\hline $4 f^{2}\left({ }^{1} \mathrm{I}_{6}\right) 6 s_{1 / 2}$ & $\left(6,{ }^{1} / 2\right)$ & $\begin{array}{l}11 / 2 \\
13 / 2\end{array}$ & $\begin{array}{l}50647.35 \\
50658.72\end{array}$ & $\begin{array}{l}100 \\
100\end{array}$ & & & \\
\hline $4 f^{2}\left({ }^{3} \mathrm{P}_{2}\right) 6 s_{1 / 2}$ & $\left(2,{ }^{1} / 2\right)$ & $\begin{array}{l}5 / 2 \\
3 / 2\end{array}$ & $\begin{array}{l}51312.8 \\
52026.9\end{array}$ & $\begin{array}{l}91 \\
90\end{array}$ & & $\begin{array}{l}9 \\
7\end{array}$ & $\left({ }^{1} \mathrm{D}_{2}\right)(2,1 / 2)$ \\
\hline $4 f^{2}\left({ }^{3} \mathrm{H}_{4}\right) 7 s_{1 / 2}$ & $\left(4,{ }^{1} / 2\right)$ & $\begin{array}{l}7 / 2 \\
9 / 2\end{array}$ & 100321.12 & & & & \\
\hline $4 f^{2}\left({ }^{3} \mathrm{H}_{4}\right) 6 d_{3 / 2}$ & $(4,3 / 2)$ & $\begin{array}{c}9 / 2 \\
11 / 2 \\
7 / 2 \\
5 / 2\end{array}$ & $\begin{array}{l}100430.29 \\
100625.04 \\
100788.95 \\
101071.43\end{array}$ & $\begin{array}{l}96 \\
96 \\
65 \\
50\end{array}$ & & $\begin{array}{l}31 \\
45\end{array}$ & $\begin{array}{l}\left({ }^{3} \mathrm{H}_{4}\right)(4,5 / 2) \\
\left({ }^{3} \mathrm{H}_{4}\right)(4,5 / 2)\end{array}$ \\
\hline $4 f^{2}\left({ }^{3} \mathrm{H}_{4}\right) 6 d_{5 / 2}$ & $(4,5 / 2)$ & $\begin{array}{c}9 / 2 \\
11 / 2 \\
7 / 2 \\
3 / 2 \\
5 / 2 \\
13 / 2\end{array}$ & $\begin{array}{r}100947.18 \\
101165.33 \\
101446.27 \\
\\
101665.58 \\
101680.38\end{array}$ & $\begin{array}{l}94 \\
94 \\
64 \\
97 \\
52 \\
84\end{array}$ & & $\begin{array}{l}28 \\
43 \\
10\end{array}$ & $\begin{array}{l}\left({ }^{3} \mathrm{H}_{4}\right)(4,3 / 2) \\
\left({ }^{3} \mathrm{H}_{4}\right)(4,3 / 2) \\
\left({ }^{3} \mathrm{H}_{5}\right)(5,3 / 2)\end{array}$ \\
\hline $4 f^{2}\left({ }^{3} \mathrm{H}_{5}\right) 7 s_{1 / 2}$ & $\left(5,{ }^{1} / 2\right)$ & $\begin{array}{l}11 / 2 \\
9 / 2\end{array}$ & $\begin{array}{l}102435.67 \\
102484.66\end{array}$ & & & & \\
\hline
\end{tabular}


Pr III, Even Parity—Continued

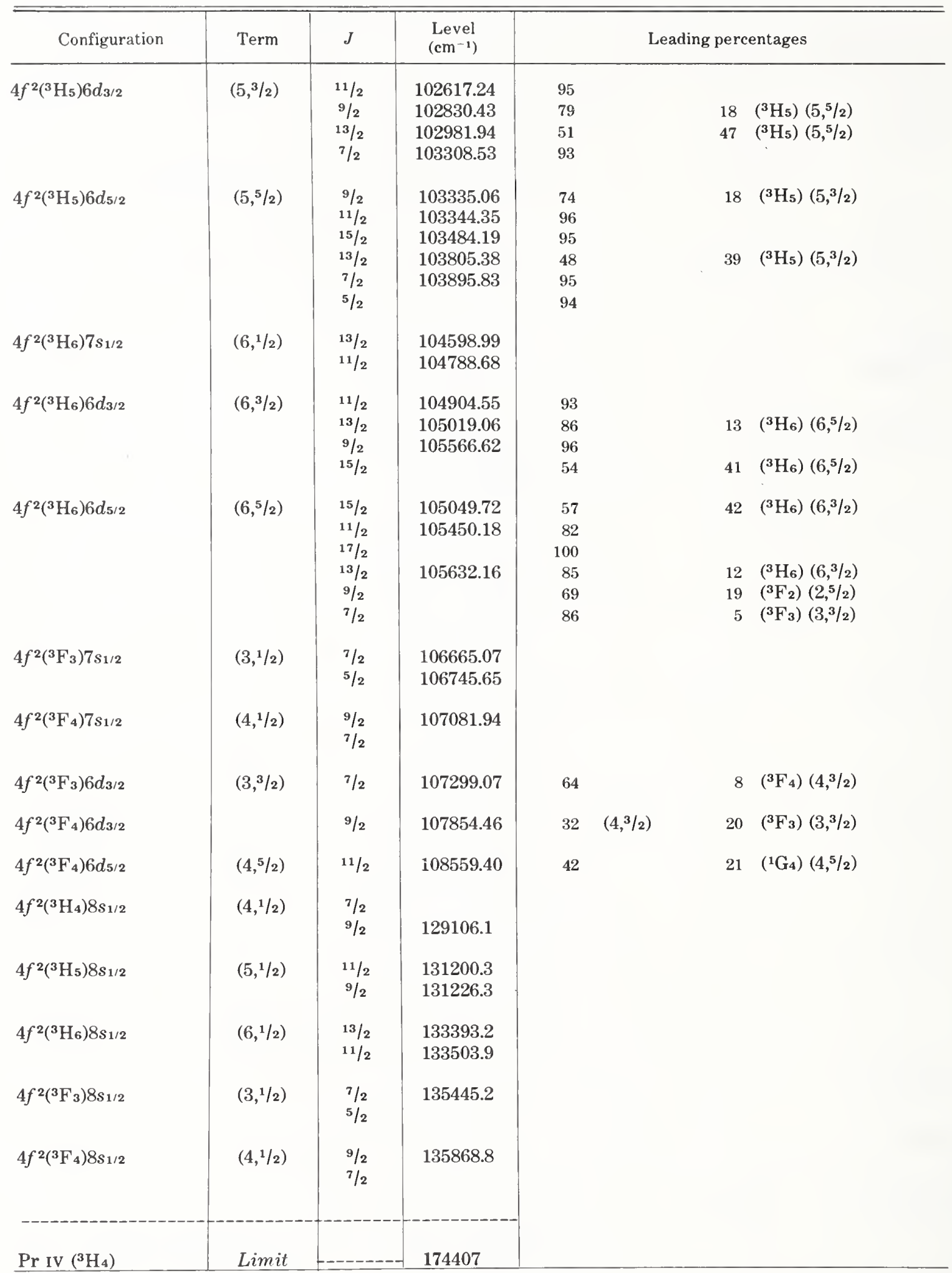


Ground state $\left(1 s^{2} 2 s^{2} 2 p^{6} 3 s^{2} 3 p^{6} 3 d^{10} 4 s^{2} 4 p^{6} 4 d^{10} 5 s^{2} 5 p^{6}\right) 4 f^{2{ }^{3}} \mathrm{H}_{4}$

Ionization energy $314400 \pm 200 \mathrm{~cm}^{-1}$

$38.98 \pm 0.02 \mathrm{eV}$

Identified even configurations $\quad 4 f^{2}, 4 f 5 f, 4 f 6 p, 5 d^{2}$

Identified odd configurations $\quad 4 f 5 d, 4 f 6 d, 4 f 6 s, 4 f 7 s, 5 d 6 p$

Sugar [1965, 1971a] and Crosswhite, Dieke, and Carter [1965] have observed this spectrum as emitted by sliding-spark discharges. The line lists in the two 1965 references overlap up to $3021 \AA$, and Sugar's additional list of classified lines [1971a] extends downward to $691 \AA$. The analysis is from these three references. The two 1965 papers are in agreement on all levels of the $4 f^{2}, 4 f 5 d, 4 f 6 s$, and $4 f 6 p$ configurations, except for $4 f^{2}{ }^{1} \mathrm{~S}_{0}$ and an interchange of designations for three pairs of $4 f 5 d$ levels. The two-place level values are from Crosswhite et al. It is not clear that these values are more accurate than the one-place values of Sugar, however, from which they differ by up to $0.3 \mathrm{~cm}^{-1}$. The $4 f^{2}{ }^{1} \mathrm{~S}_{0}$ level of Crosswhite et al. has been omitted as doubtful [Morrison and Rajnak, 1971], and Sugar's designations for the $4 f 5 d$ levels are preferred as supported by calculations. The levels of the remaining configurations are taken from Sugar [1971a], with the two possible $5 d^{2}$ levels he judged as doubtful being omitted.

The compositions of the $4 f^{2}$ levels are from Goldschmidt's thesis [1968]. Her calculation was later refined to include additional magnetic interactions, but the eigenvectors were essentially unaffected [Goldschmidt, Pasternak, and Goldschmidt, 1968; Pasternak, 1970]. Theoretical percentages for levels of the $4 f 6 s, 4 f 6 d$, and $4 f 5 f$ configurations are taken from Sugar's publications, supplemented by some of his unpublished results for alternate coupling schemes. Sugar recalculated for inclusion here the eigenvectors for the $4 f 5 d$ and $4 f 6 p$ configurations, with energy matrices including the appropriate effective interactions. Goldschmidt, Salomon, and Starkand [1974] have made similar calculations of $4 f 5 d$ in $\operatorname{Pr}$ IV, Ce III, and La II.

The average purity of the $4 f 5 f$ configuration in the $j_{1} l$ coupling scheme is only slightly greater than in the LS scheme, but the former is preferred because none of the $L S$ designations ${ }^{1} \mathrm{~F},{ }^{1} \mathrm{G}$, and ${ }^{1} \mathrm{H}$ is the leading component of any level. The $4 f 6 p$ configuration exhibits strong $j_{1} j_{2}$ coupling, and in the $4 f 6 d$ configuration the $j_{1}$ value of the $4 f$ core is defined with greater than $90 \%$ purity for every level. Thus "no strong combinations involving a change of the $4 f$ core level were observed" in the $4 f 6 p-4 f 6 d$ transition array [Sugar, 1971a].

Sugar [1971a] obtained a value for the ionization limit based on the $4 f 6 s, 7 s$ series. Sugar and Reader [1973] have revised the value using a semi-empirical method and knowledge of the system differences $4 f^{N}-4 f^{N-1} 5 d$.

\section{References}

Crosswhite, H. M., Dieke, G. H., and Carter, W. J., J. Chem. Phys. 43, 2047 (1965). EL CL W Hfs Goldschmidt, Z. B., Thesis, Hebrew Univ. Jerusalem, Israel, 487 pp. (1968). PT

Goldschmidt, Z. B., Pasternak, A., and Goldschmidt, Z. H., Phys. Lett. 28A, 265 (1968). PT

Goldschmidt, Z. B., Salomon, D., and Starkand, J., unpublished material (1974). PT

Morrison, J. C., and Rajnak, K., Phys. Rev. A 4, 536 (1971). ND AT

Pasternak, A., Thesis, Hebrew Univ. Jerusalem, Israel, 219 pp. (1970). PT

Sugar, J., J. Opt. Soc. Am. 55, 1058 (1965). EL CL W PT

Sugar, J., J. Opt. Soc. Am. 61, 727 (1971a). EL CL W IP PT

Sugar, J., unpublished calculations (1971b). PT

Sugar, J., and Reader, J., J. Chem. Phys. 59, 2083 (1973). IP

[July 1976] 
Pr IV

\begin{tabular}{|c|c|c|c|c|c|c|c|}
\hline Configuration & Term & $J$ & $\begin{array}{l}\text { Level } \\
\left(\mathrm{cm}^{-1}\right)\end{array}$ & \multicolumn{4}{|c|}{ Leading percentages } \\
\hline $4 f^{2}$ & ${ }^{3} \mathrm{H}$ & $\begin{array}{l}4 \\
5 \\
6\end{array}$ & $\begin{array}{r}0.00 \\
2152.09 \\
4389.09\end{array}$ & $\begin{array}{r}97 \\
100 \\
100\end{array}$ & & & \\
\hline $4 f^{2}$ & ${ }^{3} \mathrm{~F}$ & $\begin{array}{l}2 \\
3 \\
4\end{array}$ & $\begin{array}{l}4996.61 \\
6415.24 \\
6854.75\end{array}$ & $\begin{array}{r}98 \\
100 \\
66\end{array}$ & & 33 & ${ }^{1} \mathrm{G}$ \\
\hline $4 f^{2}$ & ${ }^{1} \mathrm{G}$ & 4 & 9921.24 & 65 & & 34 & ${ }^{3} \mathrm{~F}$ \\
\hline $4 f^{2}$ & ${ }^{1} \mathrm{D}$ & 2 & 17334.39 & 90 & & 8 & ${ }^{3 P}$ \\
\hline $4 f^{2}$ & ${ }^{3} \mathrm{P}$ & $\begin{array}{l}0 \\
1 \\
2\end{array}$ & $\begin{array}{l}21389.81 \\
22007.46 \\
23160.61\end{array}$ & $\begin{array}{r}99 \\
100 \\
92\end{array}$ & & 8 & ${ }^{1} \mathrm{D}$ \\
\hline $4 f^{2}$ & ${ }^{1} \mathrm{I}$ & 6 & 22211.54 & 100 & & & \\
\hline $4 f 5 d$ & ${ }^{1} \mathrm{G}^{\circ}$ & 4 & 61170.95 & 63 & or & 70 & $(5 / 2,3 / 2)^{\circ}$ \\
\hline $4 f 5 d$ & ${ }^{3} \mathrm{~F}^{\circ}$ & $\begin{array}{l}2 \\
3 \\
4\end{array}$ & $\begin{array}{l}61457.48 \\
64123.54 \\
66518.01\end{array}$ & $\begin{array}{l}69 \\
82 \\
73\end{array}$ & $\begin{array}{l}\text { or } \\
\text { or } \\
\text { or }\end{array}$ & $\begin{array}{l}95 \\
67 \\
91\end{array}$ & $\begin{array}{l}(5 / 2,3 / 2)^{\circ} \\
(5 / 2,5 / 2)^{\circ} \\
(7 / 2,5 / 2)^{\circ}\end{array}$ \\
\hline $4 f 5 d$ & ${ }^{3} \mathrm{G}^{\circ}$ & $\begin{array}{l}3 \\
4 \\
5\end{array}$ & $\begin{array}{l}63355.94 \\
65639.95 \\
67899.32\end{array}$ & $\begin{array}{l}77 \\
87 \\
98\end{array}$ & $\begin{array}{l}\text { or } \\
\text { or } \\
\text { or }\end{array}$ & $\begin{array}{l}85 \\
58 \\
70\end{array}$ & $\begin{array}{l}(5 / 2,3 / 2)^{\circ} \\
(7 / 2,3 / 2)^{\circ} \\
(7 / 2,5 / 2)^{\circ}\end{array}$ \\
\hline $4 f 5 d$ & ${ }^{3} \mathrm{H}^{\circ}$ & $\begin{array}{l}4 \\
5 \\
6\end{array}$ & $\begin{array}{l}63580.59 \\
65239.39 \\
68077.83\end{array}$ & $\begin{array}{r}68 \\
100 \\
100\end{array}$ & $\begin{array}{l}\text { or } \\
\text { or } \\
\text { or }\end{array}$ & $\begin{array}{r}40 \\
52 \\
100\end{array}$ & $\begin{array}{l}(5 / 2,5 / 2)^{\circ} \\
(5 / 2,5 / 2)^{\circ} \\
(7 / 2,5 / 2)^{\circ}\end{array}$ \\
\hline $4 f 5 d$ & ${ }^{1} \mathrm{D}^{\circ}$ & 2 & 65321.67 & 61 & or & 58 & $(5 / 2,5 / 2)^{\circ}$ \\
\hline $4 f 5 d$ & ${ }^{3} \mathrm{D}^{\circ}$ & $\begin{array}{l}1 \\
2 \\
3\end{array}$ & $\begin{array}{l}66967.72 \\
68411.51 \\
68495.57\end{array}$ & $\begin{array}{l}95 \\
92 \\
67\end{array}$ & $\begin{array}{l}\text { or } \\
\text { or } \\
\text { or }\end{array}$ & $\begin{array}{l}63 \\
55 \\
59\end{array}$ & $\begin{array}{l}(5 / 2,3 / 2)^{\circ} \\
\left({ }^{7} / 2,3 / 2\right)^{\circ} \\
\left({ }^{7} / 2,3 / 2\right)^{\circ}\end{array}$ \\
\hline $4 f 5 d$ & ${ }^{3} \mathrm{P}^{\circ}$ & $\begin{array}{l}1 \\
0 \\
2\end{array}$ & $\begin{array}{l}70755.33 \\
70842.93 \\
72185.10\end{array}$ & $\begin{array}{r}93 \\
100 \\
94\end{array}$ & $\begin{array}{l}\text { or } \\
\text { or } \\
\text { or }\end{array}$ & $\begin{array}{r}70 \\
100 \\
54\end{array}$ & $\begin{array}{l}(5 / 2,5 / 2)^{\circ} \\
(5 / 2,5 / 2)^{\circ} \\
(7 / 2,5 / 2)^{\circ}\end{array}$ \\
\hline $4 f 5 d$ & ${ }^{1} \mathrm{~F}^{0}$ & 3 & 71724.77 & 65 & or & 95 & $(7 / 2,5 / 2)^{\circ}$ \\
\hline $4 f 5 d$ & ${ }^{1} \mathrm{H}^{\circ}$ & 5 & 75265.66 & 98 & or & 35 & $(5 / 2,5 / 2)^{\circ}$ \\
\hline $4 f 5 d$ & ${ }^{1} \mathrm{P}^{\circ}$ & 1 & 78776.38 & 92 & or & 83 & $\left({ }^{7} / 2,5 / 2\right)^{\circ}$ \\
\hline $4 f\left({ }^{2} \mathrm{~F}_{5 / 2}^{\circ}\right) 6 s_{1 / 2}$ & $(5 / 2,1 / 2)^{\circ}$ & $\begin{array}{l}2 \\
3\end{array}$ & $\begin{array}{l}100258.48 \\
100543.85\end{array}$ & $\begin{array}{r}100 \\
99\end{array}$ & $\begin{array}{l}\text { or } \\
\text { or }\end{array}$ & $\begin{array}{r}100 \\
69\end{array}$ & $\begin{array}{l}{ }^{3} \mathrm{~F}^{\circ} \\
{ }^{3} \mathrm{~F}^{\circ}\end{array}$ \\
\hline $4 f\left({ }^{2} \mathrm{~F}_{7 / 2}^{\circ}\right) 6 s_{1 / 2}$ & $\left(7 / 2,{ }^{1} / 2\right)^{\circ}$ & $\begin{array}{l}4 \\
3\end{array}$ & $\begin{array}{l}103271.38 \\
103753.75\end{array}$ & $\begin{array}{r}100 \\
99\end{array}$ & $\begin{array}{l}\text { or } \\
\text { or }\end{array}$ & $\begin{array}{r}100 \\
69\end{array}$ & $\begin{array}{l}{ }^{3} \mathrm{~F}^{\circ} \\
{ }^{1} \mathrm{~F}^{\circ}\end{array}$ \\
\hline $4 f\left({ }^{2} \mathrm{~F}_{5 / 2}^{\circ}\right) 6 p_{1 / 2}$ & $(5 / 2,1 / 2)$ & $\begin{array}{l}3 \\
2\end{array}$ & $\begin{array}{l}136850.85 \\
137175.04\end{array}$ & $\begin{array}{l}98 \\
94\end{array}$ & $\begin{array}{l}\text { or } \\
\text { or }\end{array}$ & $\begin{array}{l}59 \\
69\end{array}$ & ${ }^{3} \mathrm{G}$ \\
\hline $5 d^{2}$ & ${ }^{3} \mathrm{~F} ?$ & $\begin{array}{l}2 ? \\
3 \\
4\end{array}$ & $\begin{array}{l}139711.8 \\
145362.7\end{array}$ & & & & \\
\hline $4 f\left({ }^{2} \mathrm{~F}_{7 / 2}^{\circ}\right) 6 p_{1 / 2}$ & $\left(7 / 2,{ }^{1} / 2\right)$ & $\begin{array}{l}3 \\
4\end{array}$ & $\begin{array}{l}139875.31 \\
140225.92\end{array}$ & $\begin{array}{l}93 \\
96\end{array}$ & $\begin{array}{l}\text { or } \\
\text { or }\end{array}$ & $\begin{array}{l}53 \\
41\end{array}$ & $\begin{array}{l}{ }^{3} \mathrm{~F} \\
{ }^{3} \mathrm{G}\end{array}$ \\
\hline $4 f\left({ }^{2} \mathrm{~F}_{5 / 2}^{\circ}\right) 6 p_{3 / 2}$ & $(5 / 2,3 / 2)$ & $\begin{array}{l}3 \\
2 \\
4 \\
1\end{array}$ & $\begin{array}{l}141254.01 \\
142331.59 \\
142565.94 \\
142997.32\end{array}$ & $\begin{array}{r}94 \\
95 \\
93 \\
100\end{array}$ & $\begin{array}{l}\text { or } \\
\text { or } \\
\text { or } \\
\text { or }\end{array}$ & $\begin{array}{r}39 \\
54 \\
56 \\
100\end{array}$ & $\begin{array}{l}{ }^{3} \mathrm{G} \\
{ }^{3} \mathrm{D} \\
{ }^{3} \mathrm{G} \\
{ }^{3} \mathrm{D}\end{array}$ \\
\hline
\end{tabular}


Pr IV-Continued

\begin{tabular}{|c|c|c|c|c|c|c|c|}
\hline Configuration & Term & $J$ & $\begin{array}{l}\text { Level } \\
\left(\mathrm{cm}^{-1}\right)\end{array}$ & \multicolumn{4}{|c|}{ Leading percentages } \\
\hline $4 f\left({ }^{2} \mathrm{~F}_{7 / 2}^{\circ}\right) 6 p_{3 / 2}$ & $(7 / 2,3 / 2)$ & $\begin{array}{l}4 \\
3 \\
5 \\
2\end{array}$ & $\begin{array}{l}144925.33 \\
144943.27 \\
145281.20 \\
146577.13\end{array}$ & $\begin{array}{r}90 \\
94 \\
100 \\
99\end{array}$ & $\begin{array}{l}\text { or } \\
\text { or } \\
\text { or } \\
\text { or }\end{array}$ & $\begin{array}{r}55 \\
65 \\
100 \\
64\end{array}$ & $\begin{array}{l}{ }^{1} \mathrm{G} \\
{ }^{3} \mathrm{D} \\
{ }^{3} \mathrm{G} \\
{ }^{1} \mathrm{D}\end{array}$ \\
\hline $4 f\left({ }^{2} \mathrm{~F}_{5 / 2}^{\circ}\right) 6 d_{3 / 2}$ & $(5 / 2,3 / 2)^{\circ}$ & $\begin{array}{l}2 \\
4 \\
3 \\
1\end{array}$ & $\begin{array}{l}193330.5 \\
193601.8 \\
193805.1\end{array}$ & $\begin{array}{l}93 \\
98 \\
83 \\
55\end{array}$ & $\begin{array}{l}\text { or } \\
\text { or } \\
\text { or } \\
\text { or }\end{array}$ & $\begin{array}{l}75 \\
72 \\
92 \\
92\end{array}$ & $\begin{array}{l}{ }^{3} \mathrm{~F}^{\circ} \\
{ }^{3} \mathrm{H}^{\circ} \\
{ }^{3} \mathrm{G}^{\circ} \\
{ }^{3} \mathrm{D}^{\circ}\end{array}$ \\
\hline $4 f\left({ }^{2} F_{5 / 2}^{\circ}\right) 6 d 5 / 2$ & $(5 / 2,5 / 2)^{\circ}$ & $\begin{array}{l}4 \\
3 \\
2 \\
5 \\
0 \\
1\end{array}$ & 194022.2 & $\begin{array}{r}98 \\
80 \\
90 \\
91 \\
100 \\
56\end{array}$ & $\begin{array}{l}\text { or } \\
\text { or } \\
\text { or } \\
\text { or } \\
\text { or } \\
\text { or }\end{array}$ & $\begin{array}{r}44 \\
71 \\
44 \\
78 \\
100 \\
84\end{array}$ & $\begin{array}{l}{ }^{3} \mathrm{G}^{\circ} \\
{ }^{3} \mathrm{~F}^{\circ} \\
{ }^{3} \mathrm{D}^{\circ} \\
{ }^{3} \mathrm{H}^{\circ} \\
{ }^{3} \mathrm{P}^{\circ} \\
{ }^{3} \mathrm{P}^{\circ}\end{array}$ \\
\hline $5 d 6 p$ & ${ }^{3} \mathrm{~F}^{\circ}$ & $\begin{array}{l}2 \\
3 \\
4\end{array}$ & 195917.0 & & & & \\
\hline $4 f\left({ }^{2} \mathrm{~F}_{7 / 2}^{\circ}\right) 6 d_{3 / 2}$ & $(7 / 2,3 / 2)^{\circ}$ & $\begin{array}{l}4 \\
3 \\
2 \\
5\end{array}$ & 198304.7 & $\begin{array}{l}99 \\
95 \\
54 \\
48\end{array}$ & $\begin{array}{l}\text { or } \\
\text { or } \\
\text { or } \\
\text { or }\end{array}$ & $\begin{array}{l}51 \\
38 \\
54 \\
81\end{array}$ & $\begin{array}{l}{ }^{3} \mathrm{G}^{\circ} \\
{ }^{3} \mathrm{D}^{\circ} \\
{ }^{3} \mathrm{D}^{\circ} \\
{ }^{1} \mathrm{H}^{\circ}\end{array}$ \\
\hline $4 f\left({ }^{2} \mathrm{~F}_{7 / 2}^{\circ}\right) 6 d_{5 / 2}$ & $(7 / 2,5 / 2)^{\circ}$ & $\begin{array}{l}4 \\
5 \\
6 \\
2 \\
3 \\
1\end{array}$ & $\begin{array}{l}196800.0 \\
197103.7 \\
197196.9 \\
198155.4 ?\end{array}$ & $\begin{array}{r}100 \\
55 \\
100 \\
54 \\
99 \\
96\end{array}$ & $\begin{array}{l}\text { or } \\
\text { or } \\
\text { or } \\
\text { or } \\
\text { or } \\
\text { or }\end{array}$ & $\begin{array}{r}63 \\
85 \\
100 \\
88 \\
53 \\
77\end{array}$ & $\begin{array}{l}{ }^{3} \mathrm{~F}^{\circ} \\
{ }^{3} \mathrm{G}^{\circ} \\
{ }^{3} \mathrm{H}^{\circ} \\
{ }^{3} \mathrm{P}^{\circ} \\
{ }^{3} \mathrm{D}^{\circ} \\
{ }^{1} \mathrm{P}^{\circ}\end{array}$ \\
\hline $4 f\left({ }^{2} \mathrm{~F}_{5 / 2}^{\circ}\right) 5 f$ & ${ }^{2}\left[{ }^{7} / 2\right]$ & $\begin{array}{l}3 \\
4\end{array}$ & $\begin{array}{l}199191.0 \\
200202.6\end{array}$ & $\begin{array}{l}86 \\
69\end{array}$ & $\begin{array}{l}\text { or } \\
\text { or }\end{array}$ & $\begin{array}{l}70 \\
87\end{array}$ & $\begin{array}{l}{ }^{3} \mathrm{G} \\
{ }^{3} \mathrm{G}\end{array}$ \\
\hline$"$ & ${ }^{2}[11 / 2]$ & $\begin{array}{l}5 \\
6\end{array}$ & $\begin{array}{l}199595.8 \\
200893.8\end{array}$ & $\begin{array}{l}68 \\
79\end{array}$ & $\begin{array}{l}\text { or } \\
\text { or }\end{array}$ & $\begin{array}{l}57 \\
90\end{array}$ & $\begin{array}{l}{ }^{3} I \\
{ }^{3} I\end{array}$ \\
\hline $4 f\left({ }^{2} \mathrm{~F}_{5 / 2}^{\circ}\right) 7 s_{1 / 2}$ & $\left(5 / 2,{ }^{1} / 2\right)^{\circ}$ & $\begin{array}{l}2 \\
3\end{array}$ & 199727.7 & & & & \\
\hline $4 f\left({ }^{2} \mathrm{~F}_{5 / 2}\right) 5 f$ & ${ }^{2}[3 / 2]$ & $\begin{array}{l}1 \\
2\end{array}$ & $\begin{array}{l}199815.5 \\
200697.1\end{array}$ & $\begin{array}{l}87 \\
55\end{array}$ & $\begin{array}{l}\text { or } \\
\text { or }\end{array}$ & $\begin{array}{l}64 \\
89\end{array}$ & $\begin{array}{l}{ }^{3} \mathrm{D} \\
{ }^{3} \mathrm{D}\end{array}$ \\
\hline$"$ & $2[9 / 2]$ & $\begin{array}{l}5 \\
4\end{array}$ & $\begin{array}{l}200235.1 \\
201985.3\end{array}$ & $\begin{array}{l}57 \\
85\end{array}$ & $\begin{array}{l}\text { or } \\
\text { or }\end{array}$ & $\begin{array}{l}39 \\
82\end{array}$ & $\begin{array}{l}{ }^{3} \mathrm{I} \\
{ }^{3} \mathrm{H}\end{array}$ \\
\hline$"$ & $2[5 / 2]$ & $\begin{array}{l}3 \\
2\end{array}$ & $\begin{array}{l}200417.0 \\
202819.0\end{array}$ & $\begin{array}{l}73 \\
74\end{array}$ & $\begin{array}{l}\text { or } \\
\text { or }\end{array}$ & $\begin{array}{l}36 \\
71\end{array}$ & $\begin{array}{l}{ }^{3} \mathrm{D} \\
{ }^{3} \mathrm{~F}\end{array}$ \\
\hline$"$ & ${ }^{2}[1 / 2]$ & $\begin{array}{l}1 \\
0\end{array}$ & 201365.2 & $\begin{array}{l}70 \\
89\end{array}$ & $\begin{array}{l}\text { or } \\
\text { or }\end{array}$ & $\begin{array}{l}55 \\
87\end{array}$ & $\begin{array}{l}{ }^{3} \mathrm{~S} \\
{ }^{3} \mathrm{P}\end{array}$ \\
\hline $4 f\left({ }^{2} \mathrm{~F}_{7 / 2}^{\circ}\right) 5 f$ & ${ }^{2}[9 / 2]$ & $\begin{array}{l}5 \\
4\end{array}$ & $\begin{array}{l}202327.9 \\
204541.7\end{array}$ & $\begin{array}{l}84 \\
55\end{array}$ & $\begin{array}{l}\text { or } \\
\text { or }\end{array}$ & $\begin{array}{l}73 \\
43\end{array}$ & ${ }^{3} \mathrm{G}$ \\
\hline $5 d 6 p$ & ${ }^{1} \mathrm{~F}^{\circ}$ & 3 & 202487.0 & & & & \\
\hline $4 f\left({ }^{2} \mathrm{~F}_{7 / 2}^{\circ}\right) 5 f$ & $2[5 / 2]$ & $\begin{array}{l}3 \\
2\end{array}$ & $\begin{array}{l}202614.7 \\
205541.6\end{array}$ & $\begin{array}{l}67 \\
74\end{array}$ & $\begin{array}{l}\text { or } \\
\text { or }\end{array}$ & $\begin{array}{l}54 \\
50\end{array}$ & $\begin{array}{l}{ }^{3} \mathrm{D} \\
{ }^{1} \mathrm{D}\end{array}$ \\
\hline$" \prime$ & $2[13 / 2]$ & $\begin{array}{l}7 \\
6\end{array}$ & $\begin{array}{l}202948.9 \\
206299.4\end{array}$ & $\begin{array}{r}100 \\
70\end{array}$ & $\begin{array}{l}\text { or } \\
\text { or }\end{array}$ & $\begin{array}{r}100 \\
88\end{array}$ & $\begin{array}{l}{ }^{3} \mathrm{I} \\
{ }^{1} \mathrm{I}\end{array}$ \\
\hline$" \prime$ & $2[3 / 2]$ & $\begin{array}{l}1 \\
2\end{array}$ & $\begin{array}{l}203265.4 \\
207046.3\end{array}$ & $\begin{array}{l}52 \\
84\end{array}$ & $\begin{array}{l}\text { or } \\
\text { or }\end{array}$ & $\begin{array}{l}51 \\
72\end{array}$ & $\begin{array}{l}{ }^{1} \mathrm{P} \\
{ }^{3} \mathrm{P}\end{array}$ \\
\hline
\end{tabular}


Pr IV-Continued

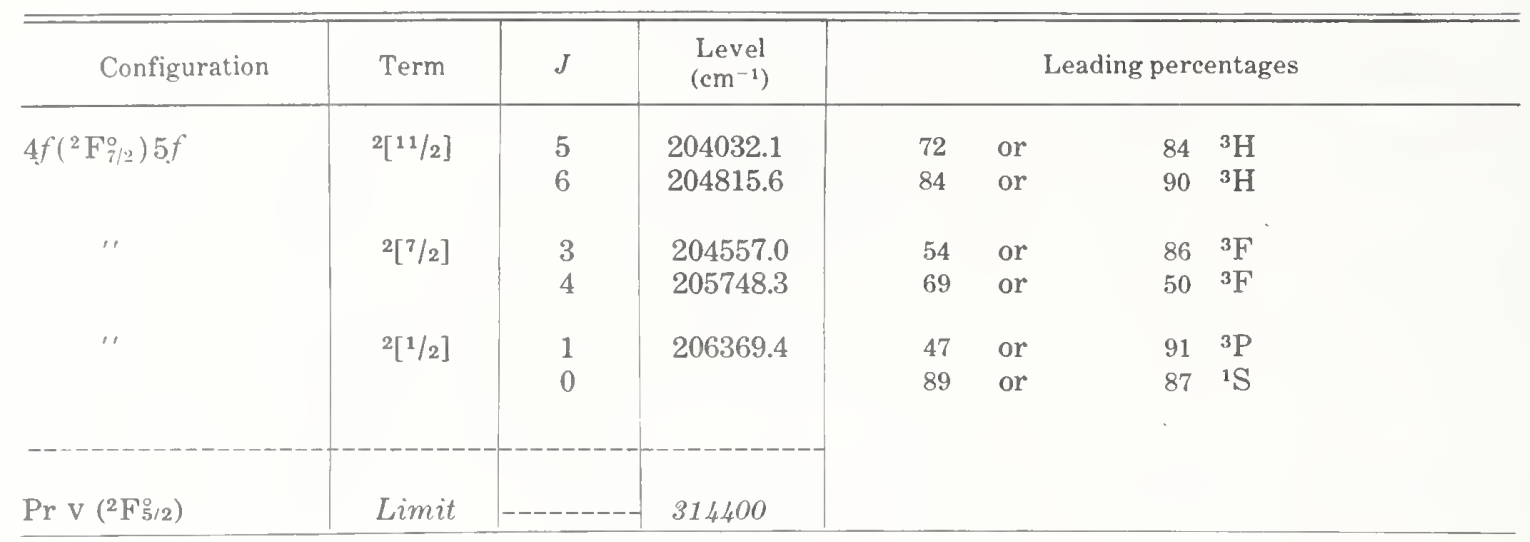




\section{$\operatorname{Pr} \mathrm{V}$}

(Cs I sequence; 55 electrons)

Ground state $\left(1 s^{2} 2 s^{2} 2 p^{6} 3 s^{2} 3 p^{6} 3 d^{10} 4 s^{2} 4 p^{6} 4 d^{10} 5 s^{2} 5 p^{6}\right) 4 f^{2} \mathrm{~F}_{5 / 2}^{\circ}$

Ionization energy $464000 \pm 400 \mathrm{~cm}^{-1}$

$57.53 \pm 0.05 \mathrm{eV}$

Kaufman and Sugar observed 12 lines of $\operatorname{Pr} \mathrm{V}$ in the region 840-2250 $\AA$, with accuracies such that the uncertainties of the resulting levels are from 1 to $2 \mathrm{~cm}^{-1}$. The term assignments are supported by comparisons along the isoelectronic sequence, the authors noting that the "quantum defect, doublet splitting, and term energy intervals were found to vary in a regular way through the Cs I sequence."

The hyperfine splitting of the $6 s{ }^{2} \mathrm{~S}_{1 / 2}$ level was resolved. The component levels are given as $178969.4 \mathrm{~cm}^{-1}(F=2)$ and $178972.3 \mathrm{~cm}^{-1}(F=3)$, with the separation of $2.95 \mathrm{~cm}^{-1}$ being accurate to about $\pm 0.05 \mathrm{~cm}^{-1}$.

Kaufman and Sugar derived the ionization potential from the $6 s$ and $7 s$ levels. A slightly different value obtained from the same data is quoted here [Sugar, 1975]. The relatively high

accuracy derives from a regularity in the quantum defects of such levels that holds throughout the rare-earth group.

\section{References}

Kaufman, V., and Sugar, J., J. Res. Nat. Bur. Stand. (U.S.) 71A, 583 (1967). E L CL W Hfs IP Sugar, J., J. Opt. Soc. Am. 65, 1366 (1975). IP

[July 1976]

\begin{tabular}{|c|c|c|c|}
\hline Configuration & Term & $J$ & $\begin{array}{l}\text { Level } \\
\left(\mathrm{cm}^{-1}\right)\end{array}$ \\
\hline $5 p^{6}\left({ }^{1} \mathrm{~S}\right) 4 f$ & ${ }^{2} \mathrm{~F}^{\circ}$ & $\begin{array}{l}5 / 2 \\
7 / 2\end{array}$ & $\begin{array}{r}0.0 \\
3027.4\end{array}$ \\
\hline $5 p^{6}\left({ }^{1} \mathrm{~S}\right) 5 d$ & ${ }^{2} \mathrm{D}$ & $\begin{array}{l}3 / 2 \\
5 / 2\end{array}$ & $\begin{array}{l}115052.3 \\
118513.8\end{array}$ \\
\hline $5 p^{6}\left({ }^{1} \mathrm{~S}\right) 6 s$ & ${ }^{2} \mathrm{~S}$ & $1 / 2$ & 178971.1 \\
\hline $5 p^{6}\left({ }^{1} \mathrm{~S}\right) 6 p$ & ${ }^{2} \mathrm{P}^{\circ}$ & $\begin{array}{l}1 / 2 \\
3 / 2\end{array}$ & $\begin{array}{l}223.478 .1 \\
230039.5\end{array}$ \\
\hline $5 p^{6}\left({ }^{1} \mathrm{~S}\right) 7 \mathrm{~s}$ & ${ }^{2} \mathrm{~S}$ & $1 / 2$ & 304511.5 \\
\hline $\operatorname{Pr}$ VI $\left({ }^{1} \mathrm{So}\right)$ & Limit & & 464000 \\
\hline
\end{tabular}




\section{NEODYMIUM}

\section{$\mathrm{Nd}$ I}

60 electrons

$Z=60$

Ground state $\left(1 s^{2} 2 s^{2} 2 p^{6} 3 s^{2} 3 p^{6} 3 d^{10} 4 s^{2} 4 p^{6} 4 d^{10} 5 s^{2} 5 p^{6}\right) 4 f^{4} 6 s^{2}{ }^{5} \mathrm{I}_{4}$

Ionization energy $44562 \pm 5 \mathrm{~cm}^{-1}$

$5.5250 \pm 0.0006 \mathrm{eV}$

Identified even configurations

$4 f^{4} 6 s^{2}, 4 f^{4} 5 d 6 s, 4 f^{3} 5 d 6 s 6 p, 4 f^{3} 5 d^{2} 6 p, 4 f^{4} 5 d^{2}$, $4 f^{4} 6 s 7 s$

Identified odd configurations

$4 f^{4} 6 s 6 p, 4 f^{3} 5 d 6 s^{2}, 4 f^{3} 5 d^{2} 6 s, 4 f^{4} 5 d 6 p$

\section{Spectrum Observations, Early Analysis}

The wavelengths and temperature classifications for $2863 \mathrm{Nd}$ lines (2963-7005 $\AA$ ) published by A. S. King in 1933 are still useful, especially since a complete list of lines based on the more recent observations has not been published.

Van de Vliet [1939] began a series of investigations of $\mathrm{Nd}$ spectra in the Zeeman Laboratory, Amsterdam, with his observations of the Zeeman effect. In 1946 Schuurmans gave the $4 f^{4} 6 s^{2}{ }^{5} \mathrm{I}$ levels of $\mathrm{Nd}$ I, which he had found with the aid of King's and van de Vliet's data. Klinkenberg [1946, 1955] and Hassan [1962, 1963] extended the observations. Klinkenberg located about 100 upper odd levels, but the analysis and interpretation were limited by the lack of sufficiently accurate wavelengths and Zeeman data [Hassan and Klinkenberg, 1963].

The more recent observations of $\mathrm{Nd}$ I and $\mathrm{Nd}$ II have yielded wavelengths for a total of more than 27000 lines in the range from $2450 \AA$ to $4.1 \mu \mathrm{m}$. About 25000 lines were photographed at the Argonne National Laboratory and measured with an automatic comparator at the Zeeman Laboratory [Hoekstra, 1969]. Wyart's thesis [1968] gives wavelengths from these measurements for about 2170 lines (4662-8713 $\AA$ ) for which he measured the Zeeman effect; the complete line list has not been published. Blaise, Chevillard, Verges, and Wyart [1970], at the Aimé Cotton Laboratory, Orsay, measured $2049 \mathrm{Nd}$ lines in the infrared region from 0.8 to $2.5 \mu \mathrm{m}$, and Morillon's observations [1970] from 2.3 to $4.1 \mu \mathrm{m}$ gave wavelengths for 470 lines. Since 1965 the work on Nd I and Nd II at Amsterdam and Orsay has been carried out "in close and stimulating collaboration" [Blaise, Wyart, Hoekstra, and Kruiver, 1971].

\section{Levels, $g$ Values, and Theoretical Interpretation}

The theses of Wyart [1968] and Hoekstra [1969] reported revisions and large extensions of the analysis of $\mathrm{Nd}$ I. About 700 energy levels were known by 1970. Most of the levels and $g$ values given here are from the paper by Blaise, Chevillard, Verges, and Wyart [1970], with some additions and corrections from Blaise et al. [1971, 1976]. Many of the three-place level values are actually given to the nearest $0.005 \mathrm{~cm}^{-1}$; the probable error for these levels is presumably somewhat larger than for the other three-place levels.

Blaise et al. [1971, 1976] found two low levels and three upper levels comprising a system not yet connected with the other levels. They tentatively assigned the two low levels to $4 f^{4} 6 s^{2}{ }^{5} \mathrm{~F}_{1}$ and ${ }^{5} \mathrm{~F}_{2}$. We have adjusted the levels to a value of $10200.00 \mathrm{~cm}^{-1}$ for the ${ }^{5} \mathrm{~F}_{1}$ level, approximately in agreement with the value they calculated for $4 f^{4} 6 s^{2}{ }^{5} \mathrm{~F}_{1}$. The lack of a connection for the system is indicated by the notation " $+x$ " following each of the five levels.

Hassan, Abbas, and Turki [1975] list 45 levels for Nd I. Several of their odd levels are confirmed by the more complete analyses used for this compilation. 
Wyart derived most of the $g$ values from his measurements of Zeeman-effect spectrograms (2900-11200 $\AA$ ) obtained by M. Fred at the Argonne Laboratory [Wyart, 1968; Hoekstra, 1969; Blaise et al., 1970]. Most of these values are given to the nearest 0.005 units. Childs and Goodman [1972] measured the $g$ values of the 5 ground-term levels to within uncertainties of two or three units in the fifth decimal place.

The available eigenvector percentages are limited to the four lowest configurations (two odd and two even). The percentages for the ${ }^{5} \mathrm{I}$ ground-term levels are from Conway and Wybourne [1963], and the percentages for the $4 f^{4} 5 d 6 s, 4 f^{3} 5 d 6 s^{2}$, and $4 f^{3} 5 d^{2} 6 s$ levels are from Blaise et al. [1971]. The $4 f^{3} 5 d^{2} 6 s$ percentages are based on the "real" $4 f^{3}\left({ }^{4} I^{\circ}\right)$ grandparent term (not pure $L S$-coupling basis states), and all $4 f^{3} 5 d^{2} 6 s$ states based on higher $4 f^{3}$ terms were neglected. Some details of the basic methods used for the calculations of $4 f^{4} 5 d 6 \mathrm{~s}$ and $4 f^{3} 5 d 6 s^{2}$ are given in Wyart's thesis [1968]. No configuration interaction has been included.

No calculations are available for two of the identified odd configurations nor for four of the identified even configurations listed above. Blaise et al. [1971] have outlined some of the criteria for the assignments of levels to these configurations. Only the configuration and final term assignments are given in the most complete list of levels [Blaise et al., 1970]. Following these assignments, we have grouped some of the levels into terms and also in some cases have supplied parentages. The designations of several of the resulting terms are followed by question marks to indicate that all of the grouped levels may not belong to the same term.

\section{Isotope Shifts, Ionization Energy}

Isotope-shift data have played an important role in the interpretation of the $\mathrm{Nd}$ I spectrum [see, e.g., Rao and Gluck, 1964; Wyart, 1968; Blaise et al., 1971]; only a few of the pertinent references are listed below.

Worden, Conway, Paisner, and Solarz [1977] have observed high members of a series in Nd I by using laser techniques. These data became available too late for inclusion here, but the quoted ionization energy was determined by Worden et al. from the new series.

\section{References}

Blaise, J., Chevillard, J., Verges, J., and Wyart, J. F., Spectrochim. Acta, Part B 25, 333 (1970). EL CL W ZE

Blaise, J., Wyart, J. F., Hoekstra, R., and Kruiver, P. J. G., J. Opt. Soc. Am. 61, 1335 (1971); unpublished additions and corrections (1976). EL ND ZE PT

Childs, W. J., and Goodman, L. S., Phys. Rev. A 6, 1772 (1972). ZE H fs

Conway, J. G., and Wybourne, B. G., Phys. Rev. 130, 2325 (1963). PT

Gerstenkorn, S., Helbert, J. M., and Chabbal, R., C. R. Acad. Sci. 261, 1232 (1965). IS

Hassan, G. E. M. A., Thesis, Univ. Amsterdam, 175 pp. (1962). EL CL W ZE IS IP

Hassan, G. E. M. A., Physica (Utrecht) 29, 1119 (1963). EL ZE IP

Hassan, G. E. M. A., Abbas, A., and Turki, A. H., Indian J. Phys. 49, 729 (1975). EL ZE

Hassan, G. E. M. A., and Klinkenberg, P. F. A., Physica (Utrecht) 29, 1133 (1963). CL ZE IS

Held, S., Israel Atomic Energy Commission, IA-927, 37 pp. (1964). W

Hoekstra, R., Thesis, Univ. Amsterdam, 64 pp. (1969). EL W ZE

King, A. S., Astrophys. J. 78, 9 (1933). W

Klinkenberg, P. F. A., Physica (Utrecht) 12, 33 (1946). EL ZE

Klinkenberg, P. F. A., Thesis, Univ. Amsterdam (1955). EL CL W ZE

Morillon, C., Spectrochim. Acta, Part B 25, 513 (1970). CL W

Nöldeke, G., and Steudel, A., Z. Phys. 137, 632 (1954). IS Hfs

Paul, F. W., Phys. Rev. 49, 156 (1936). W

Rao, P. R., and Gluck, G., Proc. R. Soc. London, Ser. A 277, 540 (1964). IS

Schuurmans, Ph., Physica (Utrecht) 11, 419 (1946). EL CL

Smith, K. F., and Spalding, I. J., Proc. R. Soc. London, Ser. A 265, 133 (1961). ZE

van de Vliet, H. J., Thesis, Univ. Amsterdam, 85 pp. (1939). ZE

Worden, E. F., Conway, J. G., Paisner, J. A., and Solarz, R. W., unpublished material (1977). IP

Wyart, J. F., Thesis (Third Cycle), Univ. Paris, Orsay, 106 pp. (1968). EL ND CL W ZE IS PT

Wyart, J. F., J. Phys. (Paris) Suppl. Colloq. C 1 30, 50 (1969). EL ZE PT

[October 1976] 
Nd I, Even Parity

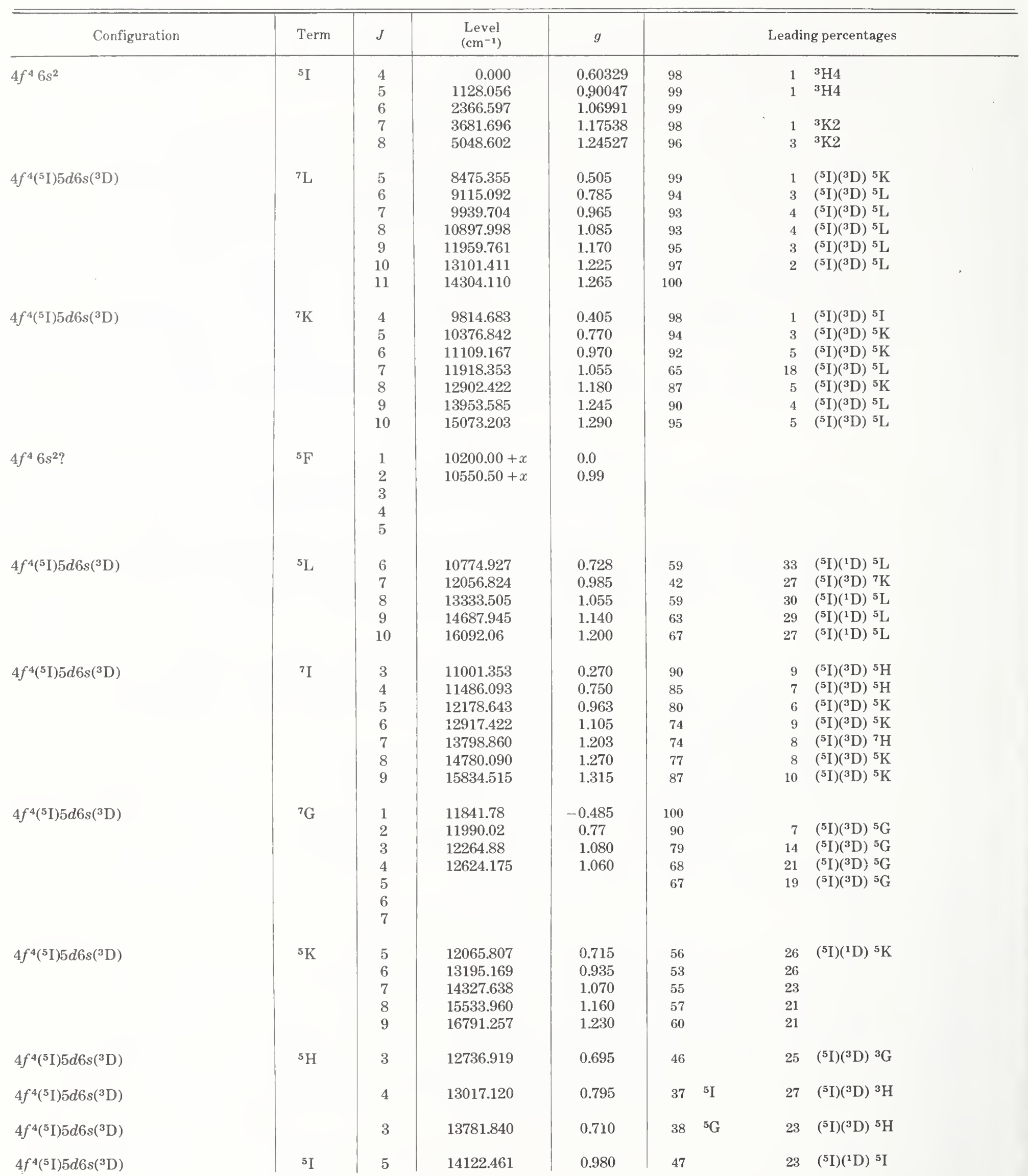


Nd I, Even Parity-Continued

Nd I, Even Parity-Continued

\begin{tabular}{|c|c|c|c|c|c|c|c|c|c|}
\hline Configuration & Term & $J$ & $\begin{array}{l}\text { Level } \\
\left(\mathrm{cm}^{-1}\right)\end{array}$ & $g$ & Configuration & Term & $J$ & $\begin{array}{l}\text { Level } \\
\left(\mathrm{em}^{-1}\right)\end{array}$ & $g$ \\
\hline \multirow[t]{5}{*}{$4 f^{3}\left({ }^{4} \mathrm{I}^{\circ}\right) 5 d\left({ }^{5} \mathrm{~L}^{\circ}\right) 6 s 6 p\left({ }^{3} \mathrm{P}^{\circ}\right)$} & \multirow[t]{5}{*}{${ }^{7} \mathrm{M}$} & \multirow{5}{*}{$\begin{array}{c}6 \\
7 \\
8 \\
9 \\
10 \\
11 \\
12\end{array}$} & \multirow{5}{*}{$\begin{array}{l}20271.673 \\
21240.345 \\
22680.963 \\
24264.991\end{array}$} & \multirow{5}{*}{$\begin{array}{l}0.595 \\
0.830 \\
0.975 \\
1.065\end{array}$} & \multirow[t]{23}{*}{$4 f^{3}\left({ }^{4} I^{\circ}\right) 5 d^{2}\left({ }^{3} \mathrm{~F}\right)\left({ }^{6} \mathrm{M}^{\circ}\right) 6 p$} & \multirow[t]{23}{*}{${ }^{7} \mathrm{~N}$} & 7 & 24856.360 & 0.850 \\
\hline & & & & & & & 6 & 24926.767 & 0.955 \\
\hline & & & & & & & 7 & 25005.315 & 0.905 \\
\hline & & & & & & & 7 & 25026.390 & 1.020 \\
\hline & & & & & & & 8 & 25046.797 & 1.025 \\
\hline $4 f^{3} 5 d 6 s 6 p$ & ${ }^{7} \mathrm{~L}$ & 5 & 20486.822 & 0.545 & & & 8 & 25189.930 & 1.110 \\
\hline $4 f^{3} 5 d 6 s 6 p$ & ${ }^{7} \mathrm{~K}$ & 4 & 21257.363 & 0.465 & & & 6 & 25207.070 & 0.905 \\
\hline $4 f^{3} 5 d 6 s 6 p$ & ${ }^{7} \mathrm{~L}$ & 5 & 21361.414 & 0.570 & & & 7 & 25383.854 & 0.971 \\
\hline $4 f^{3} 5 d 6 s 6 p$ & ${ }^{7} \mathrm{~L}$ & 6 & 21388.164 & 0.820 & & & 5 & 25450.400 & 0.820 \\
\hline $4 f^{4} 5 d^{2}$ & ${ }^{7} \mathrm{M}$ & 6 & 21889.659 & 0.580 & & & 8 & 25529.411 & 0.990 \\
\hline $4 f^{3} 5 d 6 s 6 p$ & ${ }^{\top} \mathrm{K}$ & 5 & 21924.137 & 0.780 & & & 7 & 25534.316 & 0.905 \\
\hline $4 f^{3} 5 d 6 s 6 p$ & ${ }^{7} \mathrm{~L}$ & 6 & 22121.676 & 0.765 & & & 5 & 25619.648 & 0.965 \\
\hline $4 f^{3} 5 d 6 s 6 p$ & ${ }^{7} \mathrm{~L}$ & 7 & 22615.179 & 0.845 & & & 7 & 25623.570 & 1.050 \\
\hline \multirow[t]{2}{*}{$4 f^{4} 5 d^{2}$} & \multirow[t]{2}{*}{${ }^{7} \mathrm{M}$} & 7 & 22705.580 & 0.810 & & & 6 & 25674.250 & 0.915 \\
\hline & & 6 & 22874.654 & 0.900 & & & 8 & 25703.655 & 1.095 \\
\hline \multirow[t]{7}{*}{$4 f^{3} 5 d 6 s 6 p$} & \multirow[t]{6}{*}{${ }^{7} \mathrm{I}$} & 3 & 22909.965 & 0.395 & & & 8 & 25740.206 & 1.090 \\
\hline & & 5 & 23065.496 & 0.835 & & & 6 & 25746.414 & 0.860 \\
\hline & & 6 & 23108.948 & 0.895 & & & 3 & 25822.628 & 0.660 \\
\hline & & 5 & 23175.246 & 0.785 & & & 6 & 26014.144 & 1.090 \\
\hline & & 6 & 23221.966 & 0.965 & & & 6 & 26027.860 & 1.080 \\
\hline & & 6 & 23242.421 & 1.115 & & & 2 & 26288.475 & 0.465 \\
\hline & ${ }^{7} \mathrm{I}$ & 3 & 23248.393 & 0.290 & & & 6 & 26303.870 & 0.990 \\
\hline \multirow[t]{8}{*}{$4 f^{3} 5 d 6 s 6 p$} & \multirow[t]{3}{*}{${ }^{7} \mathrm{~L}$} & 7 & 23269.485 & 0.905 & & & 5 & 26331.356 & 0.835 \\
\hline & & 4 & 23275.398 & 0.580 & \multirow[t]{21}{*}{$4 f^{3}\left({ }^{4} \mathrm{I}^{\circ}\right) 5 d^{2}\left({ }^{3} \mathrm{~F}\right)\left({ }^{6} \mathrm{M}^{\circ}\right) 6 p$} & ${ }^{7} \mathrm{~N}$ & 8 & 26333.118 & 0.890 \\
\hline & & 5 & 23281.089 & 0.545 & & & 5 & 26357.250 & 0.875 \\
\hline & ${ }^{7} \mathbf{I}$ & 4 & 23346.750 & 0.740 & & & 8 & 26425.327 & 1.135 \\
\hline & & 5 & 23565.210 & 0.760 & & & 7 & 26515.707 & 1.085 \\
\hline & & 4 & 23808.534 & 0.610 & & & 6 & 26668.475 & 1.020 \\
\hline & & 3 & 23866.080 & 0.450 & & & 4 & 26694.655 & 0.805 \\
\hline & & 8 & 23952.890 & 1.053 & & & 7 & 26835.359 & 0.860 \\
\hline \multirow[t]{4}{*}{$4 f^{4} 5 d^{2}$} & \multirow[t]{4}{*}{${ }^{7} \mathrm{~L}$} & 6 & 23964.137 & 0.815 & & & 6 & 26892.543 & 0.910 \\
\hline & & 6 & 23969.072 & 0.840 & & & 7 & 26898.411 & 1.040 \\
\hline & & 6 & 24146.624 & 0.890 & & & 9 & 26987.075 & 1.140 \\
\hline & & 5 & 24198.530 & 0.800 & & & 7 & 27000.108 & 1.055 \\
\hline \multirow[t]{4}{*}{$4 f^{3} 5 d 6 s 6 p$} & \multirow[t]{4}{*}{${ }^{5} \mathrm{M}$} & 7 & 24217.847 & 0.870 & & & 6 & 27056.090 & 0.990 \\
\hline & & 4 & 24225.650 & 0.875 & & & 4 & 27222.174 & 0.860 \\
\hline & & 8 & 24231.280 & 1.065 & & & 7 & 27223.630 & 1.095 \\
\hline & & 7 & 24260.091 & 1.010 & & & 9 & 27265.575 & 1.040 \\
\hline $4 f^{3} 5 d 6 s 6 p$ & ${ }^{7} \mathrm{M}$ & 9 & 24264.991 & 1.065 & & & 8 & 27324.410 & 1.040 \\
\hline \multirow[t]{3}{*}{$4 f^{4} 5 d^{2}$} & \multirow[t]{3}{*}{${ }^{7} \mathrm{M}$} & 9 & 24558.778 & 1.098 & & & 7 & 27381.805 & 1.005 \\
\hline & & 2 & 24559.635 & 0.245 & & & 6 & 27423.635 & 1.025 \\
\hline & & 5 & 24577.981 & 0.870 & & & 7 & 27502.595 & 1.087 \\
\hline \multirow[t]{2}{*}{$4 f^{4} 5 d^{2}$} & \multirow[t]{2}{*}{${ }^{7} \mathrm{~L}$} & 7 & 24793.359 & 0.965 & & & 7 & 27554.118 & 0.995 \\
\hline & & 7 & 24821.218 & 1.060 & & & 7 & 27567.908 & 1.000 \\
\hline
\end{tabular}




\begin{tabular}{|c|c|c|c|c|c|c|c|c|c|}
\hline Configuration & Term & $J$ & $\begin{array}{l}\text { Level } \\
\left(\mathrm{cm}^{-1}\right)\end{array}$ & $g$ & Configuration & Term & $J$ & $\begin{array}{l}\text { Level } \\
\left(\mathrm{cm}^{-1}\right)\end{array}$ & $g$ \\
\hline \multirow{43}{*}{$4 f^{4}\left({ }^{5}\right) 6 s 7 s\left({ }^{3} S\right)$} & \multirow{43}{*}{${ }^{7} \mathrm{I}$} & 4 & 27588.440 & 0.790 & & & 5 & 29310.196 & 0.870 \\
\hline & & 6 & 27589.409 & 1.080 & & & 6 & 29314.777 & 0.995 \\
\hline & & 6 & 27633.729 & 0.975 & & & 9 & 29365.082 & 1.055 \\
\hline & & 6 & 27679.543 & 1.130 & & & 8 & 29391.756 & 1.130 \\
\hline & & 6 & 27711.985 & 0.945 & & & 5 & 29493.945 & 0.830 \\
\hline & & 6 & 27755.134 & 1.045 & & & 9 & 29494.836 & 1.100 \\
\hline & & 9 & 27777.530 & 1.085 & & & 5 & 29535.427 & 0.970 \\
\hline & & 7 & 27805.365 & 1.020 & & & 8 & 29537.338 & 1.075 \\
\hline & & 8 & 27815.384 & 1.015 & & & 10 & 29570.770 & 1.060 \\
\hline & & 4 & 27852.748 & 0.845 & & & 8 & 29685.852 & 1.035 \\
\hline & & 9 & 27881.244 & 1.140 & & & 8 & 29802.070 & 0.995 \\
\hline & & 8 & 27922.081 & 1.125 & & & 6 & 29853.580 & 1.086 \\
\hline & & 5 & 27926.240 & 0.880 & & & 8 & 29873.308 & 1.110 \\
\hline & & 9 & 27961.248 & 0.975 & & & 7 & 30011.635 & 1.055 \\
\hline & & 5 & 27988.786 & 1.000 & & & 5 & 30148.057 & 0.950 \\
\hline & & 3 & 28027.403 & 0.345 & & & 5 & 30200.650 & 0.880 \\
\hline & & 4 & 28229.220 & 0.710 & & & 8 & 30319.660 & 1.165 \\
\hline & & 5 & 28578.959 & 0.945 & & & 6 & 30405.300 & 1.035 \\
\hline & & $\begin{array}{l}6 \\
7\end{array}$ & $\begin{array}{l}29522.78 \\
30630.89\end{array}$ & $\begin{array}{l}1.080 \\
1.200\end{array}$ & & & 3 & 30475.205 & 0.685 \\
\hline & & 8 & 31838.70 & 1.255 & & & 8 & 30489.865 & 1.110 \\
\hline & & 9 & 33106.00 & 1.315 & & & 9 & 30644.708 & 1.044 \\
\hline & & 6 & 28040.725 & 0.840 & & & 5 & 31129.734 & 1.000 \\
\hline & & 5 & 28086.830 & 0.860 & & & 5 & 31151.150 & 0.835 \\
\hline & & 7 & 28105.805 & 1.045 & & & 4 & 31182.990 & 0.520 \\
\hline & & 5 & 28160.209 & 0.895 & & & 8 & 31235.494 & 1.086 \\
\hline & & 5 & 28220.326 & 0.895 & & & 7 & 31242.740 & 0.940 \\
\hline & & 7 & 28354.540 & 1.060 & & & 7 & 31381.920 & 1.055 \\
\hline & & 6 & 28356.954 & 0.980 & & & 7 & 31524.204 & 0.980 \\
\hline & & 7 & 28412.288 & 0.995 & & & 6 & 31529.965 & 1.135 \\
\hline & & 4 & 28438.783 & 0.880 & & & 4 & 31541.980 & 0.860 \\
\hline & & 8 & 28440.275 & 0.935 & & & 5 & 31555.724 & 0.735 \\
\hline & & 5 & 28525.930 & 1.075 & & & 7 & 31801.150 & 0.955 \\
\hline & & 5 & 28665.120 & 0.920 & & & 3 & 32226.230 & 0.840 \\
\hline & & 4 & 28695.460 & 1.085 & & & 4 & 32265.520 & 0.935 \\
\hline & & 5 & 28731.205 & 0.870 & & & 2 & 32430.985 & 0.700 \\
\hline & & 6 & 28758.742 & 1.020 & & & 4 & 32617.700 & 0.620 \\
\hline & & 8 & 28839.355 & 1.070 & & & 7 & 33170.042 & 0.975 \\
\hline & & 6 & 28858.270 & 0.855 & & & 6 & 33634.150 & 0.925 \\
\hline & & 5 & 28885.798 & 0.825 & & & 6 & 34302.090 & 0.950 \\
\hline & & 7 & 28934.040 & 0.945 & & & 3 & 34867.271 & 0.670 \\
\hline & & 6 & 28950.824 & 0.735 & & & & & \\
\hline & & 8 & 29060.068 & 0.950 & & & & & \\
\hline & & 6 & 29280.696 & 0.720 & $\operatorname{Nd~II~}\left({ }^{6} I_{7 / 2}\right)$ & Limit & & 44562 & \\
\hline
\end{tabular}


Nd I, Odd Parity_Continued

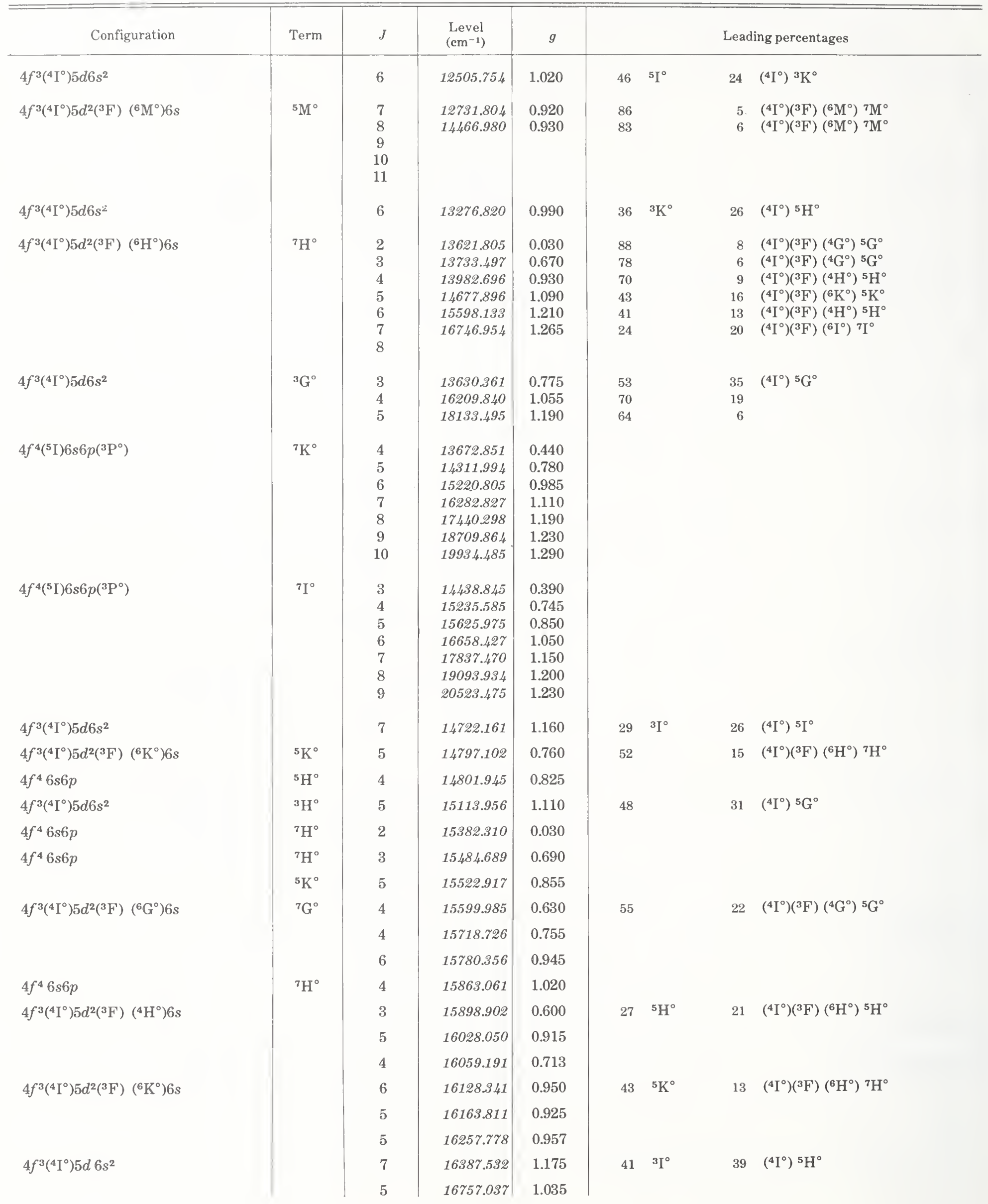


Nd I, Odd Parity-Continued

\begin{tabular}{|c|c|c|c|c|c|c|c|}
\hline Configuration & Term & $J$ & $\begin{array}{l}\text { Level } \\
\left(\mathrm{cm}^{-1}\right)\end{array}$ & $g$ & \multicolumn{3}{|c|}{ Leading percentages } \\
\hline \multirow[t]{5}{*}{$4 f^{3}\left({ }^{4} \mathrm{I}^{\circ}\right) 5 d^{2}\left({ }^{3} \mathrm{P}\right)\left({ }^{6} \mathrm{~K}^{\circ}\right) 6 s$} & ${ }^{7} \mathrm{~K}^{\circ}$ & 6 & 16796.783 & 0.955 & \multirow[t]{5}{*}{59} & \multirow[t]{5}{*}{8} & \multirow{5}{*}{$\left({ }^{4} \mathrm{I}^{\circ}\right)\left({ }^{3} \mathrm{~F}\right)\left({ }^{4} \mathrm{~L}^{\circ}\right){ }^{5} \mathrm{~L}^{\circ}$} \\
\hline & & 5 & 16844.843 & 1.015 & & & \\
\hline & & 7 & 16845.388 & 1.120 & & & \\
\hline & & 3 & 16979.352 & 0.520 & & & \\
\hline & & 4 & 17032.146 & 1.020 & & & \\
\hline $4 f^{3}\left({ }^{4} I^{\circ}\right) 5 d 6 s^{2}$ & ${ }^{3} \mathrm{H}^{\circ}$ & 6 & 17085.070 & 1.215 & 46 & 25 & $\left({ }^{4} \mathrm{I}^{\circ}\right){ }^{5} \mathrm{G}^{\circ}$ \\
\hline $4 f^{4} 6 s 6 p$ & & 5 & 17162.930 & 1.135 & & & \\
\hline \multirow[t]{4}{*}{$4 f^{3}\left({ }^{4} \mathrm{I}^{\circ}\right) 5 d^{2}\left({ }^{3} \mathrm{~F}\right)\left({ }^{6} \mathrm{H}^{\circ}\right) 6 s$} & & 6 & 17237.450 & 1.190 & \multirow[t]{4}{*}{${ }^{7} \mathrm{H}^{\circ}$} & \multirow[t]{4}{*}{17} & \multirow[t]{4}{*}{$\left({ }^{4} \mathrm{I}^{\circ}\right)\left({ }^{3} \mathrm{~F}\right)\left({ }^{6} \mathrm{G}^{\circ}\right){ }^{7} \mathrm{G}^{\circ}$} \\
\hline & & 7 & 17289.640 & 1.070 & & & \\
\hline & & 4 & 17319.761 & 0.865 & & & \\
\hline & & 5 & 17387.528 & 0.735 & & & \\
\hline \multirow[t]{2}{*}{$4 f^{3}\left({ }^{4} I^{\circ}\right) 5 d^{2}\left({ }^{3} \mathrm{P}\right)\left({ }^{6} \mathrm{I}^{\circ}\right) 6 s$} & ${ }^{7} \mathrm{I}^{\circ}$ & 4 & 17652.848 & 0.755 & \multirow[t]{2}{*}{73} & 9 & $\left({ }^{4} I^{\circ}\right)\left({ }^{3} F\right)\left({ }^{4} I^{\circ}\right){ }^{5} I^{\circ}$ \\
\hline & & 6 & 17748.814 & 1.090 & & & \\
\hline \multirow[t]{13}{*}{$4 f^{3}\left({ }^{4} \mathrm{~F}^{\circ}\right) 5 d 6 s^{2}$} & ${ }^{5} \mathrm{H}^{\circ}$ & 3 & 17786.992 & 0.600 & 88 & 5 & $\left({ }^{2} \mathrm{D}^{\circ}\right)^{3} \mathrm{G}^{\circ}$ \\
\hline & & 4 & 18741.337 & 0.930 & $\begin{array}{l}90 \\
78\end{array}$ & 3 & $\left({ }^{2} \mathrm{D}^{\circ}\right)^{3} \mathrm{G}^{\circ}$ \\
\hline & & 5 & 19815.981 & 1.110 & 78 & 3 & $\left({ }^{2} \mathrm{H}^{\circ}\right){ }^{3} \mathrm{I}^{\circ}$ \\
\hline & & $\begin{array}{l}6 \\
7\end{array}$ & 20673.022 & 1.185 & 70 & \multirow{2}{*}{\multicolumn{2}{|c|}{$\left({ }^{2} \mathrm{G}^{\circ}\right){ }^{3} \mathrm{I}^{\circ}$}} \\
\hline & & & & & & & \\
\hline & & 4 & 17790.018 & 0.920 & & & \\
\hline & & 5 & 17790.613 & 1.005 & & & \\
\hline & & 8 & 17973.309 & 1.200 & & & \\
\hline & & 3 & 17976.888 & 0.900 & & & \\
\hline & & 5 & 18029.780 & 0.970 & & & \\
\hline & & 5 & 18067.942 & 0.920 & & & \\
\hline & & 7 & 18143.686 & 1.090 & & & \\
\hline & & 6 & 18171.819 & 1.080 & & & \\
\hline \multirow[t]{8}{*}{$4 f^{3}\left({ }^{4} I^{\circ}\right) 5 d^{2}\left({ }^{3} \mathrm{~F}\right)\left({ }^{4} \mathrm{~K}^{\circ}\right) 6 s$} & & 6 & 18249.275 & 0.945 & \multirow[t]{8}{*}{$13{ }^{5} \mathrm{~K}^{\circ}$} & \multirow[t]{8}{*}{11} & \multirow[t]{8}{*}{$\left({ }^{4} \mathrm{I}^{\circ}\right)\left({ }^{3} \mathrm{P}\right)\left({ }^{6} \mathrm{I}^{\circ}\right){ }^{7} \mathrm{I}^{\circ}$} \\
\hline & & 5 & 18249.784 & 1.010 & & & \\
\hline & & 7 & 18256.805 & 0.955 & & & \\
\hline & & 5 & 18304.400 & 0.845 & & & \\
\hline & & 4 & 18436.007 & 1.075 & & & \\
\hline & & 6 & 18446.541 & 1.180 & & & \\
\hline & & 3 & 18589.901 & 0.660 & & & \\
\hline & & 5 & 18627.302 & 0.945 & & & \\
\hline \multirow[t]{6}{*}{$4 f^{3}\left({ }^{4} \mathrm{I}^{\circ}\right) 5 d^{2}\left({ }^{3} \mathrm{~F}\right)\left({ }^{6} \mathrm{I}^{\circ}\right) 6 s$} & & 6 & 18679.139 & 1.080 & \multirow[t]{6}{*}{$17{ }^{7} \mathrm{I}^{\circ}$} & 16 & $\left({ }^{4} I^{\circ}\right)\left({ }^{3} P\right)\left({ }^{6} I^{\circ}\right){ }^{7} I^{\circ}$ \\
\hline & & 5 & 18732.556 & 0.940 & & & \\
\hline & & 6 & 19152.080 & 0.930 & & & \\
\hline & & 4 & 19209.262 & 0.990 & & & \\
\hline & & 9 & 19218.140 & 1.270 & & & \\
\hline & & 5 & 19226.443 & 0.925 & & & \\
\hline $4 f^{3}\left({ }^{4} I^{\circ}\right) 5 d^{2}\left({ }^{3} F\right)\left({ }^{6} G^{\circ}\right) 6 s$ & ${ }^{7} \mathrm{G}^{\circ}$ & 7 & 19271240 & 1.260 & 54 & 16 & $\left({ }^{4} \mathrm{I}^{\circ}\right)\left({ }^{3} \mathrm{~F}\right)\left({ }^{4} \mathrm{H}^{\circ}\right){ }^{5} \mathrm{H}^{\circ}$ \\
\hline & & 6 & 19281.068 & 1.055 & & & \\
\hline & & 6 & 19428.540 & 1.040 & & & \\
\hline & & 4 & 19590.241 & 0.785 & & & \\
\hline & & 5 & 19648.022 & 1.070 & & & \\
\hline
\end{tabular}


Nd I, Odd Parity—Continued

\begin{tabular}{|c|c|c|c|c|c|c|}
\hline Configuration & Term & $J$ & $\begin{array}{c}\text { Level } \\
\left(\mathrm{cm}^{-1}\right)\end{array}$ & $g$ & & Leading percentages \\
\hline & & 5 & 19700.856 & 0.560 & & \\
\hline & & 7 & 19746.204 & 1.090 & & . \\
\hline & & 4 & 19769.507 & 0.920 & & \\
\hline & & 8 & 19862.845 & 1.290 & & \\
\hline & & 4 & 19956.814 & 0.910 & & \\
\hline & & 6 & 19994.716 & 0.920 & & \\
\hline & & 4 & 20046.654 & 0.900 & & \\
\hline \multirow[t]{3}{*}{$4 f^{3}\left({ }^{2} \mathrm{H}^{\circ}\right) 5 d 6 s^{2}$} & & 6 & 20118.994 & 1.015 & $23 \quad{ }^{3} \mathbf{K}^{\circ}$ & $\left({ }^{2} \mathrm{H}^{\circ}\right){ }^{3} \mathrm{I}^{\circ}$ \\
\hline & & 5 & 20176.912 & 0.960 & & \\
\hline & & 3 & 20281.957 & 0.895 & & \\
\hline \multirow[t]{5}{*}{$4 f^{4} 6 s 6 p$} & ${ }^{5} \mathrm{~K}^{0}$ & 5 & 20300.875 & 0.775 & & \\
\hline & & 6 & 21543.326 & 0.900 & & \\
\hline & & 7 & 22761.440 & 1.035 & & \\
\hline & & 8 & 24121.478 & 1.135 & & \\
\hline & & 9 & 25518.700 & 1.220 & & \\
\hline \multirow[t]{5}{*}{$4 f^{4} 6 s 6 p$} & ${ }^{5} I^{\circ}$ & 4 & 20360.673 & 0.735 & & \\
\hline & & 6 & 20432.386 & 1.040 & & \\
\hline & & 4 & 20478.962 & 0.895 & & \\
\hline & & 5 & 20541.833 & 0.990 & & \\
\hline & & 7 & 20583.890 & 1.070 & & \\
\hline \multirow[t]{7}{*}{$4 f^{3}\left({ }^{2} \mathrm{H}^{\circ}\right) 5 d 6 s^{2}$} & & 3 & 20594.915 & 0.910 & $32{ }^{3} \mathrm{G}^{\circ}$ & $\left({ }^{4} \mathrm{~F}^{\circ}\right){ }^{5} \mathrm{G}^{\circ}$ \\
\hline & & 6 & 20703.113 & 1.035 & & \\
\hline & & 5 & 20827.652 & 0.985 & & \\
\hline & & 6 & 20839.206 & 0.940 & & \\
\hline & & 4 & 20859.563 & 1.080 & & \\
\hline & & 6 & 20918.059 & 0.840 & & \\
\hline & & 5 & 20963.071 & 0.990 & & \\
\hline \multirow[t]{16}{*}{$4 f^{4} 6 s 6 p$} & ${ }^{5} \mathrm{I}^{\circ}$ & 5 & 21005.439 & 0.960 & & \\
\hline & & 4 & 21009.134 & 1.280 & & \\
\hline & & 7 & 21025.517 & 1.235 & & \\
\hline & & 4 & 21184.881 & 0.920 & & \\
\hline & & 3 & 21227.550 & 1.032 & & \\
\hline & & 7 & 21239.427 & 1.250 & & \\
\hline & & 5 & 21271.524 & 1.040 & & \\
\hline & & 7 & 21285.976 & 1.050 & & \\
\hline & & 6 & 21314.254 & 1.060 & & \\
\hline & & 4 & 21314.439 & 0.985 & & \\
\hline & & 6 & 21345.572 & 0.975 & & \\
\hline & & 4 & 21345.837 & 0.880 & & \\
\hline & & 7 & 21411.523 & 1.067 & & \\
\hline & & 5 & 21469.140 & 1.220 & & \\
\hline & & 4 & 21488.390 & 0.910 & & \\
\hline & & 5 & 21558.796 & 0.835 & & \\
\hline
\end{tabular}


Nd I, Odd Parity—Continued

Nd I, Odd Parity_Continued

\begin{tabular}{|c|c|c|c|c|c|c|c|c|c|}
\hline Configuration & Term & $J$ & $\begin{array}{l}\text { Level } \\
\left(\mathrm{cm}^{-1}\right)\end{array}$ & $g$ & Configuration & Term & $J$ & $\begin{array}{l}\text { Level } \\
\left(\mathrm{cm}^{-1}\right)\end{array}$ & $g$ \\
\hline \multirow[t]{44}{*}{$4 f^{4} 6 s 6 p$} & \multirow[t]{44}{*}{${ }^{5} \mathrm{H}^{\circ}$} & 3 & 21572.610 & 0.650 & \multirow{44}{*}{$4 f^{4}\left({ }^{5} \mathrm{I}\right) 5 d\left({ }^{6} \mathrm{~L}\right) 6 p$} & \multirow{44}{*}{${ }^{7} \mathbf{M}^{\circ}$} & 6 & 23040.538 & 1.075 \\
\hline & & 7 & 21640.050 & 0.980 & & & 5 & 23049.760 & 1.060 \\
\hline & & 6 & 21718.328 & 0.960 & & & 4 & 23088.680 & 1.250 \\
\hline & & 5 & 21726.771 & 1.000 & & & 5 & 23198.130 & 1.085 \\
\hline & & 4 & 21758278 & 0.735 & & & 4 & 23203.810 & 1.200 \\
\hline & & 6 & 21797.900 & 0.990 & & & 3 & 23217.930 & 1.060 \\
\hline & & 7 & 21908.639 & 0.970 & & & 5 & 23241.915 & 0.775 \\
\hline & & 4 & 21926.903 & 0.860 & & & 6 & 23283.635 & 1.040 \\
\hline & & 3 & 21951.870 & 1.070 & & & 6 & 23324.278 & 1.070 \\
\hline & & 4 & 22010.340 & 0.815 & & & 6 & 23350.793 & 0.875 \\
\hline & & 7 & 22041.570 & 1.020 & & & 5 & 23433.840 & 0.965 \\
\hline & & 6 & 22049.736 & 1.040 & & & 4 & 23438.440 & 0.885 \\
\hline & & 4 & 22076.640 & 1.035 & & & 8 & 23474.023 & 1.123 \\
\hline & & 6 & 22123.898 & 1.170 & & & 3 & 23487.080 & 0.683 \\
\hline & & 5 & 22128.600 & 1.060 & & & 6 & 23496.003 & 0.930 \\
\hline & & 5 & 22192.297 & 1.090 & & & 7 & 23517.752 & 1.052 \\
\hline & & 1 & $22215.05+x$ & 1.395 & & & 6 & 23553.851 & 0.730 \\
\hline & & 3 & 22222240 & 1.245 & & & 7 & 24529.411 & 0.820 \\
\hline & & 3 & 22229.000 & 0.705 & & & $\begin{array}{l}8 \\
9\end{array}$ & $\begin{array}{l}25596.944 \\
26740268\end{array}$ & $\begin{array}{l}0.975 \\
1.080\end{array}$ \\
\hline & & 5 & 22241.911 & 0.770 & & & 10 & 27970.520 & 1.170 \\
\hline & & 5 & 22255.855 & 1.030 & & & $\begin{array}{l}11 \\
12\end{array}$ & 29165.380 & 1.205 \\
\hline & & 6 & 22303.048 & 1.080 & & & & & \\
\hline & & 7 & 22320.290 & 1.128 & & & 4 & 23562.766 & 0.940 \\
\hline & & 5 & 22367.268 & 1.085 & & & 5 & 23573.049 & 1.080 \\
\hline & & 5 & 22406.550 & 0.885 & & & 6 & 23578.372 & 0.985 \\
\hline & & 4 & 22471.210 & 0.995 & & & 8 & 23652.731 & 1.128 \\
\hline & & 7 & 22482.764 & 1.120 & & & 7 & 23744.797 & 1.040 \\
\hline & & 3 & 22490.970 & 0.830 & & & 6 & 23755.750 & 1.075 \\
\hline & & 5 & 22530.372 & 0.850 & & & 3 & 23761.870 & 1.190 \\
\hline & & 6 & 22560.071 & 1.135 & & & 5 & 23801.447 & 1.065 \\
\hline & & 5 & 22605.665 & 1.000 & & & 5 & 23829.550 & 1.165 \\
\hline & & 4 & 22622.000 & 1.280 & & & 4 & 23845.890 & 1.175 \\
\hline & & 4 & 22622.790 & 1.135 & & & 7 & 23876.922 & 1.075 \\
\hline & & 3 & 22631.490 & 1.130 & & & 6 & 23889.268 & 1.030 \\
\hline & & 4 & 22677.810 & 0.885 & & & 4 & 23953.397 & 0.830 \\
\hline & & 5 & 22736.693 & 1.070 & & & 5 & 23968.310 & 0.980 \\
\hline & & 6 & 22738.850 & 0.985 & & & 6 & 23985.826 & 1.100 \\
\hline & & 4 & 22814.600 & 0.975 & & & 7 & 23991.098 & 1.225 \\
\hline & & 5 & 22814.888 & 0.755 & & & 6 & 23996.513 & 1.045 \\
\hline & & 6 & 22870.686 & 1.164 & & & 4 & 24001.071 & 1.110 \\
\hline & & 3 & 22929.940 & 0.765 & & & 4 & 24070241 & 0.825 \\
\hline & & 7 & 22938.734 & 1.065 & & & 8 & 24077.780 & 1.070 \\
\hline & & 3 & 22956.500 & 0.990 & & & 8 & 24168.695 & 1.100 \\
\hline & & 4 & 23016.512 & 0.810 & & & 4 & 24175.88 & 0.965 \\
\hline
\end{tabular}




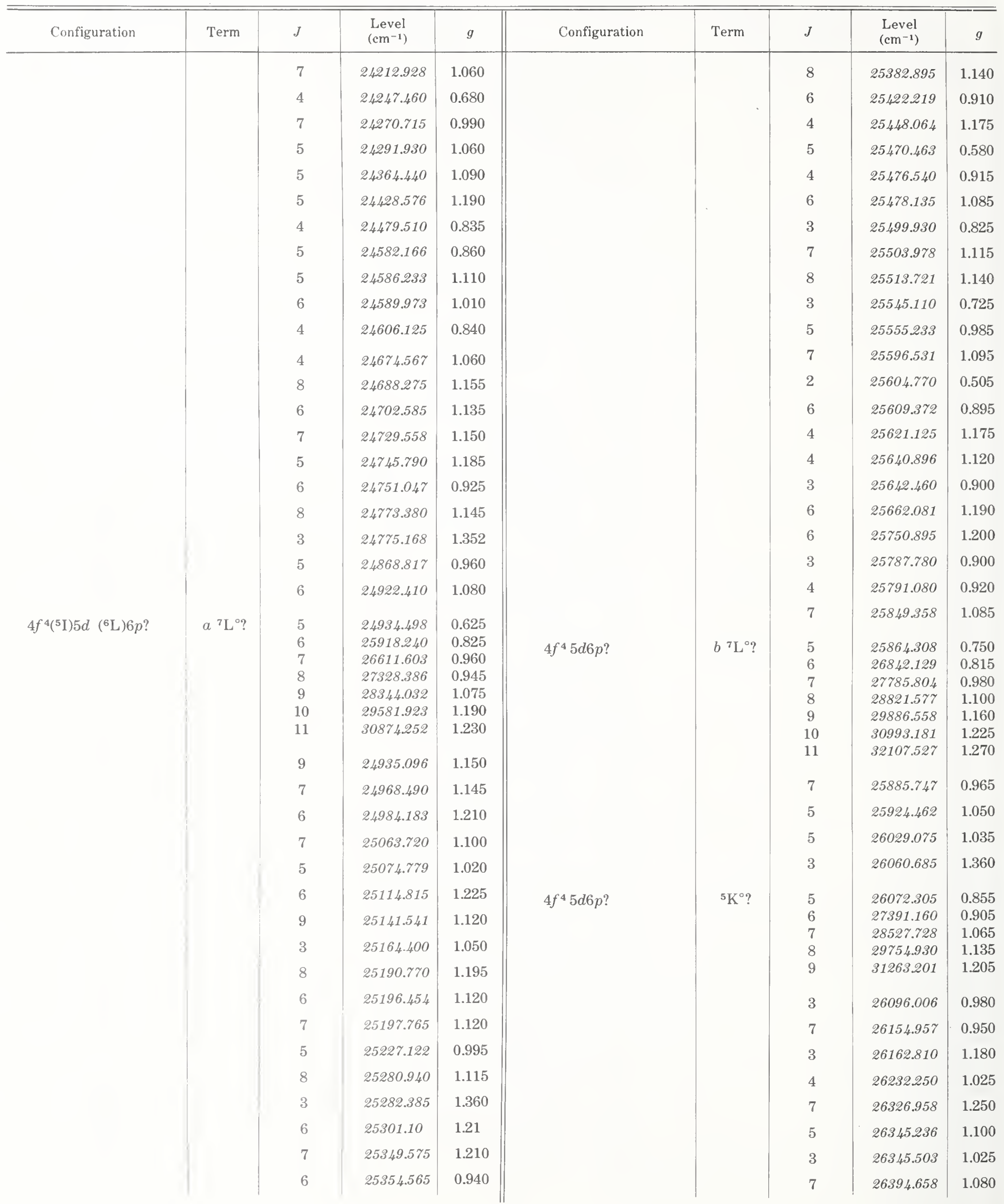




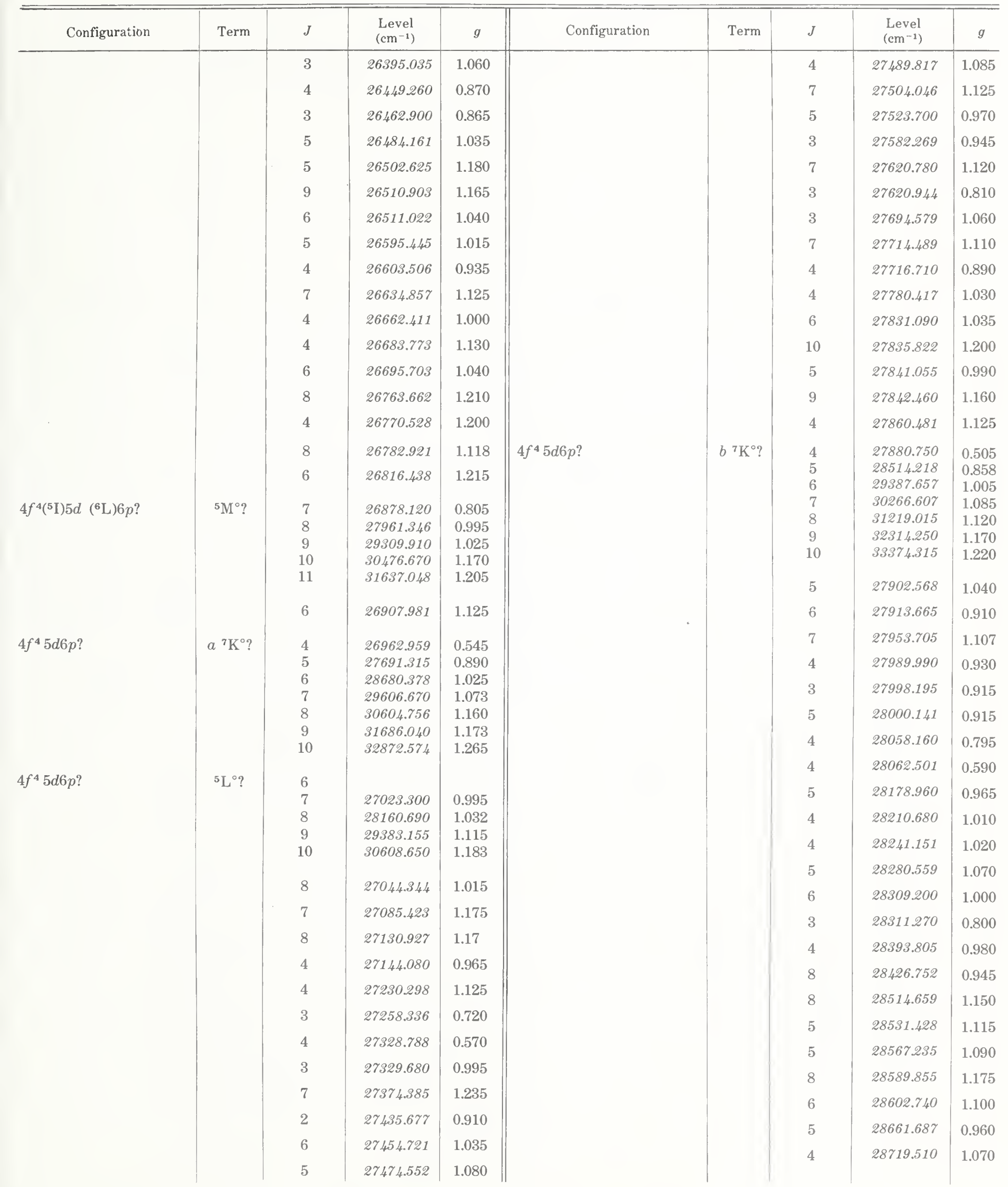




\begin{tabular}{|c|c|c|c|c|c|c|c|c|c|}
\hline Configuration & Term & $J$ & $\begin{array}{c}\text { Level } \\
\left(\mathrm{cm}^{-1}\right)\end{array}$ & $g$ & Configuration & Term & $J$ & $\begin{array}{l}\text { Level } \\
\left(\mathrm{cm}^{-1}\right)\end{array}$ & $g$ \\
\hline & & 5 & 28722.845 & 0.895 & & & 3 & 29998232 & 1.089 \\
\hline & & 3 & 28759.995 & 0.935 & & & 5 & 30014.270 & 1.037 \\
\hline & & 9 & 28781.120 & 1.215 & & . & 5 & 30060.045 & 0.985 \\
\hline & & 8 & 28787.140 & 1.175 & & & 5 & 30090.733 & 0.962 \\
\hline & & 3 & 28825.468 & 1.090 & & & 5 & 30099285 & 1.050 \\
\hline & & 5 & 28843.988 & 1.100 & & & 5 & 30139.860 & 1.070 \\
\hline & & 5 & 28862.723 & 0.945 & & & 5 & 30169.707 & 1.025 \\
\hline & & 6 & 28946.028 & 1.208 & & & 4 & 30202.415 & 1.020 \\
\hline & & 7 & 28947.443 & 1.090 & & & 2 & 30238.3 & 0.770 \\
\hline & & 7 & 29008.809 & 1.120 & $4 f^{4} 5 d 6 p ?$ & & 10 & 30295.030 & 1.243 \\
\hline & & 9 & 29060.965 & 1.015 & & & 3 & 30399.600 & 0.890 \\
\hline & & 6 & 29177.585 & 0.960 & & & 4 & 30494264 & 1.030 \\
\hline & & 4 & 29319.949 & 0.910 & & & 8 & 30726.324 & 1.145 \\
\hline & & 10 & 29340.570 & 1.217 & & & 8 & 30805.309 & 1.188 \\
\hline & & 5 & 29425.514 & 0.965 & & & 2 & 30875.395 & 0.795 \\
\hline & & 5 & 29473.175 & 1.120 & & & 6 & 31000.583 & 1.007 \\
\hline & & 6 & 29497.260 & 1.268 & $4 f^{4} 5 d 6 p ?$ & & 10 & 31260.190 & 1.245 \\
\hline & & 6 & 29510.442 & & & & 2 & $31331.36+x$ & 0.875 \\
\hline & & 6 & 29564.876 & 1.047 & $4 f^{4} 5 d 6 p ?$ & & 8 & 31412.145 & 1.065 \\
\hline & & 9 & 29764.815 & 1.185 & $4 f^{4} 5 d 6 p ?$ & & 11 & 31666.660 & 1.107 \\
\hline & & 5 & 29766.715 & 0.893 & & & 9 & 31756.355 & 1.200 \\
\hline & & 7 & 29766.974 & 1.090 & & & 9 & 31904270 & 1.240 \\
\hline & & 10 & 29826.357 & 1.020 & & & & & \\
\hline & & 9 & 29956.145 & 1.138 & & & & & \\
\hline & & 8 & 29980.378 & 1.100 & $\mathrm{Nd}$ II $\left({ }^{6} I_{z / 2}\right)$ & Limit & & 44562 & \\
\hline
\end{tabular}


Ground state $\left(1 s^{2} 2 s^{2} 2 p^{6} 3 s^{2} 3 p^{6} 3 d^{10} 4 s^{2} 4 p^{6} 4 d^{10} 5 s^{2} 5 p^{6}\right) 4 f^{4} 6 s^{6} \mathbf{I}_{7 / 2}$

Ionization energy $86500 \pm 600 \mathrm{~cm}^{-1}$

$10.73 \pm 0.08 \mathrm{eV}$

Identified even configurations

Identified odd configurations
$4 f^{4} 6 s, 4 f^{4} 5 d, 4 f^{3} 5 d 6 p$

$4 f^{3} 5 d^{2}, 4 f^{3} 5 d 6 s, 4 f^{4} 6 p$

The original analysis of this spectrum by Albertson, Harrison, and McNally [1942] gave the $4 f^{4}\left({ }^{5} \mathrm{I}\right) 6 \mathrm{~s}$ levels, 20 of the low $4 f^{4}\left({ }^{5} \mathrm{I}\right) 5 d$ levels belonging to the 5 sextet terms, and 57 upper odd levels. The analysis was based on wavelengths measured for the MIT Wavelength Tables [1939], supplemented by King's temperature classifications of Nd lines, and on Zeemaneffect data obtained at fields up to $8.7 \mathrm{~T}$. In his work at Amsterdam, Schuurmans [1946] independently duplicated part of the earlier analysis. His published data included the $4 f^{4}\left({ }^{5} I\right) 6 s$ levels and the levels of the two lowest $4 f^{4}\left({ }^{5} \mathrm{I}\right) 5 d$ terms.

The more recent progress on $\mathrm{Nd}$ II at Amsterdam and at Orsay has paralleled the Nd I work [see Nd I]. Since the wavelength and Zeeman-effect observations include both spectra, the summary of these need not be repeated. The remarks concerning the three-place level values and $g$ values for $\mathrm{Nd}$ I also apply to $\mathrm{Nd}$ II.

The main references for the levels of $\mathrm{Nd}$ II are the theses of Wyart [1968] and Hoekstra [1969], the 1970 paper by Blaise, Chevillard, Verges, and Wyart, and the report by Blaise, Wyart, Hoekstra, and Kruiver [1971]. About 500 odd levels and somewhat less than 200 even levels are now known. The positions and $g$ values given here are mainly from the tables of Blaise et al. [1970], with a few additional levels [Hoekstra, 1969; Wyart, private correspondence, 1972] and some corrections kindly supplied by Blaise and Wyart.

In his 1973 thesis, Wyart extended his original calculations [1970] of Nd II $4 f^{4} 6 s$ and $4 f^{4} 5 d$ to include effective interactions and configuration interaction. The eigenvector percentages for 78 levels of these configurations are taken from the thesis.

The $104 f^{4}\left({ }^{5} \mathrm{I}\right) 6 s$ levels fall distinctly into $5 J_{1} j$-coupling pairs [Schuurmans, 1946; Wybourne, 1965], and the experimental $g$ values agree well with the theoretical values for $J_{1} j$ coupling. The structure and $g$ values for the levels of $4 f^{4} 6 s$ based on higher core terms do not lead to unambiguous $J_{1} j$ designations, however, and no calculation in this scheme is available. We have thus listed $J_{1} j$ designations only for the $104 f^{4}\left({ }^{5} \mathrm{I}\right) 6 \mathrm{~s}$ levels, as alternates to the usual ${ }^{6} \mathrm{I},{ }^{4} \mathrm{I}$ designations of $L S$ coupling. The $J_{1} j$ names appear in the last column, following the purities in the LS scheme.

About 100 high even levels have been found, beginning with a ${ }^{6} \mathrm{~K}_{9 / 2}$ level at $34608 \mathrm{~cm}^{-1}$, but most of these have not been interpreted.

Some preliminary calculations for the odd configurations $4 f^{3}(5 d+6 s)^{2}$ and $4 f^{4}\left({ }^{5} \mathrm{I}\right) 6 p$ have been carried out [Blaise et al., 1971], but the details have not been published. In their discussion of the odd levels, Blaise et al. state that "the theoretical interpretation ... has not yielded satisfactory results. Some of the difficulty must almost undoubtedly be attributed to the use of a truncated basis set $f^{3} d s+f^{3}\left({ }^{4} \mathrm{I}\right) d^{2}$, the basis of $f^{3} d s$ being complete." They were able, however, to assign a number of the odd levels to low terms of the three identified odd configurations. Since overlapping terms of the same type occur, most of the particular groupings are given as questionable.

No levels have yet been assigned to the $4 f^{3} 6 s^{2}$ configuration, which Blaise et al. [1971] suggest may begin around $14000 \mathrm{~cm}^{-1}$.

The ionization energy is from Sugar and Reader. 


\section{References}

Albertson, W. E., Harrison, G. R., and MeNally, J. R., Jr., Phys. Rev. 61, 167 (1942). EL CL W ZE

Blaise, J., Chevillard, J., Verges, J., and Wyart, J. F., Spectrochim. Acta, Part B 25, 333 (1970). EL CL W ZE

Blaise, J., Wyart, J. F., Hoekstra, R., and Kruiver, P. J. G., J. Opt. Soc. Am. 61, 1335 (1971). E L ZE PT

Harrison, G. R., Massachusetts Institute Tech. Wavelength Tables, 429 pp. (John Wiley \& Sons, New York, 1939; MIT Press, Cambridge, 1969). W

Hassan, G. E. M. A., Thesis, Univ. Amsterdam, 175 pp. (1962). EL CL W ZE IS

Held, S., Israel Atomic Energy Commission, IA-927, 37 pp. (1964). W

Hoekstra, R., Thesis, Univ. Amsterdam, 64 pp. (1969). EL W ZE PT

King, A. S., Astrophys. J. 78, 9 (1933). W

Klinkenberg, P. F. A., Physica (Utrecht) 12, 33 (1946). ZE

Klinkenberg, P. F. A., Thesis, Univ. Amsterdam (1955). EL CL W ZE

Morillon, C., Spectrochim. Acta, Part B 25, 513 (1970). CL W

Schuurmans, Ph., Physica (Utrecht) 11, 419 (1946). EL CL ZE

Sugar, J., and Reader, J., J. Opt. Soc. Am. 55, 1286 (1965). IP

van de Vliet, H. J., Thesis, Univ. Amsterdam, 85 pp. (1939). ZE

Wyart, J. F., Thesis (Third Cycle), Univ. Paris, Orsay, 106 pp. (1968). EL CL W ZE PT

Wyart, J. F., J. Phys. (Paris) 31, 559 (1970). EL ZE PT

Wyart, J. F., Thesis, Univ. Paris-Sud, Orsay, 194 pp. (1973). EL ND ZE PT [Parameter values for the Nd II calculation have been published by Wyart, J. F., Blaise, J., and Camus, P., Phys. Scr. 9, 325 (1974). PT]

Wybourne, B. G., Spectroscopic Properties of Rare Earths, pp. 50-53 (John Wiley \& Sons Inc., New York, 1965). PT

[July 1976]

Nd II, Even Parity

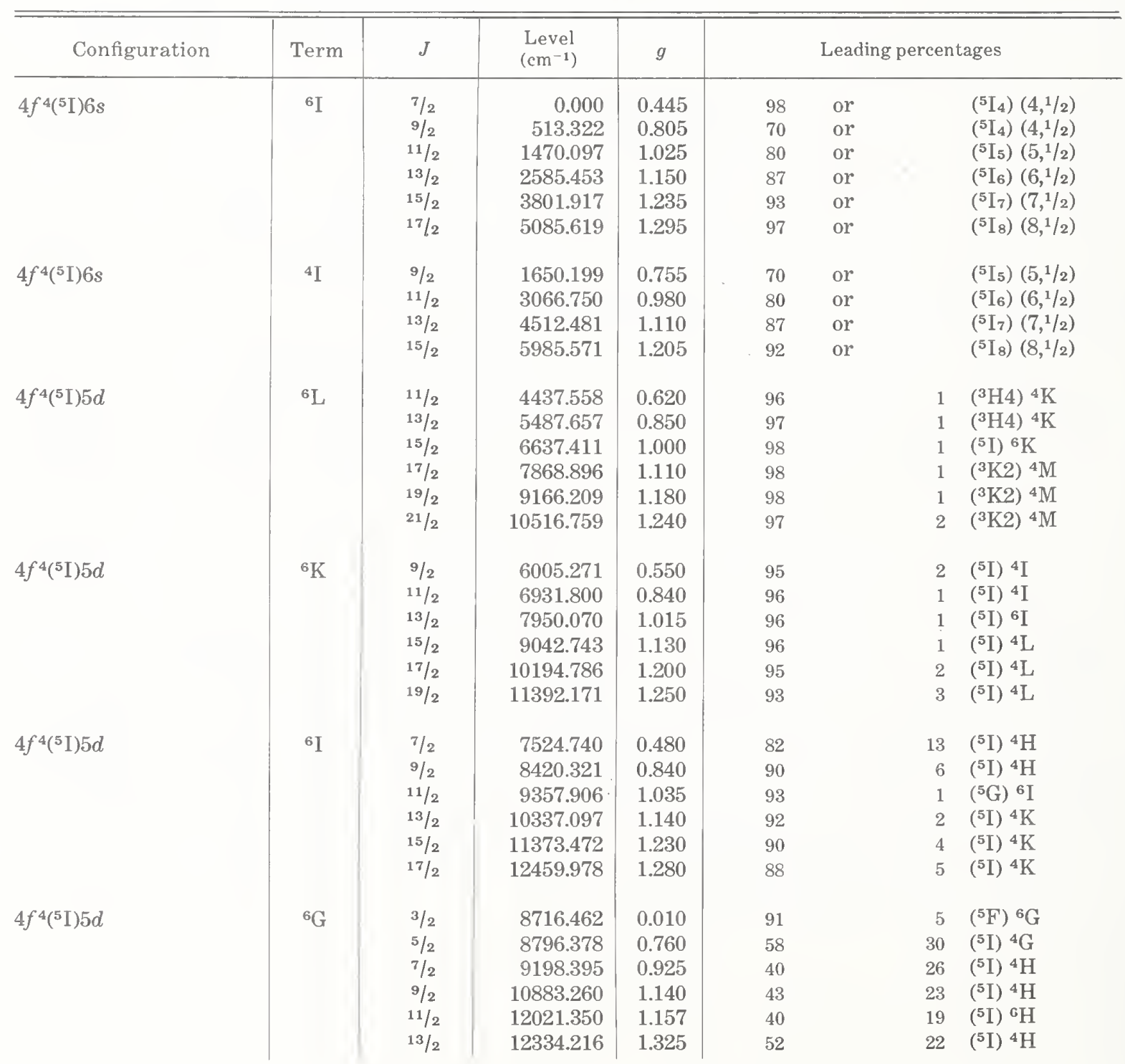




\begin{tabular}{|c|c|c|c|c|c|c|c|c|c|}
\hline Configuration & Term & $J$ & $\begin{array}{l}\text { Level } \\
\left(\mathrm{cm}^{-1}\right)\end{array}$ & $g$ & Configuration & Term & $J$ & $\begin{array}{l}\text { Level } \\
\left(\mathrm{cm}^{-1}\right)\end{array}$ & $g$ \\
\hline \multirow{44}{*}{$4 f^{3} 5 d 6 p$} & \multirow{11}{*}{${ }^{6} \mathrm{~L}$} & $13 / 2$ & 34851.551 & 0.982 & & & $5 / 2$ & 39901.515 & 0.450 \\
\hline & & $11 / 2$ & 34889.574 & 0.905 & & & $17 / 2$ & 40234.325 & 1.130 \\
\hline & & $11 / 2$ & 34915.157 & 0.960 & & & $15 / 2$ & 40247.310 & 1.086 \\
\hline & & $9 / 2$ & 35827.550 & 0.705 & & & $3 / 2$ & 40447.803 & 0.195 \\
\hline & & $11 / 2$ & 36352.460 & 0.735 & & & $9 / 2$ & 40525.585 & 0.910 \\
\hline & & $13 / 2$ & 37531.565 & 0.845 & & & $15 / 2$ & 40616.180 & 0.960 \\
\hline & & $\begin{array}{l}15 / 2 \\
17 / 2\end{array}$ & $\begin{array}{l}38486.455 \\
40136.516\end{array}$ & $\begin{array}{l}1.010 \\
1.097\end{array}$ & & & $5 / 2$ & 40647.585 & 0.605 \\
\hline & & $19 / 2$ & & & & & $9 / 2$ & 40783.030 & 0.907 \\
\hline & & & & & & & $7 / 2$ & 40837.590 & 0.765 \\
\hline & & $11 / 2$ & 36434.621 & 1.085 & & & $17 / 2$ & 40857.160 & 1.114 \\
\hline & & $13 / 2$ & 36722.170 & 0.920 & & & $9 / 2$ & 40982.115 & 0.905 \\
\hline & \multirow[t]{10}{*}{${ }^{6} \mathrm{I}$} & $7 / 2$ & 37305.750 & 0.500 & & & $15 / 2$ & 41176.480 & 1.125 \\
\hline & & $7 / 2$ & 37431.863 & 0.645 & & & $5 / 2$ & 41257.665 & 0.715 \\
\hline & & $11 / 2$ & 37449.465 & 0.935 & & & $5 / 2$ & 41277.210 & 0.755 \\
\hline & & $9 / 2$ & 37664.875 & 0.825 & & & $17 / 2$ & 41352.438 & 1.120 \\
\hline & & $13 / 2$ & 37724.558 & 1.070 & & & $3 / 2$ & 41532.990 & 0.035 \\
\hline & & $11 / 2$ & 37741.662 & 0.900 & & & $17 / 2$ & 41751.770 & 1.170 \\
\hline & & $9 / 2$ & 37799.820 & 0.820 & & & $19 / 2$ & 41912.860 & 1.162 \\
\hline & & $11 / 2$ & 37836.990 & 0.823 & & & $9 / 2$ & 41944.192 & 0.930 \\
\hline & & $13 / 2$ & 37890.200 & 0.945 & & & $15 / 2$ & 42079.900 & 1.117 \\
\hline & & $9 / 2$ & 37935.155 & 0.825 & & & $15 / 2$ & 42205.475 & 1.075 \\
\hline & \multirow[t]{9}{*}{${ }^{6} \mathrm{H}$} & $5 / 2$ & 37979.815 & 0.560 & & & $7 / 2$ & 42271.825 & 0.820 \\
\hline & & $9 / 2$ & 37985.185 & 0.840 & & & $5 / 2$ & 42424.880 & 1.050 \\
\hline & & $13 / 2$ & 38033.720 & 0.990 & & & $5 / 2$ & 42475.500 & 1.020 \\
\hline & & $9 / 2$ & 38060.876 & 1.020 & & & $9 / 2$ & 42695.670 & 1.020 \\
\hline & & $9 / 2$ & 38111.085 & 0.985 & & & $9 / 2$ & 42750.345 & 0.995 \\
\hline & & $9 / 2$ & 38134.666 & 0.875 & & & $19 / 2$ & 42816.300 & 1.191 \\
\hline & & $11 / 2$ & 38275.035 & 0.933 & & & $17 / 2$ & 42931.930 & 1.015 \\
\hline & & $7 / 2$ & 38431.770 & 0.590 & & & $19 / 2$ & 42945.320 & 1.195 \\
\hline & & $17 / 2$ & 38629.635 & 1.170 & & & $9 / 2$ & 43014.770 & 0.905 \\
\hline & \multirow[t]{14}{*}{${ }^{6} \mathrm{~K}$} & $9 / 2$ & 38644.140 & 0.615 & & & $9 / 2$ & 43062.054 & 0.890 \\
\hline & & $9 / 2$ & 38698.658 & 0.810 & & & $9 / 2$ & 43176.118 & 0.830 \\
\hline & & $11 / 2$ & 38736.080 & 0.915 & & & $7 / 2$ & 43212.630 & 0.762 \\
\hline & & $13 / 2$ & 38803.795 & 0.965 & & & $9 / 2$ & 43291.858 & 1.130 \\
\hline & & $11 / 2$ & 38852.450 & 1.150 & & & $7 / 2$ & 43326.700 & 0.750 \\
\hline & & $9 / 2$ & 38883.812 & 0.870 & & & $9 / 2$ & 43354.940 & 0.888 \\
\hline & & $15 / 2$ & 38920.650 & 1.030 & & & $9 / 2$ & 43422.595 & 0.900 \\
\hline & & $15 / 2$ & 38995.110 & 1.070 & & & $19 / 2$ & 43499.935 & 1.235 \\
\hline & & $15 / 2$ & 39136.695 & 1.083 & & & $9 / 2$ & 43883.572 & 1.005 \\
\hline & & $7 / 2$ & 39442.825 & 0.805 & & & $17 / 2$ & 43938.545 & 1.185 \\
\hline & & $7 / 2$ & 39542.970 & 0.910 & & & $17 / 2$ & 44103.625 & 1.200 \\
\hline & & $15 / 2$ & 39602.625 & 1.050 & & & $11 / 2$ & 44104.990 & 0.945 \\
\hline & & $11 / 2$ & 39657.295 & 0.985 & & & $11 / 2$ & 44205.836 & 1.005 \\
\hline & & $15 / 2$ & 39685.135 & 1.050 & & & $11 / 2$ & 44596.330 & 1.195 \\
\hline
\end{tabular}


Nd II, Even Parity-Continued

Nd II, Even Parity-Continued

\begin{tabular}{|c|c|c|c|c|c|c|c|c|c|}
\hline Configuration & Term & $J$ & $\begin{array}{l}\text { Level } \\
\left(\mathrm{cm}^{-1}\right)\end{array}$ & $g$ & Configuration & Term & $J$ & $\begin{array}{c}\text { Level } \\
\left(\mathrm{cm}^{-1}\right)\end{array}$ & $g$ \\
\hline & \multirow{5}{*}{${ }^{6} \mathrm{H}$} & $11 / 2$ & 44803.040 & 1.085 & & & $13 / 2$ & 45802.216 & 1.055 \\
\hline & & $5 / 2$ & 45039.200 & 0.355 & & & $15 / 2$ & 47020.370 & 1.154 \\
\hline & & $7 / 2$ & 45097.375 & 0.766 & & & & & \\
\hline & & $9 / 2$ & 45149.510 & 1.065 & & & & & \\
\hline & & $9 / 2$ & 45338.830 & 0.950 & $\mathrm{Nd}$ III $\left({ }^{5} \mathrm{I}_{4}\right)$ & Limit & & 86500 & \\
\hline
\end{tabular}

Nd II, Odd Parity

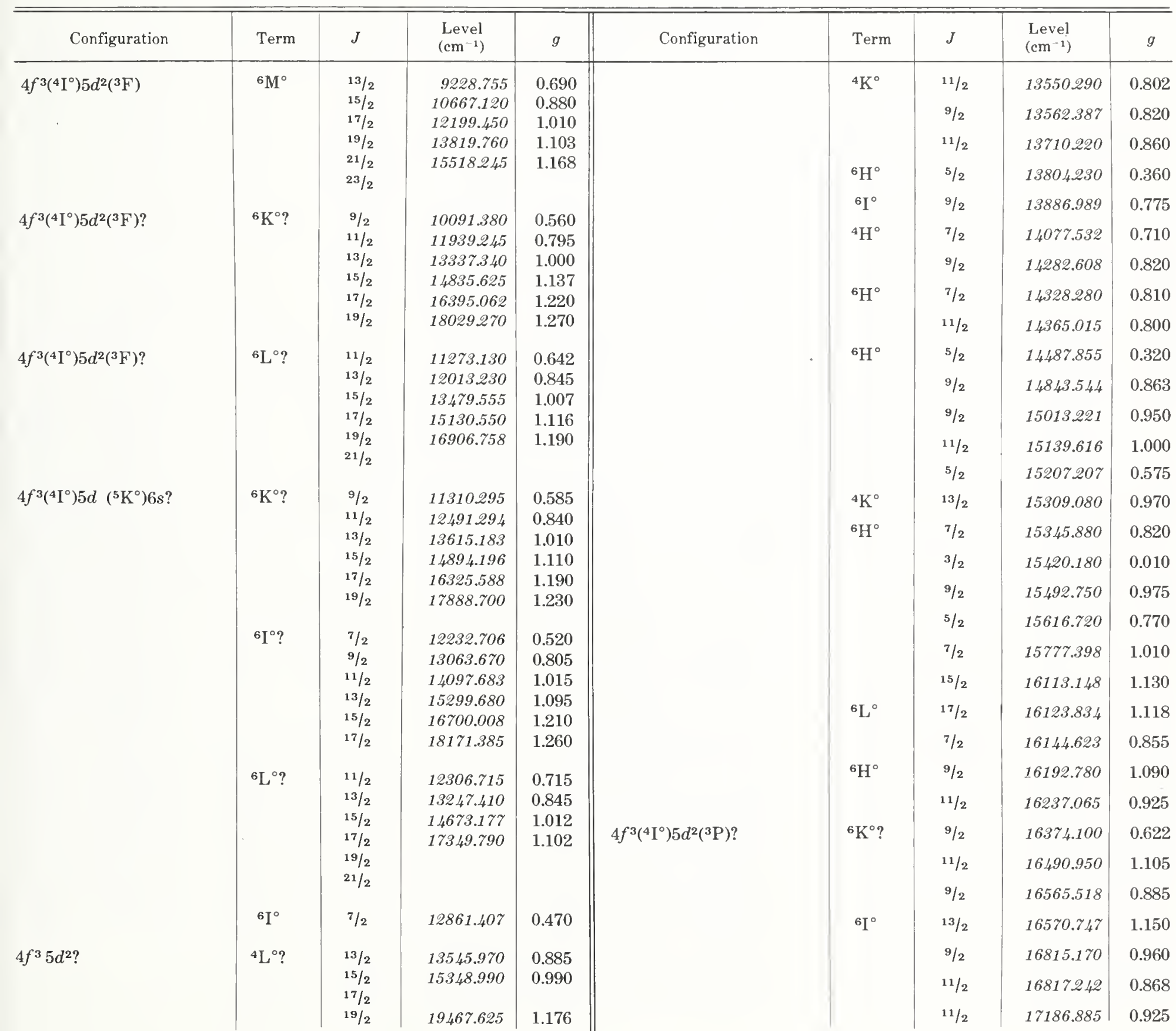

Nd II, Odd Parity 
Nd II, Odd Parity_Continued

Nd II, Odd Parity_Continued

\begin{tabular}{|c|c|c|c|c|c|c|c|c|c|}
\hline Configuration & Term & $J$ & $\begin{array}{c}\text { Level } \\
\left(\mathrm{cm}^{-1}\right)\end{array}$ & $g$ & Configuration & Term & $J$ & $\begin{array}{l}\text { Level } \\
\left(\mathrm{cm}^{-1}\right)\end{array}$ & $g$ \\
\hline & & $7 / 2$ & 17201.292 & 0.825 & \multirow{44}{*}{$4 f^{4}\left({ }^{5} I\right) 6 p$} & \multirow{10}{*}{${ }^{6} \mathrm{~K}^{\circ}$} & $7 / 2$ & 22850.552 & 0.755 \\
\hline & & $9 / 2$ & 17356.849 & 1.045 & & & $11 / 2$ & 23127.457 & 1.105 \\
\hline & & $13 / 2$ & 17465.321 & 1.045 & & & $11 / 2$ & 23159.979 & 1.065 \\
\hline & ${ }^{4} \mathrm{~K}^{\circ}$ & $17 / 2$ & 1754.4 .545 & 1.205 & & & $9 / 2$ & 23171.114 & 1.080 \\
\hline & ${ }^{6} \mathrm{~K}^{\circ}$ & $11 / 2$ & 17606.010 & 0.855 & & & $7 / 2$ & 23197.787 & 1.015 \\
\hline & ${ }^{6} \mathrm{~L}^{\circ}$ & $19 / 2$ & 17701.145 & 1.191 & & & $9 / 2$ & 23229.991 & 0.780 \\
\hline & \multirow{7}{*}{${ }^{6} \mathrm{H}^{\circ}$} & $11 / 2$ & 17848.005 & 1.230 & & & $11 / 2$ & 24445.389 & 0.915 \\
\hline & & $13 / 2$ & 18007.940 & 1.205 & & & $13 / 2$ & 25524.485 & 1.030 \\
\hline & & $7 / 2$ & 18120.909 & 1.075 & & & $\begin{array}{l}15 / 2 \\
17 / 2\end{array}$ & $\begin{array}{l}26912.765 \\
28418965\end{array}$ & $\begin{array}{l}1.130 \\
1.200\end{array}$ \\
\hline & & $5 / 2$ & 18354.613 & 0.340 & & & $19 / 2$ & 30002.302 & 1.260 \\
\hline & & $11 / 2$ & 18362.237 & 1.175 & & \multirow[t]{22}{*}{${ }^{6} \mathrm{~K}^{\circ}$} & $19 / 2$ & 23292.475 & 1.240 \\
\hline & & $15 / 2$ & 18527.325 & 1.320 & & & $11 / 2$ & 23378.327 & 1.035 \\
\hline & & $13 / 2$ & 18757.106 & 1.030 & & & $7 / 2$ & 23397.385 & 0.845 \\
\hline & \multirow[t]{3}{*}{${ }^{6} \mathrm{~K}^{\circ}$} & $9 / 2$ & 18831.219 & 1.090 & & & $9 / 2$ & 23409.537 & 1.010 \\
\hline & & $7 / 2$ & 19026.065 & 0.985 & & & $11 / 2$ & 23458.954 & 1.030 \\
\hline & & $13 / 2$ & 19113.633 & 1.085 & & & $9 / 2$ & 23537.387 & 0.945 \\
\hline & \multirow[t]{5}{*}{${ }^{6} \mathrm{H}^{\circ}$} & $15 / 2$ & 19232.234 & 1.230 & & & $7 / 2$ & 23636.759 & 1.130 \\
\hline & & $9 / 2$ & 19698.640 & 1.145 & & & $5 / 2$ & 23706.705 & 0.785 \\
\hline & & $11 / 2$ & 19703.017 & 0.965 & & & $15 / 2$ & 23737.463 & 1.060 \\
\hline & & $9 / 2$ & .19758 .554 & 1.055 & & & $9 / 2$ & 23771.040 & 1.010 \\
\hline & & $9 / 2$ & 20058.494 & 1.152 & & & $13 / 2$ & 23802.190 & 1.075 \\
\hline & \multirow[t]{3}{*}{${ }^{6} \mathrm{~K}^{\circ}$} & $15 / 2$ & 20297.802 & 1.152 & & & $11 / 2$ & 23857.278 & 1.010 \\
\hline & & $11 / 2$ & 20617.814 & 1.175 & & & $13 / 2$ & 23991.408 & 1.070 \\
\hline & & $13 / 2$ & 20646.951 & 1.153 & & & $9 / 2$ & 24003.714 & 1.050 \\
\hline & ${ }^{4} \mathbf{I}^{\circ}$ & $9 / 2$ & 20672.581 & 0.780 & & & $9 / 2$ & 24053.354 & 0.965 \\
\hline & ${ }^{4} \mathrm{H}^{\circ}$ & $7 / 2$ & 20830.048 & 0.690 & & & $7 / 2$ & 24130.300 & 1.194 \\
\hline & \multirow[t]{2}{*}{${ }^{4} \mathrm{~K}^{\circ}$} & $11 / 2$ & 20907.332 & 0.890 & & & $11 / 2$ & 24134.095 & 1.020 \\
\hline & & $9 / 2$ & 21050.626 & 1.070 & & & $13 / 2$ & 24243.228 & 1.165 \\
\hline & \multirow[t]{4}{*}{${ }^{6} \mathrm{H}^{\circ}$} & $5 / 2$ & 21241.085 & 0.310 & & & $7 / 2$ & 24316.040 & 1.140 \\
\hline & & $7 / 2$ & 21291.761 & 0.825 & & & $9 / 2$ & 24321.262 & 0.900 \\
\hline & & $7 / 2$ & 21411.258 & 0.895 & & & $13 / 2$ & 24418.720 & 1.130 \\
\hline & & $11 / 2$ & 21506.550 & 1.080 & & & $7 / 2$ & 24468.033 & 0.945 \\
\hline & \multirow[t]{3}{*}{${ }^{6} \mathrm{~K}^{\circ}$} & $17 / 2$ & 21729.555 & 1.195 & & \multirow[t]{3}{*}{${ }^{4} \mathrm{~K}^{\circ}$} & $15 / 2$ & 24547.470 & 1.120 \\
\hline & & $13 / 2$ & 21870.565 & 1.095 & & & $9 / 2$ & 24569.784 & 1.185 \\
\hline & & $7 / 2$ & 21871.522 & 0.630 & & & $11 / 2$ & 24685.721 & 1.122 \\
\hline & \multirow[t]{6}{*}{${ }^{4} \mathrm{H}^{\circ}$} & $9 / 2$ & 21918.068 & 0.940 & & \multirow[t]{9}{*}{${ }^{4} \mathrm{I}^{\circ}$} & $13 / 2$ & 24721.059 & 1.050 \\
\hline & & $9 / 2$ & 22187.658 & 0.980 & & & $9 / 2$ & 24797.387 & 0.975 \\
\hline & & $11 / 2$ & 22212.745 & 1.195 & & & $11 / 2$ & 24842.878 & 0.975 \\
\hline & & $9 / 2$ & 22358.081 & 1.080 & & & $7 / 2$ & 24913.863 & 0.860 \\
\hline & & $7 / 2$ & 22389.854 & 0.755 & & & $13 / 2$ & 24955.215 & 1.130 \\
\hline & & $9 / 2$ & 22455.624 & 1.000 & & & $11 / 2$ & 25014.930 & 1.120 \\
\hline & \multirow[t]{2}{*}{${ }^{4} \mathrm{~K}^{\circ}$} & $13 / 2$ & 22578.175 & 0.995 & & & & & \\
\hline & & $9 / 2$ & 22663.728 & 0.870 & & & & & \\
\hline & ${ }^{4} \mathrm{I}^{\circ}$ & $11 / 2$ & 22696.885 & 0.965 & & & & & \\
\hline
\end{tabular}


Nd II, Odd Parity_Continued

Nd III, Odd Parity_Continued

\begin{tabular}{|c|c|c|c|c|c|c|c|c|c|}
\hline Configuration & Term & $J$ & $\begin{array}{l}\text { Level } \\
\left(\mathrm{cm}^{-1}\right)\end{array}$ & $g$ & Configuration & Term & $J$ & $\begin{array}{l}\text { Level } \\
\left(\mathrm{cm}^{-1}\right)\end{array}$ & $g$ \\
\hline \multirow[t]{47}{*}{$4 f^{4}(5 \mathrm{I}) 6 p$} & \multirow[t]{29}{*}{${ }^{6} I^{\circ}$} & $7 / 2$ & 25044.675 & 0.820 & \multirow{47}{*}{$4 f^{4}\left({ }^{5} \mathrm{I}\right) 6 p$} & \multirow{28}{*}{${ }^{4} \mathrm{~K}^{\circ}$} & $13 / 2$ & 26328.010 & 1.083 \\
\hline & & $9 / 2$ & 25877.176 & 0.950 & & & $7 / 2$ & 26369.065 & 1.285 \\
\hline & & $\begin{array}{l}11 / 2 \\
13 / 2\end{array}$ & $\begin{array}{l}26772.093 \\
27744.196\end{array}$ & $\begin{array}{l}1.020 \\
1.080\end{array}$ & & & $9 / 2$ & 26380.727 & 1.035 \\
\hline & & $15 / 2$ & 28856.898 & 1.160 & & & 71 & & 1920 \\
\hline & & $17 / 2$ & 30246.771 & 1.220 & & & 12 & 26422.560 & 1.230 \\
\hline & & $11 / 2$ & 95000000 & 0990 & & & $11 / 2$ & 26455.191 & 1.090 \\
\hline & & $11 / 2$ & 25080.880 & 0.990 & & & $13 / 2$ & 26459.602 & 1.250 \\
\hline & & $15 / 2$ & 25120.324 & 1.165 & & & $15 / 2$ & 26473.775 & 1.231 \\
\hline & & $7 / 2$ & 25138.556 & 1.200 & & & $13 / 2$ & 26489.150 & 1.150 \\
\hline & & $9 / 2$ & 25190.458 & 1.150 & & & $9 / 2$ & 26500.422 & 1.085 \\
\hline & & $11 / 2$ & 25200.913 & 1.135 & & & $17 / 2$ & 26583.980 & 1.223 \\
\hline & & $5 / 2$ & 25218.025 & 1.345 & & & $9 / 2$ & 26670.590 & 1.155 \\
\hline & & $15 / 2$ & 25235.677 & 1.110 & & & $7 / 2$ & 26702.003 & 1.290 \\
\hline & & $17 / 2$ & 25286.074 & 1.185 & & & $13 / 2$ & 26738.805 & 1.090 \\
\hline & & $7 / 2$ & 25295.288 & 1.035 & & & $9 / 2$ & 26759238 & 1.025 \\
\hline & & $11 / 2$ & 25352.384 & 1.150 & & & $11 / 2$ & 26761.110 & 1.130 \\
\hline & & $15 / 2$ & 25353.631 & 1.190 & & & $7 / 2$ & 26793.331 & 1.095 \\
\hline & & $9 / 2$ & 25389.217 & 1.030 & & & $7 / 2$ & 26907.528 & 1.460 \\
\hline & & $13 / 2$ & 25394.612 & 1.138 & & & $9 / 2$ & 26926.997 & 0.990 \\
\hline & & $11 / 2$ & 25481274 & 1.025 & & & $11 / 2$ & 26991.889 & 1.200 \\
\hline & & $7 / 2$ & 25519.085 & 1.170 & & & $9 / 2$ & 27014265 & 1.105 \\
\hline & & $11 / 2$ & 25561.194 & 1.360 & & & $9 / 2$ & 27069.997 & 1.095 \\
\hline & & $7 / 2$ & 25648.870 & 1.230 & & & $13 / 2$ & 27124.095 & 1.110 \\
\hline & & $17 / 2$ & 25678.424 & 1.135 & & & $11 / 2$ & 27146.571 & 1.225 \\
\hline & & $5 / 2$ & 25745.016 & 1.200 & & & $9 / 2$ & 27179.602 & 0.915 \\
\hline & & $9 / 2$ & 25771.527 & 0.985 & & & $9 / 2$ & 27233.527 & 1130 \\
\hline & & $7 / 2$ & 25844.366 & 1.125 & & & $7 / 9$ & 27211020 & 190 \\
\hline & & $11 / 2$ & 25873.144 & 1.175 & & & & $21 \sim 44.0 \times 0$ & 1.100 \\
\hline & & $5 / 2$ & 25876.559 & & & \multirow[t]{19}{*}{${ }^{4} \mathrm{~K}^{\circ}$} & $11 / 2$ & 27245.453 & 0.955 \\
\hline & \multirow{8}{*}{${ }^{6} \mathrm{H}^{\circ}$} & $7 / 2$ & 26640.085 & $\begin{array}{l}0.655 \\
0.970\end{array}$ & & & $\begin{array}{l}13 / 2 \\
15 / 2\end{array}$ & $\begin{array}{l}28563.485 \\
30094850\end{array}$ & 1.044 \\
\hline & & $9 / 2$ & 27425.015 & 1.170 & & & $17 / 2$ & 31675295 & 1.180 \\
\hline & & $11 / 2$ & 28285.619 & 1.085 & & & & & \\
\hline & & $13 / 2$ & 29434270 & 1.215 & & & $7 / 2$ & 27299.543 & 1.015 \\
\hline & & $15 / 2$ & 30707282 & 1.205 & & & $13 / 2$ & 27308.940 & 1.090 \\
\hline & & $13 / 2$ & 25918.386 & 1.228 & & & $11 / 2$ & 27352.280 & 1.035 \\
\hline & & $15 / 2$ & 26031.487 & 1.140 & & & $7 / 2$ & 27444.545 & 1.070 \\
\hline & & $5 / 2$ & 26041212 & 0.940 & & & $15 / 2$ & 27445.854 & 1.110 \\
\hline & \multirow[t]{10}{*}{${ }^{4} I^{\circ}$} & $15 / 2$ & 26055.177 & 1.160 & & & $13 / 2$ & 27448.715 & 1.135 \\
\hline & & $9 / 2$ & 26108.345 & 1.055 & & & $7 / 2$ & 27504.112 & 1.168 \\
\hline & & $5 / 2$ & 26116.051 & 1.250 & & & $11 / 2$ & 27518.405 & 1.020 \\
\hline & & $5 / 2$ & 26170.820 & 1.250 & & & $9 / 2$ & 27536.610 & 1.325 \\
\hline & & $11 / 2$ & 26182.478 & 1.020 & & & $9 / 2$ & 27553.523 & 1.125 \\
\hline & & $7 / 2$ & 26206.821 & 0.925 & & & $15 / 2$ & 27611.719 & 1.100 \\
\hline & & $13 / 2$ & 26210.748 & 1.105 & & & $5 / 2$ & 27614.956 & 0.730 \\
\hline & & $9 / 2$ & 26227.110 & 1.050 & & & $7 / 2$ & 27638.675 & 1.150 \\
\hline & & $11 / 2$ & 26274.095 & 1.025 & & & $7 / 2$ & 27694.620 & 0.725 \\
\hline & & $7 / 2$ & 26292.502 & 1.215 & & & $13 / 2$ & 27721.442 & 1.175 \\
\hline
\end{tabular}




\begin{tabular}{|c|c|c|c|c|c|c|c|c|c|}
\hline Configuration & Term & $J$ & $\begin{array}{l}\text { Level } \\
\left(\mathrm{cm}^{-1}\right)\end{array}$ & $g$ & Configuration & Term & $J$ & $\begin{array}{l}\text { Level } \\
\left(\mathrm{cm}^{-1}\right)\end{array}$ & $g$ \\
\hline \multirow{18}{*}{$4 f^{4}\left({ }^{5} I\right) 6 p$} & \multirow{18}{*}{${ }^{4} I^{\circ}$} & $11 / 2$ & 27769.432 & 1.102 & & & $15 / 2$ & 29362.528 & 1.140 \\
\hline & & $9 / 2$ & 27774.135 & 1.025 & & & $11 / 2$ & 29425.150 & 1.085 \\
\hline & & $11 / 2$ & 27781.770 & 1.130 & & & $9 / 2$ & 29521.740 & 1.075 \\
\hline & & $11 / 2$ & 27798.540 & 1.065 & & & $11 / 2$ & 29581.890 & 1.105 \\
\hline & & $7 / 2$ & 27805.385 & 0.970 & & & $15 / 2$ & 29622.172 & 1.035 \\
\hline & & $15 / 2$ & 27816.793 & 1.160 & & & $13 / 2$ & 29701.474 & 1.090 \\
\hline & & $9 / 2$ & 27921.395 & 1.035 & & & $11 / 2$ & 29763.763 & 1.077 \\
\hline & & $11 / 2$ & 29484.600 & 1.022 & & & $13 / 2$ & 29813.432 & 1.065 \\
\hline & & $\begin{array}{l}13 / 2 \\
15 / 2\end{array}$ & $\begin{array}{l}31153.870 \\
32464.640\end{array}$ & $\begin{array}{l}1.115 \\
1.130\end{array}$ & & & $9 / 2$ & 29864.657 & 0.993 \\
\hline & & $11 / 2$ & 024040.040 & (1) & & & $7 / 2$ & 29878.830 & 1.215 \\
\hline & & $9 / 2$ & 27934544 & 1.070 & & & $5 / 2$ & 29882.190 & 1.195 \\
\hline & & $9 / 2$ & 27990.040 & 1.285 & & & $15 / 2$ & 29955.418 & 1.120 \\
\hline & & $11 / 2$ & 27992.437 & 1.174 & & & $5 / 2$ & 30037.077 & 0.800 \\
\hline & & $17 / 2$ & 27993.254 & 1.175 & & & $11 / 2$ & 30161.616 & 1.047 \\
\hline & & $13 / 2$ & 28089.482 & 1.025 & & & $9 / 2$ & 30196.783 & 1.052 \\
\hline & & $11 / 2$ & 28129.850 & 1.070 & & & $11 / 2$ & 30210.725 & 1.150 \\
\hline & & $9 / 2$ & 28170.456 & 1.025 & & & $5 / 2$ & 30292.992 & 0.798 \\
\hline & & $13 / 2$ & 28196.156 & 1.070 & & & $15 / 2$ & 30306.150 & 1.130 \\
\hline \multirow[t]{17}{*}{$4 f^{4}\left({ }^{5} I\right) 6 p$} & \multirow[t]{17}{*}{${ }^{4} \mathrm{H}^{\circ}$} & $7 / 2$ & 28213.885 & 0.720 & & & $7 / 2$ & 30321.425 & 1.116 \\
\hline & & $9 / 2$ & 28298.462 & 1.072 & & & $10 / 2$ & 30348.340 & 1.125 \\
\hline & & $9 / 2$ & 28340.549 & 1.050 & & & $1 / 2$ & 30393.800 & 1.105 \\
\hline & & $13 / 2$ & 28354.410 & 1.130 & & & $5 / 2$ & 30405.583 & 1.120 \\
\hline & & $7 / 2$ & 28520.155 & 1.172 & & & $7 / 2$ & 30431.849 & 0.963 \\
\hline & & $15 / 2$ & 28540.957 & 1.145 & & & $13 / 2$ & 30467.356 & 0.990 \\
\hline & & $11 / 2$ & 28582.619 & 1.180 & & & $7 / 2$ & 30552.490 & 1.005 \\
\hline & & $1 / 2$ & 28597.560 & 1.140 & & & $13 / 2$ & 30570.222 & 1.014 \\
\hline & & 72 & 28602.040 & 1.265 & & & $7 / 2$ & 30571.874 & 1.070 \\
\hline & & $15 / 2$ & 20820.930 & 1.120 & & & $9 / 2$ & 30659.962 & 0.925 \\
\hline & & $17 / 2$ & 28748.524 & 1.120 & & & $7 / 2$ & 30681.265 & 0.980 \\
\hline & & $9 / 2$ & 28785.075 & 1.155 & & & $11 / 2$ & 30691.068 & 1.005 \\
\hline & & $11 / 2$ & 28860.164 & 1.110 & & & $13 / 2$ & 30700.780 & 1.155 \\
\hline & & $13 / 2$ & 28899.080 & 1.040 & & & $17 / 2$ & 30781.493 & 1.210 \\
\hline & & $9 / 2$ & 28926.250 & 0.890 & & & $5 / 2$ & 30795.119 & 1.046 \\
\hline & & $17 / 2$ & 29027.543 & 1.200 & & & $7 / 2$ & 30805.535 & 1.080 \\
\hline & & $7 / 2$ & 29036.650 & 1.045 & & & $13 / 2$ & 30813.108 & 1.137 \\
\hline \multirow{8}{*}{$4 f^{4}\left({ }^{5} I\right) 6 p$} & \multirow[t]{8}{*}{${ }^{4} \mathrm{H}^{\circ}$} & $9 / 2$ & 29043.459 & 0.981 & & & $9 / 2$ & 30818.335 & 1.255 \\
\hline & & $11 / 2$ & 29079.868 & 1.107 & & & $5 / 2$ & 30890.990 & 1.000 \\
\hline & & $13 / 2$ & 29088.724 & 1.126 & & & $7 / 2$ & 30893.730 & 1.005 \\
\hline & & $9 / 2$ & 29220.567 & 1.160 & & & $7 / 2$ & 30919.630 & 1.090 \\
\hline & & $11 / 2$ & 29239.381 & 1.119 & & & $9 / 2$ & 30927.645 & 1.165 \\
\hline & & $15 / 2$ & 29260.740 & 1.223 & & & $13 / 2$ & 30952.181 & 1.190 \\
\hline & & $9 / 2$ & 29298.632 & 0.900 & & & $9 / 2$ & 30990.485 & 1.095 \\
\hline & & $11 / 2$ & 29336.734 & 1.025 & & & $11 / 2$ & 30995.579 & 1.190 \\
\hline
\end{tabular}


Nd II, Odd Parity_Continued

\begin{tabular}{|c|c|c|c|c|c|c|c|c|c|}
\hline Configuration & Term & $J$ & $\begin{array}{l}\text { Level } \\
\left(\mathrm{cm}^{-1}\right)\end{array}$ & $g$ & Configuration & Term & $J$ & $\begin{array}{l}\text { Level } \\
\left(\mathrm{cm}^{-1}\right)\end{array}$ & $g$ \\
\hline & & $15 / 2$ & 31069.500 & 1.040 & & & $11 / 2$ & 32759.589 & 1.030 \\
\hline & & $11 / 2$ & 31085.770 & 1.055 & & & $9 / 2$ & 32798.423 & 1.200 \\
\hline & & $7 / 2$ & 31128.940 & 1.020 & & & $13 / 2$ & 32864.841 & 0.950 \\
\hline & & $9 / 2$ & 31179.755 & 1.000 & & & $15 / 2$ & 32905.325 & 1.080 \\
\hline & & $7 / 2$ & 31186.580 & 1.050 & & & $9 / 2$ & 32918.176 & 1.120 \\
\hline & & $13 / 2$ & 31256.788 & 1.135 & & & $3 / 2$ & 32964.210 & 1.140 \\
\hline & & $11 / 2$ & 31274.810 & 1.145 & & & $11 / 2$ & 33003.220 & 1.115 \\
\hline & & $9 / 2$ & 31335.807 & 1.125 & & & $17 / 2$ & 33097.724 & 1.230 \\
\hline & & $15 / 2$ & 31362.117 & 1.020 & & & $11 / 2$ & 33120.090 & 1.015 \\
\hline & & $13 / 2$ & 31405.086 & 1.120 & & & $11 / 2$ & 33192.940 & 1.050 \\
\hline & & $11 / 2$ & 31438.956 & 1.165 & & & $17 / 2$ & 33215.040 & 1.015 \\
\hline & & $9 / 2$ & 31451299 & 1.048 & & & $3 / 2$ & 33239.917 & 0.950 \\
\hline & & $15 / 2$ & 31489.109 & 1.110 & & & $13 / 2$ & 33260.386 & 1.245 \\
\hline & & $7 / 2$ & 31514.079 & 1.050 & & & $3 / 2$ & 33301.335 & 1.210 \\
\hline & & $11 / 2$ & 31528.633 & 1.000 & & & $7 / 2$ & 33313.870 & 1.200 \\
\hline & & $9 / 2$ & 31618213 & 1.250 & & & $13 / 2$ & 33452.835 & 1.065 \\
\hline & & $9 / 2$ & 31692.997 & 1.237 & & & $3 / 2$ & 33464.640 & 0.755 \\
\hline & & $17 / 2$ & 31818.517 & 1.180 & & & $1 / 2$ & 33520220 & 0.750 \\
\hline & & $9 / 2$ & 31834.827 & 1.089 & & & $17 / 2$ & 33526.500 & 1.110 \\
\hline & & $7 / 2$ & 31844.813 & 0.945 & & & $5 / 2$ & 33542.165 & 0.810 \\
\hline & & $13 / 2$ & 31857.296 & 1.180 & & & $13 / 2$ & 33556.696 & 1.015 \\
\hline & & $15 / 2$ & 31878.132 & 1.178 & & & $1 / 2$ & 33640.336 & 1.050 \\
\hline & & $11 / 2$ & 31900.596 & 1.185 & & & $11 / 2$ & 33719.088 & 1.110 \\
\hline & & $9 / 2$ & 31944.650 & 1.071 & & & $13 / 2$ & 33759.632 & 1.063 \\
\hline & & $9 / 2$ & 31993.640 & 0.957 & & & $11 / 2$ & 33792.430 & 1.210 \\
\hline & & $7 / 2$ & 32008.576 & 1.083 & & & $13 / 2$ & 33809.180 & 1.190 \\
\hline & & $15 / 2$ & 32093.646 & 1.130 & & & $1 / 2$ & 33820.330 & 0.750 \\
\hline & & $7 / 2$ & 32123.441 & 1.022 & & & $5 / 2$ & 33861.325 & 1.170 \\
\hline & & $11 / 2$ & 32144.445 & 1.190 & & & $17 / 2$ & 33884.238 & 1.210 \\
\hline & & $9 / 2$ & 32165.264 & 0.970 & & & $3 / 2$ & 33940.640 & 0.850 \\
\hline & & $9 / 2$ & 32192.051 & 1.060 & & & $13 / 2$ & 34001.428 & 1.090 \\
\hline & & $5 / 2$ & 32194.110 & 0.990 & & & $13 / 2$ & 34087.960 & 1.150 \\
\hline & & $5 / 2$ & 32211.650 & 1.135 & & & $17 / 2$ & 34119.145 & 1.165 \\
\hline & & $15 / 2$ & 32221.573 & 1.139 & & & $11 / 2$ & 34127.822 & 1.050 \\
\hline & & $9 / 2$ & 32253.730 & 0.985 & & & $3 / 2$ & 34133.460 & 1.295 \\
\hline & & $11 / 2$ & 32375.051 & 1.095 & & & $3 / 2$ & 34226.090 & 1.700 \\
\hline & & $7 / 2$ & 32394.087 & 1.240 & & & $11 / 2$ & 34227.182 & 1.160 \\
\hline & & $13 / 2$ & 32397.555 & 1.080 & & & $5 / 2$ & 34241.915 & 1.060 \\
\hline & & $15 / 2$ & 32423.943 & 1.030 & & & $1 / 2$ & 34323.300 & 0.450 \\
\hline & & $11 / 2$ & 32444.090 & 1.095 & & & $13 / 2$ & 34399.930 & 1.165 \\
\hline & & $9 / 2$ & 32482.730 & 1.160 & & & $3 / 2$ & 34515.860 & 1.095 \\
\hline & & $11 / 2$ & 32522.136 & 0.910 & & & $9 / 2$ & 34526.890 & 1.125 \\
\hline & & $11 / 2$ & 32650.760 & 1.110 & & & $15 / 2$ & 34555.732 & 1.238 \\
\hline & & $9 / 2$ & 32671.367 & 1.185 & & & $3 / 2$ & 34563.810 & 0.920 \\
\hline
\end{tabular}


Nd II, Odd Parity_Continued

Nd II, Odd Parity-Continued

\begin{tabular}{|c|c|c|c|c|c|c|c|c|c|}
\hline Configuration & Term & $J$ & $\begin{array}{l}\text { Level } \\
\left(\mathrm{cm}^{-1}\right)\end{array}$ & $g$ & Configuration & Term & $J$ & $\begin{array}{l}\text { Level } \\
\left(\mathrm{cm}^{-1}\right)\end{array}$ & $g$ \\
\hline & & $5 / 2$ & 34632230 & 1.150 & & & $7 / 2$ & 36393.740 & 0.970 \\
\hline & & $15 / 2$ & 34671.738 & 1.115 & & & $9 / 2$ & 36417.060 & 1.235 \\
\hline & & $13 / 2$ & 34689.408 & 1.085 & & & $1 / 2$ & 36434.470 & 1.300 \\
\hline & & $5 / 2$ & 34695.523 & 1.440 & & & $13 / 2$ & 36444.422 & 1.138 \\
\hline & & $7 / 2$ & 34709.105 & 1.095 & & & $11 / 2$ & 36512.740 & 1.015 \\
\hline & & $7 / 2$ & 34746.565 & 1.130 & & & $1 / 2$ & 36553.810 & 2.280 \\
\hline & & $7 / 2$ & 34817.220 & 0.835 & & & $7 / 2$ & 36571.480 & 1.070 \\
\hline & & $15 / 2$ & 34841.070 & 1.130 & & & $3 / 2$ & 36577.063 & 1.280 \\
\hline & & $1 / 2$ & 34856.400 & 0.935 & & & $7 / 2$ & 36621.025 & 1.160 \\
\hline & & $5 / 2$ & 34870.455 & 1.040 & & & $3 / 2$ & 36706.520 & 1.520 \\
\hline & & $15 / 2$ & 34895.820 & 1.175 & & & $9 / 2$ & 36707.400 & 1.205 \\
\hline & & $5 / 2$ & 34938.750 & 1.100 & & & $7 / 2$ & 36711.050 & 1.090 \\
\hline & & $7 / 2$ & 34982.943 & 1.170 & & & $13 / 2$ & 36728.888 & 1.085 \\
\hline & & $7 / 2$ & 35056.130 & 0.990 & & & $5 / 2$ & 36785.935 & 1.130 \\
\hline & & $7 / 2$ & 35094.035 & 1.085 & & & $5 / 2$ & 36814.090 & 1.215 \\
\hline & & $5 / 2$ & 35100.115 & 1.135 & & & $3 / 2$ & 36838.640 & 1.220 \\
\hline & & $3 / 2$ & 35124.315 & 0.885 & & & $9 / 2$ & 37041.760 & 1.100 \\
\hline & & $5 / 2$ & 35165.905 & 0.970 & & & $5 / 2$ & 37041.905 & 1.230 \\
\hline & & $9 / 2$ & 35169.610 & 1.175 & & & $11 / 2$ & 37128.550 & 1.300 \\
\hline & & $11 / 2$ & 35225210 & 1.240 & & & $13 / 2$ & 37149.107 & 0.988 \\
\hline & & $13 / 2$ & 35241.818 & 1.095 & & & $13 / 2$ & 37262.720 & 1.023 \\
\hline & & $7 / 2$ & 35280.020 & 1.060 & & & $13 / 2$ & 37294.270 & 1.135 \\
\hline & & $9 / 2$ & 35331.480 & 1.030 & & & $9 / 2$ & 37354290 & 1.135 \\
\hline & & $1 / 2$ & 35344.395 & 0.435 & & & $7 / 2$ & 37366.520 & 1.285 \\
\hline & & $13 / 2$ & 35403.450 & 1.175 & & & $5 / 2$ & 37406.260 & 1.045 \\
\hline & & $7 / 2$ & 35444.230 & 1.300 & & & $7 / 2$ & 37434.915 & 1.610 \\
\hline & & $3 / 2$ & 35447.030 & 1.125 & & & $11 / 2$ & 37485.698 & 1.330 \\
\hline & & $5 / 2$ & 35496.685 & 1.005 & & & $13 / 2$ & 37495.400 & 1.055 \\
\hline & & $9 / 2$ & 35606.691 & 1.035 & & & $5 / 2$ & 37635.835 & 0.985 \\
\hline & & $15 / 2$ & 35609.220 & 1.240 & & & $11 / 2$ & 37637.360 & 1.055 \\
\hline & & $7 / 2$ & 35651.980 & 0.940 & & & $13 / 2$ & 37640.530 & 1.035 \\
\hline & & $3 / 2$ & 35681.400 & 1.200 & & & $5 / 2$ & 37720.755 & 1.355 \\
\hline & & $3 / 2$ & 35734.450 & 1.435 & & & $7 / 2$ & 37722.765 & 1.285 \\
\hline & & $5 / 2$ & 35819.425 & 0.905 & & & $5 / 2$ & 37741260 & 0.800 \\
\hline & & $9 / 2$ & 35871.490 & 1.015 & & & $7 / 2$ & 37764.315 & 1.078 \\
\hline & & $7 / 2$ & 35882.880 & 1.138 & & & $9 / 2$ & 38152.895 & 1.195 \\
\hline & & $5 / 2$ & 35962.900 & 1.375 & & & $9 / 2$ & 38179.875 & 1.240 \\
\hline & & $9 / 2$ & 35985.780 & 1.070 & & & $11 / 2$ & 38253.770 & 1.100 \\
\hline & & $5 / 2$ & 35996.995 & 0.990 & & & $15 / 2$ & 38283.040 & 1.040 \\
\hline & & $9 / 2$ & 36035.510 & 1.155 & & & $13 / 2$ & 38297.145 & 0.955 \\
\hline & & $5 / 2$ & 36138.560 & 1.375 & & & $11 / 2$ & 38318.970 & 0.910 \\
\hline & & $15 / 2$ & 36169.872 & 1.165 & & & $13 / 2$ & 38429.465 & 1.330 \\
\hline & & $7 / 2$ & 36262.706 & 1.115 & & & $15 / 2$ & 38432.360 & 1.120 \\
\hline & & $5 / 2$ & 36384.680 & 1.210 & & & $11 / 2$ & 38484.820 & 1.170 \\
\hline & & & & & & & $11 / 2$ & 38787.450 & 1.085 \\
\hline
\end{tabular}


Nd II, Odd Parity—Continued

Nd II, Odd Parity_Continued

\begin{tabular}{|c|c|c|c|c|c|c|c|c|c|}
\hline Configuration & Term & $J$ & $\begin{array}{l}\text { Level } \\
\left(\mathrm{cm}^{-1}\right)\end{array}$ & $g$ & Configuration & Term & $J$ & $\begin{array}{l}\text { Level } \\
\left(\mathrm{cm}^{-1}\right)\end{array}$ & $g$ \\
\hline & & $15 / 2$ & 38790.275 & 1.045 & & & $9 / 2$ & 39603.150 & 1.220 \\
\hline & & $11 / 2$ & 38867.790 & 1.190 & & & $13 / 2$ & 39825.830 & 1.040 \\
\hline & & $13 / 2$ & 39057.860 & 1.033 & & & $15 / 2$ & 39858.695 & 1.004 \\
\hline & & $.15 / 2$ & 39172.735 & 0.968 & & & $15 / 2$ & 39953.440 & 1.007 \\
\hline & & $13 / 2$ & 39176.490 & 1.058 & & & $5 / 2$ & 40269.060 & 1.200 \\
\hline & & $1 / 2$ & 39290.645 & 1.450 & & & $1 / 2$ & 40508.920 & 1.340 \\
\hline & & $11 / 2$ & 39366.377 & 1.195 & & & $15 / 2$ & 40919.565 & 1.052 \\
\hline & & $15 / 2$ & 39464.210 & 1.140 & & & & & \\
\hline & & $13 / 2$ & 39574.030 & 1.010 & $\mathrm{Nd}$ III $\left({ }^{5} \mathrm{I}_{4}\right)$ & Limit & & 86500 & \\
\hline
\end{tabular}


(Ce 1 sequence; 58 electrons)

Ground state $\left(1 s^{2} 2 s^{2} 2 p^{6} 3 s^{2} 3 p^{6} 3 d^{10} 4 s^{2} 4 p^{6} 4 d^{10} 5 s^{2} 5 p^{6}\right) 4 f^{45} \mathbf{I}_{4}$

Ionization energy $178600 \pm 2400 \mathrm{~cm}^{-1}$

$22.1 \pm 0.3 \mathrm{eV}$

The levels and eigenvector percentages are from unpublished material furnished by Crosswhite, who is continuing her analysis of this spectrum. The line list for Nd III has about 9500 lines extending from $1810 \AA$ to $8715 \AA$. Transitions from upper $4 f^{3} 5 d$ levels to the $4 f^{45} \mathrm{I}$ ground-term levels account for all the lines now classified.

Sugar and Reader derived the ionization energy.

\section{References}

Crosswhite, H., unpublished material (1975, 1976). EL PT

Sugar, J., and Reader, J., J. Chem. Phys. 59, 2083 (1973). 1P

[January 1977]

\section{Nd III}

\begin{tabular}{|c|c|c|c|c|c|c|}
\hline Configuration & Term & $J$ & $\begin{array}{l}\text { Level } \\
\left(\mathrm{cm}^{-1}\right)\end{array}$ & \multicolumn{3}{|c|}{ Leading percentages } \\
\hline $4 f^{4}$ & ${ }^{5} \mathrm{I}$ & $\begin{array}{l}4 \\
5 \\
6 \\
7 \\
8\end{array}$ & $\begin{array}{r}0.0 \\
1137.8 \\
2387.6 \\
3714.9 \\
5093.3\end{array}$ & $\begin{array}{l}98 \\
99 \\
99 \\
99 \\
97\end{array}$ & & \\
\hline $4 f^{3}\left({ }^{4} \mathrm{I}^{0}\right) 5 d$ & ${ }^{5} \mathrm{~K}^{\circ}$ & $\begin{array}{l}5 \\
6 \\
7 \\
8 \\
9\end{array}$ & $\begin{array}{l}15262.2 \\
16938.1 \\
18656.3 \\
20410.9 \\
22197.0\end{array}$ & $\begin{array}{l}88 \\
94 \\
98 \\
98 \\
96\end{array}$ & & 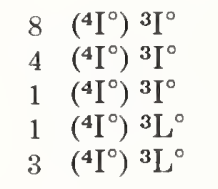 \\
\hline $4 f^{3}\left({ }^{4} \mathrm{I}^{\circ}\right) 5 d$ & ${ }^{5} \mathrm{I}^{\circ}$ & $\begin{array}{l}4 \\
5 \\
6 \\
7 \\
8\end{array}$ & $\begin{array}{l}18883.7 \\
20388.9 \\
22047.8 \\
22702.9 \\
24686.4\end{array}$ & $\begin{array}{l}83 \\
79 \\
71 \\
52 \\
83\end{array}$ & & 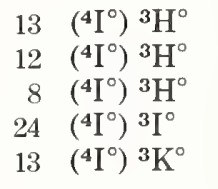 \\
\hline $4 f^{3}\left({ }^{4} I^{\circ}\right) 5 d$ & ${ }^{5} \mathrm{H}^{\circ}$ & $\begin{array}{l}3 \\
4 \\
5 \\
6 \\
7\end{array}$ & $\begin{array}{l}19211.0 \\
20144.3 \\
21886.8 \\
23819.3\end{array}$ & $\begin{array}{l}69 \\
55 \\
62 \\
45\end{array}$ & & 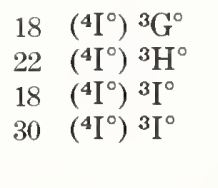 \\
\hline $4 f^{3}\left({ }^{4} \mathrm{I}^{\circ}\right) 5 d$ & & 7 & 240032 & 35 & $5 I^{\circ}$ & $27 \quad\left({ }^{4} I^{0}\right){ }^{3} I^{\circ}$ \\
\hline $4 f^{3}\left({ }^{4} \mathrm{I}^{\circ}\right) 5 d$ & & 6 & 265032 & 38 & ${ }^{5} \mathrm{G}^{\circ}$ & $32 \quad\left({ }^{4} I^{\circ}\right){ }^{3} \mathrm{H}^{\circ}$ \\
\hline $4 f^{3}\left({ }^{4} I^{\circ}\right) 5 d$ & ${ }^{3} \mathrm{~K}^{\circ}$ & 8 & 27391.4 & 67 & & $13 \quad\left({ }^{4} \mathrm{I}^{0}\right){ }^{5} \mathrm{I}^{\circ}$ \\
\hline $4 f^{3}\left({ }^{4} \mathrm{~F}^{\circ}\right) 5 d$ & & 3 & 27569.8 & 36 & ${ }^{5} \mathrm{H}^{\circ}$ & $15\left({ }^{4} \mathrm{~F}^{\circ}\right){ }^{5} \mathrm{D}^{\circ}$ \\
\hline $4 f^{3}\left({ }^{4} \mathrm{~F}^{\circ}\right) 5 d$ & ${ }^{5} \mathrm{H}^{\circ}$ & $\begin{array}{l}3 \\
4 \\
5 \\
6 \\
7\end{array}$ & $\begin{array}{l}27788.2 \\
28745.3 \\
30232.3 \\
31394.6 \\
32832.6\end{array}$ & $\begin{array}{l}49 \\
83 \\
58 \\
46 \\
44\end{array}$ & & $\begin{aligned} 15 & \left({ }^{4} \mathrm{~F}^{\circ}\right){ }^{5} \mathrm{D}^{\circ} \\
7 & \left({ }^{4} \mathrm{I}^{\circ}\right){ }^{5} \mathrm{H}^{\circ} \\
11 & \left({ }^{4} \mathrm{~F}^{\circ}\right){ }^{5} \mathrm{G}^{\circ} \\
18 & \left({ }^{4} \mathrm{~F}^{\circ}\right){ }^{5} \mathrm{G}^{\circ} \\
32 & \left({ }^{2} \mathrm{H}^{\circ} 2\right){ }^{3} \mathrm{~K}^{\circ}\end{aligned}$ \\
\hline $4 f^{3}\left({ }^{4} \mathrm{~F}^{\circ}\right) 5 d$ & & 5 & 29397.3 & 27 & ${ }^{5} \mathrm{H}^{\circ}$ & $17\left({ }^{2} \mathrm{H}^{\circ} 2\right){ }^{3} \mathrm{I}^{\circ}$ \\
\hline Nd IV $\left({ }^{4} I_{9 / 2}^{\circ}\right)$ & Limit & & 178600 & & & \\
\hline
\end{tabular}


[Nd IV ]

(La I sequence; 57 electrons)

$Z=60$

Ground state $\left(1 s^{2} 2 s^{2} 2 p^{6} 3 s^{2} 3 p^{6} 3 d^{10} 4 s^{2} 4 p^{6} 4 d^{10} 5 s^{2} 5 p^{6}\right) 4 f^{3}{ }^{4} \mathrm{I}_{9 / 2}^{\circ}$

Ionization energy $326000 \pm 3000 \mathrm{~cm}^{-1}$

$40.4 \pm 0.4 \mathrm{eV}$

No analysis of the spectrum of the free ion has been published. Irwin [1968] lists about 1000 of the strongest $\mathrm{Nd}$ lines he obtained using a spark source (1271-3109 $\AA$ ). A number of lines were classified as $4 f^{3}{ }^{4} \mathrm{I}^{\circ}-4 f^{2} 5 d, \quad 4 f^{2}\left({ }^{3} \mathrm{H}\right) 6 s-4 f^{2}\left({ }^{3} \mathrm{H}\right) 6 p$, or $4 f^{2}\left({ }^{3} \mathrm{H}\right) 5 d-4 f^{2}\left({ }^{3} \mathrm{H}\right) 6 p$ transitions in $\mathrm{Nd}$ IV, but no connection between any of the three resulting systems was found. We have not given the levels of these systems, pending confirmation by a more complete analysis.

The levels of the $4 f^{3}$ configuration listed here are based on analysis of the spectrum of triply ionized $\mathrm{Nd}$ in a $\mathrm{LaCl}_{3}$ host crystal [Crosswhite, Crosswhite, Kaseta, and Sarup, 1976]. Crosswhite et al. give the $4 f^{3}$ levels to almost $40000 \mathrm{~cm}^{-1}$; the list here is complete to 20000 $\mathrm{cm}^{-1}$, with the levels being adjusted to a value of zero for the ground level and rounded off to the nearest $10 \mathrm{~cm}^{-1}$. Dieke [1968] gives references for much of the work on the crystal spectra and analyses.

Crosswhite et al. calculated the eigenvector percentages.

The ionization energy is from Sugar and Reader, with the value rounded off to three significant figures and the estimated error doubled [Spector and Sugar, 1976].

\section{References}

Carnall, W. T., Fields, P. R., and Rajnak, K., J. Chem. Phys. 49, 4424 (1968). [EL] [CL] [W] PT

Crosswhite, H. M., Crosswhite, H., Kaseta, F. W., and Sarup, R., J. Chem. Phys. 64, 1981 (1976). [EL] [ND] [CL] [W] PT

Dieke, G. H., Spectra and Energy Levels of Rare Earth Ions in Crystals, Ed. H. M. Crosswhite and H. Crosswhite, pp. 202-232 (Interscience Publishers, New York, 1968). [EL] [CL] [W] [ZE]

Irwin, D. J. G., Thesis, Johns Hopkins Univ., Baltimore, 102 pp. (1968); (Univ. Microfilms, Ann Arbor, Mich., No. 6921084). EL CL W PT

Rajnak, K., J. Chem. Phys. 43, 847 (1965). [N D] PT

Spector, N., and Sugar, J., J. Opt. Soc. Am. 66, 436 (1976).

Sugar, J., and Reader, J., J. Chem. Phys. 59, 2083 (1973). IP

Wybourne, B. G., J. Chem. Phys. 34, 279 (1961). PT

[October 1976] 


\section{[Nd IV]}

\begin{tabular}{|c|c|c|c|c|c|c|}
\hline Configuration & Term & $J$ & $\begin{array}{l}\text { Level } \\
\left(\mathrm{cm}^{-1}\right)\end{array}$ & \multicolumn{3}{|c|}{ Leading percentages } \\
\hline $4 f^{3}$ & ${ }^{4} \mathrm{I}^{\circ}$ & $\begin{array}{c}9 / 2 \\
11 / 2 \\
13 / 2 \\
15 / 2\end{array}$ & $\begin{array}{c}0 \\
{[1880]} \\
{[3860]} \\
{[5910]}\end{array}$ & $\begin{array}{r}97 \\
99 \\
100 \\
99\end{array}$ & $\begin{array}{l}3 \\
1\end{array}$ & $\begin{array}{l}{ }^{2} \mathrm{H}^{\circ} 2 \\
{ }^{2} \mathrm{H}^{\circ} 2 \\
{ }^{2} \mathrm{~K}^{\circ}\end{array}$ \\
\hline $4 f^{3}$ & ${ }^{4} \mathrm{~F}^{\circ}$ & $\begin{array}{l}3 / 2 \\
5 / 2 \\
7 / 2 \\
9 / 2\end{array}$ & $\begin{array}{l}{[11290]} \\
{[12320]} \\
{[13280]} \\
{[145 \% 0]}\end{array}$ & $\begin{array}{l}94 \\
98 \\
93 \\
75\end{array}$ & $\begin{array}{r}5 \\
2 \\
4 \\
20\end{array}$ & $\begin{array}{l}{ }^{2} \mathrm{D}^{\circ} 1 \\
{ }^{2} \mathrm{D}^{\circ} 1 \\
{ }^{2} \mathrm{G}^{\circ} 1 \\
{ }^{2} \mathrm{H}^{\circ} 2\end{array}$ \\
\hline $4 f^{3}$ & ${ }^{2} \mathrm{H}^{\circ} 2$ & $\begin{array}{l}9 / 2 \\
11 / 2\end{array}$ & $\begin{array}{l}{[12470]} \\
{[15800]}\end{array}$ & $\begin{array}{l}54 \\
80\end{array}$ & $\begin{array}{l}14 \\
13\end{array}$ & $\begin{array}{l}{ }^{4} \mathrm{~F}^{\circ} \\
{ }^{2} \mathrm{H}^{\circ} 1\end{array}$ \\
\hline $4 f^{3}$ & ${ }^{4} \mathrm{~S}^{\circ}$ & $3 / 2$ & [13370] & 95 & 5 & ${ }^{2} \mathrm{P}^{\circ}$ \\
\hline $4 f^{3}$ & ${ }^{4} \mathrm{G}^{\circ}$ & $\begin{array}{l}5 / 2 \\
7 / 2 \\
9 / 2 \\
11 / 2\end{array}$ & $\begin{array}{l}{[16980]} \\
{[18890]} \\
{[19290]} \\
{[21280]}\end{array}$ & $\begin{array}{l}99 \\
59 \\
76 \\
92\end{array}$ & $\begin{array}{r}1 \\
24 \\
7 \\
4\end{array}$ & $\begin{array}{l}{ }^{2} \mathrm{~F}^{\circ} 2 \\
{ }^{2} \mathrm{G}^{\circ} 1 \\
{ }^{2} \mathrm{G}^{\circ} 1 \\
{ }^{2} \mathrm{H}^{\circ} 1\end{array}$ \\
\hline $4 f^{3}$ & & $7 / 2$ & {$[17100]$} & $41{ }^{4} \mathrm{G}^{\circ}$ & 31 & ${ }^{2} G^{\circ} 1$ \\
\hline $4 f^{3}$ & ${ }^{2} \mathrm{~K}^{\circ}$ & $\begin{array}{l}13 / 2 \\
15 / 2\end{array}$ & $\begin{array}{l}{[19440]} \\
{[21430]}\end{array}$ & $\begin{array}{l}99 \\
94\end{array}$ & $\begin{array}{l}1 \\
5\end{array}$ & $\begin{array}{l}{ }^{2} \mathrm{I}^{\circ} \\
{ }^{2} \mathrm{~L}^{\circ}\end{array}$ \\
\hline $\mathrm{Nd} \mathrm{V}\left({ }^{3} \mathrm{H}_{4}\right)$ & Limit & & 326000 & & & \\
\hline
\end{tabular}




\section{PROMETHIUM}

\section{Pm I}

Ground state $\left(1 s^{2} 2 s^{2} 2 p^{6} 3 s^{2} 3 p^{6} 3 d^{10} 4 s^{2} 4 p^{6} 4 d^{10} 5 s^{2} 5 p^{6}\right) 4 f^{5} 6 s^{2}{ }^{6} \mathrm{H}_{5 / 2}^{\circ}$

All known isotopes of Pm have relatively short half-lives, and the element first became available after Marinsky, Glendenin, and Coryell [1947] separated and identified two of its isotopes from other fission products of uranium [Marinsky and Glendenin, 1948]. The various observations of Pm spectra have been carried out with sources containing the ${ }^{147} \mathrm{Pm}$ isotope, which is produced in nuclear reactors and has a half-life of 2.6 years.

In 1951 Meggers, Scribner, and Bozman published wavelengths and estimated relative intensities for more than $2200 \mathrm{Pm}$ lines excited in arc and spark sources (2337-6873 $\AA$ ). An experimental separation of the lines according to ionization stage was not feasible with these sources, but the authors thought it probable that most of the lines belonged to Pm II. They pointed out the futility of attempting an analysis until more complete and accurate data could be obtained.

In 1967 Reader and Davis published "the first results of our investigation to determine the electronic structure of neutral promethium," noting that "our experimental investigation of the spectrum of promethium has extended over several years, and has included the taking of hundreds of spectrograms on several instruments, under many different conditions." This first analysis of Pm I yielded the 2 low $4 f^{5} 6 s^{2}$ terms and 209 upper even levels classifying 714 lines. No more complete list of Pm I lines has yet been published.

The levels and all except two of the $g$ values given here are from Reader and Davis. The even level at $27083.92 \mathrm{~cm}^{-1}(J=15 / 2)$ was given incorrectly as $27383.92 \mathrm{~cm}^{-1}$ in their 1967 paper. The estimated uncertainty of the low odd levels is $\pm 0.01 \mathrm{~cm}^{-1}$, and the even levels are accurate to about $\pm 0.03 \mathrm{~cm}^{-1}$.

The $g$ values quoted for the $4 f^{5} 6 s^{2}{ }^{6} \mathrm{H}_{7 / 2}^{\circ}$ and ${ }^{6} \mathrm{H}_{9 / 2}^{\circ}$ levels were obtained from atomic-beam magnetic-resonance measurements by Budick and Marrus [1963] and by Cabezas, Lindgren, and Marrus [1961], respectively. The respective uncertainties are 0.0004 and 0.004 . Cabezas, Lindgren, and Marrus obtained $J$ and $g$ values for both the ${ }^{6} \mathrm{H}_{7 / 2}^{\circ}$ and ${ }^{6} \mathrm{H}_{9 / 2}^{\circ}$ levels, and were able to conclude that the ground level was almost certainly $4 f^{5} 6 s^{2}{ }^{6} \mathrm{H}_{5 / 2}^{\circ}$.

The eigenvector percentages for the levels of the two low $4 f^{5} 6 s^{2}$ terms are from a calculation by Conway [1965]. He essentially repeated the calculation by Conway and Wybourne [1963], whose published results included eigenvectors only for levels below 5000 $\mathrm{cm}^{-1}$. (In table III of Conway and Wybourne, the last of the four ${ }^{4} \mathrm{G}^{\circ}$ components in the eigenvector for the $4 f^{5} 6 s^{2}{ }^{6} \mathrm{H}_{7 / 2}^{\circ}$ level should have seniority 3 ; and the four ${ }^{4} \mathrm{~F}^{\circ}$ components listed for the ${ }^{6} \mathrm{H}_{11 / 2}^{\circ}$ level are ${ }^{4} \mathrm{G}^{\circ}$ components, in the same order as for the lower ${ }^{6} \mathrm{H}^{\circ}$ levels.) Judd, Crosswhite, and Crosswhite [1968] made an improved calculation of Pm I $4 f^{5} 6 s^{2}$, based partially on the experimental levels of the two low terms. They included spin-spin and spinother-orbit interactions, as well as the Trees $\alpha$ parameter. The resulting values of the main (electrostatic and spin-orbit) parameters are significantly larger than the values assumed by Conway and Wybourne.

Reader and Davis [1967] note that most of the upper levels found by them must belong mainly to $4 f^{5} 6 s 6 p$ or $4 f^{4} 5 d 6 s^{2}$, and conclude on the basis of certain arguments that the latter configuration is preponderant.

The ionization energy is from Reader and Sugar. 


\section{References}

Budick, B., and Marrus, R., Phys. Rev. 132, 723 (1963). ZE Hfs

Cabezas, A. Y., Lindgren, I., and Marrus, R., Phys. Rev. 122, 1796 (1961). ZE Hfs

Conway, J. G., unpublished material (1965). PT

Conway, J. G., and Wybourne, B. G., Phys. Rev. 130, 2325 (1963). PT

Judd, B. R., Crosswhite, H. M., and Crosswhite, H., Phys. Rev. 169, 130 (1968). PT

Klinkenberg, P. F. A., and Tomkins, F. S., Physica (Utrecht) 26, 103 (1960). Hfs

Marinsky, J. A., and Glendenin, L. E., Chem. Eng. News 26, 2346 (1948).

Marinsky, J. A., Glendenin, L. E., and Coryell, C. D., J. Am. Chem. Soc. 69, 2781 (1947).

Meggers, W. F., Scribner, B. F., and Bozman, W. R., J. Res. Nat. Bur. Stand. (U.S.) 46, 85 (1951). W

Reader, J., and Davis, S. P., J. Opt. Soc. Am. 53, 431 (1963). Hfs

Reader, J., and Davis, S. P., J. Res. Nat. Bur. Stand. (U.S.) 71A, 587 (1967). EL CL ZE

Reader, J., and Davis, S. P., unpublished material (1974). EL

Reader, J., and Sugar, J., J. Opt. Soc. Am. 56, 1189 (1966). IP

[October 1976]

Pm I

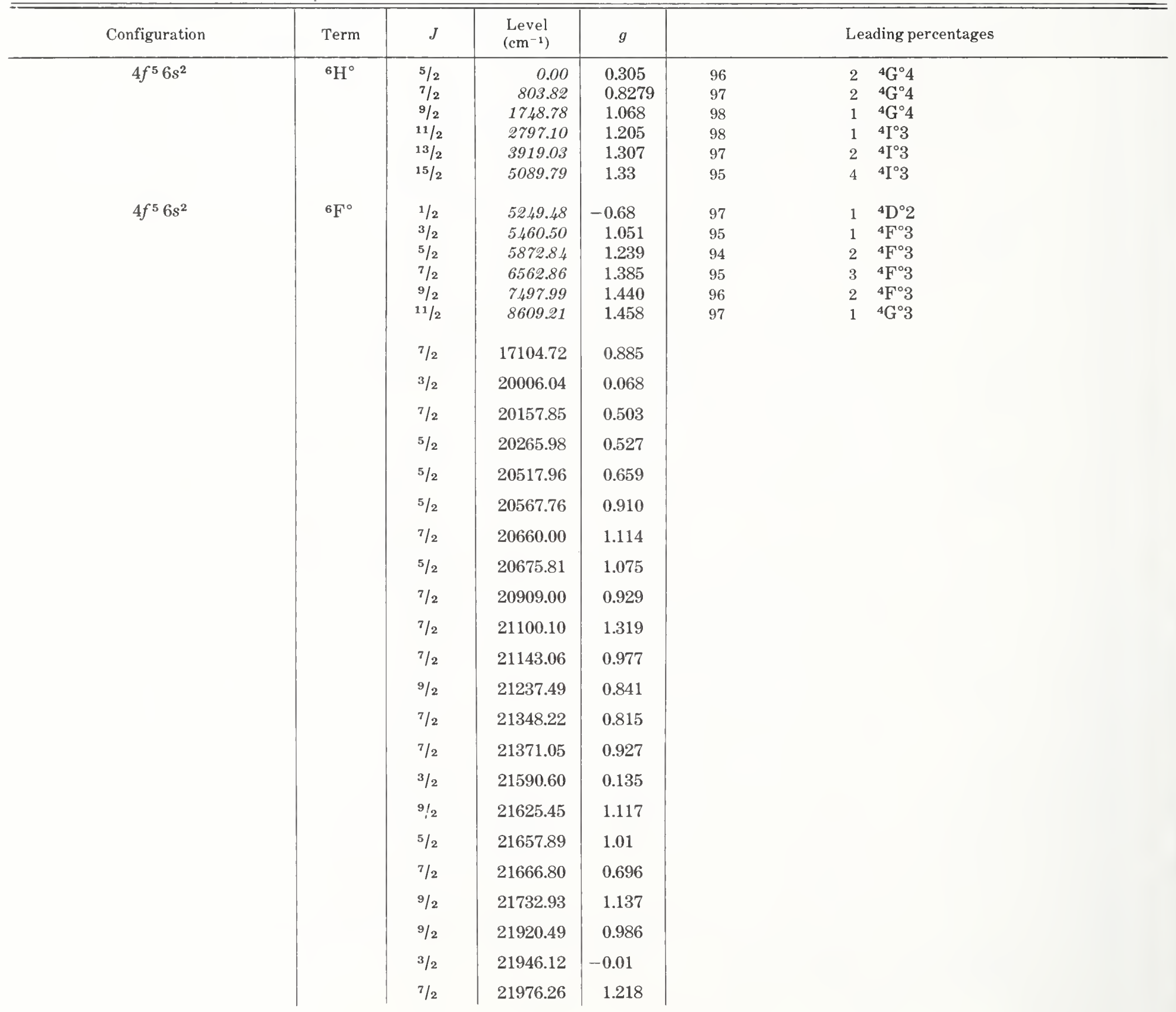


Pm I-Continued

\begin{tabular}{|c|c|c|c|c|c|c|c|c|c|}
\hline Configuration & Term & $J$ & $\begin{array}{l}\text { Level } \\
\left(\mathrm{cm}^{-1}\right)\end{array}$ & $g$ & Configuration & Term & $J$ & $\begin{array}{l}\text { Level } \\
\left(\mathrm{cm}^{-1}\right)\end{array}$ & $g$ \\
\hline & & $3 / 2$ & 22013.40 & 0.887 & & & $7 / 2$ & 23743.96 & 1.111 \\
\hline & & $5 / 2$ & 22080.08 & 0.571 & & & $11 / 2$ & 23760.57 & \\
\hline & & $7 / 2$ & 22084.65 & 0.858 & & & $3 / 2$ & 23926.91 & 1.754 \\
\hline & & $9 / 2$ & 22205.44 & 0.974 & & & $13 / 2$ & 23938.76 & 1.044 \\
\hline & & $1 / 2$ & 22259.21 & -0.32 & & & $13 / 2$ & 24013.29 & 1.29 \\
\hline & & $9 / 2$ & 22294.96 & 1.245 & & & $11 / 2$ & 24038.82 & 1.08 \\
\hline & & $5 / 2$ & 22301.24 & 0.976 & & & $9 / 2$ & 24071.03 & 1.12 \\
\hline & & $7 / 2$ & 22309.94 & 0.850 & & & $3 / 2$ & 24091.39 & 1.395 \\
\hline & & $9 / 2$ & 22355.68 & 1.374 & & & $13 / 2$ & 24122.41 & \\
\hline & & $3 / 2$ & 22388.06 & 0.84 & & & $13 / 2$ & 24180.80 & 1.28 \\
\hline & & $11 / 2$ & 22414.17 & 1.12 & & & $7 / 2$ & 24204.37 & 0.670 \\
\hline & & $5 / 2$ & 22425.58 & 0.83 & & & $9 / 2$ & 24234.42 & \\
\hline & & $11 / 2$ & 22446.20 & 1.531 & & & $13 / 2$ & 24245.66 & 1.14 \\
\hline & & $9 / 2$ & 22456.72 & 0.936 & & & $9 / 2$ & 24338.33 & 1.17 \\
\hline & & $5 / 2$ & 22522.90 & 0.735 & & & $7 / 2$ & 24418.44 & 1.124 \\
\hline & & $9 / 2$ & 22586.77 & 1.283 & & & $13 / 2$ & 24443.15 & 1.17 \\
\hline & & $7 / 2$ & 22654.34 & 0.84 & & & $9 / 2$ & 24443.57 & 1.037 \\
\hline & & $5 / 2$ & 22656.68 & 0.936 & & & $7 / 2$ & 24471.10 & 0.83 \\
\hline & & $11 / 2$ & 22761.33 & 1.296 & & & $11 / 2$ & 24503.45 & 1.22 \\
\hline & & $11 / 2$ & 22817.13 & 1.134 & & & $13 / 2$ & 24520.23 & 1.34 \\
\hline & & $5 / 2$ & 22905.24 & 1.16 & & & $11 / 2$ & 24533.27 & 1.183 \\
\hline & & $7 / 2$ & 22934.70 & 1.237 & & & $9 / 2$ & 24558.56 & 1.39 \\
\hline & & $11 / 2$ & 23006.35 & & & & $9 / 2$ & 24627.53 & 0.961 \\
\hline & & $11 / 2$ & 23033.95 & 1.0 & & & $11 / 2$ & 24681.68 & 0.895 \\
\hline & & $7 / 2$ & 23178.13 & 1.150 & & & $15 / 2$ & 24705.25 & 1.102 \\
\hline & & $5 / 2$ & 23188.54 & 1.48 & & & $9 / 2$ & 24754.58 & 0.888 \\
\hline & & $11 / 2$ & 23198.33 & 1.12 & & & $13 / 2$ & 24770.04 & 1.17 \\
\hline & & $9 / 2$ & 23276.10 & 0.83 & & & $9 / 2$ & 24789.86 & 1.08 \\
\hline & & $7 / 2$ & 23278.90 & 1.0 & & & $11 / 2$ & 24884.90 & \\
\hline & & $5 / 2$ & 23334.10 & 0.571 & & & $11 / 2$ & 24912.34 & 1.29 \\
\hline & & $7 / 2$ & 23337.53 & 1.257 & & & $11 / 2$ & 25104.27 & 1.15 \\
\hline & & $11 / 2$ & 23345.07 & 1.323 & & & $15 / 2$ & 25306.07 & 1.21 \\
\hline & & $9 / 2$ & 23435.40 & 1.00 & & & $3 / 2$ & 25351.46 & 0.58 \\
\hline & & $5 / 2$ & 23443.79 & 0.784 & & & $11 / 2$ & 25357.24 & \\
\hline & & $9 / 2$ & 23480.63 & 1.16 & & & $11 / 2$ & 25402.61 & 1.211 \\
\hline & & $11 / 2$ & 23501.57 & 1.283 & & & $13 / 2$ & 25405.29 & 1.034 \\
\hline & & $5 / 2$ & 23538.86 & 0.780 & & & $9 / 2$ & 25448.28 & 1.14 \\
\hline & & $11 / 2$ & 23550.60 & 1.170 & & & $15 / 2$ & 25474.46 & \\
\hline & & $9 / 2$ & 23571.27 & 1.26 & & & $9 / 2$ & 25521.55 & 0.910 \\
\hline & & $7 / 2$ & 23584.31 & 1.123 & & & $13 / 2$ & 25537.36 & 1.39 \\
\hline & & $13 / 2$ & 23629.06 & 1.09 & & & $15 / 2$ & 25618.77 & 1.13 \\
\hline & & $7 / 2$ & 23712.56 & 1.181 & & & $11 / 2$ & 25755.17 & 1.016 \\
\hline & & $1 / 2$ & 23732.57 & 3.24 & & & $13 / 2$ & 25919.50 & 1.26 \\
\hline & & $13 / 2$ & 23740.42 & & & & $11 / 2$ & 26015.94 & \\
\hline
\end{tabular}


Pm I-Continued

Pm I-Continued

\begin{tabular}{|c|c|c|c|c|c|c|c|c|c|}
\hline Configuration & Term & $J$ & $\begin{array}{l}\text { Level } \\
\left(\mathrm{cm}^{-1}\right)\end{array}$ & $g$ & Configuration & Term & $J$ & $\begin{array}{l}\text { Level } \\
\left(\mathrm{cm}^{-1}\right)\end{array}$ & $g$ \\
\hline & & $1 / 2$ & 26065.63 & -0.36 & & & $3 / 2$ & 27919.29 & 1.31 \\
\hline & & $17 / 2$ & 26080.99 & 1.29 & & & $5 / 2$ & 27923.37 & 0.869 \\
\hline & & $17 / 2$ & 26096.75 & 1.25 & & & $9 / 2$ & 27939.87 & 1.025 \\
\hline & & $13 / 2$ & 26101.28 & & & & $7 / 2$ & 28008.09 & \\
\hline & & $11 / 2$ & 26103.56 & 1.2 & & & $13 / 2$ & 28030.99 & 1.39 \\
\hline & & $13 / 2$ & 26181.98 & 1.55 & & & $9 / 2$ & 28075.94 & 1.155 \\
\hline & & $7 / 2$ & 26211.44 & 0.715 & & & $5 / 2$ & 28084.28 & 0.904 \\
\hline & & $9 / 2$ & 26237.84 & 1.43 & & & $11 / 2$ & 28086.21 & 1.150 \\
\hline & & $13 / 2$ & 26282.20 & 0.95 & & & $7 / 2$ & 28150.73 & 1.071 \\
\hline & & $7 / 2$ & 26285.02 & 0.877 & & & $5 / 2$ & 28153.69 & 0.632 \\
\hline & & $1 / 2$ & 26300.30 & 0.955 & & & $7 / 2$ & 28169.71 & 0.9 \\
\hline & & $13 / 2$ & 26456.26 & & & & $7 / 2$ & 28186.31 & 1.98 \\
\hline & & $1 / 2$ & 26468.80 & -0.45 & & & $1 / 2$ & 28196.56 & 2.44 \\
\hline & & $3 / 2$ & 26479.61 & 0.731 & & & $7 / 2$ & 28273.52 & 0.764 \\
\hline & & $3 / 2$ & 26522.35 & 1.135 & & & $9 / 2$ & 28274.21 & 1.055 \\
\hline & & $17 / 2$ & 26545.85 & 1.14 & & & $7 / 2$ & 28325.13 & 1.38 \\
\hline & & $13 / 2$ & 26555.44 & 1.07 & & & $5 / 2$ & 28338.98 & 0.963 \\
\hline & & $13 / 2$ & 26591.40 & 1.10 & & & $7 / 2$ & 28467.52 & 0.87 \\
\hline & & $5 / 2$ & 26609.39 & 0.56 & & & $9 / 2$ & 28490.35 & 1.123 \\
\hline & & $5 / 2$ & 26630.56 & 0.32 & & & $9 / 2$ & 28565.66 & 1.0 \\
\hline & & $11 / 2$ & 26694.38 & 1.095 & & & $9 / 2$ & 28607.33 & 1.079 \\
\hline & & $5 / 2$ & 26695.79 & & & & $3 / 2$ & 28608.57 & 1.740 \\
\hline & & $13 / 2$ & 26703.97 & 1.30 & & & $1 / 2$ & 28657.02 & 0.29 \\
\hline & & $3 / 2$ & 26725.52 & 0.65 & & & $7 / 2$ & 28680.26 & 0.88 \\
\hline & & $3 / 2$ & 26830.74 & 0.794 & & & $11 / 2$ & 28994.90 & 1.30 \\
\hline & & $5 / 2$ & 26841.36 & 1.38 & & & $3 / 2$ & 29002.94 & 0.99 \\
\hline & & $15 / 2$ & 26955.22 & & & & $9 / 2$ & 29074.03 & \\
\hline & & $5 / 2$ & 27036.66 & 0.931 & & & $9 / 2$ & 29129.60 & 1.162 \\
\hline & & $15 / 2$ & 27042.18 & & & & $9 / 2$ & 29161.96 & 1.0 \\
\hline & & $15 / 2$ & 27083.92 & & & & $11 / 2$ & 29242.64 & 1.259 \\
\hline & & $15 / 2$ & 27109.75 & & & & $11 / 2$ & 29585.21 & 1.2 \\
\hline & & $5 / 2$ & 27245.99 & 0.761 & & & $9 / 2$ & 29595.58 & 1.04 \\
\hline & & $7 / 2,9 / 2$ & 27272.46 & 0.8 & & & $9 / 2$ & 29648.42 & \\
\hline & & $15 / 2$ & 27304.15 & 1.44 & & & $11 / 2$ & 29705.77 & \\
\hline & & $7 / 2$ & 27319.28 & 1.274 & & & $11 / 2$ & 29757.69 & \\
\hline & & $5 / 2$ & 27334.48 & 0.947 & & & $9 / 2$ & 29784.08 & \\
\hline & & $7 / 2$ & 27351.42 & 0.92 & & & $11 / 2$ & 29856.72 & 1.10 \\
\hline & & $3 / 2$ & 27468.45 & 0.21 & & & $7 / 2$ & 29883.87 & 1.199 \\
\hline & & $7 / 2$ & 27476.28 & 1.077 & & & $9 / 2$ & 29908.90 & \\
\hline & & $5 / 2$ & 27512.95 & 0.913 & & & $11 / 2$ & 29960.42 & 1.18 \\
\hline & & $13 / 2$ & 27596.27 & 1.29 & & & $11 / 2$ & 30008.40 & 1.28 \\
\hline & & $7 / 2$ & 27621.74 & & & & $9 / 2$ & 30063.62 & 1.224 \\
\hline & & $3 / 2$ & 27685.89 & 2.049 & & & $11 / 2,{ }^{13} / 2$ & 30251.50 & 1.2 \\
\hline & & $9 / 2$ & 27829.89 & 1.024 & & & $13 / 2$ & 30281.98 & \\
\hline
\end{tabular}


Pm I-Continued

Pm I-Continued

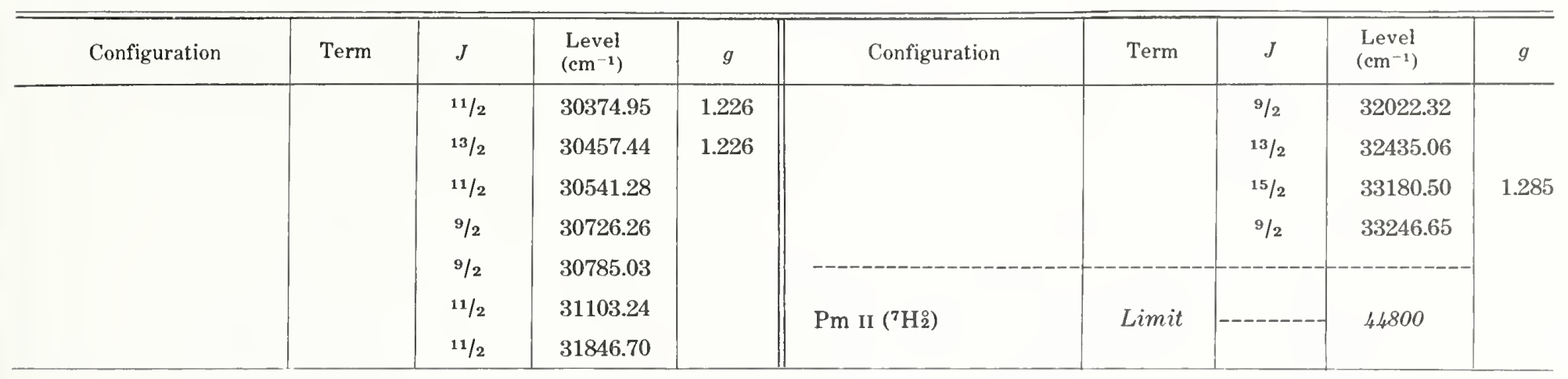


Ground state $\left(1 s^{2} 2 s^{2} 2 p^{6} 3 s^{2} 3 p^{6} 3 d^{10} 4 s^{2} 4 p^{6} 4 d^{10} 5 s^{2} 5 p^{6}\right) 4 f^{5} 6 s^{7} \mathrm{H}_{2}^{\circ}$

Ionization energy $87900 \pm 600 \mathrm{~cm}^{-1}$

$10.90 \pm 0.08 \mathrm{eV}$

Davis and Reader have reobserved the Pm spectrum and "have prepared a line list with about 17500 entries from 3100 to $10000 \AA$, including lines from both neutral and singly ionized species. Most of the lines are not clearly separated according to ionization state" [1972a]. Some 2000 lines have been classified by energy levels, but the new line list has not been published. The 1951 list of Meggers, Scribner, and Bozman (described under Pm I) thus remains the most complete published table of wavelengths for Pm II. (Davis and Reader have confirmed that practically all of the strong lines in this list belong to Pm II.)

The levels, $g$ values, and eigenvector percentages are from the unpublished and as yet incomplete analysis by Davis and Reader [1972b]. The calculation of the $4 f^{5} 6 s$ levels was carried out with matrices that included off-diagonal spin-orbit elements only between (quintet and septet) states having the same $4 f^{5}$ sextet parent term. The resulting purity (leading percentage) is given for each level in both the $L S$ and $J_{1} s$ coupling schemes. Although these levels have sometimes been assigned $J_{1} s$ designations, it is apparent that $L S$ coupling is more appropriate.

No attempt has yet been made to interpret the upper even levels, about 150 of which have been found.

The ionization energy is from Sugar and Reader.

\section{References}

Davis, S. P., and Reader, J., J. Opt. Soc. Am. 62, 1370A (1972a). EL

Davis, S. P., and Reader, J., unpublished material (1972b). EL CL W ZE PT

Klinkenberg, P. F. A., and Tomkins, F. S., Physica (Utrecht) 26, 103 (1960). Hfs

Meggers, W. F., Scribner, B. F., and Bozman, W. R., J. Res. Nat. Bur. Stand. (U.S.) 46, 85 (1951). W

Reader, J., Phys. Rev. 141, 1123 (1966). Hfs

Reader, J., and Davis, S. P., J. Opt. Soc. Am. 53, 431 (1963). Hfs

Sugar, J., and Reader, J., J. Opt. Soc. Am. 55, 1286 (1965). IP

[October 1976]

Pm II

\begin{tabular}{|c|c|c|c|c|c|c|c|c|}
\hline Configuration & Term & $J$ & $\begin{array}{l}\text { Level } \\
\left(\mathrm{cm}^{-1}\right)\end{array}$ & $g$ & \multicolumn{4}{|c|}{ Leading percentages } \\
\hline $4 f^{5}\left({ }^{6} \mathrm{H}^{\circ}\right) 6 s$ & ${ }^{7} \mathrm{H}^{\circ}$ & $\begin{array}{l}2 \\
3 \\
4 \\
5 \\
6 \\
7 \\
8\end{array}$ & $\begin{array}{r}0.00 \\
446.45 \\
1133.45 \\
1983.52 \\
2950.31 \\
4000.15\end{array}$ & $\begin{array}{l}0.000 \\
0.728 \\
1.038 \\
1.190 \\
1.29 \\
1.34\end{array}$ & $\begin{array}{r}100 \\
89 \\
89 \\
92 \\
95 \\
97 \\
100\end{array}$ & $\begin{array}{l}\text { or } \\
\text { or } \\
\text { or } \\
\text { or } \\
\text { or } \\
\text { or } \\
\text { or }\end{array}$ & $\begin{array}{r}100 \\
62 \\
79 \\
88 \\
93 \\
97 \\
100\end{array}$ & $\begin{array}{l}\left({ }^{6} \mathrm{H}_{5 / 2}^{\circ}\right)(5 / 2,1 / 2)^{\circ} \\
\left({ }^{6} \mathrm{H}_{5 / 2}^{\circ}\right)(5 / 2,1 / 2)^{\circ} \\
\left({ }^{6} \mathrm{H}_{7 / 2}^{\circ}\right)\left({ }^{7} / 2,{ }^{1} / 2\right)^{\circ} \\
\left({ }^{6} \mathrm{H}_{9 / 2}^{\circ}\right)(9 / 2,1 / 2)^{\circ} \\
\left({ }^{6} \mathrm{H}_{11 / 2}^{\circ}\right)\left({ }^{11} / 2,{ }^{1} / 2\right)^{\circ} \\
\left({ }^{6} \mathrm{H}_{13 / 2}^{\circ}\right)(13 / 2,1 / 2)^{\circ} \\
\left({ }^{6} \mathrm{H}^{\circ} 5 / 2\right)\left({ }^{15} / 2,1 / 2\right)^{\circ}\end{array}$ \\
\hline $4 f^{5}\left({ }^{6} \mathrm{H}^{\circ}\right) 6 s$ & ${ }^{5} \mathrm{H}^{\circ}$ & $\begin{array}{l}3 \\
4 \\
5 \\
6 \\
7\end{array}$ & $\begin{array}{l}1602.97 \\
2666.76 \\
3812.31 \\
5017.77\end{array}$ & $\begin{array}{l}0.531 \\
0.920 \\
1.10 \\
1.20\end{array}$ & $\begin{array}{l}89 \\
89 \\
92 \\
95 \\
97\end{array}$ & $\begin{array}{l}\text { or } \\
\text { or } \\
\text { or } \\
\text { or } \\
\text { or }\end{array}$ & $\begin{array}{l}62 \\
79 \\
88 \\
93 \\
97\end{array}$ & $\begin{array}{l}\left({ }^{6} \mathrm{H}_{7 / 2}^{\circ}\right)\left({ }^{7} / 2,1 / 2\right)^{\circ} \\
\left({ }^{6} \mathrm{H}_{9 / 2}^{\circ}\right)\left({ }^{9} / 2,1 / 2\right)^{\circ} \\
\left({ }^{6} \mathrm{H}_{11 / 2}^{\circ}\right)\left({ }^{11} / 2,{ }^{1} / 2\right)^{\circ} \\
\left({ }^{6} \mathrm{H}_{13 / 2}^{\circ}\right)\left({ }^{13} / 2,{ }^{1} / 2\right)^{\circ} \\
\left({ }^{6} \mathrm{H}_{15 / 2}^{\circ}\right)\left({ }^{\circ} / 2,2, / 2\right)^{\circ}\end{array}$ \\
\hline
\end{tabular}


Pm II-Continued

\begin{tabular}{|c|c|c|c|c|c|c|c|c|}
\hline Configuration & Term & $J$ & $\begin{array}{l}\text { Level } \\
\left(\mathrm{cm}^{-1}\right)\end{array}$ & $g$ & \multicolumn{4}{|c|}{ Leading percentages } \\
\hline $4 f^{5}\left({ }^{6} \mathrm{~F}^{\circ}\right) 6 s$ & ${ }^{7} \mathrm{~F}^{\circ}$ & $\begin{array}{l}0 \\
1 \\
2 \\
3 \\
4 \\
5 \\
6\end{array}$ & $\begin{array}{l}5280.88 \\
5391.46 \\
5632.40 \\
6048.50 \\
6705.18\end{array}$ & $\begin{array}{l}1.493 \\
1.481 \\
1.482 \\
1.50\end{array}$ & $\begin{array}{r}100 \\
99 \\
98 \\
96 \\
96 \\
98 \\
100\end{array}$ & $\begin{array}{l}\text { or } \\
\text { or } \\
\text { or } \\
\text { or } \\
\text { or } \\
\text { or } \\
\text { or }\end{array}$ & $\begin{array}{r}100 \\
53 \\
74 \\
87 \\
94 \\
97 \\
100\end{array}$ & $\begin{array}{l}\left({ }^{6} \mathrm{~F}_{1 / 2}^{\circ}\right)\left(1 / 2,{ }^{1} / 2\right)^{\circ} \\
\left({ }^{6} \mathrm{~F}_{1 / 2}^{\circ}\right)\left(1 / 2,{ }^{1} / 2\right)^{\circ} \\
\left({ }^{6} \mathrm{~F}_{3 / 2}^{\circ}\right)\left(3 / 2,{ }^{1} / 2\right)^{\circ} \\
\left({ }^{6} \mathrm{~F}_{5 / 2}^{\circ}\right)\left({ }^{5} / 2,{ }^{1} / 2\right)^{\circ} \\
\left({ }^{6} \mathrm{~F}_{7 / 2}^{\circ}\right)\left({ }^{7} / 2,{ }^{1} / 2\right)^{\circ} \\
\left({ }^{6} \mathrm{~F}_{9 / 2}^{\circ}\right)(9 / 2,1 / 2)^{\circ} \\
\left({ }^{6} \mathrm{~F}_{11 / 2}^{\circ}\right)\left({ }^{11} / 2,{ }_{1} / 2\right)^{\circ}\end{array}$ \\
\hline $4 f^{5}\left({ }^{6} \mathrm{H}^{\circ}\right) 5 d$ & ${ }^{7} \mathrm{~K}^{\circ}$ & $\begin{array}{c}4 \\
5 \\
6 \\
7 \\
8 \\
9 \\
10\end{array}$ & 5332.36 & 0.412 & & & & \\
\hline $4 f^{5}\left({ }^{6} \mathrm{~F}^{\circ}\right) 6 s$ & ${ }^{5} \mathrm{~F}^{\circ}$ & $\begin{array}{l}1 \\
2 \\
3 \\
4 \\
5\end{array}$ & $\begin{array}{l}6629.40 \\
7012.87 \\
7701.09\end{array}$ & $\begin{array}{l}0.00 \\
1.010 \\
1.23\end{array}$ & $\begin{array}{l}99 \\
98 \\
96 \\
96 \\
98\end{array}$ & $\begin{array}{l}\text { or } \\
\text { or } \\
\text { or } \\
\text { or } \\
\text { or }\end{array}$ & $\begin{array}{l}53 \\
74 \\
87 \\
94 \\
97\end{array}$ & $\begin{array}{l}\left({ }^{6} F_{3 / 2}^{\circ}\right)\left(3 / 2,{ }^{1} / 2\right)^{\circ} \\
\left({ }^{6} F_{5 / 2}^{\circ}\right)(5 / 2,1 / 2)^{\circ} \\
\left({ }^{6} F_{7 / 2}^{\circ}\right)\left({ }^{7} / 2,{ }^{1} / 2\right)^{\circ} \\
\left({ }^{6} F_{9 / 2}^{\circ}\right)\left(9 / 2,{ }^{1} / 2\right)^{\circ} \\
\left({ }^{6} F_{11 / 2}^{\circ}\right)\left(11 / 2,{ }^{\circ} / 2\right)^{\circ}\end{array}$ \\
\hline & & 3 & 15543.14 & & & & & \\
\hline & & 2 & 15968.09 & 0.365 & & & & \\
\hline & & 5 & 17561.95 & & & & & \\
\hline & & 5 & 17609.00 & & & & & \\
\hline & & 6 & 17610.00 & & & & & \\
\hline & & 5 & 17612.84 & & & & & \\
\hline & & 5 & 17712.15 & & & & & \\
\hline & & 6 & 17834.96 & & & 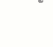 & & \\
\hline & & 5 & 18419.45 & & & & & \\
\hline & & 5 & 18635.29 & & & & & \\
\hline & & 6 & 18687.53 & 1.09 & & & & \\
\hline & & 6 & 18736.10 & & & & & \\
\hline & & 6 & 19114.25 & & & & & \\
\hline & & 6 & 19750.03 & 1.1 & & & & \\
\hline & & 6 & 20134.63 & & & & & \\
\hline & & 7 & 21139.14 & & & & & \\
\hline & & 2 & 22377.66 & 1.62 & & & & \\
\hline & & 2 & 22774.24 & 0.571 & & & & \\
\hline & & 3 & 23261.30 & 0.617 & & & & \\
\hline & & 5 & 23302.12 & & & & & \\
\hline & & 2 & 23499.82 & 0.467 & & & & \\
\hline & & 4 & 23541.00 & 1.168 & & & & \\
\hline & & 3 & 23761.98 & 0.840 & & & & \\
\hline & & 2 & 24023.04 & 0.36 & & & & \\
\hline & & 3 & 24024.75 & 0.962 & & & & \\
\hline & & 4 & 24157.20 & 0.855 & & & & \\
\hline & & 4 & 24311.93 & 0.693 & & & & \\
\hline & & 2 & 24404.51 & 1.073 & & & & \\
\hline & & 4 & 24464.73 & 1.039 & & & & \\
\hline & & 3 & 24466.32 & 0.743 & & & & \\
\hline
\end{tabular}


Pm II-Continued

Pm II-Continued

\begin{tabular}{|c|c|c|c|c|c|c|c|c|c|}
\hline Configuration & Term & $J$ & $\begin{array}{l}\text { Level } \\
\left(\mathrm{cm}^{-1}\right)\end{array}$ & $g$ & Configuration & Term & $J$ & $\begin{array}{l}\text { Level } \\
\left(\mathrm{cm}^{-1}\right)\end{array}$ & $g$ \\
\hline & & 2 & 24505.57 & 0.961 & & & 1 & 27616.10 & 2.863 \\
\hline & & 3 & 24527.89 & 0.533 & & & 3 & 27622.53 & 1.271 \\
\hline & & 4 & 24762.58 & 0.990 & & & 6 & 27645.16 & 1.1 \\
\hline & & 5 & 24771.46 & 1.160 & & & 7 & 27678.03 & \\
\hline & & 3 & 25006.51 & 0.806 & & & 3 & 27765.61 & 0.906 \\
\hline & & 5 & 25015.69 & 1.110 & & & 3 & 27848.71 & 1.164 \\
\hline & & 6 & 25094.33 & 1.17 & & & 4 & 27894.03 & 0.69 \\
\hline & & 4 & 25099.22 & 0.74 & & & 3 & 27991.45 & 1.161 \\
\hline & & 4 & 25213.82 & 1.008 & & & 5 & 28061.10 & \\
\hline & & 5 & 25258.71 & & & & 7 & 28066.15 & 1.23 \\
\hline & & 4 & 25264.46 & 0.912 & & & 4 & 28133.56 & 1.037 \\
\hline & & 2 & 25323.09 & 0.09 & & & 3 & 28166.72 & 1.040 \\
\hline & & 5 & 25365.66 & 1.14 & & & 4 & 28227.39 & 1.19 \\
\hline & & 1 & 25447.33 & 1.194 & & & 6 & 28271.15 & \\
\hline & & 5 & 25598.60 & & & & 5 & 28296.71 & \\
\hline & & 2 & 25726.08 & 1.317 & & & 5 & 28324.75 & \\
\hline & & 3 & 25737.07 & 0.883 & & & 4 & 28401.54 & \\
\hline & & 2 & 26017.92 & 0.861 & & & 5 & 28444.27 & \\
\hline & & 6 & 26027.56 & 1.16 & & & 6 & 28487.33 & 0.98 \\
\hline & & 5 & 26038.04 & 0.95 & & & 2 & 28499.09 & 1.803 \\
\hline & & 4 & 26067.53 & 1.156 & & & 5 & 28507.50 & \\
\hline & & 5 & 26132.87 & 0.943 & & & 1 & 28507.58 & 1.88 \\
\hline & & 6 & 26169.85 & 1.20 & & & 7 & 28516.85 & 1.20 \\
\hline & & 5 & 26309.89 & 1.116 & & & 5 & 28642.00 & 1.178 \\
\hline & & 2 & 26320.95 & 0.714 & & & 4 & 28765.64 & \\
\hline & & 6 & 26392.53 & & & & 1 & 28819.06 & 0.74 \\
\hline & & 3 & 26460.58 & 1.273 & & & 4 & 28930.06 & 1.024 \\
\hline & & 5 & 26574.58 & & & & 5 & 29057.65 & 1.13 \\
\hline & & 3 & 26771.13 & 0.622 & & & 5 & 29368.84 & \\
\hline & & 6 & 26834.20 & 1.11 & & & 4 & 29400.84 & \\
\hline & & 4 & 27005.58 & 1.073 & & & 4 & 29468.44 & 0.96 \\
\hline & & 2 & 27094.25 & 0.226 & & & 6 & 29586.34 & 1.11 \\
\hline & & 6 & 27097.38 & 1.18 & & & 6 & 29662.69 & 1.13 \\
\hline & & 7 & 27110.08 & & & & 5 & 29693.40 & 0.99 \\
\hline & & 4 & 27115.95 & 1.26 & & & 1 & 29802.52 & 0.12 \\
\hline & & 3 & 27126.19 & 1.053 & & & 5 & 29863.62 & \\
\hline & & 4 & 27230.36 & 0.97 & & & 5,6 & 29967.46 & \\
\hline & & 2 & 27256.71 & 0.88 & & & 6 & 29987.92 & 1.22 \\
\hline & & 5 & 27356.20 & & & & 6 & 30089.49 & \\
\hline & & 6 & 27379.79 & 1.0 & & & 2 & 30142.21 & 1.110 \\
\hline & & 4 & 27399.87 & & & & 1 & 30172.01 & 0.534 \\
\hline & & 5 & 27438.30 & & & & 2 & 30216.86 & 1.015 \\
\hline & & 3 & 27526.77 & & & & 1 & 30402.81 & 1.123 \\
\hline & & 2 & 27587.88 & 0.98 & & & 3 & 30477.48 & 1.374 \\
\hline
\end{tabular}


Pm II-Continued

Pm II--Continued

\begin{tabular}{|c|c|c|c|c|c|c|c|c|c|}
\hline Configuration & Term & $J$ & $\begin{array}{l}\text { Level } \\
\left(\mathrm{em}^{-1}\right)\end{array}$ & $g$ & Configuration & Term & $J$ & $\begin{array}{l}\text { Level } \\
\left(\mathrm{em}^{-1}\right)\end{array}$ & $g$ \\
\hline & & 5 & 30532.47 & & & & 5 & 32551.40 & 1.2 \\
\hline & & 5 & 30547.86 & & & & 2 & 32700.82 & 1.198 \\
\hline & & 1 & 30612.37 & 0.32 & & & 3 & 32755.89 & 1.457 \\
\hline & & 6 & 30688.69 & 1.28 & & & 2 & 32847.03 & 1.47 \\
\hline & & 3 & 30736.73 & 1.117 & & & 2 & 32947.27 & 1.49 \\
\hline & & 7 & 30814.52 & & & & 1 & 32973.73 & 0.95 \\
\hline & & 2 & 30996.26 & 0.901 & & & 6 & 32974.29 & \\
\hline & & 3 & 31085.12 & 1.11 & & & 1 & 33067.34 & 1.44 \\
\hline & & 5 & 31251.94 & & & & 3 & 33079.50 & 1.536 \\
\hline & & $6 ?$ & 31367.37 & & & & 2 & 33111.77 & 0.71 \\
\hline & & 3 & 31474.36 & 1.081 & & & 1 & 33272.56 & 0.990 \\
\hline & & 4 & 31558.10 & 0.94 & & & 2 & 33380.55 & 1.23 \\
\hline & & 2 & 31632.30 & 1.05 & & & 1 & 33551.90 & 1.322 \\
\hline & & 3 & 31778.84 & 1.137 & & & 1 & 33641.58 & 1.980 \\
\hline & & 4 & 31805.86 & 1.02 & & & & & \\
\hline & & 7 & 31851.14 & & & & & & \\
\hline & & 2 & 32321.10 & 1.581 & $\operatorname{Pm~III~}\left({ }^{2} \mathrm{H}_{5 / 2}^{\circ}\right)$ & Limit & & 87900 & \\
\hline & & 2 & 32420.83 & 1.18 & & & & & \\
\hline
\end{tabular}




\section{Pm III}

(Pr I sequence; 59 electrons)

Ground state $\left(1 s^{2} 2 s^{2} 2 p^{6} 3 s^{2} 3 p^{6} 3 d^{10} 4 s^{2} 4 p^{6} 4 d^{10} 5 s^{2} 5 p^{6}\right) 4 f^{5}{ }^{6} \mathrm{H}_{5 / 2}^{\circ}$

Ionization energy $180000 \pm 3000 \mathrm{~cm}^{-1}$

$22.3 \pm 0.4 \mathrm{eV}$

Although this spectrum has not been analyzed, the above designation of the ground state is not in significant doubt. The uncertainty of the ionization energy given by Sugar and Reader has been rounded off to one significant figure.

\section{Reference}

Sugar, J., and Reader, J., J. Chem. Phys. 59, 2083 (1973). IP

[October 1976] 
Ground state $\left(1 s^{2} 2 s^{2} 2 p^{6} 3 s^{2} 3 p^{6} 3 d^{10} 4 s^{2} 4 p^{6} 4 d^{10} 5 s^{2} 5 p^{6}\right) 4 f^{45} \mathbf{I}_{4}$

Ionization energy $331000 \pm 5000 \mathrm{~cm}^{-1}$

$41.1 \pm 0.6 \mathrm{eV}$

The spectrum of the free ion has not been analyzed. Carnall, Crosswhite, Crosswhite, and Conway have given the most complete interpretation of the spectrum of the $\mathrm{Pm}^{3+}$ ion in a $\mathrm{LaCl}_{3}$ host crystal. Using both absorption and fluorescence spectra, they derived the $4 f^{4}$ levels up to $31690 \mathrm{~cm}^{-1}$. Only the levels of the three lowest terms are given here, the positions listed by Carnall et al. [1976] having been adjusted to a value of zero for the ground level and rounded off to the nearest $10 \mathrm{~cm}^{-1}$. Carnall et al. evaluated the levels by first fitting a calculation to the experimental sublevels and then recalculating the levels with the crystalfield parameters set equal to zero. (Shifts of the levels due to the nephelauxetic effect are, of course, not removed by this procedure.) The eigenvector percentages are also from their calculation.

Carnall et al. [1976] took most of the energy levels based on absorption spectra from the measurements of Baer, Conway, and Davis [1973]. Gruber and Conway [1960] first observed the absorption spectrum using solutions, and the analysis by Carnall, Fields, and Rajnak [1968] was also based on solution absorption data.

Sugar and Reader derived the ionization energy, which has been rounded off to three significant figures. Following the suggestion of Spector and Sugar, we have approximately doubled the estimated uncertainty.

\section{References}

Baer, W., Conway, J. G., and Davis, S. P., J. Chem. Phys. 59, 2294 (1973). [EL] [CL] [W] PT

Carnall, W. T., Crosswhite, H., Crosswhite, H. M., and Conway, J. G., J. Chem. Phys. 64, 3582 (1976). [EL] [CL] [W] PT Carnall, W. T., Fields, P. R., and Rajnak, K., J. Chem. Phys. 49, 4424 (1968). [EL] [CL] [W] PT

Crozier, M. H., and Runciman, W. A., J. Chem. Phys. 35, 1392 (1961). ND PT

Gruber, J. B., and Conway, J. G., J. Inorg. Nucl. Chem. 14, 303 (1960). [W]

Hüfner, S., Z. Phys. 165, 397 (1961). ND PT

Spector, N., and Sugar, J., J. Opt. Soc. Am. 66, 436 (1976).

Sugar, J., and Reader, J., J. Chem. Phys. 59, 2083 (1973). IP

[October 1976]

\section{[Pm IV]}

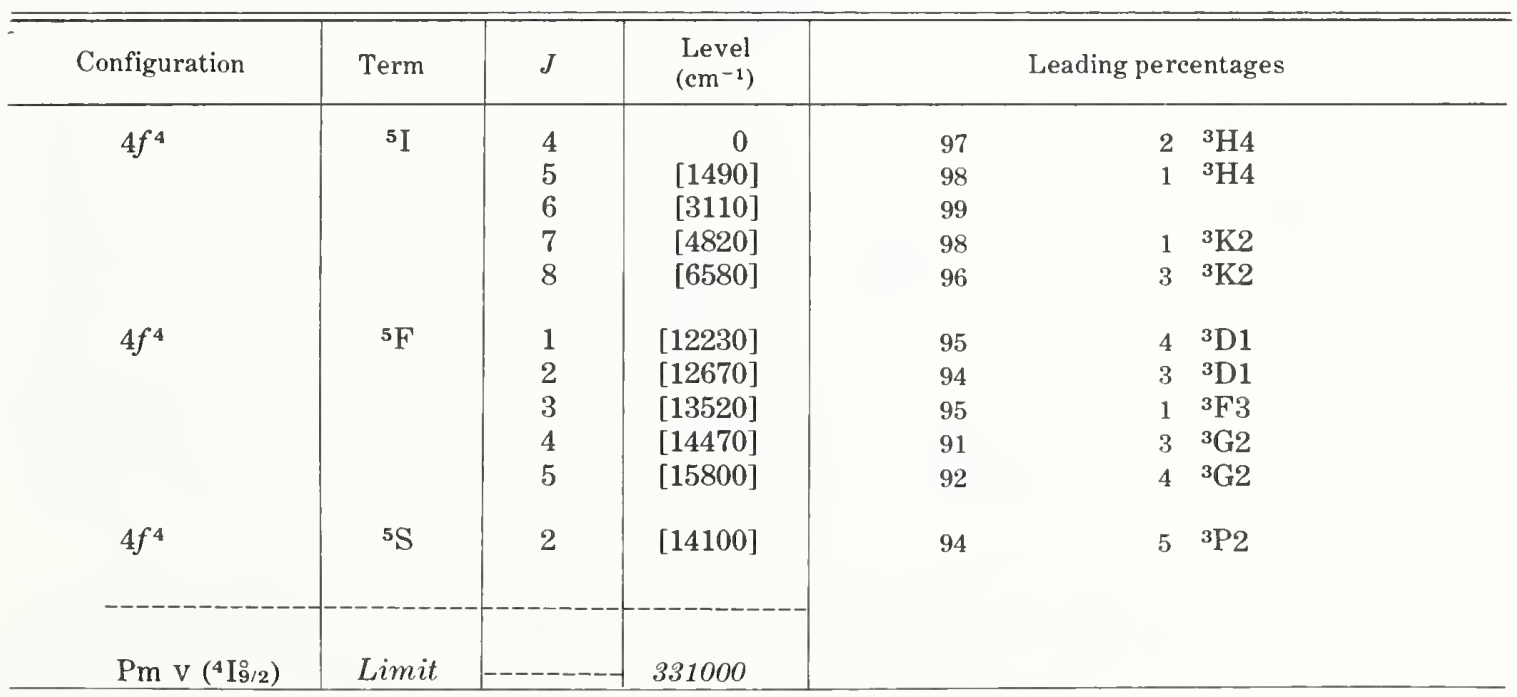




\section{SAMARIUM}

\section{Sm I}

62 electrons

Ground state $\left(1 s^{2} 2 s^{2} 2 p^{6} 3 s^{2} 3 p^{6} 3 d^{10} 4 s^{2} 4 p^{6} 4 d^{10} 5 s^{2} 5 p^{6}\right) 4 f^{6} 6 s^{2}{ }^{7} \mathrm{~F}_{0}$

Ionization energy $45519 \pm 8 \mathrm{~cm}^{-1}$

$5.6437 \pm 0.0010 \mathrm{eV}$

Identified even configurations

$4 f^{6} 6 s^{2}, 4 f^{6} 5 d 6 s, 4 f^{6} 6 s 7 s$

Identified odd configurations

$4 f^{6} 6 s 6 p, 4 f^{5} 5 d 6 s^{2}, 4 f^{5} 5 d^{2} 6 s ?, 4 f^{6} 5 d 6 p ?$

Albertson gave the levels of the $4 f^{6} 6 s^{2} 7 \mathrm{~F}$ ground term in his important 1935 paper, which included 175 levels. The analysis was extended somewhat in his 1937 article and again in the 1949 paper by Brix. Racah and Ganiel gave a theoretical interpretation for 70 of the $4 f^{6}\left({ }^{7} \mathrm{~F}\right) 6 s 6 p$ levels.

Carlier, Blaise, and Schweighofer [1968], Blaise, Morillon, Schweighofer, and Verges [1969], and Henny-Schweighofer [1970] have revised and extended the analysis to include 65 even levels and about 400 odd levels. The levels and $g$ values are taken from this work at Orsay, with the exception of the $g$ values for the ground-term levels. Blaise [1976] has supplied a few additions and revisions.

The five-place $g$ values for the $4 f^{6} 6 s^{2}{ }^{7} \mathrm{~F}$ levels are weighted averages of atomic-beam magnetic-resonance measurements by Pichanick and Woodgate [1961] and Childs and Goodman [1972], as given by the latter authors. The uncertainty is three or four units in the last place. The leading percentages for these levels are from Conway and Wybourne [1963]. (Childs and Goodman list detailed eigenvectors from Conway's refinement of this calculation.) The next lowest predicted term of $4 f^{6} 6 s^{2}$, the lowest of the three ${ }^{5} \mathrm{D}$ terms, is expected to begin above $14000 \mathrm{~cm}^{-1}$ (see Sm III $4 f^{6}$ ). None of the higher $4 f^{6} 6 s^{2}$ terms has been identified, nor have any terms (of other configurations) based on higher $4 f^{6}$ terms been identified.

The level compositions for $4 f^{6}\left({ }^{7} \mathrm{~F}\right) 5 d 6 \mathrm{~s}$ and $4 f^{6}\left({ }^{7} \mathrm{~F}\right) 6 s 7 \mathrm{~s}$ are from Carlier's thesis [1967], and the compositions for $\left(4 f^{5}\left({ }^{6} \mathrm{H}^{\circ},{ }^{6} \mathrm{~F}^{\circ}\right) 5 d 6 s^{2}+4 f^{6}\left({ }^{7} \mathrm{~F}\right) 6 s 6 p\right)$ were calculated by Carlier et al. Sugar transposed the eigenvectors from the latter calculation by recoupling to the $f^{6}\left({ }^{7} \mathrm{~F}\right) \operatorname{sp}\left({ }^{3} \mathrm{P}^{\circ}, \mathrm{P}^{\circ}\right)$ scheme given here. Designations assigned by Blaise et al. to levels that have not been calculated are listed with question marks, pending a more complete theoretical interpretation of this spectrum. Most of these tentative designations are for odd levels thought to belong to the $\left(4 f^{6} 5 d 6 p+4 f^{5} 5 d^{2} 6 s\right)$ group. A few unpublished designations or revisions for the odd levels are included here [Blaise, 1976].

Albertson's analysis was based on the extensive furnace spectrum data of A. S. King [1935], which includes wavelengths and temperature classifications for about $2800 \mathrm{Sm}$ I lines in the range of 2910-8718 $\AA$, and on the absorption lines measured by Paul (1500 lines, 2503-8301 $\AA$ ). Henny-Schweighofer's thesis gives more than $5000 \mathrm{Sm}$ I and Sm II lines between 2628 and $9789 \AA$, about three-fourths of them being classified. Zeeman data or the $g$ and $J$ values derived from such data are listed for the lines. Isotope shift measurements are also given in this thesis, and in several other references listed below. Blaise et al. list 1812 lines for the spectrum of an electrodeless lamp between 8186 and $24558 \AA$, with 859 of the lines being classified by transitions in Sm I and Sm II. Morillon [1970] used a grid spectrometer to extend the infrared observations, obtaining $370 \mathrm{Sm}$ lines (electrodeless lamp source) between 23820 and $41061 \AA$.

Parr and Inghram [1975] observed photoionization spectra of samarium vapor at different temperatures. Their paper gives the positions of 43 resonances $\left(42650-51070 \mathrm{~cm}^{-1}\right)$ due to autoionizing levels observed at a source temperature of $1270 \mathrm{~K}$. A number of low levels were 
significantly populated at the source temperatures, and no definite analyses of the spectra were possible. We give three tentative odd levels above the limit, based on the authors' statement that the first three lines they list are probably due to transitions from the $4 f^{6} 6 s^{2}{ }^{7} \mathrm{~F}_{5}$ level. (The high levels are followed by the letter " $a$ " for autoionization.) Several of the other resonances have wavenumbers corresponding to a transition from a low level to one of these three tentative autoionizing levels. The authors discuss the probable configurations of the autoionizing levels involved in the stronger resonances.

Worden, Conway, Paisner, and Solarz [1977] have observed high members of a series in Sm I by using laser techniques. These data became available too late for inclusion here, but the quoted ionization energy was determined by Worden et al. from the new series.

\title{
References
}

\author{
Albertson, W., Phys. Rev. 47, 370 (1935); 52, 644 (1937). EL CL IP \\ Blaise, J., unpublished material (1976). EL ND ZE \\ Blaise, J., Morillon, C., Schweighofer, M. G., and Verges, J., Spectrochim. Acta, Part B 24, 405 (1969). EL CL, W ZE \\ Brix, P., Z. Phys. 126, 431 (1949). EL, CL IS \\ Carlier, A., 'Thesis (Third Cycle), Univ. Paris, Orsay, 128 pp. (1967). EL P'T \\ Carlier, A., Blaise, J., and Schweighofer, M. G., J. Phys. (Paris) 29, 729 (1968). EL ZE P'T \\ Childs, W. J., and Goodman, L. S., Phys. Rev. A 6, 2011 (1972). ZE Hfs PT \\ Conway, J. G., and Wybourne, B. G., Phys. Rev. 130, 2325 (1963). P'T \\ Handrich, E., Steudel, A., Wallenstein, R., and Walther, H., J. Phys. (Paris), Colloq. Cl 30, 18 (1969). Hfs \\ Hansen, J. E., Steudel, A., and Walther, H., Z. Phys. 203, 296 (1967). IS \\ Heilig, K., Sauthoff, G., and Steudel, A., Z. Phys. 196, 39 (1966). ZE \\ Henny-Schweighofer, M. G, Thesis (Third Cycle), Univ. Paris, Orsay, 174 pp. (1970). EL CL W ZE IS \\ Kiess, C. C., Sci. Papers Bur. Stand. (U.S.) 18, 201 (1922). W \\ King, A. S., Astrophys. J. 82, 140 (1935). W \\ King, W. H., J. Opt. Soc. Am. 53, 638 (1963). IS \\ Martin, N. J., Sandars, P. G. H., and Woodgate, G. K., Proc. R. Soc. London, Ser. A 305, 139 (1968). SE Hfs \\ Morillon, C., Spectrochim. Acta, Part B 25, 513 (1970). CL W \\ Murakawa, K., Phys. Rev. 93, 1232 (1954). Hfs \\ Parr, A. C., and Inghram, M. G., J. Opt. Soc. Am. 65, 613 (1975). EL CL W \\ Paul, F. W., Phys. Rev. 49, 156 (1936). W \\ Pichanick, F. M. J., and Woodgate, G. K., Proc. R. Soc. London, Ser. A 263, 89 (1961). ZE \\ Racah, G., and Ganiel, U., J. Opt. Soc. Am. 56, 893 (1966). EL PT \\ Robertson, R. G. H., Waddington, J. C., and Summers-Gill, R. G., Can. J. Phys. 46, 2499 (1968). Hfs \\ Smith, K. F., and Spalding, I. J., Proc. R. Soc. London, Ser. A 265, 133 (1961). ZE \\ Striganov, A. R., Katulin, V. A., and Eliseev, V. V., Opt. Spektrosk. 12, 171 (1962). CL IS \\ Sugar, J., unpublished material (1971). P'T \\ Worden, E. F., Conway, J. G., Paisner, J. A., and Solarz, R. W., unpublished material (1977). IP
}

[July 1976]

Sm I, Even Parity

\begin{tabular}{|c|c|c|c|c|c|c|}
\hline Configuration & Term & $J$ & $\begin{array}{l}\text { Level } \\
\left(\mathrm{cm}^{-1}\right)\end{array}$ & $g$ & & Leading percentages \\
\hline $4 f^{6} 6 s^{2}$ & ${ }^{7} \mathrm{~F}$ & $\begin{array}{l}0 \\
1 \\
2 \\
3 \\
4 \\
5 \\
6\end{array}$ & $\begin{array}{r}0.00 \\
292.58 \\
811.92 \\
1489.55 \\
2273.09 \\
3125.46 \\
4020.66\end{array}$ & $\begin{array}{l}1.49839 \\
1.49779 \\
1.49707 \\
1.49625 \\
1.49532 \\
1.49417\end{array}$ & $\begin{array}{l}94 \\
95 \\
97 \\
98 \\
98 \\
98 \\
97\end{array}$ & $\begin{array}{ll}3 & { }^{5} \mathrm{D} 1 \\
2 & { }^{5} \mathrm{D} 1 \\
2 & { }^{5} \mathrm{D} 1 \\
1 & { }^{5} \mathrm{D} 1 \\
1 & { }^{5} \mathrm{~F} 2 \\
1 & { }^{5} \mathrm{G} 1 \\
1 & { }^{5} \mathrm{G} 1\end{array}$ \\
\hline $4 f^{6}\left({ }^{7} \mathrm{~F}\right) 5 d\left({ }^{8} \mathrm{H}\right) 6 s$ & ${ }^{9} \mathrm{H}$ & $\begin{array}{l}1 \\
2 \\
3 \\
4 \\
5 \\
6 \\
7 \\
8 \\
9\end{array}$ & $\begin{array}{l}10801.10 \\
11044.90 \\
11406.50 \\
11877.50 \\
12445.35 \\
13095.75 \\
13814.90 \\
14591.70 \\
15418.65\end{array}$ & $\begin{array}{c}-0.98 \\
0.67 \\
1.08 \\
1.25 \\
1.33 \\
1.385 \\
1.410 \\
1.430 \\
1.445\end{array}$ & $\begin{array}{r}99 \\
98 \\
98 \\
97 \\
97 \\
98 \\
99 \\
99 \\
100\end{array}$ & \\
\hline
\end{tabular}


Sm I, Even Parity-Continued

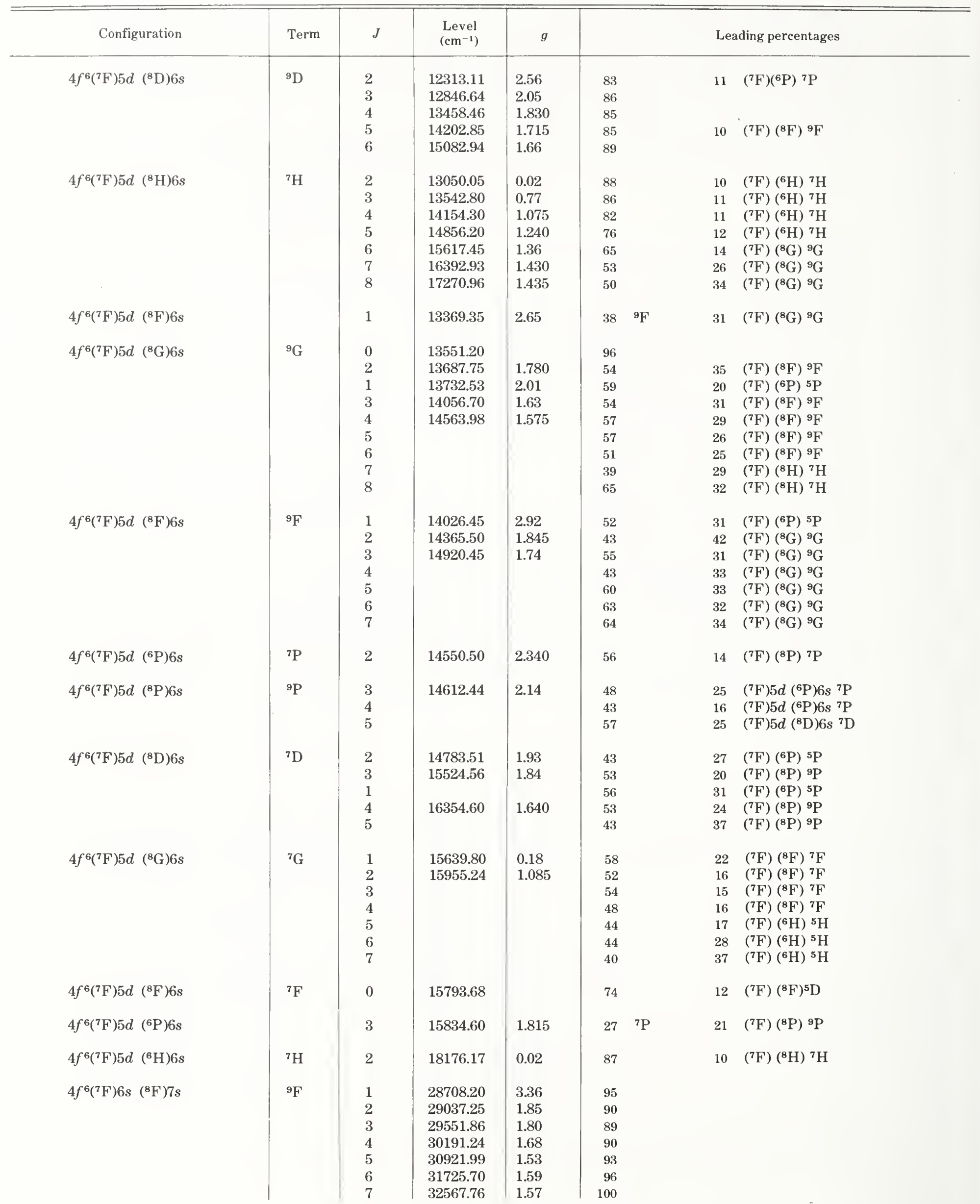


Sm I, Even Parity-Continued

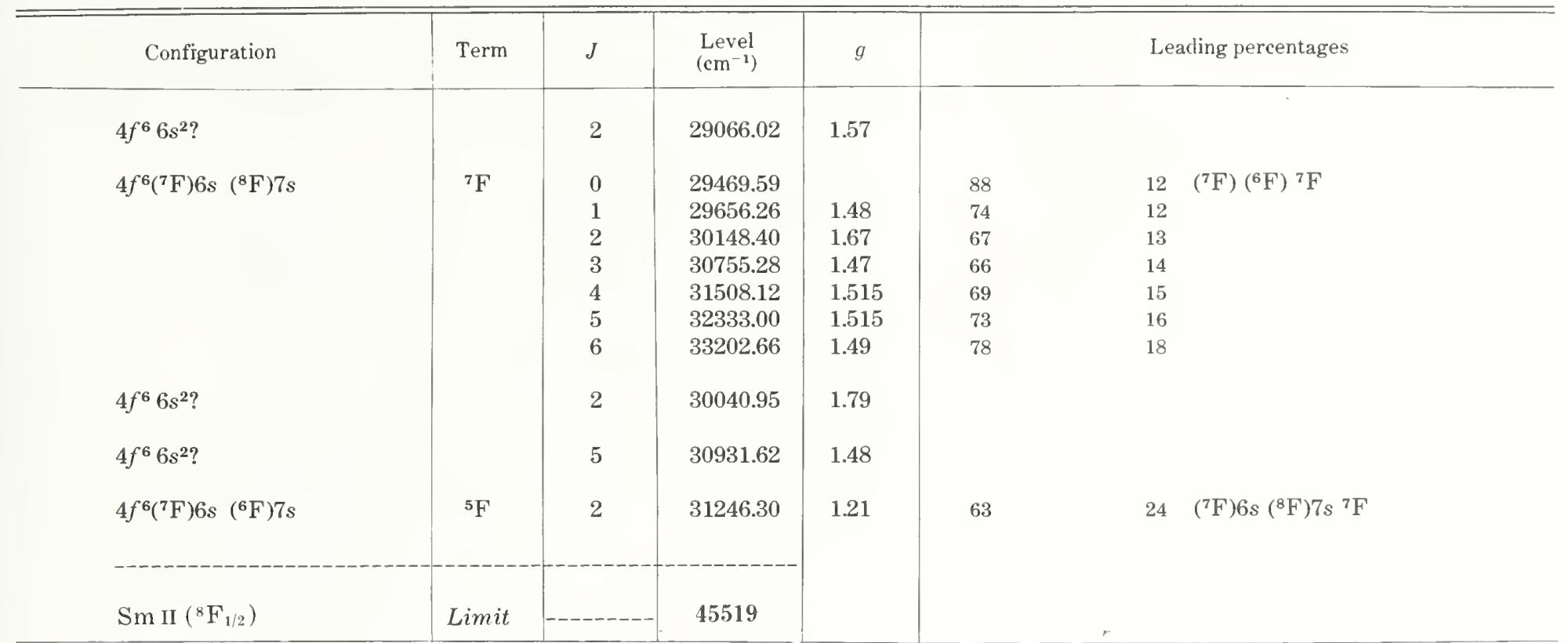

Sm I, Odd Parity

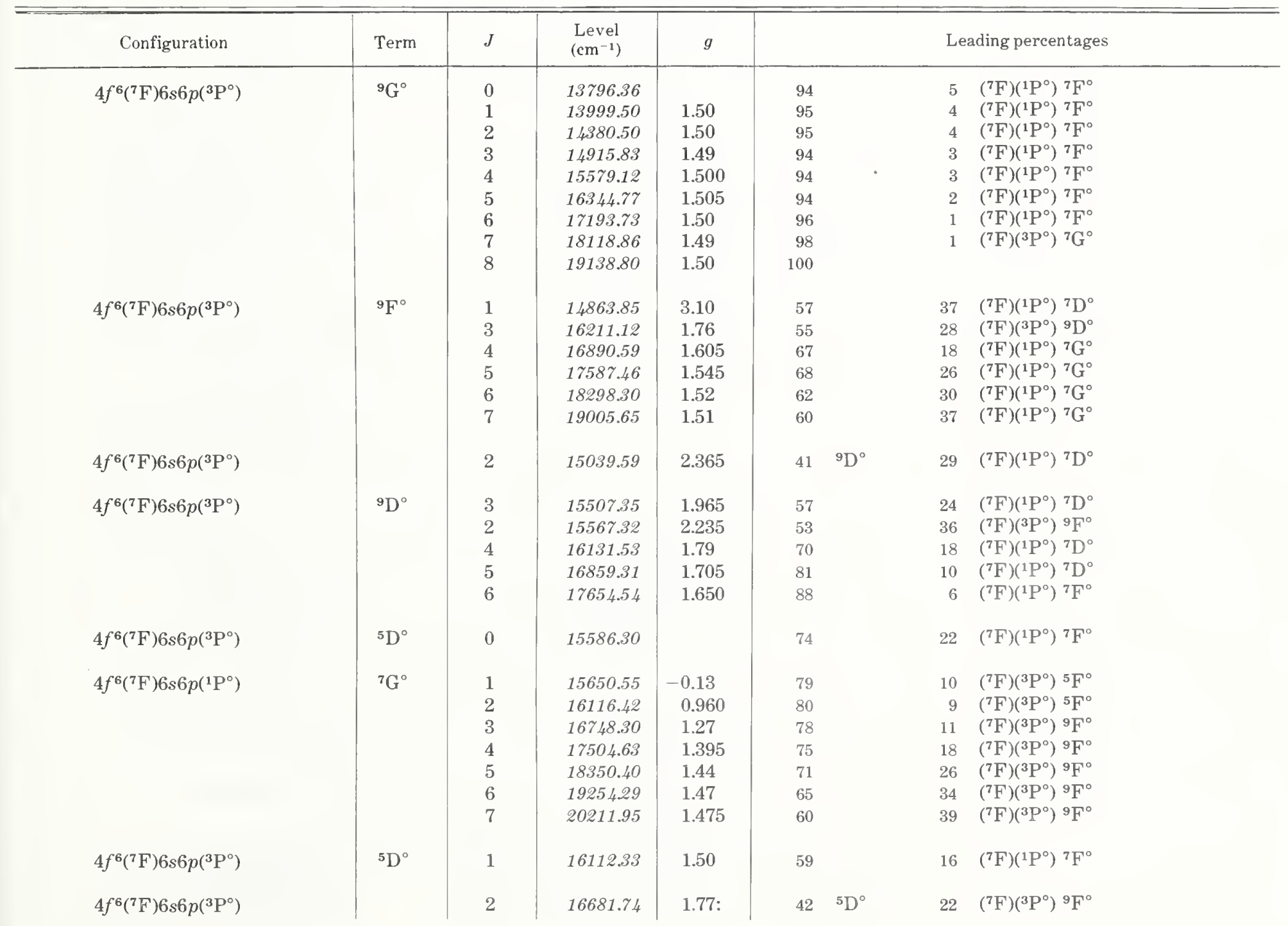


Sm I, Odd Parity—Continued

\begin{tabular}{|c|c|c|c|c|c|c|c|c|}
\hline Configuration & Term & $J$ & $\begin{array}{l}\text { Level } \\
\left(\mathrm{cm}^{-1}\right)\end{array}$ & $g$ & \multicolumn{4}{|c|}{ Leading percentages } \\
\hline $4 f^{6}\left({ }^{7} \mathrm{~F}\right) 6 s 6 p\left({ }^{1} \mathrm{P}^{\circ}\right)$ & ${ }^{7} \mathrm{D}^{\circ}$ & 1 & 16690.76 & 2.83: & 53 & & 30 & $\left({ }^{7} \mathrm{~F}\right)\left({ }^{3} \mathrm{P}^{\circ}\right){ }^{9} \mathrm{~F}^{\circ}$ \\
\hline $4 f^{6}\left({ }^{7} \mathrm{~F}\right) 6 s 6 p\left({ }^{1} \mathrm{P}^{\circ}\right)$ & ${ }^{7} \mathrm{~F}^{\circ}$ & $\begin{array}{l}2 \\
0 \\
3 \\
4 \\
5 \\
6\end{array}$ & $\begin{array}{l}17190.20 \\
17810.85 \\
17830.80 \\
19191.64 \\
20153.47 \\
21055.76\end{array}$ & $\begin{array}{l}1.425 \\
1.215 \\
1.27: \\
1.44 \\
1.455\end{array}$ & $\begin{array}{l}46 \\
65 \\
41 \\
60 \\
68 \\
65\end{array}$ & & $\begin{array}{l}25 \\
20 \\
37 \\
20 \\
19 \\
26\end{array}$ & $\begin{array}{l}\left({ }^{7} \mathrm{~F}\right)\left({ }^{1} \mathrm{P}^{\circ}\right){ }^{7} \mathrm{D}^{\circ} \\
\left({ }^{7} \mathrm{~F}\right)\left({ }^{3} \mathrm{P}^{\circ}\right){ }^{5} \mathrm{D}^{\circ} \\
\left({ }^{7} \mathrm{~F}\right)\left({ }^{3} \mathrm{P}^{\circ}\right){ }^{5} \mathrm{G}^{\circ} \\
\left({ }^{7} \mathrm{~F}\right)\left({ }^{3} \mathrm{P}^{\circ}\right){ }^{5} \mathrm{G}^{\circ} \\
\left({ }^{7} \mathrm{~F}\right)\left({ }^{3} \mathrm{P}^{\circ}\right){ }^{5} \mathrm{G}^{\circ} \\
\left({ }^{7} \mathrm{~F}\right)\left({ }^{3} \mathrm{P}^{\circ}\right){ }^{5} \mathrm{G}^{\circ}\end{array}$ \\
\hline $4 f^{6}\left({ }^{7} \mathrm{~F}\right) 6 s 6 p\left({ }^{1} \mathrm{P}^{\circ}\right)$ & & 3 & 17243.55 & 1.62 & 35 & ${ }^{7} \mathrm{D}^{\circ}$ & 21 & $\left({ }^{7} \mathrm{~F}\right)\left({ }^{3} \mathrm{P}^{\circ}\right){ }^{5} \mathrm{D}^{\circ}$ \\
\hline $4 f^{6}\left({ }^{7} \mathrm{~F}\right) 6 s 6 p\left({ }^{3} \mathrm{P}^{\circ}\right)$ & ${ }^{5} \mathrm{G}^{\circ}$ & $\begin{array}{l}2 \\
3 \\
4 \\
5 \\
6\end{array}$ & $\begin{array}{l}17462.37 \\
18209.04 \\
18503.49 \\
18811.11 \\
19712.41\end{array}$ & $\begin{array}{l}0.755 \\
1.39 \\
1.365 \\
1.45 \\
1.38\end{array}$ & $\begin{array}{l}74 \\
36 \\
41 \\
47 \\
70\end{array}$ & & $\begin{array}{r}8 \\
27 \\
27 \\
17 \\
23\end{array}$ & $\begin{array}{l}\left({ }^{7} \mathrm{~F}\right)\left({ }^{3} \mathrm{P}^{\circ}\right){ }^{5} \mathrm{~F}^{\circ} \\
\left({ }^{7} \mathrm{~F}\right)\left({ }^{1} \mathrm{P}^{\circ}\right){ }^{7} \mathrm{~F}^{\circ} \\
\left({ }^{7} \mathrm{~F}\right)\left({ }^{1} \mathrm{P}^{\circ}\right)^{7} \mathrm{D}^{\circ} \\
\left({ }^{7} \mathrm{~F}\right)\left({ }^{1} \mathrm{P}^{\circ}\right)^{7} \mathrm{~F}^{\circ} \\
\left({ }^{7} \mathrm{~F}\right)\left({ }^{1} \mathrm{P}^{\circ}\right){ }^{7} \mathrm{~F}^{\circ}\end{array}$ \\
\hline $4 f^{6}\left({ }^{7} \mathrm{~F}\right) 6 s 6 p\left({ }^{1} \mathrm{P}^{\circ}\right)$ & & 1 & 17769.71 & $0.80:$ & 26 & ${ }^{7} \mathrm{~F}^{\circ}$ & 17 & $\left({ }^{7} \mathrm{~F}\right)\left({ }^{3} \mathrm{P}^{\circ}\right){ }^{5} \mathrm{~F}^{\circ}$ \\
\hline $4 f^{6}\left({ }^{7} \mathrm{~F}\right) 6 s 6 p\left({ }^{1} \mathrm{P}^{\circ}\right)$ & & 4 & 17959.27 & 1.52 & 30 & ${ }^{7} \mathrm{D}^{\circ}$ & 21 & $\left({ }^{7} \mathrm{~F}\right)\left({ }^{3} \mathrm{P}^{\circ}\right){ }^{5} \mathrm{G}^{\circ}$ \\
\hline $4 f^{5}\left({ }^{6} \mathrm{H}^{\circ}\right) 5 d 6 s^{2}$ & ${ }^{7} \mathrm{H}^{\circ}$ & $\begin{array}{l}2 \\
3 \\
4 \\
5 \\
6 \\
7 \\
8\end{array}$ & $\begin{array}{l}18075.67 \\
18948.78 \\
19990.25 \\
21062.82 \\
22160.84 \\
23337.40\end{array}$ & $\begin{array}{l}0.41 \\
0.94 \\
1.115 \\
1.21 \\
1.305 \\
1.36\end{array}$ & $\begin{array}{l}56 \\
45 \\
53 \\
59 \\
52 \\
53 \\
74\end{array}$ & & $\begin{array}{l}11 \\
11 \\
10 \\
12 \\
15 \\
13 \\
14\end{array}$ & $\begin{array}{l}4 f^{6}\left({ }^{7} \mathrm{~F}\right) 6 s 6 p\left({ }^{1} \mathrm{P}^{\circ}\right){ }^{7} \mathrm{~F}^{\circ} \\
4 f^{6}\left({ }^{7} \mathrm{~F}\right) 6 s 6 p\left({ }^{3} \mathrm{P}^{\circ}\right){ }^{5} \mathrm{D}^{\circ} \\
\left({ }^{6} \mathrm{H}^{\circ}\right){ }^{7} \mathrm{I}^{\circ} \\
\left({ }^{6} \mathrm{H}^{\circ}\right){ }^{7} \mathrm{G}^{\circ} \\
\left({ }^{6} \mathrm{H}^{\circ}\right){ }^{7} \mathrm{G}^{\circ} \\
\left({ }^{6} \mathrm{H}^{\circ}\right){ }^{7} \mathrm{G}^{\circ} \\
\left({ }^{6} \mathrm{~F}^{\circ}\right){ }^{7} \mathrm{H}^{\circ}\end{array}$ \\
\hline $4 f^{6}\left({ }^{7} \mathrm{~F}\right) 6 s 6 p\left({ }^{1} \mathrm{P}^{\circ}\right)$ & & 1 & 18225.13 & 0.695 & 31 & ${ }^{7} \mathrm{~F}^{\circ}$ & 21 & $4 f^{5}\left({ }^{6} \mathrm{H}^{\circ}\right) 5 d 6 s^{2}{ }^{7} \mathrm{G}^{\circ}$ \\
\hline $4 f^{5}\left({ }^{6} \mathrm{H}^{\circ}\right) 5 d 6 s^{2}$ & ${ }^{7} \mathrm{~F}^{\circ}$ & $\begin{array}{l}0 \\
1 \\
2 \\
3 \\
4 \\
5 \\
6\end{array}$ & $\begin{array}{l}18309.02 \\
18475.28 \\
18788.08 \\
19501.27 \\
20163.00 \\
21458.89 \\
22944.38\end{array}$ & $\begin{array}{l}0.96 \\
1.280 \\
1.365 \\
1.390 \\
1.425 \\
1.475\end{array}$ & $\begin{array}{l}74 \\
65 \\
31 \\
49 \\
30 \\
42 \\
61\end{array}$ & & $\begin{array}{r}13 \\
13 \\
14 \\
8 \\
14 \\
18 \\
23\end{array}$ & $\begin{array}{l}4 f^{6}\left({ }^{7} \mathrm{~F}\right) 6 s 6 p\left({ }^{3} \mathrm{P}^{\circ}\right){ }^{7} \mathrm{~F}^{\circ} \\
4 f^{6}\left({ }^{7} \mathrm{~F}\right) 6 s 6 p\left({ }^{3} \mathrm{P}^{\circ}\right){ }^{5} \mathrm{~F}^{\circ} \\
4 f^{6}\left({ }^{7} \mathrm{~F}\right) 6 s 6 p\left({ }^{1} \mathrm{P}^{\circ}\right) \\
7 \mathrm{~F}^{\circ} \\
4 f^{6}\left({ }^{7} \mathrm{~F}\right) 6 s 6 p\left({ }^{3} \mathrm{P}^{\circ}\right){ }^{5} \mathrm{D}^{\circ} \\
4 f^{6}\left({ }^{7} \mathrm{~F}\right) 6 s 6 p\left({ }^{3} \mathrm{P}^{\circ}\right)^{5} \mathrm{~F}^{\circ} \\
\left({ }^{6} \mathrm{H}^{\circ}\right)^{7} \mathrm{G}^{\circ} \\
4 f^{6}\left({ }^{7} \mathrm{~F}\right) 6 s 6 p\left({ }^{3} \mathrm{P}^{\circ}\right){ }^{7} \mathrm{~F}^{\circ}\end{array}$ \\
\hline $4 f^{5}\left({ }^{6} \mathrm{H}^{\circ}\right) 5 d 6 s^{2}$ & ${ }^{7} \mathrm{I}^{\circ}$ & $\begin{array}{l}3 \\
4 \\
5 \\
6 \\
7 \\
8 \\
9\end{array}$ & $\begin{array}{l}18328.64 \\
19174.84 \\
20183.35 \\
21296.45 \\
22468.87\end{array}$ & $\begin{array}{l}0.33 \\
0.96: \\
1.03 \\
1.12 \\
1.235\end{array}$ & $\begin{array}{l}88 \\
86 \\
84 \\
82 \\
82 \\
83 \\
89\end{array}$ & & $\begin{array}{r}7 \\
9 \\
11 \\
12 \\
12 \\
8 \\
11\end{array}$ & $\begin{array}{l}\left({ }^{6} \mathrm{H}^{\circ}\right){ }^{7} \mathrm{H}^{\circ} \\
\left({ }^{6} \mathrm{H}^{\circ}\right){ }^{7} \mathrm{H}^{\circ} \\
\left({ }^{6} \mathrm{H}^{\circ}\right){ }^{7} \mathrm{H}^{\circ} \\
\left({ }^{6} \mathrm{H}^{\circ}\right){ }^{7} \mathrm{H}^{\circ} \\
\left({ }^{6} \mathrm{H}^{\circ}\right){ }^{7} \mathrm{H}^{\circ} \\
\left({ }^{6} \mathrm{H}^{\circ}\right){ }^{7} \mathrm{H}^{\circ} \\
\left({ }^{6} \mathrm{H}^{\circ}\right){ }^{7} \mathrm{~K}^{\circ}\end{array}$ \\
\hline $4 f^{5}\left({ }^{6} \mathrm{H}^{\circ}\right) 5 d 6 s^{2}$ & & 2 & 18416.62 & 1.08 & 15 & ${ }^{7} \mathrm{H}^{\circ}$ & 13 & $\left({ }^{6} \mathrm{H}^{\circ}\right){ }^{7} \mathrm{~F}^{\circ}$ \\
\hline $4 f^{6}\left({ }^{7} \mathrm{~F}\right) 6 s 6 p\left({ }^{3} \mathrm{P}^{\circ}\right)$ & ${ }^{5} \mathrm{~F}^{\circ}$ & $\begin{array}{l}1 \\
2 \\
3 \\
5\end{array}$ & $\begin{array}{l}18985.70 \\
19677.46 \\
20459.30 \\
21809.74\end{array}$ & $\begin{array}{l}0.18 \\
1.08 \\
1.295 \\
1.41\end{array}$ & $\begin{array}{l}58 \\
59 \\
55 \\
56\end{array}$ & & $\begin{array}{l}16 \\
14 \\
26 \\
14\end{array}$ & $\begin{array}{l}4 f^{5}\left({ }^{6} \mathrm{H}^{\circ}\right) 5 d 6 s^{2}{ }^{7} \mathrm{G}^{\circ} \\
\left({ }^{7} \mathrm{~F}\right)\left({ }^{3} \mathrm{P}^{\circ}\right){ }^{5} \mathrm{D}^{\circ} \\
\left({ }^{7} \mathrm{~F}\right)\left({ }^{3} \mathrm{P}^{\circ}\right){ }^{5} \mathrm{D}^{\circ} \\
4 f^{5}\left({ }^{6} \mathrm{H}^{\circ}\right) 5 d 6 s^{2}{ }^{7} \mathrm{~F}^{\circ}\end{array}$ \\
\hline $4 f^{5}\left({ }^{6} \mathrm{H}^{\circ}\right) 5 d 6 s^{2}$ & & 2 & 19009.52 & 0.945 & 37 & ${ }^{7} \mathrm{G}^{\circ}$ & 28 & $\left({ }^{6} \mathrm{H}^{\circ}\right){ }^{7} \mathrm{~F}^{\circ}$ \\
\hline $4 f^{5}\left({ }^{6} \mathrm{H}^{\circ}\right) 5 d 6 s^{2}$ & & 3 & 19210.09 & 1.27 & 22 & ${ }^{7} \mathrm{H}^{\circ}$ & 16 & $\left({ }^{6} \mathrm{H}^{\circ}\right){ }^{7} \mathrm{~F}^{\circ}$ \\
\hline $4 f^{6}\left({ }^{7} \mathrm{~F}\right) 6 s 6 p\left({ }^{1} \mathrm{P}^{\circ}\right)$ & ${ }^{7} \mathrm{D}^{\circ}$ & 5 & 19264.63 & 1.485 & 64 & & 23 & $\left({ }^{7} \mathrm{~F}\right)\left({ }^{3} \mathrm{P}^{\circ}\right){ }^{5} \mathrm{G}^{\circ}$ \\
\hline $4 f^{5}\left({ }^{6} \mathrm{H}^{\circ}\right) 5 d 6 s^{2}$ & ${ }^{7} \mathrm{G}^{\circ}$ & 3 & 19776.97 & 1.15 & 52 & & 22 & $4 f^{6}\left({ }^{7} \mathrm{~F}\right) 6 s 6 p\left({ }^{3} \mathrm{P}^{\circ}\right){ }^{7} \mathrm{G}^{\circ}$ \\
\hline $4 f^{5}\left({ }^{6} \mathrm{H}^{\circ}\right) 5 d 6 s^{2}$ & ${ }^{5} \mathrm{~F}^{0}$ & $\begin{array}{l}1 \\
2 \\
3 \\
4 \\
5\end{array}$ & $\begin{array}{l}20091.03 \\
20762.82 \\
21700.89 \\
22893.05\end{array}$ & $\begin{array}{l}0.09 \\
0.99 \\
1.235 \\
1.345\end{array}$ & $\begin{array}{l}55 \\
53 \\
46 \\
32 \\
28\end{array}$ & & $\begin{array}{l}12 \\
11 \\
15 \\
20 \\
20\end{array}$ & $\begin{array}{l}\left({ }^{6} \mathrm{H}^{\circ}\right){ }^{7} \mathrm{G}^{\circ} \\
4 f^{6}\left({ }^{7} \mathrm{~F}\right) 6 s 6 p\left({ }^{3} \mathrm{P}^{\circ}\right){ }^{7} \mathrm{G}^{\circ} \\
\left({ }^{6} \mathrm{H}^{\circ}\right){ }^{7} \mathrm{G}^{\circ} \\
\left({ }^{6} \mathrm{H}^{\circ}\right){ }^{5} \mathrm{G}^{\circ} \\
\left({ }^{6} \mathrm{H}^{\circ}\right)^{5} \mathrm{I}^{\circ}\end{array}$ \\
\hline
\end{tabular}


Sm I, Odd Parity_Continued

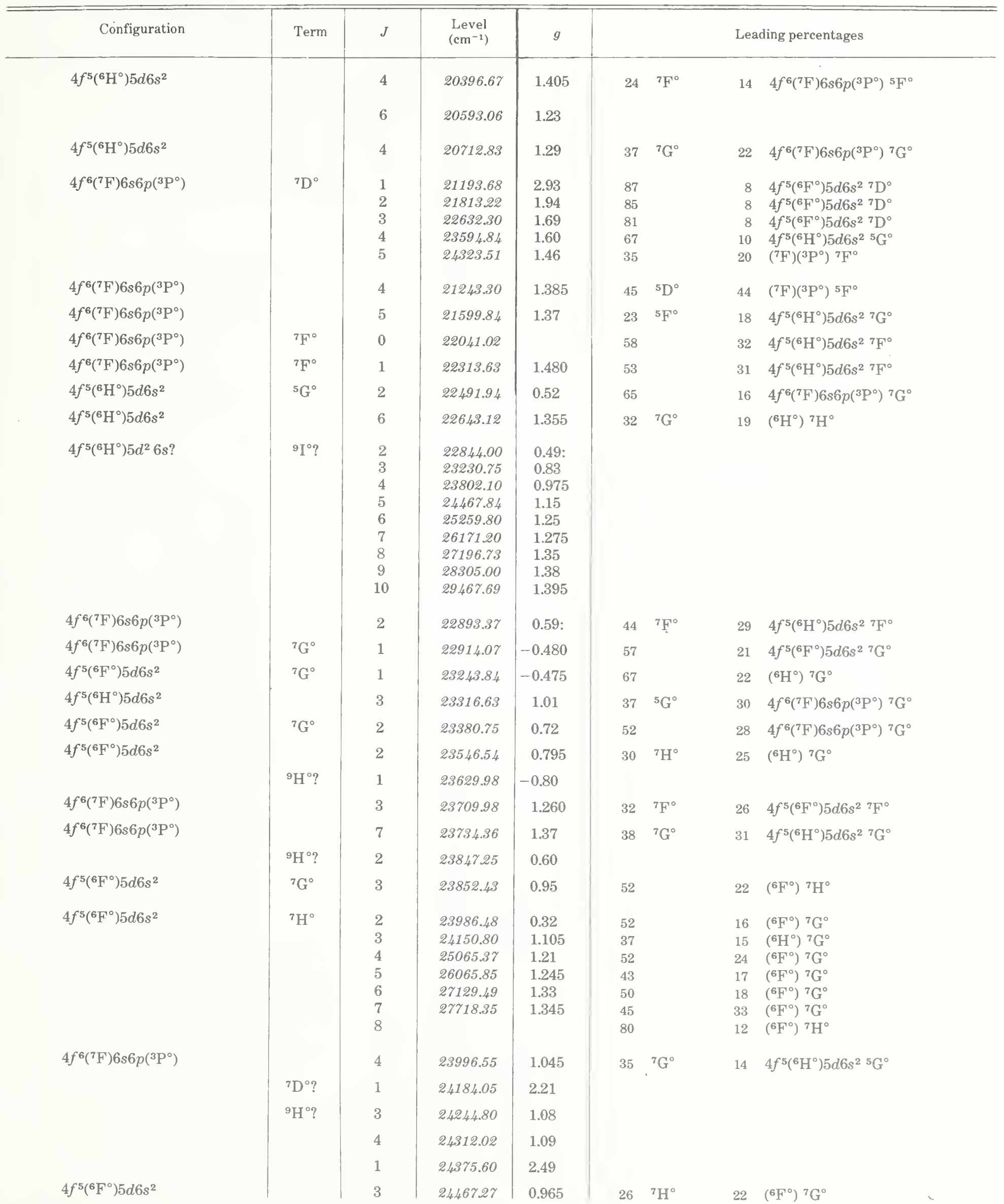


Sm I, Odd Parity-Continued

\begin{tabular}{|c|c|c|c|c|c|c|c|c|}
\hline \multirow[t]{3}{*}{ Configuration } & \multirow{2}{*}{$\begin{array}{l}\text { Term } \\
{ }^{7} \mathrm{D}^{\circ} ?\end{array}$} & \multirow{2}{*}{$\frac{J}{2}$} & \multirow{2}{*}{$\begin{array}{c}\begin{array}{c}\text { Level } \\
\left(\mathrm{cm}^{-1}\right)\end{array} \\
24498.96\end{array}$} & \multirow{2}{*}{$\frac{g}{1.73}$} & \multicolumn{4}{|c|}{ Leading percentages } \\
\hline & & & & & & & & \\
\hline & & 4 & 24570.03 & 1.04 & & & & \\
\hline $4 f^{5}\left({ }^{6} \mathrm{~F}^{\circ}\right) 5 d 6 s^{2}$ & & 4 & 24633.75 & 1.325 & 20 & ${ }^{7} \mathrm{~F}^{\circ}$ & 19 & $\left({ }^{6} \mathrm{~F}^{\circ}\right){ }^{7} \mathrm{G}^{\circ}$ \\
\hline $4 f^{6}\left({ }^{7} \mathrm{~F}\right) 6 s 6 p\left({ }^{3} \mathrm{P}^{\circ}\right)$ & & 5 & 24668.79 & 1.53 & 32 & ${ }^{7} \mathrm{D}^{\circ}$ & 17 & $\left({ }^{7} \mathrm{~F}\right)\left({ }^{3} \mathrm{P}^{\circ}\right)^{7} \mathrm{G}^{\circ}$ \\
\hline \multirow[t]{4}{*}{$4 f^{5}\left(6 \mathrm{~F}^{\circ}\right) 5 d 6 s^{2}$} & & 4 & 24772.74 & 1.25 & 27 & ${ }^{7} \mathrm{G}^{\circ}$ & 23 & $\left({ }^{6} \mathrm{~F}^{\circ}\right){ }^{7} \mathrm{H}^{\circ}$ \\
\hline & & 2 & 24913.28 & 1.770 & & & & \\
\hline & ${ }^{7} \mathrm{D}^{\circ} ?$ & 3 & 24967.14 & 1.56 & & & & \\
\hline & & 5 & 24977.83 & 1.20 & & & & \\
\hline $4 f^{5}\left({ }^{6} \mathrm{H}^{\circ}\right) 5 d 6 s^{2}$ & & 4 & 25387.17 & 1.055 & 26 & ${ }^{5} I^{\circ}$ & 18 & $\left({ }^{6} \mathrm{H}^{\circ}\right){ }^{5} \mathrm{G}^{\circ}$ \\
\hline \multirow[t]{4}{*}{$4 f^{5}\left({ }^{6} \mathrm{~F}^{\circ}\right) 5 d 6 s^{2}$} & & 5 & 25390.91 & 1.29 & 25 & ${ }^{7} \mathrm{H}^{\circ}$ & 24 & $\left({ }^{6} \mathrm{~F}^{\circ}\right){ }^{7} \mathrm{G}^{0}$ \\
\hline & & 5 & 25453.11 & 1.32 & & & & \\
\hline & ${ }^{7} \mathrm{D}^{\circ} ?$ & 4 & 25572.10 & 1.40 & & & & \\
\hline & & 3 & 25604.76 & 1.645 & & & & \\
\hline \multirow[t]{2}{*}{$4 f^{5}\left({ }^{6} \mathrm{H}^{\circ}\right) 5 d 6 s^{2}$} & & 5 & 25615.55 & 1.39 & 15 & ${ }^{7} \mathrm{G}^{\circ}$ & 14 & $\left({ }^{6} \mathrm{H}^{\circ}\right){ }^{5} \mathrm{~F}^{\circ}$ \\
\hline & & 4 & 25663.60 & 0.705 & & & & \\
\hline $4 f^{5}\left({ }^{6} \mathrm{H}^{\circ}\right) 5 d 6 s^{2}$ & & 5 & 25675.50 & 1.12 & 38 & $5 \mathrm{I}^{\circ}$ & 25 & $\left({ }^{6} \mathrm{~F}^{\circ}\right){ }^{7} \mathrm{G}^{\circ}$ \\
\hline $4 f^{6}\left({ }^{7} \mathrm{~F}\right) 5 d 6 p\left({ }^{3} \mathrm{~F}^{\circ}\right) ?$ & ${ }^{9} \mathrm{I}^{\circ} ?$ & $\begin{array}{c}2 \\
3 \\
4 \\
5 \\
6 \\
7 \\
8 \\
9 \\
10\end{array}$ & $\begin{array}{l}25808.90 \\
26214.75 \\
26752.26 \\
27852.78 \\
28864.89 \\
29948.38 \\
31129.79 \\
32129.89 \\
33808.29\end{array}$ & $\begin{array}{c}-0.22 \\
0.55 \\
0.94 \\
1.16 \\
1.25 \\
1.325 \\
1.360 \\
1.43 \\
1.42\end{array}$ & & & & \\
\hline $4 f^{6}\left({ }^{7} \mathrm{~F}\right) 6 s 6 p\left({ }^{3} \mathrm{P}^{\circ}\right)$ & & 6 & 25840.31 & 1.48 & 40 & ${ }^{7} \mathrm{G}^{\circ}$ & 17 & $4 f^{5}\left({ }^{6} \mathrm{H}^{\circ}\right) 5 d 6 s^{2}{ }^{7} \mathrm{G}^{\circ}$ \\
\hline \multirow[t]{6}{*}{$4 f^{5}\left({ }^{6} \mathrm{~F}^{\circ}\right) 5 d 6 s^{2}$} & ${ }^{7} \mathrm{P}^{\circ}$ & 2 & 25979.30 & 2.535 & 95 & & 2 & $\left({ }^{6} \mathrm{~F}^{\circ}\right){ }^{5} \mathrm{P}^{\circ}$ \\
\hline & & 2 & 26054.05 & 2.34 & & & & \\
\hline & & 1 & 26146.01 & & & & & \\
\hline & & 6 & 26180.92 & 1.29 & & & & \\
\hline & ${ }^{7} \mathrm{H}^{0} ?$ & 2 & 26220.86 & 0.09 & & & & \\
\hline & ${ }^{7} I^{\circ} ?$ & 3 & 26267.14 & 0.39 & & & & \\
\hline \multirow[t]{9}{*}{$4 f^{5}\left({ }^{6} \mathrm{~F}^{\circ}\right) 5 d 6 s^{2}$} & ${ }^{7} \mathbf{F}^{\circ}$ & 0 & & & 59 & & 25 & $4 f^{6}\left({ }^{7} \mathrm{~F}\right) 6 s 6 p\left({ }^{3} \mathrm{P}^{\circ}\right){ }^{7} \mathrm{~F}^{\circ}$ \\
\hline & & 1 & 26281.09 & 1.50 & 58 & & 25 & \\
\hline & & $\begin{array}{l}2 \\
3\end{array}$ & 26786.80 & $\begin{array}{l}1.34: \\
1.49\end{array}$ & $\begin{array}{l}58 \\
59\end{array}$ & & $\begin{array}{l}25 \\
24\end{array}$ & \\
\hline & & 4 & 28180.95 & 1.50 & 58 & & 24 & \\
\hline & & 5 & 29023.96 & 1.405 & 54 & & 23 & \\
\hline & & 6 & 30092.53 & 1.455 & 49 & & 20 & \\
\hline & ${ }^{7} \mathrm{D}^{\circ} ?$ & 5 & 26311.45 & 1.50 & & & & \\
\hline & & 4 & 2638225 & 1.57 & & & & \\
\hline & ${ }^{9} \mathrm{H}^{\circ} ?$ & 1 & 26438.25 & -0.81 & & & & \\
\hline \multirow[t]{5}{*}{$4 f^{5}\left({ }^{6} \mathrm{~F}^{\circ}\right) 5 d 6 s^{2}$} & & 6 & 26471.33 & 1.355 & 28 & ${ }^{7} \mathrm{H}^{\circ}$ & 25 & $\left({ }^{6} \mathrm{~F}^{\circ}\right){ }^{7} \mathrm{G}^{\circ}$ \\
\hline & & 5 & 26507.31 & 0.935 & & & & \\
\hline & & 3 & 26619.16 & 1.935 & & & & - \\
\hline & & 4 & 26657.10 & 0.91 & & & & \\
\hline & & 3 & 26703.85 & 1.900 & & & & \\
\hline
\end{tabular}


Sm I, Odd Parity-Continued

\begin{tabular}{|c|c|c|c|c|c|c|c|}
\hline Configuration & Term & $J$ & $\begin{array}{l}\text { Level } \\
\left(\mathrm{cm}^{-1}\right)\end{array}$ & $g$ & \multicolumn{3}{|c|}{ Leading percentages } \\
\hline & & 4 & 28401.00 & 1.42 & & & \\
\hline & ${ }^{7} \mathrm{H}^{\circ} ?$ & 4 & 28425.30 & 1.01 & & & \\
\hline & & 2 & 28445.02 & 1.54 & & & \\
\hline & & 4 & 28465.30 & 1.140 & & & \\
\hline & & 2 & 28496.57 & 1.53 & & & \\
\hline & & 3 & 28505.72 & 1.56 & & & \\
\hline & ${ }^{9} \mathrm{H}^{\circ} ?$ & 5 & 28534.04 & 1.300 & & & \\
\hline & & 3 & 28585.07 & 0.935 & & & \\
\hline & ${ }^{7} \mathrm{I}^{\circ} ?$ & 6 & 28600.02 & 1.165 & & & \\
\hline & ${ }^{7} \mathrm{D}^{\circ} ?$ & 1 & 28613.22 & 2.125: & & & \\
\hline & & 5 & 28694.06 & 1.67 & & & \\
\hline & & 1 & 28704.25 & 1.725: & & & \\
\hline & & 4 & 28705.58 & 1.53 & & & \\
\hline & & 3 & 28744.99 & 1.425 & & $\cdot$ & \\
\hline & & 4 & 28752.34 & 0.96 & & & \\
\hline & & 4 & 2885325 & 1.19 & & & \\
\hline & & 3 & 28855.76 & 1.37 & & & \\
\hline & & 1 & 28913.97 & 0.27 & & & \\
\hline & & 3 & 28942.00 & 1.02 & & & \\
\hline & & 3 & 28998.13 & 1.48 & & & \\
\hline & & 2 & 29041.31 & 1.36 & & & \\
\hline & & 5 & 29069.90 & 1.33 & & & \\
\hline & & 1 & 29130.03 & 0.495 & & & \\
\hline & ${ }^{7} \mathrm{H}^{\circ} ?$ & 5 & 29151.15 & 1.23 & & & \\
\hline & & 4 & 29152.26 & 1.45 & & & \\
\hline & & 2 & 29156.00 & 1.79 & & & \\
\hline & & 2 & 29200.62 & 1.33 & & & \\
\hline & & 4 & 29210.73 & 1.13 & & & \\
\hline & & 3 & 29226.71 & 1.68 & & & \\
\hline & & 3 & 29282.28 & 2.00 & & & \\
\hline \multirow[t]{14}{*}{$4 f^{5}\left({ }^{6} \mathrm{H}^{\circ}\right) 5 d 6 s^{2}$} & & 6 & 29379.80 & 1.215 & $42{ }^{5} \mathrm{H}^{\circ}$ & $19\left({ }^{6} \mathrm{~F}^{0}\right){ }^{5} \mathrm{H}^{\circ}$ & \\
\hline & & 5 & 29475.54 & 1.26 & & & \\
\hline & & 2 & 29478.74 & 2.06 & & & \\
\hline & & 4 & 29481.25 & 1.10 & & & \\
\hline & & 5 & 29490.40 & 1.20 & & & \\
\hline & & 2 & 29506.40 & 1.11 & & & • \\
\hline & & 4 & 29521.75 & 1.36 & & & \\
\hline & & 4 & 29557.87 & 0.93 & & & \\
\hline & ${ }^{9} \mathrm{H}^{\circ}$ ? & 9 & 29564.24 & 1.435 & & & \\
\hline & & 5 & 29602.09 & 1.20 & & & \\
\hline & ${ }^{7} I^{\circ} ?$ & 7 & 29627.02 & 1.25 & & & \\
\hline & & 2 & 29639.45 & 0.305 & & & \\
\hline & & 3 & 29648.85 & 1.230 & & & \\
\hline & & 4 & 29651.20 & 1.20 & & & \\
\hline
\end{tabular}


Sm I, Odd Parity-Continued

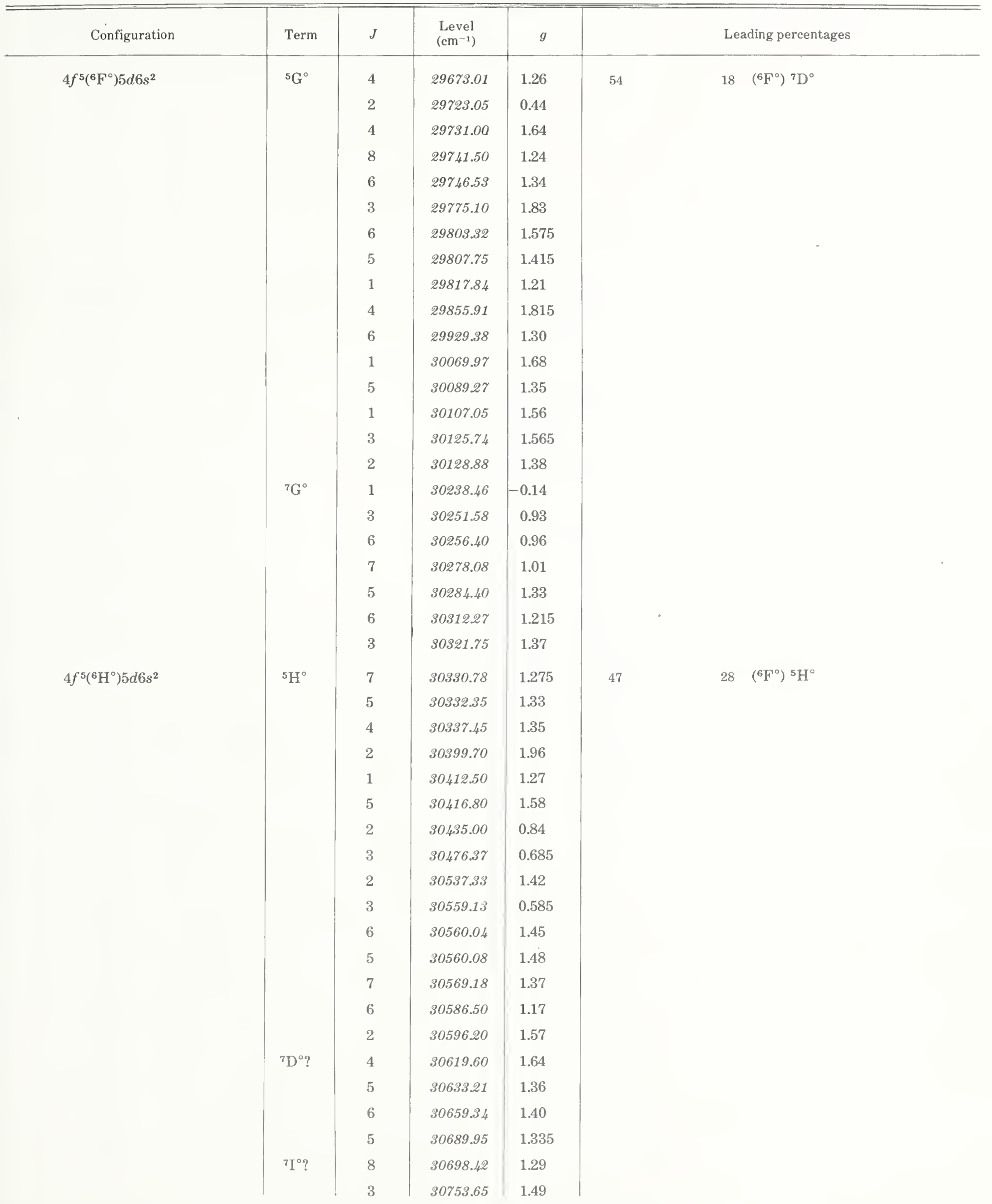




\begin{tabular}{|c|c|c|c|c|c|c|c|c|c|}
\hline Configuration & Term & $J$ & $\begin{array}{l}\text { Level } \\
\left(\mathrm{cm}^{-1}\right)\end{array}$ & $g$ & Configuration & Term & $J$ & $\begin{array}{l}\text { Level } \\
\left(\mathrm{cm}^{-1}\right)\end{array}$ & $g$ \\
\hline & & 1 & 30753.72 & 1.32 & & & 2 & 31786.74 & 1.25 \\
\hline & & 7 & 30770.00 & 1.37 & & & 3 & 31820.91 & 1.25 \\
\hline & & 4 & 30775.70 & 0.68 & & & 6 & 31845.65 & 1.33: \\
\hline & & 3 & 30788.75 & 0.75 & & & 6 & 31865.83 & 1.42: \\
\hline & & 2 & 30796.27 & 1.32 & & & 3 & 31893.78 & 1.34 \\
\hline & & 2 & 30823.54 & 1.72 & & & 4 & 31904.93 & 1.55 \\
\hline & & 3 & 30836.69 & 1.47 & & & 0 & 31923.80 & \\
\hline & & 2 & 30901.40 & 1.17 & & & 5 & 31924.70 & 1.29 \\
\hline & & 1 & 30911.22 & 1.54 & & & 2 & 31934.03 & 1.81 \\
\hline & & 3 & 30963.07 & 1.49 & & & 7 & 31960.69 & 1.495 \\
\hline & & 4 & 31009.20 & 1.425 & & & 5 & 32028.11 & 1.475 \\
\hline & & 6 & 31015.20 & 1.415 & & & 1 & 32086.66 & 1.42 \\
\hline & & 5 & 31024.75 & 1.41: & & & 4 & 32125.53 & 1.17 \\
\hline & & 1 & 31041.00 & 0.60 & & & 4 & 32149.28 & 1.565 \\
\hline & & 5 & 31047.10 & 1.11: & & & 4 & 32188.17 & 1.43 \\
\hline & & 2 & 31062.30 & 1.43 & & & 5 & 32197.35 & 1.35 \\
\hline & & 6 & 31108.42 & 1.545 & & & 8 & 32242.45 & 1.47 \\
\hline & & 4 & 31156.40 & 1.64 & & & 3 & 32252.30 & 1.47 \\
\hline & & 6 & 31174.68 & 1.29 & & & 5 & 32260.63 & 1.13 \\
\hline & & 3 & 31185.20 & 1.50 & & & 6 & 32284.49 & 1.46 \\
\hline & & 7 & 31207.22 & 1.435 & & & 4 & 32289.85 & 1.22 \\
\hline & & 5 & 31210.33 & 1.27 & & & 2 & 32294.20 & 1.73 \\
\hline & & 1 & 31219.70 & 2.66 & & & 5 & 32340.69 & 1.41 \\
\hline & & 4 & 31234.62 & 1.21 & & & 4 & 32346.24 & 1.66 \\
\hline & & 4 & 31322.84 & 1.29 & & & 2 & 32401.65 & 1.58 \\
\hline & & 3 & 31342.95 & 1.57 & & & 1 & 32402.62 & 1.93 \\
\hline & & 3 & 31352.05 & 1.50 & & & 2 & 32558.70 & 1.76 \\
\hline & & 4 & 31364.15 & 1.20 & & & 5 & 32626.35 & 1.36 \\
\hline & & 1 & 31391.80 & 0.19 & & & 6 & 82696.63 & 1.34 \\
\hline & & 4 & 31421.00 & 1.32 & & & 5 & 32735.62 & 1.26 \\
\hline & & 2 & 31439.15 & 0.765 & & & 3 & 32822.82 & 1.50 \\
\hline & & 3 & 31483.52 & 1.43 & & & 5 & 32831.50 & 1.355 \\
\hline & & 4 & 31493.41 & 1.16 & & & 1 & 32865.57 & 0.84 \\
\hline & & 1 & 31503.45 & 1.12 & & & 5 & 32868.71 & 1.42 \\
\hline & & 6 & 31503.83 & 1.42 & & & 3 & 32907.64 & 1.49 \\
\hline & & 8 & 31534.12 & 1.40 & & & 1 & 33030.91 & 0.00 \\
\hline & & 5 & 31568.40 & 1.04 & & & 6 & 33081.45 & 1.28 \\
\hline & & 1 & 31584.91 & 2.77 & & & 2 & 33199.40 & 1.96 \\
\hline & & 3 & 31597.53 & 1.73 & & & 8 & 33318.35 & 1.345 \\
\hline & & 4 & 31665.43 & 1.52 & & & 4 & 33329.35 & 1.37 \\
\hline & ${ }^{9} \mathrm{H}^{\circ} ?$ & 8 & 31699.20 & 1.400 & & & 2 & 33382.06 & 0.90 \\
\hline & & 2 & 31716.41 & 1.950 & & & 6 & 33384.92 & 1.35 \\
\hline & ${ }^{7} \mathrm{I}^{\circ} ?$ & 9 & 31746.67 & 1.35 & & & 7 & 33408.80 & 1.32 \\
\hline & & 5 & 31754.23 & 1.38 & & & 4 & 33411.09 & 1.52 \\
\hline
\end{tabular}


Sm I, Odd Parity—Continued

Sm I, Odd Parity-Continued

\begin{tabular}{|c|c|c|c|c|c|c|c|c|c|}
\hline Configuration & Term & $J$ & $\begin{array}{l}\text { Level } \\
\left(\mathrm{cm}^{-1}\right)\end{array}$ & $g$ & Configuration & Term & $J$ & $\begin{array}{l}\text { Level } \\
\left(\mathrm{cm}^{-1}\right)\end{array}$ & $g$ \\
\hline & & 4 & 33458.94 & 1.600 & \multirow{10}{*}{$\operatorname{Sm~II~}\left({ }^{6} F_{1 / 2}\right)$} & \multirow{5}{*}{${ }^{9} \mathrm{H}^{\circ}$ ? } & 4 & 34514.74 & 1.45 \\
\hline & & 7 & 33507.28 & 1.350 & & & 6 & 34723.16 & 1.35 \\
\hline & & 2 & 33634.82 & 2.22 & & & 8 & 34761.78 & 1.430 \\
\hline & & 3 & 33733.00 & 1.505 & & & 9 & 35176.51 & \multirow[t]{7}{*}{1.43} \\
\hline & & 7 & 33739.36 & 1.460 & & & & & \\
\hline & & 3 & 33838.15 & 1.35 & & \multirow{5}{*}{ Limit } & & \multirow[b]{2}{*}{45519} & \\
\hline & & 4 & 33983.40 & 1.48 & & & & & \\
\hline & & 2 & 34044.11 & 2.03 & & & $4 ?$ & $45775 a ?$ & \\
\hline & & 3 & 34189.36 & 1.88 & & & $4,5,6$ & $45865 a ?$ & \\
\hline & & 5 & 34224.44 & & & & $4,5,6$ & $46015 a ?$ & \\
\hline
\end{tabular}


(Pm I sequence; 61 electrons)

Ground state $\left(1 s^{2} 2 s^{2} 2 p^{6} 3 s^{2} 3 p^{6} 3 d^{10} 4 s^{2} 4 p^{6} 4 d^{10} 5 s^{2} 5 p^{6}\right) 4 f^{6} 6 s^{8} \mathrm{~F}_{1 / 2}$

Ionization energy $89300 \pm 600 \mathrm{~cm}^{-1}$

$11.07 \pm 0.08 \mathrm{eV}$

Identified even configurations $\quad 4 f^{6} 6 s, 4 f^{6} 5 d$

Identified odd configurations $\quad 4 f^{7}, 4 f^{6} 6 p ?, 4 f^{5} 5 d 6 s ?$

Albertson's analysis of this spectrum yielded about 40 low even levels, which he assigned to terms of the $4 f^{6}\left({ }^{7} \mathrm{~F}\right) 6 s$ and $4 f^{6}\left({ }^{7} \mathrm{~F}\right) 5 d$ subconfigurations, and more than 200 upper odd levels. The group at the Laboratoire Aime Cotton, Orsay, has revised and extended the analysis to include 70 even levels and more than 300 upper odd levels. We have taken the levels and $g$ values from their work as given in the article by Blaise, Morillon, Schweighofer, and Verges [1969] and in the theses of Carlier [1967] and Henny-Schweighofer [1970]. Carlier's calculation of the $\left(4 f^{6}\left({ }^{7} \mathrm{~F}\right) 6 s+4 f^{6}\left({ }^{7} \mathrm{~F}\right) 5 d\right)$ configurations is the source of the percentage compositions.

Blaise et al. found the $4 f^{7}{ }^{8} \mathrm{~S}_{7 / 2}^{\circ}$ term, which is the lowest known odd level. Most of the odd levels are expected to belong to the group of four configurations $\left(4 f^{6} 6 p+4 f^{5}(5 d+6 s)^{2}\right)$; a calculation including these configurations and their interactions is needed. Although singleconfiguration assignments will no doubt be meaningless for some of these levels, the tentative assignments listed here may prove useful in correlating experimental and calculated levels. Albertson made most of the tentative assignments to $4 f^{6}\left({ }^{7} \mathrm{~F}\right) 6 p$. Blaise [1976] has furnished additional tentative assignments, mostly to $4 f^{5} 5 d 6 s$, and has revised some of Albertson's designations. A few odd levels having low $J$ values and distinctive $g$ values were assigned octet designations by Blaise et al. ( $\mathrm{Six}^{8} \mathrm{~F}_{1 / 2}^{\circ}$ and five ${ }^{8} \mathrm{G}_{1 / 2}^{\circ}$ levels, for example, are known.)

The ionization potential is from Sugar and Reader.

The various observations of Sm spectra mentioned in the preceding text for Sm I included measurements of Sm II, and the description of these lists need not be repeated. King's 1935 list, which gives almost 1800 lines of Sm II, was extended somewhat for Sm II by King and Albertson, and was also supplemented by Zeeman data [Albertson, 1936]. Albertson's list of about 1200 classified Sm II lines has been extended by Henny-Schweighofer [1967], Blaise et al. [1969], and Morillon [1970].

In his 1936 paper Albertson also gave a noteworthy description of rare-earth spectra in general. He divided these into classes by character and noted that the order in which they were being analyzed corresponded to an increasing complexity. Using the different characters of the spectra and the known ground configurations for La I, Sm I, Eu I, Gd I, and Yb I, Albertson gave a table of ground configurations for all the neutral rare earths that has proved correct except for two elements.

\section{References}

Albertson, W., Astrophys. J. 84, 26 (1936). EL CL W ZE IP

Albertson, W., and King, A. S., Phys. Rev. 49, 209 (1936). ZE

Becher, J., Thesis, Johns Hopkins Univ., Baltimore, 135 pp. (1965); (Univ. Microfilms, Ann Arbor, Mich., No. 66-4956). PT

Blaise, J., private communication (1976). ND

Blaise, J., Morillon, C., Schweighofer, M. G., and Verges, J., Spectrochim. Acta, Part B 24, 405 (1969). EL CL W ZE

Carlier, A., Thesis (Third Cycle), Univ. Paris, Orsay, 128 pp. (1967). EL ND PT

Henny-Schweighofer, M. G., Thesis (Third Cycle), Univ. Paris, Orsay, 174 pp. (1970). EL CL W ZE IS

Kiess, C. C., Sci. Papers Bur. Stand. (U.S.) 18, 201 (1922). W

King, A. S., Astrophys. J. 82, 140 (1935). W

Morillon, C., Spectrochim. Acta, Part B 25, 513 (1970). CL W

Sugar, J., and Reader, J., J. Opt. Soc. Am. 55, 1286 (1965). IP

[July 1976] 
Sm II, Even Parity

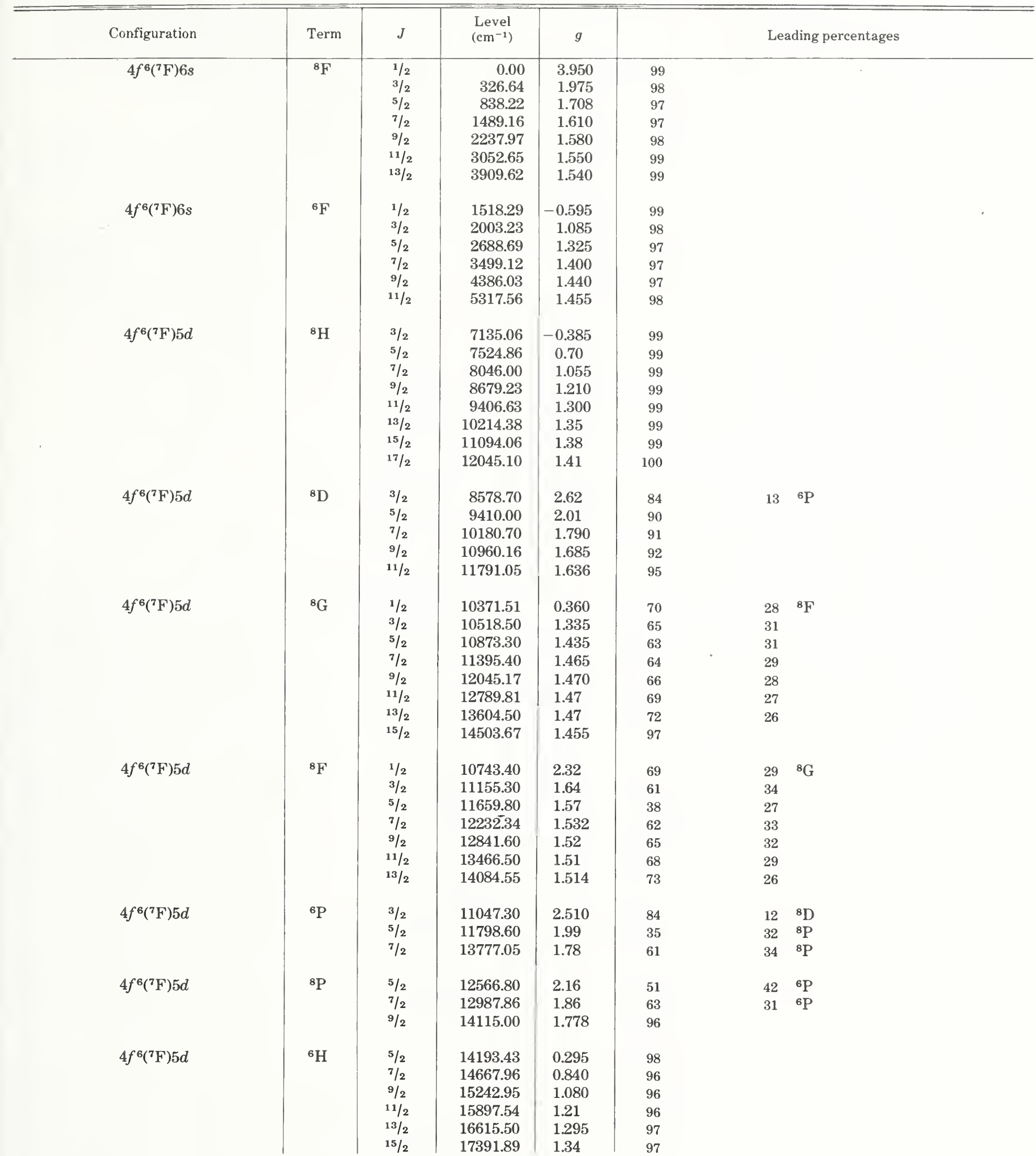


Sm II, Even Parity_Continued

\begin{tabular}{|c|c|c|c|c|c|c|}
\hline Configuration & Term & $J$ & $\begin{array}{l}\text { Level } \\
\left(\mathrm{cm}^{-1}\right)\end{array}$ & $g$ & & Leading percentages \\
\hline $4 f^{6}\left({ }^{7} \mathrm{~F}\right) 5 d$ & ${ }^{6} \mathrm{~F}$ & $\begin{array}{l}3 / 2 \\
1 / 2 \\
7 / 2 \\
9 / 2 \\
11 / 2\end{array}$ & $\begin{array}{l}16077.75 \\
16162.32 \\
17005.30 \\
17717.86 \\
18506.80\end{array}$ & $\begin{array}{l}1.35 \\
0.300 \\
1.405 \\
1.52\end{array}$ & $\begin{array}{l}46 \\
88 \\
43 \\
46 \\
70\end{array}$ & $\begin{array}{cc}26 & { }^{6} \mathrm{D} \\
10 & { }^{6} \mathrm{D} \\
41 & { }^{6} \mathrm{D} \\
42 & { }^{6} \mathrm{D} \\
27 & { }^{6} \mathrm{G}\end{array}$ \\
\hline $4 f^{6}\left({ }^{7} \mathrm{~F}\right) 5 d$ & ${ }^{6} \mathrm{D}$ & $\begin{array}{l}5 / 2 \\
1 / 2 \\
3 / 2 \\
7 / 2 \\
9 / 2\end{array}$ & $\begin{array}{l}16428.70 \\
17054.60 \\
17568.38 \\
18807.69 \\
19400.27\end{array}$ & $\begin{array}{l}1.355 \\
1.58 \\
1.52\end{array}$ & $\begin{array}{l}50 \\
87 \\
66 \\
43 \\
48\end{array}$ & $\begin{array}{ll}38 & { }^{6} \mathrm{~F} \\
10 & { }^{6} \mathrm{~F} \\
27 & { }^{6} \mathrm{~F} \\
35 & { }^{6} \mathrm{G} \\
33 & { }^{6} \mathrm{G}\end{array}$ \\
\hline $4 f^{6}\left({ }^{7} \mathrm{~F}\right) 5 d$ & & $5 / 2$ & 18050.94 & 1.54 & $37 \quad{ }^{6} \mathrm{D}$ & ${ }^{6} \mathrm{G}$ \\
\hline $4 f^{6}\left({ }^{7} \mathrm{~F}\right) 5 d$ & ${ }^{6} \mathrm{G}$ & $\begin{array}{l}3 / 2 \\
5 / 2 \\
7 / 2 \\
9 / 2 \\
13 / 2 \\
11 / 2\end{array}$ & $\begin{array}{l}18478.13 \\
19035.41 \\
19627.90 \\
20179.48 \\
20582.56 \\
20648.02\end{array}$ & $\begin{array}{l}0.01 \\
\\
1.30 \\
1.35 \\
\\
1.35\end{array}$ & $\begin{array}{l}71 \\
59 \\
54 \\
55 \\
97 \\
70\end{array}$ & $\begin{array}{cc}25 & { }^{6} \mathrm{~F} \\
33 & { }^{6} \mathrm{~F} \\
36 & { }^{6} \mathrm{~F} \\
36 & { }^{6} \mathrm{~F} \\
& \\
28 & { }^{6} \mathrm{~F}\end{array}$ \\
\hline $\mathrm{Sm}$ III $\left({ }^{7} \mathrm{~F}_{0}\right)$ & Limit & 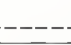 & 89300 & & & \\
\hline
\end{tabular}

Sm II, Odd Parity

Sm II, Odd Parity

\begin{tabular}{|c|c|c|c|c|c|c|c|c|c|}
\hline Configuration & Term & $J$ & $\begin{array}{l}\text { Level } \\
\left(\mathrm{cm}^{-1}\right)\end{array}$ & $g$ & Configuration & Term & $J$ & $\begin{array}{l}\text { Level } \\
\left(\mathrm{cm}^{-1}\right)\end{array}$ & $g$ \\
\hline $4 f^{7}$ & ${ }^{8} \mathrm{~S}^{\circ}$ & $7 / 2$ & 18288.85 & 2.01 & & \multirow{22}{*}{${ }^{8} \mathrm{~F}^{\circ}$} & $5 / 2$ & 24194.38 & 0.275 \\
\hline $4 f^{6}\left({ }^{7} \mathrm{~F}\right) 6 p ?$ & ${ }^{8} \mathrm{G}^{\circ}$ & $1 / 2$ & 21250.75 & -1.155 & & & $1 / 2$ & 24221.81 & 2.41 \\
\hline \multirow[t]{2}{*}{$4 f^{5}\left({ }^{6} \mathrm{H}^{\circ}\right) 5 d 6 s ?$} & & $3 / 2$ & 21507.87 & 0.19 & $4 f^{6}\left({ }^{7} \mathrm{~F}\right) 6 p ?$ & & $9 / 2$ & 24257.37 & 1.445 \\
\hline & ${ }^{8} \mathrm{~F}^{\circ}$ & $1 / 2$ & 21655.42 & 3.735 & & & $7 / 2$ & 24358.12 & 0.75 \\
\hline $4 f^{6}\left({ }^{7} \mathrm{~F}\right) 6 p ?$ & & $3 / 2$ & 21702.33 & 0.515 & $4 f^{6}\left({ }^{7} \mathrm{~F}\right) 6 p ?$ & & $3 / 2$ & 24429.52 & 1.030 \\
\hline $4 f^{5}\left({ }^{6} \mathrm{H}^{\circ}\right) 5 d 6 s ?$ & & $5 / 2$ & 21813.61 & 0.255 & $4 f^{6}\left({ }^{7} \mathrm{~F}\right) 6 p ?$ & & $5 / 2$ & 24582.59 & 1.29 \\
\hline $4 f^{6}\left({ }^{7} \mathrm{~F}\right) 6 p ?$ & & $3 / 2$ & 21904.12 & 1.81 & $4 f^{6}\left({ }^{7} \mathrm{~F}\right) 6 p ?$ & & $11 / 2$ & 24588.00 & 1.39 \\
\hline $4 f^{5}\left({ }^{6} \mathrm{H}^{\circ}\right) 5 d 6 s ?$ & & $5 / 2$ & 22039.98 & 0.70 & & & $3 / 2$ & 24685.53 & 0.360 \\
\hline $4 f^{6}\left({ }^{7} \mathrm{~F}\right) 6 p ?$ & & $5 / 2$ & 22248.32 & 1.360 & $4 f^{6}\left({ }^{7} \mathrm{~F}\right) 6 p ?$ & & $7 / 2$ & 24689.84 & 1.41 \\
\hline $4 f^{6}\left({ }^{7} \mathrm{~F}\right) 6 p ?$ & & $5 / 2$ & 22429.49 & 1.30 & $4 f^{5}\left({ }^{6} \mathrm{H}^{\circ}\right) 5 d 6 s ?$ & & $11 / 2$ & 24816.28 & 1.36 \\
\hline $4 f^{5}\left({ }^{6} \mathrm{H}^{\circ}\right) 5 d 6 s ?$ & & $7 / 2$ & 22788.68 & 1.045 & & & $5 / 2$ & 24848.47 & 1.08 \\
\hline $4 f^{6}\left({ }^{7} \mathrm{~F}\right) 6 p ?$ & & $7 / 2$ & 22875.41 & 1.46 & & & $7 / 2$ & 24897.91 & 0.835 \\
\hline $4 f^{6}\left({ }^{7} \mathrm{~F}\right) 6 p ?$ & & $3 / 2$ & 23177.49 & 0.70 & $4 f^{5}\left({ }^{6} \mathrm{H}^{\circ}\right) 5 d 6 s ?$ & & $1 / 2$ & 24919.90 & -0.85 \\
\hline \multirow[t]{3}{*}{$4 f^{6}\left({ }^{7} \mathrm{~F}\right) 6 p ?$} & & $7 / 2$ & 23260.95 & 1.355 & $4 f^{6}\left({ }^{7} \mathrm{~F}\right) 6 p ?$ & & $5 / 2$ & 24928.80 & 1.220 \\
\hline & & $5 / 2$ & 23321.24 & 0.37 & & & $3 / 2$ & 25055.54 & 1.21 \\
\hline & & $1 / 2$ & 23352.41 & -0.505 & & & $1 / 2$ & 25115.55 & 3.52 \\
\hline \multirow[t]{2}{*}{$4 f^{6}\left({ }^{7} \mathrm{~F}\right) 6 p ?$} & & $9 / 2$ & 23646.90 & 1.35 & & & $5 / 2$ & 25175.32 & 0.945 \\
\hline & & $1 / 2$ & 23659.99 & 0.235 & $4 f^{5}\left({ }^{6} \mathrm{H}^{\circ}\right) 5 d 6 s ?$ & & $3 / 2$ & 25178.45 & 0.98 \\
\hline $4 f^{5}\left({ }^{6} \mathrm{H}^{\circ}\right) 5 d 6 s ?$ & & $9 / 2$ & 23752.70 & 1.32 & $4 f^{6}\left({ }^{7} \mathrm{~F}\right) 6 p ?$ & & $7 / 2$ & 25304.09 & 1.26 \\
\hline $4 f^{6}\left({ }^{7} \mathrm{~F}\right) 6 p ?$ & & $5 / 2$ & 2384220 & 1.125 & & & $9 / 2$ & 25336.52 & 1.010 \\
\hline \multirow[t]{2}{*}{$4 f^{6}\left({ }^{7} \mathrm{~F}\right) 6 p ?$} & & $3 / 2$ & 2396225 & 1.25 & $4 f^{6}\left({ }^{7} \mathrm{~F}\right) 6 p ?$ & & $3 / 2$ & 25361.45 & 1.49 \\
\hline & & $3 / 2$ & 24013.56 & 0.83 & $4 f^{6}\left({ }^{7} \mathrm{~F}\right) 6 p ?$ & & $11 / 2$ & 25385.36 & 1.49 \\
\hline
\end{tabular}


Sm II, Odd Parity-Continued

Sm II, Odd Parity-Continued

\begin{tabular}{|c|c|c|c|c|c|c|c|c|c|}
\hline Configuration & Term & $J$ & $\begin{array}{l}\text { Level } \\
\left(\mathrm{cm}^{-1}\right)\end{array}$ & $g$ & Configuration & Term & $J$ & $\begin{array}{l}\text { Level } \\
\left(\mathrm{cm}^{-1}\right)\end{array}$ & $g$ \\
\hline & & $5 / 2$ & 25417.14 & 0.82 & $4 f^{6}\left({ }^{7} \mathrm{~F}\right) 6 p ?$ & & $7 / 2$ & 2746420 & 1.206 \\
\hline & & $5 / 2$ & 25546.03 & 0.77 & & ${ }^{8} \mathrm{H}^{\circ}$ & $3 / 2$ & 27492.95 & -0.225 \\
\hline & & $3 / 2$ & 25552.80 & 1.86 & & & $7 / 2$ & 27552.45 & 1.30 \\
\hline $4 f^{6}\left({ }^{7} \mathrm{~F}\right) 6 p ?$ & & $7 / 2$ & 25565.97 & 1.33 & $4 f^{5} 5 d 6 s ?$ & & $5 / 2$ & 27631.18 & 1.165 \\
\hline $4 f^{6}\left({ }^{7} \mathrm{~F}\right) 6 p ?$ & & $9 / 2$ & 25597.70 & 1.535 & $4 f^{6}\left({ }^{7} \mathrm{~F}\right) 6 p ?$ & & $11 / 2$ & 27638.83 & 1.315 \\
\hline \multirow[t]{2}{*}{$4 f^{6}\left({ }^{7} \mathrm{~F}\right) 6 p ?$} & & $13 / 2$ & 25664.97 & 1.415 & & & $13 / 2$ & 27639.40 & $1.26:$ \\
\hline & & $7 / 2$ & 25790.15 & 1.25 & $4 f^{5} 5 d 6 s ?$ & & $13 / 2$ & 27695.96 & 1.36 \\
\hline $4 f^{5}\left({ }^{6} \mathrm{H}^{\circ}\right) 5 d 6 s ?$ & & $13 / 2$ & 25939.87 & 1.405 & & & $9 / 2$ & 27824.78 & 1.105 \\
\hline $4 f^{5}\left({ }^{6} \mathrm{H}^{\circ}\right) 5 d 6 s ?$ & & $5 / 2$ & 25980.32 & 1.33 & & & $3 / 2$ & 27829.77 & 1.185 \\
\hline \multirow[t]{10}{*}{$4 f^{6}\left({ }^{7} \mathrm{~F}\right) 6 p ?$} & & $9 / 2$ & 26046.35 & 1.34 & & & $9 / 2$ & 27849.30 & 1.505 \\
\hline & & $7 / 2$ & 26086.63 & 1.30 & & & $5 / 2$ & 27923.96 & 0.760 \\
\hline & & $7 / 2$ & 26159.60 & 1.28 & & & $3 / 2$ & 27942.33 & 2.18 \\
\hline & & $5 / 2$ & 26190.92 & $1.32:$ & & & $11 / 2$ & 27987.24 & 1.35 \\
\hline & & $5 / 2$ & 26214.05 & $0.645:$ & & & $7 / 2$ & 2799725 & 1.170 \\
\hline & ${ }^{8} \mathrm{~F}^{\circ}$ & $1 / 2$ & 26253.55 & 3.59 & & & $3 / 2$ & 28011.88 & 0.155 \\
\hline & & $5 / 2$ & 26357.90 & 1.20 & & & $9 / 2$ & 28022.50 & 1.10 \\
\hline & & $11 / 2$ & 2641329 & 1.15 & $4 f^{5} 5 d 6 s ?$ & & $7 / 2$ & 28072.33 & 1.375 \\
\hline & ${ }^{8} \mathrm{G}^{\circ}$ & $1 / 2$ & 26442.18 & -1.21 & & & $1 / 2$ & 28142.79 & -0.470 \\
\hline & & $3 / 2$ & 26484.66 & 0.25 & & & $11 / 2$ & 28151.40 & 1.49 \\
\hline \multirow[t]{2}{*}{$4 f^{6}\left({ }^{7} \mathrm{~F}\right) 6 p ?$} & & $11 / 2$ & 26505.53 & 1.535 & & & $9 / 2$ & 28191.96 & 1.46 \\
\hline & & $7 / 2$ & 26509.67 & 0.835 & & ${ }^{8} \mathrm{H}^{\circ}$ & $3 / 2$ & 28239.54 & -0.025 \\
\hline $4 f^{6}\left({ }^{7} \mathrm{~F}\right) 6 p ?$ & & $13 / 2$ & 26540.12 & 1.51 & & & $7 / 2$ & 28256.32 & 1.085 \\
\hline \multirow[t]{2}{*}{$4 f^{6}\left({ }^{7} \mathrm{~F}\right) 6 p ?$} & & $9 / 2$ & 26565.61 & 1.28 & & & $9 / 2$ & 28314.18 & 1.30 \\
\hline & & $3 / 2$ & 26599.08 & 1.630 & & & $5 / 2$ & 28394.04 & 0.865 \\
\hline \multirow[t]{3}{*}{$4 f^{5} 5 d 6 s ?$} & ${ }^{8} \mathrm{G}^{\circ}$ & $1 / 2$ & 26690.30 & -1.100 & & & $5 / 2$ & 28429.38 & 1.775 \\
\hline & & $3 / 2$ & 26723.87 & 1.05 & & & $7 / 2$ & 28445.43 & 1.225 \\
\hline & & $7 / 2$ & 26771.62 & 0.785 & $4 f^{6}\left({ }^{7} \mathrm{~F}\right) 6 p ?$ & & $11 / 2$ & 28540.12 & 1.46 \\
\hline $4 f^{5} 5 d 6 s ?$ & & $9 / 2$ & 26820.81 & 1.415 & & & $11 / 2$ & 28554.98 & 0.775 \\
\hline \multirow[t]{2}{*}{$4 f^{6}\left({ }^{7} \mathrm{~F}\right) 6 p ?$} & & $11 / 2$ & 26828.29 & 1.315 & & & $5 / 2$ & 28573.13 & 0.74 \\
\hline & & $11 / 2$ & 26880.60 & 1.29 & & & $5 / 2$ & 28672.08 & 1.215 \\
\hline \multirow[t]{4}{*}{$4 f^{5} 5 d 6 s ?$} & & $15 / 2$ & 26889.18 & 1.395 & & & $9 / 2$ & 28725.53 & 1.315 \\
\hline & & $7 / 2$ & 26938.42 & 1.50 & & & $7 / 2$ & 28730.14 & 1.11 \\
\hline & & $5 / 2$ & 26974.67 & 0.980 & & & $3 / 2$ & 28812.92 & 0.06 \\
\hline & & $9 / 2$ & 27001.20 & 1.32 & & & $11 / 2$ & 28850.60 & 1.50 \\
\hline \multirow[t]{4}{*}{$4 f^{5} 5 d 6 s ?$} & & $3 / 2$ & 27063.30 & 0.860 & & & $9 / 2$ & 28913.99 & 1.380 \\
\hline & & $5 / 2$ & 27078.30 & 0.84 & & & $5 / 2$ & 28929.72 & 0.565 \\
\hline & & $7 / 2$ & 27107.62 & 1.11 & & & $3 / 2$ & 28938.55 & 0.92 \\
\hline & & $5 / 2$ & 27165.35 & 0.92 & & & $1 / 2$ & 28980.58 & 3.130 \\
\hline \multirow[t]{2}{*}{$4 f^{6}\left({ }^{7} \mathrm{~F}\right) 6 p ?$} & & $7 / 2$ & 27188.30 & 1.20 & & & $11 / 2$ & 28988.79 & 1.31 \\
\hline & & $1 / 2$ & 27210.12 & -0.485 & $4 f^{5} 5 d 6 s ?$ & & $9 / 2$ & 28997.14 & 1.39 \\
\hline \multirow[t]{2}{*}{$4 f^{6}\left({ }^{7} \mathrm{~F}\right) 6 p ?$} & & $15 / 2$ & 27263.25 & 1.445 & & & $13 / 2$ & 29123.57 & 1.38 \\
\hline & & $5 / 2$ & 27284.69 & 1.47 & & & $11 / 2$ & 29137.23 & 1.210 \\
\hline \multirow[t]{2}{*}{$4 f^{5} 5 d 6 s ?$} & & $9 / 2$ & 27309.73 & 1.335 & & & $5 / 2$ & 29238.56 & 0.645 \\
\hline & & $9 / 2$ & 27386.69 & 1.060 & & & $7 / 2$ & 29246.00 & 1.470 \\
\hline
\end{tabular}




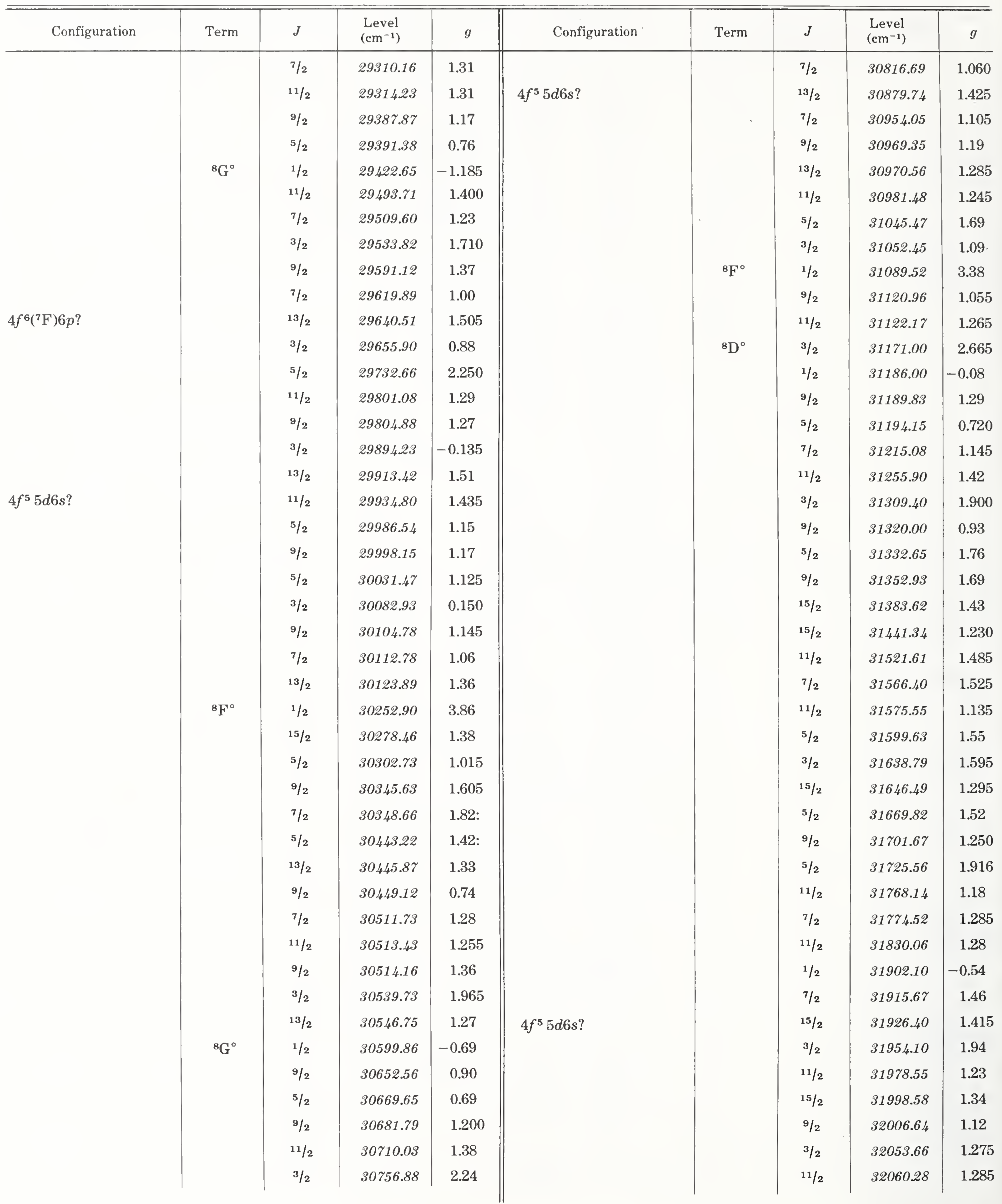


Sm II, Odd Parity-Continued

Sm II, Odd Parity-Continued

\begin{tabular}{|c|c|c|c|c|c|c|c|c|c|}
\hline Configuration & Term & $J$ & $\begin{array}{l}\text { Level } \\
\left(\mathrm{cm}^{-1}\right)\end{array}$ & $g$ & Configuration & Term & $J$ & $\begin{array}{l}\text { Level } \\
\left(\mathrm{cm}^{-1}\right)\end{array}$ & $g$ \\
\hline & & $1 / 2$ & 32067.40 & -0.150 & & & $9 / 2$ & 33772.54 & 1.14 \\
\hline & & $13 / 2$ & 32130.57 & 1.33 & & & $11 / 2$ & 33775.84 & 1.41 \\
\hline & & $7 / 2$ & 32204.31 & 1.47 & & & $13 / 2$ & 33809.85 & 1.310 \\
\hline & & $3 / 2$ & 32223.45 & 1.285 & & & $9 / 2$ & 33852.93 & 1.11 \\
\hline & & $13 / 2$ & 32286.05 & 1.27 & & & $7 / 2$ & 33881.94 & 1.44 \\
\hline & & $1 / 2$ & 32296.32 & 2.74 & & & $15 / 2$ & 33934.48 & 1.280 \\
\hline & & $9 / 2$ & 32299.89 & 1.355 & & & $9 / 2$ & 34040.37 & 1.35 \\
\hline & & $7 / 2$ & 32344.44 & 1.310 & & & $5 / 2$ & 34066.75 & 1.58 \\
\hline & & $9 / 2$ & 32345.32 & 1.510 & & & $3 / 2$ & 34068.41 & 1.540 \\
\hline & & $3 / 2$ & 32358.56 & 1.42 & & & $11 / 2$ & 34133.81 & 1.27 \\
\hline & & $7 / 2$ & 32380.78 & 1.52 & & & $13 / 2$ & 34145.44 & 1.440 \\
\hline & & $5 / 2$ & 32397.48 & 1.06 & & & $11 / 2$ & 34188.47 & 1.395 \\
\hline & & $9 / 2$ & 32434.70 & 1.455 & & & $11 / 2$ & 34205.91 & 1.26 \\
\hline & & $7 / 2$ & 32451.66 & 1.22 & & & $11 / 2$ & 34330.90 & 1.33 \\
\hline & & $3 / 2$ & 32492.65 & 2.07 & & & $9 / 2$ & 34374.70 & 1.21 \\
\hline & & $11 / 2$ & 32513.09 & 1.230 & & & $11 / 2$ & 34418.95 & 1.46 \\
\hline & & $7 / 2$ & 32549.64 & 1.60 & & & $5 / 2$ & 34453.83 & 0.95 \\
\hline & & $11 / 2$ & 32589.29 & 1.230 & & & $13 / 2$ & 34457.57 & 1.23 \\
\hline & & $5 / 2$ & 32603.65 & 1.44 & & & $9 / 2$ & 34496.00 & 1.090 \\
\hline & & $5 / 2$ & 32675.17 & 1.16 & & & $7 / 2$ & 34505.80 & 0.89 \\
\hline & & $5 / 2$ & 32685.70 & 1.665 & & & $9 / 2$ & 34722.26 & 1.43 \\
\hline & & $11 / 2$ & 32690.37 & 1.240 & & & $13 / 2$ & 34745.47 & 1.02: \\
\hline & & $13 / 2$ & 32726.78 & 1.205 & & & $13 / 2$ & 34768.41 & 1.35: \\
\hline & & $13 / 2$ & 32852.81 & 1.360 & & & $13 / 2$ & 34890.85 & 1.33 \\
\hline & & $9 / 2$ & 32857.54 & 1.36 & & & $3 / 2$ & 34910.60 & 0.58 \\
\hline & & $5 / 2$ & 32935.43 & 1.175 & & & $5 / 2$ & 34913.49 & 0.945 \\
\hline & & $9 / 2$ & 32945.19 & 1.39 & & & $9 / 2$ & 34951.90 & 1.320 \\
\hline & & $3 / 2$ & 33069.10 & 1.83 & & & $7 / 2$ & 35101.70 & 0.80 \\
\hline & & $5 / 2$ & 33107.10 & 0.84 & & & $11 / 2$ & 35192.21 & 1.09 \\
\hline & & $11 / 2$ & 33153.69 & 1.42 & & & $15 / 2$ & 35261.20 & 1.32 \\
\hline & & $5 / 2$ & 33218.75 & 1.500 & & & $7 / 2$ & 35303.60 & 1.12 \\
\hline & & $3 / 2$ & 33227.9 & 1.73 & & & $9 / 2$ & 35348.60 & 1.36 \\
\hline & & $9 / 2$ & 33252.45 & 1.22 & & & $9 / 2$ & 35463.91 & 1.295 \\
\hline & & $11 / 2$ & 33286.30 & 1.25 & & & $1 / 2$ & 35535.52 & -1.19 \\
\hline & & $7 / 2$ & 33333.40 & 1.43 & & & $11 / 2$ & 35547.50 & 1.415 \\
\hline & & $9 / 2$ & 33364.81 & 1.64 & & & $5 / 2$ & 35583.40 & 0.98 \\
\hline & & $5 / 2$ & 33539.60 & 1.55 & & & $3 / 2$ & 35966.59 & 1.20 \\
\hline & & $7 / 2$ & 33576.60 & 1.07 & & & $9 / 2$ & 36107.66 & 1.270 \\
\hline & & $11 / 2$ & 33598.70 & 1.39 & & & $1 / 2$ & 37488.95 & -0.40 \\
\hline & & $9 / 2$ & 33613.43 & 1.12 & & & $3 / 2$ & 37569.11 & 0.58 \\
\hline & & $7 / 2$ & 33630.20 & 1.07 & & & $3 / 2$ & 37586.27 & 0.55 \\
\hline & ${ }^{8} \mathrm{~F}^{\circ}$ & $1 / 2$ & 33661.26 & 3.00 & & & $19 / 2$ & 38505.66 & 1.36 \\
\hline & & $5 / 2$ & 33689.50 & 1.47 & & & & & \\
\hline & & $15 / 2$ & 33763.45 & 1.34 & $\mathrm{Sm}$ III $\left({ }^{7} \mathrm{~F}_{0}\right)$ & Limit & L--- & 89300 & \\
\hline
\end{tabular}


Ground state $\left(1 s^{2} 2 s^{2} 2 p^{6} 3 s^{2} 3 p^{6} 3 d^{10} 4 s^{2} 4 p^{6} 4 d^{10} 5 s^{2} 5 p^{6}\right) 4 f^{6}{ }^{7} \mathrm{~F}_{0}$

All the levels except $4 f^{65} \mathrm{D}_{0} 3$ are from Dupont's analysis of spark emission spectra [1967]. His list of approximately 15000 lines (1800-9500 $\AA$ ) assigned to Sm III has not been published. The levels given here classify about 100 lines in the region $1935-4033 \AA$ as $4 f^{6}{ }^{7} \mathrm{~F}-$ $4 f^{5}\left({ }^{6} \mathrm{H}^{\circ},{ }^{6} \mathrm{~F}^{\circ},{ }^{6} \mathrm{P}^{\circ}\right) 5 d$ transitions [Dupont, 1966]. A number of tentative levels listed in Dupont's thesis but not included in the 1967 publication were also omitted here.

The spectra of $\mathrm{Sm}^{2+}$ ions in several different types of crystals have been observed [see compilation in Dupont, 1967]. We have listed only the lowest of the $4 f^{65} \mathrm{D}$ levels from the $\mathrm{BaClF}: \mathrm{Sm}^{2+}$ data of Kiss and Weakliem [1965] in order to approximately locate this important term. The position of this level in the free ion is probably higher; i.e., " $x$ " is probably positive and may be as great as several hundred $\mathrm{cm}^{-1}$. The ${ }^{5} \mathrm{D}$ term extends to above $20000 \mathrm{~cm}^{-1}$.

The leading percentages for the $4 f^{6}{ }^{7} \mathrm{~F}$ and ${ }^{5} \mathrm{D}$ terms are from Ofelt's calculation [1963]. Dupont based the identifications of the $4 f^{5} 5 d$ levels on the calculations of Becher [1965], who did not list the eigenvectors. The levels have been arranged into terms following Dupont's names; a number of the analogous Sm I $4 f^{5} 5 d 6 s^{2}$ levels have eigenvector purities less than $50 \%$.

The lowest predicted terms of the $4 f^{5} 5 d$ configuration are $\left({ }^{6} \mathrm{H}^{\circ}\right)^{7} \mathrm{~K}^{\circ}$ and ${ }^{7} \mathrm{I}^{\circ}$, with the ${ }^{7} \mathrm{~K}_{4}^{\circ}$ level predicted a little above $24000 \mathrm{~cm}^{-1}$. These and some of the other missing terms of the $4 f^{5} 5 d$ configuration can probably be found after the analysis is extended to include the $4 f^{5} 6 s$ and $4 f^{5} 6 p$ configurations.

Sugar and Reader obtained the ionization energy.

\section{References}

Becher, J., Thesis, Johns Hopkins Univ., Baltimore, 135 pp. (1965); (Univ. Microfilms, Ann Arbor, Mich., No. 66-4956). $\mathrm{PT}$

Dupont, A., Thesis, Johns Hopkins Univ., Baltimore, 98 pp. (1966); (Univ. Microfilms, Ann Arbor, Mich., No. 66-7025). EL CL W

Dupont, A., J. Opt. Soc. Am. 57, 867 (1967). EL

Kiss, Z. J., and Weakliem, H. A., Phys. Rev. Lett. 15, 457 (1965). [EL]

Ofelt, G. S., J. Chem. Phys. 38, 2171 (1963). PT

Sugar, J., and Reader, J., J. Chem. Phys. 59, 2083 (1973). IP

[July 1976] 
Sm III

\begin{tabular}{|c|c|c|c|c|c|}
\hline Configuration & Term & $J$ & $\begin{array}{l}\text { Level } \\
\left(\mathrm{cm}^{-1}\right)\end{array}$ & & percentages \\
\hline $4 f^{6}$ & ${ }^{7} \mathrm{~F}$ & $\begin{array}{l}0 \\
1 \\
2 \\
3 \\
4 \\
5 \\
6\end{array}$ & $\begin{array}{r}0.00 \\
293.45 \\
813.55 \\
1492.66 \\
2277.88 \\
3131.54 \\
4026.99\end{array}$ & $\begin{array}{l}94 \\
95 \\
96 \\
94 \\
98 \\
98 \\
97\end{array}$ & $\begin{array}{ll}3 & { }^{5} \mathrm{D} 1 \\
2 & { }^{5} \mathrm{D} 1 \\
2 & { }^{5} \mathrm{D} 1 \\
1 & { }^{5} \mathrm{D} 3 \\
1 & { }^{5} \mathrm{~F} 2 \\
1 & { }^{5} \mathrm{G} 1 \\
1 & { }^{5} \mathrm{G} 1\end{array}$ \\
\hline $4 f^{6}$ & ${ }^{5} \mathrm{D} 3$ & $\begin{array}{l}0 \\
1 \\
2 \\
3 \\
4\end{array}$ & {$[14533]+x$} & $\begin{array}{l}48 \\
52 \\
56 \\
56 \\
55\end{array}$ & $\begin{array}{ll}29 & 5 \mathrm{D} 1 \\
31 & \\
33 & \\
34 & \\
34 & \end{array}$ \\
\hline $4 f^{5}\left({ }^{6} \mathrm{H}^{\circ}\right) 5 d$ & ${ }^{7} \mathrm{H}^{\circ}$ & $\begin{array}{l}2 \\
3 \\
4 \\
5 \\
6 \\
7 \\
8\end{array}$ & $\begin{array}{l}26283.55 \\
27361.55 \\
\\
29377.26\end{array}$ & & \\
\hline $4 f^{5}\left({ }^{6} \mathrm{H}^{\circ}\right) 5 d$ & ${ }^{7} \mathrm{~F}^{\circ}$ & $\begin{array}{l}0 \\
1 \\
2 \\
3 \\
4 \\
5 \\
6\end{array}$ & $\begin{array}{l}27885.89 \\
28228.81 \\
29121.36 \\
30198.89 \\
31376.56 \\
32868.01\end{array}$ & & \\
\hline $4 f^{5}\left({ }^{6} \mathrm{H}^{\circ}\right) 5 d$ & ${ }^{7} \mathrm{G}^{\circ}$ & $\begin{array}{l}1 \\
2 \\
3 \\
4 \\
5 \\
6 \\
7\end{array}$ & $\begin{array}{l}28634.34 \\
29763.82 \\
30608.25 \\
31556.56 \\
32341.80 \\
33710.00\end{array}$ & & \\
\hline $4 f^{5}\left({ }^{6} \mathrm{~F}^{\circ}\right) 5 d$ & ${ }^{7} \mathrm{G}^{\circ}$ & $\begin{array}{l}1 \\
2 \\
3 \\
4 \\
5 \\
6 \\
7\end{array}$ & $\begin{array}{l}30048.48 \\
30948.50 \\
31657.02 \\
32598.82 \\
33771.89\end{array}$ & & \\
\hline $4 f^{5}\left({ }^{6} \mathrm{~F}^{\circ}\right) 5 d$ & ${ }^{7} \mathrm{~F}^{\circ}$ & $\begin{array}{l}0 \\
1 \\
2 \\
3 \\
4 \\
5 \\
6\end{array}$ & $\begin{array}{l}34846.60 \\
35314.66 \\
35928.00 \\
36826.12 \\
37738.44 \\
38758.03\end{array}$ & & \\
\hline $4 f^{5}\left({ }^{6} \mathbf{F}^{\circ}\right) 5 d$ & ${ }^{7} \mathrm{D}^{\circ}$ & $\begin{array}{l}1 \\
2 \\
3 \\
4 \\
5\end{array}$ & $\begin{array}{l}36347.54 \\
36931.88 \\
37715.91 \\
38714.36 \\
39666.78\end{array}$ & & \\
\hline $4 f^{5}\left({ }^{6} \mathrm{P}^{\circ}\right) 5 d$ & ${ }^{7} \mathrm{D}^{\circ}$ & $\begin{array}{l}1 \\
2 \\
3 \\
4 \\
5\end{array}$ & $\begin{array}{l}51160.80 \\
51432.29 \\
51940.36 \\
53149.58\end{array}$ & & \\
\hline $\mathrm{Sm} \mathrm{IV}\left({ }^{6} \mathrm{H}_{5 / 2}^{\circ}\right)$ & Limit & & 189000 & & \\
\hline
\end{tabular}


[Sm IV ]

(Pr I sequence; 59 electrons)

Ground state $\left(1 s^{2} 2 s^{2} 2 p^{6} 3 s^{2} 3 p^{6} 3 d^{10} 4 s^{2} 4 p^{6} 4 d^{10} 5 s^{2} 5 p^{6}\right) 4 f^{5}{ }^{6} \mathrm{H}_{5 / 2}^{\circ}$

Ionization energy $334000 \pm 6000 \mathrm{~cm}^{-1}$

$41.4 \pm 0.7 \mathrm{eV}$

No analysis of the free-ion spectrum has been carried out. Most of the levels here are averages of corresponding sublevels of $\mathrm{Sm}^{3+}$ in $\mathrm{LaCl}_{3}$. Both absorption and fluorescence spectra were observed and analyzed at the Johns Hopkins University [Magno, 1958; Wybourne, 1962; Magno and Dieke, 1962; Dieke, 1968]; we have relied mainly on the data in Dieke's book [1968]. His values were tabulated with zero energy at the lowest sublevel arising from the ${ }^{6} \mathrm{H}_{5 / 2}^{\circ}$ ground level, instead of at the average of the ${ }^{6} \mathrm{H}_{5 / 2}^{\circ}$ sublevels $\left(36 \mathrm{~cm}^{-1}\right.$ higher). No corresponding correction of the higher levels was made here, since overall better agreement with the solution absorption data of Carnall, Fields, and Rajnak [1968] is obtained without the correction. Four new levels not included in the earlier references are taken from Crosswhite, Crosswhite, and Oats [1976]. These levels are also based on the spectrum of $\mathrm{Sm}^{3+}$ in $\mathrm{LaCl}_{3}$.

A number of levels above the range covered here are given by Dieke [1968] (to $\sim 31500$ $\mathrm{cm}^{-1}$ ) and by Carnall et al. [1968] (to $\left.47400 \mathrm{~cm}^{-1}\right)$.

The $g$ values are from Wybourne's compilation [1962]. Some references for the original Zeeman data are given below.

Carnall, Fields, and Rajnak [1974] calculated the tabulated eigenvector percentages; their matrices included spin-orbit and effective interactions.

The ionization energy is from Sugar and Reader. The estimated uncertainty has been approximately doubled, as recommended by Spector and Sugar, and the value rounded off accordingly.

\section{References}

Carnall, W. T., Fields, P. R., and Rajnak, K., J. Chem. Phys. 49, 4424 (1968). [EL] [CL] [W] PT

Carnall, W. T., Fields, P. R., and Rajnak, K., unpublished material (1974). ND PT

Crosswhite, H., Crosswhite, H. M., and Oats, M., unpublished material (1976). [EL] PT

Dieke, G. H., Spectra and Energy Levels of Rare Earth Ions in Crystals, Ed. H. M. Crosswhite and H. Crosswhite, pp. 233-242 (Interscience Publishers, New York, 1968). [EL] [CL] [W] [ZE]

Friederich, A., Hellwege, K. H., and Lämmermann, H., Z. Phys. 159, 524 (1960). [ZE]

Judd, B. R., Proc. Phys. Soc. London, Sect. A 69, 157 (1956). PT

Lämmermann, H., Z. Phys. 160, 355 (1960). [ZE]

Magno, M. S., Thesis, Johns Hopkins Univ., Baltimore (1958). [EL] [CL] [W] [ZE]

Spector, N., and Sugar, J., J. Opt. Soc. Am. 66, 436 (1976).

Sugar, J., and Reader, J., J. Chem. Phys. 59, 2083 (1973). IP

Wybourne, B. G., J. Chem. Phys. 36, 2301 (1962). ND PT

[July 1976] 
[Sm Iv]

\begin{tabular}{|c|c|c|c|c|c|c|c|c|}
\hline Configuration & Term & $J$ & $\begin{array}{l}\text { Level } \\
\left(\mathrm{cm}^{-1}\right)\end{array}$ & $g$ & \multicolumn{4}{|c|}{ Leading percentages } \\
\hline $4 f^{5}$ & ${ }^{6} \mathrm{H}^{\circ}$ & $\begin{array}{l}5 / 2 \\
7 / 2 \\
9 / 2 \\
11 / 2 \\
13 / 2 \\
15 / 2\end{array}$ & $\begin{array}{c}0 \\
{[1080]} \\
{[2290]} \\
{[3610]} \\
{[4990]} \\
{[6470]}\end{array}$ & $\begin{array}{l}0.79 \\
1.00\end{array}$ & $\begin{array}{l}96 \\
97 \\
98 \\
98 \\
98 \\
96\end{array}$ & & $\begin{array}{l}2 \\
2 \\
1 \\
1 \\
2 \\
3\end{array}$ & $\begin{array}{l}{ }^{4} \mathrm{G}^{\circ} 4 \\
{ }^{4} \mathrm{G}^{\circ} 4 \\
{ }^{4} \mathrm{G}^{\circ} 4 \\
{ }^{4} I^{\circ} 3 \\
{ }^{4} I^{\circ} 3 \\
{ }^{4} I^{\circ} 3\end{array}$ \\
\hline $4 f^{5}$ & ${ }^{6} \mathrm{~F}^{\circ}$ & $\begin{array}{c}1 / 2 \\
3 / 2 \\
5 / 2 \\
7 / 2 \\
9 / 2 \\
11 / 2\end{array}$ & $\begin{array}{r}{[6290]} \\
{[6540]} \\
{[7050]} \\
{[7910]} \\
{[9080]} \\
{[10470]}\end{array}$ & 1.44 & $\begin{array}{l}97 \\
96 \\
95 \\
95 \\
96 \\
97\end{array}$ & & $\begin{array}{l}1 \\
1 \\
2 \\
2 \\
2 \\
1\end{array}$ & $\begin{array}{l}{ }^{4} \mathrm{D}^{\circ} 2 \\
{ }^{4} \mathrm{~F}^{\circ} 3 \\
{ }^{4} \mathrm{~F}^{\circ} 3 \\
{ }^{4} \mathrm{~F}^{\circ} 3 \\
{ }^{4} \mathrm{~F}^{\circ} 3 \\
{ }^{4} \mathrm{G}^{\circ} 3\end{array}$ \\
\hline $4 f^{5}$ & & $5 / 2$ & {$[17860]$} & 0.79 & 29 & ${ }^{4} \mathrm{G}^{\circ} 4$ & 21 & ${ }^{4} \mathrm{~F}^{\circ} 3$ \\
\hline $4 f^{5}$ & ${ }^{4} \mathrm{~F}^{\circ} 3$ & $3 / 2$ & {$[18860]$} & 0.44 & 68 & & 15 & ${ }^{4} \mathrm{~F}^{\circ} 1$ \\
\hline $4 f^{5}$ & & $7 / 2$ & [20010] & 1.08 & 34 & ${ }^{4} \mathrm{G}^{\circ} 4$ & 20 & ${ }^{4} \mathrm{~F}^{\circ} 3$ \\
\hline $4 f^{5}$ & ${ }^{4} I^{\circ} 3$ & $\begin{array}{l}9 / 2 \\
11 / 2 \\
13 / 2 \\
15 / 2\end{array}$ & $\begin{array}{l}{[20560]} \\
{[20980]} \\
{[21550]}\end{array}$ & 1.02 & $\begin{array}{l}80 \\
63 \\
46 \\
47\end{array}$ & & $\begin{array}{l}15 \\
18 \\
22 \\
15\end{array}$ & $\begin{array}{l}{ }^{4} \mathrm{I}^{\circ} 1 \\
{ }^{4} \mathrm{~K}^{\circ} 1 \\
{ }^{4} \mathrm{~K}^{\circ} 1 \\
{ }^{4} \mathrm{~K}^{\circ} 1\end{array}$ \\
\hline $4 f^{5}$ & ${ }^{4} \mathrm{M}^{\circ}$ & $\begin{array}{l}15 / 2 \\
17 / 2 \\
19 / 2 \\
21 / 2\end{array}$ & $\begin{array}{l}{[20750]} \\
{[22530]}\end{array}$ & & $\begin{array}{l}74 \\
86 \\
91 \\
95\end{array}$ & & $\begin{array}{l}8 \\
9 \\
7 \\
5\end{array}$ & $\begin{array}{l}{ }^{4} \mathrm{~L}^{\circ} \\
{ }^{4} \mathrm{~L}^{\circ} \\
{ }^{4} \mathrm{~L}^{\circ} \\
{ }^{2} \mathrm{~N}^{\circ}\end{array}$ \\
\hline $\mathrm{Sm} \mathrm{v}\left({ }^{5} I_{4}\right)$ & Limit & & 334000 & & & & & \\
\hline
\end{tabular}




\section{Sm XXXV}

(Ni I sequence; 28 electrons)

Ground state $\left(1 s^{2} 2 s^{2} 2 p^{6} 3 s^{2} 3 p^{6}\right) 3 d^{10}{ }^{1} \mathrm{~S}_{0}$

The levels are from Burkhalter, Nagel, and Whitlock, who classified the $3 d^{10}{ }^{1} \mathrm{~S}_{0}-$ $3 d^{9} 4 p(J=1)$ and $3 d^{10}{ }^{1} \mathrm{~S}_{0}-3 d^{9} 4 f(J=1)$ resonance lines of $\mathrm{Sm}$ XXXV, Gd XXXVII, and Dy XXXIX. These authors produced spectra in the region 5-15 $\AA$ by focusing light from a pulsed laser onto rare-earth targets. We have converted the levels from units of eV to units of $10^{4} \mathrm{~cm}^{-1}$ (equivalent to $1.23985 \mathrm{eV}$ ). The uncertainty of the measurements is about $2 \times 10^{4} \mathrm{~cm}^{-1}$.

Burkhalter et al. classified the transitions using regularities along the $\mathrm{Ni}$ I isoelectronic sequence and comparisons with calculated energies. The location of the $3 d^{9} 4 f(5 / 2,5 / 2)_{1}^{\circ}$ level is tentative in all three spectra.

Although the $J_{1} j$ designations assigned by Burkhalter et al. are listed for both of the excited configurations, the $3 d^{9} 4 f$ levels may also be assigned fairly appropriate $L S$ names (see Gd XXXVII).

Burkhalter et al. discuss the spectral and configurational assignments of several other groups of Sm, Gd, and Dy lines with wavelengths down to about $5 \AA$. Observations with higher resolution may be necessary before more detailed energy level classifications can be made.

\section{Reference}

Burkhalter, P. G., Nagel, D. J., and Whitlock, R. R., Phys. Rev. A 9, 2331 (1974). EL CL W

[July 1976]

Sm XXXV

\begin{tabular}{l|c|c|c}
\hline \hline \multicolumn{1}{c|}{ Configuration } & Term & $J$ & $\begin{array}{c}\text { Level } \\
\left(10^{4} \mathrm{~cm}^{-1}\right)\end{array}$ \\
\hline $3 d^{10}$ & ${ }^{10}$ & 0 & 0.0 \\
$3 d^{9}\left({ }^{2} \mathrm{D}_{3 / 2}\right) 4 p_{1 / 2}$ & $\left(3 / 2,{ }^{1} / 2\right)^{\circ}$ & 1 & 836 \\
$3 d^{9}\left({ }^{2} \mathrm{D}_{5 / 2}\right) 4 p_{3 / 2}$ & $(5 / 2,3 / 2)^{\circ}$ & 1 & 845 \\
$3 d^{9}\left({ }^{2} \mathrm{D}_{3 / 2}\right) 4 p_{3 / 2}$ & $(3 / 2,3 / 2)^{\circ}$ & 1 & 867 \\
$3 d^{9}\left({ }^{2} \mathrm{D}_{5 / 2}\right) 4 f_{5 / 2}$ & $(5 / 2,5 / 2)^{\circ}$ & 1 & $1015 ?$ \\
$3 d^{9}\left({ }^{2} \mathrm{D}_{5 / 2}\right) 4 f_{7 / 2}$ & $(5 / 2,7 / 2)^{\circ}$ & 1 & 1040 \\
$3 d^{9}\left({ }^{2} \mathrm{D}_{3 / 2}\right) 4 f_{5 / 2}$ & $(3 / 2,5 / 2)^{\circ}$ & 1 & 1069 \\
\hline
\end{tabular}




\section{EUROPIUM}

\section{Eu I}

63 electrons

$Z=63$

Ground state $\left(1 s^{2} 2 s^{2} 2 p^{6} 3 s^{2} 3 p^{6} 3 d^{10} 4 s^{2} 4 p^{6} 4 d^{10} 5 s^{2} 5 p^{6}\right) 4 f^{7} 6 s^{2}{ }^{8} \mathrm{~S}_{7 / 2}^{\circ}$

Ionization energy $45734.9 \pm 0.2 \mathrm{~cm}^{-1}$

$5.67045 \pm 0.00003 \mathrm{eV}$

Identified odd configurations

$4 f^{7} 6 s^{2}, 4 f^{7} 5 d 6 s, 4 f^{7} 6 s 7 s, 4 f^{7} 5 d^{2}, 4 f^{7} 6 s 6 d, 4 f^{7} 6 s 8 s$, $4 f^{7} 6 s 7 d, 4 f^{7} 6 p^{2}$

Identified even configurations $\quad 4 f^{7} 6 s 6 p, 4 f^{7} 5 d 6 p, 4 f^{6} 5 d 6 s^{2}, 4 f^{6} 5 d^{2} 6 s, 4 f^{7} 6 s 7 p-18 p$, $4 f^{7} 6 s 42 p-67 p, 4 f^{7} 6 s 5 f ?, 4 f^{7} 6 s 6 f^{7} ?, 4 f^{7} 6 s 14 f$ ?, $4 f^{7} 6 s 15 f ?, 4 f^{7} 5 d 7 p$ ?

\section{Spectrum Observations, Main Sources of the Levels}

King [1939] observed 3950 europium lines from 2100 to $10165 \AA$ and assigned almost 2200 lines to Eu I. More than 1100 lines were classified by Russell and King's extension [1939] of their earlier analysis of Eu I, including practically all the strong lines. In the tables here, the term designations preceded by a small letter, as well as the numerical designations assigned to some of the levels, are as given by Russell and King; their line list may be used directly to identify the combinations of these levels. The other odd levels and a number of the remaining even levels below the ionization limit were also found by Russell and King, but their original designations have been omitted or changed on the basis of more recent work (see below). Most levels with a question mark after the position were given as uncertain by Russell and King. We have omitted their $z^{6} \mathrm{~F}_{1 / 2}$ level $\left(36586.37 \mathrm{~cm}^{-1}\right)$, as there appears to be no experimental basis for its existence independent of combinations of the $y^{8} \mathrm{D}_{3 / 2}$ level $\left(36586.35 \mathrm{~cm}^{-1}\right)$.

We assigned parentages for some of the terms designated by Russell and King that have not been calculated. In the case of one or two subconfigurations of the type $4 f^{7}\left({ }^{8} \mathrm{~S}^{\circ}\right) 6 \mathrm{snl}$ for which the best coupling order of the electrons was not clear, we chose the order $4 f^{7}\left({ }^{8} \mathrm{~S}^{\circ}\right) 6 s\left({ }^{9,7} \mathrm{~S}^{\circ}\right) n l$.

Smith and Tomkins [1975a, 1975b] have observed the Eu I absorption spectrum in the region 2100-7200 $\AA$. Since all the lines were transitions from the $4 f^{7} 6 s^{2}{ }^{8} \mathrm{~S}_{7 / 2}^{\circ}$ ground level, the upper levels have even parity and one of the three $J$ values $5 / 2,7 / 2$, or $9 / 2$. Most of the extensive system of even levels above about $40000 \mathrm{~cm}^{-1}$ was found in this work. The more recent measurements begin at $27852 \mathrm{~cm}^{-1}$, with the lowest new level, at $31203 \mathrm{~cm}^{-1}$, belonging to the ${ }^{10}$ I term of the newly found $4 f^{6} 5 d^{2} 6 s$ configuration. We give an average level value wherever the wavenumber of an absorption transition to a previously known level differed by more than $\pm 0.1 \mathrm{~cm}^{-1}$ from Russell and King's value for the level. The two long $n p$ series based on the Eu II $4 f^{7}\left({ }^{8} \mathrm{~S}^{\circ}\right) 6 s\left({ }^{9} \mathrm{~S}^{\circ}\right)$ and $\left({ }^{7} \mathrm{~S}^{\circ}\right)$ limits, respectively, were measured by Smith and Tomkins [1975a]. The members of these series below $n \cong 42$ are part of the complex absorption spectrum that has not yet been fully interpreted (see below).

\section{$g$ Values}

All except a few of the $g$ values for the even levels were derived by Smith and Tomkins [1975b] from Zeeman patterns observed in absorption. The uncertainties vary from one to a few units in the last decimal place, the less accurate values being obtained from perturbed or unresolved patterns. For a few even levels, mostly belonging to the $4 f^{7}\left({ }^{8} \mathrm{~S}^{\circ}\right) 6 s 6 p$ group, we derived $g$ values from the Zeeman patterns of Russell, Albertson, and Davis by assuming (or 
correcting to) the value 1.993 for the ground-level value. These values were averaged with those from Smith and Tomkins for levels included in both sets of data. The $g$ values measured by Handrich, Kretzen, Lange, and Steudel [1968] were also used or averaged in for three of these levels.

The $g$ value for the ground level is from Pichanick and Woodgate [1961], who give the uncertainty as \pm 0.00007 . The leading percentages for this level are from Conway and Wybourne [1963], the more precise values being $97.5 \%$ and $2.4 \%$.

\section{Theoretical Interpretation, Calculations}

Several calculations of various Eu I configurations have shown the need for new observations of the emission spectrum and further extension of the analysis. In deciding whether to include possibly doubtful or incorrect former designations we relied heavily on the comments of Smith and Wybourne [1965], who have given the most complete discussion of this spectrum based on modern calculations. The odd levels given here without term or numerical designations were assigned to $4 f^{7}\left({ }^{8} \mathrm{~S}^{\circ}\right) 5 d^{2}$ and $\left({ }^{8} \mathrm{~S}^{\circ}\right) 6 s 6 d$ by Russell and King. These subconfigurations no doubt contribute strongly to the compositions of most of the undesignated odd levels around $35000 \mathrm{~cm}^{-1}$, but Smith and Wybourne showed that the specific designations omitted here were unreliable. Some even levels previously assigned to the $\left({ }^{8} \mathrm{~S}^{\circ}\right) 6 s 7 p$ or $\left({ }^{8} \mathrm{~S}^{\circ}\right) 5 d 6 p$ subconfigurations are also listed without names.

The $\left({ }^{8} \mathrm{~S}^{\circ}\right) 6 s 8 s$ subconfiguration is perturbed, as evidenced by the position of the lower of the two ${ }^{8} \mathrm{~S}_{7 / 2}^{\circ}$ levels below the ${ }^{10} \mathrm{~S}_{9 / 2}^{\circ}$ level. Smith and Wybourne suggest that the $\left({ }^{8} \mathrm{~S}^{\circ}\right) 6 s 8 s{ }^{8} \mathrm{~S}_{7 / 2}^{\circ}$ levels are perturbed by the intervening $\left({ }^{8} \mathrm{~S}^{\circ}\right) 5 d^{2}{ }^{8} \mathrm{~S}_{7 / 2}^{\circ}$ level at $37994 \mathrm{~cm}^{-1}$.

The $4 f^{7}\left({ }^{8} \mathrm{~S}^{\circ}\right) 5 d 6 s, \quad\left({ }^{8} \mathrm{~S}^{\circ}\right) 6 s 6 p$, and $\left({ }^{8} \mathrm{~S}^{\circ}\right) 5 d 6 p$ subconfigurations were calculated by Goldschmidt and Nir, and the percentages for the latter two groups are from their results. These authors also discuss the coupling order of the electrons and the type of coupling for several configurations. The leading percentages for the $\left({ }^{8} \mathrm{~S}^{\circ}\right) 5 d 6 p$ levels are given in each of two LS schemes which differ in coupling order. The second scheme the direct coupling of the two external electrons) was used by Smith and Wybourne, and is the most appropriate order for the $\left({ }^{8} \mathrm{~S}^{\circ}\right) 6 s 6 p$ levels.

Wyart has made a calculation of $4 f^{7}\left({ }^{8} \mathrm{~S}^{\circ},{ }^{6} \mathrm{P}^{\circ}\right) 5 d 6 s$ and fitted the known levels, which belong nominally to the four terms $a{ }^{10} \mathrm{D}^{\circ}, a{ }^{8} \mathrm{D}^{\circ}, b{ }^{8} \mathrm{D}^{\circ}$, and $a{ }^{6} \mathrm{D}^{\circ}$ of $\left({ }^{8} \mathrm{~S}^{\circ}\right) 5 d 6 s$. The electrons were coupled in the order shown by Goldschmidt and Nir to be most appropriate, but Wyart's inclusion of the $4 f^{7}\left({ }^{6} \mathrm{P}^{\circ}\right)$ core term gave eigenvector purities considerably lower than those previously obtained with the $4 f^{7}\left({ }^{8} \mathrm{~S}^{\circ}\right) 5 d 6 s$ approximation. The percentages given here are from Wyart. The compositions of the eight levels with $J$ values common to the $a{ }^{6} \mathrm{D}^{\circ}$ and $b^{8} \mathrm{D}^{\circ}$ terms (based on the ${ }^{7} \mathrm{D}^{\circ}$ parent) are rather strongly mixed by inclusion of the $4 f^{7}\left({ }^{6} \mathrm{P}^{\circ}\right)$ grandparent.

The leading percentages for the $\left({ }^{8} \mathrm{~S}^{\circ}\right) 6 s 6 p$ levels are given in both $L S$ coupling and in the $J_{1} J_{2}$ scheme that Goldschmidt and Nir showed to be physically best. Bordarier, Judd, and Klapisch calculated the $\left({ }^{8} \mathrm{~S}^{\circ}\right) 6 s 6 p$ group with the interaction parameters constrained to fit the experimental value of the ratio of the Eu II $\left({ }^{8} \mathrm{~S}^{\circ}\right) 6 p{ }^{9} \mathrm{P}-{ }^{7} \mathrm{P}$ term difference to the $\left({ }^{8} \mathrm{~S}^{\circ}\right) 6 s{ }^{9} \mathrm{~S}^{\circ}-$ ${ }^{7} \mathrm{~S}^{\circ}$ difference. The analysis indicated a perturbation of $\mathrm{Eu} \mathrm{I}\left({ }^{8} \mathrm{~S}^{\circ}\right) 6 s 6 p$, with the $\left({ }^{8} \mathrm{~S}^{\circ}\right)\left({ }^{1} \mathrm{P}^{\circ}\right){ }^{8} \mathrm{P}$ term being most affected, and the hfs calculation gave a large deviation from experiment for the $\left({ }^{8} \mathrm{~S}^{\circ}\right)\left({ }^{1} \mathrm{P}^{\circ}\right){ }^{8} \mathrm{P}_{9 / 2}$ level.

One known interaction of the $\left({ }^{8} \mathrm{~S}^{\circ}\right) 6 s 6 p\left({ }^{1} \mathrm{P}^{\circ}\right){ }^{8} \mathrm{P}$ term is with the $\left({ }^{8} \mathrm{~S}^{\circ}\right) 5 d\left({ }^{7} \mathrm{D}^{\circ}\right) 6 p{ }^{8} \mathrm{P}$ term found by Smith and Collins near $42000 \mathrm{~cm}^{-1}$. The latter term, which belongs mainly to $\left({ }^{8} \mathrm{~S}^{\circ}\right) 5 d 6 p\left({ }^{1} \mathrm{P}^{\circ}\right){ }^{8} \mathrm{P}$ if calculated in the alternate coupling order, is even higher than had been predicted by Smith and Wybourne. Smith and Collins explained the discrepancy as due to interaction with the $\left({ }^{8} \mathrm{~S}^{\circ}\right) 6 s 6 p\left({ }^{1} \mathrm{P}^{\circ}\right){ }^{8} \mathrm{P}$ term; they calculated a configuration admixture of about $15 \%$ between these two terms. Since the calculation of $4 f^{7}\left({ }^{8} \mathrm{~S}^{\circ}\right) 6 s 6 p+\left({ }^{8} \mathrm{~S}\right) 5 d 6 p$ is not available, the percentages given here unfortunately do not include this interaction. (Smith and Collins state that the configuration mixing of the other terms is small.)

Smith and Wilson assigned 17 even levels to terms of $4 f^{6}\left({ }^{7} \mathrm{~F}\right) 5 d 6 \mathrm{~s}^{2}$, and the tabulated percentages are from their calculation. Their changes of the $J$ values of two of the levels are accepted here. No levels of the predicted lowest term of this configuration, $4 f^{6}\left({ }^{7} \mathrm{~F}\right) 5 d 6 s^{2}{ }^{8} \mathrm{H}$, are yet known. Smith and Wilson noted that the "102" and "110" levels are based on weak lines and apparently do not belong to $4 f^{6}\left({ }^{7} \mathrm{~F}\right) 5 d 6 s^{2}$; "these may therefore be unreal." 


\section{High Even Levels Based on Absorption and Photoionization Spectra}

The configuration and term designations for the even levels in the region 31000-45200 $\mathrm{cm}^{-1}$ given without leading percentages are from Smith and Tomkins [1975b]. The interpretation of this complex system of high even levels based mainly on absorption spectra is still in progress. Overlapping configurations of the types $4 f^{6} 5 d 6 s^{2}, 4 f^{6} 5 d^{2} 6 s, 4 f^{7} 5 d n p, 4 f^{7} 6 s n p$, and $4 f^{7} 6 s n f$ are already known to contribute to the system. Many of the designations are given as tentative pending completion of the analysis.

Final term designations are omitted for the $4 f^{7}\left({ }^{8} \mathrm{~S}^{\circ}\right) 6 s\left({ }^{9} \mathrm{~S}^{\circ}\right) n p$ and $\left({ }^{7} \mathrm{~S}^{\circ}\right) n p$ series members above $10 p$, pending a determination of the appropriate coupling scheme. These series are in general perturbed in the region below the very high members listed by Smith and Tomkins [1975a]. They conclude that most of the absorption lines in the region $45200-45655 \mathrm{~cm}^{-1}$ can probably be accounted for by several highly perturbed series [Smith and Tomkins, 1975b]; only a few of the upper even levels corresponding to the stronger transitions in this region are listed here.

The levels above the Eu II $\left({ }^{9} \mathrm{~S}_{4}^{\circ}\right)$ principal limit include, in addition to the high $\left({ }^{7} \mathrm{~S}^{\circ}\right) \mathrm{np}$ series members, a group of levels followed by the letter " $a$ " (for autoionization). Parr [1971] obtained these levels from photoionization resonances. The technique involved absorption transitions from the ${ }^{8} \mathrm{~S}_{7 / 2}^{\circ}$ ground level, and Smith and Tomkins [1975a] note that "... some feature is visible in our spectra at or near most of the maxima listed by Parr." The values for the two autoionizing levels tentatively assigned to the lower ${ }^{8} \mathrm{P}$ term of $4 f^{7}\left({ }^{8} \mathrm{~S}^{\circ}\right) 5 d 7 p$ are from absorption data [Smith and Tomkins, 1975b] that may be somewhat more accurate than Parr's determinations. These term assignments were suggested by Parr and also by Smith and Tomkins [1975b]; we have omitted more specific suggestions for the $J$ values. Parr assigned eight more of these levels to $4 f^{7}\left({ }^{8} \mathrm{~S}^{\circ}\right) 5 d n p{ }^{8} \mathrm{P}$ terms $(n=7-10)$. The designations are consistent with quantum defect data, but are omitted here, pending a more detailed theoretical treatment. Configuration-interaction calculations for the ground level and for a number of high even configurations, especially those that can contribute ${ }^{8} \mathrm{P}$ components, will probably be required for interpretation of the complex absorption spectra in this region.

\section{Ionization Energy}

Smith and Tomkins [1975a] derived the value $47404.1 \pm 0.2 \mathrm{~cm}^{-1}$ for the Eu II $4 f^{7}\left({ }^{8} \mathrm{~S}^{\circ}\right) 6{ }^{7}{ }^{7} \mathrm{~S}_{3}^{\circ}$ limit from the high members of the $\left({ }^{7} \mathrm{~S}_{3}^{\circ}\right) \mathrm{np}$ series. This result gave a value of $45734.9 \pm 0.2 \mathrm{~cm}^{-1}$ for the $\mathrm{Eu}$ II $4 f^{7}\left({ }^{8} \mathrm{~S}^{\circ}\right) 6 s^{8} \mathrm{~S}_{4}^{\circ}$ limit, which is the principal ionization energy. The new determination agrees well with the less accurate value of $45740 \mathrm{~cm}^{-1}$ derived by Russell and King, and also with the value $45680 \pm 65 \mathrm{~cm}^{-1}$ obtained by Parr from his photoionization measurements.

\section{References}

Bordarier, Y., Judd, B. R., and Klapisch, M., Proc. R. Soc. London, Ser. A 289, 81 (1965). Hfs PT

Conway, J. G., and Wybourne, B. G., Phys. Rev. 130, 2325 (1963). AT

Goldschmidt, Z. B., and Nir, S., Physica (Utrecht) 51, 222 (1971a). ND PT

Goldschmidt, Z. B., and Nir, S., unpublished material (1971b). PT

Handrich, E., Kretzen, H., Lange, W., Steudel, A., Wallenstein, R., and Walther, H., Proc. Int. Conf. Optical Pumping At. Line Shape, Ed. T. Skalinski, pp. 417-432 (Warsaw, June 25-28, 1968). ZE Hfs

King, A. S., Astrophys. J. 72, 221 (1930). W Hfs

King, A. S., Astrophys. J. 89, 377 (1939). W

Parr, A. C., J. Chem. Phys. 54, 3161 (1971). EL CL W IP

Pichanick, F. M. J., and Woodgate, G. K., Proc. R. Soc. London, Ser. A 263, 89 (1961). ZE

Russell, H. N., Albertson, W., and Davis, D. N., Phys. Rev. 60, 641 (1941). ZE

Russell, H. N., and King, A. S., Phys. Rev. 46, 1023 (1934). EL CL W IP

Russell, H. N., and King, A. S., Astrophys. J. 90, 155 (1939). EL CL W IP

Smith, G., and Collins, B. S., J. Opt. Soc. Am. 60, 866 (1970). EL CL PT

Smith, G., and Tomkins, F. S., Proc. R. Soc. London, Ser. A 342, 149 (1975a). EL CL W IP

Smith, G., and Tomkins, F. S., unpublished material (1975b). [This material is included in a recent publication: see Smith, G., and Tomkins, F. S., Philos. Trans. R. Soc. London, Ser. A, 283, 345 (1976).] EL ND CL W ZE IS

Smith, G., and Wilson, M., J. Opt. Soc. Am. 60, 1527 (1970). ND PT

Smith, G., and Wybourne, B. G., J. Opt. Soc. Am. 55, 121 (1965). ND PT

Wyart, J. F., Thesis, Univ. Paris-Sud, Orsay, 194 pp. (1973). PT

[July 1976] 
Eu I, Odd Parity

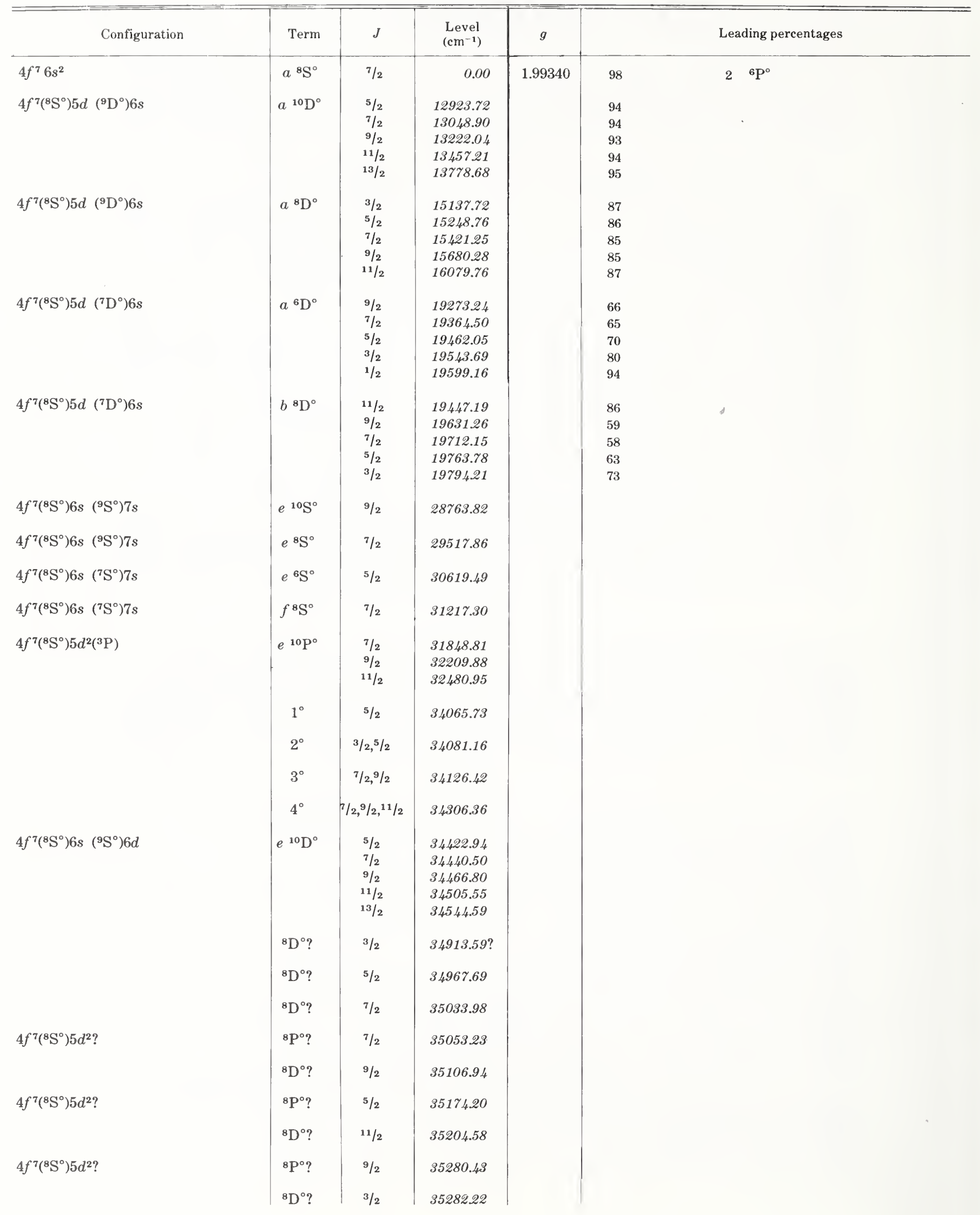


Eu I, Odd Parity-Continued

Eu I, Odd Parity-Continued

\begin{tabular}{|c|c|c|c|c|c|c|c|}
\hline Configuration & Term & $J$ & $\begin{array}{l}\text { Level } \\
\left(\mathrm{cm}^{-1}\right)\end{array}$ & Configuration & Term & $J$ & $\begin{array}{l}\text { Level } \\
\left(\mathrm{cm}^{-1}\right)\end{array}$ \\
\hline & ${ }^{8} \mathrm{D}^{\circ} ?$ & $7 / 2$ & 35377.94 & $4 f^{7}\left({ }^{8} \mathrm{~S}^{\circ}\right) 6 p^{2}\left({ }^{3} \mathrm{P}\right)$ & $f^{10} \mathrm{P}^{\circ}$ & $7 / 2$ & 40298.02 \\
\hline & ${ }^{8} \mathrm{D}^{\circ} ?$ & $5 / 2$ & 35398.16 & & & $\begin{array}{l}9 / 2 \\
11 / 2\end{array}$ & $\begin{array}{l}40854.11 \\
41443.70\end{array}$ \\
\hline & ${ }^{8} \mathrm{D}^{\circ} ?$ & $11 / 2$ & 35460.74 & & $5^{\circ}$ & $7 / 2$ & 40576.00 \\
\hline & ${ }^{8} \mathrm{D}^{\circ} ?$ & $9 / 2$ & 35813.47 & & $6^{\circ}$ & $5 / 2,7 / 2$ & 40624.10 \\
\hline \multirow[t]{3}{*}{$4 f^{7}\left({ }^{8} \mathrm{~S}^{\circ}\right) 6 s \quad\left({ }^{7} \mathrm{~S}^{\circ}\right) 6 d$} & $g^{8} \mathrm{D}^{\circ}$ & $\begin{array}{l}3 / 2 \\
5 / 2\end{array}$ & $\begin{array}{l}36045.39 \\
36072.62\end{array}$ & & $7^{\circ}$ & $7 / 2$ & 40650.51 \\
\hline & & $\begin{array}{l}7 / 2 \\
9 / 2\end{array}$ & $\begin{array}{l}36097.64 \\
3621900\end{array}$ & & $8^{\circ}$ & $9 / 2,11 / 2$ & 40764.08 \\
\hline & & $11 / 2$ & 36242.34 & & $9^{\circ}$ & $9 / 2$ & 40768.31 \\
\hline \multirow[t]{4}{*}{$4 f^{7}\left({ }^{8} \mathrm{~S}^{\circ}\right) 6 s \quad\left({ }^{7} \mathrm{~S}^{\circ}\right) 6 d$} & $e^{6} \mathrm{D}^{\circ}$ & $\begin{array}{l}9 / 2 \\
7 / 2\end{array}$ & $\begin{array}{l}36566.64 \\
36589.28\end{array}$ & & $10^{\circ}$ & $7 / 2,9 / 2$ & 40993.18 \\
\hline & & $5 / 2$ & 36608.42 & & $11^{\circ}$ & $7 / 2,9 / 2$ & $41037.58 ?$ \\
\hline & & $\begin{array}{l}3 / 2 \\
1 / 2\end{array}$ & $\begin{array}{l}36622.00 \\
36630.46\end{array}$ & $4 f^{7}\left({ }^{8} \mathrm{~S}^{\circ}\right) 6 s \quad\left({ }^{7} \mathrm{~S}^{\circ}\right) 7 d$ & & $9 / 2$ & 41174.74 \\
\hline & & & & & & $7 / 2$ & 41185.38 \\
\hline $4 f^{7}\left({ }^{8} \mathrm{~S}^{\circ}\right) 6 s \quad\left({ }^{9} \mathrm{~S}^{\circ}\right) 8 s$ & $g^{8} \mathrm{~S}^{\circ}$ & $7 / 2$ & 36659.31 & & & $\begin{array}{l}5 / 2 \\
3 / 2\end{array}$ & $\begin{array}{l}41201.21 \\
4120860\end{array}$ \\
\hline $4 f^{7}\left({ }^{8} \mathrm{~S}^{\circ}\right) 6 s\left({ }^{9} \mathrm{~S}^{\circ}\right) 8 s$ & $f^{10} \mathrm{~S}^{\circ}$ & $9 / 2$ & 37195.76 & & & $1 / 2$ & \\
\hline \multirow[t]{2}{*}{$4 f^{7}\left({ }^{8} \mathrm{~S}^{\circ}\right) 5 d^{2}\left({ }^{3} \mathrm{P}\right)$} & $e^{6} \mathrm{P}^{\circ}$ & $7 / 2$ & 37584.98 & & $12^{\circ}$ & $9 / 2,11 / 2$ & 41378.47 \\
\hline & & $\begin{array}{l}5 / 2 \\
3 / 2\end{array}$ & $\begin{array}{l}38037.34 \\
38245.66\end{array}$ & & $13^{\circ}$ & $9 / 2,11 / 2$ & 41395.92 \\
\hline $4 f^{7}\left({ }^{8} \mathrm{~S}^{\circ}\right) 5 d^{2}\left({ }^{1} \mathrm{~S}\right)$ & $h^{8} \mathrm{~S}^{\circ}$ & $7 / 2$ & 37993.98 & & $14^{\circ}$ & $7 / 2,9 / 2$ & 41631.37 \\
\hline $4 f^{7}\left({ }^{8} \mathrm{~S}^{\circ}\right) 6 s\left({ }^{7} \mathrm{~S}^{\circ}\right) 8 s$ & $f^{6} \mathrm{~S}^{\circ}$ & $5 / 2$ & 38933.74 & & $15^{\circ}$ & $9 / 2,11 / 2$ & $42721.09 ?$ \\
\hline $4 f^{7}\left({ }^{8} \mathrm{~S}^{\circ}\right) 6 s \quad\left({ }^{7} \mathrm{~S}^{\circ}\right) 8 s$ & $i^{8} \mathrm{~S}^{\circ}$ & $7 / 2$ & 39242.56 & • & $16^{\circ}$ & $7 / 2,9 / 2$ & 43100.66 \\
\hline \multirow{4}{*}{$4 f^{7}\left({ }^{8} \mathrm{~S}^{\circ}\right) 6 s \quad\left({ }^{9} \mathrm{~S}^{\circ}\right) 7 d$} & $f^{10} \mathrm{D}^{\circ}$ & $5 / 2$ & & & $17^{\circ}$ & $9 / 2,11 / 2$ & 43212.06? \\
\hline & & $7 / 2$ & 39284.73 & & $18^{\circ}$ & $7 / 2,9 / 2,11 / 2$ & 43466.82 \\
\hline & & $\begin{array}{l}9 / 2 \\
11 / 2\end{array}$ & $\begin{array}{l}39306.13 \\
39332.68\end{array}$ & & $19^{\circ}$ & $7 / 2,9 / 2$ & 43508.06 ? \\
\hline & & $13 / 2$ & 39369.19 & & & & \\
\hline \multirow[t]{2}{*}{$4 f^{7}\left({ }^{8} \mathrm{~S}^{\circ}\right) 6 s\left({ }^{9} \mathrm{~S}^{\circ}\right) 7 d$} & $h^{8} \mathrm{D}^{\circ}$ & $11 / 2$ & 39486.20 & Fu & Iimit & & $1=\times 9,0$ \\
\hline & & $\begin{array}{l}7 / 2 \\
7 / 2 \\
5 / 2 \\
3 / 2\end{array}$ & $\begin{array}{l}39491.49 \\
39496.56 \\
39500.31\end{array}$ & & & & \\
\hline
\end{tabular}


Eu I, Even Parity

\begin{tabular}{|c|c|c|c|c|c|c|c|c|}
\hline Configuration & Term & $J$ & $\begin{array}{l}\text { Level } \\
\left(\mathrm{cm}^{-1}\right)\end{array}$ & $g$ & \multicolumn{4}{|c|}{ Leading percentages } \\
\hline $4 f^{7}\left({ }^{8} \mathrm{~S}^{\circ}\right) 6 s 6 p\left({ }^{3} \mathrm{P}^{\circ}\right)$ & $z^{10} \mathrm{P}$ & $\begin{array}{c}7 / 2 \\
9 / 2 \\
11 / 2\end{array}$ & $\begin{array}{l}14067.86 \\
14563.57 \\
15581.58\end{array}$ & $\begin{array}{l}2.198 \\
1.936\end{array}$ & $\begin{array}{r}92 \\
85 \\
100\end{array}$ & $\begin{array}{l}\text { or } \\
\text { or } \\
\text { or }\end{array}$ & $\begin{array}{r}64 \\
91 \\
100\end{array}$ & $\begin{array}{l}\left({ }^{8} \mathrm{~S}_{7 / 2}^{\circ}\right)\left({ }^{3} \mathrm{P}_{0}^{\circ}\right) \\
\left({ }^{8} \mathrm{~S}_{7 / 2}^{\circ}\right)\left({ }^{3} \mathrm{P}_{1}^{\circ}\right) \\
\left({ }^{8} \mathrm{~S}_{7 / 2}^{\circ}\right)\left({ }^{3} \mathrm{P}_{2}^{\circ}\right)\end{array}$ \\
\hline $4 f^{7}\left({ }^{8} \mathrm{~S}^{\circ}\right) 6 s 6 p\left({ }^{3} \mathrm{P}^{\circ}\right)$ & $z^{8} \mathrm{P}$ & $\begin{array}{l}5 / 2 \\
7 / 2 \\
9 / 2\end{array}$ & $\begin{array}{l}15890.53 \\
15952.31 \\
16611.79\end{array}$ & $\begin{array}{l}2.227 \\
1.875 \\
1.797\end{array}$ & $\begin{array}{l}87 \\
61 \\
83\end{array}$ & $\begin{array}{l}\text { or } \\
\text { or } \\
\text { or }\end{array}$ & $\begin{array}{l}93 \\
51 \\
91\end{array}$ & $\begin{array}{l}\left({ }^{8} \mathrm{~S}_{7 / 2}^{\circ}\right)\left({ }^{3} \mathrm{P}_{1}^{\circ}\right) \\
\left({ }^{8} \mathrm{~S}_{7 / 2}^{\circ}\right)\left({ }^{3} \mathrm{P}_{i}^{\circ}\right) \\
\left({ }^{8} \mathrm{~S}_{7 / 2}^{\circ}\right)\left({ }^{3} \mathrm{P}_{2}^{\circ}\right)\end{array}$ \\
\hline $4 f^{7}\left({ }^{8} \mathrm{~S}^{\circ}\right) 6 s 6 p\left({ }^{3} \mathrm{P}^{\circ}\right)$ & $z^{6} \mathrm{P}$ & $\begin{array}{l}7 / 2 \\
5 / 2 \\
3 / 2\end{array}$ & $\begin{array}{l}17340.65 \\
17707.42 \\
17945.49\end{array}$ & $\begin{array}{l}1.787 \\
1.932\end{array}$ & $\begin{array}{r}64 \\
86 \\
100\end{array}$ & $\begin{array}{l}\text { or } \\
\text { or } \\
\text { or }\end{array}$ & $\begin{array}{r}82 \\
92 \\
100\end{array}$ & $\begin{array}{l}\left({ }^{8} \mathrm{~S}_{7 / 2}^{\circ}\right)\left({ }^{3} \mathrm{P}_{2}^{\circ}\right) \\
\left({ }^{8} \mathrm{~S}_{7 / 2}^{\circ}\right)\left({ }^{3} \mathrm{P}_{2}^{\circ}\right) \\
\left({ }^{8} \mathrm{~S}_{7 / 2}^{\circ}\right)\left({ }^{3} \mathrm{P}_{2}^{\circ}\right)\end{array}$ \\
\hline $4 f^{7}\left({ }^{8} \mathrm{~S}^{\circ}\right) 6 s 6 p\left({ }^{1} \mathrm{P}^{\circ}\right)$ & $y^{8} \mathrm{P}$ & $\begin{array}{l}5 / 2 \\
7 / 2 \\
9 / 2\end{array}$ & $\begin{array}{l}21444.58 \\
21605.17 \\
21761.26\end{array}$ & $\begin{array}{l}2.272 \\
1.929 \\
1.772\end{array}$ & $\begin{array}{l}98 \\
97 \\
96\end{array}$ & $\begin{array}{l}\text { or } \\
\text { or } \\
\text { or }\end{array}$ & $\begin{array}{l}99 \\
98 \\
98\end{array}$ & $\begin{array}{l}\left({ }^{8} \mathrm{~S}_{7 / 2}^{\circ}\right)\left({ }^{1} \mathrm{P}_{1}^{\circ}\right) \\
\left({ }^{8} \mathrm{~S}_{7 / 2}^{\circ}\right)\left({ }^{1} \mathrm{P}_{1}^{\circ}\right) \\
\left({ }^{8} \mathrm{~S}_{7 / 2}^{\circ}\right)\left({ }^{1} \mathrm{P}_{1}^{\circ}\right)\end{array}$ \\
\hline $4 f^{6}\left({ }^{7} \mathrm{~F}\right) 5 d 6 s^{2}$ & ${ }^{8} \mathrm{D}$ & $\begin{array}{c}3 / 2 \\
5 / 2 \\
7 / 2 \\
9 / 2 \\
11 / 2\end{array}$ & $\begin{array}{l}27852.90 \\
28827.83 \\
29838.59 \\
30819.44\end{array}$ & $\begin{array}{l}2.009 \\
1.811 \\
1.676\end{array}$ & $\begin{array}{l}68 \\
75 \\
76 \\
77\end{array}$ & & $\begin{array}{l}15 \\
13 \\
15 \\
17\end{array}$ & $\begin{array}{l}\left({ }^{7} \mathrm{~F}\right){ }^{6} \mathrm{P} \\
\left({ }^{7} \mathrm{~F}\right){ }^{8} \mathrm{~F} \\
\left({ }^{7} \mathrm{~F}\right){ }^{8} \mathrm{~F} \\
\left({ }^{7} \mathrm{~F}\right)^{8} \mathrm{~F}\end{array}$ \\
\hline $4 f^{7}\left({ }^{8} \mathrm{~S}^{\circ}\right) 5 d\left({ }^{9} \mathrm{D}^{\circ}\right) 6 p$ & 102 & $\begin{array}{c}3 / 2 \\
5 / 2 \\
7 / 2 \\
9 / 2 \\
11 / 2 \\
13 / 2 \\
15 / 2 \\
\\
9 / 2\end{array}$ & $\begin{array}{l}28519.97 \\
28667.39 \\
28918.17 \\
29186.32 \\
29612.69 \\
30211.09 \\
30923.71 \\
\\
29045.75 ?\end{array}$ & $\begin{array}{l}2.194 \\
1.841 \\
1.70\end{array}$ & $\begin{array}{r}94 \\
93 \\
92 \\
92 \\
93 \\
97 \\
100\end{array}$ & $\begin{array}{l}\text { or } \\
\text { or } \\
\text { or } \\
\text { or } \\
\text { or } \\
\text { or } \\
\text { or }\end{array}$ & $\begin{array}{r}94 \\
93 \\
92 \\
92 \\
93 \\
97 \\
100\end{array}$ & $\left({ }^{8} \mathrm{~S}^{\circ}\right) 5 d 6 p\left({ }^{3} \mathrm{~F}^{\circ}\right)^{10} \mathrm{~F}$ \\
\hline $4 f^{6}\left({ }^{7} \mathrm{~F}\right) 5 d 6 s^{2}$ & ${ }^{8} \mathrm{G}$ & $\begin{array}{c}1 / 2 \\
3 / 2 \\
5 / 2 \\
7 / 2 \\
9 / 2 \\
11 / 2 \\
13 / 2 \\
15 / 2\end{array}$ & $\begin{array}{l}29124.78 \\
29809.23 \\
30642.61 \\
32598.00\end{array}$ & $\begin{array}{l}1.440 \\
1.465 \\
1.470\end{array}$ & $\begin{array}{l}44 \\
56 \\
63 \\
66\end{array}$ & & $\begin{array}{l}20 \\
21 \\
19 \\
\\
25\end{array}$ & $\begin{array}{l}\left({ }^{7} \mathrm{~F}\right){ }^{8} \mathrm{~F} \\
\left({ }^{7} \mathrm{~F}\right){ }^{8} \mathrm{~F} \\
\left({ }^{7} \mathrm{~F}\right){ }^{8} \mathrm{~F} \\
\left({ }^{7} \mathrm{~F}\right){ }^{8} \mathrm{~F}\end{array}$ \\
\hline $4 f^{6}\left({ }^{7} \mathrm{~F}\right) 5 d 6 s^{2}$ & ${ }^{8} \mathrm{P}$ & $\begin{array}{l}5 / 2 \\
7 / 2 \\
9 / 2\end{array}$ & $\begin{array}{l}29982.50 \\
31116.38 \\
32130.25\end{array}$ & $\begin{array}{l}2.06 \\
1.928 \\
1.747\end{array}$ & $\begin{array}{l}51 \\
52 \\
83\end{array}$ & & $\begin{array}{r}25 \\
18 \\
9\end{array}$ & $\begin{array}{l}\left({ }^{7} \mathrm{~F}\right){ }^{6} \mathrm{P} \\
\left({ }^{7} \mathrm{~F}\right){ }^{8} \mathrm{G} \\
\left({ }^{7} \mathrm{~F}\right){ }^{8} \mathrm{D}\end{array}$ \\
\hline $4 f^{6}\left({ }^{7} \mathrm{~F}\right) 5 d 6 s^{2}$ & ${ }^{8} \mathrm{~F}$ & $\begin{array}{c}1 / 2 \\
3 / 2 \\
5 / 2 \\
7 / 2 \\
9 / 2 \\
11 / 2 \\
13 / 2\end{array}$ & $\begin{array}{l}30091.34 \\
30783.64 \\
31553.76 \\
32326.73\end{array}$ & $\begin{array}{l}1.618 \\
1.551 \\
1.522\end{array}$ & $\begin{array}{l}61 \\
50 \\
58 \\
67\end{array}$ & & $\begin{array}{l}29 \\
16 \\
28 \\
23\end{array}$ & $\begin{array}{l}\left({ }^{7} \mathrm{~F}\right){ }^{8} \mathrm{G} \\
\left({ }^{7} \mathrm{~F}\right){ }^{8} \mathrm{G} \\
\left({ }^{7} \mathrm{~F}\right){ }^{8} \mathrm{G} \\
\left({ }^{7} \mathrm{~F}\right){ }^{8} \mathrm{G}\end{array}$ \\
\hline $4 f^{7}\left({ }^{8} \mathrm{~S}^{\circ}\right) 5 d\left({ }^{9} \mathrm{D}^{\circ}\right) 6 p$ & 110 & $\begin{array}{c}5 / 2 \\
3 / 2 \\
7 / 2 \\
9 / 2 \\
11 / 2 \\
9 / 2,11 / 2\end{array}$ & $\begin{array}{l}30798.06 \\
30800.71 \\
30841.99 \\
30901.84 \\
31014.48 \\
30819.14 ?\end{array}$ & $\begin{array}{l}2.14 \\
2.80 \\
1.824 \\
1.706\end{array}$ & $\begin{array}{l}86 \\
89 \\
84 \\
81 \\
79\end{array}$ & $\begin{array}{l}\text { or } \\
\text { or } \\
\text { or } \\
\text { or } \\
\text { or }\end{array}$ & $\begin{array}{l}68 \\
69 \\
67 \\
67 \\
70\end{array}$ & $\left({ }^{8} \mathrm{~S}^{\circ}\right) 5 d 6 p\left({ }^{1} \mathrm{D}^{\circ}\right){ }^{8} \mathrm{D}$ \\
\hline $4 f^{7}\left({ }^{8} \mathrm{~S}^{\circ}\right) 5 d\left({ }^{9} \mathrm{D}^{\circ}\right) 6 p$ & $z^{10} \mathrm{D}$ & $\begin{array}{c}5 / 2 \\
7 / 2 \\
9 / 2 \\
11 / 2 \\
13 / 2\end{array}$ & $\begin{array}{l}30945.07 \\
31138.11 \\
31382.61 \\
31725.93 \\
32117.10\end{array}$ & $\begin{array}{l}2.49 \\
2.006 \\
1.860\end{array}$ & $\begin{array}{l}95 \\
94 \\
92 \\
84 \\
63\end{array}$ & $\begin{array}{l}\text { or } \\
\text { or } \\
\text { or } \\
\text { or } \\
\text { or }\end{array}$ & $\begin{array}{l}95 \\
94 \\
92 \\
84 \\
62\end{array}$ & $\left({ }^{8} \mathrm{~S}^{\circ}\right) 5 d 6 p\left({ }^{3} \mathrm{D}^{\circ}\right){ }^{10} \mathrm{D}$ \\
\hline
\end{tabular}


Eu I, Even Parity-Continued

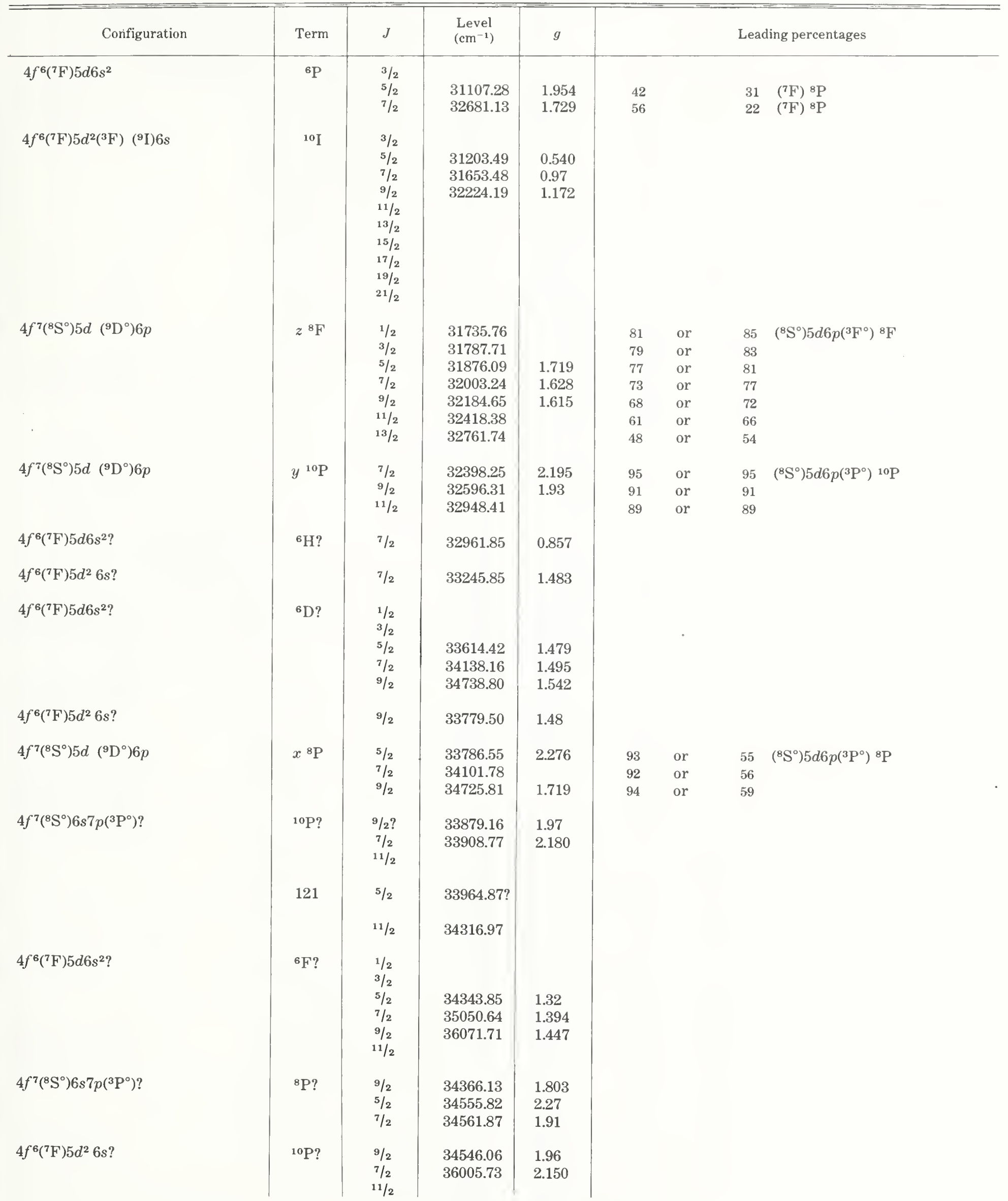


Eu I, Even Parity-Continued

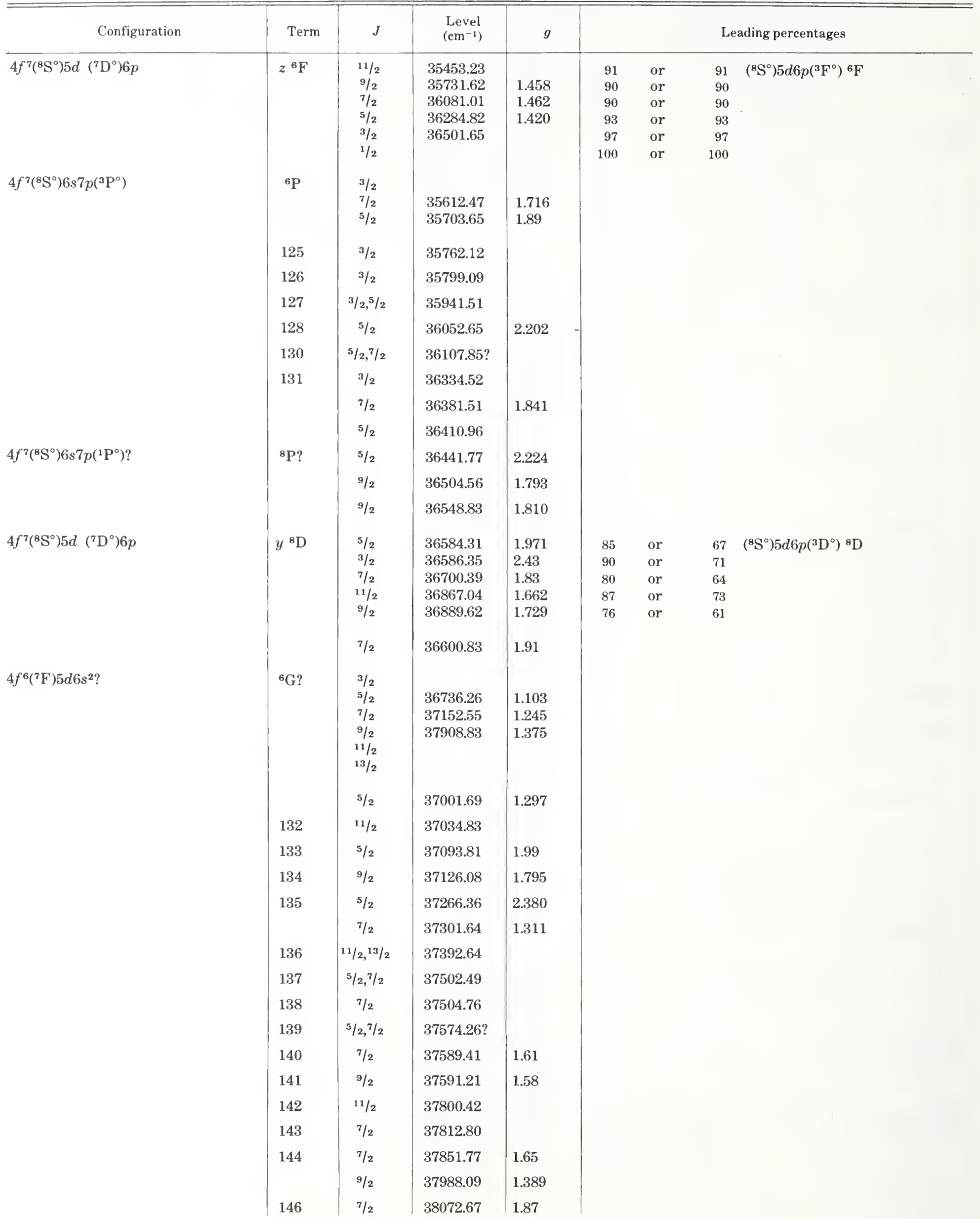


Eu I, Even Parity_Continued

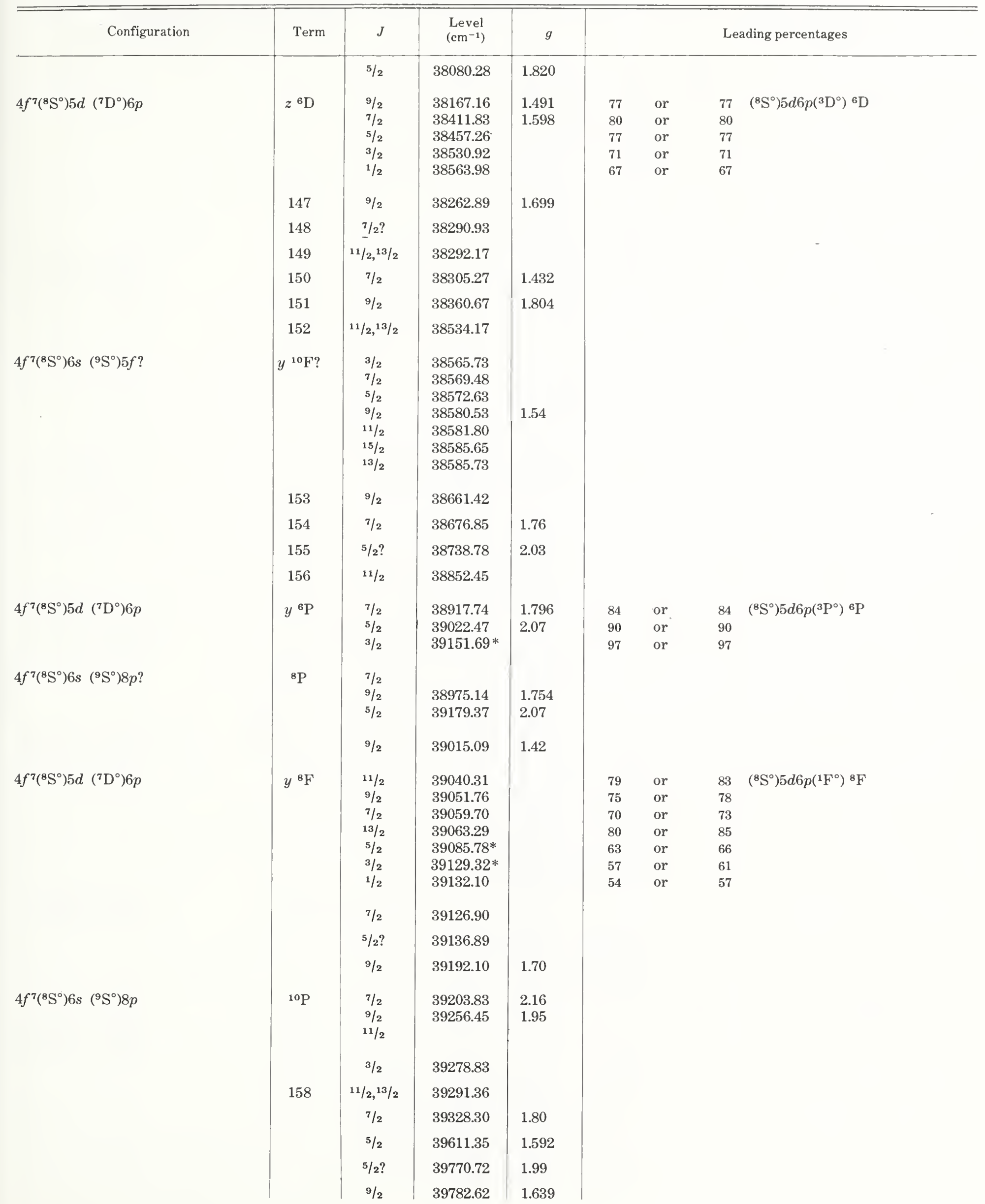


Eu I, Even Parity-Continued

\begin{tabular}{|c|c|c|c|c|c|}
\hline Configuration & Term & $J$ & $\begin{array}{l}\text { Level } \\
\left(\mathrm{cm}^{-1}\right)\end{array}$ & $g$ & Leading percentages \\
\hline & 159 & $5 / 2$ & 40073.57 & 2.305 & \\
\hline \multirow[t]{5}{*}{$4 f^{7}\left({ }^{8} \mathrm{~S}^{\circ}\right) 6 s \quad\left({ }^{7} \mathrm{~S}^{\circ}\right) 5 f ?$} & $y^{6} \mathrm{~F} ?$ & $11 / 2$ & 40198.00 & & \\
\hline & & $9 / 2$ & 40200.43 & & \\
\hline & & $7 / 2$ & 40201.14 & & \\
\hline & & $3 / 2$ & 40203.37 & & \\
\hline & & $\begin{array}{l}3 / 2 \\
1 / 2\end{array}$ & $\begin{array}{l}40203.68 \\
40206.71\end{array}$ & & \\
\hline \multirow[t]{7}{*}{$4 f^{7}\left({ }^{8} \mathrm{~S}^{\circ}\right) 6 s \quad\left({ }^{7} \mathrm{~S}^{\circ}\right) 5 f ?$} & $x^{8} \mathrm{~F} ?$ & $1 / 2$ & 40217.90 & & \\
\hline & & $3 / 2$ & 40223.66 & & \\
\hline & & $11 / 2$ & 40236.14 & & \\
\hline & & $7 / 2$ & 40236.64 & & \\
\hline & & $5 / 2$ & 40237.35 & & \\
\hline & & $\begin{array}{l}13 / 2 \\
9 / 2\end{array}$ & $\begin{array}{l}40244.66 \\
40249.68\end{array}$ & & \\
\hline & 160 & $9 / 2,{ }^{11} / 2$ & 40302.07 & & \\
\hline \multirow[t]{3}{*}{$4 f^{7}\left({ }^{6} I^{\circ}\right) 6 s 6 p ?$} & & $7 / 2$ & 40323.65 & 1.042 & \\
\hline & & $9 / 2$ & 40372.61 & 1.58 & \\
\hline & & $7 / 2$ & 40373.63 & 1.436 & \\
\hline \multirow[t]{6}{*}{$4 f^{7}\left({ }^{8} \mathrm{~S}^{\circ}\right) 6 s \quad\left({ }^{7} \mathrm{~S}^{\circ}\right) 8 p ?$} & ${ }^{8} \mathrm{P}$ & $9 / 2$ & 40455.51 & 1.768 & \\
\hline & & $7 / 2$ & 40609.30 & 1.89 & \\
\hline & & $5 / 2$ & 40629.82 & 2.264 & \\
\hline & & $7 / 2$ & 40493.09 & 1.529 & \\
\hline & & $5 / 2$ & 40792.43 & 1.605 & \\
\hline & 161 & $9 / 2,11 / 2$ & 40838.66 & & \\
\hline $4 f^{7}\left({ }^{6} I^{\circ}\right) 6 s 6 p ?$ & & $9 / 2$ & 40843.16 & 1.013 & \\
\hline \multirow[t]{6}{*}{$4 f^{7}\left({ }^{8} \mathrm{~S}^{\circ}\right) 6 s \quad\left({ }^{7} \mathrm{~S}^{\circ}\right) 8 p$} & ${ }^{6} \mathrm{P}$ & $3 / 2$ & & & \\
\hline & & $7 / 2$ & 40862.41 & 1.692 & \\
\hline & & $5 / 2$ & 40939.36 & 1.88 & \\
\hline & & $9 / 2$ & 40962.39 & 1.64 & \\
\hline & 162 & $11 / 2,13 / 2$ & $41054.83 ?$ & & \\
\hline & & $5 / 2$ & 41078.38 & 1.149 & \\
\hline \multirow[t]{9}{*}{$4 f^{7}\left({ }^{8} \mathrm{~S}^{\circ}\right) 6 s \quad\left({ }^{9} \mathrm{~S}^{\circ}\right) 6 f ?$} & $x^{10} \mathrm{~F} ?$ & $15 / 2$ & 41152.12 & & \\
\hline & & $3 / 2$ & 41166.02 & & \\
\hline & & $5 / 2$ & 41167.14 & & \\
\hline & & $7 / 2$ & 41169.20 & & \\
\hline & & $9 / 2$ & 41171.00 & & \\
\hline & & $\begin{array}{l}11 / 2 \\
13 / 2\end{array}$ & $\begin{array}{l}41176.73 \\
41184.12\end{array}$ & & \\
\hline & & $7 / 2$ & 41223.58 & 1.708 & \\
\hline & & $5 / 2,7 / 2,9 / 2$ & 41246.25 & & \\
\hline & & $5 / 2,{ }^{7} / 2,9 / 2$ & 41247.86 & & \\
\hline \multirow[t]{5}{*}{$6 f^{7}\left({ }^{8} \mathrm{~S}^{\circ}\right) 6 s\left({ }^{9} \mathrm{~S}^{\circ}\right) 9 p$} & ${ }^{8} \mathrm{P}$ & $5 / 2$ & 41283.00 & 2.256 & \\
\hline & & $9 / 2$ & 41285.58 & 1.762 & \\
\hline & & $7 / 2$ & 41335.67 & 1.775 & \\
\hline & & $5 / 2$ & 41408.47 & 1.609 & \\
\hline & & $5 / 2$ & 41497.75 & 1.357 & \\
\hline \multirow[t]{2}{*}{$4 f^{7}\left({ }^{8} \mathrm{~S}^{\circ}\right) 6 s \quad\left({ }^{9} \mathrm{~S}^{\circ}\right) 9 p$} & ${ }^{10} \mathrm{P}$ & $7 / 2$ & 41515.52 & 2.210 & \\
\hline & & $\begin{array}{c}9 / 2 \\
11 / 2\end{array}$ & 41540.43 & 1.97 & \\
\hline
\end{tabular}


Eu I, Even Parity_Continued

$4 f^{7}\left({ }^{8} \mathrm{~S}^{\circ}\right) 5 d\left({ }^{7} \mathrm{D}^{\circ}\right) 6 p$

$4 f^{7}\left({ }^{6} I^{\circ}\right) 6 s 6 p ?$

$4 f^{7}\left({ }^{6} I^{\circ}\right) 6 s 6 p ?$

$4 f^{7}\left({ }^{8} \mathrm{~S}^{\circ}\right) 6 s\left({ }^{9} \mathrm{~S}^{\circ}\right) 10 p$

$4 f^{7}\left({ }^{8} \mathrm{~S}^{\circ}\right) 6 s\left({ }^{9} \mathrm{~S}^{\circ}\right) 10 p$

$4 f^{7}\left({ }^{8} \mathrm{~S}^{\circ}\right) 6 s \quad\left({ }^{7} \mathrm{~S}^{\circ}\right) 9 p$

$4 f^{7}\left({ }^{8} \mathrm{~S}^{\circ}\right) 6 s \quad\left({ }^{7} \mathrm{~S}^{\circ}\right) 9 p$

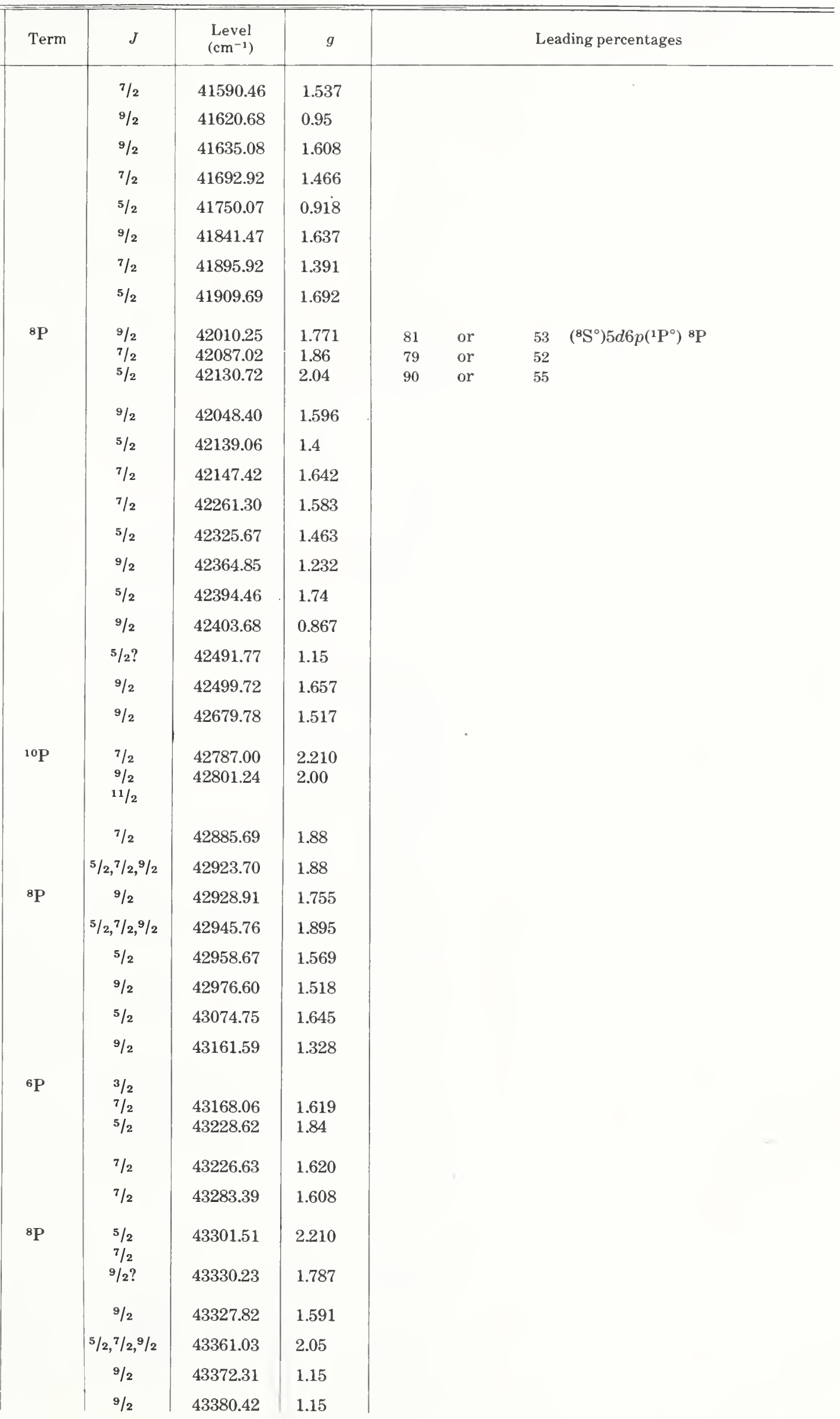




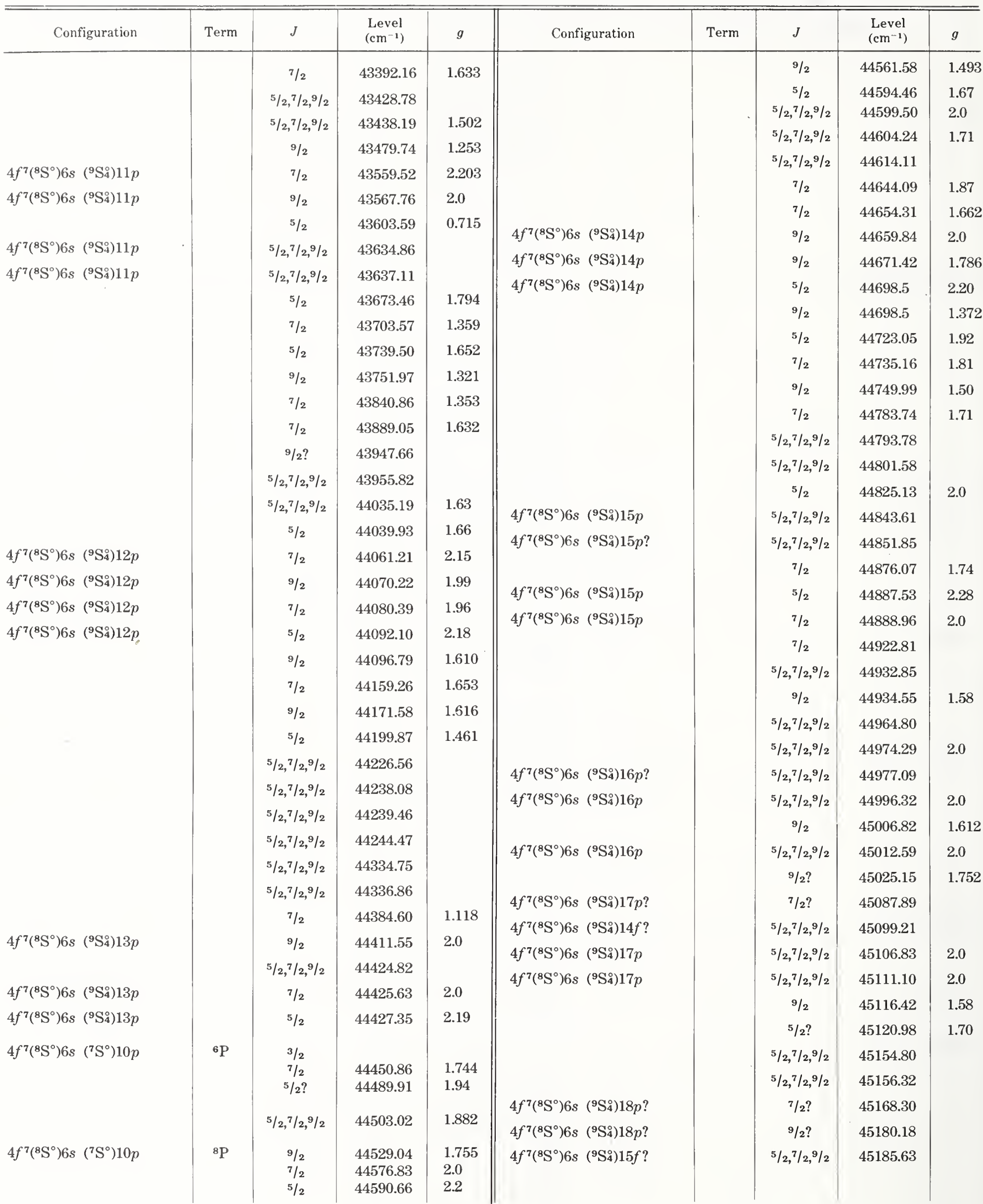


Eu I, Even Parity-Continued

Eu I, Even Parity-Continued

\begin{tabular}{|c|c|c|c|c|c|c|c|c|c|}
\hline Configuration & Term & $J$ & $\begin{array}{l}\text { Level } \\
\left(\mathrm{cm}^{-1}\right)\end{array}$ & $g$ & Configuration & Term & $J$ & $\begin{array}{c}\text { Level } \\
\left(\mathrm{cm}^{-1}\right)\end{array}$ & $g$ \\
\hline $4 f^{7}\left({ }^{8} \mathrm{~S}^{\circ}\right) 6 s \quad\left({ }^{9} \mathrm{~S}_{4}^{\circ}\right) 18 p ?$ & & $5 / 2 ?$ & 45187.63 & & $4 f^{7}\left({ }^{8} \mathrm{~S}^{\circ}\right) 6 s \quad\left({ }^{9} \mathrm{~S}_{4}\right) 57 p$ & & $5 / 2,7 / 2,9 / 2$ & 45695.50 & \\
\hline $4 f^{7}\left({ }^{8} \mathrm{~S}^{\circ}\right) 6 s \quad\left({ }^{9} \mathrm{~S}_{4}^{\circ}\right) 18 p ?$ & & $7 / 2 ?$ & 45189.42 & & $4 f^{7}\left({ }^{8} \mathrm{~S}^{\circ}\right) 6 s \quad\left({ }^{9} \mathrm{~S}_{4}{ }^{\circ}\right) 58 p$ & & $5 / 2,{ }^{7} / 2,9 / 2$ & 45696.87 & \\
\hline $4 f^{7}\left({ }^{8} \mathrm{~S}^{\circ}\right) 6 s \quad\left({ }^{9} \mathrm{~S}_{4}^{\circ}\right) 18 p ?$ & & $9 / 2 ?$ & 45191.23 & & $4 f^{7}\left({ }^{8} \mathrm{~S}^{\circ}\right) 6 s \quad\left({ }^{9} \mathrm{~S}_{4}^{\circ}\right) 59 p$ & & $5 / 2,7 / 2,9 / 2$ & 45698.16 & \\
\hline & & $5 / 2,7 / 2,9 / 2$ & 45258.76 & & $4 f^{7}\left({ }^{8} \mathrm{~S}^{\circ}\right) 6 s \quad\left({ }^{9} \mathrm{~S}_{4}^{\circ}\right) 60 p$ & & $5 / 2,7 / 2,9 / 2$ & 45699.40 & \\
\hline & & $5 / 2,{ }^{7} / 2,9 / 2$ & 45260.00 & & $4 f^{7}\left({ }^{8} \mathrm{~S}^{\circ}\right) 6 s \quad\left({ }^{9} \mathrm{~S}_{4}^{\circ}\right) 61 p$ & & $5 / 2,{ }^{7} / 2,9 / 2$ & 45700.57 & \\
\hline & & $5 / 2,7 / 2,9 / 2$ & 45262.44 & & $4 f^{7}\left({ }^{8} \mathrm{~S}^{\circ}\right) 6 s\left({ }^{9} \mathrm{~S}_{4}^{\circ}\right) 62 p$ & & $5 / 2,7 / 2,9 / 2$ & 45701.70 & \\
\hline & & $5 / 2,7 / 2,9 / 2$ & 45298.60 & & & & & & \\
\hline & & $5 / 2,7 / 2,9 / 2$ & 45304.93 & & & & & & \\
\hline & & $5 / 2,7 / 2,9 / 2$ & 45321.20 & & $\mathrm{Eu}$ II $\left({ }^{9} \mathrm{~S}_{4}^{\circ}\right)$ & Limit & ------- & 45734.9 & \\
\hline & & $5 / 2,{ }^{7} / 2,9 / 2$ & 45337.71 & & $4 f^{7}\left({ }^{8} \mathrm{~S}^{\circ}\right) 5 d\left({ }^{9} \mathrm{D}^{\circ}\right) 7 p ?$ & ${ }^{8 P}$ ? & $5 / 2,7 / 2,9 / 2$ & $45754.4 a$ & \\
\hline & & $5 / 2,{ }^{7} / 2,9 / 2$ & 45339.82 & & & & $5 / 2,7 / 2,9 / 2$ & $45975 a$ & \\
\hline & & $5 / 2,{ }^{7} / 2,9 / 2$ & 45364.35 & & $4 f^{7}\left({ }^{8} \mathrm{~S}^{\circ}\right) 5 d\left({ }^{9} \mathrm{D}^{\circ}\right) 7 p ?$ & ${ }^{8 \mathrm{P}}$ ? & $5 / 2,7 / 2,9 / 2$ & $46145 a$ & \\
\hline & & $5 / 2,7 / 2,9 / 2$ & 45367.53 & & & & $5 / 2,7 / 2,9 / 2$ & $46346 a$ & \\
\hline & & $5 / 2,7 / 2,9 / 2$ & 45403.19 & & & & $5 / 2,7 / 2,9 / 2$ & $46394 a$ & \\
\hline & & $5 / 2,{ }^{7} / 2,9 / 2$ & 45407.95 & & & & $5 / 2,{ }^{7} / 2,9 / 2$ & $46539 a$ & \\
\hline & & $5 / 2,{ }^{7} / 2,9 / 2$ & 45420.78 & & & & $5 / 2,7 / 2,9 / 2$ & $46684 a$ & \\
\hline & & $5 / 2,{ }^{7} / 2,9 / 2$ & 45423.50 & & & & $5 / 2,7 / 2,9 / 2$ & $46781 a$ & \\
\hline & & $5 / 2,{ }^{7} / 2,9 / 2$ & 45427.36 & & & & $5 / 2,7 / 2,9 / 2$ & $46983 a$ & \\
\hline & & $5 / 2,{ }^{7} / 2,9 / 2$ & 45447.46 & & & & $5 / 2,7 / 2,9 / 2$ & $47088 a$ & \\
\hline & & $5 / 2,7 / 2,9 / 2$ & 45469.87 & & & & $5 / 2,7 / 2,9 / 2$ & $47289 a$ & \\
\hline & & $5 / 2,{ }^{7} / 2,9 / 2$ & 45471.70 & & $4 f^{7}\left({ }^{8} \mathrm{~S}^{\circ}\right) 6 s \quad\left({ }^{7} \mathrm{~S}_{3}^{\circ}\right) 44 p$ & & $5 / 2,7 / 2,9 / 2$ & 47337.15 & \\
\hline & & $5 / 2,{ }^{7} / 2,9 / 2$ & 45481.97 & & $4 f^{7}\left({ }^{8} \mathrm{~S}^{\circ}\right) 6 s \quad\left({ }^{7} \mathrm{~S}_{3}^{\circ}\right) 45 p$ & & $5 / 2,7 / 2,9 / 2$ & 47340.29 & \\
\hline & & $5 / 2,7 / 2,9 / 2$ & 45490.70 & & $4 f^{7}\left({ }^{8} \mathrm{~S}^{\circ}\right) 6 s \quad\left({ }^{7} \mathrm{~S}_{3}^{\circ}\right) 46 p$ & & $5 / 2,7 / 2,9 / 2$ & 47343.21 & \\
\hline & & $5 / 2,7 / 2,9 / 2$ & 45492.79 & & $4 f^{7}\left({ }^{8} \mathrm{~S}^{\circ}\right) 6 s \quad\left({ }^{7} \mathrm{~S}_{3}\right) 47 p$ & & $5 / 2,7 / 2,9 / 2$ & 47345.98 & \\
\hline & & $5 / 2,{ }^{7} / 2,9 / 2$ & 45509.92 & & $4 f^{7}\left({ }^{8} \mathrm{~S}^{\circ}\right) 6 s \quad\left({ }^{7} \mathrm{~S}_{3}^{\circ}\right) 48 p$ & & $5 / 2,7 / 2,9 / 2$ & 47348.63 & \\
\hline & & $5 / 2,{ }^{7} / 2,9 / 2$ & 45593.85 & & $4 f^{7}\left({ }^{8} \mathrm{~S}^{\circ}\right) 6 s \quad\left({ }^{7} \mathrm{~S}_{3}^{\circ}\right) 49 p$ & & $5 / 2,7 / 2,9 / 2$ & 47351.04 & \\
\hline & & $5 / 2,{ }^{7} / 2,9 / 2$ & 45601.92 & & $4 f^{7}\left({ }^{8} \mathrm{~S}^{\circ}\right) 6 s \quad\left({ }^{7} \mathrm{~S}_{3}^{\circ}\right) 50 p$ & & $5 / 2,7 / 2,9 / 2$ & 47353.29 & \\
\hline & & $5 / 2,{ }^{7} / 2,9 / 2$ & 45607.71 & & $4 f^{7}\left({ }^{8} \mathrm{~S}^{\circ}\right) 6 s \quad\left({ }^{7} \mathrm{~S}_{3}\right) 51 p$ & & $5 / 2,7 / 2,9 / 2$ & 47355.41 & \\
\hline & & $5 / 2,{ }^{7} / 2,9 / 2$ & 45608.95 & & $4 f^{7}\left({ }^{8} \mathrm{~S}^{\circ}\right) 6 s \quad\left({ }^{7} \mathrm{~S}_{3}^{\circ}\right) 52 p$ & & $5 / 2,7 / 2,9 / 2$ & 47357.43 & \\
\hline $4 f^{7}\left({ }^{8} \mathrm{~S}^{\circ}\right) 6 s \quad\left({ }^{9} \mathrm{~S}_{4}^{\circ}\right) 42 p$ & & $5 / 2,7 / 2,9 / 2$ & 45658.74 & & $4 f^{7}\left({ }^{8} \mathrm{~S}^{\circ}\right) 63 \quad\left({ }^{7} \mathrm{~S}_{3}^{\circ}\right) 53 p$ & & $5 / 2,7 / 2,9 / 2$ & 47359.29 & \\
\hline $4 f^{7}\left({ }^{8} \mathrm{~S}^{\circ}\right) 6 s \quad\left({ }^{9} \mathrm{~S}_{4}^{\circ}\right) 43 p$ & & $5 / 2,7 / 2,9 / 2$ & 45662.57 & & $4 f^{7}\left({ }^{8} \mathrm{~S}^{\circ}\right) 6 s \quad\left({ }^{7} \mathrm{~S} 3\right) 54 p$ & & $5 / 2,{ }^{7} / 2,9 / 2$ & 47361.02 & \\
\hline $4 f^{7}\left({ }^{8} \mathrm{~S}^{\circ}\right) 6 s \quad\left({ }^{9} \mathrm{~S}_{4}^{\circ}\right) 44 p$ & & $5 / 2,7 / 2,9 / 2$ & 45666.13 & & $4 f^{7}\left({ }^{8} \mathrm{~S}^{\circ}\right) 6 s \quad\left({ }^{7} \mathrm{~S} 3\right) 55 p$ & & $5 / 2,7 / 2,9 / 2$ & 47362.69 & \\
\hline $4 f^{7}\left({ }^{8} \mathrm{~S}^{\circ}\right) 6 s \quad\left({ }^{9} \mathrm{~S}_{4}^{\circ}\right) 45 p$ & & $5 / 2,{ }^{7} / 2,9 / 2$ & 45669.43 & & $4 f^{7}\left({ }^{8} \mathrm{~S}^{\circ}\right) 6 s \quad\left({ }^{7} \mathrm{~S}_{3}\right) 56 p$ & & $5 / 2,7 / 2,9 / 2$ & 47364.27 & \\
\hline $4 f^{7}\left({ }^{8} \mathrm{~S}^{\circ}\right) 6 s \quad\left({ }^{9} \mathrm{~S}_{4}^{\circ}\right) 46 p$ & & $5 / 2,{ }^{7} / 2,9 / 2$ & 45672.53 & & $4 f^{7}\left({ }^{8} \mathrm{~S}^{\circ}\right) 6 s\left({ }^{7} \mathrm{~S}_{3}\right) 57 p$ & & $5 / 2,7 / 2,9 / 2$ & 47365.76 & \\
\hline $4 f^{7}\left({ }^{8} \mathrm{~S}^{\circ}\right) 6 s \quad\left({ }^{9} \mathrm{~S}_{4}^{\circ}\right) 47 p$ & & $5 / 2,7 / 2,9 / 2$ & 45675.37 & & $4 f^{7}\left({ }^{8} \mathrm{~S}^{\circ}\right) 6 s \quad\left({ }^{7} \mathrm{~S}_{3}\right) 58 p$ & & $5 / 2,7 / 2,9 / 2$ & 47367.15 & \\
\hline $4 f^{7}\left({ }^{8} \mathrm{~S}^{\circ}\right) 6 s \quad\left({ }^{9} \mathrm{~S}_{4}^{\circ}\right) 48 p$ & & $5 / 2,{ }^{7} / 2,9 / 2$ & 45678.00 & & $4 f^{7}\left({ }^{8} \mathrm{~S}^{\circ}\right) 6 s\left({ }^{7} \mathrm{~S} 3\right) 59 p$ & & $5 / 2,7 / 2,9 / 2$ & 47368.49 & \\
\hline $4 f^{7}\left({ }^{8} \mathrm{~S}^{\circ}\right) 6 s \quad\left({ }^{9} \mathrm{~S}_{4}^{\circ}\right) 49 p$ & & $5 / 2,7 / 2,9 / 2$ & 45680.53 & & $4 f^{7}\left({ }^{8} \mathrm{~S}^{\circ}\right) 6 s\left({ }^{7} \mathrm{~S}_{3}^{\circ}\right) 60 p$ & & $5 / 2,7 / 2,9 / 2$ & 47369.75 & \\
\hline $4 f^{7}\left({ }^{8} \mathrm{~S}^{\circ}\right) 6 s \quad\left({ }^{9} \mathrm{~S}_{4}^{\circ}\right) 50 p$ & & $5 / 2,{ }^{7} / 2,9 / 2$ & 45682.86 & & $4 f^{7}\left({ }^{8} \mathrm{~S}^{\circ}\right) 6 s\left({ }^{7} \mathrm{~S}_{3}^{\circ}\right) 61 p$ & & $5 / 2,7 / 2,9 / 2$ & 47370.90 & \\
\hline $4 f^{7}\left({ }^{8} \mathrm{~S}^{\circ}\right) 6 s \quad\left({ }^{9} \mathrm{~S}_{4}^{\circ}\right) 51 p$ & & $5 / 2,7 / 2,9 / 2$ & 45685.03 & & $4 f^{7}\left({ }^{8} \mathrm{~S}^{\circ}\right) 6 s\left({ }^{7} \mathrm{~S}_{3}^{\circ}\right) 62 p$ & & $5 / 2,7 / 2,9 / 2$ & 47372.02 & \\
\hline $4 f^{7}\left({ }^{8} \mathrm{~S}^{\circ}\right) 6 s \quad\left({ }^{9} \mathrm{~S}_{4}^{\circ}\right) 52 p$ & & $5 / 2,7 / 2,9 / 2$ & 45687.12 & & $4 f^{7}\left({ }^{8} \mathrm{~S}^{\circ}\right) 6 s\left({ }^{7} \mathrm{~S}_{3}^{\circ}\right) 63 p$ & & $5 / 2,7 / 2,9 / 2$ & 47373.10 & \\
\hline $4 f^{7}\left({ }^{8} \mathrm{~S}^{\circ}\right) 6 s \quad\left({ }^{9} \mathrm{~S}_{4}^{\circ}\right) 53 p$ & & $5 / 2,{ }^{7} / 2,9 / 2$ & 45689.05 & & $4 f^{7}\left({ }^{8} \mathrm{~S}^{\circ}\right) 6 s\left({ }^{7} \mathrm{~S}_{3}^{\circ}\right) 64 p$ & & $5 / 2,7 / 2,9 / 2$ & 47374.10 & \\
\hline $4 f^{7}\left({ }^{8} \mathrm{~S}^{\circ}\right) 6 s \quad\left({ }^{9} \mathrm{~S}_{4}^{\circ}\right) 54 p$ & & $5 / 2,{ }^{7} / 2,9 / 2$ & 45690.82 & & $4 f^{7}\left({ }^{8} \mathrm{~S}^{\circ}\right) 6 s \quad\left({ }^{7} \mathrm{~S}_{3}^{\circ}\right) 65 p$ & & $5 / 2,7 / 2,9 / 2$ & 47375.04 & \\
\hline $4 f^{7}\left({ }^{8} \mathrm{~S}^{\circ}\right) 6 s \quad\left({ }^{9} \mathrm{~S}_{4}^{\circ}\right) 55 p$ & & $5 / 2,7 / 2,9 / 2$ & 45692.50 & & $4 f^{7}\left({ }^{8} \mathrm{~S}^{\circ}\right) 6 s \quad\left({ }^{7} \mathrm{~S}_{3}^{\circ}\right) 66 p$ & & $5 / 2,7 / 2,9 / 2$ & 47375.96 & \\
\hline $4 f^{7}\left({ }^{8} \mathrm{~S}^{\circ}\right) 6 s \quad\left({ }^{9} \mathrm{~S}_{4}^{\circ}\right) 56 p$ & & $5 / 2,7 / 2,9 / 2$ & 45694.05 & & $4 f^{7}\left({ }^{8} \mathrm{~S}^{\circ}\right) 6 s \quad\left({ }^{7} \mathrm{~S}_{3}^{\circ}\right) 67 p$ & & $5 / 2,7 / 2,9 / 2$ & 47376.87 & \\
\hline
\end{tabular}


Eu I, Even Parity_Continued

\begin{tabular}{|c|c|c|c|c|c|c|c|c|c|}
\hline Configuration & Term & $J$ & $\begin{array}{l}\text { Level } \\
\left(\mathrm{cm}^{-1}\right)\end{array}$ & $g$ & Configuration & Term & $J$ & $\begin{array}{l}\text { Level } \\
\left(\mathrm{cm}^{-1}\right)\end{array}$ & $g$ \\
\hline \multirow[t]{9}{*}{ Eu II $\left({ }^{7} \mathrm{~S}_{3}^{\circ}\right)$} & Limit & --------- & 47404.1 & & & & $5 / 2,7 / 2,9 / 2$ & $51274 a$ & \\
\hline & & $5 / 2,7 / 2,9 / 2$ & $47483 a$ & & & & $5 / 2,7 / 2,9 / 2$ & $51556 a$ & \\
\hline & & $5 / 2,7 / 2,9 / 2$ & $48241 a$ & & & & $5 / 2,{ }^{7} / 2,9 / 2$ & $51806 a$ & \\
\hline & & $5 / 2,7 / 2,9 / 2$ & $48490 a$ & & & & $5 / 2,{ }^{7} / 2,9 / 2$ & $52185 a$ & \\
\hline & & $5 / 2,7 / 2,9 / 2$ & $49860 a$ & & & & $5 / 2,7 / 2,9 / 2$ & $52572 a$ & \\
\hline & & $5 / 2,7 / 2,9 / 2$ & $50040 a$ & & & & $5 / 2,7 / 2,9 / 2$ & $53000 a$ & \\
\hline & & $5 / 2,7 / 2,9 / 2$ & $50435 a$ & & & & $5 / 2,7 / 2,9 / 2$ & $53355 a$ & \\
\hline & & $5 / 2,7 / 2,9 / 2$ & $50935 a$ & & & & $5 / 2,7 / 2,9 / 2$ & $53887 a$ & \\
\hline & & $5 / 2,7 / 2,9 / 2$ & $51137 a$ & & & & $5 / 2,7 / 2,9 / 2$ & $54073 a$ & \\
\hline
\end{tabular}




\section{Eu II}

(Sm I sequence; 62 electrons)

$Z=63$

Ground state $\left(1 s^{2} 2 s^{2} 2 p^{6} 3 s^{2} 3 p^{6} 3 d^{10} 4 s^{2} 4 p^{6} 4 d^{10} 5 s^{2} 5 p^{6}\right) 4 f^{7} 6 s^{9} \mathrm{~S}_{4}^{\circ}$

Ionization energy $90665 \pm 50 \mathrm{~cm}^{-1}$

$11.241 \pm 0.006 \mathrm{eV}$

Identified odd configurations

$4 f^{7} 6 s, 4 f^{7} 5 d, 4 f^{7} 7 s, 4 f^{6} 5 d 6 p ?, 4 f^{7} 6 d, 4 f^{7} 8 s$

Identified even configurations

$4 f^{7} 6 p, 4 f^{6} 5 d 6 s, 4 f^{6} 5 d^{2}, 4 f^{\times} ?$

Albertson's 1934 note on this spectrum gave all the levels of $4 f^{7}\left({ }^{8} S^{\circ}\right) 6 s$, $\left({ }^{8} \mathrm{~S}^{\circ}\right) 6 p$, and $\left({ }^{8} \mathrm{~S}^{\circ}\right) 7 s$, as well as the $4 f^{7}\left({ }^{8} \mathrm{~S}^{\circ}\right) 5 d^{9} \mathrm{D}^{\circ}$ levels. His discovery that the Eu II ground configuration has a single external (6s) electron led to the conclusions that the Eu III ground configuration would have no such electrons ( $4 f^{7}$ ground configuration) and that the normal Eu I configuration would probably be $4 f^{7} 6 s^{2}$. Albertson's prediction for Eu I was confirmed later in the same year by Russell and King [1934, see Eu I]; these results were the first experimental contradiction of the previous usual view that all neutral rare-earth atoms had ground configurations of the type $4 f^{N} 5 d 6 s^{2}$.

The levels and $L S$-coupling designations are from the 1941 extension of the analysis by Russell, Albertson, and Davis. This analysis was based on 1861 lines (2109-10165 $\AA$ ) definitely assigned to Eu II by A. S. King [1939] and on extensive Zeeman-effect data. All of the strongest lines are classified by transitions between levels based on the $4 f^{7}\left({ }^{8} \mathrm{~S}^{\circ}\right)$ parent term; the 156 known levels classify a total of 467 lines. If all lines in King's list that probably belong to Eu II are included, more than 2200 lines remain unclassified. Russell et al. note that most of these lines must involve levels based on higher terms of $4 f^{7}$.

The $4 f^{7}\left({ }^{8} \mathrm{~S}^{\circ}\right) 5 d$ levels have high purity in the $L S$-coupling scheme, as shown by the percentages taken from the calculation of Eremin and Maryakhina. The $4 f^{7}\left({ }^{8} \mathrm{~S}^{\circ}\right) 6 p$ levels have been assigned $J_{1} j$ names by analogy with this configuration in Gd III; Callahan remarked that the corresponding levels of $4 f^{7}\left({ }^{8} S^{\circ}\right) 6 p$ in Eu II and Gd III fall very near each other on the $L S$ to- $J_{1} j$ coupling diagram. The two pairs of levels having the same $J$ value ( $J=4$ and $J=3$ ) have significantly mixed compositions in the $L S$ scheme; the percentages are from Bordarier, Judd, and Klapisch, as quoted by Guthöhrlein.

The two $4 f^{7}\left({ }^{8} S^{\circ}\right) 6 s$ levels have high purity in either $L S$ or $J_{1} j$ coupling, since the $\left({ }^{8} S^{\circ}\right)$ parent has high $L S$ purity (see Eu I). In a single-configuration pure-coupling approximation the $6 s-6 p$ lines are therefore probably best regarded as transitions from levels of the $J_{1} j$ terms $\left({ }^{8} \mathrm{~S}_{7 / 2}^{\circ}\right) 6 p_{1 / 2}$ and $\left({ }^{8} \mathrm{~S}_{7 / 2}^{\circ}\right) 6 p_{3 / 2}$ to the two $\left({ }^{8} \mathrm{~S}_{7 / 2}^{\circ}\right) 6 s_{1 / 2}$ levels. Analysis of the hyperfine structure of four of these lines (3819-4523 $\AA$ ) by Guthöhrlein and by Guthöhrlein, Himmel, and Steudel shows the $\left({ }^{8} \mathrm{~S}_{7 / 2}^{\circ}\right) 6 p$ levels to be perturbed, probably due to interaction with $4 f^{6}\left({ }^{7} \mathrm{~F}\right) 5 d 6 \mathrm{~s}$ levels.

In cases where Russell et al. assigned both a numerical and a (tentative) term designation to a level, we give here the numerical name; these were used in the list of line classifications. The even levels that were given as belonging to one of three terms $z{ }^{7} \mathrm{D}, x^{7} \mathrm{P}$, or $y{ }^{9} \mathrm{D}$ are listed here (in order of position) with tentative designations. The $L S$ assignments are strongly indicated by the observed $g$ values, but until the analysis is further developed the term groupings appear uncertain. The higher even levels probably belong mainly to $4 f^{6} 5 d 6 s$ and $4 f^{6} 5 d^{2}$, but the irregular $z^{7} \mathrm{~F}$ term $\left(\sim 56000 \mathrm{~cm}^{-1}\right)$ is tentatively assigned to $4 f^{8}$. (This term is called $z{ }^{7} \mathrm{~F}$ in the text and in tables II and $\mathrm{V}$ of Russell et al., but is given as $x^{7} \mathrm{~F}$ in tables VIII and XII.) Russell et al. state that this configuration is more probable than $4 f^{6} 5 d^{2}$ "on account of the isolation and great strength of the $\left[a^{7} \mathrm{D}^{\circ}-z^{7} \mathrm{~F}\right]$ multiplet," and the resulting position of $4 f^{8}$ is reasonable. If the level identifications are correct, the term is perturbed, since the levels should lie in the regular order for an inverted term.

Russell et al. assigned the $x^{9} \mathrm{P}$ and $y{ }^{7} \mathrm{P}$ terms to $4 f^{6}\left({ }^{7} \mathrm{~F}\right) 5 d^{2}$, the $y^{7} \mathrm{P}$ assignment being tentative. Koniordos and Winkler have observed that the hyperfine structure of the $a{ }^{7} \mathrm{~S}_{3}-$ $y{ }^{7} \mathrm{P}_{2}$ transition does not support the $4 f^{6} 5 d^{2}$ assignment for the $y^{7} \mathrm{P}_{2}$ level. 
The grouping of a few of the high odd levels into the $e^{7} \mathrm{~F}^{\circ}$ and $f^{7} \mathrm{D}^{\circ}$ terms is tentatively retained here. According to Russell et al., these terms belong to the $\left(4 f^{6} 6 s 6 p+4 f^{6} 5 d 6 p\right)$ group, with the latter configuration being more probable.

The $g$ values are based on Zeeman patterns obtained at MIT with a field of 8.785 teslas (87850 gauss). Russell et al, give the patterns for 460 lines, including "all the stronger lines and a great number of faint ones" (2317-7426 $\AA$ ). If the $g$ value of a particular level is one of two possible values, both values are listed with question marks. A colon following a $g$ value indicates that it is "doubtful." The probable error of the $g$ values ranges from about 0.001 $\left(a{ }^{9} \mathrm{~S}_{4}^{\circ}, a{ }^{7} \mathrm{~S}_{3}^{\circ}\right.$ ) to 0.006 . Wybourne pointed out that an apparent failure of the $g$-sum rule for the low terms discussed by Russell et al. could be explained by the deviation of the $g$ value of the Eu III $4 f^{78} \mathrm{~S}_{7 / 2}^{\circ}$ limit level (measured value 1.985) from the theoretical LS-coupling value (2.002). A more accurate $g$ value for the $4 f^{7}{ }^{8} \mathrm{~S}_{7 / 2}^{\circ}$ level is probably obtained by taking it equal to the accurately measured value for the $\mathrm{Eu} \mathrm{I} 4 f^{7} 6 s^{2}{ }^{8} \mathrm{~S}_{7 / 2}^{\circ}$ level (1.9934). This value would account for about half of the $g$-sum discrepancy noted by Russell et al., and the remaining discrepancy (if real) could well be due to a small error in the original field calibration, as discussed by them.

A fit of the $\left({ }^{8} \mathrm{~S}^{\circ}\right) 6 s, 7 s, 8 s,{ }^{9} \mathrm{~S}_{4}^{\circ}$ series to the Ritz formula, as was done by Russell et al., yields a value of $90713 \mathrm{~cm}^{-1}$ for the $\mathrm{Eu}$ III $4 f^{7}\left({ }^{8} \mathrm{~S}_{7 / 2}^{\circ}\right)$ limit. We have adjusted this value downward by $48 \mathrm{~cm}^{-1}$, the correction being derived from data on the systematic behavior of $n s$ series [Reader, 1973]. The uncertainty is conservatively taken as equal to the correction, although the consistency of the systematic behavior would indicate a considerably smaller error.

The identification of Eu II in the spectrum of the A-type star $\alpha^{2}$ Canum Venaticorum more than 60 years ago was one of the first indications of the peculiarity of the spectra of many stars of this type. It is now known that the light rare-earth elements are greatly overabundant (relative to the solar abundances) in the atmospheres of many "peculiar" A stars. The study of these puzzling objects furnishes one of the best examples of the need for more complete data on rare-earth spectra and energy levels.

\section{References}

Albertson, W., Phys. Rev. 45, 499 (1934). EL CL

Bordarier, Y., Judd, B. R., and Klapisch, M., unpublished results quoted by G. Guthöhrlein (1968). Hfs PT

Callahan, W. R., J. Opt. Soc. Am. 53, 695 (1963). PT

Eremin, M. V., and Maryakhina, O. I., Opt. Spectrose. (USSR) 26, 479 (1969). PT

Guthöhrlein, G., Z. Phys. 214, 332 (1968). Hfs IS PT

Guthöhrlein, G., Himmel, G., and Steudel, A., J. Phys. (Paris) Colloq. C 1 30, 66 (1969). Hfs

King, A. S., Astrophys. J. 72, 221 (1930). W Hfs

King, A. S., Astrophys. J. 89, 377 (1939). W

Koniordos, I., and Winkler, R., Phys. Lett. 27A, 198 (1968). IS

Reader, J., private communication (1973). IP

Russell, H. N., Albertson, W., and Davis, D. N., Phys. Rev. 60, 641 (1941). EL CL ZE IP

Wybourne, B. G., J. Opt. Soc. Am. 54, 267 (1964). PT

[July 1976] 
Eu II

\begin{tabular}{|c|c|c|c|c|c|c|c|c|}
\hline Configuration & Term & $J$ & $\begin{array}{c}\text { Level } \\
\left(\mathrm{cm}^{-1}\right)\end{array}$ & $g$ & \multicolumn{4}{|c|}{ Leading percentages } \\
\hline $4 f^{7}\left({ }^{8} S^{\circ}\right) 6 s$ & $a^{9} \mathrm{~S}^{\circ}$ & 4 & 0.00 & 1.984 & & or & & $\left({ }^{8} \mathrm{~S}_{7 / 2}^{\circ}\right) 6 s_{1 / 2}$ \\
\hline $4 f^{7}\left({ }^{8} S^{\circ}\right) 6 s$ & $a^{7} \mathrm{~S}^{\circ}$ & 3 & 1669.21 & 1.981 & & or & & $\left({ }^{8} \mathrm{~S}_{7 / 2}^{\circ}\right) 6 s_{1 / 2}$ \\
\hline \multirow[t]{5}{*}{$4 f^{7}\left({ }^{8} \mathrm{~S}^{\circ}\right) 5 d$} & $a^{9} \mathrm{D}^{\circ}$ & 2 & 9923.00 & 2.641 & 100 & & & \\
\hline & & 3 & 10081.65 & 2.062 & 100 & & & \\
\hline & & 4 & 10312.82 & 1.842 & 99 & & 1 & $\left({ }^{8} \mathrm{~S}^{\circ}\right)^{7} \mathrm{D}^{\circ}$ \\
\hline & & 5 & 10643.48 & 1.726 & 99 & & 1 & $\left({ }^{8} \mathrm{~S}^{\circ}\right)^{7} \mathrm{D}^{\circ}$ \\
\hline & & 6 & 11128.22 & 1.659 & 100 & & & \\
\hline \multirow[t]{5}{*}{$4 f^{7}\left({ }^{8} \mathrm{~S}^{\circ}\right) 5 d$} & $a^{7} \mathrm{D}^{\circ}$ & 5 & 16860.72 & 1.592 & 99 & & 1 & $\left({ }^{8} \mathrm{~S}^{\circ}\right)^{9} \mathrm{D}^{\circ}$ \\
\hline & & 4 & 17004.06 & 1.628 & 99 & & 1 & $\left({ }^{8} \mathrm{~S}^{\circ}\right){ }^{9} \mathrm{D}^{\circ}$ \\
\hline & & 3 & 17140.87 & 1.728 & 100 & & & \\
\hline & & 2 & 17247.67 & 1.981 & 100 & & & \\
\hline & & 1 & 17324.66 & 2.972 & 100 & & & \\
\hline \multirow[t]{2}{*}{$4 f^{7}\left({ }^{8} \mathrm{~S}_{7 / 2}^{\circ}\right) 6 p_{1 / 2}$} & $(7 / 2,1 / 2)$ & 3 & 23774.28 & 2.195 & & or & 87 & $\left({ }^{8} \mathrm{~S}^{\circ}\right) z^{9} \mathrm{P}$ \\
\hline & & 4 & 24207.86 & 1.877 & & or & 63 & \\
\hline \multirow[t]{4}{*}{$4 f^{7}\left({ }^{8} \mathrm{~S}_{7 / 2}^{\circ}\right) 6 p_{3 / 2}$} & $(7 / 2,3 / 2)$ & 5 & 26172.83 & 1.789 & & or & 100 & $\left({ }^{8} \mathrm{~S}^{\circ}\right) z^{9} \mathrm{P}$ \\
\hline & & 4 & 26838.50 & 1.801 & & or & 63 & $\left({ }^{8} \mathrm{~S}^{\circ}\right) z^{7} \mathrm{P}$ \\
\hline & & 3 & 27104.07 & 1.943 & & or & 87 & $\left({ }^{8} \mathrm{~S}^{\circ}\right) z^{7} \mathrm{P}$ \\
\hline & & 2 & 27256.35 & 2.312 & & or & 100 & $\left({ }^{8} \mathrm{~S}^{\circ}\right) z^{7} \mathrm{P}$ \\
\hline \multirow[t]{8}{*}{$4 f^{6}\left({ }^{7} \mathrm{~F}\right) 5 d\left({ }^{8} \mathrm{D}\right) 6 s$} & $z^{9} \mathrm{D}$ & 2 & 30189.31 & 2.511 & & & & \\
\hline & & 3 & 31500.82 & 2.002 & & & & \\
\hline & & 4 & 32486.10 & 1.824 & & & & \\
\hline & & 5 & 33420.20 & 1.710 & & & & \\
\hline & & 6 & 34764.97 & 1.687 & & & & \\
\hline & 101 & 4 & 33779.95 & 1.565 & & & & \\
\hline & 102 & 3 & 33919.70 & 1.681 & & & & \\
\hline & 103 & 2 & 33944.49 & 1.690 & & & & \\
\hline \multirow[t]{15}{*}{$4 f^{6}\left({ }^{7} \mathrm{~F}\right) 5 d\left({ }^{8} \mathrm{P}\right) 6 s$} & $y{ }^{9} \mathrm{P}$ & 3 & 34393.57 & 2.037 & & & & \\
\hline & & 4 & 35527.02 & 1.898 & & & & \\
\hline & & 5 & 36648.95 & 1.749 & & & & \\
\hline & 104 & 3 & 34546.10 & 1.307 & & & & \\
\hline & 105 & 4 & 34617.73 & 1.603 & & & & \\
\hline & 106 & 3 & 34923.43 & 1.669 & & & & \\
\hline & 107 & 4 & 35045.98 & 1.966 & & & & \\
\hline & 108 & 3 & 35440.88 & 2.004 & & & & \\
\hline & 109 & 3 & 35567.74 & 1.434 & & & & \\
\hline & $z^{7} \mathrm{D} ?$ & 1 & & & & & & \\
\hline & & 2 & 35846.96 & 1.931 & & & & \\
\hline & & 4 & 37010.70 & 1.702 & & & & \\
\hline & & $\begin{array}{l}3 \\
5\end{array}$ & 37167.90 & 1.733 & & & & \\
\hline & 110 & 4 & 35935.81 & 1.591 & & & & \\
\hline & 111 & 4 & 36428.89 & 1.472 & & & & \\
\hline \multirow[t]{5}{*}{$4 f^{6}\left({ }^{7} \mathrm{~F}\right) 5 d^{2}\left({ }^{3} \mathrm{~F}\right)$} & $x^{9} \mathrm{P}$ & 3 & 36628.00 & 2.163 & & & & \\
\hline & & 4 & 37223.78 & 1.892 & & & & \\
\hline & & 5 & 37849.34 & 1.715 & & & & \\
\hline & 112 & 3 & 36821.20 & 1.428 & & & & \\
\hline & 113 & 4 & 36954.00 & 1.463 & & & & \\
\hline
\end{tabular}


Eu II-Continued

Eu II-Continued

\begin{tabular}{|c|c|c|c|c|c|c|c|c|c|}
\hline Configuration & Term & $J$ & $\begin{array}{l}\text { Level } \\
\left(\mathrm{cm}^{-1}\right)\end{array}$ & $g$ & Configuration & Term & $J$ & $\begin{array}{l}\text { Level } \\
\left(\mathrm{cm}^{-1}\right)\end{array}$ & $g$ \\
\hline & 114 & 5 & 37000.00 & 1.578 & & 141 & 4,5 & 43461.07 & \\
\hline & 115 & 2 & 37003.23 & 2.151 & & 142 & 5 & 43643.12 & 1.645: \\
\hline \multirow[t]{41}{*}{$4 f^{6}\left({ }^{7} \mathrm{~F}\right) 5 d^{2} ?$} & $y^{7} \mathrm{P}$ & 2 & 37336.83 & 2.196 & & 143 & 5 & 44947.15 & \\
\hline & & 3 & 38804.98 & 1.916 & & & & & \\
\hline & & 4 & 39134.72 & 1.746 & & 144 & 4 & 46032.03 & 1.418: \\
\hline & 116 & 6 & 37583.63 & 1.775 & & 145 & 4 & 46584.45 & \\
\hline & 117 & 3 & 37605.51 & 1.735 & & 146 & 2 & 48171.51 & 1.455 \\
\hline & 118 & 3,4 & 37622.87 & & & 147 & 1,2 & 48180.68 & \\
\hline & 119 & 2 & 37799.89 & 1.744 & & 148 & 5 & 48378.03 & $\{1.748 ?$ \\
\hline & 120 & 5 & 37932.19 & 1.460 & & & & & 20. \\
\hline & 121 & 4 & 37993.64 & 1.322 & & 149 & 2 & 48522.28 & \\
\hline & 122 & & & & & 150 & 3 & 48536.61 & 1.576 \\
\hline & 122 & 4 & 38008.03 & 1.436 & & 151 & 4 & 48872.85 & \\
\hline & $x^{\mathrm{P}} \mathrm{P} ?$ & 2 & 38146.44 & 2.364 & & & & & \\
\hline & & 3 & 38463.66 & 1.716 & & 152 & 3 & 48910.95 & \\
\hline & & 4 & 39554.43 & 1.694 & & 153 & 4,5 & 49003.10 & \\
\hline & 123 & 3 & 38295.89 & 1.703 & & 154 & 2 & 49043.00 & 1.319 \\
\hline & 124 & 1 & 38582.08 & 3.155 & $4 f^{7}\left({ }^{8} \mathrm{~S}^{\circ}\right) 7 \mathrm{~s}$ & $e^{9} \mathrm{~S}^{\circ}$ & 4 & 49128.03 & 1.985 \\
\hline & 125 & 7 & 38725.32 & 1.576: & & 155 & 2 & 49254.68 & 1.558 \\
\hline & 126 & 5 & 38784.47 & 1.601 & & 156 & 3 & 49480.46 & \\
\hline & 127 & 4 & 38995.52 & 1.566 & & & & & \\
\hline & 128 & 2 & 39099.43 & 2.016 & $4 f^{7}\left({ }^{8} S^{\circ}\right) 7 s$ & $e 7 \mathrm{~S}$ & 3 & 49646.62 & 1.974 \\
\hline & & & & & & 157 & 2 & 49720.89 & \\
\hline & 129 & 6 & 39601.80 & 1.756 & & 158 & 1 & 5094746 & 007 \\
\hline & 130 & 3 & 39767.55 & $\{1.750 ?$ & & & & & 2.014 \\
\hline & & & & 2.374? & $4 f^{6} 5 d 6 p ?$ & $e^{7} \mathrm{~F}^{0}$ & $\begin{array}{l}6 \\
5\end{array}$ & 52963.40 & 1.526 \\
\hline & 131 & 6 & 40316.92 & 1.632 & & & 4 & 53150.34 & 1.552 \\
\hline & & & & & & & 3 & 53490.44 & 1.525 \\
\hline & 132 & 4 & 40870.72 & 1.892 & & & 2 & 53861.26 & 1.563 \\
\hline & 133 & 6 & 41099.11 & & & & 0 & & \\
\hline & 134 & 5 & 41223.24 & 1.661 & & $1^{\circ}$ & 4 & 53783.01 & 1.875: \\
\hline & 135 & 4 & 41438.13 & 1.694: & $4 f^{7}\left({ }^{8} \mathrm{~S}^{\circ}\right) 6 d$ & $e^{9} \mathrm{D}^{\circ}$ & 2 & 54273.98 & 2.620: \\
\hline & 136 & 4 & 41666.81 & 1.505 & & & 3 & 54320.69 & 1.912 \\
\hline & & & & & & & $\begin{array}{l}4 \\
5\end{array}$ & $\begin{array}{l}54380.30 \\
54484.27\end{array}$ & $\begin{array}{l}1.807 \\
1.507\end{array}$ \\
\hline & 137 & 4 & 41714.84 & & & & $\begin{array}{l}5 \\
6\end{array}$ & 54565.04 & 1.655 \\
\hline & 138 & & 42391.03 & 1.935 & $4 f^{7}\left({ }^{8} S^{\circ}\right) 6 d$ & $e^{7} \mathrm{D}^{\circ}$ & 4 & 54295.02 & \\
\hline & $y^{9} \mathrm{D} ?$ & 2 & 42438.56 & & & & 5 & 54428.76 & 1.414 \\
\hline & & 3 & 43193.46 & 1.987 & & & 1 & 54435.41 & 2.852 \\
\hline & & 4 & 44075.84 & 1.672 & & & 2 & 54490.63 & \\
\hline & & 5 & 45126.78 & 1.602 & & & 3 & 54589.10 & \\
\hline & & 6 & 46029.14 & 1.621 & & $2^{\circ}$ & 3,4 & 54600.07 & \\
\hline & 139 & 4 & 42710.14 & & & $3^{\circ}$ & 3 & & \\
\hline & 140 & 3,4 & 42939.45 & & & & & & \\
\hline
\end{tabular}


Eu II-Continued

\begin{tabular}{|c|c|c|c|c|c|c|c|c|c|}
\hline Configuration & Term & $J$ & $\begin{array}{l}\text { Level } \\
\left(\mathrm{cm}^{-1}\right)\end{array}$ & $g$ & Configuration & Term & $J$ & $\begin{array}{l}\text { Level } \\
\left(\mathrm{cm}^{-1}\right)\end{array}$ & $g$ \\
\hline \multirow{19}{*}{$4 f^{8} ?$} & $4^{\circ}$ & 3 & 55193.08 & & & $11^{\circ}$ & 3,4 & 57360.05 & \\
\hline & $5^{\circ}$ & 3 & 55559.02 & & & 162 & 1,2 & 57363.68 & \\
\hline & $z^{7} \mathrm{~F}$ & $\begin{array}{l}6 \\
3\end{array}$ & $\begin{array}{l}55651.82 \\
55967.88\end{array}$ & $\begin{array}{l}1.490 \\
1.460\end{array}$ & & 163 & 3 & 57388.87 & 1.736 : \\
\hline & & 0 & 55986.45 & & & 164 & 2,3 & 57415.06 & \\
\hline & & 5 & 55991.33 & 1.460 & & ser & & & \\
\hline & & $\begin{array}{l}4 \\
1\end{array}$ & $\begin{array}{l}56067.39 \\
56245.61\end{array}$ & $\begin{array}{l}1.466 \\
1.494\end{array}$ & & 165 & 3,4 & 57634.02 & \\
\hline & & 2 & 56571.04 & 1.451 & & 166 & 2 & 58920.64 & 1.502 \\
\hline & 159 & 2 & 55693.94 & 1.115 & & $12^{\circ}$ & 3 & 59045.49 & 2.061 \\
\hline & 160 & 2 & 55807.25 & 1.090 & & 167 & 1 & 59145.26 & 1.486 \\
\hline & $6^{\circ}$ & 3,4 & 56158.66 & & & 168 & 2 & 59334.70 & \\
\hline & 161 & 3 & 56229.17 & 1.381 & & 169 & 4,5 & 59343.45 & \\
\hline & $f^{7} \mathrm{D}^{\circ} ?$ & 5 & 56271.40 & 1.662 & & $13^{\circ}$ & 3 & 59765.77 & \\
\hline & & $\begin{array}{l}4 \\
3\end{array}$ & $\begin{array}{l}56293.33 \\
56309.21\end{array}$ & $1.649:$ & & 170 & 3,4 & 59995.34 & \\
\hline & & 1 & 56310.89 & 2.962 & & & & & \\
\hline & & 2 & 56321.27 & 1.792 & $4 f^{7}\left({ }^{8} S^{\circ}\right) 8 s$ & $f^{9} \mathrm{~S}^{0}$ & 4 & 66598.00 & \\
\hline & $7^{\circ}$ & 2,3 & 56421.62 & 1.844 & $4 f^{7}\left({ }^{8} S^{\circ}\right) 8 s$ & $f^{7} \mathrm{~S}^{\circ}$ & 3 & $66836.16 ?$ & \\
\hline & $8^{\circ}$ & 5 & 56781.20 & 1.454 & ---- & & & & \\
\hline & $9^{\circ}$ & 3,4 & 57127.26 & & Eu III $\left({ }^{8} \mathrm{~S}_{7 / 2}^{\circ}\right)$ & Limit & & 90665 & \\
\hline & $10^{\circ}$ & 4 & 57270.14 & & & & & & \\
\hline
\end{tabular}

Eu II-Continued 
Ground state $\left(1 s^{2} 2 s^{2} 2 p^{6} 3 s^{2} 3 p^{6} 3 d^{10} 4 s^{2} 4 p^{6} 4 d^{10} 5 s^{2} 5 p^{6}\right) 4 f^{78} \mathrm{~S}_{7 / 2}^{\circ}$

Ionization energy $201000 \pm 800 \mathrm{~cm}^{-1}$

Sugar and Spector [1974] list almost 900 lines for this spectrum in the range 2026-8582 $\AA$, with all but 22 of the lines lying below $3360 \AA$. The 105 levels obtained from their analysis, given here, classify about 300 of the observed lines.

The leading percentages for all levels except the ground level are also from Sugar and Spector $[1974,1975]$. They calculated $4 f^{6}\left({ }^{7} \mathrm{~F}\right) 6 p$ with matrices truncated to include only terms based on the ${ }^{7} \mathrm{~F}$ core term, using the "real core" approximation. The 1974 paper gives the first two percentages for each level in the $J_{1} j$ coupling scheme most appropriate for this configuration; we have omitted the second $J_{1} j$ percentages, and listed instead the leading percentages in LS coupling [Sugar and Spector, 1975].

The calculation of $\left(4 f^{6} 5 d+4 f^{6} 6 s\right)$ was expanded to include terms based on the three ${ }^{5} \mathrm{D}$ terms of $4 f^{6}$, but still more complete matrices apparently are needed to fit the observed levels, $g$ values, and intensities more accurately [Sugar and Spector, 1974]. The intensities of the transitions to the ground level indicate that the level having $J=5 / 2$ at $39769 \mathrm{~cm}^{-1}$ has a larger $4 f^{6}\left({ }^{7} \mathrm{~F}\right) 5 d^{8} \mathrm{P}$ component than the level at $40897 \mathrm{~cm}^{-1}$, contrary to the results of the 1974 calculation. We have thus followed Sugar and Spector in retaining the original ${ }^{8} \mathrm{P}$ designation for the lower of these levels [Martin and Sugar, 1973].

Baker and Williams obtained a- $g$ value of $1.9926 \pm 0.0003$ for the $4 f^{7}{ }^{8} \mathrm{~S}_{7 / 2}^{\circ}$ ground level by fitting their electron paramagnetic resonance data for $\mathrm{Eu}^{2+}$ ions (calcium fluoride host). An assumption that the observed $g$ value is due to a mixture of ${ }^{8} \mathrm{~S}_{7 / 2}^{\circ}$ and ${ }^{6} \mathrm{P}_{7 / 2}^{\circ}$ wavefunctions (see Eu I) gives a ${ }^{6} \mathrm{P}_{7 / 2}^{\circ}$ percentage of $2.9 \pm 0.1 \%$ in the eigenvector for the ground level [Baker and Williams, 1962]. The $g$ values for six upper levels are from Russell, Albertson, and Davis [1941], with some corrections noted by Martin and Sugar.

The ionization potential is from Sugar and Spector, who were able to improve the accuracy of the value obtained earlier by Sugar and Reader [1973].

\section{References}

Baker, J. M., and Williams, F. I. B., Proc. R. Soc. London, Ser. A 267, 283 (1962). ZE Hfs Martin, W. C., and Sugar, J., Astrophys. J. 184, 671 (1973). EL ND CL ZE PT Russell, H. N., Albertson, W., and Davis, D. N., Phys. Rev. 60, 641 (1941). EL CL ZE Sugar, J., and Reader, J., J. Chem. Phys. 59, 2083 (1973). IP

Sugar, J., and Spector, N., J. Opt. Soc. Am. 64, 1484 (1974). EL ND CL W IP PT

Sugar, J., and Spector, N., unpublished material (1975). PT

[July 1976] 
Eu III-Continued

\begin{tabular}{|c|c|c|c|c|c|c|c|c|}
\hline Configuration & Term & $J$ & $\begin{array}{l}\text { Level } \\
\left(\mathrm{cm}^{-1}\right)\end{array}$ & $g$ & \multicolumn{4}{|c|}{ Leading percentages } \\
\hline $4 f^{6}\left({ }^{7} \mathrm{~F}\right) 5 d$ & ${ }^{6} \mathrm{~F}$ & $\begin{array}{l}1 / 2 \\
3 / 2 \\
5 / 2 \\
7 / 2 \\
9 / 2 \\
11 / 2\end{array}$ & $\begin{array}{l}46108.79 \\
46793.38 \\
47714.74 \\
49086.13\end{array}$ & & $\begin{array}{l}55 \\
47 \\
54 \\
59 \\
64 \\
81\end{array}$ & & $\begin{array}{l}35 \\
39 \\
31 \\
25 \\
21 \\
15\end{array}$ & $\begin{array}{l}\left({ }^{7} \mathrm{~F}\right){ }^{6} \mathrm{D} \\
\left({ }^{7} \mathrm{~F}\right){ }^{6} \mathrm{D} \\
\left({ }^{7} \mathrm{~F}\right){ }^{6} \mathrm{D} \\
\left({ }^{7} \mathrm{~F}\right){ }^{6} \mathrm{D} \\
\left({ }^{7} \mathrm{~F}\right){ }^{6} \mathrm{D} \\
\left({ }^{7} \mathrm{~F}\right)^{6} \mathrm{G}\end{array}$ \\
\hline $4 f^{6}\left({ }^{7} \mathrm{~F}\right) 6 \mathrm{~s}$ & ${ }^{6} \mathrm{~F}$ & $\begin{array}{c}1 / 2 \\
3 / 2 \\
5 / 2 \\
7 / 2 \\
9 / 2 \\
11 / 2\end{array}$ & $\begin{array}{l}48259.62 \\
48828.91 \\
49610.81 \\
50805.58 \\
51848.18 \\
52960.08\end{array}$ & & $\begin{array}{l}93 \\
87 \\
78 \\
54 \\
91 \\
97\end{array}$ & & $\begin{array}{r}2 \\
8 \\
15 \\
27 \\
4 \\
1\end{array}$ & $\begin{array}{l}\left({ }^{7} \mathrm{~F}\right) 5 d^{6} \mathrm{~F} \\
\left({ }^{7} \mathrm{~F}\right) 5 d^{6} \mathrm{G} \\
\left({ }^{7} \mathrm{~F}\right) 5 d^{6} \mathrm{G} \\
\left({ }^{7} \mathrm{~F}\right) 5 d^{6} \mathrm{G} \\
\left({ }^{7} \mathrm{~F}\right) 5 d^{6} \mathrm{G} \\
\left({ }^{7} \mathrm{~F}\right)^{8} \mathrm{~F}\end{array}$ \\
\hline $4 f^{6}\left({ }^{7} \mathrm{~F}\right) 5 d$ & ${ }^{6} \mathrm{D}$ & $\begin{array}{l}1 / 2 \\
3 / 2 \\
5 / 2 \\
7 / 2 \\
9 / 2\end{array}$ & $\begin{array}{l}48496.43 \\
49292.56 \\
49956.73\end{array}$ & & $\begin{array}{l}54 \\
49 \\
54 \\
57 \\
63\end{array}$ & & $\begin{array}{l}33 \\
35 \\
21 \\
24 \\
23\end{array}$ & $\begin{array}{l}\left({ }^{7} \mathrm{~F}\right){ }^{6} \mathrm{~F} \\
\left({ }^{7} \mathrm{~F}\right){ }^{6} \mathrm{~F} \\
\left({ }^{7} \mathrm{~F}\right){ }^{6} \mathrm{~F} \\
\left({ }^{7} \mathrm{~F}\right){ }^{6} \mathrm{G} \\
\left({ }^{7} \mathrm{~F}\right)^{6} \mathrm{G}\end{array}$ \\
\hline $4 f^{6}\left({ }^{7} \mathrm{~F}\right) 5 d$ & ${ }^{6} \mathrm{G}$ & $\begin{array}{c}3 / 2 \\
5 / 2 \\
9 / 2 \\
11 / 2 \\
13 / 2\end{array}$ & $\begin{array}{l}49905.64 \\
51650.77 \\
52099.87\end{array}$ & & $\begin{array}{l}78 \\
58 \\
59 \\
80 \\
95\end{array}$ & & $\begin{array}{r}10 \\
18 \\
21 \\
15 \\
2\end{array}$ & $\begin{array}{l}\left({ }^{7} \mathrm{~F}\right){ }^{6} \mathrm{~F} \\
\left({ }^{7} \mathrm{~F}\right) 6 s^{6} \mathrm{~F} \\
\left({ }^{7} \mathrm{~F}\right){ }^{6} \mathrm{~F} \\
\left({ }^{7} \mathrm{~F}\right)^{6} \mathrm{~F} \\
\left({ }^{7} \mathrm{~F}\right)^{8} \mathrm{~F}\end{array}$ \\
\hline $4 f^{6}\left({ }^{7} \mathrm{~F}\right) 6 s$ & & $7 / 2$ & 50426.33 & & 42 & & 37 & $\left({ }^{7} \mathrm{~F}\right) 5 d^{6} \mathrm{G}$ \\
\hline $4 f^{6}\left({ }^{7} \mathrm{~F}_{0}\right) 6 p_{1 / 2}$ & $\left(0,{ }^{1} / 2\right)^{\circ}$ & $1 / 2$ & 78981.86 & & 69 & or & 88 & $\left({ }^{7} \mathrm{~F}\right){ }^{8} \mathrm{G}^{\circ}$ \\
\hline $4 f^{6}\left({ }^{7} \mathrm{~F}_{1}\right) 6 p_{1 / 2}$ & $(1,1 / 2)^{\circ}$ & $\begin{array}{l}3 / 2 \\
1 / 2\end{array}$ & $\begin{array}{l}79437.18 \\
79639.31\end{array}$ & & $\begin{array}{l}80 \\
72\end{array}$ & $\begin{array}{l}\text { or } \\
\text { or }\end{array}$ & $\begin{array}{l}84 \\
56\end{array}$ & $\begin{array}{l}\left({ }^{7} \mathrm{~F}\right){ }^{8} \mathrm{G}^{\circ} \\
\left({ }^{7} \mathrm{~F}\right)^{6} \mathrm{D}^{\circ}\end{array}$ \\
\hline $4 f^{6}\left({ }^{7} \mathrm{~F}_{2}\right) 6 p_{1 / 2}$ & $(2,1 / 2)^{\circ}$ & $\begin{array}{l}5 / 2 \\
3 / 2\end{array}$ & $\begin{array}{l}80153.47 \\
80253.58\end{array}$ & & $\begin{array}{l}85 \\
83\end{array}$ & $\begin{array}{l}\text { or } \\
\text { or }\end{array}$ & $\begin{array}{l}81 \\
48\end{array}$ & $\begin{array}{l}\left({ }^{7} \mathrm{~F}\right){ }^{8} \mathrm{G}^{\circ} \\
\left({ }^{7} \mathrm{~F}\right){ }^{6} \mathrm{D}^{\circ}\end{array}$ \\
\hline $4 f^{6}\left({ }^{7} \mathrm{~F}_{3}\right) 6 p_{1 / 2}$ & $(3,1 / 2)^{\circ}$ & $\begin{array}{l}7 / 2 \\
5 / 2\end{array}$ & $\begin{array}{l}81059.49 \\
81067.28\end{array}$ & & $\begin{array}{l}87 \\
87\end{array}$ & $\begin{array}{l}\text { or } \\
\text { or }\end{array}$ & $\begin{array}{l}77 \\
38\end{array}$ & $\begin{array}{l}\left({ }^{7} \mathrm{~F}\right){ }^{8} \mathrm{G}^{\circ} \\
\left({ }^{7} \mathrm{~F}\right){ }^{6} \mathrm{D}^{\circ}\end{array}$ \\
\hline $4 f^{6}\left({ }^{7} \mathrm{~F}_{4}\right) 6 p_{1 / 2}$ & $\left(4,{ }^{1} / 2\right)^{\circ}$ & $\begin{array}{l}7 / 2 \\
9 / 2\end{array}$ & $\begin{array}{l}81985.13 \\
82101.85\end{array}$ & & $\begin{array}{l}89 \\
89\end{array}$ & $\begin{array}{l}\text { or } \\
\text { or }\end{array}$ & $\begin{array}{l}42 \\
70\end{array}$ & $\begin{array}{l}\left({ }^{7} \mathrm{~F}\right)^{8} \mathrm{D}^{\circ} \\
\left({ }^{7} \mathrm{~F}\right)^{8} \mathrm{G}^{\circ}\end{array}$ \\
\hline $4 f^{6}\left({ }^{7} \mathrm{~F}_{5}\right) 6 p_{1 / 2}$ & $\left(5,{ }^{1} / 2\right)^{\circ}$ & $\begin{array}{l}9 / 2 \\
11 / 2\end{array}$ & $\begin{array}{l}82954.63 \\
83248.29\end{array}$ & & $\begin{array}{l}90 \\
91\end{array}$ & $\begin{array}{l}\text { or } \\
\text { or }\end{array}$ & $\begin{array}{l}60 \\
58\end{array}$ & $\begin{array}{l}\left({ }^{7} \mathrm{~F}\right){ }^{8} \mathrm{D}^{\circ} \\
\left({ }^{7} \mathrm{~F}\right)^{8} \mathrm{G}^{\circ}\end{array}$ \\
\hline $4 f^{6}\left({ }^{7} \mathrm{~F}_{1}\right) 6 p_{3 / 2}$ & $(1,3 / 2)^{\circ}$ & $\begin{array}{l}3 / 2 \\
5 / 2 \\
1 / 2\end{array}$ & $\begin{array}{l}83009.54 \\
83776.98 \\
84563.08\end{array}$ & & $\begin{array}{l}44 \\
55 \\
68\end{array}$ & $\begin{array}{l}\text { or } \\
\text { or } \\
\text { or }\end{array}$ & $\begin{array}{l}82 \\
55 \\
56\end{array}$ & $\begin{array}{l}\left({ }^{7} \mathrm{~F}\right){ }^{8} \mathrm{D}^{\circ} \\
\left({ }^{7} \mathrm{~F}\right){ }^{8} \mathrm{D}^{\circ} \\
\left({ }^{7} \mathrm{~F}\right){ }^{8} \mathrm{~F}^{\circ}\end{array}$ \\
\hline $4 f^{6}\left({ }^{7} \mathrm{~F}_{6}\right) 6 p_{1 / 2}$ & $(6,1 / 2)^{\circ}$ & $\begin{array}{l}11 / 2 \\
13 / 2\end{array}$ & $\begin{array}{l}83959.93 \\
84486.76\end{array}$ & & $\begin{array}{l}91 \\
95\end{array}$ & $\begin{array}{l}\text { or } \\
\text { or }\end{array}$ & $\begin{array}{l}79 \\
41\end{array}$ & $\begin{array}{l}\left({ }^{7} \mathrm{~F}\right){ }^{8} \mathrm{D}^{\circ} \\
\left({ }^{7} \mathrm{~F}\right)^{6} \mathrm{G}^{\circ}\end{array}$ \\
\hline $4 f^{6}\left({ }^{7} \mathrm{~F}_{0}\right) 6 p_{3 / 2}$ & $(0,3 / 2)^{\circ}$ & $3 / 2$ & 84510.34 & & 54 & or & 67 & $\left({ }^{7} \mathrm{~F}\right)^{6} \mathrm{G}^{0}$ \\
\hline $4 f^{6}\left({ }^{7} \mathrm{~F}_{2}\right) 6 p_{3 / 2}$ & $(2,3 / 2)^{\circ}$ & $\begin{array}{l}7 / 2 \\
5 / 2 \\
3 / 2 \\
1 / 2\end{array}$ & $\begin{array}{l}84640.56 \\
84938.40\end{array}$ & & $\begin{array}{l}79 \\
52 \\
61 \\
67\end{array}$ & $\begin{array}{l}\text { or } \\
\text { or } \\
\text { or } \\
\text { or }\end{array}$ & $\begin{array}{l}53 \\
62 \\
35 \\
85\end{array}$ & $\begin{array}{l}\left({ }^{7} \mathrm{~F}\right){ }^{8} \mathrm{~F}^{\circ} \\
\left({ }^{7} \mathrm{~F}\right){ }^{6} \mathrm{G}^{\circ} \\
\left({ }^{7} \mathrm{~F}\right){ }^{8} \mathrm{~F}^{\circ} \\
\left({ }^{7} \mathrm{~F}\right){ }^{6} \mathrm{~F}^{\circ}\end{array}$ \\
\hline $4 f^{6}\left({ }^{7} \mathrm{~F}_{3}\right) 6 p_{3 / 2}$ & $\left(3,{ }^{3} / 2\right)^{\circ}$ & $\begin{array}{l}9 / 2 \\
7 / 2 \\
5 / 2 \\
3 / 2\end{array}$ & $\begin{array}{l}85479.93 \\
85705.11 \\
85928.29 \\
86933.53\end{array}$ & & $\begin{array}{l}91 \\
75 \\
60 \\
72\end{array}$ & $\begin{array}{l}\text { or } \\
\text { or } \\
\text { or } \\
\text { or }\end{array}$ & $\begin{array}{l}47 \\
54 \\
37 \\
76\end{array}$ & $\begin{array}{l}\left({ }^{7} \mathrm{~F}\right){ }^{8} \mathrm{~F}^{\circ} \\
\left({ }^{7} \mathrm{~F}\right){ }^{6} \mathrm{G}^{\circ} \\
\left({ }^{7} \mathrm{~F}\right){ }^{6} \mathrm{D}^{\circ} \\
\left({ }^{7} \mathrm{~F}\right){ }^{6} \mathrm{~F}^{\circ}\end{array}$ \\
\hline $4 f^{6}\left({ }^{7} \mathrm{~F}_{4}\right) 6 p_{3 / 2}$ & $(4,3 / 2)^{\circ}$ & $\begin{array}{l}11 / 2 \\
9 / 2 \\
7 / 2 \\
5 / 2\end{array}$ & $\begin{array}{l}86282.06 \\
86760.09 \\
86944.83 \\
87820.98\end{array}$ & & $\begin{array}{l}93 \\
90 \\
58 \\
71\end{array}$ & $\begin{array}{l}\text { or } \\
\text { or } \\
\text { or } \\
\text { or }\end{array}$ & $\begin{array}{l}38 \\
46 \\
45 \\
72\end{array}$ & $\begin{array}{l}\left({ }^{7} \mathrm{~F}\right){ }^{8} \mathrm{G}^{\circ} \\
\left({ }^{7} \mathrm{~F}\right){ }^{6} \mathrm{G}^{\circ} \\
\left({ }^{7} \mathrm{~F}\right){ }^{6} \mathrm{D}^{\circ} \\
\left({ }^{7} \mathrm{~F}\right){ }^{6} \mathrm{~F}^{\circ}\end{array}$ \\
\hline
\end{tabular}


Eu III-Continued

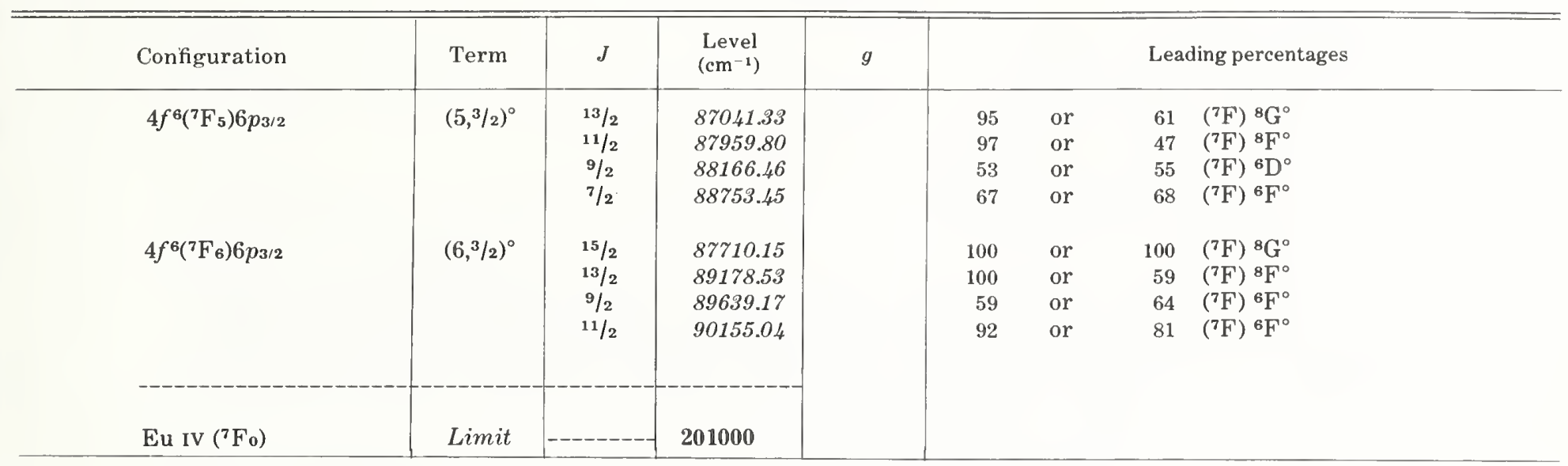


(Nd I sequence; 60 electrons)

Ground state $\left(1 s^{2} 2 s^{2} 2 p^{6} 3 s^{2} 3 p^{6} 3 d^{10} 4 s^{2} 4 p^{6} 4 d^{10} 5 s^{2} 5 p^{6}\right) 4 f^{6}{ }^{7} \mathrm{~F}_{0}$

Ionization energy $344000 \pm 5000 \mathrm{~cm}^{-1}$

$42.7 \pm 0.6 \mathrm{eV}$

The spectrum of the free ion has not been analyzed. The $4 f^{6}{ }^{7} \mathrm{~F}$ and ${ }^{5} \mathrm{D}$ levels given here are centers-of-gravity of the corresponding sublevels of the ion in hexagonal $\mathrm{LaCl}_{3}$, rounded off to the nearest $10 \mathrm{~cm}^{-1}$. DeShazer and Dieke derived the sublevels by analysis of both absorption and fluorescence spectra. Levels for higher terms are listed by Dieke [1968] and by Carnall, Fields, and Rajnak [1968] (to $41370 \mathrm{~cm}^{-1}$ ).

Ofelt has given the septet and quintet components of the eigenvectors for the $4 f^{6}$ levels up to $40000 \mathrm{~cm}^{-1}$. The percentages here are from an unpublished calculation by Carnall et al. [1974],

Sugar and Reader derived the ionization energy. Their estimate of the uncertainty has been doubled, as recommended by Spector and Sugar.

\section{References}

Carnall, W. T., Fields, P. R., and Rajnak, K., J. Chem. Phys. 49, 4450 (1968). [EL] [CL] [W] PT

Carnall, W. T., Fields, P. R., and Rajnak, K., unpublished material (1974). PT

DeShazer, L. G., and Dieke, G. H., J. Chem. Phys. 38, 2190 (1963). [EL] [CL] [W] [ZE] PT

Dieke, G. H., Spectra and Energy Levels of Rare Earth Ions in Crystals, Ed. H. M. Crosswhite and H. Crosswhite, pp. 242-249 (Interscience Publishers, New York, 1968). [EL] [CL] [W] [ZE]

Ofelt, G. S., J. Chem. Phys. 38, 2171 (1963). PT

Spector, N., and Sugar, J., J. Opt. Soc. Am. 66, 436 (1976).

Sugar, J., and Reader, J., J. Chem. Phys. 59, 2083 (1973). IP

[July 1976]

[Eu IV]

\begin{tabular}{|c|c|c|c|c|c|}
\hline Configuration & Term & $J$ & $\begin{array}{l}\text { Level } \\
\left(\mathrm{cm}^{-1}\right)\end{array}$ & \multicolumn{2}{|c|}{ Leading percentages } \\
\hline $4 f^{6}$ & ${ }^{7} \mathrm{~F}$ & $\begin{array}{l}0 \\
1 \\
2 \\
3 \\
4 \\
5 \\
6\end{array}$ & $\begin{array}{c}0 \\
{[370]} \\
{[1040]} \\
{[1890]} \\
{[2860]} \\
{[3910]} \\
{[4940]}\end{array}$ & $\begin{array}{l}93 \\
95 \\
96 \\
97 \\
98 \\
98 \\
96\end{array}$ & $\begin{array}{ll}3 & { }^{5} \mathrm{D} 1 \\
3 & { }^{5} \mathrm{D} 1 \\
2 & { }^{5} \mathrm{D} 1 \\
1 & { }^{5} \mathrm{D} 1 \\
1 & { }^{5} \mathrm{~F} 2 \\
1 & { }^{5} \mathrm{G} 1 \\
2 & { }^{5} \mathrm{G} 1\end{array}$ \\
\hline $4 f^{6}$ & ${ }^{5} \mathrm{D} 3$ & $\begin{array}{l}0 \\
1 \\
2 \\
3 \\
4\end{array}$ & $\begin{array}{l}{[17270]} \\
{[19030]} \\
{[21510]} \\
{[24390]} \\
{[27640]}\end{array}$ & $\begin{array}{l}46 \\
51 \\
55 \\
56 \\
53\end{array}$ & $\begin{array}{ll}30 & { }^{5} \mathrm{D} 1 \\
32 & \\
35 & \\
36 & \\
36 & \end{array}$ \\
\hline $\mathrm{Eu} \mathrm{v}\left({ }^{6} \mathrm{H}_{5 / 2}^{\circ}\right)$ & Limit & & 344000 & & \\
\hline
\end{tabular}




\section{GADOLINIUM}

Gd I

64 electrons

$Z=64$

Ground state $\left(1 s^{2} 2 s^{2} 2 p^{6} 3 s^{2} 3 p^{6} 3 d^{10} 4 s^{2} 4 p^{6} 4 d^{10} 5 s^{2} 5 p^{6}\right) 4 f^{7} 5 d 6 s^{2}{ }^{9} \mathrm{D}_{2}^{\circ}$

Ionization energy $49603 \pm 5 \mathrm{~cm}^{-1}$

$6.1500 \pm 0.0006 \mathrm{eV}$

Identified odd configurations

$4 f^{7} 5 d 6 s^{2}, 4 f^{7} 5 d^{2} 6 s, 4 f^{7} 5 d^{3}, 4 f^{7} 5 d 6 s 7 s, 4 f^{7} 5 d 6 s 8 s, 4 f^{7} 6 s^{2} 7 s$, $4 f^{7} 6 s 6 p^{2}, 4 f^{8} 6 s 6 p$

Identified even configurations $\quad 4 f^{8} 6 s^{2}, 4 f^{8} 5 d 6 s, 4 f^{8} 6 s 7 s, 4 f^{7} 6 s^{2} 6 p, 4 f^{7} 5 d 6 s 6 p, 4 f^{7} 5 d^{2} 6 p$

\section{Spectrum Observations}

King [1943] gave wavelengths and temperature classifications for $5775 \mathrm{Gd}$ lines in the range 2135-10667 $\AA$. By comparing intensities in the spectra from the furnace, an arc, and a spark, he separated the lines of Gd I and Gd II. A much richer spectrum has been obtained from an electrodeless lamp containing ${ }^{158} \mathrm{Gd}$. P. E. Noorman photographed the spectrum from this source with the 9-m spectrograph at the Argonne National Laboratory. The measurements of these plates by Hoekstra and Slooten [1971] gave more than $18500 \mathrm{Gd}$ lines in the range 2468-8752 $\AA$, about 11000 of which belong to Gd I. Also at Argonne, Tomkins and Camus have observed $850 \mathrm{Gd}$ I lines in absorption in the region $2200-2800 \AA$. The new lists are unpublished, but van Kleef, Blaise and Wyart [1971] used them in their analysis, and some $5300 \mathrm{Gd}$ I lines are now classified. Practically all of the 1400 strongest Gd I and Gd II lines are classified [Meggers, Corliss, and Scribner, 1975; the energy levels for the classifications in this reference were taken from Blaise, Chevillard, Verges, Wyart, and van Kleef, 1971].

Spector and Held [1970] photographed and measured 950 Gd lines between 7200 and 12315 $\AA$, and assigned about 900 lines to either Gd I or Gd II. Blaise, Chevillard, Verges, Wyart, and van Kleef [1971] give $966 \mathrm{Gd}$ lines between 8331 and $24552 \AA$, with most of the 760 classified lines belonging to $\mathrm{Gd}$ I.

\section{History of the Analysis}

In his remarkable 1935 paper Albertson gave the first analyses of both Sm I and Gd I, including the ground term and its configuration assignment in each case. In addition to the 5 levels of the Gd I $4 f^{7}\left({ }^{8} \mathrm{~S}^{\circ}\right) 5 d 6 s^{2}{ }^{9} \mathrm{D}^{\circ}$ ground term, he found 30 upper even levels and classified 71 lines in a then unpublished list of King.

The analysis was continued by Russell [1942, 1950] and greatly extended to include the $4 f^{7}\left({ }^{8} \mathrm{~S}^{\circ}\right) 5 d 6 s^{2}{ }^{8} \mathrm{D}^{\circ}$ term, the low $4 f^{7}\left({ }^{8} \mathrm{~S}^{\circ}\right) 5 d^{2} 6 s^{9} \mathrm{~F}^{\circ}$ and ${ }^{11} \mathrm{~F}^{\circ}$ terms, the high $4 f^{7}\left({ }^{8} \mathrm{~S}^{\circ}\right) 5 d 6 s 7 s^{9} \mathrm{D}^{\circ}$, ${ }^{11} \mathrm{D}^{\circ}$ and $4 f^{7}\left({ }^{8} \mathrm{~S}^{\circ}\right) 6 s^{2} 7 s^{9} \mathrm{~S}^{\circ}$ terms, and about 200 even levels. He classified 1200 lines from King's list, including most of the stronger lines.

Klinkenberg [1946] first obtained $g$ values for the levels of the ground term and for 17 upper even levels. He independently assigned several of the even levels to the two lowest ${ }^{9} \mathrm{D}$ terms of $4 f^{7}\left({ }^{8} \mathrm{~S}^{\circ}\right) 5 d 6 s 6 p$ and noted that the low even ${ }^{9} \mathrm{P}$ term belonged to $4 f^{7}\left({ }^{8} \mathrm{~S}^{\circ}\right) 6 s^{2} 6 p$. Mainly on the basis of more extensive Zeeman data (see below), Pinnington [1967] identified two $4 f^{7}\left({ }^{8} \mathrm{~S}^{\circ}\right) 6 s^{2} 6 p^{7} \mathrm{P}$ levels and assigned a number of other even levels to new $4 f^{7}\left({ }^{8} \mathrm{~S}^{\circ}\right) 5 d 6 s 6 p$ terms. Spector [1971] corrected the assignments of two low even levels and arranged the $4 f^{7}\left({ }^{8} \mathrm{~S}^{\circ}\right) 6 s^{2} 6 p$ levels into two $J_{1} j$-coupling terms.

A collaborative effort of spectroscopists at the Zeeman Laboratory, Amsterdam, and the Laboratoire Aimé Cotton, Orsay, has greatly extended the analysis to include 236 odd levels and 371 even levels [van Kleef, Slooten, Blaise, and Camus, 1970; Blaise, Wyart, and van Kleef, 1970; Blaise, Chevillard, Verges, Wyart, and van Kleef, 1971; van Kleef, Blaise, and Wyart, 
1971]. All level values are taken from the last two references; the levels are given to three decimal places, but it is apparent that many of the values were quoted only to the nearest $0.005 \mathrm{~cm}^{-1}$. In addition to the new wavelength measurements mentioned above, these authors also had available extensive new Zeeman data (see below). Their results include a number of revisions of the previous analysis.

\section{Theoretical Interpretation, Calculations}

All identified levels are based on one of the two core terms $4 f^{7}\left({ }^{8} \mathrm{~S}\right)$ or $4 f^{8}\left({ }^{7} \mathrm{~F}\right)$; the next lowest term of the $4 f^{7}$ core, the ${ }^{6} \mathrm{P}^{\circ}$ term, is predicted to be about $32500 \mathrm{~cm}^{-1}$ above the ${ }^{8} \mathrm{~S}^{\circ}$ term. The identification of the entire system based on $4 f^{8}\left({ }^{7} \mathrm{~F}\right)$ is due to the Amsterdam-Orsay group.

The eigenvector percentages for the odd levels are from Wyart's thesis [1973]. His calculations included the $\left(4 f^{7}\left({ }^{8} \mathrm{~S}^{\circ},{ }^{6} \mathrm{P}^{\circ}\right) 5 d 6 s^{2}+4 f^{7}\left({ }^{8} \mathrm{~S}^{\circ}\right) 5 d^{2} 6 \mathrm{~s}+4 f^{7}\left({ }^{8} \mathrm{~S}^{\circ}\right) 5 d^{3}\right)$ subconfigurations with their interactions, and he also calculated the $4 f^{7}\left({ }^{8} \mathrm{~S}^{\circ}\right) 5 d 6 s 7 s$ group. No calculations of configurations based on the $4 f^{8}$ core are available. Van Kleef et al. [1971] note that the $4 f^{8} 6 s 6 p$ configuration assignments, which begin at $25658 \mathrm{~cm}^{-1}$, are based mainly on strong transitions to the $4 f^{8} 6 s^{2}{ }^{7} \mathrm{~F}$ levels. These assignments have been listed tentatively above $32000 \mathrm{~cm}^{-1}$, pending a calculation of the odd configurations in this region. Van Kleef et al. did not list term assignments for the $4 f^{8} 6 s 6 p$ levels but noted the appropriateness of a $J_{1} j$ coupling scheme. An example of the scheme is the designation $4 f^{8}\left({ }^{7} \mathrm{~F}_{6}\right) 6 s 6 p\left({ }^{3} \mathrm{P}_{2}^{\circ}\right)$ for the tentative level having $J=8$ at $27797 \mathrm{~cm}^{-1}$.

Much of the interpretation of the even level system above about $26000 \mathrm{~cm}^{-1}$ appears as yet uncertain. Only the analysis of Russell was available to Nir [1969] when he calculated the group $\left(4 f^{7}\left({ }^{8} \mathrm{~S}^{\circ}\right) 6 s^{2} 6 p+4 f^{7}\left({ }^{8} \mathrm{~S}^{\circ}\right) 5 d 6 s 6 p+4 f^{7}\left({ }^{8} \mathrm{~S}^{\circ}\right) 5 d^{2} 6 p\right)$, and no more complete calculations are available. All eigenvector percentages are from $\mathrm{Nir}$, but a number of the levels belonging mainly to this group were subsequently found and assigned independently of his calculation [van Kleef et al., 1971]. The $4 f^{8}\left({ }^{7} \mathrm{~F}\right) 5 d 6 s$ subconfiguration is especially prominent among the additional even groups found by van Kleef et al. to overlap the above group. The strong configuration interaction and intermediate coupling already evident in Nir's results obviate any meaningful names for many of the levels, and these effects call only be increased if additional configurations are included. In the arrangement here we have omitted term names indicated as inappropriate by the assigned eigenvectors, and almost all of the designations of levels above $30000 \mathrm{~cm}^{-1}$ are given as questionable. In cases of disagreement we usually followed the suggested interpretation of van Kleef et al. (the data available to them being much more extensive) or omitted configuration and/or term assignments for the levels in question. The configuration assignments of a number of the levels are based partly on measured isotope shifts; some recent suggestions made by Ahmad, Saksena, and Venugopalan [1976] on this basis have been taken into account. A few of the suggested assignments of levels to Nir's eigenvectors were made by us. More complete calculations of both the even and odd levels for this spectrum are badly needed.

\section{$g$ Values, Ionization Energy}

Smith and Spalding [1961] measured $g$ values for the levels of the $4 f^{7} 5 d 6 s^{2}{ }^{9} \mathrm{D}^{\circ}$ ground term, using an atomic-beam magnetic-resonance method. Their $g$ values are given here for the lowest three levels, the uncertainties being four to six units in the fourth place. All of the three-place $g$ values and some of the two-place values represent weighted averages that include Pinnington's results [1967]. His measurements of optical Zeeman-effect patterns gave $g$ values for 118 levels of $\mathrm{Gd}$ I, with probable errors varying from 0.001 to 0.01 . The majority of the $g$ values are from the very complete measurements of the Zeeman effect by van Kleef, Blaise, and Wyart. These authors used Zeeman-effect spectrograms $(2700-11400 \quad \AA ⿻)$ photographed at Argonne by Noorman and Fred. Van Kleef et al. gave values to the nearest 0.005 , but we have truncated their values to two decimals to distinguish them from the threeplace averages. The latter are usually taken close to Pinnington's values if the probable error is $\leqslant 0.005$; the overall agreement of the two sets of measurements is satisfactory. Spector's unpublished measurements of the $g$ values for about 20 levels were also used in the averages. Some of the levels noted by van Kleef et al. or by Blaise [1976] as showing the Paschen-Back 
effect are indicated here by colons following the $g$ values.

Worden, Conway, Paisner, and Solarz [1977] observed high series members in Gd I using a stepwise laser photoionization technique. These data became available too late for inclusion here, but the quoted ionization energy was determined from the new series.

\section{References}

Ahmad, S. A., Saksena, G. D., and Venugopalan, A., Physica (Utrecht) 81C, 366 (1976). ND CL IS

Albertson, W., Phys. Rev. 47, 370 (1935). EL CL

Blaise, J., private communication (1976). ND ZE

Blaise, J., Chevillard, J., Verges, J., Wyart, J. F., and van Kleef, T. A. M., Spectrochim. Acta, Part B 26, 1 (1971). EL CL W ZE

Blaise, J., Wyart, J. F., and van Kleef, T. A. M., C. R. Acad. Sci., Ser. B 270, 261 (1970). EL ZE

Eremin, M. V., and Maryakhina, O. I., Opt. Spectrosc. (USSR) 26, 479 (1969). ND PT

Hoekstra, R., and Slooten, R., Spectrochim. Acta, Part B 26, 341 (1971). Wavelength Accuracy

King, A. S., Astrophys. J. 97, 323 (1943). W

Klinkenberg, P. F. A., Physica (Utrecht) 12, 33 (1946). EL ZE

Meggers, W. F., Corliss, C. H., and Seribner, B. F., Nat. Bur. Stand. (U.S.), Monogr. 145, Part I, 387 pp. (1975). CL

Nir, S., Thesis, Hebrew Univ. Jerusalem, Israel, 216 pp. (1969). ND PT

Pinnington, E. H., J. Opt. Soc. Am. 57, 1252 (1967). EL ND ZE

Russell, H. N., Astrophys. J. 96, 11 (1942). EL CL IP

Russell, H. N., J. Opt. Soc. Am. 40, 550 (1950). EL CL IP

Smith, K. F., and Spalding, I. J., Proc. R. Soc. London, Ser. A 265, 133 (1961). ZE

Spector, N., unpublished material (1967). ZE

Spector, N., J. Opt. Soc. Am. 61, 1350 (1971). ND

Spector, N., and Held, S., Astrophys. J. 159, 1079 (1970). W

van Kleef, T. A. M., Blaise, J., and Wyart, J. F., J. Phys. (Paris) 32, 609 (1971). EL ZE PT

van Kleef, T. A. M., Slooten, R., Blaise, J., and Camus, P., C. R. Acad. Sci., Ser. B 270, 204 (1970). EL ZE

Worden, E. F., Conway, J. G., Paisner, J. A., and Solarz, R. W., unpublished material (1977). IP

Wyart, J. F., C. R. Acad. Sci., Ser. B 271, 849 (1970). PT

Wyart, J. F., Thesis, Univ. Paris-Sud, Orsay, 194 pp. (1973). ND PT

[July 1976]

Gd I, Odd Parity

\begin{tabular}{|c|c|c|c|c|c|}
\hline Configuration & Term & $J$ & $\begin{array}{l}\text { Level } \\
\left(\mathrm{cm}^{-1}\right)\end{array}$ & $g$ & $\begin{array}{c}\text { Leading } \\
\text { percentages }\end{array}$ \\
\hline \multirow{5}{*}{$4 f^{7}\left({ }^{8} S^{\circ}\right) 5 d 6 s^{2}$} & ${ }^{9} \mathrm{D}^{\circ}$ & 2 & 0.000 & 2.6514 & 95 \\
\hline & & 3 & 215.124 & 2.0708 & 94 \\
\hline & & 4 & 532.977 & 1.8392 & 93 \\
\hline & & 5 & 999.121 & 1.720 & 92 \\
\hline & & 6 & 1719.087 & 1.660 & 95 \\
\hline \multirow[t]{7}{*}{$4 f^{7}\left({ }^{8} \mathrm{~S}^{\circ}\right) 5 d^{2}\left({ }^{3} \mathrm{~F}\right)\left({ }^{10} \mathrm{~F}^{\circ}\right) 6 s$} & ${ }^{11} \mathrm{~F}^{\circ}$ & 2 & 6378.146 & 2.980 & 98 \\
\hline & & 3 & 6550.395 & 2.237 & 98 \\
\hline & & 4 & 6786.184 & 1.935 & 98 \\
\hline & & 5 & 7103.420 & 1.781 & 98 \\
\hline & & 6 & 7480.348 & 1.72 & 98 \\
\hline & & 7 & 7947.294 & 1.695 & 98 \\
\hline & & 8 & 8498.434 & 1.62 & 99 \\
\hline \multirow[t]{5}{*}{$4 f^{7}\left({ }^{8} \mathrm{~S}^{\circ}\right) 5 d 6 s^{2}$} & ${ }^{7} \mathrm{D}^{\circ}$ & 5 & 6976.508 & 1.600 & 90 \\
\hline & & 4 & 7234.910 & 1.65 & 90 \\
\hline & & 3 & 7426.710 & 1.744 & 90 \\
\hline & & 2 & 7562.457 & 1.995: & 91 \\
\hline & & 1 & 7653.927 & 2.979: & 91 \\
\hline
\end{tabular}


Gd I, Odd Parity-Continued

\begin{tabular}{|c|c|c|c|c|c|}
\hline Configuration & Term & $J$ & $\begin{array}{l}\text { Level } \\
\left(\mathrm{cm}^{-1}\right)\end{array}$ & $g$ & $\begin{array}{l}\text { Leading } \\
\text { percentages }\end{array}$ \\
\hline \multirow[t]{7}{*}{$4 f^{7}\left({ }^{8} \mathrm{~S}^{\circ}\right) 5 d^{2}\left({ }^{3} \mathrm{~F}\right)\left({ }^{10} \mathrm{~F}^{\circ}\right) 6 s$} & ${ }^{9} \mathrm{~F}^{\circ}$ & 1 & 10222.233 & 3.466 & 79 \\
\hline & & 2 & 10359.905 & 2.144 & 78 \\
\hline & & 3 & 10576.410 & 1.814 & 77 \\
\hline & & 4 & 10883.505 & 1.684 & 75 \\
\hline & & 5 & 11296.465 & 1.619 & 74 \\
\hline & & 6 & 11830.393 & 1.58 & 70 \\
\hline & & 7 & 12486.547 & 1.56 & 70 \\
\hline \multirow[t]{3}{*}{$4 f^{7}\left({ }^{8} \mathrm{~S}^{\circ}\right) 5 d^{2}\left({ }^{3} \mathrm{P}\right)\left({ }^{10} \mathrm{P}^{\circ}\right) 6 s$} & ${ }^{11} \mathrm{P}^{\circ}$ & 4 & 11685.594 & 2.19 & 98 \\
\hline & & 5 & 12057.164 & 1.96 & 98 \\
\hline & & 6 & 12345.966 & 1.82 & 94 \\
\hline \multirow[t]{3}{*}{$4 f^{7}\left({ }^{8} \mathrm{~S}^{\circ}\right) 5 d^{2}\left({ }^{3} \mathrm{P}\right)\left({ }^{10} \mathrm{P}^{\circ}\right) 6 s$} & ${ }^{9} \mathrm{P}^{\circ}$ & 3 & 15173.639 & 2.23 & 83 \\
\hline & & 4 & 15519.180 & 1.92 & 71 \\
\hline & & 5 & 15720.715 & 1.75 & 51 \\
\hline \multirow[t]{7}{*}{$4 f^{7}\left({ }^{8} \mathrm{~S}^{\circ}\right) 5 d^{2}\left({ }^{3} \mathrm{~F}\right)\left({ }^{8} \mathrm{~F}^{\circ}\right) 6 s$} & ${ }^{9} \mathrm{~F}^{\circ}$ & 2 & 15744.826 & 2.08: & 74 \\
\hline & & 3 & 15758.277 & 1.81: & 68 \\
\hline & & 1 & 15758.651 & 3.33: & 79 \\
\hline & & 4 & 15833.797 & 1.71 & 59 \\
\hline & & 5 & 15989.463 & 1.65 & 46 \\
\hline & & 6 & 16228.822 & 1.60 & 57 \\
\hline & & 7 & 16758.940 & 1.57 & 68 \\
\hline \multirow[t]{7}{*}{$4 f^{7}\left({ }^{8} \mathrm{~S}^{\circ}\right) 5 d^{2}\left({ }^{3} \mathrm{~F}\right)\left({ }^{8} \mathrm{~F}^{\circ}\right) 6 s$} & ${ }^{7} \mathrm{~F}^{\circ}$ & 0 & 15972.270 & & 87 \\
\hline & & 1 & 16012.884 & 1.64: & 82 \\
\hline & & 2 & 16078.047 & 1.57: & 76 \\
\hline & & 3 & 16165.993 & 1.54 & 71 \\
\hline & & 4 & 16296.617 & 1.55 & 68 \\
\hline & & 5 & 16534.515 & 1.53 & 62 \\
\hline & & 6 & 17015.950 & 1.52 & 76 \\
\hline \multirow[t]{5}{*}{$4 f^{7}\left({ }^{8} \mathrm{~S}^{\circ}\right) 5 d^{2}\left({ }^{1} \mathrm{D}\right)\left({ }^{8} \mathrm{D}^{\circ}\right) 6 s$} & ${ }^{9} \mathrm{D}^{\circ}$ & 2 & 17332.222 & 2.64: & 89 \\
\hline & & 3 & 17362.198 & 2.07: & 78 \\
\hline & & 4 & 17439.747 & 1.83 & 69 \\
\hline & & 5 & 17600.818 & 1.71 & 67 \\
\hline & & 6 & 17906.737 & 1.63 & 75 \\
\hline \multirow[t]{5}{*}{$4 f^{7}\left({ }^{8} \mathrm{~S}^{\circ}\right) 5 d^{2}\left({ }^{1} \mathrm{D}\right)\left({ }^{8} \mathrm{D}^{\circ}\right) 6 s$} & ${ }^{7} \mathrm{D}^{\circ}$ & 3 & 18993.172 & 1.73 & 72 \\
\hline & & 2 & 19014.075 & 2.00 & 80 \\
\hline & & 4 & 19022.740 & 1.63 & 65 \\
\hline & & 1 & 19062.222 & 2.96 & 89 \\
\hline & & 5 & 19085.785 & 1.58 & 40 \\
\hline \multirow[t]{8}{*}{$4 f^{7}\left({ }^{8} \mathrm{~S}^{\circ}\right) 5 d^{2}\left({ }^{1} \mathrm{G}\right)\left({ }^{8} \mathrm{G}^{\circ}\right) 6 s$} & ${ }^{9} \mathrm{G}^{\circ}$ & 0 & 19361.800 & & 94 \\
\hline & & 1 & 19375.576 & 1.49 & 93 \\
\hline & & 2 & 19403.104 & 1.50 & 93 \\
\hline & & 3 & 19445.555 & 1.50: & 85 \\
\hline & & $\begin{array}{l}4 \\
5\end{array}$ & $\begin{array}{l}19507.993 \\
19592.485\end{array}$ & $\begin{array}{l}1.51 \\
1.51\end{array}$ & $\begin{array}{l}86 \\
90\end{array}$ \\
\hline & & 6 & 19682.130 & 1.50 & 92 \\
\hline & & 7 & 19781.555 & 1.51 & 94 \\
\hline & & 8 & 19850.132 & 1.50 & 96 \\
\hline \multirow[t]{3}{*}{$4 f^{7}\left({ }^{8} \mathrm{~S}^{\circ}\right) 5 d^{2}\left({ }^{3} \mathrm{P}\right)\left({ }^{8} \mathrm{P}^{\circ}\right) 6 s$} & ${ }^{9} \mathrm{P}^{\circ}$ & 3 & 19574.024 & 2.22 & 71 \\
\hline & & 4 & 19718.720 & 1.92 & 66 \\
\hline & & 5 & 19978.775 & 1.76 & 63 \\
\hline \multirow[t]{5}{*}{$4 f^{7}\left({ }^{8} \mathrm{~S}^{\circ}\right) 5 d^{2}\left({ }^{3} \mathrm{~F}\right)\left({ }^{6} \mathrm{~F}^{\circ}\right) 6 s$} & ${ }^{5} \mathrm{~F}^{\circ}$ & 3 & 20299.870 & 1.25: & 65 \\
\hline & & 2 & 20303.800 & 0.93: & 56 \\
\hline & & 1 & 20306.942 & $0.03:$ & 50 \\
\hline & & 4 & 20324.722 & 1.37 & 73 \\
\hline & & 5 & 20588.292 & 1.49 & 39 \\
\hline \multirow[t]{3}{*}{$4 f^{7}\left({ }^{8} \mathrm{~S}^{\circ}\right) 5 d^{2}\left({ }^{3} \mathrm{P}\right)\left({ }^{8} \mathrm{P}^{\circ}\right) 6 s$} & ${ }^{7} \mathrm{P}^{\circ}$ & 2 & 20565.620 & 2.27 & 79 \\
\hline & & 3 & 20759.674 & 1.86 & 68 \\
\hline & & 4 & 21152.850 & 1.72 & 66 \\
\hline
\end{tabular}


Gd I, Odd Parity-Continued

\begin{tabular}{|c|c|c|c|c|c|}
\hline Configuration & Term & $J$ & $\begin{array}{l}\text { Level } \\
\left(\mathrm{cm}^{-1}\right)\end{array}$ & $g$ & $\begin{array}{c}\text { Leading } \\
\text { percentages }\end{array}$ \\
\hline $4 f^{7}\left({ }^{8} \mathrm{~S}^{\circ}\right) 5 d^{2}\left({ }^{1} \mathrm{G}\right) \quad\left({ }^{8} \mathrm{G}^{\circ}\right) 6 s$ & ${ }^{7} \mathrm{G}^{\circ}$ & $\begin{array}{l}6 \\
5 \\
7 \\
4 \\
3 \\
2 \\
1\end{array}$ & $\begin{array}{l}21389.502 \\
21439.715 \\
21514.952 \\
21544.680 \\
21647.935 \\
21745.650 \\
21815.645\end{array}$ & $\begin{array}{r}1.42 \\
1.36 \\
1.43 \\
1.34 \\
1.21 \\
0.90 \\
-0.36\end{array}$ & $\begin{array}{l}9 \dot{9} \\
90 \\
97 \\
72 \\
66 \\
55 \\
50\end{array}$ \\
\hline $4 f^{7}\left({ }^{8} \mathrm{~S}^{\circ}\right) 5 d^{3}\left({ }^{4} \mathrm{~F}\right)$ & ${ }^{11} \mathrm{~F}^{\circ}$ & $\begin{array}{l}2 \\
3 \\
4 \\
5 \\
6 \\
7 \\
8\end{array}$ & $\begin{array}{l}22429.156 \\
22602.550 \\
2283.5 .400 \\
23128.224 \\
23479.211 \\
23883.504 \\
24332.715\end{array}$ & & $\begin{array}{l}98 \\
98 \\
98 \\
98 \\
98 \\
98 \\
98\end{array}$ \\
\hline $4 f^{8}\left({ }^{7} F\right) 6 s 6 p$ & & 6 & 25658.055 & 1.58 & \\
\hline $4 f^{8}\left({ }^{7} F\right) 6 s 6 p$ & & 7 & 25676.890 & 1.54 & \\
\hline $4 f^{8}\left({ }^{7} \mathrm{~F}\right) 6 s 6 p$ & & 5 & 26651.912 & 1.57 & \\
\hline $4 f^{7}\left({ }^{8} \mathrm{~S}^{\circ}\right) 5 d^{3}\left({ }^{4} \mathrm{P}\right)$ & ${ }^{11} \mathrm{P}^{\circ}$ & $\begin{array}{l}4 \\
5 \\
6\end{array}$ & $\begin{array}{l}27026.088 \\
27258.986 \\
27715.350\end{array}$ & $\begin{array}{l}2.18 \\
1.95 \\
1.81\end{array}$ & $\begin{array}{l}97 \\
97 \\
99\end{array}$ \\
\hline $4 f^{8}\left({ }^{7} \mathrm{~F}\right) 6 s 6 p$ & & 6 & 27343.873 & 1.48 & \\
\hline $4 f^{8}\left({ }^{7} \mathrm{~F}\right) 6 s 6 p$ & & 5 & 27628.230 & 1.50 & \\
\hline $4 f^{8}\left({ }^{7} F\right) 6 s 6 p$ & & 4 & 27648.774 & 1.66 & \\
\hline $4 f^{8}\left({ }^{7} \mathrm{~F}_{6}\right) 6 s 6 p\left({ }^{3} \mathrm{P}_{2}^{\circ}\right)$ & $(6,2)^{\circ}$ & 8 & $27797.603 ?$ & $1.50 ?$ & \\
\hline $4 f^{8}\left({ }^{7} \mathrm{~F}\right) 6 s 6 p$ & & 6 & 27829.680 & 1.55 . & \\
\hline $4 f^{8}\left({ }^{7} \mathrm{~F}\right) 6 s 6 p$ & & 7 & 27981.472 & 1.46 & \\
\hline $4 f^{8}\left({ }^{7} \mathrm{~F}\right) 6 s 6 p$ & & 6 & 28366.491 & 1.53 & \\
\hline $4 f^{7}\left({ }^{8} S^{\circ}\right) 5 d^{3}\left({ }^{4} F\right)$ & ${ }^{9} \mathrm{~F}^{\circ}$ & $\begin{array}{l}2 \\
1 \\
3 \\
4 \\
5 \\
6 \\
7\end{array}$ & $\begin{array}{l}28725.150 \\
28741.590 \\
29041.722 \\
29174.580 \\
29439.330 \\
29867.535 \\
30367.361\end{array}$ & $\begin{array}{l}2.16: \\
3.45: \\
1.80 \\
1.67 \\
1.61 \\
1.58 \\
1.56\end{array}$ & $\begin{array}{l}96 \\
98 \\
94 \\
91 \\
90 \\
90 \\
93\end{array}$ \\
\hline $4 f^{8}\left({ }^{7} \mathrm{~F}\right) 6 s 6 p$ & & 5 & 28837.240 & 1.51 & \\
\hline $4 f^{8}\left({ }^{7} \mathrm{~F}\right) 6 s 6 p$ & & 5 & 28938.287 & 1.44 & \\
\hline $4 f^{8}\left({ }^{7} \mathrm{~F}\right) 6 s 6 p$ & & 5 & 29312.770 & 1.52 & \\
\hline $4 f^{8}\left({ }^{7} \mathrm{~F}\right) 6 s 6 p$ & & 6 & 29420.675 & 1.47 & \\
\hline $4 f^{8}\left({ }^{7} \mathrm{~F}\right) 6 s 6 p$ & & 4 & 29672.515 & 1.49 & \\
\hline $4 f^{8}\left({ }^{7} \mathrm{~F}\right) 6 s 6 p$ & & 4 & 30289.386 & 1.46 & \\
\hline $4 f^{8}\left({ }^{7} \mathrm{~F}\right) 6 s 6 p$ & & 4 & 30583.740 & 1.64 & \\
\hline $4 f^{8}\left({ }^{7} \mathrm{~F}\right) 6 s 6 p$ & & 6 & 30749.194 & 1.41 & \\
\hline $4 f^{8}\left({ }^{7} \mathrm{~F}\right) 6 s 6 p$ & & 3 & 31542.413 & 1.66 & \\
\hline & & 5 & 31633.995 & 1.71 & \\
\hline $4 f^{8}\left({ }^{7} \mathrm{~F}\right) 6 s 6 p$ & & 5 & 31766.595 & 1.35 & \\
\hline
\end{tabular}


Gd I, Odd Parity-Continued

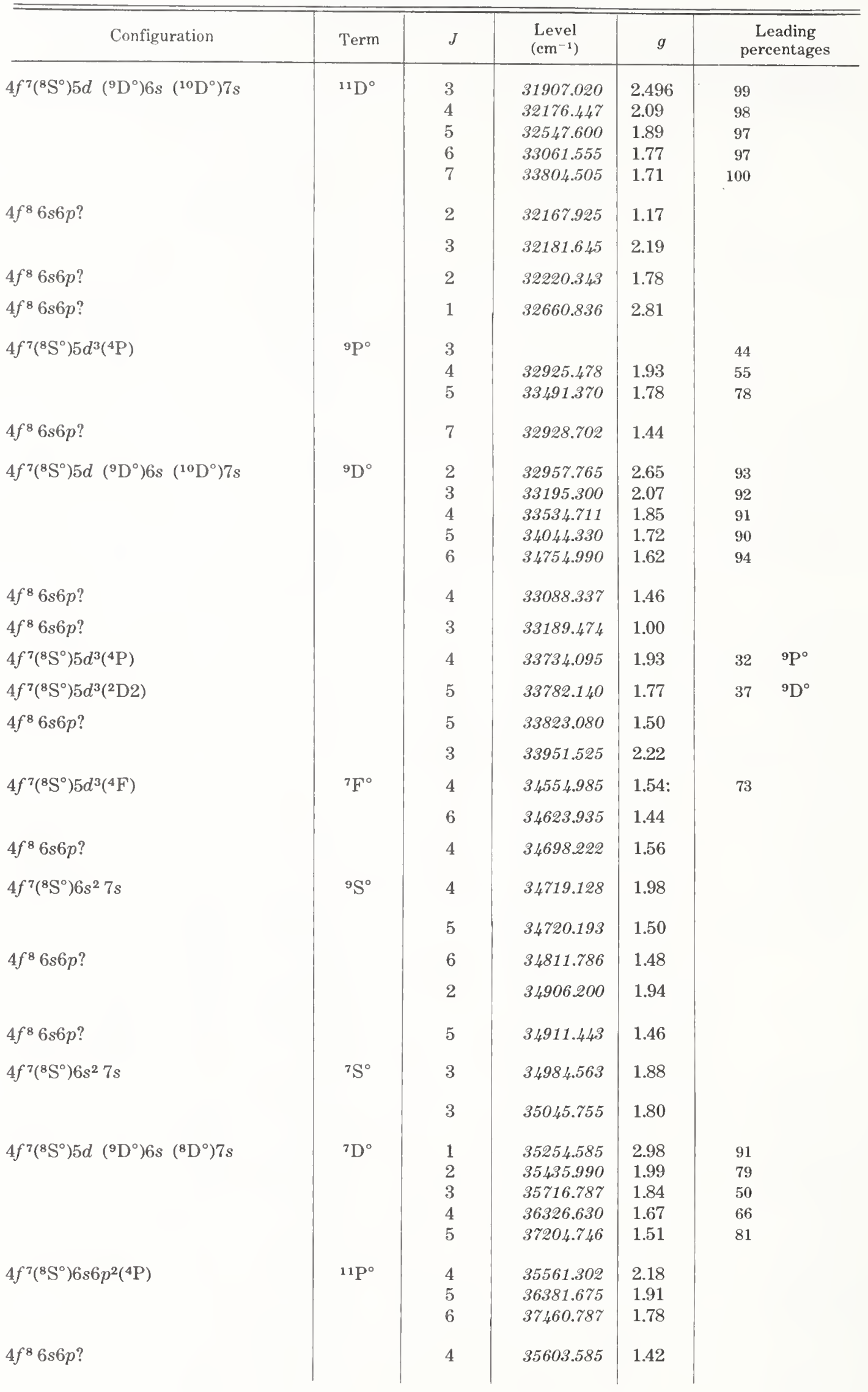


Gd I, Odd Parity-Continued

\begin{tabular}{|c|c|c|c|c|c|}
\hline Configuration & Term & $J$ & $\begin{array}{l}\text { Level } \\
\left(\mathrm{cm}^{-1}\right)\end{array}$ & $g$ & $\begin{array}{c}\text { Leading } \\
\text { percentages }\end{array}$ \\
\hline \multirow[t]{5}{*}{$4 f^{7}\left({ }^{8} \mathrm{~S}^{\circ}\right) 5 d\left({ }^{9} \mathrm{D}^{\circ}\right) 6 s\left({ }^{8} \mathrm{D}^{\circ}\right) 7 s$} & ${ }^{9} \mathrm{D}^{\circ}$ & 2 & 35606.143 & 2.61 & 74 \\
\hline & & 3 & 35860.584 & 1.99 & 46 \\
\hline & & 4 & 36060.122 & 1.74 & 65 \\
\hline & & 5 & 36541.260 & 1.68 & 72 \\
\hline & & 6 & 37150.790 & 1.65 & 66 \\
\hline \multirow[t]{3}{*}{$4 f^{8} 6 s 6 p ?$} & & 3 & 35997.029 & 1.29 & \\
\hline & & 4 & 36179.444 & 1.69: & \\
\hline & & 3 & 36182.700 & 1.78: & \\
\hline $4 f^{8} 6 s 6 p ?$ & & 2 & 36268.455 & 0.97 & \\
\hline $4 f^{8} 6 s 6 p ?$ & ${ }^{7} \mathrm{G}^{\circ}$ & 1 & 36360.088 & -0.41 & \\
\hline $4 f^{8} 6 s 6 p ?$ & & 4 & 36386.383 & 1.52 & \\
\hline \multirow[t]{2}{*}{$4 f^{8} 6 s 6 p ?$} & & 3 & 36576.972 & 1.46 & \\
\hline & & 4 & 36616.719 & 1.51 & \\
\hline $4 f^{8} 6 s 6 p ?$ & & 5 & 36653.970 & 1.58 & \\
\hline $4 f^{8} 6 s 6 p ?$ & & 5 & 36853.110 & 1.56 & \\
\hline $4 f^{8} 6 s 6 p ?$ & & 2 & 36895.845 & 1.41 & \\
\hline $4 f^{8} 6 s 6 p ?$ & & 4 & 37041.205 & 1.44 & \\
\hline $4 f^{8} 6 s 6 p ?$ & & 4 & 37123.736 & 1.58 & \\
\hline $4 f^{8} 6 s 6 p ?$ & & 3 & 37215.945 & 1.39 & \\
\hline \multirow[t]{2}{*}{$4 f^{8} 6 s 6 p ?$} & & 1 & 37246.590 & 1.04 & \\
\hline & & 3 & 37248.528 & 1.60 & \\
\hline \multirow[t]{5}{*}{$4 f^{7}\left({ }^{8} \mathrm{~S}^{\circ}\right) 5 d^{3}\left({ }^{2} \mathrm{G}\right)$} & ${ }^{7} \mathrm{G}^{\circ}$ & 6 & 37288.663 & 1.47 & \\
\hline & ${ }^{9} \mathrm{D}^{\circ} ?$ & 2 & & & \\
\hline & & 3 & 37313.720 & 2.07 & \\
\hline & & 4 & 37544.290 & 1.80 & \\
\hline & & $\begin{array}{l}5 \\
6\end{array}$ & $\begin{array}{l}37908.489 \\
38024.800\end{array}$ & $\begin{array}{l}1.68 \\
1.65\end{array}$ & \\
\hline \multirow[t]{4}{*}{$4 f^{7}\left({ }^{8} \mathrm{~S}^{\circ}\right) 5 d^{3}\left({ }^{2} \mathrm{~F}\right)$} & ${ }^{9} \mathrm{~F}^{\circ}$ & 1 & 37385210 & 3.58: & \\
\hline & & 4 & $\begin{array}{l}37463.818 \\
37590785\end{array}$ & $\begin{array}{l}1.60 \\
2.07 .\end{array}$ & 85 \\
\hline & & 3 & $\begin{array}{l}37529.785 \\
37548.383\end{array}$ & $1.80:$ & $\begin{array}{l}87 \\
88\end{array}$ \\
\hline & & $\begin{array}{l}5 \\
6 \\
7\end{array}$ & & & \\
\hline \multirow[t]{2}{*}{$4 f^{8} 6 s 6 p ?$} & & 2 & 37416.590 & 1.51 & \\
\hline & & 2 & 37424.457 & 1.97: & \\
\hline $4 f^{8} 6 s 6 p ?$ & & 0 & 37531.095 & & \\
\hline $4 f^{8} 6 s 6 p ?$ & & 1 & 37544.453 & 0.29 & \\
\hline \multirow[t]{3}{*}{$4 f^{8} 6 s 6 p ?$} & & 3 & 37604.950 & 1.82 & \\
\hline & & 3 & 37648.503 & 1.98 & \\
\hline & & 3 & 37669.207 & 2.08 & \\
\hline $4 f^{7}\left({ }^{8} \mathrm{~S}^{\circ}\right) 5 d^{3}$ & ${ }^{7} \mathrm{P}^{\circ}$ & 4 & 37702.758 & 1.74 & \\
\hline- & & 5 & 37722.087 & 1.88 & \\
\hline \multirow[t]{3}{*}{$4 f^{8} 6 s 6 p ?$} & & 2 & 37913.508 & 1.89 & \\
\hline & & 3 & 37933.446 & 1.50 & \\
\hline & & 3 & 38108.217 & 2.18 & \\
\hline \multirow[t]{2}{*}{$4 f^{8} 6 s 6 p ?$} & & 1 & 38173.340 & 2.91 & \\
\hline & & 6 & 38245.743 & 1.64: & \\
\hline
\end{tabular}


Gd I, Odd Parity-Continued

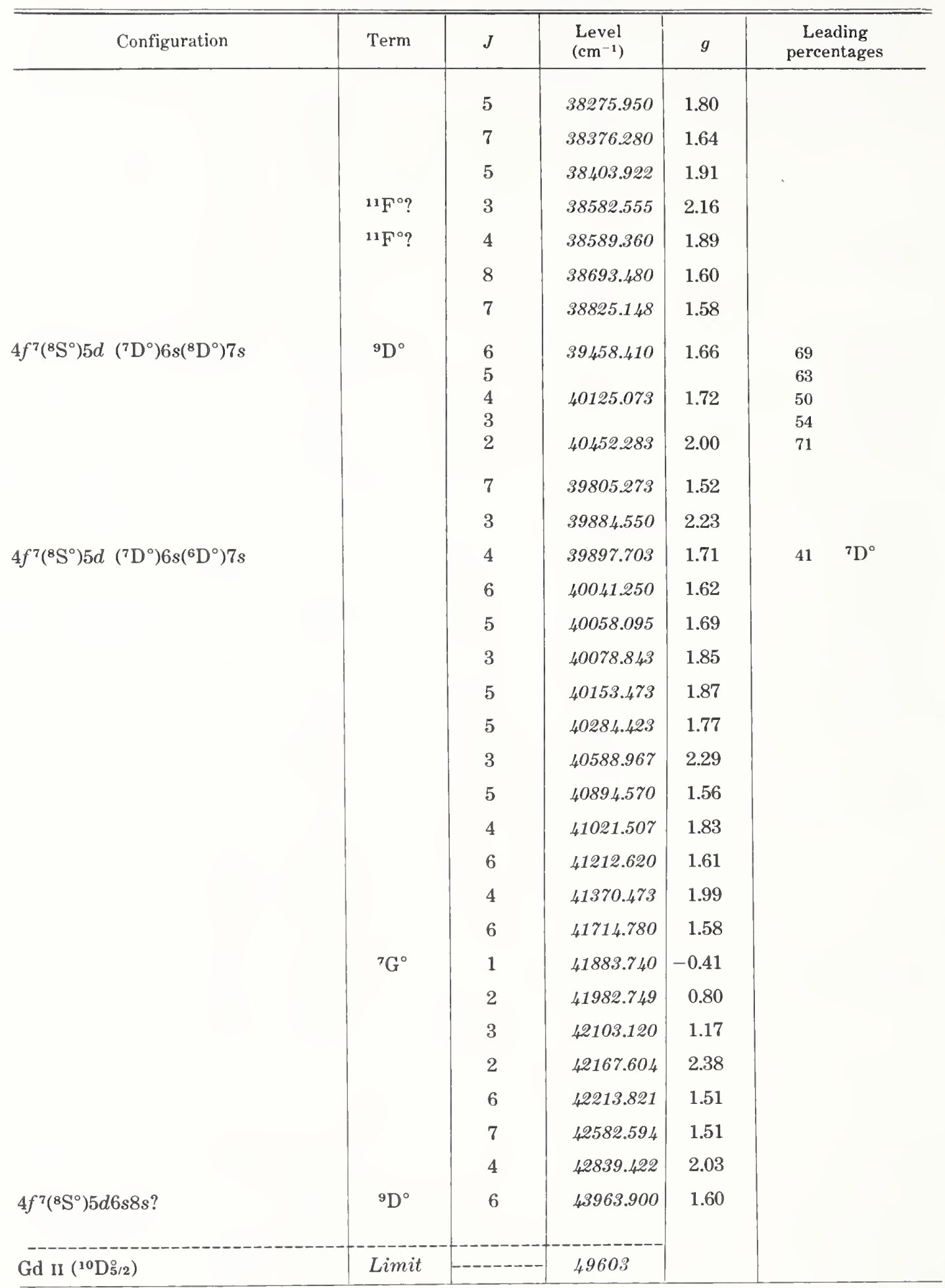


Gd I, Even Parity

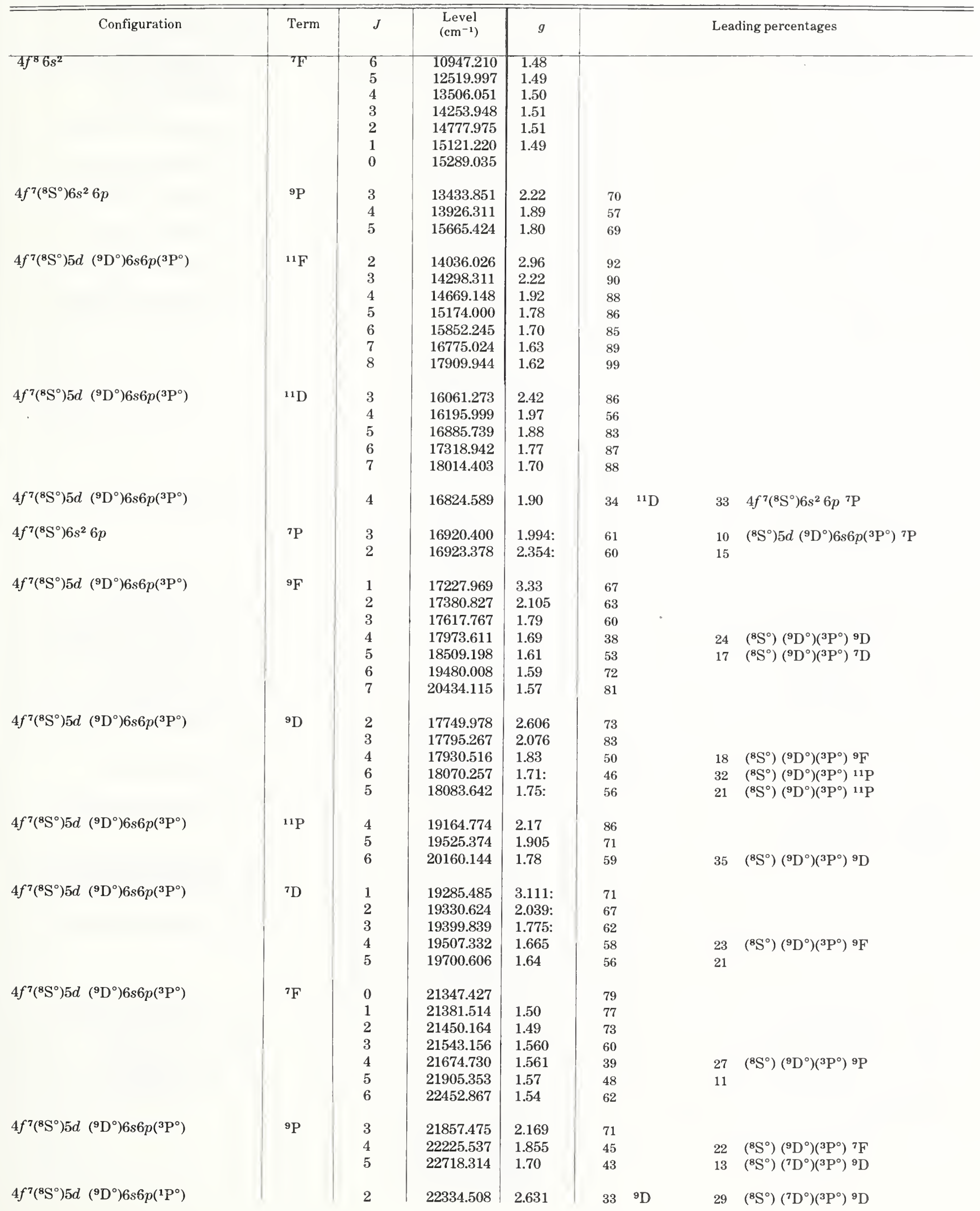


Gd I, Even Parity-Continued

\begin{tabular}{|c|c|c|c|c|c|c|c|c|}
\hline Configuration & Term & $J$ & $\begin{array}{l}\text { Level } \\
\left(\mathrm{cm}^{-1}\right)\end{array}$ & $g$ & \multicolumn{4}{|c|}{ Leading percentages } \\
\hline $4 f^{7}\left({ }^{8} \mathrm{~S}^{\circ}\right) 5 d\left({ }^{7} \mathrm{D}^{\circ}\right) 6 s 6 p\left({ }^{3} \mathrm{P}^{\circ}\right)$ & ${ }^{9} \mathrm{D}$ & $\begin{array}{l}3 \\
4 \\
5 \\
6 \\
2\end{array}$ & $\begin{array}{l}22563.824 \\
22820.895 \\
23196.410 \\
23229.298 \\
24655.639\end{array}$ & $\begin{array}{l}2.077 \\
1.849 \\
1.74 \\
1.65 \\
2.632\end{array}$ & $\begin{array}{l}40 \\
50 \\
44 \\
62 \\
45\end{array}$ & & $\begin{array}{r}27 \\
17 \\
22 \\
22\end{array}$ & $\begin{array}{l}\left({ }^{8} \mathrm{~S}^{\circ}\right)\left({ }^{9} \mathrm{D}^{\circ}\right)\left({ }^{1} \mathrm{P}^{\circ}\right){ }^{9} \mathrm{D} \\
\left({ }^{8} \mathrm{~S}^{\circ}\right)\left({ }^{9} \mathrm{D}^{\circ}\right)\left({ }^{1} \mathrm{P}^{\circ}\right) \\
\left({ }^{9} \mathrm{D}\right. \\
\left(\mathrm{S}^{\circ}\right)\left({ }^{\circ} \mathrm{D}^{\circ}\right)\left({ }^{3} \mathrm{P}^{\circ}\right){ }^{9} \mathrm{P} \\
\left({ }^{8} \mathrm{~S}^{\circ}\right) 5 d^{2}\left({ }^{3} \mathrm{~F}\right)\left({ }^{10} \mathrm{~F}^{\circ}\right) 6 p p^{9} \mathrm{D}\end{array}$ \\
\hline $4 f^{7}\left({ }^{8} \mathrm{~S}^{\circ}\right) 5 d^{2}\left({ }^{3} \mathrm{~F}\right)\left({ }^{10} \mathrm{~F}^{\circ}\right) 6 p$ & & 1 & 23103.660 & 3.549: & 29 & ${ }^{11} \mathrm{G}$ & 25 & $\left({ }^{8} \mathrm{~S}^{\circ}\right) 5 d\left({ }^{9} \mathrm{D}^{\circ}\right) 6 s 6 p\left({ }^{1} \mathrm{P}^{\circ}\right)^{9} \mathrm{~F}$ \\
\hline $4 f^{7}\left({ }^{8} \mathrm{~S}^{\circ}\right) 5 d\left({ }^{9} \mathrm{D}^{\circ}\right) 6 s 6 p\left({ }^{1} \mathrm{P}^{\circ}\right)$ & & 2 & 23215.028 & 2.176: & 25 & ${ }^{9} \mathrm{~F}$ & 25 & $\left({ }^{8} \mathrm{~S}^{\circ}\right) 5 d^{2}\left({ }^{3} \mathrm{~F}\right)\left({ }^{10} \mathrm{~F}^{\circ}\right) 6 p{ }^{11} \mathrm{G}$ \\
\hline $4 f^{7}\left({ }^{8} \mathrm{~S}^{\circ}\right) 5 d\left({ }^{9} \mathrm{D}^{\circ}\right) 6 s 6 p\left({ }^{1} \mathrm{P}^{\circ}\right)$ & & 3 & 23389.782 & 1.833 & 24 & ${ }^{9} \mathrm{~F}$ & 20 & $\left({ }^{8} \mathrm{~S}^{\circ}\right) 5 d^{2}\left({ }^{3} \mathrm{~F}\right)\left({ }^{10} \mathrm{~F}^{\circ}\right) 6 p^{11} \mathrm{G}$ \\
\hline $4 f^{7}\left({ }^{8} \mathrm{~S}^{\circ}\right) 5 d\left({ }^{9} \mathrm{D}^{\circ}\right) 6 s 6 p\left({ }^{1} \mathrm{P}^{\circ}\right)$ & & 4 & 23644.156 & 1.696 & 23 & ${ }^{9} \mathrm{~F}$ & 18 & $\left({ }^{8} \mathrm{~S}^{\circ}\right) 5 d^{2}\left({ }^{3} \mathrm{~F}\right)\left({ }^{10} \mathrm{~F}^{\circ}\right) 6 p{ }^{9} \mathrm{~F}$ \\
\hline $4 f^{7}\left({ }^{8} \mathrm{~S}^{\circ}\right) 5 d\left({ }^{9} \mathrm{D}^{\circ}\right) 6 s 6 p\left({ }^{1} \mathrm{P}^{\circ}\right)$ & & 5 & 23999.912 & 1.631 & 21 & ${ }^{9} \mathrm{~F}$ & 18 & $\left({ }^{8} \mathrm{~S}^{\circ}\right) 5 d^{2}\left({ }^{3} \mathrm{~F}\right)\left({ }^{10} \mathrm{~F}^{\circ}\right) 6 p^{9} \mathrm{~F}$ \\
\hline $4 f^{8}\left({ }^{7} \mathrm{~F}\right) 5 d\left({ }^{8} \mathrm{G}\right) 6 s$ & ${ }^{9} \mathrm{G}$ & $\begin{array}{l}7 \\
8 \\
6 \\
5 \\
4 \\
3 \\
2 \\
1 \\
0\end{array}$ & $\begin{array}{l}24255.103 \\
24854.297 \\
25380.885 \\
25820.720 \\
26247.609 \\
26588.338 \\
26834.311 \\
27089.815\end{array}$ & $\begin{array}{l}1.51 \\
1.555 \\
1.63 \\
1.580 \\
1.60 \\
1.70 \\
2.26\end{array}$ & & & & \\
\hline $4 f^{7}\left({ }^{8} \mathrm{~S}^{\circ}\right) 5 d\left({ }^{9} \mathrm{D}^{\circ}\right) 6 s 6 p\left({ }^{1} \mathrm{P}^{\circ}\right)$ & & 6 & 24430.425 & 1.580 & 20 & ${ }^{9} \mathrm{~F}$ & 17 & $\left({ }^{8} \mathrm{~S}^{\circ}\right) 5 d^{2}\left({ }^{3} \mathrm{~F}\right)\left({ }^{10} \mathrm{~F}^{\circ}\right) 6 p^{9} \mathrm{~F}$ \\
\hline $4 f^{7}\left({ }^{8} \mathrm{~S}^{\circ}\right) 5 d\left({ }^{9} \mathrm{D}^{\circ}\right) 6 s 6 p\left({ }^{3} \mathrm{P}^{\circ}\right)$ & ${ }^{7} \mathrm{P}$ & $\begin{array}{l}2 \\
3 \\
4\end{array}$ & $\begin{array}{l}24458.988 \\
24860.040 \\
25920.888\end{array}$ & $\begin{array}{l}2.31 \\
1.87 \\
1.58\end{array}$ & $\begin{array}{l}64 \\
70 \\
63\end{array}$ & & & \\
\hline $4 f^{7}\left({ }^{8} \mathrm{~S}^{\circ}\right) 5 d\left({ }^{7} \mathrm{D}^{\circ}\right) 6 s 6 p\left({ }^{3} \mathrm{P}^{\circ}\right) ?$ & & 5 & 24660.325 & 1.51 & 44 & ${ }^{5} \mathrm{~F}$ & 35 & $\left({ }^{8} S^{\circ}\right)\left({ }^{7} D^{\circ}\right)\left({ }^{3} P^{\circ}\right){ }^{7} \mathrm{D}$ \\
\hline $4 f^{7}\left({ }^{8} \mathrm{~S}^{\circ}\right) 5 d\left({ }^{7} \mathrm{D}^{\circ}\right) 6 s 6 p\left({ }^{3} \mathrm{P}^{\circ}\right)$ & & 3 & 24714.841 & 2.042 & 34 & ${ }^{9} \mathrm{D}$ & 23 & $\left({ }^{8} \mathrm{~S}^{\circ}\right) 5 d^{2}\left({ }^{3} \mathrm{~F}\right)\left({ }^{10} \mathrm{~F}^{\circ}\right) 6 p^{9} \mathrm{D}$ \\
\hline $4 f^{7}\left({ }^{8} \mathrm{~S}^{\circ}\right) 5 d\left({ }^{7} \mathrm{D}^{\circ}\right) 6 s 6 p\left({ }^{3} \mathrm{P}^{\circ}\right)$ & & 4 & 24849.514 & 1.82 & 22 & ${ }^{9} \mathrm{D}$ & 16 & $\left({ }^{8} \mathrm{~S}^{\circ}\right)\left({ }^{9} \mathrm{D}^{\circ}\right)\left({ }^{1} \mathrm{P}^{\circ}\right){ }^{9} \mathrm{D}$ \\
\hline $4 f^{7}\left({ }^{8} \mathrm{~S}^{\circ}\right) 5 d\left({ }^{7} \mathrm{D}^{\circ}\right) 6 s 6 p\left({ }^{3} \mathrm{P}^{\circ}\right)$ & ${ }^{\top} \mathrm{D}$ & $\begin{array}{l}4 \\
3 \\
2 \\
1 \\
5\end{array}$ & \begin{tabular}{l|}
24988.884 \\
25253.838 \\
25337.755 \\
25352.706 \\
$25732.850^{*}$
\end{tabular} & $\begin{array}{l}1.65 \\
1.784 \\
2.06: \\
3.07: \\
1.50\end{array}$ & $\begin{array}{l}44 \\
58 \\
65 \\
73 \\
43\end{array}$ & & $\begin{array}{r}9 \\
16\end{array}$ & $\begin{array}{l}\left({ }^{8} \mathrm{~S}^{\circ}\right)\left({ }^{9} \mathrm{D}^{\circ}\right)\left({ }^{3} \mathrm{P}^{\circ}\right){ }^{5 \mathrm{P}} \\
\left({ }^{8} \mathrm{~S}^{\circ}\right) 5 d^{2}\left({ }^{3} \mathrm{~F}\right)\left({ }^{10} \mathrm{~F}^{\circ}\right) 6 p{ }^{11} \mathrm{G} \\
\left({ }^{8} \mathrm{~S}^{\circ}\right)\left({ }^{7} \mathrm{D}^{\circ}\right)\left({ }^{3} \mathrm{P}^{\circ}\right){ }^{5} \mathrm{~F}\end{array}$ \\
\hline $4 f^{7}\left({ }^{8} \mathrm{~S}^{\circ}\right) 5 d\left({ }^{9} \mathrm{D}^{\circ}\right) 6 s 6 p\left({ }^{1} \mathrm{P}^{\circ}\right)$ & & 5 & 25043.649 & 1.65 & 30 & ${ }^{9} \mathrm{D}$ & 25 & $\left({ }^{8} \mathrm{~S}^{\circ}\right) 5 d^{2}\left({ }^{3} \mathrm{~F}\right)\left({ }^{10} \mathrm{~F}^{\circ}\right) 6 p{ }^{9} \mathrm{D}$ \\
\hline $4 f^{7}\left({ }^{8} \mathrm{~S}^{\circ}\right) 5 d^{2}\left({ }^{3} \mathrm{~F}\right)\left({ }^{10} \mathrm{~F}^{\circ}\right) 6 p$ & ${ }^{11} \mathrm{G}$ & $\begin{array}{l}1 \\
2 \\
3 \\
4 \\
5 \\
6 \\
7 \\
8 \\
9\end{array}$ & $\begin{array}{l}25069.179 \\
25164.640 \\
25403.265 \\
25571.672^{*} \\
25940.122 \\
26337.071 \\
26870.393 \\
27536.397 \\
28432.859\end{array}$ & $\begin{array}{l}3.764: \\
2.21: \\
1.857 \\
1.70 \\
1.64 \\
1.61 \\
1.57 \\
1.56 \\
1.54\end{array}$ & $\begin{array}{r}66 \\
57 \\
49 \\
35 \\
60 \\
53 \\
85 \\
92 \\
100\end{array}$ & & $\begin{array}{l}13 \\
15 \\
25 \\
11\end{array}$ & $\begin{array}{l}\left({ }^{8} \mathrm{~S}^{\circ}\right) 5 d\left({ }^{7} \mathrm{D}^{\circ}\right) 6 s 6 p\left({ }^{3} \mathrm{P}^{\circ}\right){ }^{7} \mathrm{D} \\
\left({ }^{8} \mathrm{~S}^{\circ}\right) 5 d\left({ }^{9} \mathrm{D}^{\circ}\right) 6 s 6 p\left({ }^{1} \mathrm{P}^{\circ}\right) \\
{ }^{9} \mathrm{~F} \\
\left({ }^{8} \mathrm{~S}^{\circ}\right) 5 d\left({ }^{7} \mathrm{D}^{\circ}\right) 6 s 6 p\left({ }^{3} \mathrm{P}^{\circ}\right){ }^{5} \mathrm{~F} \\
\left({ }^{8} \mathrm{~S}^{\circ}\right) 5 d\left({ }^{9} \mathrm{D}^{\circ}\right) 6 s 6 p\left({ }^{1} \mathrm{P}^{\circ}\right)^{9} \mathrm{D}\end{array}$ \\
\hline $4 f^{7}\left({ }^{8} \mathrm{~S}^{\circ}\right) 5 d\left({ }^{9} \mathrm{D}^{\circ}\right) 6 s 6 p\left({ }^{1} \mathrm{P}^{\circ}\right)$ & & 7 & 25376.313 & 1.58 & 32 & ${ }^{9} \mathrm{~F}$ & 23 & $\left({ }^{8} \mathrm{~S}^{\circ}\right) 5 d^{2}\left({ }^{3} \mathrm{~F}\right)\left({ }^{10} \mathrm{~F}^{\circ}\right) 6 p^{9} \mathrm{~F}$ \\
\hline $4 f^{7}\left({ }^{8} \mathrm{~S}^{\circ}\right) 5 d\left({ }^{7} \mathrm{D}^{\circ}\right) 6 s 6 p\left({ }^{3} \mathrm{P}^{\circ}\right) ?$ & & 4 & 25621.293 & 1.57 & 35 & ${ }^{5} \mathrm{D}$ & 30 & $\left({ }^{8} \mathrm{~S}^{\circ}\right)\left({ }^{7} \mathrm{D}^{\circ}\right)\left({ }^{3} \mathrm{P}^{\circ}\right)^{7} \mathrm{~F}$ \\
\hline $4 f^{7}\left({ }^{8} \mathrm{~S}^{\circ}\right) 5 d\left({ }^{7} \mathrm{D}^{\circ}\right) 6 s 6 p\left({ }^{3} \mathrm{P}^{\circ}\right)$ & & 6 & 25661.340 & 1.62 & 21 & ${ }^{9} \mathrm{D}$ & 21 & $\left({ }^{8} \mathrm{~S}^{\circ}\right)\left({ }^{7} \mathrm{D}^{\circ}\right)\left({ }^{3} \mathrm{P}^{\circ}\right){ }^{7} \mathrm{~F}$ \\
\hline $4 f^{8}\left({ }^{7} \mathrm{~F}\right) 5 d\left({ }^{8} \mathrm{D}\right) 6 s$ & ${ }^{9} \mathrm{D}$ & $\begin{array}{l}6 \\
5 \\
4 \\
3 \\
2\end{array}$ & $\begin{array}{l}25815.330 \\
26335.661 \\
27704.965 \\
28215.140 \\
28785.230\end{array}$ & $\begin{array}{l}1.55 \\
1.58 \\
1.73 \\
1.96 \\
2.31\end{array}$ & & & & \\
\hline
\end{tabular}


Gd I, Even Parity_Continued

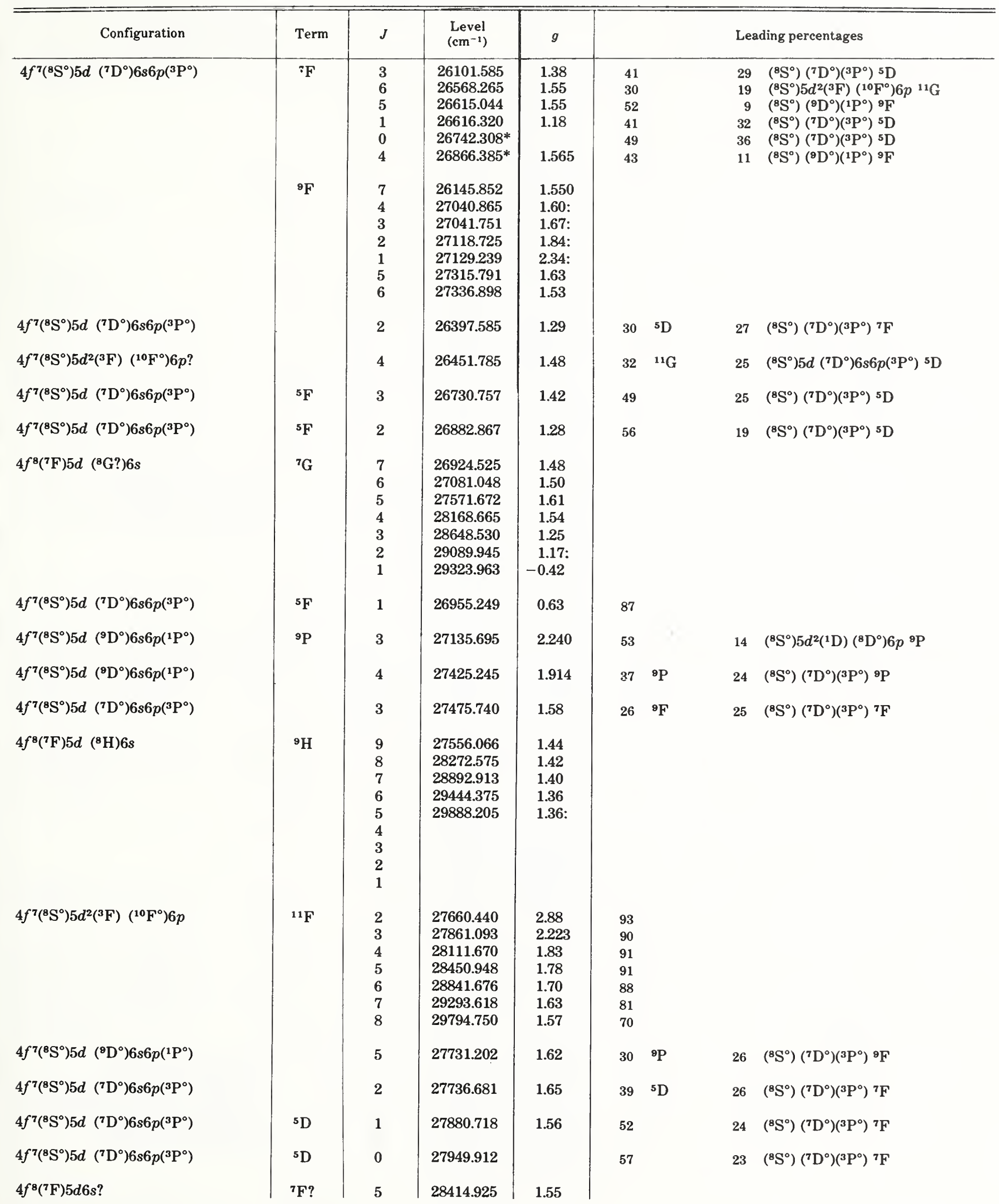


Gd I, Even Parity-Continued

\begin{tabular}{|c|c|c|c|c|c|c|}
\hline Configuration & Term & $J$ & $\begin{array}{l}\text { Level } \\
\left(\mathrm{cm}^{-1}\right)\end{array}$ & $g$ & & Leading percentages \\
\hline $4 f^{7}\left({ }^{8} \mathrm{~S}^{\circ}\right) 5 d\left({ }^{7} \mathrm{D}^{\circ}\right) 6 s 6 p\left({ }^{3} \mathrm{P}^{\circ}\right)$ & ${ }^{9} \mathrm{~F}$ ? & $\begin{array}{l}7 \\
6 \\
4 \\
5 \\
3 \\
1 \\
2\end{array}$ & $\begin{array}{l}28486.854 \\
28716.819 \\
29191.604 \\
28504.435 \\
28731.590 \\
28793.370 \\
29119.955 \\
29426.450 \\
29557.785 \\
29717.231\end{array}$ & $\begin{array}{l}1.71 \\
2.00 \\
2.74 \\
\\
1.55 \\
1.58 \\
1.60 \\
1.64 \\
1.93 \\
3.41 \\
2.24:\end{array}$ & $\begin{array}{l}64 \\
72 \\
90\end{array}$ & . \\
\hline $4 f^{7}\left({ }^{8} \mathrm{~S}^{\circ}\right) 5 d^{2}\left({ }^{3} \mathrm{~F}\right)\left({ }^{10} \mathrm{~F}^{\circ}\right) 6 p$ & ${ }^{9} \mathrm{G}$ & $\begin{array}{l}0 \\
1 \\
2 \\
3 \\
4 \\
5 \\
6 \\
7 \\
8\end{array}$ & $\begin{array}{l}28971.965 \\
28984.690 \\
29005.609 \\
29125.847 \\
29209.220^{*} \\
29628.388 \\
30007.307 \\
30505.374 \\
31171.622\end{array}$ & $\begin{array}{l}1.54: \\
1.30: \\
1.515: \\
1.603: \\
1.509 \\
1.50 \\
1.51 \\
1.50\end{array}$ & $\begin{array}{l}73 \\
73 \\
72 \\
71 \\
67 \\
66 \\
62 \\
61 \\
59\end{array}$ & \\
\hline $4 f^{8}\left({ }^{7} \mathrm{~F}\right) 5 d 6 s ?$ & ${ }^{7} \mathrm{~F}$ ? & 4 & 29141.205 & 1.49 & & \\
\hline $4 f^{7}\left({ }^{8} \mathrm{~S}^{\circ}\right) 5 d\left({ }^{7} \mathrm{D}^{\circ}\right) 6 s 6 p\left({ }^{3} \mathrm{P}^{\circ}\right) ?$ & ${ }^{7} \mathrm{P} ?$ & $\begin{array}{l}4 \\
3 \\
2\end{array}$ & $\begin{array}{l}29358.628 \\
29681.305 \\
29835.103 \\
\\
29362.593\end{array}$ & $\begin{array}{l}1.66 \\
1.86: \\
2.29 \\
1.51\end{array}$ & $\begin{array}{l}54 \\
38 \\
59\end{array}$ & 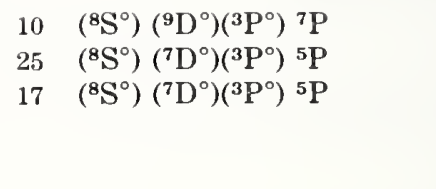 \\
\hline $4 f^{7}\left({ }^{8} \mathrm{~S}^{\circ}\right) 5 d 6 s 6 p ?$ & & 5 & $\begin{array}{l}29451.356 \\
29501.640\end{array}$ & $\begin{array}{l}1.69 \\
1.63\end{array}$ & & \\
\hline $4 f^{7}\left({ }^{8} \mathrm{~S}^{\circ}\right) 5 d^{2}\left({ }^{3} \mathrm{~F}\right)\left({ }^{10} \mathrm{~F}^{\circ}\right) 6 p$ & ${ }^{11} \mathrm{D}$ & $\begin{array}{l}3 \\
4 \\
5 \\
6 \\
7\end{array}$ & $\begin{array}{l}29631.255 \\
29876.618 \\
30242.293 \\
30652.018 \\
31146.456\end{array}$ & $\begin{array}{l}2.26 \\
2.04: \\
1.80 \\
1.78 \\
1.72\end{array}$ & $\begin{array}{l}44 \\
52 \\
67 \\
74 \\
74\end{array}$ & $\begin{array}{ll}26 & \left({ }^{8} \mathrm{~S}^{\circ}\right)\left({ }^{3} \mathrm{P}\right)\left({ }^{10} \mathrm{P}^{\circ}\right){ }^{11} \mathrm{D} \\
31 & \end{array}$ \\
\hline $4 f^{8}\left({ }^{7} \mathrm{~F}\right) 5 d 6 s ?$ & ${ }^{7} \mathrm{H} ?$ & 8 & 29754.908 & 1.39 & & \\
\hline $4 f^{7}\left({ }^{8} \mathrm{~S}^{\circ}\right) 5 d^{2} 6 p ?$ & ${ }^{11} \mathrm{D}$ ? & 3 & 29878.477 & 1.76 & & \\
\hline $4 f^{8}\left({ }^{7} \mathrm{~F}\right) 5 d 6 s ?$ & ${ }^{7} \mathrm{D} ?$ & $\begin{array}{l}5 \\
4 \\
3 \\
2 \\
1\end{array}$ & $\begin{array}{l}30197.296 \\
30834.958 \\
31327.520 \\
31549.730 \\
31602.931\end{array}$ & $\begin{array}{l}1.52 \\
1.50 \\
1.58 \\
1.71 \\
2.31\end{array}$ & & \\
\hline $4 f^{7}\left({ }^{8} \mathrm{~S}^{\circ}\right) 5 d 6 s 6 p ?$ & & 2 & $\begin{array}{l}30307.480 \\
30394.640\end{array}$ & $\begin{array}{l}1.89 \\
1.77\end{array}$ & & \\
\hline $4 f^{8}\left({ }^{7} \mathrm{~F}\right) 5 d 6 s ?$ & ${ }^{7} \mathrm{~F} ?$ & 6 & 30409.890 & 1.42 & & \\
\hline $4 f^{7}\left({ }^{8} \mathrm{~S}^{\circ}\right) 5 d^{2}\left({ }^{3} \mathrm{~F}\right)\left({ }^{10} \mathrm{~F}^{\circ}\right) 6 p$ & ${ }^{9} \mathrm{~F}$ & $\begin{array}{l}1 \\
2 \\
3 \\
4 \\
5 \\
6 \\
7\end{array}$ & $\begin{array}{l}30555.827 \\
30694.874 \\
30881.658 \\
31135.795 \\
31457.179 * \\
32091.730 \\
32729.502\end{array}$ & $\begin{array}{l}3.427 \\
2.136 \\
1.878 \\
1.685 \\
1.614 \\
1.57 \\
1.56\end{array}$ & $\begin{array}{l}62 \\
58 \\
38 \\
45 \\
42 \\
51 \\
46\end{array}$ & $\begin{array}{ll}22 & \left({ }^{8} \mathrm{~S}^{\circ}\right) 5 d\left({ }^{9} \mathrm{D}^{\circ}\right) 6 s 6 p\left({ }^{1} \mathrm{P}^{\circ}\right){ }^{9} \mathrm{~F} \\
17 & \left({ }^{8} \mathrm{~S}^{\circ}\right)\left({ }^{3} \mathrm{P}\right)\left({ }^{10} \mathrm{P}^{\circ}\right){ }^{11} \mathrm{D} \\
13 & \left({ }^{8} \mathrm{~S}^{\circ}\right) 5 d\left({ }^{9} \mathrm{D}^{\circ}\right) 6 s 6 p\left({ }^{1} \mathrm{P}^{\circ}\right){ }^{9} \mathrm{~F} \\
15 & \left({ }^{8} \mathrm{~S}^{\circ}\right) 5 d\left({ }^{9} \mathrm{D}^{\circ}\right) 6 s 6 p\left({ }^{1} \mathrm{P}^{\circ}\right){ }^{9} \mathrm{~F} \\
15 & \left({ }^{8} \mathrm{~S}^{\circ}\right) 5 d\left({ }^{9} \mathrm{D}^{\circ}\right) 6 s 6 p\left({ }^{1} \mathrm{P}^{\circ}\right){ }^{9} \mathrm{~F} \\
20 & \left({ }^{8} \mathrm{~S}^{\circ}\right)\left({ }^{\circ} \mathrm{P}\right)\left({ }^{10} \mathrm{P}^{\circ}\right){ }^{11} \mathrm{D}\end{array}$ \\
\hline $4 f^{8}\left({ }^{7} \mathrm{~F}\right) 5 d 6 \mathrm{~s} ?$ & ${ }^{5} \mathrm{~F} ?$ & 5 & 30629.495 & 1.44 & & \\
\hline
\end{tabular}


Gd I, Even Parity_Continued

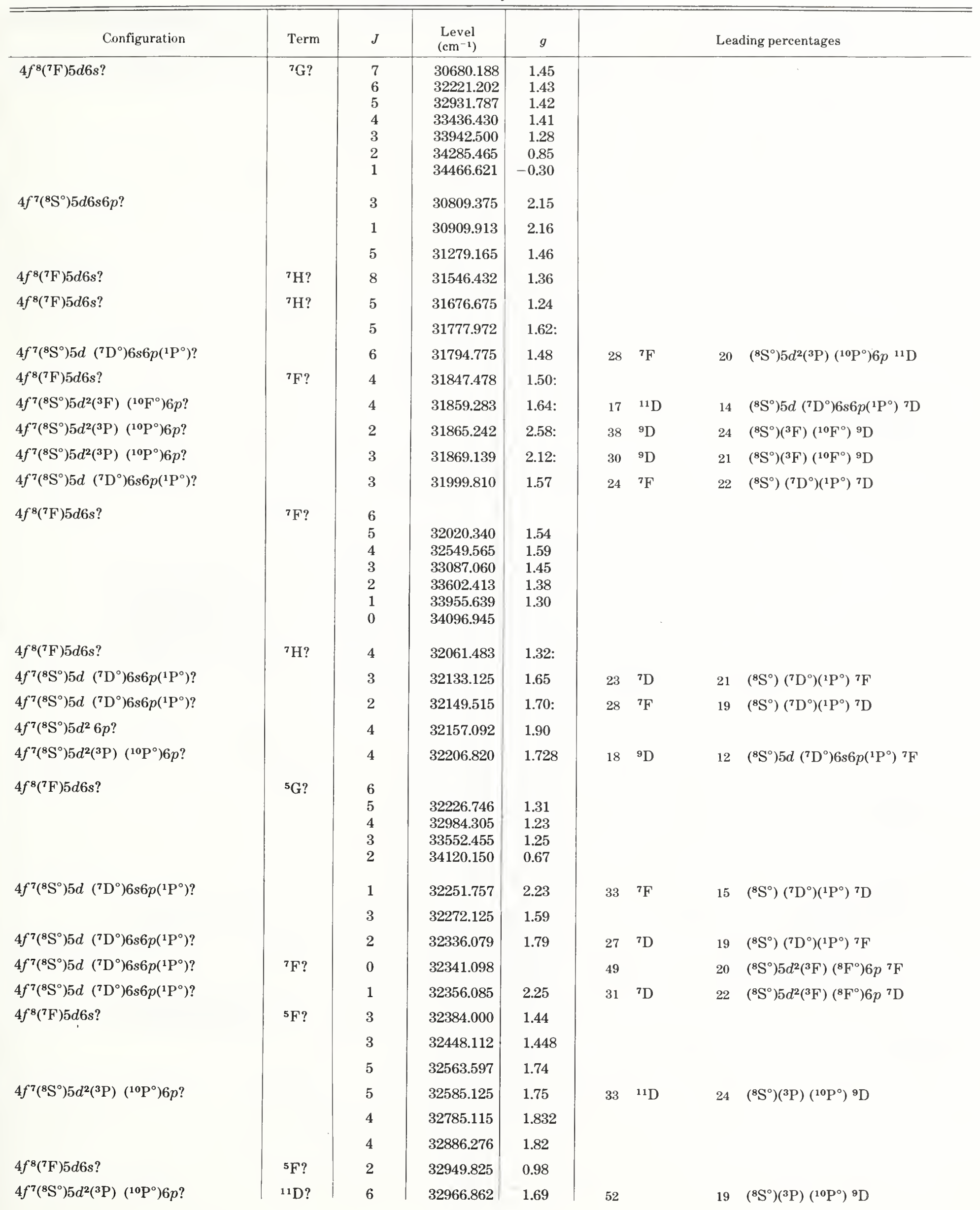


Gd I, Even Parity-Continued

\begin{tabular}{|c|c|c|c|c|c|c|}
\hline Configuration & Term & $J$ & $\begin{array}{l}\text { Level } \\
\left(\mathrm{cm}^{-1}\right)\end{array}$ & $g$ & & Leading percentages \\
\hline $4 f^{8}\left({ }^{7} \mathrm{~F}\right) 5 d 6 s ?$ & ${ }^{7} \mathrm{H} ?$ & 6 & 33013.830 & 1.26 & & \\
\hline \multirow[t]{12}{*}{$4 f^{7}\left({ }^{8} \mathrm{~S}^{\circ}\right) 5 d^{2}\left({ }^{3} \mathrm{P}\right)\left({ }^{10} \mathrm{P}^{\circ}\right) 6 p ?$} & \multirow[t]{12}{*}{${ }^{11} \mathrm{~S} ?$} & 5 & 33231.096 & 1.95 & \multirow[t]{16}{*}{84} & \\
\hline & & 5 & 33348.226 & 1.81 & & \\
\hline & & 4 & 33568.045 & 1.88 & & \\
\hline & & 3 & 33704.627 & 1.75 & & \\
\hline & & 4 & 33724.517 & 1.51 & & \\
\hline & & 5 & 33732.275 & 1.47 & & \\
\hline & & 6 & 33851.828 & 1.73 & & \\
\hline & & 5 & 33923.459 & 1.97 & & \\
\hline & & 2 & 34147.343 & 2.119 & & \\
\hline & & 7 & 34175.835 & 1.68 & & \\
\hline & & 4 & 34458.220 & 2.13: & & \\
\hline & & 3 & 34465.872 & 2.23: & & \\
\hline $4 f^{7}\left({ }^{8} \mathrm{~S}^{\circ}\right) 5 d^{2} 6 p ?$ & ${ }^{11} \mathrm{P} ?$ & 5 & 34517.671 & 1.95 & & \\
\hline \multirow[t]{3}{*}{$4 f^{8}\left({ }^{7} \mathrm{~F}\right) 5 d 6 s ?$} & \multirow[t]{3}{*}{${ }^{5} \mathrm{H}$ ? } & 4 & 34524.661 & 0.97 & & \\
\hline & & 4 & 34635.520 & 1.73 & & \\
\hline & & 4 & 34698.190 & 1.53 & & \\
\hline \multirow[t]{13}{*}{$4 f^{7}\left({ }^{8} \mathrm{~S}^{\circ}\right) 5 d^{2}\left({ }^{3} \mathrm{~F}\right)\left({ }^{8} \mathrm{~F}^{\circ}\right) 6 p ?$} & \multirow[t]{13}{*}{${ }^{9} \mathrm{G} ?$} & 0 & 34704.810 & & 67 & \\
\hline & & 1 & 34711.445 & 1.47: & 65 & \\
\hline & & 2 & 34722.925 & 1.48: & 60 & \\
\hline & & 3 & 34765.635 & 1.48: & 52 & $14 \quad\left({ }^{8} \mathrm{~S}^{\circ}\right)\left({ }^{1} \mathrm{G}\right)\left({ }^{8} \mathrm{G}^{\circ}\right)^{9} \mathrm{G}$ \\
\hline & & 4 & 34836.980 & 1.47: & 44 & $14 \quad\left({ }^{8} S^{\circ}\right)\left({ }^{1} G\right)\left({ }^{8} G^{0}\right){ }^{9} G$ \\
\hline & & 5 & 34951.016 & 1.47 & 36 & $16 \quad\left({ }^{8} S^{\circ}\right)\left({ }^{3} F\right)\left({ }^{8} F^{\circ}\right){ }^{7} G$ \\
\hline & & 6 & 35149.140 & 1.46 & 27 & $25 \quad\left({ }^{8} S^{\circ}\right)\left({ }^{3} F\right)\left({ }^{8} F^{\circ}\right){ }^{7} G$ \\
\hline & & 7 & 36482.515 & 1.48 & 45 & $33 \quad\left({ }^{8} S^{\circ}\right)\left({ }^{3} F\right)\left({ }^{8} F^{\circ}\right)^{7} G$ \\
\hline & & 8 & 36731.590 & 1.49 & 69 & \\
\hline & & 3 & 34913.620 & 1.66 & & \\
\hline & & 6 & 34938.434 & 1.80 & & \\
\hline & & 2 & 35342.327 & 2.65 & & \\
\hline & & 3 & 35360.405 & 1.89 & & \\
\hline $4 f^{7}\left({ }^{8} \mathrm{~S}^{\circ}\right) 5 d^{2} 6 p ?$ & & 3 & 35442.715 & 1.94 & & \\
\hline \multirow[t]{2}{*}{$4 f^{8}\left({ }^{7} F\right) 5 d 6 s ?$} & \multirow[t]{3}{*}{${ }^{7} \mathbf{P} ?$} & 4 & 35498.790 & 1.68 & & \\
\hline & & 3 & 35553.915 & 1.46: & & \\
\hline $4 f^{7}\left({ }^{8} \mathrm{~S}^{\circ}\right) 5 d^{2} 6 p ?$ & & 4 & 35566.145 & 1.84 & & \\
\hline \multirow[t]{7}{*}{$4 f^{7}\left({ }^{8} \mathrm{~S}^{\circ}\right) 5 d^{2}\left({ }^{3} \mathrm{~F}\right)\left({ }^{8} \mathrm{~F}^{\circ}\right) 6 p ?$} & \multirow[t]{7}{*}{${ }^{7} \mathrm{G} ?$} & 7 & 35579.553 & 1.47 & 39 & $19 \quad\left({ }^{8} S^{\circ}\right)\left({ }^{3} F\right)\left({ }^{8} F^{\circ}\right){ }^{9} G$ \\
\hline & & 1 & 35744.507 & $-0.42:$ & 84 & \\
\hline & & 2 & 35766.831 & $0.85:$ & 78 & \\
\hline & & 3 & 35811.565 & 1.22: & 71 & \\
\hline & & 4 & 35883.613 & 1.35 & 65 & \\
\hline & & 5 & 36000.475 & 1.42 & 54 & $24 \quad\left({ }^{8} \mathrm{~S}^{\circ}\right)\left({ }^{3} \mathrm{~F}\right)\left({ }^{8} \mathrm{~F}^{\circ}\right)^{9} \mathrm{G}$ \\
\hline & & 6 & 36203.224 & 1.55 & 42 & $34 \quad\left({ }^{8} \mathrm{~S}^{\circ}\right)\left({ }^{3} \mathrm{~F}\right)\left({ }^{8} \mathrm{~F}^{\circ}\right){ }^{9} \mathrm{G}$ \\
\hline \multirow[t]{3}{*}{$4 f^{8}\left({ }^{7} \mathrm{~F}\right) 5 d 6 s ?$} & \multirow[t]{3}{*}{${ }^{7} \mathrm{H} ?$} & 3 & 35593.010 & 1.03 & & \\
\hline & & 5 & 35794.634 & 1.72 & & \\
\hline & & 1 & 35908.957 & 2.71 & & \\
\hline
\end{tabular}


Gd I, Even Parity_Continued

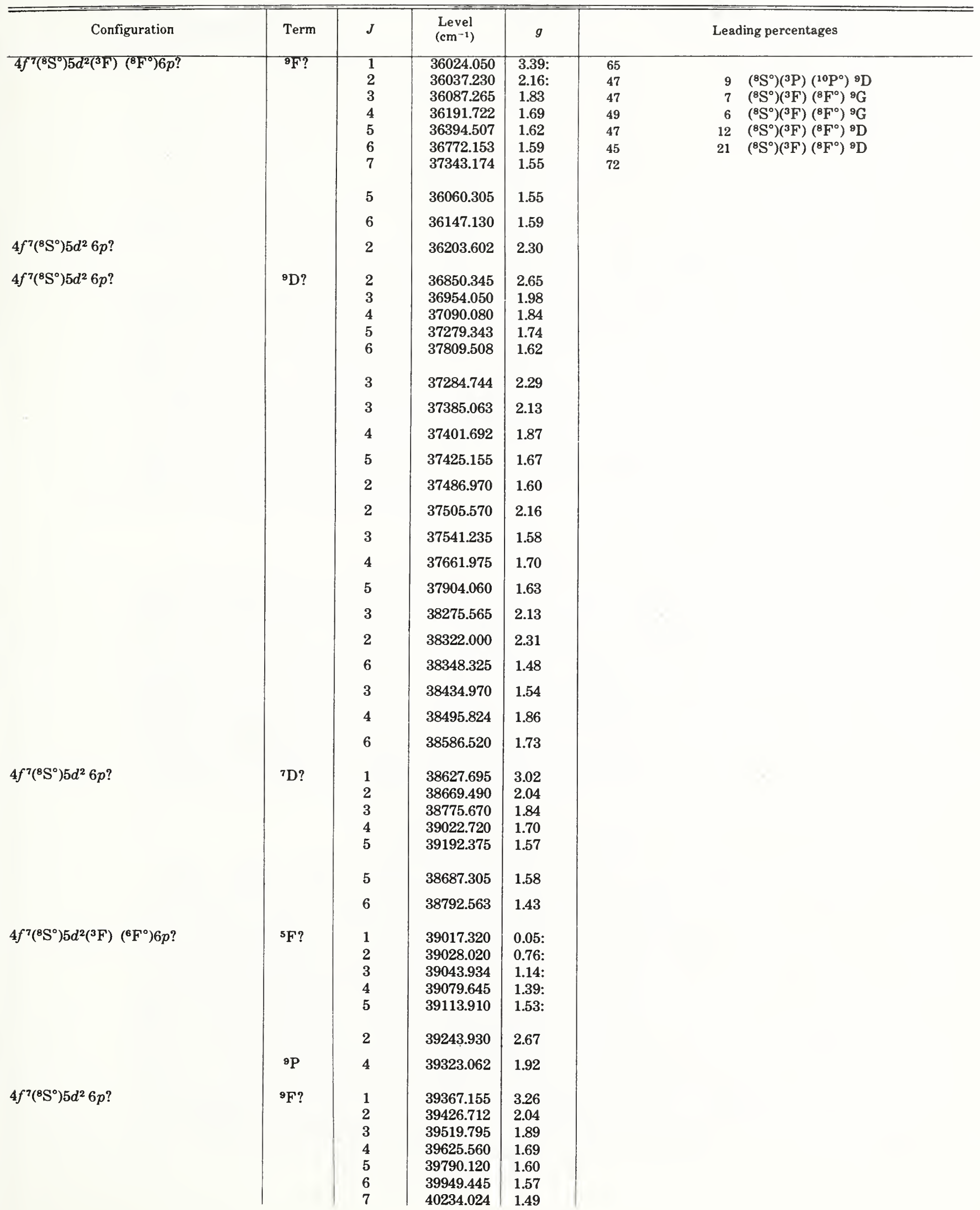


Gd I, Even Parity_Continued

\begin{tabular}{|c|c|c|c|c|c|}
\hline Configuration & Term & $J$ & $\begin{array}{c}\text { Level } \\
\left(\mathrm{cm}^{-1}\right)\end{array}$ & $g$ & Leading percentages \\
\hline \multirow{14}{*}{$4 f^{7}\left({ }^{8} \mathrm{~S}^{\circ}\right) 5 d^{2} 6 p ?$} & \multirow{14}{*}{${ }^{9} \mathrm{H} ?$} & 3 & 39404.167 & 2.00 & \\
\hline & & 5 & 39593.830 & 1.72 & \\
\hline & & 7 & 39605.305 & 1.54 & \\
\hline & & 6 & 39608.405 & 1.65 & \\
\hline & & 3 & 39668.382 & 1.94 & \\
\hline & & 4 & 39819.045 & 1.68 & \\
\hline & & 4 & 39938.046 & 1.63: & \\
\hline & & 5 & 39971.374 & 1.54 & \\
\hline & & 8 & 40127.198 & 1.45 & \\
\hline & & 6 & 40160.870 & 1.49 & \\
\hline & & 5 & 40200.540 & 1.64: & \\
\hline & & 5 & 40330.415 & 1.44 & \\
\hline & & 6 & 40382.555 & 1.54 & \\
\hline & & 4 & 40391.540 & 1.47 & \\
\hline \multirow[t]{25}{*}{$4 f^{8}\left({ }^{7} \mathrm{~F}\right) 6 s \quad\left({ }^{8} \mathrm{~F}\right) 7 s$} & \multirow[t]{25}{*}{${ }^{9} \mathrm{~F}$} & 7 & 40439.513 & 1.55 & \\
\hline & & $\begin{array}{l}6 \\
5\end{array}$ & 41069.210 & 1.52 & \\
\hline & & $\begin{array}{l}4 \\
3\end{array}$ & & & \\
\hline & & $\begin{array}{l}2 \\
1\end{array}$ & & & \\
\hline & & 9 & 40450.605 & 1.44 & \\
\hline & & 5 & 40463.758 & 1.59 & \\
\hline & & 4 & 40487.655 & 1.72 & \\
\hline & & 4 & 40554.495 & 1.21: & \\
\hline & & 6 & 40632.680 & 1.60 & \\
\hline & & 3 & 40684.910 & 1.34 & \\
\hline & & 5 & 40707.970 & 1.70 & \\
\hline & & 2 & 40786.235 & 2.32: & \\
\hline & & 3 & 40803.185 & 1.90 & \\
\hline & & 5 & 40865.900 & 1.41 & \\
\hline & & 4 & 40963.685 & 1.89 & \\
\hline & & 7 & 41097.255 & 1.47 & \\
\hline & & 4 & 41127.460 & 1.55 & \\
\hline & & 5 & 41150.915 & 1.57 & \\
\hline & & 1 & 41231.060 & 1.63 & \\
\hline & & 5 & 41283.160 & 1.38 & \\
\hline & & 4 & 41296.165 & 1.75 & \\
\hline & & 8 & 41461.330 & 1.41 & \\
\hline & & 6 & 41624.405 & 1.40 & \\
\hline & & 5 & 41665.212 & 1.46 & \\
\hline & & 5 & 41692.155 & 1.89 & \\
\hline $\mathrm{Gd}$ II $\left({ }^{10} \mathrm{D}_{5 / 2}^{\circ}\right)$ & Limit & & 49603 & & \\
\hline
\end{tabular}


Gd II

(Eu I sequence; 63 electrons)

$Z=64$

Ground state $\left(1 s^{2} 2 s^{2} 2 p^{6} 3 s^{2} 3 p^{6} 3 d^{10} 4 s^{2} 4 p^{6} 4 d^{10} 5 s^{2} 5 p^{6}\right) 4 f^{7} 5 d 6 s^{10} \mathrm{D}_{5 / 2}^{\circ}$

Ionization energy $97500 \pm 600 \mathrm{~cm}^{-1}$

$12.09 \pm 0.08 \mathrm{eV}$

Identified odd configurations $\quad 4 f^{7} 5 d 6 s, 4 f^{7} 6 s^{2}, 4 f^{7} 5 d^{2}, 4 f^{8} 6 p$

Identified even configurations $\quad 4 f^{8} 6 s, 4 f^{8} 5 d, 4 f^{7} 6 s 6 p, 4 f^{7} 5 d 6 p$

The original analysis of Gd II by Albertson, Bruynes, and Hanau yielded the $4 f^{7}\left({ }^{8} \mathrm{~S}^{\circ}\right) 5 d 6 s{ }^{10} \mathrm{D}^{\circ}$ ground term, the $\left({ }^{8} \mathrm{~S}^{\circ}\right) 5 d 6 p{ }^{10} \mathrm{~F}$ term (thus accounting for the fundamental multiplet of the spectrum), and a few other low odd and high even levels. Using King's list of more than $2600 \mathrm{Gd}$ II lines, Russell [1950] greatly extended the system based on the $4 f^{7}\left({ }^{8} \mathrm{~S}^{\circ}\right)$ core to include 137 levels classifying 1177 lines. Blaise and van Kleef [1969], Spector [1970a, 1970b], and Blaise, van Kleef, and Wyart [1971] have recently extended the analysis further and identified levels based on the $4 f^{8}$ core configuration.

More than 300 levels are now known, about equally divided between the two parities. The values here are from Blaise, van Kleef, and Wyart, who found more than half of these levels.

The principal line lists are described in the first section of the text for Gd I. More than $2200 \mathrm{Gd}$ II lines are now classified, but the most complete description of the spectrum has not been published [see Hoekstra and Slooten, 1971].

\section{$g$ Values}

Blaise, van Kleef, and Wyart also derived $g$ values for all the known Gd II levels, using Zeeman spectrograms (2700-11400 $\AA$ ) photographed at Argonne by Noorman and by M. Fred. Blaise et al. gave values rounded off to the nearest 0.005 , and most of the two-place values given here result from a further rounding-off of these values. This allows a distinction between such values and the three-place values obtained from weighted averages of the values of Desmarais and Pinnington [1967] and of Blaise, van Kleef, and Wyart. Desmarais and Pinnington derived $g$ values for 67 levels of Gd II, with estimated errors from \pm 0.001 to \pm 0.02 . The agreement between the two sets of observations is in general very good. About 30 of the two-place $g$ values given here also result from averages.

Some odd levels gave Zeeman patterns with significant Paschen-Back effects [Blaise, 1976]. The $g$ values of these levels are followed by colons.

\section{Calculations of the Odd Levels}

Zeldes [1953] noted that his calculations of the $\left(4 f^{7}\left({ }^{8} \mathrm{~S}^{\circ}\right) 5 d 6 s+\left({ }^{8} \mathrm{~S}^{\circ}\right) 5 d^{2}\right)$ odd subconfigurations and the $\left(4 f^{7}\left({ }^{8} \mathrm{~S}^{\circ}\right) 6 s 6 p+\left({ }^{8} \mathrm{~S}^{\circ}\right) 5 d 6 p\right)$ even subconfigurations could be improved by including the effects of intermediate coupling. Smith and Wybourne [1965], Goldschmidt and Nir [1965, 1971], and Wyart [1973] have since carried out such calculations.

The percentages listed here for levels of the low odd group are from Wyart's calculation of $\left(4 f^{7}\left({ }^{8} \mathrm{~S}^{\circ},{ }^{6} \mathrm{P}^{\circ}\right) 6 s^{2}+4 f^{7}\left({ }^{8} \mathrm{~S}^{\circ},{ }^{6} \mathrm{P}^{\circ}\right) 5 d 6 s+4 f^{7}\left({ }^{8} \mathrm{~S}^{\circ},{ }^{6} \mathrm{P}^{\circ}\right) 5 d^{2}\right)$. Although the ${ }^{6} \mathrm{P}^{\circ}$ term of the $4 f^{7}$ core configuration is very high, Wyart's inclusion of this core term significantly affected the eigenvectors for the levels nominally based on the $4 f^{7}\left({ }^{8} \mathrm{~S}^{\circ}\right)$ core. Wyart did not list second components for the eigenvectors, but gave total percentages from the three configurations; instead of second components we list the total percentage from a second configuration wherever a second configuration contributes most of the remaining eigenvector percentage (after the leading component). For several of the lowest terms the configuration interaction has a regularity expected for $L S$ coupling. For example, practically all of the $\sim 20 \%$ mixture of $4 f^{7} 5 d^{2}$ into the eigenvectors of the nominal $4 f^{7}\left({ }^{8} \mathrm{~S}^{\circ}\right) 5 d\left({ }^{7} \mathrm{D}^{\circ}\right) 6 s^{8} \mathrm{D}^{\circ}$ levels is due to 
$4 f^{7}\left({ }^{8} \mathrm{~S}^{\circ}\right) 5 d^{2}{ }^{8} \mathrm{D}^{\circ}$ [Smith and Wybourne, 1965; Goldschmidt and Nir, 1965]. On the other hand, the combined effects of this configuration interaction and intermediate coupling make it impossible to assemble a physically meaningful $4 f^{7}\left({ }^{8} \mathrm{~S}^{\circ}\right) 5 d^{2}{ }^{8} \mathrm{D}^{\circ}$ term from the calculated eigenvectors. Smith and Wybourne [1965] and Goldschmidt and Nir [1965] assigned several previously unidentified levels to $4 f^{7}\left({ }^{8} \mathrm{~S}^{\circ}\right) 5 d^{2}$, and the latter authors also found several new levels of this subconfiguration. Blaise, van Kleef, and Wyart [1971] found and assigned several of the highest $\left({ }^{8} \mathrm{~S}^{\circ}\right) 5 d^{2}$ levels.

A large group of uninterpreted odd levels begins only a little above the $4 f^{8}\left({ }^{7} \mathrm{~F}_{6}\right) 6 p_{1 / 2}$ pair $\left(\sim 32600 \mathrm{~cm}^{-1}\right)$, which are the lowest levels of this subconfiguration. However, most of the 37 expected levels of $4 f^{8}\left({ }^{7} \mathrm{~F}\right) 6 p$ have been found and unambiguously identified, even though the calculations included only this subconfiguration [Blaise and van Kleef, 1969; Spector, 1970a; Blaise, van Kleef, and Wyart, 1971]. The eigenvector percentages given here are from Blaise, van Kleef, and Wyart. A few of the $\left.4 f^{8}{ }^{7} \mathrm{~F}\right) 6 p$ assignments probably should not be regarded as definite until more complete calculations of the odd configurations in this region are made; three levels for which the agreement between experimental and calculated $g$ values is relatively poor are followed by asterisks. The designation of the level at $36565.69 \mathrm{~cm}^{-1}(J=9 / 2)$ as the upper level of the $4 f^{8}\left({ }^{7} \mathrm{~F}_{6}\right) 6 p_{3 / 2}$ term is also given as tentative, since Ahmad, Saksena, and Venugopalan [1976] find the isotope shift of this level to be more consistent with assignment to the $4 f^{7} 5 d 6 s$ configuration.

Spector [1970a] found that the $J_{1} j$ coupling scheme is best for the $4 f^{8}\left({ }^{7} \mathrm{~F}\right) 6 p$ configuration. He discussed the coupling in other Gd II configurations and noted that in some cases the spins of the parent terms are good quantum numbers to an unexpected degree. This explained, for example, the failure to observe any lines of the nominal $4 f^{7}\left({ }^{8} \mathrm{~S}^{\circ}\right) 5 d\left({ }^{9} \mathrm{D}^{\circ}\right) 6 s^{8} \mathrm{D}^{\circ}-4 f^{8}\left({ }^{7} \mathrm{~F}\right) 6 s^{8} \mathrm{~F}$ multiplet (in the near-infrared spectrum); the multiplet is spin forbidden because of the high purities of the respective spins of the ${ }^{9} \mathrm{D}^{\circ}$ and ${ }^{7} \mathrm{~F}$ parent terms.

\section{Calculations of the Even Levels}

The low even levels belong to the $4 f^{8}\left({ }^{7} \mathrm{~F}\right) 6 \mathrm{~s}$ and $4 f^{8}\left({ }^{7} \mathrm{~F}\right) 5 d$ subconfigurations, both of which are completely known. Spector's calculation [1970b] has been used for the $4 f^{8}\left({ }^{7} \mathrm{~F}\right) 5 d$ level percentages. The experimental $g$ values of the levels designated ${ }^{8} \mathrm{D}_{3 / 2}$ and ${ }^{8} \mathrm{~F}_{3 / 2}$ differ by only 0.05 , as compared with the calculated $g$ values of 2.57 and 2.11 , respectively. Thus the experimental $g$ values indicate a stronger mixing of ${ }^{8} \mathrm{D}$ and ${ }^{8} \mathrm{~F}$ for $J=3 / 2$ than the calculated eigenvectors show, and the assigned designations probably mean very little; in fact, the names assigned by Blaise and van Kleef [1969] are reversed from those indicated by the eigenvectors. Similar comments apply to the ${ }^{8} \mathrm{D}_{5 / 2},{ }^{8} \mathrm{~F}_{5 / 2}$ pair of levels.

The leading percentages for the levels of $\left(4 f^{7}\left({ }^{8} \mathrm{~S}^{\circ}\right) 5 d 6 p+\left({ }^{8} \mathrm{~S}^{\circ}\right) 6 s 6 p\right)$ are from Goldschmidt and Nir [1965, 1971]. Their calculations and those of Smith and Wybourne showed that previous term assignments for several $\left({ }^{8} \mathrm{~S}^{\circ}\right) 5 d 6 p$ levels were inappropriate. Zeeman-effect measurements by Desmarais and Pinnington [1967] confirmed these results and also indicated that some additional interchanges of designations should be made. Wyart's [1973] calculation of this group included the effects of the $4 f^{7}\left({ }^{6} \mathrm{P}^{\circ}\right)$ core term, but the eigenvectors are not much different from those of Goldschmidt and Nir. The assignments appear to be unambiguous except for two pairs of levels having $J=7 / 2$ (32263 and $32490 \mathrm{~cm}^{-1}$ ) and $J=9 / 2$ (39251 and 39537 $\mathrm{cm}^{-1}$ ); these levels are followed by an asterisk to indicate the possibility of interchanges of their compositions.

We have omitted any names for a number of levels of this group. The levels at $29198 \mathrm{~cm}^{-1}$ $(\bar{J}=5 / 2)$ and $29242 \mathrm{~cm}^{-1}(J=7 / 2)$, for example, have usually been designated $\left.4 f^{7}{ }^{7} \mathrm{~S}^{\circ}\right) 6 s 6 p\left({ }^{3} \mathrm{P}^{\circ}\right)^{8} \mathrm{P}$, although both levels have somewhat larger components of $\left({ }^{8} \mathrm{~S}^{\circ}\right) 5 d\left({ }^{9} \mathrm{D}^{\circ}\right) 6 p{ }^{10} \mathrm{D}$ and belong about equally to $4 f^{7} 6 s 6 p$ and $4 f^{7} 5 d 6 p$. The leading percentages for the corresponding two levels assigned to the ${ }^{10} \mathrm{D}$ term $(J=5 / 2$ and $J=7 / 2)$ are also only $\sim 40 \%$; the retention of these two not very meaningful names to complete this term is perhaps justified by the positions of the levels and the fact that they do belong mainly to $4 f^{7} 5 d 6 p$.

Goldschmidt and Nir [1971] discuss in some detail the coupling in the configurations based on the $4 f^{7}\left({ }^{8} \mathrm{~S}^{\circ}\right)$ core, including the effects of the interaction between $4 f^{7}\left({ }^{8} \mathrm{~S}^{\circ}\right) 5 d 6 p$ and $\left({ }^{8} \mathrm{~S}^{\circ}\right) 6 s 6 p$ on the coupling in these subconfigurations.

\section{Ionization Energy}

Sugar and Reader derived the quoted value for the ionization energy. 


\section{References}

Ahmad, S. A., Saksena, G. D., and Venugopalan, A., Physica (Utrecht) 81C, 366 (1976). ND CL IS

Albertson, W. E., Bruynes, H., and Hanau, R., Phys. Rev. 57, 292 (1940). EL CL ZE

Blaise, J., private communication (1976). ZE

Blaise, J., Chevillard, J., Verges, J., Wyart, J. F., and van Kleef, T. A. M., Spectrochim. Acta, Part B 26, 1 (1971). EL CL W ZE

Blaise, J., and van Kleef, T. A. M., C. R. Acad. Sci., Ser. B 268, 792 (1969). E L ZE

Blaise, J., van Kleef, T. A. M., and Wyart, J. F., J. Phys. (Paris) 32, 617 (1971). EL ZE PT

Brix, P., Z. Phys. 132, 579 (1952). ND IS

Brix, P., and Lindenberger, K. H., Z. Phys. 141, 1 (1955). Hfs IS

Desmarais, D., and Pinnington, E. H., J. Opt. Soc. Am. 57, 1245 (1967); 59, 1391 (1969). ND ZE

Goldschmidt, Z. B., and Nir, S., unpublished material (1965). ND PT

Goldschmidt, Z. B., and Nir, S., Physica (Utrecht) 51, 222 (1971). ND PT

Hoekstra, R., and Slooten, R., Spectrochim. Acta, Part B 26, 341 (1971). Wavelength Accuracy

King, A. S., Astrophys. J. 97, 323 (1943). W

Kopfermann, H., Krüger, L., and Steudel, A., Ann. Phys. (Leipzig) [61] 20, 258 (1957). IS

Meggers, W. F., Corliss, C. H., and Scribner, B. F., Nat. Bur. Stand. (U.S.), Monogr. 145, Part I, 403 pp. (1975). CL

Russell, H. N., J. Opt. Soc. Am. 40, 550 (1950). EL CL IP

Smith, G., and Wybourne, B. G., J. Opt. Soc. Am. 55, 1278 (1965). ND PT

Spector, N., J. Opt. Soc. Am. 59, 488A (1969). EL

Spector, N., J. Opt. Soc. Am. 60, 763 (1970a). EL CL PT

Spector, N., J. Phys. (Paris) Colloq. C 4 32, 173 (1970b). EL CL PT

Spector, N., Astrophys. J. 159, 1091 (1970c). CL

Spector, N., and Held, S., A strophys. J. 159, 1079 (1970). W

Sugar, J., and Reader, J., J. Chem. Phys. 59, 2083 (1973). IP

Wyart, J. F., C. R. Acad. Sci., Ser. B 271, 849 (1970). PT

Wyart, J. F., Thesis, Univ. Paris-Sud, Orsay, 194 pp. (1973). PT

Zeldes, N., Phys. Rev. 90, 413 (1953). ND PT

[July 1976]

Gd II, Odd Parity

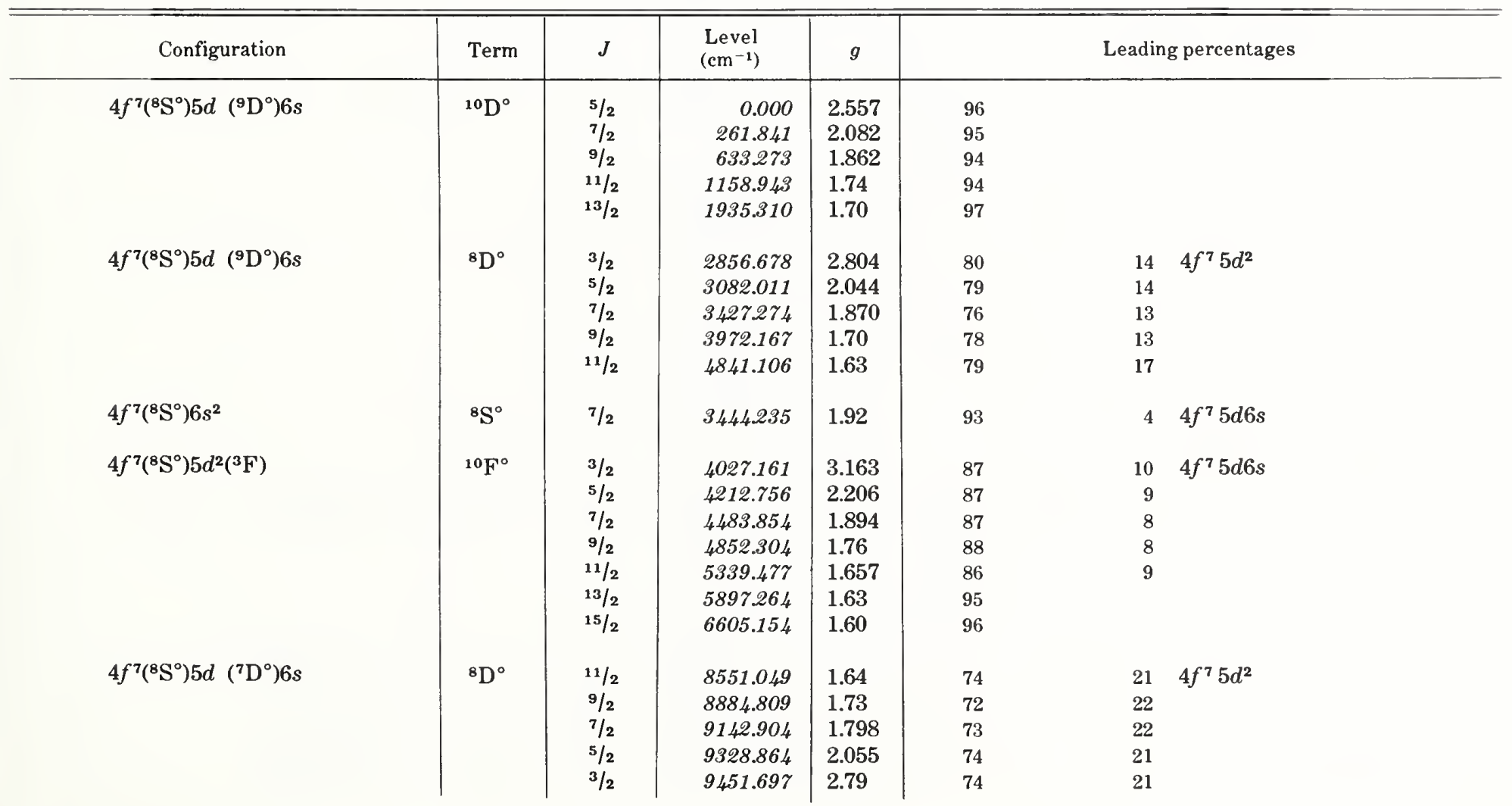


Gd II, Odd Parity-Continued

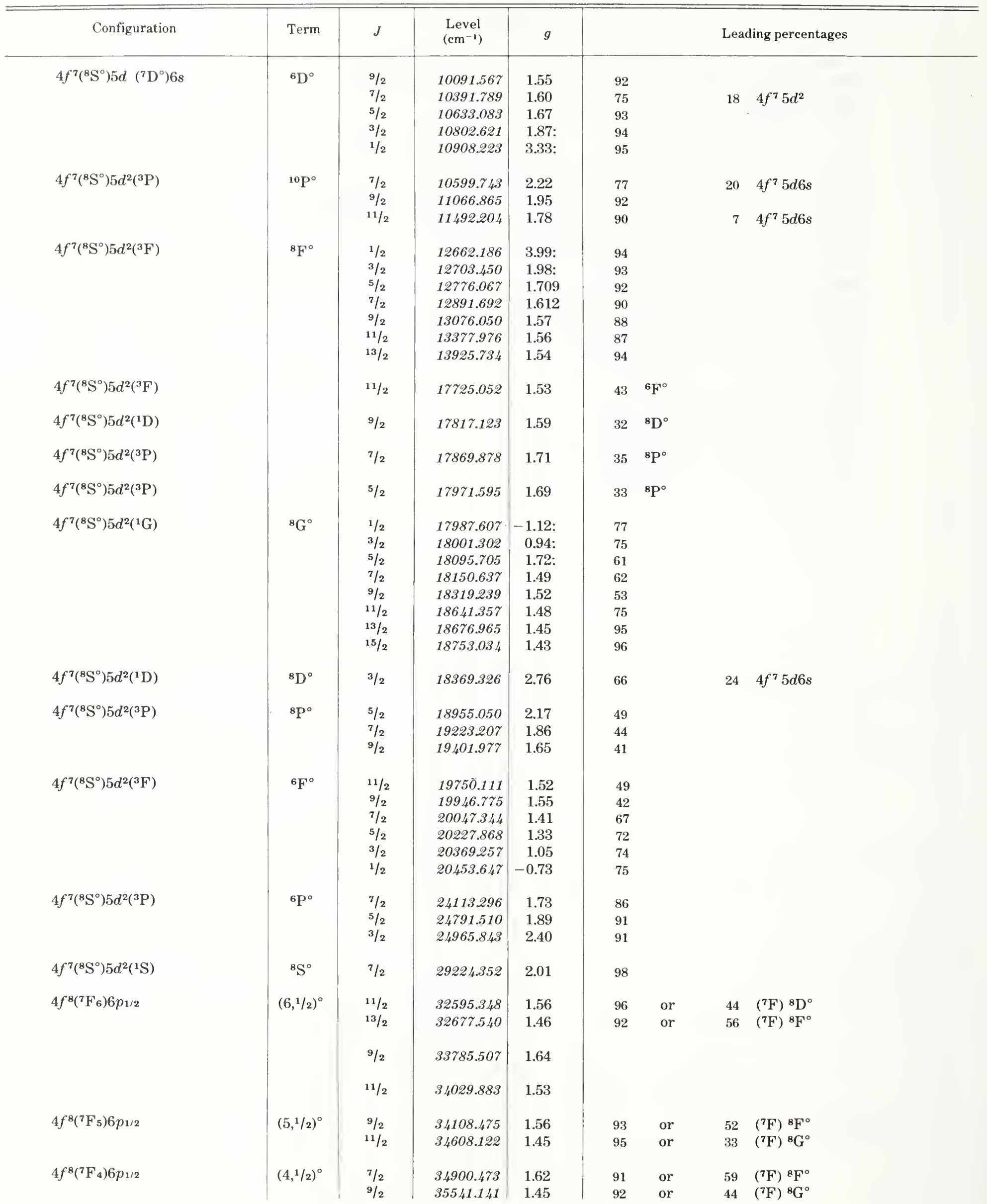


Gd II, Odd Parity_Continued

\begin{tabular}{|c|c|c|c|c|c|c|c|c|}
\hline Configuration & Term & $J$ & $\begin{array}{l}\text { Level } \\
\left(\mathrm{cm}^{-1}\right)\end{array}$ & $g$ & & & Leadi & ng percentages \\
\hline $4 f^{8}\left({ }^{7} \mathrm{~F}_{6}\right) 6 p_{3 / 2}$ & $(6,3 / 2)^{\circ}$ & $\begin{array}{l}13 / 2 \\
15 / 2 \\
11 / 2 \\
9 / 2 \\
3 / 2 \\
13 / 2 \\
5 / 2 \\
9 / 2\end{array}$ & $\begin{array}{l}35111.830 \\
35272.546 \\
35605.266 \\
36565.693^{*} \\
35298.183 \\
35362.630 \\
35404.833 \\
35822.697\end{array}$ & $\begin{array}{l}1.48 \\
1.47 \\
1.50 \\
1.58 \\
\\
2.67 \\
\\
1.50 \\
1.92 \\
\\
1.47\end{array}$ & $\begin{array}{r}88 \\
100 \\
99 \\
90\end{array}$ & $\begin{array}{l}\text { or } \\
\text { or } \\
\text { or } \\
\text { or }\end{array}$ & $\begin{array}{r}48 \\
100 \\
64 \\
87\end{array}$ & $\begin{array}{l}\left({ }^{7} \mathrm{~F}\right){ }^{8} \mathrm{G}^{\circ} \\
\left({ }^{7} \mathrm{~F}\right){ }^{8} \mathrm{G}^{\circ} \\
\left({ }^{7} \mathrm{~F}\right){ }^{6} \mathrm{~F}^{\circ} \\
\left({ }^{7} \mathrm{~F}\right){ }^{6} \mathrm{D}^{\circ}\end{array}$ \\
\hline $4 f^{8}\left({ }^{7} \mathrm{~F}_{3}\right) 6 p_{1 / 2}$ & $(3,1 / 2)^{\circ}$ & $\begin{array}{l}5 / 2 \\
7 / 2 \\
7 / 2 \\
5 / 2\end{array}$ & $\begin{array}{l}35938.545 \\
36398.500^{*} \\
35991.374 \\
36144.683\end{array}$ & $\begin{array}{l}1.62 \\
1.50 \\
\\
1.55 \\
\\
1.75\end{array}$ & $\begin{array}{l}93 \\
82\end{array}$ & $\begin{array}{l}\text { or } \\
\text { or }\end{array}$ & $\begin{array}{l}62 \\
50\end{array}$ & $\begin{array}{l}\left({ }^{7} \mathrm{~F}\right){ }^{8} \mathrm{~F}^{\circ} \\
\left({ }^{7} \mathrm{~F}\right)^{8} \mathrm{G}^{\circ}\end{array}$ \\
\hline $4 f^{8}\left({ }^{7} \mathrm{~F}_{2}\right) 6 p_{1 / 2}$ & $(2,1 / 2)^{\circ}$ & $\begin{array}{l}3 / 2 \\
5 / 2 \\
7 / 2\end{array}$ & $\begin{array}{l}36373.530 \\
36819.318 \\
36632.006\end{array}$ & $\begin{array}{l}1.78 \\
1.39 \\
1.58\end{array}$ & $\begin{array}{l}89 \\
99\end{array}$ & $\begin{array}{l}\text { or } \\
\text { or }\end{array}$ & $\begin{array}{l}64 \\
56\end{array}$ & $\begin{array}{l}\left({ }^{7} \mathrm{~F}\right){ }^{8} \mathrm{~F}^{\circ} \\
\left({ }^{7} \mathrm{~F}\right){ }^{8} \mathrm{G}^{\circ}\end{array}$ \\
\hline $4 f^{8}\left({ }^{7} \mathrm{~F}_{1}\right) 6 p_{1 / 2}$ & $(1,1 / 2)^{\circ}$ & $\begin{array}{l}1 / 2 \\
3 / 2\end{array}$ & $\begin{array}{l}36687.528 \\
37007.228\end{array}$ & $\begin{array}{l}2.80 \\
1.19\end{array}$ & $\begin{array}{l}86 \\
96\end{array}$ & $\begin{array}{l}\text { or } \\
\text { or }\end{array}$ & $\begin{array}{l}67 \\
59\end{array}$ & $\begin{array}{l}\left({ }^{7} \mathrm{~F}\right){ }^{8} \mathrm{~F}^{\circ} \\
\left({ }^{7} \mathrm{~F}\right){ }^{8} \mathrm{G}^{\circ}\end{array}$ \\
\hline $4 f^{8}\left({ }^{7} \mathrm{~F}_{5}\right) 6 p_{3 / 2}$ & $(5,3 / 2)^{\circ}$ & $\begin{array}{l}11 / 2 \\
13 / 2 \\
9 / 2 \\
7 / 2 \\
9 / 2\end{array}$ & $\begin{array}{l}36723.695 \\
36821.816 \\
37522.667 \\
36778.403\end{array}$ & $\begin{array}{l}1.47 \\
1.44 \\
\\
1.54 \\
\\
1.44\end{array}$ & $\begin{array}{l}89 \\
97 \\
85 \\
60\end{array}$ & $\begin{array}{l}\text { or } \\
\text { or } \\
\text { or } \\
\text { or }\end{array}$ & $\begin{array}{l}46 \\
72 \\
46 \\
72\end{array}$ & $\begin{array}{l}\left({ }^{7} \mathrm{~F}\right)^{8} \mathrm{~F}^{\circ} \\
\left({ }^{7} \mathrm{~F}\right)^{6} \mathrm{G}^{\circ} \\
\left({ }^{7} \mathrm{~F}\right)^{6} \mathrm{~F}^{\circ} \\
\left({ }^{7} \mathrm{~F}\right)^{6} \mathrm{D}^{\circ}\end{array}$ \\
\hline $4 f^{8}\left({ }^{7} \mathrm{~F}_{0}\right) 6 p_{1 / 2}$ & $(0,1 / 2)^{\circ}$ & $\begin{array}{l}1 / 2 \\
3 / 2\end{array}$ & $\begin{array}{l}37058.115 \\
37567.768\end{array}$ & $\begin{array}{r}-0.27 \\
1.87\end{array}$ & 90 & or & 66 & $\left({ }^{7} \mathrm{~F}\right){ }^{8} \mathrm{G}^{\circ}$ \\
\hline $4 f^{8}\left({ }^{7} \mathrm{~F}_{4}\right) 6 p_{3 / 2}$ & $(4,3 / 2)^{\circ}$ & $\begin{array}{c}11 / 2 \\
9 / 2 \\
7 / 2 \\
5 / 2 \\
3 / 2 \\
5 / 2 \\
11 / 2 \\
11 / 2 \\
9 / 2 \\
11 / 2 \\
9 / 2\end{array}$ & $\begin{array}{l}37632.423 \\
37703.968 \\
37831.032 \\
37871.723 \\
37992.923 \\
38010.603 \\
38230.334\end{array}$ & $\begin{array}{l} \\
1.99 \\
1.54 \\
0.89 \\
1.29 \\
1.45 \\
1.57 \\
1.54\end{array}$ & $\begin{array}{l}90 \\
83 \\
75 \\
68\end{array}$ & $\begin{array}{l}\text { or } \\
\text { or } \\
\text { or } \\
\text { or }\end{array}$ & $\begin{array}{l}50 \\
44 \\
50 \\
61\end{array}$ & $\begin{array}{l}\left({ }^{7} \mathrm{~F}\right){ }^{6} \mathrm{G}^{\circ} \\
\left({ }^{7} \mathrm{~F}\right){ }^{8} \mathrm{D}^{\circ} \\
\left({ }^{7} \mathrm{~F}\right){ }^{8} \mathrm{D}^{\circ} \\
\left({ }^{7} \mathrm{~F}\right){ }^{6} \mathrm{D}^{\circ}\end{array}$ \\
\hline $4 f^{8}\left({ }^{7} \mathrm{~F}_{3}\right) 6 p_{3 / 2}$ & $(3,3 / 2)^{\circ}$ & $\begin{array}{l}7 / 2 \\
9 / 2 \\
5 / 2 \\
3 / 2 \\
9 / 2 \\
9 / 2 \\
9 / 2 \\
7 / 2\end{array}$ & $\begin{array}{l}38386.557^{*} \\
38679.364 \\
39280.808 \\
38467.016 \\
38555.861 \\
38573.218 \\
38877.200\end{array}$ & $\begin{array}{l}1.39 \\
1.33 \\
1.81 \\
1.10 \\
1.20 \\
0.93 \\
1.51\end{array}$ & $\begin{array}{l}74 \\
86 \\
65 \\
68\end{array}$ & $\begin{array}{l}\text { or } \\
\text { or } \\
\text { or } \\
\text { or }\end{array}$ & $\begin{array}{l}36 \\
61 \\
62 \\
48\end{array}$ & $\begin{array}{l}\left({ }^{7} \mathrm{~F}\right){ }^{6} \mathrm{~F}^{\circ} \\
\left({ }^{7} \mathrm{~F}\right){ }^{6} \mathrm{G}^{\circ} \\
\left({ }^{7} \mathrm{~F}\right){ }^{8} \mathrm{D}^{\circ} \\
\left({ }^{7} \mathrm{~F}\right){ }^{6} \mathrm{D}^{\circ}\end{array}$ \\
\hline
\end{tabular}


Gd II, Odd Parity—Continued

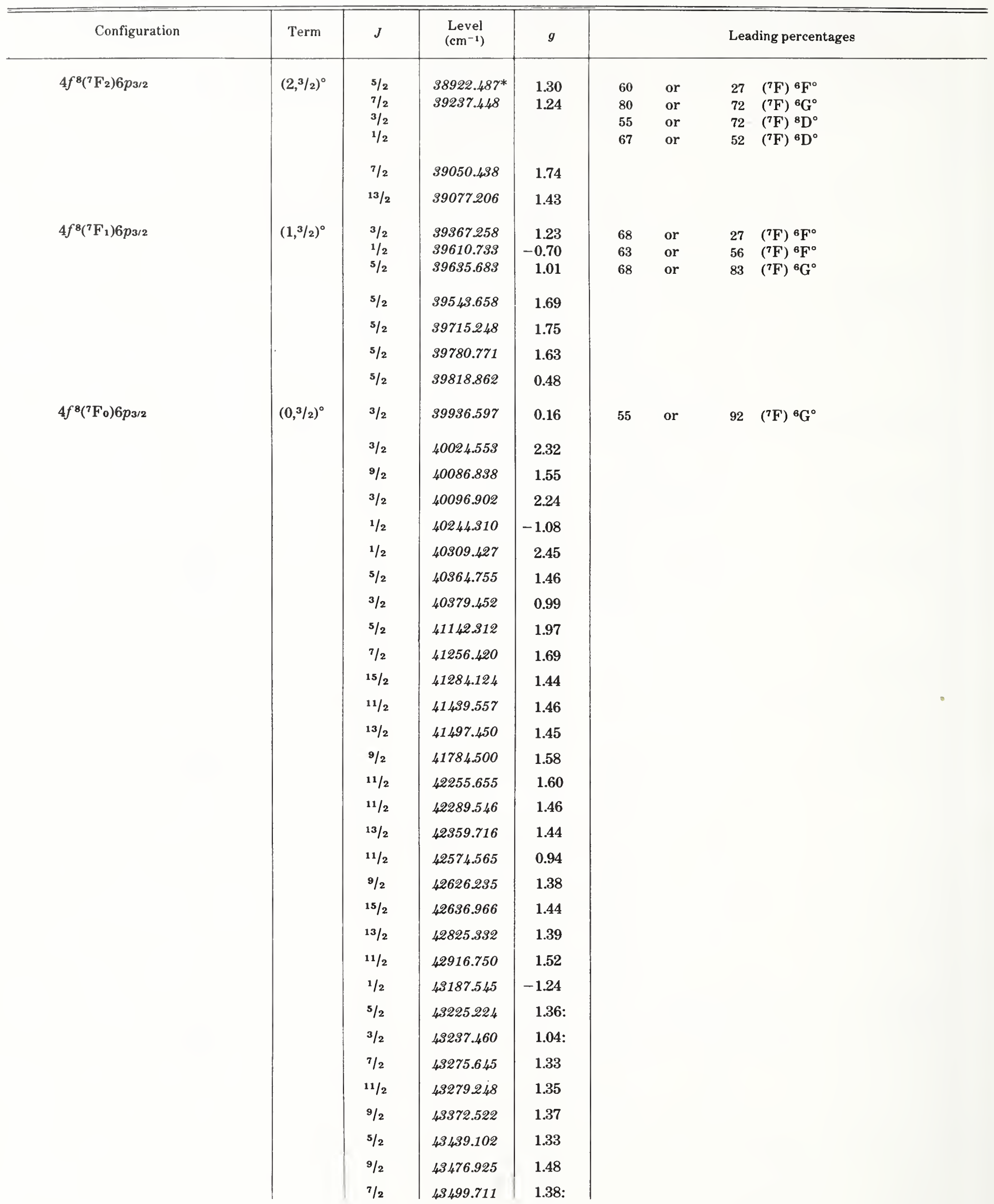


Gd II, Odd Parity-Continued

\begin{tabular}{|c|c|c|c|c|c|}
\hline Configuration & Term & $J$ & $\begin{array}{l}\text { Level } \\
\left(\mathrm{cm}^{-1}\right)\end{array}$ & $g$ & Leading percentages \\
\hline & & $13 / 2$ & 49518.298 & 1.48 & \\
\hline & & $7 / 2$ & 49673.490 & 1.30 & \\
\hline & & $9 / 2$ & 43744.110 & 1.40 & \\
\hline & & $13 / 2$ & 44197.741 & 1.40 & \\
\hline & & $9 / 2$ & 45064.987 & 1.44 & \\
\hline & & $9 / 2$ & 45170.039 & 1.60 & \\
\hline & & $9 / 2$ & 45185.749 & 1.59 & \\
\hline & & $3 / 2$ & 45212.690 & 0.04 & \\
\hline & & $5 / 2$ & 45214.658 & 1.87 & \\
\hline & & $5 / 2$ & 45394.925 & 1.09 & \\
\hline & & $7 / 2$ & 45563.276 & 1.24 & \\
\hline $\mathrm{Gd}$ III $\left({ }^{9} \mathrm{D}_{2}^{\circ}\right)$ & Limit & & 97500 & & \\
\hline
\end{tabular}

Gd II, Even Parity

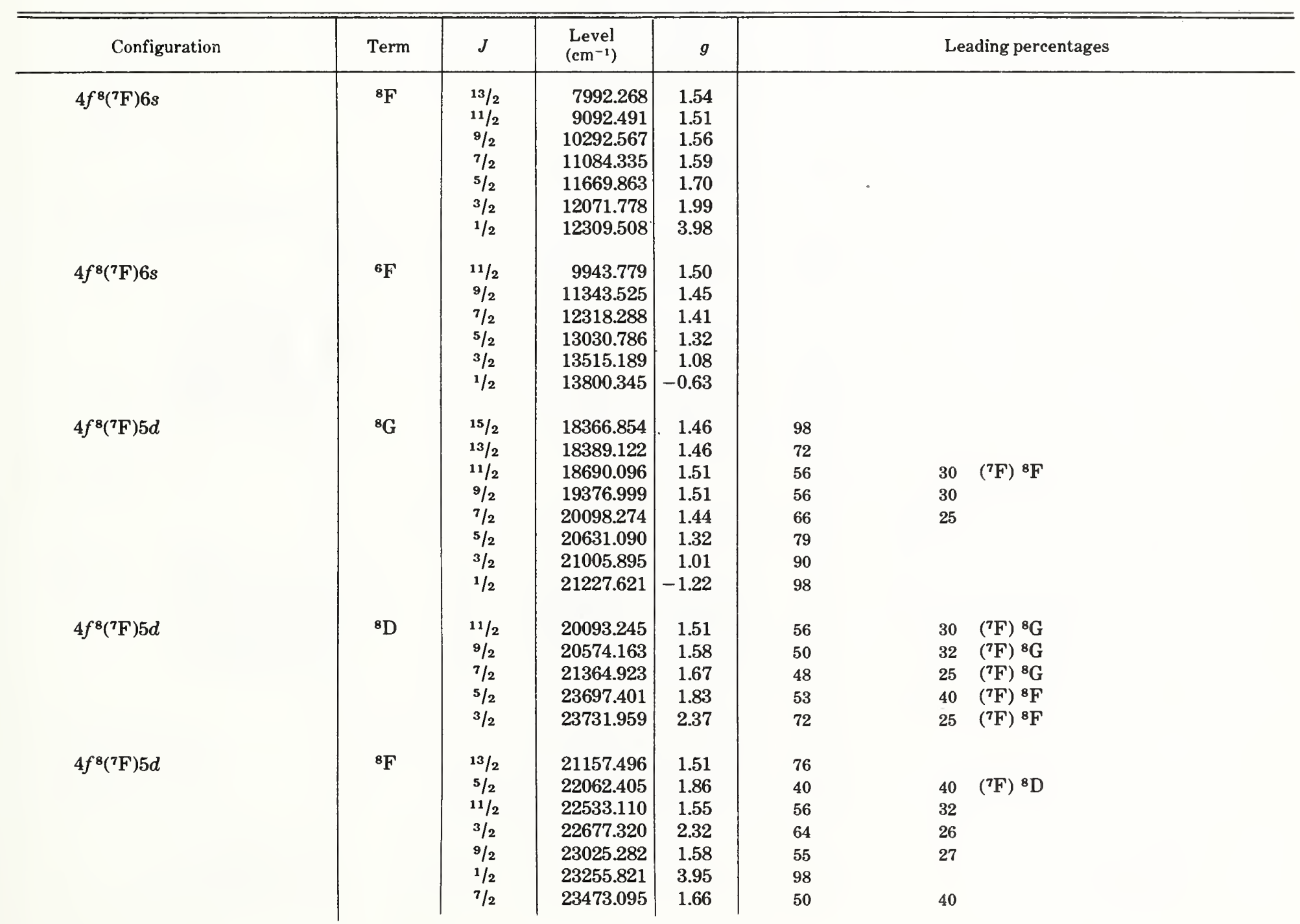


Gd II, Even Parity-Continued

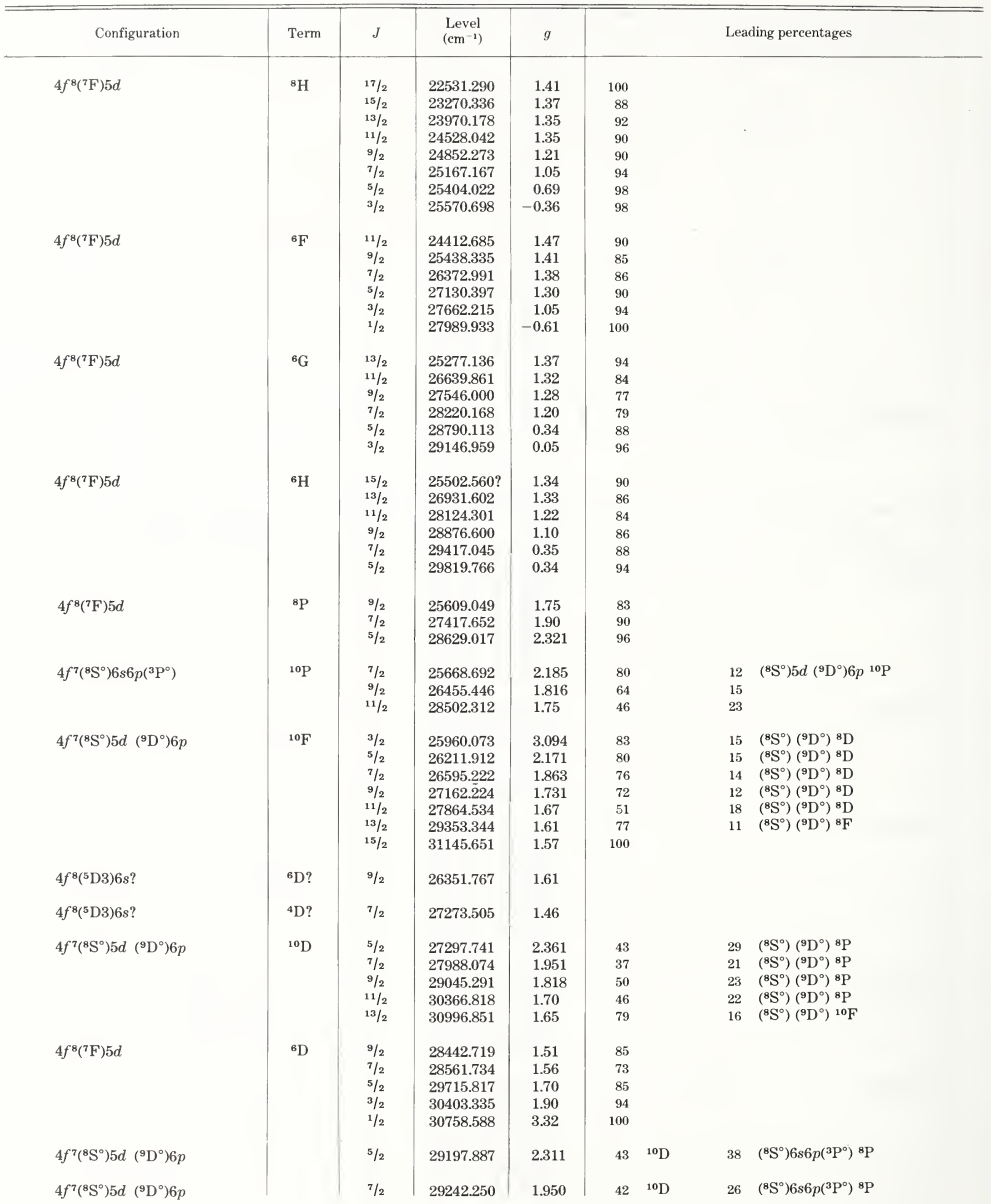


Gd II, Even Parity-Continued

\begin{tabular}{|c|c|c|c|c|c|c|c|c|}
\hline Configuration & Term & $J$ & $\begin{array}{l}\text { Level } \\
\left(\mathrm{cm}^{-1}\right)\end{array}$ & $g$ & \multicolumn{4}{|c|}{ Leading percentages } \\
\hline \multirow[t]{4}{*}{$4 f^{7}\left({ }^{8} \mathrm{~S}^{\circ}\right) 5 d\left({ }^{9} \mathrm{D}^{\circ}\right) 6 p$} & ${ }^{8} \mathrm{D}$ & $3 / 2$ & 29877.937 & 2.839 & \multicolumn{2}{|l|}{81} & 16 & $\left({ }^{8} \mathrm{~S}^{\circ}\right)\left({ }^{9} \mathrm{D}^{\circ}\right)^{10} \mathrm{~F}$ \\
\hline & & $5 / 2$ & 29965.752 & 2.040 & \multicolumn{2}{|l|}{73} & 17 & \\
\hline & & $7 / 2$ & 30008.894 & 1.832 & \multicolumn{2}{|l|}{62} & 19 & \\
\hline & & $9 / 2$ & 30027.378 & 1.78 & \multicolumn{2}{|l|}{39} & 35 & \\
\hline $4 f^{7}\left({ }^{8} \mathrm{~S}^{\circ}\right) 5 d\left({ }^{9} \mathrm{D}^{\circ}\right) 6 p$ & & $11 / 2$ & 30101.366 & 1.75 & 41 & ${ }^{10} \mathrm{~F}$ & 36 & $\left({ }^{8} \mathrm{~S}^{\circ}\right)\left({ }^{9} \mathrm{D}^{\circ}\right){ }^{8} \mathrm{D}$ \\
\hline \multirow[t]{3}{*}{$4 f^{8}\left({ }^{7} \mathrm{~F}\right) 5 d$} & \multirow[t]{3}{*}{${ }^{6} \mathbf{P}$} & $7 / 2$ & 30144.962 & 1.65 & \multicolumn{2}{|l|}{86} & & \\
\hline & & $5 / 2$ & 31237.931 & 1.84 & \multirow{2}{*}{\multicolumn{2}{|c|}{$\begin{array}{l}92 \\
98\end{array}$}} & & \\
\hline & & $3 / 2$ & 31915.795 & 2.34 & & & & \\
\hline $4 f^{7}\left({ }^{8} \mathrm{~S}^{\circ}\right) 6 s 6 p\left({ }^{3} \mathrm{P}^{\circ}\right)$ & ${ }^{8} \mathrm{P}$ & $9 / 2$ & 30849.648 & 1.784 & \multicolumn{2}{|l|}{67} & 11 & $\left({ }^{8} \mathrm{~S}^{\circ}\right) 5 d\left({ }^{9} \mathrm{D}^{\circ}\right) 6 p^{8} \mathrm{D}$ \\
\hline \multirow[t]{3}{*}{$4 f^{7}\left({ }^{8} \mathrm{~S}^{\circ}\right) 6 s 6 p\left({ }^{3} \mathrm{P}^{\circ}\right)$} & ${ }^{6} \mathrm{P}$ & $7 / 2$ & 31908.123 & 1.86 & \multicolumn{2}{|l|}{57} & 28 & $\left({ }^{8} \mathrm{~S}^{\circ}\right)\left({ }^{3} \mathrm{P}^{\circ}\right){ }^{8} \mathrm{P}$ \\
\hline & & $5 / 2$ & 32750.423 & 1.94 & \multirow{2}{*}{\multicolumn{2}{|c|}{$\begin{array}{l}79 \\
93\end{array}$}} & & \\
\hline & & $3 / 2$ & 33181.598 & 2.35 & & & & \\
\hline \multirow[t]{7}{*}{$4 f^{7}\left({ }^{8} \mathrm{~S}^{\circ}\right) 5 d\left({ }^{9} \mathrm{D}^{\circ}\right) 6 p$} & ${ }^{8} \mathrm{~F}$ & $1 / 2$ & 31977.393 & 3.97 & \multicolumn{2}{|l|}{85} & 14 & $\left({ }^{8} \mathrm{~S}^{\circ}\right)\left({ }^{7} \mathrm{D}^{\circ}\right){ }^{8} \mathrm{~F}$ \\
\hline & & $3 / 2$ & 32048.837 & 2.00 & \multicolumn{2}{|l|}{83} & 14 & $\left({ }^{8} \mathrm{~S}^{\circ}\right)\left({ }^{7} \mathrm{D}^{\circ}\right)^{8} \mathrm{~F}$ \\
\hline & & $5 / 2$ & 32150.143 & 1.81 & \multicolumn{2}{|l|}{77} & 13 & $\left({ }^{8} \mathrm{~S}^{\circ}\right)\left({ }^{7} \mathrm{D}^{\circ}\right){ }^{8} \mathrm{~F}$ \\
\hline & & $7 / 2$ & $32262.787^{*}$ & 1.743 & & 11 & $\left({ }^{8} \mathrm{~S}^{\circ}\right)\left({ }^{7} \mathrm{D}^{\circ}\right){ }^{8} \mathrm{~F}$ \\
\hline & & $9 / 2$ & 32684.712 & 1.623 & \multirow{2}{*}{\multicolumn{2}{|c|}{$\begin{array}{l}68 \\
67\end{array}$}} & 10 & $\left({ }^{8} \mathrm{~S}^{\circ}\right)\left({ }^{7} \mathrm{D}^{\circ}\right){ }^{8} \mathrm{~F}$ \\
\hline & & $11 / 2$ & 32946.196 & 1.55 & & & 10 & $\left({ }^{8} \mathrm{~S}^{\circ}\right)\left({ }^{7} \mathrm{D}^{\circ}\right)^{8} \mathrm{~F}$ \\
\hline & & $13 / 2$ & 33557.951 & 1.53 & \multicolumn{2}{|l|}{74} & 11 & $\left({ }^{8} \mathrm{~S}^{\circ}\right)\left({ }^{9} \mathrm{D}^{\circ}\right){ }^{10} \mathrm{D}$ \\
\hline $4 f^{7}\left({ }^{8} \mathrm{~S}^{\circ}\right) 5 d\left({ }^{9} \mathrm{D}^{\circ}\right) 6 p$ & & $5 / 2$ & 32260.120 & 2.172 & 37 & ${ }^{8} \mathrm{P}$ & 23 & $\left({ }^{8} \mathrm{~S}^{\circ}\right) 6 s 6 p\left({ }^{3} \mathrm{P}^{\circ}\right){ }^{8} \mathrm{P}$ \\
\hline $4 f^{7}\left({ }^{8} \mathrm{~S}^{\circ}\right) 5 d\left({ }^{9} \mathrm{D}^{\circ}\right) 6 p$ & & $7 / 2$ & $32490.510^{*}$ & 1.791 & 36 & ${ }^{8} \mathbf{P}$ & 17 & $\left({ }^{8} \mathrm{~S}^{\circ}\right) 6 s 6 p\left({ }^{1} \mathrm{P}^{\circ}\right){ }^{8} \mathrm{P}$ \\
\hline $4 f^{7}\left({ }^{8} \mathrm{~S}^{\circ}\right) 5 d\left({ }^{9} \mathrm{D}^{\circ}\right) 6 p$ & & $9 / 2$ & 32304.409 & 1.747 & 42 & ${ }^{8 P}$ & 25 & $\left({ }^{8} \mathrm{~S}^{\circ}\right) 6 s 6 p\left({ }^{3} \mathrm{P}^{\circ}\right){ }^{8} \mathrm{P}$ \\
\hline \multirow[t]{3}{*}{$4 f^{7}\left({ }^{8} \mathrm{~S}^{\circ}\right) 5 d\left({ }^{9} \mathrm{D}^{\circ}\right) 6 p$} & 10P & $7 / 2$ & 33211.481 & 2.155 & \multicolumn{2}{|l|}{80} & & \\
\hline & & $9 / 2$ & 33596.027 & 1.93 & 75 & & & \\
\hline & & $11 / 2$ & 34178.776 & 1.81 & 68 & . & & \\
\hline $4 f^{7}\left({ }^{8} \mathrm{~S}^{\circ}\right) 5 d\left({ }^{7} \mathrm{D}^{\circ}\right) 6 p$ & ${ }^{8} \mathrm{D}$ & $11 / 2$ & 36461.156 & 1.58 & 46 & & 37 & $\left({ }^{8} \mathrm{~S}^{\circ}\right)\left({ }^{7} \mathrm{D}^{\circ}\right){ }^{6} \mathrm{~F}$ \\
\hline & & $9 / 2$ & 36647.241 & 1.67 & 64 & & & \\
\hline & & $7 / 2$ & 36711.176 & 1.79 & 74 & & & \\
\hline & & $5 / 2$ & 36845.366 & 2.030 & 83 & & & \\
\hline & & $3 / 2$ & 36995.778 & 2.745 & 90 & & & \\
\hline & & $9 / 2$ & 37845.943 & 1.50 & & & & \\
\hline $4 f^{7}\left({ }^{8} \mathrm{~S}^{\circ}\right) 5 d\left({ }^{7} \mathrm{D}^{\circ}\right) 6 p$ & & $9 / 2$ & 38029.848 & 1.43 & 37 & ${ }^{6} \mathrm{~F}$ & 36 & $\left({ }^{8} \mathrm{~S}^{\circ}\right)\left({ }^{7} \mathrm{D}^{\circ}\right){ }^{6} \mathrm{D}$ \\
\hline & & $9 / 2$ & 38057.954 & 1.14 & & & & \\
\hline $4 f^{7}\left({ }^{8} \mathrm{~S}^{\circ}\right) 5 d\left({ }^{7} \mathrm{D}^{\circ}\right) 6 p$ & & $7 / 2$ & 38320.173 & 1.54 & 35 & ${ }^{6} \mathrm{~F}$ & 33 & $\left({ }^{8} \mathrm{~S}^{\circ}\right)\left({ }^{7} \mathrm{D}^{\circ}\right){ }^{6} \mathrm{D}$ \\
\hline & & $11 / 2$ & 38509.608 & 1.17 & & & & \\
\hline $4 f^{7}\left({ }^{8} \mathrm{~S}^{\circ}\right) 5 d\left({ }^{7} \mathrm{D}^{\circ}\right) 6 p$ & ${ }^{6} \mathrm{~F}$ & $11 / 2$ & 38553.210 & 1.48 & 53 & & 43 & $\left({ }^{8} \mathrm{~S}^{\circ}\right)\left({ }^{7} \mathrm{D}^{\circ}\right){ }^{8} \mathrm{D}$ \\
\hline & & $9 / 2$ & $39250.737^{*}$ & 1.66 & 42 & & 21 & $\left({ }^{8} \mathrm{~S}^{\circ}\right)\left({ }^{7} \mathrm{D}^{\circ}\right){ }^{8} \mathrm{D}$ \\
\hline & & $7 / 2$ & 39777.217 & 1.50 & 43 & & 40 & $\left({ }^{8} \mathrm{~S}^{\circ}\right)\left({ }^{7} \mathrm{D}^{\circ}\right){ }^{6} \mathrm{D}$ \\
\hline & & $5 / 2$ & 39971.223 & 1.44 & 68 & & 23 & $\left({ }^{8} \mathrm{~S}^{\circ}\right)\left({ }^{7} \mathrm{D}^{\circ}\right){ }^{6} \mathrm{D}$ \\
\hline & & $3 / 2$ & 40091.597 & 1.19 & 85 & & 10 & $\left({ }^{8} \mathrm{~S}^{\circ}\right)\left({ }^{7} \mathrm{D}^{\circ}\right){ }^{6} \mathrm{D}$ \\
\hline & & $1 / 2$ & 40162.389 & -0.43 & 96 & & & \\
\hline $4 f^{7}\left({ }^{8} \mathrm{~S}^{\circ}\right) 5 d\left({ }^{7} \mathrm{D}^{\circ}\right) 6 p$ & & $5 / 2$ & 38628.604 & 1.66 & 35 & ${ }^{6} \mathrm{D}$ & 25 & $\left({ }^{8} \mathrm{~S}^{\circ}\right)\left({ }^{7} \mathrm{D}^{\circ}\right){ }^{6} \mathrm{~F}$ \\
\hline $4 f^{7}\left({ }^{8} \mathrm{~S}^{\circ}\right) 5 d\left({ }^{7} \mathrm{D}^{\circ}\right) 6 p$ & & $3 / 2$ & 38828.402 & 1.83 & 39 & ${ }^{6} \mathrm{D}$ & 38 & $\left({ }^{8} \mathrm{~S}^{\circ}\right)\left({ }^{7} \mathrm{D}^{\circ}\right){ }^{8} \mathrm{~F}$ \\
\hline
\end{tabular}


Gd II, Even Parity-Continued

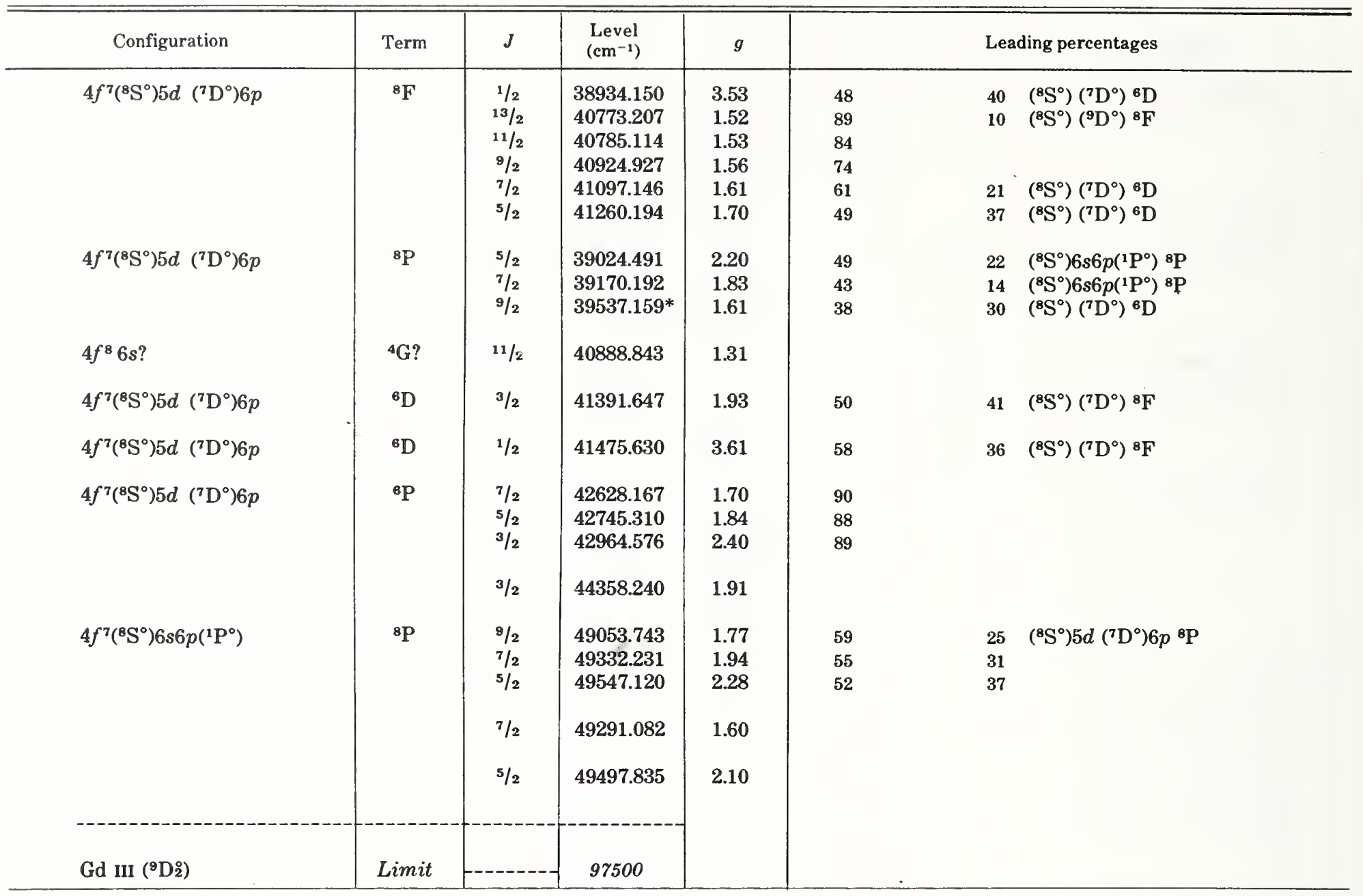




\section{Gd III}

(Sm I sequence; 62 electrons)

Ground state $\left(1 s^{2} 2 s^{2} 2 p^{6} 3 s^{2} 3 p^{6} 3 d^{10} 4 s^{2} 4 p^{6} 4 d^{10} 5 s^{2} 5 p^{6}\right) 4 f^{7} 5 d^{9} \mathrm{D}_{2}^{\circ}$

Ionization energy $166400 \pm 800 \mathrm{~cm}^{-1}$

$20.63 \pm 0.10 \mathrm{eV}$

Callahan $[1962,1963]$ observed this spectrum over the range 1900-11000 $\AA$ using a mild spark discharge. His thesis has about 8200 lines assigned to Gd III, with the "largest concentration" of the lines lying in the region 2100-3200 $\AA$. Kielkopf [1969] also measured thousands of Gd lines in connection with his investigation of Gd IV, and his thesis gives the stronger Gd III lines from 1720 to $6000 \AA$. The line lists of Callahan and Kielkopf differ considerably with regard to particular spectra in some regions. Both lists have many lines experimentally assigned to Gd III that are now known to belong to Gd II.

Callahan's analysis of the $4 f^{7}\left({ }^{8} \mathrm{~S}^{\circ}\right) 5 d-6 p$ and $4 f^{7}\left({ }^{8} \mathrm{~S}^{\circ}\right) 6 s-6 p$ transitions in Gd III classified 42 lines $\left(2043-3177 \AA\right.$ ) and yielded all the $4 f^{7}\left({ }^{8} \mathrm{~S}^{\circ}\right) 5 d, 6 s$, and $6 p$ levels [Callahan, 1963]. Johansson and Litzén [1973] found the $4 f^{8}{ }^{7} \mathrm{~F}$ levels by observing and classifying eight lines of the ${ }^{7} \mathrm{~F}-{ }^{7} \mathrm{D}^{\circ}$ multiplet in the infrared $(1.43-2.56 \mu \mathrm{m})$. Kielkopf [1976] has reevaluated the Gd III levels, taking his own wavelength determinations into account. He supplied the values given here except for the two $4 f^{7}\left({ }^{8} S^{\circ}\right) 7 s$ levels, which were recently located by Wyart [1976].

The eigenvector percentages, which are from Callahan [1963], are based on the assumption of a pure $4 f^{7}\left({ }^{8} \mathrm{~S}^{\circ}\right)$ core state. As he remarks, the actual purity of this state is about $97 \%$.

The ionization energy is from Sugar and Reader.

\section{References}

Callahan, W. R., Thesis, Johns Hopkins Univ., Baltimore, 117 pp. (1962). EL CL W PT

Callahan, W. R., J. Opt. Soc. Am. 53, 695 (1963). EL CL W PT

Johansson, S., and Litzén, U., Phys. Ser. 8, 43 (1973). EL CL W

Kielkopf, J., Thesis, Johns Hopkins Univ., Baltimore, 121 pp. (1969). W

Kielkopf, J., unpublished material (1976). EL

Sugar, J., and Reader, J., J. Chem. Phys. 59, 2083 (1973). IP

Wyart, J. F., private communication (1976). EL

[July 1976]

Gd III

\begin{tabular}{|c|c|c|c|c|c|}
\hline Configuration & Term & $J$ & $\begin{array}{l}\text { Level } \\
\left(\mathrm{cm}^{-1}\right)\end{array}$ & & ercentages \\
\hline $4 f^{7}\left({ }^{8} \mathrm{~S}^{\circ}\right) 5 d$ & ${ }^{9} \mathrm{D}^{\circ}$ & $\begin{array}{l}2 \\
3 \\
4 \\
5 \\
6\end{array}$ & $\begin{array}{r}0.00 \\
279.32 \\
694.37 \\
1310.13 \\
2282.83\end{array}$ & $\begin{array}{r}99 \\
98 \\
98 \\
97 \\
100\end{array}$ & $\begin{array}{ll}1 & \left({ }^{8} \mathrm{~S}^{\circ}\right){ }^{7} \mathrm{D}^{\circ} \\
2 & \\
2 & \\
3 & \end{array}$ \\
\hline $4 f^{8}$ & ${ }^{7} \mathbf{F}$ & $\begin{array}{l}6 \\
5 \\
4 \\
3 \\
2 \\
1 \\
0\end{array}$ & $\begin{array}{l}2381.24 \\
3996.71 \\
5015.20 \\
5789.97 \\
6334.17\end{array}$ & & \\
\hline $4 f^{7}\left({ }^{8} S^{\circ}\right) 6 s$ & ${ }^{9} \mathrm{~S}^{\circ}$ & 4 & 9195.04 & & \\
\hline
\end{tabular}


Gd III-Continued

\begin{tabular}{|c|c|c|c|c|c|c|}
\hline Configuration & Term & $J$ & $\begin{array}{l}\text { Level } \\
\left(\mathrm{cm}^{-1}\right)\end{array}$ & \multicolumn{3}{|c|}{ Leading percentages } \\
\hline $4 f^{7}\left({ }^{8} \mathrm{~S}^{\circ}\right) 5 d$ & ${ }^{7} \mathrm{D}^{\circ}$ & $\begin{array}{l}5 \\
4 \\
3 \\
2 \\
1\end{array}$ & $\begin{array}{r}9356.30 \\
9717.70 \\
10014.75 \\
10234.49 \\
10387.30\end{array}$ & $\begin{array}{r}97 \\
98 \\
98 \\
99 \\
100\end{array}$ & $\begin{array}{l}3 \\
2 \\
2 \\
1\end{array}$ & $\left({ }^{8} \mathrm{~S}^{\circ}\right)^{9} \mathrm{D}^{\circ}$ \\
\hline $4 f^{7}\left({ }^{8} \mathrm{~S}^{\circ}\right) 6 s$ & ${ }^{7} \mathrm{~S}^{\circ}$ & 3 & 11549.50 & & & \\
\hline $4 f^{7}\left({ }^{8} \mathrm{~S}_{7 / 2}\right) 6 p_{1 / 2}$ & $\left({ }^{7} / 2,1 / 2\right)$ & $\begin{array}{l}3 \\
4\end{array}$ & $\begin{array}{l}43019.99 \\
43611.69\end{array}$ & $\begin{array}{l}98 \\
95\end{array}$ & $\begin{array}{l}2 \\
5\end{array}$ & $\left({ }^{8} \mathrm{~S}_{7 / 2}^{\circ}\right)\left(7 / 2,{ }^{3} / 2\right)$ \\
\hline $4 f^{7}\left({ }^{8} \mathrm{~S}_{7 / 2}^{\circ}\right) 6 p_{3 / 2}$ & $(7 / 2,3 / 2)$ & $\begin{array}{l}5 \\
4 \\
3 \\
2\end{array}$ & $\begin{array}{l}47233.93 \\
48339.14 \\
48859.62 \\
49194.70\end{array}$ & $\begin{array}{r}100 \\
95 \\
98 \\
100\end{array}$ & $\begin{array}{l}5 \\
2\end{array}$ & $\left({ }^{8} \mathrm{~S}^{\circ} / 2\right)\left({ }^{7} / 2,1 / 2\right)$ \\
\hline $4 f^{7}\left({ }^{8} \mathrm{~S}^{\circ}\right) 7 s$ & ${ }^{9} \mathrm{~S}^{\circ}$ & 4 & 87754.4 & & & \\
\hline $4 f^{7}\left({ }^{8} \mathrm{~S}^{\circ}\right) 7 \mathrm{~s}$ & ${ }^{7} \mathrm{~S}^{\circ}$ & 3 & 88563.2 & & & \\
\hline Gd IV $\left({ }^{8} \mathrm{~S}_{7 / 2}^{0}\right)$ & Limit & & 166400 & & & \\
\hline
\end{tabular}


[Gd IV]

(Pm I sequence; 61 electrons)

$Z=64$

Ground state $\left(1 s^{2} 2 s^{2} 2 p^{6} 3 s^{2} 3 p^{6} 3 d^{10} 4 s^{2} 4 p^{6} 4 d^{10} 5 s^{2} 5 p^{6}\right) 4 f^{78} \mathrm{~S}_{7 / 2}^{\circ}$

Ionization energy $355000 \pm 6000 \mathrm{~cm}^{-1}$

$44.0 \pm 0.7 \mathrm{eV}$

Kielkopf [1969] observed the Gd spark spectra using a pulsed hollow-cathode source. The line list in his thesis includes the strongest lines experimentally assigned to Gd IV in the region 1265-6000 $\AA$, with most such lines lying below $2500 \AA$. The levels from a preliminary analysis of Gd IV by Kielkopf and Crosswhite [1970] are omitted here, pending confirmation by further work on this spectrum [Kielkopf and Crosswhite, 1976].

Some of the research on the spectra of the $\mathrm{Gd}^{3+}$ ion in crystal hosts is summarized in Dieke's book [1968]. Absorption transitions from the $4 f^{7}{ }^{8} \mathrm{~S}_{7 / 2}^{\circ}$ ground level to levels of several upper $4 f^{7}$ terms have been identified. Only the lowest excited term, ${ }^{6} \mathrm{P}^{\circ}$, is given here; the levels are averages of the corresponding sublevels of the ion in a $\mathrm{LaCl}_{3}$ host crystal [Piksis, Dieke, and Crosswhite, 1967], rounded off to the nearest $10 \mathrm{~cm}^{-1}$. Crosswhite [1976] furnished the eigenvector percentages from a new calculation of this configuration.

In deriving the quoted ionization energy, Sugar and Reader [1973] used a value of 91200 $\mathrm{cm}^{-1}$ for the lowest level of the $4 f^{6} 5 d$ configuration. Most of the uncertainty given for the ionization energy here arises from the uncertainty in this $4 f^{7}-4 f^{6} 5 d$ energy difference [Spector and Sugar, 1976].

\section{References}

Crosswhite, H. M., unpublished material (1976). PT

Dieke, G. H., Spectra and Energy Levels of Rare Earth Ions in Crystals, Ed. H. M. Crosswhite and H. Crosswhite, pp. 249-253 (Interscience Publishers, New York, 1968). [EL] [CL] [W]

Kielkopf, J. F., Jr., Thesis, Johns Hopkins Univ., Baltimore (1969). EL CL W PT

Kielkopf, J. F., and Crosswhite, H. M., J. Opt. Soc. Am. 60, 347 (1970). E L ND CL W

Kielkopf, J. F., and Crosswhite, H. M., private communication (1976).

Piksis, A. H., Dieke, G. H., and Crosswhite, H. M., J. Chem. Phys. 47, 5083 (1967). [E L] [CL] [W] PT

Spector, N., and Sugar, J., J. Opt. Soc. Am. 66, 436 (1976).

Sugar, J., and Reader, J., J. Chem. Phys. 59, 2083 (1973). IP

[July 1976]

[Gd IV]

\begin{tabular}{|c|c|c|c|c|c|}
\hline Configuration & Term & $J$ & $\begin{array}{c}\text { Level } \\
\left(\mathrm{cm}^{-1}\right)\end{array}$ & \multicolumn{2}{|c|}{ Leading percentages } \\
\hline $4 f^{7}$ & ${ }^{8} \mathrm{~S}^{\circ}$ & $7 / 2$ & 0 & 97 & $3{ }^{6} \mathrm{P}^{\circ}$. \\
\hline $4 f^{7}$ & ${ }^{6} \mathrm{P}^{\circ}$ & $\begin{array}{l}7 / 2 \\
5 / 2 \\
3 / 2\end{array}$ & $\begin{array}{l}{[32120]} \\
{[32720]} \\
{[33290]}\end{array}$ & $\begin{array}{l}75 \\
82 \\
90\end{array}$ & $\begin{array}{rr}13 & { }^{6} \mathrm{D}^{\circ} \\
14 & \\
8 & \end{array}$ \\
\hline $\mathrm{Gd} v\left({ }^{7} \mathrm{~F}_{0}\right)$ & Limit & & 355000 & & \\
\hline
\end{tabular}




\section{Gd XXXVII}

(Ni I sequence; 28 electrons)

Ground state $\left(1 s^{2} 2 s^{2} 2 p^{6} 3 s^{2} 3 p^{6}\right) 3 d^{10}{ }^{1} \mathrm{~S}_{0}$

Burkhalter, Nagel, and Whitlock excited this spectrum in the region $5-15 \AA$ by focusing light from a pulsed laser onto a Gd target. They classified the $3 d^{10}{ }^{1} \mathrm{~S}_{0}-3 d^{9} 4 p(J=1)$ and $3 d^{10}{ }^{1} \mathrm{~S}_{0}-3 d^{9} 4 f(J=1)$ resonance lines by comparison with calculations and by use of isoelectronic regularities. The levels listed by Burkhalter et al. have been converted to units of $10^{4} \mathrm{~cm}^{-1}$ (equivalent to $1.23985 \mathrm{eV}$ ).

Cowan has also calculated these configurations, and the leading percentages are from his results. The levels of both excited configurations are listed with $J_{1} j$-coupling designations, with alternative $L S$ names and percentages shown for the $3 d^{10} 4 f(J=1)$ levels.

Burkhalter et al. note that the line classified as $3 d^{10}{ }^{1} \mathrm{~S}_{0}-3 d^{9} 4 p(3 / 2,1 / 2)_{1}^{\circ}$ is too strong to belong entirely to Gd XXXVII; following a suggestion of Burkhalter we list the corresponding upper level as tentative. Burkhalter also supplied the tentative level at $1129 \times 10^{4} \mathrm{~cm}^{-1}(1400$ $\mathrm{eV})$ as being a more probable position for $3 d^{9} 4 f(5 / 2,5 / 2)_{1}^{\circ}$ than the published tentative value of $1376 \mathrm{eV}$.

\section{References}

Burkhalter, P. G., private communication (1975). EL CL

Burkhalter, P. G., Nagel, D. J., and Whitlock, R. R., Phys. Rev. A 9, 2331 (1974). EL CL W

Cowan, R. D., unpublished material (1975). AT

[July 1976]

Gd XXXVII

\begin{tabular}{|c|c|c|c|c|c|c|}
\hline Configuration & Term & $J$ & $\begin{array}{c}\text { Level } \\
\left(10^{4} \mathrm{~cm}^{-1}\right)\end{array}$ & \multicolumn{3}{|c|}{ Leading percentages } \\
\hline $3 d^{10}$ & ${ }^{1} \mathrm{~S}$ & 0 & 0.0 & & & \\
\hline $3 d^{9}\left({ }^{2} \mathrm{D}_{3 / 2}\right) 4 p_{1 / 2}$ & $(3 / 2,1 / 2)^{\circ}$ & 1 & $917 ?$ & 99 & & \\
\hline $3 d^{9}\left({ }^{2} \mathrm{D}_{5 / 2}\right) 4 p_{3 / 2}$ & $(5 / 2,3 / 2)^{\circ}$ & 1 & 930 & 98 & & \\
\hline $3 d^{9}\left({ }^{2} \mathrm{D}_{3 / 2}\right) 4 p_{3 / 2}$ & $(3 / 2,3 / 2)^{\circ}$ & 1 & 955 & 99 & & \\
\hline $3 d^{9}\left({ }^{2} \mathrm{D}_{5 / 2}\right) 4 f_{5 / 2}$ & $(5 / 2,5 / 2)^{\circ}$ & 1 & $1129 ?$ & 90 & or & $77^{3} \mathrm{P}^{\circ}$ \\
\hline $3 d^{9}\left({ }^{2} \mathrm{D}_{5 / 2}\right) 4 f_{7 / 2}$ & $\left(5 / 2,,^{7} / 2\right)^{\circ}$ & 1 & 1140 & 72 & or & $64{ }^{3} \mathrm{D}^{\circ}$ \\
\hline $3 d^{9}\left({ }^{2} \mathrm{D}_{3 / 2}\right) 4 f_{5 / 2}$ & $(3 / 2,5 / 2)^{\circ}$ & 1 & 1171 & 81 & or & $82{ }^{1} \mathrm{P}^{\circ}$ \\
\hline
\end{tabular}




\section{TERBIUM}

Tb I

65 electrons

$Z=65$

Ground state $\left(1 s^{2} 2 s^{2} 2 p^{6} 3 s^{2} 3 p^{6} 3 d^{10} 4 s^{2} 4 p^{6} 4 d^{10} 5 s^{2} 5 p^{6}\right) 4 f^{9} 6 s^{2}{ }^{6} \mathrm{H}_{15 / 2}^{\circ}$

Ionization energy $47295 \pm 5 \mathrm{~cm}^{-1}$

$5.8639 \pm 0.0006 \mathrm{eV}$

Identified odd configurations

$4 f^{9} 6 s^{2}, 4 f^{9} 5 d 6 s ?, 4 f^{8} 6 s^{2} 6 p, 4 f^{8} 5 d 6 s 6 p, 4 f^{8} 5 d^{2} 6 p ?, 4 f^{9} 6 s 7 s$

Identified even configurations $\quad 4 f^{8} 5 d 6 s^{2}, 4 f^{8} 5 d^{2} 6 s, 4 f^{9} 6 s 6 p$

Main References for Wavelengths, Energy Levels, and $g$ Values

The most complete description of this spectrum has been obtained by Klinkenberg [1966], whose original unpublished list had about $30000 \mathrm{~Tb}$ I and $\mathrm{Tb}$ II lines over the region 2335-9285 $\AA$. The wavelengths were derived from spectrograms of electrodeless-discharge sources photographed at the Argonne National Laboratory. The analysis of the Tb I spectrum at the Zeeman Laboratory, Amsterdam, is described in the papers of Klinkenberg $[1964 ; 1966 ; 1967$; 1972a], Klinkenberg and Meinders [1966; 1969], Meinders and Klinkenberg [1968], and Klinkenberg and van Kleef [1970]. The measurements were extended photographically to 11650 $\AA$, and later to $2.4 \mu \mathrm{m}$ by Verges at Orsay using the SISAM technique [Klinkenberg, 1972a]. Most of the above references include an extensive list of classified lines; by 1972 some 5400 $\mathrm{Tb}$ I lines had been assigned as transitions between 451 high odd levels and 46 low even levels (the "A" system) [Klinkenberg, 1972a].

The energy levels in the papers of Klinkenberg and his collaborators are given with respect to a value of $0.000 \mathrm{~cm}^{-1}$ for the lowest level of the " $\mathrm{A}$ " system, $4 f^{8}\left({ }^{7} \mathrm{~F}\right) 5 d 6 s^{2}{ }^{8} \mathrm{G}_{13 / 2}$. In 1970 Klinkenberg and van Kleef determined that this level lay $285.58 \mathrm{~cm}^{-1}$ above the ground level of $\mathrm{Tb} \mathrm{I}, 4 f^{9} 6 s^{2}{ }^{6} \mathrm{H}_{15 / 2}^{\circ}$. The value of this connection between the "A" and "B" systems has recently been more accurately determined as $285.500 \pm 0.005 \mathrm{~cm}^{-1}$ [Blaise and Verges, 1976]. The great majority of the levels given here are from a list furnished by Klinkenberg [1972b], adjusted to the new value for the connection. Most of the levels are given to two decimal places only, with some low levels being given to the nearest $0.005 \mathrm{~cm}^{-1}$ as in the original lists. Some additional levels and $g$ values, as well as a few revisions, are from recent work at Laboratoire Aimé Cotton, Orsay, and at Amsterdam [Blaise and Verges, 1976, 1977; Klinkenberg, 1976, 1977; some of the recent results for $\mathrm{Tb}$ I are described in the compilation by Blaise, Camus, and Wyart, 1976]. New measurements of the Zeeman effect [Klinkenberg, 1976, 1977] and of the infrared spectrum by means of Fourier spectrometry [Blaise and Verges, 1976] have been carried out, and the analysis is being continued.

The main observations of the optical Zeeman effect for $\mathrm{Tb}$ were made by Davis [1960] and by Klinkenberg and his coworkers [Klinkenberg, 1966; Meinders and Klinkenberg, 1968]. Klinkenberg and collaborators derived most of the approximately $450 \mathrm{~g}$ values given here, many of which have not previously been published [Klinkenberg, 1977]. The accuracy of most of the three-place values (usually given to the nearest 0.005 ) and two-place values is limited by hyperfine structure. The 1964 paper by Bender, Penselin, and Schlüpmann gave $J$ and $g$ values for five low levels of $\mathrm{Tb}$ I as determined by the atomic-beam magnetic-resonance technique. The five-place $g$ values listed here for the three lowest odd levels and 14 lowest even levels are from the magnetic-resonance measurements of Childs [1970], who extended the earlier work on hyperfine structure in Tb I by Childs and Goodman [1969]. The uncertainty of most of these values is two to four units in the fifth place, the main exception being an uncertainty of three units in the fourth place for the $4 f^{8}\left({ }^{7} \mathrm{~F}\right) 5 d 6 s^{2}{ }^{8} \mathrm{G}_{1 / 2}$ level. 
Worden, Conway, Paisner, and Solarz [1977] observed high series members in Tb I using a stepwise laser photoionization technique. These data became available too late for inclusion here, but the quoted ionization energy was determined from the new series.

\section{Theoretical Interpretation}

Details of the steps in the analysis of this spectrum are given in the papers of Klinkenberg and his coworkers. Their interpretation began with the identification of the low even terms, and this complex group constitutes the best understood portion of the known structure. Arnoult and Gerstenkorn [1966] and Spector [1970] calculated the $4 f^{8}\left({ }^{7} \mathrm{~F}\right) 5 d 6 s^{2}$ subconfiguration. The $4 f^{8} 5 d 6 s^{2}$ eigenvector percentages given here are from the recent calculation by Bauche-Arnoult, Sinzelle, and Bachelier [1977], who constructed the matrices for the complete configuration. Our arrangement of the lower even levels into term groups of $4 f^{8}\left({ }^{7} \mathrm{~F}\right) 5 d 6 \mathrm{~s}^{2}$ excludes only the $J=9 / 2$ level at $1371 \mathrm{~cm}^{-1}$, but it should be noticed that several of the levels included in the three lowest terms have no really satisfactory designations: particular examples are the nominal ${ }^{8} \mathrm{G}_{11 / 2},{ }^{8} \mathrm{D}_{11 / 2},{ }^{8} \mathrm{G}_{9 / 2},{ }^{8} \mathrm{D}_{3 / 2}$, and ${ }^{8} \mathrm{~F}_{3 / 2}$ levels. The designations of a few levels have been changed to conform better with the new and more accurate eigenvectors.

Klinkenberg and Meinders [1969] were able to extract the levels of a $4 f^{8} 5 d^{2} 6 s{ }^{10} \mathrm{G}$ term beginning at $8190 \mathrm{~cm}^{-1}$, and some other assignments to higher even terms have been made or suggested [Klinkenberg, 1972a, 1976; Blaise and Verges, 1976, 1977]. A number of designations are given tentatively, pending calculations of the complex $\left(4 f^{8} 5 d 6 s^{2}+4 f^{8} 5 d^{2} 6 s\right)$ group. The eigenvector compositions of many of these levels are certain to be very mixed in any coupling scheme. The levels assigned to the important upper even group $4 f^{9}\left({ }^{6} \mathrm{H}^{\circ}\right) 6 s 6 p$ are designated in a $J_{1} J_{2}$ coupling scheme [Klinkenberg and van Kleef, 1970]. Sugar [1962] classified three of the strongest $\mathrm{Tb}$ I lines as resonance transitions from three of these levels to the $4 f^{9} 6 s^{2}{ }^{6} \mathrm{H}_{15 / 2}^{\circ}$ level, but the correct configurational assignment and extension of the upper even group was accomplished by Klinkenberg and van Kleef with much more extensive data.

The development and interpretation of the system of lower odd levels is less complete than for the even group, and interpretation of the extensive group of high odd levels has hardly begun [Klinkenberg, 1971]. Conway and Wybourne [1963] gave eigenvectors for the lowest three levels of the $4 f^{9} 6 s^{2}{ }^{6} \mathrm{H}^{\circ}$ ground term; the leading percentages here are from a more recent calculation by Crosswhite [1976].

Klinkenberg [1972a] and Spector [1971] have discussed the identification of the $4 f^{8}\left({ }^{7} \mathrm{~F}\right) 6 s^{2} 6 p$ levels, some designations of which are given here in the $J_{1} j$ coupling scheme considered by both authors. Some additional assignments to the $4 f^{8}(5 d+6 s)^{2} 6 p$ group or to $4 f^{9}\left({ }^{6} \mathrm{H}^{\circ}\right) 5 d 6 s$ are listed tentatively, pending calculation of the important odd configurations [Klinkenberg, 1976; Blaise and Verges, 1976, 1977; see Blaise, Camus, and Wyart, 1976].

\section{References}

Arnoult, C., and Gerstenkorn, S., J. Opt. Soc. Am. 56, 177 (1966). Hfs PT

Bauche-Arnoult, C., Sinzelle, J., and Bachelier, A., J. Opt. Soc. Am., in press (1977). Hfs PT

Bender, I., Penselin, S., and Schlüpmann, K., Z. Phys. 179, 4 (1964). ZE

Blaise, J., Camus, P., and Wyart, J. F., in Gmelin Handbuch der Anorganischen Chemie, Vol. 39, Part B4 (SpringerVerlag, Berlin, 1976). EL ND CL ZE Hfs

Blaise, J., and Verges, J., unpublished material (1976, 1977). EL ZE

Childs, W. J., Phys. Rev. A 2, 316 (1970). ZE Hfs

Childs, W. J., and Goodman, L. S., J. Opt. Soc. Am. 59, 875 (1969). ZE Hfs

Conway, J. G., and Wybourne, B. G., Phys. Rev. 130, 2325 (1963). PT

Crosswhite, H. M., unpublished material (1976). PT

Davis, S. P., Astrophys. J. 132, 486 (1960). ZE Hfs

King, A. S., Astrophys. J. 72, 221 (1930). W Hfs

Klinkenberg, P. F. A., Z. Phys. 180, 174 (1964). EL ZE

Klinkenberg, P. F. A., Physica (Utrecht) 32, 1113 (1966). EL CL W ZE

Klinkenberg, P. F. A., Physica (Utrecht) 37, 197 (1967). EL CL W ZE

Klinkenberg, P. F. A., Physica (Utrecht) 57, 594 (1972a). EL CL W ZE

Klinkenberg, P. F. A., unpublished material (1972b, 1976, 1977). EL ZE

Klinkenberg, P. F. A., and Meinders, E., Physica (Utrecht) 32, 1617 (1966). EL CL W ZE

Klinkenberg, P. F. A., and Meinders, E., Physica (Utrecht) 42, 213 (1969). EL CL W ZE

Klinkenberg, P. F. A., and van Kleef, T. A. M., Physica (Utrecht) 50, 625 (1970). EL CL W ZE

Meinders, E., and Klinkenberg, P. F. A., Physica (Utrecht) 38, 253 (1968). EL CL W ZE

Spector, N., J. Phys. (Paris) Colloq. C 4 31, 173 (1970). PT 
Spector, N., J. Opt. Soc. Am. 61, 1350 (1971). ND

Sugar, J., J. Res. Nat. Bur. Stand. (U.S.) 66A, 321 (1962). CL

Worden, E. F., Conway, J. G., Paisner, J. A., and Solarz, R. W., unpublished material (1977). IP

[March 1977]

Tb I, Odd Parity

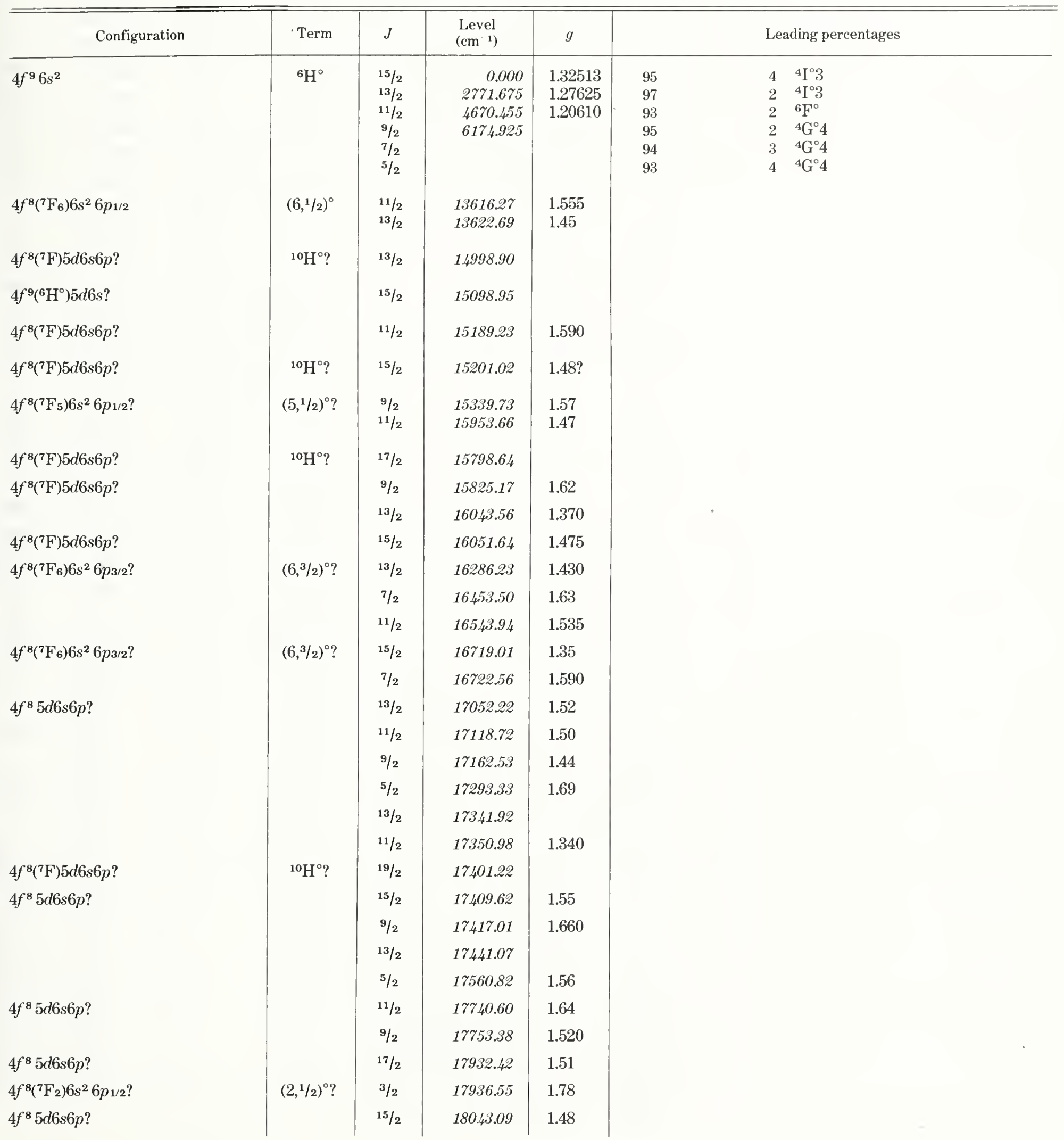


Tb I, Odd Parity-Continued

\begin{tabular}{|c|c|c|c|c|c|c|c|c|c|}
\hline Configuration & Term & $J$ & $\begin{array}{l}\text { Level } \\
\left(\mathrm{cm}^{-1}\right)\end{array}$ & $g$ & Configuration & Term & $J$ & $\begin{array}{l}\text { Level } \\
\left(\mathrm{cm}^{-1}\right)\end{array}$ & $g$ \\
\hline \multirow{6}{*}{$4 f^{8} 5 d 6 s 6 p ?$} & \multirow{43}{*}{$(1,1 / 2)^{\circ} ?$} & $7 / 2$ & 18120.77 & 1.56 & & & $7 / 2$ & 20032.52 & 1.4 \\
\hline & & $13 / 2$ & 18135.88 & 1.46 & & & $9 / 2$ & 20035.06 & 1.513 \\
\hline & & $11 / 2$ & 18182.98 & 1.47 & & & $5 / 2$ & 20042.63 & 1.398 \\
\hline & & $3 / 2$ & 18192.91 & 1.480 & & & $13 / 2$ & 20140.04 & \\
\hline & & $7 / 2$ & 18260.38 & 1.48 & & & $11 / 2$ & 20250.48 & \\
\hline & & $11 / 2$ & 18343.68 & 1.45 & & & $13 / 2$ & 20306.01 & \\
\hline $4 f^{8}\left({ }^{7} F_{1}\right) 6 s^{2} 6 p_{1 / 2} ?$ & & $1 / 2$ & 18397.56 & 2.35 & & & $11 / 2$ & 20373.50 & 1.540 \\
\hline $4 f^{9} 5 d 6 s ?$ & & $15 / 2$ & 18410.55 & & & & $9 / 2$ & 20435.91 & 1.414 \\
\hline \multirow[t]{3}{*}{$4 f^{8} 5 d 6 s 6 p ?$} & & $11 / 2$ & 18461.87 & 1.555 & & & $7 / 2$ & 20473.43 & 1.73 \\
\hline & & $9 / 2$ & 18497.20 & 1.465 & & & $17 / 2$ & 20534.69 & 1.47 \\
\hline & & $1 / 2$ & 18572.92 & 0.51 & & & $5 / 2$ & 20606.69 & 1.205 \\
\hline \multirow[t]{10}{*}{$4 f^{8} 5 d 6 s 6 p ?$} & & $13 / 2$ & 1860724 & 1.48 & & & $9 / 2$ & 20626.26 & 1.42 \\
\hline & & $9 / 2$ & 18611.47 & 1.38 & & & $7 / 2$ & 20684.03 & 1.44 \\
\hline & & $9 / 2$ & 18645.50 & 1.54 & & & $3 / 2$ & 20688.02 & 1.065 \\
\hline & & $5 / 2$ & 18709.99 & 1.596 & & & $15 / 2$ & 20700.64 & 1.42 \\
\hline & & $13 / 2$ & 18805.02 & 1.435 & $4 f^{8} 5 d 6 s 6 p ?$ & ${ }^{10} I^{\circ} ?$ & $19 / 2$ & 20750.70 & 1.39 \\
\hline & & $9 / 2$ & 18850.90 & 1.310 & & & $7 / 2$ & 20762.60 & 1.675 \\
\hline & & $15 / 2$ & 18881.74 & 1.46 & & & $13 / 2$ & 20793.11 & 1.53 \\
\hline & & $11 / 2$ & 18882.27 & 1.44 & & & $11 / 2$ & 20796.05 & 1.45 \\
\hline & & $5 / 2$ & 18915.43 & 1.385 & & & $5 / 2$ & 20823.35 & 1.99 \\
\hline & & $7 / 2$ & 19025.06 & 1.54 & & & $13 / 2$ & 20928.12 & 1.43 \\
\hline \multirow[t]{2}{*}{$4 f^{8} 5 d 6 s 6 p ?$} & & $15 / 2$ & 19080.13 & 1.48 & & & $13 / 2$ & 20989.87 & \\
\hline & & $3 / 2$ & 19108.85 & 1.75 & & & $5 / 2$ & 21004.78 & 1.668 \\
\hline \multirow[t]{2}{*}{$4 f^{8} 5 d 6 s 6 p ?$} & & $17 / 2$ & 19131.45 & 1.41 & $4 f^{8} 5 d 6 s 6 p ?$ & & $15 / 2$ & 21083.72 & 1.525 \\
\hline & & $9 / 2$ & 19155.06 & 1.600 & & & $9 / 2$ & 21130.59 & 1.708 \\
\hline \multirow[t]{6}{*}{$4 f^{8} 5 d 6 s 6 p ?$} & & $17 / 2$ & 19162.31 & 1.30 & & & $11 / 2$ & 21197.15 & 1.30 \\
\hline & & $13 / 2$ & 19269.98 & 1.35 & & & $13 / 2$ & 21217.93 & 1.45 \\
\hline & & $3 / 2$ & 19276.50 & 1.065 & & & $7 / 2$ & 21223.58 & 1.70 \\
\hline & & $7 / 2$ & 19310.33 & 1.584 & & & $5 / 2$ & 21282.61 & 1.79 \\
\hline & & $1 / 2$ & 19313.20 & 3.50 & $4 f^{9} 5 d 6 s ?$ & & $17 / 2$ & 21324.37 & \\
\hline & & $11 / 2$ & 1931728 & 1.36 & & & $15 / 2$ & 21397.91 & 1.45 \\
\hline \multirow[t]{4}{*}{$4 f^{8} 5 d 6 s 6 p ?$} & & $11 / 2$ & 19332.48 & 1.52 & & & $7 / 2$ & 21422.05 & \\
\hline & & $1 / 2$ & 19421.90 & -1.06 & & & $5 / 2$ & $\begin{array}{l}21479.41 \\
9110699\end{array}$ & 1.40 \\
\hline & & $9 / 2$ & 19450.45 & 1.58 & & & $9 / 2$ & 21518.77 & 1.40 \\
\hline & & $15 / 2$ & 19598.19 & 1.40 & & & $11 / 2$ & 21673.72 & 1.422 \\
\hline \multirow[t]{2}{*}{$4 f^{8} 5 d 6 s 6 p ?$} & & $13 / 2$ & 19606.52 & 1.54 & & & $5 / 2$ & 21679.30 & 1.69 \\
\hline & & $13 / 2$ & 19658.41 & 1.455 & & & $7 / 2$ & 21729.64 & 1.28 \\
\hline \multirow[t]{6}{*}{$4 f^{8} 5 d 6 s 6 p ?$} & & $9 / 2$ & 19691.12 & 1.650 & & & $3 / 2$ & 21731.92 & 1.99 \\
\hline & & $7 / 2$ & 19694.70 & 1.422 & & & $7 / 2$ & 21772.73 & 1.67 \\
\hline & & $5 / 2$ & 19759.83 & 1.90 & & & $15 / 2$ & 21812.08 & 1.43 \\
\hline & & $11 / 2$ & 19781.23 & 1.43 & $4 f^{8} 5 d 6 s 6 p ?$ & & $17 / 2$ & 21837.92 & 1.43 \\
\hline & & $7 / 2$ & 19901.15 & 1.52 & & & $13 / 2$ & 21854.83 & 1.35 \\
\hline & & $\begin{array}{l}7 / 2 \\
11 / 2\end{array}$ & $\begin{array}{l}19941.34 \\
19958.55\end{array}$ & $\begin{array}{l}1.46 \\
1.508\end{array}$ & & & $11 / 2$ & 21872.93 & 1.44 \\
\hline
\end{tabular}


Tb I, Odd Parity - Continued

Tb I, Odd Parity-Continued

\begin{tabular}{|c|c|c|c|c|c|c|c|c|c|}
\hline Configuration & Term & $J$ & $\begin{array}{l}\text { Level } \\
\left(\mathrm{cm}^{-1}\right)\end{array}$ & $g$ & Configuration & Term & $J$ & $\begin{array}{l}\text { Level } \\
\left(\mathrm{cm}^{-1}\right)\end{array}$ & $g$ \\
\hline \multirow{13}{*}{$4 f^{8} 5 d 6 s 6 p ?$} & \multirow{13}{*}{${ }^{10} I^{\circ} ?$} & $5 / 2$ & 21879.38 & 2.20 & \multirow[t]{44}{*}{$4 f^{8} 5 d 6 s 6 p ?$} & & $15 / 2$ & 23464.78 & 1.43 \\
\hline & & $9 / 2$ & 21887.16 & 1.355 & & & $5 / 2$ & 23487.88 & 2.05 \\
\hline & & $21 / 2$ & $21946.46 ?$ & & & & $5 / 2$ & 23492.50 & \\
\hline & & $11 / 2$ & 21987.34 & 1.58 & & & $9 / 2$ & 23519.94 & \\
\hline & & $9 / 2$ & 22011.12 & 1.33 & & & $13 / 2$ & 23689.81 & 1.36 \\
\hline & & $15 / 2$ & 22084.34 & 1.490 & & & $13 / 2$ & 23720.34 & 1.48 \\
\hline & & $3 / 2$ & 22178.66 & 2.345 & & & $9 / 2$ & 23725.23 & 1.40 \\
\hline & & $5 / 2$ & 22183.77 & 1.37 & & & $3 / 2$ & 23756.97 & 1.50 \\
\hline & & $9 / 2$ & 22202.52 & 1.634 & & & $17 / 2$ & 23797.22 & \\
\hline & & $13 / 2$ & 22222.58 & & & & $13 / 2$ & 23847.54 & 1.33 \\
\hline & & $15 / 2$ & 22223.87 & 1.375 & & & $15 / 2$ & 23889.75 & 1.38 \\
\hline & & $1 / 2$ & 22264.59 & 3.620 & & & $15 / 2$ & 23907.28 & 1.42 \\
\hline & & $11 / 2$ & 22278.93 & & & & $11 / 2$ & 23951.55 & 1.49 \\
\hline \multirow[t]{31}{*}{$4 f^{8} 5 d 6 s 6 p ?$} & \multirow[t]{31}{*}{${ }^{8} \mathbf{I}^{\circ} ?$} & $19 / 2$ & 22311.58 & 1.41 & & & $9 / 2$ & 23957.26 & 1.59 \\
\hline & & $9 / 2$ & 22327.98 & 1.45 & & & $13 / 2$ & 24003.73 & 1.44 \\
\hline & & $13 / 2$ & 22382.12 & 1.36 & & & $7 / 2$ & 24032.11 & 1.50 \\
\hline & & $5 / 2$ & 22394.69 & 1.910 & & & $13 / 2$ & 24049.29 & \\
\hline & & $13 / 2$ & 22492.98 & 1.44 & & & $7 / 2$ & 24075.69 & 1.32 \\
\hline & & $11 / 2$ & 22524.15 & & & & $15 / 2$ & 24085.58 & 1.35 \\
\hline & & $7 / 2$ & 22556.78 & 1.69 & & & $13 / 2$ & 24088.51 & 1.38 \\
\hline & & $3 / 2$ & 22617.87 & 1.78 & & & $9 / 2$ & 24092.09 & \\
\hline & & $13 / 2$ & 22644.55 & & & & $17 / 2$ & 24188.61 & \\
\hline & & $9 / 2$ & 22663.07 & 1.34 & & & $13 / 2$ & 24243.36 & 1.38 \\
\hline & & $5 / 2$ & 22706.51 & 1.515 & & & $5 / 2$ & 24271.04 & 1.28 \\
\hline & & $13 / 2$ & 22727.88 & & & & $15 / 2$ & 24325.67 & 1.397 \\
\hline & & $17 / 2$ & 22736.76 & 1.42 & & & $9 / 2$ & 2436328 & 1.31 \\
\hline & & $11 / 2$ & 22754.99 & & & & $11 / 2$ & 24392.79 & 1.335 \\
\hline & & $11 / 2$ & 22830.55 & & & & $3 / 2$ & 24420.32 & 1.528 \\
\hline & & $15 / 2$ & 22838.79 & & & & $11 / 2$ & 24438.71 & 1.08 \\
\hline & & $9 / 2$ & 22842.94 & 1.41 & & & $3 / 2$ & 24443.57 & 1.816 \\
\hline & & $3 / 2$ & 22868.81 & 1.950 & & & $9 / 2$ & 24481.11 & 1.49 \\
\hline & & $5 / 2$ & 22927.63 & 1.452 & & & $11 / 2$ & 24490.95 & 1.24 \\
\hline & & $7 / 2$ & 22933.38 & 1.155 & & & $3 / 2$ & 24551.64 & 1.38 \\
\hline & & $11 / 2$ & 22959.01 & 1.50 & & & $9 / 2$ & 24557.68 & 1.45 \\
\hline & & $9 / 2$ & 23019.01 & & & & $5 / 2$ & 24613.87 & 1.80 \\
\hline & & $11 / 2$ & 23103.41 & 1.52 & & & $13 / 2$ & 24636.94 & 1.403 \\
\hline & & $9 / 2$ & 23191.65 & & & & $15 / 2$ & 24638.54 & 1.38 \\
\hline & & $15 / 2$ & 23213.87 & 1.38 & & & $11 / 2$ & 24658.90 & 1.40 \\
\hline & & $13 / 2$ & 23248.17 & & & & $11 / 2$ & 24701.62 & 1.38 \\
\hline & & $1 / 2$ & 23248.96 & 3.555 & & & $13 / 2$ & 24769.12 & 1.32 \\
\hline & & $11 / 2$ & 23394.47 & 1.47 & & & $9 / 2$ & 24775.29 & 1.49 \\
\hline & & $11 / 2$ & 23425.83 & 1.46 & & & $11 / 2$ & 24819.21 & 1.36 \\
\hline & & $11 / 2$ & 23438.36 & 1.44 & & & $13 / 2$ & 24824.89 & \\
\hline & & $5 / 2$ & 23455.34 & 1.98 & & & $9 / 2$ & 24843.50 & \\
\hline
\end{tabular}




\begin{tabular}{|c|c|c|c|c|c|c|c|c|c|}
\hline Configuration & Term & $J$ & $\begin{array}{l}\text { Level } \\
\left(\mathrm{cm}^{-1}\right)\end{array}$ & $g$ & Configuration & Term & $J$ & $\begin{array}{l}\text { Level } \\
\left(\mathrm{cm}^{-1}\right)\end{array}$ & $g$ \\
\hline & & $17 / 2$ & 24891.99 & & \multirow{44}{*}{1} & \multirow{44}{*}{ - } & $11 / 2$ & 26297.75 & 1.27 \\
\hline & & $15 / 2$ & 24938.29 & 1.539 & & & $9 / 2$ & 26301.35 & 1.469 \\
\hline & & $9 / 2$ & 25014.66 & 1.28 & & & $13 / 2$ & 26318.00 & \\
\hline & & $7 / 2$ & 25045.93 & 1.35 & & & $11 / 2$ & 26334.67 & 1.48 \\
\hline & & $9 / 2$ & 25065.78 & 1.45 & & & $13 / 2$ & 26385.99 & \\
\hline & & $15 / 2$ & 25076.26 & 1.42 & & & $7 / 2$ & 26394.48 & 1.40 \\
\hline & & $13 / 2$ & 25122.34 & 1.374 & & & $3 / 2$ & 26403.01 & 1.35 \\
\hline & & $11 / 2$ & 25131.17 & 1.42 & & & $11 / 2$ & 26411.89 & 1.24 \\
\hline & & $7 / 2$ & 25134.81 & 1.42 & & & $11 / 2$ & 26438.75 & 1.44 \\
\hline & & $13 / 2$ & 25210.80 & & & & $9 / 2$ & 26441.33 & 1.75 \\
\hline & & $11 / 2$ & 25211.61 & 1.41 & & & $13 / 2$ & 26488.10 & \\
\hline & & $9 / 2$ & 25226.69 & 1.40 & & & $11 / 2$ & 26521.43 & \\
\hline & & $7 / 2$ & 25241.44 & 1.73 & & & $7 / 2$ & 26522.48 & 1.87 \\
\hline & & $3 / 2$ & 25340.54 & & & & $9 / 2$ & 26580.61 & 1.27 \\
\hline & & $9 / 2$ & 25348.99 & 1.38 & & & $13 / 2$ & 26584.75 & 1.36 \\
\hline & & $13 / 2$ & 25393.16 & 1.28 & & & $7 / 2$ & 26594.05 & 1.67 \\
\hline & & $7 / 2$ & 25433.81 & 1.045 & & & $7 / 2$ & 26668.76 & 1.35 \\
\hline & & $5 / 2$ & 25476.12 & & & & $9 / 2$ & 26673.59 & 1.40 \\
\hline & & $9 / 2$ & 25509.79 & 1.29 & & & $15 / 2$ & 26708.32 & \\
\hline & & $9 / 2$ & 25584.72 & 1.41 & & & $5 / 2$ & 26728.63 & 1.21 \\
\hline & & $7 / 2$ & 25652.48 & 1.38 & & & $9 / 2$ & 26757.16 & 1.25 \\
\hline & & $5 / 2$ & 25676.81 & 0.74 & & & $13 / 2$ & 26787.51 & 1.60 \\
\hline & & $13 / 2$ & 25724.41 & & & & $7 / 2$ & 26792.54 & \\
\hline & & $11 / 2$ & 25753.40 & 1.12 & & & $11 / 2$ & 26808.33 & 1.54 \\
\hline & & $5 / 2$ & 25782.97 & 1.14 & & & $1 / 2$ & 26858.68 & -0.400 \\
\hline & & $13 / 2$ & 25806.10 & 1.54 & & & $11 / 2$ & 26916.91 & 1.31 \\
\hline & & $11 / 2$ & 25818.28 & 1.45 & & & $13 / 2$ & 26950.31 & \\
\hline & & $11 / 2$ & 25859.31 & & & & $9 / 2$ & 26966.53 & 1.695 \\
\hline & & $9 / 2$ & 25859.83 & 1.39 & & & $13 / 2$ & 26973.81 & 1.38 \\
\hline & & $7 / 2$ & 25908.59 & 1.46 & & & $11 / 2$ & 27019.91 & 1.200 \\
\hline & & $9 / 2$ & 26006.65 & 1.37 & & & $9 / 2$ & 27023.13 & 1.39 \\
\hline & & $13 / 2$ & 26043.00 & 1.44 & & & $5 / 2$ & 27025.22 & 1.45 \\
\hline & & $7 / 2$ & 26051.19 & 1.37 & & & $13 / 2$ & 27046.18 & \\
\hline & & $13 / 2$ & 26086.25 & 1.3 & & & $11 / 2$ & 27061.52 & \\
\hline \multirow[t]{10}{*}{$4 f^{8} 5 d 6 s 6 p ?$} & & $17 / 2$ & 26087.13 & 1.406 & & & $9 / 2$ & 27102.94 & \\
\hline & & $11 / 2$ & 26091.79 & 1.30 & & & $9 / 2$ & 27152.53 & 1.34 \\
\hline & & $15 / 2$ & 26097.92 & 1.31 & & & $15 / 2$ & 27154.07 & 1.26 \\
\hline & & $9 / 2$ & 26118.30 & 1.192 & & & $11 / 2$ & 27220.46 & 1.37 \\
\hline & & $7 / 2$ & 26123.52 & 1.59 & & & $7 / 2$ & 27259.08 & 1.44 \\
\hline & & $13 / 2$ & 26157.32 & 1.32 & & & $13 / 2$ & 27295.14 & 1.36 \\
\hline & & $5 / 2$ & 26231.14 & 1.66 & & & $3 / 2$ & 27308.16 & 0.08 \\
\hline & & $11 / 2$ & 26240.55 & 1.54 & & & $9 / 2$ & 27314.12 & 1.34 \\
\hline & & $13 / 2$ & 26253.55 & & & & $13 / 2$ & 27344.14 & 1.27 \\
\hline & & $7 / 2$ & 26280.29 & 1.38 & & & $5 / 2$ & 27355.22 & 1.275 \\
\hline
\end{tabular}


Tb I, Odd Parity-Continued

Tb I, Odd Parity—Continued

\begin{tabular}{|c|c|c|c|c|c|c|c|c|c|}
\hline Configuration & Term & $J$ & $\begin{array}{c}\text { Level } \\
\left(\mathrm{cm}^{-1}\right)\end{array}$ & $g$ & Configuration & Term & $J$ & $\begin{array}{l}\text { Level } \\
\left(\mathrm{cm}^{-1}\right)\end{array}$ & $g$ \\
\hline \multirow[t]{4}{*}{$4 f^{8} 5 d^{2} 6 p ?$} & & $13 / 2$ & 27399.10 & 1.43 & & & $3 / 2$ & 28425.93 & 0.39 \\
\hline & & $11 / 2$ & 27402.68 & 1.38 & & & $1 / 2$ & 28453.45 & 3.14 \\
\hline & & $15 / 2$ & 27420.09 & 1.406 & & & $5 / 2$ & 28456.91 & \\
\hline & & $9 / 2$ & 27450.52 & 1.38 & & & $15 / 2$ & 28488.40 & 1.34 \\
\hline \multirow[t]{8}{*}{$4 f^{8} 5 d^{2} 6 p ?$} & & $17 / 2$ & 27458.75 & 1.365 & & & $11 / 2$ & 28500.52 & \\
\hline & & $15 / 2$ & 27473.13 & 1.39 & & & $7 / 2$ & 28503.79 & 1.32 \\
\hline & & $11 / 2$ & 27492.32 & 1.41 & & & $5 / 2$ & 28508.80 & 1.58 \\
\hline & & $5 / 2$ & 27525.58 & 1.21 & & & $13 / 2$ & 28518.76 & 1.32 \\
\hline & & $13 / 2$ & 27528.34 & 1.61 & & & $9 / 2$ & 28522.59 & 1.57 \\
\hline & & $7 / 2$ & 27531.99 & 1.26 & & & $11 / 2$ & 28553.97 & 1.37 \\
\hline & & $11 / 2$ & 27576.77 & 1.42 & & & $5 / 2$ & 28585.93 & 1.78 \\
\hline & & $9 / 2$ & 27581.87 & 1.32 & & & $7 / 2$ & 28605.10 & 1.27 \\
\hline \multirow[t]{9}{*}{$4 f^{8} 5 d^{2} 6 p ?$} & & $15 / 2$ & 27611.74 & 1.42 & & & $3 / 2$ & 28670.03 & 1.40 \\
\hline & & $3 / 2$ & 27633.57 & 1.805 & & & $5 / 2$ & 28683.95 & 1.65 \\
\hline & & $13 / 2$ & 27664.77 & 1.20 & & & $7 / 2$ & 28759.89 & 1.64 \\
\hline & & $3 / 2$ & 27684.64 & 1.52 & & & $9 / 2$ & 28781.76 & 1.38 \\
\hline & & $13 / 2$ & 27698.95 & 1.29 & & & $9 / 2$ & 28860.49 & 1.7 \\
\hline & & $5 / 2$ & 27758.18 & 1.18 & & & $13 / 2$ & 28890.24 & 1.500 \\
\hline & & $13 / 2$ & 27833.58 & & & & $9 / 2$ & 28916.53 & \\
\hline & & $13 / 2$ & 27848.42 & 1.32 & & & $11 / 2$ & 28919.14 & 1.48 \\
\hline & & $7 / 2$ & 27914.33 & 1.42 & & & $11 / 2$ & 28971.23 & \\
\hline \multirow[t]{13}{*}{$4 f^{8} 5 d^{2} 6 p ?$} & & $9 / 2$ & 27923.11 & 1.54 & & & $11 / 2$ & 28971.64 & 1.41 \\
\hline & & $13 / 2$ & 27935.09 & 1.39 & & & $9 / 2$ & 28989.47 & \\
\hline & & $13 / 2$ & 27941.95 & & & & $7 / 2$ & 29041.65 & 1.77 \\
\hline & & $9 / 2$ & 27952.26 & & & & $1 / 2$ & 29064.13 & 1.79 \\
\hline & & $11 / 2$ & 27959.07 & 1.24 & & & $13 / 2$ & 29103.40 & \\
\hline & & $3 / 2$ & 27964.82 & 1.25 & & & $9 / 2$ & 29126.41 & 1.400 \\
\hline & & $11 / 2$ & 27991.54 & & & & $5 / 2$ & 29204.26 & 1.46 \\
\hline & & $9 / 2$ & 28048.09 & 1.32 & & & $9 / 2$ & 29210.56 & 1.46 \\
\hline & & $11 / 2$ & 28065.44 & 1.42 & & & $11 / 2$ & 29244.64 & 1.370 \\
\hline & & $5 / 2$ & 28070.33 & 1.08 & & & $17 / 2$ & 29306.28 & \\
\hline & & $13 / 2$ & 28096.73 & & & & $11 / 2$ & 29326.10 & \\
\hline & & $5 / 2$ & 28156.97 & 1.54 & & & $7 / 2$ & 29366.03 & 1.40 \\
\hline & & $11 / 2$ & 28188.70 & & & & $15 / 2$ & 29388.78 & 1.385 \\
\hline \multirow[t]{10}{*}{$4 f^{8} 5 d^{2} 6 p ?$} & & $17 / 2$ & 28193.17 & 1.40 & & & $19 / 2$ & 29391.66 & \\
\hline & & $7 / 2$ & 2822024 & 1.28 & & & $5 / 2$ & 29399.99 & 1.13 \\
\hline & & $7 / 2$ & 28306.08 & 1.81 & & & $9 / 2$ & 29433.01 & 1.41 \\
\hline & & $11 / 2$ & 28315.50 & & & & $9 / 2$ & 29465.99 & 1.46 \\
\hline & & $9 / 2$ & 28318.45 & 1.35 & & & $5 / 2$ & 29483.46 & 1.32 \\
\hline & & $7 / 2$ & 28358.13 & 1.36 & & & $13 / 2$ & 29543.01 & 1.40 \\
\hline & & $13 / 2$ & 28372.18 & & & & $7 / 2$ & 29577.08 & 1.340 \\
\hline & & $11 / 2$ & 28417.42 & & & & $11 / 2$ & 29613.14 & \\
\hline & & $9 / 2$ & 28420.95 & 1.46 & & & $11 / 2$ & 29638.28 & 1.39 \\
\hline & & $7 / 2$ & 28421.89 & 1.42 & & & $13 / 2$ & 29702.72 & 1.48 \\
\hline
\end{tabular}


Tb I, Odd Parity-Continued

Tb I, Odd Parity-Continued

\begin{tabular}{|c|c|c|c|c|c|c|c|c|c|}
\hline Configuration & Term & $J$ & $\begin{array}{l}\text { Level } \\
\left(\mathrm{cm}^{-1}\right)\end{array}$ & $g$ & Configuration & Term & $J$ & $\begin{array}{l}\text { Level } \\
\left(\mathrm{cm}^{-1}\right)\end{array}$ & $g$ \\
\hline & & $9 / 2$ & 29726.86 & & & & $11 / 2$ & 31221.87 & \\
\hline & & $11 / 2$ & 29727.09 & 1.42 & & & $5 / 2$ & 31307.25 & \\
\hline & & $7 / 2$ & 29729.33 & 1.610 & & & $7 / 2$ & 31333.25 & 1.44 \\
\hline & & $11 / 2$ & 29737.61 & 1.50 & & & $9 / 2$ & 31366.57 & 1.40 \\
\hline & & $3 / 2$ & 29769.46 & 0.42 & & & $11 / 2$ & 31400.97 & 1.39 \\
\hline & & $3 / 2$ & 29831.99 & 1.16 & & & $15 / 2$ & 31406.00 & 1.32 \\
\hline & & $15 / 2$ & 29859.73 & & & & $9 / 2$ & 31477.50 & 1.41 \\
\hline & & $11 / 2$ & 29861.37 & 1.37 & & & $5 / 2$ & 31480.38 & \\
\hline & & $13 / 2$ & 29867.24 & 1.36 & & & $7 / 2$ & 31492.90 & \\
\hline & & $7 / 2$ & 29920.10 & 1.49 & & & $13 / 2$ & 31542.01 & $1.43 ?$ \\
\hline & & $11 / 2$ & 30054.07 & 1.35 & & & $15 / 2$ & 31563.57 & 1.56 \\
\hline & & $17 / 2$ & 30058.22 & & & & $9 / 2$ & 31576.16 & \\
\hline $4 f^{8} 5 d^{2} 6 p ?$ & & $15 / 2$ & 30068.35 & 1.43 & & & $5 / 2$ & 31595.48 & \\
\hline $4 f^{9}\left({ }^{6} \mathrm{H}^{\circ}\right) 6 s 7 s$ & & $17 / 2$ & 30095.53 & & & & $19 / 2$ & 31613.90 & \\
\hline & & $9 / 2$ & 30248.43 & 1.37 & & & $11 / 2$ & 31695.19 & 1.41 \\
\hline & & $5 / 2$ & 30253.95 & 1.590 & & & $5 / 2$ & 31706.41 & \\
\hline & & $7 / 2$ & 30272.65 & 1.23 & & & $9 / 2$ & 31715.80 & 1.325 \\
\hline & & $19 / 2$ & 30358.22 & & & & $13 / 2$ & 31800.43 & \\
\hline & & $13 / 2$ & 30405.08 & & & & $9 / 2$ & 31859.31 & 1.45 \\
\hline & & $7 / 2$ & 30410.44 & 1.2 & & & $7 / 2$ & 31901.42 & 1.34 \\
\hline & & $7 / 2$ & 30437.06 & 1.33 & & & $3 / 2$ & 31967.40 & 1.46 \\
\hline & & $9 / 2$ & 30546.75 & 1.37 & & & $15 / 2$ & 32015.99 & 1.91 \\
\hline & & $13 / 2$ & 30550.07 & 1.66 & & & $5 / 2$ & 32071.19 & 1.352 \\
\hline & & $15 / 2$ & 30577.26 & & & & $7 / 2$ & 32158.93 & 1.59 \\
\hline & & $9 / 2$ & 30598.84 & 1.36 & & & $9 / 2$ & 32163.47 & 1.37 \\
\hline $4 f^{9}\left({ }^{6} \mathrm{H}^{\circ}\right) 6 s 7 s$ & & $15 / 2$ & 30607.47 & & & & $5 / 2$ & 32217.55 & 1.375 \\
\hline & & $9 / 2$ & 30643.49 & 1.27 & & & $5 / 2$ & 32269.95 & \\
\hline & & $5 / 2$ & 30647.81 & 1.51 & & & $5 / 2$ & 32516.84 & 1.330 \\
\hline & & $17 / 2$ & 30666.00 & & & & $7 / 2$ & 32583.24 & 1.41 \\
\hline & & $7 / 2$ & 30680.26 & 1.41 & & & $13 / 2$ & 32605.35 & 1.5 \\
\hline & & $11 / 2$ & 30695.07 & 1.355 & & & $5 / 2$ & 32643.88 & 1.370 \\
\hline & & $9 / 2$ & 30698.72 & 1.35 & & & $11 / 2$ & 32656.68 & 1.55 \\
\hline & & $3 / 2$ & 30774.74 & 1.740 & & & $7 / 2$ & 32749.98 & 1.405 \\
\hline & & $9 / 2$ & 30789.68 & 1.07 & & & $5 / 2$ & 32787.16 & \\
\hline & & $13 / 2$ & 30825.57 & 1.41 & & & $5 / 2$ & 32829.70 & \\
\hline & & $3 / 2$ & 30852.95 & & & & $11 / 2$ & 32832.09 & 1.53 \\
\hline & & $11 / 2$ & 30916.01 & & & & $11 / 2$ & 32911.90 & \\
\hline & & $1 / 2$ & 30928.77 & 1.685 & & & $5 / 2$ & 32961.21 & \\
\hline & & $13 / 2$ & 30947.95 & 1.5 & & & $23 / 2$ & 33051.16 & \\
\hline & & $15 / 2$ & 30958.07 & & & & $11 / 2$ & 33151.06 & \\
\hline & & $5 / 2$ & 30969.15 & & & & $13 / 2$ & 33293.14 & \\
\hline & & $11 / 2$ & 30976.92 & 1.51 & & & $19 / 2$ & 33307.42 & \\
\hline & & $7 / 2$ & 31093.42 & 1.5 & & & $3 / 2$ & 33348.70 & \\
\hline & & $5 / 2$ & 31131.43 & 1.31 & & & $11 / 2$ & 33454.94 & \\
\hline
\end{tabular}


Tb I, Odd Parity-Continued

Tb 1, Odd Parity-Continued

\begin{tabular}{|c|c|c|c|c|c|c|c|c|c|}
\hline Configuration & Term & $J$ & $\begin{array}{l}\text { Level } \\
\left(\mathrm{cm}^{-1}\right)\end{array}$ & $g$ & Configuration & Term & $J$ & $\begin{array}{l}\text { Level } \\
\left(\mathrm{cm}^{-1}\right)\end{array}$ & $g$ \\
\hline & & $11 / 2$ & 33568.03 & 1.52 & & & $9 / 2$ & 34139.40 & \\
\hline & & $9 / 2$ & 33638.32 & 1.53 & & & $13 / 2$ & 34150.86 & \\
\hline & & $21 / 2$ & 33669.32 & & & & $21 / 2$ & 34223.95 & \\
\hline & & $1 / 2$ & 33696.38 & 3.880 & & & $7 / 2$ & 3444721 & 1.76 \\
\hline & & $7 / 2$ & 33727.01 & & & & $19 / 2$ & 3461623 & \\
\hline & & $9 / 2$ & 33771.57 & 1.46 & & & $19 / 2$ & 34999.92 & \\
\hline & & $11 / 2$ & 33839.15 & 1.60 & & & & & \\
\hline & & $5 / 2$ & 34114.89 & 1.638 & $\mathrm{~Tb}$ II $\left({ }^{6} \mathrm{H}^{\circ}{ }^{5} / 2\right) 6 s_{1 / 2}(15 / 2,1 / 2)_{8}^{\circ}$ & Limit & & 47295 & \\
\hline
\end{tabular}

Tb I, Even Parity

\begin{tabular}{|c|c|c|c|c|c|c|c|}
\hline Configuration & Term & $J$ & $\begin{array}{l}\text { Level } \\
\left(\mathrm{cm}^{-1}\right)\end{array}$ & $g$ & \multicolumn{3}{|c|}{ Leading percentages } \\
\hline $4 f^{8}\left({ }^{7} \mathrm{~F}\right) 5 d 6 s^{2}$ & ${ }^{8} \mathrm{G}$ & $\begin{array}{l}13 / 2 \\
15 / 2 \\
11 / 2 \\
7 / 2 \\
9 / 2 \\
5 / 2 \\
3 / 2 \\
1 / 2\end{array}$ & $\begin{array}{r}285.500 \\
462.080 \\
509.845 \\
2419.480 \\
2840.170 \\
3174.575 \\
3705.820 \\
4018.210\end{array}$ & $\begin{array}{r}1.46448 \\
1.45624 \\
1.51654 \\
1.47678 \\
1.54375 \\
1.35525 \\
1.02220 \\
-1.19125\end{array}$ & $\begin{array}{l}65 \\
91 \\
35 \\
56 \\
47 \\
73 \\
86 \\
92\end{array}$ & $\begin{array}{r}24 \\
4 \\
30 \\
25 \\
38 \\
16 \\
7 \\
3\end{array}$ & $\begin{array}{l}\left({ }^{7} \mathrm{~F}\right){ }^{8} \mathrm{~F} \\
\left({ }^{7} \mathrm{~F}\right){ }^{8} \mathrm{H} \\
\left({ }^{7} \mathrm{~F}\right){ }^{8} \mathrm{~F} \\
\left({ }^{7} \mathrm{~F}\right){ }^{8} \mathrm{~F} \\
\left({ }^{7} \mathrm{~F}\right){ }^{8} \mathrm{D} \\
\left({ }^{7} \mathrm{~F}\right){ }^{8} \mathrm{~F} \\
\left({ }^{7} \mathrm{~F}\right){ }^{8} \mathrm{~F} \\
\left({ }^{5} \mathrm{D} 1\right)^{6} \mathrm{~F}\end{array}$ \\
\hline $4 f^{8}\left({ }^{7} F\right) 5 d 6 s^{2}$ & & $9 / 2$ & 1371.045 & 1.54110 & $33 \quad{ }^{8} \mathrm{G}$ & 29 & $\left({ }^{7} \mathrm{~F}\right){ }^{8} \mathrm{~F}$ \\
\hline $4 f^{8}\left({ }^{7} F\right) 5 d 6 s^{2}$ & ${ }^{8} \mathrm{D}$ & $\begin{array}{l}11 / 2 \\
7 / 2 \\
5 / 2 \\
3 / 2\end{array}$ & $\begin{array}{l}2310.090 \\
3819.850 \\
4695.505 \\
5483.980\end{array}$ & $\begin{array}{l}1.53020 \\
1.64184 \\
1.83129 \\
2.32\end{array}$ & $\begin{array}{l}48 \\
52 \\
56 \\
51\end{array}$ & $\begin{array}{l}43 \\
29 \\
21 \\
37\end{array}$ & $\begin{array}{l}\left({ }^{7} \mathrm{~F}\right){ }^{8} \mathrm{G} \\
\left({ }^{7} \mathrm{~F}\right){ }^{8} \mathrm{G} \\
\left({ }^{7} \mathrm{~F}\right){ }^{8} \mathrm{~F} \\
\left({ }^{7} \mathrm{~F}\right){ }^{8} \mathrm{~F}\end{array}$ \\
\hline $4 f^{8}\left({ }^{7} \mathrm{~F}\right) 5 d 6 s^{2}$ & ${ }^{8} \mathrm{~F}$ & $\begin{array}{l}13 / 2 \\
11 / 2 \\
9 / 2 \\
1 / 2 \\
7 / 2 \\
5 / 2 \\
3 / 2\end{array}$ & $\begin{array}{l}3719.705 \\
5353.370 \\
5829.860 \\
6259.090 \\
6488.280 \\
6801.190 \\
6849.720\end{array}$ & $\begin{array}{l}1.50473 \\
1.545 \\
1.58 \\
3.84 \\
1.635 \\
1.80 \\
2.335\end{array}$ & $\begin{array}{l}71 \\
54 \\
56 \\
91 \\
58 \\
55 \\
48\end{array}$ & $\begin{array}{r}22 \\
21 \\
13 \\
3 \\
21 \\
31 \\
42\end{array}$ & $\begin{array}{l}\left({ }^{7} \mathrm{~F}\right){ }^{8} \mathrm{G} \\
\left({ }^{7} \mathrm{~F}\right){ }^{8} \mathrm{D} \\
\left({ }^{7} \mathrm{~F}\right){ }^{8} \mathrm{P} \\
\left({ }^{5} \mathrm{D} 1\right)^{6} \mathrm{D} \\
\left({ }^{7} \mathrm{~F}\right){ }^{8} \mathrm{D} \\
\left({ }^{7} \mathrm{~F}\right){ }^{8} \mathrm{D} \\
\left({ }^{7} \mathrm{~F}\right){ }^{8} \mathrm{D}\end{array}$ \\
\hline $4 f^{8}\left({ }^{7} F\right) 5 d 6 s^{2}$ & ${ }^{8} \mathrm{H}$ & $\begin{array}{l}17 / 2 \\
15 / 2 \\
13 / 2 \\
11 / 2 \\
9 / 2 \\
7 / 2 \\
5 / 2 \\
3 / 2\end{array}$ & $\begin{array}{l}4646.830 \\
5425.060 \\
6351.750 \\
6988.820 \\
7441.030 \\
7839.850 \\
8130.680 \\
8336.310\end{array}$ & $\begin{array}{l}1.40626 \\
1.370 \\
1.350 \\
1.315 \\
1.240 \\
1.05 \\
0.705 \\
-0.36\end{array}$ & $\begin{array}{l}95 \\
64 \\
74 \\
74 \\
74 \\
82 \\
87 \\
89\end{array}$ & $\begin{array}{r}2 \\
27 \\
10 \\
7 \\
8 \\
5 \\
5 \\
4\end{array}$ & $\begin{array}{l}\left({ }^{5} \mathrm{G} 1\right)^{6} \mathrm{I} \\
\left({ }^{7} \mathrm{~F}\right){ }^{6} \mathrm{H} \\
\left({ }^{7} \mathrm{~F}\right){ }^{6} \mathrm{H} \\
\left({ }^{7} \mathrm{~F}\right){ }^{6} \mathrm{H} \\
\left({ }^{7} \mathrm{~F}\right){ }^{6} \mathrm{G} \\
\left({ }^{7} \mathrm{~F}\right){ }^{6} \mathrm{G} \\
\left({ }^{7} \mathrm{~F}\right){ }^{6} \mathrm{G} \\
\left({ }^{7} \mathrm{~F}\right){ }^{6} \mathrm{G}\end{array}$ \\
\hline $4 f^{8}\left({ }^{7} \mathrm{~F}\right) 5 d 6 s^{2}$ & ${ }^{6} \mathrm{~F}$ & $\begin{array}{l}11 / 2 \\
9 / 2 \\
7 / 2 \\
5 / 2 \\
3 / 2 \\
1 / 2\end{array}$ & $\begin{array}{r}6674.155 \\
7824.190 \\
8994.660 \\
10030.350\end{array}$ & $\begin{array}{l}1.32 \\
1.433 \\
1.414 \\
1.305\end{array}$ & $\begin{array}{l}71 \\
66 \\
71 \\
79 \\
85 \\
91\end{array}$ & $\begin{array}{r}12 \\
12 \\
12 \\
6 \\
4 \\
3\end{array}$ & $\begin{array}{l}\left({ }^{7} \mathrm{~F}\right){ }^{6} \mathrm{G} \\
\left({ }^{7} \mathrm{~F}\right){ }^{6} \mathrm{D} \\
\left({ }^{7} \mathrm{~F}\right){ }^{6} \mathrm{D} \\
\left({ }^{7} \mathrm{~F}\right) \\
\left({ }^{6} \mathrm{D}\right. \\
\left({ }^{7} \mathrm{~F}\right){ }^{6} \mathrm{G} \\
\left({ }^{5} \mathrm{D} 1\right)^{4} \mathrm{D}\end{array}$ \\
\hline
\end{tabular}


Tb I, Even Parity-Continued

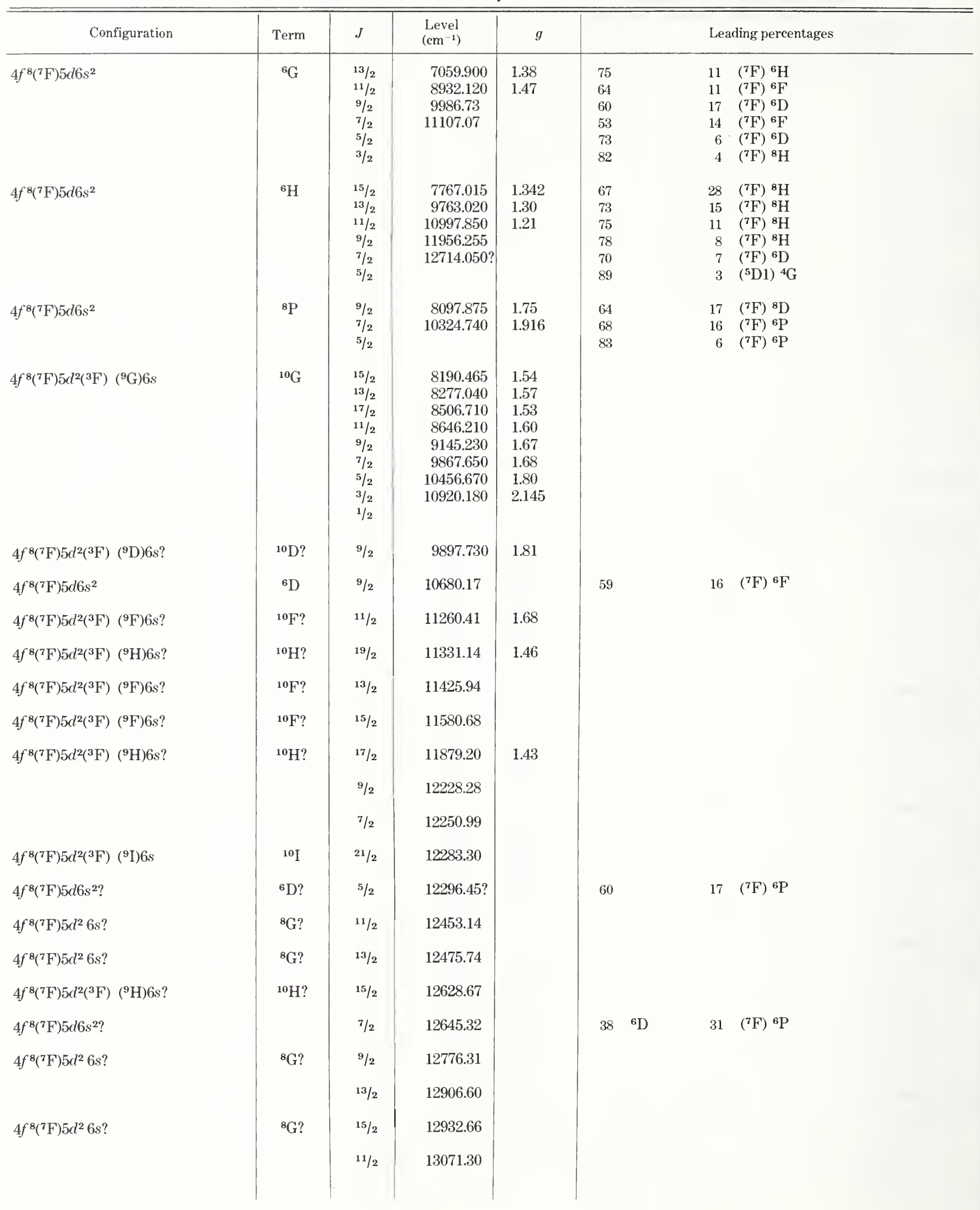


Tb I, Even Parity-Continued

\begin{tabular}{|c|c|c|c|c|c|}
\hline Configuration & Term & $J$ & $\begin{array}{l}\text { Level } \\
\left(\mathrm{cm}^{-1}\right)\end{array}$ & $g$ & Leading percentages \\
\hline & & $13 / 2$ & 13116.48 & & \\
\hline $4 f^{8}\left({ }^{7} \mathrm{~F}\right) 5 d^{2} 6 s ?$ & ${ }^{8} \mathrm{G}$ ? & $7 / 2$ & 13277.23 & & \\
\hline \multirow[t]{4}{*}{$4 f^{8}\left({ }^{7} \mathrm{~F}\right) 5 d^{2}\left({ }^{3} \mathrm{~F}\right)\left({ }^{9} \mathrm{I}\right) 6 s ?$} & ${ }^{10} \mathrm{I} ?$ & $19 / 2$ & 13398.40 & & \\
\hline & & $11 / 2$ & 13666.46 & & \\
\hline & & $7 / 2$ & 13729.12 & & \\
\hline & & $9 / 2$ & 13751.41 & & \\
\hline $4 f^{8} 5 d^{2} 6 s ?$ & & $17 / 2$ & 14016.91 & & \\
\hline $4 f^{8} 5 d^{2} 6 s ?$ & & $15 / 2$ & $14569.67 ?$ & & \\
\hline $4 f^{8} 5 d^{2} 6 s ?$ & & $17 / 2$ & 14718.11 & 1.400 & \\
\hline $4 f^{9}\left({ }^{6} \mathrm{H}_{15 / 2}^{\circ}\right) 6 s 6 p\left({ }^{3} \mathrm{P}_{0}^{\circ}\right) ?$ & $(15 / 2,0) ?$ & $15 / 2$ & 14888.11 & 1.391 & \\
\hline \multirow{3}{*}{$4 f^{8} 5 d^{2} 6 s ?$} & ${ }^{8} \mathrm{H} ?$ & $17 / 2$ & 15189.26 & 1.409 & \\
\hline & & $15 / 2$ & 15387.79 & 1.367 & \\
\hline & & $15 / 2$ & 16343.30 & 1.397 & \\
\hline $4 f^{9} 6 s 6 p ?$ & & $15 / 2$ & 16431.13 & $1.46 ?$ & \\
\hline $4 f^{9}\left({ }^{6} \mathrm{H}_{15 / 2}^{\circ}\right) 6 s 6 p\left({ }^{3} \mathrm{P}_{2}^{\circ}\right) ?$ & $(15 / 2,2) ?$ & $17 / 2$ & 17249.59 & & \\
\hline \multirow[t]{3}{*}{$4 f^{9}\left({ }^{6} \mathrm{H}_{15 / 2}^{\circ}\right) 6 s 6 p\left({ }^{3} \mathrm{P}_{2}^{\circ}\right) ?$} & $(15 / 2,2) ?$ & $13 / 2$ & 17875.98 & & \\
\hline & & $15 / 2$ & 19920.41 & & - \\
\hline & & $15 / 2$ & 23031.84 & 1.24 & \\
\hline \multirow[t]{2}{*}{$4 f^{9}\left({ }^{6} \mathrm{H}_{15 / 2}^{\circ}\right) 6 s 6 p\left({ }^{1} \mathrm{P}_{1}^{\circ}\right)$} & $(15 / 2,1)$ & $13 / 2$ & 23043.43 & $\begin{array}{l}1.391 \\
1.289\end{array}$ & \\
\hline & & $\begin{array}{l}17 / 2 \\
15 / 2\end{array}$ & $\begin{array}{l}23107.25 \\
23147.92\end{array}$ & $\begin{array}{l}1.289 \\
1.339\end{array}$ & \\
\hline \multirow[t]{3}{*}{$4 f^{9}\left({ }^{6} \mathrm{H}_{13 / 2}^{\circ}\right) 6 s 6 p\left({ }^{3} \mathrm{P}_{2}^{\circ}\right) ?$} & $(13 / 2,2) ?$ & $15 / 2$ & 23112.35 & & \\
\hline & & $13 / 2$ & 25373.85 & 1.354 & \\
\hline & & $13 / 2$ & 25553.46 & 1.328 & \\
\hline \multirow[t]{3}{*}{$4 f^{9}\left({ }^{6} \mathrm{H}_{13 / 2}^{\circ}\right) 6 s 6 p\left({ }^{1} \mathrm{P}_{1}^{\circ}\right)$} & $(13 / 2,1)$ & $\begin{array}{l}11 / 2 \\
13 / 2 \\
15 / 2\end{array}$ & $\begin{array}{l}25637.87 \\
25717.68 \\
25825.53\end{array}$ & $\begin{array}{l}1.334 \\
1.300 \\
1.246\end{array}$ & \\
\hline & & $11 / 2$ & 26553.26 & & \\
\hline & & $13 / 2$ & 26592.90 & & \\
\hline $\mathrm{Tb}$ II $\left({ }^{6} \mathrm{H}_{15 / 2}^{\circ}\right) 6 s_{1 / 2}\left(15 / 2,{ }^{1} / 2\right)_{8}^{\circ}$ & Limit & & 47295 & & \\
\hline
\end{tabular}


(Gd I sequence; 64 electrons)

Ground state $\left(1 s^{2} 2 s^{2} 2 p^{6} 3 s^{2} 3 p^{6} 3 d^{10} 4 s^{2} 4 p^{6} 4 d^{10} 5 s^{2} 5 p^{6}\right) 4 f^{9}\left({ }^{6} \mathrm{H}_{15 / 2}^{\circ}\right) 6 s\left({ }^{15 / 2,1 / 2}\right)_{8}^{\circ}$

Ionization energy $92900 \pm 600 \mathrm{~cm}^{-1}$

$11.52 \pm 0.08 \mathrm{eV}$

Identified odd configurations

Identified even configurations
$4 f^{9} 6 s, 4 f^{9} 5 d, 4 f^{8} 6 s 6 p ?, 4 f^{8} 5 d 6 p ?$

$4 f^{8} 5 d 6 s, 4 f^{8} 6 s^{2}, 4 f^{8} 5 d^{2} ?, 4 f^{9} 6 p$

Meinders carried out the basic analysis of this spectrum using a list of 8550 lines (2850$7600 \AA$ ) obtained from observations made at the Argonne National Laboratory and at the Zeeman Laboratory, Amsterdam [Meinders, 1969, 1970]. Relying more on hyperfine-structure regularities than on the Zeeman data, she discovered two systems of energy levels based respectively on the $4 f^{8} 5 d 6 s$ and $4 f^{9} 6 s$ configurations. Verges recorded the infrared spectrum $(0.72-3.3 \mu \mathrm{m})$ at the Laboratoire Aimé Cotton, Orsay, and Blaise classified several infrared transitions connecting the two systems [Blaise, Camus, and Wyart, 1976]. The connection has been confirmed in further work by Meinders and Klinkenberg [1975], who furnished their unpublished list of levels and $g$ values for this compilation. Some additional data and a few revisions due to Blaise and Verges (Orsay) and to Meinders and Klinkenberg (Amsterdam) have been taken from the article by Blaise et al. [1976]. Levels given in the original lists to the nearest $0.005 \mathrm{~cm}^{-1}$ have been truncated to two decimal places.

The wavelength lists for $\mathrm{Tb}$ II mentioned above have not been published, but Meinders' 1969 paper gives about 140 lines (2809-5348 $\AA$ ) with energy-level classifications. The $g$ values are from unpublished Zeeman-effect observations over the range 2850-7700 [Meinders, 1970]. The accuracies are in general limited by hyperfine broadening.

The lowest few $4 f^{9} 6 s$ levels occur in $J_{1} s$-coupling pairs [Meinders, 1970], and we have also listed the leading $L S$-coupling eigenvector percentages for these levels. The percentages for both the $4 f^{9} 6 s$ and $4 f^{9} 5 d$ levels are from preliminary calculations by Crosswhite [1977]. The four lowest $4 f^{9} 5 d$ levels also form a good $J_{1} j$-coupling term, $4 f^{9}\left({ }^{6} \mathrm{H}_{15 / 2}^{\circ}\right) 5 d_{3 / 2}$, as was noted by Wyart, Blaise, and Camus [1974]; these authors located the $J=9$ level of this term, the other three levels having been given by Meinders. The identified $4 f^{9} 5 d$ levels are here designated in the LS scheme, which may be as appropriate for a larger part of this configuration. Second percentages $\leqslant 5 \%$ are omitted for these levels.

No definite interpretation of the upper odd levels yet exists. A few assignments to the $4 f^{8} 6 s 6 p$ and $4 f^{8} 5 d 6 p$ configurations are given tentatively [Blaise, Camus, and Wyart, 1976].

Meinders [1970] established that the lowest even levels belong to $4 f^{8}\left({ }^{7} \mathrm{~F}\right) 5 d 6 s{ }^{9} \mathrm{G},{ }^{9} \mathrm{D},{ }^{7} \mathrm{G}$, and ${ }^{7} \mathrm{~F}$ terms, or to mixtures of these, and she also deduced that the ${ }^{7} \mathrm{~F}_{6}$ level at $5898.35 \mathrm{~cm}^{-1}$ belongs to either $4 f^{8} 6 s^{2}$ or $4 f^{8} 5 d^{2}$. The eigenvectors from a preliminary calculation of $4 f^{8}\left({ }^{7} \mathrm{~F}\right) 5 d 6 s$ by Crosswhite [1977] give no meaningful term designations for several of these levels; no ordering by terms has been attempted here. A few additional levels and tentative assignments to $4 f^{8} 6 s^{2}$ and $4 f^{8} 5 d^{2}$, from recent work at Amsterdam and Orsay, have been included [Blaise et al., 1976]. A calculation of the $4 f^{8}(5 d+6 s)^{2}$ configurations with their interactions would no doubt be helpful in extending the analysis of the even levels. Meinders [1970] has interpreted several of the upper even levels as belonging to $J_{1} j$-coupling terms of the $4 f^{9}\left({ }^{6} \mathrm{H}^{\circ}\right) 6 p$ subconfiguration.

Sugar and Reader derived the ionization energy.

\section{References}

Blaise, J., Camus, P., and Wyart, J. F., in Gmelin Handbuch der Anorganischen Chemie, Vol. 39, Part B4 (SpringerVerlag, Berlin, 1976). EL ND CL ZE Hfs

Crosswhite, H. M., unpublished material (1977). PT

Davis, S. P., Astrophys. J. 132, 486 (1960). ZE Hfs

Dekker, J. W. M., Bloemhof, H. F., Brouwer, J. H., and Klinkenberg, P. F. A., Physica (Utrecht) 46, 119 (1970). Hfs 
King, A. S., Astrophys. J. 72, 221 (1930). W Hfs

Meinders, E., Physica (Utrecht) 42, 427 (1969). EL CL W ZE HfS

Meinders, E., Thesis, Amsterdam, 75 pp. (1970). EL ND ZE Hfs PT

Meinders, E., and Klinkenberg, P. F. A., unpublished material (1975). EL ND ZE Hfs

Sugar, J., and Reader, J., J. Opt. Soc. Am. 55, 1286 (1965). IP

Wyart, J. F., Blaise, J., and Camus, P., Phys. Ser. 9, 322 (1974). EL

[May 1977]

Tb II, Odd Parity

\begin{tabular}{|c|c|c|c|c|c|c|c|c|}
\hline Configuration & Term & $J$ & $\begin{array}{l}\text { Level } \\
\left(\mathrm{cm}^{-1}\right)\end{array}$ & $g$ & \multicolumn{4}{|c|}{ Leading percentages } \\
\hline $4 f^{9}\left({ }^{6} \mathrm{H}_{15 / 2}^{\circ}\right) 6 s_{1 / 2}$ & $(15 / 2,1 / 2)^{\circ}$ & $\begin{array}{l}8 \\
7\end{array}$ & $\begin{array}{r}0.00 \\
1016.38\end{array}$ & 1.37 & $\begin{array}{l}94 \\
90\end{array}$ & $\begin{array}{l}\text { or } \\
\text { or }\end{array}$ & $\begin{array}{l}94 \\
69\end{array}$ & $\begin{array}{l}\left({ }^{6} \mathrm{H}^{\circ}\right){ }^{7} \mathrm{H}^{\circ} \\
\left({ }^{6} \mathrm{H}^{\circ}\right){ }^{5} \mathrm{H}^{\circ}\end{array}$ \\
\hline $4 f^{9}\left({ }^{6} \mathrm{H}_{13 / 2}^{\circ}\right) 6 s_{1 / 2}$ & $(13 / 2,1 / 2)^{\circ}$ & $\begin{array}{l}7 \\
6\end{array}$ & $\begin{array}{l}3010.03 \\
3542.40\end{array}$ & 1.286 & $\begin{array}{l}93 \\
85\end{array}$ & $\begin{array}{l}\text { or } \\
\text { or }\end{array}$ & $\begin{array}{l}71 \\
54\end{array}$ & $\begin{array}{l}\left({ }^{6} \mathrm{H}^{\circ}\right){ }^{7} \mathrm{H}^{\circ} \\
\left({ }^{6} \mathrm{H}^{\circ}\right){ }^{7} \mathrm{H}^{\circ}\end{array}$ \\
\hline $4 f^{9}\left({ }^{6} \mathrm{H}_{11 / 2}\right) 6 s_{1 / 2}$ & $(11 / 2,1 / 2)^{\circ}$ & $\begin{array}{l}6 \\
5\end{array}$ & $\begin{array}{l}5171.76 \\
5234.98\end{array}$ & & $\begin{array}{l}80 \\
80\end{array}$ & $\begin{array}{l}\text { or } \\
\text { or }\end{array}$ & $\begin{array}{l}52 \\
70\end{array}$ & $\begin{array}{l}\left({ }^{6} \mathrm{H}^{\circ}\right){ }^{5} \mathrm{H}^{\circ} \\
\left({ }^{6} \mathrm{H}^{\circ}\right){ }^{7} \mathrm{H}^{\circ}\end{array}$ \\
\hline $4 f^{9}\left({ }^{6} \mathrm{~F}_{11 / 2}^{\circ}\right) 6 s_{1 / 2}$ & $(11 / 2,1 / 2)^{\circ}$ & $\begin{array}{l}6 \\
5\end{array}$ & 6372.95 & 1.40 & $\begin{array}{l}92 \\
55\end{array}$ & $\begin{array}{l}\text { or } \\
\text { or }\end{array}$ & $\begin{array}{l}92 \\
61\end{array}$ & $\begin{array}{l}\left({ }^{6} \mathrm{~F}^{\circ}\right){ }^{7} \mathrm{~F}^{\circ} \\
\left({ }^{6} \mathrm{~F}^{\circ}\right){ }^{7} \mathrm{~F}^{\circ}\end{array}$ \\
\hline $4 f^{9}\left({ }^{6} \mathrm{H}_{9 / 2}^{\circ}\right) 6 s_{1 / 2}$ & $\left(9 / 2,{ }^{1} / 2\right)^{\circ}$ & $\begin{array}{l}4 \\
5\end{array}$ & 6912.49 & 1.10 & $\begin{array}{l}80 \\
77\end{array}$ & $\begin{array}{l}\text { or } \\
\text { or }\end{array}$ & $\begin{array}{l}81 \\
65\end{array}$ & $\begin{array}{l}\left({ }^{6} \mathrm{H}^{\circ}\right){ }^{7} \mathrm{H}^{\circ} \\
\left({ }^{6} \mathrm{H}^{\circ}\right){ }^{5} \mathrm{H}^{\circ}\end{array}$ \\
\hline $4 f^{9}\left({ }^{6} \mathrm{H}^{\circ}\right) 5 d$ & ${ }^{7} \mathrm{H}^{\circ}$ & $\begin{array}{l}8 \\
7 \\
6 \\
5 \\
4 \\
3 \\
2\end{array}$ & $\begin{array}{l}11261.56 \\
12200.40 \\
13605.22 \\
15212.59 \\
16475.05 \\
17475.20\end{array}$ & $\begin{array}{l}1.368 \\
1.339 \\
1.330 \\
1.221 \\
1.058 \\
0.780\end{array}$ & $\begin{array}{l}79 \\
65 \\
54 \\
66 \\
72 \\
77 \\
81\end{array}$ & & $\begin{array}{r}10 \\
15 \\
18 \\
13 \\
8 \\
10 \\
12\end{array}$ & $\begin{array}{l}\left({ }^{6} \mathrm{H}^{\circ}\right){ }^{7} \mathrm{I}^{\circ} \\
\left({ }^{6} \mathrm{H}^{\circ}\right){ }^{7} \mathrm{G}^{\circ} \\
\left({ }^{6} \mathrm{H}^{\circ}\right){ }^{7} \mathrm{G}^{\circ} \\
\left({ }^{6} \mathrm{H}^{\circ}\right){ }^{7} \mathrm{G}^{\circ} \\
\left({ }^{6} \mathrm{H}^{\circ}\right){ }^{7} \mathrm{G}^{\circ} \\
\left({ }^{6} \mathrm{~F}^{\circ}\right){ }^{7} \mathrm{H}^{\circ} \\
\left({ }^{6} \mathrm{~F}^{\circ}\right){ }^{7} \mathrm{H}^{\circ}\end{array}$ \\
\hline $4 f^{9}\left({ }^{6} \mathrm{H}^{\circ}\right) 5 d$ & ${ }^{7} \mathrm{I}^{\circ}$ & $\begin{array}{l}9 \\
8 \\
7 \\
6 \\
5 \\
4 \\
3\end{array}$ & $\begin{array}{l}13518.25 \\
15289.16 \\
17125.82 \\
17811.61\end{array}$ & $\begin{array}{l}1.324 \\
1.290 \\
1.282 \\
1.230\end{array}$ & $\begin{array}{l}88 \\
61 \\
48 \\
62 \\
73 \\
78 \\
85\end{array}$ & & $\begin{array}{l}15 \\
17 \\
13\end{array}$ & $\begin{array}{l}\left({ }^{6} \mathrm{H}^{\circ}\right){ }^{5} \mathrm{I}^{\circ} \\
\left({ }^{6} \mathrm{H}^{\circ}\right){ }^{7} \mathrm{G}^{\circ} \\
\left({ }^{6} \mathrm{H}^{\circ}\right){ }^{7} \mathrm{G}^{\circ}\end{array}$ \\
\hline $4 f^{9}\left({ }^{6} \mathrm{H}^{\circ}\right) 5 d$ & ${ }^{7} \mathrm{~F}^{\circ}$ & 6 & 15023.80 & 1.442 & 61 & & 17 & $\left({ }^{6} \mathrm{H}^{\circ}\right){ }^{7} \mathrm{H}^{\circ}$ \\
\hline $4 f^{9}\left({ }^{6} \mathrm{H}^{\circ}\right) 5 d$ & ${ }^{7} \mathrm{G}^{\circ}$ & 7 & 15844.44 & 1.37 & 62 & & 24 & $\left({ }^{6} \mathrm{H}^{\circ}\right)^{7} \mathrm{I}^{\circ}$ \\
\hline $4 f^{9}\left({ }^{6} \mathrm{H}^{\circ}\right) 5 d$ & ${ }^{7} \mathrm{~F}^{\circ}$ & 5 & 17061.53 & 1.445 & 54 & & 15 & $\left({ }^{6} \mathrm{~F}^{\circ}\right)^{7} \mathrm{~F}^{\circ}$ \\
\hline $4 f^{9}\left({ }^{6} \mathrm{H}^{\circ}\right) 5 d$ & ${ }^{5} \mathrm{I}^{\circ}$ & 8 & 17926.11 & 1.250 & 73 & & 15 & $\left({ }^{6} \mathrm{H}^{\circ}\right)^{7} \mathrm{I}^{\circ}$ \\
\hline $4 f^{9}\left({ }^{6} \mathrm{H}^{\circ}\right) 5 d$ & ${ }^{5} \mathrm{H}^{\circ}$ & 7 & 18790.52 & 1.25 & 51 & & 32 & $\left({ }^{6} \mathrm{H}^{\circ}\right)^{5} \mathrm{I}^{\circ}$ \\
\hline $4 f^{8} 6 s 6 p ?$ & & 7 & 29100.78 & 1.58 & & & & \\
\hline $4 f^{8} 5 d 6 p ?$ & & 6 & 29912.92 & & & & & \\
\hline $4 f^{8} 5 d 6 p ?$ & & $\begin{array}{l}7 \\
6\end{array}$ & $\begin{array}{l}30225.87 \\
30764.04\end{array}$ & & & & & \\
\hline $4 f^{8} 5 d 6 p ?$ & & 8 & 30809.64 & & & & & \\
\hline $4 f^{8} 5 d 6 p ?$ & & $\begin{array}{l}7 \\
5 \\
6 \\
6 \\
6\end{array}$ & $\begin{array}{l}31794.62 \\
32296.44 \\
32646.47 \\
33800.91 \\
33841.71\end{array}$ & & & & & \\
\hline
\end{tabular}


Tb II, Odd Parity_Continued

\begin{tabular}{|c|c|c|c|c|c|}
\hline Configuration & Term & $J$ & $\begin{array}{l}\text { Level } \\
\left(\mathrm{cm}^{-1}\right)\end{array}$ & $g$ & Leading percentages \\
\hline \multirow[t]{2}{*}{$4 f^{8} 5 d 6 p ?$} & & 7 & 33873.19 & & \\
\hline & & 6 & 33906.62 & & \\
\hline \multirow[t]{2}{*}{$4 f^{8} 5 d 6 p ?$} & & 7 & 34090.44 & & \\
\hline & & 5 & 34265.59 & & \\
\hline \multirow[t]{2}{*}{$4 f^{8} 5 d 6 p ?$} & & 8 & 34282.35 & 1.42 & \\
\hline & & 5 & 34465.42 & 1.555 & \\
\hline \multirow[t]{34}{*}{$4 f^{8} 5 d 6 p ?$} & & 8 & 34480.63 & 1.44 & \\
\hline & & 6 & 34658.50 & & \\
\hline & & 8 & 34683.53 & & \\
\hline & & 6 & 34788.42 & & \\
\hline & & 6 & 35075.68 & & \\
\hline & & 5,6 & 35232.47 & & \\
\hline & & 5 & 35373.35 & & \\
\hline & & 6 & 35451.55 & & \\
\hline & & 5,6 & 35468.02 & & \\
\hline & & 7 & 35486.83 & & \\
\hline & & 6 & 35590.71 & & \\
\hline & & 7 & 35798.38 & & \\
\hline & & 6 & 36000.42 & & \\
\hline & & 5,6 & 36055.04 & & \\
\hline & & 6 & 36381.24 & & \\
\hline & & 7 & 36685.66 & & \\
\hline & & 7 & 37182.22 & & \\
\hline & & 6 & 37365.20 & & \\
\hline & & 5,6 & 37423.07 & & \\
\hline & & 6 & 37526.84 & & \\
\hline & & 6 & 37565.23 & & \\
\hline & & 7 & 37721.41 & & \\
\hline & & 7 & 37754.98 & & \\
\hline & & 7 & 37773.92 & & \\
\hline & & 8 & 37926.24 & & \\
\hline & & 6 & 38096.48 & & \\
\hline & & 6 & 38260.92 & & \\
\hline & & 7 & 38331.30 & & \\
\hline & & 6 & 38592.88 & & \\
\hline & & 5 & 39035.12 & & \\
\hline & & 6 & 39335.01 & & \\
\hline & & 5,6 & 39612.10 & & \\
\hline & & 6 & 40328.21 & & \\
\hline & & 7 & 43282.56 & & \\
\hline $\mathrm{Tb}$ III $\left({ }^{6} \mathrm{H}_{15 / 2}^{\circ}\right)$ & Limit & & 92900 & & \\
\hline
\end{tabular}


Tb II, Even Parity

\begin{tabular}{|c|c|c|c|c|c|c|c|}
\hline Configuration & Term & $J$ & $\begin{array}{l}\text { Level } \\
\left(\mathrm{cm}^{-1}\right)\end{array}$ & $g$ & & & Leading percentages \\
\hline $4 f^{8}\left({ }^{7} \mathrm{~F}\right) 5 d\left({ }^{8} \mathrm{G}\right) 6 s$ & ${ }^{9} \mathrm{G}$ & 7 & 3235.18 & & 64 & & $28 \quad\left({ }^{7} \mathrm{~F}\right)\left({ }^{8} \mathrm{~F}\right){ }^{9} \mathrm{~F}$ \\
\hline $4 f^{8}\left({ }^{7} \mathrm{~F}\right) 5 d\left({ }^{8} \mathrm{G}\right) 6 s$ & ${ }^{9} \mathrm{G}$ & 8 & 3423.28 & & 92 & & $4 \quad\left({ }^{7} \mathrm{~F}\right)\left({ }^{8} \mathrm{H}\right){ }^{9} \mathrm{H}$ \\
\hline $4 f^{8}\left({ }^{7} \mathrm{~F}\right) 5 d\left({ }^{8} \mathrm{G}\right) 6 s$ & & 6 & 3440.81 & 1.53 & 36 & ${ }^{9} \mathrm{G}$ & $34 \quad\left({ }^{7} \mathrm{~F}\right)\left({ }^{8} \mathrm{~F}\right){ }^{9} \mathrm{~F}$ \\
\hline $4 f^{8}\left({ }^{7} \mathrm{~F}\right) 5 d\left({ }^{8} \mathrm{G}\right) 6 s$ & & 5 & 4158.72 & 1.54 & 33 & ${ }^{9} \mathrm{G}$ & $32 \quad\left({ }^{7} \mathrm{~F}\right)\left({ }^{8} \mathrm{~F}\right){ }^{9} \mathrm{~F}$ \\
\hline $4 f^{8}\left({ }^{7} \mathrm{~F}\right) 5 d\left({ }^{8} \mathrm{D}\right) 6 s$ & & 6 & 5147.23 & 1.593 & 48 & ${ }^{9 D}$ & $\begin{array}{ll}37 & \left({ }^{7} \mathrm{~F}\right)\left({ }^{8} \mathrm{G}\right){ }^{9} \mathrm{G}\end{array}$ \\
\hline $4 f^{8}\left({ }^{7} \mathrm{~F}\right) 5 d\left({ }^{8} \mathrm{G}\right) 6 s$ & & 5 & 5761.28 & 1.59 & 41 & ${ }^{9} \mathrm{G}$ & $40 \quad\left({ }^{7} \mathrm{~F}\right)\left({ }^{8} \mathrm{D}\right){ }^{9} \mathrm{D}$ \\
\hline $4 f^{8} 6 s^{2}$ & ${ }^{7} \mathrm{~F}$ & 6 & 5898.35 & 1.46 & & & \\
\hline $4 f^{8}\left({ }^{7} \mathrm{~F}\right) 5 d\left({ }^{8} \mathrm{G}\right) 6 s ?$ & ${ }^{7} \mathrm{G} ?$ & 7 & 6223.39 & 1.43 & 69 & & $12 \quad\left({ }^{7} \mathrm{~F}\right)\left({ }^{8} \mathrm{~F}\right){ }^{9} \mathrm{~F}$ \\
\hline $4 f^{8}\left({ }^{7} \mathrm{~F}\right) 5 d\left({ }^{8} \mathrm{G}\right) 6 s$ & ${ }^{7} \mathrm{G}$ & 6 & 6428.73 & 1.456 & 53 & & $27 \quad\left({ }^{7} \mathrm{~F}\right)\left({ }^{8} \mathrm{~F}\right){ }^{7} \mathrm{~F}$ \\
\hline $4 f^{8}\left({ }^{7} \mathrm{~F}\right) 5 d\left({ }^{8} \mathrm{G}\right) 6 s$ & & 5 & 6582.89 & 1.531 & 32 & ${ }^{7} \mathrm{~F}$ & $26 \quad\left({ }^{7} \mathrm{~F}\right)\left({ }^{8} \mathrm{D}\right){ }^{7} \mathrm{D}$ \\
\hline $4 f^{8}\left({ }^{7} \mathrm{~F}\right) 5 d\left({ }^{8} \mathrm{~F}\right) 6 s ?$ & ${ }^{9} \mathrm{~F}$ ? & 7 & 7187.85 & & 56 & & $18 \quad\left({ }^{7} \mathrm{~F}\right)\left({ }^{8} \mathrm{G}\right){ }^{7} \mathrm{G}$ \\
\hline $4 f^{8} 6 s^{2} ?$ & ${ }^{7} \mathrm{~F} ?$ & 5 & 8278.04 & 1.464 & & & \\
\hline $4 f^{8}(7 \mathrm{~F}) 5 d^{2} ?$ & ${ }^{\mathrm{g}} \mathrm{G} ?$ & 7 & 8903.62 & 1.498 & & & \\
\hline $4 f^{8}\left({ }^{7} \mathrm{~F}\right) 5 d^{2} ?$ & ${ }^{9} \mathrm{G} ?$ & 6 & 8991.10 & & & & \\
\hline \multirow[t]{2}{*}{$4 f^{8}\left({ }^{7} \mathrm{~F}\right) 5 d^{2} ?$} & ${ }^{9} \mathrm{G} ?$ & 8 & 9273.06 & & & & \\
\hline & & 8 & 11160.18 & 1.38 & & & \\
\hline $4 f^{8}\left({ }^{7} F\right) 5 d 6 s ?$ & & 6 & 11638.64 & 1.53 & & & \\
\hline $4 f^{8}\left({ }^{7} \mathrm{~F}\right) 5 d 6 s ?$ & & 7 & 11813.17 & & & & \\
\hline $4 f^{8}\left({ }^{7} \mathrm{~F}\right) 5 d\left({ }^{6} \mathrm{H}\right) 6 s ?$ & ${ }^{7} \mathrm{H}$ ? & 8 & 13593.44 & 1.38 & 77 & & $17 \quad\left({ }^{7} \mathrm{~F}\right)\left({ }^{8} \mathrm{H}\right){ }^{7} \mathrm{~F}$ \\
\hline \multirow[t]{9}{*}{$4 f^{8}\left({ }^{7} \mathrm{~F}\right) 5 d 6 s ?$} & & 6 & 14964.95 & 1.644 & & & \\
\hline & & 8 & 19710.26 & 1.30 & & & \\
\hline & & 8 & 21035.56 & & & & \\
\hline & & 7 & 21521.06 & & & & \\
\hline & & 7 & 22008.54 & & & & \\
\hline & & 7 & 23653.66 & 1.38 & & & \\
\hline & & 6 & 23678.13 & & & & \\
\hline & & 8 & 25138.49 & 1.34 & & & \\
\hline & & 7 & 25376.50 & 1.29 & & & \\
\hline \multirow[t]{4}{*}{$4 f^{9}\left({ }^{6} \mathrm{H}_{15 / 2}^{\circ}\right) 6 p_{1 / 2}$} & $(15 / 2,1 / 2)$ & $\begin{array}{l}7 \\
8\end{array}$ & $\begin{array}{l}25804.68 \\
25975.20\end{array}$ & $\begin{array}{l}1.37 \\
1.30\end{array}$ & & & \\
\hline & & 7 & 26385.71 & 1.358 & & & \\
\hline & & 6 & 26731.50 & 1.40 & & & \\
\hline & & 7 & 27244.25 & & & & \\
\hline
\end{tabular}


Tb II, Even Parity-Continued

\begin{tabular}{|c|c|c|c|c|c|}
\hline Configuration & Term & $J$ & $\begin{array}{l}\text { Level } \\
\left(\mathrm{cm}^{-1}\right)\end{array}$ & $g$ & Leading percentages \\
\hline $4 f^{9}\left({ }^{6} \mathrm{H}^{\circ}{ }^{\circ} / 2\right) 6 p_{3 / 2}$ & $\left(15 / 2,{ }^{3} / 2\right)$ & $\begin{array}{l}8 \\
7 \\
6 \\
9 \\
\\
8\end{array}$ & $\begin{array}{l}28014.90 \\
28209.47 \\
28339.32 \\
28488.83 \\
\\
28560.96\end{array}$ & $\begin{array}{l}1.29 \\
1.35 \\
1.32 \\
1.326\end{array}$ & \\
\hline $4 f^{9}\left({ }^{6} \mathrm{H}_{13 / 2}^{\circ}\right) 6 p_{1 / 2}$ & $(13 / 2,1 / 2)$ & $\begin{array}{l}7 \\
7 \\
6 \\
5 \\
6\end{array}$ & $\begin{array}{l}28649.13 \\
29407.02 \\
29803.36 \\
29995.37 \\
30068.29\end{array}$ & 1.26 & \\
\hline $4 f^{9}\left({ }^{6} \mathrm{H}_{1 / 2}^{\circ}\right) 6 p_{1 / 2}$ & $(11 / 2,1 / 2)$ & $\begin{array}{l}5 \\
6 \\
7 \\
7 \\
\\
3 \\
7\end{array}$ & $\begin{array}{l}30148.67 \\
30639.48 \\
30364.74 \\
30476.12 \\
30703.63 \\
30895.47\end{array}$ & $\begin{array}{l}1.27 \\
1.20 \\
1.34 \\
\\
1.30\end{array}$ & \\
\hline $4 f^{9}\left({ }^{6} \mathrm{H}^{\circ}{ }^{13 / 2}\right) 6 p_{3 / 2}$ & $(13 / 2,3 / 2)$ & $\begin{array}{l}8 \\
7\end{array}$ & $\begin{array}{l}31078.49 \\
31365.86\end{array}$ & 1.28 & \\
\hline $4 f^{9}\left({ }^{6} \mathrm{H}_{13 / 2}\right) 6 p_{3 / 2}$ & $(13 / 2,3 / 2)$ & $\begin{array}{c}6 \\
6 \\
5 \\
5,6 \\
6 \\
6 \\
6 \\
5 \\
6 \\
7 \\
6 \\
7 \\
8 \\
7 \\
5 \\
4 \\
8 \\
4 \\
8 \\
7 \\
6,7\end{array}$ & $\begin{array}{l}31566.42 \\
31879.15 \\
32023.68 \\
32369.10 \\
32687.20 \\
32853.61 \\
33488.95 \\
34318.93 \\
34577.23 \\
34804.92 \\
34875.62 \\
35007.90 \\
35080.26 \\
35196.20 \\
35227.65 \\
36074.16 \\
36602.00 \\
37109.47 \\
37193.86 \\
37210.37 \\
38030.16\end{array}$ & $\begin{array}{l}1.31 \\
1.14\end{array}$ & \\
\hline Tb III ( $\left.{ }^{6} \mathrm{H}_{15 / 2}^{\circ}\right)$ & Limit & & 92900 & & \\
\hline
\end{tabular}




\section{Tb III}

(Eu I sequence; 63 electrons)

$Z=65$

Ground state $\left(1 s^{2} 2 s^{2} 2 p^{6} 3 s^{2} 3 p^{6} 3 d^{10} 4 s^{2} 4 p^{6} 4 d^{10} 5 s^{2} 5 p^{6}\right) 4 f^{9}{ }^{6} \mathrm{H}_{15 / 2}^{\circ}$

Ionization energy $176700 \pm 800 \mathrm{~cm}^{-1}$

$21.91 \pm 0.10 \mathrm{eV}$

Identified odd configurations $\quad 4 f^{9}$ and $4 f^{8} 6 p$

Identified even configurations $\quad 4 f^{8} 5 d$ and $4 f^{8} 6 s$

Meinders, van Kleef, and Wyart have analyzed the Tb III spectrum obtained from a pulsed hollow-cathode source. The spectrum was photographed over the range 2000-6800 $\AA$ at the Argonne National Laboratory; most of the approximately $2500 \mathrm{~Tb}$ III lines in the unpublished list are in the range 2000-3220 $\AA$. The authors classified 442 lines in this region, including most of the 100 strongest lines, and an additional 43 lines above $5000 \AA$.

All eigenvector percentages are from Meinders et al. In calculating the $4 f^{8}\left({ }^{7} \mathrm{~F}\right) n l$ subconfigurations, they used the "real core" ${ }^{7} \mathrm{~F}$ levels in matrices truncated to include only terms based on the ${ }^{7} \mathrm{~F}$ parent.

Most of the $4 f^{8}\left({ }^{7} \mathrm{~F}\right) 6 p$ levels have appropriate names in $J_{1} j$ coupling. A few of the higher known or predicted levels of $4 f^{\times}\left({ }^{7} \mathrm{~F}\right) 6 \mathrm{p}$ having low eigenvector purities are listed with names in order to complete the $J_{1} j$ terms or to indicate missing levels. The authors omitted the odd $J=5 / 2$ level at $55706 \mathrm{~cm}^{-1}$ in fitting the theoretical parameters to the experimental levels of $4 f^{8}\left({ }^{7} \mathrm{~F}\right) 6 p$; they suggest this level may be perturbed by $4 f^{7} 5 d^{2}$.

The terms of $4 f^{8}\left({ }^{7} \mathrm{~F}\right) 6 \mathrm{~s}$ and $4 f^{8}\left({ }^{7} \mathrm{~F}\right) 5 d$ are complete except for five levels of the latter subconfiguration. The calculations of these subconfigurations included configuration interaction and "all two-particle operators acting only on the electron orbit." We have interchanged the $4 f^{8}\left({ }^{7} \mathrm{~F}\right) 5 d{ }^{8} \mathrm{D}_{3 / 2}$ and ${ }^{8} \mathrm{~F}_{3 / 2}$ designations from those of Meinders et al. to conform with the assigned eigenvectors.

Sugar and, Reader obtained the ionization energy.

\section{References}

Becher, J., Thesis, Johns Hopkins Univ., Baltimore, 135 pp. (1965). PT

Meinders, E., van Kleef, T. A. M., and Wyart, J. F., Physica (Utrecht) 61, 443 (1972). EL ND PT

Sugar, J., and Reader, J., J. Chem. Phys. 59, 2083 (1973), IP

[January 1977] 
Tb III

\begin{tabular}{|c|c|c|c|c|c|}
\hline Configuration & Term & $J$ & $\begin{array}{l}\text { Level } \\
\left(\mathrm{cm}^{-1}\right)\end{array}$ & \multicolumn{2}{|c|}{ Leading percentages } \\
\hline $4 f^{9}$ & ${ }^{6} \mathrm{H}^{\circ}$ & $\begin{array}{l}15 / 2 \\
13 / 2 \\
11 / 2 \\
9 / 2 \\
7 / 2 \\
5 / 2\end{array}$ & $\begin{array}{r}0.00 \\
2804.67 \\
4734.52 \\
6258.08 \\
7422.21 \\
8285.885\end{array}$ & $\begin{array}{l}94 \\
97 \\
94 \\
94 \\
94 \\
93\end{array}$ & $\begin{array}{ll}4 & { }^{4} \mathrm{I}^{\circ} 3 \\
2 & { }^{4} \mathrm{I}^{\circ} 3 \\
1 & { }^{6} \mathrm{~F}^{\circ} \\
2 & { }^{4} \mathrm{G}^{\circ} 4 \\
3 & { }^{4} \mathrm{G}^{\circ} 4 \\
4 & { }^{4} \mathrm{G}^{\circ} 4\end{array}$ \\
\hline $4 f^{9}$ & ${ }^{6} \mathrm{~F}^{\circ}$ & $\begin{array}{l}11 / 2 \\
9 / 2 \\
7 / 2 \\
5 / 2 \\
3 / 2 \\
1 / 2\end{array}$ & $\begin{array}{c}6470.11 \\
7621.565 \\
9144.98 \\
10220.38\end{array}$ & $\begin{array}{l}95 \\
90 \\
93 \\
93 \\
92 \\
92\end{array}$ & $\begin{array}{ll}2 & { }^{6} \mathrm{H}^{\circ} \\
6 & { }^{4} \mathrm{~F}^{\circ} 3 \\
4 & { }^{4} \mathrm{~F}^{\circ} 3 \\
2 & { }^{4} \mathrm{~F}^{\circ} 3 \\
3 & { }^{4} \mathrm{D}^{\circ} 2 \\
4 & { }^{4} \mathrm{D}^{\circ} 2\end{array}$ \\
\hline $4 f^{8}\left({ }^{7} F\right) 5 d$ & ${ }^{8} \mathrm{G}$ & $\begin{array}{l}13 / 2 \\
11 / 2 \\
15 / 2 \\
9 / 2 \\
7 / 2 \\
5 / 2 \\
3 / 2 \\
1 / 2\end{array}$ & $\begin{array}{c}8972.29 \\
9218.63 \\
9275.42 \\
10070.38 \\
10996.01 \\
11678.865 \\
12165.155 \\
12454.365\end{array}$ & $\begin{array}{l}73 \\
47 \\
96 \\
51 \\
70 \\
83 \\
92 \\
97\end{array}$ & $\begin{array}{ll}23 & \left({ }^{7} \mathrm{~F}\right){ }^{8} \mathrm{~F} \\
30 & \left({ }^{7} \mathrm{~F}\right){ }^{8} \mathrm{~F} \\
& \\
28 & \left({ }^{7} \mathrm{~F}\right){ }^{8} \mathrm{~F} \\
21 & \left.{ }^{7} \mathrm{~F}\right)^{8} \mathrm{~F} \\
13 & \left({ }^{7} \mathrm{~F}\right)^{8} \mathrm{~F}\end{array}$ \\
\hline $4 f^{8}\left({ }^{7} F\right) 5 d$ & ${ }^{8} \mathrm{D}$ & $\begin{array}{l}11 / 2 \\
9 / 2 \\
7 / 2 \\
5 / 2 \\
3 / 2\end{array}$ & $\begin{array}{l}11217.785 \\
11752.295 \\
12775.40 \\
13689.615\end{array}$ & $\begin{array}{l}54 \\
50 \\
55 \\
52 \\
57\end{array}$ & $\begin{array}{ll}38 & \left({ }^{7} \mathrm{~F}\right){ }^{8} \mathrm{G} \\
36 & \left({ }^{7} \mathrm{~F}\right)^{8} \mathrm{G} \\
21 & \left({ }^{7} \mathrm{~F}\right)^{8} \mathrm{G} \\
34 & \left({ }^{7} \mathrm{~F}\right){ }^{8} \mathrm{~F} \\
40 & \left({ }^{7} \mathrm{~F}\right)^{8} \mathrm{~F}\end{array}$ \\
\hline $4 f^{8}\left({ }^{7} F\right) 5 d$ & $8 \mathrm{~F}$ & $\begin{array}{c}13 / 2 \\
3 / 2 \\
11 / 2 \\
1 / 2 \\
9 / 2 \\
7 / 2 \\
5 / 2\end{array}$ & $\begin{array}{l}12986.505 \\
14515.35 \\
14771.12 \\
\\
15278.90 \\
15820.87 \\
16066.485\end{array}$ & $\begin{array}{l}76 \\
53 \\
61 \\
97 \\
61 \\
57 \\
51\end{array}$ & 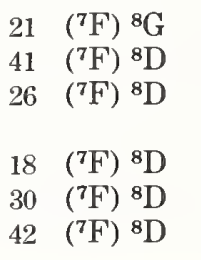 \\
\hline $4 f^{8}\left({ }^{7} \mathrm{~F}\right) 5 d$ & ${ }^{8} \mathrm{H}$ & $\begin{array}{l}17 / 2 \\
15 / 2 \\
13 / 2 \\
11 / 2 \\
9 / 2 \\
7 / 2 \\
5 / 2 \\
3 / 2\end{array}$ & $\begin{array}{l}14890.375 \\
15516.735 \\
16261.835 \\
16757.805 \\
17169.285 \\
17499.43 \\
17755.55\end{array}$ & $\begin{array}{r}100 \\
76 \\
83 \\
85 \\
89 \\
93 \\
95 \\
98\end{array}$ & $\begin{array}{ll}19 & \left({ }^{7} \mathrm{~F}\right){ }^{6} \mathrm{H} \\
11 & \left({ }^{7} \mathrm{~F}\right){ }^{6} \mathrm{H}\end{array}$ \\
\hline $4 f^{8}\left({ }^{7} F\right) 5 d$ & ${ }^{6} \mathrm{~F}$ & $\begin{array}{l}11 / 2 \\
9 / 2 \\
7 / 2 \\
5 / 2 \\
3 / 2 \\
1 / 2\end{array}$ & $\begin{array}{l}17437.515 \\
18776.53 \\
20012.85 \\
20968.37 \\
21640.59\end{array}$ & $\begin{array}{l}80 \\
80 \\
83 \\
87 \\
89 \\
92\end{array}$ & $10 \quad\left({ }^{7} \mathrm{~F}\right) 6 s^{6 \mathrm{~F}}$ \\
\hline $4 f^{8}\left({ }^{7} \mathrm{~F}\right) 6 s$ & ${ }^{8 \mathrm{~F}}$ & $\begin{array}{l}13 / 2 \\
11 / 2 \\
9 / 2 \\
7 / 2 \\
5 / 2 \\
3 / 2 \\
1 / 2\end{array}$ & $\begin{array}{l}17676.265 \\
19181.10 \\
20648.99 \\
21641.865 \\
22384.795 \\
22898.245 \\
23203.55\end{array}$ & $\begin{array}{l}99 \\
62 \\
88 \\
92 \\
97 \\
98 \\
99\end{array}$ & $28 \quad\left({ }^{7} F\right)^{6} F$ \\
\hline $4 f^{8}\left({ }^{7} \mathrm{~F}\right) 5 d$ & ${ }^{6} \mathrm{G}$ & $\begin{array}{l}13 / 2 \\
11 / 2 \\
9 / 2 \\
7 / 2 \\
5 / 2 \\
3 / 2\end{array}$ & $\begin{array}{l}18158.91 \\
19902.04 \\
21142.53 \\
22067.435 \\
22774.735 \\
23234.585\end{array}$ & $\begin{array}{l}94 \\
76 \\
86 \\
89 \\
81 \\
96\end{array}$ & $\begin{array}{l}12 \quad\left({ }^{7} \mathrm{~F}\right) 6 s^{8} \mathrm{~F} \\
12 \quad\left({ }^{7} \mathrm{~F}\right)^{8 \mathrm{P}}\end{array}$ \\
\hline
\end{tabular}


Tb III-Continued

\begin{tabular}{|c|c|c|c|c|c|c|}
\hline Configuration & Term & $J$ & $\begin{array}{l}\text { Level } \\
\left(\mathrm{cm}^{-1}\right)\end{array}$ & \multicolumn{3}{|c|}{ Leading percentages } \\
\hline $4 f^{8}\left({ }^{7} \mathrm{~F}\right) 5 d$ & ${ }^{8} \mathrm{P}$ & $\begin{array}{l}9 / 2 \\
7 / 2 \\
5 / 2\end{array}$ & $\begin{array}{l}18761.675 \\
21296.02 \\
22980.56\end{array}$ & $\begin{array}{l}80 \\
89 \\
83\end{array}$ & 15 & $\begin{array}{l}\left({ }^{7} \mathrm{~F}\right){ }^{8} \mathrm{D} \\
\left({ }^{7} \mathrm{~F}\right){ }^{6} \mathrm{G}\end{array}$ \\
\hline $4 f^{8}\left({ }^{7} \mathrm{~F}\right) 5 d$ & ${ }^{6} \mathrm{H}$ & $\begin{array}{l}15 / 2 \\
13 / 2 \\
11 / 2 \\
9 / 2 \\
7 / 2 \\
5 / 2\end{array}$ & $\begin{array}{l}18796.25 \\
20940.41 \\
22216.87 \\
23239.99 \\
24011.27 \\
24592.495\end{array}$ & $\begin{array}{l}80 \\
85 \\
88 \\
91 \\
94 \\
97\end{array}$ & $\begin{array}{l}20 \\
12\end{array}$ & $\begin{array}{l}\left({ }^{7} \mathrm{~F}\right){ }^{8} \mathrm{H} \\
\left({ }^{7} \mathrm{~F}\right){ }^{8} \mathrm{H}\end{array}$ \\
\hline $4 f^{8}\left({ }^{7} \mathrm{~F}\right) 6 s$ & ${ }^{6} \mathrm{~F}$ & $\begin{array}{l}11 / 2 \\
9 / 2 \\
7 / 2 \\
5 / 2 \\
3 / 2 \\
1 / 2\end{array}$ & $\begin{array}{l}20317.60 \\
22475.34 \\
23357.625 \\
24307.445 \\
24862.595 \\
25241.355\end{array}$ & $\begin{array}{l}60 \\
57 \\
74 \\
54 \\
61 \\
87\end{array}$ & $\begin{array}{l}33 \\
35\end{array}$ & $\begin{array}{l}\left({ }^{7} \mathrm{~F}\right){ }^{8} \mathrm{~F} \\
\left({ }^{7} \mathrm{~F}\right) 5 d{ }^{6} \mathrm{D} \\
\left({ }^{7} \mathrm{~F}\right) 5 d d^{6} \mathrm{D} \\
\left({ }^{7} \mathrm{~F}\right) 5 d^{6} \mathrm{D}\end{array}$ \\
\hline $4 f^{8}\left({ }^{7} \mathrm{~F}\right) 5 d$ & ${ }^{6} \mathrm{D}$ & $\begin{array}{l}9 / 2 \\
7 / 2 \\
5 / 2 \\
3 / 2 \\
1 / 2 \\
1 / 2\end{array}$ & $\begin{array}{l}22115.795 \\
22913.07 \\
24189.895 \\
24924.195\end{array}$ & $\begin{array}{l}66 \\
69 \\
58 \\
62 \\
91\end{array}$ & $\begin{array}{l}26 \\
13 \\
37 \\
31\end{array}$ & $\begin{array}{l}\left({ }^{7} \mathrm{~F}\right) 6 s^{6} \mathrm{~F} \\
\left({ }^{7} \mathrm{~F}\right) 6 s^{6} \mathrm{~F} \\
\left({ }^{7} \mathrm{~F}\right) 6 s^{6} \mathrm{~F} \\
\left({ }^{7} \mathrm{~F}\right) 6 s^{6} \mathrm{~F}\end{array}$ \\
\hline $4 f^{8}\left({ }^{7} \mathrm{~F}\right) 5 d$ & ${ }^{6} \mathrm{P}$ & $\begin{array}{l}7 / 2 \\
5 / 2 \\
3 / 2\end{array}$ & $\begin{array}{l}24981.97 \\
26632.53 \\
27945.64\end{array}$ & $\begin{array}{l}85 \\
95 \\
98\end{array}$ & 13 & $\left({ }^{7} \mathrm{~F}\right){ }^{6} \mathrm{D}$ \\
\hline $4 f^{8}\left({ }^{7} \mathrm{~F}_{6}\right) 6 p_{1 / 2}$ & $\left(6,{ }^{1} / 2\right)^{\circ}$ & $\begin{array}{l}11 / 2 \\
13 / 2\end{array}$ & $\begin{array}{l}52039.185 \\
52252.635\end{array}$ & $\begin{array}{l}96 \\
92\end{array}$ & & \\
\hline $4 f^{8}\left({ }^{7} \mathrm{~F}_{5}\right) 6 p_{1 / 2}$ & $\left(5,{ }^{1} / 2\right)^{\circ}$ & $\begin{array}{c}9 / 2 \\
11 / 2\end{array}$ & $\begin{array}{l}53831.125 \\
54632.46\end{array}$ & $\begin{array}{l}93 \\
95\end{array}$ & & \\
\hline $4 f^{8}\left({ }^{7} \mathrm{~F}_{4}\right) 6 p_{1 / 2}$ & $\left(4,{ }^{1} / 2\right)^{\circ}$ & $\begin{array}{l}7 / 2 \\
9 / 2\end{array}$ & $\begin{array}{l}54965.075 \\
55932.24\end{array}$ & $\begin{array}{l}93 \\
96\end{array}$ & & \\
\hline $4 f^{8}\left({ }^{7} \mathrm{~F}_{3}\right) 6 p_{1 / 2}$ & $\left(3,{ }^{1} / 2\right)^{\circ}$ & $\begin{array}{l}5 / 2 \\
7 / 2 \\
3 / 2 \\
7 / 2 \\
5 / 2\end{array}$ & $\begin{array}{l}55705.84^{*} \\
56857.39 \\
56025.375 \\
56474.84 \\
56694.12\end{array}$ & $\begin{array}{l}93 \\
97\end{array}$ & & \\
\hline $4 f^{8}\left({ }^{7} \mathrm{~F}_{6}\right) 6 p_{3 / 2}$ & $\left(6,{ }^{3} / 2\right)^{\circ}$ & $\begin{array}{c}13 / 2 \\
15 / 2 \\
11 / 2 \\
9 / 2 \\
3 / 2\end{array}$ & $\begin{array}{l}56779.125 \\
57036.08 \\
57620.57 \\
58722.65 \\
\\
57134.15\end{array}$ & $\begin{array}{r}84 \\
100 \\
99 \\
92\end{array}$ & & \\
\hline $4 f^{8}\left({ }^{7} \mathrm{~F}_{2}\right) 6 p_{1 / 2}$ & $\left(2,{ }^{1} / 2\right)^{\circ}$ & $\begin{array}{l}3 / 2 \\
5 / 2\end{array}$ & 57433.545 & $\begin{array}{l}92 \\
97\end{array}$ & & \\
\hline $4 f^{8}\left({ }^{7} \mathrm{~F}_{1}\right) 6 p_{1 / 2}$ & $(1,1 / 2)^{\circ}$ & $\begin{array}{l}1 / 2 \\
3 / 2\end{array}$ & 57773.445 & $\begin{array}{l}87 \\
96\end{array}$ & & \\
\hline $4 f^{8}\left({ }^{7} \mathrm{~F}_{0}\right) 6 p_{1 / 2}$ & $(0,1 / 2)^{\circ}$ & $1 / 2$ & 57893.975 & 91 & & \\
\hline $4 f^{8}\left({ }^{7} \mathrm{~F}_{5}\right) 6 p_{3 / 2}$ & $\left(5,{ }^{3} / 2\right)^{\circ}$ & $\begin{array}{c}11 / 2 \\
13 / 2 \\
9 / 2 \\
7 / 2\end{array}$ & $\begin{array}{l}58811.23 \\
59189.825 \\
59712.255 \\
60440.755\end{array}$ & $\begin{array}{l}73 \\
92 \\
92 \\
76\end{array}$ & 20 & $\begin{array}{l}\left({ }^{7} \mathrm{~F}_{4}\right)\left(4,{ }^{3} / 2\right)^{\circ} \\
\left({ }^{7} \mathrm{~F}_{4}\right)\left(4,{ }^{3} / 2\right)^{\circ}\end{array}$ \\
\hline
\end{tabular}


Tb III-Continued

\begin{tabular}{|c|c|c|c|c|c|}
\hline Configuration & Term & $J$ & $\begin{array}{l}\text { Level } \\
\left(\mathrm{cm}^{-1}\right)\end{array}$ & \multicolumn{2}{|c|}{ Leading percentages } \\
\hline $4 f^{8}\left({ }^{7} \mathrm{~F}_{4}\right) 6 p_{3 / 2}$ & $(4,3 / 2)^{\circ}$ & $\begin{array}{l}9 / 2 \\
11 / 2 \\
7 / 2 \\
5 / 2\end{array}$ & $\begin{array}{l}60089.475 \\
60687.115 \\
61009.61 \\
61435.10\end{array}$ & $\begin{array}{l}65 \\
77 \\
68 \\
74\end{array}$ & $\begin{array}{lll}32 & \left({ }^{7} \mathrm{~F}_{3}\right) & \left(3,{ }^{3} / 2\right)^{\circ} \\
22 & \left({ }^{7} \mathrm{~F}_{5}\right) & \left(5,{ }^{3} / 2\right)^{\circ} \\
16 & \left({ }^{7} \mathrm{~F}_{5}\right) & \left(5,{ }^{3} / 2\right)^{\circ} \\
20 & \left({ }^{7} \mathrm{~F}_{3}\right) & \left(3,{ }^{3} / 2\right)^{\circ}\end{array}$ \\
\hline $4 f^{8}\left({ }^{7} \mathrm{~F}_{3}\right) 6 p_{3 / 2}$ & $(3,3 / 2)^{\circ}$ & $\begin{array}{l}7 / 2 \\
9 / 2 \\
5 / 2 \\
3 / 2 \\
5 / 2\end{array}$ & $\begin{array}{l}61131.515 \\
61847.625 \\
61973.18 \\
61883.195\end{array}$ & $\begin{array}{l}55 \\
66 \\
38 \\
67\end{array}$ & $\begin{array}{ll}33 & \left({ }^{7} F_{2}\right)(2,3 / 2)^{\circ} \\
31 & \left({ }^{7} F_{4}\right)(4,3 / 2)^{\circ} \\
34 & \left({ }^{7} F_{1}\right)(1,3 / 2)^{\circ} \\
24 & \left({ }^{7} F_{2}\right)(2,3 / 2)^{\circ} \\
& \end{array}$ \\
\hline $4 f^{8}\left({ }^{7} \mathrm{~F}_{2}\right) 6 p_{3 / 2}$ & $(2,3 / 2)^{\circ}$ & $\begin{array}{l}5 / 2 \\
3 / 2 \\
1 / 2 \\
7 / 2 \\
5 / 2\end{array}$ & $\begin{array}{l}62157.57 \\
62733.445 \\
62578.475\end{array}$ & $\begin{array}{l}40 \\
33 \\
62 \\
54\end{array}$ & $\begin{array}{ll}33 & \left({ }^{7} \mathrm{~F}_{3}\right)\left(3,{ }^{3} / 2\right)^{\circ} \\
32 & \left({ }^{7} \mathrm{~F}_{0}\right)\left(0,{ }^{3} / 2\right)^{\circ} \\
37 & \left({ }^{7} \mathrm{~F}_{1}\right)(1,3 / 2)^{\circ} \\
40 & \left({ }^{7} \mathrm{~F}_{3}\right)\left(3,{ }^{3} / 2\right)^{\circ}\end{array}$ \\
\hline $4 f^{8}\left({ }^{7} \mathrm{~F}_{1}\right) 6 p_{3 / 2}$ & $(1,3 / 2)^{\circ}$ & $\begin{array}{l}1 / 2 \\
5 / 2 \\
3 / 2\end{array}$ & $\begin{array}{l}63383.965 \\
63721.245\end{array}$ & $\begin{array}{l}57 \\
45 \\
48\end{array}$ & $\begin{array}{ll}31 & \left({ }^{7} \mathrm{~F}_{2}\right)\left(2,{ }^{3} / 2\right)^{\circ} \\
45 & \left({ }^{7} \mathrm{~F}_{2}\right)\left(2,{ }^{3} / 2\right)^{\circ} \\
40 & \left({ }^{7} \mathrm{~F}_{0}\right)\left(0,{ }^{3} / 2\right)^{\circ}\end{array}$ \\
\hline $\mathrm{Tb}$ IV $\left({ }^{7} \mathrm{~F}_{6}\right)$ & Limit & & 176700 & & \\
\hline
\end{tabular}


Tb IV

(Sm I sequence; 62 electrons)

Ground state $\left(1 s^{2} 2 s^{2} 2 p^{6} 3 s^{2} 3 p^{6} 3 d^{10} 4 s^{2} 4 p^{6} 4 d^{10} 5 s^{2} 5 p^{6}\right) 4 f^{8}{ }^{7} \mathrm{~F}_{6}$

Ionization energy $317500 \pm 800 \mathrm{~cm}^{-1}$

$39.37 \pm 0.10 \mathrm{eV}$

Spector and Sugar have classified 48 lines of Tb IV in the region 1176-2436 $\AA$. The terms are from their analysis; they note that "the rms error in the fit of the wavenumbers of the lines to the final level values is $0.1 \mathrm{~cm}^{-1}$."

The eigenvector percentages of the $4 f^{7}\left({ }^{8} \mathrm{~S}^{\circ}\right) 5 d$ and $4 f^{7}\left({ }^{8} \mathrm{~S}^{\circ}\right) 6 p$ levels are also from Spector and Sugar, the calculations having been carried out with matrices including only terms based on the $4 f^{7}\left({ }^{8} \mathrm{~S}^{\circ}\right)$ parent term. The six $4 f^{7}\left({ }^{8} \mathrm{~S}^{\circ}\right) 6 p$ levels comprise two $J_{1} j$-coupling terms of high purity. The less appropriate $L S$-coupling names of these levels are given with corresponding percentages after the word "or." The two $4 f^{7}\left({ }^{8} \mathrm{~S}^{\circ}\right) 6 s$ levels are designated as ${ }^{9} \mathrm{~S}^{\circ}$ and ${ }^{7} \mathrm{~S}^{\circ}$ terms, but the $J_{1} j$-coupling term which constitutes an alternate designation for this pair is also shown.

In their searches for the $4 f^{85} \mathrm{~F}$ levels, Spector and Sugar made use of the approximate positions of these levels as determined from crystal spectra [Dieke, 1968]. Absorption transitions involving levels of higher terms of $4 f^{8}$ have been observed in both crystal and solution spectra [Dieke, 1968; Carnall, Fields, and Rajnak, 1968]. The lowest $4 f^{8}$ level above the ${ }^{7} \mathrm{~F}$ term is a ${ }^{5} \mathrm{D}_{4}$ level around $20000 \mathrm{~cm}^{-1}$. We took the leading percentages for the levels of the ${ }^{7} \mathrm{~F}$ term from Ofelt's calculation.

Spector and Sugar obtained the quoted ionization energy by adjusting an earlier result of Sugar and Reader [1973] to the experimental value of $4 f^{7}\left({ }^{8} \mathrm{~S}^{\circ}\right) 6 s$ relative to the ground level.

\section{References}

Carnall, W. T., Fields, P. R., and Rajnak, K., J. Chem. Phys. 49, 4447 (1968). [EL] [CL] [W] PT

Dieke, G. H., Spectra and Energy Levels of Rare Earth Ions in Crystals, Ed. H. M. Crosswhite and H. Crosswhite, pp. 253-268 (Interscience Publishers, New York, 1968). [EL] ND

Ofelt, G. S., J. Chem. Phys. 38, 2171 (1963). ND ZE PT

Spector, N., and Sugar, J., J. Opt. Soc. Am. 66, 436 (1976). EL CL IP PT

Sugar, J., and Reader, J., J. Chem. Phys. 59, 2083 (1973). IP

[October 1976]

Tb IV

\begin{tabular}{|c|c|c|c|c|c|c|}
\hline Configuration & Term & $J$ & $\begin{array}{l}\text { Level } \\
\left(\mathrm{cm}^{-1}\right)\end{array}$ & \multicolumn{3}{|c|}{ Leading percentages } \\
\hline $4 f^{8}$ & ${ }^{7} \mathrm{~F}$ & $\begin{array}{l}6 \\
5 \\
4 \\
3 \\
2 \\
1 \\
0\end{array}$ & $\begin{array}{r}0.0 \\
2051.6 \\
3314.2 \\
4292.3 \\
4977.9 \\
5431.8 \\
5653.8\end{array}$ & $\begin{array}{l}96 \\
97 \\
95 \\
95 \\
95 \\
95 \\
95\end{array}$ & $\begin{array}{l}2 \\
1 \\
1 \\
2 \\
3 \\
3 \\
3\end{array}$ & $\begin{array}{l}{ }^{5} \mathrm{G} 1 \\
{ }^{5} \mathrm{G} 1 \\
{ }^{5} \mathrm{D} 1 \\
{ }^{5} \mathrm{D} 1 \\
{ }^{5} \mathrm{D} 1 \\
{ }^{5} \mathrm{D} 1 \\
{ }^{5} \mathrm{D} 1\end{array}$ \\
\hline $4 f^{7}\left({ }^{8} \mathrm{~S}^{\circ}\right) 5 d$ & ${ }^{9} \mathrm{D}^{\circ}$ & $\begin{array}{l}2 \\
3 \\
4 \\
5 \\
6\end{array}$ & $\begin{array}{l}51404.0 \\
51800.8 \\
52399.6 \\
53316.6 \\
54882.5\end{array}$ & $\begin{array}{r}99 \\
98 \\
97 \\
97 \\
100\end{array}$ & & \\
\hline $4 f^{7}\left({ }^{8} S^{\circ}\right) 5 d$ & ${ }^{7} \mathrm{D}^{\circ}$ & $\begin{array}{l}5 \\
4 \\
3 \\
2 \\
1\end{array}$ & $\begin{array}{l}62680.6 \\
63281.4 \\
63746.2 \\
64081.4 \\
64312.2\end{array}$ & $\begin{array}{r}97 \\
97 \\
98 \\
99 \\
100\end{array}$ & & \\
\hline
\end{tabular}


Tb IV-Continued

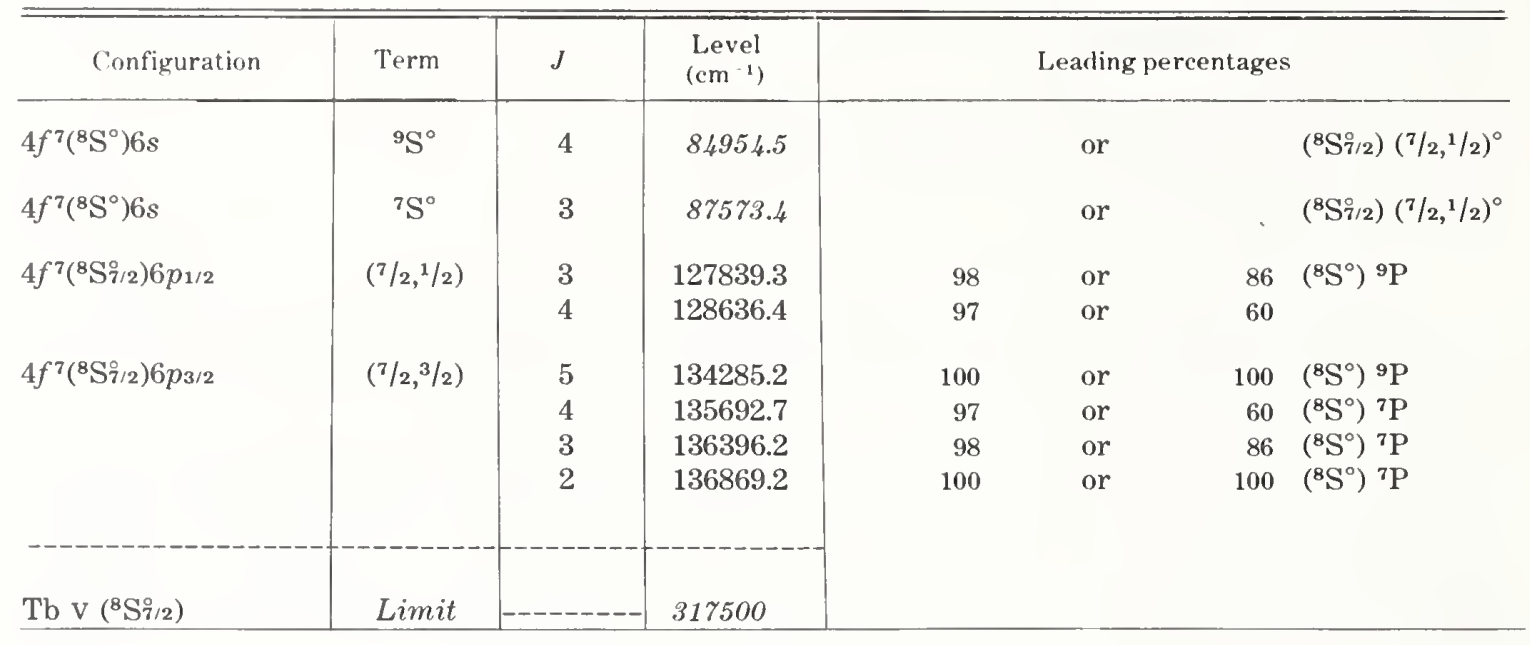




\section{DYSPROSIUM}

\section{Dy I}

66 electrons

$Z=66$

Ground state $\left(1 s^{2} 2 s^{2} 2 p^{6} 3 s^{2} 3 p^{6} 3 d^{10} 4 s^{2} 4 p^{6} 4 d^{10} 5 s^{2} 5 p^{6}\right) 4 f^{10} 6 s^{2}{ }^{5} \mathrm{I}_{8}$

Ionization energy $47900 \pm 5 \mathrm{~cm}^{-1}$

$5.9389 \pm 0.0006 \mathrm{eV}$

Identified even configurations

$4 f^{10} 6 s^{2}, 4 f^{9} 6 s^{2} 6 p, 4 f^{9} 5 d 6 s 6 p, 4 f^{10} 5 d 6 s, 4 f^{10} 6 s 7 s, 4 f^{10} 6 s 6 d$

Identified odd configurations

$4 f^{9} 5 d 6 s^{2}, 4 f^{9} 5 d^{2} 6 s, 4 f^{10} 6 s 6 p, 4 f^{10} 5 d 6 p ?, 4 f^{10} 6 s 7 p, 4 f^{9} 5 d 6 s 7 s$

Main References for Wavelengths and Energy Levels

The most complete description of the Dy I and Dy II spectra is given in the 1970 UCRL report by Conway and Worden. More than 22000 lines are listed between 2300 and $11400 \AA$, and Zeeman data are included for the range 2300 to $9000 \AA$. Sharp-line spectra were obtained by the use of electrodeless lamps containing only the isotope ${ }^{162} \mathrm{Dy}$. About $85 \%$ of the lines are assigned to either Dy I or Dy II, with almost 10000 lines being listed for Dy I. King's earlier temperature classifications of 4584 Dy lines were used in confirming and extending the separation of the two spectra [King, Conway, Worden, and Moore, 1970].

Conway and Worden $[1970,1971]$ found more than 300 levels in the first analysis of Dy I, and classified almost 2000 of the Dy I lines. The levels of the $4 f^{10} 6 s^{25} \mathrm{I}$ ground term were identified and a number of the lowest odd levels were assigned to telms of $4 f^{9} 5 d 6 \mathrm{~s}^{2}$.

Camus and Masmoudi observed 1418 Dy I lines in absorption between 2478 and $4100 \AA$, and found 81 new high levels [Masmoudi, 1972; Camus and Masmoudi, 1973]. Since the published values of the low levels are given to only two decimal places, we have for consistency rounded off the upper levels to two places.

Spector identified six upper even levels as belonging to the two $J_{1} j$-coupling terms of $4 f^{9}\left({ }^{6} \mathrm{H}_{15 / 2}^{\circ}\right) 6 s^{2} 6 p$, and Griffin, Ross, and Cowan extended the interpretation of the odd levels to include 62 assignments to eigenvectors of $4 f^{10} 6 s 6 p$ and $4 f^{9} 5 d 6 s^{2}$.

In his 1973 thesis, Wyart gives a large extension of the Dy I analysis and some revisions. He lists a total of almost 400 even levels and more than 300 odd levels. Wyart notes that a list of 9500 lines of Dy I and Dy II having energy-level classifications or Zeeman data will be given in a separate report. High-resolution infrared spectra $(0.8$ to $2.6 \mu \mathrm{m})$ obtained by Verges with a Fourier spectrometer were among the new data available to Wyart.

The levels and eigenvector percentages given here are primarily a rearrangement of the data in Wyart's thesis [1973], including some additions and revisions made later [Wyart, 1975; 1976]. A few uninterpreted levels without $g$ values and listed by only one investigator were marked as tentative.

\section{$g$ Values}

The $J$ and $g$ values of the ground level of Dy I were determined by atomic-beam magnetic-resonance techniques in 1961 [Cabezas, Lindgren, and Marrus; Smith and Spalding]; these measurements gave the first direct experimental evidence that the ground level was $4 f^{10} 6 s^{2}{ }^{5} \mathbf{I}_{8}$. The five-place $g$ values listed here are from more recent work by Childs [1970] and by Ferch, Dankwort, and Gebauer [1974]. The latter authors found the $g$ value of the ground level to be $1.2415869 \pm .0000010$, and Childs gives uncertainties of \pm 0.00003 and \pm 0.00005 for his determinations of the $g$ values of the ${ }^{5} \mathrm{I}_{7}$ and ${ }^{5} \mathrm{I}_{6}$ levels, respectively. 
The remaining $g$ values are from Conway and Worden or from Wyart $[1973,1976]$. Some values given by Wyart to the nearest 0.005 have been truncated to two places to distinguish them from the three-place values. The three-place values from Conway and Worden are accurate to about \pm 0.003 and most of the two-place values are accurate to \pm 0.01 or 0.02 .

\section{Calculations, Theoretical Interpretation}

All of Wyart's calculations of Dy I configurations except $4 f^{10} 6 s^{2}$ and $4 f^{10} 6 s 7 s$ were performed with truncated matrices. Calculation of the subconfigurations $\left(4 f^{10}\left({ }^{5} \mathrm{I}\right) 5 d 6 s+\right.$ $\left.4 f^{9}\left({ }^{6} \mathrm{H}^{\circ},{ }^{6} \mathrm{~F}^{\circ}\right) 6 s^{2} 6 p\right)$ allowed him to interpret 42 of the even levels. The $4 f^{10}\left({ }^{5} \mathrm{I}\right) 5 d 6 s$ percentages are for basis states with "real core" wavefunctions ( $4 f^{10}$ electrons in intermediate coupling); the corresponding percentages for pure-coupling basis states would in general be lower. In addition to the effect of truncation, Wyart noted that the omission of additional configurations, particularly $4 f^{9} 5 d 6 s 6 p$, from this calculation must significantly affect some of the eigenvectors. He showed that $4 f^{9} 5 d 6 s 6 p$ probably begins with the level at $23031.46 \mathrm{~cm}^{-1}(J=8)$. The configuration assignments of most even levels above this point that have not been assigned to calculated eigenvectors are given as tentative.

The eigenvector percentages for the odd levels are from Wyart's calculation [1973, 1974] of the subconfigurations $\left(4 f^{9}\left({ }^{6} \mathrm{H}^{\circ},{ }^{6} \mathrm{~F}^{\circ}\right) 5 d 6 s^{2}+4 f^{9}\left({ }^{6} \mathrm{H}^{\circ}, 6 \mathrm{~F}^{\circ}\right) 5 d^{2} 6 s+4 f^{10}\left({ }^{5} \mathrm{I}\right) 6 s 6 p\right)$. Wyart [1973] made a separate calculation of $4 f^{10}\left({ }^{5} \mathrm{I}\right) 6 s 6 p$ in $J_{1} J_{2}$ coupling, but did not transform to this scheme in his calculation including all three subconfigurations. The levels belonging mainly to $4 f^{10}\left({ }^{5} \mathrm{I}\right) 6 s 6 p$ are thus listed here in $J_{1} J_{2}$ coupling without percentages, and the leading percentages in LS coupling are given after the word "or." Murakawa and others had noted the appropriateness of $J_{1} J_{2}$ coupling for this subconfiguration, and the resultant strong effects on the intensities of the $4 f^{10}\left({ }^{5} \mathrm{I}\right) 6 s^{2}-4 f^{10}\left({ }^{5} \mathrm{I}\right) 6 s 6 p$ transitions have been mentioned by several authors. (Similar effects occur for $4 f^{N} 6 s^{2}-4 f^{N} 6 s 6 p$ transitions in general.) Cowan [1973] has calculated transition probabilities for some of these lines.

Wyart remarks that the three calculated odd subconfigurations are expected to account for the levels up to about $28000 \mathrm{~cm}^{-1}$. Interpretation of the odd levels above this point would thus require the inclusion of higher terms in the $4 f^{N}$ cores of these configurations, and also additional configurations. Wyart [1975] has tentatively assigned a number of levels above $34000 \mathrm{~cm}^{-1}$ to the $4 f^{10} 5 d 6 p$ configuration because they combine mainly with $4 f^{10} 5 d 6 \mathrm{~s}$ levels. The assignments of several levels near $36000 \mathrm{~cm}^{-1}$ to $4 f^{10} 6 s 7 p$ and the location of two $4 f^{9} 5 d 6 s 7 \mathrm{~s}$ levels above $40000 \mathrm{~cm}^{-1}$ are also due to Wyart $[1975,1976]$.

\section{Hyperfine Structure, Isotope Shifts, Ionization Potential}

Hyperfine structure and isotope shifts in Dy I have been investigated extensively; only a few of the more recent references are included below. Griffin et al. and Wyart used the isotope shifts of particular levels as a help in making configuration assignments.

Worden, Conway, Paisner, and Solarz [1977] have observed high members of two series in Dy I by using laser techniques. These data became available too late for inclusion here, but the quoted ionization energy is a new determination from these series.

\section{References}

Cabezas, A. Y., Lindgren, I., and Marrus, R., Phys. Rev. 122, 1796 (1961). ZE Hfs

Camus, P., and Masmoudi, K., Spectrochim. Acta, Part B 28, 79 (1973). EL CL W ZE

Childs, W. J., Phys. Rev. A 2, 1692 (1970). ZE Hfs

Conway, J. G., and Worden, E. F., Univ. Calif. Radiation Lab., Berkeley, UCRL-19944, 613 pp. (1970). (Available from National Technical Information Service, Springfield, Va. 22161.) EL CL W ZE PT

Conway, J. G., and Worden, E. F., J. Opt. Soc. Am. 61, 704 (1971). EL CL W ZE PT

Cowan, R. D., Nucl. Instrum. Methods 110, 173 (1973). AT

Dekker, J. W. M., Klinkenberg, P. F. A., and Langkemper, J. F., Physica (Utrecht) 39, 393 (1968). IS

Ebenhöh, W., Ehlers, V. J., and Ferch, J., Z. Phys. 200, 84 (1967). Hfs

Ferch, J., Dankwort, W., and Gebauer, H., Phys. Lett. A 49, 287 (1974). ZE Hfs

Golovin, A. F., and Striganov, A. R., Sov. Phys.-Usp. (Engl. Transl.) 10, 658 (1968). IS

Griffin, D. C., Ross, J. S., and Cowan, R. D., J. Opt. Soc. Am. 62, 571 (1972). ND IS PT

King, A. S., Astrophys. J. 72, 221 (1930). W 
King, A. S., Conway, J. G., Worden, E. F., and Moore, C. E., J. Res. Nat. Bur. Stand. (U.S.) 74A, 355 (1970). W Masmoudi, K., Thesis (Third Cycle), Univ. Paris-Sud, Orsay, 130 pp. (1972). EL CL W ZE

Miller, G. E., and Ross, J. S., J. Opt. Soc. Am. 66, 585 (1976). IS

Murakawa, K., Phys. Rev. A 7, 416 (1973). Hfs

Rosén, A., and Nyqvist, H., Phys. Scr. 6, 24 (1972). Hfs

Ross, J. S., J. Opt. Soc. Am. 62, 548 (1972). IS

Smith, K. F., and Spalding, I. J., Proc. R. Soc. London, Ser. A 265, 133 (1961). ZE

Spector, N., J. Opt. Soc. Am. 61, 1350 (1971). ND PT

Worden, E. F., Conway, J. G., Paisner, J. A., and Solarz, R. W., unpublished material (1977). IP

Wyart, J. F., C. R. Acad. Sci., Ser. B 273, 763 (1971). EL ND

Wyart, J. F., Thesis, Univ. Paris-Sud, Orsay, 194 pp. (1973). EL ND CL ZE IS IP PT

Wyart, J. F., Physica (Utrecht) 75, 371 (1974). EL ND ZE PT

Wyart, J. F., unpublished material $(1975,1976)$. EL ND ZE PT

[July 1976]

Dy I, Even Parity

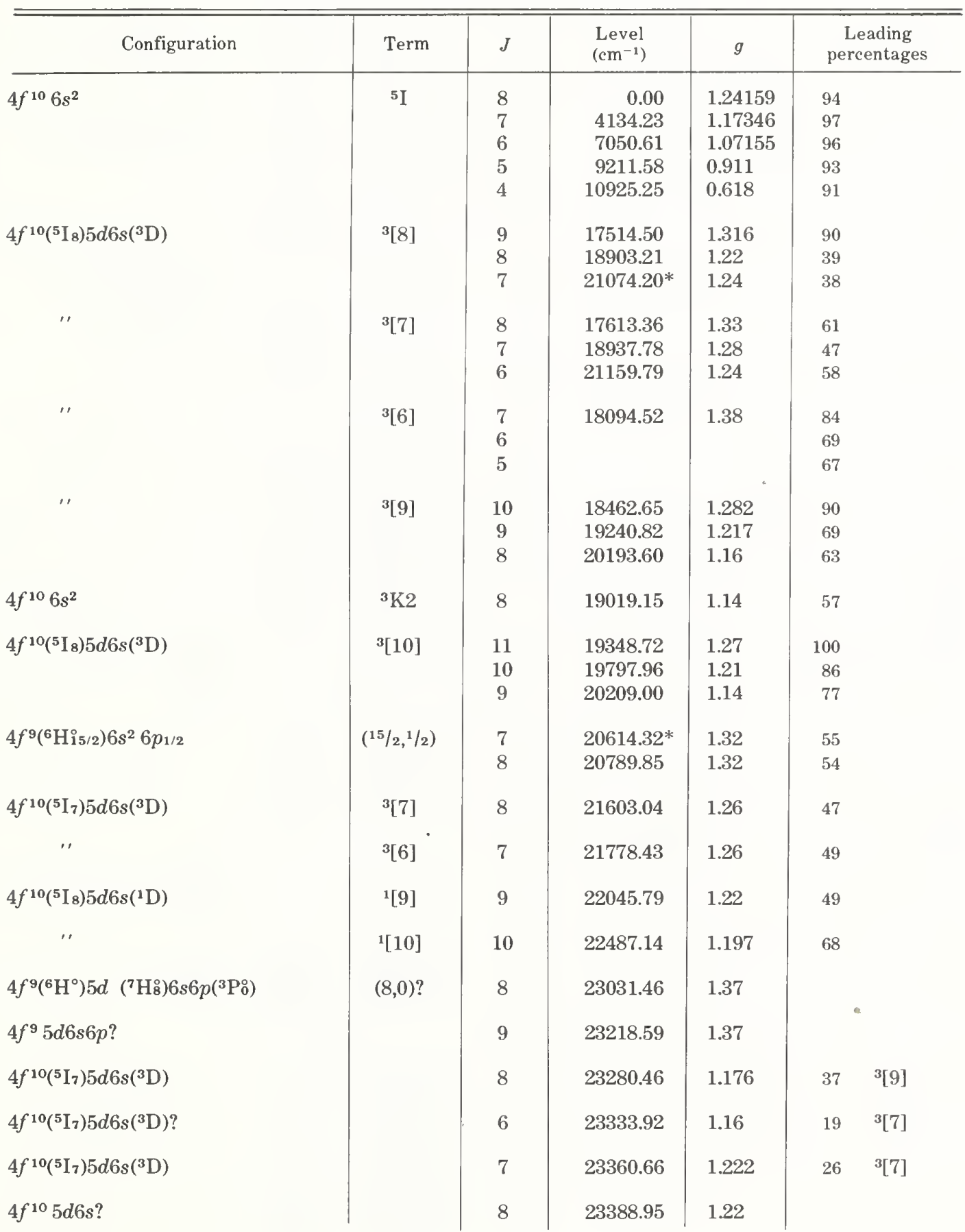


Dy I, Even Parity-Continued

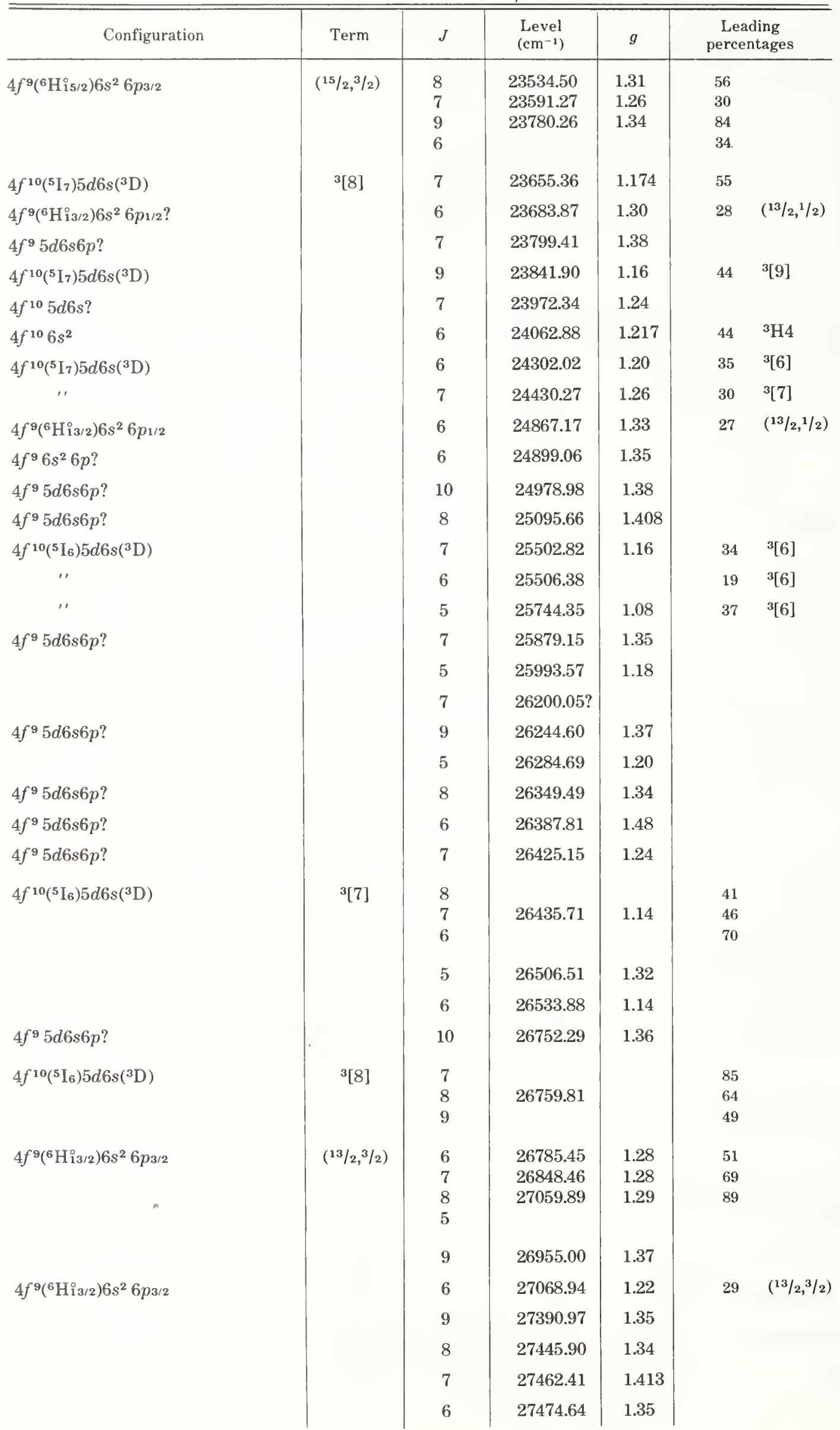


Dy I, Even Parity—Continued

\begin{tabular}{|c|c|c|c|c|c|c|}
\hline Configuration & Term & $J$ & $\begin{array}{l}\text { Level } \\
\left(\mathrm{cm}^{-1}\right)\end{array}$ & $g$ & \multicolumn{2}{|c|}{$\begin{array}{c}\text { Leading } \\
\text { percentages }\end{array}$} \\
\hline $4 f^{10}\left({ }^{5} \mathrm{I}_{5}\right) 5 d 6 s\left({ }^{3} \mathrm{D}\right)$ & & 4 & 27578.02 & 0.98 & 42 & ${ }^{3}[5]$ \\
\hline \multirow[t]{8}{*}{$4 f^{9} 6 s^{2} 6 p ?$} & & 5 & 27680.12 & 1.317 & & \\
\hline & & 8 & 27896.80 & 1.33 & & \\
\hline & & 7 & 27959.98 & 1.36 & & \\
\hline & & 6 & 27987.90 & 1.32 & & \\
\hline & & 4 & 28082.47 & 1.12 & & \\
\hline & & 9 & 28158.51 & 1.33 & & \\
\hline & & 4 & 28235.74 & 1.12 & & \\
\hline & & 5 & 28265.78 & 1.32 & & \\
\hline \multirow[t]{3}{*}{$4 f^{9} 6 s^{2} 6 p ?$} & & 5 & 28309.18 & 1.20 & & \\
\hline & & 6 & 28326.48 & 1.33 & & \\
\hline & & 7 & 28358.70 & 1.35 & & \\
\hline \multirow[t]{4}{*}{$4 f^{9}\left({ }^{6} \mathrm{~F}_{11 / 2}^{\circ}\right) 6 s^{2} 6 p_{3 / 2}$} & $(11 / 2,3 / 2)$ & 5 & 28379.82 & 1.40 & 50 & \\
\hline & & 10 & 28433.41 & 1.33 & & \\
\hline & & 8 & 28539.57 & 1.32 & & \\
\hline & & 5 & 28666.31 & 1.30 & & \\
\hline \multirow[t]{10}{*}{$4 f^{9}\left({ }^{6} \mathrm{~F}_{11 / 2}^{\circ}\right) 6 s^{2} 6 p_{1 / 2}$} & $(11 / 2,1 / 2)$ & 6 & 28849.06 & 1.36 & 69 & \\
\hline & & 6 & 28909.39 & 1.38 & & \\
\hline & & 8 & 28971.42 & 1.35 & & \\
\hline & & 5 & 28987.02 & 1.317 & & \\
\hline & & 6 & 29159.93 & 1.20 & & \\
\hline & & 7 & 29169.98 & 1.313 & & \\
\hline & & 8 & 29291.32 & 1.36 & & \\
\hline & & 9 & 29465.04 & 1.32 & & \\
\hline & & 5 & 29496.33 & 1.18 & & \\
\hline & & 7 & 29512.27 & 1.36 & & \\
\hline \multirow[t]{17}{*}{$4 f^{9} 6 s^{2} 6 p ?$} & & 7 & 29532.42 & 1.24 & & \\
\hline & & 3 & 29626.20 & 0.82 & & \\
\hline & & 6 & 29682.16 & 1.36 & & \\
\hline & & 10 & 29706.72 & 1.32 & & \\
\hline & & 9 & 29714.72 & 1.31 & & \\
\hline & & 11 & 29742.49 & 1.34 & & \\
\hline & & 8 & 29841.69 & 1.32 & & \\
\hline & & 7 & 29877.37 & 1.38 & & \\
\hline & & 6 & 30015.44 & 1.287 & & \\
\hline & & 5 & 30033.06 & 1.34 & & \\
\hline & & 6 & 30102.59 & 1.29 & & \\
\hline & & 10 & 30106.65 & 1.28 & & \\
\hline & & 7 & 30163.33 & 1.36 & & \\
\hline & & 7 & 30444.88 & 1.30 & & \\
\hline & & 9 & 30459.64 & 1.26 & & \\
\hline & & 6 & 30475.95 & 1.34 & & \\
\hline & & 5 & 30512.73 & 1.29 & & \\
\hline
\end{tabular}


Dy I, Even Parity-Continued

\begin{tabular}{|c|c|c|c|c|c|}
\hline Configuration & Term & $J$ & $\begin{array}{l}\text { Level } \\
\left(\mathrm{cm}^{-1}\right)\end{array}$ & $g$ & $\begin{array}{l}\text { Leading } \\
\text { percentages }\end{array}$ \\
\hline \multirow[t]{44}{*}{$4 f^{10}\left({ }^{5} \mathrm{I}_{8}\right) 6 s 7 s\left({ }^{3} \mathrm{~S}_{1}\right)$} & $(8,1)$ & 9 & 30560.56 & 1.32 & 99 \\
\hline & & 8 & 30979.53 & 1.26 & 92 \\
\hline & & 7 & 31509.12 & 1.18 & 99 \\
\hline & & 8 & 30600.15 & 1.31 & \\
\hline & & 7 & 30621.87 & 1.27 & \\
\hline & & 4 & 30662.79 & 1.25 & \\
\hline & & 9 & 30716.06 & 1.26 & \\
\hline & & 8 & 30739.79 & 1.32 & \\
\hline & & 4 & 30896.57 & 1.48 & \\
\hline & & 6 & 30988.25 & 1.332 & \\
\hline & & 8 & 31061.18 & 1.306 & \\
\hline & & 5 & 31079.52 & 1.223 & \\
\hline & & 7 & 31124.80 & 1.34 & \\
\hline & & 6 & 31180.01 & 1.26 & \\
\hline & & 8 & 31233.57 & 1.34 & \\
\hline & & 9 & 31287.04 & 1.35 & \\
\hline & & 7 & 31362.62 & 1.26 & \\
\hline & & 7 & 31423.04 & 1.32 & \\
\hline & & 8 & 31469.00 & 1.42 & \\
\hline & & 10 & 31489.64 & 1.276 & \\
\hline & & 6 & 31529.68 & 1.42 & \\
\hline & & 5 & 31545.99 & 1.38 & \\
\hline & & 7 & 31674.08 & 1.31 & \\
\hline & & 5 & 31742.14 & 1.393 & \\
\hline & & 9 & 31775.65 & 1.27 & \\
\hline & & 8 & 31820.28 & 1.204 & \\
\hline & & 10 & 31838.24 & 1.307 & \\
\hline & & 7 & 32036.51 & 1.32 & \\
\hline & & 9 & 32206.27 & 1.24 & \\
\hline & & 7 & 32382.29 & 1.37 & \\
\hline & & 6 & 32392.59 & 1.32 & \\
\hline & & 7 & 32411.25 & 1.32 & \\
\hline & & 10 & 32428.08 & 1.35 & \\
\hline & & 8 & 32428.66 & 1.32 & \\
\hline & & 7 & 32470.81 & 1.32 & \\
\hline & & 8 & 32554.86 & 1.27 & \\
\hline & & 8 & 32675.52 & 1.27 & \\
\hline & & 3 & 32712.54 & 1.26 & \\
\hline & & 7 & 32722.87 & 1.33 & \\
\hline & & 9 & 32763.21 & 1.29 & \\
\hline & & 8 & 32940.47 & 1.35 & \\
\hline & & 6 & 32945.30 & 1.33 & \\
\hline & & 9 & 33086.26 & 1.29 & \\
\hline & & 7 & 33110.16 & 1.28 & \\
\hline
\end{tabular}


Dy I, Even Parity_Continued

\begin{tabular}{|c|c|c|c|c|c|}
\hline Configuration & Term & $J$ & $\begin{array}{l}\text { Level } \\
\left(\mathrm{cm}^{-1}\right)\end{array}$ & $g$ & $\begin{array}{c}\text { Leading } \\
\text { percentages }\end{array}$ \\
\hline & & 6 & 33210.13 & 1.32 & \\
\hline & & 8 & 33246.13 & 1.24 & \\
\hline & & 9 & 33252.28 & 1.31 & \\
\hline & & 4 & 33358.79 & 1.07 & \\
\hline & & 5 & 33381.16 & 1.382 & \\
\hline & & 8 & 33406.06 & 1.31 & \\
\hline & & 6 & 33474.30 & 1.37 & \\
\hline & & 7 & 33475.72 & 1.33 & \\
\hline & & 7 & 33552.39 & 1.28 & \\
\hline & & 6 & 33656.96 & 1.28 & \\
\hline & & 7 & 33746.82 & 1.27 & \\
\hline & & 8 & 33753.11 & 1.31 & \\
\hline & & 7 & 33806.12 & 1.204 & \\
\hline & & 6 & 33911.02 & 1.30 & \\
\hline & & 7 & 34027.70 & 1.26 & \\
\hline & & 7 & 34060.16 & 1.13 & \\
\hline & & 7 & 34131.12 & 1.29 & \\
\hline & & 9 & 34174.66 & 1.31 & \\
\hline & & 6 & 34179.68 & 1.190 & \\
\hline & & 7 & 34196.55 & 1.32 & \\
\hline & & 6 & 34296.69 & 1.36 & \\
\hline & & 7 & 34324.66 & 1.35 & \\
\hline & & 6 & 34547.46 & 1.31 & \\
\hline & & 5 & 34573.07 & 1.30 & \\
\hline & & 8 & 34676.95 & 1.24 & \\
\hline & & 6 & 34679.75 & 1.488 & \\
\hline & & 9 & 34689.19 & 1.28 & \\
\hline & & 5 & 34742.71 & 1.24 & \\
\hline & & 7 & 34742.84 & 1.34 & \\
\hline & & 10 & 34776.04 & 1.278 & \\
\hline & & 9 & 34829.30 & 1.27 & \\
\hline & & 5 & 34841.48 & 1.36 & \\
\hline & & 7 & 34843.11 & 1.29 & \\
\hline \multirow[t]{11}{*}{$4 f^{10}\left({ }^{5} \mathrm{I}_{7}\right) 6 s 7 s\left({ }^{3} \mathrm{~S}_{1}\right)$} & $(7,1)$ & 8 & 34922.08 & 1.27 & 97 \\
\hline & & 7 & 35135.33 & 1.20 & 96 \\
\hline & & 6 & 35421.17 & 1.10 & 97 \\
\hline & & 7 & 35003.75 & 1.31 & \\
\hline & & 8 & 35053.56 & 1.32 & \\
\hline & & 3 & 35141.43 & 0.98 & \\
\hline & & 5 & 35184.56 & 1.30 & \\
\hline & & 9 & 35219.85 & 1.28 & \\
\hline & & 7 & 35221.98 & 1.32 & \\
\hline & & 8 & 35249.13 & 1.32 & \\
\hline & & 5 & 35354.27 & 1.38 & \\
\hline
\end{tabular}


Dy I, Even Parity-Continued

\begin{tabular}{|c|c|c|c|c|c|}
\hline Configuration & Term & $J$ & $\begin{array}{l}\text { Level } \\
\left(\mathrm{cm}^{-1}\right)\end{array}$ & $g$ & $\begin{array}{c}\text { Leading } \\
\text { percentages }\end{array}$ \\
\hline & & 8 & 35377.51 & 1.28 & \\
\hline & & 10 & 35385.78 & 1.27 & \\
\hline & & 5 & 35523.35 & 1.16 & \\
\hline & & 5 & 35578.47 & 1.21 & \\
\hline & & 7 & 35695.77 & 1.34 & \\
\hline & & 7 & 35737.77 & 1.20 & \\
\hline & & 6 & 35744.63 & 1.32 & \\
\hline & & 9 & 35762.55 & 1.32 & \\
\hline & & 7 & 35866.60 & 1.24 & \\
\hline & & 8 & 35938.74 & 1.35 & \\
\hline & & 9 & 35940.35 & 1.32 & \\
\hline & & 6 & 35970.10 & 1.40 & \\
\hline & & 6 & 36093.54 & 1.27 & \\
\hline & & 7 & 36119.46 & 1.25 & \\
\hline & & 8 & 36308.08 & 1.27 & \\
\hline & & 6 & 36365.09 & & \\
\hline & & 8 & 36392.11 & 1.33 & \\
\hline & & 7 & 36417.25 & 1.29 & \\
\hline & & 9 & 36487.20 & 1.31 & \\
\hline & & 10 & 36490.07 & 1.25 & \\
\hline & & 7 & 36491.05 & & \\
\hline & & 7 & 36508.79 & 1.34 & \\
\hline & & 8 & 36553.84 & 1.38 & \\
\hline & & 8 & 36599.44 & 1.29 & \\
\hline & & 7 & 36608.28 & 1.18 & \\
\hline & & 6 & 36612.84 & 1.262 & \\
\hline \multirow[t]{3}{*}{$4 f^{10}\left({ }^{5} \mathrm{I}_{7}\right) 6 s 7 s\left({ }^{1} \mathrm{~S}_{0}\right)$} & $(7,0)$ & 7 & 36667.78 & & 91 \\
\hline & & 9 & 36708.15 & 1.32 & \\
\hline & & 9 & 36717.57 & 1.29 & \\
\hline \multirow[t]{4}{*}{$4 f^{10} 6 s 6 d ?$} & & 8 & 36760.64 & 1.24 & \\
\hline & & 8 & 36807.39 & 1.34 & \\
\hline & & 9 & 36822.27 & 1.25 & \\
\hline & & 7 & 36865.40 & 1.31 & \\
\hline \multirow[t]{3}{*}{$4 f^{10} 6 s 6 d ?$} & & 10 & 36905.44 & 1.25 & \\
\hline & & 7 & 36924.54 & 1.22 & \\
\hline & & 8 & 36954.35 & 1.30 & \\
\hline $4 f^{10} 6 s 6 d ?$ & & 9 & 36964.32 & 1.21 & \\
\hline \multirow[t]{2}{*}{$4 f^{10} 6 s 6 d ?$} & & 10 & 37007.58 & 1.22 & \\
\hline & & 7 & 37015.22 & 1.27 & \\
\hline \multirow[t]{4}{*}{$4 f^{10}\left({ }^{5} \mathrm{I}_{8}\right) 6 s 6 d\left({ }^{3} \mathrm{D}\right) ?$} & & 11 & 37039.00 & 1.28 & \\
\hline & & 6 & 37058.60 & 1.31 & \\
\hline & & 8 & 37087.47 & 1.33 & \\
\hline & & 9 & 37121.97 & 1.27 & \\
\hline
\end{tabular}


Dy I, Even Parity-Continued

\begin{tabular}{|c|c|c|c|c|c|}
\hline Configuration & Term & $J$ & $\begin{array}{l}\text { Level } \\
\left(\mathrm{cm}^{-1}\right)\end{array}$ & $g$ & $\begin{array}{l}\text { Leading } \\
\text { percentages }\end{array}$ \\
\hline & & 6 & 37125.45 & 1.33 & \\
\hline & & 7 & 37135.33 & 1.20 & \\
\hline & & 6 & 37163.16 & 1.29 & \\
\hline & & 5 & 37182.19 & 1.26 & \\
\hline & & 7 & 37212.06 & 1.30 & \\
\hline & & 5 & 37231.26 & 1.26 & \\
\hline & & 7 & 37295.97 & 1.28 & \\
\hline & & 11 & 37299.36 & 1.26 & \\
\hline & & 6 & 37324.62 & 1.24 & \\
\hline \multirow[t]{8}{*}{$4 f^{10} 6 s 6 d ?$} & & 8 & 37339.89 & 1.25 & \\
\hline & & 7 & 37366.09 & 1.32 & \\
\hline & & 8 & 37398.46 & 1.25 & \\
\hline & & 5 & 37472.68 & 1.34 & \\
\hline & & 7 & 37501.58 & 1.243 & \\
\hline & & 8 & 37527.15 & 1.24 & \\
\hline & & 7 & 37551.19 & 1.30 & \\
\hline & & 5 & 37559.17 & 1.27 & \\
\hline \multirow[t]{6}{*}{$4 f^{10} 6 s 6 d ?$} & & 9 & 37591.83 & 1.212 & \\
\hline & & 7 & 37607.89 & 1.29 & \\
\hline & & 5 & 37646.62 & 1.20 & \\
\hline & & 6 & 37650.98 & 1.4 & \\
\hline & & 8 & 37676.89 & 1.32 & \\
\hline & & 7 & 37694.25 & 1.26 & \\
\hline \multirow[t]{3}{*}{$4 f^{10} 6 s 6 d ?$} & & 10 & 37706.12 & 1.203 & \\
\hline & & 9 & 37751.03 & 1.29 & \\
\hline & & 7 & 37751.34 & & \\
\hline \multirow[t]{7}{*}{$4 f^{10} 6 s 6 d ?$} & & 8 & 37820.22 & 1.14 & \\
\hline & & 8 & 37841.84 & 1.29 & \\
\hline & & 6 & 37856.42 & 1.247 & \\
\hline & & 9 & 37933.63 & 1.377 & \\
\hline & & 7 & 37980.03 & 1.191 & \\
\hline & & 8 & 37992.78 & 1.187 & \\
\hline & & 7 & 38054.61 & 1.333 & \\
\hline \multirow[t]{11}{*}{$4 f^{10}\left({ }^{5} \mathrm{I}_{6}\right) 6 s 7 s\left({ }^{3} \mathrm{~S}_{1}\right)$} & $(6,1)$ & 6 & 38070.03 & 1.11 & 95 \\
\hline & & 5 & 38093.85 & 1.08 & 96 \\
\hline & & 7 & 38123.30 & 1.20 & 91 \\
\hline & & 8 & 38078.12 & 1.19 & \\
\hline & & 5 & 38101.89 & 1.20 & \\
\hline & & 8 & 38150.52 & 1.38 & \\
\hline & & 5 & 38164.83 & 1.257 & \\
\hline & & 6 & 38214.81 & 1.22 & \\
\hline & & 8 & 38254.97 & 1.29 & \\
\hline & & 7 & 38264.28 & 1.34 & \\
\hline & & 9 & 38285.36 & 1.25 & \\
\hline
\end{tabular}


Dy I, Even Parity_Continued

\begin{tabular}{|c|c|c|c|c|c|}
\hline Configuration & Term & $J$ & $\begin{array}{l}\text { Level } \\
\left(\mathrm{cm}^{-1}\right)\end{array}$ & $g$ & $\begin{array}{c}\text { Leading } \\
\text { percentages }\end{array}$ \\
\hline & & 10 & 38329.91 & 1.30 & \\
\hline & & 5 & 38334.20 & & \\
\hline & & 8 & 38356.27 & 1.36 & \\
\hline & & 7 & 38366.31 & 1.333 & \\
\hline & & 4 & 38431.81 & 1.26 & \\
\hline & & 9 & 38444.35 & 1.28 & \\
\hline & & 7 & 38452.46 & 1.238 & \\
\hline & & 7 & 38515.31 & 1.298 & \\
\hline & & 8 & 38516.86 & 1.222 & \\
\hline & & 7 & 38524.52 & 1.34 & \\
\hline & & 8 & 38551.45 & 1.25 & \\
\hline & & 5 & 38673.50 & 1.30 & \\
\hline & & 9 & 38674.91 & 1.28 & \\
\hline & & 6 & 38715.04 & 1.26 & \\
\hline & & 7 & 38737.66 & 1.29 & \\
\hline & & 6 & 38852.60 & 1.19 & \\
\hline & & 7 & 38861.55 & & \\
\hline & & 8 & 38870.01 & 1.25 & \\
\hline & & 5 & 38890.92 & & \\
\hline & & 7 & 38954.01 & 1.31 & \\
\hline & & 5 & 38964.68 & 1.28 & \\
\hline & & 8 & 38973.45 & 1.24 & \\
\hline & & 9 & 39035.85 & 1.24 & \\
\hline & & 5 & 39048.18 & 1.38 & \\
\hline & & 7 & 39078.14 & 1.28 & \\
\hline & & 4 & 39084.97 & 1.33 & \\
\hline & & 8 & 39096.06 & & \\
\hline & & 7 & 39097.74 & 1.21 & \\
\hline & & 9 & 39120.61 & 1.34 & \\
\hline & & 10 & 39176.58 & 1.26 & \\
\hline & & 5 & 39201.13 & 1.10 & \\
\hline & & 9 & 39332.82 & 1.28 & \\
\hline & & 7 & 39332.97 & 1.29 & \\
\hline & & 8 & 39376.93 & 1.33 & \\
\hline & & 4 & 39378.71 & 0.98 & \\
\hline & & 5 & 39411.02 & 1.272 & \\
\hline & & 4 & 39420.80 & 1.20 & \\
\hline & & 4 & 39430.94 & 1.14 & \\
\hline & & 9 & 39513.68 & 1.30 & \\
\hline & & 7 & 39516.88 & 1.288 & \\
\hline \multirow[t]{3}{*}{$4 f^{10}\left({ }^{5} \mathrm{I}_{6}\right) 6 s 7 s\left({ }^{1} \mathrm{~S}_{0}\right)$} & $(6,0)$ & 6 & 39545.90 & & 84 \\
\hline & & 10 & 39573.04 & 1.28 & \\
\hline & & 11 & 39627.83 & 1.27 & \\
\hline
\end{tabular}


Dy I, Even Parity-Continued

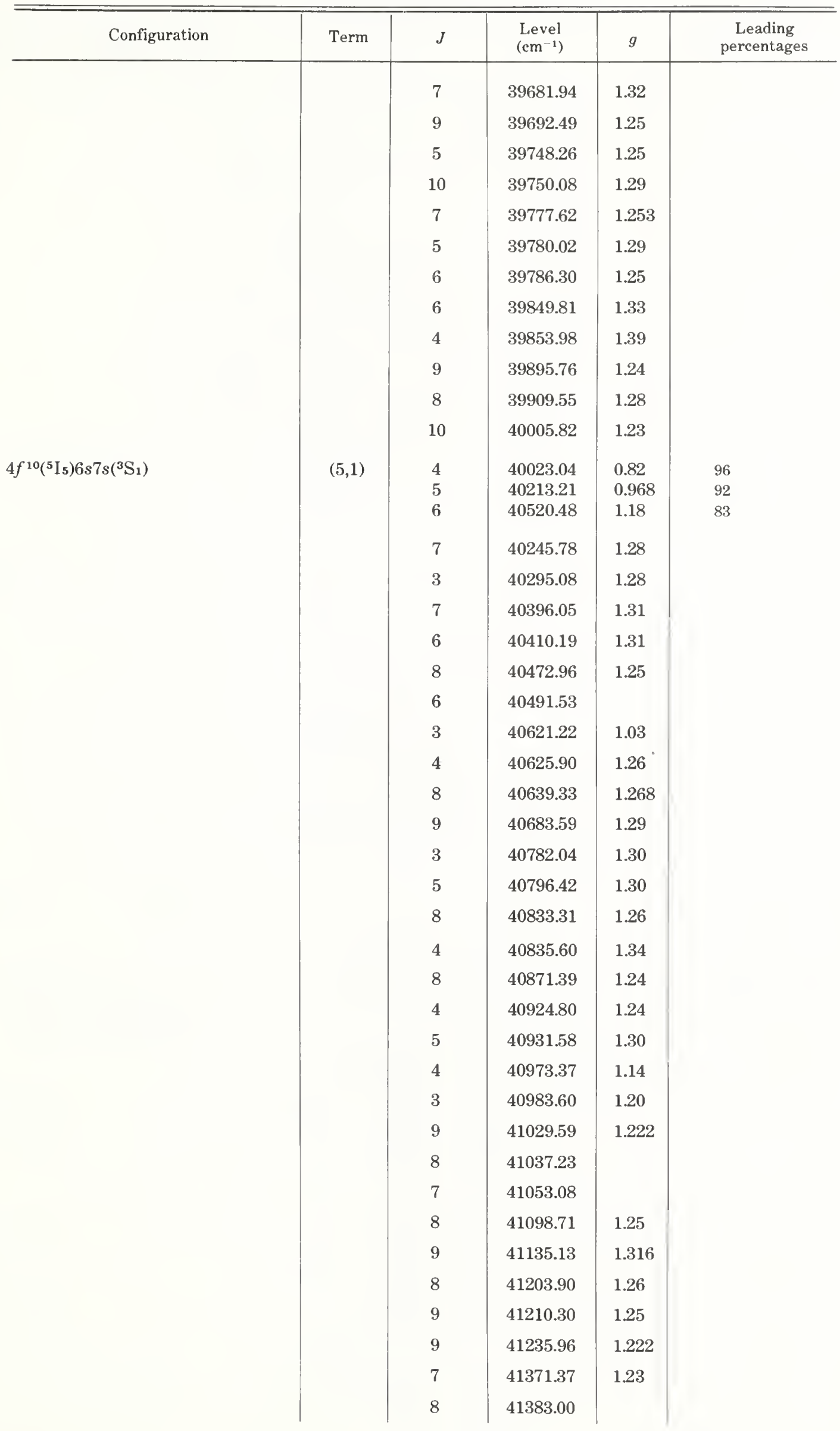


Dy I, Even Parity—Continued

\begin{tabular}{|c|c|c|c|c|c|}
\hline Configuration & Term & $J$ & $\begin{array}{l}\text { Level } \\
\left(\mathrm{cm}^{-1}\right)\end{array}$ & $g$ & $\begin{array}{l}\text { Leading } \\
\text { percentages }\end{array}$ \\
\hline & & 4 & 41458.22 & 1.22 & \\
\hline \multirow[t]{21}{*}{$4 f^{10}\left({ }^{5} \mathrm{I}_{4}\right) 6 s 7 s\left({ }^{3} \mathrm{~S}_{1}\right)$} & $(4,1)$ & 3 & 41492.41 & 0.44 & 99 \\
\hline & & 4 & 41859.44 & 0.80 & 92 \\
\hline & & 5 & 42490.50 & 1.00 & 81 \\
\hline & & 7 & 41503.84 & 1.27 & \\
\hline & & 8 & 41638.55 & & \\
\hline & & 4 & 41957.18 & 1.22 & \\
\hline & & 4 & 42146.02 & 1.22 & \\
\hline & & 3 & 42220.12 & 1.26 & \\
\hline & & 4 & 42236.05 & 1.22 & \\
\hline & & 10 & 42668.10 & 1.35 & \\
\hline & & 4 & 42892.94 & 1.12 & \\
\hline & & 8 & 42921.39 & & \\
\hline & & 4 & 42940.96 & 1.23 & \\
\hline & & 7 & 42984.71 & 1.21 & \\
\hline & & 5 & 43020.84 & 1.30 & \\
\hline & & 7 & 43222.10 & & \\
\hline & & 8 & 43728.57 & & \\
\hline & & 8 & 44487.65 & & \\
\hline & & 6 & 45703.64 & & \\
\hline & & 7 & 46391.45 & & \\
\hline & & 8 & 47354.04 & & \\
\hline Dy II $\left({ }^{5} I_{8}\right) 6 s_{1 / 2}(8,1 / 2)_{17 / 2}$ & Limit & & 47900 & & \\
\hline
\end{tabular}

Dy I, Odd Parity

\begin{tabular}{|c|c|c|c|c|c|}
\hline Configuration & Term & $J$ & $\begin{array}{l}\text { Level } \\
\left(\mathrm{cm}^{-1}\right)\end{array}$ & $g$ & Leading percentages \\
\hline $4 f^{9}\left({ }^{6} \mathrm{H}^{\circ}\right) 5 d 6 s^{2}$ & ${ }^{7} \mathrm{H}^{\circ}$ & $\begin{array}{l}8 \\
7 \\
6 \\
5 \\
4 \\
3 \\
2\end{array}$ & $\begin{array}{r}7565.60 \\
8519.20 \\
10088.80 \\
12298.56 \\
13952.00 \\
15254.94\end{array}$ & $\begin{array}{l}1.35246 \\
1.336 \\
1.36 \\
1.24 \\
1.082 \\
0.77\end{array}$ & $\begin{array}{l}81 \\
60 \\
33 \\
56 \\
66 \\
75 \\
82\end{array}$ \\
\hline $4 f^{9}\left({ }^{6} \mathrm{H}^{\circ}\right) 5 d 6 s^{2}$ & ${ }^{7} \mathrm{I}^{\circ}$ & $\begin{array}{l}9 \\
8 \\
7 \\
6 \\
5 \\
4 \\
3\end{array}$ & $\begin{array}{r}9990.95 \\
12007.10 \\
14367.80 \\
14970.70 \\
16684.73\end{array}$ & $\begin{array}{l}1.32 \\
1.28 \\
1.27 \\
1.24 \\
1.082\end{array}$ & $\begin{array}{l}89 \\
50 \\
50 \\
39 \\
67 \\
79 \\
76\end{array}$ \\
\hline $4 f^{9}\left({ }^{6} \mathrm{H}^{\circ}\right) 5 d 6 s^{2}$ & ${ }^{7} \mathrm{~F}^{\circ}$ & 6 & 11673.49 & 1.392 & 47 \\
\hline $4 f^{9}\left({ }^{6} \mathrm{H}^{\circ}\right) 5 d 6 \mathrm{~s}^{2}$ & ${ }^{7} \mathrm{G}^{\circ}$ & 7 & 12655.13 & 1.36 & 64 \\
\hline
\end{tabular}


Dy I, Odd Parity-Continued

\begin{tabular}{|c|c|c|c|c|c|c|c|}
\hline Configuration & Term & $J$ & $\begin{array}{l}\text { Level } \\
\left(\mathrm{cm}^{-1}\right)\end{array}$ & $g$ & & & Leading percentages \\
\hline $4 f^{9}\left({ }^{6} \mathrm{H}^{\circ}\right) 5 d 6 s^{2}$ & ${ }^{7} \mathrm{~K}^{\circ}$ & $\begin{array}{c}10 \\
8 \\
9 \\
7 \\
6 \\
5 \\
4\end{array}$ & $\begin{array}{l}12892.76 \\
16288.73 \\
16717.79 \\
17687.90 \\
19182.66\end{array}$ & $\begin{array}{l}1.29 \\
1.19 \\
1.24 \\
1.16 \\
1.036\end{array}$ & $\begin{array}{l}99 \\
46 \\
64 \\
44 \\
72 \\
81 \\
89\end{array}$ & & \\
\hline $4 f^{9}\left({ }^{6} \mathrm{H}^{\circ}\right) 5 d 6 s^{2}$ & ${ }^{5} \mathrm{~K}^{\circ}$ & $\begin{array}{l}9 \\
8 \\
7 \\
6 \\
5\end{array}$ & $\begin{array}{l}13495.92 \\
19688.59 \\
21783.42 \\
23464.02 \\
24881.85\end{array}$ & $\begin{array}{l}1.23 \\
1.22 \\
1.15 \\
0.96 \\
0.72\end{array}$ & $\begin{array}{l}65 \\
57 \\
70 \\
52 \\
67\end{array}$ & & . \\
\hline $4 f^{9}\left({ }^{6} \mathrm{H}^{\circ}\right) 5 d 6 s^{2}$ & ${ }^{7} \mathrm{~F}^{\circ}$ & 5 & 14153.49 & 1.42 & 51 & & \\
\hline $4 f^{9}\left({ }^{6} \mathrm{H}^{\circ}\right) 5 d 6 s^{2}$ & ${ }^{5} \mathrm{I}^{\circ}$ & $\begin{array}{l}8 \\
7 \\
6 \\
5 \\
4\end{array}$ & $\begin{array}{l}14625.64 \\
18339.80^{*} \\
20554.73 \\
22294.88 \\
23686.81\end{array}$ & $\begin{array}{l}1.25 \\
1.21 \\
1.11 \\
1.02 \\
0.767\end{array}$ & $\begin{array}{l}67 \\
26 \\
45 \\
39 \\
70\end{array}$ & & \\
\hline $4 f^{9}\left({ }^{6} \mathrm{H}^{\circ}\right) 5 d 6 s^{2}$ & ${ }^{5} \mathrm{H}^{\circ}$ & 7 & 15194.83 & 1.26 & 78 & & \\
\hline $4 f^{10}\left({ }^{5} \mathrm{I} 8\right) 6 s 6 p\left({ }^{3} \mathrm{P}_{0}^{\circ}\right)$ & $(8,0)^{\circ}$ & 8 & 15567.38 & 1.31 & & or & $58 \quad\left({ }^{5} \mathrm{I}\right)\left({ }^{3} \mathrm{P}^{\circ}\right){ }^{7} \mathrm{H}^{\circ}$ \\
\hline $4 f^{9}\left({ }^{6} \mathrm{H}^{\circ}\right) 5 d 6 s^{2}$ & & 6 & 15862.64 & 1.257 & 43 & ${ }^{5} \mathrm{G}^{\circ}$ & \\
\hline $4 f^{10}\left({ }^{5} \mathrm{I}_{8}\right) 6 s 6 p\left({ }^{3} \mathrm{P}_{1}^{\circ}\right)$ & $(8,1)^{\circ}$ & $\begin{array}{l}9 \\
7 \\
8\end{array}$ & $\begin{array}{l}15972.35 \\
16693.87 \\
16733.20\end{array}$ & $\begin{array}{l}1.29 \\
1.22 \\
1.20\end{array}$ & & $\begin{array}{l}\text { or } \\
\text { or } \\
\text { or }\end{array}$ & 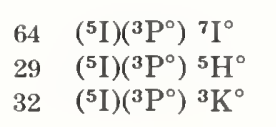 \\
\hline $4 f^{9}\left({ }^{6} \mathrm{~F}^{\circ}\right) 5 d 6 s^{2}$ & ${ }^{7} \mathrm{P}^{\circ}$ & 4 & 16069.98 & 1.62 & 47 & 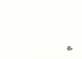 & \\
\hline $4 f^{9}\left({ }^{6} \mathrm{~F}^{\circ}\right) 5 d 6 s^{2}$ & & 4 & 16412.80 & 1.51 & 35 & ${ }^{7} \mathrm{P}^{\circ}$ & \\
\hline $4 f^{9}\left({ }^{6} \mathrm{H}^{\circ}\right) 5 d 6 s^{2}$ & & 6 & 16591.38 & 1.348 & 42 & ${ }^{7} \mathrm{G}^{\circ}$ & \\
\hline $4 f^{9}\left({ }^{6} \mathrm{~F}^{\circ}\right) 5 d 6 s^{2}$ & & 5 & 17502.89 & 1.45 & 24 & ${ }^{7} \mathrm{D}^{\circ}$ & \\
\hline $4 f^{10}\left({ }^{5} \mathrm{I} 8\right) 6 s 6 p\left({ }^{3} \mathrm{P}_{2}^{\circ}\right)$ & $(8,2)^{\circ}$ & $\begin{array}{l}10 \\
9 \\
8 \\
7 \\
6\end{array}$ & $\begin{array}{l}17513.33 \\
17727.15 \\
18021.89 \\
18433.76^{*} \\
18711.93\end{array}$ & $\begin{array}{l}1.30 \\
1.25 \\
1.23 \\
1.20 \\
1.172\end{array}$ & & $\begin{array}{l}\text { or } \\
\text { or } \\
\text { or } \\
\text { or } \\
\text { or }\end{array}$ & 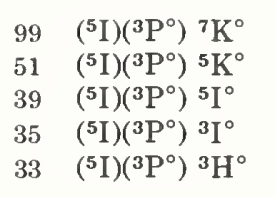 \\
\hline $4 f^{9}\left({ }^{6} \mathrm{H}^{\circ}\right) 5 d 6 s^{2}$ & ${ }^{5} \mathrm{~F}^{\circ}$ & 5 & 17804.24 & 1.322 & 54 & & \\
\hline $4 f^{9}\left({ }^{6} \mathrm{H}^{\circ}\right) 5 d 6 s^{2}$ & & 6 & 18172.87 & 1.34 & 39 & ${ }^{5} \mathrm{H}^{\circ}$ & \\
\hline $4 f^{9}\left({ }^{6} \mathrm{H}^{\circ}\right) 5 d^{2}\left({ }^{3} \mathrm{~F}\right)\left({ }^{8} \mathrm{G}^{\circ}\right) 6 s$ & ${ }^{9} \mathrm{G}^{\circ}$ & 8 & 18472.71 & 1.46 & 48 & & \\
\hline $4 f^{9}\left({ }^{6} \mathrm{H}^{\circ}\right) 5 d^{2}\left({ }^{3} \mathrm{~F}\right)\left({ }^{8} \mathrm{G}^{\circ}\right) 6 s$ & & 7 & 18528.55 & 1.467 & 25 & ${ }^{9} \mathrm{G}^{\circ}$ & \\
\hline $4 f^{9}\left({ }^{6} \mathrm{~F}^{\circ}\right) 5 d 6 s^{2}$ & & 6 & 18561.20 & 1.27 & 18 & ${ }^{7} \mathrm{G}^{\circ}$ & \\
\hline $4 f^{9}\left({ }^{6} \mathrm{~F}^{\circ}\right) 5 d 6 s^{2}$ & & 7 & 18857.04 & 1.335 & 22 & ${ }^{7} \mathrm{H}^{\circ}$ & \\
\hline $4 f^{9}\left({ }^{6} \mathrm{~F}^{\circ}\right) 5 d 6 s^{2}$ & ${ }^{7} \mathrm{H}^{\circ}$ & 8 & 19092.30 & 1.33 & 91 & & - \\
\hline $4 f^{9}\left({ }^{6} \mathrm{H}^{\circ}\right) 5 d^{2}\left({ }^{3} \mathrm{~F}\right)\left({ }^{8} \mathrm{~F}^{\circ}\right) 6 s$ & & 6 & 19304.26 & 1.54 & 36 & ${ }^{3} \mathrm{~F}^{\circ}$ & \\
\hline $4 f^{9}\left({ }^{6} \mathrm{~F}^{\circ}\right) 5 d 6 s^{2}$ & & 5 & 19480.87 & 1.35 & 29 & ${ }^{7} \mathrm{G}^{\circ}$ & \\
\hline $4 f^{9}\left({ }^{6} \mathrm{H}^{\circ}\right) 5 d^{2}\left({ }^{3} \mathrm{~F}\right)\left({ }^{8} \mathrm{I}^{\circ}\right) 6 s$ & & 9 & 19557.83 & 1.39 & 36 & ${ }^{9} \mathrm{I}^{\circ}$ & \\
\hline $4 f^{9}\left({ }^{6} \mathrm{H}^{\circ}\right) 5 d^{2}\left({ }^{3} \mathrm{~F}\right)\left({ }^{8} \mathrm{~K}^{\circ}\right) 6 s$ & & 10 & 19797.96 & 1.367 & 40 & ${ }^{9} \mathrm{~K}^{\circ}$ & \\
\hline $4 f^{9}\left({ }^{6} \mathrm{H}^{\circ}\right) 5 d 6 s^{2}$ & & 5 & 19813.98 & 1.270 & 33 & ${ }^{5} \mathrm{H}^{\circ}$ & \\
\hline $4 f^{9}\left({ }^{6} \mathrm{~F}^{\circ}\right) 5 d 6 s^{2}$ & & 6 & 19856.88 & 1.35 & 40 & ${ }^{7} \mathrm{H}^{\circ}$ & \\
\hline
\end{tabular}


Dy 1, Odd Parity-Continued

\begin{tabular}{|c|c|c|c|c|c|c|c|c|}
\hline Configuration & Term & $J$ & $\begin{array}{l}\text { Level } \\
\left(\mathrm{cm}^{-1}\right)\end{array}$ & $g$ & \multicolumn{4}{|c|}{ Leading percentages } \\
\hline $4 f^{10}\left({ }^{5} \mathrm{I}_{7}\right) 6 s 6 p\left({ }^{3} \mathrm{P} 0\right)$ & $(7,0)^{\circ}$ & 7 & 19907.51 & 1.23 & & or & 26 & $\left({ }^{5} \mathrm{I}\right)\left({ }^{3} \mathrm{P}^{\circ}\right){ }^{7} \mathrm{I}^{\circ}$ \\
\hline $4 f^{10}\left({ }^{5} \mathrm{I}_{7}\right) 6 s 6 p\left({ }^{3} \mathrm{P}_{1}^{\circ}\right)$ & $(7,1)^{\circ}$ & $\begin{array}{l}8 \\
7 \\
6\end{array}$ & $\begin{array}{l}20341.32 \\
20766.29 \\
20817.61\end{array}$ & $\begin{array}{l}1.23 \\
1.16 \\
1.13\end{array}$ & & $\begin{array}{l}\text { or } \\
\text { or } \\
\text { or }\end{array}$ & & $\begin{array}{l}\left({ }^{5} \mathrm{I}\right)\left({ }^{3} \mathrm{P}^{\circ}\right){ }^{7} \mathrm{~K}^{\circ} \\
\left({ }^{5} \mathrm{I}\right)\left({ }^{3} \mathrm{P}^{\circ}\right){ }^{5} \mathrm{~K}^{\circ} \\
\left({ }^{5} \mathrm{I}\right)\left({ }^{3} \mathrm{P}^{\circ}\right){ }^{7} \mathrm{I}^{\circ}\end{array}$ \\
\hline $4 f^{9}\left({ }^{6} \mathrm{H}^{\circ}\right) 5 d 6 s^{2}$ & & 4 & 20430.11 & 1.28 & 33 & ${ }^{7} \mathrm{~F}^{\circ}$ & & \\
\hline $4 f^{9}\left({ }^{6} \mathrm{H}^{\circ}\right) 5 d^{2}\left({ }^{3} \mathrm{~F}\right)\left({ }^{8} \mathrm{~K}^{\circ}\right) 6 s$ & ${ }^{9} \mathrm{~K}^{\circ}$ & 11 & 20448.44 & 1.354 & 87 & & & \\
\hline $4 f^{9}\left({ }^{6} \mathrm{H}^{\circ}\right) 5 d 6 s^{2}$ & & 4 & 20474.99 & 1.30 & 31 & ${ }^{5} \mathrm{G}^{\circ}$ & & \\
\hline $4 f^{9}\left({ }^{6} \mathrm{~F}^{\circ}\right) 5 d 6 s^{2}$ & ${ }^{7} \mathrm{G}^{\circ}$ & 7 & 20485.40 & 1.375 & 63 & & & \\
\hline $4 f^{9}\left({ }^{6} \mathrm{H}^{\circ}\right) 5 d^{2}\left({ }^{3} \mathrm{~F}\right)\left({ }^{8} \mathrm{I}^{\circ}\right) 6 s$ & & 8 & 20884.87 & 1.36 & 29 & ${ }^{9} \mathrm{I}^{\circ}$ & & \\
\hline $4 f^{9}\left({ }^{6} \mathrm{H}^{\circ}\right) 5 d^{2}\left({ }^{3} \mathrm{~F}\right)\left({ }^{8} \mathrm{G}^{\circ}\right) 6 s$ & & 5 & 20891.64 & 1.32 & 31 & ${ }^{9} \mathrm{G}^{\circ}$ & & \\
\hline $4 f^{9}\left({ }^{6} \mathrm{H}^{\circ}\right) 5 d 6 s^{2}$ & & 5 & 20921.55 & 1.30 & 35 & ${ }^{5} \mathrm{H}^{\circ}$ & & \\
\hline $4 f^{9}\left({ }^{6} \mathrm{H}^{\circ}\right) 5 d^{2}\left({ }^{3} \mathrm{~F}\right)\left({ }^{8} \mathrm{~F}^{\circ}\right) 6 s$ & & 7 & 20954.18 & 1.41 & 30 & $9 \mathrm{~F}^{\circ}$ & & \\
\hline $4 f^{9}\left({ }^{6} \mathrm{H}^{\circ}\right) 5 d^{2}\left({ }^{3} \mathrm{~F}\right)\left({ }^{8} \mathrm{D}^{\circ}\right) 6 s$ & & 6 & 21392.40 & 1.46 & 29 & ${ }^{9} \mathrm{D}^{\circ}$ & & \\
\hline $4 f^{9}\left({ }^{6} \mathrm{H}^{\circ}\right) 5 d^{2}\left({ }^{3} \mathrm{~F}\right)\left({ }^{8} \mathrm{H}^{\circ}\right) 6 s$ & & 9 & 21540.68 & 1.34 & 39 & ${ }^{9} \mathrm{H}^{\circ}$ & & \\
\hline $4 f^{9}\left({ }^{6} \mathrm{~F}^{\circ}\right) 5 d 6 s^{2}$ & ${ }^{5} \mathrm{H}^{\circ}$ & $\begin{array}{l}7 \\
6 \\
4 \\
3\end{array}$ & $\begin{array}{l}21675.28 \\
24040.59 \\
26440.41 \\
27601.33\end{array}$ & $\begin{array}{l}1.22 \\
1.26 \\
1.046 \\
0.84\end{array}$ & $\begin{array}{l}62 \\
44 \\
53 \\
68\end{array}$ & & & • \\
\hline $4 f^{9}\left({ }^{6} \mathrm{H}^{\circ}\right) 5 d^{2}\left({ }^{3} \mathrm{~F}\right)\left({ }^{8} \mathrm{~K}^{\circ}\right) 6 s$ & & 10 & 21788.93 & 1.34 & 33 & ${ }^{9} \mathrm{~K}^{\circ}$ & & \\
\hline $4 f^{10}\left({ }^{5} \mathrm{I}_{7}\right) 6 s 6 p\left({ }^{3} \mathrm{P}_{2}^{\circ}\right)$ & $(7,2)^{\circ}$ & $\begin{array}{l}9 \\
8 \\
7 \\
6 \\
5\end{array}$ & $\begin{array}{l}21838.55 \\
21899.22 \\
22061.29 \\
22286.87 \\
22524.21\end{array}$ & $\begin{array}{l}1.25 \\
1.20 \\
1.18 \\
1.15 \\
1.04\end{array}$ & & $\begin{array}{l}\text { or } \\
\text { or } \\
\text { or } \\
\text { or }\end{array}$ & & $\begin{array}{l}\left({ }^{5} \mathrm{I}\right)\left({ }^{3} \mathrm{P}^{\circ}\right){ }^{7} \mathrm{~K}^{\circ} \\
\left({ }^{5} \mathrm{I}\right)\left({ }^{3} \mathrm{P}^{\circ}\right){ }^{7} \mathrm{I}^{\circ} \\
\left({ }^{5} \mathrm{I}\right)\left({ }^{3} \mathrm{P}^{\circ}\right){ }^{7} \mathrm{I}^{\circ} \\
\left({ }^{5} \mathrm{I}\right)\left({ }^{3} \mathrm{P}^{\circ}\right){ }^{7} \mathrm{H}^{\circ}\end{array}$ \\
\hline $4 f^{9}\left({ }^{6} \mathrm{H}^{\circ}\right) 5 d 6 s^{2}$ & & 4 & 22099.06 & 1.059 & 41 & ${ }^{5} \mathrm{H}^{\circ}$ & & \\
\hline $4 f^{9}\left({ }^{6} \mathrm{H}^{\circ}\right) 5 d^{2}\left({ }^{3} \mathrm{~F}\right)\left({ }^{8} \mathrm{~L}^{\circ}\right) 6 s$ & ${ }^{9} \mathbf{L}^{\circ}$ & $\begin{array}{c}12 \\
11 \\
10 \\
9 \\
8 \\
7 \\
6\end{array}$ & $\begin{array}{l}22541.18 \\
23677.38 \\
24858.74 \\
25955.10 \\
\\
27556.34\end{array}$ & $\begin{array}{l}1.333 \\
1.288 \\
1.26 \\
1.27 \\
\\
1.24\end{array}$ & $\begin{array}{l}99 \\
78 \\
49 \\
34 \\
33 \\
28 \\
40\end{array}$ & & & \\
\hline $4 f^{9}\left({ }^{6} \mathrm{~F}^{\circ}\right) 5 d 6 s^{2}$ & ${ }^{5} \mathrm{G}^{\circ}$ & 6 & 22633.23 & 1.29 & 50 & & & \\
\hline $4 f^{9}\left({ }^{8} \mathrm{H}^{\circ}\right) 5 d^{2}\left({ }^{3} \mathrm{~F}\right)\left({ }^{8} \mathrm{~K}^{\circ}\right) 6 s$ & & 7 & 22647.94 & 1.34 & 22 & ${ }^{9} \mathrm{~K}^{\circ}$ & & \\
\hline $4 f^{9}\left({ }^{6} \mathrm{H}^{\circ}\right) 5 d^{2}\left({ }^{3} \mathrm{~F}\right)\left({ }^{8} \mathrm{G}^{\circ}\right) 6 s$ & & 4 & 22696.82 & 1.487 & 35 & ${ }^{9} \mathrm{G}^{\circ}$ & & \\
\hline $4 f^{9}\left({ }^{6} \mathrm{H}^{\circ}\right) 5 d^{2}\left({ }^{3} \mathrm{P}\right)\left({ }^{8} \mathrm{G}^{\circ}\right) 6 s$ & & 8 & 22767.83 & 1.34 & 43 & ${ }^{9} \mathrm{G}^{\circ}$ & & \\
\hline $4 f^{9}\left({ }^{6} \mathrm{H}^{\circ}\right) 5 d 6 s^{2}$ & & 4 & 22938.03 & 1.07 & 24 & ${ }^{5} \mathrm{H}^{\circ}$ & & \\
\hline $4 f^{10}\left({ }^{5} \mathrm{I}_{6}\right) 6 s 6 p\left({ }^{3} \mathrm{P}_{0}^{\circ}\right)$ & $(6,0)^{\circ}$ & 6 & 22956.84 & 1.06 & & or & 24 & $\left({ }^{5} \mathrm{I}\right)\left({ }^{3} \mathrm{P}^{\circ}\right){ }^{7} \mathrm{~K}^{\circ}$ \\
\hline $4 f^{9}\left({ }^{6} \mathrm{H}^{\circ}\right) 5 d^{2}\left({ }^{3} \mathrm{P}\right)\left({ }^{8} \mathrm{I}^{\circ}\right) 6 s$ & & 9 & 23271.74 & 1.33 & 41 & ${ }^{9} \mathrm{I}^{\circ}$ & & \\
\hline $4 f^{9}\left({ }^{6} \mathrm{H}^{\circ}\right) 5 d^{2}\left({ }^{3} \mathrm{~F}\right)\left({ }^{8} \mathrm{G}^{\circ}\right) 6 s$ & & 7 & 23340.12 & 1.39 & 28 & ${ }^{7} \mathrm{G}^{\circ}$ & & \\
\hline $4 f^{9}\left({ }^{6} \mathrm{H}^{\circ}\right) 5 d^{2}\left({ }^{3} \mathrm{~F}\right)\left({ }^{8} \mathrm{~F}^{\circ}\right) 6 s$ & & 6 & 23359.82 & 1.35 & 30 & ${ }^{7} \mathrm{~F}^{\circ}$ & & \\
\hline $4 f^{9}\left({ }^{6} \mathrm{H}^{\circ}\right) 5 d^{2}\left({ }^{3} \mathrm{~F}\right)\left({ }^{8} \mathrm{~F}^{\circ}\right) 6 s$ & & 5 & 23440.46 & 1.36 & 17 & ${ }^{9} \mathrm{~F}^{\circ}$ & & \\
\hline $4 f^{10}\left({ }^{5} \mathrm{I}_{6}\right) 6 s 6 p\left({ }^{3} \mathrm{P}_{1}^{\circ}\right)$ & $(6,1)^{\circ}$ & $\begin{array}{l}7 \\
5 \\
6\end{array}$ & $\begin{array}{l}23479.77 \\
23552.65 \\
23687.87\end{array}$ & $\begin{array}{l}1.13 \\
1.07 \\
1.076\end{array}$ & & $\begin{array}{l}\text { or } \\
\text { or } \\
\text { or }\end{array}$ & $\begin{array}{l}35 \\
32 \\
29\end{array}$ & $\begin{array}{l}\left({ }^{5} \mathrm{I}\right)\left({ }^{3} \mathrm{P}^{\circ}\right){ }^{7} \mathrm{~K}^{\circ} \\
\left({ }^{5} \mathrm{I}\right)\left({ }^{3} \mathrm{P}^{\circ}\right){ }^{7} \mathrm{I}^{\circ} \\
\left({ }^{5} \mathrm{I}\right)\left({ }^{3} \mathrm{P}^{\circ}\right){ }^{7} \mathrm{~K}^{\circ}\end{array}$ \\
\hline $4 f^{9}\left({ }^{6} \mathrm{H}^{\circ}\right) 5 d^{2}\left({ }^{3} \mathrm{~F}\right)\left({ }^{8} \mathrm{D}^{\circ}\right) 6 s$ & & 6 & 23529.01 & 1.41 & 20 & ${ }^{9} \mathrm{D}^{\circ}$ & & \\
\hline
\end{tabular}


Dy I, Odd Parity-Continued

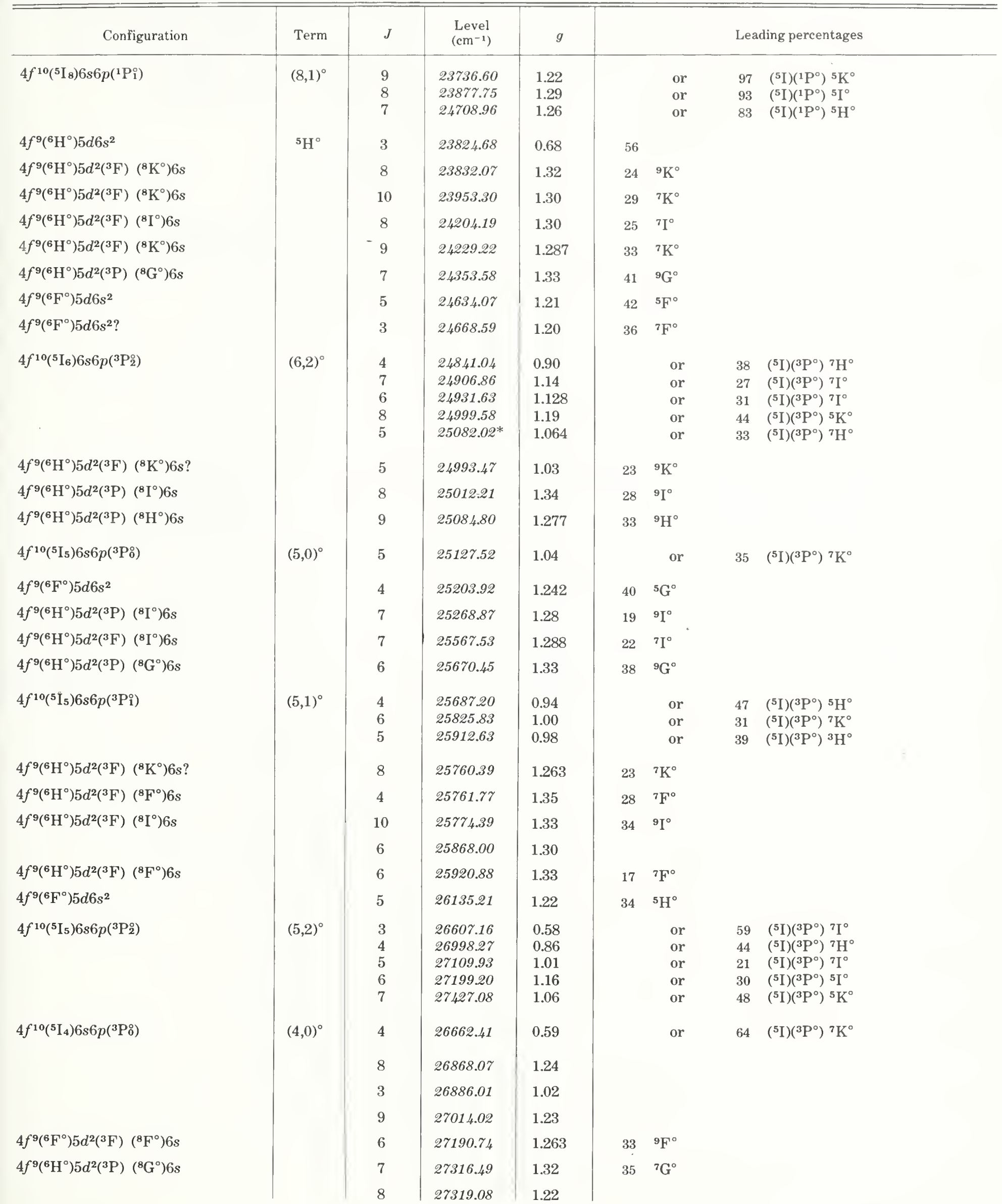


Dy I, Odd Parity-Continued

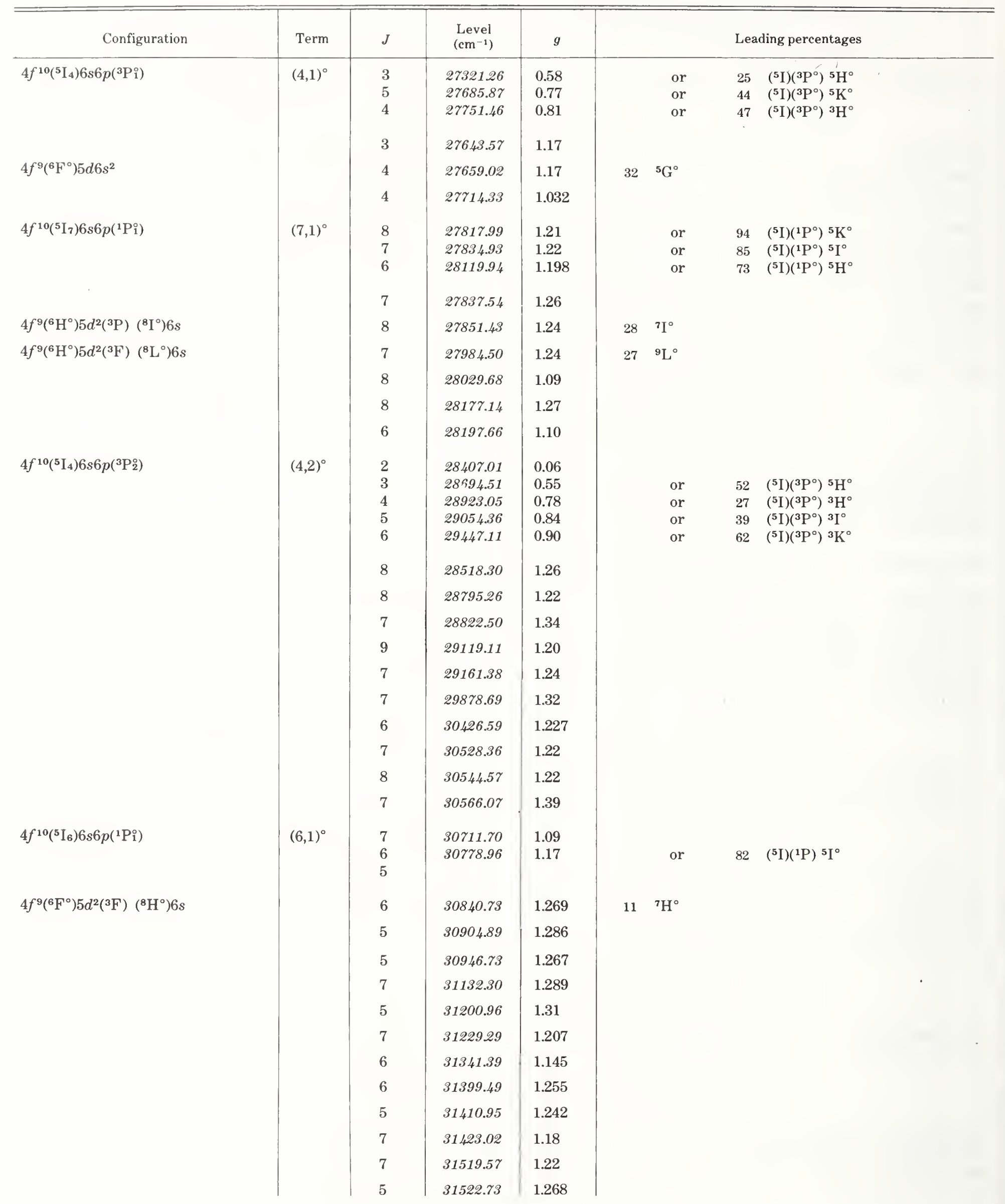


Dy I, Odd Parity_Continued

Dy I, Odd Parity_Continued

\begin{tabular}{|c|c|c|c|c|c|c|c|c|c|}
\hline Configuration & Term & $J$ & $\begin{array}{l}\text { Level } \\
\left(\mathrm{cm}^{-1}\right)\end{array}$ & $g$ & Configuration & Term & $J$ & $\begin{array}{l}\text { Level } \\
\left(\mathrm{cm}^{-1}\right)\end{array}$ & $g$ \\
\hline \multirow[t]{3}{*}{$4 f^{10}\left({ }^{5} \mathrm{I}\right) 5 d 6 p ?$} & & 10 & 36868.73 & 1.178 & $4 f^{10}\left({ }^{5} \mathrm{I}\right) 5 d 6 p ?$ & & 7 & 38362.65 & \\
\hline & & 8 & 36892.10 & 1.188 & $4 f^{10}\left({ }^{5} \mathrm{I}\right) 5 d 6 p ?$ & & 7 & 38421.29 & 1.33 \\
\hline & & 8 & 37041.02 & 1.19 & & & 8 & 38438.87 & 1.27 \\
\hline $4 f^{10}\left({ }^{5} \mathrm{I}\right) 5 d 6 p ?$ & & 7 & 37073.86 & 1.288 & $4 f^{10}\left({ }^{5} \mathrm{I}\right) 5 d 6 p ?$ & & 9 & 38563.33 & 1.254 \\
\hline $4 f^{10}\left({ }^{5} \mathrm{I}\right) 5 d 6 p ?$ & & 9 & 37090.46 & 1.208 & & & 7 & 38779.77 & \\
\hline $4 f^{10}\left({ }^{5} \mathrm{I}\right) 5 d 6 p ?$ & & 8 & 37146.54 & 1.233 & & & 8 & $38814.46 ?$ & \\
\hline $4 f^{10}\left({ }^{5} \mathrm{I}\right) 5 d 6 p ?$ & & 11 & 37182.98 & 1.23 & $4 f^{10}(5 \mathrm{I}) 5 d 6 p ?$ & & 8 & 39135.32 & 1194 \\
\hline \multirow{3}{*}{ 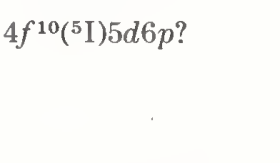 } & & 8 & 37355.00 & 1.12 & $4 f^{10}\left({ }^{5} \mathrm{I}\right) 5 d 6 p ?$ & & 10 & 39182.40 & 1.28 \\
\hline & & 7 & 37360.01 & 1.185 & & & 6 & 39188.23 & \\
\hline & & 7 & 37366.93 & & & & 7 & 3932528 & \\
\hline \multirow[t]{2}{*}{$4 f^{10}\left({ }^{5} \mathrm{I}\right) 5 d 6 p ?$} & & 7 & $37471.09 ?$ & & $4 f^{10}\left({ }^{5} \mathrm{I}\right) 5 d 6 p ?$ & & 8 & 39398.10 & 1.228 \\
\hline & & 7 & 37587.58 & 1.23 & $4 f^{10}\left({ }^{5} \mathrm{I}\right) 5 d 6 p ?$ & & 9 & 39602.47 & 1.28 \\
\hline \multirow{2}{*}{$4 f^{10}\left({ }^{5} \mathrm{I}\right) 5 d 6 p ?$} & & 8 & $3763526 ?$ & & $4 f^{10}\left({ }^{5} \mathrm{I}\right) 5 d 6 p ?$ & & 8 & 39714.20 & 1.184 \\
\hline & & 8 & 37646.28 & 1.09 & $4 f^{10}\left({ }^{5} \mathrm{I}\right) 5 d 6 p ?$ & & 9 & 39903.34 & 1.28 \\
\hline $4 f^{10}\left({ }^{5} \mathrm{I}\right) 5 d 6 p ?$ & & 8 & 37721.06 & 1.199 & $4 f^{10}\left({ }^{5} \mathrm{I}\right) 5 d 6 p ?$ & & 9 & 40030.48 & 1.192 \\
\hline $4 f^{10}\left({ }^{5} \mathrm{I}\right) 5 d 6 p ?$ & & 9 & 37836.50 & 1.18 & $4 f^{9}\left({ }^{6} \mathrm{H}^{\circ}\right) 5 d 6 s 7 s$ & & 9 & 40594.03 & 1.41 \\
\hline \multirow[t]{2}{*}{$4 f^{10}\left({ }^{5} \mathrm{I}\right) 5 d 6 p ?$} & & 8 & 37843.38 & 1.265 & & & 8 & 40605.98 & 1.11 \\
\hline & & 10 & 38019.12 & 1.152 & $4 f^{9}\left({ }^{6} \mathrm{H}^{\circ}\right) 5 d 6 s 7 s$ & & 8 & 41136.59 & 1.35 \\
\hline $4 f^{10}\left({ }^{5} \mathrm{I}\right) 5 d 6 p ?$ & & 9 & 38102.92 & 1.19 & & & 6 & 41577.18 & \\
\hline $4 f^{10}\left({ }^{5} \mathrm{I}\right) 5 d 6 p ?$ & & 8 & 38202.48 & 1.313 & & & 7 & 41642.79 & \\
\hline \multirow[t]{2}{*}{$4 f^{10}\left({ }^{5} \mathrm{I}\right) 5 d 6 p ?$} & & 10 & 38247.38 & 1.22 & $4 f^{10}\left({ }^{5} \mathrm{I}\right) 5 d 6 p ?$ & & 6 & 41656.46 & 1.21 \\
\hline & & 7 & 38251.55 & & & & 6 & 42375.03 & 1.28 \\
\hline $4 f^{10}\left({ }^{5} \mathrm{I}\right) 5 d 6 p ?$ & & 10 & 38297.52 & 1.244 & & & 5 & 42479.83 & 1.07 \\
\hline \multirow[t]{2}{*}{$4 f^{10}\left({ }^{5} \mathrm{I}\right) 5 d 6 p ?$} & & 7 & 38342.48 & 1.323 & & & & & \\
\hline & & 8 & 38358.88 & 1.095 & Dy II $\left({ }^{5} I_{8}\right) 6 s_{1 / 2}\left(8,{ }^{1 / 2}\right)_{17 / 2}$ & Limit & & 47900 & \\
\hline
\end{tabular}


Ground state $\left(1 s^{2} 2 s^{2} 2 p^{6} 3 s^{2} 3 p^{6} 3 d^{10} 4 s^{2} 4 p^{6} 4 d^{10} 5 s^{2} 5 p^{6}\right) 4 f^{10}\left({ }^{5} \mathrm{I}_{8}\right) 6 s(8,1 / 2)_{17 / 2}$

Ionization energy $94100 \pm 600 \mathrm{~cm}^{-1}$

$11.67 \pm 0.08 \mathrm{eV}$

Identified even configurations

Identified odd configurations

$$
4 f^{10} 6 s, 4 f^{10} 5 d, 4 f^{9} 6 s 6 p, 4 f^{9} 5 d 6 p
$$

$4 f^{9} 5 d 6 s, 4 f^{9} 6 s^{2}, 4 f^{9} 5 d^{2}, 4 f^{10} 6 p$

In the earlier work on this spectrum, one or more real energy differences were found or confirmed by Paulson [1914], Meggers [1942], Blank [1952], and Corliss and Corliss [1953]. Blank's analysis was greatly aided by his measurements of the Zeeman patterns of 510 lines. He classified 100 lines according to four wavenumber differences, but his Zeeman data showed that the differences involved eight separate low levels. The $J$ and $g$ values of these levels indicated ${ }^{6} \mathrm{I}$ and ${ }^{4} \mathrm{I}$ terms, as expected for a $4 f^{10} 6 s$ ground configuration. Blank concluded that the ${ }^{6} \mathrm{I}_{17 / 2}$ level was probably the ground level. Corliss and Corliss [1953] derived a single connected system consisting of the four lowest levels and a number of upper odd levels. Their analysis yielded classifications for 80 of the stronger Dy II lines tabulated by Meggers, Corliss, and Scribner [1961].

Vander Sluis and MeNally [1970] at Oak Ridge and Conway and Worden [1970, 1971] at the Lawrence Laboratory made new observations of Dy I and Dy II using electrodeless lamps containing the separated isotope ${ }^{162} \mathrm{Dy}$. The UCRL report by Conway and Worden has more than 9000 lines assigned to Dy II in the range 2300-11400 $\AA$. Conway and Worden extended the analysis to include all ten levels of the ground subconfiguration $4 f^{10}\left({ }^{5} \mathrm{I}\right) 6 s$, the two levels of $4 f^{10}\left({ }^{5} \mathrm{~F}_{5}\right) 6 s$, two other low even levels, and 214 upper odd levels. About 1000 lines were classified. The analysis was partly based on new measurements of Zeeman patterns, and $g$ values were obtained for most of the levels. In a separate investigation, Vander Sluis and McNally used Zeeman spectra photographed at MIT to derive $g$ values for the ten lowest levels of $4 f^{10} 6 \mathrm{~s}$ and for 77 upper odd levels.

Wyart has greatly extended the analysis to include about 550 levels. He worked with the line list of Conway and Worden, and with new data including additional measurements of Zeeman patterns. Wyart has also made extensive calculations that are the basis for the interpretation of the levels giver here. All eigenvector percentages are from his results.

Conway and Worden state the uncertainty of their three-place $g$ values to be \pm 0.003 , whereas the standard deviations of the $g$ values obtained for the $4 f^{10}\left({ }^{5} \mathrm{I}\right) 6 \mathrm{~s}$ levels by Vander Sluis and McNally lie between 0.001 and 0.016 . We have taken suitable averages of $g$ values included in both sets of measurements. The measurements of Vander Sluis and McNally for the high odd levels were usually weighted according to the number of determinations. The $g$ values given by Wyart to the nearest 0.005 have been rounded to two places to distinguish them from the generally more accurate three-place values.

The $J_{1} j$-coupling of the $4 f^{10} 6 s$ ground configuration, as exhibited by the pairing of the low levels, was noted by Conway and Worden. Vander Sluis and MeNally pointed out the good agreement of the experimental $g$ values with theoretical $J_{1} j$-coupling values. No eigenvector percentages in this scheme are available, but we have listed the leading percentages in $L S$ coupling after the word "or." The percentages are from Wyart's recent calculation of $\left(4 f^{10} 6 s+\right.$ $4 f^{10} 5 d$ ) including configuration interaction [1976]. The known $4 f^{10} 5 d$ levels belong mainly to the $4 f^{10}\left({ }^{5} \mathrm{I}\right) 5 d$ subconfiguration; Spector [1971] located several of the low levels of this group, but the detailed interpretation required experimental $g$ values and an expansion of the analysis [Wyart, 1971; 1973; 1976].

Wyart [1972b] has been able to assign all but a few of the 141 high even levels (36000$\left.54000 \mathrm{~cm}^{-1}\right)$ to eigenvectors of $\left(4 f^{9}\left({ }^{6} \mathrm{H}^{\circ},{ }^{6} \mathrm{~F}^{\circ}\right) 6 s 6 p+4 f^{9}\left({ }^{6} \mathrm{H}^{\circ},{ }^{6} \mathrm{~F}^{\circ}\right) 5 d 6 p\right)$. All of the assigned levels of this group have $J \geqslant 7 / 2$. Wyart notes that transitions from these levels to levels of $4 f^{9}(5 d+$ 
$6 s)^{2}$ account for the strongest lines originating on high even levels, although levels of $4 f^{10} 6 s$ and $4 f^{10} 5 d$ must also lie in this region.

The eigenvector percentages for the odd levels are from Wyart's calculation of $\left(4 f^{9}(5 d+\right.$ $6 s)^{2}+4 f^{10} 6 p$ ), the three configurations based on $4 f^{9}$ being limited to the subeonfigurations from the $4 f^{9}{ }^{6} \mathrm{H}^{\circ}$ and ${ }^{6} \mathrm{~F}^{\circ}$ terms [Wyart, 1972a]. All interpreted odd levels have $J \geqslant 9 / 2$. An extension of the calculation to include levels based on higher terms of $4 f^{9}$ would probably allow interpretation of many of the levels above $30000 \mathrm{~cm}^{-1}$; some 240 uninterpreted odd levels are listed, the bulk lying between 32000 and $42000 \mathrm{~cm}^{-1}$. Wyart notes, however, that a meaningful extension of his calculation would require matrices of order greater than 500 .

Sugar and Reader determined the quoted ionization energy.

\section{References}

Blank, J. M., Thesis, Mass. Inst. Tech., Cambridge, Mass., 93 pp. (1952). EL W ZE

Conway, J. G., and Worden, E. F., Univ. Calif. Radiation Lab., Berkeley, UCRL-19944, 613 pp. (1970). (Available from National Technical Information Service, Springfield, Va. 22161.) EL ND CL W ZE PT

Conway, J. G., and Worden, E. F., J. Opt. Soc. Am. 61, 704 (1971). EL ND CL W ZE PT

Corliss, C. H., and Corliss, E. L. R., unpublished material (1953); see Meggers et al. (1961).

King, A. S., Astrophys. J. 72, 221 (1930). W

King, A. S., Conway, J. G., Worden, E. F., and Moore, C. E., J. Res. Nat. Bur. Stand. (U.S.) 74 A, 355 (1970). W

Meggers, W. F., Rev. Mod. Phys. 14, 96 (1942). EL

Meggers, W. F., Corliss, C. H., and Scribner, B. F., Tables of Spectral Line Intensities, Nat. Bur. Stand. (U.S.), Monogr.

32, Part I, 473 pp. (1961); more recent data are given in the second edition, Nat. Bur. Stand. (U.S.), Monogr. 145, Part I, 403 pp. (1975). EL CL

Paulson, E., Astrophys. J. 40, 298 (1914). EL

Spector, N., J. Opt. Soc. Am. 61, 672A (1971). EL

Sugar, J., and Reader, J., J. Opt. Soc. Am. 55, 1286 (1965). IP

Vander Sluis, K. L., and McNally, J. R., Jr., J. Opt. Soc. Am. 60, 1209 (1970). EL CL W ZE

Wyart, J. F., C. R. Acad. Sci., Ser. B 272, 933 (1971). EL ND ZE PT

Wyart, J. F., Physica (Utrecht) 61, 182 (1972a). EL ND PT

Wyart, J. F., Physica (Utrecht) 61, 191 (1972b). EL ND ZE PT

Wyart, J. F., Thesis, Univ. Paris-Sud, Orsay, 194 pp. (1973). EL ND ZE PT

Wyart, J. F., unpublished material $(1975,1976)$. EL ZE

Wyart, J. F., Physica (Utrecht) 83C, 361 (1976). EL ND ZE PT

[July 1976]

Dy II, Even Parity

\begin{tabular}{|c|c|c|c|c|c|c|c|c|}
\hline Configuration & Term & $J$ & $\begin{array}{l}\text { Level } \\
\left(\mathrm{cm}^{-1}\right)\end{array}$ & $g$ & \multicolumn{4}{|c|}{ Leading percentages } \\
\hline $4 f^{10}\left(5 I_{8}\right) 6 s_{1 / 2}$ & $(8,1 / 2)$ & $\begin{array}{l}17 / 2 \\
15 / 2\end{array}$ & $\begin{array}{r}0.00 \\
828.31\end{array}$ & $\begin{array}{l}1.290 \\
1.203\end{array}$ & & $\begin{array}{l}\text { or } \\
\text { or }\end{array}$ & $\begin{array}{l}95 \\
72\end{array}$ & $\begin{array}{l}\left({ }^{5} I\right)^{6} I \\
\left({ }^{5} I\right)^{4} I\end{array}$ \\
\hline $4 f^{10}\left({ }^{5} I_{7}\right) 6 s_{1 / 2}$ & $(7,1 / 2)$ & $\begin{array}{l}15 / 2 \\
13 / 2\end{array}$ & $\begin{array}{l}4341.10 \\
4755.66\end{array}$ & $\begin{array}{l}1.225 \\
1.130\end{array}$ & & $\begin{array}{l}\text { or } \\
\text { or }\end{array}$ & $\begin{array}{l}75 \\
50\end{array}$ & $\begin{array}{l}\left({ }^{5} I\right)^{6} \mathrm{I} \\
\left({ }^{5} I\right)^{4} \mathrm{I}\end{array}$ \\
\hline $4 f^{10}\left({ }^{5} \mathrm{I}_{6}\right) 6 s_{1 / 2}$ & $(6,1 / 2)$ & $\begin{array}{l}11 / 2 \\
13 / 2\end{array}$ & $\begin{array}{l}7463.88 \\
7485.09\end{array}$ & $\begin{array}{l}1.015 \\
1.134\end{array}$ & & $\begin{array}{l}\text { or } \\
\text { or }\end{array}$ & $\begin{array}{l}69 \\
49\end{array}$ & $\left({ }^{5} I\right)^{6} I$ \\
\hline $4 f^{10}\left({ }^{5} I_{5}\right) 6 s_{1 / 2}$ & $(5,1 / 2)$ & $\begin{array}{c}9 / 2 \\
11 / 2\end{array}$ & $\begin{array}{l}9432.07 \\
9870.99\end{array}$ & $\begin{array}{l}0.825 \\
0.997\end{array}$ & & $\begin{array}{l}\text { or } \\
\text { or }\end{array}$ & $\begin{array}{l}82 \\
66\end{array}$ & $\begin{array}{l}\left({ }^{5} I\right){ }^{6} I \\
\left({ }^{5} I\right){ }^{4} I\end{array}$ \\
\hline $4 f^{10\left(5 \mathrm{I}_{4}\right) 6 s_{1 / 2}}$ & $(4,1 / 2)$ & $\begin{array}{l}7 / 2 \\
9 / 2\end{array}$ & $\begin{array}{l}10953.94 \\
11801.01\end{array}$ & $\begin{array}{l}0.470 \\
0.756\end{array}$ & & $\begin{array}{l}\text { or } \\
\text { or }\end{array}$ & $\begin{array}{l}92 \\
80\end{array}$ & $\begin{array}{l}\left({ }^{5} I\right){ }^{6} I \\
\left({ }^{5} I\right)\end{array}$ \\
\hline $4 f^{10}\left({ }^{5} \mathrm{~F}_{5}\right) 6 s_{1 / 2}$ & $(5,1 / 2)$ & $\begin{array}{l}11 / 2 \\
9 / 2\end{array}$ & $\begin{array}{l}13338.27 \\
14126.93\end{array}$ & $\begin{array}{l}1.413 \\
1.32\end{array}$ & & $\begin{array}{l}\text { or } \\
\text { or }\end{array}$ & $\begin{array}{l}85 \\
62\end{array}$ & $\begin{array}{l}\left({ }^{5} F\right)^{6} F \\
\left({ }^{5} F\right){ }^{4} F\end{array}$ \\
\hline $4 f^{10}\left({ }^{5} \mathrm{I}\right) 5 d$ & ${ }^{6} \mathrm{I}$ & $17 / 2$ & 14845.91 & 1.25 & 78 & & 13 & $\left({ }^{5} \mathrm{I}\right){ }^{6} \mathrm{~K}$ \\
\hline $4 f^{10}\left({ }^{5} \mathrm{I}\right) 5 d$ & ${ }^{6} \mathrm{H}$ & $15 / 2$ & 14952.14 & 1.30 & 56 & & 28 & $\left({ }^{5} I\right)^{6} I$ \\
\hline $4 f^{10}\left({ }^{5} \mathrm{I}\right) 5 d$ & ${ }^{6} \mathrm{G}$ & $13 / 2$ & 15691.99 & 1.34 & 65 & & 21 & $\left({ }^{5} \mathrm{I}\right){ }^{6} \mathrm{H}$ \\
\hline
\end{tabular}


Dy II, Even Parity_Continued

\begin{tabular}{|c|c|c|c|c|c|c|c|c|}
\hline Configuration & Term & $J$ & $\begin{array}{l}\text { Level } \\
\left(\mathrm{cm}^{-1}\right)\end{array}$ & $g$ & \multicolumn{4}{|c|}{ Leading percentages } \\
\hline $4 f^{10}\left({ }^{5} \mathrm{~S}_{2}\right) 6 s_{1 / 2}$ & $(2,1 / 2)$ & $\begin{array}{r}5 / 2 \\
3 / 2\end{array}$ & $\begin{array}{l}15889.87 \\
16758.92\end{array}$ & $\begin{array}{l}1.85 \\
1.72\end{array}$ & & $\begin{array}{l}\text { or } \\
\text { or }\end{array}$ & $\begin{array}{l}81 \\
72\end{array}$ & $\begin{array}{l}\left({ }^{5} \mathrm{~S}\right){ }^{6} \mathrm{~S} \\
\left({ }^{5} \mathrm{~S}\right){ }^{4} \mathrm{~S}\end{array}$ \\
\hline $4 f^{10}\left({ }^{5} \mathrm{~F}_{4}\right) 6 s_{1 / 2}$ & $(4,1 / 2)$ & $\begin{array}{l}9 / 2 \\
7 / 2\end{array}$ & $\begin{array}{l}15979.27 \\
16418.93\end{array}$ & $\begin{array}{l}1.40 \\
1.309\end{array}$ & & $\begin{array}{l}\text { or } \\
\text { or }\end{array}$ & $\begin{array}{l}70 \\
52\end{array}$ & $\left({ }^{5} \mathrm{~F}\right){ }^{6} \mathrm{~F}$ \\
\hline $4 f^{10}\left({ }^{5} \mathrm{I}\right) 5 d$ & ${ }^{6} \mathrm{~K}$ & $19 / 2$ & 16117.40 & 1.24 & 75 & & 12 & $\left({ }^{5} \mathrm{I}\right){ }^{6} \mathrm{~L}$ \\
\hline $4 f^{10}\left({ }^{5} \mathrm{I}\right) 5 d$ & ${ }^{6} \mathrm{~L}$ & $21 / 2$ & 17406.59 & 1.24 & 94 & & 4 & $\left({ }^{3} \mathrm{~K} 2\right)^{4} \mathrm{M}$ \\
\hline $4 f^{10}\left({ }^{5} \mathrm{I}\right) 5 d$ & ${ }^{4} \mathrm{~K}$ & $17 / 2$ & 17589.52 & 1.20 & 52 & & 23 & $\left({ }^{5} \mathrm{I}\right)^{6} \mathrm{~K}$ \\
\hline $4 f^{10}\left({ }^{5} \mathrm{~F}_{3}\right) 6 s_{1 / 2}$ & $(3,1 / 2)$ & $\begin{array}{l}5 / 2 \\
7 / 2\end{array}$ & $\begin{array}{l}17779.98 \\
17912.80\end{array}$ & $\begin{array}{l}1.29 \\
1.30\end{array}$ & & $\begin{array}{l}\text { or } \\
\text { or }\end{array}$ & $\begin{array}{l}78 \\
52\end{array}$ & $\begin{array}{l}\left({ }^{5} F\right){ }^{6} \mathrm{~F} \\
\left({ }^{5} \mathrm{~F}\right){ }^{4} \mathrm{~F}\end{array}$ \\
\hline $4 f^{10}\left({ }^{5} \mathrm{I}\right) 5 d$ & ${ }^{4} \mathrm{~L}$ & $19 / 2$ & 17788.82 & 1.17 & 62 & & 16 & $\left({ }^{5} \mathrm{I}\right)^{6} \mathrm{~K}$ \\
\hline $4 f^{10\left({ }^{5} \mathrm{I}\right) 5 d}$ & & $13 / 2$ & 18219.72 & 1.24 & 29 & ${ }^{6} \mathrm{I}$ & 25 & $\left({ }^{5} \mathrm{I}\right){ }^{6} \mathrm{G}$ \\
\hline $4 f^{10}\left({ }^{5} \mathrm{I}\right) 5 d$ & & $15 / 2$ & 18234.95 & 1.24 & 34 & ${ }^{6} \mathrm{H}$ & 33 & $\left({ }^{5} I\right)^{6} \mathrm{I}$ \\
\hline $4 f^{10}\left({ }^{5} \mathrm{I}\right) 5 d$ & ${ }^{6} \mathrm{G}$ & $11 / 2$ & 18866.51 & 1.26 & 48 & & 29 & $\left({ }^{5} \mathrm{I}\right){ }^{6} \mathrm{H}$ \\
\hline $4 f^{10}\left({ }^{5} \mathrm{I}\right) 5 d$ & & $17 / 2$ & 20700.59 & 1.16 & 36 & ${ }^{4} \mathrm{~K}$ & 27 & $\left({ }^{5} \mathrm{I}\right)^{6} \mathrm{~K}$ \\
\hline $4 f^{10}\left({ }^{5} \mathrm{I}\right) 5 d$ & & $13 / 2$ & 20748.43 & 1.17 & 35 & ${ }^{6} \mathrm{I}$ & 29 & $\left({ }^{5} \mathrm{I}\right){ }^{4} \mathrm{H}$ \\
\hline $4 f^{10}\left({ }^{5} \mathrm{I}\right) 5 d$ & ${ }^{6} \mathrm{~K}$ & $15 / 2$ & 21076.07 & 1.13 & 46 & & 16 & $\left({ }^{5} I\right)^{4} I$ \\
\hline $4 f^{10\left({ }^{5} \mathrm{I}\right) 5 d}$ & & $11 / 2$ & 21177.84 & 1.15 & 42 & ${ }^{6} \mathbf{I}$ & 23 & $\left({ }^{5} \mathrm{I}\right){ }^{6} \mathrm{G}$ \\
\hline $4 f^{10}\left({ }^{5} \mathrm{I}\right) 5 d$ & ${ }^{4} \mathbf{I}$ & $15 / 2$ & 21338.35 & 1.18 & 50 & & 24 & $\left({ }^{5} I\right)^{6} I$ \\
\hline $4 f^{10}\left({ }^{5} \mathrm{I}\right) 5 d$ & & $17 / 2$ & 21627.53 & 1.12 & 39 & ${ }^{4} \mathrm{~L}$ & 30 & $\left({ }^{5} \mathrm{I}\right){ }^{6} \mathrm{~K}$ \\
\hline $4 f^{10}\left({ }^{5} \mathrm{I}\right) 5 d$ & ${ }^{6} \mathrm{~L}$ & $19 / 2$ & 21806.92 & 1.17 & 69 & & 25 & $\left({ }^{5} \mathrm{I}\right){ }^{4} \mathrm{~L}$ \\
\hline $4 f^{10}\left({ }^{5} \mathrm{I}\right) 5 d$ & & $9 / 2$ & 21813.12 & 1.10 & 41 & ${ }^{6} \mathrm{H}-$ & 36 & $\left({ }^{5} \mathrm{I}\right){ }^{6} \mathrm{G}$ \\
\hline $4 f^{10}\left({ }^{5} \mathrm{I}\right) 5 d$ & & $13 / 2$ & 22116.32 & 1.20 & 40 & ${ }^{4} \mathrm{H}$ & 31 & $\left({ }^{5} \mathrm{I}\right)^{6} \mathrm{H}$ \\
\hline $4 f^{10}\left({ }^{5} \mathrm{I}\right) 5 d$ & & $11 / 2$ & 23309.80 & 1.105 & 32 & ${ }^{6} \mathrm{H}$ & 24 & $\left({ }^{5} I\right)^{6} I$ \\
\hline $4 f^{10}\left({ }^{5} \mathrm{I}\right) 5 d$ & & $13 / 2$ & 23375.41 & 0.994 & 29 & ${ }^{6} \mathrm{~K}$ & 20 & $\left({ }^{3} \mathrm{~K} 2\right) 6 s{ }^{4} \mathrm{~K}$ \\
\hline $4 f^{10}\left({ }^{3} \mathrm{~K} 2\right) 6 s$ & & $13 / 2$ & 23479.89 & 1.00 & 26 & ${ }^{4} \mathrm{~K}$ & 23 & $\left.{ }^{5} \mathrm{I}\right) 5 d^{6} \mathrm{~K}$ \\
\hline $4 f^{10}\left({ }^{5} \mathrm{I}\right) 5 d$ & ${ }^{6} \mathrm{I}$ & $9 / 2$ & 23495.20 & 1.00 & 48 & & 34 & $\left({ }^{5} \mathrm{I}\right){ }^{6} \mathrm{G}$ \\
\hline $4 f^{10}\left({ }^{5} \mathrm{I}\right) 5 d$ & ${ }^{6} \mathrm{~L}$ & $15 / 2$ & 23840.25 & 1.036 & 59 & & 19 & $\left({ }^{5} \mathrm{I}\right){ }^{4} \mathrm{~K}$ \\
\hline $4 f^{10}\left({ }^{5} \mathrm{I}\right) 5 d$ & ${ }^{6} \mathrm{H}$ & $7 / 2$ & 23976.76 & 0.837 & 46 & & 24 & $\left({ }^{5} \mathrm{I}\right){ }^{6} \mathrm{G}$ \\
\hline $4 f^{10}\left({ }^{5} I\right) 5 d$ & & $15 / 2$ & 24685.63 & 1.10 & 34 & ${ }^{4} \mathrm{~K}$ & 32 & $\left({ }^{5} \mathrm{I}\right){ }^{6} \mathrm{~K}$ \\
\hline $4 f^{10}\left({ }^{5} \mathrm{I}\right) 5 d$ & & $17 / 2$ & 25051.49 & 1.10 & 48 & ${ }^{4} \mathrm{~L}$ & 42 & $\left({ }^{5} \mathrm{I}\right){ }^{6} \mathrm{~L}$ \\
\hline $4 f^{10(5}\left({ }^{5}\right) 5 d$ & ${ }^{6} \mathrm{~K}$ & $11 / 2$ & 25086.95 & 0.89 & 63 & & 11 & $\left({ }^{5} I\right)^{6} I$ \\
\hline $4 f^{10}\left({ }^{5} \mathrm{I}\right) 5 d$ & & $9 / 2$ & 25214.39 & 1.01 & 38 & ${ }^{6} \mathrm{H}$ & 19 & $\left({ }^{5} \mathrm{I}\right){ }^{6} \mathrm{G}$ \\
\hline $4 f^{10}\left({ }^{5} \mathrm{I}\right) 5 d$ & ${ }^{6} \mathrm{I}$ & $7 / 2$ & 25292.49 & 0.743 & 55 & & 32 & $\left({ }^{5} \mathrm{I}\right){ }^{6} \mathrm{G}$ \\
\hline $4 f^{10}\left({ }^{5} \mathrm{I}\right) 5 d$ & ${ }^{6} \mathrm{~L}$ & $13 / 2$ & 25746.58 & 0.888 & 70 & & 7 & $\left({ }^{5} \mathrm{I}\right){ }^{4} \mathrm{~L}$ \\
\hline $\left.4 f^{10(5} \mathrm{I}\right) 5 d$ & ${ }^{6} \mathrm{H}$ & $5 / 2$ & 25933.69 & 0.45 & 62 & & 25 & $\left({ }^{5} \mathrm{I}\right){ }^{6} \mathrm{G}$ \\
\hline $4 f^{10\left({ }^{5} \mathrm{I}\right) 5 d}$ & & $7 / 2$ & 26550.05 & 0.91 & 35 & ${ }^{6} \mathrm{G}$ & 35 & $\left({ }^{5} \mathrm{I}\right){ }^{6} \mathrm{H}$ \\
\hline $4 f^{10}\left({ }^{5} \mathrm{I}\right) 5 d$ & ${ }^{6} \mathrm{G}$ & $5 / 2$ & 27494.66 & 0.75 & 66 & & 21 & $\left({ }^{5} \mathrm{I}\right){ }^{6} \mathrm{H}$ \\
\hline $\left.4 f^{10(5} \mathrm{I}\right) 5 d$ & ${ }^{4} \mathrm{~L}$ & $15 / 2$ & 27519.67 & 1.00 & 62 & & 22 & $\left({ }^{5} \mathrm{I}\right){ }^{6} \mathrm{~L}$ \\
\hline $4 f^{10}\left({ }^{5} \mathrm{I}\right) 5 d$ & ${ }^{6} \mathrm{G}$ & $3 / 2$ & 28214.92 & 0.04 & 90 & & 4 & $\left({ }^{3} \mathrm{H} 4\right){ }^{4} \mathrm{~F}$ \\
\hline $4 f^{9}\left({ }^{7} \mathrm{H}^{\circ}\right) 6 s 6 p\left({ }^{3} \mathrm{P}^{\circ}\right)$ & ${ }^{8} \mathrm{G}$ & $15 / 2$ & 36212.19 & 1.405 & 45 & & & \\
\hline $4 f^{9}\left({ }^{7} \mathrm{H}^{\circ}\right) 6 s 6 p\left({ }^{3} \mathrm{P}^{\circ}\right)$ & ${ }^{8} \mathrm{H}$ & $17 / 2$ & 36522.90 & 1.373 & 59 & & & \\
\hline $4 f^{9}\left({ }^{7} \mathrm{H}^{\circ}\right) 5 d\left({ }^{7} \mathrm{H}^{\circ}\right) 6 p$ & & $15 / 2$ & 37817.31 & 1.333 & 20 & ${ }^{8} \mathrm{G}$ & & \\
\hline $4 f^{9}\left({ }^{7} \mathrm{H}^{\circ}\right) 6 s 6 p\left({ }^{3} \mathrm{P}^{\circ}\right)$ & & $13 / 2$ & 37878.55 & 1.303 & 20 & ${ }^{6} \mathrm{H}$ & & \\
\hline
\end{tabular}


Dy II, Even Parity-Continued

\begin{tabular}{|c|c|c|c|c|c|c|}
\hline Configuration & Term & $J$ & $\begin{array}{l}\text { Level } \\
\left(\mathrm{cm}^{-1}\right)\end{array}$ & $g$ & & Leading percentages \\
\hline $4 f^{9}\left({ }^{7} \mathrm{H}^{\circ}\right) 5 d\left({ }^{7} \mathrm{H}^{\circ}\right) 6 p$ & \multirow{11}{*}{${ }^{8} \mathrm{I}$} & $17 / 2$ & 38402.40 & 1.326 & \multirow[t]{5}{*}{23} & ${ }^{8} \mathrm{H}$ \\
\hline $4 f^{9}\left({ }^{7} \mathrm{H}^{\circ}\right) 5 d\left({ }^{7} \mathrm{H}^{\circ}\right) 6 p$ & & $15 / 2$ & 38427.36 & 1.317 & & ${ }^{8} \mathbf{H}$ \\
\hline $4 f^{9}\left({ }^{7} \mathrm{H}^{\circ}\right) 5 d\left({ }^{7} \mathrm{H}^{\circ}\right) 6 p$ & & $13 / 2$ & 38939.69 & 1.381 & & ${ }^{8} \mathrm{G}$ \\
\hline \multirow[t]{2}{*}{$4 f^{9}\left({ }^{7} \mathrm{H}^{\circ}\right) 6 s 6 p\left({ }^{3} \mathrm{P}^{\circ}\right)$} & & $13 / 2$ & 39896.72 & 1.24 & & ${ }^{8} \mathrm{H}$ \\
\hline & & $13 / 2$ & 39944.24 & 1.23 & & \\
\hline $4 f^{9}\left({ }^{7} \mathrm{H}^{\circ}\right) 6 s 6 p\left({ }^{3} \mathrm{P}^{\circ}\right)$ & & $19 / 2$ & 40158.04 & 1.36 & 69 & \\
\hline $4 f^{9}\left({ }^{7} \mathrm{H}^{\circ}\right) 5 d\left({ }^{7} \mathrm{H}^{\circ}\right) 6 p$ & & $11 / 2$ & 40194.02 & 1.34 & 18 & ${ }^{8} \mathrm{H}$ \\
\hline $4 f^{9}\left({ }^{7} \mathrm{H}^{\circ}\right) 6 s 6 p\left({ }^{3} \mathrm{P}^{\circ}\right)$ & & $17 / 2$ & 40455.73 & 1.326 & 39 & ${ }^{6} \mathrm{I}$ \\
\hline $4 f^{9}\left({ }^{7} \mathrm{H}^{\circ}\right) 6 s 6 p\left({ }^{3} \mathrm{P}^{\circ}\right)$ & & $15 / 2$ & 40516.80 & 1.35 & 17 & ${ }^{8} \mathrm{I}$ \\
\hline \multirow[t]{2}{*}{$4 f^{9}\left({ }^{7} \mathrm{H}^{\circ}\right) 6 s 6 p\left({ }^{3} \mathrm{P}^{\circ}\right)$} & & $15 / 2$ & 40604.11 & 1.295 & 24 & ${ }^{4} I$ \\
\hline & & $15 / 2$ & 40626.42 & 1.093 & & \\
\hline $4 f^{9}\left({ }^{7} \mathrm{H}^{\circ}\right) 5 d\left({ }^{7} \mathrm{H}^{\circ}\right) 6 p$ & & $19 / 2$ & 40807.20 & 1.332 & 44 & ${ }^{8} \mathrm{I}$ \\
\hline $4 f^{9}\left({ }^{7} \mathrm{H}^{\circ}\right) 6 s 6 p\left({ }^{3} \mathrm{P}^{\circ}\right)$ & & $11 / 2$ & 40841.63 & 1.326 & 20 & ${ }^{8} \mathrm{H}$ \\
\hline $4 f^{9}\left({ }^{7} \mathrm{H}^{\circ}\right) 6 s 6 p\left({ }^{3} \mathrm{P}^{\circ}\right)$ & & $13 / 2$ & 41004.07 & 1.26 & 15 & ${ }^{6} \mathrm{I}$ \\
\hline $4 f^{9}\left({ }^{7} \mathrm{H}^{\circ}\right) 6 s 6 p\left({ }^{3} \mathrm{P}^{\circ}\right)$ & & $15 / 2$ & 41111.11 & 1.32 & 40 & ${ }^{6} \mathrm{H}$ \\
\hline $4 f^{9}\left({ }^{7} \mathrm{H}^{\circ}\right) 5 d\left({ }^{7} \mathrm{H}^{\circ}\right) 6 p$ & & $17 / 2$ & 41583.90 & 1.33 & 25 & ${ }^{6} \mathrm{I}$ \\
\hline $4 f^{9}\left({ }^{7} \mathrm{H}^{\circ}\right) 6 s 6 p\left({ }^{3} \mathrm{P}^{\circ}\right)$ & & $13 / 2$ & 41878.48 & 1.314 & 16 & ${ }^{4} \mathrm{H}$ \\
\hline $4 f^{9}\left({ }^{7} \mathrm{H}^{\circ}\right) 5 d\left({ }^{7} \mathrm{~F}^{\circ}\right) 6 p$ & & $11 / 2$ & 42083.59 & 1.33 & 24 & ${ }^{8 \mathrm{D}}$ \\
\hline \multirow[t]{2}{*}{$4 f^{9}\left({ }^{7} \mathrm{H}^{\circ}\right) 6 s 6 p\left({ }^{3} \mathrm{P}^{\circ}\right)$} & & $13 / 2$ & 42137.31 & 1.33 & 32 & ${ }^{6} \mathrm{G}$ \\
\hline & & $11 / 2$ & 42150.67 & 1.10 & & \\
\hline $4 f^{9}\left({ }^{7} \mathrm{H}^{\circ}\right) 5 d\left({ }^{7} \mathrm{H}^{\circ}\right) 6 p$ & & $9 / 2$ & 42256.43 & 1.23 & 27 & ${ }^{8} \mathrm{H}$ \\
\hline $4 f^{9}\left({ }^{7} \mathrm{H}^{\circ}\right) 5 d\left({ }^{7} \mathrm{H}^{\circ}\right) 6 p$ & & $13 / 2$ & 42289.33 & 1.348 & 30 & ${ }^{6} \mathrm{G}$ \\
\hline $4 f^{9}\left({ }^{7} \mathrm{H}^{\circ}\right) 5 d\left({ }^{7} \mathrm{H}^{\circ}\right) 6 p$ & & $19 / 2$ & 42478.98 & 1.31 & 35 & ${ }^{8} \mathrm{I}$ \\
\hline $4 f^{9}\left({ }^{7} \mathrm{H}^{\circ}\right) 5 d\left({ }^{7} \mathrm{H}^{\circ}\right) 6 p$ & & $15 / 2$ & 42550.09 & 1.34 & 26 & ${ }^{6} \mathrm{H}$ \\
\hline $4 f^{9}\left({ }^{7} \mathrm{H}^{\circ}\right) 5 d\left({ }^{7} \mathrm{H}^{\circ}\right) 6 p$ & & $17 / 2$ & 42741.69 & 1.32 & 12 & ${ }^{8} \mathrm{H}$ \\
\hline $4 f^{9}\left({ }^{7} \mathrm{H}^{\circ}\right) 6 s 6 p\left({ }^{3} \mathrm{P}^{\circ}\right)$ & & $11 / 2$ & 42813.08 & 1.208 & 13 & ${ }^{4} \mathrm{G}$ \\
\hline $4 f^{9}\left({ }^{7} \mathrm{H}^{\circ}\right) 5 d\left({ }^{7} \mathrm{~F}^{\circ}\right) 6 p$ & & $13 / 2$ & 42883.01 & 1.367 & 19 & ${ }^{8} \mathrm{~F}$ \\
\hline $4 f^{9}\left({ }^{7} \mathrm{H}^{\circ}\right) 6 s 6 p\left({ }^{3} \mathrm{P}^{\circ}\right)$ & & $9 / 2$ & 42911.59 & 1.21 & 33 & ${ }^{8} \mathrm{H}$ \\
\hline $4 f^{9}\left({ }^{7} \mathrm{H}^{\circ}\right) 5 d\left({ }^{7} \mathrm{H}^{\circ}\right) 6 p$ & & $15 / 2$ & 43002.98 & 1.33 & 20 & ${ }^{8} \mathrm{H}$ \\
\hline $4 f^{9}\left({ }^{6} \mathrm{H}^{\circ}\right) 5 d\left({ }^{7} \mathrm{H}^{\circ}\right) 6 p$ & & $17 / 2$ & 43066.19 & 1.343 & 35 & ${ }^{8} \mathrm{I}$ \\
\hline $4 f^{9}\left({ }^{6} \mathrm{H}^{\circ}\right) 6 s 6 p\left({ }^{3} \mathrm{P}^{\circ}\right)$ & & $13 / 2$ & 43422.06 & 1.307 & 19 & ${ }^{8} \mathrm{I}$ \\
\hline $4 f^{9}\left({ }^{6} \mathrm{~F}^{\circ}\right) 6 s 6 p\left({ }^{3} \mathrm{P}^{\circ}\right)$ & ${ }^{8} \mathrm{D}$ & $11 / 2$ & 43689.99 & 1.401 & 45 & \\
\hline $4 f^{9}\left({ }^{6} \mathrm{H}^{\circ}\right) 6 s 6 p\left({ }^{3} \mathrm{P}^{\circ}\right)$ & & $15 / 2$ & 43794.35 & 1.298 & 20 & ${ }^{8} \mathrm{I}$ \\
\hline $4 f^{9}\left({ }^{6} \mathrm{H}^{\circ}\right) 5 d\left({ }^{7} \mathrm{H}^{\circ}\right) 6 p$ & & $7 / 2$ & 43838.10 & 1.04 & 33 & ${ }^{8} \mathrm{H}$ \\
\hline $4 f^{9}\left({ }^{6} \mathrm{H}^{\circ}\right) 5 d\left({ }^{7} \mathrm{G}^{\circ}\right) 6 p$ & & $15 / 2$ & 43891.33 & 1.31 & 21 & ${ }^{6} \mathrm{H}$ \\
\hline $4 f^{9}\left({ }^{6} \mathrm{H}^{\circ}\right) 6 s 6 p\left({ }^{3} \mathrm{P}^{\circ}\right)$ & & $13 / 2$ & 44049.22 & 1.312 & 12 & ${ }^{6} \mathrm{H}$ \\
\hline $4 f^{9}\left({ }^{6} \mathrm{H}^{\circ}\right) 6 s 6 p\left({ }^{3} \mathrm{P}^{\circ}\right)$ & & $17 / 2$ & 44074.41 & 1.31 & 25 & ${ }^{8} \mathrm{I}$ \\
\hline $4 f^{9}\left({ }^{6} \mathrm{H}^{\circ}\right) 5 d\left({ }^{7} \mathrm{I}^{\circ}\right) 6 p$ & ${ }^{8} \mathrm{~K}$ & $21 / 2$ & 44156.98 & 1.317 & 73 & \\
\hline $4 f^{9}\left({ }^{6} \mathrm{H}^{\circ}\right) 5 d\left({ }^{7} \mathrm{H}^{\circ}\right) 6 p$ & & $13 / 2$ & 44189.52 & 1.31 & 20 & ${ }^{6} \mathrm{H}$ \\
\hline $4 f^{9}\left({ }^{6} \mathrm{H}^{\circ}\right) 6 s 6 p\left({ }^{3} \mathrm{P}^{\circ}\right)$ & & $11 / 2$ & 44331.36 & 1.242 & 16 & ${ }^{6} \mathrm{I}$ \\
\hline $4 f^{9}\left({ }^{6} \mathrm{H}^{\circ}\right) 5 d\left({ }^{7} \mathrm{H}^{\circ}\right) 6 p$ & & $9 / 2$ & 44352.01 & 1.17 & 22 & ${ }^{8} \mathbf{I}$ \\
\hline $4 f^{9}\left({ }^{6} \mathrm{H}^{\circ}\right) 5 d\left({ }^{7} \mathrm{I}^{\circ}\right) 6 p$ & & $19 / 2$ & 44501.98 & 1.32 & 43 & ${ }^{8} \mathrm{I}$ \\
\hline $4 f^{9}\left({ }^{6} \mathrm{H}^{\circ}\right) 6 s 6 p\left({ }^{3} \mathrm{P}^{\circ}\right)$ & & $7 / 2$ & 44529.45 & 1.00 & $?$ & ${ }^{8} \mathrm{H}$ \\
\hline $4 f^{9}\left({ }^{6} \mathrm{H}^{\circ}\right) 6 s 6 p\left({ }^{3} \mathrm{P}^{\circ}\right)$ & & $17 / 2$ & 44545.25 & 1.28 & 24 & ${ }^{8} \mathrm{I}$ \\
\hline
\end{tabular}


Dy II, Even Parity-Continued

\begin{tabular}{|c|c|c|c|c|c|c|}
\hline Configuration & Term & $J$ & $\begin{array}{l}\text { Level } \\
\left(\mathrm{cm}^{-1}\right)\end{array}$ & $g$ & & Leading percentages \\
\hline $4 f^{9}\left({ }^{6} \mathrm{H}^{\circ}\right) 5 d\left({ }^{7} \mathrm{H}^{\circ}\right) 6 p$ & & $15 / 2$ & 44653.18 & 1.318 & 12 & ${ }^{6} \mathrm{H}$ \\
\hline $4 f^{9}\left({ }^{6} \mathrm{H}^{\circ}\right) 5 d\left({ }^{7} \mathrm{H}^{\circ}\right) 6 p$ & & $9 / 2$ & 44737.52 & 1.38 & 11 & ${ }^{8} \mathrm{G}$ \\
\hline $4 f^{9}\left({ }^{6} \mathrm{H}^{\circ}\right) 5 d\left({ }^{7} \mathrm{H}^{\circ}\right) 6 p$ & & $13 / 2$ & 44812.00 & 1.39 & 14 & ${ }^{8} \mathrm{G}$ \\
\hline $4 f^{9}\left({ }^{6} \mathrm{H}^{\circ}\right) 6 s 6 p\left({ }^{3} \mathrm{P}^{\circ}\right)$ & & $11 / 2$ & 44903.87 & 1.31 & 15 & ${ }^{4} \mathrm{H}$ \\
\hline $4 f^{9}\left({ }^{6} \mathrm{~F}^{\circ}\right) 6 s 6 p\left({ }^{3} \mathrm{P}^{\circ}\right)$ & & $13 / 2$ & 45123.11 & 1.323 & 19 & ${ }^{8} \mathrm{~F}$ \\
\hline $4 f^{10}\left({ }^{1} \mathrm{~K}_{7}\right) 6 s_{1 / 2} ?$ & & $15 / 2$ & 45132.74 & 1.09 & & \\
\hline $4 f^{9}\left({ }^{6} \mathrm{H}^{\circ}\right) 5 d\left({ }^{7} \mathrm{H}^{\circ}\right) 6 p$ & & $15 / 2$ & 45139.88 & 1.24 & 26 & ${ }^{8} \mathrm{I}$ \\
\hline $4 f^{9}\left({ }^{6} \mathrm{H}^{\circ}\right) 5 d\left({ }^{7} \mathrm{I}^{\circ}\right) 6 p$ & & $17 / 2$ & 45240.02 & 1.33 & 31 & ${ }^{8} \mathrm{H}$ \\
\hline $4 f^{9}\left({ }^{6} \mathrm{H}^{\circ}\right) 5 d\left({ }^{7} \mathrm{~F}^{\circ}\right) 6 p$ & & $11 / 2$ & 45241.75 & 1.34 & 11 & ${ }^{8} \mathrm{D}$ \\
\hline $4 f^{9}\left({ }^{6} \mathrm{H}^{\circ}\right) 5 d\left({ }^{7} \mathrm{I}^{\circ}\right) 6 p$ & & $15 / 2$ & 45317.04 & 1.28 & 29 & ${ }^{6} \mathrm{I}$ \\
\hline $4 f^{9}\left({ }^{6} \mathrm{H}^{\circ}\right) 5 d\left({ }^{7} \mathrm{H}^{\circ}\right) 6 p$ & & $13 / 2$ & 45356.53 & 1.448 & 13 & ${ }^{8} \mathrm{H}$ \\
\hline $4 f^{9}\left({ }^{6} \mathrm{H}^{\circ}\right) 6 s 6 p\left({ }^{3} \mathrm{P}^{\circ}\right)$ & & $9 / 2$ & 45427.49 & 1.26 & 13 & ${ }^{8} \mathrm{D}$ \\
\hline $4 f^{9}\left({ }^{6} \mathrm{H}^{\circ}\right) 5 d\left({ }^{7} \mathrm{~F}^{\circ}\right) 6 p$ & & $11 / 2$ & 45545.68 & 1.36 & 24 & ${ }^{6} \mathrm{~F}$ \\
\hline $4 f^{9}\left({ }^{6} \mathrm{H}^{\circ}\right) 5 d\left({ }^{7} \mathrm{I}^{\circ}\right) 6 p$ & & $19 / 2$ & 45701.64 & 1.29 & 36 & ${ }^{8} \mathrm{~K}$ \\
\hline $4 f^{9}\left({ }^{6} \mathrm{H}^{\circ}\right) 5 d\left({ }^{7} \mathrm{~F}^{\circ}\right) 6 p$ & & $13 / 2$ & 45773.90 & 1.29 & 23 & ${ }^{8} \mathrm{~F}$ \\
\hline $4 f^{9}\left({ }^{6} \mathrm{H}^{\circ}\right) 5 d\left({ }^{7} \mathrm{H}^{\circ}\right) 6 p$ & & $9 / 2$ & 45800.00 & 1.282 & 28 & ${ }^{6} \mathrm{G}$ \\
\hline $4 f^{9}\left({ }^{6} \mathrm{H}^{\circ}\right) 5 d\left({ }^{7} \mathrm{~K}^{\circ}\right) 6 p$ & ${ }^{8} \mathrm{~K}$ & $21 / 2$ & 45934.94 & 1.30 & 53 & \\
\hline $4 f^{9}\left({ }^{6} \mathrm{H}^{\circ}\right) 5 d\left({ }^{7} \mathrm{~K}^{\circ}\right) 6 p$ & & $19 / 2$ & 46066.65 & 1.24 & 25 & ${ }^{8} \mathrm{~K}$ \\
\hline $4 f^{9}\left({ }^{6} \mathrm{H}^{\circ}\right) 5 d\left({ }^{7} \mathrm{G}^{\circ}\right) 6 p$ & & $15 / 2$ & 46082.83 & 1.303 & 13 & ${ }^{8} \mathrm{G}$ \\
\hline $4 f^{9}\left({ }^{6} \mathrm{H}^{\circ}\right) 6 s 6 p\left({ }^{3} \mathrm{P}^{\circ}\right)$ & & $9 / 2$ & 46091.32 & 1.278 & 19 & ${ }^{6} \mathrm{G}$ \\
\hline $4 f^{9}\left({ }^{6} \mathrm{H}^{\circ}\right) 6 s 6 p\left({ }^{3} \mathrm{P}^{\circ}\right)$ & & $9 / 2$ & 46161.85 & 1.305 & 13 & ${ }^{4} \mathrm{G}$ \\
\hline $4 f^{9}\left({ }^{6} \mathrm{H}^{\circ}\right) 5 d\left({ }^{7} \mathrm{I}^{\circ}\right) 6 p$ & & $13 / 2$ & 46220.92 & 1.310 & 16 & ${ }^{8} \mathbf{I}$ \\
\hline $4 f^{9}\left({ }^{6} \mathrm{H}^{\circ}\right) 5 d\left({ }^{7} \mathrm{I}^{\circ}\right) 6 p$ & & $17 / 2$ & 46256.77 & 1.275 & 22 & ${ }^{6} \mathrm{I}$ \\
\hline $4 f^{9}\left({ }^{6} \mathrm{H}^{\circ}\right) 5 d\left({ }^{7} \mathrm{I}^{\circ}\right) 6 p$ & & $13 / 2$ & 46490.31 & 1.229 & 23 & ${ }^{6} \mathrm{H}$ \\
\hline $4 f^{9}\left({ }^{6} \mathrm{H}^{\circ}\right) 6 s 6 p\left({ }^{3} \mathrm{P}^{\circ}\right)$ & & $11 / 2$ & 46498.62 & 1.275 & 14 & ${ }^{4} \mathrm{H}$ \\
\hline $4 f^{9}\left({ }^{6} \mathrm{H}^{\circ}\right) 5 d\left({ }^{7} \mathrm{~F}^{\circ}\right) 6 p$ & & $11 / 2$ & 46647.75 & 1.338 & 10 & ${ }^{8} \mathrm{D}$ \\
\hline $4 f^{9}\left({ }^{6} \mathrm{H}^{\circ}\right) 5 d\left({ }^{7} \mathrm{I}^{\circ}\right) 6 p$ & & $17 / 2$ & 46694.14 & 1.274 & 20 & ${ }^{8} \mathbf{I}$ \\
\hline $4 f^{9}\left({ }^{6} \mathrm{H}^{\circ}\right) 6 s 6 p\left({ }^{3} \mathrm{P}^{\circ}\right)$ & & $13 / 2$ & 46779.06 & 1.297 & 14 & ${ }^{8} \mathbf{I}$ \\
\hline $4 f^{9}\left({ }^{6} \mathrm{H}^{\circ}\right) 5 d\left({ }^{7} \mathrm{H}^{\circ}\right) 6 p$ & & $7 / 2$ & 46798.32 & 1.11 & 14 & ${ }^{6} \mathrm{G}$ \\
\hline $4 f^{9}\left({ }^{6} \mathrm{H}^{\circ}\right) 5 d\left({ }^{7} \mathrm{~F}^{\circ}\right) 6 p$ & & $15 / 2$ & 46853.71 & 1.28 & 21 & ${ }^{8} \mathrm{G}$ \\
\hline $4 f^{9}\left({ }^{6} \mathrm{H}^{\circ}\right) 5 d\left({ }^{7} \mathrm{H}^{\circ}\right) 6 p$ & & $13 / 2$ & 46943.02 & 1.23 & 27 & ${ }^{8} \mathrm{I}$ \\
\hline $4 f^{9}\left({ }^{6} \mathrm{H}^{\circ}\right) 5 d\left({ }^{7} \mathrm{H}^{\circ}\right) 6 p$ & & $11 / 2$ & 46976.07 & 1.24 & 12 & ${ }^{8} \mathrm{H}$ \\
\hline $4 f^{9}\left({ }^{6} \mathrm{H}^{\circ}\right) 5 d\left({ }^{7} \mathrm{H}^{\circ}\right) 6 p$ & & $11 / 2$ & 47023.09 & 1.32 & 6 & ${ }^{8} \mathrm{G}$ \\
\hline $4 f^{9}\left({ }^{6} \mathrm{H}^{\circ}\right) 6 s 6 p\left({ }^{3} \mathrm{P}^{\circ}\right)$ & & $15 / 2$ & 47091.91 & 1.35 & 41 & ${ }^{6} \mathrm{I}$ \\
\hline $4 f^{9}\left({ }^{6} \mathrm{H}^{\circ}\right) 5 d\left({ }^{7} \mathrm{~K}^{\circ}\right) 6 p$ & & $17 / 2$ & 47208.15 & 1.24 & 14 & ${ }^{6} \mathrm{~K}$ \\
\hline $4 f^{9}\left({ }^{6} \mathrm{H}^{\circ}\right) 5 d\left({ }^{7} \mathrm{H}^{\circ}\right) 6 p$ & & $13 / 2$ & 47357.74 & 1.22 & 17 & ${ }^{6} \mathrm{I}$ \\
\hline $4 f^{9}\left({ }^{6} \mathrm{~F}^{\circ}\right) 6 s 6 p\left({ }^{3} \mathrm{P}^{\circ}\right)$ & & $11 / 2$ & 47401.40 & 1.22 & 18 & ${ }^{8} \mathrm{G}$ \\
\hline $4 f^{9}\left({ }^{6} \mathrm{H}^{\circ}\right) 5 d\left({ }^{7} \mathrm{H}^{\circ}\right) 6 p$ & & $9 / 2$ & 47462.43 & 1.32 & 13 & ${ }^{8} \mathrm{G}$ \\
\hline $4 f^{9}\left({ }^{6} \mathrm{~F}^{\circ}\right) 6 s 6 p\left({ }^{3} \mathrm{P}^{\circ}\right)$ & & $15 / 2$ & 47597.73 & 1.262 & 33 & ${ }^{8} \mathrm{G}$ \\
\hline \multirow[t]{2}{*}{$4 f^{9}\left({ }^{6} \mathrm{H}^{\circ}\right) 5 d\left({ }^{7} \mathrm{G}^{\circ}\right) 6 p$} & \multirow{5}{*}{${ }^{8} \mathrm{~L}$} & $17 / 2$ & 47718.75 & 1.278 & 19 & ${ }^{8} \mathrm{H}$ \\
\hline & & $11 / 2$ & 47833.92 & 1.16 & & \\
\hline $4 f^{9}\left({ }^{6} \mathrm{H}^{\circ}\right) 5 d\left({ }^{7} \mathrm{~K}^{\circ}\right) 6 p$ & & $23 / 2$ & 47960.83 & 1.31 & 100 & \\
\hline $4 f^{9}\left({ }^{6} \mathrm{H}^{\circ}\right) 5 d\left({ }^{7} \mathrm{G}^{\circ}\right) 6 p$ & & $17 / 2$ & 48022.12 & 1.248 & 17 & ${ }^{8} \mathrm{H}$ \\
\hline $4 f^{9}\left({ }^{6} \mathrm{H}^{\circ}\right) 5 d\left({ }^{7} \mathrm{H}^{\circ}\right) 6 p$ & & $13 / 2$ & 48035.88 & 1.31 & 8 & ${ }^{6} \mathrm{I}$ \\
\hline
\end{tabular}


Dy II, Even Parity-Continued

\begin{tabular}{|c|c|c|c|c|c|c|}
\hline Configuration & Term & $J$ & $\begin{array}{c}\text { Level } \\
\left(\mathrm{cm}^{-1}\right)\end{array}$ & $g$ & & Leading percentages \\
\hline $4 f^{9}\left({ }^{6} \mathrm{H}^{\circ}\right) 5 d\left({ }^{7} \mathrm{I}^{\circ}\right) 6 p$ & & $15 / 2$ & 48052.16 & 1.29 & 14 & ${ }^{8} \mathrm{H}$ \\
\hline $4 f^{9}\left({ }^{6} \mathrm{H}^{\circ}\right) 5 d\left({ }^{7} \mathrm{~K}^{\circ}\right) 6 p$ & & $21 / 2$ & 48059.91 & 1.28 & 34 & ${ }^{8} \mathrm{~K}$ \\
\hline $4 f^{9}\left({ }^{6} \mathrm{H}^{\circ}\right) 5 d\left({ }^{7} \mathrm{I}^{\circ}\right) 6 p$ & & $19 / 2$ & 48069.10 & 1.273 & 34 & ${ }^{6} \mathrm{~K}$ \\
\hline $4 f^{9}\left({ }^{6} \mathrm{H}^{\circ}\right) 5 d\left({ }^{7} \mathrm{I}^{\circ}\right) 6 p$ & & $11 / 2$ & 48240.94 & 1.28 & 23 & ${ }^{8} \mathrm{~K}$ \\
\hline $4 f^{9}\left({ }^{6} \mathrm{H}^{\circ}\right) 5 d\left({ }^{5} \mathrm{~K}^{\circ}\right) 6 p$ & & $19 / 2$ & 48346.29 & 1.23 & 26 & ${ }^{4} \mathrm{~L}$ \\
\hline $4 f^{9}\left({ }^{6} \mathrm{H}^{\circ}\right) 5 d\left({ }^{7} \mathrm{I}^{\circ}\right) 6 p$ & & $11 / 2$ & 48469.08 & 1.29 & 13 & ${ }^{6} \mathrm{H}$ \\
\hline $4 f^{9}\left({ }^{6} \mathrm{~F}^{\circ}\right) 6 s 6 p\left({ }^{3} \mathrm{P}^{\circ}\right)$ & & $13 / 2$ & 48487.13 & 1.46 & 33 & ${ }^{6} \mathrm{G}$ \\
\hline $4 f^{9}\left({ }^{6} \mathrm{H}^{\circ}\right) 5 d\left({ }^{7} \mathrm{G}^{\circ}\right) 6 p$ & & $15 / 2$ & 48489.05 & 1.35 & 17 & ${ }^{8} \mathrm{G}$ \\
\hline $4 f^{9}\left({ }^{6} \mathrm{H}^{\circ}\right) 6 s 6 p\left({ }^{3} \mathrm{P}^{\circ}\right)$ & & $9 / 2$ & 48684.98 & 1.20 & 15 & ${ }^{6} \mathrm{G}$ \\
\hline \multirow[t]{2}{*}{$4 f^{9}\left({ }^{6} \mathrm{H}^{\circ}\right) 5 d\left({ }^{7} \mathrm{I}^{\circ}\right) 6 p$} & & $13 / 2$ & 48831.17 & 1.27 & 10 & ${ }^{6} \mathrm{I}$ \\
\hline & & $13 / 2$ & 48844.85 & 1.26 & & \\
\hline $4 f^{9}\left({ }^{6} \mathrm{~F}^{\circ}\right) 6 s 6 p\left({ }^{3} \mathrm{P}^{\circ}\right)$ & & $11 / 2$ & 48863.45 & 1.32 & 18 & ${ }^{6} \mathrm{~F}$ \\
\hline $4 f^{9}\left({ }^{6} \mathrm{H}^{\circ}\right) 5 d\left({ }^{5} \mathrm{I}^{\circ}\right) 6 p$ & & $15 / 2$ & 48950.09 & 1.222 & 12 & ${ }^{6} \mathrm{H}$ \\
\hline \multirow[t]{2}{*}{$4 f^{9}\left({ }^{6} \mathrm{H}^{\circ}\right) 5 d\left({ }^{7} \mathrm{G}^{\circ}\right) 6 p$} & & $15 / 2$ & 48988.85 & 1.27 & 19 & ${ }^{8} \mathrm{G}$ \\
\hline & & $11 / 2$ & 49058.03 & 1.38 & & \\
\hline $4 f^{9}\left({ }^{6} \mathrm{~F}^{\circ}\right) 5 d\left({ }^{7} \mathrm{~F}^{\circ}\right) 6 p$ & & $13 / 2$ & 49132.94 & 1.23 & 12 & ${ }^{8} \mathrm{G}$ \\
\hline $4 f^{9}\left({ }^{6} \mathrm{H}^{\circ}\right) 5 d\left({ }^{7} \mathrm{~K}^{\circ}\right) 6 p$ & & $15 / 2$ & 49233.16 & 1.23 & 15 & ${ }^{6} \mathrm{~K}$ \\
\hline \multirow[t]{2}{*}{$4 f^{9}\left({ }^{6} \mathrm{H}^{\circ}\right) 5 d\left({ }^{7} \mathrm{~K}^{\circ}\right) 6 p$} & & $13 / 2$ & 49340.48 & 1.253 & 9 & ${ }^{8} \mathrm{~K}$ \\
\hline & & $11 / 2$ & 49462.33 & 1.29 & & \\
\hline $4 f^{9}\left({ }^{6} \mathrm{H}^{\circ}\right) 5 d\left({ }^{5} \mathrm{I}^{\circ}\right) 6 p$ & & $17 / 2$ & 49537.65 & 1.222 & 27 & $6 \mathrm{I}$ \\
\hline $4 f^{9}\left({ }^{6} \mathrm{H}^{\circ}\right) 5 d\left({ }^{5} \mathrm{~K}^{\circ}\right) 6 p$ & & $19 / 2$ & 49544.70 & 1.278 & 20 & ${ }^{6} \mathrm{~K}$ \\
\hline \multirow[t]{2}{*}{$4 f^{9}\left({ }^{6} \mathrm{H}^{\circ}\right) 6 s 6 p\left({ }^{3} \mathrm{P}^{\circ}\right)$} & & $13 / 2$ & 49603.11 & 1.32 & 29 & ${ }^{6} \mathrm{I}$ \\
\hline & & $11 / 2$ & 49607.50 & 1.15 & & \\
\hline $4 f^{9}\left({ }^{6} \mathrm{H}^{\circ}\right) 5 d\left({ }^{5} \mathrm{I}^{\circ}\right) 6 p$ & & $17 / 2$ & 49675.74 & 1.19 & 18 & ${ }^{4} \mathrm{~K}$ \\
\hline $4 f^{9}\left({ }^{6} \mathrm{H}^{\circ}\right) 5 d\left({ }^{5} \mathrm{H}^{\circ}\right) 6 p$ & & $13 / 2$ & 49680.29 & 1.35 & 18 & ${ }^{6} \mathrm{G}$ \\
\hline $4 f^{9}\left({ }^{6} \mathrm{H}^{\circ}\right) 5 d\left({ }^{7} \mathrm{~K}^{\circ}\right) 6 p$ & & $15 / 2$ & 49813.64 & 1.273 & 9 & ${ }^{6} \mathrm{~K}$ \\
\hline $4 f^{9}\left({ }^{6} \mathrm{H}^{\circ}\right) 5 d\left({ }^{7} \mathrm{~K}^{\circ}\right) 6 p$ & & $13 / 2$ & 49856.57 & 1.23 & 9 & ${ }^{6} \mathrm{I}$ \\
\hline \multirow[t]{3}{*}{$4 f^{9}\left({ }^{6} \mathrm{H}^{\circ}\right) 5 d\left({ }^{7} \mathrm{~K}^{\circ}\right) 6 p$} & & $19 / 2$ & 50017.73 & 1.278 & 39 & ${ }^{8} \mathbf{I}$ \\
\hline & & $13 / 2$ & 50051.48 & 1.26 & & \\
\hline & & $13 / 2$ & 50101.90 & 1.11 & & \\
\hline $4 f^{9}\left({ }^{6} \mathrm{H}^{\circ}\right) 5 d\left({ }^{5} \mathrm{H}^{\circ}\right) 6 p$ & & $15 / 2$ & 50129.27 & 1.212 & 18 & ${ }^{4} \mathrm{I}$ \\
\hline \multirow[t]{2}{*}{$4 f^{9}\left({ }^{6} \mathrm{H}^{\circ}\right) 5 d \quad\left({ }^{7} \mathrm{G}^{\circ}\right) 6 p$} & & $15 / 2$ & 50206.50 & 1.21 & 14 & ${ }^{8} \mathrm{H}$ \\
\hline & & $13 / 2$ & 50408.821 & 1.18 & & \\
\hline $4 f^{9}\left({ }^{6} \mathrm{H}^{\circ}\right) 5 d\left({ }^{7} \mathrm{~K}^{\circ}\right) 6 p$ & & $17 / 2$ & 50457.94 & 1.28 & 20 & ${ }^{8} \mathrm{I}$ \\
\hline \multirow[t]{2}{*}{$4 f^{9}\left({ }^{6} \mathrm{H}^{\circ}\right) 5 d\left({ }^{5} \mathrm{H}^{\circ}\right) 6 p$} & & $15 / 2$ & 50521.44 & 1.244 & 17 & ${ }^{4} I$ \\
\hline & & $11 / 2$ & 50768.52 & 1.35 & & \\
\hline $4 f^{9}\left({ }^{6} \mathrm{H}^{\circ}\right) 5 d\left({ }^{7} \mathrm{G}^{\circ}\right) 6 p$ & & $15 / 2$ & 50783.98 & 1.322 & 12 & ${ }^{8} \mathrm{H}$ \\
\hline $4 f^{9}\left({ }^{6} \mathrm{H}^{\circ}\right) 5 d\left({ }^{5} \mathrm{G}^{\circ}\right) 6 p$ & & $13 / 2$ & 50858.43 & 1.30 & 19 & ${ }^{4} \mathrm{H}$ \\
\hline $4 f^{9}\left({ }^{6} \mathrm{H}^{\circ}\right) 5 d\left({ }^{7} \mathrm{G}^{\circ}\right) 6 p$ & & $13 / 2$ & 51087.71 & 1.31 & 18 & ${ }^{8} \mathrm{G}$ \\
\hline \multirow[t]{2}{*}{$4 f^{9}\left({ }^{6} \mathrm{H}^{\circ}\right) 5 d\left({ }^{5} \mathrm{I}^{\circ}\right) 6 p$} & & $15 / 2$ & 51124.24 & 1.23 & 35 & ${ }^{4} \mathrm{I}$ \\
\hline & & $17 / 2$ & 51406.04 & 1.28 & & \\
\hline $4 f^{9}\left({ }^{6} \mathrm{H}^{\circ}\right) 5 d\left({ }^{7} \mathrm{~K}^{\circ}\right) 6 p$ & ${ }^{8} \mathrm{~L}$ & $21 / 2$ & 51593.35 & 1.25 & 46 & \\
\hline $4 f^{9}\left({ }^{6} \mathrm{H}^{\circ}\right) 5 d\left({ }^{7} \mathrm{~K}^{\circ}\right) 6 p$ & & $19 / 2$ & 51640.22 & 1.25 & 25 & ${ }^{8} \mathrm{~K}$ \\
\hline $4 f^{9}\left({ }^{6} \mathrm{H}^{\circ}\right) 5 d\left({ }^{5} I^{\circ}\right) 6 p$ & & $17 / 2$ & 51716.65 & 1.232 & 18 & ${ }^{4} \mathrm{~K}$ \\
\hline
\end{tabular}


Dy II, Even Parity-Continued

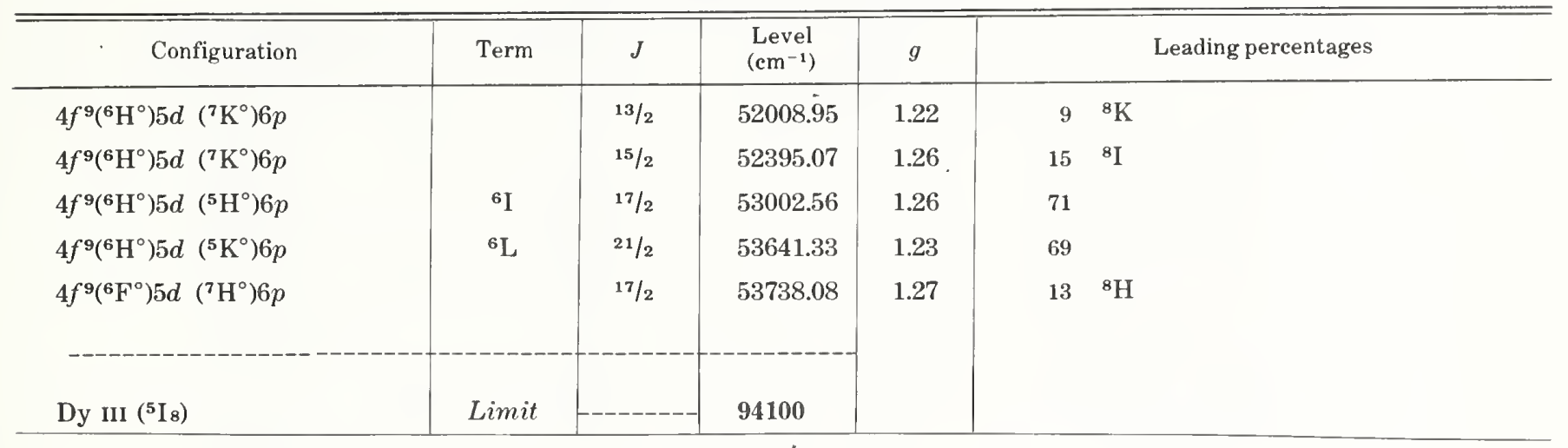

Dy II, Odd Parity

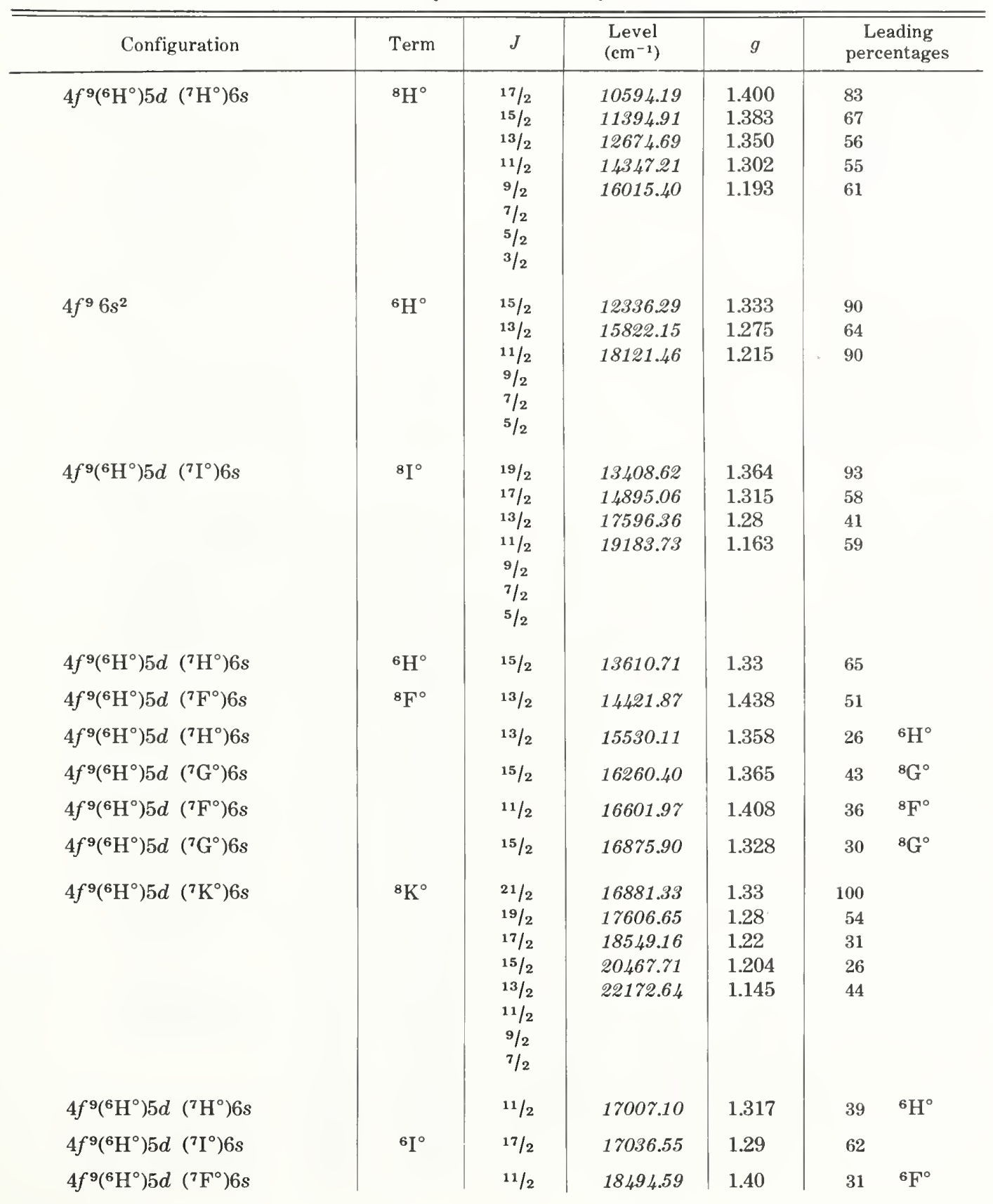


Dy II, Odd Parity_Continued

\begin{tabular}{|c|c|c|c|c|c|c|}
\hline Configuration & Term & $J$ & $\begin{array}{l}\text { Level } \\
\left(\mathrm{cm}^{-1}\right)\end{array}$ & $g$ & \multicolumn{2}{|c|}{$\begin{array}{c}\text { Leading } \\
\text { percentages }\end{array}$} \\
\hline $4 f^{9}\left({ }^{6} \mathrm{H}^{\circ}\right) 5 d\left({ }^{5} \mathrm{I}^{\circ}\right) 6 s$ & \multirow{6}{*}{${ }^{6} \mathrm{I}^{\circ}$} & $15 / 2$ & 18678.44 & 1.272 & 27 & ${ }^{4} I^{\circ}$ \\
\hline $4 f^{9}\left({ }^{6} \mathrm{H}^{\circ}\right) 5 d\left({ }^{7} \mathrm{~F}^{\circ}\right) 6 s$ & & $9 / 2$ & 18738.41 & 1.42 & 27 & ${ }^{8} \mathrm{~F}^{\circ}$ \\
\hline $4 f^{9}\left({ }^{6} \mathrm{~F}^{\circ}\right) 5 d\left({ }^{7} \mathrm{H}^{\circ}\right) 6 s$ & & $9 / 2$ & 18955.57 & 1.21 & 44 & ${ }^{6} \mathrm{H}^{\circ}$ \\
\hline $4 f^{9}\left({ }^{6} \mathrm{H}^{\circ}\right) 5 d\left({ }^{7} \mathrm{G}^{\circ}\right) 6 s$ & & $13 / 2$ & 18978.23 & 1.331 & 22 & ${ }^{8} \mathrm{G}^{\circ}$ \\
\hline $4 f^{9}\left({ }^{6} \mathrm{H}^{\circ}\right) 5 d\left({ }^{5} \mathrm{I}^{\circ}\right) 6 s$ & & $17 / 2$ & 19251.81 & 1.28 & 65 & \\
\hline $4 f^{9}\left({ }^{6} \mathrm{H}^{\circ}\right) 5 d\left({ }^{5} \mathrm{H}^{\circ}\right) 6 s$ & & $15 / 2$ & 19451.35 & 1.39 & 31 & ${ }^{6} \mathrm{H}^{\circ}$ \\
\hline $4 f^{9}\left({ }^{6} \mathrm{~F}^{\circ}\right) 5 d\left({ }^{7} \mathrm{P}^{\circ}\right) 6 s$ & \multirow[t]{2}{*}{${ }^{8} \mathrm{P}^{\circ}$} & $9 / 2$ & 1946723 & 1.660 & 58 & \\
\hline $4 f^{9}\left({ }^{6} \mathrm{H}^{\circ}\right) 5 d^{2}\left({ }^{3} \mathrm{~F}\right)$ & & $13 / 2$ & 19492.15 & 1.436 & 26 & ${ }^{8} G^{\circ}$ \\
\hline $4 f^{9}\left({ }^{6} \mathrm{H}^{\circ}\right) 5 d\left({ }^{7} \mathrm{~K}^{\circ}\right) 6 s$ & ${ }^{6} \mathrm{~K}^{\circ}$ & $19 / 2$ & 19571.75 & 1.267 & 54 & \\
\hline $4 f^{9} 6 s^{2}$ & \multirow[t]{7}{*}{${ }^{6} \mathrm{~F}^{\circ}$} & $11 / 2$ & 19927.64 & 1.445 & 84 & \\
\hline $4 f^{9}\left({ }^{6} \mathrm{H}^{\circ}\right) 5 d\left({ }^{7} \mathrm{G}^{\circ}\right) 6 s$ & & $13 / 2$ & 19956.65 & 1.310 & 19 & ${ }^{6} \mathrm{G}^{\circ}$ \\
\hline $4 f^{9}\left({ }^{6} \mathrm{H}^{\circ}\right) 5 d\left({ }^{5} \mathrm{H}^{\circ}\right) 6 s$ & & $15 / 2$ & 20166.45 & 1.313 & 43 & ${ }^{6} \mathrm{H}^{\circ}$ \\
\hline $4 f^{9}\left({ }^{6} \mathrm{H}^{\circ}\right) 5 d^{2}\left({ }^{3} \mathrm{~F}\right)$ & & $11 / 2$ & 20517.39 & 1.463 & 33 & ${ }^{8} \mathrm{~F}^{\circ}$ \\
\hline $4 f^{9}\left({ }^{6} \mathrm{H}^{\circ}\right) 5 d\left({ }^{5} \mathrm{~K}^{\circ}\right) 6 s$ & & $17 / 2$ & 20793.13 & 1.225 & 40 & ${ }^{4} \mathrm{~K}^{\circ}$ \\
\hline $4 f^{9}\left({ }^{6} \mathrm{H}^{\circ}\right) 5 d\left({ }^{7} \mathrm{G}^{\circ}\right) 6 s$ & & $11 / 2$ & 20817.16 & 1.44 & 40 & ${ }^{8} \mathrm{G}^{\circ}$ \\
\hline $4 f^{9}\left({ }^{6} \mathrm{H}^{\circ}\right) 5 d^{2}\left({ }^{3} \mathrm{~F}\right)$ & & $17 / 2$ & 20884.42 & 1.35 & 33 & ${ }^{8} \mathrm{I}^{\circ}$ \\
\hline $4 f^{9}\left({ }^{6} \mathrm{H}^{\circ}\right) 5 d\left({ }^{5} \mathrm{H}^{\circ}\right) 6 s$ & \multirow[t]{4}{*}{${ }^{4} \mathrm{H}^{\circ}$} & $13 / 2$ & 20896.37 & 1.33 & 66 & \\
\hline $4 f^{9}\left({ }^{6} \mathrm{H}^{\circ}\right) 5 d\left({ }^{7} \mathrm{~K}^{\circ}\right) 6 s$ & & $19 / 2$ & 20985.62 & 1.29 & 35 & ${ }^{8} \mathrm{~K}^{\circ}$ \\
\hline $4 f^{9}\left({ }^{6} \mathrm{H}^{\circ}\right) 5 d\left({ }^{7} \mathrm{I}^{\circ}\right) 6 s$ & & $13 / 2$ & 21134.77 & 1.24 & 19 & ${ }^{6} \mathrm{I}^{\circ}$ \\
\hline $4 f^{9}\left({ }^{6} \mathrm{H}^{\circ}\right) 5 d\left({ }^{7} \mathrm{I}^{\circ}\right) 6 s$ & & $15 / 2$ & 21272.07 & 1.21 & 28 & ${ }^{6} \mathrm{I}^{\circ}$ \\
\hline $4 f^{9}\left({ }^{6} \mathrm{H}^{\circ}\right) 5 d\left({ }^{5} \mathrm{G}^{\circ}\right) 6 s$ & \multirow[t]{2}{*}{${ }^{6} \mathrm{G}^{\circ}$} & $13 / 2$ & 21358.06 & 1.31 & 53 & \\
\hline $4 f^{9}\left({ }^{6} \mathrm{H}^{\circ}\right) 5 d^{2}\left({ }^{3} \mathrm{~F}\right)$ & & $19 / 2$ & 21464.98 & 1.316 & 36 & ${ }^{8} \mathrm{I}^{\circ}$ \\
\hline $4 f^{9}\left({ }^{6} \mathrm{H}^{\circ}\right) 5 d^{2}\left({ }^{3} \mathrm{~F}\right)$ & \multirow[t]{4}{*}{${ }^{8} \mathrm{~K}^{\circ}$} & $21 / 2$ & 22031.98 & 1.322 & 87 & \\
\hline $4 f^{9}\left({ }^{6} \mathrm{~F}^{\circ}\right) 5 d\left({ }^{7} \mathrm{G}^{\circ}\right) 6 s$ & & $13 / 2$ & 22083.31 & 1.402 & 29 & ${ }^{6} \mathrm{G}^{\circ}$ \\
\hline \multirow[t]{2}{*}{$4 f^{9}\left({ }^{6} \mathrm{~F}^{\circ}\right) 5 d\left({ }^{7} \mathrm{H}^{\circ}\right) 6 s$} & & $15 / 2$ & 22467.10 & 1.314 & 44 & ${ }^{8} \mathrm{H}^{\circ}$ \\
\hline & & $9 / 2$ & 22653.44 & 1.45 & & \\
\hline $4 f^{9}\left({ }^{6} \mathrm{H}^{\circ}\right) 5 d\left({ }^{5} \mathrm{I}^{\circ}\right) 6 s$ & \multirow{8}{*}{${ }^{8} \mathrm{H}^{\circ}$} & $15 / 2$ & 22672.54 & 1.364 & 21 & ${ }^{6} \mathrm{I}^{\circ}$ \\
\hline $4 f^{9}\left({ }^{6} \mathrm{H}^{\circ}\right) 5 d\left({ }^{5} \mathrm{I}^{\circ}\right) 6 s$ & & $15 / 2$ & 22854.69 & 1.28 & 26 & ${ }^{6} \mathrm{I}^{\circ}$ \\
\hline $4 f^{9}\left({ }^{6} \mathrm{H}^{\circ}\right) 5 d^{2}\left({ }^{3} \mathrm{~F}\right)$ & & $13 / 2$ & 2287120 & 1.387 & 29 & ${ }^{8} \mathrm{~F}^{\circ}$ \\
\hline $4 f^{9}\left({ }^{6} \mathrm{H}^{\circ}\right) 5 d\left({ }^{7} \mathrm{~K}^{\circ}\right) 6 s$ & & $17 / 2$ & 22908.05 & 1.24 & 31 & ${ }^{6} \mathrm{~K}^{\circ}$ \\
\hline $4 f^{9}\left({ }^{6} \mathrm{~F}^{\circ}\right) 5 d\left({ }^{7} \mathrm{H}^{\circ}\right) 6 s$ & & $17 / 2$ & 22990.86 & 1.40 & 91 & \\
\hline $4 f^{9}\left({ }^{6} \mathrm{H}^{\circ}\right) 5 d\left({ }^{5} \mathrm{~K}^{\circ}\right) 6 s$ & & $15 / 2$ & 23202.72 & 1.186 & 29 & ${ }^{4} \mathrm{~K}^{\circ}$ \\
\hline \multirow[t]{2}{*}{$4 f^{9}\left({ }^{6} \mathrm{H}^{\circ}\right) 5 d\left({ }^{5} \mathrm{H}^{\circ}\right) 6 s$} & & $13 / 2$ & 23303.06 & 1.31 & 31 & ${ }^{6} \mathrm{H}^{\circ}$ \\
\hline & & $11 / 2$ & 23561.58 & 1.368 & & \\
\hline $4 f^{9}\left({ }^{6} \mathrm{~F}^{\circ}\right) 5 d\left({ }^{7} \mathrm{H}^{\circ}\right) 6 s$ & \multirow{6}{*}{${ }^{6} \mathrm{~K}^{\circ}$} & $13 / 2$ & 23575.15 & 1.330 & 20 & ${ }^{8} \mathrm{H}^{\circ}$ \\
\hline $4 f^{9}\left({ }^{6} \mathrm{H}^{\circ}\right) 5 d^{2}\left({ }^{3} \mathrm{~F}\right)$ & & $19 / 2$ & 23707.18 & 1.30 & 29 & ${ }^{8} \mathrm{~K}^{\circ}$ \\
\hline $4 f^{9}\left({ }^{6} \mathrm{H}^{\circ}\right) 5 d^{2}\left({ }^{3} \mathrm{~F}\right)$ & & $17 / 2$ & 23765.80 & 1.27 & 28 & ${ }^{8} \mathrm{H}^{\circ}$ \\
\hline $4 f^{9}\left({ }^{6} \mathrm{H}^{\circ}\right) 5 d\left({ }^{5} \mathrm{I}^{\circ}\right) 6 s$ & & $13 / 2$ & 24104.44 & 1.173 & 28 & ${ }^{4} I^{\circ}$ \\
\hline $4 f^{9}\left({ }^{6} \mathrm{H}^{\circ}\right) 5 d\left({ }^{5} \mathrm{~K}^{\circ}\right) 6 s$ & & $17 / 2$ & 24316.09 & 1.23 & 45 & \\
\hline $4 f^{9}\left({ }^{6} \mathrm{H}^{\circ}\right) 5 d\left({ }^{7} \mathrm{~K}^{\circ}\right) 6 s$ & & $13 / 2$ & 24506.80 & 1.10 & 25 & ${ }^{6} \mathrm{~K}^{\circ}$ \\
\hline $4 f^{9}\left({ }^{6} \mathrm{H}^{\circ}\right) 5 d^{2}\left({ }^{3} \mathrm{~F}\right)$ & \multirow[t]{4}{*}{${ }^{8} \mathrm{~L}^{\circ}$} & $23 / 2$ & 24786.37 & 1.303 & 99 & \\
\hline $4 f^{9} 5 d^{2}$ & & $15 / 2$ & 24806.67 & 1.28 & & \\
\hline $4 f^{9} 5 d^{2}$ & & $13 / 2$ & 24818.31 & 1.303 & & \\
\hline $4 f^{9}\left({ }^{6} \mathrm{H}^{\circ}\right) 5 d^{2}\left({ }^{3} \mathrm{~F}\right)$ & & $13 / 2$ & 24967.31 & 1.283 & 16 & ${ }^{8} \mathrm{I}^{\circ}$ \\
\hline
\end{tabular}


Dy II, Odd Parity-Continued

\begin{tabular}{|c|c|c|c|c|c|c|}
\hline Configuration & Term & $J$ & $\begin{array}{l}\text { Level } \\
\left(\mathrm{cm}^{-1}\right)\end{array}$ & $g$ & \multicolumn{2}{|c|}{$\begin{array}{c}\text { Leading } \\
\text { percentages }\end{array}$} \\
\hline $4 f^{9}\left({ }^{6} \mathrm{~F}^{\circ}\right) 5 d\left({ }^{7} \mathrm{H}^{\circ}\right) 6 s$ & & $11 / 2$ & 25036.76 & 1.335 & 36 & ${ }^{8} \mathrm{H}^{\circ}$ \\
\hline $4 f^{10}\left({ }^{5} \mathrm{I}\right) 6 p$ & & $15 / 2$ & 25192.03 & 1.293 & 22 & ${ }^{6} \mathrm{H}^{\circ}$ \\
\hline $4 f^{9}\left({ }^{6} \mathrm{H}^{\circ}\right) 5 d^{2}\left({ }^{3} \mathrm{P}\right)$ & & $17 / 2$ & 25343.42 & 1.255 & 17 & ${ }^{8} \mathbf{I}^{\circ}$ \\
\hline \multirow[t]{2}{*}{$4 f^{9}\left({ }^{6} \mathrm{H}^{\circ}\right) 5 d\left({ }^{5} \mathrm{I}^{\circ}\right) 6 s$} & & $13 / 2$ & 25468.58 & 1.15 & 31 & ${ }^{6} \mathbf{I}^{\circ}$ \\
\hline & & $11 / 2$ & 25648.90 & 1.02 & & \\
\hline $4 f^{9}\left({ }^{6} \mathrm{H}^{\circ}\right) 5 d^{2}\left({ }^{3} \mathrm{~F}\right)$ & ${ }^{8} \mathrm{~L}^{\circ}$ & $21 / 2$ & 25756.29 & 1.26 & 57 & \\
\hline $4 f^{9}\left({ }^{6} \mathrm{H}^{\circ}\right) 5 d\left({ }^{7} \mathrm{G}^{\circ}\right) 6 s$ & & $13 / 2$ & 25772.86 & 1.37 & 29 & ${ }^{8} \mathrm{G}^{\circ}$ \\
\hline $4 f^{10}\left({ }^{5} \mathrm{I}\right) 6 p$ & & $17 / 2$ & 25818.43 & 1.27 & 37 & ${ }^{4} \mathrm{~K}^{\circ}$ \\
\hline \multirow[t]{2}{*}{$4 f^{9}\left({ }^{6} \mathrm{~F}^{\circ}\right) 5 d\left({ }^{7} \mathrm{H}^{\circ}\right) 6 s$} & & $15 / 2$ & 25833.38 & 1.22 & 22 & ${ }^{6} \mathrm{H}^{\circ}$ \\
\hline & & $11 / 2$ & 26047.86 & 1.15 & & \\
\hline \multirow[t]{2}{*}{$4 f^{9}\left({ }^{6} \mathrm{H}^{\circ}\right) 5 d^{2}\left({ }^{3} \mathrm{P}\right)$} & & $19 / 2$ & 26279.67 & 1.26 & 19 & ${ }^{8} \mathrm{I}^{\circ}$ \\
\hline & & $11 / 2$ & 26407.42 & 1.11 & & \\
\hline $4 f^{9}\left({ }^{6} \mathrm{H}^{\circ}\right) 5 d^{2}\left({ }^{3} \mathrm{P}\right)$ & & $15 / 2$ & 26634.20 & 1.290 & 21 & ${ }^{8} \mathrm{G}^{\circ}$ \\
\hline $4 f^{9} 5 d^{2}$ & & $13 / 2$ & 26853.72 & 1.320 & & \\
\hline $4 f^{9}\left({ }^{6} \mathrm{~F}^{\circ}\right) 5 d\left({ }^{5} \mathrm{H}^{\circ}\right) 6 s$ & & $13 / 2$ & 27193.05 & 1.295 & 15 & ${ }^{4} \mathrm{H}^{\circ}$ \\
\hline $4 f^{9}\left({ }^{6} \mathrm{H}^{\circ}\right) 5 d^{2}\left({ }^{3} \mathrm{~F}\right)$ & & $19 / 2$ & 27209.91 & 1.22 & 37 & ${ }^{8} \mathrm{~L}^{0}$ \\
\hline $4 f^{9}\left({ }^{6} \mathrm{~F}^{\circ}\right) 5 d\left({ }^{5} \mathrm{H}^{\circ}\right) 6 s$ & & $15 / 2$ & 27216.93 & 1.30 & 34 & ${ }^{6} \mathrm{H}^{\circ}$ \\
\hline $4 f^{9}\left({ }^{6} \mathrm{H}^{\circ}\right) 5 d^{2}\left({ }^{3} \mathrm{~F}\right)$ & & $17 / 2$ & 27232.69 & 1.24 & 36 & ${ }^{6} \mathrm{I}^{\circ}$ \\
\hline \multirow[t]{5}{*}{$4 f^{9}\left({ }^{6} \mathrm{H}^{\circ}\right) 5 d^{2}\left({ }^{3} \mathrm{P}\right)$} & & $15 / 2$ & 27435.12 & 1.308 & 16 & ${ }^{8} \mathrm{I}^{\circ}$ \\
\hline & & $11 / 2$ & 27462.39 & 1.18 & & \\
\hline & & $9 / 2$ & 27476.88 & 1.18 & & \\
\hline & & $13 / 2$ & 27502.93 & 1.20 & & \\
\hline & & $13 / 2$ & 27792.48 & 1.18 & & \\
\hline \multirow[t]{2}{*}{$4 f^{9}\left({ }^{6} \mathrm{H}^{\circ}\right) 5 d^{2}\left({ }^{3} \mathrm{~F}\right)$} & & $15 / 2$ & 27885.60 & 1.251 & 21 & ${ }^{6} \mathrm{I}^{\circ}$ \\
\hline & & $11 / 2$ & 27990.27 & 1.291 & & \\
\hline $4 f^{9} 5 d^{2}$ & & $19 / 2$ & 28005.87 & 1.32 & & \\
\hline $4 f^{9}\left({ }^{6} \mathrm{H}^{\circ}\right) 5 d^{2}\left({ }^{3} \mathrm{~F}\right)$ & & $13 / 2$ & 28019.70 & 1.25 & 21 & ${ }^{6} \mathrm{G}^{\circ}$ \\
\hline $4 f^{9}\left({ }^{6} \mathrm{H}^{\circ}\right) 5 d^{2}\left({ }^{1} \mathrm{G}\right)$ & & $17 / 2$ & 28252.34 & 1.235 & 15 & ${ }^{6} \mathbf{I}^{\circ}$ \\
\hline $\left.4 f^{10(5} \mathrm{I}\right) 6 p$ & ${ }^{6} \mathrm{~K}^{\circ}$ & $19 / 2$ & 28306.83 & 1.26 & 76 & \\
\hline $4 f^{9}\left({ }^{6} \mathrm{~F}^{\circ}\right) 5 d\left({ }^{7} \mathrm{H}^{\circ}\right) 6 s$ & & $13 / 2$ & 28456.12 & 1.25 & 16 & ${ }^{6} \mathrm{H}^{\circ}$ \\
\hline \multirow[t]{3}{*}{$4 f^{9}\left({ }^{6} \mathrm{~F}^{\circ}\right) 5 d\left({ }^{5} \mathrm{H}^{\circ}\right) 6 s$} & & $13 / 2$ & 28550.72 & 1.25 & 17 & ${ }^{6} \mathrm{H}^{\circ}$ \\
\hline & & $7 / 2$ & 28648.88 & 0.965 & & \\
\hline & & $13 / 2$ & 28784.49 & 1.03 & & \\
\hline \multirow[t]{2}{*}{$4 f^{10}\left({ }^{5} \mathrm{I}\right) 6 p$} & & $15 / 2$ & 28885.37 & 1.254 & 25 & ${ }^{6} \mathrm{~K}^{\circ}$ \\
\hline & & $11 / 2$ & 28908.29 & 1.23 & & \\
\hline $4 f^{9}\left({ }^{6} \mathrm{H}^{\circ}\right) 5 d^{2}\left({ }^{3} \mathrm{~F}\right)$ & & $17 / 2$ & 29014.43 & 1.256 & 15 & ${ }^{8} \mathrm{~K}^{\circ}$ \\
\hline $4 f^{9}\left({ }^{6} \mathrm{H}^{\circ}\right) 5 d^{2}\left({ }^{3} \mathrm{~F}\right)$ & & $15 / 2$ & 29109.09 & 1.230 & 16 & ${ }^{8} \mathrm{~L}^{\circ}$ \\
\hline $4 f^{10}\left({ }^{5} \mathrm{I}\right) 6 p$ & ${ }^{6} \mathrm{I}^{\circ}$ & $17 / 2$ & 29336.06 & 1.268 & 52 & \\
\hline $4 f^{9} 5 d^{2}$ & & $21 / 2$ & 29376.94 & 1.26 & & \\
\hline $4 f^{10}\left({ }^{5} \mathrm{I}\right) 6 p$ & & $15 / 2$ & 29436.60 & 1.222 & 42 & ${ }^{4} \mathrm{I}^{\circ}$ \\
\hline \multirow[t]{3}{*}{$4 f^{9}\left({ }^{6} \mathrm{H}^{\circ}\right) 5 d^{2}\left({ }^{3} \mathrm{~F}\right)$} & & $19 / 2$ & 29681.75 & 1.21 & 42 & ${ }^{4} \mathrm{~L}^{\circ}$ \\
\hline & & $11 / 2$ & 29685.14 & 1.26 & & \\
\hline & & $17 / 2$ & 29765.74 & 1.19 & & \\
\hline $4 f^{9}\left({ }^{6} \mathrm{H}^{\circ}\right) 5 d^{2}\left({ }^{3} \mathrm{~F}\right)$ & & $13 / 2$ & 29769.30 & 1.250 & 16 & ${ }^{6} \mathrm{G}^{\circ}$ \\
\hline
\end{tabular}


Dy II, Odd Parity-Continued

\begin{tabular}{|c|c|c|c|c|c|c|}
\hline Configuration & Term & $J$ & $\begin{array}{r}\text { Level } \\
\left(\mathrm{cm}^{-1}\right)\end{array}$ & $g$ & \multicolumn{2}{|c|}{$\begin{array}{c}\text { Leading } \\
\text { percentages }\end{array}$} \\
\hline $4 f^{9}\left({ }^{6} \mathrm{H}^{\circ}\right) 5 d^{2}\left({ }^{3} \mathrm{~F}\right)$ & & $13 / 2$ & 29877.52 & 1.406 & 32 & ${ }^{8} \mathrm{~F}^{\circ}$ \\
\hline $4 f^{9}\left({ }^{6} \mathrm{H}^{\circ}\right) 5 d^{2}\left({ }^{1} \mathrm{G}\right)$ & & $15 / 2$ & 30112.92 & 1.208 & 30 & ${ }^{6} \mathrm{I}^{0}$ \\
\hline $\left.4 f^{10(5} \mathrm{I}\right) 6 p$ & & $13 / 2$ & 30287.36 & 1.216 & 18 & ${ }^{6} \mathrm{H}^{\circ}$ \\
\hline $4 f^{10}\left({ }^{5} I\right) 6 p$ & ${ }^{4} \mathrm{H}^{\circ}$ & $13 / 2$ & 30361.80 & 1.194 & 53 & \\
\hline \multirow{5}{*}{$4 f^{9}\left({ }^{6} \mathrm{H}^{\circ}\right) 5 d^{2}\left({ }^{3} \mathrm{~F}\right)$} & & $15 / 2$ & 30399.10 & 1.201 & 23 & ${ }^{6} \mathrm{H}^{\circ}$ \\
\hline & & $11 / 2$ & 30553.63 & 1.31 & & \\
\hline & & $17 / 2$ & 30580.57 & 1.33 & & \\
\hline & & $13 / 2$ & 30638.55 & 1.20 & & \\
\hline & & $11 / 2$ & 30718222 & 1.300 & & \\
\hline \multirow[t]{3}{*}{$4 f^{9}\left({ }^{6} \mathrm{~F}^{\circ}\right) 5 d^{2}\left({ }^{3} \mathrm{~F}\right)$} & & $15 / 2$ & 30747.02 & 1.32 & 13 & ${ }^{8} \mathrm{H}^{\circ}$ \\
\hline & & $9 / 2$ & 30831.15 & 1.350 & & \\
\hline & & $19 / 2$ & 30894.44 & 1.32 & & \\
\hline $4 f^{9}\left({ }^{6} \mathrm{H}^{\circ}\right) 5 d^{2}\left({ }^{3} \mathrm{~F}\right)$ & & $13 / 2$ & 30973.97 & 1.139 & 35 & ${ }^{8} \mathrm{~L}^{\circ}$ \\
\hline $4 f^{9}\left({ }^{6} \mathrm{H}^{\circ}\right) 5 d^{2}\left({ }^{1} \mathrm{G}\right)$ & & $13 / 2$ & 31183.10 & 1.16 & 18 & ${ }^{6} \mathbf{I}^{\circ}$ \\
\hline \multirow[t]{3}{*}{$4 f^{9}\left({ }^{6} \mathrm{H}^{\circ}\right) 5 d^{2}\left({ }^{3} \mathrm{~F}\right)$} & & $15 / 2$ & 31306.47 & 1.22 & 30 & ${ }^{4} \mathrm{I}^{\circ}$ \\
\hline & & $11 / 2$ & 31313.36 & 1.375 & & \\
\hline & & $11 / 2$ & 31529.11 & 1.04 & & \\
\hline \multirow[t]{4}{*}{$4 f^{9}\left({ }^{6} \mathrm{H}^{\circ}\right) 5 d^{2}\left({ }^{1} \mathrm{G}\right)$} & & $19 / 2$ & 31536.69 & 1.26 & 38 & ${ }^{6} \mathrm{~K}^{\circ}$ \\
\hline & & $9 / 2$ & 31667.43 & 1.14 & & \\
\hline & & $11 / 2$ & 31701.66 & 1.075 & & \\
\hline & & $13 / 2$ & 31715.10 & 0.94 & & \\
\hline \multirow[t]{2}{*}{$4 f^{9}\left({ }^{6} \mathrm{H}^{\circ}\right) 5 d^{2}\left({ }^{1} \mathrm{D}\right)$} & & $15 / 2$ & 31826.35 & 1.33 & 23 & ${ }^{6} \mathrm{H}^{\circ}$ \\
\hline & & $9 / 2$ & 31866.89 & 1.28 & & \\
\hline $4 f^{10}\left({ }^{5} \mathrm{I}\right) 6 p$ & ${ }^{6} \mathrm{~K}^{\circ}$ & $17 / 2$ & 31879.66 & 1.17 & 46 & \\
\hline $4 f^{9}\left({ }^{6} \mathrm{H}^{\circ}\right) 5 d^{2}\left({ }^{3} \mathrm{P}\right)$ & & $13 / 2$ & 31978.85 & 1.232 & 30 & ${ }^{6} \mathrm{G}^{\circ}$ \\
\hline \multirow[t]{2}{*}{$4 f^{10}\left({ }^{5} \mathrm{I}\right) 6 p$} & & $13 / 2$ & 32218.48 & 1.157 & 40 & ${ }^{6} \mathrm{~K}^{\circ}$ \\
\hline & & $17 / 2$ & 32281.84 & 1.20 & & \\
\hline \multirow[t]{2}{*}{ - } & & $11 / 2$ & 32386.50 & 1.22 & & \\
\hline & & $13 / 2$ & 32591.49 & 1.10 & & \\
\hline \multirow[t]{2}{*}{$4 f^{9}\left({ }^{6} \mathrm{H}^{\circ}\right) 5 d^{2}\left({ }^{3} \mathrm{~F}\right)$} & & $17 / 2$ & 32613.41 & 1.19 & 24 & ${ }^{4} \mathrm{~L}^{\circ}$ \\
\hline & & $9 / 2$ & 32679.28 & 1.13 & & \\
\hline \multirow[t]{2}{*}{$4 f^{10}\left({ }^{5} \mathrm{I}\right) 6 p$} & ${ }^{6} \mathrm{I}^{\circ}$ & $15 / 2$ & 32709.97 & 1.20 & 48 & \\
\hline & & $11 / 2$ & 32903.81 & 1.10 & & \\
\hline \multirow[t]{9}{*}{$\left.4 f^{10(5} \mathrm{I}\right) 6 p$} & & $13 / 2$ & 32914.90 & 1.210 & 37 & ${ }^{4} I^{\circ}$ \\
\hline & & $9 / 2$ & 32919.48 & 1.180 & & \\
\hline & & $11 / 2$ & 33001.21 & 1.370 & & \\
\hline & & $19 / 2$ & 33035.57 & 1.232 & & \\
\hline & & $9 / 2$ & 33097.32 & 1.093 & & \\
\hline & & $11 / 2$ & 33306.71 & 1.29 & & \\
\hline & & $13 / 2$ & 33319.21 & 1.18 & & \\
\hline & & $15 / 2$ & 33465.39 & 1.273 & & \\
\hline & & $11 / 2$ & 33507.29 & 1.12 & & \\
\hline \multirow[t]{2}{*}{$4 f^{9}\left({ }^{6} \mathrm{H}^{\circ}\right) 5 d^{2}\left({ }^{1} \mathrm{G}\right)$} & & $17 / 2$ & 33529.34 & 1.30 & 18 & ${ }^{6} \mathrm{I}^{\circ}$ \\
\hline & & $15 / 2$ & 33576.32 & 1.25 & & \\
\hline
\end{tabular}


Dy II, Odd Parity-Continued

\begin{tabular}{|c|c|c|c|c|c|c|}
\hline Configuration & Term & $J$ & $\begin{array}{l}\text { Level } \\
\left(\mathrm{cm}^{-1}\right)\end{array}$ & $g$ & \multicolumn{2}{|c|}{$\begin{array}{c}\text { Leading } \\
\text { percentages }\end{array}$} \\
\hline & & $13 / 2$ & 33616.81 & 1.259 & & \\
\hline & & $11 / 2$ & 33677.88 & 1.137 & & \\
\hline & & $9 / 2$ & 33701.13 & 1.076 & & \\
\hline & & $13 / 2$ & 33817.65 & 1.24 & & \\
\hline & & $15 / 2$ & 33950.19 & 1.13 & & \\
\hline & & $13 / 2$ & 34110.17 & 1.309 & & \\
\hline & & $11 / 2$ & 34216.48 & 1.132 & & \\
\hline & & $13 / 2$ & 34255.72 & 1.272 & & \\
\hline & & $9 / 2$ & 34298.38 & 1.14 & & \\
\hline & & $19 / 2$ & 34307.62 & 1.233 & & \\
\hline \multirow[t]{34}{*}{$4 f^{9}\left({ }^{6} \mathrm{H}^{\circ}\right) 5 d^{2}\left({ }^{1} \mathrm{G}\right)$} & & $17 / 2$ & 34396.87 & 1.28 & 24 & ${ }^{6} \mathrm{~K}^{\circ}$ \\
\hline & & $15 / 2$ & 34423.22 & 1.148 & & \\
\hline & & $17 / 2$ & 34519.09 & 1.22 & & \\
\hline & & $11 / 2$ & 34524.06 & 1.102 & & \\
\hline & & $11 / 2$ & 34691.55 & 1.151 & & \\
\hline & & $15 / 2$ & 34692.39 & 1.16 & & \\
\hline & & $9 / 2$ & 34712.23 & 1.077 & & \\
\hline & & $7 / 2$ & 34730.20 & 1.184 & & \\
\hline & & $13 / 2$ & 34759.15 & 1.28 & & \\
\hline & & $9 / 2$ & 34864.90 & 1.03 & & \\
\hline & & $11 / 2$ & 34884.43 & 1.272 & & \\
\hline & & $11 / 2$ & 35000.63 & 1.30 & & \\
\hline & & $15 / 2$ & 35207.83 & 1.132 & & \\
\hline & & $13 / 2$ & 35230.58 & 1.14 & & \\
\hline & & $17 / 2$ & 35266.61 & 1.266 & & \\
\hline & & $11 / 2$ & 35268.41 & 1.20 & & \\
\hline & & $9 / 2$ & 35294.74 & 0.976 & & \\
\hline & & $15 / 2$ & 35365.10 & 1.21 & & \\
\hline & & $7 / 2$ & 35387.84 & 0.794 & & \\
\hline & & $13 / 2$ & 35434.55 & 1.13 & & \\
\hline & & $17 / 2$ & 35495.93 & 1.23 & & \\
\hline & & $15 / 2$ & 35565.89 & 1.17 & & \\
\hline & & $11 / 2$ & 35657.83 & 1.46 & & \\
\hline & & $11 / 2$ & 35685.84 & 1.229 & & \\
\hline & & $15 / 2$ & 35707.07 & 1.25 & & \\
\hline & & $9 / 2$ & 35841.78 & 1.04 & & \\
\hline & & $9 / 2$ & 3600125 & 0.96 & & \\
\hline & & $13 / 2$ & 36003.95 & 1.028 & & \\
\hline & & $11 / 2$ & 36057.03 & 1.21 & & \\
\hline & & $17 / 2$ & 36058.90 & 1.158 & & \\
\hline & & $13 / 2$ & 36173.95 & 1.14 & & \\
\hline & & $9 / 2$ & 36260.74 & 0.953 & & \\
\hline & & $11 / 2$ & 36264.62 & 1.14 & & \\
\hline & & $13 / 2$ & 36282.61 & 1.21 & & \\
\hline
\end{tabular}




\begin{tabular}{|c|c|c|c|c|c|c|c|c|c|}
\hline Configuration & Term & $J$ & $\begin{array}{l}\text { Level } \\
\left(\mathrm{cm}^{-1}\right)\end{array}$ & $g$ & Configuration & Term & $J$ & $\begin{array}{l}\text { Level } \\
\left(\mathrm{cm}^{-1}\right)\end{array}$ & $g$ \\
\hline & & $9 / 2$ & 36340.06 & 1.155 & & & $13 / 2$ & 38297.44 & 1.20 \\
\hline & & $11 / 2$ & 36350.70 & 1.07 & & & $7 / 2$ & 38322.19 & 1.37 \\
\hline & & $15 / 2$ & 36377.84 & 1.347 & & & $9 / 2$ & 38457.65 & 1.20 \\
\hline & & $13 / 2$ & 36387.03 & 1.226 & & & $11 / 2$ & 38495.04 & 1.131 \\
\hline & & $9 / 2$ & 36466.84 & 1.08 & & & $11 / 2$ & 38559.41 & 1.26 \\
\hline & & $7 / 2$ & 36643.97 & 1.26 & & & $7 / 2$ & 38583.10 & 1.197 \\
\hline & & $7 / 2$ & 36699.50 & 1.11 & & & $3 / 2$ & 38599.78 & 1.76 \\
\hline & & $15 / 2$ & 36719.64 & 1.23 & & & $9 / 2$ & 38604.25 & 1.26 \\
\hline & & $9 / 2$ & 36805.43 & 1.225 & & & $7 / 2$ & 38635.84 & 1.094 \\
\hline & & $9 / 2$ & 36859.32 & 1.37 & & & $15 / 2$ & 38656.68 & 1.06 \\
\hline & & $13 / 2$ & 36884.70 & 1.26 & & & $5 / 2$ & 38723.21 & 1.45 \\
\hline & & $11 / 2$ & 36970.68 & 1.20 & & & $9 / 2$ & 38735.83 & 1.353 \\
\hline & & $7 / 2$ & 37036.56 & 0.97 & & & $13 / 2$ & 38758.89 & 1.12 \\
\hline & & $13 / 2$ & 37060.94 & 1.28 & & & $3 / 2$ & 38774.19 & 1.35 \\
\hline & & $9 / 2$ & 37066.56 & 1.20 & & & $15 / 2$ & 38804.02 & 1.252 \\
\hline & & $11 / 2$ & 37076.28 & 1.209 & & & $7 / 2$ & 38818.29 & 1.20 \\
\hline & & $13 / 2$ & 37150.73 & 1.24 & & & $9 / 2$ & 38972.58 & 1.227 \\
\hline & & $11 / 2$ & 37187.31 & 1.397 & & & $13 / 2$ & 39021.89 & 1.09 \\
\hline & & $15 / 2$ & 37188.01 & 1.264 & & & $9 / 2$ & 39050.67 & 1.15 \\
\hline & & $7 / 2$ & 87199.97 & 1.088 & & & $7 / 2$ & 3908124 & 1.08 \\
\hline & & $11 / 2$ & 37363.99 & 1.250 & & & $11 / 2$ & 39117.83 & 1.24 \\
\hline & & $13 / 2$ & 37382.90 & 1.30 & & & $7 / 2$ & 39209.26 & 1.22 \\
\hline & & $11 / 2$ & 37517.38 & 1.24 & & & $11 / 2$ & 39215.90 & 1.268 \\
\hline & & $9 / 2$ & 37527.89 & 1.164 & & & $13 / 2$ & 39231.25 & 1.20 \\
\hline & & $15 / 2$ & 37623.10 & 1.242 & & & $13 / 2$ & 39293.76 & 1.09 \\
\hline & & $11 / 2$ & 37637.95 & 1.123 & & & $9 / 2$ & 39301.45 & 1.153 \\
\hline & & $13 / 2$ & 37671.30 & 1.320 & & & $7 / 2$ & 39308.62 & 1.021 \\
\hline & & $3 / 2$ & 37703.36 & 1.53 & & & $9 / 2$ & 39336.52 & 1.17 \\
\hline & & $7 / 2$ & 37764.84 & 1.054 & & & $5 / 2$ & 39377.02 & 0.701 \\
\hline & & $11 / 2$ & 37795.70 & 1.208 & & & $11 / 2$ & 39416.22 & 1.049 \\
\hline & & $7 / 2$ & 37839.46 & 0.76 & & & $7 / 2$ & 39499.72 & 1.214 \\
\hline & & $9 / 2$ & 37895.05 & 0.78 & & & $9 / 2$ & 39517.39 & 1.41 \\
\hline & & $13 / 2$ & 37905.71 & 1.11 & & & $11 / 2$ & 39549.11 & 0.953 \\
\hline & & $15 / 2$ & 37942.19 & 1.25 & & & $7 / 2$ & 39607.80 & 1.09 \\
\hline & & $9 / 2$ & 37958.42 & 1.165 & & & $9 / 2$ & 39691.58 & 1.017 \\
\hline & & $11 / 2$ & 38078.02 & 1.097 & & & $9 / 2$ & 39735.10 & 1.43 \\
\hline & & $13 / 2$ & 38110.71 & 1.09 & & & $11 / 2$ & 39791.15 & 1.22 \\
\hline & & $13 / 2$ & 38130.17 & 1.228 & & & $13 / 2$ & 39814.97 & 1.08 \\
\hline & & $11 / 2$ & 38156.00 & 1.177 & & & $9 / 2$ & 39838.11 & 1.233 \\
\hline & & $5 / 2$ & 38162.59 & 1.183 & & & $11 / 2$ & 39854.90 & 1.19 \\
\hline & & $9 / 2$ & 38214.89 & 1.22 & & & $5 / 2$ & 39957.42 & 1.13 \\
\hline & & $7 / 2$ & 38255.29 & 1.05 & & & $11 / 2$ & 39985.33 & 1.19 \\
\hline & & $17 / 2$ & 38266.59 & 1.152 & & & $7 / 2$ & 40036.03 & 1.101 \\
\hline & & $11 / 2$ & 38290.17 & 1.15 & & & $7 / 2$ & 40060.35 & 1.19 \\
\hline
\end{tabular}


Dy II, Odd Parity-Continued

Dy II, Odd Parity_Continued

\begin{tabular}{|c|c|c|c|c|c|c|c|c|c|}
\hline Configuration & Term & $J$ & $\begin{array}{l}\text { Level } \\
\left(\mathrm{cm}^{-1}\right)\end{array}$ & $g$ & Configuration & Term & $J$ & $\begin{array}{l}\text { Level } \\
\left(\mathrm{cm}^{-1}\right)\end{array}$ & $g$ \\
\hline & & $9 / 2$ & 40091.48 & 0.97 & & & $5 / 2$ & 42752.23 & 1.05 \\
\hline & & $5 / 2$ & 40137.54 & 1.32 & & & $9 / 2$ & 42796.25 & 1.05 \\
\hline & & $11 / 2$ & 40146.37 & 1.07 & & & $7 / 2$ & 42831.46 & 1.17 \\
\hline & & $5 / 2$ & 40209.88 & 0.832 & & & $3 / 2$ & 43048.38 & 1.16 \\
\hline & & $11 / 2$ & 40223.84 & 1.075 & & & $7 / 2$ & 43150.78 & 1.13 \\
\hline & & $5 / 2$ & 40264.90 & 1.151 & & & $7 / 2$ & 43165.66 & 1.35 \\
\hline & & $9 / 2$ & 40315.09 & 1.06 & & & $5 / 2$ & 43250.10 & 1.42 \\
\hline & & $9 / 2$ & 40343.72 & 1.19 & & & $9 / 2$ & 43534.34 & 1.24 \\
\hline & & $3 / 2$ & 40454.94 & 1.55 & & & $5 / 2$ & 43586.31 & 0.99 \\
\hline & & $7 / 2$ & 40477.77 & 1.09 & & & $7 / 2$ & 43668.47 & 1.15 \\
\hline & & $3 / 2$ & 40526.09 & 1.32 & & & $9 / 2$ & 43672.95 & 1.17 \\
\hline & & $9 / 2$ & 40541.00 & 1.21 & & & $5 / 2$ & 43828.98 & 1.02 \\
\hline & & $11 / 2$ & 40635.58 & 0.96 & & & $3 / 2$ & 43924.53 & 0.66 \\
\hline & & $7 / 2$ & 40694.78 & 1.32 & & & $5 / 2$ & 43939.40 & 1.22 \\
\hline & & $11 / 2$ & 40945.42 & 1.137 & & & $11 / 2$ & 44043.04 & 1.21 \\
\hline & & $9 / 2$ & 40977.16 & 1.148 & & & $7 / 2$ & 44119.99 & 1.18 \\
\hline & & $13 / 2$ & 41070.62 & 1.12 & & & $3 / 2$ & 44211.30 & 1.16 \\
\hline & & $5 / 2$ & 41266.26 & 1.41 & & & $5 / 2$ & 44449.17 & 1.06 \\
\hline & & $5 / 2$ & 41274.20 & 1.41 & & & $7 / 2$ & 44524.40 & 1.22 \\
\hline & & $11 / 2$ & 41305.27 & 1.12 & & & $3 / 2$ & 44532.86 & 1.42 \\
\hline & & $9 / 2$ & 41317.47 & 1.12 & & & $7 / 2$ & 44647.33 & 1.10 \\
\hline & & $7 / 2$ & 41335.44 & 1.04 & & & $5 / 2$ & 44865.63 & 1.30 \\
\hline & & $7 / 2$ & 41455.39 & 1.10 & & & $9 / 2$ & 45056.80 & 1.20 \\
\hline & & $9 / 2$ & 41472.39 & 1.10 & & & $3 / 2$ & 45066.73 & 0.97 \\
\hline & & $13 / 2$ & 41546.61 & 1.20 & & & $7 / 2$ & 45101.52 & 1.18 \\
\hline & & $9 / 2$ & 41547.15 & 1.15 & & & $9 / 2$ & 45138.51 & 1.21 \\
\hline & & $9 / 2$ & 41594.26 & 1.148 & & & $5 / 2$ & 45160.67 & 1.45 \\
\hline & & $5 / 2$ & 41638.63 & 1.346 & & & $5 / 2$ & 45210.54 & 1.18 \\
\hline & & $7 / 2$ & 41689.34 & 1.382 & & & $7 / 2$ & 45227.67 & 1.25 \\
\hline & & $7 / 2$ & 41702.39 & 1.21 & & & $7 / 2$ & 45331.71 & 1.06 \\
\hline & & $9 / 2$ & 41747.56 & 0.99 & & & $7 / 2$ & 45358.28 & 1.28 \\
\hline & & $7 / 2$ & 41808.88 & 1.01 & & & $5 / 2$ & 45500.67 & 1.26 \\
\hline & & $7 / 2$ & 41912.70 & 1.18 & & & $5 / 2$ & 45521.69 & 1.08 \\
\hline & & $5 / 2$ & 42060.49 & 1.28 & & & $7 / 2$ & 45624.03 & 1.25 \\
\hline & & $11 / 2$ & 42067.67 & 1.15 & & & $7 / 2$ & 45967.45 & 1.34 \\
\hline & & $9 / 2$ & 42286.35 & 1.14 & & & $3 / 2$ & 46010.41 & 1.46 \\
\hline & & $5 / 2$ & 42322.32 & 1.39 & & & $5 / 2$ & 47564.56 & 1.44 \\
\hline & & $13 / 2$ & 42333.66 & 1.17 & & & & & \\
\hline & & $3 / 2$ & 42451.74 & 1.59 & Dy III $\left({ }^{5} I_{8}\right)$ & Limit & & 94100 & \\
\hline & & $7 / 2$ & 42628.84 & 1.23 & 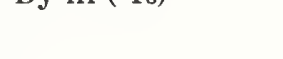 & & & & \\
\hline & & $5 / 2$ & 42662.83 & 1.46 & & & & & \\
\hline
\end{tabular}




\section{Dy III}

(Gd I sequence; 64 electrons)

Ground state $\left(1 s^{2} 2 s^{2} 2 p^{6} 3 s^{2} 3 p^{6} 3 d^{10} 4 s^{2} 4 p^{6} 4 d^{10} 5 s^{2} 5 p^{6}\right) 4 f^{10}{ }^{5} \mathrm{I}_{8}$

Ionization energy $183800 \pm 2400 \mathrm{~cm}^{-1}$

The spectrum has not been analyzed. Hussain [1973] gives the wavelengths of more than 500 lines assigned to Dy III, mostly in the region 2000-4450 $\AA$. Spector and Sugar have photographed the spectrum at the National Bureau of Standards, and an analysis is planned.

The $4 f^{105} \mathrm{I}_{8}$ designation for the ground level is practically certain. The lowest level from another configuration is expected to belong to $4 f^{9} 5 d$, beginning about $17000 \mathrm{~cm}^{-1}$ above the ground level [Brewer, 1971; Martin, 1971; Sugar and Reader, 1973].

Sugar and Reader derived the quoted ionization energy.

\section{References}

Brewer, L., J. Opt. Soc. Am. 61, 1666 (1971).

Hussain, R., Thesis, Johns Hopkins Univ., Baltimore, 142 pp. (1973); (Univ. Microfilms, Ann Arbor, Mich., No. 7410419). W

Martin, W. C., J. Opt. Soc. Am. 61, 1682 (1971).

Sugar, J., and Reader, J., J. Chem. Phys. 59, 2083 (1973). IP

[January 1977] 


\section{[Dy IV ]}

(Eu I sequence; 63 electrons)

Ground state $\left(1 s^{2} 2 s^{2} 2 p^{6} 3 s^{2} 3 p^{6} 3 d^{10} 4 s^{2} 4 p^{6} 4 d^{10} 5 s^{2} 5 p^{6}\right) 4 f^{9}{ }^{6} \mathrm{H}_{15: 2}^{\circ}$

Ionization energy $334000 \pm 3000 \mathrm{~cm}^{-1}$

$41.4 \pm 0.4 \mathrm{eV}$

Hussain [1973] lists about 300 of the strongest lines assigned to Dy IV on the basis of his observations of the spectra of pulsed sparks between Dy electrodes (1250-2510 $\AA$ ). A few of the lines are given as classified by transitions of the types of $4 f^{8} 6 s-4 f^{8} 6 p$ or $4 f^{8} 5 d-4 f^{8} 6 p$, but no connection between the resulting level system and the $4 f^{9}$ ground configuration was found; Hussain notes that measurements below $1200 \AA$ are needed. Pending a more complete analysis, we have not included any levels obtained from the spark spectrum.

Dieke [1968] has reviewed work on the absorption and fluorescence spectra of Dy ${ }^{3+}$ ions in crystal hosts. The $4 f^{9}{ }^{6} \mathrm{H}^{\circ}$ and ${ }^{6} \mathrm{~F}^{\circ}$ levels given here are from Axe and Dieke, who derived them from corresponding experimental sublevels of $\mathrm{Dy}^{3+}$ in $\mathrm{LaCl}_{3}$ [Crosswhite and Dieke; Dieke]. The levels are adjusted to a value of zero for the ground level, and rounded off to the nearest $10 \mathrm{~cm}^{-1}$. The ${ }^{6} \mathrm{H}_{9 / 2}^{\circ}$ and ${ }^{6} \mathrm{~F}_{11 / 2}^{\circ}$ levels, which are not given here, are both near $7600 \mathrm{~cm}^{-1}$; the Stark components of these levels overlap, and Axe and Dieke did not derive free-ion values. No transitions to the ${ }^{6} \mathrm{~F}_{1 / 2}^{\circ}$ level have been observed. Dieke gives additional levels to above $26000 \mathrm{~cm}^{-1}$, and the levels obtained by Carnall, Fields, and Rajnak [1968] from solution absorption spectra extend to $41700 \mathrm{~cm}^{-1}$.

Wybourne [1962] and Carnall et al. [1968] have published the results of calculations for Dy IV $4 f^{9}$. The eigenvector percentages given here are from an unpublished calculation by Carnall et al. [1974].

Sugar and Reader [1973] obtained a value of $334500 \pm 1600 \mathrm{~cm}^{-1}$ for the ionization energy. We have rounded the value to three significant figures, on the low side of the former value, based on the results obtained for Tb IV by Spector and Sugar [1976]. The estimated error has been approximately doubled, as suggested by Spector and Sugar.

\section{References}

Axe, J. D., and Dieke, G. H., J. Chem. Phys. 37, 2364 (1962). [EL] PT

Carnall, W. T., Fields, P. R., and Rajnak, K., J. Chem. Phys. 49, 4424 (1968). [EL] [CL] [W]

Carnall, W. T., Fields, P. R., and Rajnak, K., unpublished material (1974). ND PT

Crosswhite, H. M., and Dieke, G. H., J. Chem. Phys. 35, 1535 (1961). [EL] ND [CL] [ZE]

Dieke, G. H., Spectra and Energy Levels of Rare Earth Ions in Crystals, Ed. H. M. Crosswhite and H. Crosswhite, pp. 268-279 (Interscience Publishers, New York, 1968). [EL] ND [CL] [W] [ZE]

Hussain, R., Thesis, Johns Hopkins Univ., Baltimore, 142 pp. (1973); (Univ. Microfilms, Ann Arbor, Mich., No. 7410419). EL CL W PT

Spector, N., and Sugar, J., J. Opt. Soc. Am. 66, 436 (1976).

Sugar, J., and Reader, J., J. Chem. Phys. 59, 2083 (1973). IP

Wybourne, B. G., J. Chem. Phys. 36, 2301 (1962). PT

[July 1976] 
[Dy IV]

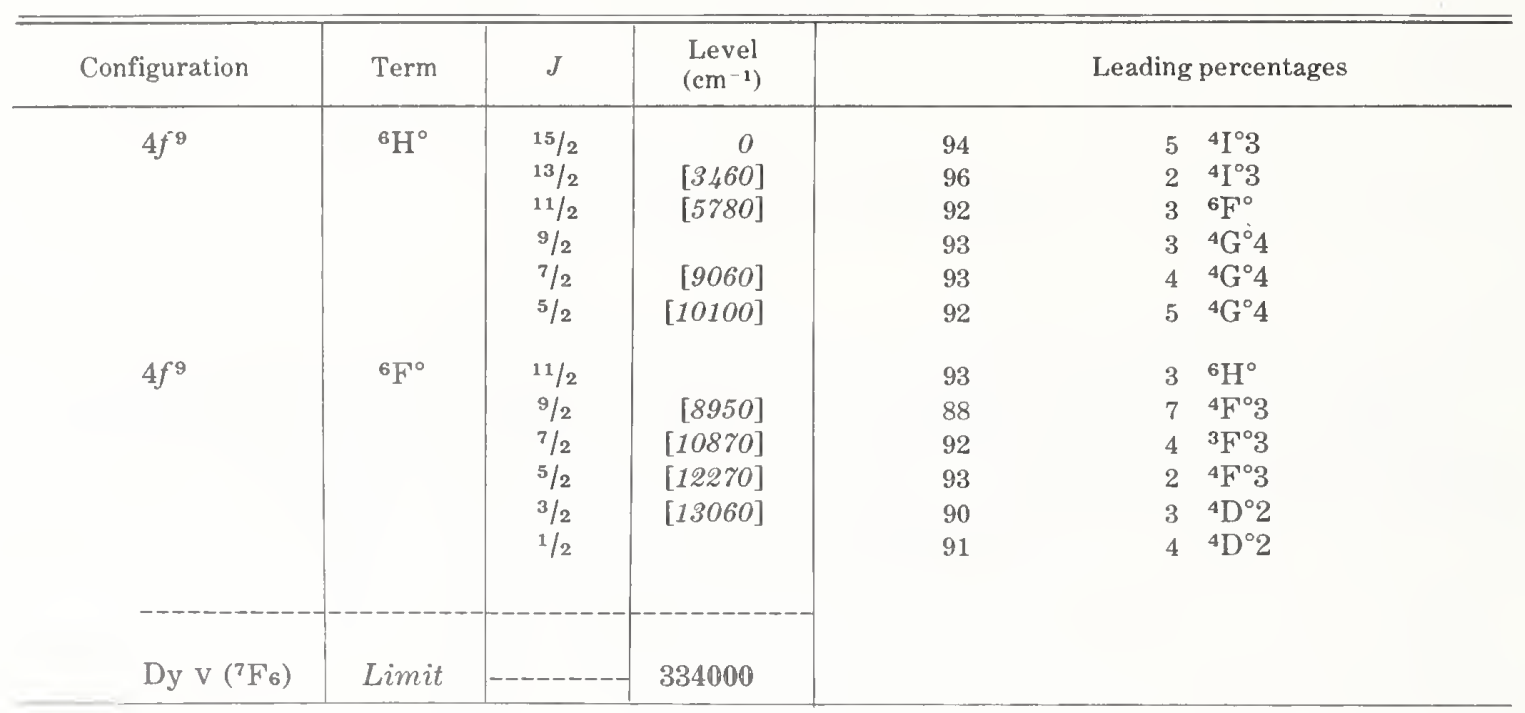




\section{Dy XXXIX}

(Ni I sequence; 28 electrons)

$Z=66$

Ground state $\left(1 s^{2} 2 s^{2} 2 p^{6} 3 s^{2} 3 p^{6}\right) 3 d^{10}{ }^{1} \mathrm{~S}_{0}$

Burkhalter, Nagel, and Whitlock excited the spectrum of this ion in the 5-15 $\AA$ region by focusing light from a pulsed laser onto a Dy target. Using isoelectronic regularities and comparisons with calculated energies, they were able to classify the $3 d^{10}{ }^{1} \mathrm{~S}_{0}-3 d^{9} 4 p(J=1)$ and $3 d^{10}{ }^{1} \mathrm{~S}_{0}-3 d^{9} 4 f(J=1)$ transitions. We have converted the levels from units of eV to units of $10^{4}$ $\mathrm{cm}^{-1}$ (equivalent to $1.23985 \mathrm{eV}$ ).

The coupling in the excited configurations should be very similar in Gd XXXVII and Dy XxxIx; leading eigenvector percentages have been calculated for the Gd case (see Gd XXXVII).

\section{Reference}

Burkhalter, P. G., Nagel, D. J., and Whitlock, R. R., Phys. Rev. A 9, 2331 (1974). EL CL ,

[July 1976]

Dy Xxxix

\begin{tabular}{l|c|c|c}
\hline \hline \multicolumn{1}{c|}{ Configuration } & Term & $J$ & $\begin{array}{c}\text { Level } \\
\left(10^{4} \mathrm{~cm}^{-1}\right)\end{array}$ \\
\hline $3 d^{10}$ & ${ }^{10}$ & 0 & 0.0 \\
$3 d^{9}\left({ }^{2} \mathrm{D}_{3 / 2}\right) 4 p_{1 / 2}$ & $\left(3 / 2,{ }^{1} / 2\right)^{\circ}$ & 1 & 1004 \\
$3 d^{9}\left({ }^{2} \mathrm{D}_{5 / 2}\right) 4 p_{3 / 2}$ & $\left(5 / 2,{ }^{3} / 2\right)^{\circ}$ & 1 & 1019 \\
$3 d^{9}\left({ }^{2} \mathrm{D}_{3 / 2}\right) 4 p_{3 / 2}$ & $(3 / 2,3 / 2)^{\circ}$ & 1 & 1049 \\
$3 d^{9}\left({ }^{2} \mathrm{D}_{5 / 2}\right) 4 f_{5 / 2}$ & $(5 / 2,5 / 2)^{\circ}$ & 1 & $1207 ?$ \\
$3 d^{9}\left({ }^{2} \mathrm{D}_{5 / 2}\right) 4 f_{7 / 2}$ & $\left(5 / 2,{ }^{7} / 2\right)^{\circ}$ & 1 & 1247 \\
$3 d^{9}\left({ }^{2} \mathrm{D}_{3 / 2}\right) 4 f_{5 / 2}$ & $(3 / 2,5 / 2)^{\circ}$ & 1 & 1278 \\
\hline
\end{tabular}




\section{HOLMIUM}

\section{Ho I}

67 electrons

Ground state $\left(1 s^{2} 2 s^{2} 2 p^{6} 3 s^{2} 3 p^{6} 3 d^{10} 4 s^{2} 4 p^{6} 4 d^{10} 5 s^{2} 5 p^{6}\right) 4 f^{11} 6 s^{24} \mathrm{I}_{15 / 2}^{\circ}$

Ionization energy $48567 \pm 5 \mathrm{~cm}^{-1}$

$6.0216 \pm 0.0006 \mathrm{eV}$

Identified odd configurations

Identified even configurations

$$
4 f^{11} 6 s^{2}, 4 f^{10} 6 s^{2} 6 p, 4 f^{11} 5 d 6 s, 4 f^{10} 5 d 6 s 6 p, 4 f^{11} 6 s 7 s
$$

$$
4 f^{10} 5 d 6 s^{2}, 4 f^{10} 5 d^{2} 6 s, 4 f^{11} 6 s 6 p, 4 f^{11} 6 s 7 p
$$

\section{Levels, Ionization Energy, Wavelength Observations}

Extensive data on the level structure of most neutral rare-earth atoms had been obtained before the analysis of Ho I by Blaise, Camus, Guelachvili, Verges, and Wyart [1972a, 1972b]. The remarkable achievement of these authors in identifying at least six of the above configurations very early in the analysis of this complex spectrum was no doubt aided by the available data from other spectra. The levels, identifications, and eigenvector percentages given here are from unpublished material communicated by Wyart [1975, 1976]; many new data from the continuing analysis are included, as well as some revised level values. Wyart [1976] notes that 20 of the even levels originally based on absorption lines [Blaise et al., 1972a] have been definitely confirmed by additional emission transitions. These levels are included here.

Worden, Conway, Paisner, and Solarz [1977] have observed high members of two series in Ho I by using laser techniques. These data became available too late for inclusion here, but the quoted ionization energy is a new determination from these series.

Meggers, Corliss, and Scribner list about 950 lines assigned to either Ho I or Ho II (2502$8916 \AA$ ), with Ho I classifications from the earlier lists of energy levels [Blaise et al., 1972a; 1972b]. Spector's [1977] list of 460 Ho lines in the near infrared $(0.759-1.2 \mu \mathrm{m})$ also has spectrum assignments to Ho I or Ho II. Unpublished infrared spectra obtained at Orsay (0.8$4.2 \mu \mathrm{m}$ ) have yielded many resolved hyperfine structures [Camus, Verges, and Wyart, 1975]. The absorption spectrum was photographed at Argonne over the range 2400-4000 [unpublished data mentioned by Blaise et al., 1972a].

\section{$g$ Values}

The $g$ value for the ground level is from the atomic-beam magnetic-resonance data of Haberstroh, Moran, and Penselin [1972], rounded off to five places. Dankwort and Ferch [1974] have reevaluated these measurements to obtain a value of $1.1951429 \pm 0.0000025$. The original determinations of the $g$ and $J$ values of this level were consistent with the $4 f^{11} 6 s^{2}{ }^{4} I_{15 / 2}^{\circ}$ designation [Childs and Goodman, 1961; Cabezas, Lindgren, and Marrus, 1961; Goodman, Kopfermann, and Schlüpmann, 1962]. Livingston and Pinnington [1971] tentatively classified a transition to the ground level on the basis of their optical Zeeman-effect data. We have listed $g$ values for two other levels of the ground term and for six upper levels, as derived from these data and the known levels. More recent observations of the Zeeman effect by Camus and Fred at Argonne have not been published. 


\section{Interpretation of Even Levels}

Some levels of the low even configurations $4 f^{10} 5 d 6 s^{2}$ and $4 f^{11} 6 s 6 p$ are designated in the $J_{1} j$ and $J_{1} J_{2}$ coupling schemes, respectively [Blaise et al., 1972a; 1972b; Wyart, 1973; 1975; 1976]. No eigenvector percentages are given in these schemes, but leading percentages in $L S$ coupling from Wyart's [1975] calculation of $\left(4 f^{10} 5 d 6 s^{2}+4 f^{11} 6 s 6 p\right)$ are listed for most of these levels. No $L S$ percentages from $4 f^{11}\left({ }^{4} I^{\circ}\right) 6 s 6 p$ quartet terms are given, since Wyart did not use the $6 s 6 p\left({ }^{3} \mathrm{P}^{\circ},{ }^{1} \mathrm{P}^{\circ}\right)$ parentage scheme in his calculation. In some cases, total percentages from one or both configurations are given instead of term percentages. The $J_{1} j$-coupling percentages given by Wyart [1973] for the lower $4 f^{10} 5 d 6 s^{2}$ levels are not listed because the earlier calculation did not include interaction with $4 f^{11} 6 s 6 \mathrm{p}$.

Wyart [1975] calculated the $4 f^{10}\left({ }^{5} \mathrm{I}\right) 5 d^{2} 6 s$ group of levels separately, with matrices truncated to include only this subconfiguration. The twelve known levels of $4 f^{11} 6 s 7 p$ are arranged into four complete $4 f^{11} 6 s\left(J_{1}\right) 7 p(j)$ terms, built on the lowest two levels of Ho II $\left(J_{1}=\right.$ $8,7)$ [Wyart, 1975]. We list the two $J_{1}$ parent levels as belonging to the $4 f^{11}\left({ }^{4} \mathrm{I}_{15 / 2}^{\circ}\right) 6 \mathrm{~s}(15 / 2,1 / 2)_{8,7}^{\circ}$ ground term of Ho II, in accordance with the coupling in this parent configuration (see Ho II).

\section{Interpretation of Odd Levels}

Aside from the $4 f^{11} 6 s^{2}$ ground configuration, the most important configurations in accounting for the known odd levels are $4 f^{10} 6 s^{2} 6 p, 4 f^{10} 5 d 6 s 6 p$, and $4 f^{11} 5 d 6 s$. The eigenvector percentages are from Wyart's [1976] calculation of $\left(4 f^{10} 6 s^{2} 6 p+4 f^{11} 5 d 6 s\right)$; no detailed interpretation of the $4 f^{10} 5 d 6 s 6 p$ levels has yet been made. A calculation including the interactions between all three configurations may be needed. The $4 f^{10} 6 s^{2} 6 p$ levels are designated in $J_{1} j$ coupling [Blaise et al., 1972b; Spector, 1974; Wyart, 1976], with the leading percentages in $L S$ coupling listed after the word "or."

\section{References}

Belyanin, V. B., Opt. Spektrosk. 3, 322 (1957). Hfs

Blaise, J., Camus, P., Guelachvili, G., Verges, J., and Wyart, J. F., C. R. Acad. Sci. Ser. B 274, 1302 (1972a). EL PT Blaise, J., Camus, P., Guelachvili, G., Verges, J., and Wyart, J. F., C. R. Acad. Sci. Ser. B 275, 81 (1972b). EL ND PT Cabezas, A. Y., Lindgren, I., and Marrus, R., Phys. Rev. 122, 1796 (1961). ZE Hfs

Camus, P., Verges, J., and Wyart, J. F., unpublished material (1975). CL W Hfs

Childs, W. J., and Goodman, L. S., Phys. Rev. 122, 591 (1961). ZE

Dankwort, W., and Ferch, J., Z. Phys. 267, 239 (1974). ZE Hfs

Goodman, L. S., Kopfermann, H., and Schlüpmann, K., Naturwiss. 49, 101 (1962). Hfs and $J$ value

Goodman, L. S., and Schlïpmann, K., Z. Phys. 178, 235 (1964). ZE Hfs

Haberstroh, R. A., Moran, T. I., and Penselin, S., Z. Phys. 252, 421 (1972). ZE Hfs

King, A. S., Astrophys. J. 72, 221 (1930). W

Livingston, A. E., Jr., and Pinnington, E. H., J. Opt. Soc. Am. 61, 1429 (1971). ZE

Meggers, W. F., Corliss, C. H., and Scribner, B. F., Tables of Spectral-Line Intensities, Nat. Bur. Stand. (U.S.), Mono. 145, Part I, 403 pp. (1975). CL W

Spector, N., Phys. Scr. 9, 313 (1974). ND

Spector, N., Astrophys. J. 211, 600 (1977). W

Worden, E. F., Conway, J. G., Paisner, J. A., and Solarz, R. W., unpublished material (1977). IP

Wyart, J. F., Thesis, Univ, Paris-Sud, Orsay, 194 pp. (1973). PT

Wyart, J. F., unpublished material $(1975,1976)$. EL ND PT

[October 1976] 
Ho I, Odd Parity

\begin{tabular}{|c|c|c|c|c|c|c|c|c|}
\hline Configuration & Term & $J$ & $\begin{array}{l}\text { Level } \\
\left(\mathrm{cm}^{-2}\right)\end{array}$ & $g$ & \multicolumn{4}{|c|}{ Leading percentages } \\
\hline $4 f^{11} 6 s^{2}$ & ${ }^{4} I^{\circ}$ & $\begin{array}{l}15 / 2 \\
13 / 2 \\
11 / 2 \\
9 / 2\end{array}$ & $\begin{array}{r}0.00 \\
5419.70 \\
8605.16 \\
10695.75\end{array}$ & $\begin{array}{l}1.19514 \\
1.012 \\
0.866\end{array}$ & $\begin{array}{l}97 \\
99 \\
86 \\
61\end{array}$ & & $\begin{array}{r}3 \\
1 \\
12 \\
16\end{array}$ & $\begin{array}{l}{ }^{2} \mathrm{~K}^{\circ} \\
{ }^{2} \mathrm{~K}^{\circ} \\
{ }^{2} \mathrm{H}^{\circ} 2 \\
{ }^{2} \mathrm{H}^{\circ} 2\end{array}$ \\
\hline $\left.4 f^{10(5} \mathrm{I}_{8}\right) 6 s^{2} 6 p_{1 / 2}$ & $\left(8,{ }^{1} / 2\right)^{\circ}$ & $\begin{array}{l}15 / 2 \\
17 / 2\end{array}$ & $\begin{array}{l}18572.28 \\
18737.79\end{array}$ & & & $\begin{array}{l}\text { or } \\
\text { or }\end{array}$ & $\begin{array}{l}57 \\
42\end{array}$ & $\begin{array}{l}\left({ }^{5} \mathrm{I}\right){ }^{6} \mathrm{H}^{\circ} \\
\left({ }^{5} \mathrm{I}\right)^{6} \mathrm{I}^{\circ}\end{array}$ \\
\hline $4 f^{11}\left({ }^{4} \mathrm{I}^{\circ}\right) 5 d 6 s\left({ }^{3} \mathrm{D}\right)$ & ${ }^{6} \mathrm{G}^{\circ}$ & $13 / 2$ & 18867.40 & & 82 & & 13 & $\left({ }^{4} \mathrm{I}^{\circ}\right)\left({ }^{3} \mathrm{D}\right){ }^{6} \mathrm{H}^{0}$ \\
\hline $4 f^{11}\left({ }^{4} \mathrm{I}^{\circ}\right) 5 d 6 s\left({ }^{3} \mathrm{D}\right)$ & ${ }^{6} \mathrm{H}^{-}$ & $15 / 2$ & 19276.94 & & 82 & & 11 & $\left({ }^{4} I^{\circ}\right)\left({ }^{3} \mathrm{D}\right){ }^{6} \mathrm{I}^{\circ}$ \\
\hline $4 f^{11}\left({ }^{4} \mathrm{I}^{\circ}\right) 5 d 6 s\left({ }^{3} \mathrm{D}\right)$ & & $11 / 2$ & 20060.76 & & 37 & ${ }^{4} \mathrm{G}^{\circ}$ & 30 & $\left({ }^{4} \mathrm{I}^{\circ}\right)\left({ }^{3} \mathrm{D}\right){ }^{6} \mathrm{G}^{\circ}$ \\
\hline $4 f^{11}\left({ }^{4} \mathrm{I}^{\circ}\right) 5 d 6 s\left({ }^{3} \mathrm{D}\right)$ & & $19 / 2$ & 20124.25 & & 37 & ${ }^{6} \mathrm{~L}^{\circ}$ & 31 & $\left({ }^{4} \mathrm{I}^{\circ}\right)\left({ }^{3} \mathrm{D}\right){ }^{4} \mathrm{~L}^{\circ}$ \\
\hline $4 f^{11}\left({ }^{4} \mathrm{I}^{\circ}\right) 5 d 6 s\left({ }^{3} \mathrm{D}\right)$ & ${ }^{6} \mathrm{~L}^{0}$ & $21 / 2$ & 20210.60 & & 97 & & 3 & $\left({ }^{2} \mathrm{~K}^{\circ}\right)\left({ }^{3} \mathrm{D}\right){ }^{4} \mathrm{M}^{\circ}$ \\
\hline $4 f^{11}\left({ }^{4} \mathrm{I}^{\circ}\right) 5 d 6 s\left({ }^{3} \mathrm{D}\right)$ & & $17 / 2$ & 20315.89 & & 25 & ${ }^{4} \mathrm{~L}^{\circ}$ & 23 & $\left({ }^{4} \mathrm{I}^{\circ}\right)\left({ }^{3} \mathrm{D}\right){ }^{6} \mathrm{~K}^{\circ}$ \\
\hline $4 f^{11}\left({ }^{4} \mathrm{I}^{\circ}\right) 5 d 6 s\left({ }^{3} \mathrm{D}\right)$ & & $13 / 2$ & 20493.77 & & 35 & ${ }^{4} \mathrm{H}^{\circ}$ & 23 & $\left({ }^{4} \mathrm{I}^{\circ}\right)\left({ }^{3} \mathrm{D}\right){ }^{6} \mathrm{H}^{\circ}$ \\
\hline $4 f^{11}\left({ }^{4} \mathrm{I}^{\circ}\right) 5 d 6 s\left({ }^{3} \mathrm{D}\right)$ & & $17 / 2$ & 20613.81 & & 34 & ${ }^{6} \mathrm{I}^{\circ}$ & 27 & $\left({ }^{4} \mathrm{I}^{\circ}\right)\left({ }^{3} \mathrm{D}\right){ }^{2} \mathrm{~L}^{\circ}$ \\
\hline $4 f^{11}\left({ }^{4} \mathrm{I}^{\circ}\right) 5 d 6 s\left({ }^{3} \mathrm{D}\right)$ & ${ }^{6} \mathrm{~K}^{\circ}$ & $19 / 2$ & 20831.18 & & 60 & & 31 & $\left({ }^{4} \mathrm{I}^{0}\right)\left({ }^{3} \mathrm{D}\right){ }^{4} \mathrm{~L}^{0}$ \\
\hline $4 f^{11}\left({ }^{4} \mathrm{I}^{\circ}\right) 5 d 6 s\left({ }^{3} \mathrm{D}\right)$ & & $15 / 2$ & 21069.22 & & 27 & ${ }^{4} \mathrm{~K}^{0}$ & 18 & $\left({ }^{4} \mathrm{I}^{\circ}\right)\left({ }^{3} \mathrm{D}\right){ }^{2} \mathrm{~K}^{\circ}$ \\
\hline $\left.4 f^{10(5} \mathrm{I}_{8}\right) 6 s^{2} 6 p_{3 / 2}$ & $(8,3 / 2)^{\circ}$ & $\begin{array}{l}17 / 2 \\
19 / 2 \\
15 / 2 \\
13 / 2\end{array}$ & $\begin{array}{l}21378.54 \\
21485.02 \\
21682.90 \\
22024.20\end{array}$ & & & $\begin{array}{l}\text { or } \\
\text { or } \\
\text { or } \\
\text { or }\end{array}$ & $\begin{array}{l}38 \\
91 \\
48 \\
52\end{array}$ & $\begin{array}{l}\left({ }^{5} \mathrm{I}\right){ }^{6} \mathrm{I}^{\circ} \\
\left({ }^{5} \mathrm{I}\right){ }^{6} \mathrm{~K}^{\circ} \\
\left({ }^{5} \mathrm{I}\right){ }^{4} \mathrm{I}^{\circ} \\
\left({ }^{5} \mathrm{I}\right){ }^{4} \mathrm{H}^{\circ}\end{array}$ \\
\hline $4 f^{11}\left({ }^{4} \mathrm{I}^{0}\right) 5 d 6 s\left({ }^{3} \mathrm{D}\right)$ & & $17 / 2$ & 21552.02 & & 34 & ${ }^{4} \mathrm{~K}^{\circ}$ & 28 & $\left({ }^{4} \mathrm{I}^{\circ}\right)\left({ }^{3} \mathrm{D}\right){ }^{6} \mathrm{I}^{\circ}$ \\
\hline $4 f^{11}\left({ }^{4} \mathrm{I}^{\circ}\right) 5 d 6 s\left({ }^{3} \mathrm{D}\right)$ & & $15 / 2$ & 22014.13 & & 30 & ${ }^{4} \mathrm{I}^{\circ}$ & 27 & $\left({ }^{4} \mathrm{I}^{\circ}\right)\left({ }^{3} \mathrm{D}\right){ }^{2} \mathrm{~K}^{\circ}$ \\
\hline $4 f^{11}\left({ }^{4} \mathrm{I}^{\circ}\right) 5 d 6 s\left({ }^{3} \mathrm{D}\right)$ & & $9 / 2$ & 22157.88 & & 36 & ${ }^{4} \mathrm{G}^{\circ}$ & 31 & $\left({ }^{4} \mathrm{I}^{\circ}\right)\left({ }^{3} \mathrm{D}\right){ }^{2} \mathrm{G}^{\circ}$ \\
\hline $4 f^{11}\left({ }^{4} \mathrm{I}^{\circ}\right) 5 d 6 s\left({ }^{3} \mathrm{D}\right)$ & & $13 / 2$ & 22392.88 & & 36 & ${ }^{2} \mathrm{I}^{\circ}$ & 25 & $\left({ }^{4} \mathrm{I}^{\circ}\right)\left({ }^{3} \mathrm{D}\right){ }^{4} \mathrm{I}^{\circ}$ \\
\hline $4 f^{11}\left({ }^{4} I^{\circ}\right) 5 d 6 s\left({ }^{3} \mathrm{D}\right)$ & & $11 / 2$ & 22518.59 & & 28 & ${ }^{2} \mathrm{H}^{\circ}$ & 28 & $\left({ }^{4} \mathrm{I}^{\circ}\right)\left({ }^{3} \mathrm{D}\right){ }^{4} \mathrm{H}^{\circ}$ \\
\hline $4 f^{11}\left({ }^{4} \mathrm{G}^{\circ}\right) 6 s^{2}$ & ${ }^{4} \mathrm{G}^{0}$ & $11 / 2$ & 22593.53 & & 55 & & 32 & ${ }^{2} \mathrm{H}^{\circ} 2$ \\
\hline $4 f^{11}\left({ }^{4} \mathrm{I}^{\circ}\right) 5 d 6 s\left({ }^{1} \mathrm{D}\right)$ & & $13 / 2$ & 23585.31 & & 38 & ${ }^{4} \mathrm{H}^{\circ}$ & 15 & $4 f^{10}\left({ }^{5} \mathrm{I}\right) 6 s^{2} 6 p^{6} \mathrm{H}^{\circ}$ \\
\hline $4 f^{10}\left({ }^{5} \mathrm{I}_{7}\right) 6 s^{2} 6 p_{1 / 2}$ & $(7,1 / 2)^{\circ}$ & $\begin{array}{l}15 / 2 \\
13 / 2\end{array}$ & $\begin{array}{c}23818.54 \\
23942.71 \\
-\end{array}$ & & & $\begin{array}{l}\text { or } \\
\text { or }\end{array}$ & $\begin{array}{l}31 \\
26\end{array}$ & $\begin{array}{l}\left({ }^{5} \mathrm{I}\right){ }^{6} \mathrm{~K}^{\circ} \\
\left({ }^{5} \mathrm{I}\right){ }^{4} \mathrm{H}^{\circ}\end{array}$ \\
\hline $4 f^{11}\left({ }^{4} I^{0}\right) 5 d 6 s\left({ }^{1} \mathrm{D}\right)$ & ${ }^{4} \mathrm{~K}^{\circ}$ & $17 / 2$ & 23885.74 & & 89 & & 6 & $\left({ }^{4} \mathrm{I}^{0}\right)\left({ }^{1} \mathrm{D}\right){ }^{4} \mathrm{~L}^{\circ}$ \\
\hline $\left.4 f^{10(5} \mathrm{I}\right) 5 d 6 s 6 p$ & & $15 / 2$ & 24112.04 & & & & & \\
\hline $4 f^{10}\left({ }^{5} \mathrm{I}\right) 5 d 6 s 6 p$ & & $17 / 2$ & 24115.17 & & & & & \\
\hline $4 f^{11}\left({ }^{4} \mathrm{I}^{\circ}\right) 5 d 6 s\left({ }^{1} \mathrm{D}\right)$ & ${ }^{4} I^{\circ}$ & $15 / 2$ & 24357.90 & & 80 & & 10 & $\left({ }^{4} \mathrm{I}^{\circ}\right)\left({ }^{1} \mathrm{D}\right){ }^{4} \mathrm{~K}^{\circ}$ \\
\hline $\left.4 f^{10(5} \mathrm{I}\right) 5 d 6 s 6 p$ & & $13 / 2$ & 24594.75 & & & & & \\
\hline $4 f^{10}(5) 5 d 6 s 6 p$ & & $19 / 2$ & 24678.25 & & & & & \\
\hline $4 f^{11}\left({ }^{4} I^{\circ}\right) 5 d 6 s\left({ }^{3} \mathrm{D}\right)$ & & $15 / 2$ & 25591.43 & & 37 & ${ }^{4} \mathrm{~L}^{\circ}$ & 37 & $\left({ }^{4} \mathrm{I}^{\circ}\right)\left({ }^{3} \mathrm{D}\right){ }^{6} \mathrm{~L}^{0}$ \\
\hline $4 f^{11}\left({ }^{4} I^{\circ}\right) 5 d 6 s\left({ }^{3} \mathrm{D}\right)$ & & $11 / 2$ & 25616.46 & & 44 & ${ }^{4} \mathrm{G}^{\circ}$ & 17 & $\left({ }^{4} \mathrm{I}^{\circ}\right)\left({ }^{3} \mathrm{D}\right){ }^{6} \mathrm{G}^{\circ}$ \\
\hline $4 f^{11}\left({ }^{4} \mathrm{I}^{\circ}\right) 5 d 6 s\left({ }^{3} \mathrm{D}\right)$ & ${ }^{6} \mathrm{~L}^{\circ}$ & $17 / 2$ & 25660.40 & & 58 & & 21 & $\left({ }^{4} \mathrm{I}^{0}\right)\left({ }^{3} \mathrm{D}\right){ }^{2} \mathrm{~L}^{\circ}$ \\
\hline $4 f^{11}\left({ }^{4} \mathrm{I}^{\circ}\right) 5 d 6 s\left({ }^{3} \mathrm{D}\right)$ & & $9 / 2$ & 25805.04 & & 34 & ${ }^{2} \mathrm{G}^{\circ}$ & 33 & $\left({ }^{4} \mathrm{I}^{\circ}\right)\left({ }^{3} \mathrm{D}\right){ }^{6} \mathrm{G}^{\circ}$ \\
\hline $\left.4 f^{10(5} \mathrm{I}\right) 5 d 6 s 6 p$ & & $21 / 2$ & 25980.35 & & & & & \\
\hline $4 f^{10}\left({ }^{5} \mathrm{I}\right) 5 d 6 s 6 p$ & & $15 / 2$ & 26023.52 & & & & & \\
\hline $4 f^{10}\left({ }^{5} \mathrm{I}\right) 5 d 6 s 6 p$ & & $13 / 2$ & 26127.22 & & & & & \\
\hline $4 f^{10}\left({ }^{5} \mathrm{I}\right) 5 d 6 s 6 p$ & & $17 / 2$ & 26143.57 & & & & & \\
\hline $4 f^{11}\left({ }^{4} \mathrm{I}^{\circ}\right) 5 d 6 s\left({ }^{3} \mathrm{D}\right)$ & & $15 / 2$ & 26198.39 & & 37 & ${ }^{6} \mathrm{~K}^{\circ}$ & 28 & $\left({ }^{4} \mathrm{I}^{\circ}\right)\left({ }^{3} \mathrm{D}\right){ }^{4} \mathrm{I}^{\circ}$ \\
\hline $4 f^{11}\left({ }^{4} \mathrm{I}^{\circ}\right) 5 d 6 s\left({ }^{3} \mathrm{D}\right)$ & & $13 / 2$ & 26225.76 & & 24 & ${ }^{6} \mathrm{~K}^{\circ}$ & 17 & $\left({ }^{4} \mathrm{~J}^{\circ}\right)\left({ }^{3} \mathrm{D}\right){ }^{4} \mathrm{H}^{\circ}$ \\
\hline $4 f^{11}\left({ }^{4} \mathrm{I}^{\circ}\right) 5 d 6 s\left({ }^{3} \mathrm{D}\right)$ & & $13 / 2$ & 25288.10 & & 26 & ${ }^{4} \mathrm{H}^{\circ}$ & 16 & $\left({ }^{4} \mathrm{I}^{\circ}\right)\left({ }^{3} \mathrm{D}\right){ }^{6} \mathrm{H}^{\circ}$ \\
\hline
\end{tabular}


Ho I, Odd Parity-Continued

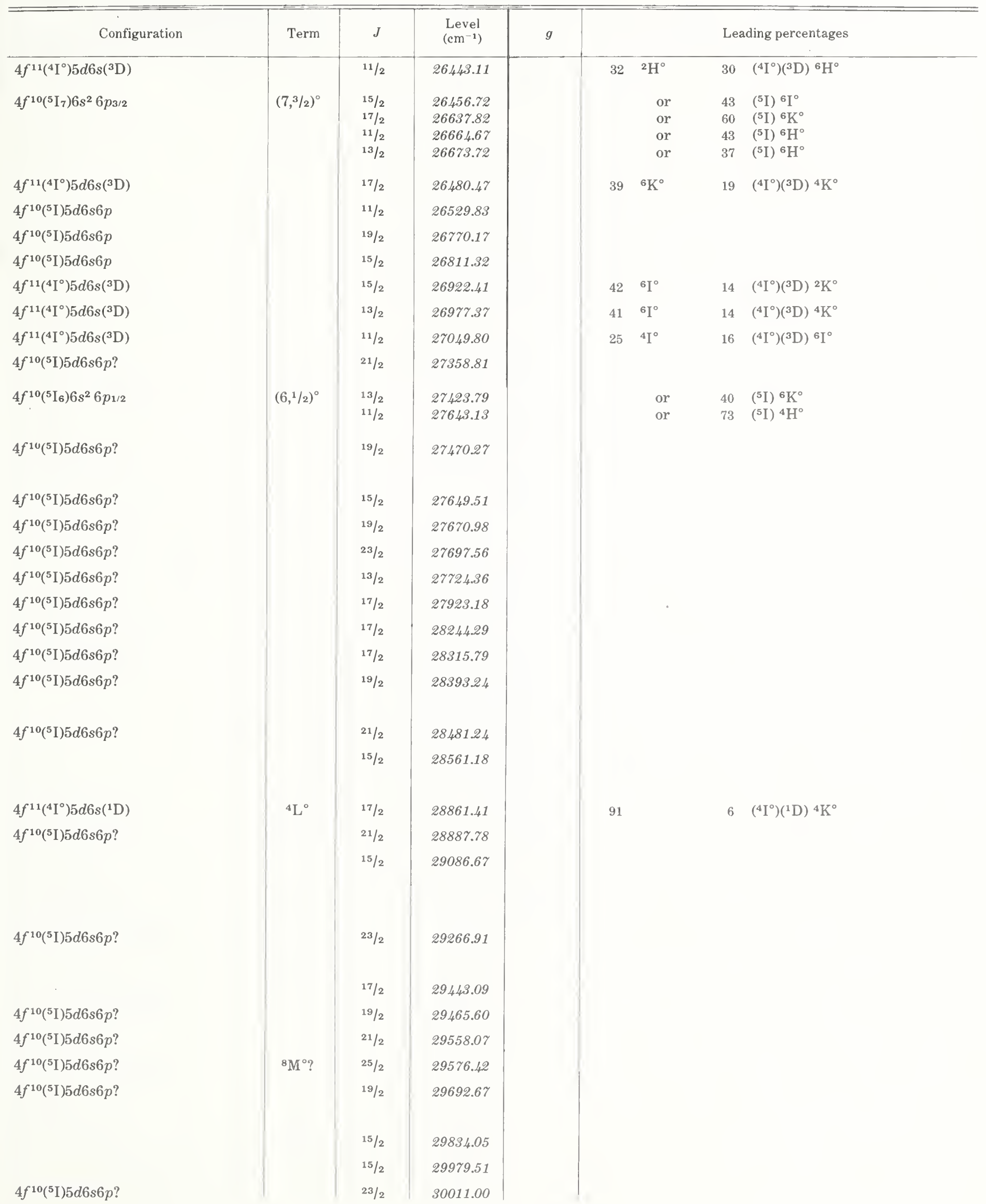


Ho I, Odd Parity_Continued

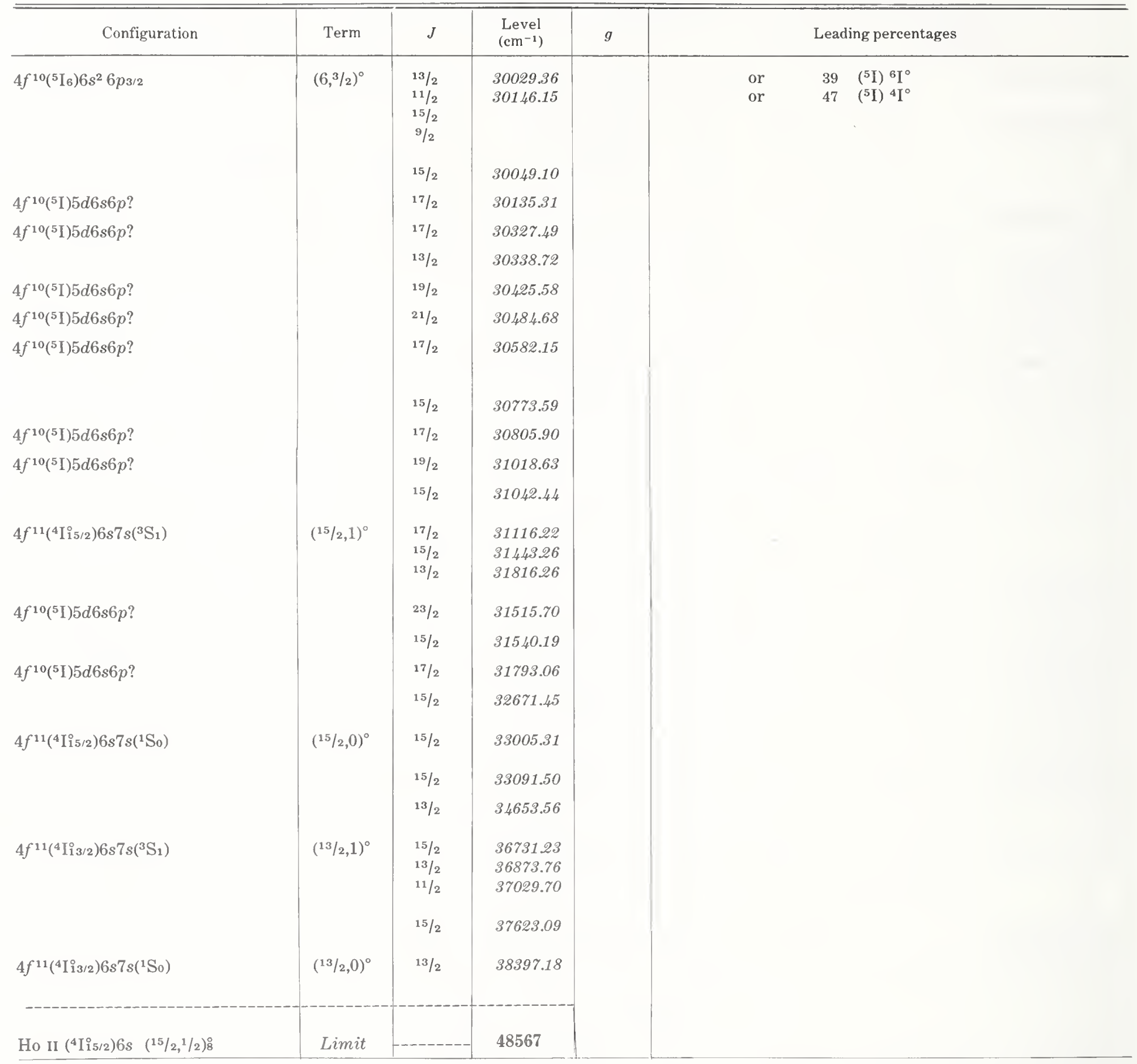


Ho I, Even Parity

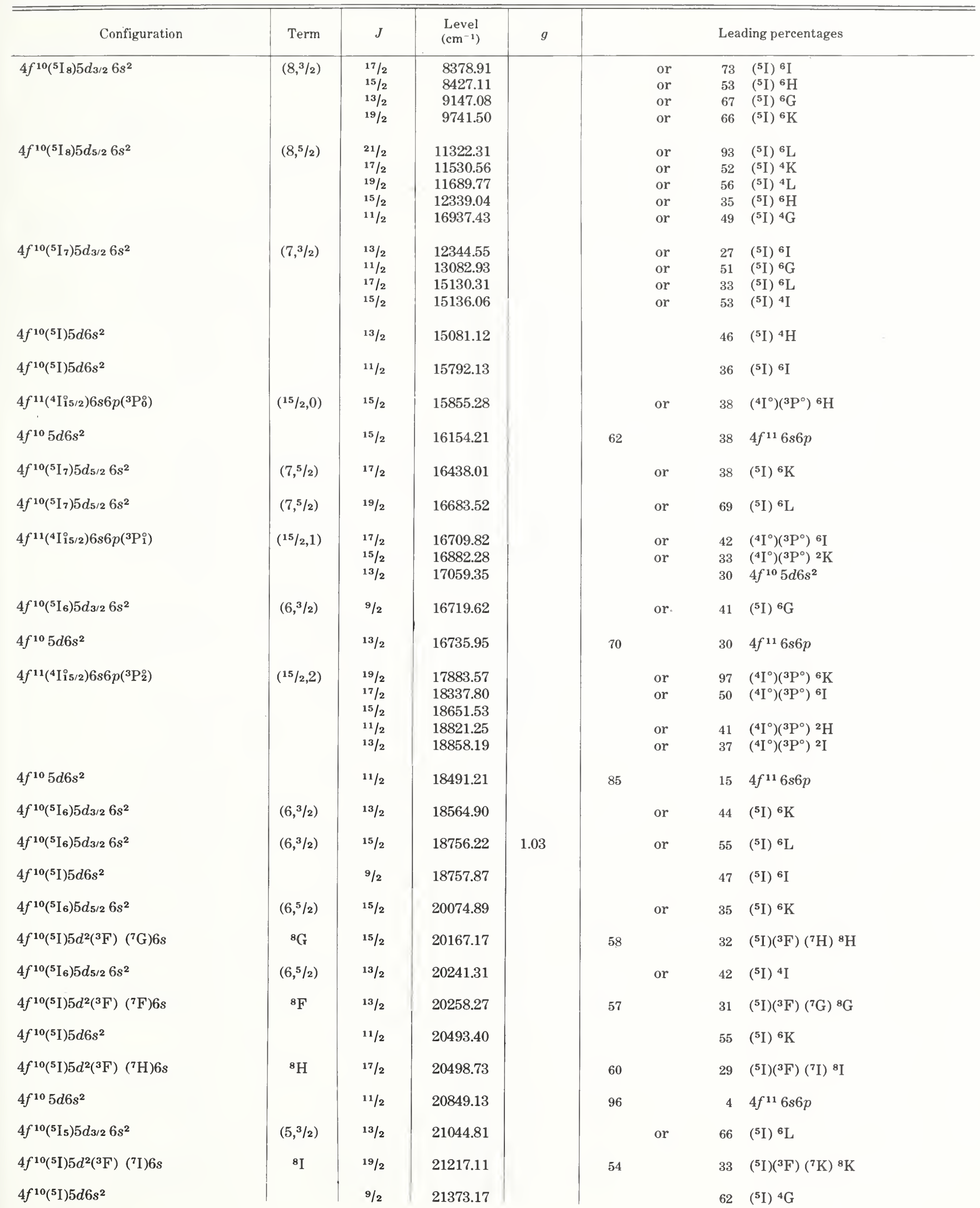


Ho I, Even Parity-Continued

\begin{tabular}{|c|c|c|c|c|c|c|c|c|}
\hline Configuration & Term & $J$ & $\begin{array}{l}\text { Level } \\
\left(\mathrm{cm}^{-1}\right)\end{array}$ & $g$ & & & \multicolumn{2}{|c|}{ Leading percentages } \\
\hline $4 f^{10}\left({ }^{5} \mathrm{I}\right) 5 d^{2}\left({ }^{3} \mathrm{~F}\right)\left({ }^{7} \mathrm{~K}\right) 6 s$ & ${ }^{8} \mathrm{~K}$ & $21 / 2$ & 21494.89 & & 56 & & 34 & $\left({ }^{5} \mathrm{I}\right)\left({ }^{3} \mathrm{~F}\right)\left({ }^{7} \mathrm{~L}\right){ }^{8} \mathrm{~L}$ \\
\hline $4 f^{11}\left({ }^{4} \mathrm{I}_{13 / 2}\right) 6 s 6 p\left({ }^{3} \mathrm{P}_{0}^{\circ}\right)$ & $(13 / 2,0)$ & $13 / 2$ & 21584.89 & & & & & \\
\hline $4 f^{10}\left({ }^{5} \mathrm{I}\right) 5 d^{2}\left({ }^{3} \mathrm{~F}\right)\left({ }^{7} \mathrm{~L}\right) 6 \mathrm{~s}$ & ${ }^{8} \mathrm{~L}$ & $23 / 2$ & 21980.67 & & 84 & & 14 & $\left({ }^{5} \mathrm{I}\right)\left({ }^{3} \mathrm{~F}\right)\left({ }^{7} \mathrm{M}\right){ }^{8} \mathrm{M}$ \\
\hline $4 f^{11}\left({ }^{4} \mathrm{I}_{13 / 2}^{\circ}\right) 6 s 6 p\left({ }^{3} \mathrm{P}_{1}^{\circ}\right)$ & $(13 / 2,1)$ & $\begin{array}{l}13 / 2 \\
15 / 2 \\
11 / 2\end{array}$ & $\begin{array}{l}22157.86 \\
22227.34 \\
22413.14\end{array}$ & & & or & 43 & $\left({ }^{4} \mathrm{I}^{\circ}\right)\left({ }^{3} \mathrm{P}^{\circ}\right){ }^{6} \mathrm{~K}$ \\
\hline $4 f^{10}\left({ }^{5} \mathrm{I}\right) 5 d^{2}\left({ }^{3} \mathrm{~F}\right)\left({ }^{7} \mathrm{~F}\right) 6 s$ & & $13 / 2$ & 22414.04 & & 24 & ${ }^{8} \mathrm{~F}$ & 20 & $\left({ }^{5} \mathrm{I}\right)\left({ }^{3} \mathrm{~F}\right)\left({ }^{7} \mathrm{H}\right){ }^{8} \mathrm{H}$ \\
\hline $4 f^{10}\left({ }^{5} \mathrm{I}\right) 5 d^{2}\left({ }^{3} \mathrm{~F}\right)\left({ }^{7} \mathrm{~K}\right) 6 s$ & & $17 / 2$ & 22476.89 & & 22 & ${ }^{8} \mathrm{~K}$ & 18 & $\left({ }^{5} \mathrm{I}\right)\left({ }^{3} \mathrm{~F}\right)\left({ }^{7} \mathrm{H}\right){ }^{8} \mathrm{H}$ \\
\hline $4 f^{10}\left({ }^{5} \mathrm{I}\right) 5 d^{2}\left({ }^{3} \mathrm{~F}\right) \quad\left({ }^{7} \mathrm{G}\right) 6 s$ & & $15 / 2$ & 22500.62 & & 21 & ${ }^{8} \mathrm{G}$ & 20 & $\left({ }^{5} I\right)\left({ }^{3} F\right)\left({ }^{7} I\right){ }^{8} I$ \\
\hline $4 f^{10}\left({ }^{5} \mathrm{I}\right) 5 d^{2}\left({ }^{3} \mathrm{~F}\right)\left({ }^{7} \mathrm{I}\right) 6 s$ & & $19 / 2$ & 22866.55 & & 25 & ${ }^{8} \mathrm{I}$ & 20 & $\left({ }^{5} \mathrm{I}\right)\left({ }^{3} \mathrm{~F}\right)\left({ }^{7} \mathrm{~L}\right){ }^{8} \mathrm{~L}$ \\
\hline $4 f^{10}\left({ }^{5} \mathrm{I}_{5}\right) 5 d_{5 / 2} 6 s^{2}$ & $(5,5 / 2)$ & $13 / 2$ & 22978.19 & & & or & 41 & $\left({ }^{5} \mathrm{I}\right){ }^{4} \mathrm{~K}$ \\
\hline $4 f^{10}\left({ }^{5} \mathrm{I}\right) 5 d^{2}\left({ }^{3} \mathrm{~F}\right)\left({ }^{7} \mathrm{~K}\right) 6 s$ & & $21 / 2$ & 23366.71 & & 27 & ${ }^{8} \mathrm{~K}$ & 17 & $\left({ }^{5} \mathrm{I}\right)\left({ }^{3} \mathrm{~F}\right)\left({ }^{5} \mathrm{~L}\right){ }^{6} \mathrm{~L}$ \\
\hline $4 f^{10}\left({ }^{5} \mathrm{I}\right) 5 d^{2}\left({ }^{3} \mathrm{~F}\right)\left({ }^{7} \mathrm{M}\right) 6 s$ & ${ }^{8} \mathrm{M}$ & $25 / 2$ & 23375.28 & & 100 & & & \\
\hline $4 f^{10}\left({ }^{5} I_{5}\right) 5 d_{5 / 2} 6 s^{2}$ & $(5,5 / 2)$ & $11 / 2$ & 23379.31 & & & or & 24 & $\left({ }^{5} \mathrm{I}\right){ }^{4} \mathrm{I}$ \\
\hline $4 f^{10}\left({ }^{5} I_{5}\right) 5 d_{5 / 2} 6 s^{2}$ & $(5,5 / 2)$ & $15 / 2$ & 23445.28 & 1.012 & & or & 59 & $\left({ }^{5} \mathrm{I}\right){ }^{4} \mathrm{~L}$ \\
\hline $4 f^{11}\left({ }^{4} \mathrm{I}_{13 / 2}\right) 6 s 6 p\left({ }^{3} \mathrm{P}_{2}^{\circ}\right)$ & $(13 / 2,2)$ & $\begin{array}{c}17 / 2 \\
15 / 2 \\
9 / 2 \\
11 / 2 \\
13 / 2 \\
7 / 2\end{array}$ & $\begin{array}{l}23498.57 \\
23834.94 \\
23861.17 \\
23946.16 \\
23955.69\end{array}$ & & & $\begin{array}{l}\text { or } \\
\text { or }\end{array}$ & $\begin{array}{l}59 \\
43\end{array}$ & $\begin{array}{l}\left({ }^{4} \mathrm{I}^{\circ}\right)\left({ }^{3} \mathrm{P}^{\circ}\right){ }^{6} \mathrm{~K} \\
\left({ }^{4} \mathrm{I}^{\circ}\right)\left({ }^{3} \mathrm{P}^{\circ}\right){ }^{6} \mathrm{I}\end{array}$ \\
\hline $4 f^{10}\left({ }^{5} \mathrm{I}\right) 5 d^{2}\left({ }^{3} \mathrm{~F}\right)\left({ }^{7} \mathrm{M}\right) 6 s$ & & $23 / 2$ & 24006.23 & & 41 & ${ }^{8} \mathrm{M}$ & 37 & $\left({ }^{5} \mathrm{I}\right)\left({ }^{3} \mathrm{~F}\right)\left({ }^{5} \mathrm{M}\right){ }^{6} \mathrm{M}$ \\
\hline $4 f^{11}\left({ }^{4} \mathrm{I}^{\circ} 5 / 2\right) 6 s 6 p\left({ }^{1} \mathrm{P}_{i}^{\circ}\right)$ & $(15 / 2,1)$ & $\begin{array}{l}13 / 2 \\
17 / 2 \\
15 / 2\end{array}$ & $\begin{array}{l}24014.22 \\
24360.81 \\
24660.80\end{array}$ & & & $\begin{array}{l}\text { or } \\
\text { or }\end{array}$ & & $\begin{array}{l}\left({ }^{4} I^{0}\right)(\quad)^{4} \mathrm{~K} \\
\left({ }^{4} I^{0}\right)(\quad)^{4} \mathrm{I}\end{array}$ \\
\hline $4 f^{10} 5 d 6 s^{2}$ & & $11 / 2$ & 24141.21 & & 95 & & 5 & $4 f^{11} 6 s 6 p$ \\
\hline $4 f^{10}\left({ }^{5} \mathrm{I}\right) 5 d^{2}\left({ }^{3} \mathrm{P}\right)\left({ }^{7} \mathrm{H}\right) 6 s$ & & $17 / 2$ & 24263.96 & & 27 & ${ }^{8} \mathrm{H}$ & 14 & $\left({ }^{5} \mathrm{I}\right)\left({ }^{3} \mathrm{~F}\right)\left({ }^{7} \mathrm{I}\right){ }^{8} \mathrm{I}$ \\
\hline $4 f^{10} 5 d 6 s^{2}$ & & $9 / 2$ & 24355.64 & & 96 & & 4 & $4 f^{11} 6 s 6 p$ \\
\hline $4 f^{10}\left({ }^{5} \mathrm{I}\right) 5 d^{2}\left({ }^{3} \mathrm{~F}\right)\left({ }^{7} \mathrm{H}\right) 6 s$ & & $15 / 2$ & 24376.91 & & 16 & ${ }^{8} \mathrm{H}$ & 10 & $\left({ }^{5} \mathrm{I}\right)\left({ }^{3} \mathrm{~F}\right)\left({ }^{5} \mathrm{~K}\right){ }^{6} \mathrm{~K}$ \\
\hline $4 f^{10}\left({ }^{5} \mathrm{I}\right) 5 d^{2}\left({ }^{3} \mathrm{~F}\right)\left({ }^{5} \mathrm{M}\right) 6 s$ & & $19 / 2$ & 24404.19 & & 12 & ${ }^{6} \mathrm{M}$ & 11 & $\left({ }^{5} \mathrm{I}\right)\left({ }^{3} \mathrm{~F}\right)\left({ }^{7} \mathrm{~K}\right){ }^{8} \mathrm{~K}$ \\
\hline $4 f^{10}\left({ }^{5} \mathrm{I}\right) 5 d^{2}\left({ }^{3} \mathrm{~F}\right)\left({ }^{5} \mathrm{M}\right) 6 s$ & & $21 / 2$ & 24714.34 & & 24 & ${ }^{6} \mathrm{M}$ & 14 & $\left({ }^{5} \mathrm{I}\right)\left({ }^{3} \mathrm{~F}\right)\left({ }^{3} \mathrm{M}\right){ }^{4} \mathrm{M}$ \\
\hline $4 f^{10} 5 d 6 s^{2}$ & & $13 / 2$ & 24740.52 & & 82 & & 18 & $4 f^{11} 6 s 6 p$ \\
\hline $4 f^{10}\left({ }^{5} \mathrm{I}\right) 5 d^{2}\left({ }^{3} \mathrm{~F}\right)\left({ }^{7} \mathrm{G}\right) 6 s$ & & $13 / 2$ & 24760.13 & & 18 & ${ }^{8} \mathrm{G}$ & 10 & $\left({ }^{5} \mathrm{I}\right)\left({ }^{3} \mathrm{~F}\right)\left({ }^{7} \mathrm{I}\right){ }^{8} \mathrm{I}$ \\
\hline $4 f^{10}\left({ }^{5} \mathrm{I}\right) 5 d^{2}\left({ }^{3} \mathrm{P}\right)\left({ }^{7} \mathrm{H}\right) 6 s$ & ${ }^{8} \mathrm{H}$ & $17 / 2$ & 24795.58 & & 64 & & 6 & $\left({ }^{5} \mathrm{I}\right)\left({ }^{3} \mathrm{~F}\right)\left({ }^{7} \mathrm{H}\right){ }^{8} \mathrm{H}$ \\
\hline $4 f^{10} 5 d 6 s^{2}$ & & $11 / 2$ & 25261.55 & 0.90 & 63 & & 37 & $4 f^{11} 6 s 6 p$ \\
\hline $4 f^{10} 5 d 6 s^{2}$ & & $15 / 2$ & 25272.63 & & & & 76 & $\left({ }^{5} \mathrm{~F}\right){ }^{8} \mathrm{H}$ \\
\hline $4 f^{10}\left({ }^{5} \mathrm{I}\right) 5 d^{2}\left({ }^{3} \mathrm{P}\right)\left({ }^{7} \mathrm{~K}\right) 6 s$ & ${ }^{8} \mathrm{~K}$ & $21 / 2$ & 25545.07 & & 86 & & 6 & $\left({ }^{5} \mathrm{I}\right)\left({ }^{1} \mathrm{D}\right)\left({ }^{5} \mathrm{~L}\right){ }^{8} \mathrm{~L}$ \\
\hline $4 f^{10}\left({ }^{5} \mathrm{I}\right) 5 d^{2}\left({ }^{3} \mathrm{~F}\right)\left({ }^{3} \mathrm{M}\right) 6 s$ & & $19 / 2$ & 25548.26 & & 22 & ${ }^{2} \mathrm{M}$ & 11 & $\left({ }^{5} \mathrm{I}\right)\left({ }^{3} \mathrm{~F}\right)\left({ }^{5} \mathrm{~L}\right){ }^{6} \mathrm{~L}$ \\
\hline $4 f^{10}\left({ }^{5} I_{4}\right) 5 d_{5 / 2} 6 s^{2}$ & $(4,5 / 2)$ & $13 / 2$ & 25930.66 & & & or & 75 & $\left({ }^{5} \mathrm{I}\right){ }^{4} \mathrm{~L}$ \\
\hline $4 f^{10}\left({ }^{5} \mathrm{I}\right) 5 d^{2}\left({ }^{3} \mathrm{~F}\right)\left({ }^{7} \mathrm{H}\right) 6 s$ & & $15 / 2$ & 25967.69 & & 35 & ${ }^{8} \mathrm{H}$ & 11 & $\left({ }^{5} I\right)\left({ }^{3} F\right)\left({ }^{7} I\right){ }^{8} I$ \\
\hline
\end{tabular}


Ho I, Even Parity-Continued

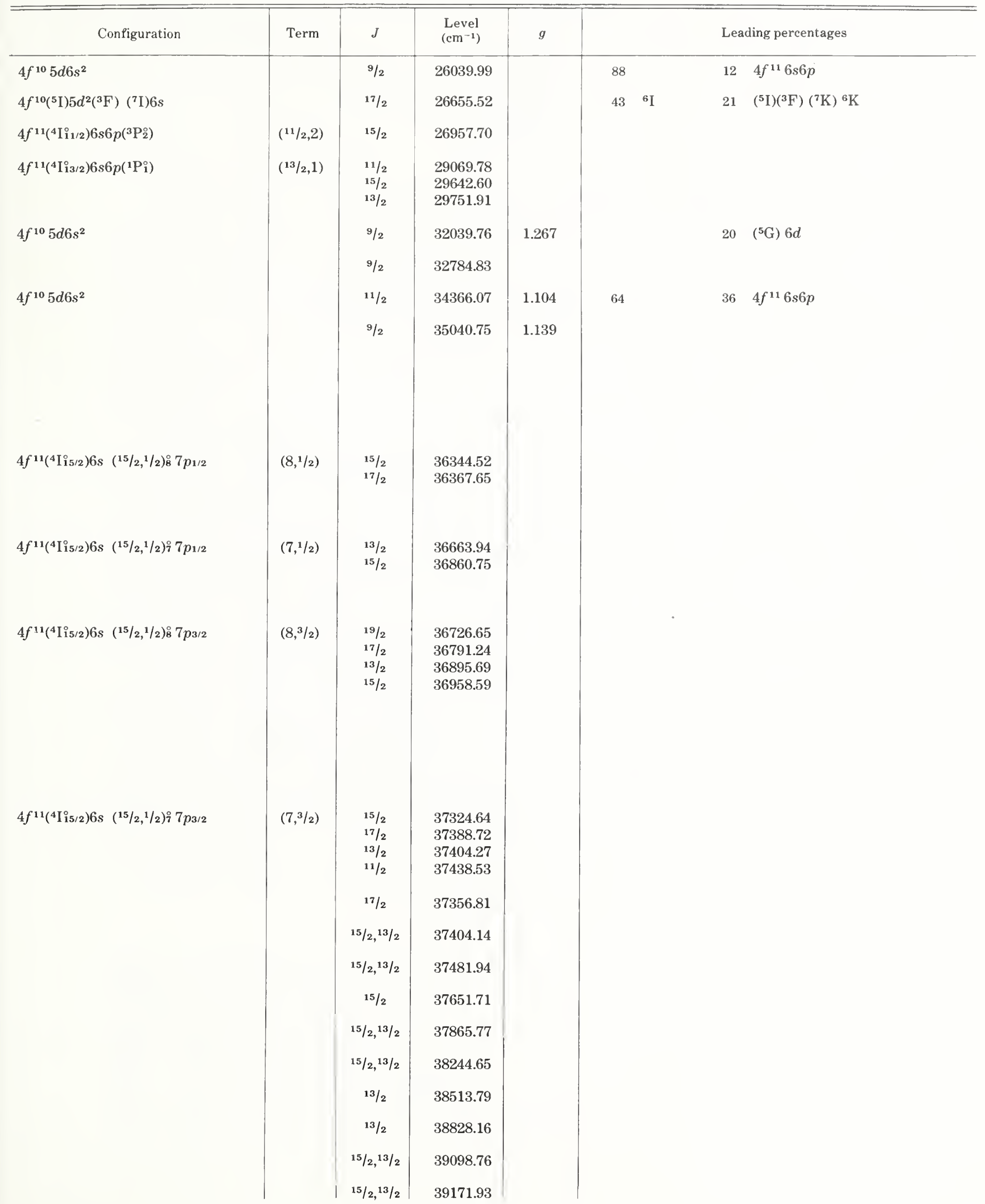


Ho I, Even Parity-Continued

\begin{tabular}{c|c|c|c|c|c}
\hline \hline Configuration & Term & $J$ & $\begin{array}{c}\text { Level } \\
\left(\mathrm{cm}^{-1}\right)\end{array}$ & $g$ & Leading percentages \\
\hline & & $13 / 2$ & 39554.49 & & \\
& & $15 / 2$ & 39676.57 & & \\
& & $15 / 2$ & 39904.45 & & \\
\hline
\end{tabular}




\section{Ho II}

(Dy I sequence; 66 electrons)

Ground state $\left(1 s^{2} 2 s^{2} 2 p^{6} 3 s^{2} 3 p^{6} 3 d^{10} 4 s^{2} 4 p^{6} 4 d^{10} 5 s^{2} 5 p^{6}\right) 4 f^{11}\left({ }^{4} \mathrm{I}_{15 / 2}^{\circ}\right) 6 s(15 / 2,1 / 2)_{8}^{\circ}$

Ionization energy $95200 \pm 600 \mathrm{~cm}^{-1}$

$11.80 \pm 0.08 \mathrm{eV}$

Sugar and Spector identified the ground-term pair of levels in Ho II by classifying "several pairs of lines originating from common upper levels and terminating on the two lowest levels of the $4 f^{11} 6 s$ configuration..." [Sugar, 1968]. Only two upper even levels were listed in connection with Sugar's study of the hyperfine structure, but Livingston and Pinnington [1971] gave eight additional upper levels and the corresponding pairs of lines. The identification of the excited configurations was begun by Wyart, Blaise, and Camus [1974], who designated several $4 f^{11}\left({ }^{4} \mathrm{I}_{15 / 2}^{\circ}\right) 5 d$ and $4 f^{11}\left({ }^{4} \mathrm{I}_{15 / 2}^{\circ}\right) 6 p$ levels that classified a group of infrared lines. Wyart found the three upper pairs of levels that complete the $4 f^{11}\left({ }^{4} \mathrm{I}^{\circ}\right) 6 \mathrm{~s}$ subconfiguration, as well as additional $4 f^{11}\left({ }^{4} \mathrm{I}^{\circ}\right) 5 d$ and $4 f^{11}\left({ }^{4} \mathrm{I}^{\circ}\right) 6 p$ levels [Wyart, Koot, and van Kleef, 1974; Wyart, 1975; 1976]. The term designations for all three configurations are in $J_{1} j$ coupling schemes. Spector [1976] gave several new levels to extend the $4 f^{11}\left({ }^{4} I^{\circ}\right) 5 d$ and $4 f^{11}\left({ }^{4} \mathrm{I}^{\circ}\right) 6 p$ structures.

The analysis is still at an early stage. Although the Zeeman effect for this spectrum is complicated by hyperfine structure, Wyart has made use of some Zeeman data in his analysis. The levels and interpretation as given here include several additions and corrections by Wyart $[1975,1976]$. The hyperfine widths of some of the even levels indicate large contributions from the $4 f^{10} 5 d 6 s$ configuration [Livingston and Pinnington; Wyart, Blaise, and Camus], which is expected to begin below $12000 \mathrm{~cm}^{-1}$. A satisfactory analysis will probably require the calculation of the $\left(4 f^{11} 6 p+4 f^{10}(5 d+6 s)^{2}\right)$ group [Wyart, Koot, and van Kleef]. The leading percentages listed for some of the even levels are from a calculation of the $4 f^{11} 6 p$ configuration alone [Wyart, Koot, and van Kleef]. Some of these levels are known to be perturbed, and two of the $4 f^{11}\left({ }^{4} \mathrm{I}_{15 / 2}^{\circ}\right) 6 p_{3 / 2}$ assignments are given specifically as uncertain (asterisks after levels) [Wyart, 1976].

Sugar gave eigenvectors for the ground-term pair of levels in the $J_{1} j$ coupling scheme. No percentages in this scheme are available for the other $4 f^{11}\left({ }^{4} \mathrm{I}^{\circ}\right) 6 s$ and $4 f^{11}\left({ }^{4} \mathrm{I}^{\circ}\right) 5 d$ even levels. The leading percentages for these levels in the LS scheme (given after the word "or") are from eigenvectors calculated by Wyart [1975].

Several of the published lists of Ho lines are noted in the text for Ho I. More complete lists for Ho I and Ho II based on recent observations are not yet available. Wyart is using measurements from spectrograms obtained at Argonne and, for the infrared region, Fouriertransform spectra observed by Verges at Orsay. Spector [1977] prepared a list of Ho lines for the infrared region $0.759-1.2 \mu \mathrm{m}$. His 1976 paper gives 40 classified Ho II lines, mostly in this region.

Sugar and Reader derived the ionization energy.

\section{References}

King, A. S., Astrophys. J. 72, 221 (1930). W

Livingston, A. E., Jr., and Pinnington, E. H., J. Opt. Soc. Am. 61, 1429 (1971). EL CL Hfs

Meggers, W. F., Corliss, C. H., and Scribner, B. F., Tables of Spectral-Line Intensities, Nat. Bur. Stand. (U.S.), Monogr. 145, Part I, 403 pp. (1975). CL W

Spector, N., Phys. Scr. 13, 181 (1976). EL ND CL W PT

Spector, N., Astrophys. J. 211, 600 (1977). W

Sugar, J., and Reader, J., J. Opt. Soc. Am. 55, 1286 (1965). IP

Sugar, J., J. Opt. Soc. Am. 58, 1519 (1968). EL CL Hfs PT

Wyart, J. F., unpublished material $(1975,1976)$. EL ND PT

Wyart, J. F., Blaise, J., and Camus, P., Phys. Scr. 9, 322 (1974). EL ND CL W

Wyart, J. F., Koot, J. J. A., and van Kleef, T. A. M., Physica (Utrecht) 77, 159 (1974). EL PT

[October 1.976] 
Ho II

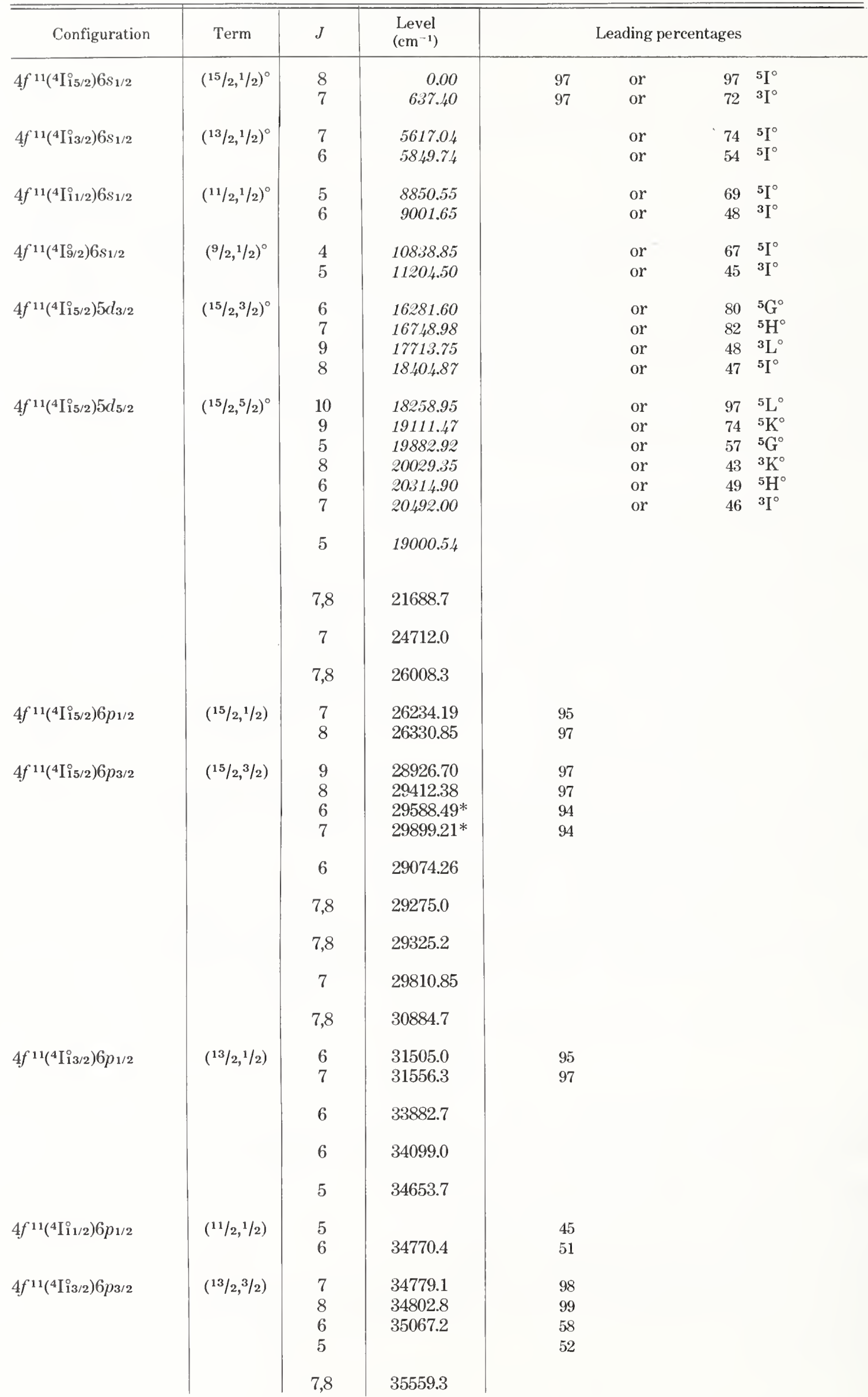


Ho II-Continued

\begin{tabular}{|c|c|c|c|c|}
\hline Configuration & Term & $J$ & $\begin{array}{l}\text { Level } \\
\left(\mathrm{cm}^{-1}\right)\end{array}$ & Leading percentages \\
\hline $4 f^{11}\left({ }^{4} \mathrm{I}_{11 / 2}^{\circ}\right) 6 p_{3 / 2}$ & $(11 / 2,3 / 2)$ & $\begin{array}{l}4 \\
5 \\
5 \\
7 \\
4 \\
5 \\
6 \\
5\end{array}$ & $\begin{array}{l}36055.8 \\
36884.1 \\
37159.2 \\
37499.0 \\
37779.1 \\
40332.8\end{array}$ & $\begin{array}{l}85 \\
79 \\
80 \\
83\end{array}$ \\
\hline Ho III $\left({ }^{4} \mathrm{I}_{15 / 2}^{0}\right)$ & Limit & --1 & 95200 & \\
\hline
\end{tabular}


Ground state $\left(1 s^{2} 2 s^{2} 2 p^{6} 3 s^{2} 3 p^{6} 3 d^{10} 4 s^{2} 4 p^{6} 4 d^{10} 5 s^{2} 5 p^{6}\right) 4 f^{11}{ }^{4} \mathrm{I}_{\mathbf{1 5 / 2}}^{\circ}$

Ionization energy $184200 \pm 800 \mathrm{~cm}^{-1}$

$22.84 \pm 0.10 \mathrm{eV}$

Identified odd configurations

Identified even configurations

$$
4 f^{11} \text { and } 4 f^{10} 6 p
$$

$4 f^{10} 5 d$ and $4 f^{10} 6 s$

The original analysis of this spectrum by McElaney [1964, 1967] gave 42 levels, including the $4 f^{11}{ }^{4} \mathrm{I}^{\circ}$ ground-term levels and levels assigned to the other three known configurations. The designations were based in part on calculations made by Becher [1965]. Hussain [1973] added several levels to the analysis.

The levels here are from a recent extension of the analysis by Wyart, Crosswhite, and Hussain [1977]. Some 80 of the 120 known levels were found in their investigation, with most of the new levels belonging to the $4 f^{10} 5 d$ configuration. The additions to $4 f^{11}$ include the ${ }^{4} \mathrm{~F}^{\circ}$ term, and the new $4 f^{10} 6 s$ and $4 f^{10} 6 p$ levels incorporate some revisions of Hussain's analysis.

The eigenvector percentages are from Crosswhite and Wyart [1976], whose calculation for the even group $\left(4 f^{10} 5 d+4 f^{10} 6 s\right)$ included configuration interaction. Some of the percentages differ slightly from the values published by Wyart, Crosswhite, and Hussain because of insignificant differences in the energy parameters used.

The known portions of the $4 f^{10} 6 s$ and $4 f^{10} 6 p$ configurations have clear $J_{1} j$-coupling structures. A number of the $4 f^{10} 5 d$ levels have no meaningful names in either the $J_{1} j$ or $L S$ coupling schemes, but some of the lower levels of this configuration have been grouped into $J_{1} j$ terms. The leading percentages in $L S$ coupling are given for the $4 f^{10} 6 s$ and $4 f^{10} 5 d$ levels, instead of second percentages in $J_{1} j$ coupling. Only second percentages $\geqslant 10 \%$ in the $J_{1} j$ scheme are listed for the $4 f^{10} 6 p$ levels.

McElaney's thesis [1964] has about 9000 lines (1920-10877 $\AA$ ) assigned to the first four Ho spectra. Electrodeless-discharge spectra $(2500 \AA-4 \mu \mathrm{m})$ obtained at the Laboratoire Aimé Cotton enable a more reliable separation of the Ho II and Ho III lines, but the newly classified Ho III lines have not been published. About 660 Ho III lines are now classified as transitions between the known levels. The lines typically have wide hyperfine structures, especially the $4 f^{10} 6 s-4 f^{10} 6 p$ transitions. The positions of the $4 f^{10} 6 s$ levels are based on the third diagonal hyperfine component of such transitions, the second decimal place for these levels having little significance otherwise.

Wyart, Crosswhite, and Hussain note that attempts to find levels based on higher terms of the $4 f^{10}$ core have been mostly unsuccessful, and searches for the lowest $4 f^{10} 6 d$ and $4 f^{10} 7 \mathrm{~s}$ levels were inconclusive. They attribute most of the unclassified lines to configurations based on the $4 f^{9}$ core $\left(4 f^{9} 5 d^{2}, 4 f^{9} 5 d 6 s, 4 f^{9} 5 d 6 p, 4 f^{9} 6 s 6 p\right)$ and to transitions between higher levels of $4 f^{11}$ and $4 f^{10} 5 d$.

The ionization energy is from Sugar and Reader.

\section{References}

Becher, J., Thesis, Johns Hopkins Univ., Baltimore, 135 pp. (1965). PT

Crosswhite, H. M., and Wyart, J. F., unpublished material (1976). PT

Hussain, R., Thesis, Johns Hopkins Univ., Baltimore, 142 pp. (1973). EL CL Hfs PT

McElaney, J. H., Thesis, Johns Hopkins Univ., Baltimore (1964). EL CL W Hfs PT

McElaney, J. H., J. Opt. Soc. Am. 57, 870 (1976). EL

Sugar, J., and Reader, J., J. Chem. Phys. 59, 2083 (1973). IP

Wyart, J. F., Crosswhite, H. M., and Hussain, R., Physica (Utrecht) 85C, 386 (1976). EL ND PT

[February 1977] 
Ho III

\begin{tabular}{|c|c|c|c|c|c|c|c|c|c|}
\hline . Configuration & Term & $J$ & $\begin{array}{l}\text { Level } \\
\left(\mathrm{cm}^{-1}\right)\end{array}$ & \multicolumn{6}{|c|}{ Leading percentages } \\
\hline $4 f^{11}$ & ${ }^{4} \mathrm{I}^{\circ}$ & $\begin{array}{l}15 / 2 \\
13 / 2 \\
11 / 2 \\
9 / 2\end{array}$ & $\begin{array}{r}0.00 \\
5438.53 \\
8644.59 \\
10770.40\end{array}$ & $\begin{array}{l}97 \\
99 \\
85 \\
61\end{array}$ & & & $\begin{array}{r}3 \\
1 \\
12 \\
16\end{array}$ & $\begin{array}{l}{ }^{2} \mathrm{~K}^{\circ} \\
{ }^{2} \mathrm{~K}^{\circ} \\
{ }^{2} \mathrm{H}^{\circ} 2 \\
{ }^{2} \mathrm{H}^{\circ} 2\end{array}$ & \\
\hline $4 f^{11}$ & ${ }^{4} \mathrm{~F}^{\circ}$ & $\begin{array}{l}9 / 2 \\
7 / 2 \\
5 / 2 \\
3 / 2\end{array}$ & $\begin{array}{l}13329.42 \\
17868.42 \\
19375.33\end{array}$ & $\begin{array}{l}66 \\
93 \\
86 \\
66\end{array}$ & & & $\begin{array}{r}18 \\
4 \\
11 \\
17\end{array}$ & $\begin{array}{l}{ }^{4} \mathrm{I}^{\circ} \\
{ }^{2} \mathrm{G}^{\circ} 1 \\
{ }^{2} \mathrm{D}^{\circ} 1 \\
{ }^{2} \mathrm{D}^{\circ} 1\end{array}$ & \\
\hline $4 f^{11}$ & ${ }^{2} \mathrm{H}^{\circ} 2$ & $11 / 2$ & 16891.20 & 48 & & & 38 & ${ }^{4} \mathrm{G}^{\circ}$ & \\
\hline $4 f^{10}\left({ }^{5} \mathrm{I}_{8}\right) 5 d d_{3 / 2}$ & $(8,3 / 2)$ & $\begin{array}{l}15 / 2 \\
17 / 2 \\
13 / 2 \\
19 / 2\end{array}$ & $\begin{array}{l}18033.40 \\
18099.14 \\
19010.09 \\
19940.47\end{array}$ & $\begin{array}{l}83 \\
67 \\
87 \\
62\end{array}$ & $\begin{array}{l}\text { or } \\
\text { or } \\
\text { or } \\
\text { or }\end{array}$ & & $\begin{array}{l}52 \\
73 \\
62 \\
64\end{array}$ & $\begin{array}{l}\left({ }^{5} \mathrm{I}\right){ }^{6} \mathrm{H} \\
\left({ }^{5} \mathrm{I}\right){ }^{6} \mathrm{I} \\
\left({ }^{5} \mathrm{I}\right){ }^{6} \mathrm{G} \\
\left({ }^{5} \mathrm{I}\right){ }^{6} \mathrm{~K}\end{array}$ & \\
\hline $4 f^{11}$ & & $9 / 2$ & 21533.75 & 22 & ${ }^{4} \mathrm{~F}^{\circ}$ & & 19 & ${ }^{2} \mathrm{G}^{\circ} 1$ & \\
\hline $4 f^{10}\left({ }^{5} \mathrm{I}_{8}\right) 6 s_{1 / 2}$ & $(8,1 / 2)$ & $\begin{array}{l}17 / 2 \\
15 / 2\end{array}$ & $\begin{array}{l}21824.15 \\
22993.98\end{array}$ & $\begin{array}{l}94 \\
92\end{array}$ & $\begin{array}{l}\text { or } \\
\text { or }\end{array}$ & & $\begin{array}{l}79 \\
69\end{array}$ & $\begin{array}{l}\left({ }^{5} I\right)^{6} I \\
\left({ }^{5} I\right){ }^{4} I\end{array}$ & \\
\hline $4 f^{10}\left({ }^{5} \mathrm{I}_{8}\right) 5 d_{5 / 2}$ & $(8,5 / 2)$ & $21 / 2$ & 22153.79 & 93 & or & & 93 & $\left({ }^{5} \mathrm{I}\right){ }^{6} \mathrm{~L}$ & A \\
\hline $4 f^{10}\left({ }^{5} \mathrm{I}_{8}\right) 5 d_{5 / 2}$ & $(8,5 / 2)$ & $17 / 2$ & 22243.06 & 58 & or & & 36 & $\left({ }^{5} \mathrm{I}\right){ }^{4} \mathrm{~K}$ & \\
\hline $4 f^{10}\left({ }^{5} \mathrm{I}_{7}\right) 5 d_{3 / 2}$ & $(7,3 / 2)$ & $\begin{array}{l}13 / 2 \\
11 / 2 \\
17 / 2 \\
15 / 2\end{array}$ & $\begin{array}{l}22431.10 \\
23212.29 \\
25557.75 \\
26081.47\end{array}$ & $\begin{array}{l}58 \\
67 \\
79 \\
45\end{array}$ & $\begin{array}{l}\text { or } \\
\text { or } \\
\text { or } \\
\text { or }\end{array}$ & & $\begin{array}{l}35 \\
45 \\
35 \\
41\end{array}$ & $\begin{array}{l}\left({ }^{5} \mathrm{I}\right){ }^{6} \mathrm{I} \\
\left({ }^{5} \mathrm{I}\right){ }^{6} \mathrm{G} \\
\left({ }^{5} \mathrm{I}\right){ }^{6} \mathrm{~L} \\
\left({ }^{5} \mathrm{I}\right){ }^{6} \mathrm{~K}\end{array}$ & \\
\hline $4 f^{10}\left({ }^{5} \mathrm{I}_{8}\right) 5 d_{5 / 2}$ & $(8,5 / 2)$ & $19 / 2$ & 22504.08 & 60 & or & & 52 & $\left({ }^{5} \mathrm{I}\right){ }^{4} \mathrm{~L}$ & \\
\hline $4 f^{10}\left({ }^{5} \mathrm{I}_{7}\right) 5 d_{3 / 2}$ & & $15 / 2$ & 22637.87 & 41 & $(7,3 / 2)$ & or & 33 & $\left({ }^{5} \mathrm{I}\right){ }^{6} \mathrm{H}$ & \\
\hline $4 f^{11}$ & ${ }^{4} \mathrm{G}^{\circ}$ & $\begin{array}{l}11 / 2 \\
9 / 2 \\
7 / 2 \\
5 / 2\end{array}$ & $\begin{array}{l}23884.67 \\
24648.07 \\
28960.42\end{array}$ & $\begin{array}{l}59 \\
81 \\
45 \\
93\end{array}$ & & & $\begin{array}{r}29 \\
14 \\
24 \\
3\end{array}$ & $\begin{array}{l}{ }^{2} \mathrm{H}^{\circ} 2 \\
{ }^{2} \mathrm{H}^{\circ} 2 \\
{ }^{2} \mathrm{G}^{\circ} 1 \\
{ }^{2} \mathrm{~F}^{\circ} 2\end{array}$ & \\
\hline $4 f^{10}\left({ }^{5} \mathrm{I}_{6}\right) 5 d_{3 / 2}$ & & $13 / 2$ & 25699.23 & 25 & $(6,3 / 2)$ & or & 27 & $\left({ }^{5} I\right)^{6} I$ & \\
\hline $4 f^{10}\left({ }^{5} \mathrm{I}_{6}\right) 5 d_{3 / 2}$ & $(6,3 / 2)$ & $\begin{array}{c}11 / 2 \\
9 / 2 \\
13 / 2 \\
15 / 2\end{array}$ & $\begin{array}{l}25973.40 \\
26603.07 \\
28867.24 \\
29162.57\end{array}$ & $\begin{array}{l}55 \\
59 \\
40 \\
86\end{array}$ & $\begin{array}{l}\text { or } \\
\text { or } \\
\text { or } \\
\text { or }\end{array}$ & & $\begin{array}{l}45 \\
42 \\
42 \\
57\end{array}$ & $\begin{array}{l}\left({ }^{5} \mathrm{I}\right){ }^{6} \mathrm{I} \\
\left({ }^{5} \mathrm{I}\right){ }^{6} \mathrm{H} \\
\left({ }^{5} \mathrm{I}\right){ }^{6} \mathrm{~K} \\
\left({ }^{5} \mathrm{I}\right){ }^{6} \mathrm{~L}\end{array}$ & \\
\hline $4 f^{10}\left({ }^{5} \mathrm{I}_{7}\right) 5 d_{5 / 2}$ & $(7,5 / 2)$ & $15 / 2$ & 27074.39 & 50 & or & & 44 & $\left({ }^{5} \mathrm{I}\right){ }^{4} \mathrm{I}$ & \\
\hline $4 f^{10}\left({ }^{5} \mathrm{I}_{7}\right) 6 s_{1 / 2}$ & $(7,1 / 2)$ & $\begin{array}{l}15 / 2 \\
13 / 2\end{array}$ & $\begin{array}{l}27180.12 \\
27735.94\end{array}$ & $\begin{array}{l}95 \\
93\end{array}$ & $\begin{array}{l}\text { or } \\
\text { or }\end{array}$ & & $\begin{array}{l}70 \\
50\end{array}$ & $\begin{array}{l}\left({ }^{5} \mathrm{I}\right){ }^{6} \mathrm{I} \\
\left({ }^{5} \mathrm{I}\right) \\
{ }^{6} \mathrm{I}\end{array}$ & \\
\hline $4 f^{10}\left(5 I_{7}\right) 5 d_{5 / 2}$ & $(7,5 / 2)$ & $17 / 2$ & 27260.26 & 76 & or & & 42 & $\left({ }^{5} \mathrm{I}\right){ }^{6} \mathrm{~K}$ & \\
\hline $4 f^{10}\left({ }^{5} I_{7}\right) 5 d d_{5 / 2}$ & $(7,5 / 2)$ & $19 / 2$ & 27666.77 & 94 & or & & 67 & $\left({ }^{5} \mathrm{I}\right){ }^{6} \mathrm{~L}$ & \\
\hline $4 f^{10}\left({ }^{5} \mathrm{I}_{8}\right) 5 d d_{5 / 2}$ & & $13 / 2$ & 27913.71 & 34 & $(8,5 / 2)$ & or & 49 & $\left({ }^{5} \mathrm{I}\right){ }^{4} \mathrm{H}$ & \\
\hline $4 f^{10}\left({ }^{5} I_{5}\right) 5 d_{3 / 2}$ & $(5,3 / 2)$ & $\begin{array}{c}9 / 2 \\
7 / 2 \\
11 / 2 \\
13 / 2\end{array}$ & $\begin{array}{l}28683.36 \\
29148.88 \\
30618.22 \\
31313.51\end{array}$ & $\begin{array}{l}51 \\
48 \\
53 \\
81\end{array}$ & $\begin{array}{l}\text { or } \\
\text { or } \\
\text { or } \\
\text { or }\end{array}$ & & $\begin{array}{l}45 \\
42 \\
55 \\
61\end{array}$ & $\begin{array}{l}\left({ }^{5} \mathrm{I}\right){ }^{6} \mathrm{I} \\
\left({ }^{5} \mathrm{I}\right) \\
{ }^{6} \mathrm{H} \\
\left({ }^{5} \mathrm{I}\right){ }^{6} \mathrm{~K} \\
\left({ }^{5} \mathrm{I}\right){ }^{6} \mathrm{~L}\end{array}$ & \\
\hline $4 f^{10}\left({ }^{5} \mathrm{I}_{6}\right) 5 d_{5 / 2}$ & & $11 / 2$ & 28724.89 & 32 & $(6,5 / 2)$ & or & 32 & $\left({ }^{5} \mathrm{I}\right){ }^{6} \mathrm{H}$ & \\
\hline $4 f^{10}\left(5 I_{8}\right) 5 d 5 / 2$ & $(8,5 / 2)$ & $11 / 2$ & 28809.29 & 49 & or & & 67 & $\left({ }^{5} \mathrm{I}\right){ }^{4} \mathrm{G}$ & \\
\hline $4 f^{10}\left(5 \mathrm{I}_{4}\right) 5 d_{3 / 2}$ & & $7 / 2$ & 30887.77 & 42 & $(4,3 / 2)$ & or & 50 & $\left({ }^{5} \mathrm{I}\right){ }^{6} \mathrm{I}$ & \\
\hline
\end{tabular}


Ho III-Continued

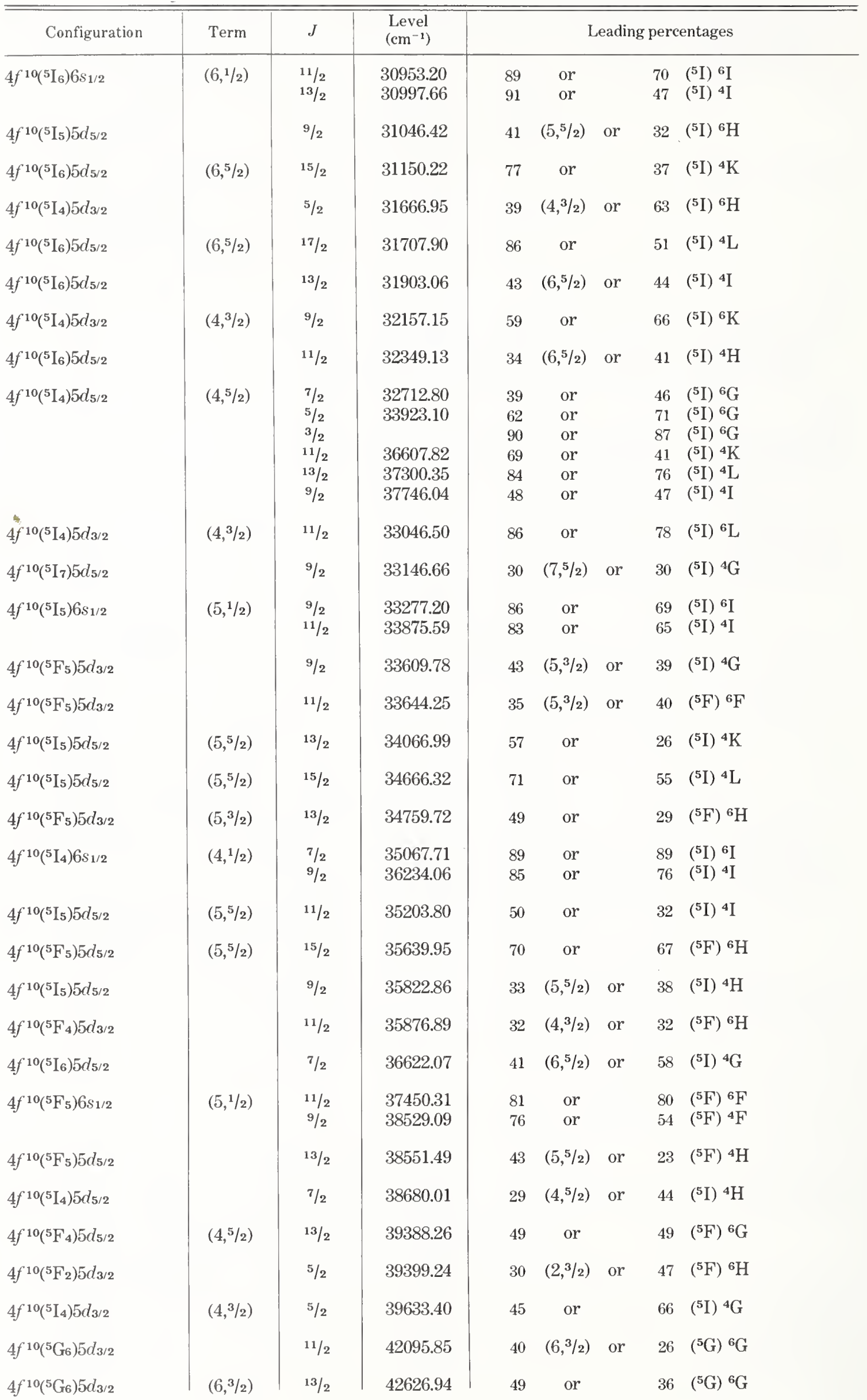


Ho III-Continued

\begin{tabular}{|c|c|c|c|c|c|c|c|}
\hline Configuration & Term & $J$ & $\begin{array}{l}\text { Level } \\
\left(\mathrm{cm}^{-1}\right)\end{array}$ & \multicolumn{4}{|c|}{ Leading percentages } \\
\hline $4 f^{10}\left({ }^{5} \mathrm{G}_{6}\right) 6 s_{1 / 2}$ & $(6,1 / 2)$ & $13 / 2$ & 44242.39 & 81 & or & 81 & $\left({ }^{5} \mathrm{G}\right){ }^{6} \mathrm{G}$ \\
\hline $4 f^{10}\left({ }^{5} \mathrm{I}_{8}\right) 6 p_{1 / 2}$ & $(8,1 / 2)^{\circ}$ & $\begin{array}{l}15 / 2 \\
17 / 2\end{array}$ & $\begin{array}{l}57497.72 \\
57853.14\end{array}$ & $\begin{array}{l}93 \\
92\end{array}$ & & & \\
\hline $4 f^{10}\left({ }^{5} I_{7}\right) 6 p_{1 / 2}$ & $(7,1 / 2)^{\circ}$ & $\begin{array}{l}13 / 2 \\
15 / 2\end{array}$ & $\begin{array}{l}62484.48 \\
62844.11\end{array}$ & $\begin{array}{l}84 \\
77\end{array}$ & & $\begin{array}{l}12 \\
18\end{array}$ & $\left({ }^{5} I_{8}\right)(8,3 / 2)^{\circ}$ \\
\hline $4 f^{10}\left({ }^{5} \mathrm{I}_{8}\right) 6 p_{3 / 2}$ & $(8,3 / 2)^{\circ}$ & $\begin{array}{l}17 / 2 \\
19 / 2 \\
15 / 2 \\
13 / 2\end{array}$ & $\begin{array}{l}62804.69 \\
62912.63 \\
63468.62 \\
64115.86\end{array}$ & $\begin{array}{l}92 \\
93 \\
74 \\
80\end{array}$ & & $\begin{array}{l}19 \\
12\end{array}$ & $\begin{array}{l}\left({ }^{5} I_{7}\right)(7,1 / 2)^{\circ} \\
\left({ }^{5} I_{7}\right)(7,1 / 2)^{\circ}\end{array}$ \\
\hline $4 f^{10}\left({ }^{5} \mathrm{I}_{6}\right) 6 p_{1 / 2}$ & $\left(6,{ }^{1} / 2\right)^{\circ}$ & $\begin{array}{l}11 / 2 \\
13 / 2\end{array}$ & $\begin{array}{l}66128.33 \\
66493.62\end{array}$ & $\begin{array}{l}89 \\
92\end{array}$ & & & \\
\hline $4 f^{10}\left(5 I_{7}\right) 6 p_{3 / 2}$ & $(7,3 / 2)^{\circ}$ & $\begin{array}{l}15 / 2 \\
17 / 2 \\
13 / 2 \\
11 / 2\end{array}$ & $\begin{array}{l}67896.30 \\
68104.87 \\
68266.03 \\
68755.93\end{array}$ & $\begin{array}{l}95 \\
97 \\
93 \\
70\end{array}$ & & 20 & $\left({ }^{5} I_{5}\right)\left(5,{ }^{1} / 2\right)^{\circ}$ \\
\hline $4 f^{10}\left(5 I_{5}\right) 6 p_{1 / 2}$ & $\left(5,{ }^{1} / 2\right)^{\circ}$ & $\begin{array}{l}9 / 2 \\
11 / 2\end{array}$ & $\begin{array}{l}68788.48 \\
69148.27\end{array}$ & $\begin{array}{l}85 \\
69\end{array}$ & & 20 & $\left({ }^{5} I_{7}\right)\left(7,{ }^{3} / 2\right)^{\circ}$ \\
\hline $4 f^{10}\left({ }^{5} \mathrm{I}_{4}\right) 6 p_{1 / 2}$ & $\left(4,{ }^{1} / 2\right)^{\circ}$ & $\begin{array}{l}7 / 2 \\
9 / 2\end{array}$ & $\begin{array}{l}70979.23 \\
71052.23\end{array}$ & $\begin{array}{l}85 \\
88\end{array}$ & & & \\
\hline $4 f^{10}\left(5 I_{6}\right) 6 p_{3 / 2}$ & $(6,3 / 2)^{\circ}$ & $\begin{array}{l}13 / 2 \\
11 / 2 \\
15 / 2 \\
9 / 2\end{array}$ & $\begin{array}{l}71499.30 \\
71611.84 \\
71784.40 \\
72090.65\end{array}$ & $\begin{array}{l}92 \\
91 \\
94 \\
86\end{array}$ & & & \\
\hline $4 f^{10}\left({ }^{5} \mathrm{~F}_{5}\right) 6 p_{1 / 2}$ & $(5,1 / 2)^{\circ}$ & $\begin{array}{l}9 / 2 \\
11 / 2\end{array}$ & $\begin{array}{l}72978.94 \\
73420.46\end{array}$ & $\begin{array}{l}77 \\
76\end{array}$ & & . 10 & $\left({ }^{3} \mathrm{G}_{5} 2\right)(5,1 / 2)^{\circ}$ \\
\hline $4 f^{10}\left({ }^{5} I_{5}\right) 6 p_{3 / 2}$ & $(5,3 / 2)^{\circ}$ & $\begin{array}{c}9 / 2 \\
11 / 2 \\
7 / 2 \\
13 / 2\end{array}$ & $\begin{array}{l}74140.80 \\
74209.17 \\
74280.00 \\
74530.99\end{array}$ & $\begin{array}{l}84 \\
86 \\
83 \\
89\end{array}$ & & & \\
\hline $4 f^{10}\left({ }^{5} \mathrm{I}_{4}\right) 6 p_{3 / 2}$ & $(4,3 / 2)^{\circ}$ & $\begin{array}{c}5 / 2 \\
7 / 2 \\
9 / 2 \\
11 / 2\end{array}$ & $\begin{array}{l}75867.06 \\
76237.80 \\
76355.20 \\
76810.88\end{array}$ & $\begin{array}{l}89 \\
83 \\
87 \\
88\end{array}$ & & & \\
\hline $4 f^{10}\left({ }^{5} \mathrm{~F}_{5}\right) 6 p_{3 / 2}$ & $(5,3 / 2)^{\circ}$ & $\begin{array}{l}13 / 2 \\
11 / 2 \\
9 / 2 \\
7 / 2\end{array}$ & $\begin{array}{l}78048.95 \\
78290.61 \\
79047.71\end{array}$ & $\begin{array}{l}76 \\
55 \\
71 \\
77\end{array}$ & & $\begin{array}{l}26 \\
10 \\
10\end{array}$ & $\begin{array}{l}\left({ }^{5} \mathrm{G}_{6}\right)\left(6,{ }^{1 / 2}\right)^{\circ} \\
\left({ }^{3} \mathrm{G}_{5} 2\right)(5,3 / 2)^{\circ} \\
\left({ }^{3} \mathrm{G}_{5} 2\right)(5,3 / 2)^{\circ}\end{array}$ \\
\hline $4 f^{10}\left({ }^{5} \mathrm{G}_{6}\right) 6 p_{1 / 2}$ & $(6,1 / 2)^{\circ}$ & $\begin{array}{l}11 / 2 \\
13 / 2\end{array}$ & $\begin{array}{l}80190.52 \\
80360.21\end{array}$ & $\begin{array}{l}50 \\
77\end{array}$ & & 21 & $\left({ }^{5} F_{5}\right)\left(5,{ }^{3} / 2\right)^{\circ}$ \\
\hline Ho IV $\left({ }^{5} I_{8}\right)$ & Limit & & 184200 & & & & \\
\hline
\end{tabular}


[Ho IV]

(Gd I sequence; 64 electrons)

Ground state $\left(1 s^{2} 2 s^{2} 2 p^{6} 3 s^{2} 3 p^{6} 3 d^{10} 4 s^{2} 4 p^{6} 4 d^{10} 5 s^{2} 5 p^{6}\right) 4 f^{10}{ }^{5} \mathrm{I}_{8}$

Ionization energy $343000 \pm 5000 \mathrm{~cm}^{-1}$

$42.5 \pm 0.6 \mathrm{eV}$

No analysis of the Ho IV free-ion spectrum is available. The $4 f^{10}$ levels given here are based on the absorption and fluorescence spectra of $\mathrm{Ho}^{3+}$ in $\mathrm{LaCl}_{3}$, mainly as observed by Dieke and Pandey. All but three of the values are centers-of-gravity of the corresponding sublevels in the crystal [Rajnak and Krupke], adjusted to a value of zero for the c.g. of the ground level. The ${ }^{5} \mathrm{~F}_{1},{ }^{3} \mathrm{~K}_{6} 2$, and ${ }^{5} \mathrm{G}_{2}$ levels are from Crosswhite, Crosswhite, Edelstein, and Rajnak [1976], who have extended the interpretation of the $\mathrm{Ho}^{3+}: \mathrm{LaCl}_{3}$ spectrum. They derived the level positions by first fitting a calculation to the observed sublevels. All levels are rounded off to the nearest $10 \mathrm{~cm}^{-1}$.

Transitions to other $4 f^{10}$ levels, mostly above those given here, have been observed in crystal spectra [Dieke, 1968, and references therein; Crosswhite et al., 1976] and in solution absorption spectra [Carnall, Fields, and Rajnak, 1968].

Crozier and Runciman interpreted the levels of the ${ }^{5} \mathrm{I},{ }^{5} \mathrm{~F}$, and ${ }^{5} \mathrm{~S}$ terms, as well as several higher levels, in 1961. Eigenvector percentages may be found in their paper and in the papers of Rajnak and Krupke [1967], Carnall et al. [1968], and Crosswhite et al. [1976]. The percentages given here are from unpublished calculations made by Carnall et al. [1974] with matrices including effective interactions.

Sugar and Reader obtained the ionization energy, which is rounded off to three significant figures. The estimated uncertainty has been approximately doubled, in accordance with the suggestion of Spector and Sugar.

\section{References}

Carnall, W. T., Fields, P. R., and Rajnak, K., J. Chem. Phys. 49, 4424 (1968). [EL] ND [CL] [W]

Carnall, W. T., Fields, P. R., and Rajnak, K., unpublished material (1974). PT

Crosswhite, H., Crosswhite, H. M., Edelstein, N., and Rajnak, K., unpublished material (1976). [EL] ND [CL] [W] PT

Crozier, M. H., and Runciman, W. A., J. Chem. Phys. 35, 1392 (1961). [EL] ND [CL] [W] PT

Dieke, G. H., Spectra and Energy Levels of Rare Earth Ions in Crystals, Ed. H. M. Crosswhite and H. Crosswhite, pp. 279-294 (Interscience Publishers, New York, 1968). [EL] ND [W] [CL]

Dieke, G. H., and Pandey, B., J. Chem. Phys. 41, 1952 (1964). [EL] [CL] [W]

Rajnak, K., and Krupke, W. F., J. Chem. Phys. 46, 3532 (1967). [EL] ND PT

Spector, N., and Sugar, J., J. Opt. Soc. Am. 66, 436 (1976).

Sugar, J., and Reader, J., J. Chem. Phys. 59, 2083 (1973). IP

[October 1976] 
[Ho IV]

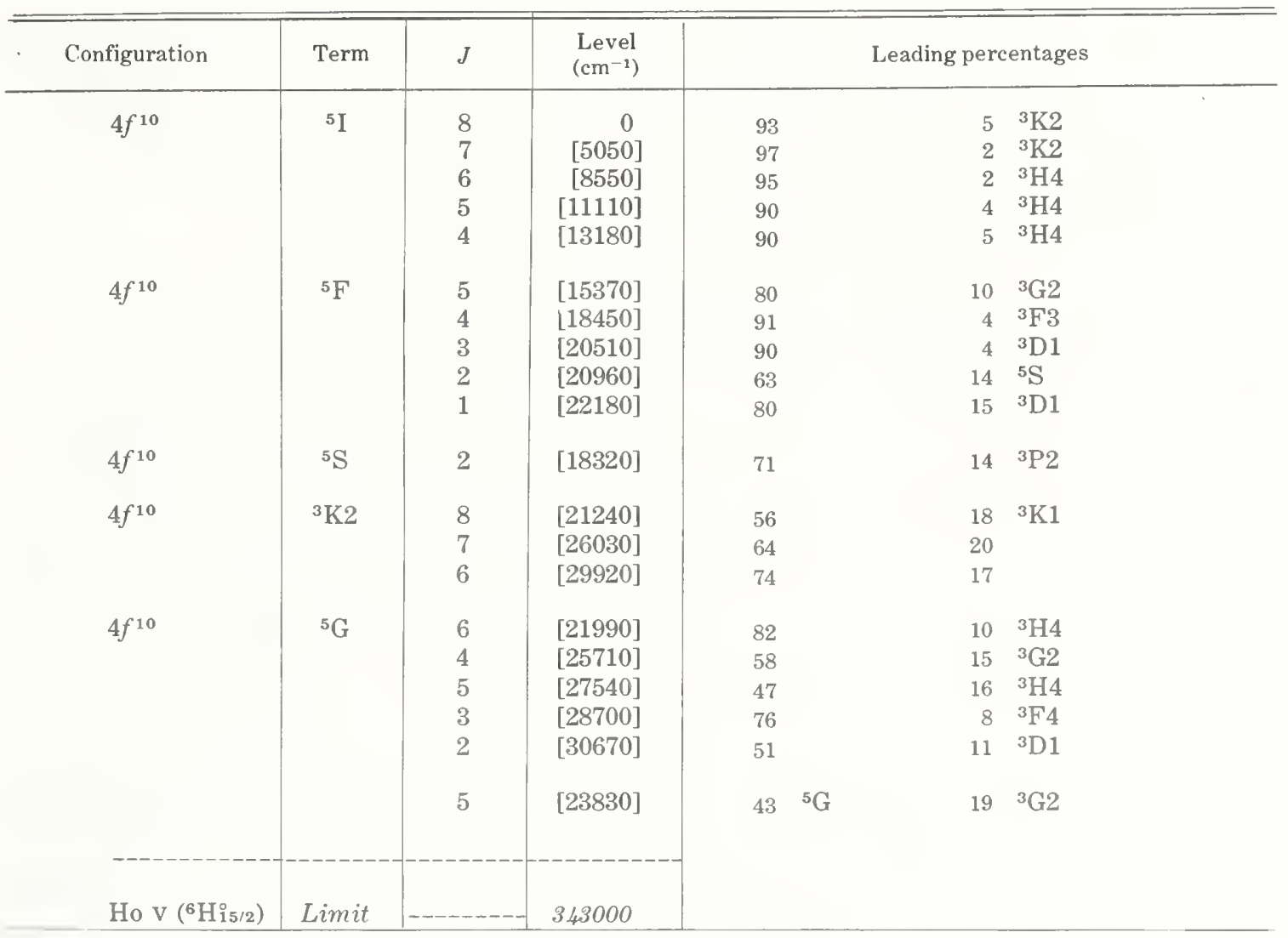




\section{ERBIUM}

\section{Er I}

68 electrons

$Z=68$

Ground state $\left(1 s^{2} 2 s^{2} 2 p^{6} 3 s^{2} 3 p^{6} 3 d^{10} 4 s^{2} 4 p^{6} 4 d^{10} 5 s^{2} 5 p^{6}\right) 4 f^{12} 6 s^{2}{ }^{3} \mathrm{H}_{6}$

Ionization energy $49262 \pm 5 \mathrm{~cm}^{-1}$

$6.1078 \pm 0.0006 \mathrm{eV}$

Identified even configurations

$4 f^{12} 6 s^{2}, 4 f^{12} 5 d 6 s, 4 f^{11} 6 s^{2} 6 p, 4 f^{11} 5 d 6 s 6 p, 4 f^{12} 6 s 7 s$, $4 f^{12} 6 s 8 s, 4 f^{12} 6 s 6 d$

Identified odd configurations

$4 f^{11} 5 d 6 s^{2}, 4 f^{11} 5 d^{2} 6 s, 4 f^{12} 6 s 6 p, 4 f^{11} 5 d 6 s 7 s$

\section{Energy Levels, Lists of Lines}

The first analysis of Er I by Marquet and Davis [1965] was based on accurate wavelength measurements of about $8000 \mathrm{Er}$ I and Er II lines [Marquet, 1964] and on the Zeeman-effect data of Lindner and Davis [1958]. The levels of the $4 f^{12} 6 s^{2}{ }^{3} \mathrm{H}$ ground term and of the $4 f^{12} 6 s^{2}{ }^{3} \mathrm{~F}$ term were among those identified. Marquet's thesis [1964] gives the energy-level classifications for $172 \mathrm{Er}$ I lines $(2780-6781 \AA)$. Several investigators soon extended the analysis and interpretation to include the $4 f^{11} 5 d 6 s^{2}$ and $4 f^{12} 6 s 6 p$ configurations [Marquet and Behring, 1965; Spector, 1965; 1966; 1967; Racah, Goldschmidt, and Toaff, 1965]. Spector's 1966 paper gives classifications for more than 300 Er I lines (2724-9927 $\AA$ ), and a few more are given in his 1967 paper. The most complete available lists of Er I and Er II lines with experimental separation of the two spectra are given in Held's 1966 report; a total of about 4500 lines are divided between the spectra over the range 2260-6900 $\AA$. Spector and Held [1971] published wavelengths for 980 lines of $\operatorname{Er}$ I and Er II in the range 6614-11962 $\AA$, and Held [1971] classified twelve of the Er I lines as transitions from three new even levels.

Heilig and Kircher [1968] and Vander Sluis and McNally [1969] observed the Zeeman effect, and their additional $g$ values in general confirmed the analysis. Vander Sluis and McNally give classifications and Zeeman patterns for about 75 lines (3181-4745 $\AA$ ) and list all levels for which $g$ values had been determined.

The energy levels here are from unpublished lists of van Kleef and Koot, who are continuing the analyses of $\mathrm{Er} \mathrm{I}$ and $\mathrm{Er}$ II in collaboration with Wyart [van Kleef and Koot, 1973; Wyart, Koot, and van Kleef, 1974; van Kleef and Koot, 1975]. Much of the work is based on extensive new measurements of the spectra by van Kleef and Koot, but their lists of lines and classifications have not been published. Unpublished contributions to the Er I and Er II analyses as available in 1973 were, however, used in the preparation of the Er line list given by Meggers, Corliss, and Scribner [1975]; classifications are included for more than 900 of the stronger Er I and Er II lines (2341-8870 §). Spector's 1974 paper gives 45 Er I lines (3794$11657 \AA$ ) classified as transitions from the $4 f^{12}\left({ }^{3} \mathrm{H}_{6}\right) 6 s 7 \mathrm{~s}$ levels, which he had identified.

\section{$g$ Values, Ionization Energy}

Most of the $g$ values are from van Kleef and Koot [1975], who give their results to the nearest 0.005 units. Other investigators of the optical Zeeman effect in Er have determined $g$ values for a smaller number of levels, and the determinations for several of the lowest levels of each parity have stated probable errors as small as 0.001 to 0.003 [see especially the summary tables of Vander Sluis and McNally]. We have tried to take suitable averages wherever more than one determination was available. Smith and Spalding [1961] and Cabezas, Lindgren, and Marrus [1961] first determined the $J$ and $g$ values of the $4 f^{12} 6 s^{2}{ }^{3} \mathrm{H}_{6}$ ground 
level by the atomic-beam magnetic-resonance technique. Doyle and Marrus [1963] used this technique to obtain the $g$ value quoted here for this level; they give the estimated error as \pm 0.00005 .

Worden, Conway, Paisner, and Solarz [1977] have observed high members of two series in Er I by using laser techniques. These data became available too late for inclusion here, but the quoted ionization energy is a new determination from the series.

\section{Theoretical Interpretation of the Even Levels}

The eigenvector percentages for the $4 f^{12} 6 s^{2}{ }^{3} \mathrm{H}$ and ${ }^{3} \mathrm{~F}$ levels are from Goldschmidt's thesis, second percentages less than $10 \%$ having been omitted for the even levels throughout. Goldschmidt independently located the ${ }^{3} \mathrm{~F}_{3}$ and ${ }^{3} \mathrm{~F}_{2}$ levels; no higher terms of the ground configuration are yet known.

Wyart et al. [1974] noted that their calculation of the $4 f^{11} 6 s^{2} 6 p$ levels "represents a first step of a progressive theoretical study of the even energy levels." The percentages here are from their later calculation of $\left(4 f^{11} 6 s^{2} 6 p+4 f^{12} 5 d 6 s\right)$ [Wyart, Koot, and van Kleef, 1976]. No levels of this group having $J \leqslant 2$ have as yet been confirmed by the calculation. Wyart points out that the $4 f^{11} 5 d 6 s 6 p$ configuration should be added in a more complete calculation. Some assignments to $4 f^{11} 5 d 6 s 6 p$ are listed [van Kleef and Koot, 1975], but some preliminary assignments to configurations and terms that have not yet been calculated are not given here. A few of the assignments to eigenvectors of the $\left(4 f^{11} 6 s^{2} 6 p+4 f^{12} 5 d 6 s\right)$ group are listed as tentative; these involve levels for which experimental $g$ values have not yet been obtained.

The assignments of the $4 f^{12} 6 s 7 s$ and $4 f^{12} 6 s 8 s$ levels to terms in the $J_{1} J_{2}$-coupling scheme are from van Kleef and Koot [1975]. Wyart et al. [1976] supplied the percentages for the $4 f^{12} 6 s 7 s$ levels in this scheme. Some assignments to the $4 f^{12}\left({ }^{3} \mathrm{H}\right) 6 s 6 d$ subconfiguration are included tentatively in the region above $38000 \mathrm{~cm}^{-1}$ [van Kleef and Koot, 1975]; the $4 f^{11} 5 d 6 s 6 p$, $4 f^{11} 5 d^{2} 6 p$, and $4 f^{12} 6 p^{2}$ configurations are also known or expected to contribute to the levels in this region.

\section{Theoretical Interpretation of the Odd Levels}

The lower odd levels belong to the $4 f^{11}\left({ }^{4} \mathrm{I}^{\circ}\right) 5 d 6 s^{2}$ and $4 f^{12}\left({ }^{3} \mathrm{H}\right) 6 s 6 p\left({ }^{3} \mathrm{P}^{\circ}\right)$ subconfigurations. The term structures in this region clearly exhibit $J_{1} j$ and $J_{1} J_{2}$ coupling, respectively, as was noted by Spector [1965; 1966; 1967] and by Racah et al. [1966]. Above about $21000 \mathrm{~cm}^{-1}$, however, very little term structure in any coupling scheme is apparent, and the system is further complicated by the $4 f^{11}\left({ }^{4} \mathrm{I}^{\circ}\right) 5 d^{2} 6 s$ subconfiguration beginning with the ${ }^{7} \mathrm{~F}_{6}^{\circ}$ level at $20166 \mathrm{~cm}^{-1}$ [van Kleef and Koot, 1975]. Thus all levels following the $4 f^{11}\left({ }^{4} \mathrm{I}^{\circ}{ }_{11 / 2}\right) 5 d_{5 / 2} 6 s$ term are given here simply in order of position.

A major advance in the interpretation has been achieved with the calculation of $\left(4 f^{12} 6 s 6 p+\right.$ $\left.4 f^{11} 5 d 6 s^{2}+4 f^{11}\left({ }^{4} \mathrm{I}^{\circ}\right) 5 d^{2} 6 s\right)$ by Wyart et al. [1976]. All percentages given here are from this calculation, which at its present stage accounts for most of the odd levels up to about $35000 \mathrm{~cm}^{-1}$. The eigenvectors from the calculation are so far available only in $L S$ coupling. The leading percentages in $L S$ coupling for the low $4 f^{11}\left({ }^{4} \mathrm{I}^{\circ}\right) 5 d 6 s^{2}$ and $4 f^{12}\left({ }^{3} \mathrm{H}\right) 6 s 6 p\left({ }^{3} \mathrm{P}^{\circ}\right)$ levels are given following the word "or," since these levels are grouped into $J_{1} j$ and $J_{1} J_{2}$ terms. Pending a coupling transformation to these latter schemes, we have not entered any term designations for the remaining levels of $4 f^{11} 5 d 6 s^{2}$ and $4 f^{12} 6 s 6 p$; the $L S$ term notations for the leading percentages are given under "Leading Percentages," regardless of the percentage. LS-coupling names are listed for some of the $4 f^{11}\left({ }^{4} I^{\circ}\right) 5 d^{2} 6 s$ levels, however, according to our usual convention. Wyart et al. coupled the electrons in the $4 f^{12} 6 s 6 p$ configuration in a different order from that shown here, with the result that we do not list a percentage for any $4 f^{12} 6 s 6 p$ term having the same multiplicity as its $4 f^{12}$ grandparent term. Depending on the possible relative sizes of such components in the eigenvectors, only partial information on the leading components is given for many of the assigned levels; a number of such levels are listed entirely without configuration or term notations. Preliminary designations for levels not yet included in the calculations are also omitted here.

Van Kleef and Koot [1975] have assigned several of the highest known odd levels, beginning at $41724 \mathrm{~cm}^{-1}$, to the $4 f^{11}\left({ }^{4} \mathrm{I}^{\circ}\right) 5 d 6 s 7 \mathrm{~s}$ subconfiguration. The identifications are based on the relative strength and character of transitions from these levels down to $4 f^{11}\left({ }^{4} \mathrm{I}^{\circ}\right) 5 d 6 s 6 p$ levels. 


\section{References}

Cabezas, A. Y., Lindgren, I., and Marrus, R., Phys. Rev. 122, 1796 (1961). ZE

Doyle, W. M., and Marrus, R., Phys. Rev. 131, 1586 (1963). ZE Hfs

Goldschmidt, Z. B., Thesis, Hebrew Univ. Jerusalem, Israel, 487 pp. (1968). EL PT

Heilig, K., and Kircher, O., Z. Phys. 212, 243 (1968). ZE

Held, S., Israel, A.E.C. Report IA-1037, 163 pp. (1966). W

Held, S., Astrophys. J. 167, 203 (1971). EL CL

Lindner, J. W., and Davis, S. P., J. Opt. Soc. Am. 48, 542 (1958). ZE W

Marquet, L. C., Thesis, Univ. California, Berkeley, 130 pp. (1964). EL CL W ZE PT

Marquet, L. C., and Behring, W. E., J. Opt. Soc. Am. 55, 576 (1965). EL

Marquet, L. C., and Davis, S. P., J. Opt. Soc. Am. 55, 471 (1965). EL ZE

Meggers, W. F., Corliss, C. H., and Scribner, B. F., Nat. Bur. Stand. (U.S.) Monogr. 145, Pạrt I, 403 pp. (1975). CL W

Racah, G., Goldschmidt, Z. B., and Toaff, S., J. Opt. Soc. Am. 56, 407 (1966). EL

Smith, K. F., and Spalding, I. J., Proc. R. Soc. London, Ser. A 265, 133 (1961). ZE

Spector, N., J. Opt. Soc. Am. 55, 576 (1965). EL

Spector, N., J. Opt. Soc. Am. 56, 341 (1966). EL CL PT

Spector, N., J. Opt. Soc. Am. 57, 308 (1967). EL CL

Spector, N., J. Opt. Soc. Am. 61, 1350 (1971). EL CL W PT

Spector, N., Phys. Ser. 9, 313 (1974). EL ND

Spector, N., and Held, S., Astrophys. J. 167, 193 (1971). W

van Kleef, T. A. M., and Koot, J. J. A., J. Opt. Soc. Am. 63, 1315A (1973).

van Kleef, T. A. M., and Koot, J. J. A., unpublished material (1975). EL ND CL ZE

Vander Sluis, K. L., and MeNally, Jr., J. R., J. Opt. Soc. Am. 59, 1202 (1969). EL CL ZE

Worden, E. F., Conway, J. G., Paisner, J. A., and Solarz, R. W., unpublished material (1977). IP

Wyart, J. F., Koot, J. J. A., and van Kleef, T. A. M., Physica (Utrecht) 77, 159 (1974). EL ND ZE PT

Wyart, J. F., Koot, J. J. A., and van Kleef, T. A. M., unpublished material (1976). PT

[May 1977]

\section{Er I, Even Parity}

\begin{tabular}{|c|c|c|c|c|c|c|}
\hline Configuration & Term & $J$ & $\begin{array}{l}\text { Level } \\
\left(\mathrm{cm}^{-1}\right)\end{array}$ & $g$ & & Leading percentages \\
\hline $4 f^{12} 6 s^{2}$ & ${ }^{3} \mathrm{H}$ & $\begin{array}{l}6 \\
5 \\
4\end{array}$ & $\begin{array}{r}0.000 \\
6958.329 \\
10750.982\end{array}$ & $\begin{array}{l}1.16381 \\
1.031 \\
0.936\end{array}$ & $\begin{array}{r}99 \\
100 \\
65\end{array}$ & $23 \quad{ }^{3} \mathrm{~F}$ \\
\hline $4 f^{12} 6 s^{2}$ & ${ }^{3} \mathrm{~F}$ & $\begin{array}{l}4 \\
3 \\
2\end{array}$ & $\begin{array}{r}5035.193 \\
12377.534 \\
13097.906\end{array}$ & $\begin{array}{l}1.147 \\
1.065 \\
0.750\end{array}$ & $\begin{array}{r}68 \\
100 \\
79\end{array}$ & $\begin{array}{ll}25 & { }^{1} \mathrm{G} \\
20 & { }^{1} \mathrm{D}\end{array}$ \\
\hline $4 f^{11}\left({ }^{4} \mathrm{Y}_{15 / 2}^{\circ}\right) 6 s^{2} 6 p_{1 / 2}$ & $\left(15 / 2,{ }^{1} / 2\right)$ & $\begin{array}{l}7 \\
8\end{array}$ & $\begin{array}{l}16464.934 \\
16727.479\end{array}$ & $\begin{array}{l}1.237 \\
1.175\end{array}$ & $\begin{array}{l}95 \\
97\end{array}$ & \\
\hline $4 f^{11}\left({ }^{4} \mathrm{I}_{15 / 2}^{\circ}\right) 6 s^{2} 6 p_{3 / 2}$ & $(15 / 2,3 / 2)$ & $\begin{array}{l}9 \\
8 \\
6 \\
7\end{array}$ & $\begin{array}{l}19355.149 \\
19723.271 \\
19816.936 \\
19915.789\end{array}$ & $\begin{array}{l}1.225 \\
1.210 \\
1.165 \\
1.185\end{array}$ & $\begin{array}{l}97 \\
97 \\
96 \\
94\end{array}$ & \\
\hline $4 f^{12}\left({ }^{3} \mathrm{H}_{6}\right) 5 d 6 s\left({ }^{3} \mathrm{D}\right)$ & $3[4]$ & $\begin{array}{l}5 \\
4 \\
3\end{array}$ & $\begin{array}{l}19362.105 \\
20497.641 \\
22391.431\end{array}$ & $\begin{array}{l}1.390 \\
1.280\end{array}$ & $\begin{array}{l}92 \\
76 \\
89\end{array}$ & $12 \quad\left({ }^{3} \mathrm{H}_{6}\right)\left({ }^{1} \mathrm{D}\right){ }^{1}[4]$ \\
\hline $4 f^{12\left({ }^{3} \mathrm{H}_{13 / 2}\right) 5 d 6 s\left({ }^{3} \mathrm{D}\right)}$ & $3[5]$ & $\begin{array}{l}6 \\
5 \\
4\end{array}$ & $\begin{array}{l}21035.201 \\
22056.785 \\
23300.042\end{array}$ & $\begin{array}{l}1.325 \\
1.195 \\
1.125\end{array}$ & $\begin{array}{l}92 \\
72 \\
57\end{array}$ & $\begin{array}{ll}12 & \left({ }^{3} \mathrm{H}_{6}\right)\left({ }^{3} \mathrm{D}\right) \\
17 & \left({ }^{3} \mathrm{H}_{6}\right)(6] \\
\left.{ }^{1} \mathrm{D}\right) & { }^{1}[4]\end{array}$ \\
\hline $4 f^{12}\left({ }^{3} \mathrm{H}_{6}\right) 5 d 6 s\left({ }^{3} \mathrm{D}\right)$ & $3[8]$ & $\begin{array}{l}8 \\
7 \\
9\end{array}$ & $\begin{array}{l}21069.365 \\
21101.510 \\
21204.465\end{array}$ & $\begin{array}{l}1.155 \\
1.025 \\
1.215\end{array}$ & $\begin{array}{l}93 \\
95 \\
99\end{array}$ & \\
\hline $4 f^{12}\left({ }^{3} \mathrm{H}_{6}\right) 5 d 6 s\left({ }^{3} \mathrm{D}\right)$ & ${ }^{3}[6]$ & $\begin{array}{l}7 \\
5 \\
6\end{array}$ & $\begin{array}{l}22521.790 \\
23314.115 \\
23432.428\end{array}$ & $\begin{array}{l}1.215 \\
1.050 \\
1.100\end{array}$ & $\begin{array}{l}52 \\
81 \\
49\end{array}$ & 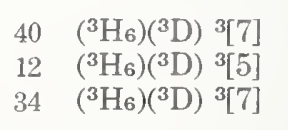 \\
\hline $4 f^{12}\left({ }^{3} \mathrm{H}_{6}\right) 5 d 6 s\left({ }^{3} \mathrm{D}\right)$ & $3[7]$ & $\begin{array}{l}6 \\
8 \\
7\end{array}$ & $\begin{array}{l}22691.855 \\
22810.531 \\
23336.111\end{array}$ & $\begin{array}{l}1.095 \\
1.210 \\
1.185\end{array}$ & $\begin{array}{l}53 \\
92 \\
46\end{array}$ & $\begin{array}{ll}38 & \left({ }^{3} \mathrm{H}_{6}\right)\left({ }^{3} \mathrm{D}\right){ }^{3}[6] \\
27 & \left({ }^{3} \mathrm{H}_{6}\right)\left({ }^{3} \mathrm{D}\right){ }^{3}[6]\end{array}$ \\
\hline
\end{tabular}


Er I, Even Parity-Continued

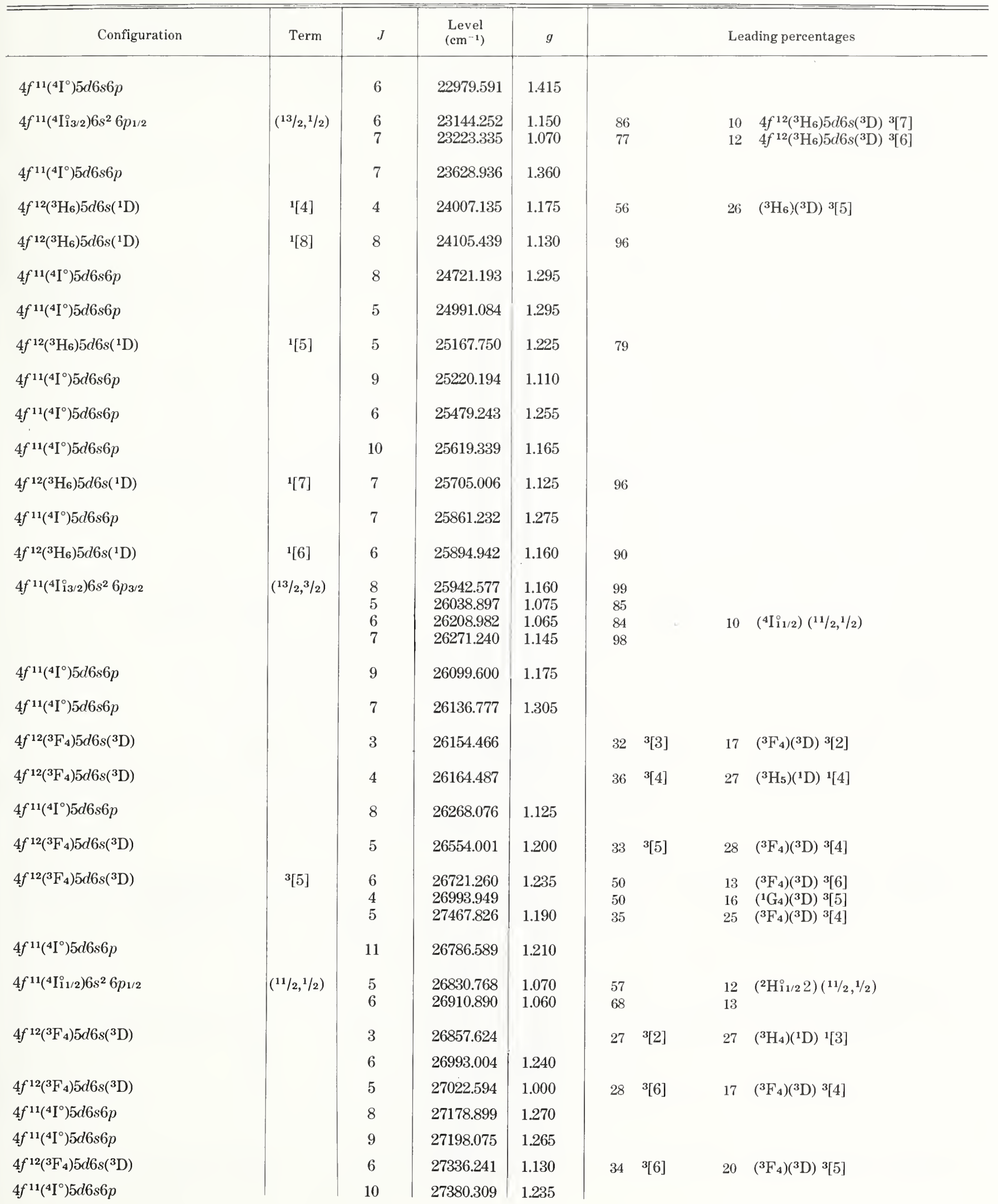


Er II, Even Parity-Continued

\begin{tabular}{|c|c|c|c|c|c|c|c|c|}
\hline Configuration & Term & $J$ & $\begin{array}{l}\text { Level } \\
\left(\mathrm{cm}^{-1}\right)\end{array}$ & $g$ & \multicolumn{4}{|c|}{ Leading percentages } \\
\hline $4 f^{12}\left({ }^{3} \mathrm{H}_{5}\right) 5 d 6 s\left({ }^{3} \mathrm{D}\right) ?$ & & 4 & 27391.885 & & 44 & 3[3] & 12 & $\left({ }^{3} \mathrm{~F}_{4}\right)\left({ }^{3} \mathrm{D}\right){ }^{3}[4]$ \\
\hline $4 f^{11}\left({ }^{4} I^{\circ}\right) 5 d 6 s 6 p$ & & 7 & 27459.437 & 1.250 & & & & \\
\hline $4 f^{11}\left({ }^{4} I^{\circ}\right) 5 d 6 s 6 p$ & & 8 & 27578.745 & 1.280 & & & & \\
\hline \multirow[t]{2}{*}{$4 f^{12}\left({ }^{3} \mathrm{~F}_{4}\right) 5 d 6 s\left({ }^{3} \mathrm{D}\right)$} & ${ }^{3}[6]$ & 7 & 27625.541 & 1.225 & 52 & & 20 & $\left({ }^{1} \mathrm{G}_{4}\right)\left({ }^{3} \mathrm{D}\right){ }^{3}[6]$ \\
\hline & & 5 & 27747.206 & 1.240 & & & & \\
\hline \multirow[t]{2}{*}{$4 f^{12}\left({ }^{3} \mathrm{H}_{5}\right) 5 d 6 s\left({ }^{3} \mathrm{D}\right)$} & $3[7]$ & 6 & 27819.334 & 1.030 & 91 & & & \\
\hline & & 8 & 28579.006 & 1.120 & 100 & & & \\
\hline \multirow{2}{*}{$4 f^{12}\left({ }^{3} \mathrm{~F}_{4}\right) 5 d 6 s\left({ }^{3} \mathrm{D}\right) ?$} & & 3 & 27839.291 & & 15 & ${ }^{3}[3]$ & 14 & $\left({ }^{3} \mathrm{H}_{4}\right)\left({ }^{1} \mathrm{D}\right){ }^{1}[3]$ \\
\hline & & 6 & 27932.038 & 1.105 & & & & \\
\hline $4 f^{11}\left({ }^{4} I^{\circ}\right) 5 d 6 s 6 p$ & & 10 & 27977.129 & 1.205 & & & & \\
\hline $4 f^{11}\left({ }^{4} I^{\circ}\right) 5 d 6 s 6 p$ & & 9 & 28043.271 & 1.230 & & & & \\
\hline $4 f^{12}\left({ }^{3} \mathrm{H}_{5}\right) 5 d 6 s\left({ }^{3} \mathrm{D}\right) ?$ & ${ }^{3}[3] ?$ & 3 & 28109.637 & & 53 & & 13 & $\left({ }^{3} \mathrm{H}_{5}\right)\left({ }^{3} \mathrm{D}\right){ }^{3}[4]$ \\
\hline $4 f^{11}\left({ }^{4} I^{\circ}\right) 5 d 6 s 6 p$ & & 7 & 28288.072 & 1.180 & & & & \\
\hline $4 f^{11}\left({ }^{4} I^{\circ}\right) 5 d 6 s 6 p$ & & 8 & 28397.139 & 1.185 & & & & \\
\hline $4 f^{12}\left({ }^{3} \mathrm{H}_{s}\right) 5 d 6 s\left({ }^{3} \mathrm{D}\right) ?$ & & 4 & 28405.430 & & 36 & ${ }^{3}[3]$ & 16 & $\left({ }^{3} \mathrm{~F}_{4}\right)\left({ }^{3} \mathrm{D}\right){ }^{3}[4]$ \\
\hline $4 f^{11}\left({ }^{4} \mathrm{I}^{\circ}\right) 5 d 6 s 6 p$ & & 9 & 28406.458 & 1.195 & & & & \\
\hline $4 f^{11} 5 d 6 s 6 p$ & & 5 & 28516.337 & 1.175 & & & & \\
\hline $4 f^{11}\left({ }^{4} I^{\circ}\right) 5 d 6 s 6 p$ & & 10 & 28598.169 & 1.235 & & & & \\
\hline $4 f^{11} 5 d 6 s 6 p$ & & 8 & 28699.849 & 1.180 & & & & \\
\hline $4 f^{11}\left({ }^{4} \mathrm{I}_{9 / 2}^{\circ}\right) 6 s^{2} 6 p_{1 / 2} ?$ & $(9 / 2,1 / 2) ?$ & $\begin{array}{l}4 \\
5\end{array}$ & $\begin{array}{l}28754.019 \\
28980.740\end{array}$ & & $\begin{array}{l}39 \\
44\end{array}$ & & $\begin{array}{l}17 \\
19\end{array}$ & $\left({ }^{2} \mathrm{H}_{9 / 2} 2\right)(9 / 2,1 / 2)$ \\
\hline $4 f^{11} 5 d 6 s 6 p$ & & $\begin{array}{lllll}9 & & & \end{array}$ & 28839.974 & 1.160 & & & & \\
\hline $4 f^{11} 5 d 6 s 6 p$ & & 7 & 28918.439 & 1.140 & & & & \\
\hline $4 f^{11}\left({ }^{4} \mathrm{I}^{\circ}\right) 5 d 6 s 6 p$ & & 11 & 29013.821 & 1.230 & & & & \\
\hline \multirow[t]{4}{*}{$4 f^{12}\left({ }^{3} \mathrm{H}_{5}\right) 5 d 6 s\left({ }^{3} \mathrm{D}\right)$} & ${ }^{3}[4]$ & 5 & 29069.482 & 1.195 & 76 & & & \\
\hline & & $\begin{array}{l}4 \\
3\end{array}$ & $\begin{array}{l}29269.579 \\
29422.769\end{array}$ & 1.110 & $\begin{array}{l}64 \\
53\end{array}$ & & 10 & $\left({ }^{3} \mathrm{H}_{5}\right)\left({ }^{3} \mathrm{D}\right){ }^{3}[3]$ \\
\hline & & 6 & 29138.739 & 1.135 & & & & \\
\hline & & 9 & 29201.811 & 1.150 & & & & \\
\hline $4 f^{11}\left({ }^{4} I^{\circ}\right) 5 d 6 s 6 p$ & & 12 & 29280.254 & & & & & \\
\hline \multirow[t]{3}{*}{$4 f^{12}\left({ }^{3} \mathrm{H}_{5}\right) 5 d 6 s\left({ }^{3} \mathrm{D}\right)$} & ${ }^{3}[6]$ & 5 & 29372.716 & 0.955 & 54 & & & $\left({ }^{3} \mathrm{H}_{5}\right)\left({ }^{3} \mathrm{D}\right){ }^{3}[5]$ \\
\hline & & $\begin{array}{l}6 \\
7\end{array}$ & $\begin{array}{l}29445.385 \\
30091.528\end{array}$ & $\begin{array}{l}1.130 \\
1.165\end{array}$ & $\begin{array}{l}43 \\
92\end{array}$ & & & $\left({ }^{3} \mathrm{H}_{5}\right)\left({ }^{3} \mathrm{D}\right){ }^{3}[5]$ \\
\hline & & 8 & 29451.894 & 1.270 & & & & \\
\hline $4 f^{11}\left({ }^{4} I^{\circ}\right) 5 d 6 s 6 p$ & & 10 & 29648.807 & 1.205 & & & & \\
\hline \multirow[t]{5}{*}{$4 f^{11}\left({ }^{4} I_{11 / 2}^{\circ}\right) 6 s^{2} 6 p_{3 / 2}$} & $\left(11 / 2,{ }^{3} / 2\right)$ & 7 & 29681.661 & 1.070 & 83 & & 15 & $\left({ }^{2} \mathrm{H}_{11 / 2}^{\circ} 2\right)(11 / 2,3 / 2)$ \\
\hline & & $\begin{array}{l}4 \\
5\end{array}$ & $\begin{array}{l}29682.975 \\
29829.740\end{array}$ & 1.020 & $\begin{array}{l}71 \\
71\end{array}$ & & $\begin{array}{l}11 \\
13\end{array}$ & \\
\hline & & 6 & 29989.842 & 1.140 & 74 & & 14 & \\
\hline & & 8 & 29760.553 & 1.115 & & & & \\
\hline & & 6 & 29801.226 & 1.185 & & & & \\
\hline $4 f^{12}\left({ }^{3} \mathrm{~F}_{4}\right) 5 d 6 s\left({ }^{1} \mathrm{D}\right)$ & ${ }^{1}[5]$ & 5 & 29907.781 & 1.095 & 66 & & 18 & $\left({ }^{1} \mathrm{G}_{4}\right)\left({ }^{1} \mathrm{D}\right){ }^{1}[5]$ \\
\hline
\end{tabular}


Er I, Even Parity-Continued

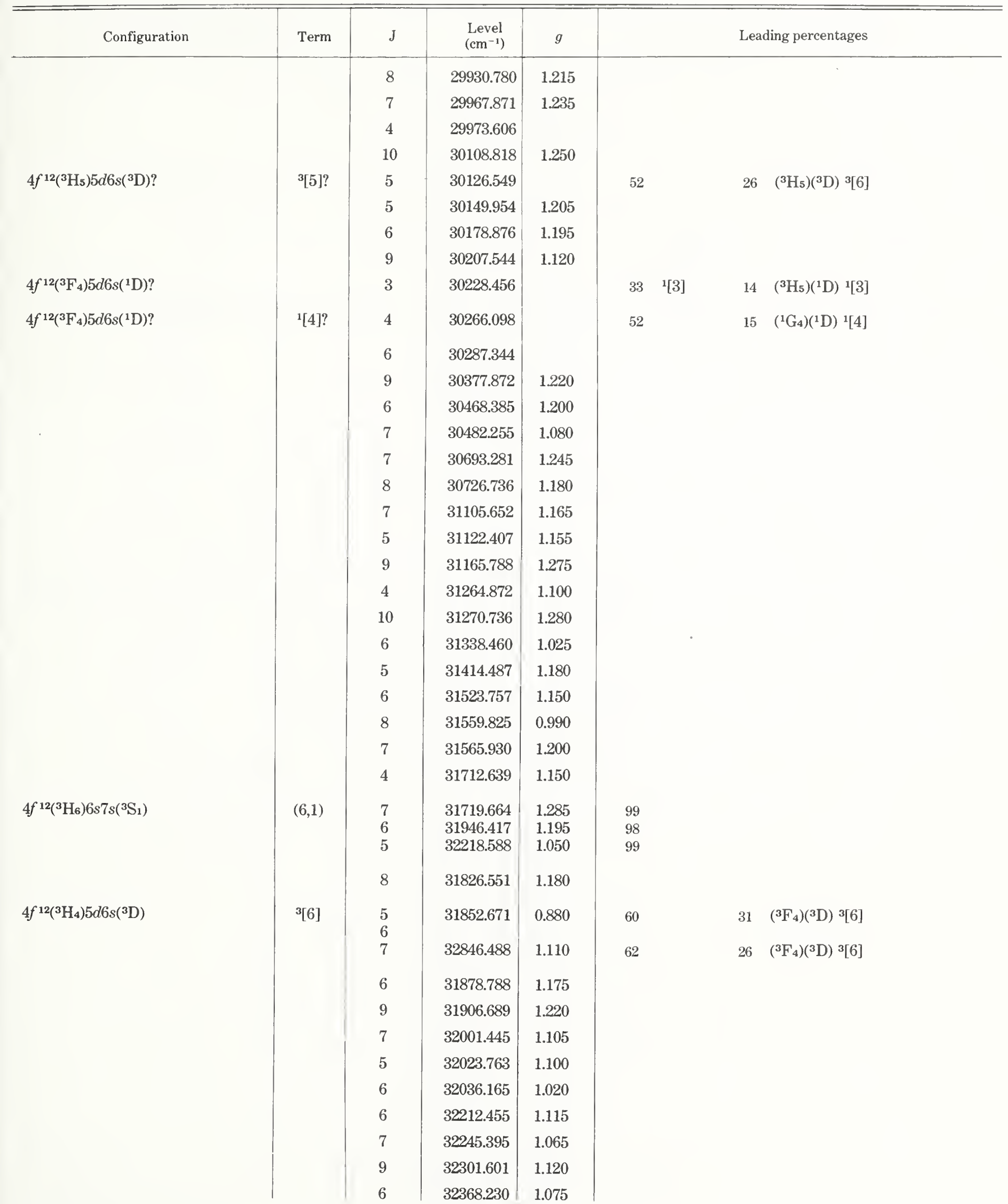


Er I, Even Parity_Continued

\begin{tabular}{|c|c|c|c|c|c|c|}
\hline Configuration & Term & $J$ & $\begin{array}{l}\text { Level } \\
\left(\mathrm{cm}^{-1}\right)\end{array}$ & $g$ & & Leading percentages \\
\hline \multirow{6}{*}{$4 f^{12}\left({ }^{3} \mathrm{H}_{4}\right) 5 d 6 s\left({ }^{3} \mathrm{D}\right)$} & \multirow{8}{*}{$(6,0)$} & 8 & 32525.666 & 1.170 & & \\
\hline & & 7 & 32799.498 & 1.075 & & \\
\hline & & 8 & 32884.867 & 1.145 & & \\
\hline & & 5 & 32962.601 & 1.130 & & \\
\hline & & 5 & 33034.654 & 1.050 & $35 \quad 3[5]$ & $14 \quad\left({ }^{3} \mathrm{H}_{4}\right)\left({ }^{3} \mathrm{D}\right)^{3}[4]$ \\
\hline & & 6 & 33139.808 & 1.065 & & \\
\hline \multirow[t]{2}{*}{$4 f^{12}\left({ }^{3} \mathrm{H}_{6}\right) 6 s 7 s\left({ }^{1} \mathrm{~S}_{0}\right)$} & & 6 & 33519.739 & 1.180 & 98 & \\
\hline & & 10 & 33619.332 & 1.160 & & \\
\hline \multirow[t]{34}{*}{$4 f^{12}\left({ }^{3} \mathrm{H}_{4}\right) 5 d 6 s\left({ }^{3} \mathrm{D}\right)$} & \multirow[t]{34}{*}{${ }^{3}[5]$} & 6 & 33750.645 & 1.100 & 52 & $20 \quad\left({ }^{1} \mathrm{G}_{4}\right)\left({ }^{3} \mathrm{D}\right){ }^{3}[5]$ \\
\hline & & 6 & 33853.872 & 1.240 & & \\
\hline & & 10 & 33942.292 & 1.118 & & \\
\hline & & 6 & 34055.264 & 1.190 & & \\
\hline & & 7 & 34153.760 & 1.160 & & \\
\hline & & 9 & 34224.049 & 1.205 & & \\
\hline & & 8 & 34288.437 & 1.190 & & \\
\hline & & 6 & 34313.100 & 1.235 & & \\
\hline & & 5 & 34393.833 & 1.070 & & \\
\hline & & 5 & 34447.758 & 1.090 & & \\
\hline & & 6 & 34457.999 & 1.183 & & \\
\hline & & 9 & 34587.802 & 1.135 & & \\
\hline & & 7 & 34596.396 & 1.025 & & \\
\hline & & 9 & 34724.897 & 1.165 & & \\
\hline & & 7 & 34733.243 & 1.110 & & \\
\hline & & 8 & 34756.580 & 1.140 & & \\
\hline & & 8 & 34990.233 & 1.135 & & \\
\hline & & 7 & 35014.743 & 1.190 & & \\
\hline & & 5 & 35185.409 & 1.140 & & \\
\hline & & 7 & 35191.459 & 0.965 & & \\
\hline & & 6 & 35218.609 & 1.080 & & \\
\hline & & 8 & 35300.412 & 1.125 & & \\
\hline & & 5 & 35328.671 & 1.125 & & \\
\hline & & 7 & 35394.592 & 1.130 & & \\
\hline & & 9 & 35402.733 & 1.170 & & \\
\hline & & 4 & 35428.605 & 1.105 & & \\
\hline & & 8 & 35493.301 & 1.170 & & \\
\hline & & 6 & 35557.173 & 1.085 & & \\
\hline & & 8 & 35632.606 & 1.180 & & \\
\hline & & 6 & 35667.077 & 1.060 & & \\
\hline & & 5 & 35680.994 & 1.155 & & \\
\hline & & 7 & 35717.098 & 1.210 & & \\
\hline & & 8 & 35794.481 & 1.220 & & \\
\hline & & 7 & 35833.788 & 1.050 & & \\
\hline
\end{tabular}


Er I, Even Parity_Continued

\begin{tabular}{|c|c|c|c|c|c|c|}
\hline Configuration & Term & $J$ & $\begin{array}{l}\text { Level } \\
\left(\mathrm{cm}^{-1}\right)\end{array}$ & $g$ & & Leading percentages \\
\hline & \multirow{43}{*}{$(4,1)$} & 6 & 35918.454 & 1.095 & & \\
\hline & & 5 & 35952.787 & 1.005 & & \\
\hline & & 6 & 35963.366 & 1.085 & & \\
\hline & & 6 & 36026.864 & 1.185 & & \\
\hline & & 6 & 36051.449 & 1.195 & & \\
\hline & & 7 & 36123.034 & 1.180 & & \\
\hline & & 8 & 36130.860 & 1.050 & & \\
\hline & & 9 & 36136.191 & 1.165 & & \\
\hline & & 6 & 36306.253 & 0.975 & & \\
\hline & & 9 & 36321.915 & 1.130 & & \\
\hline & & 7 & 36418.402 & 1.130 & & \\
\hline & & 10 & 36461.845 & 1.180 & & \\
\hline & & 9 & 36494.167 & 1.140 & & \\
\hline & & 6 & 36511.221 & 1.175 & & \\
\hline & & 5 & 36516.831 & 1.155 & & \\
\hline & & 5 & 36710.827 & 1.150 & & \\
\hline & & 10 & 36712.578 & 1.120 & & \\
\hline & & 7 & 36735.742 & 1.195 & & \\
\hline & & 6 & 36876.457 & 1.160 & & \\
\hline \multirow[t]{25}{*}{$4 f^{12}\left({ }^{3} \mathrm{~F}_{4}\right) 6 s 7 s\left({ }^{3} \mathrm{~S}_{1}\right)$} & & 5 & 36883.886 & 1.255 & 70 & $\left({ }^{1} \mathrm{G}_{4}\right)\left({ }^{3} \mathrm{~S}_{1}\right)$ \\
\hline & & 4 & 37014.608 & 1.135 & 67 & 22 \\
\hline & & 3 & 37144.092 & 0.890 & 66 & 24 \\
\hline & & 5 & 36898.613 & 1.110 & & \\
\hline & & 6 & 36979.594 & 0.940 & & \\
\hline & & 4 & 36997.475 & 1.055 & & \\
\hline & & 8 & 37021.273 & 1.170 & & \\
\hline & & 6 & 37044.419 & 1.125 & & \\
\hline & & 9 & 37062.822 & 1.170 & & \\
\hline & & 7 & 37178.437 & 1.180 & & \\
\hline & & 6 & 37199.912 & 1.070 & & \\
\hline & & 9 & 37271.015 & 1.095 & & \\
\hline & & 8 & 37378.443 & 1.195 & & \\
\hline & & 7 & 37391.232 & 1.100 & & \\
\hline & & 6 & 37542.792 & 1.150 & & \\
\hline & & 10 & 37593.886 & 1.205 & & \\
\hline & & 8 & 37645.754 & 1.200 & & \\
\hline & & 7 & 37714.613 & 1.205 & & \\
\hline & & 7 & 37806.983 & 1.090 & & \\
\hline & & 8 & 37843.845 & 1.170 & & \\
\hline & & 8 & 38164.906 & 1.225 & & \\
\hline & & 5 & 38169.753 & 1.085 & & \\
\hline & & 9 & 38172.809 & 1.190 & & \\
\hline & & 7 & 38195.326 & 0.970 & & \\
\hline & & 5 & 38225.984 & 1.370 & & \\
\hline
\end{tabular}


Er I, Even Parity—Continued

\begin{tabular}{|c|c|c|c|c|c|c|}
\hline Configuration & Term & $J$ & $\begin{array}{l}\text { Level } \\
\left(\mathrm{cm}^{-1}\right)\end{array}$ & $g$ & & Leading percentages \\
\hline & & 8 & 38253.685 & 1.160 & & \\
\hline & & 7 & 38301.613 & 1.095 & & \\
\hline & & 8 & 38343.693 & 1.135 & & \\
\hline $4 f^{12}\left({ }^{3} \mathrm{H}\right) 6 s 6 d ?$ & & 6 & 38407.470 & 1.320 & & \\
\hline \multirow[t]{3}{*}{$4 f^{12\left({ }^{3} \mathrm{H}\right) 6 s 6 d ?}$} & & 9 & 38424.655 & 1.210 & & \\
\hline & & 6 & 38457.832 & 1.130 & & \\
\hline & & 9 & 38487.253 & 1.220 & & \\
\hline $4 f^{12}\left({ }^{3} \mathrm{H}\right) 6 s 6 d ?$ & & 7 & 38545.334 & 1.130 & & \\
\hline \multirow[t]{2}{*}{$4 f^{12}\left({ }^{3} \mathrm{H}\right) 6 s 6 d ?$} & & 8 & 38556.751 & 1.180 & & \\
\hline & . & 7 & 38560.986 & 1.100 & & \\
\hline \multirow[t]{4}{*}{$4 f^{12}\left({ }^{3} \mathrm{H}\right) 6 s 6 d ?$} & & 4 & 38587.912 & 1.210 & & \\
\hline & & 6 & 38604.588 & 1.095 & & \\
\hline & & 4 & 38657.464 & 1.120 & & \\
\hline & & 7 & 38664.249 & 1.170 & & \\
\hline $4 f^{12\left({ }^{3} \mathrm{H}\right) 6 s 6 d ?}$ & & 6 & 38667.799 & 1.150 & & \\
\hline \multirow[t]{3}{*}{$\left.4 f^{12(}{ }^{3} \mathrm{H}\right) 6 s 6 d ?$} & & 8 & 38668.310 & 1.195 & & \\
\hline & & 5 & 38703.037 & 1.110 & & \\
\hline & & 8 & 38734.243 & 1.135 & & \\
\hline \multirow[t]{2}{*}{$4 f^{12}\left({ }^{3} \mathrm{H}\right) 6 s 6 d ?$} & & 7 & 38751.622 & 1.180 & & \\
\hline & & 6 & 38786.542 & 1.005 & & \\
\hline $4 f^{12}\left({ }^{3} \mathrm{H}\right) 6 s 6 d ?$ & & 5 & 38795.477 & 1.165 & & \\
\hline \multirow[t]{2}{*}{$4 f^{12}\left({ }^{3} \mathrm{H}\right) 6 s 6 d ?$} & & 7 & 38797.084 & 1.075 & & \\
\hline & & 5 & 38831.967 & 1.170 & & \\
\hline \multirow[t]{2}{*}{$4 f^{12}\left({ }^{3} \mathrm{H}\right) 6 s 6 d ?$} & & 8 & 38834.142 & 1.130 & & \\
\hline & & 7 & 38857.639 & 1.150 & & \\
\hline \multirow[t]{2}{*}{$4 f^{12}\left({ }^{3} \mathrm{H}_{5}\right) 6 s 7 s\left({ }^{3} \mathrm{~S}_{1}\right)$} & $(5,1)$ & 6 & 38870.770 & 1.180 & 100 & \\
\hline & & $\begin{array}{l}5 \\
4\end{array}$ & $\begin{array}{l}38893.172 \\
38921.185\end{array}$ & $\begin{array}{l}1.045 \\
0.855\end{array}$ & $\begin{array}{r}100 \\
98\end{array}$ & \\
\hline \multirow[t]{5}{*}{$4 f^{12}\left({ }^{3} \mathrm{H}\right) 6 s 6 d ?$} & & 6 & 38923.586 & 1.105 & & \\
\hline & & 7 & 38986.765 & 1.075 & & \\
\hline & & 6 & 39010.445 & 1.050 & & \\
\hline & & 8 & 39049.481 & 1.180 & & \\
\hline & & 10 & 39062.614 & 1.255 & & \\
\hline \multirow[t]{2}{*}{$\left.4 f^{12(}{ }^{3} \mathrm{H}\right) 6 s 6 d ?$} & & 7 & 39070.645 & 1.145 & & \\
\hline & & 6 & 39075.883 & 1.145 & & \\
\hline \multirow[t]{3}{*}{$4 f^{12\left({ }^{3} \mathrm{H}\right) 6 s 6 d ?}$} & & 5 & 39125.605 & 1.040 & & \\
\hline & & 7 & 39145.450 & 1.130 & & \\
\hline & & 6 & 39164.268 & 1.140 & & \\
\hline $4 f^{12}\left({ }^{3} \mathrm{H}\right) 6 s 6 d ?$ & & 6 & 39193.564 & 1.115 & & \\
\hline \multirow[t]{5}{*}{$4 f^{12}\left({ }^{3} \mathrm{H}\right) 6 s 6 d ?$} & & 4 & 39237.878 & 1.040 & & \\
\hline & & 6 & 39256.456 & 1.140 & & \\
\hline & & 6 & 39350.505 & 1.155 & & \\
\hline & & 7 & 39359.683 & 1.175 & & \\
\hline & & 8 & 39432.657 & 1.160 & & \\
\hline
\end{tabular}


Er I, Even Parity_Continued

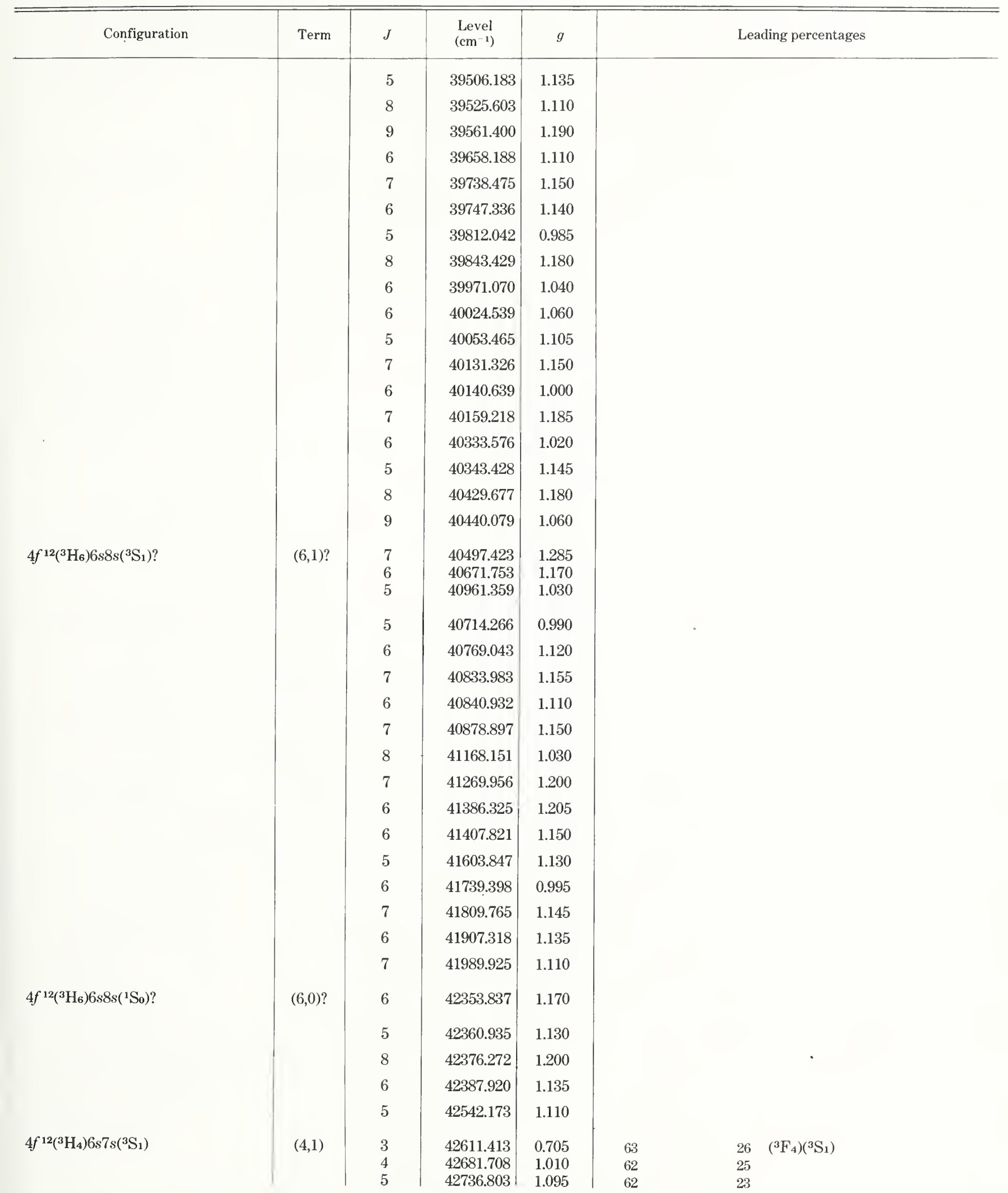


Er I, Even Parity_Continued

\begin{tabular}{|c|c|c|c|c|c|c|}
\hline Configuration & Term & $J$ & $\begin{array}{l}\text { Level } \\
\left(\mathrm{cm}^{-1}\right)\end{array}$ & $g$ & & Leading percentages \\
\hline & & 6 & 42627.566 & 1.165 & & \\
\hline & & 8 & 42632.533 & 1.170 & & \\
\hline & & 7 & 42671.747 & 1.130 & & \\
\hline & & 8 & 42797.550 & 1.130 & & \\
\hline & & 9 & 42806.697 & 1.200 & & \\
\hline & & 6 & 42882.350 & 1.110 & & \\
\hline & & 6 & 43191.617 & 1.105 & & \\
\hline & & 6 & 43298.210 & 1.135 & & \\
\hline & & 7 & 43518.518 & 1.205 & & \\
\hline & & 8 & 43537.586 & 1.140 & & \\
\hline & & 8 & 43586.058 & 1.130 & & \\
\hline & & 7 & 43826.668 & 1.090 & & \\
\hline & & 6 & 43870.496 & 1.170 & & \\
\hline & & 6 & 43934.136 & 1.255 & & \\
\hline & & 9 & 43982.879 & 1.215 & & \\
\hline & & 7 & 44039.685 & 1.160 & & \\
\hline & & 8 & 44041.430 & 1.135 & & \\
\hline & & 10 & 44112.202 & 1.095 & & \\
\hline & & 6 & 44194.771 & 1.190 & & \\
\hline & & 8 & 44201.047 & 1.030 & & \\
\hline & & 8 & 44301.508 & 1.120 & & \\
\hline \multirow[t]{7}{*}{$4 f^{12}\left({ }^{3} \mathrm{~F}_{3}\right) 6 s 7 s\left({ }^{3} \mathrm{~S}_{1}\right)$} & $(3,1)$ & 3 & 44308.935 & 1.150 & 93 & \\
\hline & & $\begin{array}{l}2 \\
4\end{array}$ & 44347.866 & 0.870 & 93 & \\
\hline & & 7 & 44394.215 & 1.125 & & \\
\hline & & 8 & 44410.891 & 1.150 & & \\
\hline & & 9 & 44525.705 & 1.140 & & \\
\hline & & 7 & 44827.907 & 1.100 & & \\
\hline & & 12 & 44872.599 & 1.190 & & \\
\hline \multirow[t]{7}{*}{$4 f^{12}\left({ }^{3} \mathrm{~F}_{2}\right) 6 s 7 s\left({ }^{3} \mathrm{~S}_{1}\right)$} & $(2,1)$ & 1 & 44886.482 & 0.105 & 82 & $17 \quad\left({ }^{1} \mathrm{D}_{2}\right)\left({ }^{3} \mathrm{~S}_{1}\right)$ \\
\hline & & 2 & 45060.489 & 0.850 & 75 & 16 \\
\hline & & 年 & $402+0.001$ & 1.100 & 13 & \\
\hline & & 11 & 45195.732 & 1.195 & & \\
\hline & & 7 & 45325.988 & 1.125 & & \\
\hline & & 7 & 45874.406 & 1.050 & & \\
\hline & & 7 & 46970.808 & 1.145 & & \\
\hline Er II $\left({ }^{3} \mathrm{H}_{6}\right) 6 s_{1 / 2}\left(6,{ }^{1} / 2\right)_{13 / 2}$ & Limit & & 49262 & & & \\
\hline
\end{tabular}


Er I, Odd Parity

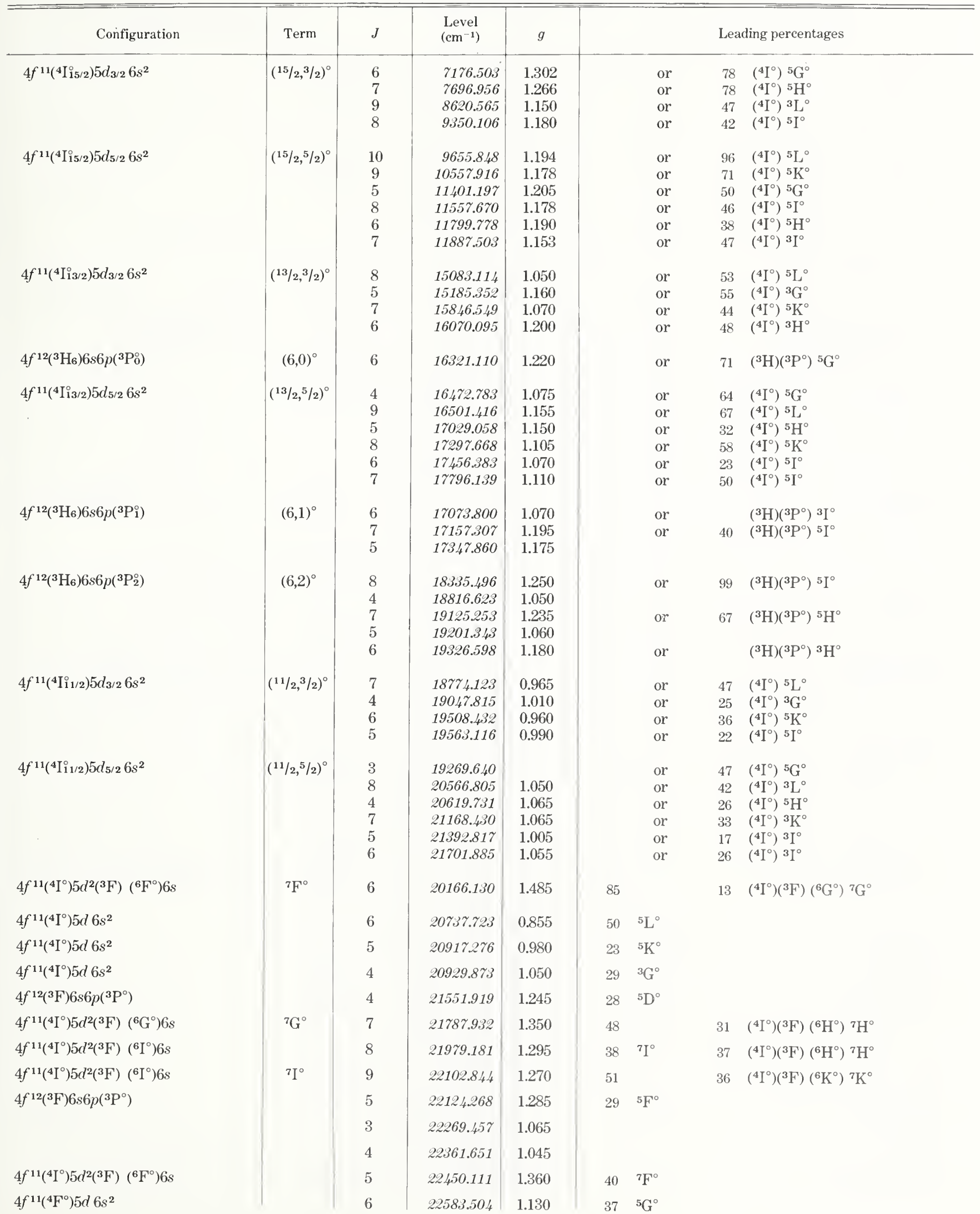


Er I, Odd Parity-Continued

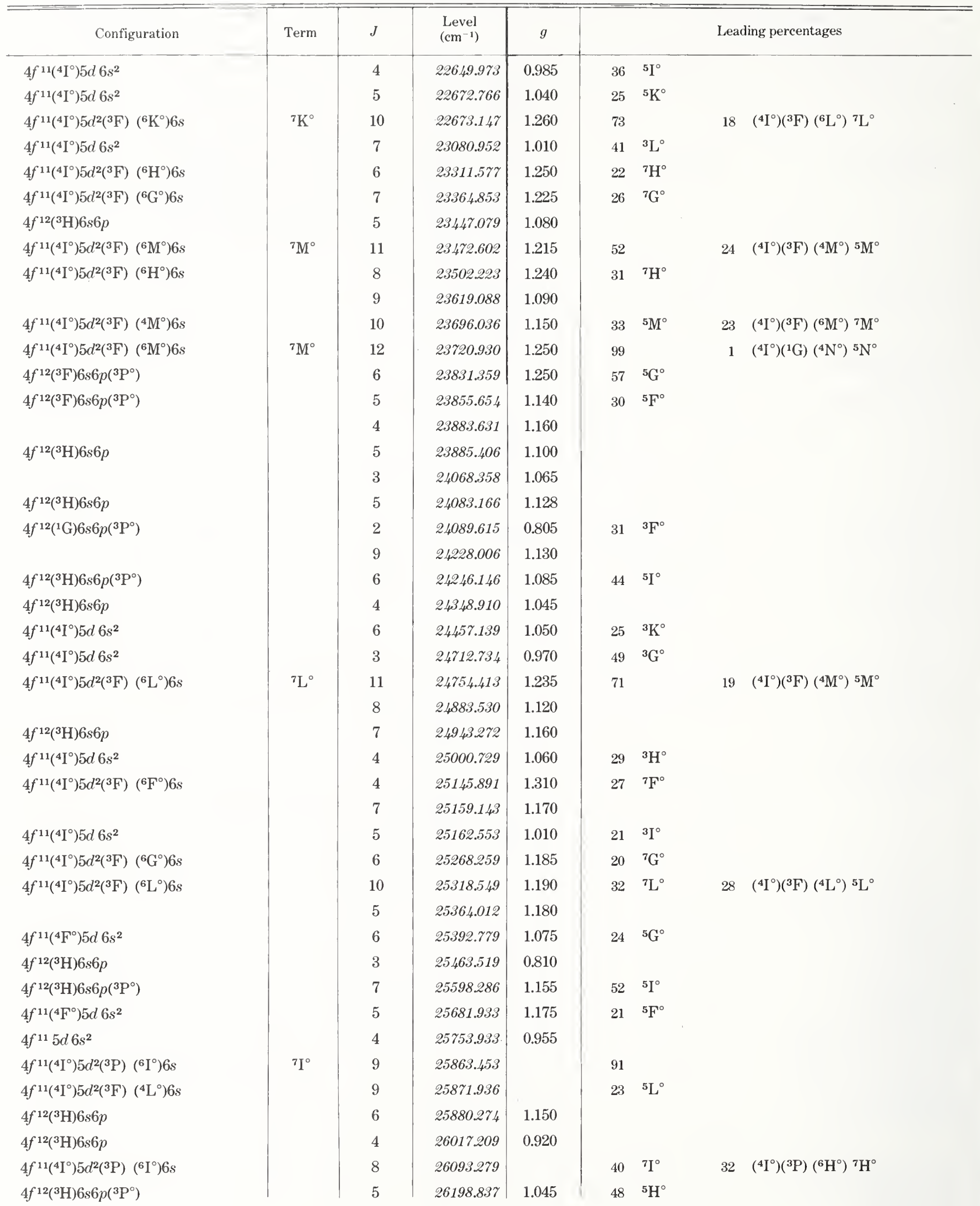


Er I, Odd Parity-Continued

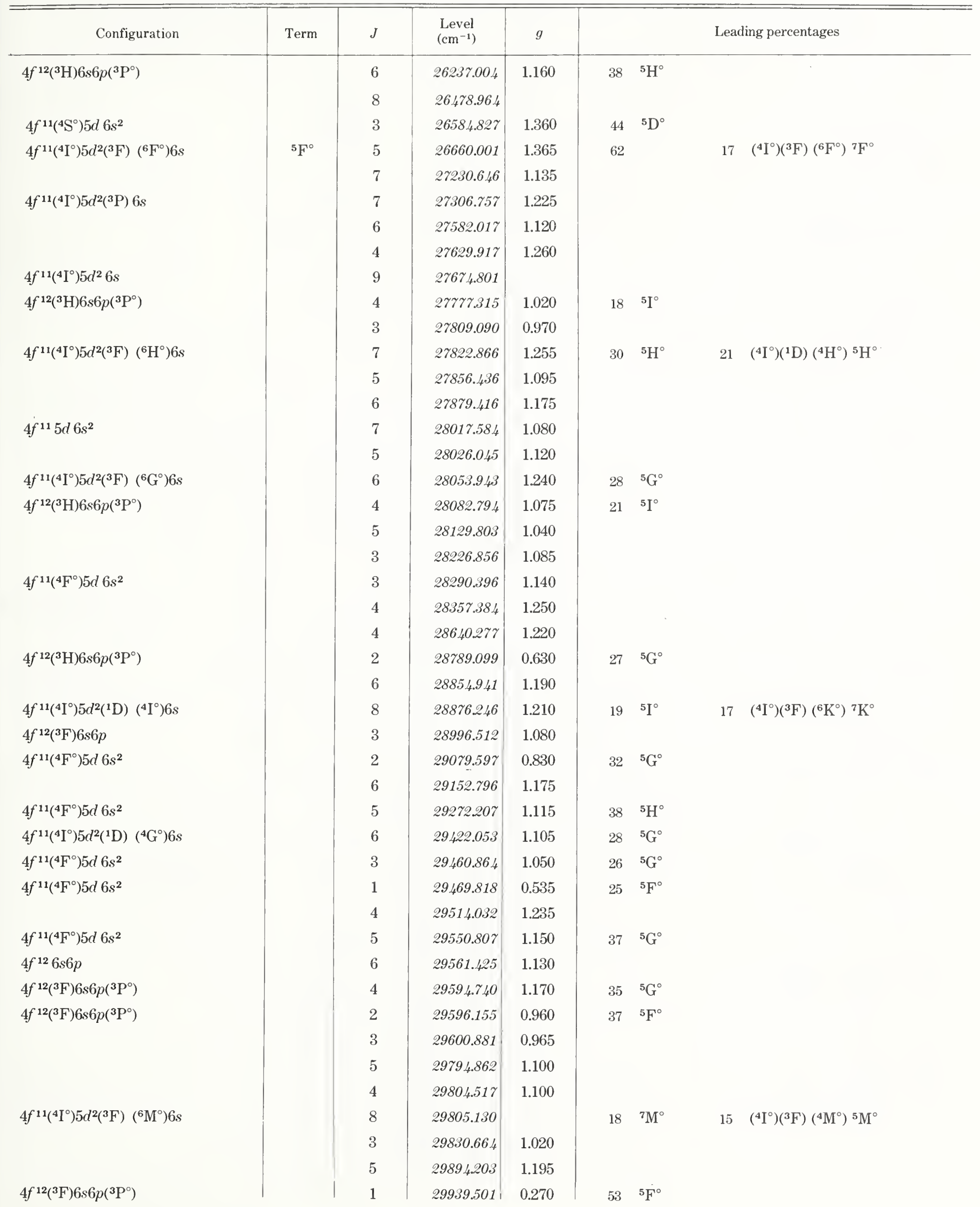


Er I, Odd Parity-Continued

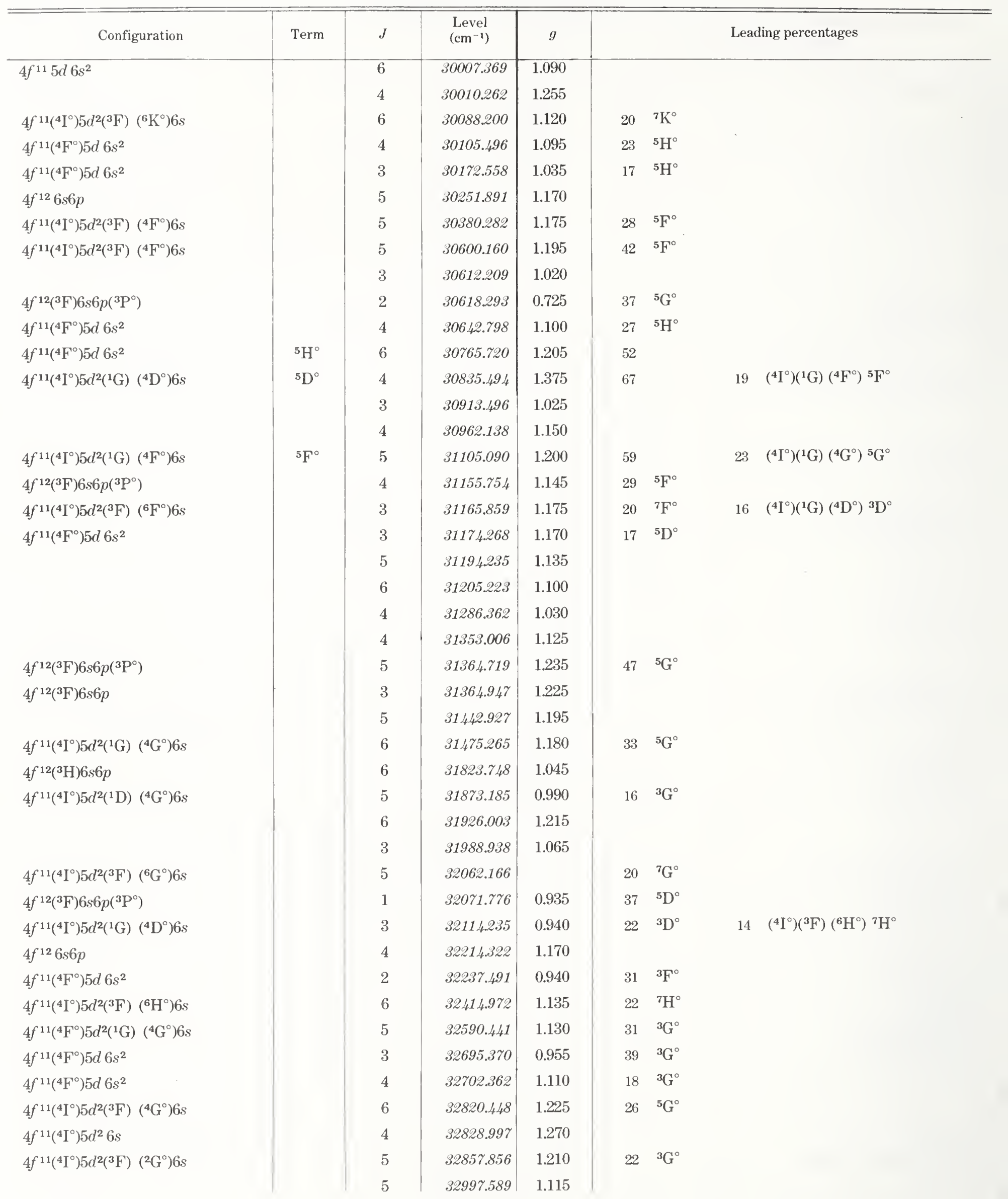


Er I, Odd Parity-Continued

\begin{tabular}{|c|c|c|c|c|c|c|}
\hline Configuration & Term & $J$ & $\begin{array}{l}\text { Level } \\
\left(\mathrm{cm}^{-1}\right)\end{array}$ & $g$ & & Leading percentages \\
\hline \multirow[t]{2}{*}{$4 f^{11}\left({ }^{4} I^{\circ}\right) 5 d^{2}\left({ }^{3} F\right)\left({ }^{4} F^{\circ}\right) 6 s$} & & 3 & 37075.570 & 1.150 & $23{ }^{5} \mathrm{~F}^{\circ}$ & $19\left({ }^{4} I^{\circ}\right)\left({ }^{1} G\right)\left({ }^{4} D^{\circ}\right){ }^{5} D^{\circ}$ \\
\hline & & 4 & 37083.198 & 0.970 & & \\
\hline \multirow[t]{22}{*}{$4 f^{12}\left({ }^{1} \mathrm{G}\right) 6 s 6 p\left({ }^{3} \mathrm{P}^{\circ}\right)$} & & 3 & 37125.206 & 0.935 & $26{ }^{3} \mathrm{~F}^{\circ}$ & · \\
\hline & & 4 & 37210.947 & 1.140 & & \\
\hline & & 5 & 37249.090 & & & \\
\hline & & 4 & 37265.129 & 1.050 & & \\
\hline & & -3 & 37289.592 & 1.090 & & \\
\hline & & 4 & 37319.434 & 1.035 & & \\
\hline & & 6 & 37323.593 & 1.235 & & \\
\hline & & 8 & 37361.513 & 1.210 & & \\
\hline & & 5 & 37398.878 & & & \\
\hline & & 6 & 37435.116 & 1.265 & & \\
\hline & & 3 & 37492.247 & 1.105 & & \\
\hline & & 5 & 37535.325 & 1.125 & & \\
\hline & & 7 & 37567.222 & 1.195 & & \\
\hline & & 4 & 37582.298 & 1.045 & & \\
\hline & & 8 & 37621.885 & 1.155 & & \\
\hline & & 7 & 37637.311 & 1.130 & & \\
\hline & & 3 & 37649.344 & 0.895 & & \\
\hline & & 6 & 37745.276 & 1.070 & & \\
\hline & & 5 & 37778.256 & 1.065 & & \\
\hline & & 4 & 37853.673 & 1.015 & & \\
\hline & & 4 & 37893.246 & 1.025 & & \\
\hline & & 4 & 37970.621 & 1.020 & & \\
\hline \multirow[t]{20}{*}{$4 f^{11}\left({ }^{4} \mathrm{G}^{\circ}\right) 5 d 6 s^{2}$} & & 2 & 37989.003 & 1.120 & $24{ }^{5} \mathrm{D}^{\circ}$ & \\
\hline & & 5 & 38054.883 & 1.165 & & \\
\hline & & 2 & 38168.987 & 1.086 & & \\
\hline & & 7 & 38175.040 & 1.135 & & \\
\hline & & 5 & 38175.430 & 1.115 & & \\
\hline & & 4 & 38183.254 & 1.110 & & \\
\hline & & 2 & 38263.263 & 1.085 & & \\
\hline & & 8 & 38344.055 & 1.170 & & \\
\hline & & 4 & 38376.825 & 1.190 & & \\
\hline & & 7 & 38377.547 & 1.115 & & \\
\hline & & 3 & 38397.715 & 0.960 & & \\
\hline & & 2 & 38400.908 & 0.880 & & \\
\hline & & 6 & 38410.587 & 1.120 & & \\
\hline & & 5 & 38415.612 & 1.135 & & \\
\hline & & 4 & 38487.704 & 1.090 & & \\
\hline & & 8 & 38527.562 & 1.120 & & \\
\hline & & 7 & 38580.582 & 1.045 & & \\
\hline & & 3 & 38667.387 & 1.025 & & \\
\hline & & 7 & 38724.252 & 1.165 & & \\
\hline & & 3 & 38836.476 & 0.970 & & \\
\hline
\end{tabular}


Er I, Odd Parity-Continued

\begin{tabular}{|c|c|c|c|c|c|}
\hline Configuration & Term & $J$ & $\begin{array}{l}\text { Level } \\
\left(\mathrm{cm}^{-1}\right)\end{array}$ & $g$ & Leading percentages \\
\hline & & 8 & 38974.873 & 1.085 & \\
\hline & & 4 & 38982.003 & 1.075 & \\
\hline & & 7 & 39009.204 & 1.030 & \\
\hline & & 7 & 39221.603 & 1.070 & \\
\hline & & 8 & 39225.503 & 1.055 & \\
\hline & & 3 & 39467.934 & 1.155 & \\
\hline & & 6 & 39535.919 & 1.150 & \\
\hline & & 6 & 39608.064 & 1.135 & \\
\hline & & 3 & 39688.151 & 1.105 & \\
\hline & & 7 & 39702.568 & 1.130 & \\
\hline & & 7 & 39855.032 & 1.210 & \\
\hline & & 5 & 40137.476 & 1.170 & \\
\hline & & 7 & 40149.125 & 1.115 & \\
\hline & & 4 & 40155.872 & 1.130 & \\
\hline & & 2 & 40202.083 & 1.100 & \\
\hline & & 7 & 40751.909 & 1.050 & \\
\hline & & 6 & 40859.924 & 1.140 & \\
\hline & & 8 & 40877.166 & 1.120 & \\
\hline & & 6 & 41101.214 & 1.255 & \\
\hline & & 8 & 41382.319 & 1.135 & \\
\hline & & 7 & 41432.910 & 1.135 & \\
\hline & & 6 & 41445.371 & 1.050 & . \\
\hline & & 5 & 41516.515 & 1.130 & \\
\hline $4 f^{11}\left({ }^{4} \mathrm{I}^{\circ}\right) 5 d 6 s 7 s$ & & 7 & 41794.387 & 1.400 & \\
\hline $4 f^{11}\left({ }^{4} I^{\circ}\right) 5 d 6 s 7 s$ & & 6 & 42153.614 & 1.225 & \\
\hline \multirow[t]{7}{*}{$4 f^{11}\left({ }^{4} I^{\circ}\right) 5 d 6 s 7 s$} & & 8 & 42360.312 & 1.340 & \\
\hline & & 8 & 43040.275 & 1.180 & \\
\hline & & 7 & 43056.149 & 1.150 & \\
\hline & & 9 & 43201.126 & 1.165 & \\
\hline & & 8 & 43373.966 & 1.160 & \\
\hline & & 7 & 43575.279 & 1.140 & \\
\hline & & 8 & 43607.066 & 1.140 & \\
\hline $4 f^{11}\left({ }^{4} \mathrm{I}^{\circ}\right) 5 d 6 s 7 s$ & & 10 & 43981.088 & 1.220 & \\
\hline \multirow[t]{3}{*}{$4 f^{11}\left({ }^{4} \mathrm{I}^{\circ}\right) 5 d 6 s 7 s$} & & 9 & 44012.041 & 1.175 & \\
\hline & & 9 & 44217.464 & 1.115 & \\
\hline & & 7 & 44247.852 & 1.125 & \\
\hline $4 f^{11}\left({ }^{4} I^{\circ}\right) 5 d 6 s 7 s$ & & 8 & 44270.569 & 1.045 & \\
\hline $4 f^{11}\left({ }^{4} I^{\circ}\right) 5 d 6 s 7 s$ & & 10 & 44379.781 & 1.195 & \\
\hline $\operatorname{Er} \amalg\left({ }^{3} \mathrm{H}_{6}\right) 6 s_{1 / 2} \quad\left(6,{ }^{1} / 2\right)_{13 / 2}$ & Limit & & 49262 & & \\
\hline
\end{tabular}


(Ho I sequence; 67 electrons)

Ground state $\left(1 s^{2} 2 s^{2} 2 p^{6} 3 s^{2} 3 p^{6} 3 d^{10} 4 s^{2} 4 p^{6} 4 d^{10} 5 s^{2} 5 p^{6}\right) 4 f^{12}\left({ }^{3} \mathrm{H}_{6}\right) 6 s(6,1 / 2)_{13 / 2}$

Ionization energy $96200 \pm 600 \mathrm{~cm}^{-1}$

$11.93 \pm 0.08 \mathrm{eV}$

Identified even configurations

$4 f^{12} 6 s, 4 f^{12} 5 d, 4 f^{11} 6 s 6 p, 4 f^{11} 5 d 6 p$

Identified odd configurations

$4 f^{11} 6 s^{2}, 4 f^{11} 5 d 6 s, 4 f^{11} 5 d^{2} ?, 4 f^{12} 6 p$

\section{Energy Levels, Wavelengths, $g$ Values, Ionization Energy}

McNally and Vander Sluis [1959] used the Zeeman data of Lindner and Davis [1958] in their first analysis of Er II. They identified the lowest levels as belonging to $4 f^{12}\left({ }^{3} \mathrm{H}\right) 6 \mathrm{~s}$ and $4 f^{12}\left({ }^{3} \mathrm{~F}\right) 6 \mathrm{~s}$ terms, and found more than forty upper odd levels. The wavelengths given in Marquet's thesis [see Er I] were adopted in the succeeding investigations of Er II by Marquet and Davis [1965] and Vander Sluis and McNally [1970]. The references to several Er line lists described in the text for Er I need not be repeated here; additional published lists for some of the classified Er II lines are given by Vander Sluis and McNally (3172-4385 $\AA$ ) and Spector (7367-11238 $\AA$ ) [1971a]. Vander Sluis and McNally also gave the most complete published tables of energy levels and Zeeman data. Spector [1971b] later found the $4 f^{12}\left({ }^{3} \mathrm{H}_{6}\right) 5 d$ levels as comprising two $J_{1} j$-coupling terms.

The levels here are from an unpublished extension of the analysis by van Kleef and Koot [1975], which is still in progress. Many new levels of both parities have been located; about 120 even levels and 250 odd levels are now known. Most of the $g$ values are also from van Kleef and Koot, who give the values to the nearest 0.005 units. The $g$ values for several of the lower even levels are averages from the measurements of Vander Sluis and McNally, Lindner and Davis, and van Kleef and Koot; the estimated errors in the $g$-value determinations by Vander Sluis and McNally for some of these levels are significantly less than 0.005 units.

Sugar and Reader derived the ionization energy.

\section{Theoretical Interpretation}

The levels of the $4 f^{12} 6 s$ ground configuration are paired in $J_{1} j$-coupling terms. The $L S$ designations of the $4 f^{12}$ parent levels are nominal; the "real core" wavefunctions for some of the parent levels have very mixed LS compositions [Judd and Marquet, 1962; Goldschmidt, 1963]. The lowest $4 f^{12} 5 d$ levels are also grouped here into three $J_{1} j$-coupling terms [Spector, 1971b; van Kleef and Koot, 1975]. Leading percentages in the LS-coupling scheme are given for the $4 f^{12} 6 s$ and $4 f^{12} 5 d$ levels, the $L S$ percentages for levels of $J_{1} j$-coupling terms being preceded by the word "or." The percentages are from a calculation by Wyart and Koot [1975] that included the interaction between the two configurations.

Van Kleef and Koot have established that many of the even levels above $32000 \mathrm{~cm}^{-1}$ belong to the $4 f^{11} 6 s 6 p$ and $4 f^{11} 5 d 6 p$ configurations. The assignments for the low $J_{1} J_{2}$-coupling terms of $4 f^{11} 6 s 6 p$ and the other assignments to these configurations are from their recent work; no calculation of the even levels in this region is yet available [van Kleef and Koot, 1975].

The discovery and identification of low odd levels belonging to the $4 f^{11} 6 s^{2}{ }^{4} \mathrm{I}^{\circ}$ term and to the $4 f^{11}\left({ }^{4} I^{\circ}\right) 5 d 6 s$ subconfiguration is a notable part of the analysis by van Kleef and Koot. They have also identified levels of the $4 f^{12} 6 p$ configuration, which overlaps the $4 f^{11}(5 d+6 s)^{2}$ group above $25000 \mathrm{~cm}^{-1}$. The assignments of the odd levels are from their analysis. More definite term identifications will be possible after the completion of calculations for the four odd configurations already known; it can be anticipated, however, that the effects of intermediate coupling and configuration interaction will result in low eigenvector purities for many of these levels. 


\section{References}

Goldschmidt, Z. B., J. Opt. Soc. Am. 53, 594 (1963). PT

Judd, B. R., and Marquet, L. C., J. Opt. Soc. Am. 52, 504 (1962). PT

Lindner, J. W., and Davis, S. P., J. Opt. Soc. Am. 48, 542 (1958). W ZE

Marquet, L. C., and Davis, S. P., J. Opt. Soc. Am. 55, 471 (1965). EL ZE

MeNally, Jr., J. R., and Vander Sluis, K. L., J. Opt. Soc. Am. 49, 200 (1959). EL ZE

Spector, N., Astrophys. J. 167, 205 (1971a). EL CL

Spector, N., J. Opt. Soc. Am. 61, 672 (1971b). EL

Spector, N., and Held, S., Astrophys. J. 167, 193 (1971). W

Sugar, J., and Reader, J., J. Opt. Soc. Am. 55, 1286 (1965). IP

van Kleef, T. A. M., and Koot, J. J. A., unpublished material (1975). EL ND CL ZE

Vander Sluis, K. L., and MeNally, Jr., J. R., J. Opt. Soc. Am. 60, 94 (1970). EL CL ZE

Wyart, J. F., and Koot, J. J. A., unpublished material (1975). PT

[May 1977]

Er II, Even Parity

\begin{tabular}{|c|c|c|c|c|c|c|c|c|}
\hline Configuration & Term & $J$ & $\begin{array}{l}\text { Level } \\
\left(\mathrm{cm}^{-1}\right)\end{array}$ & $g$ & \multicolumn{4}{|c|}{ Leading percentages } \\
\hline $4 f^{12}\left({ }^{3} \mathrm{H}_{6}\right) 6 s_{1 / 2}$ & $(6,1 / 2)$ & $\begin{array}{l}13 / 2 \\
11 / 2\end{array}$ & $\begin{array}{r}0.000 \\
440.434\end{array}$ & $\begin{array}{l}1.230 \\
1.101\end{array}$ & & $\begin{array}{l}\text { or } \\
\text { or }\end{array}$ & $\begin{array}{l}99 \\
67\end{array}$ & $\begin{array}{l}{ }^{4} \mathrm{H} \\
{ }^{2} \mathrm{H}\end{array}$ \\
\hline $4 f^{12}\left({ }^{3} \mathrm{~F}_{4}\right) 6 s_{1 / 2}$ & $(4,1 / 2)$ & $\begin{array}{l}9 / 2 \\
7 / 2\end{array}$ & $\begin{array}{l}5132.608 \\
5403.688\end{array}$ & $\begin{array}{l}1.245 \\
1.042\end{array}$ & & $\begin{array}{l}\text { or } \\
\text { or }\end{array}$ & $\begin{array}{l}65 \\
43\end{array}$ & $\begin{array}{l}{ }^{4} \mathrm{~F} \\
{ }^{2} \mathrm{~F}\end{array}$ \\
\hline $4 f^{12\left({ }^{3} \mathrm{H}_{5}\right) 6 s_{1 / 2}}$ & $(5,1 / 2)$ & $\begin{array}{c}11 / 2 \\
9 / 2\end{array}$ & $\begin{array}{l}7149.630 \\
7195.355\end{array}$ & $\begin{array}{l}1.120 \\
0.948\end{array}$ & & $\begin{array}{l}\text { or } \\
\text { or }\end{array}$ & $\begin{array}{l}68 \\
64\end{array}$ & $\begin{array}{l}{ }^{4} \mathrm{H} \\
{ }^{4} \mathrm{H}\end{array}$ \\
\hline $4 f^{12}\left({ }^{3} \mathrm{H}_{4}\right) 6 s_{1 / 2}$ & $(4,1 / 2)$ & $\begin{array}{l}7 / 2 \\
9 / 2\end{array}$ & $\begin{array}{l}10893.936 \\
11042.640\end{array}$ & $\begin{array}{l}0.833 \\
1.050\end{array}$ & & $\begin{array}{l}\text { or } \\
\text { or }\end{array}$ & $\begin{array}{l}61 \\
41\end{array}$ & $\begin{array}{l}{ }^{4} \mathrm{H} \\
{ }^{2} \mathrm{H}\end{array}$ \\
\hline $4 f^{\mathbf{1 2}}\left({ }^{3} \mathrm{~F}_{3}\right) 6 s_{1 / 2}$ & $\left(3,{ }^{1} / 2\right)$ & $\begin{array}{l}7 / 2 \\
5 / 2\end{array}$ & $\begin{array}{l}12587.998 \\
12600.093\end{array}$ & $\begin{array}{l}1.204 \\
1.000\end{array}$ & & $\begin{array}{l}\text { or } \\
\text { or }\end{array}$ & $\begin{array}{l}68 \\
79\end{array}$ & $\begin{array}{l}{ }^{4} \mathrm{~F} \\
{ }^{4} \mathrm{~F}\end{array}$ \\
\hline $4 f^{12}\left({ }^{3} \mathrm{~F}_{2}\right) 6 s_{1 / 2}$ & $(2,1 / 2)$ & $\begin{array}{l}3 / 2 \\
5 / 2\end{array}$ & $\begin{array}{l}13188.467 \\
13558.333\end{array}$ & $\begin{array}{l}0.487 \\
0.948\end{array}$ & & $\begin{array}{l}\text { or } \\
\text { or }\end{array}$ & $\begin{array}{l}80 \\
69\end{array}$ & $\begin{array}{l}{ }^{4} \mathrm{~F} \\
{ }^{2} \mathrm{~F}\end{array}$ \\
\hline $4 f^{12}\left({ }^{3} \mathrm{H}_{6}\right) 5 d_{3 / 2}$ & $\left(6,{ }^{3} / 2\right)$ & $\begin{array}{l}9 / 2 \\
15 / 2 \\
11 / 2 \\
13 / 2\end{array}$ & $\begin{array}{l}16552.871 \\
18617.495 \\
18889.101 \\
20728.050\end{array}$ & $\begin{array}{l}1.315 \\
1.075 \\
1.255 \\
1.140\end{array}$ & & $\begin{array}{l}\text { or } \\
\text { or } \\
\text { or } \\
\text { or }\end{array}$ & $\begin{array}{l}83 \\
58 \\
85 \\
35\end{array}$ & $\begin{array}{l}\left({ }^{3} \mathrm{H}\right){ }^{4} \mathrm{~F} \\
\left({ }^{3} \mathrm{H}\right){ }^{2} \mathrm{~K} \\
\left({ }^{3} \mathrm{H}\right){ }^{4} \mathrm{G} \\
\left({ }^{3} \mathrm{H}\right){ }^{4} \mathrm{H}\end{array}$ \\
\hline $4 f^{12}\left({ }^{3} \mathrm{H}_{6}\right) 5 d_{5 / 2}$ & $(6,5 / 2)$ & $\begin{array}{c}17 / 2 \\
7 / 2 \\
15 / 2 \\
11 / 2 \\
9 / 2 \\
13 / 2\end{array}$ & $\begin{array}{l}19302.832 \\
19903.107 \\
21533.153 \\
21697.852 \\
21894.055 \\
22141.355\end{array}$ & $\begin{array}{l}1.175 \\
1.185 \\
1.185 \\
1.100 \\
1.165 \\
1.145\end{array}$ & & $\begin{array}{l}\text { or } \\
\text { or } \\
\text { or } \\
\text { or } \\
\text { or } \\
\text { or }\end{array}$ & $\begin{array}{l}99 \\
51 \\
86 \\
54 \\
41 \\
42\end{array}$ & $\begin{array}{l}\left({ }^{3} \mathrm{H}\right){ }^{4} \mathrm{~K} \\
\left({ }^{3} \mathrm{H}\right){ }^{4} \mathrm{~F} \\
\left({ }^{3} \mathrm{H}\right){ }^{4} \mathrm{I} \\
\left({ }^{3} \mathrm{H}\right){ }^{2} \mathrm{H} \\
\left({ }^{3} \mathrm{H}\right){ }^{4} \mathrm{G} \\
\left({ }^{3} \mathrm{H}\right){ }^{4} \mathrm{H}\end{array}$ \\
\hline $4 f^{12}\left({ }^{3} \mathrm{~F}_{4}\right) 5 d_{3 / 2}$ & $(4,3 / 2)$ & $\begin{array}{c}5 / 2 \\
7 / 2 \\
9 / 2 \\
11 / 2\end{array}$ & $\begin{array}{l}23883.022 \\
23962.378 \\
24563.288 \\
24745.034\end{array}$ & $\begin{array}{l}1.105 \\
1.195 \\
1.140 \\
1.085\end{array}$ & & $\begin{array}{l}\text { or } \\
\text { or } \\
\text { or } \\
\text { or }\end{array}$ & $\begin{array}{l}22 \\
20 \\
24 \\
26\end{array}$ & $\begin{array}{l}\left({ }^{1} \mathrm{G}\right){ }^{2} \mathrm{~F} \\
\left({ }^{3} \mathrm{~F}\right){ }^{4} \mathrm{~F} \\
\left({ }^{3} \mathrm{~F}\right){ }^{4} \mathrm{G} \\
\left({ }^{3} \mathrm{~F}\right){ }^{4} \mathrm{G}\end{array}$ \\
\hline $4 f^{12}\left({ }^{3} \mathrm{H}\right) 5 d$ & & $7 / 2$ & 25041.268 & 1.130 & 28 & ${ }^{2} \mathrm{~F}$ & & \\
\hline $4 f^{12}\left({ }^{3} \mathrm{H}\right) 5 d$ & & $13 / 2$ & 25532.352 & & 63 & ${ }^{4} \mathrm{~K}$ & & \\
\hline $4 f^{12}\left({ }^{3} \mathrm{~F}\right) 5 d$ & & $11 / 2$ & 25586.376 & 1.095 & 38 & ${ }^{4} \mathrm{G}$ & & \\
\hline $4 f^{12}\left({ }^{3} \mathrm{~F}\right) 5 d$ & & $9 / 2$ & 26131.105 & 1.155 & 33 & ${ }^{4} \mathrm{~F}$ & & \\
\hline $4 f^{12}\left({ }^{3} \mathrm{~F}\right) 5 d$ & & $13 / 2$ & 26414.450 & 1.125 & 41 & ${ }^{4} \mathrm{H}$ & & \\
\hline $4 f^{12}\left({ }^{3} \mathrm{H}\right) 5 d$ & & $7 / 2$ & 26949.099 & 1.060 & 24 & ${ }^{4} \mathrm{~F}$ & & \\
\hline $4 f^{12}\left({ }^{3} \mathrm{H}\right) 5 d$ & & $11 / 2$ & 27540.195 & 1.030 & 43 & ${ }^{4} \mathrm{I}$ & & \\
\hline
\end{tabular}


Er II, Even Parity-Continued

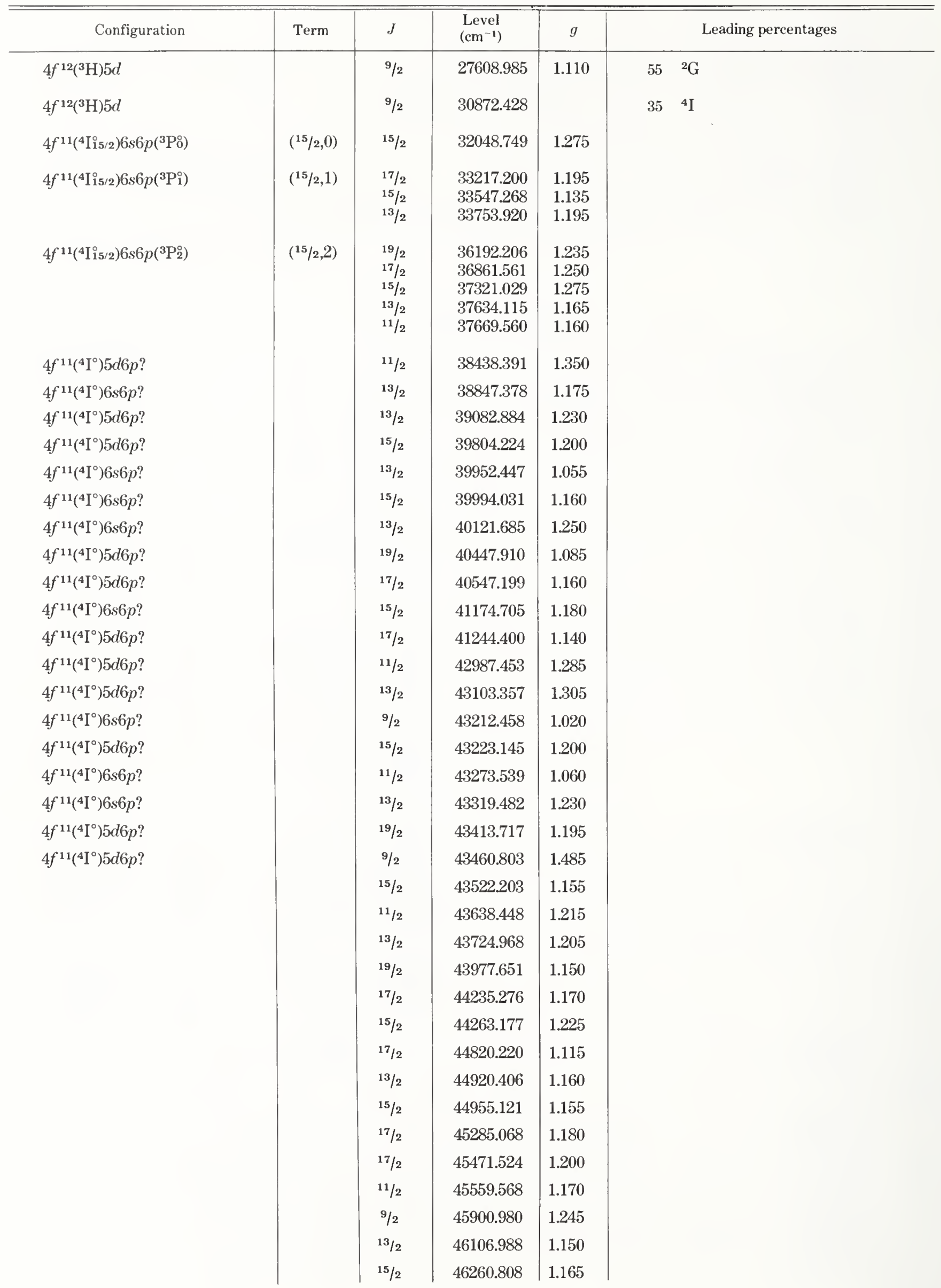


Er II, Even Parity-Continued

Er II, Even Parity-Continued

\begin{tabular}{|c|c|c|c|c|c|c|c|c|c|}
\hline Configuration. & Term & $J$ & $\begin{array}{l}\text { Level } \\
\left(\mathrm{cm}^{-1}\right)\end{array}$ & $g$ & Configuration & Term & $J$ & $\begin{array}{l}\text { Level } \\
\left(\mathrm{cm}^{-1}\right)\end{array}$ & $g$ \\
\hline & & $11 / 2$ & 46525.882 & 1.195 & & & $15 / 2$ & 49660.227 & 1.195 \\
\hline & & $21 / 2$ & 46560.615 & 1.265 & & & $13 / 2$ & 50115.664 & 1.150 \\
\hline & & $15 / 2$ & 46757.391 & 1.220 & & & $15 / 2$ & 50246.395 & 1.080 \\
\hline & & $19 / 2$ & 46757.780 & 1.200 & & & $11 / 2$ & 50387.772 & 1.160 \\
\hline & & $17 / 2$ & 46915.510 & 1.050 & & & $15 / 2$ & 50451.958 & 1.135 \\
\hline & & $13 / 2$ & 47050.810 & 1.220 & & & $13 / 2$ & 50457.727 & 1.030 \\
\hline & & $15 / 2$ & 47162.334 & 1.075 & & & $13 / 2$ & 50850.345 & 1.015 \\
\hline & & $17 / 2$ & 47174.273 & 1.205 & & & $15 / 2$ & 51010.412 & 1.040 \\
\hline & & $19 / 2$ & 47426.638 & 1.215 & & & $11 / 2$ & 51037.663 & 1.070 \\
\hline & & $15 / 2$ & 47466.969 & 1.190 & & & $15 / 2$ & 51103.871 & 1.100 \\
\hline & & $19 / 2$ & 47786.901 & 1.155 & & & $13 / 2$ & 51574.835 & 1.075 \\
\hline & & $13 / 2$ & 47840.962 & 1.125 & & & $15 / 2$ & 51606.662 & 1.045 \\
\hline & & $11 / 2$ & 47847.615 & 1.050 & & & $13 / 2$ & 52247.929 & 0.980 \\
\hline & & $15 / 2$ & 48151.388 & 1.075 & & & $17 / 2$ & 52392.832 & 1.135 \\
\hline & & $13 / 2$ & 48525.666 & 1.165 & & & $15 / 2$ & 52996.133 & 1.130 \\
\hline & & $9 / 2$ & 48607.416 & 1.195 & & & $13 / 2$ & 53891.761 & 1.120 \\
\hline & & $17 / 2$ & 48676.243 & 1.205 & & & $13 / 2$ & 54387.255 & 1.175 \\
\hline & & $19 / 2$ & 48788.988 & 1.230 & & & $15 / 2$ & 55316.342 & 1.145 \\
\hline & & $13 / 2$ & 49005.981 & 1.185 & & & & & \\
\hline & & $13 / 2$ & 49091.098 & 1.035 & & & & & \\
\hline & & $15 / 2$ & 49378.255 & 1.175 & $\operatorname{Er}$ III $\left({ }^{3} \mathrm{H}_{6}\right)$ & Limit & & 96200 & \\
\hline
\end{tabular}

Er II, Odd Parity

Er II, Odd Parity

\begin{tabular}{|c|c|c|c|c|c|c|c|c|c|}
\hline Configuration & Term & $J$ & $\begin{array}{l}\text { Level } \\
\left(\mathrm{cm}^{-1}\right)\end{array}$ & $g$ & Configuration & Term & $J$ & $\begin{array}{l}\text { Level } \\
\left(\mathrm{cm}^{-1}\right)\end{array}$ & $g$ \\
\hline \multirow[t]{3}{*}{$4 f^{11} 6 s^{2}$} & \multirow[t]{19}{*}{${ }^{4} I^{\circ}$} & $15 / 2$ & 6824.774 & 1.200 & $4 f^{11}\left({ }^{4} I^{\circ}\right) 5 d 6 s$ & & $11 / 2$ & 17063.735 & 1.150 \\
\hline & & $\begin{array}{l}13 / 2 \\
11 / 2\end{array}$ & 13338.777 & 1.120 & $4 f^{11}\left({ }^{4} \mathrm{I}^{\circ}\right) 5 d 6 \mathrm{~s}$ & & $13 / 2$ & 17378.917 & 1.135 \\
\hline & & $9 / 2$ & & & $4 f^{11}\left({ }^{4} \mathrm{I}^{\circ}\right) 5 d 6 s$ & & $17 / 2$ & 17842.682 & 1.140 \\
\hline $4 f^{11}\left({ }^{4} I^{\circ}\right) 5 d 6 s$ & & $13 / 2$ & 10667.186 & 1.345 & $4 f^{11}\left({ }^{4} \mathrm{I}^{\circ}\right) 5 d 6 s$ & & $15 / 2$ & 18463.347 & 1.180 \\
\hline $4 f^{11}\left({ }^{4} \mathrm{I}^{\circ}\right) 5 d 6 s$ & & $15 / 2$ & 11309.180 & 1.285 & $4 f^{11}\left({ }^{4} I^{\circ}\right) 5 d 6 s$ & & $15 / 2$ & 19375.584 & 1.020 \\
\hline $4 f^{11}\left({ }^{4} I^{\circ}\right) 5 d 6 s$ & & $11 / 2$ & 12388.090 & 1.240 & $4 f^{11}\left({ }^{4} I^{\circ}\right) 5 d 6 s$ & & $17 / 2$ & 19606.715 & 1.035 \\
\hline $4 f^{11}\left({ }^{4} \mathrm{I}^{\circ}\right) 5 d 6 s$ & & $19 / 2$ & 12815.068 & 1.180 & $4 f^{11\left({ }^{4} I^{\circ}\right) 5 d 6 s}$ & & $9 / 2$ & 19920.613 & 1.095 \\
\hline $4 f^{11}\left({ }^{4} I^{\circ}\right) 5 d 6 s$ & & $17 / 2$ & 13027.927 & 1.105 & $4 f^{11\left({ }^{4} \mathrm{I}^{\circ}\right) 5 d 6 s}$ & & $11 / 2$ & 19992.895 & 1.260 \\
\hline $4 f^{11}\left({ }^{4} I^{\circ}\right) 5 d 6 s$ & & $13 / 2$ & 13060.715 & 1.200 & $4 f^{11}\left({ }^{4} I^{\circ}\right) 5 d 6 s$ & & $13 / 2$ & 20319.934 & 1.035 \\
\hline $4 f^{11}\left({ }^{4} \mathrm{I}^{\circ}\right) 5 d 6 s$ & & $21 / 2$ & 13572.118 & 1.235 & $4 f^{11}\left({ }^{4} I^{\circ}\right) 5 d 6 s$ & & $15 / 2$ & 20517.717 & 1.130 \\
\hline $4 f^{11}\left({ }^{4} \mathrm{I}^{\circ}\right) 5 d 6 \mathrm{~s}$ & & $17 / 2$ & 13719.584 & 1.145 & $4 f^{11}\left({ }^{4} \mathrm{I}^{\circ}\right) 5 d 6 s$ & & $13 / 2$ & 21004.060 & 1.195 \\
\hline $4 f^{11}\left({ }^{4} \mathrm{I}^{\circ}\right) 5 d 6 s$ & & $15 / 2$ & 14280.723 & 1.180 & $4 f^{11}\left({ }^{4} \mathrm{I}^{\circ}\right) 5 d 6 s$ & & $11 / 2$ & 21040.001 & 1.130 \\
\hline $4 f^{11}\left({ }^{4} I^{\circ}\right) 5 d 6 s$ & & $19 / 2$ & 14649.277 & 1.265 & $4 f^{11}\left({ }^{4} I^{\circ}\right) 5 d 6 s ?$ & & $17 / 2$ & 21629.159 & 1.160 \\
\hline $4 f^{11}\left({ }^{4} \mathrm{I}^{\circ}\right) 5 d 6 s$ & & $17 / 2$ & 15732.917 & 1.205 & $4 f^{11}\left({ }^{4} I^{\circ}\right) 5 d 6 s ?$ & & $11 / 2$ & 21656.831 & 1.060 \\
\hline $4 f^{11}\left({ }^{4} \mathrm{I}^{\circ}\right) 5 d 6 s$ & & $9 / 2$ & 16241.348 & 1.150 & $4 f^{11}\left({ }^{4} \mathrm{I}^{\circ}\right) 5 d^{2} ?$ & & $11 / 2$ & 21819.914 & 1.375 \\
\hline $4 f^{11}\left({ }^{4} I^{\circ}\right) 5 d 6 s$ & & $15 / 2$ & 16473.871 & 1.160 & $4 f^{11}\left(4 I^{\circ}\right) 5 d 6 s ?$ & & $13 / 2$ & 22120.446 & 1.085 \\
\hline $4 f^{11}\left({ }^{4} I^{\circ}\right) 5 d 6 s$ & & $11 / 2$ & 16529.413 & 1.220 & $4 f^{11}\left({ }^{4} I^{\circ}\right) 5 d 6 s ?$ & & $15 / 2$ & 22200.015 & 1.220 \\
\hline $4 f^{11}\left({ }^{4} \mathrm{I}^{\circ}\right) 5 d 6 s$ & & $13 / 2$ & 16643.237 & 1.160 & $4 f^{11}\left({ }^{4} I^{\circ}\right) 5 d 6 s ?$ & & $13 / 2$ & 22859.510 & 0.920 \\
\hline $4 f^{11}\left({ }^{4} I^{\circ}\right) 5 d 6 s$ & & $19 / 2$ & 16935.832 & 1.145 & & & $11 / 2$ & 23240.649 & 1.085 \\
\hline
\end{tabular}


Er II, Odd Parity-Continued

Er II, Odd Parity_Continued

\begin{tabular}{|c|c|c|c|c|c|c|c|c|c|}
\hline Configuration & Term & $J$ & $\begin{array}{l}\text { Level } \\
\left(\mathrm{cm}^{-1}\right)\end{array}$ & $g$ & Configuration & Term & $J$ & $\begin{array}{l}\text { Level } \\
\left(\mathrm{cm}^{-1}\right)\end{array}$ & $g$ \\
\hline \multirow{11}{*}{$4 f^{11}\left({ }^{4} I^{\circ}\right) 5 d^{2 ?}$} & \multirow{25}{*}{$(6,1 / 2)^{\circ} ?$} & $17 / 2$ & 23539.702 & 1.085 & \multirow{44}{*}{$4 f^{12\left({ }^{3} \mathrm{H}_{5}\right)} 6 p_{1 / 2} ?$} & & $9 / 2$ & 30917.436 & 1.070 \\
\hline & & $13 / 2$ & 23669.096 & 1.285 & & & $7 / 2$ & 31312.019 & 1.095 \\
\hline & & $11 / 2$ & 23820.327 & 0.910 & & & $9 / 2$ & 31381.779 & 1.095 \\
\hline & & $15 / 2$ & 23973.877 & 1.220 & & & $11 / 2$ & 31385.667 & 1.055 \\
\hline & & $17 / 2$ & 24053.517 & 1.175 & & & $13 / 2$ & 31418.481 & 1.175 \\
\hline & & $11 / 2$ & 24415.265 & 1.100 & & & $11 / 2$ & 31801.102 & 1.135 \\
\hline & & $15 / 2$ & 24431.042 & 1.030 & & & $9 / 2$ & 31844.124 & 1.170 \\
\hline & & $11 / 2$ & 24687.523 & 0.790 & & & $7 / 2$ & 31902.682 & 1.100 \\
\hline & & $19 / 2$ & 24708.113 & 1.190 & & & $11 / 2$ & 32073.360 & 1.340 \\
\hline & & $13 / 2$ & 24935.855 & 1.070 & & & $9 / 2$ & 32267246 & 1.305 \\
\hline & & $11 / 2$ & 25382.379 & 1.160 & & & $9 / 2$ & 32502.680 & 1.230 \\
\hline \multirow{14}{*}{$4 f^{12}\left({ }^{3} \mathrm{H}_{6}\right) 6 p_{1 / 2} ?$} & & $11 / 2$ & 25592.343 & 1.200 & & & $13 / 2$ & 32528.401 & 1.165 \\
\hline & & $13 / 2$ & 26098.972 & 1.115 & & & $11 / 2$ & 32618.753 & 1.115 \\
\hline & & $13 / 2$ & 25604.266 & 1.015 & & & $9 / 2$ & 32753.468 & 1.200 \\
\hline & & $11 / 2$ & 25971.476 & 1.125 & & & $7 / 2$ & 32790.085 & 1.150 \\
\hline & & $9 / 2$ & 25996.174 & 1.250 & & & $11 / 2$ & 32811.006 & 1.090 \\
\hline & & $11 / 2$ & 26399.775 & 1.085 & & $\left(5,{ }^{1} / 2\right)^{\circ} ?$ & $9 / 2$ & 32896.371 & 1.220 \\
\hline & & $13 / 2$ & 26769.141 & 1.165 & & & $11 / 2$ & 33105.534 & 1.065 \\
\hline & & $11 / 2$ & 26805.448 & 1.215 & & & $13 / 2$ & 33012.493 & 1.060 \\
\hline & & $9 / 2$ & 27454.514 & 0.980 & & & $9 / 2$ & 33028.394 & 1.105 \\
\hline & & $13 / 2$ & 27513.555 & 1.105 & & & $9 / 2$ & 33129.912 & 1.195 \\
\hline & & $11 / 2$ & 27642.658 & 1.050 & & & $9 / 2$ & 33307.365 & 1.250 \\
\hline & & $9 / 2$ & 27890.690 & 0.950 & & & $7 / 2$ & 33539.273 & 0.915 \\
\hline & & $13 / 2$ & 28082.701 & 1.020 & & & $9 / 2$ & 33565.895 & 1.100 \\
\hline & & $11 / 2$ & 28361.386 & 1.030 & & & $9 / 2$ & 33649.964 & 1.030 \\
\hline \multirow{18}{*}{$4 f^{12\left({ }^{3} \mathrm{H}_{6}\right) 6 p} p_{3 / 2} ?$} & \multirow{18}{*}{$(6,3 / 2)^{\circ} ?$} & $9 / 2$ & 29011.015 & 1.110 & & & $11 / 2$ & 33659.536 & 1.175 \\
\hline & & $11 / 2$ & 29492.329 & 1.095 & & & $13 / 2$ & 33674.250 & 1.135 \\
\hline & & $15 / 2$ & 29640.863 & 1.185 & & & $11 / 2$ & 33721.545 & 1.190 \\
\hline & & $13 / 2$ & 30621.102 & 1.170 & & & $9 / 2$ & 33804.465 & 1.180 \\
\hline & & $13 / 2$ & 29119.606 & 1.095 & & & $11 / 2$ & 33988.301 & 1.160 \\
\hline & & $7 / 2$ & 29263.402 & 1.165 & & & $9 / 2$ & 34074.875 & 1.245 \\
\hline & & $15 / 2$ & 29472.789 & 1.145 & & & $7 / 2$ & 34148.162 & 1.255 \\
\hline & & $13 / 2$ & 29628.405 & 1.165 & & & $9 / 2$ & 34196.388 & 1.165 \\
\hline & & $7 / 2$ & 29780.571 & & & & $7 / 2$ & 34203.251 & 1.200 \\
\hline & & $9 / 2$ & 29783.733 & 1.160 & & & $11 / 2$ & 34341.611 & 1.135 \\
\hline & & $11 / 2$ & 29858.739 & 1.125 & & & $9 / 2$ & 34397.143 & 1.150 \\
\hline & & $13 / 2$ & 29892.370 & 1.135 & & & $13 / 2$ & 34545.248 & 1.140 \\
\hline & & $15 / 2$ & 29972.408 & 1.190 & & & $11 / 2$ & 34563.257 & 1.145 \\
\hline & & $13 / 2$ & 30008.331 & 1.120 & & & $9 / 2$ & 34674.173 & 1.135 \\
\hline & & $9 / 2$ & 30028.618 & 1.050 & & & $7 / 2$ & 34902.323 & 1.145 \\
\hline & & $11 / 2$ & 30122.939 & 1.125 & & & $11 / 2$ & 34972.437 & 1.155 \\
\hline & & $7 / 2$ & 30130.762 & 1.090 & & & $9 / 2$ & 35063.892 & 1.170 \\
\hline & & $11 / 2$ & 30157.742 & 1.070 & & & $11 / 2$ & 35215.487 & 1.150 \\
\hline $4 f^{12\left({ }^{3} \mathrm{~F}_{4}\right) 6 p_{1 / 2} ?}$ & $(4,1 / 2)^{\circ} ?$ & $\begin{array}{l}9 / 2 \\
7 / 2\end{array}$ & $\begin{array}{l}30317.974 \\
30894.447\end{array}$ & $\begin{array}{l}1.130 \\
1.265\end{array}$ & & & $13 / 2$ & 35276.531 & 1.180 \\
\hline
\end{tabular}


Er II, Odd Parity-Continued

Er II, Odd Parity-Continued

\begin{tabular}{|c|c|c|c|c|c|c|c|c|c|}
\hline Configuration & Term & $J$ & $\begin{array}{l}\text { Level } \\
\left(\mathrm{cm}^{-1}\right)\end{array}$ & $g$ & Configuration & Term & $J$ & $\begin{array}{l}\text { Level } \\
\left(\mathrm{cm}^{-1}\right)\end{array}$ & $g$ \\
\hline & & $7 / 2$ & 35359.885 & 1.195 & & & $11 / 2$ & 38642.554 & 1.140 \\
\hline & & $11 / 2$ & 35617.906 & 1.150 & & & $7 / 2$ & 38651.814 & 1.030 \\
\hline & & $13 / 2$ & 35671.599 & 1.190 & & & $9 / 2$ & 38847.186 & 1.090 \\
\hline & & $7 / 2$ & 35819.939 & 1.185 & & & $7 / 2$ & 39021.546 & 1.060 \\
\hline & & $9 / 2$ & 35877.083 & 1.155 & & & $5 / 2$ & 39036.642 & 1.155 \\
\hline & & $11 / 2$ & 35885.232 & 0.975 & & & $7 / 2$ & 39053.063 & 0.980 \\
\hline & & $9 / 2$ & 35899.232 & 1.050 & & & $7 / 2$ & 39140.561 & 1.180 \\
\hline & & $7 / 2$ & 35927.604 & 0.915 & & & $11 / 2$ & 39242.082 & 1.095 \\
\hline & & $9 / 2$ & 36007.182 & 1.020 & & & $7 / 2$ & 39277.605 & 1.215 \\
\hline & & $11 / 2$ & 36147.709 & 1.155 & & & $7 / 2$ & 39304.952 & 1.025 \\
\hline & & $9 / 2$ & 36322.233 & 1.075 & & & $9 / 2$ & 39392.224 & 1.095 \\
\hline & & $3 / 2$ & 36464.008 & 1.070 & & & $5 / 2$ & 39447.909 & 0.945 \\
\hline & & $7 / 2$ & 36470.944 & 0.800 & & & $9 / 2$ & 39509.332 & 1.245 \\
\hline & & $7 / 2$ & 36471.984 & 1.105 & & & $11 / 2$ & 39525.696 & 1.125 \\
\hline & & $11 / 2$ & 36643.232 & 1.095 & & & $5 / 2$ & 39643.159 & 1.175 \\
\hline & & $7 / 2$ & 36738.247 & 1.080 & & & $9 / 2$ & 39653.816 & 1.085 \\
\hline & & $9 / 2$ & 36761.150 & 1.000 & & & $11 / 2$ & 39766.447 & 1.105 \\
\hline & & $7 / 2$ & 36825.194 & 1.160 & & & $3 / 2$ & 39833.544 & 0.920 \\
\hline & & $11 / 2$ & 36863.932 & 1.055 & & & $9 / 2$ & 39845.276 & 1.080 \\
\hline & & $5 / 2$ & 36932.273 & 1.495 & & & $7 / 2$ & 39975.403 & 1.035 \\
\hline & & $9 / 2$ & 37038.764 & 1.145 & & & $9 / 2$ & 40000.774 & 1.115 \\
\hline & & $7 / 2$ & 37057.724 & 1.150 & & & $5 / 2$ & 40123.804 & 0.790 \\
\hline & & $11 / 2$ & 37098.956 & 1.250 & & & $11 / 2$ & 40124.080 & 1.070 \\
\hline & & $9 / 2$ & 37110.402 & 1.065 & & & $9 / 2$ & 40303.477 & 1.105 \\
\hline & & $5 / 2$ & 37126.953 & 1.205 & & & $5 / 2$ & 40368.926 & 0.700 \\
\hline & & $11 / 2$ & 37146.674 & 1.150 & & & $11 / 2$ & 40435.615 & 1.115 \\
\hline & & $3 / 2$ & 37357.383 & 0.755 & & & $9 / 2$ & 40497.076 & 1.055 \\
\hline & & $9 / 2$ & 37438.656 & 1.060 & & & $5 / 2$ & 40642.960 & 1.110 \\
\hline & & $9 / 2$ & 37483.090 & 1.175 & & & $3 / 2$ & 40687.445 & 1.060 \\
\hline & & $7 / 2$ & 37527.159 & 1.150 & & & $5 / 2$ & 40723.759 & 1.060 \\
\hline & & $9 / 2$ & 37617.073 & 0.920 & & & $7 / 2$ & 40747.134 & 1.015 \\
\hline & & $7 / 2$ & 37698.823 & 1.115 & & & $11 / 2$ & 40756.773 & 1.045 \\
\hline & & $5 / 2$ & 37712.008 & 1.245 & & & $9 / 2$ & 40853.950 & 1.000 \\
\hline & & $9 / 2$ & 37736.569 & 1.020 & & & $9 / 2$ & 40905.867 & 0.955 \\
\hline & & $7 / 2$ & 37747.938 & 0.915 & & & $7 / 2$ & 40943.527 & 1.045 \\
\hline & & $9 / 2$ & 37959.624 & 1.115 & & & $9 / 2$ & 40959.837 & 1.045 \\
\hline & & $5 / 2$ & 38204.486 & 0.990 & & & $3 / 2$ & 41003.728 & 1.340 \\
\hline & & $11 / 2$ & 38310.987 & 1.075 & & & $5 / 2$ & 41013.774 & 0.960 \\
\hline & & $7 / 2$ & 38318.204 & 0.925 & & & $7 / 2$ & 41029.308 & 1.145 \\
\hline & & $9 / 2$ & 38363.814 & 1.075 & & & $9 / 2$ & 41067.403 & 1.065 \\
\hline & & $3 / 2$ & 38470.551 & 1.380 & & & $9 / 2$ & 41170.514 & 0.950 \\
\hline & & $9 / 2$ & 38477.809 & 1.110 & & & $7 / 2$ & 41206.903 & 0.965 \\
\hline & & $5 / 2$ & 38580.914 & 0.935 & & & $9 / 2$ & 41268.620 & 0.975 \\
\hline & & $5 / 2$ & 38617.345 & 0.990 & & & $11 / 2$ & 41415.615 & 1.070 \\
\hline
\end{tabular}


Er II, Odd Parity-Continued

Er II, Odd Parity_Continued

\begin{tabular}{|c|c|c|c|c|c|c|c|c|c|}
\hline Configuration & Term & $J$ & $\begin{array}{l}\text { Level } \\
\left(\mathrm{cm}^{-1}\right)\end{array}$ & $g$ & Configuration & Term & $J$ & $\begin{array}{l}\text { Level } \\
\left(\mathrm{cm}^{-1}\right)\end{array}$ & $g$ \\
\hline & & $9 / 2$ & 41486.824 & 1.170 & & & $3 / 2$ & 42862.753 & 0.920 \\
\hline & & $7 / 2$ & 41505.714 & 1.045 & & & $5 / 2$ & 43161.997 & 1.020 \\
\hline & & $9 / 2$ & 41617.792 & 1.075 & & - & $9 / 2$ & 43221.660 & 1.060 \\
\hline & & $5 / 2$ & 41682.421 & 0.990 & & & $7 / 2$ & 43302.725 & 1.120 \\
\hline & & $7 / 2$ & 41876.127 & 1.030 & & & $5 / 2$ & 43553.435 & 1.035 \\
\hline & & $5 / 2$ & 42064.826 & 1.105 & & & $7 / 2$ & 43925.092 & 1.185 \\
\hline & & $7 / 2$ & 42149.596 & 0.955 & & & $3 / 2$ & 43929.434 & 0.810 \\
\hline & & $5 / 2$ & 42177.581 & 1.040 & & & $7 / 2$ & 43939.914 & 1.225 \\
\hline & & $5 / 2$ & 42264.318 & 1.125 & & & $7 / 2$ & 44148.073 & 1.065 \\
\hline & & $7 / 2$ & 42414.845 & 1.040 & & & $3 / 2$ & 44162.153 & 0.770 \\
\hline & & $7 / 2$ & 42461.068 & 1.000 & & & $3 / 2$ & 44848.559 & 1.095 \\
\hline & & $7 / 2$ & 42527.318 & 1.020 & & & $7 / 2$ & 45011.044 & 0.990 \\
\hline & & $7 / 2$ & 42626.606 & 1.100 & & & & & \\
\hline & & $3 / 2$ & 42650.795 & 0.845 & Er III $\left({ }^{3} \mathrm{H}_{6}\right)$ & Limit & & 96200 & \\
\hline & & $5 / 2$ & 42747.673 & 0.935 & & & & & \\
\hline & & $7 / 2$ & 42769.495 & 1.135 & & & & & \\
\hline
\end{tabular}




\section{Er III}

(Dy I sequence; 66 electrons)

Ground state $\left(1 s^{2} 2 s^{2} 2 p^{6} 3 s^{2} 3 p^{6} 3 d^{10} 4 s^{2} 4 p^{6} 4 d^{10} 5 s^{2} 5 p^{6}\right) 4 f^{12}{ }^{3} \mathrm{H}_{6}$

Ionization energy $183400 \pm 800 \mathrm{~cm}^{-1}$

$22.74 \pm 0.10 \mathrm{eV}$

Identified even configurations

$4 f^{12}$ and $4 f^{11} 6 p$

Identified odd configurations

$4 f^{11} 6 s$ and $4 f^{11} 5 d$

Becher [1965] began the analysis of this spectrum by finding nine levels belonging to the $4 f^{11}\left({ }^{4} \mathrm{I}_{15 / 2}^{\circ}\right) 5 d, 4 f^{11}\left({ }^{4} \mathrm{I}_{15 / 2}^{\circ}\right) 6 s$, and $4 f^{11}\left({ }^{4} \mathrm{I}_{15 / 2}^{\circ}\right) 6 p$ groups. He noted the $J_{1} j$ coupling in these subconfigurations, and assigned levels to all five $J_{1} j$ terms based on the $4 f^{11}\left({ }^{4} I_{15 / 2}^{0}\right)$ parent level.

Most of the levels and term assignments given here are from Spector [1973]. He independently found a more extensive system of excited levels and located them relative to three levels of the $4 f^{12}$ ground configuration, including the ground level. Wyart, Blaise, and Camus [1974] in their study of $\left(4 f^{N} 5 d+4 f^{N} 6 s\right)$ configurations calculated this group for Er III $(N=11)$. They added two levels and suggested the deletion of two other levels from Spector's list. The leading percentages in $L S$ coupling are taken from their paper. Three levels of this group could not be unambiguously assigned to calculated eigenvectors.

Wyart, Koot, and van Kleef [1974] calculated the percentages in $J_{1} j$ coupling for the $4 f^{11}\left({ }^{4} I^{\circ}\right) 6 p$ group of levels. These authors also located an additional experimental level of this group and found two new levels of $4 f^{11}\left({ }^{4} I^{\circ}\right) 5 d$. Van Kleef [1975] has supplied three unpublished levels, including the $4 f^{12}{ }^{3} \mathrm{H}_{4}$ level to complete the ground term.

Spector's published line list gives about 150 classified lines in the range $2165-5903 \AA$. He states that his complete list included $800 \mathrm{Er}$ III lines. Becher's thesis has some 3800 lines believed to belong to Er III and Er II, with wavelengths from $2036 \AA$ to $8725 \AA$.

Sugar and Reader obtained the quoted ionization potential.

\section{References}

Becher, J., Thesis, Johns Hopkins Univ., 134 pp. (1966). EL CL W PT

Spector, N., J. Opt. Soc. Am. 63, 358 (1973). EL CL W PT

Sugar, J., and Reader, J., J. Chem. Phys. 59, 2083 (1973). IP

van Kleef, Th. A. M., private communication (1975). EL

Wyart, J. F., Blaise, J., and Camus, P., Phys. Scr. 9, 325 (1974). EL PT

Wyart, J. F., Koot, J. J. A., and van Kleef, Th. A. M., Physica (Utrecht) 77, 159 (1974). EL PT

[October 1976]

\section{Er III}

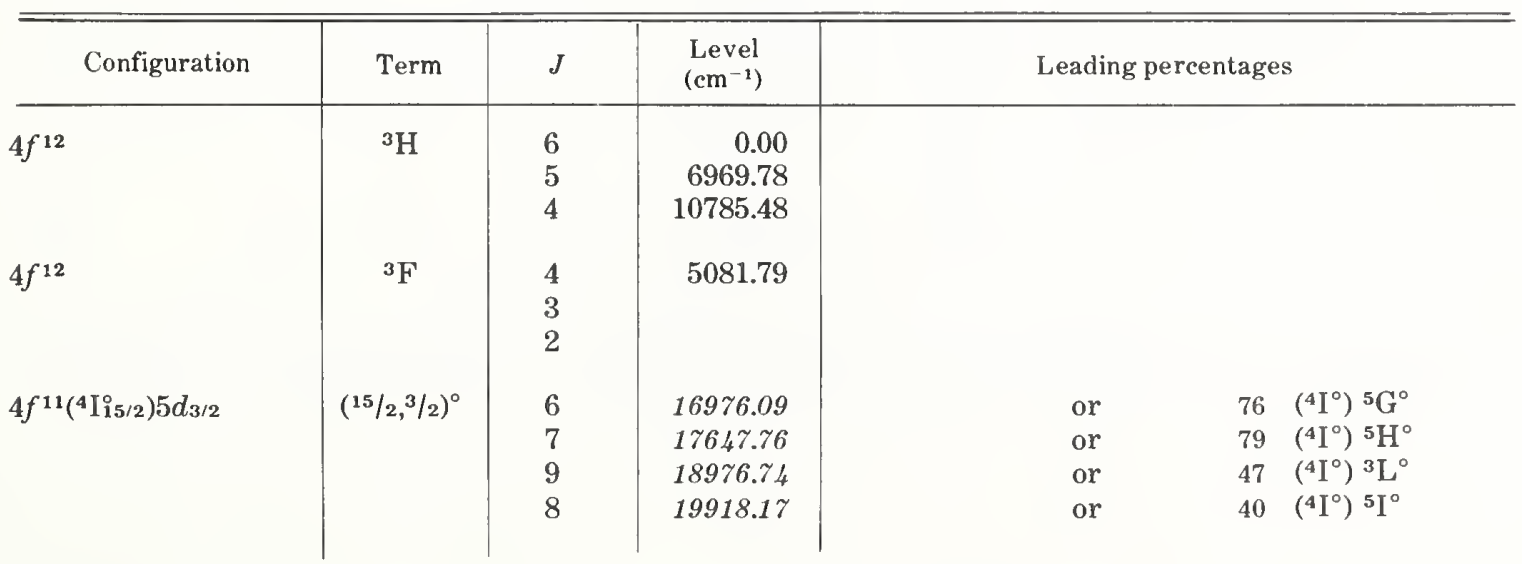


Er III-Continued

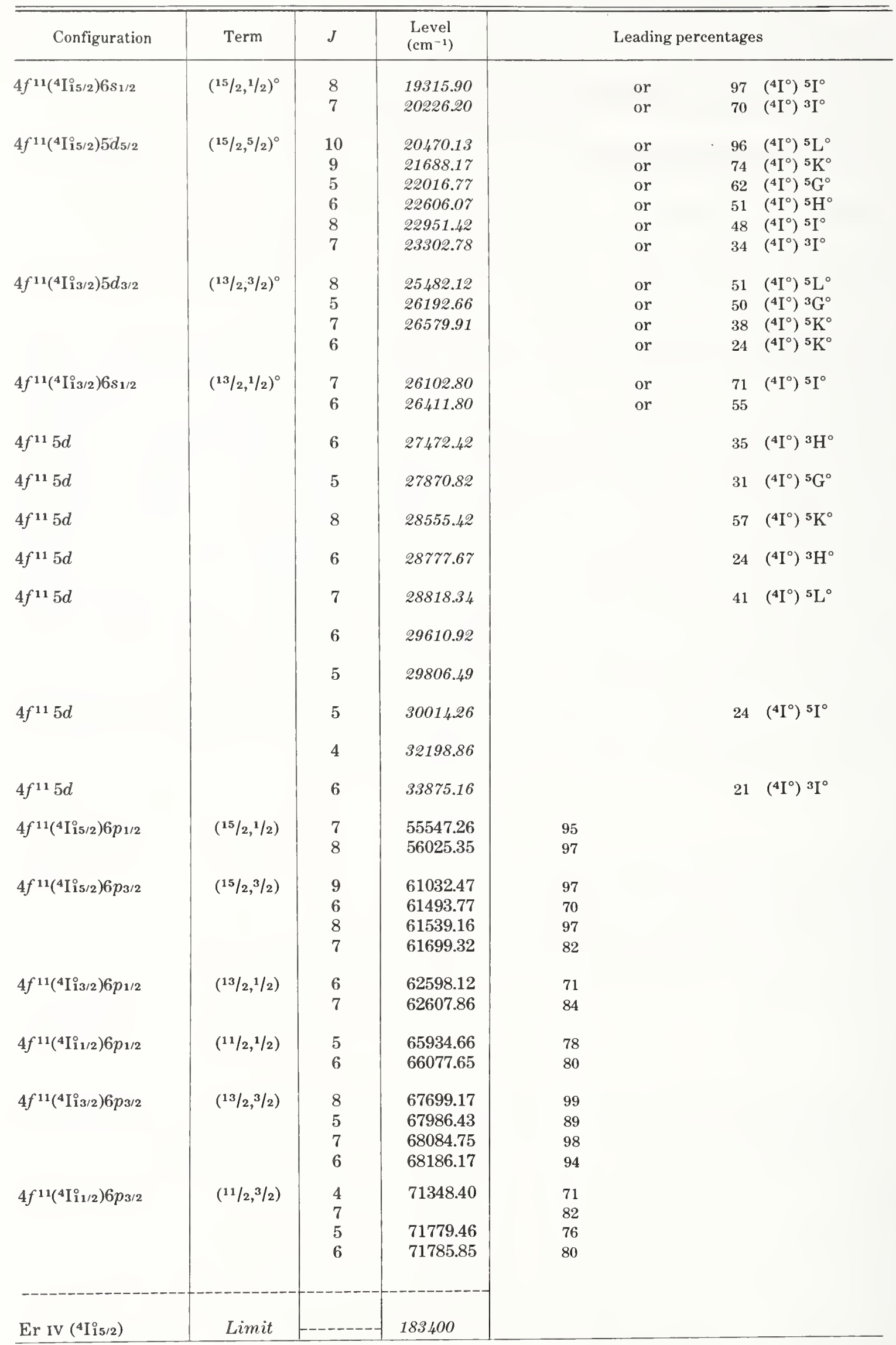




\section{[Er IV]}

(Tb I sequence; 65 electrons)

Ground state $\left(1 s^{2} 2 s^{2} 2 p^{6} 3 s^{2} 3 p^{6} 3 d^{10} 4 s^{2} 4 p^{6} 4 d^{10} 5 s^{2} 5 p^{6}\right) 4 f^{11}{ }^{4} \mathrm{I}_{15 / 2}^{0}$

Ionization energy $344000 \pm 3000 \mathrm{~cm}^{-1}$

$42.7 \pm 0.4 \mathrm{eV}$

Carter's thesis [1966] has a list of about 1200 lines assigned to $\operatorname{Er}$ IV from 1225 to $5609 \AA$, but only lines regarded as classified in the analysis were included. Unfortunately, the $4 f^{11}-$ $4 f^{10} 5 d$ transition array given by Carter proved inconsistent with the energy separation derived later from the systematic behavior of such configurations in the rare earths [Sugar and Reader, 1973]. Carter's analysis also included a number of $4 f^{10} 6 s-4 f^{10} 6 p$ transitions. We have not given any of the levels, pending further work on the free-ion spectrum.

The levels of the three $4 f^{11}$ terms here were derived from the spectrum of $\mathrm{Er}^{3+}$ in $\mathrm{LaCl}_{3}$ [Dieke, 1968]. The positions are the mean values of the corresponding sublevels, rounded off to the nearest $10 \mathrm{~cm}^{-1}$. Carnall, Fields, and Rajnak [1974] supplied the eigenvector percentages from their unpublished calculations. Dieke gives the $4 f^{11}$ energy structure of this ion in several other crystals, and lists additional levels up to $34000 \mathrm{~cm}^{-1}$.

Sugar and Reader derived the ionization energy. The uncertainty has been approximately doubled, in accordance with the suggestion by Spector and Sugar [1976].

\section{References}

Carnall, W. T., Fields, P. R., and Rajnak, K., J. Chem. Phys. 49, 4424 (1968). [EL]

Carnall, W. T., Fields, P. R., and Rajnak, K., unpublished material (1974). PT

Carter, W. J., Thesis, Johns Hopkins Univ., Baltimore, 65 pp. (1966). EL CL W PT

Dieke, G. H., Spectra and Energy Levels of Rare Earth Ions in Crystals, Ed. H. M. Crosswhite and Hे. Crosswhite, pp. 294-309 (Interscience Publishers, New York, 1968). [EL] ND [CL] [W]

Spector, N., and Sugar, J., J. Opt. Soc. Am. 66, 436 (1976).

Sugar, J., and Reader, J., J. Chem. Phys. 59, 2083 (1973). IP

Wybourne, B. G., J. Chem. Phys. 34, 279 (1961). PT

[March 1977]

[Er IV]

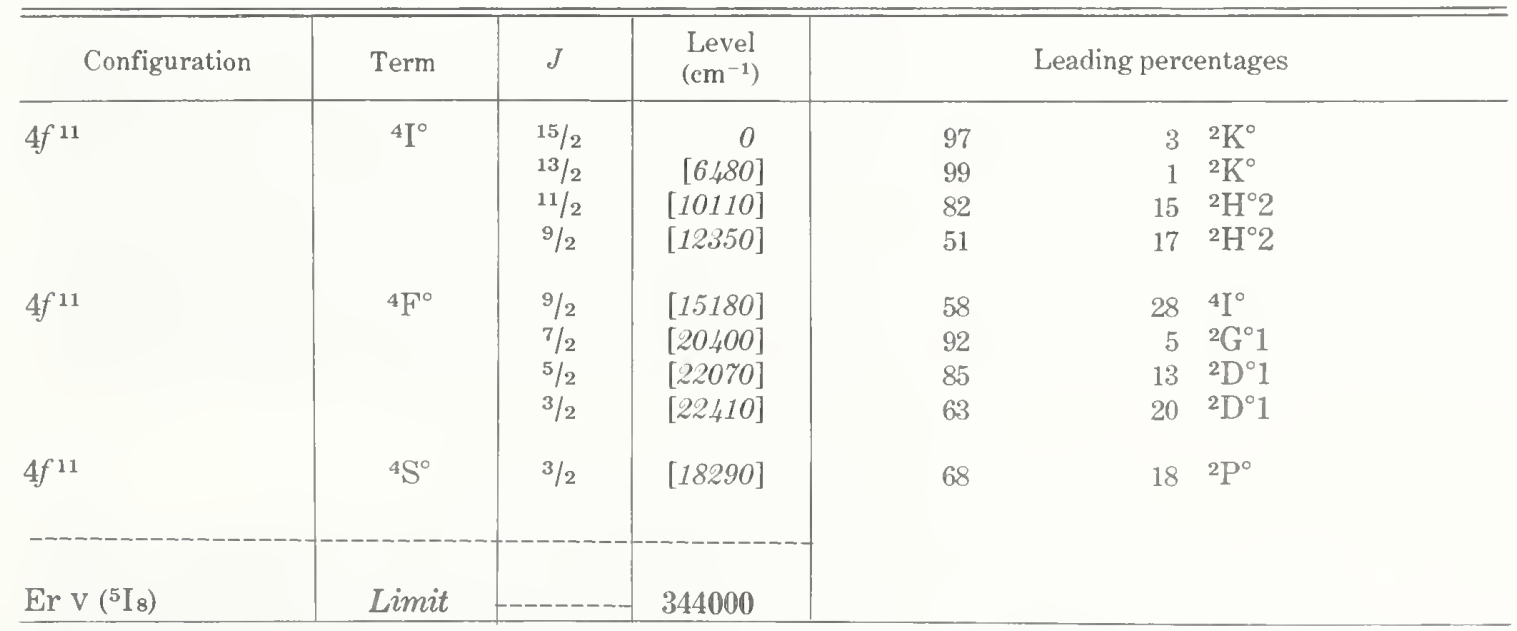




\section{THULIUM}

\section{Tm I}

69 electrons

Ground state $\left(1 s^{2} 2 s^{2} 2 p^{6} 3 s^{2} 3 p^{6} 3 d^{10} 4 s^{2} 4 p^{6} 4 d^{10} 5 s^{2} 5 p^{6}\right) 4 f^{13} 6 s^{2}{ }^{2} \mathrm{~F}_{7 / 2}^{\circ}$

Ionization energy $49879.8 \pm 0.5 \mathrm{~cm}^{-1}$

$6.18436 \pm 0.00006 \mathrm{eV}$

Identified odd configurations

$4 f^{13} 6 s^{2}, 4 f^{13} 5 d 6 s, 4 f^{12} 6 s^{2} 6 p, 4 f^{12} 5 d 6 s 6 p, 4 f^{13} 6 s 7 s$, $4 f^{13} 6 s 6 d, 4 f^{13} 6 s 8 s, 4 f^{13} 6 p^{2}$

Identified even configurations

$$
4 f^{12} 5 d 6 s^{2}, 4 f^{13} 6 s 6 p-8 p, 4 f^{13} 6 s 17 p-48 p, 4 f^{13} 5 d 6 p
$$

Meggers' start on this analysis in 1942 gave the interval between the levels of the $4 f^{13} 6 s^{2}{ }^{2} \mathrm{~F}^{\circ}$ ground term, along with 16 upper even levels. In 1963 Blaise and his collaborators published extensions of the analysis, and by 1965 Camus, Blaise, and others had obtained Zeeman and infrared wavelength data that allowed a further extension of both the odd and even level systems and gave $J$ and $g$ values for nearly all known levels. The $4 f^{12} 5 d 6 s^{2}$ and $4 f^{13} 6 s 6 p$ even configurations and the $4 f^{12} 6 s^{2} 6 p$ and $4 f^{13} 5 d 6 s$ odd configurations were interpreted theoretically, and the $4 f^{13} 6 s 7 s$ and $4 f^{13} 6 s 8 s$ series had been identified.

The paper by Sugar, Meggers, and Camus [1973] included additional levels belonging to the above configurations, and many new levels assigned to the $4 f^{13} 5 d 6 p, 4 f^{12} 5 d 6 s 6 p, 4 f^{13} 6 s 6 d$, and $4 f^{13} 6 p^{2}$ configurations. This collaboration of the Laboratoire Aime Cotton and the National Bureau of Standards was based in part on new measurements of the spectrum made at NBS. The list of about 4400 emission lines (2500-11750 $\AA$ ) includes more than 2700 classified lines. Practically all of the energy levels below $46000 \mathrm{~cm}^{-1}$ are taken from this paper, which included a total of almost 500 levels. The level values were obtained by least-squares adjustment to the measured lines; "for levels given to three decimal places the standard deviation varies from 0.004 to $0.010 \mathrm{~cm}^{-1}$ [and] for those given to two it varies from 0.010 to $0.02 \mathrm{~cm}^{-1}$."

\section{$g$ Values}

Most of the $g$ values are from Camus' thesis [1971], and are based on Zeeman-effect plates obtained by Tomkins and Fred at the Argonne National Laboratory. A few revisions have been supplied by Camus and Wyart [1976]. The line list of Sugar, Meggers, and Camus includes the Zeeman-effect measurements for the lines. The $g$ value for the ground level is from the atomic-beam magnetic-resonance measurement by Giglberger and Penselin, who gave this quantity as $1.141189 \pm 0.000003$. Budick and Simievic obtained the quoted $g$ values for two of the $4 f^{13}\left({ }^{2} \mathrm{~F}_{7 / 2}^{\circ}\right) 6 s 6 p\left({ }^{3} \mathrm{P}_{1}^{\circ}\right)$ levels, $J=7 / 2$ and $9 / 2$, with respective uncertainties of 0.00006 and 0.00010 . The $g$ value for the even level at $21121 \mathrm{~cm}^{-1}$ is given with an uncertainty of \pm 0.00003 by Steudel, Wallenstein, and Walther. Steudel et al. also measured the quoted four-place $g$ values with uncertainties between 0.0005 and 0.0012 .

\section{Theoretical Interpretation, Calculations}

All leading percentages are taken from Camus' thesis [1971]. The interpretation of the levels of both parities is complete up to above $30000 \mathrm{~cm}^{-1}$, with most of the configurations being given in a $J_{1} j$ or $J_{1} J_{2}$ coupling scheme. In order to shorten the notations for second percentages from configurations coupled in those schemes, we have omitted the final $\left(J_{1}, j\right)$ or $\left(J_{1}, J_{2}\right)$ term symbols wherever they would be redundant. Leading percentages for the 
$4 f^{13} 6 s 6 d$ levels are given in both the preferred $J_{1} L_{2}$ scheme and in a $J_{1} J_{2}$ scheme (following the word "or"), the final $\left(J_{1}, J_{2}\right)$ term symbols being omitted in this case also.

The complexity of the spectrum is now known to arise mainly from configurations based on the $4 f^{12}$ core; for example, the bulk of the 284 known odd levels belong to the $4 f^{12} 5 d 6 s 6 p$ configuration. The average purity of 356 levels of $4 f^{12} 5 d 6 s 6 p$ calculated in an $L S$ scheme was only $16 \%$ [Camus, 1971]. The scheme preferred by Camus for this configuration is an "intermediate" $J_{1} J_{2}$ coupling scheme illustrated by the lowest term, $4 f \cdot{ }^{12}\left({ }^{3} \mathrm{H}_{6}\right) 5 d 6 s 6 p\left({ }^{4} \mathrm{~F}_{3 / 2}^{\circ}\right)$, beginning at $29309 \mathrm{~cm}^{-1}$. (The scheme is intermediate in the sense that the two $5 d 6 s 6 p$ parent doublet terms of each type $\left({ }^{2} \mathrm{~F}^{\circ},{ }^{2} \mathrm{D}^{\circ},{ }^{2} \mathrm{P}^{\circ}\right)$ are mixtures of corresponding terms having pure parentages within the $5 d 6 s 6 p$ group; for example, $5 d\left({ }^{2} \mathrm{D}\right) 6 s 6 p\left({ }^{3} \mathrm{P}^{\circ}\right)\left({ }^{2} \mathrm{~F}^{\circ},{ }^{2} \mathrm{D}^{\circ},{ }^{2} \mathrm{P}^{\circ}\right)$ and $5 d\left({ }^{2} \mathrm{D}\right) 6 s 6 p\left({ }^{1} \mathrm{P}^{\circ}\right)\left({ }^{2} \mathrm{~F}^{\circ},{ }^{2} \mathrm{D}^{\circ},{ }^{2} \mathrm{P}^{\circ}\right)$ would be obtained in a pure coupling scheme based on vanishing $5 d-6 s$ and $5 d-6 p$ interactions.) For technical reasons, Camus transformed the $4 f^{12} 5 d 6 s 6 p$ eigenvectors to the intermediate $J_{1} J_{2}$ scheme only for $J \geqslant 15 / 2$. We have thus given the leading percentages in the $J_{1} J_{2}$ scheme for levels having $J \geqslant 15 / 2$ if $J_{2}$ (in the leading $J_{1} J_{2}$ component) belongs to a $5 d 6 s 6 p$ quartet level. The $J_{1} J_{2}$ names for a few levels $(J \geqslant 15 / 2)$ based on $5 d 6 s 6 p$ doublet levels are given, but without percentages; these levels have leading percentages $>50 \%$ in the intermediate $J_{1} J_{2}$ scheme. In some cases we include tentative guesses as to the most appropriate parentages for the doublets in a corresponding pure coupling scheme; for example, $4 f^{12}\left({ }^{3} \mathrm{H}_{6}\right) 5 d\left({ }^{2} \mathrm{D}\right) 6 s 6 p\left({ }^{3} \mathrm{P}^{\circ}\right.$ ?) $\left({ }^{2} \mathrm{D}_{5 / 2}^{\circ}\right)$. Camus was able to assign $J_{1} J_{2}$ designations to some levels with $J<15 / 2$, and these are listed for several such levels belonging to low $J_{1} J_{2}$ terms. Pending a more complete calculation and transformation to $J_{1} J_{2}$ coupling, only the configuration assignment is given for many of the $4 f^{12} 5 d 6 s 6 p$ levels.

None of the even levels have been assigned to a configuration as complex as $4 f^{12} 5 d 6 s 6 p$, but Sugar, Meggers, and Camus note that many of the unidentified even levels (which begin above $35000 \mathrm{~cm}^{-1}$ ) probably belong to the as yet unlocated configuration $4 f^{12} 5 d^{2} 6 s$. Camus and Wyart [1976] have kindly supplied a few additional levels and revised assignments for the even group.

\section{Absorption and Photoionization Spectra, Ionization Energy}

Camus [1971] observed the absorption spectrum of thulium vapor over the region 2000$2750 \AA$. He noted that at the low temperature used $\left(1200{ }^{\circ} \mathrm{C}\right)$, only transitions from the ${ }^{2} \mathrm{~F}_{7 / 2}^{\circ}$ ground level would be expected. Some even levels based on this work begin at $37557 \mathrm{~cm}^{-1}$, and the long $4 f^{13}\left({ }^{2} \mathrm{~F}_{7 / 2}^{\circ}\right) 6 s n p$ series were observed in absorption. The assignments from $17 p$ through $20 p$ are given as tentative because of the density of the spectrum in this region and possible ambiguities in the $J$ values of particular levels within the terms. The absorption spectrum in the range $45500-49500 \mathrm{~cm}^{-1}$ is largely uninterpreted; Camus states that configurations of the type $4 f^{12} 6 s^{2} n d$ and (through configuration interaction) $4 f^{12} 5 d 6 s m s$ probably account for many of the lines.

The positions of the photoionization resonances observed by Parr are followed by the letter " $a$ " (autoionization). A number of the high $4 f^{13}\left({ }^{2} \mathrm{~F}_{7 / 2}^{\circ}\right) 6 s(7 / 2,1 / 2)_{3}^{\circ} n p$ series members observed by Camus apparently are superimposed on the lowest of the broad photoionization resonances, at $49975 \mathrm{~cm}^{-1}$. Parr's tentative assignments of a few of the autoionizing levels to terms of $4 f^{13} 5 d n p$ configurations are omitted, pending a more detailed theoretical treatment of this complex spectrum above the limit.

Camus [1971] determined the quoted value for the ionization energy by fitting the $4 f^{13}\left({ }^{2} \mathrm{~F}_{7 / 2}^{\circ}\right) 6 s\left(7 / 2,{ }^{1 / 2}\right)_{3}^{\circ} n p_{3 / 2}$ series $(17 \leqslant n \leqslant 48)$. Parr's value of $6.180 \pm 0.008 \mathrm{eV}$, determined directly from the threshold for photoionization, agrees well with the more accurate spectroscopic value.

\section{References}

Allen, L., United Kingdom Atomic Energy Research Establishment AERE-R 4029, 34 pp. (1962). W ZE

Blaise, J., and Vetter, R., C. R. Acad. Sci. 256, 630 (1963). EL Hfs IP

Blaise, J., and Camus, P., C. R. Acad. Sci. 260, 4693 (1965). EL ZE

Bordarier, Y., Vetter, R., and Blaise, J., J. Phys. (Paris) 24, 1107 (1963). EL CL W Hfs

Bovey, L. F. H., and Garton, W. R. S., Proc. Phys. Soc. London, Sect. A 67, 476 (1954). W

Budick, B., and Simievic, A., Spectroscopic and Group Theoretical Methods in Physics, Ed. F. Bloch et al., pp. 161-165 (John Wiley \& Sons, N.Y., 1968). ZE Hfs 
Camus, P., J. Phys. (Paris) 27, 717 (1966). EL ZE PT

Camus, P., J. Phys. (Paris) 31, 985 (1970). PT

Camus, P., Thesis, Univ. Paris, Orsay, 265 pp. (1971). EL ND CL W ZE IP SF PT

Camus, P., J. Phys. (Paris) 33, 203 (1972). EL ND ZE PT

Camus, P., and Blaise, J., C. R. Acad. Sci. 261, 4359 (1965). EL ZE IP

Camus, P., Guelachvili, G., and Verges, J., Spectrochim. Acta, Part B 24, 373 (1969). EL CL W ZE Hfs

Camus, P., and Wyart, J. F., unpublished material (1976). EL ND ZE

Giglberger, D., and Penselin, S., Z. Phys. 199, 244 (1967). ZE Hfs

King, A. S., Astrophys. J. 94, 226 (1941). Temperature Classification

Meggers, W. F., Rev. Mod. Phys. 14, 96 (1942). EL CL

Parr, A. C., J. Chem. Phys. 54, 3161 (1971). EL CL W IP

Ritter, G. J., Phys. Rev. 128, 2238 (1962). ZE Hfs

Steudel, A., Wallenstein, R., and Walther, H., Phys. Lett. A 31, 433 (1970). ZE

Sugar, J., Meggers, W. F., and Camus, P., J. Res. Nat. Bur. Stand. (U.S.) 77A, 1 (1973): EL CL W ZE IP

[October 1976]

Tm I, Odd Parity

\begin{tabular}{|c|c|c|c|c|c|c|}
\hline Configuration & Term & $J$ & $\begin{array}{l}\text { Level } \\
\left(\mathrm{cm}^{-1}\right)\end{array}$ & $g$ & & Leading percentages \\
\hline $4 f^{13}\left({ }^{2} \mathrm{~F}^{\circ}\right) 6 s^{2}$ & ${ }^{2} \mathrm{~F}^{\circ}$ & $\begin{array}{l}7 / 2 \\
5 / 2\end{array}$ & $\begin{array}{r}0.000 \\
8771.243\end{array}$ & $\begin{array}{l}1.14119 \\
0.855\end{array}$ & & \\
\hline $4 f^{13}\left({ }^{2} \mathrm{~F}_{7 / 2}^{\circ}\right) 5 d 6 s\left({ }^{3} \mathrm{D}\right)$ & $3[3 / 2]^{\circ}$ & $\begin{array}{l}5 / 2 \\
3 / 2 \\
1 / 2\end{array}$ & $\begin{array}{l}20406.840 \\
21799.380 \\
24160.63\end{array}$ & $\begin{array}{l}1.58 \\
1.45 \\
1.010\end{array}$ & $\begin{array}{l}97 \\
72 \\
93\end{array}$ & $\begin{array}{rll}1 & \left({ }^{2} \mathrm{~F}_{7 / 2}^{\circ}\right)\left({ }^{3} \mathrm{D}\right){ }^{3}\left[{ }^{5} / 2\right]^{\circ} \\
22 & \left({ }^{2} \mathrm{~F}^{\circ} / 2\right)\left({ }^{1} \mathrm{D}\right) & { }^{13}[/ 2]^{\circ} \\
4 & \left({ }^{2} \mathrm{~F}_{5 / 2}^{\circ}\right)\left({ }^{1} \mathrm{D}\right) & { }^{1}[1 / 2]^{\circ}\end{array}$ \\
\hline $4 f^{13}\left({ }^{2} \mathrm{~F}_{7 / 2}^{\circ}\right) 5 d 6 s\left({ }^{3} \mathrm{D}\right)$ & $3[11 / 2]^{\circ}$ & $\begin{array}{c}9 / 2 \\
11 / 2 \\
13 / 2\end{array}$ & $\begin{array}{l}22419.764 \\
22559.502 \\
22542.797\end{array}$ & $\begin{array}{l}0.934 \\
1.15 \\
1.198\end{array}$ & $\begin{array}{l}98 \\
79 \\
90\end{array}$ & 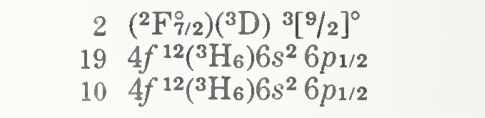 \\
\hline $4 f^{12}\left({ }^{3} \mathrm{H}_{6}\right) 6 s^{2} 6 p_{1 / 2}$ & $(6,1 / 2)^{\circ}$ & $\begin{array}{l}11 / 2 \\
13 / 2\end{array}$ & $\begin{array}{l}22468.046 \\
22902.12 \pi\end{array}$ & $\begin{array}{l}1.21 \\
1.138\end{array}$ & $\begin{array}{l}74 \\
88\end{array}$ & $\begin{array}{l}184 f^{13}\left({ }^{2} \mathrm{~F}_{7 / 2}^{\circ}\right) 5 d 6 s\left({ }^{3} \mathrm{D}\right){ }^{3}\left[{ }^{11} / 2\right] \\
10\end{array}$ \\
\hline $4 f^{13}\left({ }^{2} \mathrm{~F}_{7 / 2}^{\circ}\right) 5 d 6 s\left({ }^{3} \mathrm{D}\right)$ & $3[5 / 2]^{\circ}$ & $\begin{array}{l}7 / 2 \\
5 / 2 \\
3 / 2\end{array}$ & $\begin{array}{l}23335.111 \\
23431.844 \\
235 \% 4.413\end{array}$ & $\begin{array}{l}1.362 \\
1.22 \\
0.83\end{array}$ & $\begin{array}{l}79 \\
82 \\
94\end{array}$ & $\begin{array}{rr}20 & \left({ }^{2} \mathrm{~F}_{7 / 2}^{\circ}\right)\left({ }^{3} \mathrm{D}\right){ }^{3}\left[{ }^{7} / 2\right]^{\circ} \\
16 & \left({ }^{2} \mathrm{~F}_{7 / 2}^{\circ}\right)\left({ }^{3} \mathrm{D}\right){ }^{3}\left[{ }^{7} / 2\right]^{\circ} \\
5 & \left({ }^{2} \mathrm{~F}_{7 / 2}^{\circ}\right)\left({ }^{3} \mathrm{D}\right){ }^{3}\left[{ }^{3} / 2\right]^{\circ}\end{array}$ \\
\hline $4 f^{13}\left({ }^{2} \mathrm{~F}_{7 / 2}^{\circ}\right) 5 d 6 s\left({ }^{3} \mathrm{D}\right)$ & $3[7 / 2]^{\circ}$ & $\begin{array}{l}9 / 2 \\
5 / 2 \\
7 / 2\end{array}$ & $\begin{array}{l}23941.071 \\
24611.303 \\
24 \% 08.041\end{array}$ & $\begin{array}{l}1.29 \\
0.92 \\
1.08\end{array}$ & $\begin{array}{l}82 \\
77 \\
49\end{array}$ & $\begin{array}{lll}16 & \left({ }^{2} \mathrm{~F}_{7 / 2}^{\circ}\right)\left({ }^{3} \mathrm{D}\right) & { }^{3}\left[{ }^{9} / 2\right]^{\circ} \\
16 & \left({ }^{2} \mathrm{~F}_{7 / 2}^{\circ}\right)\left({ }^{3} \mathrm{D}\right) & { }^{3}\left[{ }^{5} / 2\right]^{\circ} \\
42 & \left({ }^{2} \mathrm{~F}_{7 / 2}^{\circ}\right)\left({ }^{3} \mathrm{D}\right) & { }^{3}\left[{ }^{9} / 2\right]^{\circ}\end{array}$ \\
\hline $4 f^{13}\left({ }^{2} \mathrm{~F}_{7 / 2}^{\circ}\right) 5 d 6 s\left({ }^{3} \mathrm{D}\right)$ & $3[9 / 2]^{\circ}$ & $\begin{array}{c}7 / 2 \\
9 / 2 \\
11 / 2\end{array}$ & $\begin{array}{l}24246.425 \\
24701.058 \\
24957.469\end{array}$ & $\begin{array}{l}1.02 \\
1.145 \\
1.235\end{array}$ & $\begin{array}{l}55 \\
75 \\
75\end{array}$ & 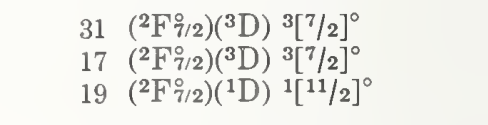 \\
\hline $4 f^{13}\left({ }^{2} \mathrm{~F}_{7 / 2}^{\circ}\right) 5 d 6 s\left({ }^{1} \mathrm{D}\right)$ & ${ }^{1}[3 / 2]^{\circ}$ & $3 / 2$ & 25207.732 & 1.365 & 72 & $16\left({ }^{2} \mathrm{~F}_{7 / 2}^{\circ}\right)\left({ }^{3} \mathrm{D}\right){ }^{3}\left[{ }^{3} / 2\right]^{\circ}$ \\
\hline $4 f^{13}\left({ }^{2} \mathrm{~F}_{7 / 2}^{\circ}\right) 5 d 6 s\left({ }^{1} \mathrm{D}\right)$ & ${ }^{1}[11 / 2]^{\circ}$ & $11 / 2$ & 25520.987 & 1.10 & 78 & $18\left({ }^{2} \mathrm{~F}_{7 / 2}^{\circ}\right)\left({ }^{3} \mathrm{D}\right){ }^{3}\left[{ }^{9} / 2\right]^{\circ}$ \\
\hline $4 f^{12}\left({ }^{3} \mathrm{H}_{6}\right) 6 s^{2} 6 p_{3 / 2}$ & $(6,3 / 2)^{\circ}$ & $\begin{array}{c}15 / 2 \\
9 / 2 \\
13 / 2 \\
11 / 2\end{array}$ & $\begin{array}{l}25536.116 \\
25699.714 \\
26357.736 \\
26368.412\end{array}$ & $\begin{array}{l}1.20 \\
1.12 \\
1.188 \\
1.125\end{array}$ & $\begin{array}{l}99 \\
88 \\
98 \\
91\end{array}$ & $\begin{array}{ll}1 & \left({ }^{1} \mathrm{I}_{6}\right)\left(6,{ }^{3} / 2\right)^{\circ} \\
5 & 4 f^{13}\left({ }^{2} \mathrm{~F}^{\circ} / 2\right) 5 d 6 s\left({ }^{3} \mathrm{D}\right){ }^{3}\left[{ }^{9} / 2\right]^{\circ} \\
1 & \left({ }^{1} \mathrm{I}_{6}\right)\left(6,{ }^{3} / 2\right)^{\circ} \\
3 & \left({ }^{3} \mathrm{H}_{6}\right)\left(6,{ }^{1} / 2\right)^{\circ}\end{array}$ \\
\hline $4 f^{13}\left({ }^{2} \mathrm{~F}^{\circ} / 2\right) 5 d 6 s\left({ }^{1} \mathrm{D}\right)$ & $1[5 / 2]^{\circ}$ & $5 / 2$ & $26448.2 \pi 4$ & 1.14 & 93 & $6\left({ }^{2} \mathrm{~F}_{7 / 2}^{\circ}\right)\left({ }^{3} \mathrm{D}\right)^{3}\left[{ }^{7} / 2\right]^{\circ}$ \\
\hline $4 f^{13}\left({ }^{2} \mathrm{~F}_{7 / 2}^{\circ}\right) 5 d 6 s\left({ }^{1} \mathrm{D}\right)$ & $1[7 / 2]^{\circ}$ & $7 / 2$ & 27037.468 & 1.11 & 97 & $1\left({ }^{2} \mathrm{~F}_{7 / 2}^{\circ}\right)\left({ }^{3} \mathrm{D}\right){ }^{3}[5 / 2]^{\circ}$ \\
\hline $4 f^{13}\left({ }^{2} \mathrm{~F}_{7 / 2}^{\circ}\right) 5 d 6 s\left({ }^{1} \mathrm{D}\right)$ & $1[9 / 2]^{\circ}$ & $9 / 2$ & 27440.858 & & 95 & $2\left({ }^{2} \mathrm{~F}^{\circ} / 2\right)\left({ }^{3} \mathrm{D}\right){ }^{3}\left[{ }^{9} / 2\right]^{\circ}$ \\
\hline $4 f^{12}\left({ }^{3} \mathrm{~F}_{4}\right) 6 s^{2} 6 p_{1 / 2}$ & $(4,1 / 2)^{\circ}$ & $\begin{array}{l}7 / 2 \\
9 / 2\end{array}$ & $\begin{array}{l}28340.29 \\
28514.423\end{array}$ & 1.085 & $\begin{array}{l}62 \\
60\end{array}$ & $\begin{array}{l}29 \\
28\end{array}\left({ }^{1} \mathrm{G}_{4}\right)\left(4,{ }^{1} / 2\right)^{\circ}$ \\
\hline $4 f^{12}\left({ }^{3} \mathrm{H}_{6}\right) 5 d 6 s 6 p\left({ }^{4} \mathrm{~F}_{3 / 2}^{\circ}\right)$ & $(6,3 / 2)^{\circ}$ & $\begin{array}{c}9 / 2 \\
11 / 2 \\
15 / 2 \\
13 / 2\end{array}$ & $\begin{array}{l}29308.69 \\
31080.846 \\
32112.98 \\
32950.10\end{array}$ & $\begin{array}{l}1.49 \\
1.37 \\
0.98 \\
1.31\end{array}$ & 80 & \\
\hline $4 f^{13}\left({ }^{2} \mathrm{~F}_{5}^{\circ} / 2\right) 5 d 6 s\left({ }^{3} \mathrm{D}\right)$ & $3[9 / 2]^{\circ}$ & $\begin{array}{r}7 / 2 \\
9 / 2 \\
11 / 2\end{array}$ & $\begin{array}{l}30921.58 \\
31388.303 \\
32107.268\end{array}$ & $\begin{array}{l}0.69 \\
0.98 \\
1.115\end{array}$ & $\begin{array}{l}98 \\
88 \\
99\end{array}$ & $\begin{array}{lll}1 & \left({ }^{2} \mathrm{~F}_{7 / 2}^{\circ}\right)\left({ }^{3} \mathrm{D}\right) & { }^{3}\left[{ }^{9} / 2\right]^{\circ} \\
3 & \left({ }^{2} \mathrm{~F}_{5 / 2}^{\circ}\right)\left({ }^{1} \mathrm{D}\right) & { }^{1}[9 / 2]^{\circ}\end{array}$ \\
\hline
\end{tabular}


Tm I, Odd Parity-Continued

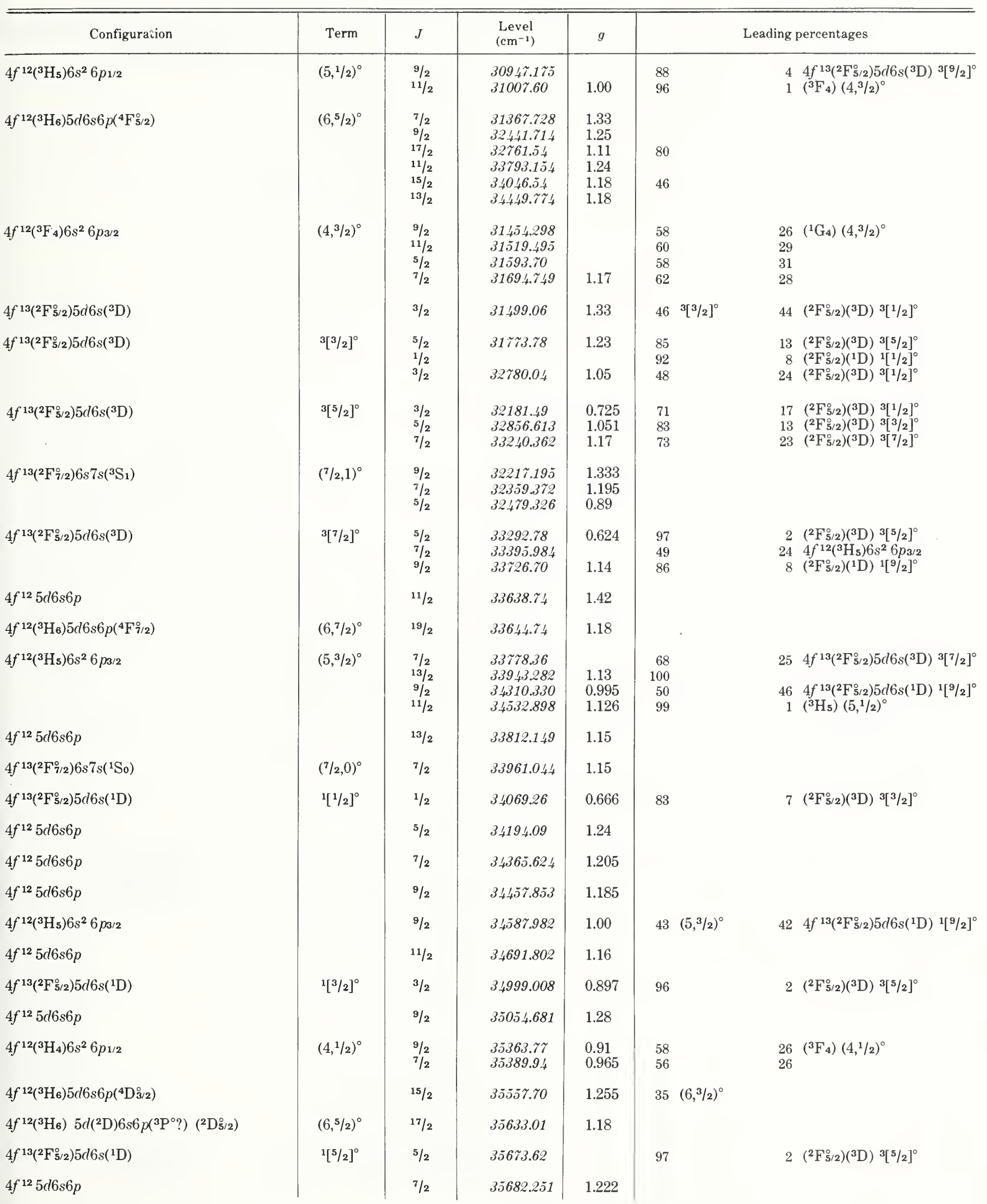


Tm I, Odd Parity_Continued

\begin{tabular}{|c|c|c|c|c|c|c|c|}
\hline Configuration & Term & $J$ & $\begin{array}{l}\text { Level } \\
\left(\mathrm{cm}^{-1}\right)\end{array}$ & $g$ & & & Leading percentages \\
\hline $4 f^{12} 5 d 6 s 6 p$ & & $13 / 2$ & 35958.880 & 1.134 & & & \\
\hline $4 f^{12}\left({ }^{3} \mathrm{H}_{6}\right) 5 d 6 s 6 p\left({ }^{4} \mathrm{D}_{5 / 2}\right)$ & $(6,5 / 2)^{\circ}$ & $15 / 2$ & 36179.60 & 1.155 & 47 & & \\
\hline $4 f^{12} 5 d 6 s 6 p$ & & $13 / 2$ & 36223.628 & 1.12 & & & \\
\hline $4 f^{12}\left({ }^{3} \mathrm{H}_{6}\right) 5 d 6 s 6 p\left({ }^{4} \mathrm{~F}_{7 / 2}^{\circ}\right)$ & & $15 / 2$ & 36273.94 & 1.13 & 29 & $\left(6,{ }^{7} / 2\right)^{\circ}$ & \\
\hline $4 f^{12} 5 d 6 s 6 p$ & & $11 / 2$ & 36329.786 & 1.035 & & & \\
\hline $4 f^{12} 5 d 6 s 6 p$ & & $9 / 2$ & 36377.733 & 1.21 & & & \\
\hline $4 f^{13}\left({ }^{2} \mathrm{~F}^{\circ} / 2\right) 5 d 6 s\left({ }^{1} \mathrm{D}\right)$ & ${ }^{1}\left[{ }^{7} / 2\right]^{\circ}$ & $7 / 2$ & 36435.78 & & & & \\
\hline $4 f^{12}\left({ }^{3} \mathrm{H}_{6}\right) 5 d\left({ }^{2} \mathrm{D}\right) 6 s 6 p\left({ }^{3} \mathrm{P}^{\circ} ?\right)\left({ }^{2} \mathrm{D}_{3 / 2}^{\circ}\right)$ & $(6,3 / 2)^{\circ}$ & $15 / 2$ & 36653.924 & 1.13 & & & \\
\hline $4 f^{12} 5 d 6 s 6 p$ & & $11 / 2$ & 36656.724 & 1.16 & & & \\
\hline $4 f^{12} 5 d 6 s 6 p$ & & $7 / 2$ & 36934.216 & 1.285 & & & \\
\hline $4 f^{12} 5 d 6 s 6 p$ & & $9 / 2$ & 37010.534 & 1.11 & & & \\
\hline $4 f^{12} 5 d 6 s 6 p$ & & $15 / 2$ & 37159.51 & 1.225 & & & \\
\hline $4 f^{12}\left({ }^{3} \mathrm{~F}_{3}\right) 6 s^{2} 6 p_{1 / 2}$ & $\left(3,{ }^{1} / 2\right)^{\circ}$ & $\begin{array}{l}5 / 2 \\
7 / 2\end{array}$ & 37221.455 & & $\begin{array}{l}82 \\
96\end{array}$ & & $\begin{array}{ll}6 & \left({ }^{3} \mathrm{~F}_{2}\right) \\
3 & (2,1 / 2)^{\circ} \\
3 & \left({ }^{3} \mathrm{H}_{5}\right)\left(5,{ }^{3} / 2\right)^{\circ}\end{array}$ \\
\hline $4 f^{12\left({ }^{3} \mathrm{H}_{6}\right) 5 d 6 s 6 p\left({ }^{4} \mathrm{D}_{7 / 2}\right)}$ & & $17 / 2$ & 37252.03 & 1.20 & 27 & $(6,7 / 2)^{\circ}$ & \\
\hline $4 f^{12} 5 d 6 s 6 p$ & & $11 / 2$ & 37276.778 & 1.25 & & & \\
\hline $4 f^{12} 5 d 6 s 6 p$ & & $13 / 2$ & 37365.13 & 1.20 & & & \\
\hline $4 f^{12} 5 d 6 s 6 p$ & & $7 / 2$ & 37519.484 & 1.09 & & & \\
\hline $4 f^{12} 5 d 6 s 6 p$ & & $13 / 2$ & 37646.186 & 1.175 & & & \\
\hline $4 f^{12} 5 d 6 s 6 p$ & & $11 / 2$ & 37657.928 & 1.01 & & & \\
\hline $4 f^{12} 5 d 6 s 6 p$ & & $9 / 2$ & 37768.662 & 1.215 & & & \\
\hline $4 f^{12\left({ }^{3} \mathrm{H}_{6}\right) 5 d 6 s 6 p\left({ }^{4} \mathrm{D}_{7 / 2}^{\circ}\right)}$ & $\left(6,{ }^{7} / 2\right)^{\circ}$ & $15 / 2$ & 37858.62 & 1.20 & 53 & & \\
\hline $4 f^{12} 5 d 6 s 6 p$ & & $13 / 2$ & 38012.795 & 1.09 & & & \\
\hline $4 f^{12} 5 d 6 s 6 p$ & & $11 / 2$ & 38161.255 & 1.125 & & & \\
\hline $4 f^{12} 5 d 6 s 6 p$ & & $7 / 2$ & 38211.625 & 1.175 & & & \\
\hline $4 f^{12\left({ }^{3} \mathrm{H}_{4}\right) 6 s^{2} 6 p_{3 / 2}}$ & $(4,3 / 2)^{\circ}$ & $\begin{array}{l}11 / 2 \\
5 / 2 \\
7 / 2 \\
9 / 2\end{array}$ & $\begin{array}{l}38347.875 \\
38664.184 \\
38685.555\end{array}$ & $\begin{array}{l}1.07 \\
1.042 \\
1.043\end{array}$ & $\begin{array}{l}59 \\
41 \\
57 \\
57\end{array}$ & & 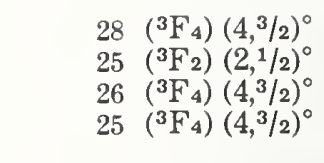 \\
\hline $4 f^{12} 5 d 6 s 6 p$ & & $9 / 2$ & 38384.926 & 1.134 & & & \\
\hline $4 f^{12} 5 d 6 s 6 p$ & & $7 / 2$ & 38482.584 & 1.25 & & & \\
\hline $4 f^{12\left({ }^{3} \mathrm{H}_{6}\right) 5 d 6 s 6 p\left({ }^{4} \mathrm{~F}_{9 / 2}^{\circ}\right)}$ & $(6,9 / 2)^{\circ}$ & $15 / 2$ & 38485.85 & 1.145 & 45 & & \\
\hline $4 f^{12} 5 d 6 s 6 p$ & & $9 / 2$ & 38.499 .016 & 1.064 & & & \\
\hline $4 f^{12} 5 d 6 s 6 p$ & & $11 / 2$ & 38529.686 & 1.17 & & & \\
\hline $4 f^{12} 5 d 6 s 6 p$ & & $13 / 2$ & 38588.46 & 1.212 & & & \\
\hline $4 f^{12} 5 d 6 s 6 p$ & & $5 / 2$ & 38751.812 & 1.215 & & & \\
\hline $4 f^{13}\left({ }^{2} \mathrm{~F}_{7 / 2}^{\circ}\right) 6 s 6 d\left({ }^{3} \mathrm{D}\right)$ & $3\left[^{3} / 2\right]^{\circ}$ & $\begin{array}{l}5 / 2 \\
3 / 2 \\
1 / 2\end{array}$ & $\begin{array}{l}38861.93 \% \\
39602.902 \\
39737.08\end{array}$ & $\begin{array}{l}1.565 \\
1.30 \\
0.81\end{array}$ & $\begin{array}{r}96 \\
57 \\
100\end{array}$ & $\begin{array}{l}\text { or } \\
\text { or } \\
\text { or }\end{array}$ & $\begin{array}{rr}57 & \left({ }^{2} \mathrm{~F}_{7 / 2}^{\circ}\right)\left({ }^{3} \mathrm{D}_{1}\right) \\
64 & \left({ }^{2} \mathrm{~F}_{7 / 2}^{\circ}\right)\left({ }^{3} \mathrm{D}_{3}\right) \\
100 & \left({ }^{2} \mathrm{~F}_{7 / 2}^{\circ}\right)\left({ }^{3} \mathrm{D}_{3}\right)\end{array}$ \\
\hline
\end{tabular}


Tm I, Odd Parity-Continued

\begin{tabular}{|c|c|c|c|c|c|c|c|}
\hline Configuration & Term & $J$ & $\begin{array}{l}\text { Level } \\
\left(\mathrm{cm}^{-1}\right)\end{array}$ & $g$ & & & Leading percentages \\
\hline $4 f^{12} 5 d 6 s 6 p$ & & $11 / 2$ & 38877.211 & 1.065 & & & \\
\hline $4 f^{12} 5 d 6 s 6 p$ & & $13 / 2$ & 38915.90 & 1.22 & & & \\
\hline $4 f^{13}\left({ }^{2} \mathrm{~F}_{7 / 2}^{\circ}\right) 6 s 6 d\left({ }^{1} \mathrm{D}\right)$ & $1[3 / 2]^{\circ}$ & $3 / 2$ & 39061.819 & 1.34 & 57 & or & $57 \quad\left({ }^{2} \mathrm{~F}_{7 / 2}^{\circ}\right)\left({ }^{1} \mathrm{D}_{2}\right)$ \\
\hline $4 f^{13}\left({ }^{2} \mathrm{~F}_{7 / 2}^{\circ}\right) 6 s 6 d\left({ }^{3} \mathrm{D}\right)$ & $3[11 / 2]^{\circ}$ & $\begin{array}{c}11 / 2 \\
13 / 2 \\
9 / 2\end{array}$ & $\begin{array}{l}39089.533 \\
3915 \% .5 \% \\
39220.035\end{array}$ & $\begin{array}{l}1.09 \\
1.24 \\
1.12\end{array}$ & $\begin{array}{r}65 \\
100 \\
78\end{array}$ & $\begin{array}{l}\text { or } \\
\text { or } \\
\text { or }\end{array}$ & $\begin{aligned} 64 & \left({ }^{2} \mathrm{~F}_{7 / 2}^{\circ}\right)\left({ }^{3} \mathrm{D}_{2}\right) \\
100 & \left({ }^{2} \mathrm{~F}_{7 / 2}^{\circ}\right)\left({ }^{3} \mathrm{D}_{3}\right) \\
97 & \left({ }^{2} \mathrm{~F}_{7 / 2}^{\circ}\right)\left({ }^{3} \mathrm{D}_{1}\right)\end{aligned}$ \\
\hline $4 f^{13}\left({ }^{2} \mathrm{~F}_{7 / 2}^{\circ}\right) 6 s 6 d\left({ }^{3} \mathrm{D}\right)$ & $\left.3{ }^{5} / 2\right]^{\circ}$ & $\begin{array}{l}7 / 2 \\
5 / 2 \\
3 / 2\end{array}$ & $\begin{array}{l}39187.473 \\
39277.087 \\
39419.90\end{array}$ & $\begin{array}{l}1.336 \\
1.184 \\
0.916\end{array}$ & $\begin{array}{l}76 \\
68 \\
86\end{array}$ & $\begin{array}{l}\text { or } \\
\text { or } \\
\text { or }\end{array}$ & $\begin{array}{ll}49 & \left({ }^{2} \mathrm{~F}_{7 / 2}^{\circ}\right)\left({ }^{3} \mathrm{D}_{1}\right) \\
37 & \left({ }^{2} \mathrm{~F}^{\mathrm{c}}{ }^{\circ}\right)\left({ }^{3} \mathrm{D}_{3}\right) \\
67 & \left({ }^{2} \mathrm{~F}_{7 / 2}{ }^{\circ}\right)\left({ }^{3} \mathrm{D}_{2}\right)\end{array}$ \\
\hline $4 f^{12} 5 d 6 s 6 p$ & & $9 / 2$ & 39244.584 & 1.05 & & & \\
\hline $4 f^{13}\left({ }^{2} \mathrm{~F}_{7 / 2}^{\circ}\right) 6 s 6 d\left({ }^{3} \mathrm{D}\right)$ & $3\left[{ }^{7} / 2\right]^{\circ}$ & $\begin{array}{l}9 / 2 \\
7 / 2 \\
5 / 2\end{array}$ & $\begin{array}{l}39322.014 \\
39462.782 \\
39658.852\end{array}$ & $\begin{array}{l}1.153 \\
1.12 \\
0.96\end{array}$ & $\begin{array}{l}67 \\
40 \\
72\end{array}$ & $\begin{array}{l}\text { or } \\
\text { or } \\
\text { or }\end{array}$ & $\begin{array}{ll}84 & \left({ }^{2} \mathrm{~F}_{7 / 2}^{\circ}\right)\left({ }^{3} \mathrm{D}_{2}\right) \\
38 & \left({ }^{2} \mathrm{~F}_{7 / 2}^{\circ}\right)\left({ }^{3} \mathrm{D}_{1}\right) \\
54 & \left({ }^{2} \mathrm{~F}_{7 / 2}^{\circ}\right)\left({ }^{3} \mathrm{D}_{3}\right)\end{array}$ \\
\hline $4 f^{13}\left({ }^{2} \mathrm{~F}_{7 / 2}^{\circ}\right) 6 s 6 d\left({ }^{1} \mathrm{D}\right)$ & $1[11 / 2]^{\circ}$ & $11 / 2$ & 39362.651 & 1.13 & 66 & or & $66 \quad\left({ }^{2} \mathrm{~F}_{7 / 2}^{\circ}\right)\left({ }^{1} \mathrm{D}_{2}\right)$ \\
\hline $4 f^{12} 5 d 6 s 6 p$ & & $5 / 2$ & 39386.959 & 0.982 & & & \\
\hline $4 f^{12} 5 d 6 s 6 p$ & & $7 / 2$ & 39413.672 & 1.165 & & & \\
\hline $4 f^{12} 5 d 6 s 6 p$ & & $13 / 2$ & 39434.151 & 1.15 & & & \\
\hline $4 f^{13}\left({ }^{2} \mathrm{~F}_{7 / 2}^{\circ}\right) 6 s 6 d\left({ }^{1} \mathrm{D}\right)$ & & $9 / 2$ & 39444.295 & 1.13 & 38 & ${ }^{1}[9 / 2]^{\circ}$ & $59\left({ }^{2} \mathrm{~F}_{7 / 2}^{\circ}\right)\left({ }^{3} \mathrm{D}_{3}\right)$ \\
\hline $4 f^{13}\left({ }^{2} \mathrm{~F}_{7 / 2}^{\circ}\right) 6 s 6 d\left({ }^{1} \mathrm{D}\right)$ & $1[5 / 2]^{\circ}$ & $5 / 2$ & 39470.489 & 1.04 & 70 & or & $70 \quad\left({ }^{2} \mathrm{~F}_{7 / 2}^{\circ}\right)\left({ }^{1} \mathrm{D}_{2}\right)$ \\
\hline $4 f^{13}\left({ }^{2} \mathrm{~F}_{7 / 2}^{\circ}\right) 6 s 6 d\left({ }^{3} \mathrm{D}\right)$ & $3[9 / 2]^{\circ}$ & $11 / 2$ & 39479.873 & 1.21 & 87 & or & $95 \quad\left({ }^{2} \mathrm{~F}_{7 / 2}^{\circ}\right)\left({ }^{3} \mathrm{D}_{3}\right)$ \\
\hline $4 f^{12} 5 d 6 s 6 p$ & & $7 / 2$ & 39480.272 & 1.17 & & & \\
\hline $4 f^{12} 5 d 6 s 6 p$ & & $9 / 2$ & 39488.236 & 1.095 & ' & & \\
\hline $4 f^{13}\left({ }^{2} \mathrm{~F}_{7 / 2}^{\circ}\right) 6 s 6 d\left({ }^{1} \mathrm{D}\right)$ & $1[7 / 2]^{\circ}$ & $7 / 2$ & 39542.165 & 1.06 & 81 & or & $81 \quad\left({ }^{2} \mathrm{~F}^{\circ} / 2\right)\left({ }^{1} \mathrm{D}_{2}\right)$ \\
\hline $4 f^{12} 5 d 6 s 6 p$ & & $11 / 2$ & 39563.003 & 1.11 & & & \\
\hline $4 f^{13}\left({ }^{2} \mathrm{~F}_{\mathrm{7} / 2}^{\circ}\right) 6 s 6 d\left({ }^{3} \mathrm{D}\right)$ & & $7 / 2$ & 39627.996 & 1.04 & 45 & ${ }^{3}[9 / 2]^{\circ}$ & $59\left({ }^{2} \mathrm{~F}_{7 / 2}^{\circ}\right)\left({ }^{3} \mathrm{D}_{3}\right)$ \\
\hline $4 f^{12} 5 d 6 s 6 p$ & & $11 / 2$ & 39709.038 & 1.17 & & & \\
\hline $4 f^{13}\left({ }^{2} \mathrm{~F}_{7 / 2}^{\circ}\right) 6 s 6 d\left({ }^{1} \mathrm{D}\right)$ & $1[9 / 2]^{\circ}$ & $9 / 2$ & 39741.913 & 1.112 & 58 & or & $58\left({ }^{2} \mathrm{~F}_{7 / 2}^{\circ}\right)\left({ }^{1} \mathrm{D}_{2}\right)$ \\
\hline $4 f^{12}\left({ }^{3} F_{3}\right) 6 s^{2} 6 p_{3 / 2}$ & $(3,3 / 2)^{\circ}$ & $\begin{array}{l}7 / 2 \\
5 / 2 \\
9 / 2 \\
3 / 2\end{array}$ & $\begin{array}{l}40061.324 \\
40208.499\end{array}$ & $\begin{array}{l}1.13 \\
1.18\end{array}$ & $\begin{array}{l}97 \\
95 \\
98 \\
94\end{array}$ & & 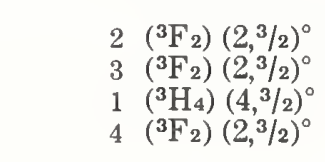 \\
\hline $4 f^{12} 5 d 6 s 6 p$ & & $13 / 2$ & 40113.75 & & & & \\
\hline $4 f^{12} 5 d 6 s 6 p$ & & $9 / 2$ & 40322.536 & 1.115 & & & \\
\hline $4 f^{12} 5 d 6 s 6 p$ & & $5 / 2$ & 40347.267 & 1.14 & & & \\
\hline $4 f^{12} 5 d 6 s 6 p$ & & $13 / 2$ & 40376.20 & & & & \\
\hline $4 f^{12} 5 d 6 s 6 p$ & & $9 / 2$ & 40517.661 & 1.153 & & & \\
\hline $4 f^{12} 5 d 6 s 6 p$ & & $7 / 2$ & 40533.825 & 1.24 & & & \\
\hline $4 f^{12} 5 d 6 s 6 p$ & & $7 / 2$ & 40596.302 & 1.305 & & & \\
\hline $\begin{array}{l}4 f^{12} 5 d 6 s 6 p \\
4 f^{12} 5 d 6 s 6 p\end{array}$ & & $\begin{array}{l}11 / 2 \\
7 / 2\end{array}$ & $\begin{array}{l}40663.129 \\
40763.892\end{array}$ & 1.178 & & & \\
\hline $4 f^{12} 5 d 6 s 6 p$ & & $13 / 2$ & 40780.904 & 1.22 & & & \\
\hline $4 f^{12} 5 d 6 s 6 p$ & & $9 / 2$ & 40855.580 & 1.08 & & & \\
\hline $4 f^{12} 5 d 6 s 6 p$ & & $5 / 2$ & 40938.61 & 1.10 & & & \\
\hline
\end{tabular}


Tm I, Odd Parity-Continued

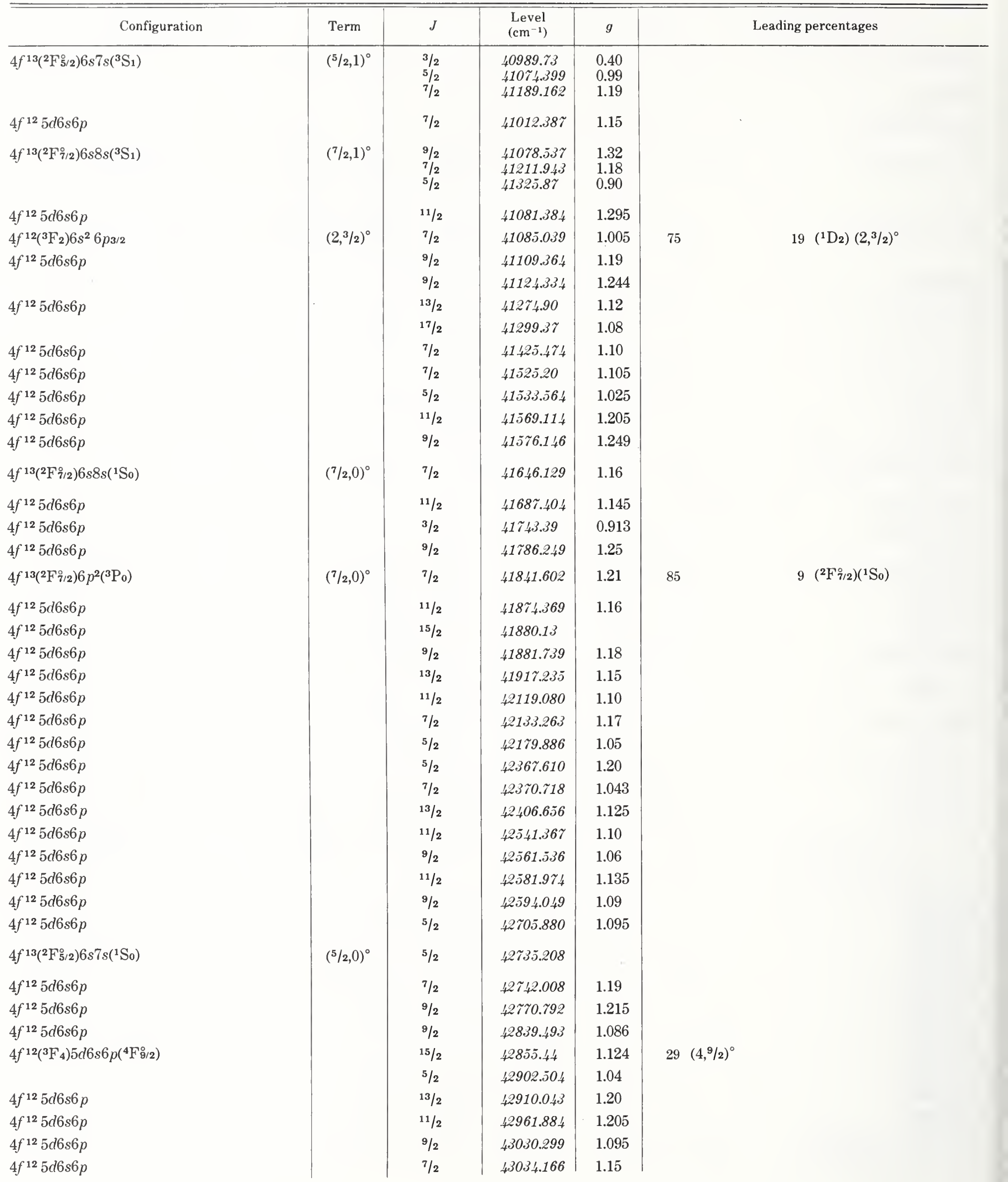


Tm I, Odd Parity—Continued

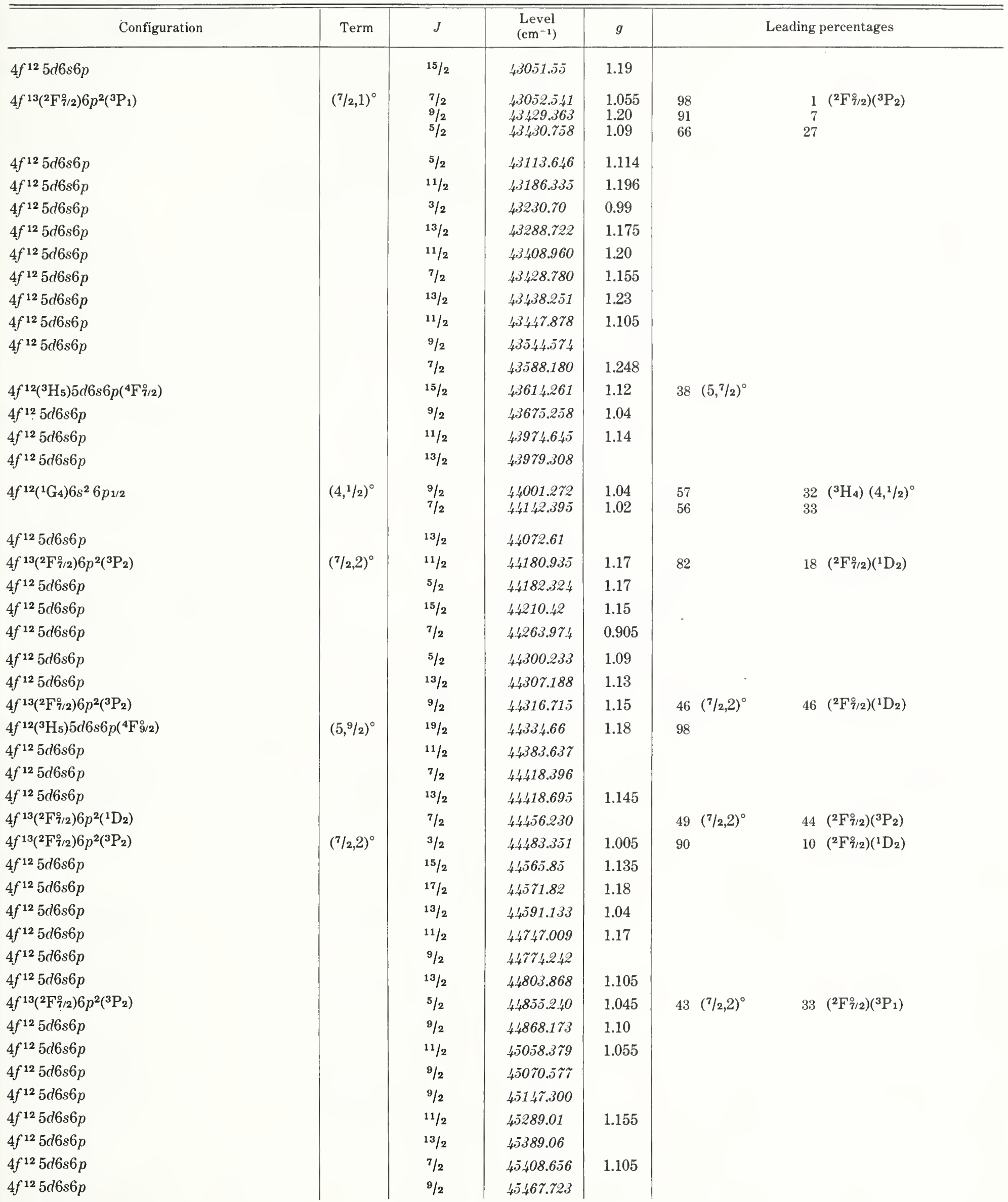


Tm I, Odd Parity_Continued

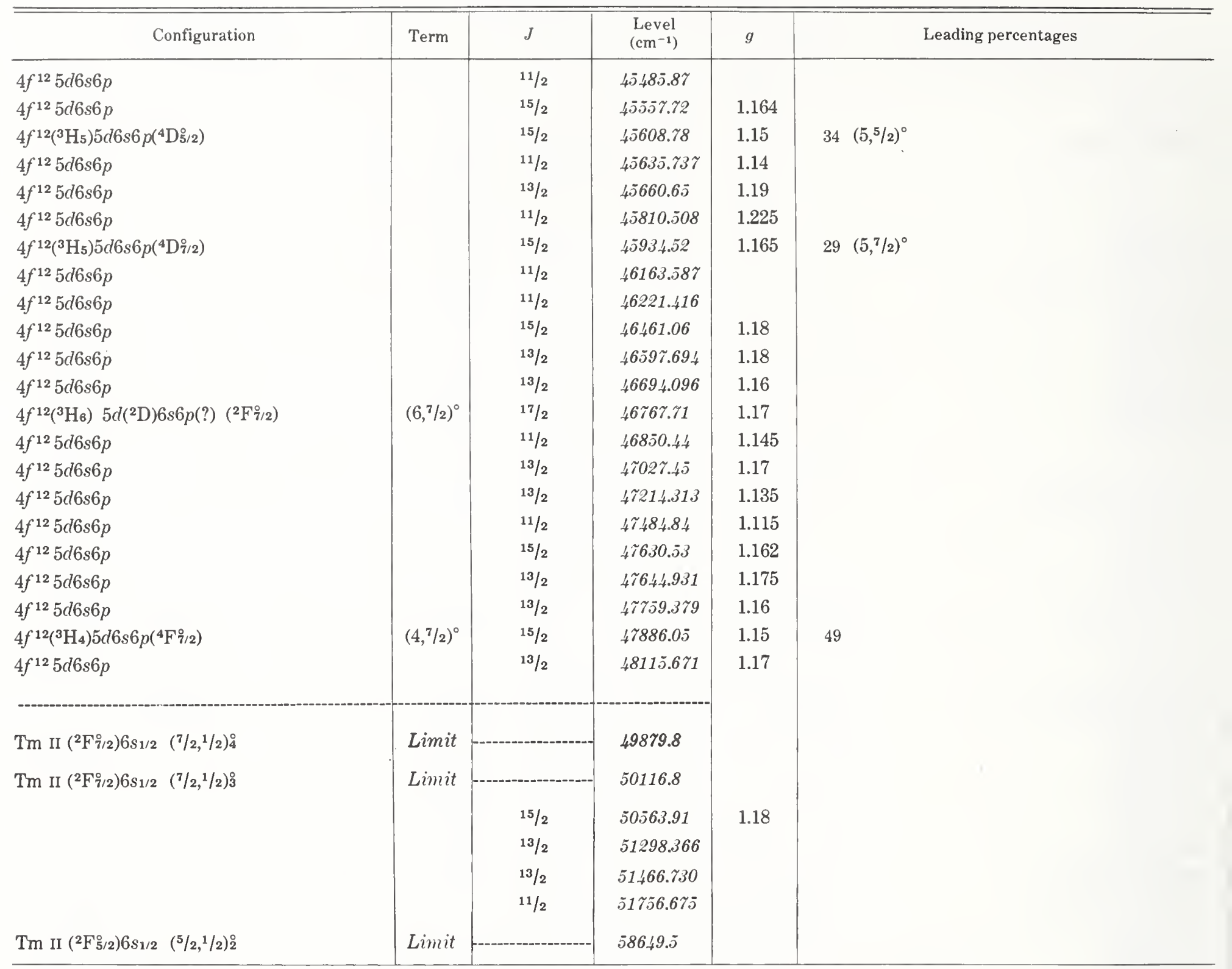


Tm I, Even Parity

\begin{tabular}{|c|c|c|c|c|c|c|}
\hline Configuration & Term & $J$ & $\begin{array}{l}\text { Level } \\
\left(\mathrm{cm}^{-1}\right)\end{array}$ & $g$ & \multicolumn{2}{|c|}{ Leading percentages } \\
\hline $4 f^{12}\left({ }^{3} \mathrm{H}_{6}\right) 5 d_{3 / 2} 6 s^{2}$ & $(6,3 / 2)$ & $\begin{array}{l}9 / 2 \\
15 / 2 \\
11 / 2 \\
13 / 2\end{array}$ & $\begin{array}{l}13119.610 \\
15271.002 \\
15587.811 \\
17454.818\end{array}$ & $\begin{array}{l}1.305 \\
1.08 \\
1.255 \\
1.15\end{array}$ & $\begin{array}{l}86 \\
96 \\
76 \\
95\end{array}$ & 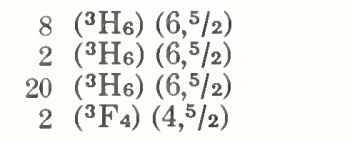 \\
\hline $4 f^{12\left({ }^{3} \mathrm{H}_{6}\right) 5 d_{5 / 2} 6 s^{2}}$ & $(6,5 / 2)$ & $\begin{array}{l}17 / 2 \\
7 / 2 \\
15 / 2 \\
9 / 2 \\
11 / 2 \\
13 / 2\end{array}$ & $\begin{array}{l}16456.913 \\
16957.006 \\
18693.074 \\
18837.385 \\
18853.823 \\
19466.663\end{array}$ & $\begin{array}{l}1.175 \\
1.1722 \\
1.18 \\
1.1318 \\
1.15 \\
1.15\end{array}$ & $\begin{array}{l}99 \\
70 \\
97 \\
76 \\
48 \\
85\end{array}$ & $\begin{array}{rl}7 & \left({ }^{3} \mathrm{~F}_{4}\right)\left(4,{ }^{3} / 2\right) \\
2 & \left({ }^{3} \mathrm{H}_{6}\right)\left(6,{ }^{3} / 2\right) \\
6 & \left({ }^{3} \mathrm{H}_{6}\right)\left(6,{ }^{3} / 2\right) \\
32 & 4 f^{13}\left({ }^{2} \mathrm{~F}_{7 / 2}^{\circ}\right) 6 s 6 p\left({ }^{3} \mathrm{P}_{2}^{\circ}\right) \\
9 & \left({ }^{3} \mathrm{~F}_{4}\right)(4,5 / 2)\end{array}$ \\
\hline $4 f^{13}\left({ }^{2} \mathrm{~F}_{7 / 2}^{\circ}\right) 6 s 6 p\left({ }^{3} \mathrm{P}_{0}^{\circ}\right)$ & $\left({ }^{7} / 2,0\right)$ & $7 / 2$ & 16742.237 & 1.325 & 78 & $17\left({ }^{2} \mathrm{~F}_{7 / 2}^{\circ}\right)\left({ }^{3} \mathrm{P}_{1}^{\circ}\right)$ \\
\hline $4 f^{13}\left({ }^{2} \mathrm{~F}_{7 / 2}^{\circ}\right) 6 s 6 p\left({ }^{3} \mathrm{P}_{1}^{\circ}\right)$ & $(7 / 2,1)$ & $\begin{array}{l}7 / 2 \\
9 / 2 \\
5 / 2\end{array}$ & $\begin{array}{l}17343.374 \\
17613.659 \\
17752.634\end{array}$ & $\begin{array}{l}1.02153 \\
1.18598 \\
1.186\end{array}$ & $\begin{array}{l}77 \\
94 \\
88\end{array}$ & $\begin{aligned} 16 & \left({ }^{2} \mathrm{~F}_{7 / 2}^{\circ}\right)\left({ }^{3} \mathrm{P}_{0}^{\circ}\right) \\
3 & \left({ }^{2} \mathrm{~F}_{7 / 2}^{\circ}\right)\left({ }^{3} \mathrm{P}_{2}^{\circ}\right) \\
9 & \left({ }^{2} \mathrm{~F}_{7 / 2}^{\circ}\right)\left({ }^{3} \mathrm{P}_{2}^{\circ}\right)\end{aligned}$ \\
\hline $4 f^{13}\left({ }^{2} \mathrm{~F}_{7 / 2}^{\circ}\right) 6 s 6 p\left({ }^{3} \mathrm{P}_{2}^{\circ}\right)$ & $(7 / 2,2)$ & $\begin{array}{l}11 / 2 \\
3 / 2 \\
5 / 2 \\
9 / 2 \\
7 / 2\end{array}$ & $\begin{array}{l}18990.406 \\
19132.245 \\
19548.834 \\
19748.543 \\
19753.830\end{array}$ & $\begin{array}{l}1.215 \\
0.88 \\
0.983 \\
1.29 \\
1.1839\end{array}$ & $\begin{array}{l}68 \\
99 \\
90 \\
97 \\
95\end{array}$ & $\begin{array}{ll}21 & 4 f^{12}\left({ }^{3} \mathrm{H}_{6}\right) 5 d_{5 / 2} 6 s^{2} \\
& \\
9 & \left({ }^{2} \mathrm{~F}_{7 / 2}^{\circ}\right)\left({ }^{3} \mathrm{P}_{1}^{\circ}\right) \\
3 & \left({ }^{2} \mathrm{~F}_{7 / 2}^{\circ}\right)\left({ }^{3} \mathrm{P}_{1}^{\circ}\right) \\
2 & \left({ }^{2} \mathrm{~F}_{7 / 2}^{\circ}\right)\left({ }^{3} \mathrm{P}_{0}^{\circ}\right)\end{array}$ \\
\hline $4 f^{12}\left({ }^{3} \mathrm{~F}_{4}\right) 5 d_{3 / 2} 6 s^{2}$ & $(4,3 / 2)$ & $\begin{array}{l}7 / 2 \\
5 / 2 \\
9 / 2 \\
11 / 2\end{array}$ & $\begin{array}{l}21120.836 \\
21161.401 \\
21737.685 \\
21997.473\end{array}$ & $\begin{array}{l}1.17372 \\
1.14 \\
1.1210 \\
1.06\end{array}$ & $\begin{array}{l}54 \\
49 \\
61 \\
40\end{array}$ & $\begin{array}{ll}21 & \left({ }^{1} \mathrm{G}_{4}\right)\left(4,{ }^{3} / 2\right) \\
29 & \\
25 & \\
26 & \end{array}$ \\
\hline $4 f^{12}\left({ }^{3} \mathrm{H}_{5}\right) 5 d_{3 / 2} 6 s^{2}$ & $(5,3 / 2)$ & $\begin{array}{c}7 / 2 \\
13 / 2 \\
9 / 2 \\
11 / 2\end{array}$ & $\begin{array}{l}22791.176 \\
23374.681 \\
24348.692 \\
25488.545\end{array}$ & $\begin{array}{l}1.1427 \\
0.98 \\
1.12\end{array}$ & $\begin{array}{l}75 \\
87 \\
44 \\
91\end{array}$ & $\begin{array}{rl}5 & \left({ }^{3} \mathrm{~F}_{4}\right)(4,5 / 2) \\
4 & \left({ }^{3} \mathrm{H}_{6}\right)(6,5 / 2) \\
28 & 4 f^{13}\left({ }^{2} \mathrm{~F}^{3} / 2\right) 6 s 6 p\left({ }^{1} \mathrm{P}_{1}^{\circ}\right) \\
2 & \left({ }^{3} \mathrm{~F}_{4}\right)\left(4,{ }^{3} / 2\right)\end{array}$ \\
\hline $4 f^{12}\left({ }^{3} \mathrm{H}_{5}\right) 5 d_{5 / 2} 6 s^{2}$ & & $5 / 2$ & 22929.717 & 1.152 & $27(5,5 / 2)$ & $20\left({ }^{3} F_{4}\right)(4,5 / 2)$ \\
\hline $4 f^{12}\left({ }^{3} \mathrm{~F}_{4}\right) 5 d_{5 / 2} 6 s^{2}$ & $(4,5 / 2)$ & $11 / 2$ & 23309.979 & 1.11 & 56 & $25 \quad\left({ }^{1} \mathrm{G}_{4}\right)(4,5 / 2)$ \\
\hline $4 f^{12}\left({ }^{3} \mathrm{~F}_{4}\right) 5 d_{5 / 2} 6 s^{2}$ & $(4,5 / 2)$ & $9 / 2$ & 23781.698 & 1.1499 & 49 & $21\left({ }^{1} \mathrm{G}_{4}\right)\left(4,{ }^{5} / 2\right)$ \\
\hline $4 f^{12}\left({ }^{3} \mathrm{~F}_{4}\right) 5 d_{5 / 2} 6 s^{2}$ & & $7 / 2$ & 23873.207 & 1.135 & $37(4,5 / 2)$ & $224 f^{13}\left({ }^{2} \mathrm{~F}_{7 / 2}^{\circ}\right) 6 s 6 p\left({ }^{1} \mathrm{P}_{1}^{\circ}\right)$ \\
\hline $4 f^{12}\left({ }^{3} \mathrm{~F}_{4}\right) 5 d_{5 / 2} 6 s^{2}$ & & $3 / 2$ & 23882.41 & & $37(4,5 / 2)$ & $34 \quad\left({ }^{1} \mathrm{G}_{4}\right)(4,5 / 2)$ \\
\hline $4 f^{12}\left({ }^{3} \mathrm{~F}_{4}\right) 5 d_{5 / 2} 6 s^{2}$ & & $13 / 2$ & 24137.196 & 1.128 & $43(4,5 / 2)$ & $28\left({ }^{1} \mathrm{G}_{4}\right)(4,5 / 2)$ \\
\hline $4 f^{13}\left({ }^{2} \mathrm{~F}_{7 / 2}^{\circ}\right) 6 s 6 p\left({ }^{1} \mathrm{P}_{\mathrm{i}}^{\circ}\right)$ & $(7 / 2,1)$ & $5 / 2$ & 24418.018 & 1.06 & 50 & $324 f^{12}\left({ }^{3} \mathrm{H}_{5}\right) 5 d_{5 / 2} 6 s^{2}$ \\
\hline $4 f^{12}\left({ }^{3} \mathrm{H}_{5}\right) 5 d_{5 / 2} 6 s^{2}$ & $(5,5 / 2)$ & $\begin{array}{l}15 / 2 \\
7 / 2 \\
9 / 2 \\
13 / 2 \\
11 / 2\end{array}$ & $\begin{array}{l}25130.453 \\
25717.197 \\
26889.125 \\
27377.13 \\
27491.31\end{array}$ & $\begin{array}{l}1.10 \\
1.02 \\
1.11 \\
1.12\end{array}$ & $\begin{array}{l}99 \\
70 \\
52 \\
92 \\
88\end{array}$ & $\begin{array}{rl}12 & 4 f^{13}\left({ }^{2} \mathrm{~F}^{\circ} / 2\right) 6 s 6 p\left({ }^{1} \mathrm{P}_{1}^{\circ}\right) \\
31 & 4 f^{13}\left({ }^{2} \mathrm{~F}^{\circ} / 2\right) 6 s 6 p\left({ }^{1} \mathrm{P}_{1}^{\circ}\right) \\
5 & \left({ }^{3} \mathrm{H}_{5}\right)(5,3 / 2) \\
9 & \left({ }^{3} \mathrm{~F}_{3}\right)(3,5 / 2)\end{array}$ \\
\hline $4 f^{13}\left({ }^{2} \mathrm{~F}_{5 / 2}^{\circ}\right) 6 s 6 p\left({ }^{3} \mathrm{P}_{0}^{\circ}\right)$ & $(5 / 2,0)$ & $5 / 2$ & 25656.019 & 0.724 & 86 & $6\left({ }^{2} \mathrm{~F}_{5 / 2}^{\circ}\right)\left({ }^{3} \mathrm{P}_{1}^{\circ}\right)$ \\
\hline $4 f^{13}\left({ }^{2} \mathrm{~F}_{7 / 2}^{\circ}\right) 6 s 6 p\left({ }^{1} \mathrm{P}_{1}^{\circ}\right)$ & & $5 / 2$ & 25745.117 & 1.09 & $28(7 / 2,1)$ & $114 f^{12}\left({ }^{3} \mathrm{~F}_{4}\right) 5 d_{3 / 2} 6 s^{2}$ \\
\hline $4 f^{13}\left({ }^{2} \mathrm{~F}_{5 / 2}^{\circ}\right) 6 s 6 p\left({ }^{3} \mathrm{P}_{1}^{\circ}\right)$ & $(5 / 2,1)$ & $\begin{array}{l}5 / 2 \\
7 / 2 \\
3 / 2\end{array}$ & $\begin{array}{l}26126.907 \\
26439.491 \\
26488.70\end{array}$ & $\begin{array}{l}1.10 \\
1.000 \\
0.85\end{array}$ & $\begin{array}{l}87 \\
92 \\
86\end{array}$ & $\begin{aligned} 7 & \left({ }^{2} \mathrm{~F}_{5 / 2}^{\circ}\right)\left({ }^{3} \mathrm{P}_{\circ}^{\circ}\right) \\
4 & \left({ }^{2} \mathrm{~F}_{5 / 2}^{\circ}\right)\left({ }^{3} \mathrm{P}_{2}^{\circ}\right) \\
11 & \left({ }^{2} \mathrm{~F}_{5 / 2}^{\circ}\right)\left({ }^{3} \mathrm{P}_{2}^{\circ}\right)\end{aligned}$ \\
\hline $4 f^{12}\left({ }^{3} \mathrm{H}_{5}\right) 5 d_{3 / 2} 6 s^{2}$ & & $9 / 2$ & 26646.214 & 0.961 & $34 \quad(5,3 / 2)$ & $25\left({ }^{3} \mathrm{H}_{5}\right)\left(5,{ }^{5} / 2\right)$ \\
\hline $4 f^{13}\left({ }^{2} \mathrm{~F}_{7 / 2}^{\circ}\right) 6 s 6 p\left({ }^{1} \mathrm{P}_{1}^{\circ}\right)$ & & $7 / 2$ & 26701.325 & 1.076 & $39(7 / 2,1)$ & $134 f^{12}\left({ }^{3} \mathrm{H}_{4}\right) 5 d_{3 / 2} 6 s^{2}$ \\
\hline $4 f^{13}\left({ }^{2} \mathrm{~F}_{5 / 2}^{\circ}\right) 6 s 6 p\left({ }^{3} \mathrm{P}_{2}^{\circ}\right)$ & $(5 / 2,2)$ & $\begin{array}{l}1 / 2 \\
9 / 2 \\
3 / 2 \\
5 / 2 \\
7 / 2\end{array}$ & $\begin{array}{l}27314.56 \\
28024.01 \\
28143.67 \\
28448.585 \\
28555.799\end{array}$ & $\begin{array}{l}-0.015 \\
1.15 \\
0.66 \\
1.000 \\
1.157\end{array}$ & $\begin{array}{r}100 \\
99 \\
60 \\
71 \\
96\end{array}$ & $\begin{array}{rl}10 & 4 f^{12}\left({ }^{3} \mathrm{H}_{4}\right) 5 d_{5 / 2} 6 s^{2} \\
7 & 4 f^{12}\left({ }^{3} \mathrm{H}_{4}\right) 5 d_{3 / 2} 6 s^{2} \\
4 & \left({ }^{2} \mathrm{~F}_{5 / 2}^{\circ}\right)\left({ }^{3} \mathrm{P}_{1}^{\circ}\right)\end{array}$ \\
\hline $4 f^{13}\left({ }^{2} \mathrm{~F}_{5 / 2}^{\circ}\right) 6 s 6 p\left({ }^{3} \mathrm{P}_{2}^{\circ}\right)$ & & $5 / 2$ & 28051.37 & 0.96 & $25 \quad(5 / 2,2)$ & $184 f^{12}\left({ }^{3} \mathrm{H}_{4}\right) 5 d_{3 / 2} 6 s^{2}$ \\
\hline
\end{tabular}


Tm I, Even Parity-Continued

\begin{tabular}{|c|c|c|c|c|c|c|}
\hline Configuration & Term & $J$ & $\begin{array}{l}\text { Level } \\
\left(\mathrm{cm}^{-1}\right)\end{array}$ & $g$ & \multicolumn{2}{|c|}{ Leading percentages } \\
\hline $4 f^{12}\left({ }^{3} \mathrm{~F}_{3}\right) 5 d_{3 / 2} 6 s^{2}$ & & $7 / 2$ & 29260.59 & 0.928 & $46 \quad(3,3 / 2)$ & $11\left({ }^{3} \mathrm{H}_{4}\right)\left(4,{ }^{3} / 2\right)$ \\
\hline $4 f^{12}\left({ }^{3} \mathrm{H}_{4}\right) 5 d_{3 / 2} 6 s^{2}$ & $\left(4,{ }^{3} / 2\right)$ & $9 / 2$ & 29316.690 & 0.978 & 50 & $21 \quad\left({ }^{3} \mathrm{~F}_{4}\right)\left(4,{ }^{3} / 2\right)$ \\
\hline $4 f^{12}\left({ }^{3} \mathrm{~F}_{2}\right) 5 d_{3 / 2} 6 s^{2}$ & & $5 / 2$ & 30082.18 & 0.81 & $29(2,3 / 2)$ & $20\left({ }^{3} \mathrm{H}_{4}\right)(4,5 / 2)$ \\
\hline $4 f^{12}\left({ }^{3} \mathrm{H}_{4}\right) 5 d_{5 / 2} 6 s^{2}$ & & $7 / 2$ & 30124.02 & 0.955 & $24(4,5 / 2)$ & $15\left({ }^{3} F_{4}\right)\left(4,{ }^{5} / 2\right)$ \\
\hline $4 f^{12\left({ }^{3} \mathrm{H}_{4}\right) 5 d_{5 / 2} 6 s^{2}}$ & $(4,5 / 2)$ & $13 / 2$ & 30125.61 & & 58 & $32\left({ }^{3} \mathrm{~F}_{4}\right)(4,5 / 2)$ \\
\hline $4 f^{12}\left({ }^{3} \mathrm{H}_{4}\right) 5 d_{5 / 2} 6 s^{2}$ & & $5 / 2$ & 30302.42 & 0.91 & $22(4,5 / 2)$ & $18\left({ }^{3} \mathrm{~F}_{2}\right)(2,3 / 2)$ \\
\hline $4 f^{12\left({ }^{3} \mathrm{~F}_{3}\right) 5 d_{3 / 2} 6 s^{2}}$ & & $3 / 2$ & 30585.65 & 1.085 & $32 \quad(3,3 / 2)$ & $31 \quad\left({ }^{3} \mathrm{~F}_{2}\right)\left(2,{ }^{3} / 2\right)$ \\
\hline $4 f^{12\left(3 \mathrm{~F}_{3}\right) 5 d_{3 / 2} 6 s^{2}}$ & & $9 / 2$ & 30915.02 & 0.99 & $28 \quad(3,3 / 2)$ & $27\left({ }^{3} \mathrm{H}_{4}\right)(4,5 / 2)$ \\
\hline $4 f^{12\left({ }^{3} \mathrm{H}_{4}\right) 5 d_{5 / 2} 6 s^{2}}$ & $(4,5 / 2)$ & $11 / 2$ & 30972.46 & & 46 & $19\left({ }^{3} F_{4}\right)\left(4,{ }^{5} / 2\right)$ \\
\hline $4 f^{12}\left({ }^{3} \mathrm{~F}_{3}\right) 5 d_{3 / 2} 6 s^{2}$ & & $5 / 2$ & 31431.88 & 1.07 & $42(3,3 / 2)$ & $16 \quad\left({ }^{3} \mathrm{~F}_{2}\right)(2,5 / 2)$ \\
\hline $4 f^{12}\left({ }^{3} \mathrm{~F}_{3}\right) 5 d_{3 / 2} 6 s^{2}$ & & $9 / 2$ & 31440.54 & 1.13 & $37 \quad(3,3 / 2)$ & $30 \quad\left({ }^{3} F_{3}\right)(3,5 / 2)$ \\
\hline $4 f^{12}\left({ }^{3} \mathrm{~F}_{3}\right) 5 d_{5 / 2} 6 s^{2}$ & & $7 / 2$ & 31510.24 & 1.11 & $22(3,5 / 2)$ & $17\left({ }^{3} F_{2}\right)(2,5 / 2)$ \\
\hline $4 f^{12}\left({ }^{3} \mathrm{~F}_{3}\right) 5 d_{3 / 2} 6 s^{2}$ & & $3 / 2$ & 31520.98 & 0.79 & $40 \quad(3,3 / 2)$ & $20 \quad\left({ }^{3} \mathrm{H}_{4}\right)(4,5 / 2)$ \\
\hline $4 f^{12}\left({ }^{3} \mathrm{~F}_{3}\right) 5 d d_{5 / 2} 6 s^{2}$ & $(3,5 / 2)$ & $\begin{array}{c}5 / 2 \\
9 / 2 \\
7 / 2 \\
3 / 2 \\
11 / 2 \\
1 / 2\end{array}$ & $\begin{array}{l}32174.49 \\
32407.78 \\
32811.02 \\
32928.76\end{array}$ & $\begin{array}{l}1.16 \\
1.04 \\
0.935\end{array}$ & $\begin{array}{l}72 \\
58 \\
69 \\
48 \\
79 \\
63\end{array}$ & $\begin{array}{rl}6 & \left({ }^{3} \mathrm{~F}_{3}\right)\left(3,{ }^{3} / 2\right) \\
18 & \left({ }^{3} \mathrm{~F}_{3}\right)\left(3,{ }^{3} / 2\right) \\
8 & \left({ }^{3} \mathrm{H}_{4}\right)\left(4,{ }^{3 / 2}\right) \\
40 & 4 f^{13}\left({ }^{2} \mathrm{~F}_{5 / 2}^{0}\right) 6 s 6 p\left({ }^{1} \mathrm{P}_{1}^{\circ}\right) \\
7 & \left({ }^{3} \mathrm{H}_{5}\right)\left(5,{ }^{5} / 2\right) \\
17 & \left({ }^{3} \mathrm{~F}_{2}\right)\left(2,{ }^{3} / 2\right)\end{array}$ \\
\hline $4 f^{12}\left({ }^{3} \mathrm{~F}_{2}\right) 5 d_{3 / 2} 6 s^{2}$ & & $7 / 2$ & 32446.26 & 0.89 & $41 \quad(2,3 / 2)$ & $10\left({ }^{3} \mathrm{H}_{4}\right)(4,5 / 2)$ \\
\hline $4 f^{12}\left({ }^{3} \mathrm{~F}_{2}\right) 5 d_{5 / 2} 6 s^{2}$ & $(5 / 2,2)$ & $3 / 2$ & 33489.36 & 1.468 & 45 & $18\left({ }^{3} \mathrm{~F}_{3}\right)(3,5 / 2)$ \\
\hline $4 f^{12}\left({ }^{3} \mathrm{~F}_{2}\right) 5 d_{5 / 2} 6 s^{2}$ & & $7 / 2$ & 33623.78 & 0.92 & $39 \quad(2,5 / 2)$ & $11 \quad\left({ }^{3} F_{3}\right)(3,3 / 2)$ \\
\hline $4 f^{13}\left({ }^{2} \mathrm{~F}_{5 / 2}^{\circ}\right) 6 s 6 p\left({ }^{1} \mathrm{P}_{1}^{\circ}\right)$ & $(5 / 2,1)$ & $5 / 2$ & 34085.20 & 0.95 & 45 & $20 \quad 4 f^{12}\left({ }^{3} \mathrm{~F}_{2}\right) 5 d d_{5 / 2} 6 s^{2}$ \\
\hline $4 f^{13}\left({ }^{2} \mathrm{~F}_{5 / 2}^{\circ}\right) 6 s 6 p\left({ }^{1} \mathrm{P}_{1}^{\circ}\right)$ & $(5 / 2,1)$ & $7 / 2$ & 34297.17 & 0.915 & 74 & $44 f^{12}\left({ }^{1} \mathrm{G}_{4}\right) 5 d_{3 / 2} 6 s^{2}$ \\
\hline $4 f^{12}\left({ }^{3} \mathrm{~F}_{2}\right) 5 d_{5 / 2} 6 s^{2}$ & $(2,5 / 2)$ & $9 / 2$ & 34446.22 & & 58 & $14\left({ }^{1} \mathrm{D}_{2}\right)(2,5 / 2)$ \\
\hline $4 f^{13}\left({ }^{2} \mathrm{~F}_{5 / 2}^{\circ}\right) 6 s 6 p\left({ }^{1} \mathrm{P}_{1}^{\circ}\right)$ & & $5 / 2$ & 35026.22 & 1.022 & $26(5 / 2,1)$ & $234 f^{12}\left({ }^{3} \mathrm{~F}_{2}\right) 5 d_{5 / 2} 6 s^{2}$ \\
\hline $4 f^{13}\left({ }^{2} \mathrm{~F}_{5 / 2}^{\circ}\right) 6 s 6 p\left({ }^{1} \mathrm{P}_{1}^{\circ}\right)$ & & $3 / 2$ & 35089.67 & 0.85 & $33(5 / 2,1)$ & $214 f^{12}\left({ }^{3} \mathrm{~F}_{3}\right) 5 d_{5 / 2} 6 s^{2}$ \\
\hline & & $5 / 2$ & 35261.762 & 1.346 & & \\
\hline & & $5 / 2$ & 37064.18 & 1.42 & & \\
\hline & & $3 / 2$ & 37138.57 & 1.56 & & \\
\hline $4 f^{13}\left({ }^{2} \mathrm{~F}_{7 / 2}^{\circ}\right) 6 s 7 p$ & & $7 / 2$ & 37557.25 & 1.313 & & \\
\hline $4 f^{\mathbf{1 3}}\left({ }^{\mathbf{2}} \mathrm{F}_{7 / 2}^{\circ}\right) 6 s 7 p$ & & $9 / 2$ & 37576.866 & 1.19 & & \\
\hline & & $9 / 2$ & 37711.074 & 1.14 & & \\
\hline $4 f^{13}\left({ }^{2} \mathrm{~F}_{7 / 2}^{\circ}\right) 6 s 7 p$ & & $5 / 2$ & 37724.84 & 1.19 & & \\
\hline $4 f^{13}\left({ }^{2} \mathrm{~F}_{7 / 2}^{\circ}\right) 6 s 7 p$ & & $7 / 2$ & 37781.90 & 0.97 & & \\
\hline $4 f^{13}\left({ }^{2} \mathrm{~F}_{7 / 2}^{0}\right) 6 s 7 p$ & & $9 / 2$ & 37946.39 & & & \\
\hline & & $5 / 2,7 / 2$ & 37980.69 & & & \\
\hline $4 f^{12}\left({ }^{1} \mathrm{G}_{4}\right) 5 d_{5 / 2} 6 s^{2}$ & & $5 / 2$ & 38014.37 & 1.11 & $40 \quad(4,5 / 2)$ & $21 \quad\left({ }^{3} \mathrm{H}_{4}\right)(4,5 / 2)$ \\
\hline $4 f^{12}\left({ }^{1} \mathrm{G}_{4}\right) 5 d_{3 / 2} 6 s^{2}$ & $(4,3 / 2)$ & $9 / 2$ & 38120.71 & & 50 & $38\left({ }^{3} \mathrm{H}_{4}\right)(4,3 / 2)$ \\
\hline $4 f^{13}\left({ }^{12} F_{7 / 2}^{\circ}\right) 6 s 7 p$ & & $7 / 2$ & 38123.02 & 1.14 & & \\
\hline & & $5 / 2$ & 38128.37 & 1.05 & & \\
\hline $4 f^{13}\left({ }^{2} \mathrm{~F}_{7 / 2}^{\circ}\right) 6 s 7 p$ & & $3 / 2$ & 38237.89 & 1.04 & & \\
\hline $4 f^{13}\left({ }^{2} \mathrm{~F}_{7 / 2}^{\circ}\right) 5 d 6 p\left({ }^{3} \mathrm{~F}_{2}^{\circ}\right)$ & $(7 / 2,2)$ & $\begin{array}{l}3 / 2 \\
5 / 2 \\
11 / 2 \\
7 / 2 \\
9 / 2\end{array}$ & $\begin{array}{l}38318.62 \\
39547.31 \\
40128.384 \\
40802.56 \\
41074.45\end{array}$ & $\begin{array}{l}1.195 \\
1.285 \\
1.10 \\
1.185 \\
1.07\end{array}$ & $\begin{array}{l}80 \\
52 \\
74 \\
65 \\
45\end{array}$ & 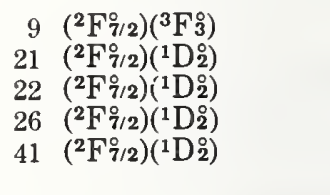 \\
\hline $4 f^{12}\left({ }^{1} \mathrm{G}_{4}\right) 5 d_{5 / 2} 6 s^{2}$ & & $7 / 2$ & 38342.57 & 1.108 & $30(4,5 / 2)$ & $27\left({ }^{3} \mathrm{H}_{4}\right)(4,5 / 2)$ \\
\hline $4 f^{13}\left({ }^{2} \mathrm{~F}_{7 / 2}^{\circ}\right) 6 s 7 p$ & & $5 / 2$ & 38433.92 & & & \\
\hline $4 f^{13}\left({ }^{2} \mathrm{~F}_{7 / 2}^{\circ}\right) 6 s 7 p$ & & $9 / 2$ & 38502.00 & 1.10 & & \\
\hline & & $5 / 2$ & 38696.79 & & & \\
\hline
\end{tabular}


Tm I, Even Parity-Continued

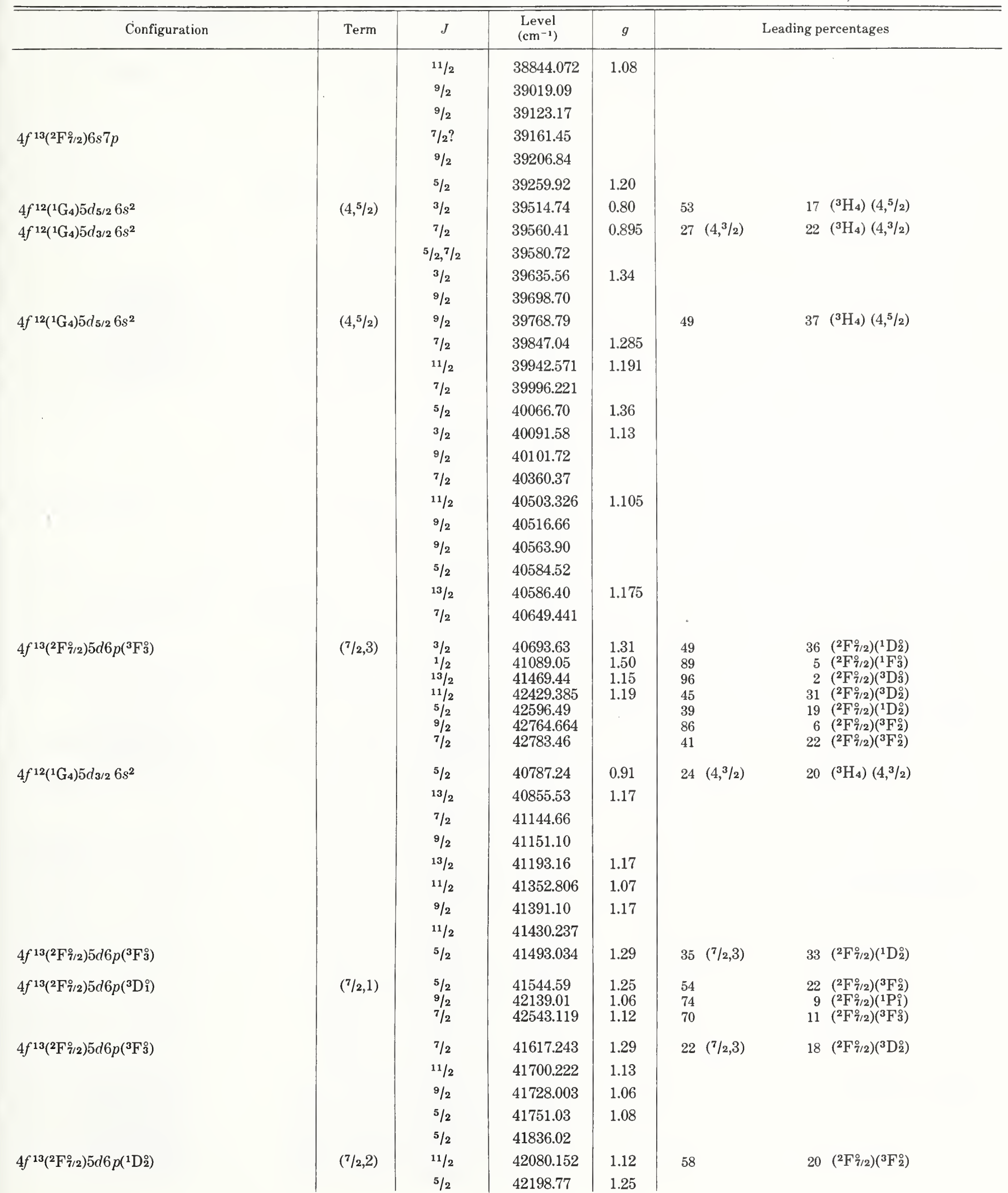


Tm I, Even Parity-Continued

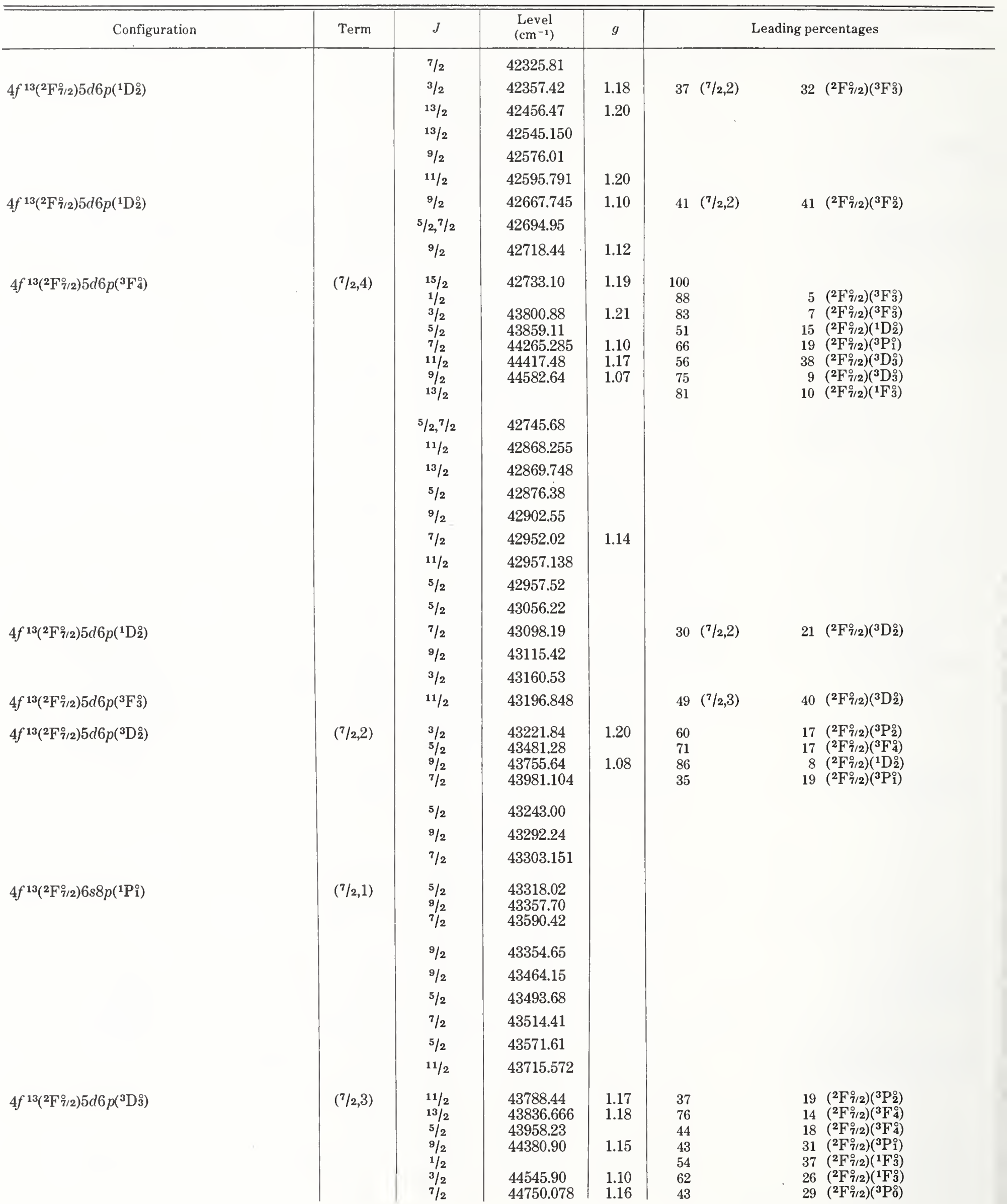


Tm I, Even Parity-Continued

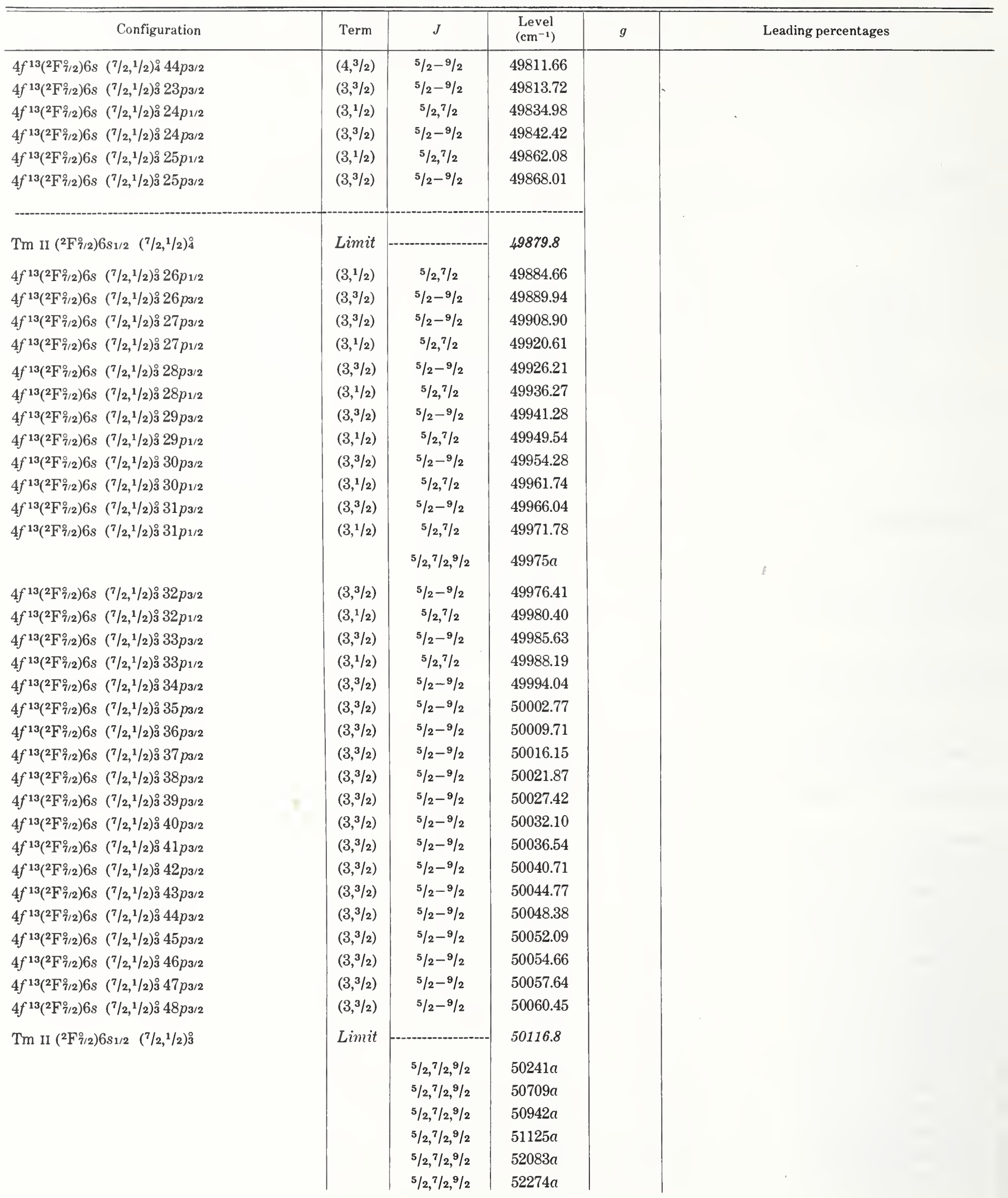


Tm I, Even Parity-Continued

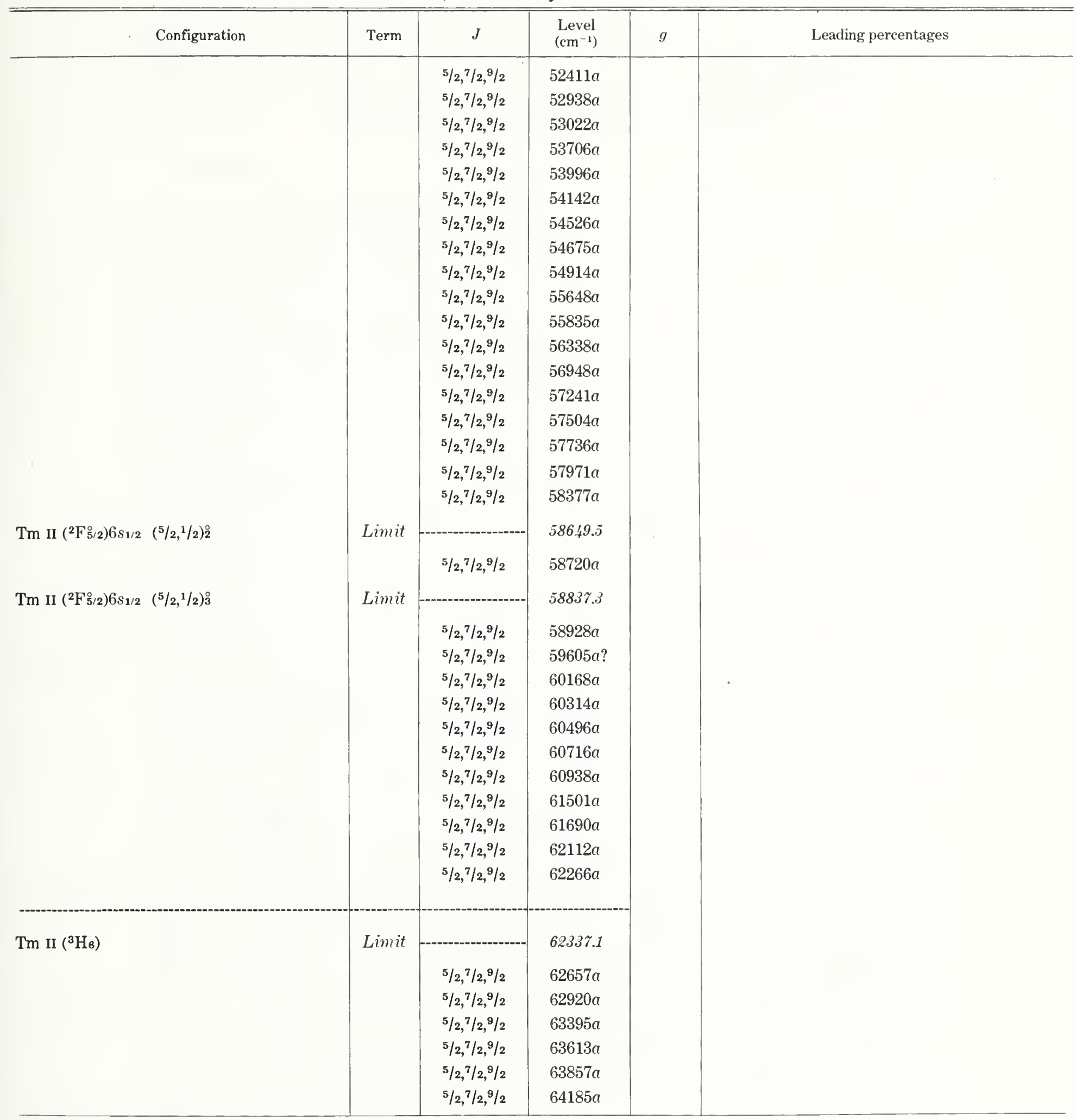


(Er I sequence; 68 electrons)

Ground state $\left(1 s^{2} 2 s^{2} 2 p^{6} 3 s^{2} 3 p^{6} 3 d^{10} 4 s^{2} 4 p^{6} 4 d^{10} 5 s^{2} 5 p^{6}\right) 4 f^{13}\left({ }^{2} \mathrm{~F}_{7 / 2}^{\circ}\right) 6 s(7 / 2,1 / 2)_{4}^{\circ}$

Ionization energy $97200 \pm 600 \mathrm{~cm}^{-1}$

$12.05 \pm 0.08 \mathrm{eV}$

Identified odd configurations

$4 f^{13} 6 s, 4 f^{13} 5 d, 4 f^{12} 6 s 6 p, 4 f^{12} 5 d 6 p, 4 f^{13} 7 s$

Identified even configurations

$$
4 f^{12} 6 s^{2}, 4 f^{12} 5 d 6 s, 4 f^{13} 6 p, 4 f^{12} 5 d^{2}
$$

Meggers first identified the four levels of the $4 f^{13} 6 s$ ground configuration, and he also published their combinations with 66 upper even levels [1942]. Spector [1962, 1967] and Blaise and Camus [1965] extended the analysis, the latter authors having new Zeeman data and a list of infrared lines to $2.4 \mu \mathrm{m}$. Theoretical calculations allowed assignment of most of the even levels to $4 f^{12} 5 d 6 s, 4 f^{12} 6 s^{2}$, and $4 f^{13} 6 p$, and the odd levels were extended to include $4 f^{13} 5 d$ and $4 f^{12} 6 s 6 p$.

The further extension and revision of the analysis as given here results mainly from a collaboration between the Laboratoire Aimé Cotton (Orsay, France) and NBS [Camus and Sugar, 1971a; 1971b]. Much of the detail of Camus' work is given in his thesis [1971]. The level values are mainly from Camus [1971], with some slightly revised values being supplied by Sugar [1973]. The complete line list for the region 2000-10000 $\AA$ has not been published; Spector lists about 200 classified lines (2421-6733 $\AA$ ), and Meggers, Corliss, and Scribner [1975] give 576 of the stronger Tm II lines in the region $2280-8566 \AA$, most of which are classified. The list of $537 \mathrm{Tm}$ infrared lines (8014-24483 $\AA$ ) measured by Camus, Guelachvili, and Verges [1969] includes a number of Tm II lines. Zeeman data for some strong infrared lines were also obtained by these authors.

The eigenvector percentages for $\left(4 f^{13} 6 s+4 f^{13} 5 d\right)$ are from Camus' thesis. The $J_{1} j$ coupling scheme is most appropriate for both configurations, although calculated percentages for the $4 f^{13} 6 s$ levels in this scheme were not available. The leading percentages in $L S$ coupling are given following the word "or." Wyart, Blaise, and Camus [1974] have recalculated Tm II $\left(4 f^{13} 6 s+4 f^{13} 5 d\right)$ with matrices including effective interactions. They found two new $4 f^{13} 5 d$ levels, at 23934.73 and $28874.14 \mathrm{~cm}^{-1}$.

Except for the $4 f^{13} 7 \mathrm{~s}$ levels and a few isolated higher odd levels, the known odd levels above the $4 f^{13} 6 s$ and $4 f^{13} 5 d$ groups are accounted for by the $\left(4 f^{12} 6 s 6 p+4 f^{12} 5 d 6 p\right)$ configurations [Camus and Sugar, 1971b]. Spector showed that the $J_{1} J_{2}$ coupling scheme was best for $4 f^{12} 6 s 6 p$, and Camus arranged additional levels of $\left(4 f^{12} 6 s 6 p+4 f^{12} 5 d 6 p\right)$ into $J_{1} J_{2}$ terms. Such term names as appear meaningful on the basis of the percentages given by Camus and Sugar [1971b] are retained here. Most of the term-designated levels of this group are given without percentages, since the appropriate percentages from the calculation including configuration interaction were not available. In the case of many levels having term purities significantly below 50\%, only the total eigenvector percentages from each of the two configurations are listed [Camus and Sugar, 1971b].

The calculation of the even levels included all four identified even configurations (with interaction) and allowed the assignment of 155 levels [Camus and Sugar, 1971a]. Most of the level assignments to the four $J_{1} L_{2}$-coupling terms of $4 f^{12}\left({ }^{3} \mathrm{H}_{6}\right) 5 d 6 \mathrm{~s}$ were originally made by Spector. In the arrangement here, three of these terms each have a level "missing" because of low eigenvector purities, and a couple of interchanged designations were made by Camus. Approximate percentages in the $J_{1} L_{2}$ scheme are listed for a number of higher $4 f^{12} 5 d 6 \mathrm{~s}$ levels, but only two additional term groups are retained for this configuration on the basis of the percentages given by Camus and Sugar. Some designated levels of $4 f^{12} 6 s^{2}$ and $4 f^{12} 5 d 6 s$ are given without leading percentages; such levels have significant percentages from more than one configuration, and the published term percentages did not include configuration-interaction effects [Camus and Sugar, 1971a]. Many levels having low term purities are given here without designations, but with the total percentages from one or both of the two main 
configurations. Such total percentages are also listed for all levels belonging mainly to $4 f^{12} 5 d^{2}$ or $4 f^{13} 6 p$, since term percentages from the calculation including configuration interactions were not available.

The ionization energy is from Sugar and Reader. Their estimate of the position of the $4 f^{13} 7 s$ configuration was confirmed very closely by the later experimental value [Camus and Sugar, 1971b].

\section{References}

Allen, L., United Kingdom Atomic Energy Research Establishment AERE-R 4029, 34 pp. (1962). W ZE

Blaise, J., and Camus, P., C. R. Acad. Sci. 260, 4693 (1965). EL ND ZE

Bovey, L. F. H., and Garton, W. R. S., Proc. Phys. Soc. London, Sect. A 67, 476 (1954). W

Camus, P., and Blaise, J., C. R. Acad. Sci. 261, 4359 (1965). EL ZE

Camus, P., Guelachvili, G., and Verges, J., Spectrochim. Acta, Part B 24, 373 (1969). EL CL W ZE

Camus, P., Thesis, Univ. Paris Orsay, 265 pp. (1971). E L ND CL W ZE IP PT

Camus, P., and Sugar, J., Phys. Scr. 4, 257 (1971a). EL ND ZE PT

Camus, P., and Sugar, J., Phys. Scr. 4, 263 (1971b). EL ND ZE IP PT

Goldschmidt, Z. B., Thesis, Hebrew Univ. Jerusalem, 487 pp. (1968). PT

King, A. S., Astrophys. J. 94, 226 (1941). Temperature Classification

Meggers, W. F., Rev. Mod. Phys. 14, 96 (1942). EL CL

Meggers, W. F., Corliss, C. H., and Scribner, B. F., Nat. Bur. Stand. (U.S.), Monogr. 145, Part 1, 403 pp. (1975).

Spector, N., Thesis, Hebrew Univ. Jerusalem (1962). EL ND CL PT

Spector, N., J. Opt. Soc. Am. 57, 312 (1967). EL CL PT

Sugar, J., unpublished material (1973). EL

Sugar, J., and Reader, J., J. Opt. Soc. Am. 55, 1286 (1965). IP

Wyart, J. F., Blaise, J., and Camus, P., Phys. Scr. 9, 325 (1974). EL CL PT

[October 1976]

Tm II, Odd Parity

\begin{tabular}{|c|c|c|c|c|c|c|c|c|}
\hline Configuration & Term & $J$ & $\begin{array}{l}\text { Level } \\
\left(\mathrm{cm}^{-1}\right)\end{array}$ & $g$ & \multicolumn{4}{|c|}{ Leading percentages } \\
\hline $4 f^{13}\left({ }^{2} \mathrm{~F}_{7 / 2}^{\circ}\right) 6 s_{1 / 2}$ & $(7 / 2,1 / 2)^{\circ}$ & $\begin{array}{l}4 \\
3\end{array}$ & $\begin{array}{r}0.00 \\
236.95\end{array}$ & $\begin{array}{l}1.250 \\
1.035\end{array}$ & 100 & $\begin{array}{l}\text { or } \\
\text { or }\end{array}$ & $\begin{array}{r}100 \\
59\end{array}$ & ${ }^{3} \mathrm{~F}^{\circ}$ \\
\hline $4 f^{13}\left({ }^{2} \mathrm{~F}_{5 / 2}^{\circ}\right) 6 s_{1 / 2}$ & $(5 / 2,1 / 2)^{\circ}$ & $\begin{array}{l}2 \\
3\end{array}$ & $\begin{array}{l}8769.68 \\
8957.47\end{array}$ & $\begin{array}{l}0.667 \\
1.055\end{array}$ & 100 & $\begin{array}{l}\text { or } \\
\text { or }\end{array}$ & $\begin{array}{r}100 \\
59\end{array}$ & $\begin{array}{l}{ }^{3} \mathrm{~F}^{\circ} \\
{ }^{1} \mathrm{~F}^{\circ}\end{array}$ \\
\hline $4 f^{13}\left({ }^{2} \mathrm{~F}_{7 / 2}^{\circ}\right) 5 d_{3 / 2}$ & $\left({ }^{7} / 2,{ }^{3} / 2\right)^{\circ}$ & $\begin{array}{l}2 \\
5 \\
3 \\
4\end{array}$ & $\begin{array}{l}17624.65 \\
20228.75 \\
21713.74 \\
22457.51\end{array}$ & $\begin{array}{l}1.48 \\
1.020 \\
1.22\end{array}$ & $\begin{array}{l}75 \\
95 \\
84 \\
93\end{array}$ & $\begin{array}{l}\text { or } \\
\text { or } \\
\text { or } \\
\text { or }\end{array}$ & $\begin{array}{l}91 \\
55 \\
70 \\
47\end{array}$ & $\begin{array}{l}\left({ }^{2} \mathrm{~F}^{\circ}\right){ }^{3} \mathrm{P}^{\circ} \\
\left({ }^{2} \mathrm{~F}^{\circ}\right){ }^{3} \mathrm{H}^{\circ} \\
\left({ }^{2} \mathrm{~F}^{\circ}\right){ }^{3} \mathrm{D}^{\circ} \\
\left({ }^{2} \mathrm{~F}^{\circ}\right){ }^{3} \mathrm{~F}^{\circ}\end{array}$ \\
\hline $4 f^{13}\left({ }^{2} \mathrm{~F}_{7 / 2}^{\circ}\right) 5 d_{5 / 2}$ & $(7 / 2,5 / 2)^{\circ}$ & $\begin{array}{l}6 \\
2 \\
1 \\
4 \\
3 \\
5\end{array}$ & $\begin{array}{l}21133.68 \\
21978.77 \\
22141.96 \\
23524.09 \\
23934.73 \\
24273.20\end{array}$ & $\begin{array}{l}1.167 \\
1.02 \\
1.325\end{array}$ & $\begin{array}{r}100 \\
76 \\
72 \\
93 \\
79 \\
95\end{array}$ & $\begin{array}{l}\text { or } \\
\text { or } \\
\text { or } \\
\text { or } \\
\text { or } \\
\text { or }\end{array}$ & $\begin{array}{r}100 \\
58 \\
78 \\
44 \\
34 \\
86\end{array}$ & $\begin{array}{l}\left({ }^{2} \mathrm{~F}^{\circ}\right){ }^{3} \mathrm{H}^{\circ} \\
\left({ }^{2} \mathrm{~F}^{\circ}\right){ }^{1} \mathrm{D}^{\circ} \\
\left({ }^{2} \mathrm{~F}^{\circ}\right){ }^{3} \mathrm{P}^{\circ} \\
\left({ }^{2} \mathrm{~F}^{\circ}\right){ }^{3} \mathrm{~F}^{\circ} \\
\left({ }^{2} \mathrm{~F}^{\circ}\right){ }^{1} \mathrm{~F}^{\circ} \\
\left({ }^{2} \mathrm{~F}^{\circ}\right){ }^{3} \mathrm{G}^{\circ}\end{array}$ \\
\hline $4 f^{13}\left({ }^{2} \mathrm{~F}_{5 / 2}^{\circ}\right) 5 d_{3 / 2}$ & $(5 / 2,3 / 2)^{\circ}$ & $\begin{array}{l}4 \\
2 \\
3 \\
1\end{array}$ & 28874.14 & & $\begin{array}{l}94 \\
86 \\
67 \\
76\end{array}$ & $\begin{array}{l}\text { or } \\
\text { or } \\
\text { or } \\
\text { or }\end{array}$ & $\begin{array}{l}85 \\
41 \\
87 \\
86\end{array}$ & $\begin{array}{l}\left({ }^{2} \mathrm{~F}^{\circ}\right){ }^{3} \mathrm{H}^{\circ} \\
\left({ }^{2} \mathrm{~F}^{\circ}\right){ }^{1} \mathrm{D}^{\circ} \\
\left({ }^{2} \mathrm{~F}^{\circ}\right){ }^{3} \mathrm{G}^{\circ} \\
\left({ }^{2} \mathrm{~F}^{\circ}\right){ }^{1} \mathrm{P}^{\circ}\end{array}$ \\
\hline $4 f^{12}\left({ }^{3} \mathrm{H}_{6}\right) 6 s 6 p\left({ }^{3} \mathrm{P}_{0}^{\circ}\right)$ & $(6,0)^{\circ}$ & 6 & 38225.46 & 1.273 & & & & \\
\hline $4 f^{12}\left({ }^{3} \mathrm{H}_{6}\right) 6 s 6 p\left({ }^{3} \mathrm{P}_{1}^{\circ}\right)$ & $(6,1)^{\circ}$ & $\begin{array}{l}6 \\
7 \\
5\end{array}$ & $\begin{array}{l}39477.60 \\
39638.41 \\
39863.81\end{array}$ & $\begin{array}{l}1.12 \\
1.185 \\
1.189\end{array}$ & & & & \\
\hline $4 f^{12}\left({ }^{3} \mathrm{H}_{6}\right) 6 s 6 p\left({ }^{3} \mathrm{P}_{2}^{\circ}\right)$ & $(6,2)^{\circ}$ & $\begin{array}{l}8 \\
4 \\
7 \\
5 \\
6\end{array}$ & $\begin{array}{l}42570.92 \\
43217.64 \\
43717.30 \\
43840.82 \\
43998.81\end{array}$ & $\begin{array}{l}1.25 \\
1.07 \\
1.25 \\
1.10 \\
1.20\end{array}$ & & & & \\
\hline
\end{tabular}


Tm II, Odd Parity_Continued

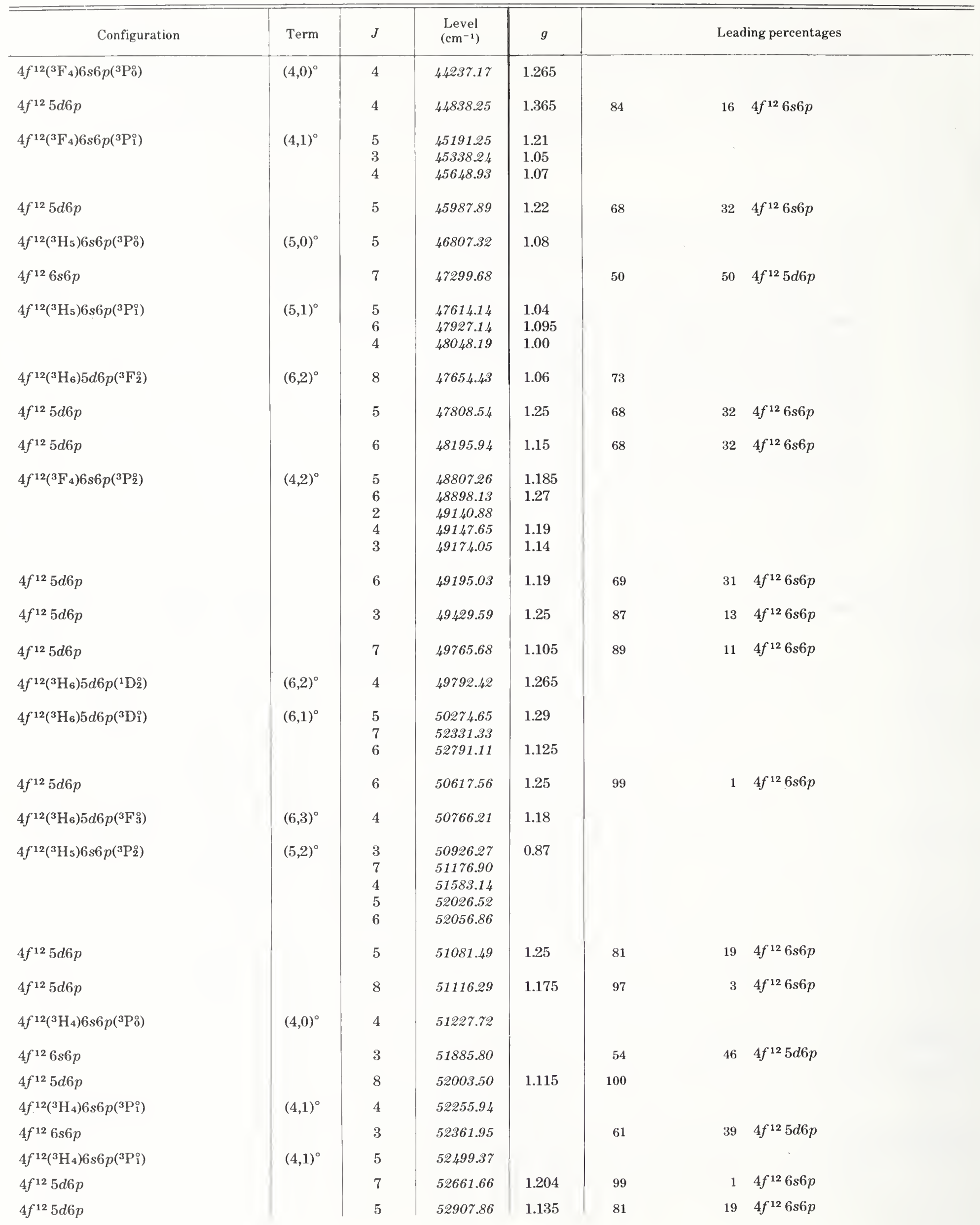


Tm II, Odd Parity-Continued

\begin{tabular}{|c|c|c|c|c|c|c|c|}
\hline Configuration & Term & $J$ & $\begin{array}{l}\text { Level } \\
\left(\mathrm{cm}^{-1}\right)\end{array}$ & $g$ & \multicolumn{3}{|c|}{ Leading percentages } \\
\hline $4 f^{12} 5 d 6 p$ & & 4 & 53002.40 & & 82 & 18 & $4 f^{12} 6 s 6 p$ \\
\hline $4 f^{12} 5 d 6 p$ & & 6 & 53013.80 & 1.19 & 95 & 5 & $4 f^{12} 6 s 6 p$ \\
\hline $4 f^{12}\left({ }^{3} \mathrm{~F}_{3}\right) 6 s 6 p\left({ }^{3} \mathrm{P}_{0}^{\circ}\right)$ & $(3,0)^{\circ}$ & 3 & 53150.82 & & & & \\
\hline $4 f^{12} 5 d 6 p$ & & 7 & 53304.88 & & 97 & 3 & $4 f^{12} 6 s 6 p$ \\
\hline $4 f^{12} 5 d 6 p$ & & 5 & 53336.89 & & 77 & 23 & $4 f^{12} 6 s 6 p$ \\
\hline $4 f^{12} 5 d 6 p$ & & 2 & 53411.92 & & 82 & 18 & $4 f^{12} 6 s 6 p$ \\
\hline $4 f^{12} 5 d 6 p$ & & 3 & 53494.12 & & 71 & 29 & $4 f^{12} 6 s 6 p$ \\
\hline $4 f^{12} 5 d 6 p$ & & 4 & 53576.56 & & 56 & 44 & $4 f^{12} 6 s 6 p$ \\
\hline $4 f^{13}\left({ }^{2} \mathrm{~F}_{7 / 2}^{\circ}\right) 7 s_{1 / 2}$ & $\left(7 / 2,{ }^{1} / 2\right)^{\circ}$ & $\begin{array}{l}4 \\
3\end{array}$ & $\begin{array}{l}53673.03 \\
53733.50\end{array}$ & $\begin{array}{l}1.24 \\
1.03\end{array}$ & & & \\
\hline $4 f^{12\left({ }^{3} \mathrm{H}_{6}\right) 5 d 6 p\left({ }^{3} \mathrm{D}_{2}^{\circ}\right)}$ & $(6,2)^{\circ}$ & 7 & 53738.88 & & & & \\
\hline $4 f^{12} 6 s 6 p$ & & 2 & 53888.54 & & 66 & 34 & $4 f^{12} 5 d 6 p$ \\
\hline $4 f^{12} 5 d 6 p$ & & 6 & 53896.44 & & 93 & 7 & $4 f^{12} 6 s 6 p$ \\
\hline $4 f^{12} 6 s 6 p$ & & 4 & 53955.64 & & 65 & 35 & $4 f^{12} 5 d 6 p$ \\
\hline $4 f^{12} 5 d 6 p$ & & 5 & 54317.06 & & 77 & 23 & $4 f^{12} 6 s 6 p$ \\
\hline $4 f^{12}\left({ }^{3} \mathrm{~F}_{3}\right) 6 s 6 p\left({ }^{3} \mathrm{P}_{1}^{\circ}\right)$ & $(3,1)^{\circ}$ & 3 & 54354.01 & & & & \\
\hline $4 f^{12} 5 d 6 p$ & & 4 & 54419.68 & & 79 & 21 & $4 f^{12} 6 s 6 p$ \\
\hline $4 f^{12} 5 d 6 p$ & & 5 & 54523.58 & & 98 & 2 & $4 f^{12} 6 s 6 p$ \\
\hline $4 f^{12} 6 s 6 p$ & & 3 & 54536.94 & & 71 & 29 & $4 f^{12} 5 d 6 p$ \\
\hline $4 f^{12} 5 d 6 p$ & & 4 & 54583.82 & & 83 & 17 & $4 f^{12} 6 s 6 p$ \\
\hline $4 f^{12} 5 d 6 p$ & & 8 & 54636.26 & & 99 & 1 & $4 f^{12} 6 s 6 p$ \\
\hline $4 f^{12} 5 d 6 p$ & & 3 & 54679.43 & & 90 & 10 & $4 f^{12} 6 s 6 p$ \\
\hline $4 f^{12} 5 d 6 p$ & & 6 & 54710.56 & & 94 & 6 & $4 f^{12} 6 s 6 p$ \\
\hline $4 f^{12} 5 d 6 p$ & & 7 & 54988.96 & & 99 & 1 & $4 f^{12} 6 s 6 p$ \\
\hline $4 f^{12} 5 d 6 p$ & & 3 & 55138.18 & & 76 & 24 & $4 f^{12} 6 s 6 p$ \\
\hline $4 f^{12} 5 d 6 p$ & & 6 & 55157.61 & & 90 & 10 & $4 f^{12} 6 s 6 p$ \\
\hline $4 f^{12} 5 d 6 p$ & & 5 & 55167.69 & & 95 & 5 & $4 f^{12} 6 s 6 p$ \\
\hline $4 f^{12} 5 d 6 p$ & & 6 & 55476.18 & & 72 & 28 & $4 f^{12} 6 s 6 p$ \\
\hline $4 f^{12} 5 d 6 p$ & & 6 & 55753.84 & & 53 & 47 & $4 f^{12} 6 s 6 p$ \\
\hline $4 f^{12} 5 d 6 p$ & & 7 & 55758.31 & 1.03 & 100 & & \\
\hline $4 f^{12} 5 d 6 p$ & & 4 & 55833.29 & & 57 & 43 & $4 f^{12} 6 s 6 p$ \\
\hline $4 f^{12} 6 s 6 p$ & & 6 & 55972.37 & & 54 & 46 & $4 f^{12} 5 d 6 p$ \\
\hline $4 f^{12}\left({ }^{3} \mathrm{H}_{4}\right) 6 s 6 p\left({ }^{3} \mathrm{P}_{2}^{\circ}\right)$ & $(4,2)^{\circ}$ & 3 & 56042.07 & & & & \\
\hline $4 f^{12\left({ }^{3} \mathrm{H}_{4}\right) 6 s 6 p\left({ }^{3} \mathrm{P}_{2}^{\circ}\right)}$ & $(4,2)^{\circ}$ & 5 & 56066.86 & & & & \\
\hline $4 f^{12} 5 d 6 p$ & & 7 & 56157.29 & & 99 & 1 & $4 f^{12} 6 s 6 p$ \\
\hline $4 f^{12} 6 s 6 p$ & & 4 & 56351.99 & & 59 & 41 & $4 f^{12} 5 d 6 p$ \\
\hline $4 f^{12} 5 d 6 p$ & & 3 & 56356.92 & & 92 & 8 & $4 f^{12} 6 s 6 p$ \\
\hline $4 f^{12} 5 d 6 p$ & & 5 & 56506.46 & & 94 & 6 & $4 f^{12} 6 s 6 p$ \\
\hline $4 f^{12} 5 d 6 p$ & & 6 & 56642.72 & & 95 & 5 & $4 f^{12} 6 s 6 p$ \\
\hline $4 f^{12} 5 d 6 p$ & & 4 & 56714.21 & & 92 & 8 & $4 f^{12} 6 s 6 p$ \\
\hline $4 f^{12} 5 d 6 p$ & & 5 & 56853.76 & & 97 & 3 & $4 f^{12} 6 s 6 p$ \\
\hline $4 f^{12} 6 s 6 p$ & & 5 & 57050.32 & , & 52 & 48 & $4 f^{12} 5 d 6 p$ \\
\hline $4 f^{12} 5 d 6 p$ & & 3 & 57253.02 & & 64 & 36 & $4 f^{12} 6 s 6 p$ \\
\hline $4 f^{12} 6 s 6 p$ & & 3 & 57465.55 & & 62 & 38 & $4 f^{12} 5 d 6 p$ \\
\hline
\end{tabular}


Tm II, Odd Parity_Continued

\begin{tabular}{|c|c|c|c|c|c|c|}
\hline Configuration & Term & $J$ & $\begin{array}{l}\text { Level } \\
\left(\mathrm{cm}^{-1}\right)\end{array}$ & $g$ & & Leading percentages \\
\hline $4 f^{12} 6 s 6 p$ & & 4 & 57520.46 & & 81 & $19 \quad 4 f^{12} 5 d 6 p$ \\
\hline $4 f^{12} 5 d 6 p$ & & 6 & 57526.40 & & 97 & $3 \quad 4 f^{12} 6 s 6 p$ \\
\hline $4 f^{12} 5 d 6 p$ & & 3 & 57547.98 & & 89 & $11 \quad 4 f^{12} 6 s 6 p$ \\
\hline $4 f^{12} 5 d 6 p$ & & 7 & 57573.34 & & 98 & $2 \quad 4 f^{12} 6 s 6 p$ \\
\hline $4 f^{12} 6 s 6 p$ & & 5 & 57599.35 & & 73 & $27 \quad 4 f^{12} 5 d 6 p$ \\
\hline $4 f^{12} 5 d 6 p$ & & 4 & 57741.72 & & 91 & $9 \quad 4 f^{12} 6 s 6 p$ \\
\hline $4 f^{12} 5 d 6 p$ & & 6 & 57829.34 & & 99 & $14 f^{12} 6 s 6 p$ \\
\hline $4 f^{12} 5 d 6 p$ & & 4 & 57859.31 & & 84 & $16 \quad 4 f^{12} 6 s 6 p$ \\
\hline $4 f^{12} 5 d 6 p$ & & 3 & 57919.15 & & 86 & $14 \quad 4 f^{12} 6 s 6 p$ \\
\hline $4 f^{12} 5 d 6 p$ & & 6 & 57961.53 & & 95 & $5 \quad 4 f^{12} 6 s 6 p$ \\
\hline $4 f^{12} 5 d 6 p$ & & 7 & 58051.31 & & 98 & $24 f^{12} 6 s 6 p$ \\
\hline $4 f^{12} 5 d 6 p$ & & 5 & 58107.79 & & 88 & $124 f^{12} 6 s 6 p$ \\
\hline $4 f^{12} 5 d 6 p$ & & 6 & 58236.52 & & 98 & $24 f^{12} 6 s 6 p$ \\
\hline $4 f^{12} 5 d 6 p$ & & 4 & 58351.88 & & 51 & $49 \quad 4 f^{12} 6 s 6 p$ \\
\hline $4 f^{12} 6 s 6 p$ & & 3 & 58379.52 & & 80 & $20 \quad 4 f^{12} 5 d 6 p$ \\
\hline $4 f^{12} 5 d 6 p$ & & 5 & 58383.36 & & 98 & $24 f^{12} 6 s 6 p$ \\
\hline $4 f^{12} 5 d 6 p$ & & 6 & 58500.70 & & 95 & $5 \quad 4 f^{12} 6 s 6 p$ \\
\hline $4 f^{12} 5 d 6 p$ & & 5 & 58538.86 & & 96 & $4 \quad 4 f^{12} 6 s 6 p$ \\
\hline $4 f^{12} 5 d 6 p$ & & 3 & 58644.27 & & 96 & $4 \quad 4 f^{12} 6 s 6 p$ \\
\hline $4 f^{12} 5 d 6 p$ & & 5 & 58698.22 & & 98 & $24 f^{12} 6 s 6 p$ \\
\hline $4 f^{12} 5 d 6 p$ & & 3 & 58742.98 & & 90 & $10 \quad 4 f^{12} 6 s 6 p$ \\
\hline $4 f^{12\left({ }^{3} \mathrm{~F}_{4}\right) 5 d 6 p\left({ }^{3} \mathrm{~F}_{3}^{\circ}\right)}$ & $(4,3)^{\circ}$ & 7 & 58978.88 & & & \\
\hline \multirow[t]{2}{*}{$4 f^{12} 5 d 6 p$} & & 4 & 5906728 & & 88 & $12 \quad 4 f^{12} 6 s 6 p$ \\
\hline & & 2 & 59135.89 & & & \\
\hline $4 f^{12} 5 d 6 p$ & & 2 & 59156.94 & & 95 & $5 \quad 4 f^{12} 6 s 6 p$ \\
\hline $4 f^{12} 5 d 6 p$ & & 4 & 59297.28 & & 80 & $20 \quad 4 f^{12} 6 s 6 p$ \\
\hline $4 f^{12} 5 d 6 p$ & & 5 & 59607.45 & & 85 & $15 \quad 4 f^{12} 6 s 6 p$ \\
\hline $4 f^{12} 5 d 6 p$ & & 4 & 59663.34 & & 94 & $6 \quad 4 f^{12} 6 s 6 p$ \\
\hline $4 f^{12} 5 d 6 p$ & & 3 & 59763.33 & & 79 & $21 \quad 4 f^{12} 6 s 6 p$ \\
\hline $4 f^{12} 5 d 6 p$ & & 3 & 59815.73 & & 96 & $4 \quad 4 f^{12} 6 s 6 p$ \\
\hline $4 f^{12} 5 d 6 p$ & & 4 & 59889.74 & & 59 & $41 \quad 4 f^{12} 6 s 6 p$ \\
\hline $4 f^{12} 5 d 6 p$ & & 4 & 60004.92 & & 73 & $27 \quad 4 f^{12} 6 s 6 p$ \\
\hline $4 f^{12} 5 d 6 p$ & & 3 & 60116.36 & & 82 & $18 \quad 4 f^{12} 6 s 6 p$ \\
\hline $4 f^{12} 5 d 6 p$ & & 5 & 60255.39 & & 79 & $21 \quad 4 f^{12} 6 s 6 p$ \\
\hline $4 f^{12}\left({ }^{3} \mathrm{H}_{5}\right) 5 d 6 p\left({ }^{3} \mathrm{D}_{\mathrm{i}}\right)$ & $(5,1)^{\circ}$ & 6 & 60262.99 & & & \\
\hline $4 f^{12} 5 d 6 p$ & & 4 & 60278.73 & & 86 & $14 \quad 4 f^{12} 6 s 6 p$ \\
\hline $4 f^{12} 5 d 6 p$ & & 7 & 60325.70 & & 100 & \\
\hline $4 f^{12\left({ }^{3} \mathrm{H}_{6}\right) 5 d 6 p\left({ }^{1} \mathrm{~F}_{3}^{\circ}\right)}$ & $(6,3)^{\circ}$ & 8 & 60396.70 & & 71 & \\
\hline \multirow[t]{2}{*}{$4 f^{12} 5 d 6 p$} & & 6 & 60418.58 & & 99 & $1 \quad 4 f^{12} 6 s 6 p$ \\
\hline & & 3 & 60503.23 & & & \\
\hline $4 f^{12} 5 d 6 p$ & & 7 & 60539.57 & & 99 & $1 \quad 4 f^{12} 6 s 6 p$ \\
\hline $4 f^{12} 5 d 6 p$ & & 4 & 60539.76 & & 75 & $25 \quad 4 f^{12} 6 s 6 p$ \\
\hline $4 f^{12} 5 d 6 p$ & & 3 & 60572.87 & & 92 & $8 \quad 4 f^{12} 6 s 6 p$ \\
\hline $4 f^{12} 5 d 6 p$ & & 6 & 60658.68 & & 99 & $14 f^{12} 6 s 6 p$ \\
\hline
\end{tabular}


Tm II, Odd Parity-Continued

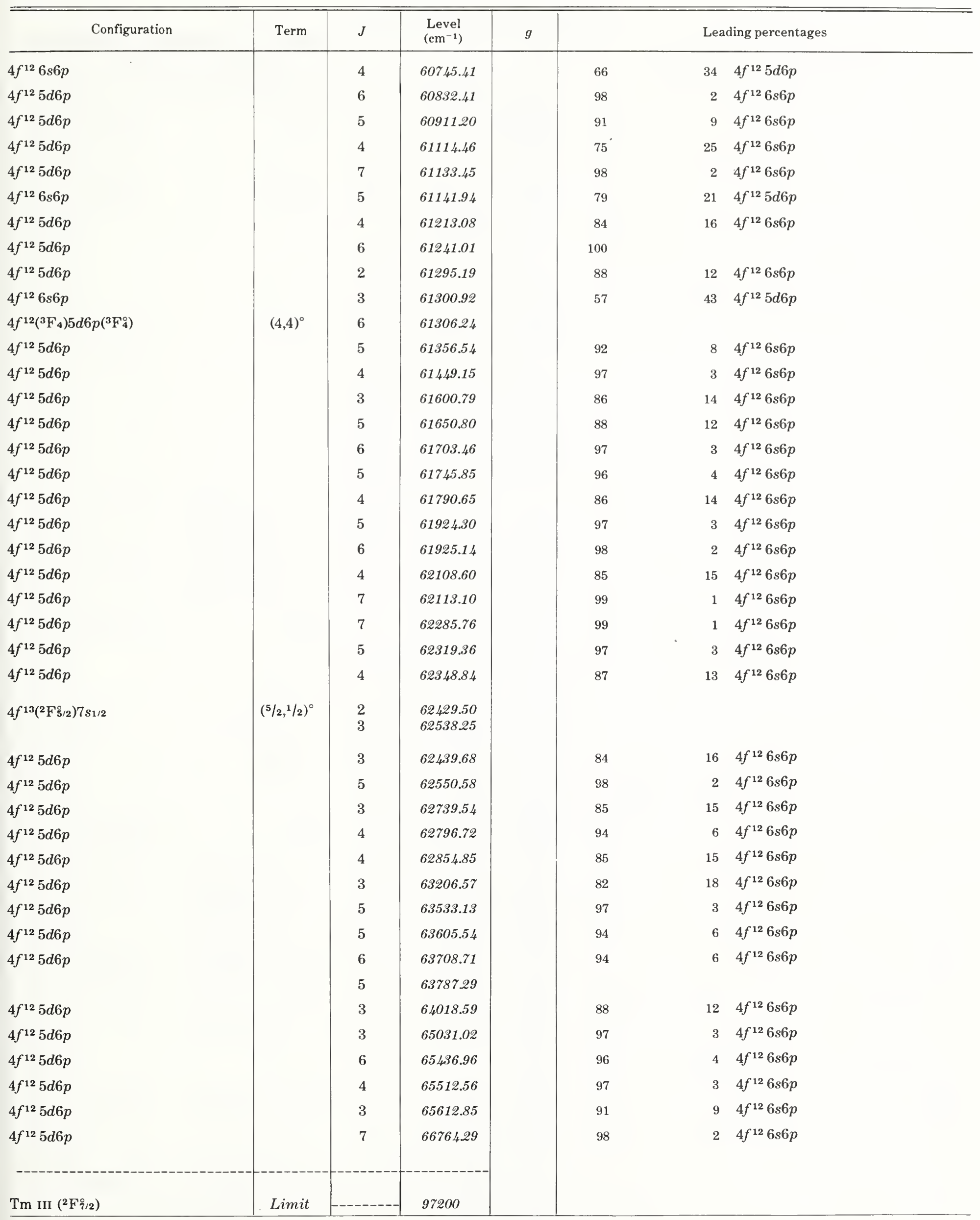


Tm II, Even Parity

\begin{tabular}{|c|c|c|c|c|c|c|}
\hline Configuration & Term & $J$ & $\begin{array}{l}\text { Level } \\
\left(\mathrm{cm}^{-1}\right)\end{array}$ & $g$ & & Leading percentages \\
\hline $4 f^{12}\left({ }^{3} \mathrm{H}\right) 6 s^{2}$ & ${ }^{3} \mathrm{H}$ & $\begin{array}{l}6 \\
5 \\
4\end{array}$ & $\begin{array}{l}12457.29 \\
20619.05 \\
24932.00\end{array}$ & 1.165 & $\begin{array}{l}96 \\
57\end{array}$ & \\
\hline $4 f^{12}\left({ }^{3} \mathrm{H}_{6}\right) 5 d 6 s\left({ }^{3} \mathrm{D}_{1}\right)$ & $(6,1)$ & $\begin{array}{l}5 \\
7 \\
6\end{array}$ & $\begin{array}{l}16567.47 \\
19619.08 \\
22355.43\end{array}$ & $\begin{array}{l}1.374 \\
1.02 \\
1.085\end{array}$ & $\begin{array}{l}64 \\
86 \\
55\end{array}$ & \\
\hline $4 f^{12}\left({ }^{3} \mathrm{~F}\right) 6 s^{2}$ & ${ }^{3} \mathrm{~F}$ & $\begin{array}{l}4 \\
3 \\
2\end{array}$ & $\begin{array}{l}17974.30 \\
26578.77\end{array}$ & $\begin{array}{l}1.175 \\
1.069\end{array}$ & $\begin{array}{l}94 \\
71\end{array}$ & \\
\hline $4 f^{12}\left({ }^{3} \mathrm{H}_{6}\right) 5 d 6 s\left({ }^{3} \mathrm{D}_{2}\right)$ & $(6,2)$ & $\begin{array}{l}4 \\
6 \\
8 \\
7\end{array}$ & $\begin{array}{l}18291.37 \\
19526.82 \\
19682.97 \\
22052.39\end{array}$ & $\begin{array}{l}1.25 \\
1.313 \\
1.09 \\
1.209\end{array}$ & $\begin{array}{l}64 \\
44 \\
91 \\
79\end{array}$ & \\
\hline $4 f^{12}\left({ }^{3} \mathrm{H}_{6}\right) 5 d 6 s\left({ }^{3} \mathrm{D}_{3}\right)$ & $(6,3)$ & $\begin{array}{l}9 \\
3 \\
8 \\
5 \\
6 \\
7\end{array}$ & $\begin{array}{l}20465.82 \\
21608.26 \\
23024.29 \\
23768.84 \\
23904.43 \\
23961.60\end{array}$ & $\begin{array}{l}1.225 \\
1.10 \\
1.21 \\
1.04 \\
1.125 \\
1.20\end{array}$ & $\begin{array}{r}100 \\
69 \\
89 \\
59 \\
64 \\
83\end{array}$ & \\
\hline $4 f^{12} 5 d 6 s$ & & 5 & 21021.98 & 1.178 & 88 & $114 f^{12} 6 s^{2}$ \\
\hline $4 f^{12} 5 d 6 s$ & & 4 & 22308.82 & 1.24 & 96 & $24 f^{13} 6 p$ \\
\hline $4 f^{12} 5 d 6 s$ & & 4 & 23803.28 & 1.08 & 89 & $10 \quad 4 f^{13} 6 p$ \\
\hline $4 f^{12}\left({ }^{3} \mathrm{H}_{6}\right) 5 d 6 s\left({ }^{1} \mathrm{D}_{2}\right)$ & $(6,2)$ & $\begin{array}{l}8 \\
5 \\
7 \\
6\end{array}$ & $\begin{array}{l}24059.08 \\
25014.75 \\
26256.26 \\
26478.28 \\
\\
25219.51\end{array}$ & $\begin{array}{l}1.147 \\
1.185 \\
1.127\end{array}$ & 48 & \\
\hline $4 f^{12}\left({ }^{3} \mathrm{~F}_{4}\right) 5 d 6 s\left({ }^{3} \mathrm{D}_{1}\right)$ & $(4,1)$ & $\begin{array}{l}3 \\
4 \\
5\end{array}$ & $\begin{array}{l}25257.52 \\
25696.21 \\
26188.47\end{array}$ & $\begin{array}{l}1.225 \\
1.25 \\
1.20\end{array}$ & $\begin{array}{l}76 \\
56 \\
43\end{array}$ & \\
\hline $4 f^{12}\left({ }^{3} \mathrm{~F}_{4}\right) 5 d 6 s\left({ }^{3} \mathrm{D}_{2}\right)$ & $(4,2)$ & $\begin{array}{l}2 \\
6 \\
3 \\
5\end{array}$ & $\begin{array}{l}25498.77 \\
26709.43 \\
26837.58 \\
26867.70\end{array}$ & $\begin{array}{l}0.89 \\
1.19 \\
1.085 \\
0.91\end{array}$ & $\begin{array}{l}74 \\
55\end{array}$ & \\
\hline $4 f^{13} 6 p$ & & 3 & 25980.02 & 1.26 & 55 & $45 \quad 4 f^{12} 5 d 6 s$ \\
\hline $4 f^{12} 5 d 6 s$ & & 4 & 26574.66 & 1.057 & 55 & $45 \quad 4 f^{13} 6 p$ \\
\hline $4 f^{12} 5 d 6 s$ & & 4 & 27009.39 & 1.19 & 80 & $19 \quad 4 f^{13} 6 p$ \\
\hline $4 f^{12} 5 d 6 s$ & & 4 & 27254.42 & 1.084 & 83 & $15 \quad 4 f^{13} 6 p$ \\
\hline $4 f^{12} 5 d 6 s$ & & 2 & 27294.79 & 1.045 & 98 & $24 f^{13} 6 p$ \\
\hline $4 f^{12}\left({ }^{3} \mathrm{H}_{5}\right) 5 d 6 s\left({ }^{3} \mathrm{D}_{1}\right)$ & $(5,1)$ & 6 & 27598.57 & 0.955 & 67 & \\
\hline $4 f^{12} 5 d 6 s$ & & 3 & 27702.42 & 1.10 & 90 & $9 \quad 4 f^{13} 6 p$ \\
\hline $4 f^{12}\left({ }^{3} \mathrm{H}_{5}\right) 5 d 6 s\left({ }^{3} \mathrm{D}_{2}\right)$ & $(5,2)$ & 7 & 28095.31 & & 51 & \\
\hline $4 f^{12}\left({ }^{3} \mathrm{~F}_{4}\right) 5 d 6 s\left({ }^{3} \mathrm{D}_{3}\right)$ & $(4,3)$ & 6 & 28096.17 & & 45 & \\
\hline $4 f^{12} 5 d 6 s$ & & 3 & 28267.88 & 1.11 & 94 & $5 \quad 4 f^{13} 6 p$ \\
\hline $4 f^{12}\left({ }^{3} \mathrm{~F}_{4}\right) 5 d 6 s\left({ }^{3} \mathrm{D}_{3}\right)$ & $(4,3)$ & 5 & 28276.32 & 1.21 & 81 & \\
\hline $4 f^{12} 5 d 6 s$ & & 5 & 28875.12 & 1.18 & 65 & $34 \quad 4 f^{13} 6 p$ \\
\hline $4 f^{12}\left({ }^{3} \mathrm{~F}_{4}\right) 5 d 6 s\left({ }^{3} \mathrm{D}_{3}\right)$ & $(4,3)$ & 7 & 28974.93 & & 67 & \\
\hline $4 f^{12} 5 d 6 s$ & & 4 & 29183.39 & 1.15 & 81 & $18 \quad 4 f^{13} 6 p$ \\
\hline $4 f^{13} 6 p$ & & 2 & 29285.72 & 0.96 & 55 & $45 \quad 4 f^{12} 5 d 6 s$ \\
\hline
\end{tabular}


Tm II, Even Parity_Continued

\begin{tabular}{|c|c|c|c|c|c|c|c|}
\hline Configuration & Term & $J$ & $\begin{array}{l}\text { Level } \\
\left(\mathrm{cm}^{-1}\right)\end{array}$ & $g$ & \multicolumn{3}{|c|}{ Leading percentages } \\
\hline $4 f^{12} 5 d 6 s$ & & 3 & 29424.98 & 1.05 & 49 & 48 & $4 f^{13} 6 p$ \\
\hline $4 f^{12\left({ }^{3} \mathrm{H}_{5}\right) 5 d 6 s\left({ }^{3} \mathrm{D}_{3}\right)}$ & $(5,3)$ & 8 & 29671.40 & & 98 & & \\
\hline $4 f^{12} 5 d 6 s$ & & 2 & 29967.17 & 1.05 & 72 & 26 & $4 f^{13} 6 p$ \\
\hline $4 f^{12}\left({ }^{3} \mathrm{H}_{5}\right) 5 d 6 s\left({ }^{3} \mathrm{D}_{1}\right)$ & $(5,1)$ & 5 & 30197.45 & 0.95 & 49 & & \\
\hline $4 f^{12}\left({ }^{3} \mathrm{H}_{5}\right) 5 d 6 s\left({ }^{3} \mathrm{D}_{2}\right)$ & $(5,2)$ & 6 & 30361.06 & & 67 & & \\
\hline $4 f^{12} 5 d 6 s$ & & 4 & 30377.16 & 1.12 & 91 & 8 & $4 f^{13} 6 p$ \\
\hline $4 f^{12} 5 d 6 s$ & & 4 & 30508.76 & 1.25 & 40 & 19 & $4 f^{13} 6 p$ \\
\hline $4 f^{12} 5 d 6 s$ & & 5 & 30627.58 & 1.125 & 89 & & \\
\hline $4 f^{12} 5 d 6 s$ & & 3 & 30684.43 & 1.05 & 90 & 9 & $4 f^{13} 6 p$ \\
\hline $4 f^{12} 5 d^{2}$ & & 4 & 30840.77 & 1.29 & 60 & 32 & $4 f^{12} 5 d 6 s$ \\
\hline $4 f^{12}\left({ }^{3} \mathrm{H}_{4}\right) 5 d 6 s\left({ }^{3} \mathrm{D}_{1}\right)$ & $(4,1)$ & 3 & 31036.61 & 1.08 & 46 & & \\
\hline $4 f^{12} 5 d 6 s$ & & 2 & 31090.09 & 1.03 & 89 & 6 & $4 f^{13} 6 p$ \\
\hline $4 f^{12} 5 d 6 s$ & & 4 & 31135.73 & 0.91 & 93 & 2 & $4 f^{13} 6 p$ \\
\hline $4 f^{12} 5 d 6 s$ & & 6 & 31323.73 & & 85 & & \\
\hline $4 f^{12} 5 d 6 s$ & & 5 & 31726.40 & 1.055 & 85 & 14 & $4 f^{13} 6 p$ \\
\hline $4 f^{12} 5 d 6 s$ & & 3 & 31745.49 & 0.95 & 75 & 23 & $4 f^{13} 6 p$ \\
\hline $4 f^{12}\left({ }^{3} \mathrm{H}_{5}\right) 5 d 6 s\left({ }^{3} \mathrm{D}_{3}\right)$ & $(5,3)$ & 7 & 31836.90 & & 83 & & \\
\hline $4 f^{12} 5 d 6 s$ & & 4 & 31900.00 & 1.17 & 72 & 25 & $4 f^{13} 6 p$ \\
\hline $4 f^{12} 5 d 6 s$ & & 5 & 31926.82 & 1.175 & 63 & 32 & $4 f^{13} 6 p$ \\
\hline $4 f^{12}\left({ }^{3} \mathrm{H}_{5}\right) 5 d 6 s\left({ }^{3} \mathrm{D}_{3}\right)$ & $(5,3)$ & 6 & 32289.22 & & 80 & & \\
\hline $4 f^{12} 5 d 6 s$ & & 2 & 32500.26 & 0.98 & 90 & 6 & $4 f^{13} 6 p$ \\
\hline $4 f^{12}\left({ }^{3} \mathrm{H}_{5}\right) 5 d 6 s\left({ }^{1} \mathrm{D}_{2}\right)$ & $(5,2)$ & 7 & 32590.14 & & 85 & & \\
\hline $4 f^{12} 5 d^{2}$ & & 6 & 32917.50 & & 97 & 3 & $4 f^{12} 5 d 6 s$ \\
\hline $4 f^{12} 5 d^{2}$ & & 5 & 33036.56 & 1.265 & 95 & 4 & $4 f^{12} 5 d 6 s$ \\
\hline $4 f^{12} 5 d 6 s$ & & 2 & 33096.78 & 0.73 & 98 & 2 & $4 f^{13} 6 p$ \\
\hline $4 f^{12} 5 d 6 s$ & & 6 & 33150.48 & & 99 & & \\
\hline $4 f^{12} 5 d^{2}$ & & 7 & 33182.00 & & 97 & 3 & $4 f^{12} 5 d 6 s$ \\
\hline $4 f^{12} 5 d 6 s$ & & 4 & 33391.55 & 1.03 & 79 & 8 & $4 f^{13} 6 p$ \\
\hline $4 f^{12} 5 d 6 s$ & & 3 & 33398.70 & 1.07 & 93 & 4 & $4 f^{13} 6 p$ \\
\hline $4 f^{12}\left({ }^{3} \mathrm{~F}_{3}\right) 5 d 6 s\left({ }^{3} \mathrm{D}_{1}\right)$ & $(3,1)$ & 3 & 33565.12 & 0.88 & 45 & & \\
\hline $4 f^{12} 5 d 6 s$ & & 4 & 33665.99 & 0.908 & 87 & 9 & $4 f^{12} 6 s^{2}$ \\
\hline $4 f^{12}\left({ }^{3} \mathrm{H}_{4}\right) 5 d 6 s\left({ }^{3} \mathrm{D}_{2}\right)$ & $(4,2)$ & 5 & 34157.64 & 1.06 & 57 & & \\
\hline $4 f^{12} 5 d 6 s$ & & 4 & 34307.52 & 0.98 & 97 & 1 & $4 f^{13} 6 p$ \\
\hline $4 f^{12} 5 d 6 s$ & & 3 & 34398.30 & 0.98 & 82 & 17 & $4 f^{13} 6 p$ \\
\hline $4 f^{12}\left({ }^{3} \mathrm{~F}_{2}\right) 5 d 6 s\left({ }^{3} \mathrm{D}_{1}\right)$ & $(2,1)$ & 2 & 34404.26 & 0.79 & 50 & & \\
\hline $4 f^{12}\left({ }^{3} \mathrm{H}_{5}\right) 5 d 6 s\left({ }^{1} \mathrm{D}_{2}\right)$ & $(5,2)$ & 5 & 34580.75 & 1.055 & 65 & & \\
\hline $4 f^{12} 5 d^{2} ?$ & & $7 ?$ & 34685.75 & & 94 & 6 & $4 f^{12} 5 d 6 s$ \\
\hline $4 f^{12} 5 d^{2}$ & & 8 & 34766.81 & & 100 & & \\
\hline $4 f^{12} 5 d 6 s$ & & 4 & 34871.37 & 1.05 & 96 & 3 & $4 f^{13} 6 p$ \\
\hline $4 f^{12} 5 d^{2}$ & & 3 & 34913.84 & 1.377 & 98 & 2 & $4 f^{12} 5 d 6 s$ \\
\hline $\left.4 f^{12}\left({ }^{3} \mathrm{~F}_{2}\right) 5 d 6 s^{3} \mathrm{D}_{2}\right)$ & $(2,2)$ & 1 & 34984.88 & 0.887 & 48 & & \\
\hline $4 f^{12} 5 d 6 s$ & & 2 & 35003.98 & 0.91 & 76 & 22 & $4 f^{13} 6 p$ \\
\hline $4 f^{12} 5 d^{2}$ & & 4 & 35122.91 & & 92 & 6 & $4 f^{12} 5 d 6 s$ \\
\hline $4 f^{12}\left({ }^{3} \mathrm{H}_{4}\right) 5 d 6 s\left({ }^{3} \mathrm{D}_{3}\right)$ & $(4,3)$ & 7 & 35195.12 & & 93 & & \\
\hline $4 f^{12} 5 d^{2}$ & & 5 & 35227.68 & & 94 & 5 & $4 f^{12} 5 d 6 s$ \\
\hline $4 f^{12} 5 d 6 s$ & & 2 & 35274.43 & 1.15 & 64 & 33 & $4 f^{13} 6 p$ \\
\hline
\end{tabular}


Tm II, Even Parity—Continued

\begin{tabular}{|c|c|c|c|c|c|c|}
\hline Configuration & Term & $J$ & $\begin{array}{c}\text { Level } \\
\left(\mathrm{cm}^{-1}\right)\end{array}$ & $g$ & & Leading percentages \\
\hline $4 f^{12} 5 d 6 s$ & & 3 & 35380.12 & 0.92 & 80 & $18 \quad 4 f^{13} 6 p$ \\
\hline $4 f^{12} 5 d 6 s$ & & 3 & 35753.72 & 1.152 & 93 & $7 \quad 4 f^{13} 6 p$ \\
\hline $4 f^{12} 5 d 6 s$ & & 5 & 35772.82 & 1.12 & 97 & $2 \quad 4 f^{13} 6 p$ \\
\hline $4 f^{12} 5 d 6 s$ & & 1 & 35806.78 & 0.877 & 97 & $3 \quad 4 f^{13} 6 p$ \\
\hline $4 f^{12} 5 d 6 s$ & & 2 & 35833.62 & 1.05 & 73 & $2 \quad 4 f^{13} 6 p$ \\
\hline $4 f^{12} 5 d 6 s$ & & 4 & 35966.41 & 1.21 & 97 & $1 \quad 4 f^{13} 6 p$ \\
\hline $4 f^{12} 5 d^{2}$ & & 6 & 35979.94 & & 86 & $14 \quad 4 f^{12} 5 d 6 s$ \\
\hline $4 f^{12} 5 d 6 s$ & & 3 & 36041.02 & 1.145 & 92 & $4 \quad 4 f^{13} 6 p$ \\
\hline $4 f^{12} 5 d 6 s$ & & 3 & 36132.08 & 0.915 & 57 & $42 \quad 4 f^{13} 6 p$ \\
\hline $4 f^{12(}\left({ }^{3} \mathrm{H}_{4}\right) 5 d 6 s\left({ }^{3} \mathrm{D}_{3}\right)$ & $(4,3)$ & 6 & 36145.30 & & 63 & \\
\hline $4 f^{12} 5 d 6 s$ & & 2 & 36394.64 & 1.02 & 67 & $30 \quad 4 f^{13} 6 p$ \\
\hline $4 f^{12} 5 d 6 s$ & & 4 & 36547.84 & 0.89 & 98 & $1 \quad 4 f^{13} 6 p$ \\
\hline $4 f^{12} 5 d^{2}$ & & 7 & 36805.72 & & 97 & $3 \quad 4 f^{12} 5 d 6 s$ \\
\hline $4 f^{12} 5 d 6 s$ & & 3 & 36868.99 & 1.11 & 89 & $7 \quad 4 f^{13} 6 p$ \\
\hline $4 f^{12} 5 d^{2}$ & & 2 & 37151.09 & 1.273 & 68 & $30 \quad 4 f^{12} 5 d 6 s$ \\
\hline $4 f^{12} 5 d 6 s$ & & 2 & 37482.67 & 1.11 & 85 & $10 \quad 4 f^{13} 6 p$ \\
\hline $4 f^{12} 5 d 6 s$ & & 4 & 37581.47 & 1.06 & 93 & $4 \quad 4 f^{13} 6 p$ \\
\hline $4 f^{12} 5 d 6 s$ & & 4 & 37775.23 & & 63 & $32 \quad 4 f^{12} 5 d^{2}$ \\
\hline $4 f^{12} 5 d 6 s$ & & 2 & 37841.25 & 1.387 & 94 & $4 \quad 4 f^{13} 6 p$ \\
\hline $4 f^{13} 6 p$ & & 1 & 37916.11 & 0.58 & 65 & $34 \quad 4 f^{12} 5 d 6 s$ \\
\hline $4 f^{12} 5 d^{2}$ & & 3 & 37957.72 & 0.915 & 70 & $28 \quad 4 f^{12} 5 d 6 s$ \\
\hline $4 f^{12} 5 d^{2}$ & & 6 & 37968.84 & & 97 & $3 \quad 4 f^{12} 5 d 6 s$ \\
\hline $4 f^{12} 5 d 6 s$ & & 4 & 38093.53 & 1.06 & 77 & $4 \quad 4 f^{13} 6 p$ \\
\hline $4 f^{12} 5 d 6 s$ & & 5 & 38200.36 & & 62 & \\
\hline $4 f^{12} 5 d 6 s$ & & 3 & 38261.05 & & 77 & $3 \quad 4 f^{13} 6 p$ \\
\hline $4 f^{12} 5 d 6 s$ & & 4 & 38361.24 & 1.035 & 34 & $20 \quad 4 f^{13} 6 p$ \\
\hline $4 f^{12}\left({ }^{3} \mathrm{~F}_{2}\right) 5 d 6 s\left({ }^{3} \mathrm{D}_{3}\right)$ & $(2,3)$ & 1 & 38533.56 & 1.81 & 74 & \\
\hline $4 f^{12}\left({ }^{3} \mathrm{~F}_{3}\right) 5 d 6 s\left({ }^{3} \mathrm{D}_{3}\right)$ & $(3,3)$ & 6 & 38537.96 & & 52 & \\
\hline $4 f^{12} 5 d^{2}$ & & 3 & 38582.95 & 1.00 & 62 & $33 \quad 4 f^{12} 5 d 6 s$ \\
\hline $4 f^{12} 5 d^{2}$ & & 7 & 38981.51 & & 100 & \\
\hline $4 f^{12} 5 d 6 s$ & & 1 & 38990.65 & 1.37 & 86 & $13 \quad 4 f^{13} 6 p$ \\
\hline $4 f^{12} 5 d 6 s$ & & 3 & 38991.96 & & 66 & $9 \quad 4 f^{13} 6 p$ \\
\hline $4 f^{12} 5 d 6 s$ & & 2 & 39000.76 & 1.16 & 83 & $3 \quad 4 f^{13} 6 p$ \\
\hline $4 f^{12} 5 d 6 s$ & & 4 & 39025.63 & & 62 & $24 f^{13} 6 p$ \\
\hline $4 f^{12} 5 d^{2}$ & & 5 & 39092.22 & & 92 & $8 \quad 4 f^{12} 5 d 6 s$ \\
\hline $4 f^{12} 5 d^{2}$ & & 6 & 39149.06 & & 96 & $4 \quad 4 f^{12} 5 d 6 s$ \\
\hline $4 f^{12} 5 d 6 s$ & & 4 & 39162.07 & 1.05 & 53 & $3 \quad 4 f^{13} 6 p$ \\
\hline $4 f^{12} 5 d^{2}$ & & 2 & 39196.70 & 1.03 & 82 & $13 \quad 4 f^{12} 5 d 6 s$ \\
\hline $4 f^{12} 6 s^{2}$ & & 2 & 39514.68 & 1.247 & 41 & $27 \quad 4 f^{13} 6 p$ \\
\hline $4 f^{12} 5 d 6 s$ & & 3 & 39554.05 & 1.10 & 55 & $17 \quad 4 f^{13} 6 p$ \\
\hline $4 f^{12} 5 d 6 s$ & & 4 & 39636.53 & & 41 & $37 \quad 4 f^{13} 6 p$ \\
\hline $4 f^{12} 5 d^{2}$ & & 6 & 39713.04 & & 95 & $5 \quad 4 f^{12} 5 d 6 s$ \\
\hline $4 f^{12} 6 s^{2}$ & & 2 & 39828.63 & & 40 & $27 \quad 4 f^{12} 5 d 6 s$ \\
\hline $4 f^{12} 5 d 6 s$ & & 4 & 39843.24 & 1.11 & 46 & $22 \quad 4 f^{13} 6 p$ \\
\hline $4 f^{12} 5 d 6 s$ & & 3 & 39893.87 & 1.006 & 60 & $31 \quad 4 f^{13} 6 p$ \\
\hline
\end{tabular}


Tm III

(Ho I sequence; 67 electrons)

$Z=69$

Ground state $\left(1 s^{2} 2 s^{2} 2 p^{6} 3 s^{2} 3 p^{6} 3 d^{10} 4 s^{2} 4 p^{6} 4 d^{10} 5 s^{2} 5 p^{6}\right) 4 f^{13}{ }^{2} \mathrm{~F}_{7 / 2}^{\circ}$

Ionization energy $191000 \pm 800 \mathrm{~cm}^{-1}$

$23.68 \pm 0.10 \mathrm{eV}$

Identified odd configurations $\quad 4 f^{13}, 4 f^{12} 6 p$

Identified even configurations $\quad 4 f^{12} 5 d, 4 f^{12} 6 s$

Sugar has classified more than 450 lines of this spectrum in the region $1977-5868 \AA$. His analysis was based on a list of 848 sliding-spark lines assigned to Tm III, but only the classified lines are given in the 1970 paper.

The levels are mainly from Sugar's paper, which gave 108 levels, but include several new even levels from his unpublished extension of this work [1973].

The eigenvector percentages for $\left(4 f^{12} 5 d+4 f^{12} 6 s\right)$ are from an unpublished calculation by Sugar [1973] that included configuration interaction and appropriate effective operators. The coupling in these configurations is highly intermediate, the purity being somewhat higher in the $J_{1} j$ scheme used for the designations. The eigenvectors for three of the known levels of this group have a large second component belonging to the configuration alternate to that for the first component. The second percentages are listed for these three levels to show the strong configuration interaction; for all other levels of the group, the leading percentage in the $L S$-coupling scheme is given instead of the second $J_{1} j$ component.

Wyart, Blaise, and Camus [1974] give the parameter values obtained from a calculation of $\left(4 f^{12} 5 d+4 f^{12} 6 s\right)$ including configuration interaction.

The percentages in $J_{1} j$ coupling for the $4 f^{12} 6 p$ levels are taken from Sugar's 1970 paper. Sugar has also supplied from this same calculation the leading percentages in $L S$ coupling for inclusion here.

The ionization energy is from Sugar and Reader [1973]. According to Sugar, observation of the spectrum with a higher-excitation sliding spark could be expected to give a considerable extension of the line list and analysis.

\section{References}

Sugar, J., J. Opt. Soc. Am. 60, 454 (1970). EL CL W IP PT

Sugar, J., private communication (1973). EL PT

Sugar, J., and Reader, J., J. Chem. Phys. 59, 2083 (1973). IP

Wyart, J. F., Blaise, J., and Camus, P., Phys. Scr. 9, 325 (1974). PT

[July 1976] 
Tm III

\begin{tabular}{|c|c|c|c|c|c|c|c|c|}
\hline \multirow[t]{2}{*}{ Configuration } & \multirow{2}{*}{$\begin{array}{c}\text { Term } \\
{ }^{2} \mathrm{~F}^{\circ}\end{array}$} & \multirow{2}{*}{$\begin{array}{c}J \\
7 / 2 \\
5 / 2\end{array}$} & \multirow{2}{*}{$\begin{array}{r}\begin{array}{r}\text { Level } \\
\left(\mathrm{cm}^{-1}\right)\end{array} \\
0.00 \\
8774.02\end{array}$} & \multicolumn{5}{|c|}{ Leading percentages } \\
\hline & & & & & & & & \\
\hline \multirow[t]{4}{*}{$4 f^{12}\left({ }^{3} \mathrm{H}_{6}\right) 5 d_{3 / 2}$} & $(6,3 / 2)$ & $9 / 2$ & 22897.47 & 84 & or & & 81 & $\left({ }^{3} \mathrm{H}\right){ }^{4} \mathrm{~F}$ \\
\hline & & $15 / 2$ & 25878.84 & 96 & or & & 55 & $\left({ }^{3} \mathrm{H}\right)^{2} \mathrm{~K}$ \\
\hline & & $11 / 2$ & 26252.40 & 72 & or & & 78 & $\left({ }^{3} \mathrm{H}\right){ }^{4} \mathrm{G}$ \\
\hline & & $13 / 2$ & 28720.87 & 92 & or & & 33 & $\left({ }^{3} \mathrm{H}\right){ }^{4} \mathrm{I}$ \\
\hline \multirow[t]{2}{*}{$4 f^{12\left({ }^{3} \mathrm{H}_{6}\right) 6 s_{1 / 2}}$} & $\left(6,{ }^{1} / 2\right)$ & $13 / 2$ & 25302.65 & 99 & or & & 99 & $\left({ }^{3} \mathrm{H}\right){ }^{4} \mathrm{H}$ \\
\hline & & $11 / 2$ & 25920.78 & 94 & or & & 64 & $\left({ }^{3} \mathrm{H}\right){ }^{2} \mathrm{H}$ \\
\hline \multirow[t]{6}{*}{$4 f^{12}\left({ }^{3} \mathrm{H}_{6}\right) 5 d 5 / 2$} & $(6,5 / 2)$ & $7 / 2$ & 27547.25 & 53 & or & & 51 & $\left({ }^{3} \mathrm{H}\right){ }^{4} \mathrm{~F}$ \\
\hline & & $17 / 2$ & 27557.98 & 92 & or & & 99 & $\left({ }^{3} \mathrm{H}\right){ }^{4} \mathrm{~K}$ \\
\hline & & $11 / 2$ & 30532.34 & 61 & or & & 41 & $\left({ }^{3} \mathrm{H}\right){ }^{2} \mathrm{H}$ \\
\hline & & $9 / 2$ & 30535.69 & 60 & or & & 39 & $\left({ }^{3} \mathrm{H}\right){ }^{4} \mathrm{G}$ \\
\hline & & $15 / 2$ & 30664.98 & 96 & or & & 84 & $\left({ }^{3} \mathrm{H}\right){ }^{4} \mathrm{I}$ \\
\hline & & $13 / 2$ & 31323.46 & 72 & or & & 40 & $\left({ }^{3} \mathrm{H}\right){ }^{4} \mathrm{H}$ \\
\hline \multirow[t]{2}{*}{$4 f^{12}\left({ }^{3} \mathrm{~F}_{4}\right) 6 s_{1 / 2}$} & $\left(4,{ }^{1} / 2\right)$ & $9 / 2$ & 31037.52 & 63 & or & & 63 & $\left({ }^{3} \mathrm{~F}\right){ }^{4} \mathrm{~F}$ \\
\hline & & $7 / 2$ & 31368.59 & 58 & or & & 42 & $\left({ }^{3} \mathrm{~F}\right)^{2} \mathrm{~F}$ \\
\hline \multirow[t]{4}{*}{$4 f^{12}\left({ }^{3} \mathrm{~F}_{4}\right) 5 d_{3 / 2}$} & $(4,3 / 2)$ & $5 / 2$ & 31570.50 & 44 & or & & 22 & $\left({ }^{1} \mathrm{G}\right){ }^{2} \mathrm{~F}$ \\
\hline & & $7 / 2$ & 32262.88 & 47 & or & & 22 & $\left({ }^{3} \mathrm{~F}\right){ }^{4} \mathrm{D}$ \\
\hline & & $9 / 2$ & 32797.68 & 52 & or & & 22 & $\left({ }^{3} \mathrm{~F}\right){ }^{4} \mathrm{G}$ \\
\hline & & $11 / 2$ & 33236.12 & 30 & or & & 19 & $\left({ }^{3} F\right){ }^{4} \mathrm{G}$ \\
\hline \multirow[t]{6}{*}{$4 f^{12}\left({ }^{3} \mathrm{H}_{5}\right) 5 d_{5 / 2}$} & $(5,5 / 2)$ & $5 / 2$ & 33605.40 & 42 & or & & 61 & $\left({ }^{3} \mathrm{H}\right){ }^{4} \mathrm{~F}$ \\
\hline & & $7 / 2$ & 36405.98 & 41 & or & & 16 & $\left({ }^{3} \mathrm{H}\right){ }^{4} \mathrm{~F}$ \\
\hline & & $15 / 2$ & 36466.44 & 99 & or & & 59 & $\left({ }^{3} \mathrm{H}\right){ }^{4} \mathrm{~K}$ \\
\hline & & $9 / 2$ & 38610.32 & 43 & & & 22 & $\left({ }^{3} \mathrm{H}_{4}\right) 6 s_{1 / 2}$ \\
\hline & & $13 / 2$ & 39496.32 & 90 & or & & 57 & $\left({ }^{3} \mathrm{H}\right){ }^{4} \mathrm{I}$ \\
\hline & & $11 / 2$ & 39604.23 & 79 & or & & 45 & $\left({ }^{3} \mathrm{H}\right){ }^{4} \mathrm{H}$ \\
\hline \multirow[t]{4}{*}{$4 f^{12\left({ }^{3} \mathrm{H}_{5}\right) 5 d_{3 / 2}}$} & $(5,3 / 2)$ & $7 / 2$ & 33630.68 & 55 & or & & 45 & $\left({ }^{3} \mathrm{H}\right){ }^{2} \mathrm{~F}$ \\
\hline & & $13 / 2$ & 34124.20 & 86 & or & & 56 & $\left({ }^{3} \mathrm{H}\right){ }^{4} \mathrm{~K}$ \\
\hline & & $11 / 2$ & 36810.12 & 85 & or & & 42 & $\left({ }^{3} \mathrm{H}\right){ }^{4} \mathrm{I}$ \\
\hline & & $9 / 2$ & 37028.88 & 65 & or & & 48 & $\left({ }^{3} \mathrm{H}\right){ }^{2} \mathrm{G}$ \\
\hline \multirow[t]{2}{*}{$4 f^{12\left({ }^{3} \mathrm{H}_{5}\right) 6 s_{1 / 2}}$} & $\left(5,{ }^{1} / 2\right)$ & $11 / 2$ & 33802.80 & 95 & or & & 64 & $\left({ }^{3} \mathrm{H}\right){ }^{4} \mathrm{H}$ \\
\hline & & $9 / 2$ & 33831.91 & 99 & or & & 65 & \\
\hline $4 f^{12}\left({ }^{3} \mathrm{~F}_{4}\right) 5 d 5 / 2$ & $(4,5 / 2)$ & $11 / 2$ & 34826.83 & 56 & or & & 46 & $\left({ }^{3} F\right){ }^{4} G$ \\
\hline $4 f^{12}\left({ }^{3} \mathrm{~F}_{4}\right) 5 d 5 / 2$ & $(4,5 / 2)$ & $9 / 2$ & 35751.15 & 57 & or & & 34 & $\left({ }^{3} \mathrm{~F}\right){ }^{4} \mathrm{~F}$ \\
\hline $4 f^{12}\left({ }^{3} \mathrm{~F}_{4}\right) 5 d 5 / 2$ & & $13 / 2$ & 36369.12 & 33 & $(4,5 / 2)$ & or & 33 & $\left({ }^{3} \mathrm{~F}\right){ }^{4} \mathrm{H}$ \\
\hline $4 f^{12}\left({ }^{3} \mathrm{~F}_{4}\right) 5 d_{3 / 2}$ & & $5 / 2$ & 36391.74 & 23 & $(4,3 / 2)$ & or & 28 & $\left({ }^{3} \mathrm{~F}\right){ }^{4} \mathrm{P}$ \\
\hline $4 f^{12\left({ }^{3} \mathrm{H}_{5}\right) 5 d 5 / 2}$ & & $7 / 2$ & 37367.92 & 30 & $(5,5 / 2)$ & or & 31 & $\left({ }^{3} \mathrm{~F}\right){ }^{4} \mathrm{D}$ \\
\hline $4 f^{12\left({ }^{3} \mathrm{H}_{4}\right) 6 s_{1 / 2}}$ & & $9 / 2$ & 38003.36 & 36 & $\left(4,{ }^{1} / 2\right)$ & & 29 & $\left({ }^{3} \mathrm{H}_{5}\right) 5 d_{5 / 2}$ \\
\hline $4 f^{12\left({ }^{3} \mathrm{H}_{4}\right) 6 s_{1 / 2}}$ & $\left(4,{ }^{1} / 2\right)$ & $7 / 2$ & 38055.53 & 58 & or & & 58 & $\left({ }^{3} \mathrm{H}\right){ }^{4} \mathrm{H}$ \\
\hline $4 f^{12}\left({ }^{3} \mathrm{~F}_{4}\right) 5 d_{5 / 2}$ & & $3 / 2$ & 38175.36 & 35 & $(4,5 / 2)$ & or & 30 & $\left({ }^{3} \mathrm{~F}\right){ }^{4} \mathrm{~F}$ \\
\hline $4 f^{12}\left({ }^{3} \mathrm{H}_{4}\right) 5 d 3 / 2$ & $(4,3 / 2)$ & $11 / 2$ & 39142.50 & 51 & or & & 42 & $\left({ }^{3} \mathrm{H}\right){ }^{4} \mathrm{~K}$ \\
\hline $4 f^{12\left({ }^{3} \mathrm{H}_{5}\right) 5 d 5 / 2}$ & & $5 / 2$ & 39278.57 & 25 & $(5,5 / 2)$ & or & 33 & $\left({ }^{3} \mathrm{H}\right){ }^{2} \mathrm{~F}$ \\
\hline $4 f^{12}\left({ }^{3} \mathrm{~F}_{3}\right) 6 s_{1 / 2}$ & $(3,1 / 2)$ & $5 / 2$ & 39882.62 & 79 & or & & 84 & $\left({ }^{3} \mathrm{~F}\right){ }^{4} \mathrm{~F}$ \\
\hline & & $7 / 2$ & 39934.71 & 94 & or & & 64 & \\
\hline $4 f^{12}\left({ }^{3} \mathrm{~F}_{3}\right) 5 d 3 / 2$ & $(3,3 / 2)$ & $7 / 2$ & 40070.58 & 48 & or & & 34 & $\left({ }^{3} F\right){ }^{4} \mathrm{G}$ \\
\hline $4 f^{12}\left({ }^{3} \mathrm{H}_{4}\right) 5 d_{3 / 2}$ & $(4,3 / 2)$ & $9 / 2$ & 40400.90 & 50 & or & & 30 & $\left({ }^{3} \mathrm{H}\right){ }^{4} \mathrm{I}$ \\
\hline $4 f^{12}\left({ }^{3} \mathrm{~F}_{2}\right) 6 s_{1 / 2}$ & $\left(2,{ }^{1} / 2\right)$ & $3 / 2$ & 40403.59 & 78 & or & & 78 & $\left({ }^{3} \mathrm{~F}\right){ }^{4} \mathrm{~F}$ \\
\hline
\end{tabular}


Tm III-Continued

\begin{tabular}{|c|c|c|c|c|c|c|c|c|}
\hline Configuration & Term & $J$ & $\begin{array}{l}\text { Level } \\
\left(\mathrm{cm}^{-1}\right)\end{array}$ & \multicolumn{5}{|c|}{ Leading percentages } \\
\hline $4 f^{12}\left({ }^{3} \mathrm{~F}_{2}\right) 6 s_{1 / 2}$ & & $5 / 2$ & 40754.18 & 38 & $\left(2,{ }^{1} / 2\right)$ & & 23 & $\left({ }^{3} \mathrm{~F}_{2}\right) 5 d_{3 / 2}$ \\
\hline $4 f^{12}\left({ }^{3} \mathrm{~F}_{2}\right) 5 d_{3 / 2}$ & & $7 / 2$ & 41233.38 & 22 & $(2,3 / 2)$ & or & 21 & $\left({ }^{3} \mathrm{~F}\right)^{2} \mathrm{~F}$ \\
\hline $4 f^{12}\left({ }^{3} \mathrm{~F}_{3}\right) 5 d_{3 / 2}$ & & $3 / 2$ & 41595.66 & 30 & $\left(3,{ }^{3} / 2\right)$ & or & 35 & $\left({ }^{3} \mathrm{~F}\right){ }^{2} \mathrm{P}$ \\
\hline $4 f^{12}\left({ }^{3} \mathrm{H}_{4}\right) 5 d_{5 / 2}$ & $(4,5 / 2)$ & $13 / 2$ & 41864.33 & 55 & or & & 38 & $\left({ }^{3} \mathrm{H}\right){ }^{2} \mathrm{~K}$ \\
\hline $4 f^{12}\left({ }^{3} \mathrm{~F}_{3}\right) 5 d_{3 / 2}$ & & $9 / 2$ & 42669.92 & 36 & $\left(3,{ }^{3} / 2\right)$ & or & 36 & $\left({ }^{3} \mathrm{~F}\right){ }^{4} \mathrm{H}$ \\
\hline $4 f^{12}\left({ }^{3} \mathrm{~F}_{3}\right) 5 d_{3 / 2}$ & & $5 / 2$ & 42915.07 & 39 & $\left(3,{ }^{3} / 2\right)$ & or & 36 & $\left({ }^{3} F\right){ }^{4} F$ \\
\hline $4 f^{12}\left({ }^{3} \mathrm{H}_{4}\right) 5 d_{5 / 2}$ & & $11 / 2$ & 42952.97 & 44 & $(4,5 / 2)$ & or & 34 & $\left({ }^{3} \mathrm{H}\right){ }^{2} \mathrm{I}$ \\
\hline $4 f^{12}\left({ }^{3} \mathrm{~F}_{3}\right) 5 d_{5 / 2}$ & & $9 / 2$ & 43022.30 & 33 & $(3,5 / 2)$ & or & 29 & $\left({ }^{3} F\right){ }^{4} \mathrm{G}$ \\
\hline $4 f^{12}\left({ }^{3} \mathrm{~F}_{3}\right) 5 d_{5 / 2}$ & $(3,5 / 2)$ & $\begin{array}{c}7 / 2 \\
5 / 2 \\
9 / 2 \\
3 / 2 \\
11 / 2 \\
1 / 2\end{array}$ & $\begin{array}{l}43139.50 \\
43768.40 \\
44495.79\end{array}$ & $\begin{array}{l}38 \\
64 \\
52 \\
56 \\
72 \\
59\end{array}$ & $\begin{array}{l}\text { or } \\
\text { or } \\
\text { or } \\
\text { or } \\
\text { or } \\
\text { or }\end{array}$ & & $\begin{array}{l}45 \\
32 \\
41 \\
81 \\
54 \\
52\end{array}$ & $\begin{array}{l}\left({ }^{3} \mathrm{~F}\right){ }^{4} \mathrm{~F} \\
\left({ }^{3} \mathrm{~F}\right){ }^{4} \mathrm{D} \\
\left({ }^{3} \mathrm{~F}\right){ }^{2} \mathrm{H} \\
\left({ }^{3} \mathrm{~F}\right){ }^{4} \mathrm{P} \\
\left({ }^{3} \mathrm{~F}\right){ }^{4} \mathrm{H} \\
\left({ }^{3} \mathrm{~F}\right){ }^{2} \mathrm{P}\end{array}$ \\
\hline $4 f^{12}\left({ }^{3} \mathrm{~F}_{3}\right) 5 d_{3 / 2}$ & & $3 / 2$ & 43160.58 & 34 & $(3,3 / 2)$ & or & 31 & $\left({ }^{3} \mathrm{H}\right){ }^{4} \mathrm{~F}$ \\
\hline $4 f^{12}\left({ }^{3} \mathrm{~F}_{3}\right) 5 d 5 / 2$ & & $7 / 2$ & 44496.38 & 27 & $(3,5 / 2)$ & or & 64 & $\left({ }^{3} \mathrm{~F}\right){ }^{2} \mathrm{G}$ \\
\hline $4 f^{12}\left({ }^{3} \mathrm{H}_{4}\right) 5 d_{3 / 2}$ & & $7 / 2$ & 45732.62 & 26 & $(4,3 / 2)$ & or & 38 & $\left({ }^{3} \mathrm{~F}\right){ }^{2} \mathrm{~F}$ \\
\hline $4 f^{12}\left({ }^{1} \mathrm{G}_{4}\right) 5 d_{5 / 2}$ & & $7 / 2$ & 45794.62 & 24 & $(4,5 / 2)$ & or & 27 & $\left({ }^{3} \mathrm{~F}\right){ }^{4} \mathrm{H}$ \\
\hline $4 f^{12}\left({ }^{1} \mathrm{G}_{4}\right) 6 s_{1 / 2}$ & $(4,1 / 2)$ & $\begin{array}{l}7 / 2 \\
9 / 2\end{array}$ & $\begin{array}{l}46786.94 \\
46816.79\end{array}$ & $\begin{array}{l}55 \\
54\end{array}$ & $\begin{array}{l}\text { or } \\
\text { or }\end{array}$ & & $\begin{array}{l}55 \\
54\end{array}$ & $\left({ }^{1} \mathrm{G}\right){ }^{2} \mathrm{G}$ \\
\hline $4 f^{12}\left({ }^{3} \mathrm{~F}_{2}\right) 5 d_{5 / 2}$ & $(2,5 / 2)$ & $9 / 2$ & 47070.54 & 54 & or & & 32 & $\left({ }^{3} \mathrm{~F}\right){ }^{2} \mathrm{H}$ \\
\hline $4 f^{12}\left({ }^{1} \mathrm{G}_{4}\right) 5 d_{3 / 2}$ & $\left(4,{ }^{3} / 2\right)$ & $11 / 2$ & 47603.51 & 55 & or & & 55 & $\left({ }^{1} \mathrm{G}\right){ }^{2} \mathrm{I}$ \\
\hline $4 f^{12}\left({ }^{3} \mathrm{~F}_{2}\right) 5 d_{5 / 2}$ & & $5 / 2$ & 47624.18 & 43 & $(2,5 / 2)$ & or & 52 & $\left({ }^{3} \mathrm{~F}\right)^{2} \mathrm{D}$ \\
\hline $4 f^{12}\left({ }^{1} \mathrm{G}_{4}\right) 5 d_{3 / 2}$ & $(4,3 / 2)$ & $9 / 2$ & 49206.69 & 47 & or & & 42 & $\left({ }^{1} \mathrm{G}\right){ }^{2} \mathrm{H}$ \\
\hline $4 f^{12}\left({ }^{1} \mathrm{G}_{4}\right) 5 d 5 / 2$ & & $7 / 2$ & 50562.58 & 35 & $(4,5 / 2)$ & or & 43 & $\left({ }^{1} \mathrm{G}\right){ }^{2} \mathrm{~F}$ \\
\hline $4 f^{12}\left({ }^{1} \mathrm{G}_{4}\right) 5 d_{3 / 2}$ & & $7 / 2$ & 51071.25 & 33 & $(4,3 / 2)$ & or & 40 & $\left({ }^{1} G\right){ }^{2} G$ \\
\hline $4 f^{12}\left({ }^{1} \mathrm{G}_{4}\right) 5 d_{5 / 2}$ & $(4,5 / 2)$ & $9 / 2$ & 51634.22 & 47 & or & & 37 & $\left({ }^{1} \mathrm{G}\right){ }^{2} \mathrm{G}$ \\
\hline $4 f^{12}\left({ }^{1} \mathrm{D}_{2}\right) 5 d_{3 / 2}$ & & $5 / 2$ & 52679.70 & 20 & $(2,3 / 2)$ & or & 27 & $\left({ }^{1} \mathrm{G}\right){ }^{2} \mathrm{~F}$ \\
\hline $4 f^{12}\left({ }^{3} \mathrm{P}_{2}\right) 6 s_{1 / 2}$ & & $5 / 2$ & 53565.42 & 41 & $\left(2,{ }^{1} / 2\right)$ & or & 41 & $\left({ }^{3} \mathrm{P}\right){ }^{4} \mathrm{P}$ \\
\hline $4 f^{12}\left({ }^{1} \mathrm{D}_{2}\right) 6 s_{1 / 2}$ & & $3 / 2$ & 53778.35 & 44 & $\left(2,{ }^{1} / 2\right)$ & or & 44 & $\left({ }^{1} \mathrm{D}\right){ }^{2} \mathrm{D}$ \\
\hline $4 f^{12}\left({ }^{3} \mathrm{P}_{2}\right) 5 d 5 / 2$ & & $7 / 2$ & 55586.87 & 22 & $(2,5 / 2)$ & or & 36 & $\left({ }^{3} \mathrm{P}\right){ }^{4} \mathrm{D}$ \\
\hline $4 f^{12\left({ }^{3} \mathrm{P}_{2}\right) 5 d 3 / 2}$ & $(2,3 / 2)$ & $3 / 2$ & 55735.51 & 47 & or & & 40 & $\left({ }^{3} \mathrm{P}\right){ }^{4} \mathrm{D}$ \\
\hline $4 f^{12}\left({ }^{3} \mathrm{P}_{2}\right) 5 d_{3 / 2}$ & & $5 / 2$ & 56217.70 & 22 & $(2,3 / 2)$ & or & 28 & $\left({ }^{3} \mathrm{~F}\right)^{2} \mathrm{~F}$ \\
\hline $4 f^{12}\left({ }^{1} \mathrm{D}_{2}\right) 5 d_{3 / 2}$ & & $7 / 2$ & 57779.95 & 21 & $(2,3 / 2)$ & or & 31 & $\left({ }^{1} \mathrm{D}\right){ }^{2} \mathrm{G}$ \\
\hline $4 f^{12}\left({ }^{1} \mathrm{D}_{2}\right) 5 d_{5 / 2}$ & & $5 / 2$ & 59029.55 & 37 & $(2,5 / 2)$ & or & 24 & $\left({ }^{1} \mathrm{D}\right)^{2} \mathrm{~F}$ \\
\hline $4 f^{12}\left({ }^{1} \mathrm{I}_{6}\right) 5 d_{3 / 2}$ & $\left(6,{ }^{3} / 2\right)$ & $9 / 2$ & 60640.80 & 79 & or & & 91 & $\left({ }^{1} \mathrm{I}\right){ }^{2} \mathrm{G}$ \\
\hline $4 f^{12}\left({ }^{3} \mathrm{H}_{6}\right) 6 p_{1 / 2}$ & $(6,1 / 2)^{\circ}$ & $\begin{array}{l}11 / 2 \\
13 / 2\end{array}$ & $\begin{array}{l}62063.57 \\
62572.76\end{array}$ & $\begin{array}{l}96 \\
99\end{array}$ & $\begin{array}{l}\text { or } \\
\text { or }\end{array}$ & & $\begin{array}{l}72 \\
46\end{array}$ & $\begin{array}{l}\left({ }^{3} \mathrm{H}\right){ }^{4} \mathrm{G}^{\circ} \\
\left({ }^{3} \mathrm{H}\right){ }^{2} \mathrm{I}^{\circ}\end{array}$ \\
\hline $4 f^{12}\left({ }^{3} \mathrm{P}_{0}\right) 5 d_{5 / 2}$ & & $5 / 2$ & 63527.40 & 32 & $(0,5 / 2)$ & or & 44 & $\left({ }^{3} \mathrm{P}\right){ }^{4} \mathrm{D}$ \\
\hline
\end{tabular}


Tm III-Continued

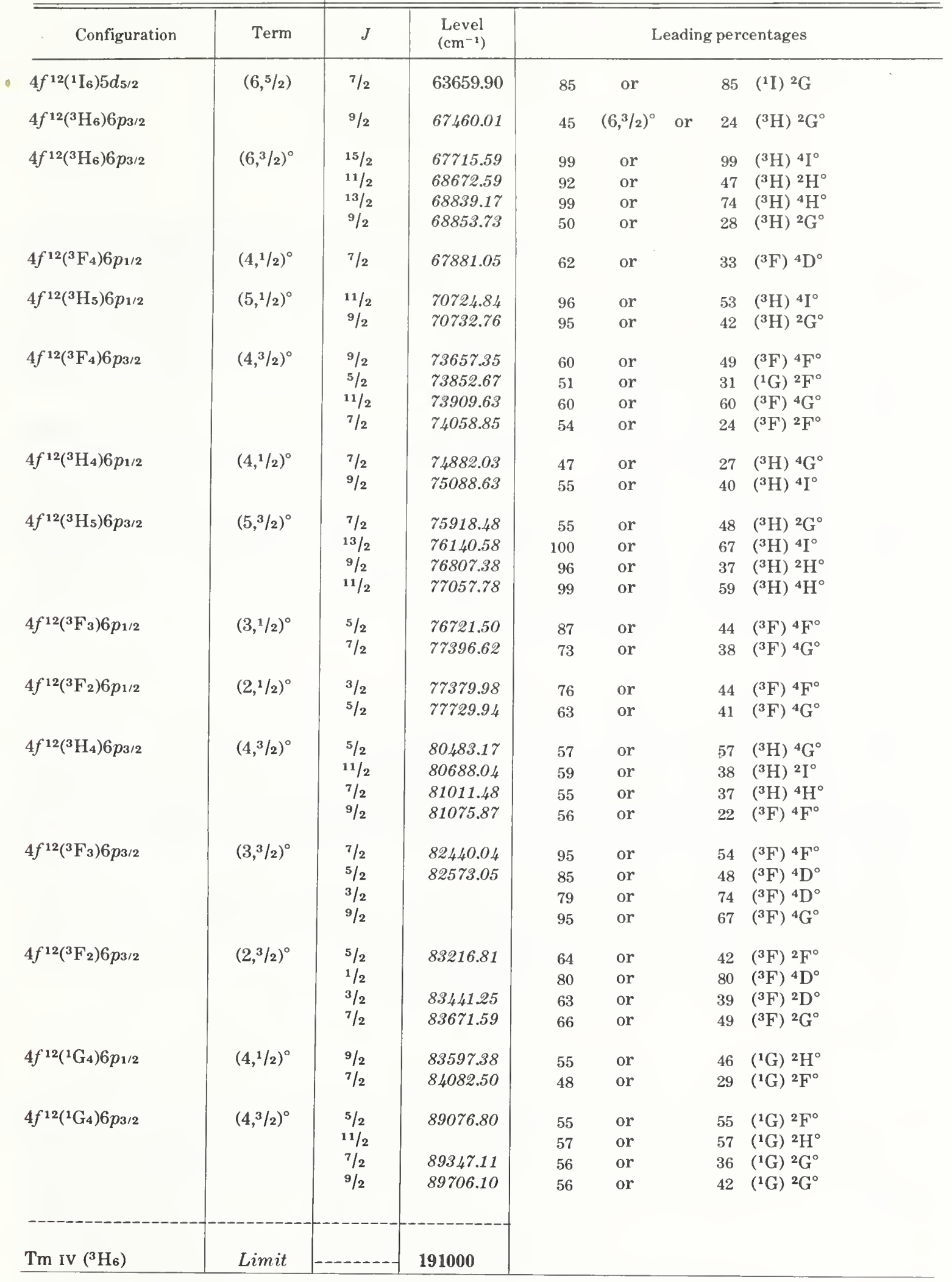




\section{[Tm IV]}

(Dy I sequence; 66 electrons)

Ground state $\left(1 s^{2} 2 s^{2} 2 p^{6} 3 s^{2} 3 p^{6} 3 d^{10} 4 s^{2} 4 p^{6} 4 d^{10} 5 s^{2} 5 p^{6}\right) 4 f^{12}{ }^{3} \mathrm{H}_{6}$

Ionization energy $344000 \pm 3000 \mathrm{~cm}^{-1}$

$42.7 \pm 0.4 \mathrm{eV}$

The spectrum of the free ion has not been analyzed. The positions given here are centers of gravity of the Stark components of the corresponding levels of the ion in $\mathrm{Y}_{2} \mathrm{O}_{3}$. The values (rounded off to the nearest $10 \mathrm{~cm}^{-1}$ ) are from the paper by Gruber, Krupke, and Poindexter, who observed absorption and fluorescence spectra. The $4 f^{12}$ levels have also been evaluated for the ion in other crystals, e.g., thulium ethyl sulfate [Johnsen; Gruber and Conway; Wong and Richman; Krupke and Gruber], and from solution absorption spectra [Carnall, Fields, and Rajnak, 1968]. Absorption transitions to the ${ }^{1} \mathrm{D}$, ${ }^{1} \mathrm{I}$, and ${ }^{3} \mathrm{P}$ levels of $4 f^{12}$ have also been observed, but we omit these higher levels.

Krupke and Gruber have published eigenvectors for Tm IV $4 f^{12}$. The percentages given here are from unpublished results of Carnall et al. [1974]. Several calculations of Tm IV $4 f^{12}$ published since about 1959 yield a value of $\sim 6$ for the intermediate coupling parameter $\zeta / F_{2}$ [see Morrison and Wortman]. All such calculations give roughly the same eigenvectors, and the old reversal of the ${ }^{3} \mathrm{H}_{4}$ and ${ }^{3} \mathrm{~F}_{4}$ designations from the correct respective levels should be discontinued; the ${ }^{3} \mathrm{H}$ percentage of the lowest level having $J=4\left(\sim 5640 \mathrm{~cm}^{-1}\right)$ is about $8 \%$, as compared with the leading percentage of about $63 \%{ }^{3} \mathrm{~F}$ for this level.

Sugar and Reader obtained the ionization energy.

\section{References}

Carnall, W. T., Fields, P. R., and Rajnak, K., J. Chem. Phys. 49, 4424 (1968). [EL] [CL] [W] PT

Carnall, W. T., Fields, P. R., and Rajnak, K., unpublished material (1974). PT

Dieke, G. H., Spectra and Energy Levels of Rare Earth Ions in Crystals, Ed. H. M. Crosswhite and H. Crosswhite, pp. 309-312 (Interscience Publishers, New York, 1968). [EL] [CL] [W]

Gruber, J. B., and Conway, J. G., J. Chem. Phys. 32, 1178 (1960). [EL] [CL] [W] PT

Gruber, J. B., Krupke, W. F., and Poindexter, J. M., J. Chem. Phys. 41, 3363 (1964). [EL] [CL] [W] PT

Jenssen, H. P., Linz, A., Leavitt, R. P., Morrison, C. A., and Wortman, D. E., Phys. Rev. B 11, 92 (1975). [EL] [CL] [W] PT

Johnsen, U., Z. Phys. 152, 454 (1958). [EL] [CL] [W] [ZE]

Krupke, W. F., and Gruber, J. B., Phys. Rev. 139, A2008 (1965). [EL] [CL] [W] PT

Morrison, C. A., and Wortman, D. E., U.S. Nat. Tech. Inform. Serv., AD Rep. No. 735319, 29 pp. (1971). PT

Runciman, W. A., and Wybourne, B. G., J. Chem. Phys. 31, 1149 (1959). PT

Sugar, J., and Reader, J., J. Chem. Phys. 59, 2083 (1973). IP

Wong, E. Y., and Richman, I., J. Chem. Phys. 34, 1182 (1961). [EL] [CL] [W] [ZE] PT

[July 1976]

[Tm IV]

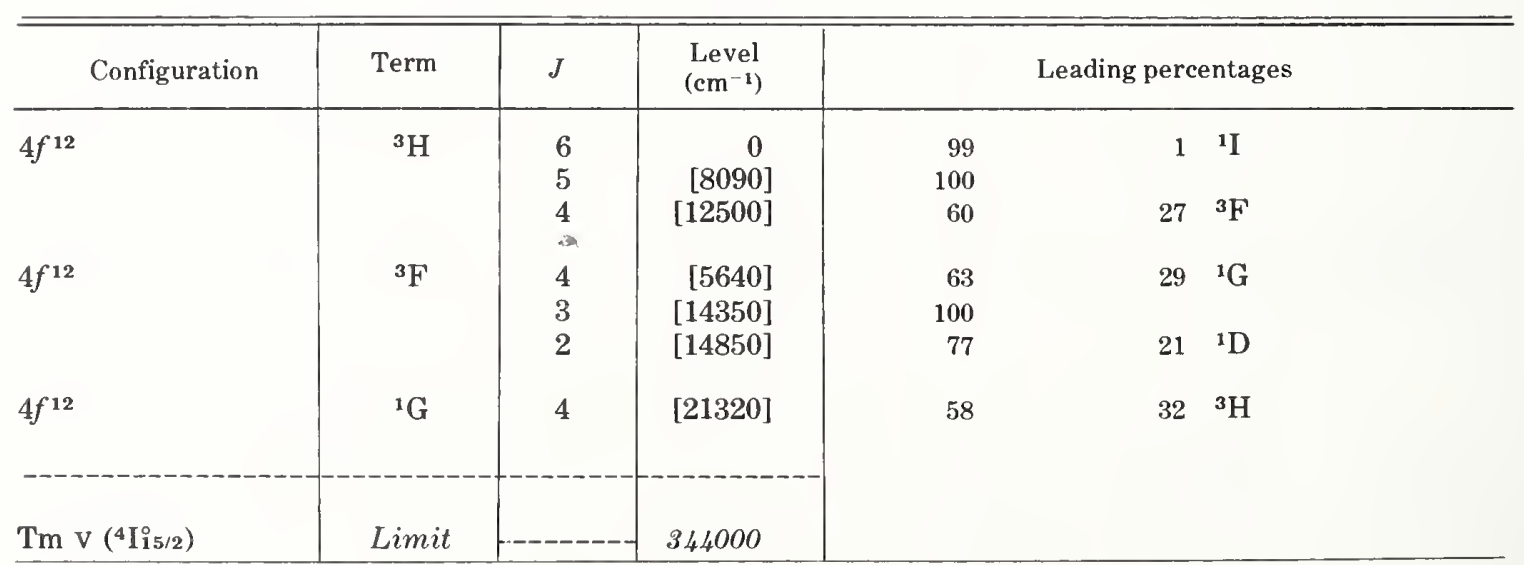




\section{YTTERBIUM}

\section{Yb I}

70 electrons

$Z=70$

Ground state $\left(1 s^{2} 2 s^{2} 2 p^{6} 3 s^{2} 3 p^{6} 3 d^{10} 4 s^{2} 4 p^{6} 4 d^{10} 5 s^{2} 5 p^{6}\right) 4 f^{14} 6 s^{2}{ }^{1} \mathrm{~S}_{0}$

Ionization energy $50441.0 \pm 0.2 \mathrm{~cm}^{-1}$

$6.25394 \pm 0.00003 \mathrm{eV}$

Identified even configurations

$4 f^{14} 6 s^{2}, 4 f^{14} 5 d 6 s, 4 f^{14} 6 s 7 s-13 s, 4 f^{14} 6 s 6 d-9 d, 4 f^{14} 6 p^{2}$, $4 f^{14} 5 d^{2} ?, 4 f^{13} 6 s^{2} 6 p, 4 f^{13} 5 d 6 s 6 p$

Identified odd configurations

$$
\begin{aligned}
& 4 f^{14} 6 s 6 p-48 p, 4 f^{14} 6 s 5 f-7 f, 4 f^{14} 5 d 6 p ?, 4 f^{14} 5 d 7 p ? \\
& 4 f^{13} 5 d 6 s^{2}, 4 f^{13} 5 d^{2} 6 s ?
\end{aligned}
$$

A short history of the research on this spectrum by Meggers and others is given in a recent report [Meggers and Tech, 1977], the published version of which can be consulted for details. Almost 1800 lines ( $2155 \AA$ to $3.13 \mu \mathrm{m}$ ) are listed for $\mathrm{Yb}$ I in this paper, with nearly 800 emission lines classified by the known energy levels. In addition to the levels derived mainly from Meggers' analysis, we have tabulated many high odd-parity $J=1$ levels obtained from absorption spectra (see below).

\section{$g$ Values}

Meggers and Corliss [1966] describe observations of the Zeeman effect for the Yb spectra, and Meggers and Tech [1977] give Zeeman data for about 250 lines. Most of the $g$ values were derived by Meggers, with some additions due to C. E. Moore also being included in the new tables [Meggers and Tech, 1977]. The $g$ values for the three lowest odd levels having $J=1$ are from optical double-resonance determinations. The value given for the $4 f^{14}\left({ }^{1} \mathrm{~S}\right) 6 s 6 p{ }^{3} \mathrm{P}_{1}^{\circ}$ level is an average of the results of Budick and Snir [1967] and Baumann and Wandel [1968], which agree to within 5 units in the fifth place. Baumann and Wandel also measured the $g$ value listed for the $4 f^{14}\left({ }^{1} \mathrm{~S}\right) 6 s 6 p^{1} \mathrm{P}_{1}^{\circ}$ level (stated error \pm 0.005 ), and the value for the $4 f^{13} 5 d 6 s^{2}$ $(7 / 2,5 / 2)_{1}^{\circ}$ level is from Budick and Snir (stated error \pm 0.0009 ).

\section{Theoretical Interpretation}

The levels of the $4 f^{14} 6 s^{2}, 4 f^{14} 5 d 6 s$, and $4 f^{14} 6 s 6 p$ configurations were given by Meggers and Corliss in their 1960 abstract, by which time some 70 higher levels had also been found and most of the strong lines classified. The interpretation of the system of levels based on the $4 f^{13}\left({ }^{2} \mathrm{~F}^{\circ}\right)$ core was aided by calculations by Spector, by Racah, and by Nir and Goldschmidt. Spector collaborated with Meggers on the first searches for levels of this system, and his 1971 paper gives levels and the results of his calculations for the $4 f^{13} 5 d 6 s^{2}$ and $4 f^{13} 6 s^{2} 6 p$ configurations. Miller and Ross [1976] independently assigned 17 even levels to the $4 f^{13} 5 d 6 s 6 p$ configuration on the basis of isotope-shift measurements.

Nir and Goldschmidt [1976] have given the detailed interpretation of the even levels belonging mainly to the $4 f^{13} 5 d 6 s 6 p$ configuration. The eigenvectors for the even levels are from their calculations of the $\left(4 f^{14} 6 s^{2}+4 f^{14} 5 d 6 s+4 f^{14} 5 d^{2}+4 f^{13} 6 s^{2} 6 p+4 f^{13} 5 d 6 s 6 p\right)$ and $4 f^{14} 6 s 6 d$ configurations. Second percentages less than $5 \%$ are omitted. The $4 f^{13} 6 s^{2} 6 p$ and $4 f^{13} 5 d 6 s 6 p$ configurations were calculated in $J_{1} j$ and $J_{1} J_{2}$ coupling schemes, respectively. In listing second percentages from the latter configuration, we usually give only the $4 f^{13}{ }^{2} \mathrm{~F}^{\circ}$ core level and the final parent level from the $5 d 6 s 6 p$ electrons; the intermediate $6 s 6 p^{3} \mathrm{P}^{\circ}$ or ${ }^{1} \mathrm{P}^{\circ}$ parent term is given only if it is different from the corresponding parent in the first percentage. 
The interpretation of several even levels from 47341 to $47673 \mathrm{~cm}^{-1}$ having $J=1,2$, or 3 appears uncertain; the assignments are given tentatively, and percentages from the eigenvectors belonging mainly to $4 f^{14} 5 d^{2}$ are omitted, pending the determination of more levels and $g$ factors for this configuration. The $4 f^{14} 6 p^{2}$ configuration probably interacts significantly with one or more of the other even configurations, but the assignments to the $4 f^{14} 6 p^{2}{ }^{3} \mathrm{P}^{\circ}$ and ${ }^{1} \mathrm{D}^{\circ}$ terms appear to be the most likely designations for the four levels involved. These designations are also consistent with isotope-shift data [Miller and Ross, 1976].

Nir and Goldschmidt [1974] supplied the eigenvector percentages for the two lowest odd configurations, from their calculation of $\left(4 f^{14} 6 s 6 p+4 f^{13} 5 d 6 s^{2}\right)$. The $4 f^{13} 5 d 6 s^{2}$ configuration was calculated in $J_{1} j$ coupling; leading percentages for these levels in the $J_{1} l$ coupling scheme are given by Spector [1971]. Most of the remaining unidentified odd levels probably belong to the $4 f^{13} 5 d^{2} 6 s$ configuration, which has not yet been calculated [Camus and Tomkins, 1969; Meggers and Tech, 1977]. This has been indicated by giving the two lowest of the otherwise unidentified odd levels beginning near $37000 \mathrm{~cm}^{-1}$ tentatively assigned to this configuration.

\section{Series, Ionization Energy, Autoionizing Levels}

The identifications of some members of $4 f^{14} 6 s n s, 4 f^{14} 6 s n d$, and $4 f^{14} 6 s n p$ series were made by Nir [1970]. Tech has extended some of these series and assigned the $4 f^{14} 6 s n f$ levels [Meggers and Tech, 1977]. Camus and Tomkins [1969] observed the two $4 f^{14} 6 s^{2}{ }^{1} \mathrm{~S}_{0}-4 f^{14} 6 s n p$ $(J=1)$ series in absorption over the region $1980-2300 \AA$. The structure of the $4 f^{14} 6 s n p$ configurations arising from a $4 f^{14} 6 s\left({ }^{2} \mathrm{~S}_{1 / 2}\right) n p_{j}$ coupling scheme is apparent for $n=10$, and we have designated the higher series members according to this scheme. Camus and Tomkins derived the quoted value for the $4 f^{14} 6 s\left({ }^{2} \mathrm{~S}_{1 / 2}\right)$ ionization limit from the $\left({ }^{2} \mathrm{~S}_{1 / 2}\right) n p_{3 / 2}\left({ }^{1 / 2},{ }^{3 / 2}\right)_{1}^{\circ}$ series (the ${ }^{1} \mathrm{P}_{1}^{\circ}$ series in $L S$ coupling), which they observed to $n=48$.

Most of the levels given above the $4 f^{14} 6 s\left({ }^{2} \mathrm{~S}_{1 / 2}\right)$ principal limit were obtained from photoionization resonances [Parr and Elder, 1968] or from optical absorption spectra [Kozlov, Kotochigova, and Nikolaev, 1976]. The letter " $a$ " is given after the positions of these $J=1$ levels, to indicate autoionization broadening. The levels from Parr and Elder are rounded off to the nearest 10 or $100 \mathrm{~cm}^{-1}$, depending on the width. The value of Kozlov et al. for the very wide level near $55400 \mathrm{~cm}^{-1}$ is also rounded off, and the level they derive as a separate component of this resonance (at $54700 \mathrm{~cm}^{-1}$ ) is given tentatively. Parr and Elder give some additional levels derived from weaker resonances that are not listed here. The tentative assignments to $4 f^{14} 5 d 6 p$ and $4 f^{14} 5 d 7 p$ terms are due to Parr and Elder; additional assignments are omitted here, pending calculation of the complex absorption structure in this region.

\section{References}

Baumann, M., and Wandel, G., Phys. Lett. 28A, 200 (1968). ZE

Budick, B., and Snir, J., Phys. Lett. 24A, 689(1967). Hfs ZE

Camus, P., and Tomkins, F. S., J. Phys. (Paris) 30, 545 (1969). EL CL IP

Humphreys, C. J., and Paul, E., Jr., Quarterly Report Foundational Res. Projects, NAVoRD Report 5970, NOLC Report 473, 57 pp. (1959). W

King, A. S., Astrophys. J. 74, 328 (1931). W

Kozlov, M. G., Kotochigova, S. A., and Nikolaev, V. N., Opt. Spectrose. (USSR) 41, 4 (1976). EL CL W

Meggers, W. F., and Corliss, C. H., J. Opt. Soc. Am. 50, 1136A (1960). EL ZE

Meggers, W. F., and Corliss, C. H., J. Res. Nat. Bur. Stand. (U.S.) 70A, 63 (1966). W

Meggers, W. F., Corliss, C. H., and Scribner, B. F., Tables of Spectral-Line Intensities, Nat. Bur. Stand. (U.S.) Monogr. 145, Part 1, 403 pp. (1975). CL

Meggers, W. F., and Scribner, B. F., J. Res. Nat. Bur. Stand. (U.S.) 19, 651 (1937). EL CL W

Meggers, W. F., and Tech, J. L., preprint entitled "The First Spectrum of Ytterbium (Yb I)" (1977). This paper will be submitted for publication in J. Res. Nat. Bur. Stand. (U.S.), EL CL W ZE

Miller, G. E., and Ross, J. S., J. Opt. Soc. Am. 66, 585 (1976). ND IS

Nir, S., J. Opt. Soc. Am. 60, 354 (1970). EL ND

Nir, S., and Goldschmidt, Z. B., unpublished material $(1974,1976)$. ND PT

Parr. A. C., and Elder, F. A., J. Chem. Phys. 49, 2665 (1968). EL CL

Reader, J., and Sugar, J., J. Opt. Soc. Am. 56, 1189 (1966). IP

Spector, N., J. Opt. Soc. Am. 61, 1350 (1971). EL ZE PT

[May 1977] 
Yb I, Even Parity

\begin{tabular}{|c|c|c|c|c|c|c|}
\hline Configuration & Term & $J$ & $\begin{array}{l}\text { Level } \\
\left(\mathrm{cm}^{-1}\right)\end{array}$ & $g$ & \multicolumn{2}{|c|}{ Leading percentages } \\
\hline $4 f^{14}\left({ }^{1} \mathrm{~S}\right) 6 s^{2}$ & ${ }^{1} \mathrm{~S}$ & 0 & 0.000 & & 99 & \\
\hline $4 f^{14}\left({ }^{1} \mathrm{~S}\right) 5 d 6 s$ & ${ }^{3} \mathrm{D}$ & $\begin{array}{l}1 \\
2 \\
3\end{array}$ & $\begin{array}{l}24489.102 \\
24751.948 \\
25270.902\end{array}$ & $\begin{array}{l}0.50 \\
1.16 \\
1.34\end{array}$ & $\begin{array}{l}99 \\
97 \\
99\end{array}$ & \\
\hline $4 f^{14}\left({ }^{1} \mathrm{~S}\right) 5 d 6 s$ & ${ }^{1} \mathrm{D}$ & 2 & 27677.665 & 1.01 & 95 & \\
\hline $4 f^{13}\left({ }^{2} \mathrm{~F}_{7 / 2}^{\circ}\right) 6 s^{2} 6 p_{1 / 2}$ & $(7 / 2,1 / 2)$ & $\begin{array}{l}3 \\
4\end{array}$ & $\begin{array}{l}32065.282 \\
32273.597\end{array}$ & 1.23 & $\begin{array}{l}89 \\
92\end{array}$ & \\
\hline $4 f^{14}\left({ }^{1} \mathrm{~S}\right) 6 s 7 s$ & ${ }^{3} \mathrm{~S}$ & 1 & 32694.692 & 2.01 & & \\
\hline $4 f^{14}\left({ }^{1} \mathrm{~S}\right) 6 s 7 s$ & ${ }^{1} \mathrm{~S}$ & 0 & 34350.65 & & & \\
\hline $4 f^{13}\left({ }^{2} \mathrm{~F}_{7 / 2}^{\circ}\right) 6 s^{2} 6 p 3 / 2$ & $(7 / 2,3 / 2)$ & $\begin{array}{l}5 \\
2 \\
3 \\
4\end{array}$ & $\begin{array}{l}35178.78 \\
35196.98 \\
35807.52 \\
36060.98\end{array}$ & $\begin{array}{l}1.05 \\
1.08\end{array}$ & $\begin{array}{l}93 \\
92 \\
88 \\
92\end{array}$ & $5\left({ }^{2} \mathrm{~F}_{7 / 2}^{\circ}\right)\left({ }^{7} / 2,{ }^{1} / 2\right)$ \\
\hline $4 f^{14}\left({ }^{1} \mathrm{~S}\right) 6 s 6 d$ & ${ }^{3} \mathrm{D}$ & $\begin{array}{l}1 \\
2 \\
3\end{array}$ & $\begin{array}{l}39808.72 \\
39838.04 \\
39966.09\end{array}$ & $\begin{array}{l}0.50 \\
1.16 \\
1.33\end{array}$ & $\begin{array}{r}100 \\
86 \\
100\end{array}$ & $14^{1} \mathrm{D}$ \\
\hline $4 f^{13}\left({ }^{2} \mathrm{~F}_{7 / 2}^{\circ}\right) 5 d\left({ }^{2} \mathrm{D}\right) 6 s 6 p\left({ }^{3} \mathrm{P}^{\circ}\right)\left({ }^{4} \mathrm{~F}_{3 / 2}^{\circ}\right)$ & $(7 / 2,3 / 2)$ & $\begin{array}{l}2 \\
5 \\
4 \\
3\end{array}$ & $\begin{array}{l}39880.26 \\
42935.78 \\
44984.75 \\
45462.54\end{array}$ & $\begin{array}{l}1.83 \\
0.89 \\
1.08 \\
1.20\end{array}$ & $\begin{array}{l}61 \\
80 \\
54 \\
41\end{array}$ & $\begin{aligned} 25 & \left({ }^{2} \mathrm{~F}_{7 / 2}^{\circ}\right)\left({ }^{4} \mathrm{~F}_{5 / 2}^{\circ}\right)\left({ }^{7} / 2,5 / 2\right) \\
9 & \left({ }^{2} \mathrm{~F}_{7 / 2}^{\circ}\right)\left({ }^{4} \mathrm{~F}_{5 / 2}^{\circ}\right)\left({ }^{7} / 2,{ }^{7} / 2\right) \\
12 & \left({ }^{2} \mathrm{~F}_{7 / 2}^{\circ}\right)\left({ }^{2} \mathrm{D}_{3 / 2}^{\circ}\right)\left({ }^{7} / 2,{ }^{3} / 2\right) \\
17 & \left({ }^{2} \mathrm{~F}_{7 / 2}^{\circ}\right)\left({ }^{2} \mathrm{D}_{1 / 2}^{\circ}\right)\left({ }^{7} / 2,{ }^{1} / 2\right)\end{aligned}$ \\
\hline $4 f^{14}\left({ }^{1} \mathrm{~S}\right) 6 s 6 d$ & ${ }^{1} \mathrm{D}$ & 2 & 40061.51 & 1.03 & 86 & $14^{3} \mathrm{D}$ \\
\hline $4 f^{14}\left({ }^{1} \mathrm{~S}\right) 6 s 8 s$ & ${ }^{3} \mathrm{~S}$ & 1 & 41615.04 & 2.02 & & \\
\hline $4 f^{13}\left({ }^{2} \mathrm{~F}_{7 / 2}^{\circ}\right) 5 d\left({ }^{2} \mathrm{D}\right) 6 s 6 p\left({ }^{3} \mathrm{P}^{\circ}\right)\left({ }^{4} \mathrm{~F}_{5 / 2}^{\circ}\right)$ & & 3 & 41827.30 & 1.52 & $30 \quad(7 / 2,5 / 2)$ & $28\left({ }^{2} \mathrm{~F}_{7 / 2}^{\circ}\right)\left({ }^{4} \mathrm{~F}_{3 / 2}^{\circ}\right)\left({ }^{7} / 2,{ }^{3 / 2}\right)$ \\
\hline $4 f^{14}\left({ }^{1} \mathrm{~S}\right) 6 s 8 s$ & ${ }^{1} \mathrm{~S}$ & 0 & 41939.90 & & . & \\
\hline $4 f^{13}\left({ }^{2} \mathrm{~F}_{7 / 2}^{\circ}\right) 5 d\left({ }^{2} \mathrm{D}\right) 6 s 6 p\left({ }^{3} \mathrm{P}^{\circ}\right)\left({ }^{4} \mathrm{~F}_{5 / 2}^{\circ}\right)$ & $(7 / 2,5 / 2)$ & 1 & 42436.70 & 1.73 & 57 & $11\left({ }^{2} \mathrm{~F}_{7 / 2}^{\circ}\right)\left({ }^{2} \mathrm{D}_{5 / 2}^{\circ}\right)\left({ }^{7} / 2,5 / 2\right)$ \\
\hline $4 f^{14}\left({ }^{1} \mathrm{~S}\right) 6 p^{2}$ & ${ }^{3} \mathrm{P}$ & $\begin{array}{l}0 \\
1 \\
2\end{array}$ & $\begin{array}{l}42436.91 \\
43805.42 \\
44760.37\end{array}$ & $\begin{array}{l}1.47 \\
1.34\end{array}$ & & \\
\hline $4 f^{13}\left({ }^{2} \mathrm{~F}_{5 / 2}^{\circ}\right) 6 s^{2} 6 p_{1 / 2}$ & $(5 / 2,1 / 2)$ & $\begin{array}{l}3 \\
2\end{array}$ & 42531.87 & 1.01 & $\begin{array}{l}92 \\
89\end{array}$ & \\
\hline $4 f^{13}\left({ }^{2} \mathrm{~F}_{7 / 2}^{\circ}\right) 5 d\left({ }^{2} \mathrm{D}\right) 6 s 6 p\left({ }^{3} \mathrm{P}^{\circ}\right)\left({ }^{4} \mathrm{~F}_{5 / 2}^{\circ}\right)$ & & 2 & 43224.78 & 1.34 & $22(7 / 2,5 / 2)$ & $18\left({ }^{2} \mathrm{~F}_{7 / 2}^{\circ}\right)\left({ }^{4} \mathrm{~F}^{\circ} / 2\right)\left({ }^{7} / 2,{ }^{7} / 2\right)$ \\
\hline $4 f^{13}\left({ }^{2} \mathrm{~F}_{7 / 2}^{\circ}\right) 5 d\left({ }^{2} \mathrm{D}\right) 6 s 6 p\left({ }^{3} \mathrm{P}^{\circ}\right) \quad\left({ }^{4} \mathrm{~F}_{5 / 2}^{\circ}\right)$ & $(7 / 2,5 / 2)$ & 6 & 43814.11 & 1.08 & 78 & $7\left({ }^{2} \mathrm{~F}_{7 / 2}^{\circ}\right)\left({ }^{2} \mathrm{D}_{5 / 2}^{\circ}\right)\left({ }^{7} / 2,5 / 2\right)$ \\
\hline $4 f^{13}\left({ }^{2} \mathrm{~F}^{\circ} / 2\right) 5 d\left({ }^{2} \mathrm{D}\right) 6 s 6 p\left({ }^{3} \mathrm{P}^{\circ}\right) \quad\left({ }^{4} \mathrm{~F}_{5 / 2}^{\circ}\right)$ & & 4 & 44232.66 & 1.48 & $20(7 / 2,5 / 2)$ & $16\left({ }^{2} \mathrm{~F}_{7 / 2}^{\circ}\right)\left({ }^{4} \mathrm{~F}_{7 / 2}^{\circ}\right)\left({ }^{7} / 2,{ }^{7} / 2\right)$ \\
\hline $4 f^{14}\left({ }^{1} \mathrm{~S}\right) 6 s 7 d$ & ${ }^{3} \mathrm{D}$ & $\begin{array}{l}1 \\
2 \\
3\end{array}$ & $\begin{array}{l}44311.38 \\
44313.05 \\
44380.82\end{array}$ & 1.32 & & \\
\hline $4 f^{14}\left({ }^{1} \mathrm{~S}\right) 6 s 7 d$ & ${ }^{1} \mathrm{D}$ & 2 & 44357.60 & 1.10 & & \\
\hline $4 f^{13}\left({ }^{2} \mathrm{~F}_{7 / 2}^{\circ}\right) 5 d\left({ }^{2} \mathrm{D}\right) 6 s 6 p\left({ }^{3} \mathrm{P}^{\circ}\right)\left({ }^{4} \mathrm{~F}_{7 / 2}^{\circ}\right)$ & & 3 & 44713.12 & 1.39 & $24\left({ }^{7} / 2,7 / 2\right)$ & $13\left({ }^{2} \mathrm{~F}_{7 / 2}^{\circ}\right)\left({ }^{4} \mathrm{~F}_{9 / 2}^{\circ}\right)\left({ }^{7} / 2,{ }^{9} / 2\right)$ \\
\hline $4 f^{13}\left({ }^{2} \mathrm{~F}_{5 / 2}^{\circ}\right) 6 s^{2} 6 p_{3 / 2}$ & $(5 / 2,3 / 2)$ & $\begin{array}{l}1 \\
4 \\
2 \\
3\end{array}$ & $\begin{array}{l}44834.61 \\
45497.62 \\
45913.86\end{array}$ & 0.66 & $\begin{array}{l}87 \\
89 \\
88 \\
88\end{array}$ & \\
\hline $4 f^{14}\left({ }^{1} \mathrm{~S}\right) 6 s 9 s$ & ${ }^{3} \mathrm{~S}$ & 1 & 45121.37 & 1.98 & & \\
\hline $4 f^{13}\left({ }^{2} \mathrm{~F}_{7 / 2}^{\circ}\right) 5 d\left({ }^{2} \mathrm{D}\right) 6 s 6 p\left({ }^{3} \mathrm{P}^{\circ}\right)\left({ }^{2} \mathrm{D}_{5 / 2}^{\circ}\right)$ & & 2 & 45338.53 & 1.30 & $38 \quad(7 / 2,5 / 2)$ & $23 \quad\left({ }^{2} \mathrm{~F}_{7 / 2}^{\circ}\right)\left({ }^{4} \mathrm{~F}_{7 / 2}^{\circ}\right)\left({ }^{7} / 2,{ }^{7 / 2}\right)$ \\
\hline $4 f^{13}\left({ }^{2} \mathrm{~F}_{7 / 2}^{\circ}\right) 5 d\left({ }^{2} \mathrm{D}\right) 6 s 6 p\left({ }^{3} \mathrm{P}^{\circ}\right) \quad\left({ }^{4} \mathrm{~F}_{5 / 2}^{\circ}\right)$ & & 5 & 45410.91 & 1.17 & $40(7 / 2,5 / 2)$ & $18\left({ }^{2} \mathrm{~F}_{7 / 2}^{\circ}\right)\left({ }^{4} \mathrm{D}_{3 / 2}^{\circ}\right)(7 / 2,3 / 2)$ \\
\hline
\end{tabular}


Yb I, Even Parity_Continued

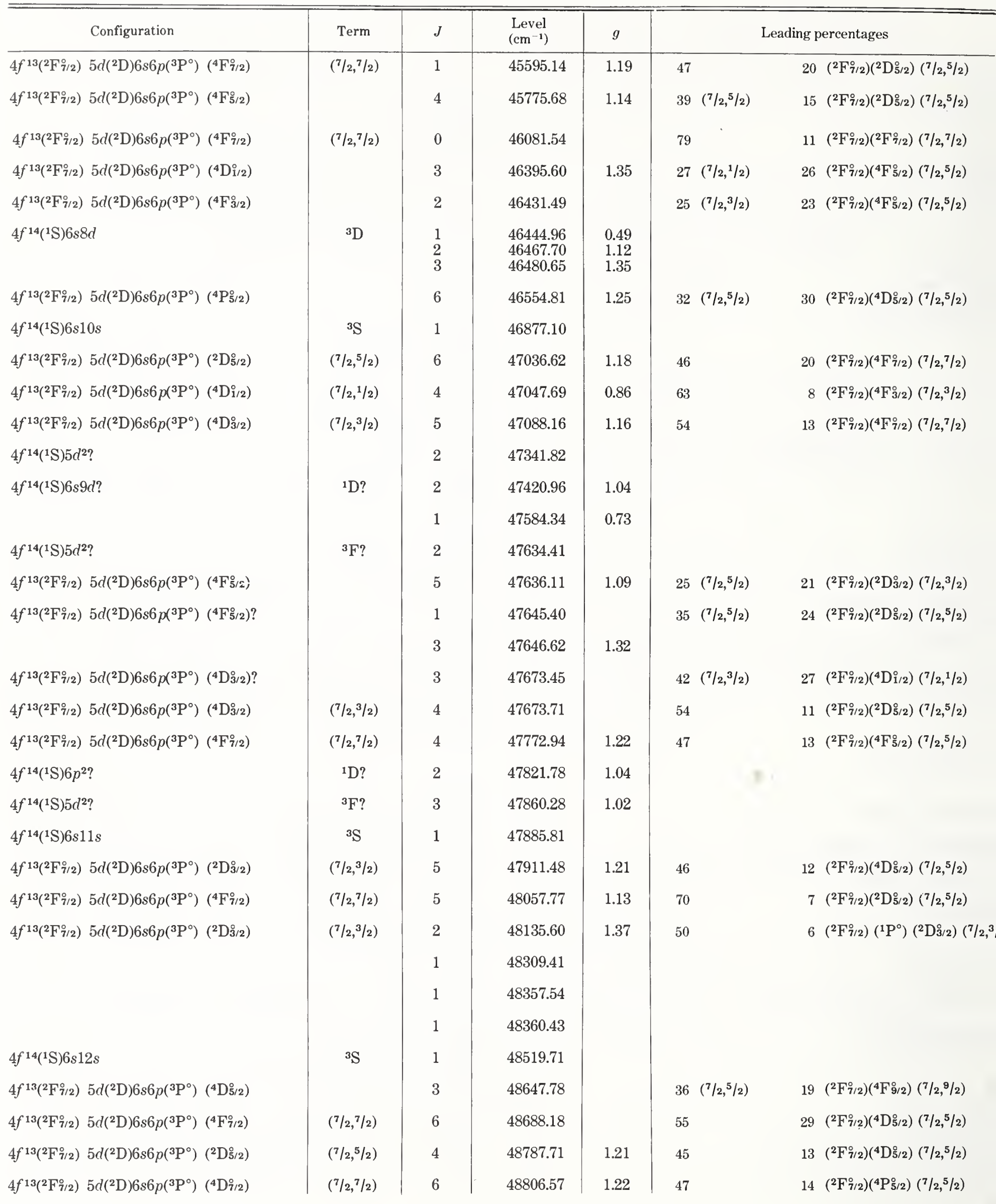


Yb I, Even Parity-Continued

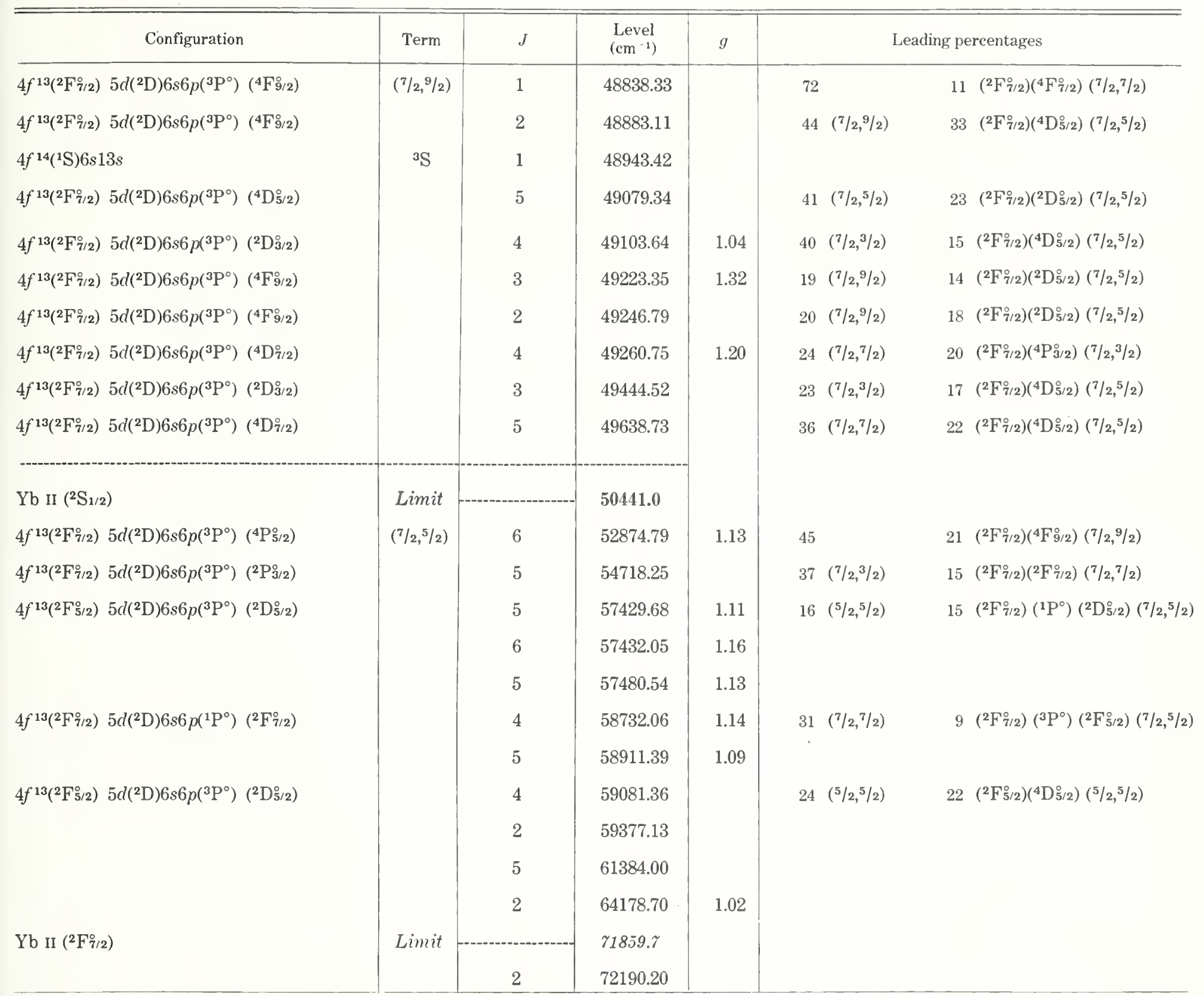


Yb I, Odd Parity

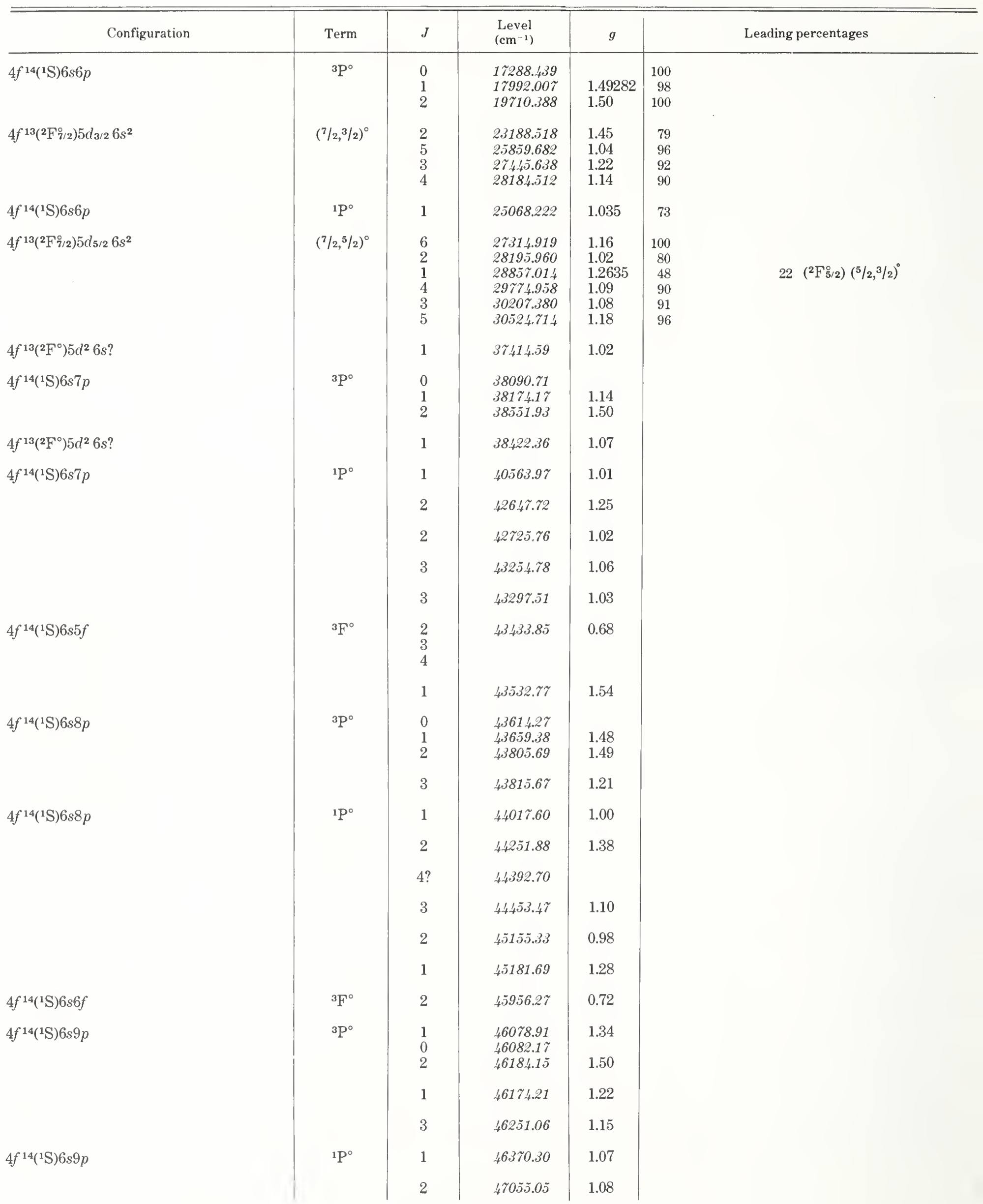


Yb I, Odd Parity—Continued

\begin{tabular}{|c|c|c|c|c|c|}
\hline Configuration & Term & $J$ & $\begin{array}{l}\text { Level } \\
\left(\mathrm{cm}^{-1}\right)\end{array}$ & $g$ & Leading percentages \\
\hline & & $3 ?$ & $4 \% 325.19$ & & \\
\hline \multirow[t]{2}{*}{$4 f^{14}\left({ }^{1} \mathrm{~S}\right) 6 s 7 f$} & ${ }^{3} \mathrm{~F}^{\circ}$ & 2 & $4 \pi 326.65$ & & \\
\hline & & 1 & 47332.26 & 0.95 & \\
\hline $4 f^{14}\left({ }^{1} \mathrm{~S}\right) 6 s\left({ }^{2} \mathrm{~S}_{1 / 2}\right) 10 p_{1 / 2}$ & $(1 / 2,1 / 2)^{\circ}$ & $\begin{array}{l}0 \\
1\end{array}$ & $\begin{array}{l}4 \pi 409.82 \\
4 \% 420.93\end{array}$ & 1.36 & \\
\hline \multirow[t]{5}{*}{$4 f^{14}\left({ }^{1} \mathrm{~S}\right) 6 s\left({ }^{2} \mathrm{~S}_{1 / 2}\right) 10 p_{3 / 2}$} & $(1 / 2,3 / 2)^{\circ}$ & $\begin{array}{l}2 \\
1\end{array}$ & $\begin{array}{l}47471.10 \\
47498.83\end{array}$ & 1.03 & \\
\hline & & $3 ?$ & 47843.13 & & \\
\hline & & 1 & 47859.31 & 1.30 & \\
\hline & & 4 & $4 \pi 939.10$ & & \\
\hline & & 3 & $48154 . \pi 1$ & & \\
\hline $4 f^{14}\left({ }^{1} \mathrm{~S}\right) 6 s\left({ }^{2} \mathrm{~S}_{1 / 2}\right) 11 p_{1 / 2}$ & $(1 / 2,1 / 2)^{\circ}$ & $\begin{array}{l}0 \\
1\end{array}$ & 48212.10 & & \\
\hline \multirow[t]{7}{*}{$4 f^{14}\left({ }^{1} \mathrm{~S}\right) 6 s\left({ }^{2} \mathrm{~S}_{1 / 2}\right) 11 p_{3 / 2}$} & $\left(1 / 2,{ }^{3} / 2\right)^{\circ}$ & $\begin{array}{l}2 \\
1\end{array}$ & 48258.47 & & \\
\hline & & 2 & 48234.12 & & \\
\hline & & 3 & 48237.39 & 1.24 & \\
\hline & & 2 & 48324.89 & & \\
\hline & & 2 & 484.49 .27 & 1.15 & \\
\hline & & 3 & 48487.98 & 1.23 & \\
\hline & & 2 & 48701.60 & & \\
\hline $4 f^{14}\left({ }^{1} \mathrm{~S}\right) 6 s\left({ }^{2} \mathrm{~S}_{1 / 2}\right) 12 p_{1 / 2}$ & $(1 / 2,1 / 2)^{\circ}$ & 1 & 48719.03 & & $v^{\circ}$ \\
\hline \multirow[t]{3}{*}{$4 f^{14}\left({ }^{1} \mathrm{~S}\right) 6 s\left({ }^{2} \mathrm{~S}_{1 / 2}\right) 12 p 3 / 2$} & $(1 / 2,3 / 2)^{\circ}$ & 1 & 48761.84 & & \\
\hline & & 2 & 48965.16 & & \\
\hline & & 1 & $49005 . \tilde{4}$ & & \\
\hline $4 f^{14}\left({ }^{1} \mathrm{~S}\right) 6 s\left({ }^{2} \mathrm{~S}_{1 / 2}\right) 13 p_{1 / 2}$ & $(1 / 2,1 / 2)^{0}$ & 1 & 49110.07 & & \\
\hline \multirow[t]{2}{*}{$1 f^{14}\left({ }^{1} \mathrm{~S}\right) 6 s\left({ }^{2} \mathrm{~S}_{1 / 2}\right) 13 p_{3 / 2}$} & $(1 / 2,3 / 2)^{\circ}$ & 1 & $4912 \pi .38$ & & \\
\hline & & 4 & 49282.36 & & \\
\hline $4 f^{14}\left({ }^{1} \mathrm{~S}\right) 6 s\left({ }^{2} \mathrm{~S}_{1 / 2}\right) 14 p_{1 / 2}$ & $(1 / 2,1 / 2)^{\circ}$ & 1 & $49352.4 \pi$ & & \\
\hline $4 f^{14}\left({ }^{1} \mathrm{~S}\right) 6 s\left({ }^{2} \mathrm{~S}_{1 / 2}\right) 14 p_{3 / 2}$ & $(1 / 2,3 / 2)^{\circ}$ & 1 & 49360.60 & & \\
\hline $4 f^{14}\left({ }^{1} \mathrm{~S}\right) 6 s\left({ }^{2} \mathrm{~S}_{1 / 2}\right) 15 p_{1 / 2}$ & $(1 / 2,1 / 2)^{\circ}$ & 1 & 49536.43 & & \\
\hline $4 f^{14}\left({ }^{1} \mathrm{~S}\right) 6 s\left({ }^{2} \mathrm{~S}_{1 / 2}\right) 15 p_{3 / 2}$ & $(1 / 2,3 / 2)^{\circ}$ & 1 & 495.46 .55 & & \\
\hline $4 f^{14}\left({ }^{1} \mathrm{~S}\right) 6 s\left({ }^{2} \mathrm{~S}_{1 / 2}\right) 16 p_{1 / 2}$ & $(1 / 2,1 / 2)^{\circ}$ & 1 & 49677.59 & & \\
\hline $4 f^{14}\left({ }^{1} \mathrm{~S}\right) 6 s\left({ }^{2} \mathrm{~S}_{1 / 2}\right) 16 p_{3 / 2}$ & $(1 / 2,3 / 2)^{\circ}$ & 1 & 49688.55 & & \\
\hline $4 f^{14}\left({ }^{1} \mathrm{~S}\right) 6 s\left({ }^{2} \mathrm{~S}_{1 / 2}\right) 17 p_{1 / 2}$ & $(1 / 2,1 / 2)^{\circ}$ & 1 & 49786.39 & & \\
\hline $4 f^{14}\left({ }^{1} \mathrm{~S}\right) 6 s\left({ }^{2} \mathrm{~S}_{1 / 2}\right) 17 p_{3 / 2}$ & $(1 / 2,3 / 2)^{\circ}$ & 1 & 49799.20 & & \\
\hline \multirow[t]{2}{*}{$4 f^{14}\left({ }^{1} \mathrm{~S}\right) 6 s\left({ }^{2} \mathrm{~S}_{1 / 2}\right) 18 p_{3 / 2}$} & $(1 / 2,3 / 2)^{\circ}$ & 1 & $49867.13 ?$ & & \\
\hline & & 1 & 49920.15 & & \\
\hline $4 f^{14}\left({ }^{1} \mathrm{~S}\right) 6 s\left({ }^{2} \mathrm{~S}_{1 / 2}\right) 19 p_{3 / 2}$ & $(1 / 2,3 / 2)^{\circ}$ & 1 & 49969.10 & & \\
\hline $4 f^{14}\left({ }^{1} \mathrm{~S}\right) 6 s\left({ }^{2} \mathrm{~S}_{1 / 2}\right) 20 p_{1 / 2}$ & $(1 / 2,1 / 2)^{\circ}$ & 1 & 50016.08 & & \\
\hline $4 f^{14}\left({ }^{1} \mathrm{~S}\right) 6 s\left({ }^{2} \mathrm{~S}_{1 / 2}\right) 20 p_{3 / 2}$ & $(1 / 2,3 / 2)^{\circ}$ & 1 & 50021.23 & & \\
\hline
\end{tabular}


Yb I, Odd Parity-Continued

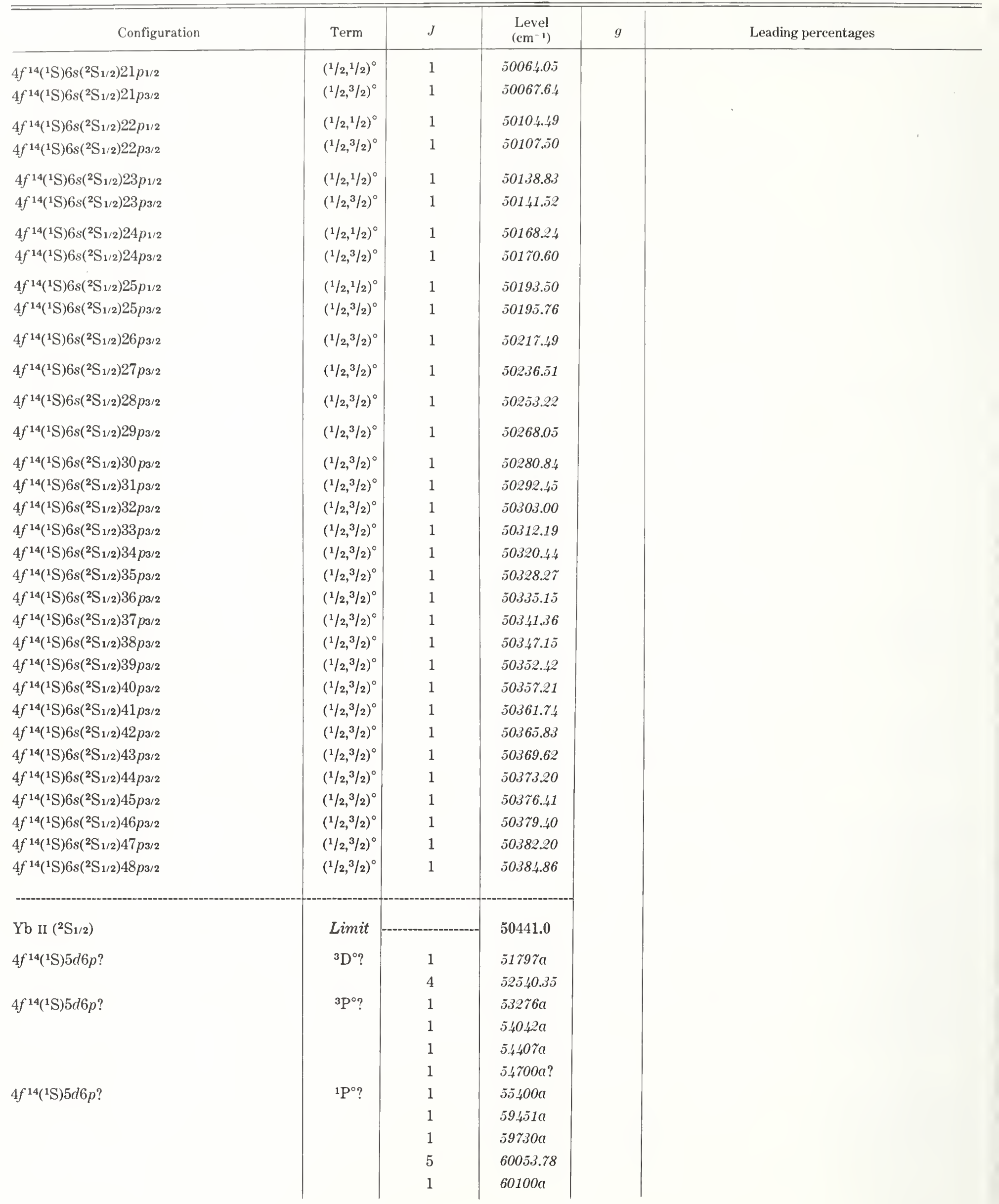


Yb I, Odd Parity-Continued

\begin{tabular}{|c|c|c|c|c|c|}
\hline Configuration & Term & $J$ & $\begin{array}{l}\text { Level } \\
\left(\mathrm{cm}^{-1}\right)\end{array}$ & $g$ & Leading percentages \\
\hline $\begin{array}{l} \\
4 f^{14}\left({ }^{1} \mathrm{~S}\right) 5 d 7 p ? \\
4 f^{14}\left({ }^{1} \mathrm{~S}\right) 5 d 7 p ? \\
4 f^{14}\left({ }^{1} \mathrm{~S}\right) 5 d 7 p ?\end{array}$ & $\begin{array}{l}{ }^{3} \mathrm{D}^{\circ} ? \\
{ }^{3} \mathrm{P}^{\circ} ? \\
{ }^{1} \mathrm{P}^{\circ} ?\end{array}$ & $\begin{array}{l}1 \\
1 \\
5 \\
5 \\
6 \\
1 \\
1 \\
5 \\
1 \\
1 \\
6 \\
1 \\
1\end{array}$ & $\begin{array}{l}61420 a \\
62200 a \\
62308.46 \\
62395.51 \\
62687.40 \\
63050 a \\
63650 a \\
63672.48 \\
64500 a \\
67310 a \\
67844.34 \\
68790 a \\
69900 a\end{array}$ & 1.10 & \\
\hline
\end{tabular}


(Tm I sequence; 69 electrons)

Ground state $\left(1 s^{2} 2 s^{2} 2 p^{6} 3 s^{2} 3 p^{6} 3 d^{10} 4 s^{2} 4 p^{6} 4 d^{10} 5 s^{2} 5 p^{6}\right) 4 f^{14} 6 s^{2} \mathrm{~S}_{1 / 2}$

Ionization energy $98269 \pm 50 \mathrm{~cm}^{-1}$

$12.184 \pm 0.006 \mathrm{eV}$

Identified even configurations

$4 f^{14} 6 s-8 s, 10 s, 4 f^{14} 5 d-11 d, 4 f^{13} 6 s 6 p, 4 f^{13} 5 d 6 p, 4 f^{14} 5 g, 6 g$

Identified odd configurations

$4 f^{13} 6 s^{2}, 4 f^{13} 5 d 6 s, 4 f^{14} 6 p, 7 p, 4 f^{13} 5 d^{2}, 4 f^{14} 5 f-14 f$

The papers by Meggers and Corliss [1966] and by Meggers [1967, edited by C. E. Moore] give detailed histories of work on this spectrum. The levels are mainly from Meggers' tables [1967], supplemented by the $4 f^{14} \mathrm{nl}$ series members analyzed by Kaufman and Sugar [1973]. The latter authors also derived the quoted ionization energy.

The relative completeness of the analysis and theoretical interpretation is due to a full collaboration between Meggers and coworkers at NBS and Racah and his group at the Hebrew University, Jerusalem. The first calculations of Yb II by Racah and his students [Racah, 1954; 1960] were important not only for extension of the analysis but as early examples of $J_{1} J_{2}$ and $J_{1} L_{2}$ coupling schemes also appropriate for some other rare-earth spectra. The eigenvector percentages given here are from later calculations by Racah and Goldschmidt including configuration interactions: $\left(4 f^{13} 5 d 6 p+4 f^{13} 6 s 6 p\right)$ and $\left(4 f^{13} 6 s^{2}+4 f^{13} 5 d 6 s+4 f^{13} 5 d^{2}\right)$.

Spector [1968] discusses the $4 f^{13} 5 d^{2}$ configuration, including theoretical results in the single-configuration approximation. One of the levels previously assigned to this configuration is now designated $4 f^{14} 7 p^{2} \mathrm{P}_{3 / 2}^{\circ}\left(65594 \mathrm{~cm}^{-1}\right)$, following Kaufman and Sugar.

Kaufman and Sugar's reassignments of two even levels near $76500 \mathrm{~cm}^{-1}$ to $4 f^{14} 7 d^{2} \mathrm{D}$ are given tentatively, as are our correlations of the two levels formerly designated $7 d^{2} \mathrm{D}$ with calculated levels of $4 f^{13} 5 d 6 p$. No calculations that include the one-electron series have been carried out for either parity.

The $\mathrm{Yb}$ line list of Meggers and Corliss [1966] has more than 5000 lines assigned to $\mathrm{Yb}$ II (2018-11559 $\AA$ ), and Meggers [1967] was able to classify about 4000 of these, including practically all of the strong lines. Kaufman and Sugar classified about 30 additional lines from the list as belonging to one of the $4 f^{14} \mathrm{nl}$ series. The extension of the observations down to $1377 \AA$ gave about 280 additional Yb II lines, a number of which also belong to the $n l$ series [Kaufman and Sugar, 1973].

Meggers and Corliss describe the main observations of the Zeeman effect for Yb. The $g$ values of more than 250 levels have been derived from the measurements of more than 1000 Zeeman patterns [Meggers, 1967].

\section{References}

Allen, L., United Kingdom Atomic Energy Authority, Res. Group Report, AERE-R 4029, 34 pp. (1962). W ZE Goldschmidt, Z. B., Thesis, Hebrew Univ., Jerusalem, Israel, 487 pp. (1968). ND PT

Humphreys, C. J., and Paul, E., Jr., Quarterly Report Foundational Res. Projects, NAVORD Report 5970, NOLC Report 473, 57 (1959). W

Kaufman, V., and Sugar, J., J. Opt. Soc. Am. 63, 1168 (1973). EL ND CL W ZE IP

King, A. S., Astrophys. J. 74, 328 (1931). W

Meggers, W. F., J. Opt. Soc. Am. 37, 988A (1947). EL IP

Meggers, W. F., (Edited by C. E. Moore), J. Res. Nat. Bur. Stand. (U.S.) 71A, 396 (1967). EL CL W ZE IP

Meggers, W. F., and Corliss, C. H., J. Res. Nat. Bur. Stand. (U.S.) 70A, 63 (1966). W ZE

Meggers, W. F., and Scribner, B. F., J. Res. Nat. Bur. Stand. (U.S.) 19, 651 (1937). EL CL W

Parr, A. C., and Inghram, M. G., J. Chem. Phys. 52, 4916 (1970). IP

Racah, G., Lunds Univ. Arssk., Avd. 2, 50, 31 (1954).

Racah, G., J. Opt. Soc. Am. 50, 408 (1960). EL ND ZE

Racah, G., Goldschmidt, Z. B., and Bordarier, Y., unpublished calculations (1965). (Obtained from Goldschmidt, private communication, 1974.) ND PT

Spector, N., J. Opt. Soc. Am. 58, 837 (1968). EL ND ZE PT

[October 1976] 
Yb II, Even Parity

\begin{tabular}{|c|c|c|c|c|c|c|c|c|}
\hline Configuration & Term & $J$ & $\begin{array}{l}\text { Level } \\
\left(\mathrm{cm}^{-1}\right)\end{array}$ & $g$ & & & \multicolumn{2}{|c|}{ Leading percentages } \\
\hline $4 f^{14}\left({ }^{1} \mathrm{~S}\right) 6 s$ & ${ }^{2} \mathrm{~S}$ & $1 / 2$ & 0.00 & 1.998 & & & & \\
\hline \multirow[t]{2}{*}{$4 f^{14}\left({ }^{1} \mathrm{~S}\right) 5 d$} & ${ }^{2} \mathrm{D}$ & $3 / 2$ & 22960.80 & 1.802 & & & & \\
\hline & & $5 / 2$ & 24332.69 & 1.202 & & & & \\
\hline $4 f^{13}\left({ }^{2} \mathrm{~F}_{7 / 2}^{\circ}\right) 6 s 6 p\left({ }^{3} \mathrm{P}_{0}^{\circ}\right)$ & $(7 / 2,0)$ & $7 / 2$ & 47912.31 & 1.280 & 85 & & 10 & $\left({ }^{2} \mathrm{~F}_{7 / 2}^{\circ}\right)\left({ }^{3} \mathrm{P}_{1}^{\circ}\right)$ \\
\hline \multirow[t]{3}{*}{$4 f^{13}\left({ }^{2} \mathrm{~F}_{7 / 2}^{\circ}\right) 6 s 6 p\left({ }^{3} \mathrm{P}_{1}^{\circ}\right)$} & $(7 / 2,1)$ & $7 / 2$ & 48900.41 & 1.0 & 82 & & 11 & $\left({ }^{2} \mathrm{~F}_{7 / 2}^{\circ}\right)\left({ }^{3} \mathrm{P}_{0}^{\circ}\right)$ \\
\hline & & $9 / 2$ & 49301.16 & 1.187 & 89 & & 5 & $\left({ }^{2} \mathrm{~F}_{\mathrm{T} / 2}^{\circ}\right)\left({ }^{1} \mathrm{P}_{\mathrm{i}}^{\circ}\right)$ \\
\hline & & $5 / 2$ & 49498.04 & 1.145 & 83 & & 6 & $\left({ }^{2} \mathrm{~F}_{7 / 2}^{\circ}\right)\left({ }^{1} \mathrm{P}_{1}^{\circ}\right)$ \\
\hline \multirow[t]{5}{*}{$4 f^{13}\left({ }^{2} \mathrm{~F}_{7 / 2}^{\circ}\right) 6 s 6 p\left({ }^{3} \mathrm{P}_{2}^{\circ}\right)$} & $(7 / 2,2)$ & $11 / 2$ & 52517.35 & 1.27 & 95 & & 4 & $\left({ }^{2} \mathrm{~F}_{7 / 2}^{\circ}\right) 5 d 6 p\left({ }^{3} \mathrm{P}_{2}^{\circ}\right)$ \\
\hline & & $3 / 2$ & 52938.01 & 0.877 & 95 & & 4 & $\left({ }^{2} \mathrm{~F}_{7 / 2}^{\circ}\right) 5 d 6 p\left({ }^{3} \mathrm{P}_{2}^{\circ}\right)$ \\
\hline & & $5 / 2$ & 53404.96 & 1.033 & 89 & & 5 & $\left({ }^{2} \mathrm{~F}_{7 / 2}^{\circ}\right)\left({ }^{3} \mathrm{P}_{1}^{\circ}\right)$ \\
\hline & & $7 / 2$ & 53715.26 & 1.181 & 93 & & 4 & $\left({ }^{2} \mathrm{~F}_{7 / 2}^{\circ}\right) 5 d 6 p\left({ }^{3} \mathrm{P}_{2}^{\circ}\right)$ \\
\hline & & $9 / 2$ & 53720.71 & 1.26 & 95 & & 4 & $\left({ }^{2} \mathrm{~F}_{7 / 2}^{\circ}\right) 5 d 6 p\left({ }^{3} \mathrm{P}_{2}^{\circ}\right)$ \\
\hline $4 f^{14}\left({ }^{1} \mathrm{~S}\right) 7 s$ & ${ }^{2} \mathrm{~S}$ & $1 / 2$ & 54304.30 & 2.001 & & & & \\
\hline \multirow[t]{5}{*}{$4 f^{13}\left({ }^{2} \mathrm{~F}_{7 / 2}^{\circ}\right) 5 d 6 p\left({ }^{3} \mathrm{~F}_{2}^{\circ}\right)$} & $\left({ }^{7} / 2,2\right)$ & $3 / 2$ & 55702.07 & 1.693 & 76 & & 8 & $\left({ }^{2} \mathrm{~F}_{7 / 2}^{\circ}\right)\left({ }^{3} \mathrm{~F}_{3}^{\circ}\right)$ \\
\hline & & $5 / 2$ & 58823.58 & 1.266 & 36 & & 20 & $\left({ }^{2} \mathrm{~F}_{7 / 2}^{\circ}\right) 6 s 6 p\left({ }^{1} \mathrm{P}_{\mathrm{i}}^{\circ}\right)$ \\
\hline & & $11 / 2$ & 58961.37 & 0.974 & 74 & & 21 & $\left({ }^{2} \mathrm{~F}_{7 / 2}^{\circ}\right)\left({ }^{1} \mathrm{D}_{2}^{\circ}\right)$ \\
\hline & & $7 / 2$ & 61051.06 & 1.18 & 64 & & 18 & $\left({ }^{2} \mathrm{~F}_{7 / 2}^{\circ}\right)\left({ }^{1} \mathrm{D}_{2}^{\circ}\right)$ \\
\hline & & $9 / 2$ & 61214.66 & 1.07 & 54 & & 30 & $\left({ }^{2} \mathrm{~F}_{7 / 2}^{\circ}\right)\left({ }^{1} \mathrm{D}_{2}^{\circ}\right)$ \\
\hline $4 f^{13}\left({ }^{2} \mathrm{~F}_{7 / 2}^{\circ}\right) 5 d 6 p\left({ }^{1} \mathrm{P}_{\mathrm{i}}^{\circ}\right)$ & & $5 / 2$ & 56375.91 & 1.226 & 24 & $\left({ }^{7} / 2,1\right)$ & 22 & $\left({ }^{2} \mathrm{~F}_{7 / 2}^{\circ}\right) 6 s 6 p\left({ }^{1} \mathrm{P}_{\mathrm{i}}^{\circ}\right)$ \\
\hline $4 f^{13}\left({ }^{2} \mathrm{~F}_{7 / 2}^{\circ}\right) 6 s 6 p\left({ }^{1} \mathrm{P}_{1}^{\circ}\right)$ & $(7 / 2,1)$ & $9 / 2$ & 57765.32 & 1.12 & 45 & & 36 & $\left({ }^{2} \mathrm{~F}_{7 / 2}^{\circ}\right) 5 d 6 p\left({ }^{1} \mathrm{P}_{1}^{\circ}\right)$ \\
\hline $4 f^{13}\left({ }^{2} \mathrm{~F}_{5 / 2}^{\circ}\right) 6 s 6 p\left({ }^{3} \mathrm{P}_{0}^{\circ}\right)$ & $(5 / 2,0)$ & $5 / 2$ & 58283.91 & 0.77 & 87 & & 5 & $\left({ }^{2} \mathrm{~F}_{5 / 2}^{\circ}\right)\left({ }^{3} \mathrm{P}_{\mathrm{i}}^{\circ}\right)$ \\
\hline $4 f^{13}\left({ }^{2} \mathrm{~F}_{7 / 2}^{\circ}\right) 6 s 6 p\left({ }^{1} \mathrm{P}_{1}^{\circ}\right)$ & & $7 / 2$ & 59090.13 & 1.122 & 36 & $(7 / 2,1)$ & 25 & $\left({ }^{2} \mathrm{~F}_{7 / 2}^{\circ}\right) 5 d 6 p\left({ }^{1} \mathrm{P}_{1}^{\circ}\right)$ \\
\hline \multirow[t]{3}{*}{$4 f^{13}\left({ }^{2} \mathrm{~F}_{5 / 2}^{\circ}\right) 6 s 6 p\left({ }^{3} \mathrm{P}_{1}^{\circ}\right)$} & $(5 / 2,1)$ & $5 / 2$ & 59259.24 & 1.105 & 78 & & 6 & $\left({ }^{2} \mathrm{~F}_{5 / 2}^{\circ}\right)\left({ }^{3} \mathrm{P}_{0}^{\circ}\right)$ \\
\hline & & $7 / 2$ & 59618.90 & 1.00 & 65 & & 15 & $\left({ }^{2} \mathrm{~F}_{7 / 2}^{\circ}\right)\left({ }^{1} \mathrm{P}_{1}^{\circ}\right)$ \\
\hline & & $3 / 2$ & 59710.65 & 0.82 & 82 & & 5 & $\left({ }^{2} \mathrm{~F}_{5 / 2}^{\circ}\right)\left({ }^{1} \mathrm{P}_{i}^{\circ}\right)$ \\
\hline \multirow[t]{7}{*}{$4 f^{13}\left({ }^{2} \mathrm{~F}_{7 / 2}^{\circ}\right) 5 d 6 p\left({ }^{3} \mathrm{~F}_{3}^{\circ}\right)$} & $(7 / 2,3)$ & $3 / 2$ & 60586.46 & 1.323 & 45 & & 33 & $\left({ }^{2} \mathrm{~F}_{7 / 2}^{\circ}\right)\left({ }^{1} \mathrm{D}_{2}^{\circ}\right)$ \\
\hline & & $1 / 2$ & 61120.49 & 1.661 & 75 & & 6 & $\left({ }^{2} \mathrm{~F}_{5 / 2}^{\circ}\right)\left({ }^{3} \mathrm{~F}_{2}^{\circ}\right)$ \\
\hline & & $13 / 2$ & 61873.40 & 1.10 & 91 & & 6 & $\left({ }^{2} \mathrm{~F}_{7 / 2}^{\circ}\right)\left({ }^{3} \mathrm{D}_{3}^{\circ}\right)$ \\
\hline & & $5 / 2$ & 64055.82 & 1.108 & 35 & & 25 & $\left({ }^{2} \mathrm{~F}_{7 / 2}^{\circ}\right)\left({ }^{3} \mathrm{D}_{1}^{\circ}\right)$ \\
\hline & & $9 / 2$ & 64923.11 & 1.10 & 83 & & 6 & $\left({ }^{2} \mathrm{~F}_{7 / 2}^{\circ}\right)\left({ }^{3} \mathrm{P}_{1}^{\circ}\right)$ \\
\hline & & $7 / 2$ & 65093.14 & 0.973 & 37 & & 20 & $\left({ }^{2} \mathrm{~F}_{7 / 2}^{\circ}\right)\left({ }^{3} \mathrm{D}_{2}^{\circ}\right)$ \\
\hline & & $11 / 2$ & 66082.25 & 1.141 & 55 & & 36 & $\left({ }^{2} \mathrm{~F}_{7 / 2}^{\circ}\right)\left({ }^{3} \mathrm{D}_{2}^{\circ}\right)$ \\
\hline $4 f^{13}\left({ }^{2} \mathrm{~F}_{7 / 2}^{\circ}\right) 5 d 6 p\left({ }^{1} \mathrm{D}_{2}^{\circ}\right)$ & & $5 / 2$ & 61374.49 & 1.299 & 32 & $\left({ }^{7} / 2,2\right)$ & 21 & $\left({ }^{2} F_{7 / 2}^{\circ}\right)\left({ }^{3} F_{3}^{\circ}\right)$ \\
\hline $4 f^{13}\left({ }^{2} \mathrm{~F}_{7 / 2}^{\circ}\right) 5 d 6 p\left({ }^{3} \mathrm{D}_{2}^{\circ}\right)$ & & $7 / 2$ & 61822.58 & 1.327 & 23 & $(7 / 2,2)$ & 13 & $\left({ }^{2} \mathrm{~F}_{7 / 2}^{\circ}\right)\left({ }^{1} \mathrm{D}_{2}^{\circ}\right)$ \\
\hline \multirow[t]{5}{*}{$4 f^{13}\left({ }^{2} \mathrm{~F}_{5 / 2}^{\circ}\right) 6 s 6 p\left({ }^{3} \mathrm{P}_{2}^{\circ}\right)$} & $(5 / 2,2)$ & $1 / 2$ & 62136.94 & 0.08 & 90 & & 4 & $\left({ }^{2} \mathrm{~F}_{5 / 2}^{\circ}\right) 5 d 6 p\left({ }^{3} \mathrm{P}_{2}^{\circ}\right)$ \\
\hline & & $3 / 2$ & 63011.85 & 0.79 & 77 & & 5 & $\left({ }^{2} \mathrm{~F}_{5 / 2}^{\circ}\right) 5 d 6 p\left({ }^{3} \mathrm{P}_{2}^{\circ}\right)$ \\
\hline & & $9 / 2$ & 63163.79 & 1.12 & 91 & & 4 & $\left({ }^{2} \mathrm{~F}_{5 / 2}^{\circ}\right) 5 d 6 p\left({ }^{3} \mathrm{P}_{2}^{\circ}\right)$ \\
\hline & & $5 / 2$ & 63702.32 & 1.07 & 84 & & 4 & $\left({ }^{2} \mathrm{~F}_{7 / 2}^{\circ}\right) 5 d 6 p\left({ }^{3} \mathrm{~F}_{3}^{\circ}\right)$ \\
\hline & & $7 / 2$ & 63957.80 & 1.148 & 90 & & 4 & $\left({ }^{2} \mathrm{~F}_{5 / 2}^{\circ}\right) 5 d 6 p\left({ }^{3} \mathrm{P}_{2}^{\circ}\right)$ \\
\hline \multirow[t]{2}{*}{$4 f^{14}\left({ }^{1} \mathrm{~S}\right) 6 d$} & ${ }^{2} \mathrm{D}$ & $3 / 2$ & 62174.10 & 0.80 & & & & \\
\hline & & $5 / 2$ & 62559.02 & 1.22 & & & & \\
\hline $4 f^{13}\left({ }^{2} \mathrm{~F}_{7 / 2}^{\circ}\right) 5 d 6 p\left({ }^{1} \mathrm{D}_{2}^{\circ}\right)$ & & $11 / 2$ & 62861.27 & 1.140 & 44 & $(7 / 2,2)$ & 18 & $\left({ }^{2} \mathrm{~F}_{7 / 2}^{\circ}\right)\left({ }^{3} \mathrm{P}_{2}^{\circ}\right)$ \\
\hline \multirow[t]{3}{*}{$4 f^{13}\left({ }^{2} \mathrm{~F}_{7 / 2}^{\circ}\right) 5 d 6 p\left({ }^{3} \mathrm{D}_{i}^{\circ}\right)$} & $(7 / 2,1)$ & $5 / 2$ & 63234.11 & 1.208 & 31 & & 24 & $\left({ }^{2} \mathrm{~F}_{7 / 2}^{\circ}\right)\left({ }^{3} \mathrm{~F}_{2}^{\circ}\right)$ \\
\hline & & $9 / 2$ & 63726.83 & 1.005 & 68 & & 14 & $\left({ }^{2} \mathrm{~F}_{7 / 2}^{\circ}\right)\left({ }^{1} \mathrm{D}_{2}^{\circ}\right)$ \\
\hline & & $7 / 2$ & 64598.28 & 1.223 & 44 & & 19 & $\left({ }^{2} F_{7 / 2}\right)\left({ }^{3} F_{3}^{\circ}\right)$ \\
\hline $4 f^{13}\left({ }^{2} \mathrm{~F}_{7 / 2}^{\circ}\right) 5 d 6 p\left({ }^{1} \mathrm{D}_{2}^{\circ}\right)$ & & $3 / 2$ & 63647.73 & 1.149 & 29 & $(7 / 2,2)$ & 15 & $\left({ }^{2} \mathrm{~F}_{7 / 2}^{\circ}\right)\left({ }^{3} \mathrm{~F}_{3}^{\circ}\right)$ \\
\hline
\end{tabular}


Yb II, Even Parity-Continued

\begin{tabular}{|c|c|c|c|c|c|c|c|c|c|}
\hline Configuration & Term & $J$ & $\begin{array}{l}\text { Level } \\
\left(\mathrm{cm}^{-1}\right)\end{array}$ & $g$ & \multicolumn{5}{|c|}{ Leading percentages } \\
\hline $4 f^{13}\left({ }^{2} \mathrm{~F}_{7 / 2}^{\circ}\right) 5 d 6 p\left({ }^{3} \mathrm{D}_{2}^{\circ}\right)$ & $(7 / 2,2)$ & $\begin{array}{l}11 / 2 \\
3 / 2 \\
9 / 2 \\
5 / 2 \\
7 / 2\end{array}$ & $\begin{array}{l}63944.18 \\
64461.08 \\
65950.95 \\
66351.21 \\
66558.00\end{array}$ & $\begin{array}{l}1.115 \\
1.176 \\
1.09 \\
1.125 \\
1.100\end{array}$ & $\begin{array}{l}33 \\
51 \\
65 \\
72 \\
30\end{array}$ & & $\begin{array}{r}33 \\
10 \\
14 \\
7 \\
24\end{array}$ & $\begin{array}{l}\left({ }^{2} \mathrm{~F}_{7 / 2}^{\circ}\right)\left({ }^{3} \mathrm{~F}_{3}^{\circ}\right) \\
\left({ }^{2} \mathrm{~F}_{7 / 2}^{\circ}\right)\left({ }^{3} \mathrm{~F}_{3}^{\circ}\right) \\
\left({ }^{2} \mathrm{~F}_{7 / 2}^{\circ}\right)\left({ }^{1} \mathrm{D}_{2}^{\circ}\right) \\
\left({ }^{2} \mathrm{~F}_{7 / 2}^{\circ}\right)\left({ }^{3} \mathrm{~F}_{3}^{\circ}\right) \\
\left({ }^{2} \mathrm{~F}_{7 / 2}^{\circ}\right)\left({ }^{3} \mathrm{D}_{3}^{\circ}\right)\end{array}$ & \\
\hline $4 f^{13}\left({ }^{2} \mathrm{~F}_{7 / 2}^{\circ}\right) 5 d 6 p\left({ }^{3} \mathrm{~F}_{4}^{\circ}\right)$ & $(7 / 2,4)$ & $\begin{array}{c}15 / 2 \\
3 / 2 \\
5 / 2 \\
1 / 2 \\
11 / 2 \\
7 / 2 \\
9 / 2 \\
13 / 2\end{array}$ & $\begin{array}{l}64891.30 \\
65888.46 \\
66395.72 \\
66462.86 \\
68148.88 \\
68450.11 \\
68549.23 \\
68720.44\end{array}$ & $\begin{array}{l}1.19 \\
1.206 \\
1.235 \\
1.11 \\
1.20 \\
1.08 \\
1.157 \\
1.157\end{array}$ & $\begin{array}{r}100 \\
45 \\
39 \\
68 \\
59 \\
48 \\
56 \\
55\end{array}$ & & $\begin{array}{r}9 \\
20 \\
12 \\
25 \\
24 \\
13 \\
40\end{array}$ & $\begin{array}{l}\left({ }^{2} \mathrm{~F}_{7 / 2}^{\circ}\right)\left({ }^{1} \mathrm{D}_{2}^{\circ}\right) \\
\left({ }^{2} \mathrm{~F}_{7 / 2}^{\circ}\right)\left({ }^{1} \mathrm{D}_{2}^{\circ}\right) \\
\left({ }^{2} \mathrm{~F}_{5 / 2}^{\circ}\right)\left({ }^{3} \mathrm{~F}_{3}^{\circ}\right) \\
\left({ }^{2} \mathrm{~F}_{7 / 2}^{\circ}\right)\left({ }^{3} \mathrm{D}_{3}^{\circ}\right) \\
\left({ }^{2} \mathrm{~F}_{7 / 2}^{\circ}\right)\left({ }^{3} \mathrm{P}_{1}^{\circ}\right) \\
\left({ }^{2} \mathrm{~F}_{5 / 2}^{\circ}\right)\left({ }^{3} \mathrm{~F}_{2}^{\circ}\right) \\
\left({ }^{2} \mathrm{~F}_{7 / 2}^{\circ}\right)\left({ }^{1} \mathrm{~F}_{3}^{\circ}\right)\end{array}$ & \\
\hline $4 f^{13}\left({ }^{2} \mathrm{~F}_{7 / 2}^{\circ}\right) 5 d 6 p\left({ }^{3} \mathrm{~F}_{2}^{\circ}\right)$ & & $9 / 2$ & 65199.54 & 1.08 & 34 & $(7 / 2,2)$ & 26 & $\left({ }^{2} \mathrm{~F}_{7 / 2}^{\circ}\right)\left({ }^{1} \mathrm{D}_{2}^{\circ}\right)$ & \\
\hline $4 f^{13}\left({ }^{2} \mathrm{~F}_{7 / 2}^{\circ}\right) 5 d 6 p\left({ }^{1} \mathrm{~F}_{3}^{\circ}\right)$ & $(7 / 2,3)$ & $\begin{array}{c}1 / 2 \\
3 / 2 \\
13 / 2 \\
5 / 2 \\
7 / 2 \\
9 / 2 \\
11 / 2\end{array}$ & $\begin{array}{l}65566.72 \\
69443.52 \\
70136.24 \\
71468.70 \\
72779.99 \\
73283.35 \\
73291.95\end{array}$ & $\begin{array}{l}1.43 \\
1.15 \\
1.12 \\
1.02 \\
1.10 \\
1.04 \\
1.09\end{array}$ & $\begin{array}{l}54 \\
27 \\
46 \\
33 \\
67 \\
49 \\
75\end{array}$ & & $\begin{array}{r}19 \\
18 \\
27 \\
19 \\
11 \\
24 \\
9\end{array}$ & $\begin{array}{l}\left({ }^{2} \mathrm{~F}_{5 / 2}^{\circ}\right)\left({ }^{3} \mathrm{~F}_{2}^{\circ}\right) \\
\left({ }^{2} \mathrm{~F}_{7 / 2}^{\circ}\right)\left({ }^{3} \mathrm{D}_{3}^{\circ}\right) \\
\left({ }^{2} \mathrm{~F}_{7 / 2}^{\circ}\right)\left({ }^{3} \mathrm{~F}_{4}^{\circ}\right) \\
\left({ }^{2} \mathrm{~F}_{5 / 2}^{\circ}\right)\left({ }^{3} \mathrm{~F}_{2}^{\circ}\right) \\
\left({ }^{2} \mathrm{~F}_{7 / 2}^{\circ}\right)\left({ }^{3} \mathrm{~F}_{3}^{\circ}\right) \\
\left({ }^{2} \mathrm{~F}_{5 / 2}^{\circ}\right)\left({ }^{1} \mathrm{D}_{2}^{\circ}\right) \\
\left({ }^{2} \mathrm{~F}_{7 / 2}^{\circ}\right)\left({ }^{3} \mathrm{D}_{3}^{\circ}\right)\end{array}$ & \\
\hline $4 f^{13}\left({ }^{2} \mathrm{~F}_{7 / 2}^{\circ}\right) 5 d 6 p\left({ }^{1} \mathrm{D}_{2}^{\circ}\right)$ & & $7 / 2$ & 65577.11 & 1.055 & 38 & $(7 / 2,2)$ & 18 & $\left({ }^{2} \mathrm{~F}_{7 / 2}^{\circ}\right)\left({ }^{3} \mathrm{~F}_{2}^{\circ}\right)$ & \\
\hline $4 f^{13}\left({ }^{2} \mathrm{~F}_{7 / 2}^{\circ}\right) 5 d 6 p\left({ }^{3} \mathrm{D}_{3}^{\circ}\right)$ & $(7 / 2,3)$ & $\begin{array}{c}13 / 2 \\
11 / 2 \\
3 / 2 \\
9 / 2 \\
1 / 2\end{array}$ & $\begin{array}{l}65875.77 \\
66571.58 \\
67204.97 \\
67416.03 \\
67955.23\end{array}$ & $\begin{array}{l}1.205 \\
1.189 \\
1.04 \\
1.26 \\
0.895\end{array}$ & $\begin{array}{l}68 \\
55 \\
29 \\
52 \\
68\end{array}$ & & $\begin{array}{l}15 \\
19 \\
25 \\
21 \\
14\end{array}$ & $\begin{array}{l}\left({ }^{2} \mathrm{~F}_{7 / 2}^{\circ}\right)\left({ }^{3} \mathrm{~F}_{4}^{\circ}\right) \\
\left({ }^{2} \mathrm{~F}_{7 / 2}^{\circ}\right)\left({ }^{3} \mathrm{P}_{2}^{\circ}\right) \\
\left({ }^{2} \mathrm{~F}_{7 / 2}^{\circ}\right)\left({ }^{1} \mathrm{~F}_{3}^{\circ}\right) \\
\left({ }^{2} \mathrm{~F}_{7 / 2}^{\circ}\right)\left({ }^{3} \mathrm{P}_{1}^{\circ}\right) \\
\left({ }^{2} \mathrm{~F}_{7 / 2}^{\circ}\right)\left({ }^{1} \mathrm{~F}_{3}^{\circ}\right)\end{array}$ & \\
\hline $4 f^{13}\left({ }^{2} \mathrm{~F}_{5}^{\circ} / 2\right) 6 s 6 p\left({ }^{1} \mathrm{P}_{1}^{\circ}\right)$ & & $7 / 2$ & 67938.68 & 0.960 & 30 & $(5 / 2,1)$ & 24 & $\left({ }^{2} \mathrm{~F}_{5}^{\circ} / 2\right) 5 d 6 p\left({ }^{1} \mathrm{P}_{1}^{\circ}\right)$ & \\
\hline $4 f^{13}\left({ }^{1} \mathrm{~F}_{7 / 2}^{\circ}\right) 5 d 6 p\left({ }^{3} \mathrm{~F}_{4}^{\circ}\right)$ & & $5 / 2$ & 68135.00 & 1.087 & 38 & $(7 / 2,4)$ & 24 & $\left({ }^{2} \mathrm{~F}_{7 / 2}^{\circ}\right)\left({ }^{3} \mathrm{D}_{3}^{\circ}\right)$ & \\
\hline $4 f^{13}\left({ }^{2} \mathrm{~F}_{5}^{\circ} / 2\right) 6 s 6 p\left({ }^{1} \mathrm{P}_{1}^{\circ}\right)$ & & $3 / 2$ & 68150.57 & 0.928 & 32 & $(5 / 2,1)$ & 20 & $\left({ }^{2} \mathrm{~F}_{5 / 2}^{\circ}\right) 5 d 6 p\left({ }^{1} \mathrm{P}_{1}^{\circ}\right)$ & \\
\hline $4 f^{13}\left({ }^{2} \mathrm{~F}_{7 / 2}^{\circ}\right) 5 d 6 p\left({ }^{3} \mathrm{P}_{0}^{\circ}\right)$ & & $7 / 2$ & 68756.04 & 1.32 & 35 & $(7 / 2,0)$ & 29 & $\left({ }^{2} \mathrm{~F}_{7 / 2}^{\circ}\right)\left({ }^{3} \mathrm{D}_{3}^{\circ}\right)$ & \\
\hline $4 f^{13}\left({ }^{2} \mathrm{~F}_{5 / 2}^{\circ}\right) 5 d 6 p\left({ }^{3} \mathrm{~F}_{2}^{\circ}\right)$ & $(5 / 2,2)$ & $\begin{array}{l}9 / 2 \\
5 / 2 \\
7 / 2 \\
3 / 2 \\
1 / 2\end{array}$ & $\begin{array}{l}68943.65 \\
71270.01 \\
72202.03 \\
73116.71 \\
75905.77\end{array}$ & $\begin{array}{l}0.89 \\
0.91 \\
0.86 \\
0.863 \\
1.28\end{array}$ & $\begin{array}{l}53 \\
33 \\
43 \\
34 \\
40\end{array}$ & & $\begin{array}{l}18 \\
18 \\
29 \\
23 \\
20\end{array}$ & $\begin{array}{l}\left({ }^{2} \mathrm{~F}_{7 / 2}^{\circ}\right)\left({ }^{3} \mathrm{~F}_{4}^{\circ}\right) \\
\left({ }^{2} \mathrm{~F}_{7 / 2}^{\circ}\right)\left({ }^{3} \mathrm{P}_{2}^{\circ}\right) \\
\left({ }^{2} \mathrm{~F}_{5 / 2}^{\circ}\right)\left({ }^{1} \mathrm{D}_{2}^{\circ}\right) \\
\left({ }^{2} \mathrm{~F}_{5 / 2}^{\circ}\right)\left({ }^{3} \mathrm{~F}_{3}^{\circ}\right) \\
\left({ }^{2} \mathrm{~F}_{7 / 2}^{\circ}\right)\left({ }^{1} \mathrm{~F}_{3}^{\circ}\right)\end{array}$ & 、 \\
\hline $4 f^{13}\left({ }^{2} \mathrm{~F}^{\circ} / 2\right) 6 s 6 p\left({ }^{1} \mathrm{P}_{1}^{\circ}\right)$ & & $5 / 2$ & 69079.88 & 0.89 & 30 & $(5 / 2,1)$ & 21 & $\left({ }^{2} \mathrm{~F}_{5 / 2}^{\circ}\right) 5 d 6 p\left({ }^{1} \mathrm{P}_{1}^{\circ}\right)$ & \\
\hline $4 f^{13}\left({ }^{2} \mathrm{~F}_{7 / 2}^{\circ}\right) 5 d 6 p\left({ }^{3} \mathrm{P}_{0}^{\circ}\right)$ & & $7 / 2$ & 69395.20 & 0.94 & 25 & $(7 / 2,0)$ & 21 & $\left({ }^{2} \mathrm{~F}_{7 / 2}^{\circ}\right)\left({ }^{3} \mathrm{P}_{1}^{\circ}\right)$ & \\
\hline $4 f^{13}\left({ }^{2} \mathrm{~F}_{7 / 2}^{\circ}\right) 5 d 6 p\left({ }^{3} \mathrm{P}_{1}^{\circ}\right)$ & & $5 / 2$ & 69607.54 & 1.210 & 24 & $(7 / 2,1)$ & 18 & $\left({ }^{2} \mathrm{~F}_{7 / 2}^{\circ}\right)\left({ }^{3} \mathrm{P}_{2}^{\circ}\right)$ & \\
\hline $4 f^{13}\left({ }^{2} \mathrm{~F}_{7 / 2}^{\circ}\right) 5 d 6 p\left({ }^{3} \mathrm{P}_{1}^{\circ}\right)$ & & $9 / 2$ & 69957.49 & 1.112 & 34 & $(7 / 2,1)$ & 18 & $\left({ }^{3} \mathrm{~F}_{7 / 2}^{\circ}\right)\left({ }^{3} \mathrm{D}_{3}^{\circ}\right)$ & \\
\hline $4 f^{13}\left({ }^{2} \mathrm{~F}_{5 / 2}^{\circ}\right) 5 d 6 p\left({ }^{3} \mathrm{~F}_{3}^{\circ}\right)$ & $(5 / 2,3)$ & $\begin{array}{c}1 / 2 \\
11 / 2 \\
3 / 2 \\
5 / 2 \\
7 / 2 \\
9 / 2\end{array}$ & $\begin{array}{l}70029.04 \\
72615.74 \\
73657.29 \\
74270.48 \\
75640.06 \\
76516.11\end{array}$ & $\begin{array}{l}0.68 \\
0.99 \\
1.04 \\
0.92 \\
0.81 \\
1.01\end{array}$ & $\begin{array}{l}28 \\
85 \\
32 \\
72 \\
51 \\
46\end{array}$ & & $\begin{array}{r}19 \\
4 \\
25 \\
7 \\
7 \\
19 \\
27\end{array}$ & $\begin{array}{l}\left({ }^{2} \mathrm{~F}_{5 / 2}^{\circ}\right)\left({ }^{1} \mathrm{D}_{2}^{\circ}\right) \\
\left({ }^{2} \mathrm{~F}_{7 / 2}^{\circ}\right)\left({ }^{1} \mathrm{~F}_{3}^{\circ}\right) \\
\left({ }^{2} \mathrm{~F}_{5 / 2}^{\circ}\right)\left({ }^{3} \mathrm{~F}_{4}^{\circ}\right) \\
\left({ }^{2} \mathrm{~F}_{5 / 2}^{\circ}\right)\left({ }^{3} \mathrm{D}_{3}^{\circ}\right) \\
\left({ }^{2} \mathrm{~F}_{5 / 2}^{\circ}\right)\left({ }^{3} \mathrm{~F}_{2}^{\circ}\right) \\
\left({ }^{2} \mathrm{~F}_{5 / 2}^{\circ}\right)\left({ }^{3} \mathrm{D}_{2}^{\circ}\right)\end{array}$ & \\
\hline $4 f^{13}\left({ }^{2} \mathrm{~F}_{7 / 2}^{\circ}\right) 5 d 6 p\left({ }^{3} \mathrm{P}_{2}^{\circ}\right)$ & $(7 / 2,2)$ & $\begin{array}{l}11 / 2 \\
9 / 2 \\
3 / 2 \\
7 / 2\end{array}$ & $\begin{array}{l}70793.96 \\
70858.18 \\
71113.84 \\
71222.36\end{array}$ & $\begin{array}{l}1.20 \\
1.28 \\
0.967 \\
1.15\end{array}$ & $\begin{array}{l}41 \\
70 \\
22 \\
52\end{array}$ & & $\begin{array}{l}28 \\
13 \\
21 \\
16\end{array}$ & $\begin{array}{l}\left({ }^{2} \mathrm{~F}_{7 / 2}^{\circ}\right)\left({ }^{3} \mathrm{~F}_{4}^{\circ}\right) \\
\left({ }^{2} \mathrm{~F}_{7 / 2}^{\circ}\right)\left({ }^{3} \mathrm{P}_{1}^{\circ}\right) \\
\left({ }^{2} \mathrm{~F}_{5 / 2}^{\circ}\right)\left({ }^{3} \mathrm{~F}_{2}^{\circ}\right) \\
\left({ }^{2} \mathrm{~F}_{7 / 2}^{\circ}\right)\left({ }^{1} \mathrm{~F}_{3}^{\circ}\right)\end{array}$ & \\
\hline
\end{tabular}


Yb II, Even Parity-Continued

\begin{tabular}{|c|c|c|c|c|c|c|c|c|}
\hline Configuration & Term & $J$ & $\begin{array}{l}\text { Level } \\
\left(\mathrm{cm}^{-1}\right)\end{array}$ & $g$ & \multicolumn{4}{|c|}{ Leading percentages } \\
\hline $4 f^{13}\left({ }^{2} \mathrm{~F}_{7 / 2}^{\circ}\right) 5 d 6 p\left({ }^{3} \mathrm{P}_{1}^{\circ}\right)$ & \multirow{5}{*}{${ }^{2} \mathrm{~S}$} & $5 / 2$ & 70954.27 & 1.014 & 27 & $(7 / 2,1)$ & 20 & $\left({ }^{3} \mathrm{~F}_{7 / 2}^{\circ}\right)\left({ }^{3} \mathrm{P}_{2}^{\circ}\right)$ \\
\hline $4 f^{13}\left({ }^{2} \mathrm{~F}_{5 / 2}^{\circ}\right) 5 d 6 p\left({ }^{1} \mathrm{D}_{2}^{\circ}\right)$ & & $3 / 2$ & 71763.67 & 1.11 & 20 & $(5 / 2,2)$ & 18 & $\left({ }^{2} \mathrm{~F}_{5 / 2}^{\circ}\right)\left({ }^{3} \mathrm{~F}_{4}^{\circ}\right)$ \\
\hline $4 f^{14}\left({ }^{1} \mathrm{~S}\right) 8 s$ & & $1 / 2$ & 73039.61 & & & & & \\
\hline $4 f^{13}\left({ }^{2} \mathrm{~F}_{5 / 2}^{\circ}\right) 5 d 6 p\left({ }^{1} \mathrm{D}_{2}^{\circ}\right)$ & & $1 / 2$ & 73636.83 & 0.70 & 24 & $(5 / 2,1)$ & 21 & $\left({ }^{2} \mathrm{~F}_{5 / 2}^{\circ}\right)\left({ }^{3} \mathrm{D}_{2}^{\circ}\right)$ \\
\hline $4 f^{13}\left({ }^{2} \mathrm{~F}_{5 / 2}^{\circ}\right) 5 d 6 p\left({ }^{1} \mathrm{D}_{2}^{\circ}\right)$ & & $9 / 2$ & 73750.90 & 1.07 & 39 & $(5 / 2,2)$ & 31 & $\left({ }^{2} \mathrm{~F}_{5 / 2}^{\circ}\right)\left({ }^{1} \mathrm{~F}_{3}^{\circ}\right)$ \\
\hline \multirow[t]{3}{*}{$4 f^{13}\left({ }^{2} \mathrm{~F}_{5 / 2}^{\circ}\right) 5 d 6 p\left({ }^{3} \mathrm{D}_{1}^{\circ}\right)$} & \multirow[t]{3}{*}{$(5 / 2,1)$} & $7 / 2$ & 73966.81 & 0.763 & 63 & & 16 & $\left({ }^{2} \mathrm{~F}_{5 / 2}^{\circ}\right)\left({ }^{1} \mathrm{D}_{2}^{\circ}\right)$ \\
\hline & & $5 / 2$ & 75058.11 & 0.76 & 53 & & 8 & $\left({ }^{2} \mathrm{~F}_{5 / 2}^{\circ}\right)\left({ }^{3} \mathrm{~F}_{2}^{\circ}\right)$ \\
\hline & & $3 / 2$ & 77284.02 & 0.90 & 41 & & 12 & $\left({ }^{2} \mathrm{~F}_{5 / 2}^{\circ}\right)\left({ }^{3} \mathrm{D}_{3}^{\circ}\right)$ \\
\hline $4 f^{13}\left({ }^{2} \mathrm{~F}_{5 / 2}^{\circ}\right) 5 d 6 p\left({ }^{3} \mathrm{D}_{2}^{\circ}\right)$ & \multirow[t]{4}{*}{$(5 / 2,2)$} & $9 / 2$ & 74568.58 & 1.04 & 55 & & 25 & $\left({ }^{2} \mathrm{~F}_{5 / 2}^{\circ}\right)\left({ }^{3} \mathrm{~F}_{3}^{\circ}\right)$ \\
\hline $4 f^{13}\left({ }^{2} \mathrm{~F}_{5 / 2}^{\circ}\right) 5 d 6 p\left({ }^{3} \mathrm{~F}_{3}^{\circ}\right)$ & & $7 / 2$ & 74973.41 & 1.07 & 35 & $(5 / 2,3)$ & 26 & $\left({ }^{2} \mathrm{~F}_{5 / 2}^{\circ}\right)\left({ }^{3} \mathrm{~F}_{2}^{\circ}\right)$ \\
\hline $4 f^{13}\left({ }^{2} \mathrm{~F}_{5 / 2}^{\circ}\right) 5 d 6 p\left({ }^{1} \mathrm{D}_{2}^{\circ}\right)$ & & $1 / 2$ & 74989.49 & 0.52 & 35 & $(5 / 2,2)$ & 27 & $\left({ }^{2} \mathrm{~F}_{5 / 2}^{\circ}\right)\left({ }^{3} \mathrm{D}_{2}^{\circ}\right)$ \\
\hline $4 f^{13}\left({ }^{2} \mathrm{~F}_{5 / 2}^{\circ}\right) 5 d 6 p\left({ }^{3} \mathrm{D}_{2}^{\circ}\right)$ & & $3 / 2$ & 74991.36 & 1.05 & 35 & $(5 / 2,2)$ & 19 & $\left({ }^{2} \mathrm{~F}_{5 / 2}^{\circ}\right)\left({ }^{1} \mathrm{D}_{2}^{\circ}\right)$ \\
\hline $4 f^{13}\left({ }^{2} \mathrm{~F}_{5 / 2}^{\circ}\right) 5 d 6 p\left({ }^{1} \mathrm{D}_{2}^{\circ}\right)$ & $(5 / 2,2)$ & $5 / 2$ & 75550.94 & 0.91 & 45 & & 18 & $\left({ }^{2} \mathrm{~F}_{5 / 2}^{\circ}\right)\left({ }^{3} \mathrm{D}_{2}^{\circ}\right)$ \\
\hline \multirow[t]{5}{*}{$4 f^{13}\left({ }^{2} \mathrm{~F}_{5 / 2}^{\circ}\right) 5 d 6 p\left({ }^{3} \mathrm{~F}_{4}^{\circ}\right)$} & \multirow[t]{5}{*}{$(5 / 2,4)$} & $5 / 2$ & 76170.25 & 1.12 & 35 & & 24 & $\left({ }^{2} \mathrm{~F}_{5 / 2}^{\circ}\right)\left({ }^{3} \mathrm{D}_{2}^{\circ}\right)$ \\
\hline & & $13 / 2$ & 76233.60 & 1.07 & 98 & & 1 & $\left({ }^{2} \mathrm{~F}_{7 / 2}^{\circ}\right)\left({ }^{3} \mathrm{~F}_{4}^{\circ}\right)$ \\
\hline & & $7 / 2$ & 77882.04 & 1.13 & 71 & & 7 & $\left({ }^{2} \mathrm{~F}_{5 / 2}^{\circ}\right)\left({ }^{3} \mathrm{P}_{1}^{\circ}\right)$ \\
\hline & & $9 / 2$ & 79126.83 & $1.15 ?$ & 71 & & 15 & $\left({ }^{2} \mathrm{~F}_{5 / 2}^{\circ}\right)\left({ }^{3} \mathrm{D}_{3}^{\circ}\right)$ \\
\hline & & $11 / 2$ & & & 74 & & 21 & $\left({ }^{2} \mathrm{~F}_{5 / 2}^{\circ}\right)\left({ }^{1} \mathrm{~F}_{3}^{\circ}\right)$ \\
\hline $4 f^{13}\left({ }^{2} \mathrm{~F}_{5 / 2}^{\circ}\right) 5 d 6 p\left({ }^{3} \mathrm{D}_{2}^{\circ}\right)$ & $(5 / 2,2)$ & $7 / 2$ & 76323.49 & 1.00 & 56 & & 18 & $\left({ }^{2} \mathrm{~F}_{5 / 2}^{\circ}\right)\left({ }^{1} \mathrm{D}_{2}^{\circ}\right)$ \\
\hline \multirow[t]{2}{*}{$4 f^{14}\left({ }^{1} \mathrm{~S}\right) 7 d ?$} & \multirow[t]{2}{*}{${ }^{2} \mathrm{D} ?$} & $3 / 2$ & 76517.21 & 0.85 & & & & \\
\hline & & $5 / 2$ & 76676.31 & 1.10 & & & & \\
\hline \multirow[t]{5}{*}{$4 f^{13}\left({ }^{2} \mathrm{~F}_{5 / 2}^{\circ}\right) 5 d 6 p\left({ }^{3} \mathrm{D}_{3}^{\circ}\right)$} & \multirow[t]{8}{*}{$(5 / 2,3)$} & $11 / 2$ & 76649.80 & 1.08 & 65 & & 20 & $\left({ }^{2} \mathrm{~F}_{5 / 2}^{\circ}\right)\left({ }^{1} \mathrm{~F}_{3}^{\circ}\right)$ \\
\hline & & $7 / 2$ & 77449.32 & 1.16 & 55 & & 12 & $\left({ }^{2} \mathrm{~F}_{5 / 2}^{\circ}\right)\left({ }^{3} \mathrm{D}_{2}^{\circ}\right)$ \\
\hline & & $9 / 2$ & 77747.40 & 1.07 & 41 & & 19 & $\left({ }^{2} \mathrm{~F}_{5 / 2}^{\circ}\right)\left({ }^{3} \mathrm{P}_{2}^{\circ}\right)$ \\
\hline & & $1 / 2$ & 77887.30 & 2.20 & 69 & & 12 & $\left({ }^{2} \mathrm{~F}_{5 / 2}^{\circ}\right)\left({ }^{3} \mathrm{~F}_{3}^{\circ}\right)$ \\
\hline & & $5 / 2$ & 78814.86 & 1.22 & 32 & & 24 & $\left({ }^{2} \mathrm{~F}_{5 / 2}^{\circ}\right)\left({ }^{3} \mathrm{P}_{1}^{\circ}\right)$ \\
\hline $4 f^{13}\left({ }^{2} \mathrm{~F}_{5 / 2}^{\circ}\right) 5 d 6 p\left({ }^{3} \mathrm{D}_{3}^{\circ}\right) ?$ & & $3 / 2$ & 76799.07 & 0.84 & 23 & $(5 / 2,3)$ & 23 & $\left({ }^{2} \mathrm{~F}_{5 / 2}^{\circ}\right)\left({ }^{3} \mathrm{D}_{2}^{\circ}\right)$ \\
\hline $4 f^{13}\left({ }^{2} \mathrm{~F}_{5 / 2}^{\circ}\right) 5 d 6 p\left({ }^{3} \mathrm{D}_{1}^{\circ}\right) ?$ & & $5 / 2$ & 76839.00 & 1.11 & 18 & $(5 / 2,1)$ & 18 & $\left({ }^{2} \mathrm{~F}_{5 / 2}^{\circ}\right)\left({ }^{3} \mathrm{~F}_{4}^{\circ}\right)$ \\
\hline $4 f^{13}\left({ }^{2} \mathrm{~F}_{5 / 2}^{\circ}\right) 5 d 6 p\left({ }^{3} \mathrm{~F}_{4}^{\circ}\right)$ & & $5 / 2$ & 77606.58 & 1.14 & 30 & $(5 / 2,4)$ & 22 & $\left({ }^{2} \mathrm{~F}_{7 / 2}^{\circ}\right)\left({ }^{1} \mathrm{P}_{1}^{\circ}\right)$ \\
\hline \multirow[t]{2}{*}{$4 f^{13}\left({ }^{2} \mathrm{~F}_{7 / 2}^{\circ}\right) 5 d 6 p\left({ }^{1} \mathrm{P}_{\mathrm{i}}^{\circ}\right)$} & \multirow[t]{3}{*}{$(7 / 2,1)$} & $9 / 2$ & 78070.19 & 1.10 & 52 & & 26 & $\left({ }^{2} \mathrm{~F}_{7 / 2}^{\circ}\right) 6 s 6 p\left({ }^{1} \mathrm{P}_{1}^{\circ}\right)$ \\
\hline & & $7 / 2$ & 79628.71 & 1.11 & 55 & & 26 & \\
\hline $4 f^{13}\left({ }^{2} \mathrm{~F}_{5 / 2}^{\circ}\right) 5 d 6 p\left({ }^{1} \mathrm{~F}_{3}^{\circ}\right)$ & & $3 / 2$ & 78464.76 & 1.18 & 23 & $(5 / 2,2)$ & 18 & $\left({ }^{2} \mathrm{~F}_{5 / 2}^{\circ}\right)\left({ }^{3} \mathrm{P}_{2}^{\circ}\right)$ \\
\hline $4 f^{13}\left({ }^{2} \mathrm{~F}_{5 / 2}^{\circ}\right) 5 d 6 p\left({ }^{3} \mathrm{P}_{0}^{\circ}\right)$ & & $5 / 2$ & 79583.11 & 0.68 & 27 & $(5 / 2,0)$ & 20 & $\left({ }^{2} \mathrm{~F}_{5 / 2}^{\circ}\right)\left({ }^{3} \mathrm{P}_{1}^{\circ}\right)$ \\
\hline $4 f^{13}\left({ }^{2} \mathrm{~F}_{5 / 2}^{\circ}\right) 5 d 6 p\left({ }^{3} \mathrm{P}_{\mathrm{i}}^{\circ}\right)$ & & $3 / 2$ & 79605.95 & 1.16 & 29 & $(5 / 2,1)$ & 24 & $\left({ }^{2} \mathrm{~F}_{5 / 2}^{\circ}\right)\left({ }^{3} \mathrm{D}_{3}^{\circ}\right)$ \\
\hline $4 f^{13}\left({ }^{2} \mathrm{~F}_{5 / 2}^{\circ}\right) 5 d 6 p\left({ }^{3} \mathrm{P}_{1}^{\circ}\right)$ & & $7 / 2$ & 80457.71 & 0.99 & 33 & $(5 / 2,1)$ & 19 & $\left({ }^{2} \mathrm{~F}_{5 / 2}^{\circ}\right)\left({ }^{3} \mathrm{D}_{3}^{\circ}\right)$ \\
\hline $4 f^{14}\left({ }^{1} \mathrm{~S}\right) 5 g$ & ${ }^{2} \mathrm{G}$ & $7 / 2,9 / 2$ & 80607.45 & & & & & \\
\hline \multirow[t]{6}{*}{$4 f^{13}\left({ }^{2} \mathrm{~F}_{5 / 2}^{\circ}\right) 5 d 6 p\left({ }^{1} \mathrm{~F}_{3}^{\circ}\right)$} & \multirow[t]{6}{*}{$(5 / 2,3)$} & $11 / 2$ & 80679.18 & $1.00 ?$ & 55 & & 30 & $\left({ }^{2} \mathrm{~F}_{5 / 2}^{\circ}\right)\left({ }^{3} \mathrm{D}_{3}^{\circ}\right)$ \\
\hline & & $1 / 2$ & 82091.32 & & 65 & & 11 & $\left({ }^{2} \mathrm{~F}_{7 / 2}^{\circ}\right)\left({ }^{3} \mathrm{~F}_{4}^{\circ}\right)$ \\
\hline & & $3 / 2$ & 82398.98 & 1.042 & 60 & & 9 & $\left({ }^{2} \mathrm{~F}_{5 / 2}^{\circ}\right)\left({ }^{3} \mathrm{D}_{2}^{\circ}\right)$ \\
\hline & & $5 / 2$ & 82891.80 & $1.03 ?$ & 54 & & 10 & $\left({ }^{2} \mathrm{~F}_{5 / 2}^{\circ}\right)\left({ }^{3} \mathrm{P}_{2}^{\circ}\right)$ \\
\hline & & $\begin{array}{l}7 / 2 \\
9 / 2\end{array}$ & 83363.88 & 0.97 & 86 & & 8 & $\left({ }^{2} \mathrm{~F}_{5 / 2}^{\circ}\right)\left({ }^{3} \mathrm{D}_{3}^{\circ}\right)$ \\
\hline & & $9 / 2$ & & & 85 & & 10 & $\left({ }^{2} \mathrm{~F}_{5 / 2}^{\circ}\right)\left({ }^{3} \mathrm{D}_{3}^{\circ}\right)$ \\
\hline
\end{tabular}


Yb II, Even Parity-Continued

\begin{tabular}{|c|c|c|c|c|c|c|}
\hline Configuration & Term & $J$ & $\begin{array}{l}\text { Level } \\
\left(\mathrm{cm}^{-1}\right)\end{array}$ & $g$ & & Leading percentages \\
\hline $4 f^{13}\left({ }^{2} \mathrm{~F}_{5 / 2}^{\circ}\right) 5 d 6 p\left({ }^{3} \mathrm{P}_{2}^{\circ}\right)$ & $(5 / 2,2)$ & $\begin{array}{l}1 / 2 \\
9 / 2 \\
5 / 2 \\
7 / 2\end{array}$ & $\begin{array}{l}81205.27 \\
81312.65 \\
81692.92\end{array}$ & $\begin{array}{l}1.09 \\
1.11 ? \\
1.16\end{array}$ & $\begin{array}{l}61 \\
54 \\
38 \\
55\end{array}$ & $\begin{array}{ll}12 & \left({ }^{2} \mathrm{~F}_{5 / 2}^{\circ}\right)\left({ }^{3} \mathrm{D}_{2}^{\circ}\right) \\
15 & \left({ }^{2} \mathrm{~F}_{5 / 2}^{\circ}\right)\left({ }^{3} \mathrm{~F}_{4}^{\circ}\right) \\
28 & \left({ }^{2} \mathrm{~F}_{5 / 2}^{\circ}\right)\left({ }^{1} \mathrm{P}_{3}^{\circ}\right) \\
22 & \left({ }^{2} \mathrm{~F}_{5 / 2}^{\circ}\right)\left({ }^{3} \mathrm{P}_{1}^{\circ}\right)\end{array}$ \\
\hline $4 f^{13}\left({ }^{2} \mathrm{~F}_{5 / 2}^{\circ}\right) 5 d 6 p\left({ }^{3} \mathrm{P}_{1}^{\circ}\right)$ & $(5 / 2,1)$ & $3 / 2$ & 81512.62 & 0.48 & 46 & $31 \quad\left({ }^{2} \mathrm{~F}_{5 / 2}^{\circ}\right)\left({ }^{3} \mathrm{P}_{2}^{\circ}\right)$ \\
\hline $4 f^{13}\left({ }^{2} \mathrm{~F}_{5 / 2}^{\circ}\right) 5 d 6 p\left({ }^{3} \mathrm{P}_{0}^{\circ}\right)$ & & $5 / 2$ & 81657.13 & & $31 \quad(5 / 2,0)$ & $17 \quad\left({ }^{2} \mathrm{~F}_{5 / 2}^{\circ}\right)\left({ }^{3} \mathrm{P}_{2}^{\circ}\right)$ \\
\hline $4 f^{14}\left({ }^{1} \mathrm{~S}\right) 8 d$ & ${ }^{2} \mathrm{D}$ & $\begin{array}{l}3 / 2 \\
5 / 2\end{array}$ & $\begin{array}{l}83839.86 \\
84015.98\end{array}$ & 1.20 & & \\
\hline $4 f^{14}\left({ }^{1} \mathrm{~S}\right) 6 g$ & ${ }^{2} \mathrm{G}$ & $7 / 2,9 / 2$ & 85994.92 & & & \\
\hline $4 f^{14}\left({ }^{1} \mathrm{~S}\right) 10 s$ & ${ }^{2} \mathrm{~S}$ & $1 / 2$ & 86768.26 & & & \\
\hline $4 f^{14}\left({ }^{1} \mathrm{~S}\right) 9 d$ & ${ }^{2} \mathrm{D}$ & $\begin{array}{l}3 / 2 \\
5 / 2\end{array}$ & $\begin{array}{l}87804.88 \\
87980.96\end{array}$ & & & \\
\hline $4 f^{13}\left({ }^{2} \mathrm{~F}_{5 / 2}^{\circ}\right) 5 d \grave{o} p\left({ }^{1} \mathrm{P}_{1}^{\circ}\right)$ & $(5 / 2,1)$ & $\begin{array}{l}3 / 2 \\
7 / 2 \\
5 / 2\end{array}$ & 89713.17 & & $\begin{array}{l}62 \\
58 \\
63\end{array}$ & $\begin{array}{ll}31 & \left({ }^{2} \mathrm{~F}_{5 / 2}^{\circ}\right) 6 s 6 p\left({ }^{1} \mathrm{P}_{1}^{\circ}\right) \\
30 & \\
31 & \end{array}$ \\
\hline $4 f^{14}\left({ }^{1} \mathrm{~S}\right) 10 d$ & ${ }^{2} \mathrm{D}$ & $\begin{array}{l}3 / 2 \\
5 / 2\end{array}$ & $\begin{array}{l}90414.35 \\
90519.48\end{array}$ & & & \\
\hline $4 f^{14}\left({ }^{1} \mathrm{~S}\right) 11 d$ & ${ }^{2} \mathrm{D}$ & $5 / 2$ & 92219.53 & & & \\
\hline $\mathrm{Yb}$ III $\left({ }^{1} \mathrm{So}\right)$ & Limit & & 98269 & & & \\
\hline
\end{tabular}

Yb II, Odd Parity

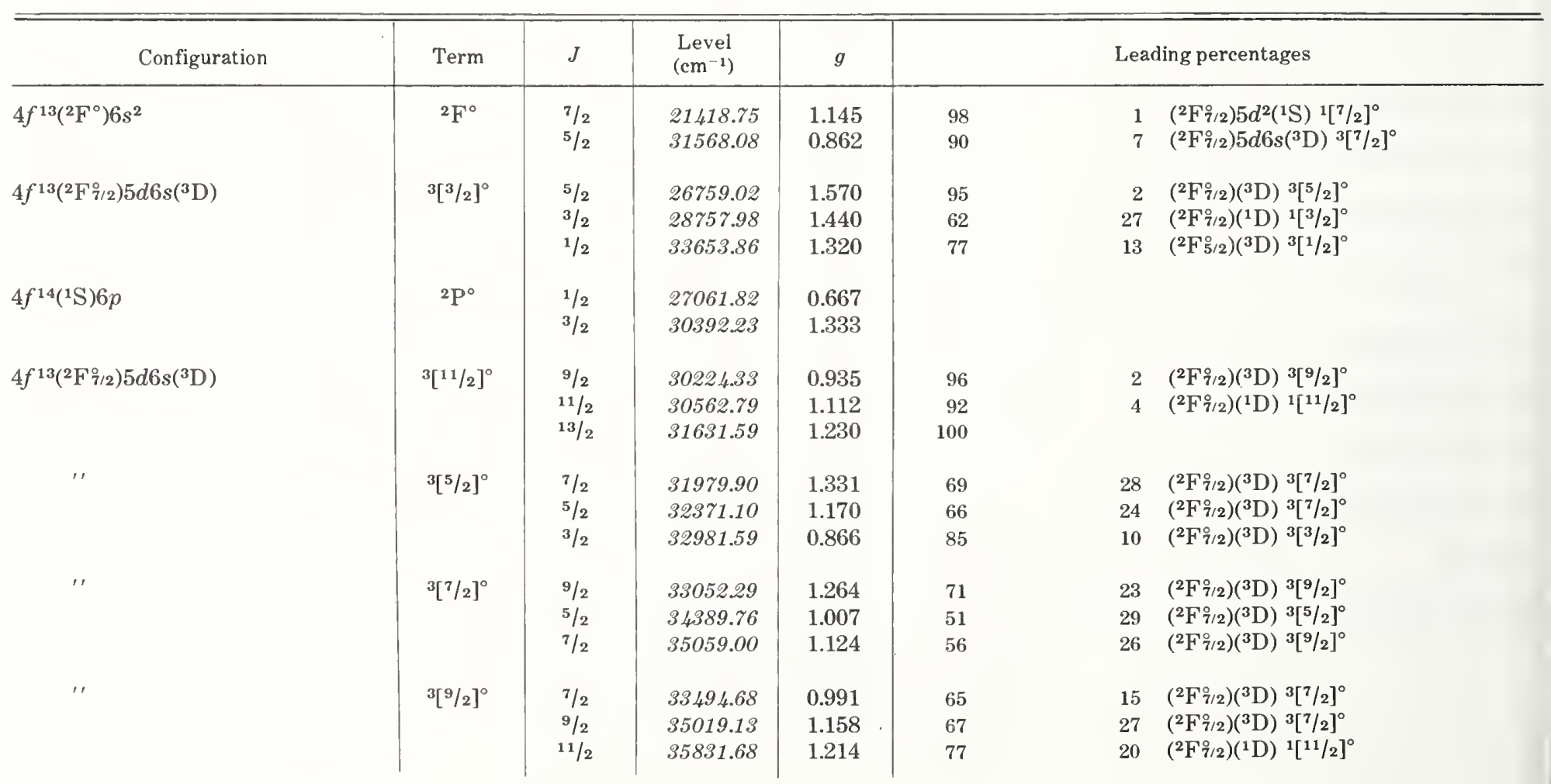


Yb II, Odd Parity_Continued

\begin{tabular}{|c|c|c|c|c|c|c|c|c|}
\hline Configuration & Term & $J$ & $\begin{array}{l}\text { Level } \\
\left(\mathrm{cm}^{-1}\right)\end{array}$ & $g$ & \multicolumn{4}{|c|}{ Leading percentages } \\
\hline $4 f^{13}\left({ }^{2} \mathrm{~F}_{7 / 2}^{\circ}\right) 5 d 6 s\left({ }^{1} \mathrm{D}\right)$ & ${ }^{1}[3 / 2]^{\circ}$ & $3 / 2$ & 34575.37 & 1.434 & 53 & & 27 & $\left({ }^{2} \mathrm{~F}_{5 / 2}^{\circ}\right)\left({ }^{3} \mathrm{D}\right){ }^{3}\left[{ }^{1} / 2\right]^{\circ}$ \\
\hline ", & ${ }^{1}[11 / 2]^{\circ}$ & $11 / 2$ & 34784.95 & 1.119 & 70 & & 18 & $\left({ }^{2} \mathrm{~F}_{7 / 2}^{\circ}\right)\left({ }^{3} \mathrm{D}\right){ }^{3}\left[{ }^{9} / 2\right]^{\circ}$ \\
\hline$" \prime$ & ${ }^{1}[5 / 2]^{\circ}$ & $5 / 2$ & 37077.59 & 1.113 & 74 & & 17 & $\left({ }^{2} \mathrm{~F}^{\circ} / 2\right)\left({ }^{3} \mathrm{D}\right){ }^{3}\left[{ }^{7} / 2\right]^{\circ}$ \\
\hline$" \prime$ & ${ }^{1}\left[{ }^{7} / 2\right]^{\circ}$ & $7 / 2$ & 37516.59 & 1.119 & 80 & & 9 & $\left({ }^{2} \mathrm{~F}_{7 / 2}^{\circ}\right) 5 d^{2}\left({ }^{1} \mathrm{D}\right){ }^{1}\left[{ }^{7} / 2\right]^{0}$ \\
\hline$\prime \prime$ & ${ }^{1}[9 / 2]^{\circ}$ & $9 / 2$ & 38342.02 & 1.093 & 81 & & 11 & $\left({ }^{2} \mathrm{~F}_{7 / 2}^{\circ}\right) 5 d^{2}\left({ }^{1} \mathrm{D}\right){ }^{1}\left[{ }^{9} / 2\right]^{\circ}$ \\
\hline $4 f^{13}\left({ }^{2} \mathrm{~F}^{\circ} / 2\right) 5 d 6 s\left({ }^{3} \mathrm{D}\right)$ & ${ }^{3}[1 / 2]^{\circ}$ & $1 / 2$ & 39378.89 & 1.850 & 80 & & 13 & $\left({ }^{2} \mathrm{~F}_{5 / 2}^{\circ}\right)\left({ }^{1} \mathrm{D}\right){ }^{1}[1 / 2]^{\circ}$ \\
\hline$\prime \prime$ & ${ }^{3}[9 / 2]^{\circ}$ & $\begin{array}{l}7 / 2 \\
9 / 2 \\
11 / 2\end{array}$ & $\begin{array}{l}40035.82 \\
40917.91 \\
42915.13\end{array}$ & $\begin{array}{l}0.720 \\
0.967 \\
1.115\end{array}$ & $\begin{array}{l}94 \\
87 \\
98\end{array}$ & & $\begin{array}{l}4 \\
8 \\
1\end{array}$ & $\begin{array}{l}\left({ }^{2} \mathrm{~F}_{7 / 2}^{\circ}\right)\left({ }^{3} \mathrm{D}\right){ }^{3}[9 / 2]^{\circ} \\
\left({ }^{2} \mathrm{~F}_{5 / 2}^{\circ}\right)\left({ }^{1} \mathrm{D}\right){ }^{1}\left[{ }^{9} / 2\right]^{\circ} \\
\left({ }^{2} \mathrm{~F}_{7 / 2}^{\circ}\right)\left({ }^{3} \mathrm{D}\right)^{3}\left[{ }^{9} / 2\right]^{\circ}\end{array}$ \\
\hline$" \prime$ & $3[3 / 2]^{\circ}$ & $\begin{array}{l}5 / 2 \\
3 / 2 \\
1 / 2\end{array}$ & $\begin{array}{l}41678.73 \\
41688.45 \\
43007.79\end{array}$ & $\begin{array}{l}1.193 \\
0.784 \\
0.15\end{array}$ & $\begin{array}{l}68 \\
46 \\
91\end{array}$ & & $\begin{array}{r}26 \\
47 \\
9\end{array}$ & $\begin{array}{l}\left({ }^{2} \mathrm{~F}_{5 / 2}^{\circ}\right)\left({ }^{3} \mathrm{D}\right){ }^{3}[5 / 2]^{\circ} \\
\left({ }^{2} \mathrm{~F}_{5 / 2}^{\circ}\right)\left({ }^{3} \mathrm{D}\right){ }^{3}\left[{ }^{5} / 2\right]^{\circ} \\
\left({ }^{2} \mathrm{~F}_{5 / 2}^{\circ}\right)\left({ }^{1} \mathrm{D}\right){ }^{1}\left[{ }^{1} / 2\right]^{\circ}\end{array}$ \\
\hline$" \prime$ & $3[5 / 2]^{\circ}$ & $\begin{array}{l}3 / 2 \\
5 / 2 \\
7 / 2\end{array}$ & $\begin{array}{l}43075.09 \\
43956.41 \\
44438.03\end{array}$ & $\begin{array}{l}0.96 \\
0.99 \\
1.10\end{array}$ & $\begin{array}{l}43 \\
46 \\
53\end{array}$ & & $\begin{array}{l}34 \\
33 \\
40\end{array}$ & $\begin{array}{l}\left({ }^{2} \mathrm{~F}_{5 / 2}^{\circ}\right)\left({ }^{3} \mathrm{D}\right){ }^{3}\left[{ }^{3} / 2\right]^{\circ} \\
\left({ }^{2} \mathrm{~F}_{5 / 2}^{\circ}\right)\left({ }^{3} \mathrm{D}\right){ }^{3}\left[{ }^{7} / 2\right]^{\circ} \\
\left({ }^{2} \mathrm{~F}_{5 / 2}^{\circ}\right)\left({ }^{\circ} \mathrm{D}\right){ }^{3}\left[{ }^{7} / 2\right]^{\circ}\end{array}$ \\
\hline "' & ${ }^{3}\left[{ }^{7} / 2\right]^{\circ}$ & $\begin{array}{l}5 / 2 \\
7 / 2 \\
9 / 2\end{array}$ & $\begin{array}{l}44497.51 \\
45429.82 \\
46169.78\end{array}$ & $\begin{array}{l}0.734 \\
1.150 \\
1.10\end{array}$ & $\begin{array}{l}63 \\
48 \\
77\end{array}$ & & $\begin{array}{l}22 \\
41 \\
20\end{array}$ & $\begin{array}{l}\left({ }^{2} \mathrm{~F}_{5 / 2}^{\circ}\right)\left({ }^{3} \mathrm{D}\right){ }^{3}\left[{ }^{5} / 2\right]^{\circ} \\
\left({ }^{2} \mathrm{~F}_{5 / 2}^{\circ}\right)\left({ }^{3} \mathrm{D}\right){ }^{3}\left[{ }^{5} / 2\right]^{\circ} \\
\left({ }^{2} \mathrm{~F}_{5 / 2}^{\circ}\right)\left({ }^{1} \mathrm{D}\right){ }^{1}\left[{ }^{9} / 2\right]^{\circ}\end{array}$ \\
\hline $\left.4 f^{13}\left({ }^{2} \mathrm{~F}_{5 / 2}^{\circ}\right) 5 d 6 s^{3} \mathrm{D}\right)$ & & $3 / 2$ & 44940.61 & 1.244 & 29 & ${ }^{3}[1 / 2]^{\circ}$ & 17 & $\left({ }^{2} \mathrm{~F}_{7 / 2}^{\circ}\right) 5 d^{2}\left({ }^{3} \mathrm{~F}\right){ }^{3}\left[{ }^{3} / 2\right]^{\circ}$ \\
\hline $4 f^{13}\left({ }^{2} \mathrm{~F}_{7 / 2}^{\circ}\right) 5 d^{2}\left({ }^{3} \mathrm{~F}\right)$ & $3[5 / 2]^{\circ}$ & $5 / 2$ & 45012.79 & 1.281 & 48 & & 34 & $\left({ }^{2} \mathrm{~F}_{7 / 2}^{\circ}\right)\left({ }^{3} \mathrm{~F}\right){ }^{3}\left[{ }^{3} / 2\right]^{\circ}$ \\
\hline $4 f^{13}\left({ }^{2} \mathrm{~F}_{7 / 2}^{\circ}\right) 5 d^{2}\left({ }^{3} \mathrm{~F}\right)$ & & $7 / 2$ & 45273.02 & 1.18 & 34 & ${ }^{3}\left[{ }^{7} / 2\right]^{\circ}$ & 34 & $\left({ }^{2} \mathrm{~F}^{\circ} / 2\right)\left({ }^{3} \mathrm{~F}\right){ }^{3}[5 / 2]^{\circ}$ \\
\hline $4 f^{13}\left({ }^{2} \mathrm{~F}_{5 / 2}^{\circ}\right) 5 d 6 s\left({ }^{1} \mathrm{D}\right)$ & ${ }^{1}[9 / 2]^{\circ}$ & $9 / 2$ & 45335.03 & 1.01 & 65 & & 20 & $\left({ }^{2} \mathrm{~F}_{5 / 2}^{\circ}\right)\left({ }^{3} \mathrm{D}\right){ }^{3}\left[{ }^{7} / 2\right]^{\circ}$ \\
\hline $4 f^{13}\left({ }^{2} \mathrm{~F}^{\circ} / 2\right) 5 d^{2}\left({ }^{3} \mathrm{~F}\right)$ & & $3 / 2$ & 4573729 & 1.333 & 34 & $3\left[^{3} / 2\right]^{\circ}$ & 22 & $\left({ }^{2} \mathrm{~F}_{7 / 2}^{\circ}\right)\left({ }^{3} \mathrm{~F}\right){ }^{3}\left[{ }^{1} / 2\right]^{\circ}$ \\
\hline $4 f^{13}\left({ }^{2} \mathrm{~F}^{\circ} / 2\right) 5 d^{2}\left({ }^{3} \mathrm{~F}\right)$ & $3[7 / 2]^{\circ}$ & $9 / 2$ & 46354.55 & 1.29 & 54 & & 28 & $\left({ }^{2} \mathrm{~F}^{\circ} / 2\right)\left({ }^{3} \mathrm{P}\right){ }^{3}\left[{ }^{7} / 2\right]^{\circ}$ \\
\hline$" \prime$ & $3[13 / 2]^{\circ}$ & $\begin{array}{l}11 / 2 \\
13 / 2 \\
15 / 2\end{array}$ & $\begin{array}{l}46547.72 \\
47680.40 \\
48923.30\end{array}$ & $\begin{array}{l}0.97 \\
1.12 \\
1.20\end{array}$ & $\begin{array}{l}82 \\
85 \\
96\end{array}$ & & $\begin{array}{r}14 \\
15 \\
4\end{array}$ & $\begin{array}{l}\left({ }^{2} \mathrm{~F}_{7 / 2}^{\circ}\right)\left({ }^{3} \mathrm{~F}\right){ }^{3}\left[{ }^{11} / 2\right]^{\circ} \\
\left({ }^{2} \mathrm{~F}^{\circ} / 2\right)\left({ }^{3} \mathrm{~F}\right){ }^{3}\left[{ }^{11} / 2\right]^{\circ} \\
\left({ }^{2} \mathrm{~F}^{\circ} / 2\right)\left({ }^{1} \mathrm{G}\right){ }^{1}\left[{ }^{15} / 2\right]^{\circ}\end{array}$ \\
\hline $4 f^{13}\left({ }^{2} \mathrm{~F}_{7 / 2}^{\circ}\right) 5 d^{2}\left({ }^{3} \mathrm{~F}\right)$ & $3[3 / 2]^{\circ}$ & $1 / 2$ & 46902.71 & 1.30 & 48 & & 24 & $\left({ }^{2} \mathrm{~F}^{\circ}{ }^{\circ} / 2\right)\left({ }^{3} \mathrm{~F}\right){ }^{3}\left[{ }^{1} / 2\right]^{\circ}$ \\
\hline $4 f^{13}\left({ }^{2} \mathrm{~F}_{5 / 2}^{\circ}\right) 5 d 6 s\left({ }^{1} \mathrm{D}\right)$ & $1[3 / 2]^{\circ}$ & $3 / 2$ & 47005.46 & 0.990 & 77 & & 8 & $\left({ }^{2} \mathrm{~F}_{5 / 2}^{\circ}\right)\left({ }^{3} \mathrm{D}\right){ }^{3}\left[{ }^{1 / 2}\right]^{\circ}$ \\
\hline$" \prime$ & $1[1 / 2]^{\circ}$ & $1 / 2$ & 47228.96 & 0.74 & 50 & & 14 & $\left({ }^{2} \mathrm{~F}_{7 / 2}^{\circ}\right)\left({ }^{3} \mathrm{D}\right){ }^{3}\left[{ }^{3} / 2\right]^{\circ}$ \\
\hline "' & $1[5 / 2]^{\circ}$ & $5 / 2$ & 47329.44 & 0.990 & 58 & & 12 & $\left({ }^{2} \mathrm{~F}_{7 / 2}^{\circ}\right) 5 d^{2}\left({ }^{3} \mathrm{~F}\right){ }^{3}\left[{ }^{3} / 2\right]^{\circ}$ \\
\hline $4 f^{13}\left({ }^{2} \mathrm{~F}_{7 / 2}^{\circ}\right) 5 d^{2}\left({ }^{3} \mathrm{~F}\right)$ & ${ }^{3}[11 / 2]^{\circ}$ & $\begin{array}{c}9 / 2 \\
11 / 2 \\
13 / 2\end{array}$ & $\begin{array}{l}47663.52 \\
48503.82 \\
49727.19\end{array}$ & $\begin{array}{l}0.98 \\
1.10 \\
1.20\end{array}$ & $\begin{array}{l}62 \\
54 \\
84\end{array}$ & & $\begin{array}{l}19 \\
28 \\
15\end{array}$ & $\begin{array}{l}\left({ }^{2} \mathrm{~F}_{7 / 2}^{\circ}\right)\left({ }^{3} \mathrm{~F}\right){ }^{3}\left[{ }^{9} / 2\right]^{\circ} \\
\left({ }^{2} \mathrm{~F}^{\circ} / 2\right)\left({ }^{3} \mathrm{~F}\right){ }^{3}\left[{ }^{9} / 2\right]^{\circ} \\
\left({ }^{2} \mathrm{~F}^{\circ} \mathrm{\gamma} / 2\right)\left({ }^{3} \mathrm{~F}\right){ }^{3}\left[{ }^{13 / 2}\right]^{\circ}\end{array}$ \\
\hline $4 f^{13}\left({ }^{2} \mathrm{~F}_{7 / 2}^{\circ}\right) 5 d^{2}\left({ }^{3} \mathrm{~F}\right)$ & & $7 / 2$ & 47758.54 & 1.08 & 41 & $3[9 / 2]^{\circ}$ & 38 & $\left({ }^{2} \mathrm{~F}_{7 / 2}^{\circ}\right)\left({ }^{3} \mathrm{~F}\right){ }^{3}\left[{ }^{5} / 2\right]^{\circ}$ \\
\hline $4 f^{13}\left({ }^{2} \mathrm{~F}_{7 / 2}^{\circ}\right) 5 d^{2}\left({ }^{3} \mathrm{~F}\right)$ & ${ }^{3}[1 / 2]^{\circ}$ & $\begin{array}{l}3 / 2 \\
1 / 2\end{array}$ & $\begin{array}{l}48024.70 \\
49419.13\end{array}$ & $\begin{array}{l}1.38 \\
1.41\end{array}$ & $\begin{array}{l}53 \\
67\end{array}$ & & $\begin{array}{l}30 \\
22\end{array}$ & $\begin{array}{l}\left.\left({ }^{2} \mathrm{~F}_{7 / 2}^{\circ}\right)\left({ }^{3} \mathrm{~F}\right)^{3}{ }^{3}{ }^{5} / 2\right]^{\circ} \\
\left({ }^{2} \mathrm{~F}_{7 / 2}^{\circ}\right)\left({ }^{3} \mathrm{D}\right)^{3}\left[{ }^{3} / 2\right]^{\circ}\end{array}$ \\
\hline $4 f^{13}\left({ }^{2} \mathrm{~F}_{7 / 2}^{\circ}\right) 5 d^{2}\left({ }^{3} \mathrm{~F}\right)$ & & $5 / 2$ & 48272.77 & 1.14 & 36 & $\left.3{ }^{3} / 2\right]^{\circ}$ & 22 & $\left({ }^{2} \mathrm{~F}_{5}^{\circ} / 2\right) 5 d 6 s\left({ }^{1} \mathrm{D}\right){ }^{1}\left[{ }^{5} / 2\right]^{\circ}$ \\
\hline $4 f^{13}\left({ }^{2} \mathrm{~F}_{5 / 2}^{\circ}\right) 5 d 6 s\left({ }^{1} \mathrm{D}\right)$ & ${ }^{1}\left[{ }^{7} / 2\right]^{\circ}$ & $7 / 2$ & 48556.57 & 0.941 & 81 & & 11 & $\left({ }^{2} \mathrm{~F}_{5}^{\circ} / 2\right) 5 d^{2}\left({ }^{1} \mathrm{D}\right){ }^{1}\left[{ }^{7} / 2\right]^{\circ}$ \\
\hline
\end{tabular}


Yb II, Odd Parity_Continued

\begin{tabular}{|c|c|c|c|c|c|c|c|c|}
\hline Configuration & Term & $J$ & $\begin{array}{l}\text { Level } \\
\left(\mathrm{cm}^{-1}\right)\end{array}$ & $g$ & \multicolumn{4}{|c|}{ Leading percentages } \\
\hline $4 f^{13}\left({ }^{2} \mathrm{~F}_{7 / 2}^{\circ}\right) 5 d^{2}\left({ }^{3} \mathrm{~F}\right)$ & $3[9 / 2]^{\circ}$ & $\begin{array}{c}7 / 2 \\
9 / 2 \\
11 / 2\end{array}$ & $\begin{array}{l}49008.93 \\
49916.50 \\
50468.05\end{array}$ & $\begin{array}{l}1.03 \\
1.090 \\
1.213\end{array}$ & $\begin{array}{l}43 \\
52 \\
49\end{array}$ & & $\begin{array}{l}15 \\
31 \\
28\end{array}$ & $\begin{array}{l}\left({ }^{2} \mathrm{~F}_{7 / 2}^{\circ}\right)\left({ }^{3} \mathrm{~F}\right){ }^{3}\left[{ }^{5} / 2\right]^{\circ} \\
\left({ }^{2} \mathrm{~F}_{7 / 2}^{\circ}\right)\left({ }^{3} \mathrm{~F}\right){ }^{3}[11 / 2]^{\circ} \\
\left({ }^{2} \mathrm{~F}_{7 / 2}^{\circ}\right)\left({ }^{3} \mathrm{~F}\right){ }^{3}[11 / 2]^{0}\end{array}$ \\
\hline $4 f^{13}\left({ }^{2} \mathrm{~F}_{7 / 2}^{\circ}\right) 5 d^{2}\left({ }^{3} \mathrm{~F}\right)$ & & $3 / 2$ & 50832.65 & 0.956 & 35 & $3[5 / 2]^{\circ}$ & 30 & $\left({ }^{2} \mathrm{~F}^{\circ} / 2\right)\left({ }^{3} \mathrm{~F}\right){ }^{3}\left[{ }^{3} / 2\right]^{\circ}$ \\
\hline $4 f^{13}\left({ }^{2} \mathrm{~F}_{7 / 2}^{\circ}\right) 5 d^{2}\left({ }^{3} \mathrm{~F}\right)$ & & $5 / 2$ & 51248.86 & 1.034 & 40 & $\left.3{ }^{7} / 2\right]^{\circ}$ & 25 & $\left({ }^{2} \mathrm{~F}_{7 / 2}^{\circ}\right)\left({ }^{3} \mathrm{~F}\right){ }^{3}\left[{ }^{5} / 2\right]^{\circ}$ \\
\hline $4 f^{13}\left({ }^{2} \mathrm{~F}_{7 / 2}^{\circ}\right) 5 d^{2}\left({ }^{1} \mathrm{G}\right)$ & $1[1 / 2]^{\circ}$ & $1 / 2$ & 52067.87 & 0.54 & 67 & & 20 & $\left({ }^{2} \mathrm{~F}_{7 / 2}^{\circ}\right)\left({ }^{3} \mathrm{~F}\right){ }^{3}\left[{ }^{3} / 2\right]^{\circ}$ \\
\hline $4 f^{13}\left({ }^{2} \mathrm{~F}_{7 / 2}^{\circ}\right) 5 d^{2}\left({ }^{3} \mathrm{P}\right)$ & $3\left[{ }^{7} / 2\right]^{\circ}$ & $\begin{array}{l}9 / 2 \\
7 / 2 \\
5 / 2\end{array}$ & $\begin{array}{l}52880.75 \\
52921.62 \\
53120.58\end{array}$ & $\begin{array}{l}1.26 \\
1.15 \\
0.944\end{array}$ & $\begin{array}{l}45 \\
51 \\
67\end{array}$ & & $\begin{array}{l}35 \\
36 \\
10\end{array}$ & $\left({ }^{2} \mathrm{~F}_{7 / 2}^{\circ}\right)\left({ }^{3} \mathrm{~F}\right){ }^{3}\left[{ }^{7} / 2\right]^{\circ}$ \\
\hline $4 f^{13}\left({ }^{2} \mathrm{~F}^{\circ} / 2\right) 5 d^{2}\left({ }^{1} \mathrm{G}\right)$ & $1[3 / 2]^{0}$ & $3 / 2$ & 52987.76 & 1.131 & 61 & & 12 & $\left({ }^{2} \mathrm{~F}_{5 / 2}^{\circ}\right)\left({ }^{3} \mathrm{~F}\right){ }^{3}\left[{ }^{3} / 2\right]^{\circ}$ \\
\hline$"$ & ${ }^{1}[15 / 2]^{\circ}$ & $15 / 2$ & 53322.89 & 1.04 & 96 & & 4 & $\left({ }^{2} \mathrm{~F}_{7 / 2}^{\circ}\right)\left({ }^{3} \mathrm{~F}\right){ }^{3}[13 / 2]^{\circ}$ \\
\hline $4 f^{13}\left({ }^{2} \mathrm{~F}_{7 / 2}^{\circ}\right) 5 d^{2}\left({ }^{1} \mathrm{D}\right)$ & $1[7 / 2]^{\circ}$ & $7 / 2$ & 53644.89 & 1.18 & 45 & & 13 & $\left({ }^{2} \mathrm{~F}_{5 / 2}^{\circ}\right)\left({ }^{3} \mathrm{~F}\right){ }^{3}\left[{ }^{5} / 2\right]^{\circ}$ \\
\hline $4 f^{13}\left({ }^{2} \mathrm{~F}_{5 / 2}^{\circ}\right) 5 d^{2}\left({ }^{3} \mathrm{~F}\right)$ & & $5 / 2$ & 53716.38 & 1.183 & 32 & ${ }^{3}\left[{ }^{5} / 2\right]^{\circ}$ & 19 & $\left({ }^{2} \mathrm{~F}^{\circ} / 2\right)\left({ }^{1} \mathrm{D}\right){ }^{1}\left[{ }^{5} / 2\right]^{\circ}$ \\
\hline $4 f^{13}\left({ }^{2} \mathrm{~F}_{7 / 2}^{\circ}\right) 5 d^{2}\left({ }^{1} \mathrm{D}\right)$ & $1\left[^{11 / 2}\right]^{\circ}$ & $11 / 2$ & 54192.51 & 1.11 & 70 & & 8 & $\left({ }^{2} \mathrm{~F}_{7 / 2}^{\circ}\right)\left({ }^{3} \mathrm{~F}\right){ }^{3}\left[{ }^{9} / 2\right]^{\circ}$ \\
\hline $4 f^{13}\left({ }^{2} \mathrm{~F}^{\circ}{ }^{\circ} / 2\right) 5 d^{2}\left({ }^{1} \mathrm{D}\right)$ & ${ }^{1}[9 / 2]^{\circ}$ & $9 / 2$ & 54640.82 & 1.13 & 60 & & 10 & $\left({ }^{2} \mathrm{~F}^{\circ} / 2\right)\left({ }^{3} \mathrm{P}\right){ }^{3}\left[{ }^{7} / 2\right]^{\circ}$ \\
\hline$" \prime$ & ${ }^{1}[3 / 2]^{\circ}$ & $3 / 2$ & 55221.46 & 1.11 & 56 & & 22 & $\left({ }^{2} \mathrm{~F}_{7 / 2}^{\circ}\right)\left({ }^{3} \mathrm{P}\right){ }^{3}\left[{ }^{5} / 2\right]^{0}$ \\
\hline $4 f^{13}\left({ }^{2} \mathrm{~F}^{\circ} / 2\right) 5 d^{2}\left({ }^{3} \mathrm{P}\right)$ & $3[9 / 2]^{\circ}$ & $\begin{array}{c}7 / 2 \\
9 / 2 \\
11 / 2\end{array}$ & $\begin{array}{l}55462.68 \\
56088.39 \\
56621.15\end{array}$ & $\begin{array}{l}0.878 \\
1.11 \\
1.16\end{array}$ & $\begin{array}{l}62 \\
63 \\
64\end{array}$ & & $\begin{array}{l}22 \\
10 \\
14\end{array}$ & $\begin{array}{l}\left({ }^{2} \mathrm{~F}_{5 / 2}^{\circ}\right)\left({ }^{3} \mathrm{~F}\right){ }^{3}\left[{ }^{9} / 2\right]^{\circ} \\
\left({ }^{2} \mathrm{~F}^{\circ}{ }^{\circ / 2}\right)\left({ }^{1} \mathrm{G}\right){ }^{1}\left[{ }^{9} / 2\right]^{\circ} \\
\left({ }^{2} \mathrm{~F}^{\circ} / 2\right)\left({ }^{1} \mathrm{G}\right){ }^{1}\left[{ }^{11 / 2}\right]^{\circ}\end{array}$ \\
\hline $4 f^{13}\left({ }^{2} \mathrm{~F}_{7 / 2}^{\circ}\right) 5 d^{2}\left({ }^{3} \mathrm{P}\right)$ & & $5 / 2$ & 56056.91 & 1.12 & 32 & $3[5 / 2]^{\circ}$ & 32 & $\left({ }^{2} \mathrm{~F}_{7 / 2}^{\circ}\right)\left({ }^{3} \mathrm{~F}\right){ }^{3}\left[{ }^{5} / 2\right]^{0}$ \\
\hline $4 f^{13}\left({ }^{2} \mathrm{~F}_{5 / 2}^{\circ}\right) 5 d^{2}\left({ }^{3} \mathrm{~F}\right)$ & $3\left[^{11} / 2\right]^{\circ}$ & $\begin{array}{c}9 / 2 \\
11 / 2 \\
13 / 2\end{array}$ & $\begin{array}{l}56480.77 \\
58051.51 \\
59632.21 ?\end{array}$ & $\begin{array}{l}0.76 \\
0.95\end{array}$ & $\begin{array}{l}94 \\
99 \\
93\end{array}$ & & 3 & $\begin{array}{l}\left({ }^{2} \mathrm{~F}_{7 / 2}^{\circ}\right)\left({ }^{3} \mathrm{P}\right){ }^{3}\left[{ }^{9} / 2\right]^{\circ} \\
\left({ }^{2} \mathrm{~F}_{5 / 2}^{\circ}\right)\left({ }^{1} \mathrm{G}\right){ }^{1}\left[{ }^{13} / 2\right]^{\circ}\end{array}$ \\
\hline $4 f^{13}\left({ }^{2} \mathrm{~F}_{7 / 2}^{\circ}\right) 5 d^{2}\left({ }^{3} \mathrm{P}\right)$ & $3[5 / 2]^{\circ}$ & $\begin{array}{l}7 / 2 \\
5 / 2 \\
3 / 2\end{array}$ & $\begin{array}{l}56500.64 \\
57798.51 \\
58162.75\end{array}$ & $\begin{array}{l}1.352 \\
1.151 \\
0.94\end{array}$ & $\begin{array}{l}77 \\
50 \\
60\end{array}$ & & $\begin{array}{r}8 \\
22 \\
18\end{array}$ & $\begin{array}{l}\left({ }^{2} \mathrm{~F}_{7 / 2}^{\circ}\right)\left({ }^{3} \mathrm{P}\right){ }^{3}\left[{ }^{7} / 2\right]^{\circ} \\
\left({ }^{2} \mathrm{~F}^{\circ} / 2\right)\left({ }^{1} \mathrm{D}\right){ }^{1}\left[{ }^{5} / 2\right]^{\circ} \\
\left({ }^{2} \mathrm{~F}_{7 / 2}^{\circ}\right)\left({ }^{1} \mathrm{D}\right){ }^{1}\left[{ }^{3} / 2\right]^{\circ}\end{array}$ \\
\hline $4 f^{13}\left({ }^{2} \mathrm{~F}_{5 / 2}^{\circ}\right) 5 d^{2}\left({ }^{3} \mathrm{~F}\right)$ & & $3 / 2$ & 56840.04 & 0.90 & 40 & $3[5 / 2]^{\circ}$ & 19 & $\left({ }^{2} \mathrm{~F}_{5 / 2}^{\circ}\right)\left({ }^{3} \mathrm{~F}\right){ }^{3}\left[{ }^{3} / 2\right]^{\circ}$ \\
\hline $4 f^{13}\left({ }^{2} \mathrm{~F}_{5 / 2}^{\circ}\right) 5 d^{2}\left({ }^{3} \mathrm{~F}\right)$ & $3[1 / 2]^{\circ}$ & $\begin{array}{l}1 / 2 \\
3 / 2\end{array}$ & $\begin{array}{l}56977.71 \\
59777.07\end{array}$ & $\begin{array}{l}2.19 \\
1.63\end{array}$ & $\begin{array}{l}90 \\
71\end{array}$ & & $\begin{array}{r}8 \\
21\end{array}$ & $\left({ }^{2} \mathrm{~F}_{5 / 2}^{\circ}\right)\left({ }^{3} \mathrm{~F}\right){ }^{3}\left[{ }^{3} / 2\right]^{\circ}$ \\
\hline $4 f^{13}\left({ }^{2} \mathrm{~F}_{7 / 2}^{\circ}\right) 5 d^{2}\left({ }^{1} \mathrm{G}\right)$ & ${ }^{1}\left[{ }^{7} / 2\right]^{\circ}$ & $7 / 2$ & 57103.02 & 1.10 & 82 & & 11 & $\left({ }^{2} \mathrm{~F}_{7 / 2}^{\circ}\right)\left({ }^{1} \mathrm{D}\right){ }^{1}\left[{ }^{7} / 2\right]^{\circ}$ \\
\hline $4 f^{13}\left({ }^{2} \mathrm{~F}_{5 / 2}^{\circ}\right) 5 d^{2}\left({ }^{3} \mathrm{~F}\right)$ & $3[9 / 2]^{\circ}$ & $\begin{array}{c}7 / 2 \\
9 / 2 \\
11 / 2\end{array}$ & $\begin{array}{l}57534.45 \\
59046.89 \\
61442.82\end{array}$ & $\begin{array}{l}0.87 \\
1.08 \\
1.11\end{array}$ & $\begin{array}{l}54 \\
57 \\
91\end{array}$ & & $\begin{array}{r}16 \\
24 \\
4\end{array}$ & $\begin{array}{l}\left({ }^{2} \mathrm{~F}_{5 / 2}^{\circ}\right)\left({ }^{3} \mathrm{~F}\right){ }^{3}\left[{ }^{7} / 2\right]^{\circ} \\
\left({ }^{2} \mathrm{~F}_{5 / 2}^{\circ}\right)\left({ }^{3} \mathrm{~F}\right){ }^{3}\left[{ }^{7} / 2\right]^{\circ} \\
\left({ }^{2} \mathrm{~F}_{7 / 2}^{\circ}\right)\left({ }^{1} \mathrm{G}\right){ }^{1}\left[{ }^{11 / 2}\right]^{\circ}\end{array}$ \\
\hline $4 f^{13}\left({ }^{2} \mathrm{~F}_{7 / 2}^{\circ}\right) 5 d^{2}\left({ }^{1} \mathrm{G}\right)$ & $1[13 / 2]^{\circ}$ & $13 / 2$ & 57561.62 & 1.04 & 94 & & 5 & $\left({ }^{2} \mathrm{~F}_{5 / 2}^{\circ}\right)\left({ }^{1} \mathrm{G}\right){ }^{1}\left[{ }^{13} / 2\right]^{\circ}$ \\
\hline $4 f^{13}\left({ }^{2} \mathrm{~F}_{7 / 2}^{\circ}\right) 5 d^{2}\left({ }^{1} \mathrm{G}\right)$ & ${ }^{1}[9 / 2]^{\circ}$ & $9 / 2$ & 58484.54 & 1.02 & 75 & & 11 & $\left({ }^{2} \mathrm{~F}_{5 / 2}^{\circ}\right)\left({ }^{3} \mathrm{~F}\right){ }^{3}\left[{ }^{9} / 2\right]^{\circ}$ \\
\hline $4 f^{13}\left({ }^{2} \mathrm{~F}_{5 / 2}^{\circ}\right) 5 d^{2}\left({ }^{3} \mathrm{~F}\right)$ & $3[7 / 2]^{c}$ & $\begin{array}{l}5 / 2 \\
7 / 2 \\
9 / 2\end{array}$ & $\begin{array}{l}58661.13 \\
59624.66 \\
60649.86\end{array}$ & $\begin{array}{l}0.86 \\
1.10\end{array}$ & $\begin{array}{l}59 \\
60 \\
57\end{array}$ & & $\begin{array}{l}14 \\
19 \\
20\end{array}$ & $\begin{array}{l}\left({ }^{2} \mathrm{~F}_{5 / 2}^{\circ}\right)\left({ }^{3} \mathrm{~F}\right){ }^{3}\left[{ }^{5} / 2\right]^{\circ} \\
\left({ }^{2} \mathrm{~F}_{5 / 2}^{\circ}\right)\left({ }^{3} \mathrm{~F}\right){ }^{3}\left[{ }^{9} / 2\right]^{\circ} \\
\left({ }^{2} \mathrm{~F}_{5 / 2}^{\circ}\right)\left({ }^{3} \mathrm{~F}\right){ }^{3}\left[{ }^{9} / 2\right]^{\circ}\end{array}$ \\
\hline "' & $3[3 / 2]^{\circ}$ & $1 / 2$ & 58672.53 & 0.538 & 69 & & 15 & $\left({ }^{2} \mathrm{~F}^{\circ} / 2\right)\left({ }^{1} \mathrm{G}\right){ }^{1}[1 / 2]^{\circ}$ \\
\hline $4 f^{13}\left({ }^{2} \mathrm{~F}_{5 / 2}^{\circ}\right) 5 d^{2}\left({ }^{3} \mathrm{~F}\right)$ & & $5 / 2$ & 59439.08 & 0.994 & 33 & $3[3 / 2]^{\circ}$ & 29 & $\left({ }^{2} \mathrm{~F}_{5 / 2}^{\circ}\right)\left({ }^{3} \mathrm{~F}\right){ }^{3}\left[{ }^{5} / 2\right]^{\circ}$ \\
\hline $4 f^{13}\left({ }^{2} \mathrm{~F}_{7 / 2}^{\circ}\right) 5 d^{2}\left({ }^{1} \mathrm{G}\right)$ & ${ }^{1}[11 / 2]^{\circ}$ & $11 / 2$ & 59753.84 & 1.11 & 73 & & 14 & $\left({ }^{2} \mathrm{~F}_{7 / 2}^{\circ}\right)\left({ }^{1} \mathrm{D}\right){ }^{1}\left[{ }^{11} / 2\right]^{\circ}$ \\
\hline
\end{tabular}


Yb II, Odd Parity-Continued

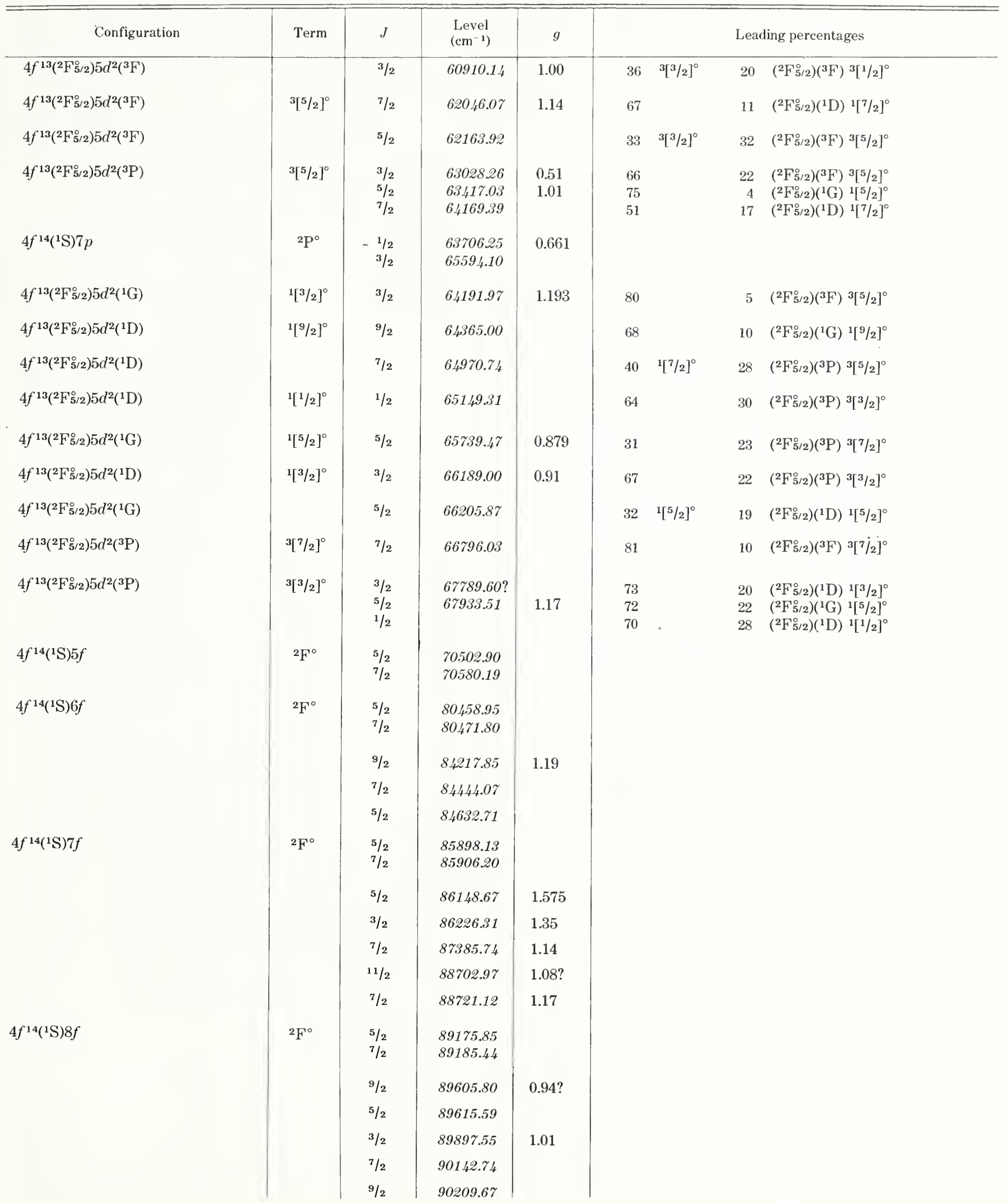


Yb II, Odd Parity_Continued

Yb II, Odd Parity-Continued

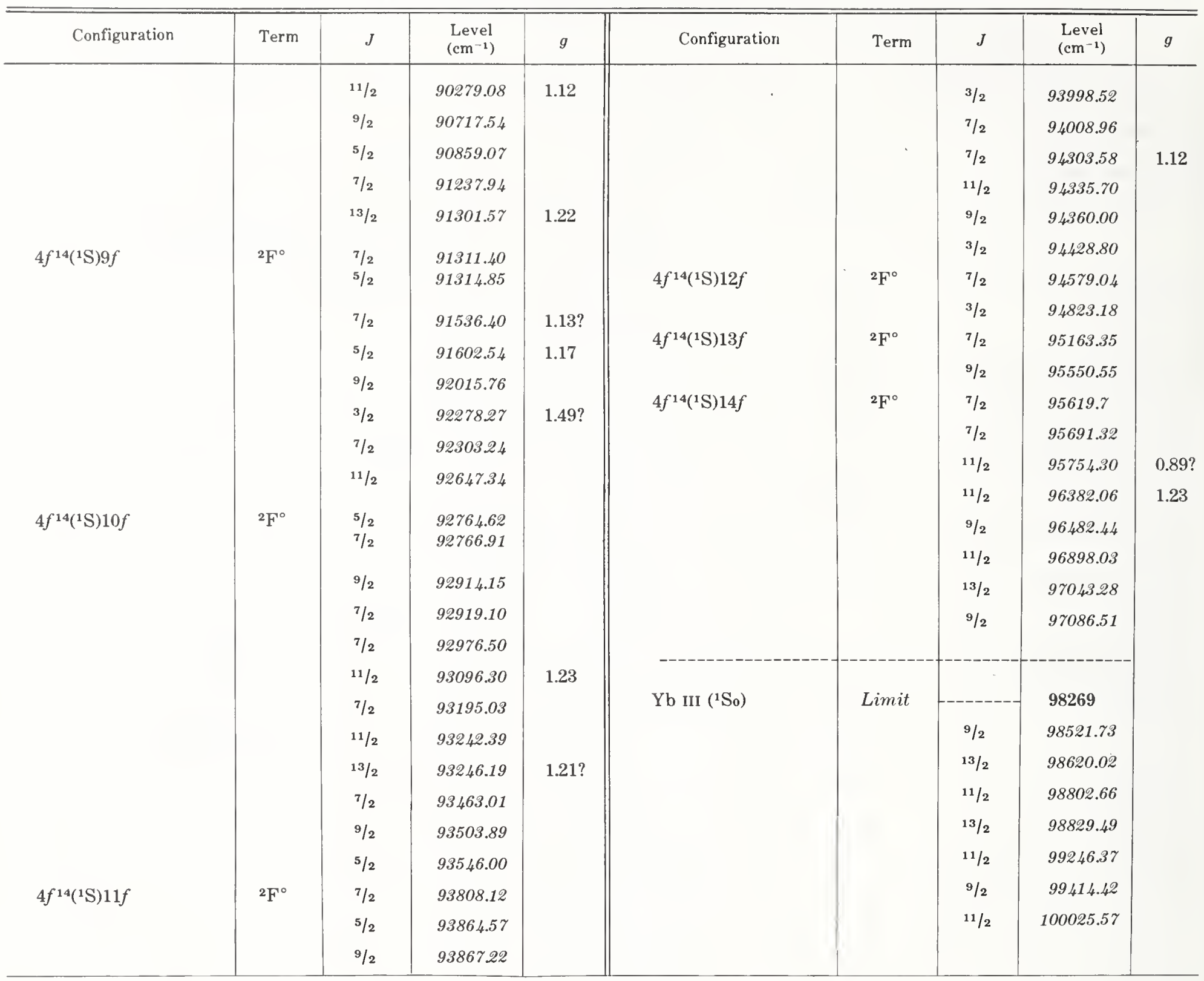


Yb III

(Er I sequence; 68 electrons)

Ground state $\left(1 s^{2} 2 s^{2} 2 p^{6} 3 s^{2} 3 p^{6} 3 d^{10} 4 s^{2} 4 p^{6} 4 d^{10} 5 s^{2} 5 p^{6}\right) 4 f^{14}{ }^{1} \mathrm{~S}_{0}$

Ionization energy $202070 \pm 200 \mathrm{~cm}^{-1}$

$25.05 \pm 0.03 \mathrm{eV}$

Identified even configurations $\quad 4 f^{14}, 4 f^{13} 6 p$

Identified odd configurations $\quad 4 f^{13} 5 d, 4 f^{13} 6 s, 4 f^{13} 7 s, 4 f^{13} 6 d$

All but one of the levels are from Bryant's analysis of this spectrum [1961, 1965]. Except for $4 f^{13} 6 d$, each of the configurations listed above is complete. The position for the $4 f^{13}\left({ }^{2} \mathrm{~F}_{5 / 2}^{\circ}\right) 5 d(5 / 2,5 / 2)_{0}^{\circ}$ level was suggested by Sugar [1970].

The basic theoretical interpretation is also due to Bryant, who noted the strong tendency toward $J_{1} j$ coupling. The leading eigenvector percentages in $L S$ coupling are also listed. All percentages are from Bryant's calculations except for $4 f^{13} 5 d$, which has more recently been calculated with the inclusion of appropriate effective interactions. The leading percentages for $4 f^{13} 5 d$ in $J_{1} j$ coupling are from Goldschmidt and Salomon [1972], and the percentages in $L S$ coupling are from a similar calculation by Sugar [1973]. A complete set of energy-parameter values derived by fitting $\left(4 f^{13} 5 d+4 f^{13} 6 s\right)$ has been published by Wyart, Blaise, and Camus [1974].

Bryant gave positions for 10 of the 20 levels expected for $4 f^{13} 6 d$, which he located by classifying a number of $4 f^{13} 6 p-4 f^{13} 6 d$ transitions in the vicinity of $2100 \AA$. His $J_{1} j$ designations for the $4 f^{13} 6 d$ levels are tentative.

Bryant's full line list [1961] gives an experimental separation of the Yb spectra from the different ions, and has lines assigned to Yb III extending from $968 \AA$ to $10830 \AA$. Meggers and Corliss [1966] attributed 430 lines to Yb III in the region above $2000 \AA$, also based on an experimental separation. The assignments of lines occurring in both lists do not always agree. Bryant's analysis gives classifications for almost $200 \mathrm{Yb}$ III lines, including practically all of the strong lines $(1798-5432 \AA)$.

The ionization potential is from Kaufman and Sugar.

\section{References}

Bryant, B. W., Johns Hopkins Spectr. Report No. 21, 82 pp. (1961). EL CL W IP PT

Bryant, B. W., J. Opt. Soc. Am. 55, 771 (1965). EL CL W IP PT

Goldschmidt, Z. B., Thesis, Hebrew Univ. Jerusalem, Israel, 487 pp. (1968). PT

Goldschmidt, Z. B., and Salomon, D., unpublished calculations (1972); see Goldschmidt, Z. B., in Atomic Physies 3, S. J. Smith and G. K. Walters, Eds., pp. 221-246 (Plenum Press, New York, 1973). ND PT

Kaufman, V., and Sugar, J., J. Opt. Soc. Am. 66, 1019 (1976). IP

Meggers, W. F., and Corliss, C. H., J. Res. Nat. Bur. Stand. (U.S.) 70A, 63 (1966). W

Sugar, J., J. Opt. Soc. Am. 60, 571 (1970). EL CL PT

Sugar, J., unpublished calculations (1973). PT

Wyart, J. F., Blaise, J., and Camus, P., Phys. Ser. 9, 325 (1974). PT

[October 1976] 
Yb III

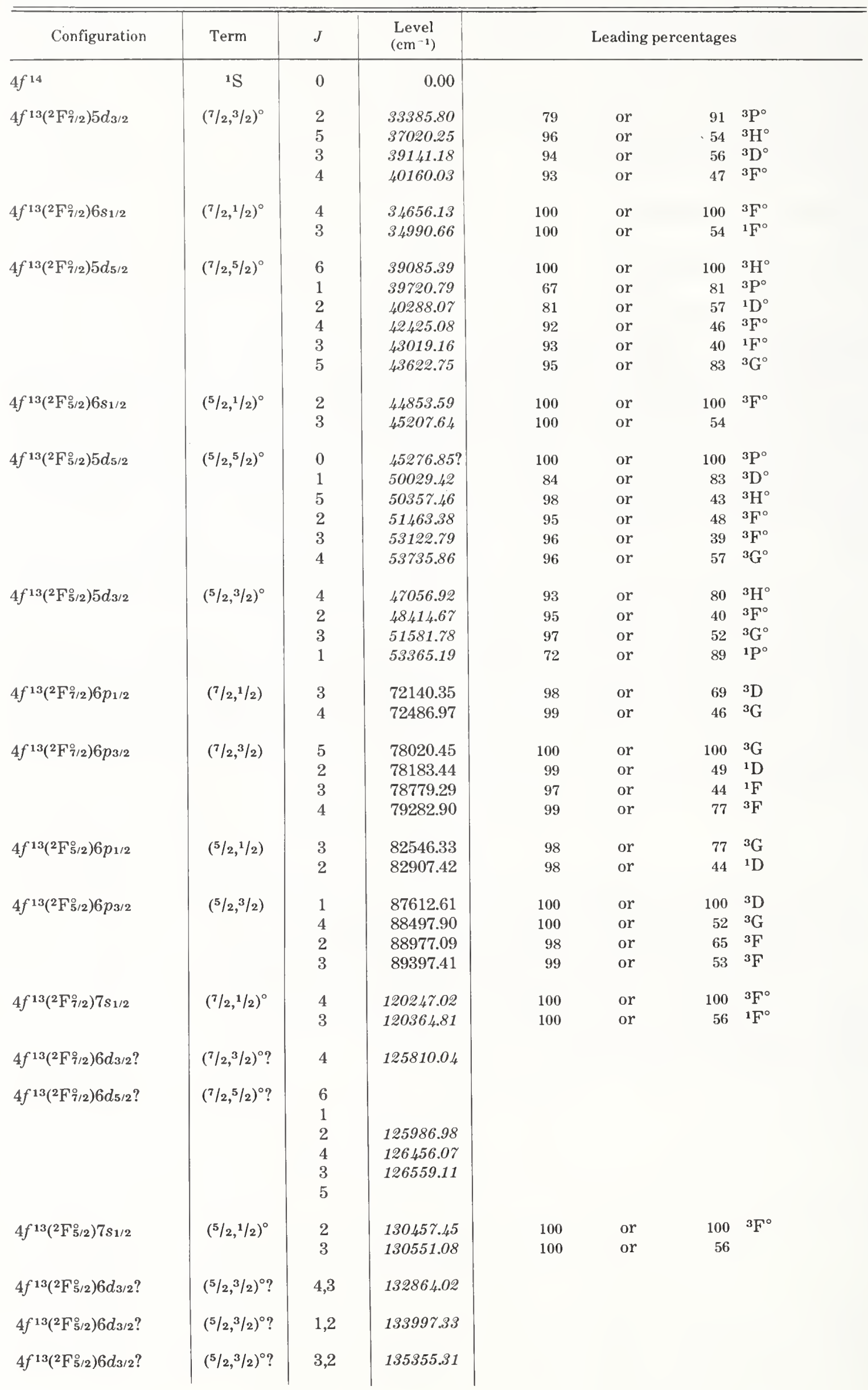


Yb III-Continued

\begin{tabular}{l|c|c|c|c}
\hline Configuration & Term & $J$ & $\begin{array}{c}\text { Level } \\
\left(\mathrm{cm}^{-1}\right)\end{array}$ & Leading percentages \\
\hline $4 f^{13}\left({ }^{2} \mathrm{~F}_{5 / 2}^{\circ}\right) 6 d_{5 / 2} ?$ & $(5 / 2,5 / 2)^{\circ} ?$ & 2 & 136350.98 & \\
$4 f^{13}\left({ }^{2} \mathrm{~F}_{5 / 2}^{\circ}\right) 6 d_{5 / 2} ?$ & $(5 / 2,5 / 2)^{\circ} ?$ & 3 & 136699.69 & \\
$4 f^{13}\left({ }^{2} \mathrm{~F}_{5 / 2}^{\circ}\right) 6 d_{5 / 2} ?$ & $(5 / 2,5 / 2)^{\circ} ?$ & 4,3 & 136849.44 & \\
-------------- & & & \\
Yb IV $\left({ }^{2} \mathrm{~F}_{7 / 2}^{\circ}\right)$ & Limit & & & \\
\hline
\end{tabular}




\section{Yb IV}

(Ho I sequence; 67 electrons)

Ground state $\left(1 s^{2} 2 s^{2} 2 p^{6} 3 s^{2} 3 p^{6} 3 d^{10} 4 s^{2} 4 p^{6} 4 d^{10} 5 s^{2} 5 p^{6}\right) 4 f^{13}{ }^{2} \mathrm{~F}_{7 / 2}^{\circ}$

Ionization energy $351300 \pm 800 \mathrm{~cm}^{-1}$

$43.56 \pm 0.10 \mathrm{eV}$

Identified odd configurations $\quad 4 f^{13}, 4 f^{12} 6 p$

Identified even configurations $\quad 4 f^{12} 5 d, 4 f^{12} 6 s$

Bryant [1961] observed the spectra of arc and spark discharges between Yb electrodes and gave an experimental separation of the lines from the different ions (see Yb III). His line list includes assignments to Yb IV from 677 to above $3700 \AA$, but the wavelengths below 2000 $\AA$ are probably not sufficiently accurate for analysis. An array of transitions to the $4 f^{132} \mathrm{~F}^{\circ}$ levels was set up, but the derived ${ }^{2} \mathrm{~F}^{\circ}$ interval was later found to be erroneous.

Kaufman and Sugar [1976] observed the spectrum of a sliding-spark source over the region 800-1500 $\AA$ and assigned about 200 lines to Yb IV. Their paper gives the wavelengths for 38 of these lines, which were classified as transitions from 19 upper $4 f^{12} 5 d$ levels to the $4 f^{13}{ }^{2} \mathrm{~F}^{\circ}$ ground doublet. The observations have recently been extended up to $2190 \AA$, and about 320 of the more than 600 lines now assigned to Yb IV are classified [Sugar, Kaufman, and Spector, 1977]. The levels here are from this new analysis, which includes transitions from the $4 f^{12} 5 d-4 f^{12} 6 p$ and $4 f^{12} 6 s-4 f^{12} 6 p$ arrays. The ${ }^{2} \mathrm{~F}_{5 / 2}^{\circ}-{ }^{2} \mathrm{~F}_{7 / 2}^{\circ}$ ground-term interval is accurate to about $\pm 0.1 \mathrm{~cm}^{-1}$, and most of the higher levels are accurate to within $\pm 0.5 \mathrm{~cm}^{-1}$.

The leading percentages for the levels of the excited configurations are from calculations by Sugar et al. [1977]. We have listed the percentages in the same coupling schemes as for the isoelectronc spectrum Tm III, with the first percentages being given in $J_{1} j$ schemes based on levels of the $4 f^{12}$ core. Leading percentages in LS coupling are listed in the last column (following the word "or") for the $4 f^{12} 6 p$ levels and for all except three of the even levels. The calculation for $\left(4 f^{12} 5 d+4 f^{12} 6 s\right)$ included the interaction of these configurations; for three levels with eigenvectors having $>20 \%$ configuration mixing, we give as the second percentage the total percentage contribution of the second configuration.

Sugar et al. [1977] obtained the quoted ionization energy by the method outlined by Sugar and Reader [1973]; the new value is more accurate because of the elimination of the uncertainty in the position of the $4 f^{12} 5 d$ configuration.

\section{References}

Bryant, B. W., Johns Hopkins Spectr. Rep. No. 21, 82 pp. (1961). W Bryant, B. W., J. Opt. Soc. Am. 55, 771 (1965).

Kaufman, V., and Sugar, J., J. Opt. Soc. Am. 66, 439 (1976). EL CL

Sugar, J., Kaufman, V., and Spector, N., unpublished material (1977). EL ND CL W IP PT

Sugar, J., and Reader, J., J. Chem. Phys. 59, 2083 (1973). IP

[May 1977] 
Yb IV

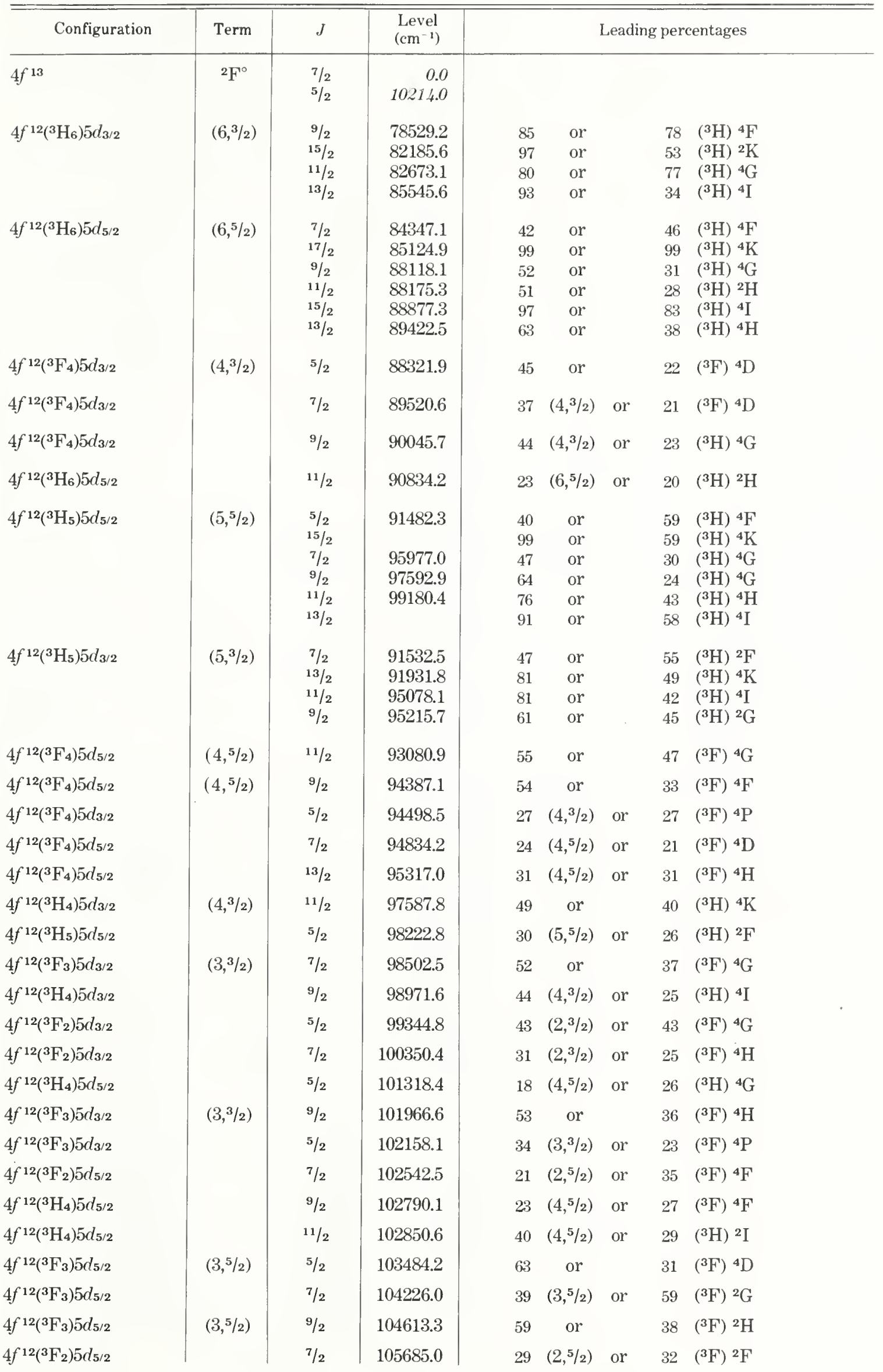


Yb IV-Continued

\begin{tabular}{|c|c|c|c|c|c|c|c|c|}
\hline Configuration & Term & $J$ & $\begin{array}{l}\text { Level } \\
\left(\mathrm{cm}^{-1}\right)\end{array}$ & \multicolumn{5}{|c|}{ Leading percentages } \\
\hline $4 f^{12}\left({ }^{3} \mathrm{H}_{6}\right) 6 s_{1 / 2}$ & $(6,1 / 2)$ & $\begin{array}{l}13 / 2 \\
11 / 2\end{array}$ & $\begin{array}{l}105978.8 \\
106801.7\end{array}$ & $\begin{array}{l}99 \\
77\end{array}$ & or & & $\begin{array}{l}99 \\
22\end{array}$ & $\begin{array}{l}\left({ }^{3} \mathrm{H}\right){ }^{4} \mathrm{H} \\
4 f^{12} 5 d\end{array}$ \\
\hline $4 f^{12}\left({ }^{3} \mathrm{~F}_{3}\right) 5 d_{5 / 2}$ & & $7 / 2$ & 106040.0 & 23 & $(3,5 / 2)$ & or & 20 & $\left({ }^{3} \mathrm{H}\right){ }^{2} \mathrm{G}$ \\
\hline $4 f^{12}\left({ }^{3} \mathrm{~F}_{3}\right) 5 d_{5 / 2}$ & $(3,5 / 2)$ & $11 / 2$ & 106557.7 & 58 & or & & 52 & $\left({ }^{3} \mathrm{~F}\right){ }^{4} \mathrm{H}$ \\
\hline $4 f^{12}\left({ }^{3} \mathrm{~F}_{2}\right) 5 d d_{5 / 2}$ & $(2,5 / 2)$ & $9 / 2$ & 107566.2 & 46 & or & & 28 & $\left({ }^{3} \mathrm{~F}\right){ }^{2} \mathrm{H}$ \\
\hline $4 f^{12}\left({ }^{3} \mathrm{~F}_{2}\right) 5 d_{5 / 2}$ & & $5 / 2$ & 107873.4 & 40 & $(2,5 / 2)$ & or & 46 & $\left({ }^{3} \mathrm{~F}\right){ }^{2} \mathrm{D}$ \\
\hline $4 f^{12}\left({ }^{3} \mathrm{H}_{4}\right) 5 d_{3 / 2}$ & & $9 / 2$ & 109025.2 & 41 & $(4,3 / 2)$ & or & 38 & $\left({ }^{1} \mathrm{G}\right){ }^{2} \mathrm{H}$ \\
\hline $\left.4 f^{12(1} \mathrm{G}_{4}\right) 5 d_{3 / 2}$ & & $7 / 2$ & 110808.7 & 35 & $(4,3 / 2)$ & or & 31 & $\left({ }^{1} \mathrm{G}\right){ }^{2} \mathrm{~F}$ \\
\hline $4 f^{12}\left({ }^{1} \mathrm{G}_{4}\right) 5 d_{5 / 2}$ & & $7 / 2$ & 111550.7 & 34 & $(4,5 / 2)$ & or & 28 & $\left({ }^{3} \mathrm{H}\right){ }^{4} \mathrm{H}$ \\
\hline $4 f^{12}\left({ }^{3} \mathrm{~F}_{4}\right) 6 s_{1 / 2}$ & $(4,1 / 2)$ & $\begin{array}{l}9 / 2 \\
7 / 2\end{array}$ & $\begin{array}{l}112189.4 \\
112772.6\end{array}$ & $\begin{array}{l}64 \\
55\end{array}$ & $\begin{array}{l}\text { or } \\
\text { or }\end{array}$ & & $\begin{array}{l}64 \\
39\end{array}$ & $\begin{array}{l}\left({ }^{3} \mathrm{~F}\right){ }^{4} \mathrm{~F} \\
\left({ }^{3} \mathrm{~F}\right)^{2} \mathrm{~F}\end{array}$ \\
\hline $4 f^{12}\left({ }^{1} \mathrm{D}_{2}\right) 5 d_{3 / 2}$ & & $5 / 2$ & 112679.1 & 21 & $(2,3 / 2)$ & or & 22 & $\left({ }^{1} \mathrm{G}\right){ }^{2} \mathrm{~F}$ \\
\hline $4 f^{12}\left({ }^{1} \mathrm{G}_{4}\right) 5 d_{5 / 2}$ & & $9 / 2$ & 112812.4 & 42 & $(4,5 / 2)$ & or & 31 & $\left({ }^{1} \mathrm{G}\right){ }^{2} \mathrm{G}$ \\
\hline $4 f^{12\left({ }^{3} \mathrm{H}_{5}\right) 6 s_{1 / 2}}$ & $(5,1 / 2)$ & $\begin{array}{l}11 / 2 \\
9 / 2\end{array}$ & $\begin{array}{l}115910.8 \\
115974.6\end{array}$ & $\begin{array}{l}98 \\
98\end{array}$ & $\begin{array}{l}\text { or } \\
\text { or }\end{array}$ & & $\begin{array}{l}66 \\
66\end{array}$ & $\left({ }^{3} \mathrm{H}\right){ }^{4} \mathrm{H}$ \\
\hline $4 f^{12}\left({ }^{3} \mathrm{~F}_{2}\right) 5 d_{3 / 2}$ & & $5 / 2$ & 117026.4 & 18 & $(2,3 / 2)$ & or & 29 & $\left({ }^{3} \mathrm{~F}\right){ }^{2} \mathrm{~F}$ \\
\hline $4 f^{12}\left({ }^{1} \mathrm{D}_{2}\right) 5 d_{5 / 2}$ & & $5 / 2$ & 120328.9 & 32 & $(2,5 / 2)$ & or & 22 & $\left({ }^{1} \mathrm{D}\right){ }^{2} \mathrm{~F}$ \\
\hline $4 f^{12\left({ }^{1} I_{6}\right) 5 d_{3 / 2}}$ & $(6,3 / 2)$ & $9 / 2$ & 120633.5 & 53 & & & 32 & $4 f^{12} 6 s$ \\
\hline $4 f^{12\left({ }^{3} \mathrm{H}_{4}\right) 6 s_{1 / 2}}$ & $\left(4,{ }^{1} / 2\right)$ & $7 / 2$ & 120667.2 & 54 & or & & 54 & $\left({ }^{3} \mathrm{H}\right){ }^{4} \mathrm{H}$ \\
\hline $4 f^{12}\left({ }^{3} \mathrm{P}_{2}\right) 5 d_{5 / 2}$ & & $9 / 2$ & 120773.8 & 34 & $(2,5 / 2)$ & or & 33 & $\left({ }^{3} \mathrm{P}\right){ }^{4} \mathrm{~F}$ \\
\hline $4 f^{12}\left({ }^{3} \mathrm{H}_{4}\right) 6 s_{1 / 2}$ & & $9 / 2$ & 121008.2 & 27 & $\left(4,{ }^{1} / 2\right)$ & & 49 & $4 f^{12} 5 d$ \\
\hline $4 f^{12}\left({ }^{3} \mathrm{~F}_{3}\right) 6 s_{1 / 2}$ & $\left(3,{ }^{1} / 2\right)$ & $\begin{array}{l}5 / 2 \\
7 / 2\end{array}$ & $\begin{array}{l}122476.4 \\
122650.4\end{array}$ & $\begin{array}{l}70 \\
95\end{array}$ & $\begin{array}{l}\text { or } \\
\text { or }\end{array}$ & & $\begin{array}{l}88 \\
63\end{array}$ & $\left({ }^{3} \mathrm{~F}\right){ }^{4} \mathrm{~F}$ \\
\hline $4 f^{12}\left({ }^{3} \mathrm{~F}_{2}\right) 6 s_{1 / 2}$ & $(2,1 / 2)$ & $\begin{array}{l}3 / 2 \\
5 / 2\end{array}$ & $\begin{array}{l}122787.4 \\
123710.5\end{array}$ & $\begin{array}{l}74 \\
47\end{array}$ & $\begin{array}{l}\text { or } \\
\text { or }\end{array}$ & & $\begin{array}{l}74 \\
73\end{array}$ & $\begin{array}{l}\left({ }^{3} \mathrm{~F}\right){ }^{4} \mathrm{~F} \\
\left({ }^{3} \mathrm{~F}\right){ }^{2} \mathrm{~F}\end{array}$ \\
\hline $4 f^{12}\left({ }^{3} \mathrm{P}_{0}\right) 5 d_{5 / 2}$ & & $5 / 2$ & 124939.3 & 24 & $(0,5 / 2)$ & or & 42 & $\left({ }^{3} \mathrm{P}\right){ }^{4} \mathrm{D}$ \\
\hline $4 f^{12}\left({ }^{3} \mathrm{P}_{1}\right) 5 d_{3 / 2}$ & $(1,3 / 2)$ & $5 / 2$ & 126399.3 & 78 & or & & 64 & $\left({ }^{3} \mathrm{P}\right){ }^{4} \mathrm{~F}$ \\
\hline $4 f^{12}\left({ }^{1} \mathrm{D}_{2}\right) 5 d_{5 / 2}$ & & $7 / 2$ & 126867.0 & 29 & $(2,5 / 2)$ & or & 39 & $\left({ }^{3} \mathrm{P}\right){ }^{4} \mathrm{D}$ \\
\hline $4 f^{12}\left({ }^{3} \mathrm{P}_{1}\right) 5 d_{5 / 2}$ & & $7 / 2$ & 128900.2 & 39 & $(1,5 / 2)$ & or & 61 & $\left({ }^{3} \mathrm{P}\right){ }^{4} \mathrm{~F}$ \\
\hline $4 f^{12}\left({ }^{1} \mathrm{G}_{4}\right) 6 s_{1 / 2}$ & $\left(4,{ }^{1} / 2\right)$ & $\begin{array}{l}7 / 2 \\
9 / 2\end{array}$ & $\begin{array}{l}130524.0 \\
130676.9\end{array}$ & $\begin{array}{l}49 \\
51\end{array}$ & $\begin{array}{l}\text { or } \\
\text { or }\end{array}$ & & $\begin{array}{l}49 \\
51\end{array}$ & $\left({ }^{1} \mathrm{G}\right){ }^{2} \mathrm{G}$ \\
\hline $4 f^{12}\left({ }^{3} \mathrm{H}_{6}\right) 6 p_{1 / 2}$ & $\left(6,{ }^{1} / 2\right)^{\circ}$ & $\begin{array}{l}11 / 2 \\
13 / 2\end{array}$ & $\begin{array}{l}152589.1 \\
153208.4\end{array}$ & $\begin{array}{l}97 \\
99\end{array}$ & $\begin{array}{l}\text { or } \\
\text { or }\end{array}$ & & $\begin{array}{l}71 \\
46\end{array}$ & $\begin{array}{l}\left({ }^{3} \mathrm{H}\right){ }^{4} \mathrm{G}^{\circ} \\
\left({ }^{3} \mathrm{H}\right){ }^{2} \mathrm{I}^{\circ}\end{array}$ \\
\hline $4 f^{12\left({ }^{3} \mathrm{~F}_{4}\right) 6 p_{1 / 2}}$ & $\left(4,{ }^{1} / 2\right)^{\circ}$ & $\begin{array}{l}7 / 2 \\
9 / 2\end{array}$ & $\begin{array}{l}158935.7 \\
158939.6\end{array}$ & $\begin{array}{l}62 \\
57\end{array}$ & $\begin{array}{l}\text { or } \\
\text { or }\end{array}$ & & $\begin{array}{l}32 \\
23\end{array}$ & $\begin{array}{l}\left({ }^{3} \mathrm{~F}\right){ }^{4} \mathrm{D}^{\circ} \\
\left({ }^{3} \mathrm{~F}\right){ }^{2} \mathrm{G}^{\circ}\end{array}$ \\
\hline $4 f^{12\left({ }^{3} \mathrm{H}_{6}\right) 6 p 3 / 2}$ & $(6,3 / 2)^{\circ}$ & $\begin{array}{c}15 / 2 \\
9 / 2 \\
11 / 2 \\
13 / 2\end{array}$ & $\begin{array}{l}161110.4 \\
162061.8 \\
162635.1\end{array}$ & $\begin{array}{l}99 \\
60 \\
55 \\
99\end{array}$ & $\begin{array}{l}\text { or } \\
\text { or } \\
\text { or } \\
\text { or }\end{array}$ & & $\begin{array}{l}99 \\
58 \\
32 \\
73\end{array}$ & $\begin{array}{l}\left({ }^{3} \mathrm{H}\right){ }^{4} \mathrm{I}^{\circ} \\
\left({ }^{3} \mathrm{H}\right){ }^{4} \mathrm{G}^{\circ} \\
\left({ }^{3} \mathrm{H}\right){ }^{4} \mathrm{I}^{\circ} \\
\left({ }^{3} \mathrm{H}\right){ }^{4} \mathrm{H}^{\circ}\end{array}$ \\
\hline $4 f^{12}\left({ }^{3} \mathrm{H}_{5}\right) 6 p_{1 / 2}$ & $(5,1 / 2)^{\circ}$ & $\begin{array}{l}11 / 2 \\
9 / 2\end{array}$ & $\begin{array}{l}162991.9 \\
163002.5\end{array}$ & $\begin{array}{l}58 \\
69\end{array}$ & $\begin{array}{l}\text { or } \\
\text { or }\end{array}$ & & $\begin{array}{l}58 \\
76\end{array}$ & $\begin{array}{l}\left({ }^{3} \mathrm{H}\right){ }^{2} \mathrm{H}^{\circ} \\
\left({ }^{3} \mathrm{H}\right){ }^{2} \mathrm{G}^{\circ}\end{array}$ \\
\hline $4 f^{12}\left({ }^{3} \mathrm{H}_{4}\right) 6 p_{1 / 2}$ & $\left(4,{ }^{1} / 2\right)^{\circ}$ & $\begin{array}{l}9 / 2 \\
7 / 2\end{array}$ & $\begin{array}{l}167196.3 \\
167413.4\end{array}$ & $\begin{array}{l}38 \\
48\end{array}$ & $\begin{array}{l}\text { or } \\
\text { or }\end{array}$ & & & $\begin{array}{l}\left({ }^{3} \mathrm{H}\right){ }^{4} \mathrm{I}^{\circ} \\
\left({ }^{3} \mathrm{H}\right){ }^{4} \mathrm{G}^{\circ}\end{array}$ \\
\hline
\end{tabular}


Yb IV-Continued

\begin{tabular}{|c|c|c|c|c|c|c|}
\hline Configuration & Term & $J$ & $\begin{array}{l}\text { Level } \\
\left(\mathrm{cm}^{-1}\right)\end{array}$ & \multicolumn{3}{|c|}{ Leading percentages } \\
\hline $4 f^{12}\left({ }^{3} \mathrm{~F}_{4}\right) 6 p_{3 / 2}$ & $(4,3 / 2)^{\circ}$ & $\begin{array}{c}5 / 2 \\
11 / 2 \\
9 / 2 \\
7 / 2\end{array}$ & $\begin{array}{l}167591.0 \\
168021.4 \\
168092.9 \\
168124.6\end{array}$ & $\begin{array}{l}40 \\
59 \\
44 \\
46\end{array}$ & $\begin{array}{l}\text { or } \\
\text { or } \\
\text { or } \\
\text { or }\end{array}$ & $\begin{array}{ll}28 & \left({ }^{1} \mathrm{G}\right){ }^{2} \mathrm{~F}^{\circ} \\
59 & \left({ }^{3} \mathrm{~F}\right) \\
{ }^{4} \mathrm{G}^{\circ} \\
34 & \left({ }^{3} \mathrm{~F}\right){ }^{2} \mathrm{G}^{\circ} \\
33 & \left({ }^{3} \mathrm{~F}\right){ }^{4} \mathrm{D}^{\circ}\end{array}$ \\
\hline $4 f^{12}\left({ }^{3} \mathrm{~F}_{3}\right) 6 p_{1 / 2}$ & $\left(3,{ }^{1} / 2\right)^{\circ}$ & $\begin{array}{l}5 / 2 \\
7 / 2\end{array}$ & $\begin{array}{l}169279.8 \\
169417.6\end{array}$ & $\begin{array}{l}84 \\
61\end{array}$ & $\begin{array}{l}\text { or } \\
\text { or }\end{array}$ & $\begin{array}{ll}43 & \left({ }^{3} \mathrm{~F}\right){ }^{4} \mathrm{~F}^{\circ} \\
44 & \left({ }^{3} \mathrm{~F}\right){ }^{2} \mathrm{~F}^{\circ}\end{array}$ \\
\hline $4 f^{12\left(3 \mathrm{~F}_{2}\right) 6 p_{1 / 2}}$ & $\left(2,{ }^{1} / 2\right)^{\circ}$ & $\begin{array}{l}3 / 2 \\
5 / 2\end{array}$ & $\begin{array}{l}169733.3 \\
170669.6\end{array}$ & $\begin{array}{l}73 \\
51\end{array}$ & $\begin{array}{l}\text { or } \\
\text { or }\end{array}$ & 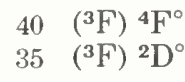 \\
\hline $4 f^{12}\left({ }^{3} \mathrm{H}_{5}\right) 6 p_{3 / 2}$ & $(5,3 / 2)^{\circ}$ & $\begin{array}{c}13 / 2 \\
7 / 2 \\
9 / 2 \\
11 / 2\end{array}$ & $\begin{array}{l}170974.7 \\
171510.4 \\
171867.0 \\
172211.3\end{array}$ & $\begin{array}{r}100 \\
76 \\
96 \\
99\end{array}$ & $\begin{array}{l}\text { or } \\
\text { or } \\
\text { or } \\
\text { or }\end{array}$ & $\begin{array}{ll}67 & \left({ }^{3} \mathrm{H}\right){ }^{4} \mathrm{I}^{\circ} \\
45 & \left({ }^{3} \mathrm{H}\right){ }^{4} \mathrm{G}^{0} \\
34 & \left({ }^{3} \mathrm{H}\right){ }^{4} \mathrm{H} \\
59 & \left({ }^{3} \mathrm{H}\right){ }^{4} \mathrm{H}\end{array}$ \\
\hline $4 f^{12}\left({ }^{3} \mathrm{H}_{4}\right) 6 p_{3 / 2}$ & $(4,3 / 2)^{\circ}$ & $\begin{array}{c}5 / 2 \\
11 / 2 \\
9 / 2 \\
7 / 2\end{array}$ & $\begin{array}{l}175765.1 \\
176127.7 \\
176478.9 \\
176542.8\end{array}$ & $\begin{array}{l}56 \\
57 \\
44 \\
51\end{array}$ & $\begin{array}{l}\text { or } \\
\text { or } \\
\text { or } \\
\text { or }\end{array}$ & 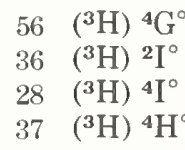 \\
\hline $4 f^{12}\left({ }^{1} \mathrm{G}_{4}\right) 6 p_{1 / 2}$ & & $7 / 2$ & 177796.3 & 45 & $(4,1 / 2)^{\circ}$ or & $29\left({ }^{1} \mathrm{G}\right){ }^{2} \mathrm{~F}^{\circ}$ \\
\hline $4 f^{12}\left({ }^{3} \mathrm{~F}_{3}\right) 6 p_{3 / 2}$ & $(3,3 / 2)^{\circ}$ & $\begin{array}{l}7 / 2 \\
5 / 2 \\
3 / 2 \\
9 / 2\end{array}$ & $\begin{array}{l}178036.7 \\
178049.9 \\
178524.6\end{array}$ & $\begin{array}{l}81 \\
68 \\
60 \\
93\end{array}$ & $\begin{array}{l}\text { or } \\
\text { or } \\
\text { or } \\
\text { or }\end{array}$ & $\begin{array}{ll}37 & \left({ }^{3} \mathrm{~F}\right){ }^{4} \mathrm{~F}^{\circ} \\
42 & \left({ }^{3} \mathrm{~F}\right) \\
79 & \left({ }^{4} \mathrm{D}^{\circ}\right. \\
60 & \left({ }^{3} \mathrm{~F}\right){ }^{4} \mathrm{D}^{\circ} \mathrm{G}^{\circ}\end{array}$ \\
\hline $4 f^{12}\left({ }^{3} \mathrm{~F}_{2}\right) 6 p_{3 / 2}$ & $(2,3 / 2)^{\circ}$ & $\begin{array}{l}5 / 2 \\
1 / 2 \\
3 / 2 \\
7 / 2\end{array}$ & 178684.9 & $\begin{array}{l}49 \\
77 \\
49 \\
68\end{array}$ & $\begin{array}{l}\text { or } \\
\text { or } \\
\text { or } \\
\text { or }\end{array}$ & 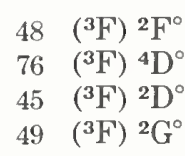 \\
\hline $4 f^{12}\left({ }^{1} \mathrm{D}_{2}\right) 6 p_{1 / 2}$ & & $5 / 2$ & 184497.6 & 34 & $(2,1 / 2)^{\circ}$ or & $30\left({ }^{1} \mathrm{D}\right){ }^{2} \mathrm{~F}^{\circ}$ \\
\hline $4 f^{12}\left({ }^{1} \mathrm{G}_{4}\right) 6 p_{3 / 2}$ & $(4,3 / 2)^{\circ}$ & $\begin{array}{c}5 / 2 \\
11 / 2 \\
7 / 2 \\
9 / 2\end{array}$ & $\begin{array}{l}185869.2 \\
185941.1 \\
185972.2 \\
186461.7\end{array}$ & $\begin{array}{l}48 \\
54 \\
54 \\
53\end{array}$ & $\begin{array}{l}\text { or } \\
\text { or } \\
\text { or } \\
\text { or }\end{array}$ & $\begin{array}{ll}47 & \left({ }^{1} \mathrm{G}\right){ }^{2} \mathrm{~F}^{\circ} \\
54 & \left({ }^{1} \mathrm{G}\right){ }^{2} \mathrm{H}^{\circ} \\
34 & \left({ }^{1} \mathrm{G}\right){ }^{2} \mathrm{G}^{\circ} \\
40 & \left({ }^{1} \mathrm{G}\right){ }^{2} \mathrm{G}^{\circ}\end{array}$ \\
\hline $\mathrm{Yb} \vee\left({ }^{3} \mathrm{H}_{6}\right)$ & Limit & & 351300 & & & \\
\hline
\end{tabular}




\section{LUTETIUM}

\section{Lu I}

71 electrons

Ground state $\left(1 s^{2} 2 s^{2} 2 p^{6} 3 s^{2} 3 p^{6} 3 d^{10} 4 s^{2} 4 p^{6} 4 d^{10} 4 f^{14} 5 s^{2} 5 p^{6}\right) 5 d 6 s^{2}{ }^{2} \mathrm{D}_{3 / 2}$

Ionization energy $43762.39 \pm 0.10 \mathrm{~cm}^{-1}$

$5.42589 \pm 0.00002 \mathrm{eV}$

Identified even configurations

$5 d 6 s^{2}, 5 d^{2} 6 s, 6 s^{2} 7 s-14 s, 6 s^{2} 6 d-34 d, 6 s 6 p^{2}, 5 d 6 s 7 s$

Identified odd configurations

$6 s^{2} 6 p-27 p, 5 d 6 s 6 p, 5 d^{2} 6 p ?, 6 s^{2} 5 f-35 f$

\section{Main References for Levels, Wavelengths, $g$ Values}

In their 1930 paper on the first three spectra of lutetium, Meggers and Scribner noted that "there is no evidence of $f$ electrons playing any part in the production of the spectra; it is concluded that the fourteen $f$ electrons in Lu form a closed shell of considerable stability." We have recognized this stability by writing the ground configurations with the $4 f^{14}$ electrons included with the other electrons of the $N$ shell, instead of with the external electrons. With regard to interactions of the $5 d, 6 s$, and $6 p$ electrons, the Lu spectra give a smooth connection between the rare earths and the Pt $(5 d)$ group. In fact Lu I and Lu II are structurally two of the simplest spectra of the $5 d$ group, rather than rare-earth spectra.

In addition to their discovery of the ground term of Lu I, Meggers and Scribner found 15 odd levels. In 1937 these authors published a more complete description of the arc and spark spectra of lutetium that includes about $250 \mathrm{Lu}$ I lines in the range 2500-10770 $\AA$. Except for the long series of absorption lines measured by Camus and Tomkins (2280-3700 $\AA$ ), this has remained the main source of Lu I wavelengths in the range covered. Klinkenberg's extension of the analysis in 1954 allowed the classification of 124 lines by 51 levels.

Most of the energy levels are taken from Klinkenberg and from Camus and Tomkins [1972], but a number of confirmations, revisions, and extensions of the analysis are based on additional observations. Zeeman-effect measurements by Anderson [1956] and by Pinnington [1963], for example, allowed the confirmation, identification, or revision of several levels, and also some additions. Bovey, Steers, and Wise [1956] observed three infrared transitions that confirmed the positions of the $6 s^{2} 6 p^{2} \mathrm{P}^{\circ}$ levels with respect to the ground term. The 1960 report by these same authors gives about 140 lines between $8578 \AA$ and $24612 \AA$, practically all of which apparently belong to Lu I. The authors were able to classify some 50 of these lines and suggest several confirmations and revisions of the analysis.

The accurate $g$ values for the two levels of the ground term were obtained by Ritter [1962] using the atomic-beam magnetic-resonance technique. The stated error limits are \pm 0.00008 for the ${ }^{2} \mathrm{D}_{3 / 2}$ level and \pm 0.00016 for ${ }^{2} \mathrm{D}_{5 / 2}$.

\section{Even Configurations}

Unfortunately, a number of ambiguities and uncertainties remain in the level systems of both parities. The levels tentatively assigned to the ${ }^{2} \mathrm{D}$ and ${ }^{2} \mathrm{P}$ terms of $5 d^{2} 6 s$ very likely belong to this configuration, but calculations of the even levels, and perhaps additional experimental $g$ values, are needed for more definite term assignments. The $J$ values of several of the $5 d^{2} 6 s$ levels were confirmed or settled by the hfs observations of Horstmann, Nöldeke, and Steudel [1963]. These authors also independently discovered the two levels having $J=5 / 2$ at $24711.19 \mathrm{~cm}^{-1}$ and $25860.76 \mathrm{~cm}^{-1}$, but apparently due to a numerical error gave each value about $3 \mathrm{~cm}^{-1}$ too high. Their value of $30747.18 \mathrm{~cm}^{-1}$ for the ${ }^{2} \mathrm{~S}_{1 / 2}$ level is given here, the wide 
hyperfine splitting of this level having led earlier to the listing of the two components as different levels. The ${ }^{2} \mathrm{~S}_{1 / 2}$ interpretation was confirmed by Pinnington, whose suggested designations for several of the $5 d^{2} 6 s$ and $6 s 6 p^{2}$ levels have been followed. Four of the $5 d^{2} 6 s$ levels and the $6 s^{2}\left({ }^{1} \mathrm{~S}\right) 7 d^{2} \mathrm{D}_{5 / 2}$ level are from Wyart and Verges [1977], who recently began new work on this spectrum.

Klinkenberg's $5 d 6 s\left({ }^{3} \mathrm{D}\right) 7 s^{2} \mathrm{D}$ designation for two levels above $42000 \mathrm{~cm}^{-1}$ is given tentatively. The ${ }^{2} \mathrm{D}$ character of these levels is evidenced by their combinations, as noted by Klinkenberg, and also by the observed perturbation of the $6 s^{2}\left({ }^{1} \mathrm{~S}\right) n d{ }^{2} \mathrm{D}_{3 / 2}$ series [Camus and Tomkins, 1972], apparently due to the level designated $5 d 6 s\left({ }^{3} \mathrm{D}\right) 7 s^{2} \mathrm{D}_{3 / 2}\left(42576.69 \mathrm{~cm}^{-1}\right)$. The finding of the other $5 d 6 s 7 \mathrm{~s}^{2} \mathrm{D}$ term would help in the confirmation of both the $5 d 6 \mathrm{~s} 7 \mathrm{~s}$ configuration assignment and the $5 d 6 s\left({ }^{3} \mathrm{D}\right)$ parental designation of these two known levels.

\section{Odd Configurations}

The eigenvector percentages and assignments for the $\left(5 d 6 s 6 p+6 s^{2} 7 p\right)$ group of levels are taken from Camus and Masmoudi. A few of these levels have total percentages of up to $10 \%$ from the $5 d^{2} 6 p$ configuration, which was also included in the calculation. The inclusion of two levels for the $5 f^{2} \mathrm{~F}^{\circ}$ term appears to have significantly affected only the eigenvector of the level designated $5 d 6 s\left({ }^{3} \mathrm{D}\right) 6 p^{2} \mathrm{~F}_{7 / 2}^{\circ}\left(31751.17 \mathrm{~cm}^{-1}\right)$; the calculated nominal $5 f^{2} \mathrm{~F}_{7 / 2}^{\circ}$ eigenvector has a $39 \%$ contribution from $5 d 6 s 6 p$, and the other eigenvectors indicate that practically all of this contribution comes from $5 d 6 s\left({ }^{3} \mathrm{D}\right) 6 p^{2} \mathrm{~F}_{7 / 2}^{\circ}$. As an estimate of the effect of eliminating the $5 f^{2} \mathrm{~F}^{\circ}$ term from the calculation (see below), we have increased the calculated $53 \%$ $5 d 6 s\left({ }^{3} \mathrm{D}\right) 6 p^{2} \mathrm{~F}_{7 / 2}^{\circ}$ leading component of the affected level by $39 \%$. (The resulting estimate of $92 \%$ purity for this level is followed by a question mark.)

The new $6 s^{2}\left({ }^{1} \mathrm{~S}\right) 7 p^{2} \mathrm{P}_{1 / 2}^{\circ}$ and $5 d 6 s\left({ }^{1} \mathrm{D}\right) 6 p^{2} \mathrm{~F}_{7 / 2}$ levels are from Wyart and Verges [1977]. The eigenvectors for the nominal $6 s^{2}\left({ }^{\mathrm{S}} \mathrm{S}\right) 7 p^{2} \mathrm{P}^{\circ}$ levels have strong admixtures from $5 d 6 s 6 p$ (not shown here). Two eigenvector assignments may not be conclusive, and the $J$ value of the level at $29607.98 \mathrm{~cm}^{-1}$ is given as questionable; Göbel's [1971] level-crossing observations gave a $J$ value of $3 / 2$ for this level whereas Pinnington interpreted the Zeeman pattern of the $3376.50 \AA$ resonance line as giving a $J$ value of $5 / 2$.

\section{Series, Ionization Energy}

All the higher $6 s^{2}\left({ }^{1} \mathrm{~S}\right) n l$ series members of both parities are from the paper by Camus and Tomkins, who photographed the absorption spectrum in the region $2280-3700 \AA$ with the Argonne 30 -foot spectrograph. The $n$ values originally assigned to the $6 s^{2} n f$ series members have been decreased by unity, and the original $5 f^{2} \mathrm{~F}^{\circ}$ levels are omitted as probably not belonging to these series [Kaufman and Sugar, 1973; Camus, 1976]. Camus and Tomkins found significant perturbations of all but the $6 s^{2} n f^{2} \mathrm{~F}_{7 / 2}^{\circ}$ series; the quoted limit was derived by them from this series.

\section{References}

Anderson, J., Thesis, Imperial College, London, 89 pp. (1956). EL ND ZE

Bovey, L. F. H., and Garton, W. R. S., Proc. Phys. Soc. London, Sect. A 67, 291 (1954). W

Bovey, L. F. H., Steers, E. B. M., and Wise, H. S., Proc. Phys. Soc. London, Sect. A 69, 783 (1956). EL CL W

Bovey, L., Steers, E. B. M., and Wise, H. S., U. K. Atomic Energy Research Establishment Rep. AERE-R 3225, Harwell, 8 pp. (1960). EL CL W

Camus, P., Thesis, Univ. Paris-Sud, Orsay, 265 pp. (1971). EL CL W IP SF

Camus, P., private communication (1976).

Camus, P., and Masmoudi, K., Physica (Utrecht) 60, 513 (1972). EL ND ZE PT

Camus, P., and Tomkins, F. S., J. Phys. (Paris) 33, 197 (1972). EL ND CL W IP

Göbel, L. H., Z. Naturforsch., Teil A 25, 1401 (1970). ZE Hfs

Göbel, L. H., Z. Naturforsch., Teil A 26, 1559 (1971). ND Hfs

Horstmann, U., Nöldeke, G., and Steudel, A., Ann. Phys. (Leipzig) [7] 12, 14 (1963). EL ND CL Hfs

Kaufman, V., and Sugar, J., J. Opt. Soc. Am. 63, 1168 (1973). EL ND

King, A. S., Astrophys. J. 74, 328 (1931). W Hfs

Klinkenberg, P. F. A., Physica (Utrecht) 21, 53 (1954). EL ND CL IP 
Masmoudi, K., Thesis, Univ. Paris-Sud, Orsay, 130 pp. (1972). ND PT

Meggers, W. F., and Scribner, B. F., J.Res. Nat. Bur. Stand. (U.S.) 5, 73 (1930). EL CL W

Meggers, W. F., and Seribner, B. F., J. Res. Nat. Bur. Stand. (U.S.) 19, 31 (1937). W

Pinnington, E. H., Can. J. Phys. 41, 1294 (1963). EL ND CL ZE Hfs

Ritter, G. J., Phys. Rev. 126, 240 (1962). ZE Hfs

Wyart, J. F., and Verges, J., unpublished material (1977). EL

[October 1976]

Lu I, Even Parity

Lu I, Even Parity

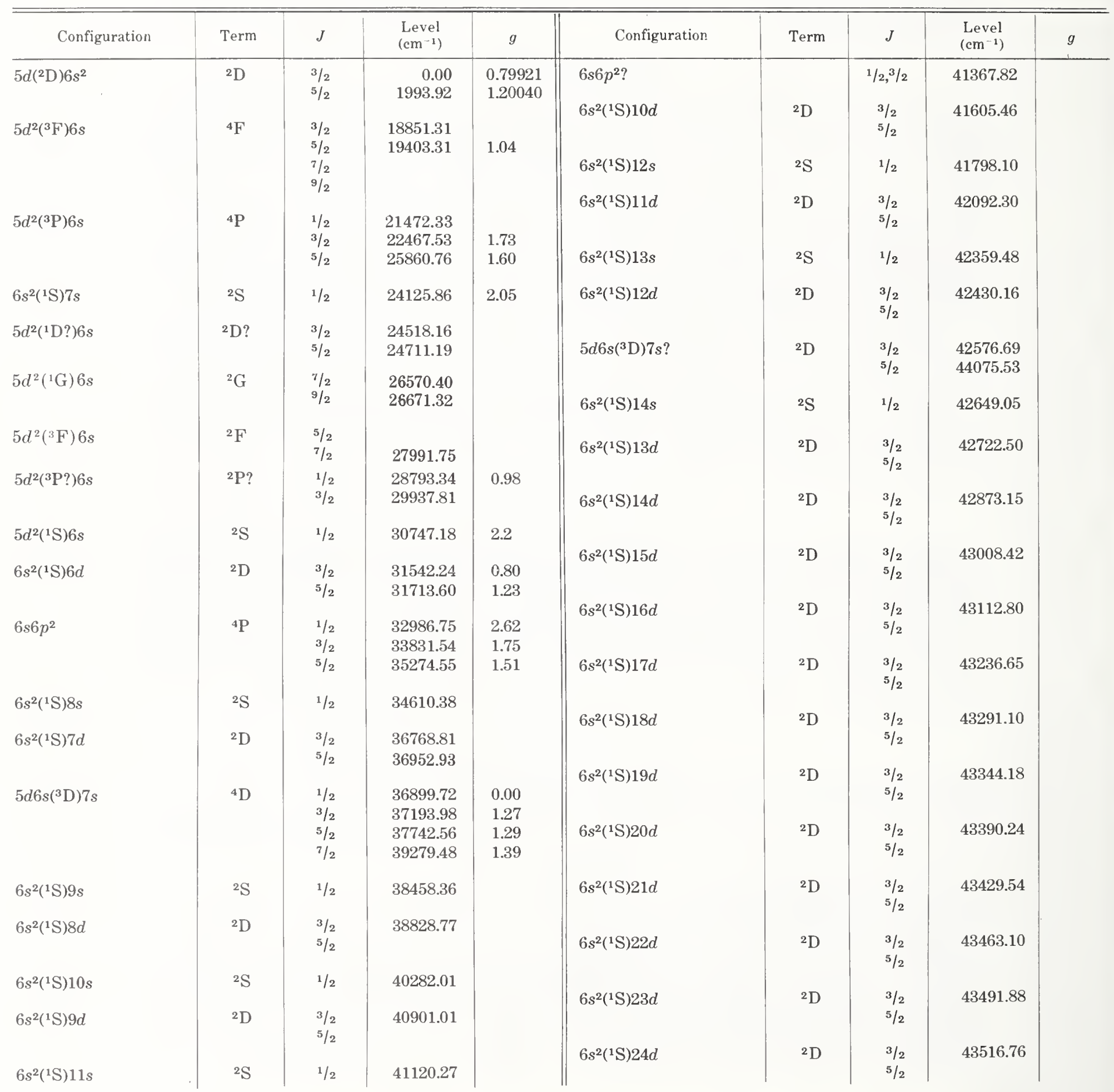


Lu I, Even Parity—Continued

Lu I, Even Parity_Continued

\begin{tabular}{|c|c|c|c|c|c|c|c|c|c|}
\hline Configuration & Term & $J$ & $\begin{array}{l}\text { Level } \\
\left(\mathrm{cm}^{-1}\right)\end{array}$ & $g$ & Configuration & Term & $J$ & $\begin{array}{l}\text { Level } \\
\left(\mathrm{cm}^{-1}\right)\end{array}$ & $g$ \\
\hline $6 s^{2}\left({ }^{1} \mathrm{~S}\right) 25 d$ & ${ }^{2} \mathrm{D}$ & $\begin{array}{l}3 / 2 \\
5 / 2\end{array}$ & 43538.42 & & $6 s^{2}\left({ }^{1} \mathrm{~S}\right) 31 d$ & ${ }^{2} \mathrm{D}$ & $\begin{array}{l}3 / 2 \\
5 / 2\end{array}$ & 43623.82 & \\
\hline $6 s^{2}\left({ }^{1} \mathrm{~S}\right) 26 d$ & ${ }^{2} \mathrm{D}$ & $\begin{array}{l}3 / 2 \\
5 / 2\end{array}$ & 43557.30 & & $6 s^{2}\left({ }^{1} \mathrm{~S}\right) 32 d$ & ${ }^{2} \mathrm{D}$ & $\begin{array}{l}3 / 2 \\
5 / 2\end{array}$ & 43633.23 & \\
\hline $6 s^{2}\left({ }^{1} \mathrm{~S}\right) 27 d$ & ${ }^{2} \mathrm{D}$ & $\begin{array}{l}3 / 2 \\
5 / 2\end{array}$ & 43573.94 & & $6 s^{2}\left({ }^{1} \mathrm{~S}\right) 33 d$ & ${ }^{2} \mathrm{D}$ & $\begin{array}{l}3 / 2 \\
5 / 2\end{array}$ & 43641.79 & \\
\hline $6 s^{2}\left({ }^{1} \mathrm{~S}\right) 28 d$ & ${ }^{2} \mathrm{D}$ & $\begin{array}{l}3 / 2 \\
5 / 2\end{array}$ & 43588.68 & & $6 s^{2}\left({ }^{1} \mathrm{~S}\right) 34 d$ & ${ }^{2} \mathrm{D}$ & $\begin{array}{l}3 / 2 \\
5 / 2\end{array}$ & 43649.10 & \\
\hline $6 s^{2}\left({ }^{1} \mathrm{~S}\right) 29 d$ & ${ }^{2} \mathrm{D}$ & $\begin{array}{l}3 / 2 \\
5 / 2\end{array}$ & 43601.73 & & --০-০-০-০- & Limit & & 4376239 & \\
\hline $6 s^{2}\left({ }^{1} \mathrm{~S}\right) 30 d$ & ${ }^{2} \mathrm{D}$ & $\begin{array}{l}3 / 2 \\
5 / 2\end{array}$ & 43613.30 & & & & & & \\
\hline
\end{tabular}

Lu I, Odd Parity

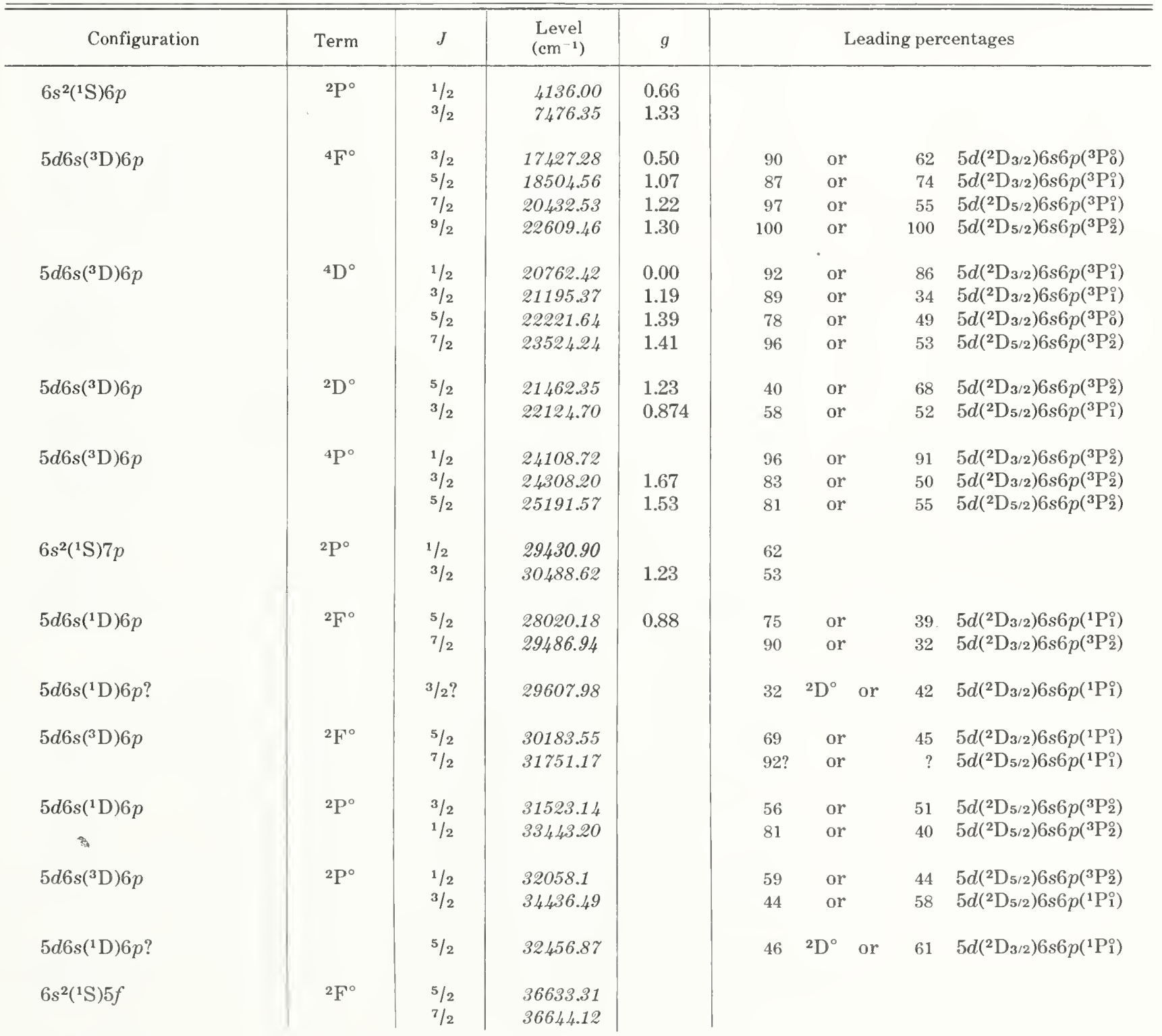


Lu I, Odd Parity-Continued

Lu I, Odd Parity-Continued

\begin{tabular}{|c|c|c|c|c|c|c|c|c|}
\hline Configuration & Term & $J$ & $\begin{array}{l}\text { Level } \\
\left(\mathrm{cm}^{-1}\right)\end{array}$ & $g$ & Configuration & Term & $J$ & $\begin{array}{l}\text { Level } \\
\left(\mathrm{cm}^{-1}\right)\end{array}$ \\
\hline $6 s^{2}\left({ }^{1} \mathrm{~S}\right) 8 p$ & ${ }^{2} \mathrm{P}^{\circ}$ & $\begin{array}{l}1 / 2 \\
3 / 2\end{array}$ & $\begin{array}{l}36808.76 \\
37131.38\end{array}$ & & $6 s^{2}\left({ }^{1} \mathrm{~S}\right) 19 p$ & ${ }^{2} \mathrm{P}^{\circ}$ & $\begin{array}{l}1 / 2 \\
3 / 2\end{array}$ & 43258.71 \\
\hline $6 s^{2}\left({ }^{1} \mathrm{~S}\right) 6 f$ & ${ }^{2} \mathrm{~F}^{\circ}$ & $\begin{array}{l}5 / 2 \\
7 / 2\end{array}$ & $\begin{array}{l}39212.61 \\
39220.17\end{array}$ & & $6 s^{2}\left({ }^{1} \mathrm{~S}\right) 16 f$ & ${ }^{2} \mathrm{~F}^{\circ}$ & $\begin{array}{l}5 / 2 \\
7 / 2\end{array}$ & 43267.57 \\
\hline $6 s^{2}\left({ }^{1} \mathrm{~S}\right) 9 p$ & ${ }^{2} \mathrm{P}^{\circ}$ & $\begin{array}{l}1 / 2 \\
3 / 2\end{array}$ & $\begin{array}{l}39321.96 \\
39424.68\end{array}$ & & $6 s^{2}\left({ }^{1} \mathrm{~S}\right) 17 f$ & ${ }^{2} \mathrm{~F}^{\circ}$ & $\begin{array}{l}7 / 2 \\
5 / 2\end{array}$ & $\begin{array}{l}43327.84 \\
43332.57\end{array}$ \\
\hline $5 d^{2} 6 p ?$ & & $5 / 2$ & 40558.63 & & $6 s^{2}\left({ }^{1} \mathrm{~S}\right) 20 p$ & ${ }^{2} \mathrm{P}^{\circ}$ & $\begin{array}{l}1 / 2 \\
3 / 2\end{array}$ & $\begin{array}{l}43328.46 \\
43336.86\end{array}$ \\
\hline $6 s^{2}\left({ }^{1} \mathrm{~S}\right) 7 f$ & ${ }^{2} \mathrm{~F}^{\circ}$ & $\begin{array}{l}7 / 2 \\
5 / 2\end{array}$ & $\begin{array}{l}40619.01 \\
40626.82\end{array}$ & & $6 s^{2}\left({ }^{1} \mathrm{~S}\right) 18 f$ & ${ }^{2} \mathrm{~F}^{\circ}$ & $\begin{array}{l}7 / 2 \\
5 / 2\end{array}$ & $\begin{array}{l}43377.74 \\
43379.31\end{array}$ \\
\hline $6 s^{2}\left({ }^{1} \mathrm{~S}\right) 10 p$ & ${ }^{2} \mathrm{P}^{\circ}$ & $\begin{array}{l}1 / 2 \\
3 / 2\end{array}$ & $\begin{array}{l}40661.02 \\
40735.33\end{array}$ & & $6 s^{2}\left({ }^{1} \mathrm{~S}\right) 21 p$ & ${ }^{2} \mathrm{P}^{\circ}$ & $\begin{array}{l}1 / 2 \\
3 / 2\end{array}$ & 43384.56 \\
\hline $6 s^{2}\left({ }^{1} \mathrm{~S}\right) 8 f$ & ${ }^{2} \mathrm{~F}^{\circ}$ & $\begin{array}{l}5 / 2 \\
7 / 2\end{array}$ & $\begin{array}{l}41456.26 \\
41460.08\end{array}$ & & $6 s^{2}\left({ }^{1} \mathrm{~S}\right) 19 f$ & ${ }^{2} F^{\circ}$ & $\begin{array}{l}7 / 2 \\
7 / 2\end{array}$ & $\begin{array}{l}43419.54 \\
43420.16\end{array}$ \\
\hline $6 s^{2}\left({ }^{1} \mathrm{~S}\right) 11 p$ & ${ }^{2} \mathrm{P}^{\circ}$ & $\begin{array}{l}1 / 2 \\
3 / 2\end{array}$ & $\begin{array}{l}41487.02 \\
41533.30\end{array}$ & & $6 s^{2}\left({ }^{1} \mathrm{~S}\right) 22 p$ & ${ }^{2} \mathrm{P}^{\circ}$ & $\begin{array}{l}1 / 2 \\
3 / 2\end{array}$ & $\begin{array}{l}43423.85 \\
43425.08\end{array}$ \\
\hline $6 s^{2}\left({ }^{1} \mathrm{~S}\right) 9 f$ & ${ }^{2} \mathrm{~F}^{\circ}$ & $\begin{array}{l}5 / 2 \\
7 / 2\end{array}$ & $\begin{array}{l}42000.72 \\
42006.00\end{array}$ & & $6 s^{2}\left({ }^{1} \mathrm{~S}\right) 20 f$ & ${ }^{2} \mathrm{~F}^{\circ}$ & $\begin{array}{l}7 / 2 \\
5 / 2\end{array}$ & $\begin{array}{l}43454.87 \\
43455.08\end{array}$ \\
\hline $6 s^{2}\left({ }^{1} \mathrm{~S}\right) 12 p$ & ${ }^{2} \mathrm{P}^{\circ}$ & $\begin{array}{l}1 / 2 \\
3 / 2\end{array}$ & $\begin{array}{l}42021.84 \\
42052.89\end{array}$ & & $6 s^{2}\left({ }^{1} \mathrm{~S}\right) 23 p$ & ${ }^{2} \mathrm{P}^{\circ}$ & $\begin{array}{l}1 / 2 \\
3 / 2\end{array}$ & $\begin{array}{r}43457.65 \\
43459.47\end{array}$ \\
\hline $6 s^{2}\left({ }^{1} \mathrm{~S}\right) 10 f$ & ${ }^{2} \mathrm{~F}^{\circ}$ & $\begin{array}{l}5 / 2 \\
7 / 2\end{array}$ & $\begin{array}{l}42372.94 \\
42376.66\end{array}$ & & $6 s^{2}\left({ }^{1} \mathrm{~S}\right) 21 f$ & ${ }^{2} \mathrm{~F}^{\circ}$ & $\begin{array}{l}5 / 2 \\
7 / 2\end{array}$ & $\begin{array}{l}43485.01 \\
43485.01\end{array}$ \\
\hline $6 s^{2}\left({ }^{1} \mathrm{~S}\right) 13 p$ & ${ }^{2} \mathrm{P}^{\circ}$ & $\begin{array}{l}1 / 2 \\
3 / 2\end{array}$ & $\begin{array}{l}42386.76 \\
42414.79\end{array}$ & & $6 s^{2}\left({ }^{1} \mathrm{~S}\right) 24 p$ & ${ }^{2} \mathrm{P}^{\circ}$ & $\begin{array}{l}1 / 2 \\
3 / 2\end{array}$ & $\begin{array}{l}43487.15 \\
43488.91\end{array}$ \\
\hline $6 s^{2}\left({ }^{1} \mathrm{~S}\right) 11 f$ & ${ }^{2} \mathrm{~F}^{\circ}$ & $\begin{array}{l}5 / 2 \\
7 / 2\end{array}$ & $\begin{array}{l}42638.48 \\
42642.05\end{array}$ & & $6 s^{2}\left({ }^{1} \mathrm{~S}\right) 22 f$ & ${ }^{2} \mathrm{~F}^{\circ}$ & $\begin{array}{l}5 / 2 \\
7 / 2\end{array}$ & $\begin{array}{l}43510.81 \\
43510.93\end{array}$ \\
\hline $6 s^{2}\left({ }^{1} \mathrm{~S}\right) 14 p$ & ${ }^{2} \mathrm{P}^{\circ}$ & $\begin{array}{l}1 / 2 \\
3 / 2\end{array}$ & $\begin{array}{l}42657.69 \\
42667.06\end{array}$ & & $6 s^{2}\left({ }^{1} \mathrm{~S}\right) 25 p$ & ${ }^{2} \mathrm{P}^{\circ}$ & $\begin{array}{l}1 / 2 \\
3 / 2\end{array}$ & $\begin{array}{l}43512.65 \\
43514.29\end{array}$ \\
\hline $6 s^{2}\left({ }^{1} \mathrm{~S}\right) 12 f$ & ${ }^{2} \mathrm{~F}^{\circ}$ & $\begin{array}{l}5 / 2 \\
7 / 2\end{array}$ & $\begin{array}{l}42834.43 \\
42837.99\end{array}$ & & $6 s^{2}\left({ }^{1} \mathrm{~S}\right) 23 f$ & ${ }^{2} \mathrm{~F}^{\circ}$ & $\begin{array}{l}5 / 2 \\
7 / 2\end{array}$ & $\begin{array}{l}43533.18 \\
43533.38\end{array}$ \\
\hline $6 s^{2}\left({ }^{1} \mathrm{~S}\right) 15 p$ & ${ }^{2} \mathrm{P}^{\circ}$ & $\begin{array}{l}1 / 2 \\
3 / 2\end{array}$ & $\begin{array}{l}42848.31 \\
42855.91\end{array}$ & & $6 s^{2}\left({ }^{1} \mathrm{~S}\right) 26 p$ & ${ }^{2} \mathrm{P}^{\circ}$ & $\begin{array}{l}1 / 2 \\
3 / 2\end{array}$ & $\begin{array}{l}43534.90 \\
43536.23\end{array}$ \\
\hline $6 s^{2}\left({ }^{1} \mathrm{~S}\right) 13 f$ & ${ }^{2} \mathrm{~F}^{\circ}$ & $\begin{array}{l}5 / 2 \\
7 / 2\end{array}$ & $\begin{array}{l}42982.79 \\
42986.69\end{array}$ & & $6 s^{2}\left({ }^{1} \mathrm{~S}\right) 24 f$ & ${ }^{2} \mathrm{~F}^{\circ}$ & $\begin{array}{l}5 / 2 \\
7 / 2\end{array}$ & $\begin{array}{l}43552.71 \\
43552.95\end{array}$ \\
\hline $6 s^{2}\left({ }^{1} \mathrm{~S}\right) 16 p$ & ${ }^{2} \mathrm{P}^{\circ}$ & $\begin{array}{l}1 / 2 \\
3 / 2\end{array}$ & 43002.55 & & $6 s^{2}\left({ }^{1} \mathrm{~S}\right) 27 p$ & ${ }^{2} \mathrm{P}^{\circ}$ & $\begin{array}{l}1 / 2 \\
3 / 2\end{array}$ & 43555.28 \\
\hline $6 s^{2}\left({ }^{1} \mathrm{~S}\right) 14 f$ & ${ }^{2} \mathrm{~F}^{\circ}$ & $\begin{array}{l}5 / 2 \\
7 / 2\end{array}$ & $\begin{array}{l}43098.15 \\
43102.31\end{array}$ & & $6 s^{2}\left({ }^{1} \mathrm{~S}\right) 25 f$ & ${ }^{2} \mathrm{~F}^{\circ}$ & $\begin{array}{l}5 / 2 \\
7 / 2\end{array}$ & $\begin{array}{l}43569.85 \\
43570.13\end{array}$ \\
\hline $6 s^{2}\left({ }^{1} \mathrm{~S}\right) 17 p$ & ${ }^{2} \mathrm{P}^{\circ}$ & $\begin{array}{l}1 / 2 \\
3 / 2\end{array}$ & 43111.69 & & $6 s^{2}\left({ }^{1} \mathrm{~S}\right) 26 f$ & ${ }^{2} \mathrm{~F}^{\circ}$ & $\begin{array}{l}5 / 2 \\
7 / 2\end{array}$ & $\begin{array}{l}43584.98 \\
43585.27\end{array}$ \\
\hline $6 s^{2}\left({ }^{1} \mathrm{~S}\right) 15 f$ & ${ }^{2} \mathrm{~F}^{\circ}$ & $\begin{array}{l}5 / 2 \\
7 / 2\end{array}$ & $\begin{array}{r}43188.28 \\
43193.85\end{array}$ & & $6 s^{2}\left({ }^{1} \mathrm{~S}\right) 27 f$ & ${ }^{2} \mathrm{~F}^{\circ}$ & $\begin{array}{l}5 / 2 \\
7 / 2\end{array}$ & $\begin{array}{l}43598.40 \\
43598.68\end{array}$ \\
\hline $6 s^{2}\left({ }^{1} \mathrm{~S}\right) 18 p$ & ${ }^{2} \mathrm{P}^{\circ}$ & $\begin{array}{l}1 / 2 \\
3 / 2\end{array}$ & 43197.07 & & $6 s^{2}\left({ }^{1} \mathrm{~S}\right) 28 f$ & ${ }^{2} \mathrm{~F}^{\circ}$ & $\begin{array}{l}5 / 2 \\
7 / 2\end{array}$ & $\begin{array}{l}43610.34 \\
43610.66\end{array}$ \\
\hline
\end{tabular}


Lu I, Odd Parity—Continued

Lu I, Odd Parity—Continued

\begin{tabular}{|c|c|c|c|c|c|c|c|c|c|}
\hline Configuration & Term & $J$ & $\begin{array}{l}\text { Level } \\
\left(\mathrm{cm}^{-1}\right)\end{array}$ & $g$ & Configuration & Term & $J$ & $\begin{array}{l}\text { Level } \\
\left(\mathrm{cm}^{-1}\right)\end{array}$ & $g$ \\
\hline $6 s^{2}\left({ }^{1} \mathrm{~S}\right) 29 f$ & ${ }^{2} \mathrm{~F}^{\circ}$ & $\begin{array}{l}5 / 2 \\
7 / 2\end{array}$ & $\begin{array}{l}43621.02 \\
43621.28\end{array}$ & & $6 s^{2}\left({ }^{1} \mathrm{~S}\right) 34 f$ & ${ }^{2} \mathrm{~F}^{\circ}$ & $\begin{array}{l}5 / 2 \\
7 / 2\end{array}$ & $\begin{array}{l}43660.81 \\
43660.95\end{array}$ & \\
\hline $6 s^{2}\left({ }^{1} \mathrm{~S}\right) 30 f$ & ${ }^{2} \mathrm{~F}^{\circ}$ & $\begin{array}{l}5 / 2 \\
7 / 2\end{array}$ & $\begin{array}{r}43630.64 \\
43630.88\end{array}$ & & $6 s^{2}\left({ }^{1} \mathrm{~S}\right) 35 f$ & ${ }^{2} \mathrm{~F}^{\circ}$ & $\begin{array}{l}5 / 2 \\
7 / 2\end{array}$ & $\begin{array}{l}43666.72 \\
43666.85\end{array}$ & \\
\hline $6 s^{2}\left({ }^{1} \mathrm{~S}\right) 31 f$ & ${ }^{2} \mathrm{~F}^{\circ}$ & $\begin{array}{l}5 / 2 \\
7 / 2\end{array}$ & $\begin{array}{r}43639.31 \\
43639.58\end{array}$ & & & & & & \\
\hline $6 s^{2}\left({ }^{1} \mathrm{~S}\right) 32 f$ & ${ }^{2} \mathrm{~F}^{\circ}$ & $\begin{array}{l}5 / 2 \\
7 / 2\end{array}$ & $\begin{array}{r}43647.13 \\
43647.34\end{array}$ & & Lu II $\left({ }^{1} \mathrm{So}_{0}\right)$ & Limit & & 43762.39 & \\
\hline $6 s^{2}\left({ }^{1} \mathrm{~S}\right) 33 f$ & ${ }^{2} \mathrm{~F}^{\circ}$ & $\begin{array}{l}5 / 2 \\
7 / 2\end{array}$ & $\begin{array}{r}43654.23 \\
43654.47\end{array}$ & & & & & & \\
\hline
\end{tabular}




\section{Lu II}

( $\mathrm{Yb}$ I sequence; 70 electrons)

$Z=71$

Ground state $\left(1 s^{2} 2 s^{2} 2 p^{6} 3 s^{2} 3 p^{6} 3 d^{10} 4 s^{2} 4 p^{6} 4 d^{10} 4 f^{14} 5 s^{2} 5 p^{6}\right) 6 s^{2}{ }^{1} \mathrm{~S}_{0}$

Ionization energy $112000 \pm 3000 \mathrm{~cm}^{-1}$

$13.9 \pm 0.4 \mathrm{eV}$

Sometime before 1935 W. F. Meggers supplied H. N. Russell at Princeton with an extension of Meggers' and Scribner's original analysis of Lu II [1930]. Most of the levels here are taken from an unpublished term list prepared in 1935 from Meggers' analysis [Meggers, 1935]. The values were given to two decimal places, except for four of the higher even levels. (The probable error for all of the levels appears to be about $\pm 0.10 \mathrm{~cm}^{-1}$.)

The remaining levels are taken from the 1956 report by Bovey and Pearse. They correctly designated the $5 d^{2}{ }^{1} \mathrm{D}_{2}$ level, added the $5 d^{2}{ }^{3} \mathrm{P}_{0}, 5 d 6 p{ }^{1} \mathrm{P}_{1}^{\circ}$, and $6 s 7 s^{3} \mathrm{~S}_{1}$ levels, and also found a number of other levels beginning above $47000 \mathrm{~cm}^{-1}$. A number of tentative levels listed by Bovey and Pearse have been omitted, pending confirmation by more complete observations of the spectrum. (The omitted levels lie in the range from $47447 \mathrm{~cm}^{-1}$ to $83994 \mathrm{~cm}^{-1}$.) Bovey and Pearse noted that these levels needed confirmation, since they were mainly based on very weak lines. The possible even level at $79546.8 \mathrm{~cm}^{-1}$ was listed tentatively by Meggers and by Bovey and Pearse. The provisional $6 s 6 d{ }^{3} \mathrm{D}$ and $5 d 7 s^{3} \mathrm{D}$ terms follow the arrangement of Bovey and Pearse.

\section{Theoretical Interpretation}

The eigenvector percentages are taken from Goldschmidt's calculations of the $(5 d+6 s)^{2}$ and $(6 s 6 p+5 d 6 p)$ configurations [1968]. The percentages for the $(5 d+6 s)^{2}$ group show that $5 d^{2}{ }^{3} \mathrm{P}_{2}$ and $5 d^{2}{ }^{1} \mathrm{D}_{2}$ have little meaning as designations because of the strong mixture of these terms in two of the eigenvectors (levels at 36098.18 and $38574.94 \mathrm{~cm}^{-1}$ ); we have retained the usual ${ }^{3} \mathrm{P}_{2}$ designation for one of the levels, mainly to fill out the $5 d^{2}{ }^{3} \mathrm{P}$ term. The experimental $g$ factors for the two levels are not sufficiently reliable to afford a test of the eigenvectors (see below).

A tentative value of $37826.8 \mathrm{~cm}^{-1}$ suggested by Anderson [1956] for the $5 d^{2}{ }^{1} \mathrm{G}_{4}$ level has been omitted. The experimental evidence for the level is not convineing, and a large perturbation would be required for the level to be so far below the position near $39000 \mathrm{~cm}^{-1}$ predicted by Goldschmidt.

Goldschmidt [1967] discusses the interaction between the $6 s 6 p$ and $5 d 6 p$ configurations. A calculated repulsion of $8500 \mathrm{~cm}^{-1}$ between the two ${ }^{1} \mathrm{P}^{\circ}$ levels is reflected in the strong mixture of configurations in the two corresponding eigenvectors (levels at 38223 and $59122 \mathrm{~cm}^{-1}$ ). Calculations for several two-electron spectra, including Lu II, were important in obtaining reliable values of theoretical parameters needed for comparison, or for use as initial values in calculations of more complex rare-earth spectra.

\section{$g$ Values}

The $g$ values are from Zeeman-effect measurements by Anderson [1956] and by Pinnington [1963], Anderson's observations having included patterns confirming all the known levels with $J=0$. Pinnington's $g$ values are listed for the seven levels involved in his measurements, the stated uncertainties being \pm 0.02 or \pm 0.03 . Three of the $g$ values are followed by colons to indicate that each is well outside the range of allowed values (for the pertinent $J$ value) unless interactions with quintet or higher multiplicity terms are significant. (Such terms would arise from excited-core configurations.) Pinnington's $g$ value of 1.41 for the $5 d 6 s^{3} \mathrm{D}_{3}$ level appears to be confirmed to about \pm 0.02 by the patterns for three different lines, and his $g$ value of $1.66 \pm 0.02$ for the $6 s 6 p{ }^{3} \mathrm{P}_{2}^{\circ}$ level was obtained from one of these same patterns; the theoretical upper limits for the $g$ values of these two levels (higher multiplicities excluded) are 1.33 and 1.50, respectively. The possible effects of hfs on these patterns should 
perhaps be investigated. Anderson's $g$ value of 0.43 for the $5 d 6 p{ }^{3} \mathrm{D}_{1}^{\circ}$ level (theoretical lower limit, 0.50) may be less accurate than Goldschmidt's calculated value of 0.58 .

The three $g$ values followed by question marks are dubious. Anderson's value of 1.37 for the $g$ values of both the $5 d^{2}$ level at $36098 \mathrm{~cm}^{-1}(J=2)$ and the $5 d 6 p{ }^{1} \mathrm{P}_{1}^{\circ}$ level was based entirely on a simple triplet Zeeman pattern observed for the transition between these levels. The $g$ value of 1.49 for the (nominal) $5 d^{2}{ }^{3} \mathrm{P}_{2}$ level was then obtained from another triplet pattern by assuming the $g$ value of 1.37 for the $5 d 6 p{ }^{1} \mathrm{P}_{1}^{\circ}$ level. Goldschmidt's calculations predict $g$ values of about 1.25 for each of the two $5 d^{2}$ levels in question, and a value of 1.01 for the $5 d 6 p{ }^{1} \mathrm{P}_{1}^{\circ}$ level. Anderson's $g$ values for the two $5 d^{2}$ levels cause a large deviation from the $g$-sum rule for the $(5 d+6 s)^{2}$ levels having $J=2$, and his $g$ value for the $5 d 6 p{ }^{1} \mathrm{P}_{1}^{\circ}$ level cannot be accurate if the level is correctly assigned.

\section{Line Lists, Ionization Energy}

New and more complete observations of Lu I and Lu II are needed. The basic line list for Lu II at present is the one published by Meggers and Scribner in 1937, which gives 370 lines in the range 2065-10758 $\AA$. Bovey and Pearse give a complete list of the classified lines, and also include tables showing the intensities of the combinations of each level.

The ionization energy is from Sugar and Reader [1965].

\section{References}

Anderson, J., Thesis, Imperial College, London, 89 pp. (1956). EL ND ZE

Bovey, L. F. H., and Pearse, R. W. B., U. K. Atomic Energy Research Establishment Rep. AERE C/R 1976, Harwell, 19 pp. (1956). EL CL IP

Goldschmidt, Z. B., Colloq. Int. C.N.R.S. No. 164, 365 (1967). PT

Goldschmidt, Z. B., Thesis, Hebrew Univ. Jerusalem, Israel, 487 pp. (1968). PT

King, A. S., Astrophys. J. 74, 328 (1931). W $\mathrm{H}$ fs

Meggers, W. F., unpublished material (1935). These data were assembled by C. E. Moore at Princeton. EL CL

Meggers, W. F., and Scribner, B. F., J. Res. Nat. Bur. Stand. (U.S.) 5, 73 (1930). EL CL W

Meggers, W. F., and Scribner, B. F., J. Res. Nat. Bur. Stand. (U.S.) 19, 31 (1937). W

Pinnington, E. H., Can. J. Phys. 41, 1305 (1963). ZE

Sugar, J., and Reader, J., J. Opt. Soc. Am. 55, 1286 (1965). IP

[July 1976]

Lu II

\begin{tabular}{|c|c|c|c|c|c|c|c|}
\hline Configuration & Term & $J$ & $\begin{array}{l}\text { Level } \\
\left(\mathrm{cm}^{-1}\right)\end{array}$ & $g$ & & \multicolumn{2}{|c|}{ Leading percentages } \\
\hline $6 s^{2}$ & ${ }^{1} \mathrm{~S}$ & 0 & 0.00 & & 98 & \multirow{11}{*}{9} & \multirow{8}{*}{$5 d^{2}{ }^{1} \mathrm{D}$} \\
\hline \multirow[t]{3}{*}{$5 d 6 s$} & ${ }^{3} \mathrm{D}$ & 1 & 11796.24 & 0.52 & 100 & & \\
\hline & & 2 & 12435.32 & 1.14 & 94 & & \\
\hline & & 3 & 14199.08 & 1.41: & 100 & & \\
\hline $5 d 6 s$ & ${ }^{1} \mathrm{D}$ & 2 & 17332.58 & 1.09 & 85 & & \\
\hline \multirow[t]{3}{*}{$6 s 6 p$} & ${ }^{3} \mathrm{P}^{\circ}$ & 0 & 27264.40 & & 99 & & \\
\hline & & 1 & 28503.16 & 1.51 & 94 & & \\
\hline & & 2 & 32453.26 & 1.66: & 98 & & \\
\hline \multirow[t]{3}{*}{$5 d^{2}$} & ${ }^{3} \mathrm{~F}$ & 2 & 29406.70 & 0.66 & 97 & & \\
\hline & & 3 & 30889.09 & 1.05 & 100 & & \\
\hline & & 4 & 32503.62 & 1.27 & 98 & & \\
\hline \multirow[t]{3}{*}{$5 d^{2}$} & ${ }^{3} \mathrm{P}$ & 0 & 35652.1 & & 98 & & \\
\hline & & 1 & 36557.05 & 1.41 & 100 & & \\
\hline & & 2 & 38574.94 & $1.49 ?$ & 51 & 44 & ${ }^{1} \mathrm{D}$ \\
\hline $5 d^{2}$ & & 2 & 36098.18 & $1.37 ?$ & $49 \quad 3 \mathrm{P}$ & 45 & ${ }^{1} \mathrm{D}$ \\
\hline $6 s 6 p$ & ${ }^{1} \mathrm{P}^{\circ}$ & 1 & 38223.49 & 0.99 & 56 & 34 & $5 d 6 p^{1} \mathrm{P}^{\circ}$ \\
\hline
\end{tabular}


Lu II-Continued

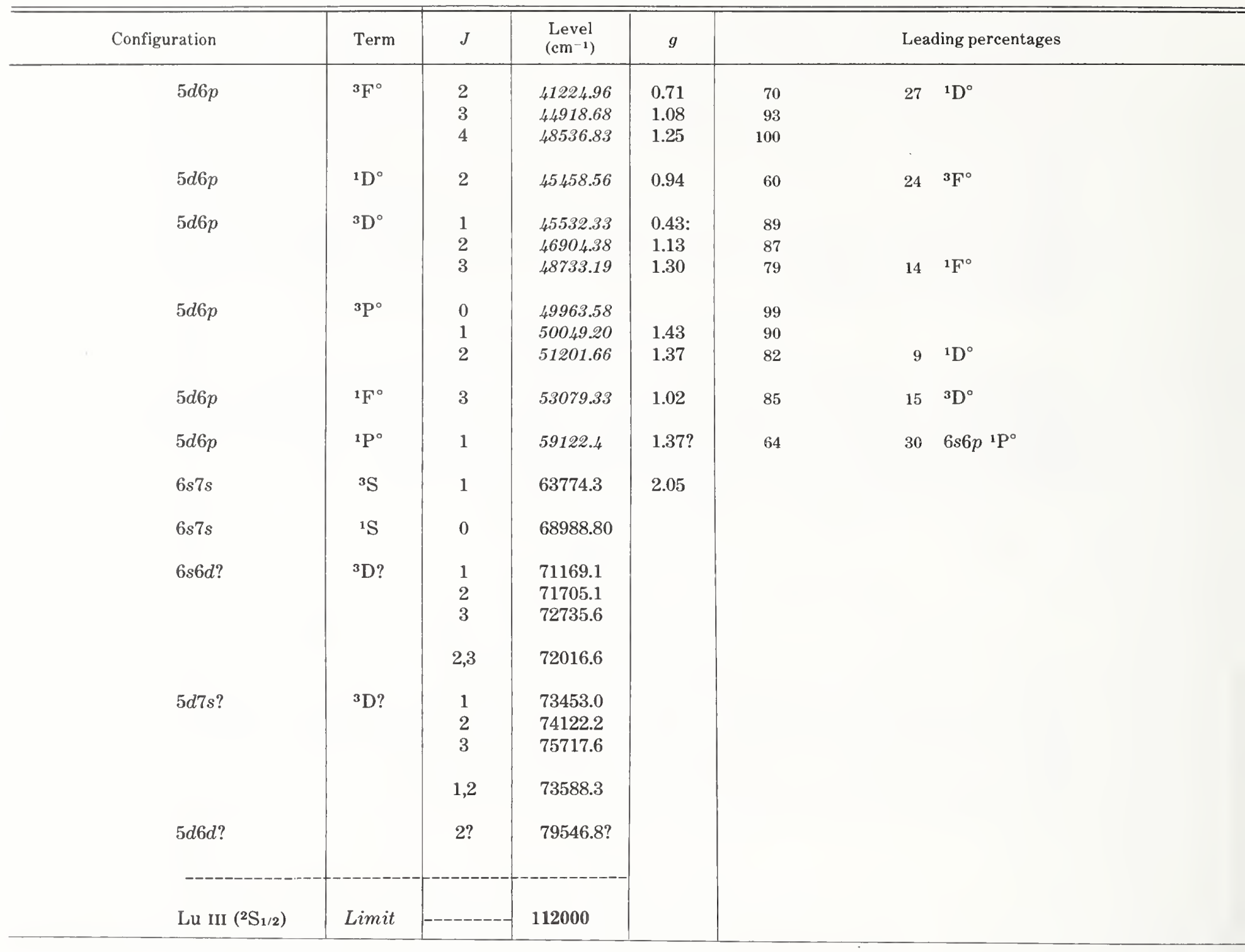




\section{Lu III}

(Tm I sequence; 69 electrons)

$\dot{Z}=71$

Ground state $\left(1 s^{2} 2 s^{2} 2 p^{6} 3 s^{2} 3 p^{6} 3 d^{10} 4 s^{2} 4 p^{6} 4 d^{10} 4 f^{14} 5 s^{2} 5 p^{6}\right) 6 s^{2} \mathrm{~S}_{1 / 2}$

Ionization energy $169049 \pm 10 \mathrm{~cm}^{-1}$

$20.9596 \pm 0.0012 \mathrm{eV}$

Kaufman and Sugar [1971] photographed this spectrum over the region 400 to $9000 \AA$. They note that the "simple one-electron spectrum is superimposed on the complex threeelectron spectrum arising from the excitation of an electron out of the $4 f$ shell." Although they found levels of the $\left(4 f^{13} 5 d^{2}+4 f^{13} 5 d 6 s+4 f^{13} 6 s^{2}\right)$ group, the published results were limited to the simple one-electron configurations given here, which classify about 70 lines from 677 to $8009 \AA$. The strongest of these lines belong to the $6 s-6 p$ and $5 d-6 p$ multiplets (2236-3058 $\AA$ ) first classified by Meggers and Scribner [1930]; their classifications of two of the lines were later correctly interchanged by Steudel [1958].

One of the levels from the more complex configurations, the odd level at $128057.3 \mathrm{~cm}^{-1}$ with $J=5 / 2$, is included here; "the inversion of the $6 f^{2} \mathrm{~F}^{\circ}$ term appears to have been caused mainly by interaction" between this level and the $6 f^{2} \mathrm{~F}_{5 / 2}^{\circ}$ level.

Kaufman and Sugar gave all levels or hyperfine-structure levels of ${ }^{175} \mathrm{Lu}$ III with respect to the ground hyperfine level, $6 s^{2} \mathrm{~S}_{\mathrm{l} / 2}(F=3)$ at $0.000 \mathrm{~cm}^{-1}$, the $F=4 \mathrm{hfs}$ level being $1.746 \pm 0.010$ $\mathrm{cm}^{-1}$ higher. Their table includes hfs intervals for the $6 s-8 s, 6 p$, and $7 p$ levels. The values given here for these levels are the baricenters of the corresponding hfs levels, with the hfs baricenter of the ground level being taken at $0.00 \mathrm{~cm}^{-1}$. The uncertainties are $0.1 \mathrm{~cm}^{-1}$ for all levels except those of $8 f$ and $9 f$, which are uncertain by $\pm 1.0 \mathrm{~cm}^{-1}$.

Kaufman and Sugar derived the quoted ionization potential from the $n s$ series.

\section{References}

Heilig, K., and Kasten, P., Naturwissenschaften 54, 338 (1967). Hfs

Kaufman, V., and Sugar, J., J. Opt. Soc. Am. 61, 1693 (1971). EL CL W Hfs IP

Meggers, W. F., and Scribner, B. F., J. Res. Nat. Bur. Stand. (U.S.) 5, 73 (1930). EL CL. W

Meggers, W. F., and Scribner, B. F., J. Res. Nat. Bur. Stand. (U.S.) 19, 31 (1937). W

Steudel, A., Z. Phys. 152, 599 (1958). EL CL Hfs

[July 1976]

Lu III

\begin{tabular}{|c|c|c|c|c|c|c|c|}
\hline Configuration & Term & $J$ & $\begin{array}{l}\text { Level } \\
\left(\mathrm{cm}^{-1}\right)\end{array}$ & Configuration & Term & $J$ & $\begin{array}{l}\text { Level } \\
\left(\mathrm{cm}^{-1}\right)\end{array}$ \\
\hline $4 f^{14}\left({ }^{1} \mathrm{~S}\right) 6 s$ & ${ }^{2} \mathrm{~S}$ & $1 / 2$ & 0.00 & $4 f^{14}\left({ }^{1} \mathrm{~S}\right) 6 f$ & ${ }^{2} \mathrm{~F}^{\circ}$ & $7 / 2$ & 128799.8 \\
\hline \multirow[t]{2}{*}{$4 f^{14}\left({ }^{1} \mathrm{~S}\right) 5 d$} & ${ }^{2} \mathrm{D}$ & $3 / 2$ & 5707.6 & & & $5 / 2$ & 129053.2 \\
\hline & & $5 / 2$ & 8647.8 & $4 f^{14}\left({ }^{1} \mathrm{~S}\right) 5 g$ & ${ }^{2} \mathrm{G}$ & $7 / 2$ & 129105.4 \\
\hline \multirow[t]{2}{*}{$4 f^{14}\left({ }^{1} \mathrm{~S}\right) 6 p$} & ${ }^{2} \mathrm{P}^{\circ}$ & $1 / 2$ & 38400.61 & & & $8 / 2$ & 129106.3 \\
\hline & & $3 / 2$ & 44705.21 & $4 f^{14}\left({ }^{1} \mathrm{~S}\right) 9 \mathrm{~s}$ & ${ }^{2} \mathrm{~S}$ & $1 / 2$ & 136209.86 \\
\hline $4 f^{14}\left({ }^{1} \mathrm{~S}\right) 7 s$ & ${ }^{2} \mathrm{~S}$ & $1 / 2$ & 86681.21 & $4 f^{14}\left({ }^{1} \mathrm{~S}\right) 7 f$ & ${ }^{2} \mathrm{~F}^{\circ}$ & $5 / 2$ & 141069.6 \\
\hline \multirow[t]{2}{*}{$4 f^{14}\left({ }^{1} \mathrm{~S}\right) 6 d$} & ${ }^{2} \mathrm{D}$ & $3 / 2$ & 92321.6 & & & $7 / 2$ & 141092.2 \\
\hline & & $5 / 2$ & 93107.6 & $4 f^{14}\left({ }^{1} \mathrm{~S}\right) 10 s$ & ${ }^{2} \mathrm{~S}$ & $1 / 2$ & 145587.3 \\
\hline $4 f^{14}\left({ }^{1} \mathrm{~S}\right) 7 p$ & ${ }^{2} \mathrm{P}^{\circ}$ & $\begin{array}{l}1 / 2 \\
3 / 2\end{array}$ & $\begin{array}{l}100357.09 \\
102810.82\end{array}$ & $4 f^{14}\left({ }^{1} \mathrm{~S}\right) 8 f$ & ${ }^{2} \mathrm{~F}^{\circ}$ & $\begin{array}{l}5 / 2 \\
7 / 2\end{array}$ & $\begin{array}{l}148513.8 \\
148530.0\end{array}$ \\
\hline $4 f^{14}\left({ }^{1} \mathrm{~S}\right) 5 f$ & ${ }^{2} \mathrm{~F}^{\circ}$ & $\begin{array}{l}5 / 2 \\
7 / 2\end{array}$ & $\begin{array}{l}105590.6 \\
105704.1\end{array}$ & $4 f^{14}\left({ }^{1} \mathrm{~S}\right) 9 f$ & ${ }^{2} \mathrm{~F}^{\circ}$ & $\begin{array}{l}5 / 2 \\
7 / 2\end{array}$ & $\begin{array}{l}153343.6 \\
153354.5\end{array}$ \\
\hline $4 f^{14}\left({ }^{1} \mathrm{~S}\right) 8 s$ & ${ }^{2} \mathrm{~S}$ & $1 / 2$ & 119784.75 & & & & \\
\hline $4 f^{14}\left({ }^{1} \mathrm{~S}\right) 7 d$ & ${ }^{2} \mathrm{D}$ & $\begin{array}{l}3 / 2 \\
5 / 2\end{array}$ & $\begin{array}{l}122622.5 \\
122981.0\end{array}$ & Lu IV ( $\left.{ }^{1} \mathrm{~S}_{0}\right)$ & Limit & & 169049 \\
\hline & & $5 / 2$ & 128057.3 & & & & \\
\hline
\end{tabular}




\section{Lu IV}

(Er I sequence; 68 electrons)

Ground state $\left(1 s^{2} 2 s^{2} 2 p^{6} 3 s^{2} 3 p^{6} 3 d^{10} 4 s^{2} 4 p^{6} 4 d^{10} 5 s^{2} 5 p^{6}\right) 4 f^{14}{ }^{1} \mathrm{~S}_{0}$

Ionization energy $364960 \pm 200 \mathrm{~cm}^{-1}$

$45.25 \pm 0.03 \mathrm{eV}$

One immediate advantage of the analysis of this spectrum was that the results could be combined with certain known regularities to give predicted values for fundamental energy differences of the type $4 f^{N}-4 f^{N-1} 5 d$ in the fourth and fifth spectra of the lanthanides [Sugar and Kaufman, 1972]. The 57 known energy levels of Lu IV include nearly all levels of the listed configurations. The line list obtained from a sliding-spark source includes 246 lines over the range $876-2130 \AA$, with about $70 \%$ of the lines being classified. "The uncertainty of the levels relative to the lowest level of $4 f^{13} 5 d$ is about $\pm 0.1 \mathrm{~cm}^{-1}$, but relative to the ground state it is $\pm 0.5 \mathrm{~cm}^{-1}$," the latter connection being based on the wavelengths of the three resonance lines from the $4 f^{13} 5 d$ levels having $J=1$.

Leading percentages in the $J_{1} j$ coupling scheme are given for all excited levels. Sugar and Kaufman found that the lowest average purity in this scheme was $91 \%$ for $4 f^{13} 5 d$, whereas the $L S$ purity of this configuration is $65 \%$. The $4 f^{13} 6 s$ and $4 f^{13} 7 s$ configurations have $100 \%$ purity in $J_{1} j$ coupling; in $L S$ coupling the two levels having $J=3$ share the ${ }^{1} \mathrm{~F}^{\circ}$ and ${ }^{3} \mathrm{~F}^{\circ}$ percentages almost equally.

Wyart, Blaise, and Camus [1974] give the parameter values obtained from a calculation of $\left(4 f^{13} 5 d+4 f^{13} 6 s\right)$ including configuration interaction.

Kaufman and Sugar [1976] obtained the $4 f^{13}{ }^{2} \mathrm{~F}_{7 / 2}^{\circ}$ limit position from the $4 f^{13} 6 s, 7 s$ series and an adopted value for the difference in the quantum defects of the two members. The uncertainty arises from their estimated uncertainty in the latter quantity.

\section{References}

Kaufman, V., and Sugar, J., J. Opt. Soc. Am. 66, 1019 (1976). IP

Sugar, J., and Kaufman, V., J. Opt. Soc. Am. 62, 562 (1972). E L CL W IP PT

Sugar, J., and Reader, J., J. Chem. Phys. 59, 2083 (1973). IP

Wyart, J. F., Blaise, J., and Camus, P., Phys. Ser. 9, 325 (1974). PT

[July 1976]

Lu IV

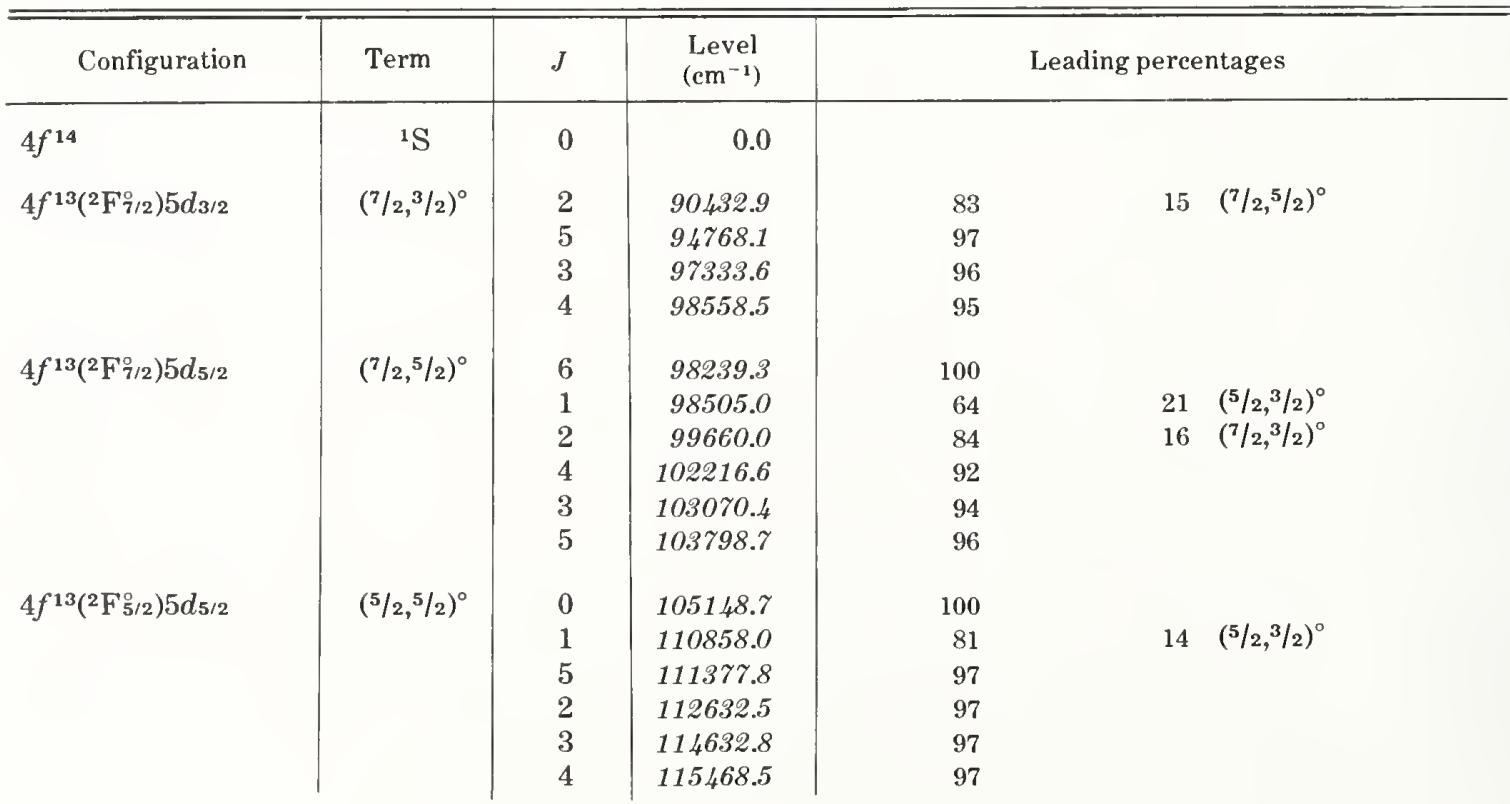


Lu IV-Continued

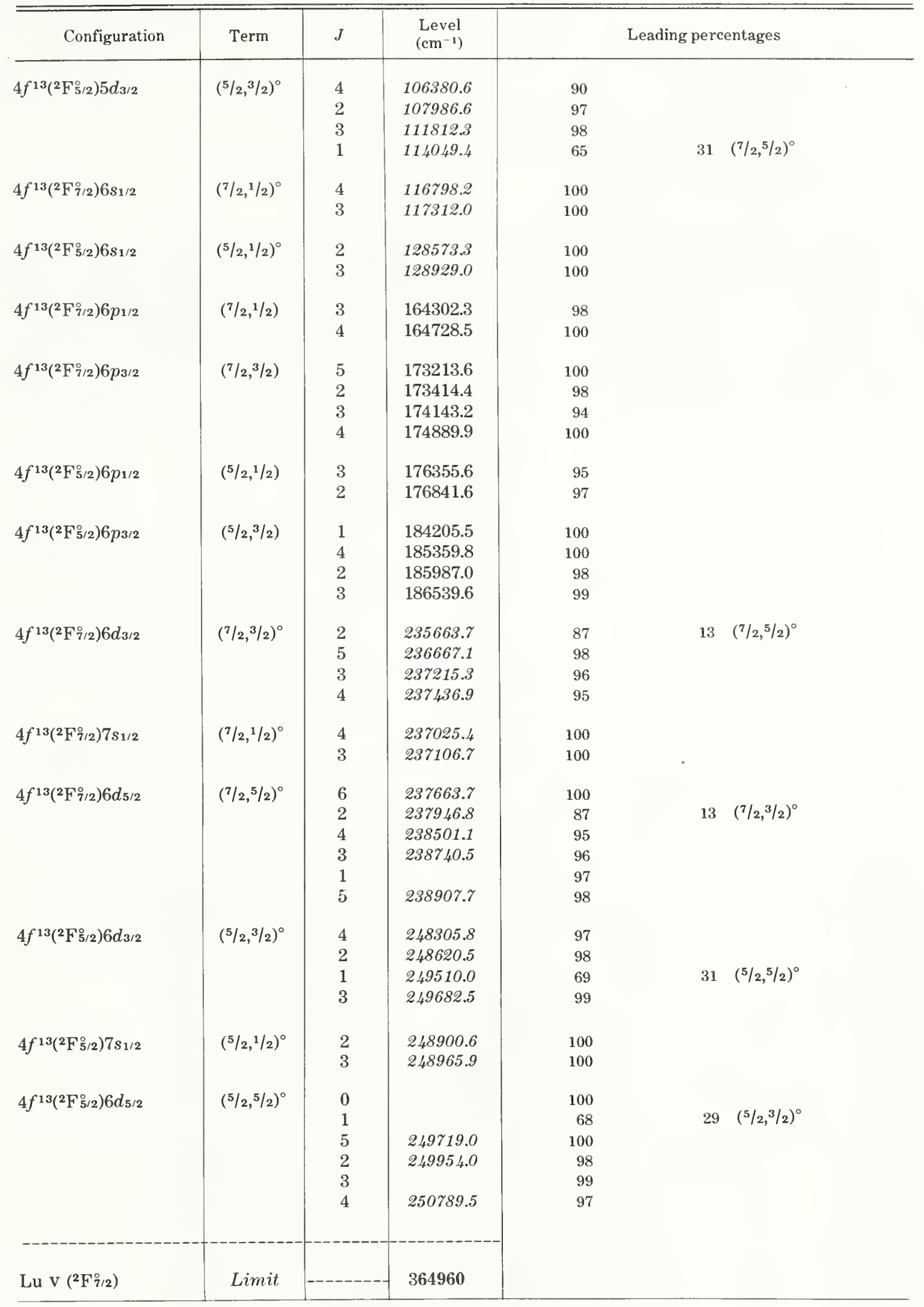




\section{Lu V}

(Ho I sequence; 67 electrons)

$Z=71$

Ground state $\left(1 s^{2} 2 s^{2} 2 p^{6} 3 s^{2} 3 p^{6} 3 d^{10} 4 s^{2} 4 p^{6} 4 d^{10} 5 s^{2} 5 p^{6}\right) 4 f^{13}{ }^{2} \mathrm{~F}_{7 / 2}^{\circ}$

Ionization energy $538700 \pm 2700 \mathrm{~cm}^{-1}$

$66.8 \pm 0.3 \mathrm{eV}$

Sugar and Kaufman [1972] gave the $4 f^{13}{ }^{2} \mathrm{~F}^{\circ}$ interval for this spectrum in their paper on Lu IV. They have classified more than $50 \mathrm{Lu} \mathrm{v}$ lines in the region $500-700 \AA$ as transitions from $4 f^{12} 5 d$ levels to the ground-term levels [Sugar and Kaufman, 1973]. The eigenvector percentages for the upper levels are also from their unpublished material. Leading percentages in both $J_{1} j$ and $L S$ coupling are given; neither scheme is a good approximation. An extension of this analysis is planned.

Sugar [1975] obtained the ionization energy by a method similar to that of Sugar and Reader [1973].

\section{References}

Sugar, J., J. Opt. Soc. Am. 65, 1366 (1975). IP

Sugar, J., and Kaufman, V., J. Opt. Soc. Am. 62, 562 (1972). EL

Sugar, J., and Kaufman, V., unpublished material (1973). EL PT

Sugar, J., and Reader, J., J. Chem. Phys. 59, 2083 (1973).

[July 1976]

Lu v

\begin{tabular}{|c|c|c|c|c|c|c|c|c|}
\hline Configuration & Term & $J$ & $\begin{array}{l}\text { Level } \\
\left(\mathrm{cm}^{-1}\right)\end{array}$ & \multicolumn{5}{|c|}{ Leading percentages } \\
\hline $4 f^{13}$ & ${ }^{2} \mathrm{~F}^{\circ}$ & $\begin{array}{l}7 / 2 \\
5 / 2\end{array}$ & $\begin{array}{r}0.0 \\
11792.8\end{array}$ & & & & & \\
\hline $4 f^{12\left(3 \mathrm{H}_{6}\right) 5 d_{5 / 2}}$ & & $7 / 2$ & 150765.0 & 34 & $(6,5 / 2)$ & or & 38 & $\left({ }^{3} \mathrm{H}\right){ }^{4} \mathrm{~F}$ \\
\hline $4 f^{12}\left({ }^{3} \mathrm{~F}_{4}\right) 5 d_{3 / 2}$ & & $9 / 2$ & 154986.5 & 39 & $(4,3 / 2)$ & or & 20 & $\left({ }^{3} \mathrm{~F}\right){ }^{4} \mathrm{G}$ \\
\hline $4 f^{12\left({ }^{3} \mathrm{H}_{6}\right) 5 d_{5 / 2}}$ & & $9 / 2$ & 156855.6 & 34 & $(6,5 / 2)$ & or & 34 & $\left({ }^{3} \mathrm{H}\right){ }^{4} \mathrm{G}$ \\
\hline $4 f^{12\left({ }^{3} \mathrm{H}_{6}\right) 5 d_{5 / 2}}$ & & $7 / 2$ & 158983.3 & 40 & $(6,5 / 2)$ & or & 72 & $\left({ }^{3} \mathrm{H}\right)^{2} \mathrm{~F}$ \\
\hline $4 f^{12\left({ }^{3} \mathrm{H}_{5}\right) 5 d_{5 / 2}}$ & & $5 / 2$ & 159035.8 & 35 & $(5,5 / 2)$ & or & 55 & $\left({ }^{3} \mathrm{H}\right){ }^{4} \mathrm{~F}$ \\
\hline $4 f^{12\left(3 \mathrm{~F}_{4}\right) 5 d_{3 / 2}}$ & & $5 / 2$ & 161973.0 & 29 & $(4,3 / 2)$ & or & 27 & $\left({ }^{3} \mathrm{~F}\right){ }^{4} \mathrm{P}$ \\
\hline $4 f^{12(}\left({ }^{3} \mathrm{~F}_{4}\right) 5 d_{5 / 2}$ & & $7 / 2$ & 162558.9 & 34 & $(4,5 / 2)$ & or & 27 & $\left({ }^{3} \mathrm{~F}\right){ }^{4} \mathrm{D}$ \\
\hline $4 f^{12\left(3 \mathrm{~F}_{4}\right) 5 d_{5 / 2}}$ & $(4,5 / 2)$ & $9 / 2$ & 162806.4 & 56 & or & & 35 & $\left({ }^{3} \mathrm{~F}\right){ }^{4} \mathrm{~F}$ \\
\hline $4 f^{12\left({ }^{3} \mathrm{H}_{5}\right)} 5 d_{5 / 2}$ & & $7 / 2$ & 164200.2 & 37 & $(5,5 / 2)$ & or & 18 & $\left({ }^{3} \mathrm{~F}\right){ }^{4} \mathrm{H}$ \\
\hline $4 f^{12(}\left({ }^{3} \mathrm{~F}_{3}\right) 5 d_{3 / 2}$ & $(3,3 / 2)$ & $3 / 2$ & 164665.8 & 47 & or & & 42 & $\left({ }^{3} \mathrm{~F}\right){ }^{4} \mathrm{D}$ \\
\hline $4 f^{12}\left({ }^{3} \mathrm{~F}_{3}\right) 5 d_{3 / 2}$ & & $7 / 2$ & 166576.0 & 33 & $(3,3 / 2)$ & or & 27 & $\left({ }^{3} \mathrm{H}\right){ }^{4} \mathrm{G}$ \\
\hline $4 f^{12}\left({ }^{3} \mathrm{H}_{5}\right) 5 d_{5 / 2}$ & & $5 / 2$ & 167222.3 & 42 & $(5,5 / 2)$ & or & 40 & $\left({ }^{3} \mathrm{H}\right){ }^{2} \mathrm{~F}$ \\
\hline $4 f^{12}\left({ }^{3} \mathrm{~F}_{2}\right) 5 d_{3 / 2}$ & & $3 / 2$ & 168013.2 & 27 & $(2,3 / 2)$ & or & 38 & $\left({ }^{3} \mathrm{~F}\right){ }^{2} \mathrm{P}$ \\
\hline $4 f^{12}\left({ }^{3} \mathrm{~F}_{2}\right) 5 d_{3 / 2}$ & & $7 / 2$ & 168669.8 & 41 & $(2,3 / 2)$ & or & 30 & $\left({ }^{3} \mathrm{~F}\right){ }^{4} \mathrm{H}$ \\
\hline $4 f^{12}\left({ }^{3} \mathrm{~F}_{3}\right) 5 d_{3 / 2}$ & & $7 / 2$ & 170023.8 & 26 & $(3,3 / 2)$ & or & 33 & $\left({ }^{3} F\right)^{2} \mathrm{~F}$ \\
\hline $4 f^{12}\left({ }^{3} \mathrm{~F}_{3}\right) 5 d_{3 / 2}$ & & $9 / 2$ & 170638.6 & 42 & $(3,3 / 2)$ & or & 24 & $\left({ }^{3} \mathrm{~F}\right){ }^{4} \mathrm{H}$ \\
\hline $4 f^{12}\left({ }^{3} \mathrm{H}_{4}\right) 5 d_{5 / 2}$ & & $5 / 2$ & 171005.9 & 42 & $(4,5 / 2)$ & or & 32 & $\left({ }^{3} \mathrm{H}\right){ }^{4} \mathrm{G}$ \\
\hline $4 f^{12}\left({ }^{3} \mathrm{H}_{4}\right) 5 d_{5 / 2}$ & & $3 / 2$ & 171573.5 & 40 & $(4,5 / 2)$ & or & 40 & $\left({ }^{3} \mathrm{H}\right){ }^{4} \mathrm{~F}$ \\
\hline $4 f^{12}\left({ }^{3} \mathrm{~F}_{2}\right) 5 d_{5 / 2}$ & & $7 / 2$ & 172807.3 & 38 & $(2,5 / 2)$ & or & 23 & $\left({ }^{3} F\right)^{2} \mathrm{G}$ \\
\hline
\end{tabular}


Lu v-Continued

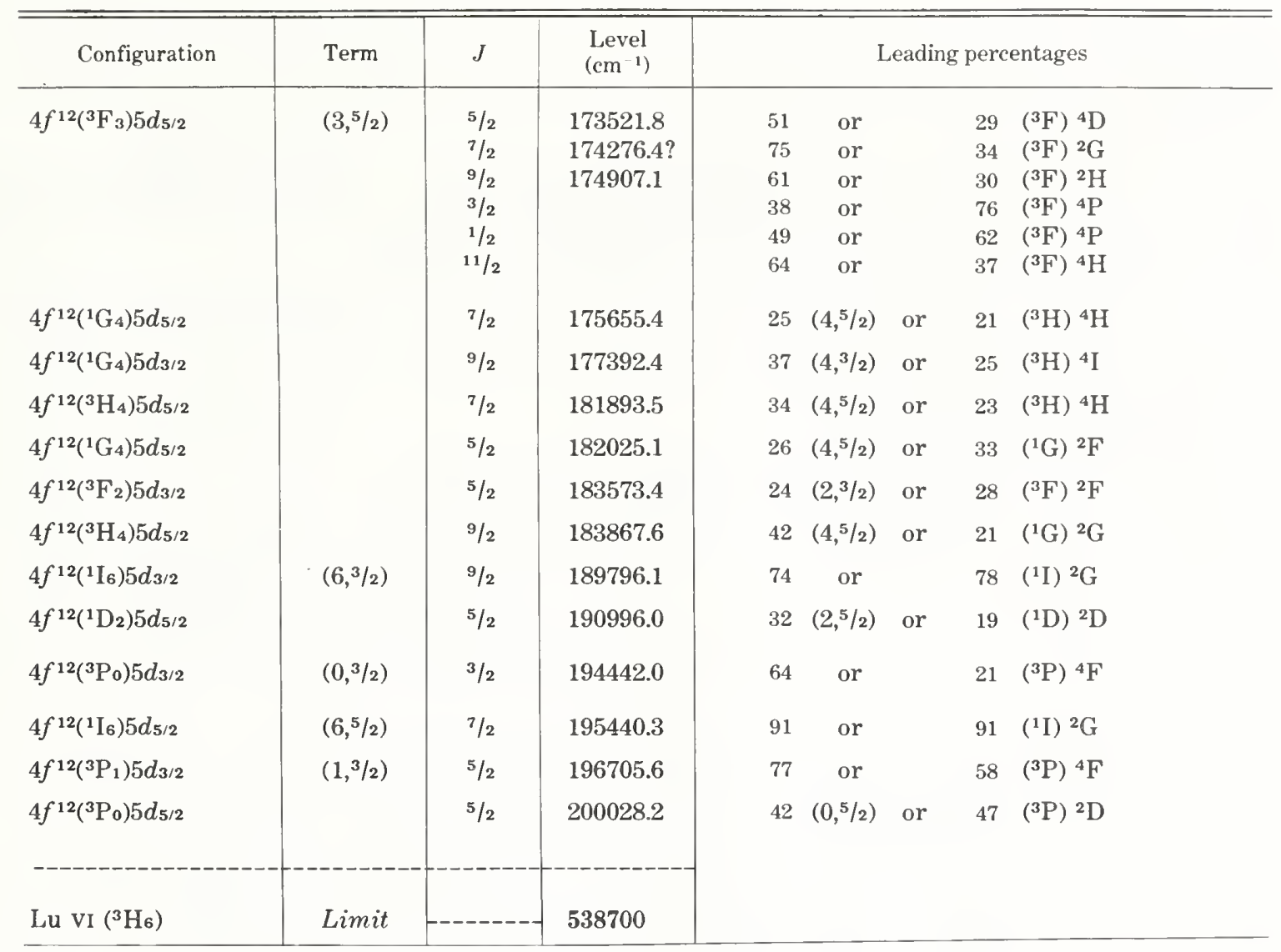


NBS-114A (REV. 7-73)

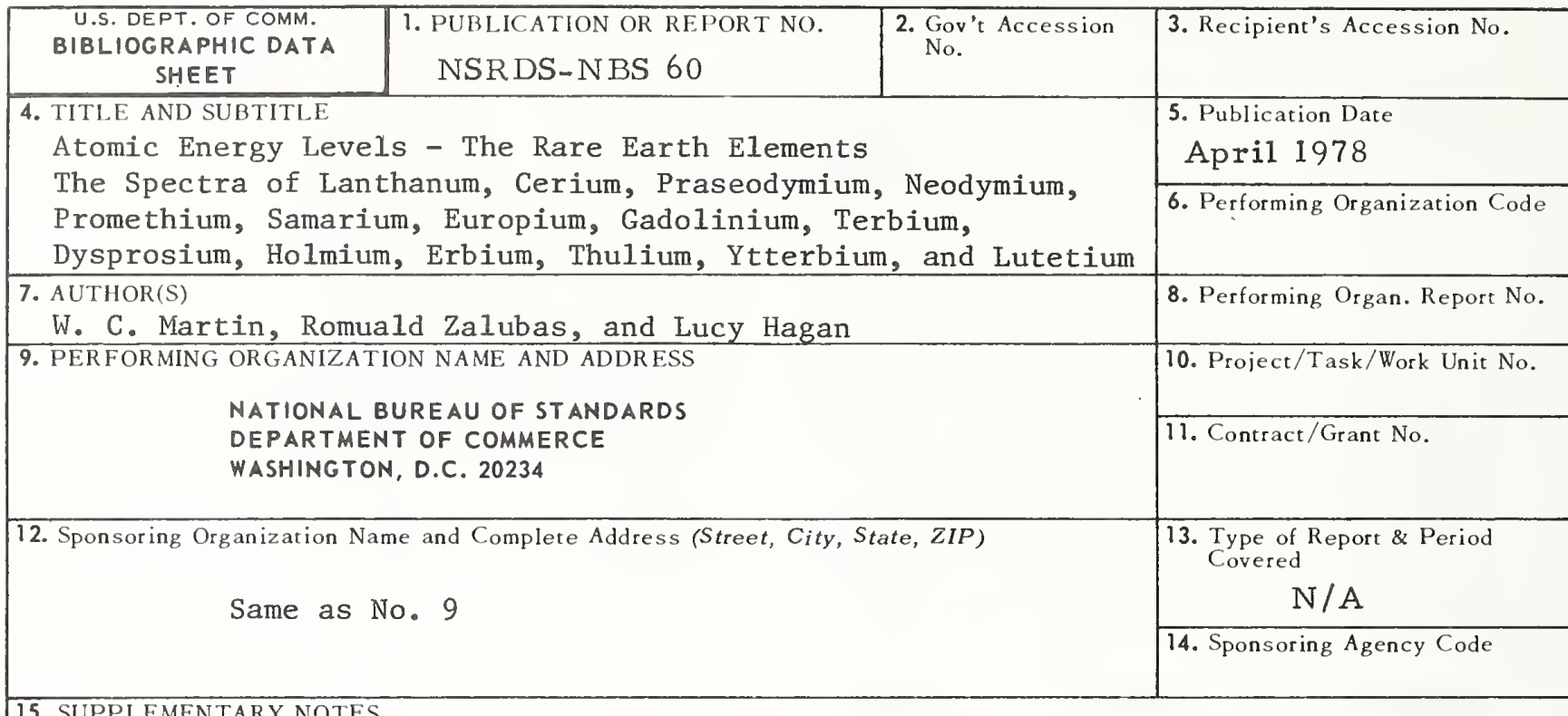

Library of Congress Catalog Card Number: 77-12195

16. ABSTRACT (A 200-word or less factual summary of most significant information. If document includes a significant bibliography or literature survey, mention it here.)

Energy level data are given for 66 atoms and atomic ions of the 15 elements lanthanum $(Z=57)$ through lutetium $(Z=71)$. The se data have been critically compiled from published and unpublished material. Only experimentally determined energy levels are included the energies being restricted to excitations of outer-shell electrons and to inner-shell excitations up to the soft $x$-ray range. The levels were taken from analyses of the spectra of atomic gases wherever possible; however, the levels for several of the triply ionized rare earths are from analyses of the spectra of the ions in crystals or solutions. In addition to the level value (usually in units of $\mathrm{cm}^{-1}$ ) and the parity, the $J$ value, configuration and term assignments, and the experimental $\mathrm{g}$ value are listed wherever available. Leading percentages from the calculated eigenvector are also tabulated for each level if available. The levels are grouped into spectroscopic terms of appropriate coupling schemes where such groups appear meaningful. Ionization potentials are tabulated for most of the spectra. Complete references for the tabulated data are given for each spectrum.

17. KEY WORDS (six to twelve entries; alphabetical order; capitalize only the first letter of the first key word unless a proper name; separated by semicolons)

Atomic energy levels; atomic spectroscopy; electron configurations; ionization potentials; lanthanides; rare earths; spectra; Zeeman effect.

18. AVAILABILITY $\quad \mathrm{X}$ Unlimited

For Official Distribution. Do Not Release to NTIS

X] Order From Sup. of Doc., U.S. Government Printing Office Washington, D.C. 20402, SD Cat. No. C13 48:60

0 Order From NationaI Technical Information Service (NTIS) Springfield, Virginia 22151

\begin{tabular}{|l|c|}
$\begin{array}{l}\text { 19. SECURITY CLASS } \\
\text { (THIS REPURT) }\end{array}$ & $\begin{array}{c}\text { 21. NO. OF PAGES } \\
\text { UNCL ASSIFIED }\end{array}$ \\
\hline $\begin{array}{l}\text { 20. SECURITY CLASS } \\
\text { (THIS PAGE) }\end{array}$ & $\begin{array}{c}\text { 22. Price } \\
\$ 9.50\end{array}$ \\
UNCLASSIFIED & USCOMM.DC 29042-P74 \\
\hline
\end{tabular}




\section{Announcement of New Publications in National Standard Reference Data Series}

Superintendent of Documents, Government Printing Office, Washington, D.C. 20402

Dear Sir:

Please add my name to the announcement list of new publications to be issued in the series: National Standard Reference Data Series-National Bureau of Standards.

Name

Company

Address

City State Zip Code

(Notification Key N-519) 



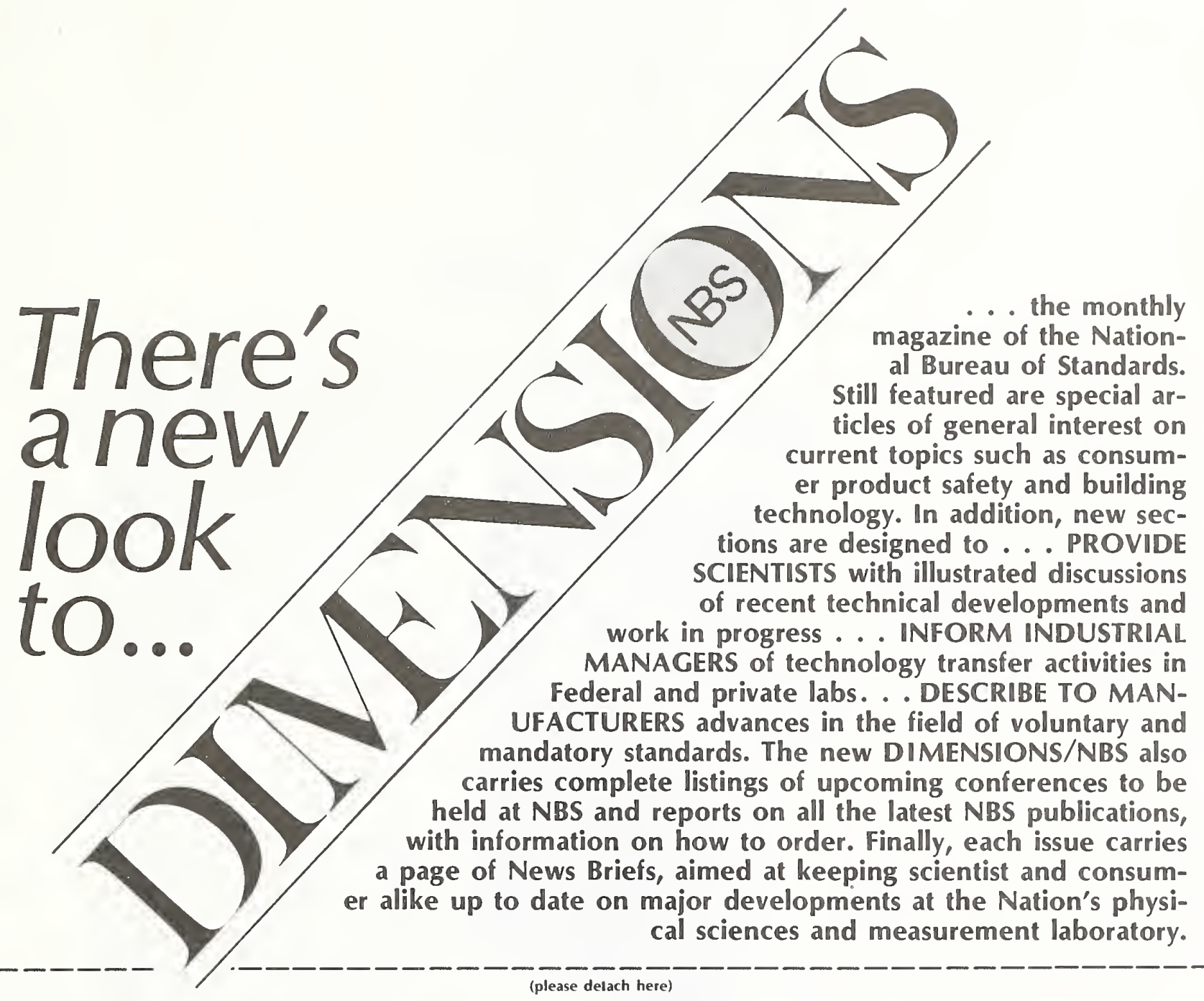

SUBSCRIPTION ORDER FORM

Enter my Subscription To DIMENSIONS/NBS at $\$ 12.50$. Add $\$ 3.15$ for foreign mailing. No additional postage is required for mailing within the United States or its possessions. Domestic remittances should be made either by postal money order, express money order, or check. Foreign remittances should be made either by international money order, draft on an American bank, or by UNESCO coupons.

Send Subscription to:

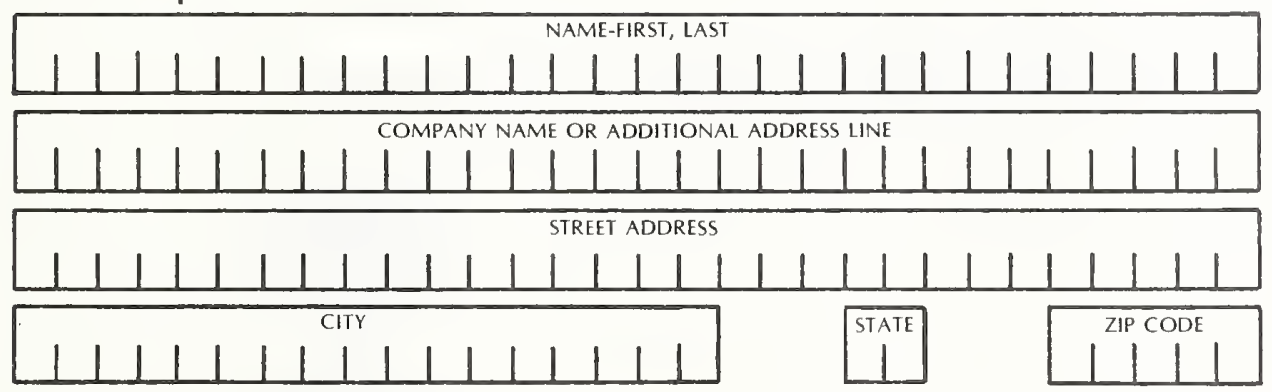

Remittance Enclosed (Make checks payable to Superintendent of Documents)

Charge to my Deposit Account No.

MAIL ORDER FORM TO: Superintendent of Documents Government Printing Office Washington, D.C. 20402 


\section{SINGLE \\ DATA \\ REVISED! UPDATED!}

In 1954, the first edition of CRYSTAL DATA (Determinative Tables and Systematic Tables) was published as Memoir 60 of the Geo. logical Society of America. In 1960, the second edition of the Determinative Tables was issued as Monograph 5 of the American Crystallographic Association, and in 1967, the Systematic Tables were issued as Monograph 6. These editions proved extremely valuable to crystallographers throughout the world. Recognizing the need for updated crystallographic in. formation, the National Bureau of Stand ards Office of Standard Reference Data has sponsored the issuance of a new edition.

This, the THIRD EDITION, should be of particular interest not only to crystal. lographers but also to chemists, mineralogists, physicists and individuals in related fields of study. The current edition which comprises two volumes, Organic and Inorganic, is a thoroughly revised and updated work, containing over 25,000 entries.

The entries are listed, within each crystal system, according to increasing values of a determinative number: $a / b$ ratio in trimetric systems, c/a ratio in dimetric systems, and cubic cell edge $a$, in the isometric system. In addition, the following information is given:

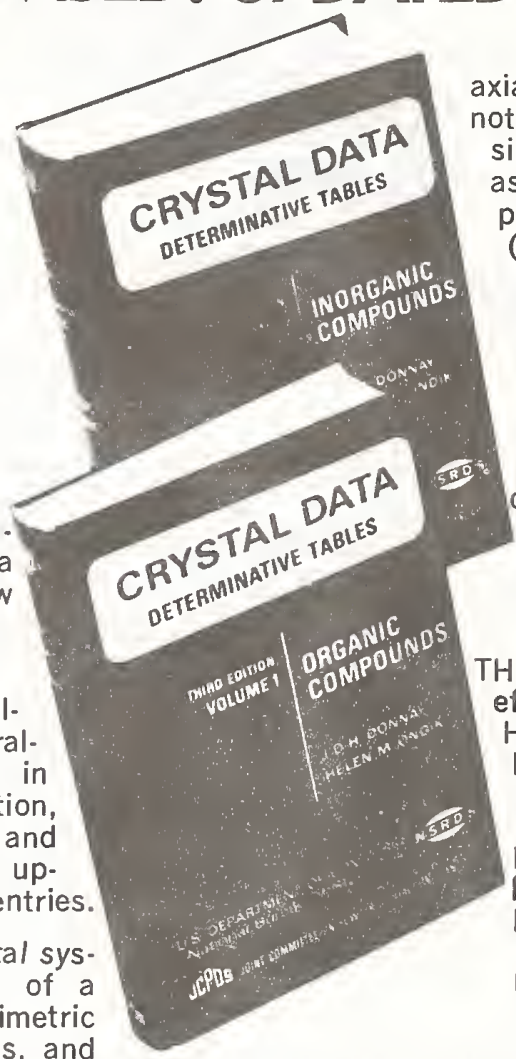

UNORGANIC VOLUME $\$ 50.00$

ORGANIC VOLUME $\$ 30.00$ axial ratio(s) and interaxial angles not fixed by symmetry, cell dimensions, space group or diffraction aspect, number of formula units per unit cell, crystal structure, (whether determined), measured density and $x$-ray calculated den. sity. Also listed is the name of the compound and synonym(s), chemical formula, literature reference and transformation matrix. When available, the crystal structure type, crystal habit, cleavages, twinning, color, optical properties, indices of refraction, optical orientation, melting point and transition point are also listed.

THIS EDITION culminates years of effort by J. D. H. Donnay, Johns Hopkins University, Helen M. Ondik, National Bureau of Standards, Sten Samson, California Institute of Technology, Quintin Johnson, Lawrence Radiation Laboratory, Melvin H. Mueller, Argonne National Laboratory, Gerard M. Wolten, Aerospace Corporation, Mary E. Mrose, U.S. Geological Survey, Olga Ken. nard and David G. Watson, Cam. bridge University, England and Murray Vernon King, Massachu. setts General Hospital.

Plus shipping and handling

Shipments are made via insured parcel post. Additional charges for shipments by air or commercial carrier.

TERMS: Domestic-30 days Foreign-prepayment required. Address all orders to:

JOINT COMMITTEE ON POWDER DIFFRACTION STANDARDS 1601 Park Lane, Swarthmore, Pennsylvania 19081

Please accept my order for CRYSTAL DATA, DETERMINATIVE TABLES, Third Edition, Donnay/Ondik.

$\square$ Organic Volume

$\square$ Inorganic Volume

Ship to:

Signature

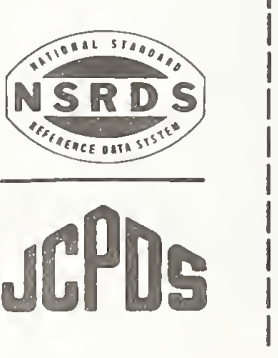






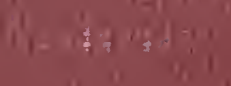

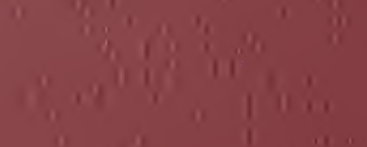

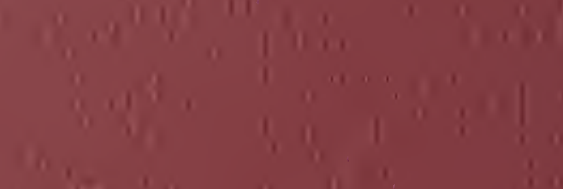

$$
\begin{aligned}
& \therefore=9 \\
& \text { in }
\end{aligned}
$$

(11.,

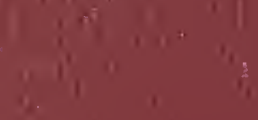

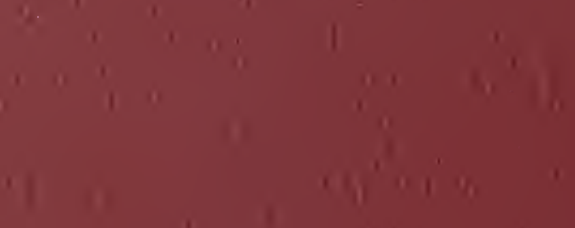

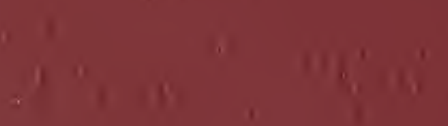

,$\times 17$,

1. 4. 